$$
\begin{aligned}
& \text { i. }
\end{aligned}
$$

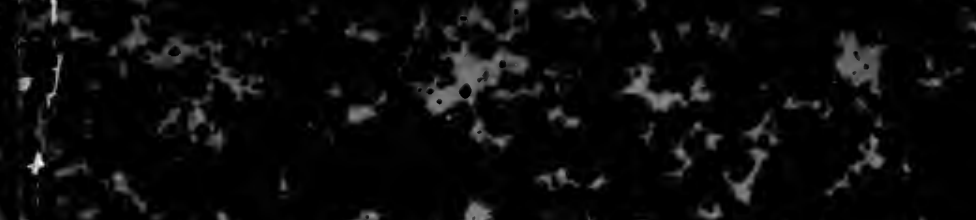

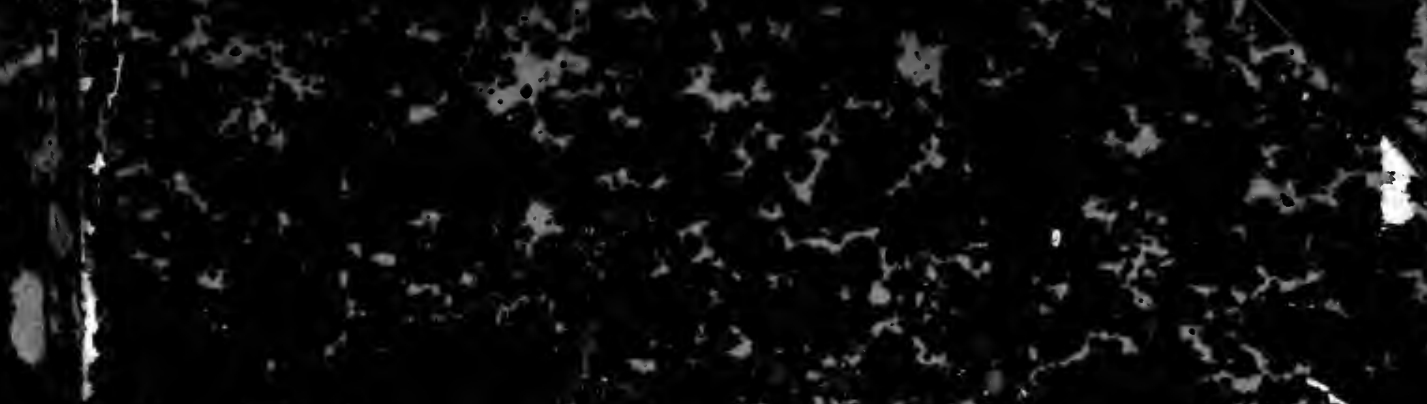

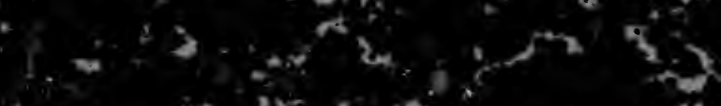

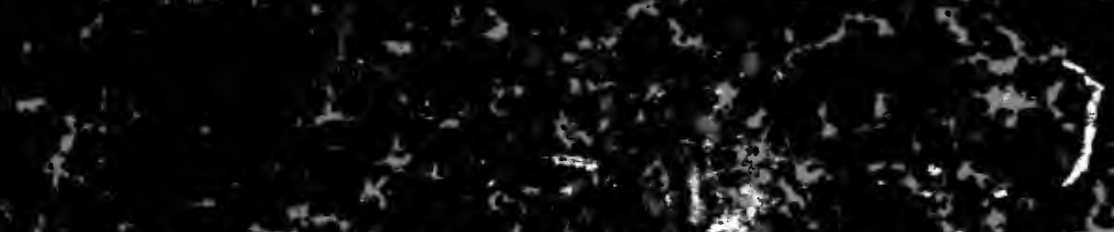

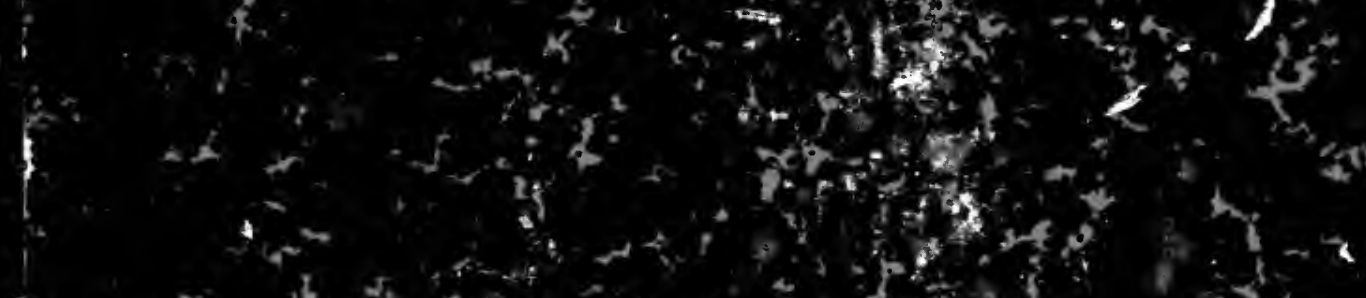

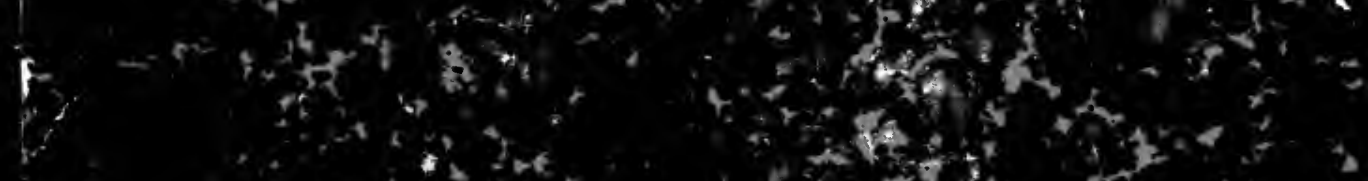

$$
\begin{aligned}
& \text { 女. }
\end{aligned}
$$

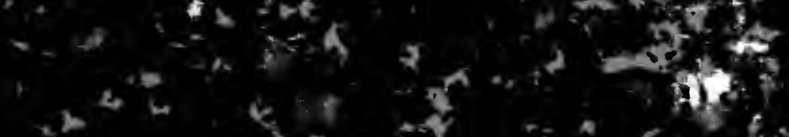

$$
\begin{aligned}
& \text {. }
\end{aligned}
$$

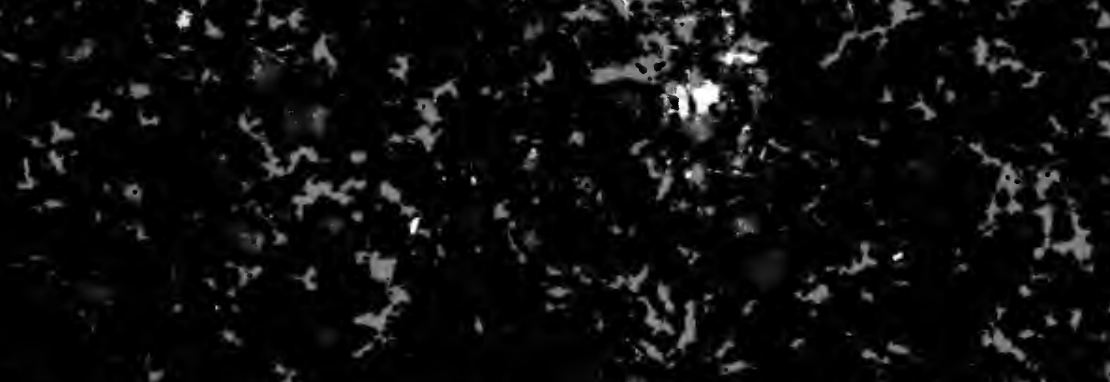

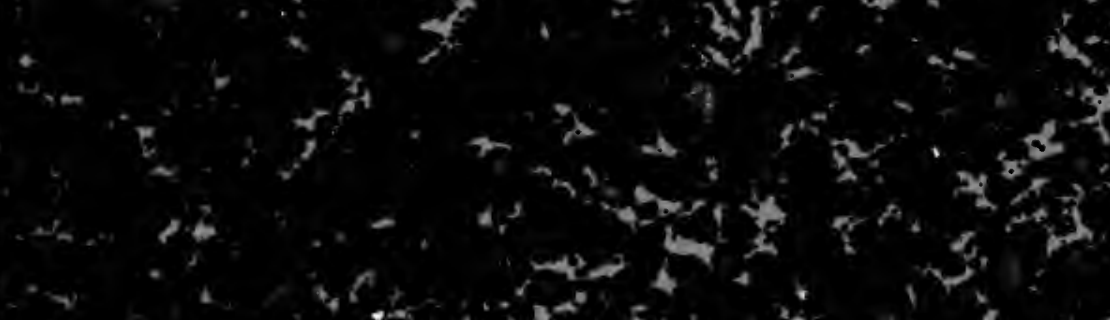

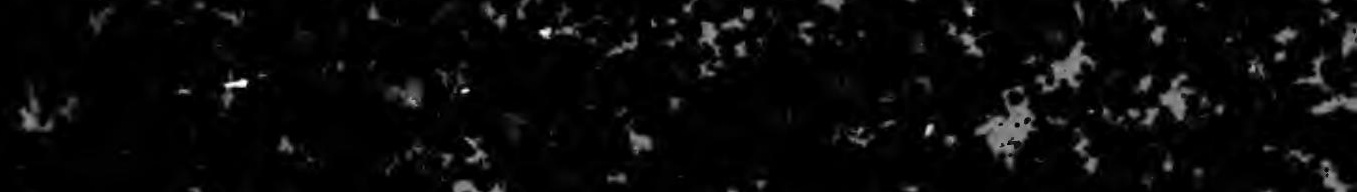

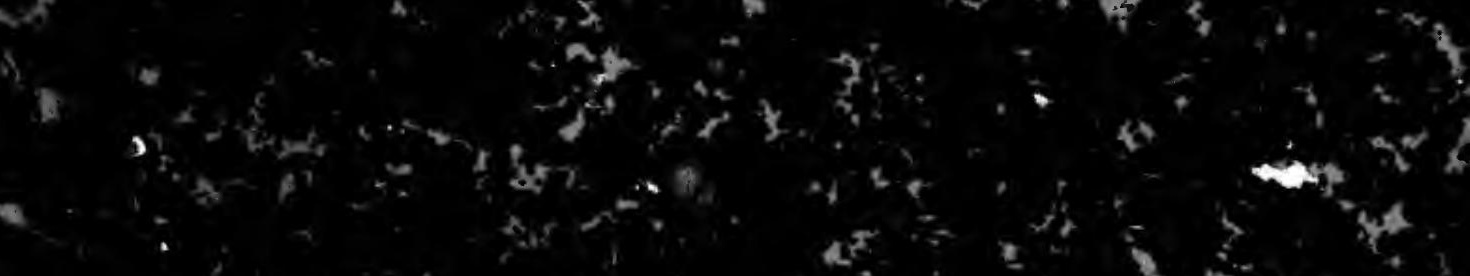

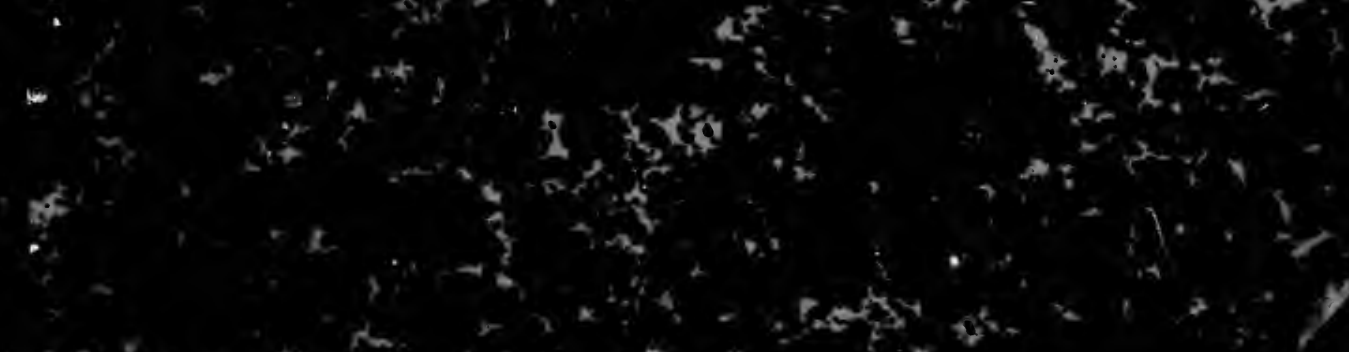




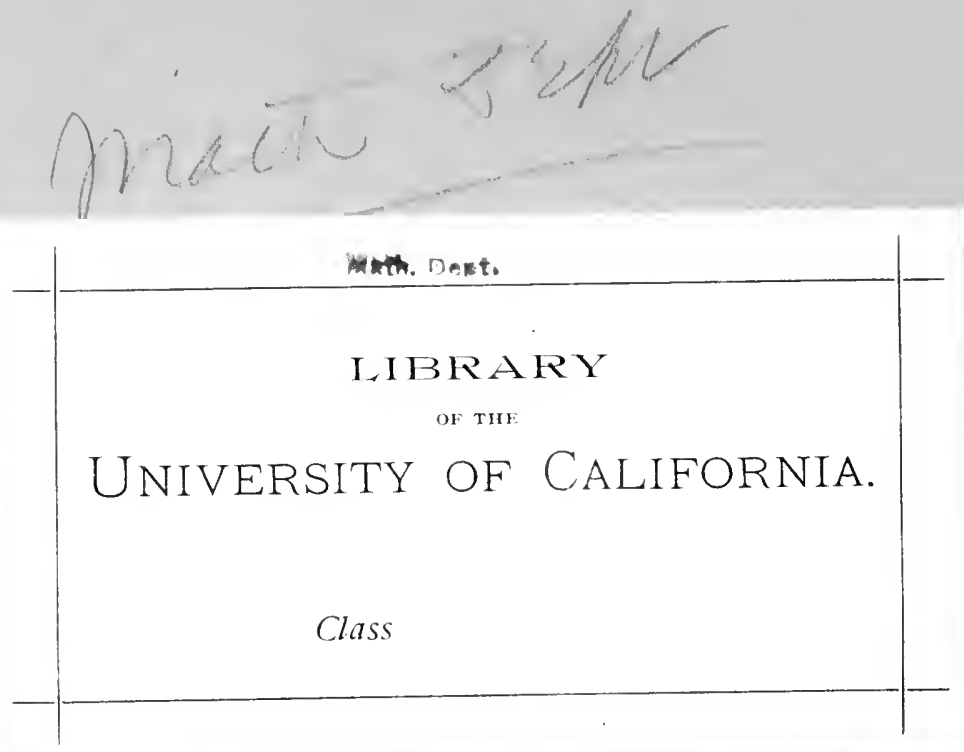





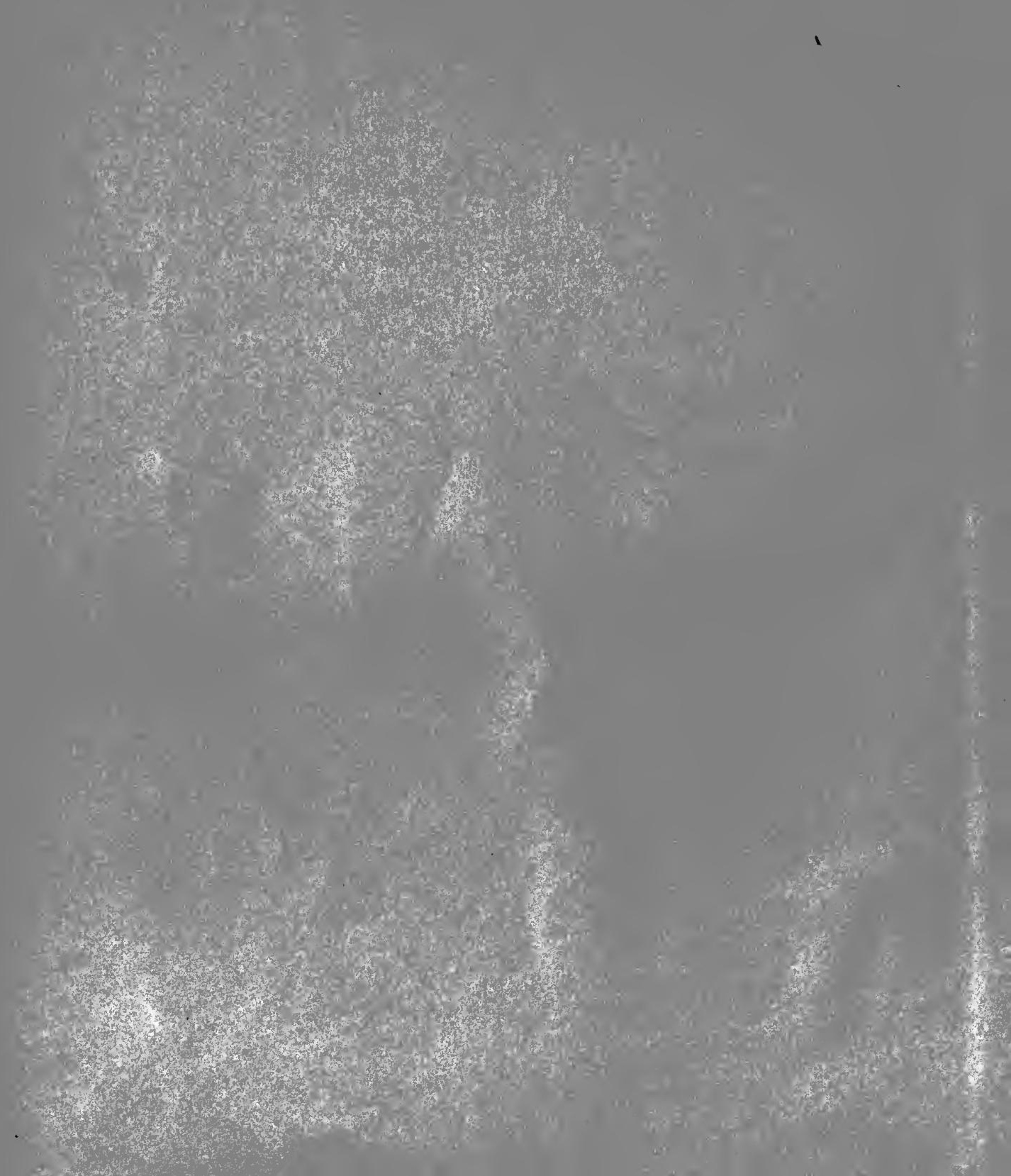




\section{R. Maseluke.}

\section{VORLESUNGEN}

AUS DER

\section{ANALYTISCHEN GEOMETRIE}

DEL

\section{KEGELSCHNITTE \\ vox}

\section{SIGMUND GUNDELFINGER.}

HERAUSGEGEBEN

Vox

FRIEDRICH DINGELDEY.

MIT IN DEN TEXT GEDRUCKTEN FIGUREN UND EINEM ANHANGE, ENTHALTEND AUFGabeN UND WEITERE AUSF̈̈HRUNGEx.

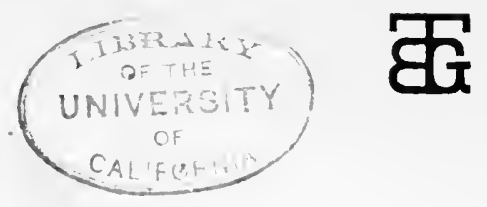

LEIPZIG, DRUCK UND VERLAG VON B. G. TEUBNER. 1895. 
$G \bar{\gamma}$

marb

ALLE RECHTE,
DES UBERSETZUNGSRECHTS, VORBEHALTEN. 


\section{Vorrede.}

Das vorliegende Buch ist eine Ausarbeitung von Vorlesungen, welche Herr Gundelfinger seit über zwanzig Jahren an der Universität Tübingen und an der technischen Hochschule zu Darmstadt gehalten hat. Bei der Ausarbeitung benutzte ich theilweise das von einem früheren Hörer nachgeschriebene Collegienheft, ferner Manuscripte von Herrn Gundelfinger selbst, sowie dessen zweites und drittes Supplement zu Hesse's Vorlesungen über analytische Geometrie des Raumes; endlicls aber war es ganz besonders ein fortwährend reger persönlicher Verkehr, während dessen mich Herr Gundelfinger in seine Methoden einführte. Diesem persönlichen Verkehr verdanke ich überhaupt die Anregung zur Herausgabe des Buches, bei der ich für die stilistische Durchführung und theilweise Anordnung des Stoffes natürlich die volle Verantwortlichkeit selbst übernehme.

Es gibt zwar eine grosse Zahl guter Lehrbücher der analytischen Geometrie der Kegelschnitte; sie sind jedoch entweder zum grössten Theil elementar gehalten, oder bieten zu wenig, wie z. B. Hesse's bekannte sieben Vorlesungen ïber dieses Gebiet, die doch eigentlich nur ein werthvolles Bruchstück darstellen. Das ausgezeichnete Werk über Kegelschnitte von Salmon-Fiedler ist zwar von grosser Vollständigkeit und von höchstem pädagogischem Werth namentlich durch den Umstand, dass mitunter ein und dasselbe Theorem an verschiedenen Stellen wiederkehrt und demgemäss von mehreren Seiten beleuchtet wird; aber gerade dieser Vorzug des Buches musste nothwendig die harmonische Einheit der Darstellung etwas Noth leiden lassen. Das vortreffliche Werk über Geometrie der Ebene von Clebsch-Lindemann endlich verfolgt ein viel weiter gehendes Ziel als dasjenige, welches einer Geometrie der Kegelschnitte gesteckt werden muss. Das Lindemann'sche Buch füllt eine wesentliche Lücke in der mathematischen Litteratur aus, indem es in seinem Hauptinhalte eine Theorie der Curven beliebig hoher Ordnung und der mit ihnen im Zusammenhang stehenden algebraischen Functionen (resp. Abel'schen Integrale) gibt. Die beiden zuletzt erwähnten Werke lösen ausserdem die wichtigsten 
metrischen Probleme über Kegelschnitte für Parallel- und Dreieckscoordinaten getrennt; für die letzteren fast immer in einer Gestalt, dass die entsprechenden Formeln in Parallelcoordinaten nicht ohne weiteres aus ihnen folgen können.

In den hier herausgegebenen Vorlesungen des Herrn Gundelfinger sind sämmtliche Probleme vermittelst der ("Staudt-Fiedler'schen") projectivischen Coordinaten behandelt, so dass die Formeln für Parallelund specielle Dreieckscoordinaten sich ohne weiteres durch besondere Annahmen ergeben.

Da für die Geometrie der geraden Linie und des Punktes Hesse's „Vorlesungen aus der analytischen Geometrie der geraden Linie, des Punktes und des Kreises in der Ebene" vorhanden sind, habe ich dieses Gebiet bei der Herausgabe von Herrn Gundelfinger's Vorlesungen ausser Acht gelassen, und nur mit Rücksicht auf spätere Paragraphen sind in $\S \S 1-3$ des Buches die endgiltigen Formeln für die Theorie der allgemeinen projectivischen Coordinaten nach einer Methode von Herrn Gundelfinger entwickelt. $\S \S 4-7$ behandelt die Curven zweiter Ordnung und zweiter Classe (Pol, Polare u.s. w.), die Classification und Kriterien der Kegelschnitte (wohl in vollständig neuer Ableitung), sowie das Kreispunktepaar. In $\S \S 8-9$ werden gewisse Gleichungen mit nur reellen Wurzeln betrachtet und der Invariantenbegriff entwickelt; $\S 10$ ist der Transformation der Curven zweiter Ordnung auf die Hauptaxen gewidmet. Es wird dabei im Anschluss an $\S 8 \mathrm{im}$ Principe die interessante Aufgabe gelöst, zwei quadratische Formen mit contragredienten Veränderlichen (im speciellen Falle den Ausdruck für die gegebene Curve zweiter Ordnung und denjenigen für das imaginäre Kreispunktepaar) gleichzeitig in eine Summe von Quadraten zu verwandeln. Die $\S \S 11$ und 12 enthalten in Kürze die elementaren Eigenschaften der nicht ausartenden Kegelschnitte, sowie das Wichtigste über die Erzeugung der Curven zweiten Grades durch projective Strahlenbüschel und Punktreihen.

Die bisher genannten $\S \S 1-12$ bilden den ersten Abschnitt des Werkes, während der zweite Abschnitt (§§ 13-26) den Kegelschnittbüscheln, Kegelschnittnetzen und dualistisch entsprechenden Gebilden gewidmet ist. In $\S \S \cdot 13-16$ werden die Schnittpunkte und gemeinsamen Tangenten zweier Kegelschnitte bestimmt und die allgemeinen Eigenschaften des Kegelschnitt-Büschels, resp. -Schaar, entwickelt, in $\S 17$ einige algebraische Formen geometrisch gedeutet. Hieran reiht sich in $\S \S 18$ und 19 eine Untersuchung der confocalen Kegelschnitte und der zwei mit ihnen in Zusammenhang stehenden Systeme doppeltberührender Kreise. $\S 20$ behandelt die wichtigsten Curven mit in- 
varianter Beziehung zu einem Kegelschnitt-Büschel, resp. -Schaar (insbesondere auch Mittelpunktskegelschnitt, Steiner'sche Parabel, die ihr reciprok entsprechende bekannte gleichseitige Hyperbel), § 21 enthält die Formeln für Mittelpunkt und Radius des Krümmungskreises, der irgend einem Punkte einer Curve $k^{\text {ter }}$ Classe oder $n^{\text {ter }}$ Ordnung zugehört, ferner die Gleichungen für die Evolute einer Curve zweiter Classe oder Ordnung. In $\S \S 22-25$ wird die Theorie der KegelschnittNetze und -Gewebe dargelegt, § 26 ist gewissen conjugirten, linearen Kegelschnittsystemen gewidmet.

Die letzteren Paragraphen enthalten vielfach nur die Grundzüge der betreffenden Theorie, soweit sie für die Anwendung in dem das Buch abschliessenden Anhange erforderlich sind. Dieser Anhang soll im wesentlichen zeigen, in welcher Weise die im Haupttexte entwickelten Methoden fruchtbar gemacht werden können zur Lösung allgemeinerer Aufgaben und zum Beweise von Sätzen; übrigens sind mitunter auch Methoden angewandt, die sich im Haupttexte nicht finden. Besonders werden zahlreiche Sätze abgeleitet, welche Steiner in seinen Abhandlungen (fast sämmtlich ohne Berveis) aufgestellt hat.

Als eine hübsche Anwendung der Lehre von den Poldreiecken und der Theorie der Kegelschnittbüschel sind am Schlusse des Anhanges zwei von Herrn Gundelfinger seit längerer Zeit vollendete Untersuchungen über specielle Classen algebraischer Integrale aufgenommen worden.

Die Zahl der im Anhange enthaltenen Aufgaben und Sätze liätte sich leicht vermehren, insbesondere auf Curven höherer Ordnung ausdehnen lassen; doch unterblieb dies, um das Buch nicht zu umfangreich zu gestalten. Die meisten Aufgaben und Sätze des Anhanges nebst Lösungen und Beweisen rühren von Herrn Gundelfinger ber, mehrere hat auch der Herausgeber theils selbständig hinzugefügt, theils auf Anregung von Herrn Gundelfinger bearbeitet.

Diejenigen Nummern des Anbanges, welche schwierigere Aufgaben behandeln, oder zu deren Erledigung noch andere als nur analytischgeometrische Hilfsmittel nöthig waren, sind durch * bezeichnet.

Um Missdeutungen zu entgehen, möge zum Schlusse noch bemerkt werden, dass die meisten Citate sich auf Steiner'sche Sätze beziehen, die mitunter wörtlich angeführt sind; die übrigen vereinzelt auftretenden Citate kamen meist zur Anwendung, wenn die ursprüngliche Quelle eines Theorems nicht als allgemein bekannt vorausgesetzt werden durfte.

Darmstadt, den 9. Januar 1895. 


\title{
Inhaltsverzeichniss.
}

\author{
I. Abschnitt. \\ Einleitende Betrachtungen. Die fundamentalen Eigenschaften \\ eines Kegelschnitts.
}

\& 1. Das Coordinatendreieck. Punkt- und Liniencoordinaten . . . . . . 1

\& 2. Elementare metrische Beziehungen zwischen Punkten uud geraden Linien ............ . . . 8

3. Die Transformation der Coordinaten . . . . . . . . . . . . . . . 14

4. Die Curven zweiter Ordnung . . . . . . . . . . . . . . . . . . 18

5. Die Curren zweiter Classe . . . . . . . . . . . . . . . . 38

\$ 6. Classification der Kegelschnitte. . . . . . . . . ... . . . . . . . 46

\$ 7. Der Kreis, die imaginären Kreispunkte und ihre Beziehung zu dem Winkel zweier Geraden . . . . . . . . . . . . . . . . . . .

$\S$ 8. Ueber einen fundamentalen Satz aus der Theorie der definiten quadratischen Formen und über zwei Gattungen von Gleichungen mit nur reellen Wurzeln . . . . . . . . . . . . . . . 65

§. 9. Ein Excurs über Invarianten . . . . . . . . . . . . . . . . 76

\& 10. Transformation der Curven zweiter Ordnung auf die Hauptaxen . . . 85

§ 11. Nähere Untersuchnng der nicht ausartenden Kegelschnitte . . . . . 109

§ 12. Erzeugung der Kegelschnitte durch projective Strahlenbüschel und Punktreihen. Sätze von Pascal und Brianchon ....... . . 121

\section{Abschnitt.}

Kegelschnittbüschel und Kegelschnittnetze, sowie die dualistisch entsprechenden Gebilde.

§ 13. Einleitende Bemerkungen . . . . . . . . . . . . . . . . .

§ 14. Bestimmung der Schnittpunkte und gemeinsamen Tangenten zweier Kegelschnitte . . . . . . . . . . . . . . . . 131

$\S$ 15. Allgemeine Eigenschaften des Kegelschnittbüschels; die Kegelschnittschaar . . . . . .

§ 16. Einführung zweckmässiger Coordinatendreiecke in den verschiedenen speciellen Fällen, welche bei einem Kegelschnittbüschel auftreten können . . . . . . . . . . . . . . . 149

§ 17. Geometrische Deutung einiger algebraischer Formen . . . . . . . 158

\& 18. Confocale Kegelschnitte . . . . . . . . . . . . . . . . 165

\& 19. Weitere Untersuchungen über die Brennpunkte: Doppeltberührende Kreise, Sätze über Brennstrahlen . . . . . . . . . . . . . . . 
$\S 20$. Ueber einige Curven, welche in invarianter Beziehung stehen zu einem Kegelschnittbüschel, resp. einer Kegelschnittschaar. . . . . . . . 195

§ 21. Krümmungskreis und Evolute

Kegelschnittnetz und Kegelschnittgewebe.

$\S 22$. Die Hessiane eines Kegelschnittnetzes. . . . . . . . . . . . . . 218

$\S$ 23. Die Cayley'sche Curve eines Kegelschnittnetzes . . . . . . . . . . 222

$\S 24$. Das Kegelschnittgewebe . . . . . . . . . . . . . . . . . . . . . 226

§ 25. Ueber einen merkwürdigen Dualismus, der zwischen der Hesse'schen und Cayley'schen Curve besteht . . . . . . . . . . . . . . . . 228

$\S 26$. Ueber conjugirte, lineare Kegelschnittsysteme . . . . . . . . . . 232

\section{Anhang. \\ Ergänzungen zur voranstehenden Theorie und Lösung von Aufgaben.}

Anwendung von $\S 1-3(\mathrm{Nr} \cdot 1-25)$.

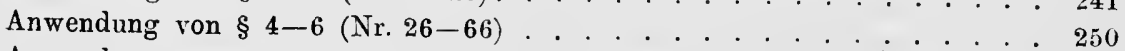

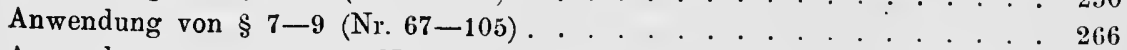

Anwendung ron $\S 10-12(\mathrm{Nr} 106-199)$. . . . . . . . . . . . . . . . . . . 281

Anwendung von $\S 13-17(\mathrm{Nr} .200-255)$. . . . . . . . . . . . . . . . . . . . . 327

Anwendung von $\S 18-19$ (Nr. 256-271) . . . . . . . . . . . . . . . . 346

Anwendung von $\S 20-21(\mathrm{Nr} .272-302)$. . . . . . . . . . . . . . . 354

Excurs über binäre kubische Formen (Nr. 282). . . . . . . . . . . 363

Zusammenhang zwischen der Combinante $\psi$, den äquianharmonischen und harmonischen Kegelschnitten, sowie dem Poldreiseit des Büschels (Nr. 286-290) . . . . . . . . . . . . . . . . . . .

Kriterien der Realität für die Schnittpunkte zweier Curven zweiter Ordnung (Nr. 291). . . . . . . . . . . . . . . . . 371

Anwendung von $\S 22-26$ (Nr. 303-361) . . . . . . . . . . . . . . . . . . . 382

Beispiel eines Kegelschnittgewebes mitzwei Doppelpunkten (Nr.331-332) 394

Ueber Kegelschnitte, die zwei andere doppelt berühren (Netze und

Gewebe mit zwei Doppelgeraden, bezw. Doppelpunkten), (Nr. 333-346)

Brennpunktscurve für die einem Vierseit eingeschriebenen Kegelschnitte

(Nr. 347-353). . . . . . . . . . . . . . . . . . . . . 404

Die Steiner'sche Curve dritter Classe (Nr. 354-361) . . . . . . . . 410

Anwendung der Poldreiecke auf ein Integral von Aronhold (Nr. 362). . . . 415

Ueber eine Verallgemeinerung der Weierstrass'schen Methode, elliptische Integrale auf die Normalform zu reduciren $(\mathrm{Nr}, 363)$. . . . . . 422 


\section{Verbesserungen.}

Seite 2, Zeile 14 von unten ist nach den Worten "des Dreieckswinkels $\boldsymbol{A}_{2}$ “ einzufügen: und $E$ im Inneren des Dreiecks.

" 23, ", 17 von oben lies ,projectiven" statt projectiven.

"51, " 2 von unten lies 1) statt 2).

" 54,,$\quad 11$ von oben lies $F(p, p)>0$ statt $F(p, p)<0$.

$" 111, " 3$ von unten lies $[a, \omega]^{2}$ statt $[a, \omega]$.

"14t, " 15 von unten sind die Worte ,nur dann“ zu streichen.

" 289. Ein etwas einfacherer Ausdruck für die Axe der Parabel ergibt sich, wenn eine der beiden Gleichungen in (122) mit $\cos w$ multiplicirt und zur anderen addirt wird. Man erhält alsdann $a_{11} x+a_{12} y+\frac{a_{13}\left(a_{11}+a_{22}-2 a_{12} \cos w\right)+A_{13}+A_{23} \cos w}{a_{11}+a_{22}-2 a_{12} \cos w}=0$ oder auch

$a_{21} x+a_{22} y+\frac{a_{23}\left(a_{11}+a_{22}-2 a_{12} \cos w\right)+A_{13} \cos w+A_{23}}{a_{11}+a_{22}-2 a_{12} \cos w}=0$.

"358, Zeile 12 von oben lies 316 statt 216.

"374, " 15 " , lies gleiche (positive) Vorzeichen statt gleiche Vorzeichen.

" 18-21 sind die Worte ,hieraus folgt, dass auch $\psi_{33}=\psi_{11} \psi_{22}-\psi_{12}{ }^{2}>0$ sein muss"zu streichen. 


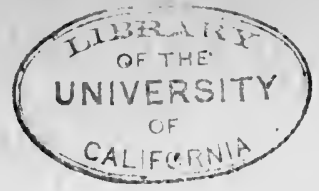

\section{Abschnitt.}

\section{Einleitende Betrachtungen. Die fundamentalen Eigenschaften eines Kegelsehnitts.}

\section{$\S 1$. \\ Das Coordinatendreieck. Punkt- und Liniencoordinaten.}

Die Grundlage der analytischen Geometrie bildet bekanntlich eine Methode, die uns in den Stand setzt, jeden beliebigen Punkt der Ebene oder des Raumes durch Zahlen der Art festzulegen, dass bei Angabe dieser Zahlen die Lage des Punktes eindeutig bestimmt ist. Ein Mittel hierzu bieten z. B. die recht- oder schiefwinkligen Coordinaten von Descartes oder die Polarcoordinaten oder irgend eine Art Dreieckscoordinaten u. s. w.

Auch im Folgenden werde ein Dreieck, das Coordinatendreieck, zu Grunde gelegt, ausserdem innerhalb desselben ein fester Punkt, der sogenannte Einheitspunkt ${ }^{1}$ ). Es seien $a_{1}, a_{2}, a_{3}$ die Geraden, welche das Dreieck bilden, $A_{1}, A_{2}, A_{3}$ die ihnen resp. gegenüberliegenden Ecken und Winkel, $E$ der Einheitspunkt. Dabei mögen $a_{1}, a_{2}, a_{3}$ je nach Bedarf bald Bezeichuungen für jene Geraden sein in ihrer unendlichen Ausdehnung, bald die absoluten Längen der Dreiecksseiten bedeuten. Bezüglich des Punktes $E$ werde angenommen, dass er von den Seiten $a_{1}, a_{2}, a_{3}$ resp. die Abstände $e_{1}, e_{2}, e_{3}$ habe, wobei die zwar willkürliche, aber zweckmässigste Verabredung getroffen werden möge, diese Abstände stets als positiv anzunehmen.

Sind nun $q_{1}, q_{2}, q_{3}$ die Abstände eines beliebigen Punktes $P$ von den Seiten des Dreiecks, so verstehen wir nach v. Staudt und Fiedler ${ }^{2}$ )

1) Davon abgesehen, dass dieser Punkt mit keiner Ecke des Dreiecks zusammenfallen darf, könnte man ihn ganz beliebig in der Ebene annehmen, doch erweist sich die Lage innerhalb des Coordinatendreiecks als die zweckmässigste, da bei ihr keine der Seiten des Dreiecks irgendwie ausgezeichnet ist.

2) v. Staudt: „Beiträge zur Geometrie der Lage“, 2. Heft, Nürnberg 1857, S. $266 \mathrm{f.}$; W. Fiedler: „Ueber die projectivischen Coordinaten“, Vierteljahrsschrift der naturforschenden Gesellschaft in Zürich, 15. Jahrgang, 1870, S. 152 -182, oder auch "Analytische Geometrie der Kegelschnitte“, nach G. Salmon 
unter den Coordinaten dieses Punktes Zahlenwerthe $x_{1}, x_{2}, x_{3}$, welche die Relation erfüllen:

$$
x_{1}: x_{2}: x_{3}=\frac{q_{1}}{e_{1}}: \frac{q_{2}}{e_{2}}: \frac{q_{3}}{e_{3}} .
$$

Die Coordinaten eines Punktes sind demnach Zahlen, welche proportional sind den Abständen des Punktes von den Seiten des Coordinatendreiecks, jeder Abstand dividirt durch die Entfernung des Einheitspunktes von denselben Seiten.

Rückt der Punkt $P$ insbesondere in den Einheitspunkt $E$, so werden die $q_{i}$ und $e_{i}(i=1,2,3)$ bezüglich gleich und (1) verwandelt sich in $x_{1}: x_{2}: x_{3}=1: 1: 1$. Weil in diesem Falle die Verhältnisse der Coordinaten' sämmtlich gleich der Einheit werden, hat man den Punkt $E$ den Einheitspunkt genannt.

In (1) sind die Abstände $q_{i}$ mit dem positiven oder negativen Vorzeichen zu versehen, je nachdem die Punkte $P$ und $E$ in Bezug auf die Gerade $a_{i}$ auf gleichen oder verschiedenen Seiten liegen.

Durch die drei Geraden $a_{i}$ entstehen in der Ebene, vom Coordinatendreieck abgesehen, noch sechs Bezirke, von denen drei begrenzt werden durch die Verlängerungen je zweier Seiten des Dreiecks über ihren Schnittpunkt hinaus und durch die unendlich ferne Gerade: sie mögen als die drei trigonalen Felder bezeichnet werden; die drei anderen, die tetragonalen Felder, werden begrenat durch eine Seite des Dreiecks, die Verlängerungen der beiden anstossenden Seiten und die unendlich ferne Gerade. Es sind daher je nach der Lage des Punktes $P$ bezüglich der Vorzeichen der Abstände $q_{i}$ im ganzen sieben verschiedene Fälle möglich. Liegt z. B. $P$ im Scheitelwinkel des Dreieckswinkels $A_{2}$, so ist $q_{2}$ positiv, $q_{1}$ und $q_{3}$ sind negativ.

Man sieht leicht, dass das Produkt $q_{1} q_{2} q_{3}$ positiv oder negativ ist, je nachdem der Punkt $P$ in einem trigonalen oder tetragonalen Felde liegt; dabei ist vorausgesetzt, dass der Einheitspunkt in einem trigonalen Felde angenommen wird. Das Umgekehrte findet statt, wenn der Einheitspunkt in einem der tetragonalen Felder liegt.

Aus (1) ersieht man, dass die Coordinaten $x_{1}, x_{2}, x_{3}$ selbst nicht völlig bestimmt sind, wohl aber ihre Verhältnisse. Mit Benutzung. eines Proportionalitätsfactors $\varrho$ lässt sich daher die laufende Proportion

frei bearbeitet von W. Fiedler, 5. Aufl., 1. Theil, S. $141 \mathrm{ff}$., 1887. Vgl. übrigens auch W. R. Hamilton: „Elements of Quaternions", edited by W. E. Hamilton, Loudon 1866, S. 27, sowie die nach dem Manuscript einer Vorlesung von Herrn Gundelfinger gegebene Darstellung bei Herrn H. Cranz: „Lehrbuch der analytischen Geometrie der Ebene", 1. Theil, Stuttgart 1892, S. 162-183. 
(1) ersetzen durch die Gleichungen:

(2)

$$
q_{1}=\varrho e_{1} x_{1}, \quad q_{2}=\varrho e_{2} x_{2}, \quad q_{3}=\rho e_{3} x_{3} .
$$

Zwischen diesen drei Abständen $q_{i}$ und den Höhen $h_{1}, h_{2}, h_{3}$ des Dreiecks besteht nun die Relation ${ }^{1}$ )

$$
\frac{q_{1}}{h_{1}}+\frac{q_{2}}{h_{2}}+\frac{q_{3}}{h_{3}}=1
$$

führt man in dieselbe die Werthe der $q_{i}$ aus (2) ein, so ergibt sich

$$
\varrho\left(\frac{e_{1} x_{1}}{h_{1}}+\frac{e_{2} x_{2}}{h_{2}}+\frac{e_{3} x_{3}}{h_{3}}\right)=1 \text {. }
$$

Für die Coefficienten $\begin{gathered}e_{i} \\ h_{i}\end{gathered}$ in dieser Gleichung wollen wir die kürzere Bezeichnungsweise anwenden:

$$
\frac{e_{1}}{h_{1}}=p_{1}, \quad \frac{e_{2}}{h_{2}}=p_{2}, \quad \frac{e_{3}}{h_{3}}=p_{3}
$$

und erhalten alsdann durch Substitution dẹs aus (4) folgenden Werthes von $\varrho$ in die drei Gleichungen (2) die Formeln

$$
q_{1}=\frac{e_{1} x_{1}}{\sum_{1}^{3} p_{i} x_{i}}, \quad q_{2}=\frac{e_{2} x_{2}}{\sum_{1}^{3} p_{i} x_{i}}, \quad q_{3}=\frac{e_{3} x_{3}}{\sum_{1}^{3} p_{i} x_{i}},
$$

mit deren Hilfe die Abstände eines Punktes von den Seiten des Coordinatendreiecks berechnet werden könıen, wenn die Verlı̈ltnisse $x_{1}: x_{2}: x_{3}$ der Coordinaten des Punktes gegeben sind.

Da für $\sum_{1}^{3} p_{i} x_{i}=0$ diese Abstände unendlich gross werden, so wird durch

$$
p_{1} x_{1}+p_{2} x_{2}+p_{3} x_{3}=0
$$

der geometrische Ort aller Punkte dargestellt, die von den Seiten des Coordinatendreiecks unendlich weit entfernt sind, d. h. es ist (7) die Gleichung der unendlich fernen Geraden.

Nachdem wir im Vorhergehenden in (1) Werthe für die Dreieckscoordinaten eines Punktes gegeben haben, handelt es sich nun noch darum, auch eine gerade Linie durch Coordinaten festzulegen.

Es sei also wieder gegeben ein Coordinatendreieck $A_{1} A_{2} A_{3}$, ferner ein beliebiger Punkt $P$ und eine beliebige Gerade $I$. Sind dann $q_{1}$,

1) Der Inhalt $\Delta$ des Dreiecks $A_{1} A_{2} A_{3}$ besitzt nämlich einerseits den Werth $\Delta=\frac{1}{2}\left(a_{1} q_{1}+a_{2} q_{2}+a_{3} q_{3}\right)$, und andrerseits ist $a_{i}=\frac{2 \Delta}{h_{i}}(i=1,2,3)$; substituirt man nun die Werthe der $a_{i}$ in diesen Ausdruck für $\Delta$ und dividirt die so entstehende Gleichung durch ihren Factor $\Delta$, so erhält man (3). Ein Beweis, der die geometrische Anschauung nicht benutzt, folgt in der Fussnote zu (11), S. 4. 
$q_{2}, q_{3}, q$ die Abstände des Punktes $P$ resp. von den Seiten des Dreiecks und vou der Geraden $\Pi$, bedeuten fernier $x, \lambda, \mu$ drei Constanten, die nur von der Gestalt des Coordinatendreiecks und von der Lage der Geraden $\Pi I$ gegen dasselbe abhängen, so ist stets:

$$
q=x q_{1}+\lambda q_{2}+\mu q_{3} \text {. }
$$

Sind nämlich $U_{1}=0, U_{2}=0, U_{3}=0, U=0$ die Gleichungen der Seiten unseres Coordinatendreiecks und der Geraden $\Pi$, bezogen auf ein beliebiges, z. B. rechtwinkliges, Coordinatensystem mit dem Anfangspunkte in $E$, so lassen sich bekanntlich drei Factoren $\varkappa, \lambda, \mu$ der Art bestimmen, dass die Identität besteht:

$$
U \equiv \varkappa U_{1}+\lambda U_{2}+\mu U_{3} \text {. }
$$

Wir dürfen auch annehmen, dass $U, U_{1}, U_{2}, U_{3}$ in der Hesse'schen Normalform gegeben sind, in welchem Falle die Ausdrücke $-U,-U_{1}$ u. s. w. bekanntlich den senkrechten Abstand ${ }^{1}$ ) des Punktes $P$ von den betreffenden Geraden ausdrücken, wenn tan an die Stelle der Variabeln die Coordinaten dieses Punktes einsetzt, $d$. h. wir erhalten

$$
q=x q_{1}+\lambda q_{2}+\mu q_{3} .
$$

Die Constanten $\varkappa, \lambda, \mu$ bestimmen sich natürlich aus den Coefficienten der Gleichungen jener Geraden, hängen also in der That nicht von der Lage des Punktes $P$, sondern nur von der Lage der Seiten des Coordinatendreiecks und der Geraden $I I$ ab. Diese Constanten lassen sich daher auch leicht bestimmen durch specielle Annahmen hinsichtlich der Lage des Punktes $P$. Wir bezeichnen zu dem Zweck die Abstände der Ecken $A_{1}, A_{2}, A_{3}$ des Coordinatendreiecks von der Geraden $\Pi$ resp. durch $\pi_{1}, \pi_{2}, \pi_{3}$ und verlegen nun den Punkt $P$ in die Ecke $A_{1}$. Alsdann ist $q=\pi_{1}, q_{1}=h_{1}, q_{2}=q_{3}=0$, und die Gleichung (8) liefert $\pi_{1}=x h_{1}$, woraus folgt

$$
x=\frac{\pi_{1}}{h_{1}}, \text { und analog wird } \lambda=\frac{\pi_{2}}{h_{2}}, \quad \mu=\frac{\pi_{3}}{h_{3}} .
$$

Mit Hilfe dieser Werthe verwandelt sich die Gleichung (8) in

$$
\left.q=\frac{\pi_{1}}{h_{1}} q_{1}+\frac{\pi_{2}}{h_{2}} q_{2}+\frac{\pi_{3}}{h_{3}} q_{3} \cdot{ }^{2}\right)
$$

Liegt nun der Punkt $P$ irgendwo auf der Geraden $\Pi$, so verschwindet

1) Die Ableitung zeigt, dass der Abstand eines Punktes $P$ von einer Geraden $\Pi$ positiv oder negativ zu nehmen ist, je nachdem die Punkte $\boldsymbol{P}$ und $\boldsymbol{E}$ auf derselben oder auf entgegengesetzten Seiten von $\Pi$ liegen.

2) Es möge noch bemerkt werden, dass sich auch aus (11) leicht die Formel (3) ergibt; dividirt man nämlich (11) durch $q$ und lässt man die Gerade $\Pi$ ins Unendliche rücken, so wird $\frac{\pi_{1}}{q}=\frac{\pi_{2}}{q}=\frac{\pi_{3}}{q}=1$, und es bleibt $1=\frac{q_{1}}{h_{1}}+\frac{q_{2}}{h_{2}}+\frac{q_{3}}{h_{3}}$. 
$q$, und mit Hilfe von (2) erhält man

$$
\frac{e_{1} \pi_{1}}{h_{1}} x_{1}+\frac{e_{2} \pi_{2}}{h_{2}} x_{2}+\frac{e_{3} \pi_{3}}{h_{3}} x_{3}=0
$$

Diese Gleichung wird nur von solchen Werthsystemen $x_{1}, x_{2}, x_{3}$ erfüllt, welche den Coordinaten eines auf $\Pi$ gelegenen Punktes zugehören, wobei die Gerade $I I$ von den Ecken des Coordinatendreiecks die Entfernungen $\pi_{1}, \pi_{2}, \pi_{3}$ hat; es kann daher (12) als die Gleichung dieser Geraden bezeichnet werden. Die Coefficienten voll $x_{1}, x_{2}, x_{3}$ nennt man die Coordinaten der Geraden, es sind dies hiernach Werthe $u_{1}, u_{2}, u_{3}$, welche die Relation erfüllen:

d. h.:

$$
u_{1}: u_{2}: u_{3}=\frac{e_{1} \pi_{1}}{h_{1}}: \frac{e_{2} \pi_{2}}{h_{3}}: \frac{e_{3} \pi_{3}}{h_{3}},
$$

Die Coordinaten einer Geraden sind Zahlen, welche proportional sind den Abständen der betreffenden Geraden von den Ecken des Coordinatendreiecks, sowie den Abständen des Einheitspunktes von den gegenüberliegenden Seiten, umgekehrt proportional den Höhen des Coordinatendreiecks.

Eine Gleichung von der Form

$$
u_{1} x_{1}+u_{2} x_{2}+u_{3} x_{3}=0
$$

drückt hiernach die vereinigte Lage des Punktes $x_{1}, x_{2}, x_{3}$ und der Geraden $u_{1}, u_{2}, u_{3}$ aus ${ }^{1}$ ); andrerseits wird (1t) für gegebene Coordinaten eines Punktes (etwa für $x_{1}: x_{2}: x_{3}=\frac{q_{1}}{e_{1}}: \frac{q_{2}}{e_{2}}: \frac{q_{3}}{e_{3}}$ ) nur dann von den Coordinaten $u_{1}, u_{2}, u_{3}$ gerader Linien erfüllt, wemn diese lurch den gegebenen Punkt hindurchgehen, man nennt daher eine Gleichung von der Form

$$
\frac{q_{3}}{e_{1}} u_{1}+\frac{q_{2}}{e_{2}} u_{2}+\frac{q_{3}}{e_{3}} u_{3}=0
$$

die Gleichung jenes Punktes.

Auch die Coordinaten $u_{1}, u_{2}, u_{3}$ einer Geraden sind nach (13) nicht völlig bestimmt, sondern nur ihre Verhältnisse. Setzen wir, mit $\sigma$ einen Proportionalitätsfactor bezeichnend,

$$
\sigma u_{1}=\frac{e_{1} \pi_{1}}{h_{1}}, \quad \sigma u_{2}=\frac{e_{2} \pi_{2}}{h_{2}}, \quad \sigma u_{3}=\frac{e_{3} \pi_{3}}{h_{3}},
$$

so lässt sich dieser Factor $\sigma$ leicht durch die Coordinaten $u_{i}$ der Geraden, $e_{i}$ des Einheitspunktes, sowie die Winkel des Coordinatendreiecks ausdrücken.

1) Wir werden im Folgenden statt Punkt mit den Coordinaten $x_{1}, x_{2}, x_{3}$ gewöhnlich kürzer sagen Punkt $x_{1}, x_{2}, x_{3}$ oder auch Punkt $x$ und in analoger Weise von einer Geraden $u_{1}, u_{2}, u_{3}$ oder einer Geraden $u$ reden. 


\section{Sind nämlich}

$$
\begin{gathered}
U \equiv x \cos \alpha+y \sin \alpha-\delta=0, \quad U_{i} \equiv x \cos \alpha_{i}+y \sin \alpha_{i}-\delta_{i}=0 \\
(i=1,2,3)
\end{gathered}
$$

die Gleichungen der Geraden $\Pi$ und der drei Seiten des Coordinatendreiecks, bezogen auf irgend ein rechtwinkliges System, so bestehen auf Grund der Identität (9) die Relationen:

(18) $\cos \alpha=x \cos \alpha_{1}+\lambda \cos \alpha_{2}+\mu \cos \alpha_{3}, \sin \alpha=x \sin \alpha_{1}+\lambda \sin \alpha_{2}+\mu \sin \alpha_{3}$, und hieraus folgt durch Quadriren und Addiren beider Gleichungen:

(19) $1=x^{2}+\lambda^{2}+\mu^{2}+2 x \lambda \cos \left(a_{1}, a_{2}\right)+2 \lambda \mu \cos \left(a_{2}, a_{3}\right)+2 \mu x \cos \left(a_{3}, a_{1}\right)$, wo $\left(a_{i}, a_{k}\right)$ den Winkel bezeichnet, den die vom Coordinatenanfang auf die Geraden $a_{i}, a_{k}$ gefällten Lothe mit einander bilden und der zugleich kleiner ist als zwei Rechte, d. h. $\left(a_{i}, a_{k}\right)$ ist derjenige Winkel der beiden Geraden, in welchem der Coordinatenanfang nicht liegt. Gehen wir nun wieder zu unserem Coordinatendreieck zurück und substituiren wir die in (10) gegebenen Werthe von $x, \lambda, \mu$ in die Gleichung (19), so verwandelt sich dieselbe in

$$
\begin{aligned}
1=\frac{\pi_{1}{ }^{2}}{h_{1}{ }^{2}}+\frac{\pi_{2}{ }^{2}}{h_{2}{ }^{2}}+\frac{\pi_{3}{ }^{2}}{h_{3}{ }^{2}}+2 \frac{\pi_{1} \pi_{2}}{h_{1} h_{2}} \cos \left(a_{1}, a_{2}\right)+2 \frac{\pi_{2} \pi_{3}}{h_{2} h_{3}} \cos \left(a_{2}, a_{3}\right) \\
+2 \frac{\pi_{3} \pi_{1}}{h_{3} h_{1}} \cos \left(a_{3}, a_{1}\right)
\end{aligned}
$$

wo unter $\left(a_{i}, a_{k}\right)$ derjenige Winkel der beiden Geraden $a_{i}, a_{k}$ zu verstehen ist, in welchem der Einheitspunkt nicht liegt. Speciell bei der früher getroffenen Verabredung den Einheitspunkt in das Innere des Dreiecks $A_{1} A_{2} A_{3}$ zu verlegen, ist daher $\left(a_{i}, a_{k}\right)$ nicht etwa der Dreieckswinkel der beiden Seiten $a_{i}, a_{k}$, sondern dessen Nebenwinkel, ein Aussenwinkel des Dreiecks.

Ferner folgt aus (20) mit Berücksichtigung von (16):

$$
\begin{gathered}
\sigma^{2}\left(\frac{u_{1}^{2}}{e_{1}{ }^{2}}+\frac{u_{2}{ }^{2}}{e_{2}{ }^{2}}+\frac{u_{3}{ }^{2}}{e_{3}{ }^{2}}+2 \frac{u_{1} u_{2}}{e_{1} e_{2}} \cos \left(a_{1}, a_{2}\right)+2 \frac{u_{2} u_{3}}{e_{2} e_{3}} \cos \left(a_{2}, a_{3}\right)\right. \\
\left.+2 \frac{u_{3} u_{1}}{e_{3} e_{1}} \cos \left(a_{3}, a_{1}\right)\right)=1
\end{gathered}
$$

und wenn man zur Abkürzung setzt

folgt:

$$
\begin{aligned}
\frac{u_{1}^{2}}{e_{1}^{2}}+\frac{u_{2}^{2}}{e_{2}^{2}}+\frac{u_{3}^{2}}{e_{3}^{2}}+2 \frac{u_{1} u_{2}}{e_{1} e_{2}} \cos \left(a_{1}, a_{2}\right)+2 \frac{u_{2} u_{3}}{e_{2} e_{3}} \cos \left(a_{2}, a_{3}\right) \\
+2 \frac{u_{3} u_{1}}{e_{3} e_{1}} \cos \left(a_{3}, a_{1}\right) \equiv \omega\left(u_{1}, u_{2}, u_{3}\right)
\end{aligned}
$$

$$
\sigma=\frac{1}{ \pm \sqrt{\omega\left(u_{1}, u_{2}, u_{3}\right)}}
$$


Hierbei ist in (22) das Vorzeichen der Quadratwurzel so zu wählen, dass der Abstand des Einheitspunktes von der Geraden $I I$ positiv wird; denn dieser Punkt hat von allen Geraden der Ebene positiven Abstand, da auch seine Abstände von den Seiten cles Coordinatendreiecks als positiv angenommen wurden. Es muss daher auf Grund von (11) stets die Ungleichung erfüllt sein:

$$
\frac{e_{1} \pi_{1}}{h_{1}}+\frac{e_{2} \pi_{2}}{h_{2}}+\frac{e_{3} \pi_{3}}{h_{3}}>0
$$

welche sich mit Benutzung von (16) verwandelt in

$$
\sigma\left(u_{1}+u_{2}+u_{3}\right)>0 \text {, }
$$

woraus hervorgeht, dass $\sigma$ stets dasselbe Vorzeichen zu erhalten hat wie die Summe $u_{1}+u_{2}+u_{3}{ }^{1}$ ).

Es möge nun noch an zwei Beispielen gezeigt werden, wie manche vielfach gebräuchliche Coordinateusysteme nur einen speciellen Fall des bisher betrachteten Systems bilden, das ein Coordinateudreieck nebst einem Einheitspunkte benutzt.

Das erste Beispiel seien die barycentrischen oder Flächencoordinaten, welche von Möbius in seinem barycentrischen Calcul vielfach benutzt wurden ${ }^{2}$ ). Man erhält dieselben dadurch, dass man den Einheitspunkt in den Schwerpunkt des Coordinatendreiecks legt; alsdann ist $e_{i}=\frac{1}{3} h_{i}(i=1,2,3)$ und für die Coordinaten $x_{i}$ eines Punktes $P$ folgt aus (1):

$$
x_{1}: x_{2}: x_{3}=\frac{q_{1}}{h_{1}}: \frac{q_{2}}{h_{2}}: \frac{q_{3}}{h_{3}} .
$$

Es sei nun $\Delta$ der Inhalt des Dreiecks $A_{1} A_{2} A_{3}, \Delta_{1}$ der Inhalt des Dreiecks $P A_{2} A_{3}$, ferner $\Delta_{2}=P A_{3} A_{1}, \Delta_{3}=P A_{1} A_{2}$; mit Hilfe von $2 \Delta=a_{i} h_{i}(i=1,2,3)$ verwandelt sich daher unsere laufende Proportion in

$x_{1}: x_{2}: x_{3}=a_{1} q_{1}: a_{2} q_{2}: a_{3} q_{3}$ oder in $x_{1}: x_{2}: x_{3}=\Delta_{1}: \Delta_{2}: \Delta_{3}$.

Jetzt sind demnach die Coordinaten eines Punktes $P$ proportional zu den Inhalten derjenigen Dreiecke, welche durch den betreffenden Punkt. $P$ und die Endpunkte der ihm gegenüberliegenden Seite des Coordinatendreiecks bestimmt sind. Man pflegt dalier in diesem Falle von den Flächencoordinaten des Punktes $P$ zu reden. Natürlich ist das Vorzeichen der Dreiecksinhalte wohl zu berücksichtigen. Es wird

1) In $\S 7$ wird auch analytisch bewiesen, dass $\omega\left(u_{1}, u_{2}, u_{3}\right)$ für reelle Werthe der $u$ niemals negativ, also $\sqrt{\omega\left(u_{1}, u_{2}, u_{3}\right)}$ nie imaginär werden kann.

2) „Der barycentrische Calcul“. Leipzig, 1827. § 23 und 31, oder auch in den „Gesammelten Werken", Bd. I, S. 45 und $51 \mathrm{f}$. 
z. B., wenn wir den Inhalt von $A_{1} A_{2} A_{3}$ als positiv voraussetzen, derjenige von $\Delta_{1}=P A_{2} A_{3}$ positiv oder negativ, je nachdem man bei einem Umlauf um $A_{1}$ von $P$ aus über $A_{2}$ nach $A_{3}$ die Fläche dieses Dreiecks auf derselben (linken oder rechten) Seite hat, wie die Fläche von $\Delta$ bei einem Umlauf von $A_{1}$ aus über $A_{2}$ nach $A_{3}$, oder je nachdem dies nicht der Fall ist.

Das zweite Beispiel seien die gewöhnlichen schiefwinkligen Parallelcoordinaten. Um diese zu erhalten, lassen wir eine Seite des Dreieclis, etwa $a_{3}$, mit der unendlich fernen Geraden zusammen-

Fig. 1.

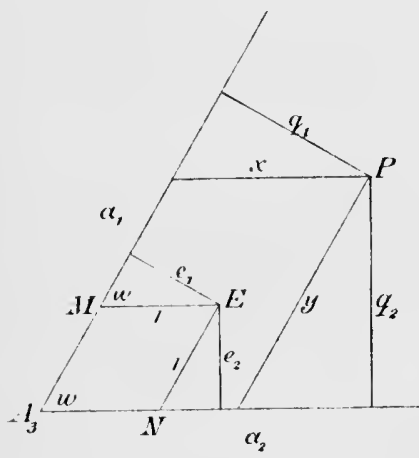
fallen und verlegen den Einheitspunkt $E$ auf die Halbirungslinie des Winkels $A_{2} A_{3} A_{1}=w$ der Art, dass die von $E$ bis an die Seite $a_{1}$ gezogene Parallele $E M$ zu $a_{2}$ die Länge 1 hat, ebenso wie die von $E$ bis an die Seite $a_{2}$ gezogene Parallele $E N$ zu $a_{1}$. Es ist alsdann

$$
\begin{gathered}
e_{1}=e_{2}=\sin w, \quad e_{3}=\infty, \frac{q_{3}}{e_{3}}=1, \\
h_{1}=h_{2}=h_{3}=\infty, \quad \frac{e_{1}}{h_{1}}=\frac{e_{2}}{h_{2}}=0, \frac{e_{3}}{h_{3}}=1 .
\end{gathered}
$$

Betrachten wir ferner $a_{2}$ und $a_{1}$ als die Abscissen- bezw. Ordinatenaxe eines Systems schiefwinkliger Parallelcoordinaten mit dem Axenwinkel $w$ und sind $x, y$ die auf dieses System bezogenen Coordinaten des Punktes $P$, so ist $q_{1}=x \sin w, q_{2}=y \sin w$ und $\frac{q_{1}}{e_{1}}=x, \frac{q_{2}}{e_{2}}=y$, so dass sich die Proportion (1) verwandelt in

$$
x_{1}: x_{2}: x_{3}=x: y: 1,
$$

und umgekehrt ist $x=x_{1}: x_{3}, y=x_{2}: x_{3}$, wir haben demnach hier die bekannten homogenen Coordinaten, die besonders von $\mathrm{Pl}$ ïcker und Hesse mit grossem Erfolg angewandt wurden.

\section{$\S 2$.}

Elementare metrische Beziehungen zwischen Punkten und geraden ' Linien.

Wir wollen uns nunmehr die Aufgabe stellen, den Abstand eines Punktes $y_{1}, y_{2}, y_{3}$ vou einer Geraden $u_{1}, u_{2}, u_{3}$ zu berechnen.

Dieser Abstand ist zunächst nach Gleichung (11) des vorhergehenden Paragraphen bestimmt durch

$$
q=\frac{\pi_{1}}{h_{1}} q_{1}+\frac{\pi_{2}}{h_{2}} q_{2}+\frac{\pi_{3}}{h_{3}} q_{3},
$$

ein Ausdruck, der sich mit Benutzung von (16) verwandelt in 


$$
q=\sigma\left(\frac{u_{1}}{e_{1}} q_{1}+\frac{u_{2}}{e_{2}} q_{2}+\frac{u_{3}}{e_{3}} q_{3}\right),
$$

und wenn man hier für die $q_{i}$ ihre Werthe aus (6) einsetzt, sowie die Gleichung (22) benutzt, ergibt sich für den gesuchten Abstand die Formel:

$$
q=\frac{u_{1} y_{1}+u_{2} y_{2}+u_{3} y_{3}}{ \pm \sqrt{\omega\left(u_{1}, u_{2}, u_{3}\right) \sum_{1}^{3}} p_{i} y_{i}} .
$$

Ertheilt man hier der Wurzel dasselbe Vorzeichen, welehes $u_{1}+u_{2}+u_{3}$ hat, so liegt der Punkt $y$ mit dem Einheitspunkt auf derselben Seite der Geraden, wenu $q$ einen positiven Werth erhält; hingegen sind der Punkt $y$ und der Einheitspunkt durch die Gerade getrennt, wenn $q$ negativ ist.

Man erkennt auch aus (1), dass fïr alle Punkte, welche anf einer und derselben Seite der Geraden $u_{1} x_{1}+u_{2} x_{2}+u_{3} x_{3}=0$ liegen, das Vorzeichen von $\begin{aligned} & u_{1} y_{1}+u_{2} y_{2}+u_{3} y_{3} \\ & p_{1} y_{1}+p_{2} y_{2}+p_{3} y_{3}\end{aligned}$ dasselbe bleibt; denn für alle liese Punkte hat $q$ dasselbe Vorzeichen, da das Zeichen bei $\sqrt{\omega\left(u_{1}, u_{2}, u_{3}\right)}$ von der Lage des Punktes $y$ unabhängig ist und nur mit dem Zeichen von $u_{1}+u_{2}+u_{3}$ übereinstimmen muss.

Eine besondere Erwähnung erfordert der Fall, dass $u_{1}+u_{2}+u_{3}=0$ ist, also die Gerade $u_{1} x_{1}+u_{2} x_{2}+u_{3} x_{3}=0$ durch den Einheitspmint lindurchgeht. Man zieht hier bei Entscheidung der Frage, auf welcher Seite der Geraden $u_{1} x_{1}+u_{2} x_{2}+u_{3} x_{3}=0$ ein gegebener Punkt $y$ liegt, die Ecken des Coordinatendreieeks zu Hilfe. Hat dimn

$$
\begin{aligned}
& u_{1} y_{1}+u_{2} y_{2}+u_{3} y_{3} \\
& p_{1} y_{1}+p_{2} y_{2}+p_{3} y_{3}
\end{aligned}
$$

dasselbe Zeichen wie $\frac{u_{i}}{p_{i}}(i=1$ oder 2 oder 3$)$, so liegt der Punkt $y$ mit der Ecke $u_{i}=0$ des Coordinatendreiecks auf derselben Seite der Geriden $u_{1} x_{1}+u_{2} x_{2}+u_{3} x_{3}=0$; hingegen werden $y$ und $u_{i}=0$ durch diese Gerade getrennt, falls $\frac{u_{1} y_{1}+u_{2} y_{2}+u_{3} y_{3}}{p_{1} y_{1}+p_{2} y_{2}+p_{3} y_{3}}$ und $\frac{u_{i}}{p_{i}}$ entgegengesetzte Vorzeichen haben.

Liegen schiefwinklige Parallelcoordinaten zu Grunde, so ist, wie bereits in $\S 1$ bemerkt wurde, $e_{1}=e_{2}=\sin w, e_{3}=\infty$,

$$
h_{1}=h_{2}=h_{3}=\infty, \quad \frac{e_{3}}{h_{3}}=1, \quad y_{1}: y_{2}: y_{3}=x: y: 1 \text {; }
$$

ferner wird $\cos \left(a_{1}, a_{2}\right)=\cos (2 R-w)=-\cos w$, denn $\left(a_{1}, a_{2}\right)$ ist derjenige Winkel der beiden Coordinatenaxen, in welchem der Einheitspunkt nicht liegt. Der Ausdruck für $\omega\left(u_{1}, u_{2}, u_{3}\right)$ (Gleichung (21) 
des vorhergehenden Paragraphen) vereinfacht sich daher im jetzigen Falle in:

$$
\omega\left(u_{1}, u_{2}, u_{3}\right)=\frac{u_{1}^{2}+u_{2}^{2}-2 u_{1} u_{2} \cos w}{\sin ^{2} w},
$$

während sich $\sum_{i}^{3} p_{i} y_{i}$ auf $y_{3}$ reducirt; für $q$ erhält man also nunmelır

$$
q=\frac{\left(u_{1} x+u_{2} y+u_{3}\right) \sin w}{ \pm \sqrt{u_{1}^{2}+u_{2}^{2}-2 u_{1} u_{2} \cos w}} .
$$

In Analogie zu der Hesse'schen Normalform der Gleichung. der geraden Linie $x \cos \alpha+y \sin \alpha-\delta=0$ bei rechtwinkligen Cartesischen Coordinaten werden wir die Gleichung einer beliebigen Geráden $u_{1} x_{1}+u_{2} x_{2}+u_{3} x_{3}=0$ bei Dreieckscoordinaten durch Division mit $\sqrt{\omega\left(\imath_{1}, u_{2}, u_{3}\right)}$ auf die Normalform bringen und wir werden sagen, die Gerade $u$ sei in ihrer Normalform gegeben, wenn

$$
\sqrt{\omega\left(u_{1}, u_{2}, u_{3}\right)}=+1
$$

wobei nach (22) und (23) in $\$ 1$ das Vorzeichen der Wurzel mit demjenigen von $u_{1}+u_{2}+u_{3}$ übereinzustimmen hat.

Die Gleichung (21), durch welche $\omega\left(u_{1}, u_{2}, u_{3}\right)$ definirt wurde, lässt sich übrigens mit Hilfe von $\omega_{i k}=\frac{1}{2} \frac{\partial^{2} \omega\left(u_{1}, u_{2}, u_{3}\right)}{\partial u_{i} \cdot \partial u_{k}}$ auch schreiben in der Form

wobei

$$
\begin{aligned}
\omega\left(u_{1}, u_{2}, u_{3}\right)=\omega_{11} u_{1}^{2} & +\omega_{22} u_{2}{ }^{2}+\omega_{33} u_{3}{ }^{2} \\
& +2 \omega_{12} u_{1} u_{2}+2 \omega_{13} u_{1} u_{3}+2 \omega_{23} u_{2} u_{3},
\end{aligned}
$$

$$
\left|\begin{array}{lll}
\omega_{11} & \omega_{12} & \omega_{13} \\
\omega_{21} & \omega_{22} & \omega_{23} \\
\omega_{31} & \omega_{32} & \omega_{33}
\end{array}\right|=0
$$

denn diese Determinante ist nach Einführung der Werthe der $\omega_{i k}$ gleich

$$
\frac{1}{{e_{1}{ }^{2} e_{2}^{2} e_{3}{ }^{2}}^{2}}\left|\begin{array}{ccc}
1 & \cos \left(a_{1}, a_{2}\right) & \cos \left(a_{1}, a_{3}\right) \\
\cos \left(a_{2}, a_{1}\right) & 1 & \cos \left(a_{2}, a_{3}\right) \\
\cos \left(a_{3}, a_{1}\right) & \cos \left(a_{3}, a_{2}\right) & 1
\end{array}\right|
$$

und verschwindet zufolge der Relation

$$
\begin{aligned}
1+ & 2 \cos \left(a_{1}, a_{2}\right) \cdot \cos \left(a_{2}, a_{3}\right) \cdot \cos \left(a_{3}, a_{1}\right) \\
& -\cos ^{2}\left(a_{1}, a_{2}\right)-\cos ^{2}\left(a_{2}, a_{3}\right)-\cos ^{2}\left(a_{3}, a_{1}\right)=0,
\end{aligned}
$$

welche besteht, so lange die Summe der Winkel $\left(a_{1}, a_{2}\right),\left(a_{2}, a_{3}\right),\left(a_{3}, a_{1}\right)$ vier Rechte beträgt, was hier der Fall ist, da diese Winkel der Seiten des Dreiecks nach unseren früheren Verabredungen über die Lage des 
Einheitspunktes diejenigen sind, in welchen der Einheitspunkt nicht liegt, also die Aussenwinkel des Dreiecks. (Vgl. auch S. 14.)

Während die Determinante (5) zwar den Werth Null hat, lässt sich andrerseits nachweisen, dass nicht alle Unterdeterminanten von (5) verschwinden können, so lauge das Coordinatendreieck ein eigentliches ist. Für die Uuterdeterminanten $\boldsymbol{\Omega}_{i i}$ der Diagonalelemente findet man nämlich die Werthe

$$
\left.\Omega_{11}=\frac{\sin ^{2}\left(a_{2}, a_{3}\right)}{e_{2}^{2} e_{3}^{2}}, \quad \Omega_{22}=\frac{\sin ^{2}\left(a_{3}, a_{1}\right)}{e_{3}{ }^{2} e_{1}{ }^{2}}, \quad \Omega_{33}=\frac{\sin ^{2}\left(a_{1}, a_{2}\right)}{e_{1}^{2} e_{2}{ }^{2}},{ }^{1}\right)
$$

und diese drei könuen niemals gleichzeitig verschwinden, so lange zwei Seiten des Dreiecks im Endlichen liegen ohne zusammenzufallen. Würde eine Seite, z. B. $a_{3}$, ins Unendliche rücken, so wär, allerdings $e_{3}=\infty$, daher $\Omega_{11}=\Omega_{22}=0$, aber $\Omega_{33}$ ist dann immer noch von Null verschieden.

Wir wollen nunmehr den Winkel bestimmen, welchen zwei durch ihre Gleichungen gegebene beliebige Geraden mit einander bilden; zuvor mögen speciell die Winkel berechnet werlen, die eine beliebige Gerade mit den Seiten des Coordinatendreiecks bildet.

Zu diesem Zweck benutzen wir die Gleichungen (18) des vorhergehenden Paragraphen, und zwar multipliciren wir, um den Cosinus des Winkels $\alpha-\alpha_{1}$ der Geraden $U=0$ mit der Seite $U_{1}=0$ des Coordinatendreiecks zu erhalten, die erste jener Gleichungen mit $\cos \alpha_{1}$, die zweite mit $\sin \alpha_{1}$ und addiren beide. Hierdurch entsteht

$$
\cos \left(\alpha-\alpha_{1}\right)=x+\lambda \cos \left(\alpha_{1}-\alpha_{2}\right)+\mu \cos \left(\alpha_{1}-\alpha_{3}\right),
$$

und unter Berücksichtigung von $\alpha-\alpha_{1}=\left(I, a_{1}\right), \alpha_{1}-\alpha_{2}=\left(a_{1}, a_{2}\right)$, $\alpha_{1}-\alpha_{3}=\left(a_{1}, a_{3}\right), \varkappa=\frac{\pi_{1}}{h_{1}}, \lambda=\frac{\pi_{9}}{h_{2}}, \mu=\frac{\pi_{3}}{h_{3}}$ wird

$$
\cos \left(\Pi, a_{1}\right)=\frac{\pi_{1}}{h_{1}}+\frac{\pi_{2}}{h_{2}} \cos \left(a_{1}, a_{2}\right)+\frac{\pi_{3}}{h_{3}} \cos \left(a_{1}, a_{3}\right) .
$$

Hier ist wieder $\cos \left(\Pi, a_{1}\right)$ der Cosinus desjenigen Winkels der beiden Geraden $\Pi$ und $a_{1}$, in welchem der Einheitspunkt nicht liegt. Ganz analog findet man

$$
\left\{\begin{array}{l}
\cos \left(I, a_{2}\right)=\frac{\pi_{1}}{h_{1}} \cos \left(a_{2}, a_{1}\right)+\frac{\pi_{2}}{h_{2}}+\frac{\pi_{3}}{h_{3}} \cos \left(a_{2}, a_{3}\right) \\
\cos \left(I, a_{3}\right)=\frac{\pi_{1}}{h_{1}} \cos \left(a_{3}, a_{1}\right)+\frac{\pi_{2}}{h_{2}} \cos \left(a_{3}, a_{2}\right)+\frac{\pi_{3}}{h_{3}}
\end{array}\right.
$$

1) Mit Rücksicht auf spätere Betrachtungen sei bemerkt, dass die ïbrigen Unterdeterminanten von (5) die Werthe besitzen:

$\Omega_{23}=\frac{\sin \left(a_{3}, a_{1}\right) \sin \left(a_{1}, a_{2}\right)}{e_{1} e_{2} e_{3} e_{3}}, \Omega_{31}=\frac{\sin \left(a_{1}, a_{2}\right) \sin \left(a_{2}, a_{3}\right)}{e_{1} e_{2}{ }^{2} e_{3}}, \Omega_{12}=\frac{\sin \left(a_{2}, a_{3}\right) \sin \left(a_{3}, a_{1}\right)}{e_{1} e_{2} e_{3}{ }^{2}}$. 
Es sei nun noch eine weitere Gerade $\Pi_{0}$ gegeben durch ihre Gleichung in der Hesse'schen Normalform

$$
U_{0} \equiv x \cos \alpha_{0}+y \sin \alpha_{0}-\delta_{0}=0 ;
$$

multiplicirt man die erste der Gleichungen (18) in $\S 1$ mit $\cos \alpha_{0}$, die zweite mit $\sin \alpha_{0}$ und addirt beide, so entsteht

$$
\cos \left(\Pi, \Pi_{0}\right)=\frac{\pi_{1}}{h_{1}} \cos \left(\Pi_{0}, a_{1}\right)+\frac{\pi_{2}}{h_{2}} \cos \left(\Pi_{0}, a_{2}\right)+\frac{\pi_{3}}{h_{3}} \cos \left(\Pi_{0}, a_{3}\right) .
$$

Hier lassen sich die rechts stehenden Cosinus leicht in anderer Weise ausdrücken; sind nämlich $x_{1}, x_{2}, x_{3}$ die Abstände der Ecken des Coordinatendreiecks von der Geraden $\Pi_{0}$, so ist nach (7)

$$
\cos \left(\Pi_{0}, a_{1}\right)=\frac{x_{1}}{h_{1}}+\frac{x_{2}}{h_{2}} \cos \left(a_{1}, a_{2}\right)+\frac{x_{3}}{h_{3}} \cos \left(a_{1}, a_{3}\right),
$$

und analoge Werthe besitzen $\cos \left(\Pi_{0}, a_{2}\right)$ und $\cos \left(\Pi_{0}, a_{3}\right)$. Durch Substitution derselben in (8) erhält man

$$
\begin{aligned}
\cos \left(\Pi, \Pi_{0}\right)= & \frac{\pi_{1} x_{1}}{h_{1}{ }^{2}}+\frac{\pi_{2} x_{2}}{h_{2}{ }^{2}}+\frac{\pi_{3} x_{3}}{h_{3}{ }^{2}}+\frac{\left(\pi_{2} x_{3}+\pi_{3} x_{2}\right)}{h_{2} h_{3}} \cos \left(a_{2}, a_{3}\right) \\
& +\frac{\left(\pi_{3} x_{1}+\pi_{1} x_{3}\right)}{h_{3} h_{1}} \cos \left(a_{3}, a_{1}\right)+\frac{\left(\pi_{1} x_{2}+\pi_{2} x_{1}\right)}{h_{1} h_{2}} \cos \left(a_{1}, a_{2}\right) .
\end{aligned}
$$

Durch diese Formel ist ein leichter Uebergang gegeben zu dem Falle, dass die Gleichungen der Geraden vorliegen in der allgemeinen Form

$$
\left\{\begin{array}{l}
I I \equiv u_{1} x_{1}+u_{2} x_{2}+u_{3} x_{3}=0 \\
I I_{0} \equiv v_{1} x_{1}+v_{2} x_{2}+v_{3} x_{3}=0
\end{array}\right.
$$

bezogen auf $A_{1} A_{2} A_{3}$ als Coordinatendreieck. Nach (16) in $\S 1$ ist nämlich

$$
\frac{\pi_{i}}{h_{i}}=\frac{\sigma u_{i}}{e_{i}}, \quad \frac{x_{i}}{h_{i}}=\frac{\sigma_{0} v_{i}}{e_{i}} \quad(i=1,2,3),
$$

wo $\sigma$ durch (22) in $\S 1$ definirt ist und $\sigma_{0}$ aus $\sigma$ dadurch hervorgeht, dass man die $u$ ersetzt durch die $v$; ferner werde noch daran erinnert, dass $\sigma$ dasselbe Vorzeichen zu erhalten hat wie $u_{1}+u_{2}+u_{3}, \sigma_{0}$ dasselbe wie $v_{1}+v_{2}+v_{3}$. Alsdann verwandelt sich (9) unter Benutzung von (11) in

$$
\begin{array}{r}
\frac{u_{1}}{e_{1}}\left(\frac{v_{1}}{e_{1}}+\frac{v_{2}}{e_{2}} \cos \left(a_{1}, a_{2}\right)+\frac{v_{3}}{e_{3}} \cos \left(a_{1}, a_{3}\right)\right)+\frac{u_{2}}{e_{2}}\left(\frac{v_{1}}{e_{1}} \cos \left(a_{2}, a_{1}\right)+\frac{v_{2}}{e_{2}}+\frac{v_{3}}{e_{3}} \cos \left(a_{2}, a_{3}\right)\right) \\
+\frac{u_{3}}{e_{3}}\left(\frac{v_{1}}{e_{1}} \cos \left(a_{3}, a_{1}\right)+\frac{v_{2}}{e_{2}} \cos \left(a_{3}, a_{2}\right)+\frac{v_{3}}{e_{3}}\right)
\end{array},
$$

wobei die $u$ offenbar mit den $v$ vertauschbar sind. 
Man kann diesen Ausdruck auch in der Form schreiben

$$
\cos \left(\Pi, \Pi_{0}\right)=\frac{1}{2} \frac{v_{1} \omega^{\prime}\left(u_{1}\right)+v_{2} \omega^{\prime}\left(u_{2}\right)+v_{3} \omega^{\prime}\left(u_{3}\right)}{\sqrt{\omega\left(u_{1}, u_{2}, \overline{\left.u_{3}\right)}\right.} \sqrt{\omega\left(v_{1}, v_{2}, v_{3}\right)}},
$$

wobei $\omega^{\prime}\left(u_{i}\right)$ zur Abkürzung gesetzt ist für $\frac{\partial \omega\left(u_{1}, u_{2}, u_{3}\right)}{\partial u_{i}}$.

Eine andere Bezeichnungsweise, die später angewandt wird, besteht darin, dass man setzt

$$
\begin{gathered}
\frac{1}{2} \omega^{\prime}\left(u_{1}\right) v_{1}+\frac{1}{2} \omega^{\prime}\left(u_{2}\right) v_{2}+\frac{1}{2} \omega^{\prime}\left(u_{3}\right) v_{3}=\omega(u, v), \\
\omega\left(u_{1}, u_{2}, u_{3}\right)=\omega(u, u), \text { sowie } \omega\left(v_{1}, v_{2}, v_{3}\right)=\omega(v, v),
\end{gathered}
$$

indem man mit den zwei Buchstaben $u$ bei $\omega(u, u)$ andeutet, dass $\omega$ die $u$ im zweiten Grade enthält; alsdann ist

$$
\left.\cos \left(\Pi, \Pi_{0}\right)=\frac{\omega(u, v)}{\sqrt{\omega(u, \bar{u})} \sqrt{\omega(v, v)}} \cdot{ }^{1}\right)
$$

Gibt man den Wurzeln dasselbe Zeichen, welches auch $u_{1}+u_{2}+u_{3}$ resp. $v_{1}+v_{2}+v_{3}$ hat, so stellt diese Formel den Cosinus desjenigen Winkels der beiden Geraden $I$ und $\Pi_{0}$ dar, in welchem der Einleitspunkt nicht liegt.

Als Bedingung dafür, dass zwei gerade Linien mit den Coordinaten $u_{1}, u_{2}, u_{3}$ resp. $v_{1}, v_{2}, v_{3}$ auf einander senkrecht stehen, folgt hieraus sofort

$$
v_{1} \omega^{\prime}\left(u_{1}\right)+v_{2} \omega^{\prime}\left(u_{2}\right)+v_{3} \omega^{\prime}\left(u_{3}\right)=0
$$

oder auch $u_{1} \omega^{\prime}\left(v_{1}\right)+u_{2} \omega^{\prime}\left(v_{2}\right)+u_{3} \omega^{\prime}\left(v_{3}\right)=0$,

wo $\omega^{\prime}\left(v_{i}\right)$ den Werth bedeutet, der aus $\omega^{\prime}\left(u_{i}\right)$ durch Vertauschen der $u$ mit den $v$ hervorgeht.

Fixirt man hier die Gerade $u$, so sind $\omega^{\prime}\left(u_{i}\right)(i=1,2,3)$ die Coordinaten eines ganz bestimmten Punktes mit der Gleichung

$$
\omega^{\prime}\left(u_{1}\right) v_{1}+\omega^{\prime}\left(u_{2}\right) v_{2}+\omega^{\prime}\left(u_{3}\right) v_{3}=0
$$

in veränderlichen Liniencoordinaten $v_{i}$, und diese Gleichung wird befriedigt durch die Coordinaten jeder Geraden $v$, die zu $u$ normal ist, es müssen daher alle Normalen von $u$ durch den betreffenden Punkt hindurchgehen, und da diese Normalen einander parallel sind, liegt ihr gemeinsamer Schnittpunkt im Unendlichen; er werde das Normalencentrum der gegebenen Geraden $u$ genannt. Dass dasselbe der umendlich fernen Geraden angehört, wird auch bestätigt, wenn man in (13) an Stelle von $v_{i}$ die Coordinaten der unendlich fernen Geraden

$$
v_{1}: v_{2}: v_{3}=\frac{e_{1}}{h_{1}}: \frac{e_{2}}{h_{2}}: \frac{e_{3}}{h_{3}},
$$

1) Andere Ausdrücke für den Winkel zweier Geraden folgen in $\S 7$. 
wofür der Kürze halber $p_{1}: p_{2}: p_{3}$ gesetzt wurde, einführt. Alsdann erhält die Gleichung (13) die Gestalt

$$
u_{1} \omega^{\prime}\left(p_{1}\right)+u_{2} \omega^{\prime}\left(p_{2}\right)+u_{3} \omega^{\prime}\left(p_{3}\right)=0
$$

oder auch $p_{1} \omega^{\prime}\left(u_{1}\right)+p_{2} \omega^{\prime}\left(u_{2}\right)+p_{3} \omega^{\prime}\left(u_{3}\right)=0$.

In der ersten Gleichung (14) verschwinden nun die drei Coefficienten $\operatorname{der} u^{1}$ ), sie wird also erfüllt, welche Werthe die $u$ haben mögen, womit ausgesagt wird, dass die unendlich ferne Gerade ïberhaupt keine bestimmte Richtung hat, sondern zu jeder Geraden der Ebene normal ist. Bei dieser Auffassungsweise erfüllen die Normalencentra sämmtlicher Geraden der Ebene die unendlich ferne Gerade.

Man könnte in vorliegendem Paragraphen noch die Ableitung der Formel für die Entfernung zweier durch ihre Coordinaten gegebenen Punkte erwarten; doch wird dieselbe erst in $\S 7$ gelegentlich der Untersuchungen über den Kreis gegeben werden, da diese Formel mit der Gleichung eines Kreises in Punktcoordinaten in offenbar engem Zusammenhange steht.

\section{$\S 3$.}

\section{Die Transformation der Coordinaten.}

Bei der Transformation der Coordinaten handelt es sich darum, aus den auf ein Dreieck $D$ bezogenen Coordinaten $x_{1}, x_{2}, x_{3}$ eines Punktes $P$ die Coordinaten $X_{1}, X_{2}, X_{3}$ desselben Punktes abzuleiten, wenn diese bezogen sind auf ein anderes Dreieck $\Delta$.

Die Einheitspunkte $e$ und $E$ mögen von den Seiten der ihnen zugehörigen Dreiecke $D$ und $\Delta$ die Abstïnde haben $e_{1}, e_{2}, e_{3}$, resp. $E_{1}, E_{2}, E_{3}$. Alsdann ist

$$
\left\{\begin{array}{c}
x_{1}: x_{2}: x_{3}=\frac{q_{1}}{e_{1}}: \frac{q_{2}}{e_{2}}: \frac{q_{3}}{e_{3}} \\
X_{1}: X_{2}: X_{3}=\frac{Q_{1}}{E_{1}}: \frac{Q_{2}}{E_{2}}: \frac{Q_{3}}{E_{3}},
\end{array}\right.
$$

wobei die $q_{i}$ die Abstände des Punktes $P$ von den Seiten des Drei-

1) In der That hat z. B. der Coefficient $\omega^{\prime}\left(p_{1}\right)$ von $u_{1}$ den Werth

$$
\frac{2}{e_{1}}\left\{\frac{1}{e_{1}} \frac{e_{1}}{h_{1}}+\frac{1}{e_{2}} \frac{e_{2}}{h_{2}} \cos \left(a_{1}, a_{2}\right)+\frac{1}{e_{3}} \frac{e_{3}}{h_{3}} \cos \left(a_{1}, a_{3}\right)\right\} ;
$$

beachtet man aber, dass $h_{i}=2 \Delta: a_{i}$, sowie dass $\left(a_{1}, a_{2}\right)$ und $\left(a_{1}, a_{3}\right)$ als Aussenwinkel des Dreiecks $A_{1} A_{2} A_{3}$ gleich $2 R-A_{3}$, resp. $2 R-A_{2}$ sind, so wird dieser Coefficient gleich $\frac{1}{\Delta e_{1}}\left(a_{1}-a_{2} \cos A_{3}-a_{8} \cos A_{2}\right)$, wobei nun der Klammeransdruck verschwindet, da $a_{1}=a_{2} \cos A_{3}+a_{3} \cos A_{2}$. Ein anderer Beweis folgt aus (7), wenn man, wie in der Anm. zu S. 4, $\Pi$ ins Unendliche rücken lässt, durch $q$ dividirt und berücksichtigt, dass $\frac{\pi_{1}}{q}=\frac{\pi_{2}}{q}=\frac{\pi_{3}}{q}=1$. 
ecks $D$, die $Q_{i}$ dessen Abstände von den Seiten des Dreiecks $\Delta$ bezeichnen.

Zwischen den $q_{i}$ und $Q_{i}$ bestehen nun, wie wir in $\S 1$ sahen, Relationen von der Form

$$
\left\{\begin{array}{l}
Q_{1}=\alpha_{1} q_{1}+\alpha_{2} q_{2}+\alpha_{3} q_{3} \\
Q_{2}=\beta_{1} q_{1}+\beta_{2} q_{2}+\beta_{3} q_{3} \\
Q_{3}=\gamma_{1} q_{1}+\gamma_{3} q_{2}+\gamma_{3} q_{3}
\end{array}\right.
$$

wobei die $\alpha$ Constanten sind, welche nur ablängen von der Gestalt des Dreiecks $D$ und von der Lage derjenigen Seite des Dreiecks $\Delta$, die von dem Punkte $P$ die Entfernung $Q_{1}$ hat, und Allaloges gilt von den $\beta$ und $\gamma$. Es ist nämlich nach (10) oder (11) in $\S 1$

$$
\left\{\begin{array}{lll}
\alpha_{1}=\frac{\pi_{1}{ }^{\prime}}{h_{1}}, & \alpha_{2}=\frac{\pi_{2}^{\prime}}{h_{2}}, & \alpha_{3}=\frac{\pi_{3}{ }^{\prime}}{h_{3}}, \\
\beta_{1}=\frac{\pi_{1}^{\prime \prime}}{h_{1}}, & \beta_{2}=\frac{\pi_{2}{ }^{\prime \prime}}{h_{2}}, & \beta_{3}=\frac{\pi_{3}{ }^{\prime \prime}}{h_{3}}, \\
\gamma_{1}=\frac{\pi_{1}^{\prime \prime \prime}}{h_{1}}, & \gamma_{2}=\frac{\pi_{2}{ }^{\prime \prime \prime}}{h_{2}}, & \gamma_{3}=\frac{\pi_{3}{ }^{\prime \prime}}{h_{3}} .
\end{array}\right.
$$

Hier sind $\pi_{1}{ }^{\prime}, \pi_{2}{ }^{\prime}, \pi_{3}{ }^{\prime}$ die Abstände der Seite $X_{1}=0$ des neuen von den Ecken des ursprünglich gegebenen Dreiecks $D$; die $\pi_{i}^{\prime \prime}$ und $\boldsymbol{x}_{i}^{\prime \prime \prime}(i=1,2,3)$ haben die analoge Bedeutung für die beiden anderen Seiten $X_{2}=0$ und $X_{3}=0$.

Ersetzt man die Gleichungen (1) mit Einführung zweier Proportionalitätsfactoren $\varrho$ und $P$ durch

$$
\varrho x_{i}=\frac{q_{i}}{e_{i}}, \quad P X_{i}=\frac{Q_{i}}{E_{i}^{\prime}} \quad(i=1,2,3)
$$

und fübrt man die hieraus folgenden Werthe der $q_{i}$ und $Q_{i}$ in (2) ein, so entstehen drei Gleichungen von der Form

$$
\left\{\begin{array}{l}
\mu X_{1}=a_{1} x_{1}+a_{2} x_{2}+a_{3} x_{3} \\
\mu X_{2}=b_{1} x_{1}+b_{2} x_{2}+b_{3} x_{3} \\
\mu X_{3}=c_{1} x_{1}+c_{2} x_{2}+c_{3} x_{3}
\end{array}\right.
$$

in welchen $\mu$ zur Abkürzung gesetzt ist für $\frac{P}{\rho}$, während die $a, b, c$ Grössen sind, die von den $e_{i}$ und $E_{i}$, sowie resp. von den $\alpha_{i}, \beta_{i}, \gamma_{i}$ in einfacher Weise abhängen. Es ist nämlich

$$
\left\{\begin{array}{l}
a_{1}=\frac{\pi_{1}{ }^{\prime} e_{1}}{E_{1} h_{1}}, \quad a_{2}=\frac{\pi_{2}{ }^{\prime} e_{2}}{E_{1} h_{2}}, \quad a_{3}=\frac{\pi_{3} e_{3}}{E_{1} h_{3}}, \\
b_{1}=\frac{\pi_{1}{ }^{\prime \prime} e_{1}}{E_{2} h_{1}}, \text { u. s. w. }
\end{array}\right.
$$

daher $a_{1}: b_{1}: c_{1}=\frac{\pi_{1}{ }^{\prime}}{E_{1}}: \frac{\pi_{1}{ }^{\prime \prime}}{E_{2}^{\prime}}: \frac{\pi_{1}^{\prime \prime \prime}}{E_{3}}$, so dass hiernach die $a_{1}, b_{1}, c_{1}$ die 
Coordinaten der Ecke $A_{1}$ des ursprünglichen Dreiecks $D$, bezogen auf das neue Dreieck $\Delta$ sind. Analoge Bedeutung haben $a_{2}: b_{2}: c_{2}$ und $a_{3}: b_{3}: c_{3}$. Will man von dem System $x_{1}, x_{2}, x_{3}$ zu dem System $X_{1}, X_{2}, X_{3}$ übergehen, so hat man die Gleichungen (4) nach $x_{1}, x_{2}, x_{3}$ aufzulösen. Man erhält:

$$
\left\{\begin{array}{l}
\frac{R}{\mu} x_{1}=A_{1} X_{1}+B_{1} X_{2}+C_{1} X_{3} \\
\frac{R}{\mu} x_{2}=A_{2} X_{1}+B_{2} X_{2}+C_{2} X_{3} \\
\frac{R}{\mu} x_{3}=A_{3} X_{1}+B_{3} X_{2}+C_{3} X_{3}
\end{array}\right.
$$

wobei

$$
R=\left|\begin{array}{ccc}
a_{1} & a_{2} & a_{3} \\
b_{1} & b_{2} & b_{3} \\
c_{1} & c_{2} & c_{3}
\end{array}\right|
$$

und die $A_{i}, B_{i}, C_{i}$ in bekannter Weise Unterdeterminanten von $R$ sind.

Die geometrische Bedeutung der von der Lage des Punktes $P$ ganz unabhängigen $A, B, C$ erhält man in einfachster Weise dadurch, dass man diesen Punkt etwa in die Ecke. $X_{2}=X_{3}=0$ des neuen Dreiecks verlegt; aus (6) folgt dann sofort $x_{1}: x_{2}: x_{3}=A_{1}: A_{2}: A_{3}$, es sind daher die $A$ die Coordinaten der Ecke $X_{2}=X_{3}=0$ des neuen Dreiecks bezogen auf das ursprüngliche, und analoge Bedeutung haben die $B$ und $C$.

Legt man eine Seite des Coordinatendreiecks $D$ ins Unendliche und bestimmt man die Lage des Einheitspunktes $e$ der Art, dass die $x_{1}, x_{2}, x_{3}$ homogene Parallelcoordinaten sind, nämlich $\frac{x_{1}}{x_{3}}=x, \frac{x_{2}}{x_{3}}=y$ (vgl. (25) in $\S 1$ ), so verwandeln sich die Gleichungen (4) nach Division durch $x_{3}$ in

$$
\left\{\begin{array}{l}
\varrho X_{1}=a_{1} x+a_{2} y+a_{3} \\
\varrho X_{2}=b_{1} x+b_{2} y+b_{3} \\
\varrho X_{3}=c_{1} x+c_{2} y+c_{3}
\end{array}\right.
$$

wobei $\varrho=\frac{\mu}{x_{3}}$. Um $x$ und $y$ durch $X_{1}, X_{2}$ und $X_{3}$ auszudrücken, denkt man sich die rechten Seiten von (8) erst wieder durch eine dritte Variabele $z$ in homogene Form gebracht, löst das System (8) nach $x, y, z$ auf und setzt dann wieder $z=1$. Man erhält auf solche Weise unter Anwendung des Proportionalitätsfactors 6:

$$
\left\{\begin{array}{l}
\sigma x=A_{1} X_{1}+B_{1} X_{2}+C_{1} X_{3} \\
\sigma y=A_{2} X_{1}+B_{2} X_{2}+C_{2} X_{3} \\
\sigma=A_{3} X_{1}+B_{3} X_{2}+C_{3} X_{3}
\end{array}\right.
$$


oder auch

$$
\left\{\begin{array}{l}
x=\frac{A_{1} X_{1}+B_{1} X_{2}+C_{1} X_{3}}{A_{3} X_{1}+B_{3} X_{2}+C_{3} X_{3}} \\
y=\frac{A_{2} X_{1}+B_{2} X_{2}+C_{2} X_{3}}{A_{3} X_{1}+B_{3} X_{2}+C_{3} X_{3}}
\end{array}\right.
$$

Es erübrigt nun noch die Transformation der Coordiuaten $u_{1}, u_{2}, u_{3}$ einer Geraden zu betrachten.

Sei $u_{1} x_{1}+u_{2} x_{2}+u_{3} x_{3}=0$ die Gleichung einer Geraden bezogen auf das Dreieck $D$ und $U_{1} X_{1}+U_{2} X_{2}+U_{3} X_{3}=0$ ihre Gleichung bezogen auf das Dreieck $\Delta$. Führt man für die $x_{i}$ ihre Werthe aus (6) in $u_{1} x_{1}+u_{2} x_{2}+u_{3} x_{3}=0$ ein, so entsteht nach Wegfall des Factors $\frac{R}{\mu}$ :

$$
\begin{aligned}
\left(u_{1} A_{1}+u_{2} A_{2}+u_{3} A_{3}\right) X_{1} & +\left(u_{1} B_{1}+u_{2} B_{2}+u_{3} B_{3}\right) X_{2} \\
& +\left(u_{1} C_{1}+u_{2} C_{2}+u_{3} C_{3}\right) X_{3}=0
\end{aligned}
$$

als Gleichung der Geraden, bezogen auf das neue System. Mit Anwendung eines Proportionalitätsfactors $\sigma$ ist daher

$$
\left\{\begin{array}{l}
\sigma U_{1}=A_{1} u_{1}+A_{2} u_{2}+A_{3} u_{3} \\
\sigma U_{2}=B_{1} u_{1}+B_{2} u_{2}+B_{3} u_{3} \\
\sigma U_{3}=C_{1} u_{1}+C_{2} u_{2}+C_{3} u_{3}
\end{array}\right.
$$

woraus nach Multiplication der drei Gleichungen resp. mit $a_{i}, b_{i}, c_{i}$ durch Auflösung nach dẹn $u_{i}$ folgt:

$$
\left\{\begin{array}{l}
\frac{R}{\sigma} u_{1}=a_{1} U_{1}+b_{1} U_{2}+c_{1} U_{3} \\
\frac{R}{\sigma} u_{2}=a_{2} U_{1}+b_{2} U_{2}+c_{2} U_{3} \\
\frac{R}{\sigma} u_{3}=a_{3} U_{1}+b_{3} U_{2}+c_{3} U_{3} .
\end{array}\right.
$$

Hier sind, wie man aus (5) sofort erkennt, die Coefficienten $a_{1}, a_{2}, a_{3}$ die Coordinaten der Seite $X_{1}=0$ des neuen Dreiecks bezogen auf das ursprüngliche, und analoge Bedeutung besitzen die $b$ und $c$. Lässt man ferner die Gerade $u_{1}, u_{2}, u_{3}$ mit der Seite $x_{1}=0$ des ursprünglichen Dreiecks zusammenfallen $\left(u_{2}=u_{3}=0\right)$, so folgt aus (11)

$$
A_{1}: B_{1}: C_{1}=U_{1}: U_{2}: U_{3} \text {, }
$$

es sind daher $A_{1}, B_{1}, C_{1}$ die Coordinaten der Seite $x_{1}=0$ des ursprünglichen Dreiecks bezogen auf das neue, und analoge Bedeutung besitzen die anderen Coefficienten in (11). 


\section{§ 4 .}

\section{Die Curven zweiter Ordnung.}

Als Curve zweiter Ordnung bezeichnet man den geometrischen Ort aller Punkte, deren Coordinaten $x_{1}, x_{2}, x_{3}$ einer homogenen Gleichung zweiten Grades in $x_{1}, x_{2}, x_{3}$, also einer Gleichung von der Form

$$
a_{11} x_{1}{ }^{2}+2 a_{12} x_{1} x_{2}+a_{22} x_{2}{ }^{2}+2 a_{13} x_{1} x_{3}+2 a_{23} x_{2} x_{3}+a_{33} x_{3}{ }^{2}=0
$$
genügen. Wir wollen die linke Seite dieses Ausdrucks kurz bezeichnen durch $f(x, x)$, wobei das doppelte $x$ anzeigen möge, dass (1) in den $x$ vom zweiten Grade ist; ausserdem lässt sich unter der für das Folgende stets giltigen Voraussetzung, dass $a_{i k}=a_{k i}$ sei, (1) ersetzen $\operatorname{durch} \sum_{1}^{3} \sum_{1}^{3} a_{i k} x_{i} x_{k}=0$.

Für die weitere Untersuchung ist ein Ausdruck von Wichtigkeit, der bereits hier erwähnt werden möge, nämlich:

$$
\begin{aligned}
f(x, y) \equiv\left(a_{11} y_{1}+a_{12} y_{2}+a_{13} y_{3}\right) x_{1} & +\left(a_{21} y_{1}+a_{22} y_{2}+a_{23} y_{3}\right) x_{2} \\
& +\left(a_{31} y_{1}+a_{32} y_{2}+a_{33} y_{3}\right) x_{3} .
\end{aligned}
$$

Derselbe ändert sich nicht bei Vertauschung der $y_{i}$ und $x_{i}$, weil $a_{i k}=a_{k i}$, es ist daher auch

(3) $f(x, y) \equiv f(y, x) \equiv\left(a_{11} x_{1}+a_{12} x_{2}+a_{23} x_{3}\right) y_{1}+\left(a_{21} x_{1}+a_{22} x_{2}+a_{23} x_{3}\right) y_{2}$

$$
+\left(a_{31} x_{1}+a_{32} x_{2}+a_{33} x_{3}\right) y_{3} \text {. }
$$

Setzt man in (2) oder (3) die $y_{i}$ gleich den $x_{i}(i=1,2,3)$, so . entsteht, wie man sofort sieht, aus $f(x, y)$ wieder die Function $f(x, x)$. Ferner sind die nach den $x_{i}$ genommenen ersten Ableitungen von $f(x, x)$ nach Multiplication mit $\frac{1}{2}$ gleich den in (3) auftretenden Klammern, oder wenn man in diesen Ableitungen die $x_{i}$ durch die $y_{i}$ ersetzt, gleich den Klammerausdrücken in (2). Es ist demgemäss

$$
\begin{aligned}
f(x, y) & \equiv \frac{1}{2}\left\{f^{\prime}\left(y_{1}\right)^{0} x_{1}+f^{\prime}\left(y_{2}\right) x_{2}+f^{\prime}\left(y_{3}\right) x_{3}\right\} \\
& \equiv \frac{1}{2}\left\{f^{\prime}\left(x_{1}\right) y_{1}+f^{\prime}\left(x_{2}\right) y_{2}+f^{\prime}\left(x_{3}\right) y_{3}\right\}
\end{aligned}
$$

und, wie auch aus dem Euler'schen Satz über homogene Functionen folgt:

$$
f(x, x) \equiv \frac{1}{2}\left\{f^{\prime}\left(x_{1}\right) x_{1}+f^{\prime}\left(x_{2}\right) x_{2}+f^{\prime}\left(x_{3}\right) x_{3}\right\} .
$$

Es wird übrigens im Folgenden statt $\frac{1}{2} f^{\prime}\left(x_{i}\right)$ zuweilen auch $f_{i}$ gesetzt werden, alsdann ist kürzer

$$
f(x, x) \equiv f_{1} x_{1}+f_{2} x_{2}+f_{3} x_{3} .
$$


Nach diesen. Bemerkungen über Bezeichnungsweisen wollen wir nun die durch (1) dargestellte Curve näher untersuchen. Zunächst sei erwähnt, dass, wie in der analytischen Geometrie des Raumes gezeigt wird, die Gleichung (1) jene Curve darstellt, die man durch den Schnitt eines Kegels zweiter. Ordnung mit einer Ebene erhält, also einen Kegelschnitt.

Da man in (1) durch einen der Coefficienten dividiren kann und alsdann noch fünf von einander unabhängige Coefficienten vorhanden sind, von denen sich keiner mehr durch Division wegbringen lässt, so ist ein Kegelschnitt im allgemeinen durch fünf Bedingungen zwischen den Coefficienten, z. B. durch füf seiner Punkte bestimmt. Sind die Bedingungen für die Coefficienten nicht linear, sondern von höherem Grade, so wird es zwar mehrere Kegelschnitte geben, welche diese Bedingungen erfüllen, aber im allgemeinen doch nur eine endliche Anzahl.

Die Gleichung eines Kegelschnitts, der durch fünf Punkte $x_{1}{ }^{(i)}, x_{2}{ }^{(i)}, x_{3}{ }^{(i)}(i=1,2, \ldots 5)$ geht, besteht in einer gleich Null gesetzten Determinante sechsten Grades, die in der ersten Reihe die sechs Quadrate und Producte enthält $x_{1}^{2}, x_{1} x_{2}, x_{2}^{2}, x_{1} x_{3}, x_{2} x_{3}, x_{3}{ }^{2}$, während die fünf anderen Reihen die entsprechenden Quadrate und Producte $x_{1}^{(i)^{2}}, x_{1}^{(i)} x_{2}^{(i)}, x_{2}^{(i)^{2}}, x_{1}^{(i)} x_{3}^{(i)}, x_{2}{ }^{(i)} x_{3}{ }^{(i)}, x_{3}{ }^{(i)^{2}}(i=1,2, \ldots 5)$ enthalten. Soll ein Kegelschnitt durch die fünf Punkte $x^{(i)}$ gehen, so muss jedenfalls diese Determinante sechsten Grades verschwinden, wenn an Stelle von $x_{1}$, $x_{2}, x_{3}$ die Coordinaten eines sechsten Punktes der Curve gesetzt werdeu. Umgekehrt, wenn die Determinante verschwindet, gibt es immer Werthe $a_{i k}$, welche die sechs Gleichungen befriedigen von der Form

$$
\sum_{1}^{3} \sum_{1}^{3} a_{i k} x_{i}^{(i)} x_{k}^{(i)}=0,((i)=1,2,3,4,5), \quad \sum_{1}^{3} \sum_{1}^{3} a_{i k} x_{i} x_{k}=0
$$

es fragt sich aber, ob es nicht vielleicht unendlich viele solcher Werthe $a_{i k}$, also unendlich viele solcher Kegelschnitte gibt, die durch die fünf Punkte gehen.

Es gibt nur einen Kegelschnitt, so lange die sechs Coefficienten von $x_{1}{ }^{2}, x_{1} x_{2}, x_{2}{ }^{2}, x_{1} x_{3}, x_{2} x_{3}, x_{3}{ }^{2}$ in jener Determinante nicht gleichzeitig Null sind. Bei beliebiger Lage der fünf Punkte (sie mögen $a, b, c, d, e$ heissen) kann dies nicht eintreten, weil schon in dem speciellen Falle, dass drei von den fünf Punkten, z. B. $a, b, c$, auf einer Geraden, die zwei übrigen nicht auf dieser Geraden liegen, der Kegelschnitt vollständig bestimmt ist, nämlich aus dem Geradenpaare $a b c$, 
de besteht ${ }^{1}$ ). Der Kegelschnitt ist daher um so mehr bestimmt, wenn die fünf Punkte keine specielle Lage haben, also von den 10 Determinanten dritten Gerades, die man aus den Coordinaten der fünf Punkte bilden kann, keine verschwindet. Eine Unbestimmtheit tritt erst ein, wenn von den fünf Punkten vier auf einer Geraden liegen, indem alsdann der Kegelschnitt aus dieser Geraden und einer beliebig durch den fünften Punkt gelegten Geraden besteht; die Gleichung des Kegelschnitts verschwindet in diesem Falle identisch. Man vergleiche hierzu auch die Fussnote zu (5) in $\S 12$.

Wir wollen ferner die Frage beantworten, in wieviel Punkten ein Kegelschnitt von einer beliebigen Geraden, z. B. von der Verbindungslinie zweier Punkte mit den Coordinaten $x_{1}, x_{2}, x_{3}$ und $y_{1}, y_{2}, y_{3}$ geschnitten wird. Zur Lösung dieser Aufgabe werde vorerst daran erinnert, dass ein jeder Punkt dieser Verbindungslinie Coordinaten hat von der Form $y_{1}+\lambda x_{1}, y_{2}+\lambda x_{2}, y_{3}+\lambda x_{3}$, wo $\lambda$ einen von Punkt zu Punkt variirenden Parameter bedeutet. Sind nämlich $U_{1}=0$ und $U_{2}=0$ die Gleichungen zweier Punkte $x$ und $y$ in Dreieckscoordinaten, so kaun, wie wir hier als bekannt voraussetzen, die Gleichung eines beliebigen anderen Punktes der betreffenden Verbindungslinie in die Form gebracht werden $\lambda U_{1}+U_{2}=0$; hierin sind alsdann die Coefficienten von $u_{1}, u_{2}, u_{3}$ die Dreieckscoordinaten des dem Parameter $\lambda$ entsprechenden Punktes; diese Coefficienten besitzen aber gerade die oben genannten Werthe.

Die Coordinaten der Schnittpunkte der Curve (1) und jener Verbindungslinie müssen nun die Gleichung der Curve erfüllen, d. h. es muss sein

$$
f\left(y_{1}+\lambda x_{1}, y_{2}+\lambda x_{2}, y_{3}+\lambda x_{3}\right)=0,
$$

und wenn man nach Potenzen von $\lambda$ entwickelt:

$$
f(y, y)+2 \lambda f(y, x)+\lambda^{2} f(x, x)=0 .
$$

Diese Gleichung ist in $\lambda$ vom zweiten Grade, woraus hervorgeht, dass die Curve (1) von der Verbindungslinie der Punkte $x$ und $y$ in zwei Punkten geschnitten wird, deren Coordinaten von der Form $y_{i}+\lambda x_{i}$ $(i=1,2,3)$ sind, wobei sich für $\lambda$ aus (8) zwei im allgemeinen verschiedene Werthe ergeben. Wir haben demnach den Satz:

(9) Jede Curve zweiter Ordnung wird von einer Geraden im allgemeinen in zwei Punkten geschnitten ${ }^{2}$ ).

1) Zufolge eines in der Fussnote zu S. 21 erwähnten Satzes muss nämlich eine Gerade, die mit einer Curve zweiter Ordnung drei Punkte gemeinsam hat, ganz der Curve angehören.

2) Wären wir von einer Gleichung $x^{\text {ten }}$ Grades in $x_{1}, x_{2}, x_{3}$ ausgegangen, 
Offenbar sind die Schnittpunkte reell, wenn

$$
f^{2}(x, y)-f(x, x) \cdot f(y, y)>0
$$

sie sind imaginär, wenn $f^{2}(x, y)-f(x, x) \cdot f(y, y)<0$; sie fallen zusammen, wenn $\left.f^{2}(x, y)-f(x, x) \cdot f(y, y)=0^{1}\right)$. In letzterem Falle ist die Gerade im allgemeinen eine Tangente der Curve, so lange nämlich die Curve einen eigentlichen, nicht in zwei gerade Linien zerfallenden Kegelschnitt darstellt. Auf den Fall, dass $f(x, x)=0$ zerfällt, werden wir später zurückkommen.

Nehmen wir an, der Punkt $y$ liege fest, während $x$ variabel ist, so stellt daher

$$
f^{2}(x, y)-f(x, x) \cdot f(y, y)=0
$$

die Bedingung dar, der die $x_{i}$ genügen müssen, um auf einer vom Punkte $y$ an den Kegelschnitt gezogenen Tangente zu liegen, d. h. (10) ist die Gleichung des vom Punkte $y$ an den Kegelschnitt gelegten Tangentenpaares und zwar eines Paares, weil (10) in den $x$ vom zweiten Grade ist.

Sind $\lambda_{1}$ und $\lambda_{2}$ die Wurzeln der quadratischen Gleichung (8), so besitzt das Doppelverhältniss des Punktepaares $x, y$ zu dem Paare $\lambda_{1} x+y, \lambda_{2} x+y$ den Werth $\alpha=\frac{\lambda_{1}}{\lambda_{2}}$ oder auch $\alpha^{\prime}=\frac{\lambda_{2}}{\lambda_{1}}$; wir berücksichtigen zunächst nur $\alpha$. Aus $\alpha=\frac{\lambda_{1}}{\lambda_{2}}$ folgt nun $\frac{\alpha+1}{\alpha-1}=\frac{\lambda_{1}+\frac{\lambda_{2}}{\lambda_{1}}-\frac{\lambda_{2}}{\lambda_{2}}}{\lambda_{1}}$ und $(\alpha+1)^{2}\left[\left(\lambda_{1}+\lambda_{2}\right)^{2}-4 \lambda_{1} \lambda_{2}\right]=(\alpha-1)^{2}\left(\lambda_{1}+\lambda_{2}\right)^{2}$; mit Rücksicht anf (8) ist aber $\lambda_{1}+\lambda_{2}=-2 \frac{f(x, y)}{f(x, x)}, \lambda_{1} \lambda_{2}=\frac{f(y, y)}{f(x, x)}$, es ergibt sich daher zur Bestimmung des Doppelverhältnisses $\alpha$ die Gleichung

$$
(\alpha+1)^{2}\left[f^{2}(x, y)-f(x, x) \cdot f(y, y)\right]-(\alpha-1)^{2} f^{2}(x, y)=0 .
$$

Dieselbe ist in $\alpha$ vom zweiten Grade, und zwar sind ihre Wurzeln von der Form $\alpha$ und $\alpha^{\prime}=\frac{1}{\alpha}$, denn (11) ändert sich nicht bei Vertauschung von $\alpha$ mit $\frac{1}{\alpha}$; hieraus geht auch hervor, dass es erlaubt war nur $\alpha$ zu berücksichtigen.

von einer sogenannten Curve $n^{\text {ter }}$ Ordnung, so würde man auf gleiche Weise finden, dass dieselbe von einer Geraden im allgemeinen in $n$ Punkten geschnitten wird. Deshalb heisst eben die Curve von der $n^{\text {ten }}$ Ordnung.

1) Man kann noch fragen, welche geometrische Bedeutung es hat, wenn in (8) $f(y, y)=0, f(y, x)=0$ und $f(x, x)=0$. Es lässt sich nun algebraisch leicht zeigen, dass die Gleichung (8) für drei verschiedene Werthe $\lambda=\lambda_{1}, \lambda=\lambda_{2}$, $\lambda=\lambda_{3}$ nur befriedigt werden kann, wenn ihre Coefficienten verschwinden und dass alsdann die Gleichung für jeden Werth von $\lambda$ befriedigt wird. Darin liegt auf Grund obiger Discussion der Satz: Wenn eine gerade Linie drei verschiedene Punkte mit dem Kegelschnitt gemeinsam hat, so muss sie demselben ganz angehören. 
Man findet aus (11) übrigens

$$
\frac{\alpha+1}{\alpha-1}=\frac{f(x, y)}{\sqrt{f^{2}(x, y)-f(x, x) \cdot f(y, y)}}
$$

und hieraus durch correspondirende Addition und Subtraction

$$
\alpha=\frac{f(x, y)+\sqrt{f^{2}(x, y)-f(x, x) \cdot f(y, y)}}{f(x, y)-\sqrt{f^{2}(x, y)-f(x, x) \cdot f(y, y)}} .
$$

Denken wir uns $y$ wieder fest gegeben, $x$ variabel und $\alpha$ einen bestimmten Zahlenwerth ertheilt, so stellt (11) einen Kegelschnitt dar, der zu der gegebenen Curve (1) in folgender Beziehung steht: (14) Legt man durch einen beliebig gegebenen Punkt $y$ der Ebene ein Strahlenbüschel und construirt man auf jedem Strahl desselben zu seinen zwei Schnittpunkten mit dem Kegelschnitt (1) und zu $y$ einen vierten Punkt $x$ der Art, dass diese vier Punkte auf jedem Strahl dasselbe Doppelverhältniss $\alpha$ bilden, so liegen alle diese vierten Punkte $x$ auf dem Kegelschnitt (11).

Bei beliebiger Aenderung von $\alpha$ ergibt sich natürlich ein ganzes System von Kegelschnitten.

Von besouderer Wichtigkeit sind hierbei die den Werthen $\alpha=+1$ und $\alpha=-1$ des Doppelverhältnisses entsprechenden Fälle. Für $\alpha=+1$ verwandelt sich (11) in (10), der Kegelschnitt (11) zerfällt dann in das rom Punkte $y$ an (1) gelegte Tangentenpaar.

Für $\alpha=-1$, wenn also die vier Punkte harmonisch liegen sollen, ergibt sich aus (11)

$$
f^{2}(x, y)=0
$$

eine doppelt zu zählende Gerade, die man als Polare des Punktes $y$ in Bezug auf den Kegelschnitt bezeichnet ${ }^{1}$ ). In (3) oder auch (4) ist die Gleichung der Polare ausführlich angegeben. Wir haben somit den Satz:

(16) Zieht man durch einen Punkt $y$ beliebig viele Strahlen und construirt man auf jedem derselben den vierten harmo-

1) Es werde bei dieser Gelegenheit darauf anfmerksam gemacht, dass der Ausdruck $f(x, y)$ der Coefficient von $2 \lambda$ ist in der durch (8) gegebenen Entwickelung von $f\left(y_{1}+\lambda x_{1}, y_{2}+\lambda x_{2}, y_{8}+\lambda x_{8}\right)$ nach Potenzen von $\lambda$. Bildet man diese Entwickelung auch in dem allgemeinen Falle, dass $f\left(x_{1}, x_{2}, x_{3}\right)=0$ die Gleichung einer Curve $n^{\text {ter }}$ Ordnung ist, so stellt der Coefficient von $\lambda k$, gleich Null gesetzt, eine dem Punkte $y$ zugeordnete Curve $k^{\text {ter }}$ Ordnung dar, die sogenannte $(n-k)^{\text {te }}$ Polare des Punktes $y$ in Bezug auf die gegebene Curve $f\left(x_{1}, x_{2}, x_{3}\right)=0$. Diese Art der Einführung der Grösse $\lambda$ findet sich wohl zuerst bei Joachimsthal in Crelle's Journal, Bd. 33, S. $373 \mathrm{f}$, 1846. 
nischen Punkt zu y und zu den beiden Schnittpunkten des betreffenden Strahles mit einer Curve zweiter Ordnung, so liegen alle diese vierten harmonischen Punkte auf einer und derselben Geraden, der Polare des Punktes $y$.

Es seien nun $t$ und $z$ irgend zwei Punkte der Polare von $y$; alsdann hat jeder Punkt dieser Polare Coordinaten von der Form $x_{i}=$ $t_{i}+\lambda z_{i}(i=1,2,3)$, und weil er auf $f(x, y)=0$ liegt, ist für ihn $f(t+\lambda z, y)=0$. Andrerseits ist $f(t+\lambda z, x)=0$ die Gleichung der Polare jenes Punktes; dieselbe wird offenbar erfüllt durch $x_{i}=y_{i}$, woraus folgt, dass die Polare irgend eines auf $f(x, y)=0$ gelegenen Punktes durch $y$ geht. Da ferner vier Punkten mit den Coordinaten $t_{i}+\lambda_{k} z_{i}(i=1,2,3 ; k=1,2,3,4)$ die Polaren zugehören $f\left(x, t+\lambda_{k} z\right)=0$ oder $f(x, t)+\lambda_{k} f(x, z)=0$ und sonach das Doppelverhältniss der vier Punkte gleich demjenigen der entsprechenden Polaren ist, folgt: (17) Durchläuft ein Punkt eine Punktreihe, so beschreibt seine Polare in Bezug auf eine Curve zweiter Ordnung einen zur Punktreihe projectiven Strahlenbüschel ${ }^{1}$ ).

Ein weiterer wichtiger Satz lautet:

(18) Die Polare eines Punktes y geht durch die Berührungrs punkte der von $y$ an den Kegelschnitt gelegten Tangenten hindurch, fällt also mit der Verbindungslinie dieser beiden Punkte zusammen.

Die Gleichung des Tangentenpaares ist nämlich nach (10)

$$
f^{2}(x, y)-f(x, x) \cdot f(y, y)=0
$$

für die Schnittpunkte der Polare $f(x, y)=0$ mit diesen Tangenten fällt nun das Glied $f^{2}(x, y)$ weg, die betreffenden Schnittpunkte sind daher dieselben, wie diejenigen der Polare mit dem Kegelschnitt $f(x, x)=0$, w. z. b. w.

Bisher wurde angenommen, dass der Punkt $y$ nicht auf seiner Polare $f(x, y)=0$ liege, also $f(y, y) \gtrless 0$ sei; wir wollen nun fragen, was für eine geometrische Bedeutung $f(x, y)=0$ hat, wenn $y$ auf seiner Polare liegt. Jedenfalls ist alsdann $f(y, y)=0$, d. h. $y$ liegt in diesem Falle auch auf $\operatorname{dem}^{\top}$ Kegelschnitt, und die Gleichung (8) zur Berechnung der Schnittpunkte einer Geraden mit einer Curve zweiter Ordnung hat zwei Wurzeln $\lambda=0$, die Gerade $f(x, y)=0$ hat also

1) Die Fassung dieses Satzes muss etwas modificirt werden, wenn der Kegelschnitt ein Geradenpaar ist, durch dessen Schnittpunkt der Träger der Punktreihe hindurchgeht; sämmtliche Strahlen des Büschels fallen dann offenbar zusammen mit dem vierten harmonischen Strahle zu dem Geradenpaar und dem Träger der Pnnktreihe. Dieser vierte harmonische Strahl ist jetzt die Polare eines jeden Punktes der Punktreihe. 
zwei zusammenfallende Punkte mit der Curve gemeinsam oder ist eine Tangente der Curve, und zwar im Punkte $y$, da dieser jetzt auf der Curve und auf $f(x, y)=0$ liegt. Man hat daher den Satz:

(19) Die Polare eines auf der Curve zweiter Ordnung $f(x, x)=0$ gelegenen Punktes $y$ fällt mit der in diesem Punkte gezogenen Tangente zusammen; die Gleichung derselben ist $f(x, y)=0$.

Wenn der Punkt $y$ in beliebiger Richtung ins Unendliche rückt; so werden die durch ihn gezogenen Strahlen einander parallel, die vierten harmonischen Punkte zu $y$ und zu den Schnittpunkten der einzelnen Strahlen mit der Curve fallen mit den Halbirungspunkten der durch die Strahlen gebildeten parallelen Sehnen zusammen und liegen natürlich auch auf der Geraden $f(x, y)=0$. Falls jene Richtung zu der Geraden $u_{1} x_{1}+u_{2} x_{2}+u_{3} x_{3}=0$ normal ist, wird der unendlich ferne Punkt $y$ zum Normalencentrum dieser Geraden und hat nach $\S 2$ (am Schluss) die Coordinaten $\omega^{\prime}\left(u_{1}\right), \omega^{\prime}\left(u_{2}\right), \omega^{\prime}\left(u_{3}\right)$; die Gleichung der zugehörigen Polare ist $\omega^{\prime}\left(u_{1}\right) f^{\prime}\left(x_{1}\right)+\omega^{\prime}\left(u_{2}\right) f^{\prime}\left(x_{2}\right)+\omega^{\prime}\left(u_{3}\right) f^{\prime}\left(x_{3}\right)=0$. Man kann somit sagen:

(20) Zieht man bei einer Curve zweiter Ordnung ein System paralleler Sehnen, so liegen deren Mittelpunkte auf einer und derselben Geraden; ihre Gleichung ist

$$
\omega^{\prime}\left(u_{1}\right) f^{\prime}\left(x_{1}\right)+\omega^{\prime}\left(u_{2}\right) f^{\prime}\left(x_{2}\right)+\omega^{\prime}\left(u_{3}\right) f^{\prime}\left(x_{3}\right)=0,
$$

wobei $\omega^{\prime}\left(u_{i}\right)(i=1,2,3)$ die Coordinaten des in Richtung der parallelen Sehnen im Unendlichen gelegenen Punktes bezeichnen.

Man nennt die Gerade (20a) den zur Richtung der parallelen Sehnen conjugirten Durchmesser der Curve $f(x, x)=0$.

Der Grund zu dieser Bezeichnungsweise liegt darin, dass jede Gerade (20a), zu welcher Richtung sie auch conjugirt sein mag, stets durch einen und denselben Punkt $z$ geht, für welchen $\frac{f^{\prime}\left(z_{1}\right)}{p_{1}}=\frac{f^{\prime}\left(z_{2}\right)}{p_{2}}=\frac{f^{\prime}\left(z_{3}\right)}{p_{3}}$, unter $p_{1}, p_{2}, p_{3}$ die Coordinaten der unendlich fernen Geraden verstanden. Ein solcher Punkt $z$ genügt nämlich immer der Gleichung $(20 \mathrm{a})$, weil nach $(14)$ in $\S 2 \boldsymbol{\omega}^{\prime}\left(u_{1}\right) \cdot p_{1}+\omega^{\prime}\left(u_{2}\right) \cdot p_{2}+\omega^{\prime}\left(u_{3}\right) \cdot p_{3}=0$.

Ausserdem hat der Punkt $z$ die Eigenschaft, dass er jede durch ihn gezogene Sehne der Curve zweiter Ordnung halbirt. Zum Beweise lege man durch $z$ irgend eine Sehne; der zur Richtung derselben conjugirte Durchmesser geht gleichfalls, wie eben gezeigt wurde, durch $z$ und halbirt nach (20) die Sehne, und zwar gilt dies für jede durch $z$ gezogene Sehne und ihre conjugirte Gerade, da die Richtung des unendlich fernen Punktes $\omega^{\prime}\left(u_{i}\right)$ ganz willkürlich war. 
Man nennt einen derartigen Punkt $z$ Mittelpunkt der Curve zweiter Ordnung, jede durch ihn gezogene Sehne einen Durchmesser der Curve, und in diesem Sinn kann man sagen:

(21) Jede zu einer gegebenen Richtung $\omega^{\prime}\left(u_{i}\right)$ in Bezug auf den Kegelschnitt $f(x, x)=0$ conjugirte Gerade (20a) ist ein Durchmesser des Kegelschnitts.

Der Punkt $z$ ist, so lange nicht als Curve $f(x, x)=0$ ein Parallelenpaar vorliegt, derjenige Punkt, in welchem sich die Polaren irgend zweier Punkte der unendlich fernen Geraden schneiden; im Falle zweier Parallelen hat man für diese Polaren zufolge der Fussnote S. 23 keinen - bestimmten Schnittpunkt, sondern der Ort der Schnittpunkte wird der vierte harmonische Strahl zu dem Parallelenpaar und zu der unendlich fernen Geraden, also die in der Mitte zwischen den beiden Parallelen verlaufende Gerade; es existirt, wie man sieht, in diesem Falle eine „Mittelpunktslinie“.

Durch die Relationen

$$
\frac{1}{2} \frac{f^{\prime}\left(z_{1}\right)}{p_{1}}=\frac{1}{2} \frac{f^{\prime}\left(z_{2}\right)}{p_{2}}=\frac{1}{2} \frac{f^{\prime \prime}\left(z_{3}\right)}{p_{3}},
$$

denen die Coordinaten $z$ des Mittelpunktes genügen, sind gewissermassen zwei Gleichungen gegeben, die in den verănderlichen Punktcoordinaten $z_{i}$ zwei gerade Linien repräsentiren. Letztere können folgende verschiedene Lagen zu einander haben:

1) sie schneiden sich in einem einzigen Punkte und zwar:

a) im Endlichen: dann existirt ein einziger im Endlichen gelegener Mittelpunkt des Kegelschnitts;

b) im Unendlichen: dann existirt ein einziger im Unendlichen gelegener Mittelpunkt;

2) sie fallen zusammen: dann existirt eine ganze Mittelpunktslinie. Wir setzen nun

$$
\frac{1}{2} f^{\prime}\left(z_{1}\right)=\mu p_{1}, \quad \frac{1}{2} f^{\prime}\left(z_{2}\right)=\mu p_{2}, \quad \frac{1}{2} f^{\prime}\left(z_{3}\right)=\mu p_{3},
$$

wo $\mu$ einen Proportionalitätsfactor bedeutet. Es lässt sich alsdann nachweisen und ist für weitere Untersuchungen von Wichtigkeit, dass die Grösse $\frac{\mu}{p_{z}}$ nicht nur in jedem der Fälle 1), sondern auch im Falle 2) einen ganz bestimmten Werth besitzt, und zwar ist es im letztgenannten Falle gleichgiltig, welchen Punkt der Mittelpunktslinie man auch als Punkt $z$ wählen mag. Hat derselbe die Coordinaten $z_{1}^{\prime}, z_{2}^{\prime}, z_{3}^{\prime}$, so gelten für ihn Gleichungen von der Form

$$
\frac{1}{2} f^{\prime}\left(z_{1}^{\prime}\right)=\nu p_{1}, \quad \frac{1}{2} f^{\prime}\left(z_{2}^{\prime}\right)=\nu p_{2}, \quad \frac{1}{2} f^{\prime}\left(z_{3}{ }^{\prime}\right)=\nu p_{3} ;
$$


durch Multiplication derselben mit $z_{1}, z_{2}, z_{3}$ und Addition erhält man mit Rücksicht auf (23)

$$
\nu p_{z}=\mu p_{z^{\prime}}, \quad \text { also } \frac{\mu}{p_{z}}=\frac{\nu}{p_{z^{\prime}}},
$$

w. z. b. w. Die Bestimmung von $\frac{\mu}{p_{z}}$ wird später (S. 31) erfolgen.

Aus den vorhergehenden Betrachtunger, insbesondere aus (18), folgt noch, dass der zur Richtung nach dem Punkte $\omega^{\prime}\left(u_{1}\right), \omega^{\prime}\left(u_{2}\right), \omega^{\prime}\left(u_{3}\right)$ conjugirte Durchmesser die Curve $f(x, x)=0$ in zwei Punkten trifft, deren Tangenten sich in $\omega^{\prime}(u)$, also im Unendlichen, schneiden; diese Tangenten sind demnach einander parallel und zwar von gleicher Richtung, wie das Sehnensystem, zu welchem der Durchmesser conjugirt ist.

Ein zu einem System paralleler Sehnen conjugirter Durchmesser bildet zusammen mit demjenigen Durchmesser, der in dem System selbst enthalten ist, ein Paar zu einander conjugirter Durchmesser. Der, eine halbirt die zu dem anderen parallel gezogenen Sehnen.

Aus der Gleichung $f(x, y)=0$ der Polare eines Punktes $y$ in Bezug auf die Curve (1) folgt durch Vergleichen mit $u_{1} x_{1}+u_{2} x_{2}$ $+u_{3} x_{3}=0$, dass die Coordinaten dieser Polare die Werthe besitzen

$$
\left\{\begin{array}{l}
\varrho u_{1}=a_{11} y_{1}+a_{12} y_{2}+a_{13} y_{3}=\frac{1}{2} f^{\prime}\left(y_{1}\right) \\
\varrho u_{2}=a_{21} y_{1}+a_{22} y_{2}+a_{23} y_{3}=\frac{1}{2} f^{\prime}\left(y_{2}\right) \\
\varrho u_{3}=a_{31} y_{1}+a_{32} y_{2}+a_{33} y_{3}=\frac{1}{2} f^{\prime}\left(y_{3}\right),
\end{array}\right.
$$

wo $\varrho$ einen Proportionalitätsfactor bedeutet. Umgekehrt entspricht auch jeder Polare mit den Coordinaten $u_{i}$ ein Punkt $y$, wenn sich die Gleichungen (26) eindeutig nach den $y_{i}$ auflösen lassen; man nennt dann $y$ den Pol der Polare $u$. Die Auflösung von (26) ist eindeutig möglich, wenn

$$
A \equiv\left|\begin{array}{lll}
a_{11} & a_{12} & a_{13} \\
a_{21} & a_{22} & a_{23} \\
a_{31} & a_{32} & a_{33}
\end{array}\right| \gtrless 0 ;
$$

diese Determinante wird die Discriminante oder Determinante des Kegelschnitts genannt. Man findet, wenn $A \gtrless 0$, für die Coordinaten des Pols die Werthe

$$
\left\{\begin{array}{l}
\sigma y_{1}=A_{11} u_{1}+A_{21} u_{2}+A_{31} u_{3} \\
\sigma y_{2}=A_{12} u_{1}+A_{22} u_{2}+A_{32} u_{3} \\
\sigma y_{3}=A_{13} u_{1}+A_{23} u_{2}+A_{33} u_{3}
\end{array},\right.
$$


wobei $A_{i k}$ die Unterdeterminante von $a_{i k}$ in $A$ bedeutet und $\sigma=\frac{A}{\rho}$ ist.

Nach (19) fällt die Polare $u$ eines Curvenpunktes $y$ mit der in $y$ gezogenen Tangente der Curve zusammen; da in diesem Falle der Punkt $y$ auf seiner Polare liegt, ist

$$
u_{1} y_{1}+u_{2} y_{2}+u_{3} y_{3}=0 \text {, }
$$

und wenn man aus dieser Gleichung und aus (26) die $y_{i}$ eliminirt, erhält man die Bedingung dafür, dass eine Gerade $u$ Tangente der Curve $f(x, x)=0$ sei in der Form

$$
\left|\begin{array}{llll}
a_{11} & a_{12} & a_{13} & u_{1} \\
a_{21} & a_{22} & a_{23} & u_{2} \\
a_{31} & a_{32} & a_{33} & u_{3} \\
u_{1} & u_{2} & u_{3} & 0
\end{array}\right|=0
$$

ein Ausdruck, der nach Multiplication mit -1 identisch ist nit

$$
\begin{gathered}
F(u, u) \equiv A_{11} u_{1}{ }^{2}+2 A_{12} u_{1} u_{2}+A_{22} u_{2}{ }^{2}+2 A_{13} u_{1} u_{3} \\
+2 A_{23} u_{2} u_{3}+A_{33} u_{3}{ }^{2}=0
\end{gathered}
$$

und als Gleichung der Curve zweiter Ordnung in Liniencoordinaten bezeichnet wird $^{1}$ ).

Dieselbe steht in einer einfachen Beziehung zu der Gleichung $f^{2}(x, y)-f(x, x) \cdot f(y, y)=0$, welche nach (10) die Bedingung dafür ausdrückt, dass die Verbindungslinie zweier Punkte $x$ und $y$ eine Tangente der Curve $f(x, x)=0$ sei. Sind nämlich

$$
u_{1}=x_{2} y_{3}-x_{3} y_{2}, \quad u_{2}=x_{3} y_{1}-x_{1} y_{3}, \quad u_{3}=x_{1} y_{2}-x_{2} y_{1}
$$

die Coordinaten der Tangente, so müssen sich diese in (10) einführen lassen, und kann alsdann (10) von dem Ausdruck (30) nur um einen Zahlenfactor verschieden sein. Man findet in der That

$$
\begin{aligned}
f(x, x) \cdot f(y, y)-f^{2}(x, y) \equiv & A_{11} u_{1}{ }^{2}+2 A_{12} u_{1} u_{2}+A_{22} u_{2}{ }^{2} \\
& +2 A_{13} u_{1} u_{3}+2 A_{23} u_{2} u_{3}+A_{33} u_{3}{ }^{2},
\end{aligned}
$$

wobei die $u_{i}$ durch (31) definirt sind.

Es möge nunmehr angenommen werden, dass zwar $A$ verschwinde, jedoch nicht sämmtliche Unterdeterminanten von $A .{ }^{2}$ )

1) Vgl. über diese Bezeichnungsweise die Fussnote zu (9) in $§ 5$.

2) Es muss dann mindestens eine der drei „Hanptunterdeterminanten“ $A_{11}, A_{22}, A_{33}$ von Null verschieden sein; denn würden diese drei verschwinden, so wäre zufolge der bekannten Relationen aus der Determinantentheorie

$$
A_{22} A_{33}-A_{23}{ }^{2}=A a_{11}, \quad A_{33} A_{11}-A_{31}{ }^{2}=A a_{23}, \quad A_{11} A_{22}-A_{12}{ }^{2}=A a_{33}
$$


In diesem Falle lassen sich die Gleichungen (26) nicht mehr eindeutig nach den $y$ auflösen; zu einem beliebigen Punkte $y$ gehört dann zwar noch im allgemeinen eine bestimmte Polare $u$, aber zu einer beliebigen Polare nicht umgekehrt ein bestimmter Pol.

Jedoch gibt es zufolge $A=0$ ein bestimmtes Werthsystem von $y_{1}: y_{2}: y_{3}$ (dasselbe werde mit $\eta_{1}: \eta_{2}: \eta_{3}$ bezeichnet), das die drei Gleichungen erfüllt:

$$
\left\{\begin{array}{l}
a_{11} \eta_{1}+a_{12} \eta_{2}+a_{13} \eta_{3}=0 \\
a_{21} \eta_{1}+a_{22} \eta_{2}+a_{23} \eta_{3}=0 \\
a_{31} \eta_{1}+a_{32} \eta_{2}+a_{33} \eta_{3}=0
\end{array}\right.
$$

für diesen Punkt $\eta$ wird auch die zugehörige Polare unbestimmt.

Berechnet man die Verhältnisse $\eta_{1}: \eta_{2}: \eta_{3}$ aus je zweien dieser drei Gleichungen, so ergibt sich aus der zweiten und dritten $\eta_{1}: \eta_{2}: \eta_{3}$ $=A_{11}: A_{12}: A_{13}$, aus der dritten und ersten $\eta_{1}: \eta_{2}: \eta_{3}=A_{21}: A_{22}: A_{23}$, aus der ersten und zweiten $\eta_{1}: \eta_{2}: \eta_{3}=A_{31}: A_{32}: A_{33}$, und wenn man die linken Seiten dieser drei laufenden Proportionen erweitert resp. durch $\eta_{1}, \eta_{2}, \eta_{3}$ und die Identitäten $A_{i k}=A_{k i}$ berücksichtigt, folgt $\eta_{1}{ }^{2}: \eta_{1} \eta_{2}: \eta_{2}{ }^{2}: \eta_{1} \eta_{3}: \eta_{2} \eta_{3}: \eta_{3}{ }^{2}=A_{11}: A_{12}: A_{22}: A_{13}: A_{23}: A_{33}$, oder unter Anwendung eines Proportionalitätsfactors $\varrho$ :

$$
\begin{cases}\varrho \eta_{1}{ }^{2}=A_{11} & \varrho \eta_{2} \eta_{3}=A_{23} \\ \varrho \eta_{2}{ }^{2}=A_{22} & \varrho \eta_{3} \eta_{1}=A_{31} \\ \varrho \eta_{3}{ }^{2}=A_{33} & \varrho \eta_{1} \eta_{2}=A_{12}\end{cases}
$$

Die Frage nach der geometrischen Bedeutung dieses ganz bestimmten Punktes $\eta$ erledigt sich zugleich mit Beantwortung der Frage, wie überhaupt die Curve $f(x, x)=0$ im Falle $A=0$ beschaffen ist. Um dies zu erfahren, beziehen wir die Curve auf ein neues Coordinatendreieck. Da nicht alle Hauptunterdeterminanten $A_{11}, A_{22}, A_{33}$ verschwinden sollen, sei etwa $A_{33}$ von Null verschieden, und unter dieser Voraussetzung werde jetzt das neue Coordinatendreieck mit dem ursprünglichen verbunden durch die Relationen:

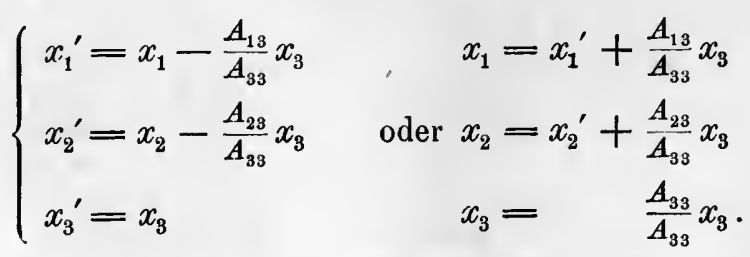

wegen $A=0$ auch $A_{23}=A_{31}=A_{12}=0$, was ausgeschlossen sein sollte. Ausserdem erkennt man, dass im Falle $A=0$ die drei Hauptunterdeterminanten gleiches Vorzeichen besitzen. 
Mit Benutzung der Relation

erhält man alsdann

$$
a_{i 1} A_{k 1}+a_{i 2} A_{k 2}+a_{i 3} A_{k 3}=0 \text { für } i \gtrless k
$$

$$
\begin{aligned}
& \frac{1}{2} f^{\prime}\left(x_{1}\right) \equiv a_{11} x_{1}+a_{12} x_{2}+a_{13} x_{3}=a_{11} x_{1}^{\prime}+a_{12} x_{2}^{\prime} \\
& \frac{1}{2} f^{\prime}\left(x_{2}\right) \equiv a_{21} x_{1}+a_{22} x_{2}+a_{23} x_{3}=a_{21} x_{1}^{\prime}+a_{22} x_{2}^{\prime} \\
& \frac{1}{2} f^{\prime}\left(x_{3}\right) \equiv a_{31} x_{1}+a_{32} x_{2}+a_{33} x_{3}=a_{31} x_{1}^{\prime}+a_{32} x_{2}{ }^{\prime}+\frac{A}{A_{33}} x_{3},
\end{aligned}
$$

und die Gleichung unseres Kegelschnitts

$$
f(x, x) \equiv \frac{1}{2}\left\{f^{\prime}\left(x_{1}\right) \cdot x_{1}+f^{\prime}\left(x_{2}\right) \cdot x_{2}+f^{\prime}\left(x_{3}\right) \cdot x_{3}\right\}=0
$$

verwandelt sich durch die Transformation (35) in

$$
a_{11} x_{1}^{\prime 2}+2 a_{12} x_{1}^{\prime} x_{2}^{\prime}+a_{22} x_{2}^{\prime 2}+\frac{A}{A_{33}} x_{3}{ }^{2}=0 .
$$

Diese Gleichung enthält im Falle $A=0$ nur noch die zwei Variabeln $x_{1}^{\prime}$ und $x_{2}^{\prime}$, in denen sie homogen ist, lässt sich daher sofort in zwei Factoren zerfällen, so dass sie von der Form wird

$$
a_{11}\left(x_{1}^{\prime}-\alpha x_{2}{ }^{\prime}\right)\left(x_{1}^{\prime}-\beta x_{2}^{\prime}\right)=0 .
$$

$\mathrm{Da}$ aber jeder dieser Factoren eine Gerade darstellt, die durch den Punkt $x_{1}^{\prime}=x_{2}{ }^{\prime}=0$ geht, artet im Falle $A=0$ der Kegelschnitt in ein Geradenpaar aus, das den Punkt $x_{1}^{\prime}=x_{2}^{\prime}=0$ zum Schuittpunkt oder zur Spitze hat, und aus den Gleichungen (35) folgt sofort, dass diese Spitze, auf das ursprüngliche Dreieck bezogen, die Coordinaten hat $x_{1}: x_{2}: x_{3}=A_{13}: A_{23}: A_{33}$, also .mit dem oben betrachteten Punkte $\eta$ identisch ist ${ }^{1}$ ). Wir können daher sagen:

(38) Sobald die Determinante $A$ des Kegelschnitts $f(x, x)=0$ verschwindet, zerfällt derselbe in ein Geradenpaar ${ }^{2}$ ).

(39) Man kann auch umgekehrt zeigen, dass die Determinante $A$ verschwindet, wenn der Kegelschnitt aus einem Geradenpaar besteht.

1) Uebrigens ist die Spitze des Geradenpaares zugleich der Mittelpunkt desselben, denn ihre Coordinaten erfüllen die Gleichungen $\frac{f^{\prime}\left(\eta_{1}\right)}{p_{1}}=\frac{f^{\prime}\left(\eta_{2}\right)}{p_{2}}=\frac{f^{\prime}\left(\eta_{3}\right)}{p_{3}}$, aus denen, wie wir oben sahen, die Coordinaten des Mittelpanktes zu berechnen wären; im vorliegenden Fall ist Null der gemeinsame Werth der drei Quotienten.

2) In dem zu vorliegendem Paragraphen gehörigen Theile des Anhangs sind die beiden Geraden einzeln dargestellt. Die Frage nach dem Winkel, den zwei durch $f(x, x)=0$ dargestellte Geraden mit einander bilden, wird in $\$ 7$ beantwortet. 
Sind nämlich $U=0, V=0$ die Gleichungen der beiden Geraden, so ist $f(x, x) \equiv U V$, daher

$$
f^{\prime}\left(x_{i}\right) \equiv U \frac{\partial V}{\partial x_{i}}+V \frac{\partial U}{\partial x_{i}} \quad(i=1,2,3)
$$

und wenn man hier für die $x$ die Coordinaten $\eta$ des Schnittpunktes der beiden Geraden $U$ und $V$ einsetzt, erhält man an Stelle der $f^{\prime}\left(x_{i}\right)$ die in (33) links stehenden Ausdrücke, während die rechten Seiten von (40) für die Coordinaten des Schnittpunktes verschwinden. Die Gleichungen (33) können aber nach einem bekannten Satze der Determinantentheorie nur dann zusammen bestehen, wenn ihre Determinante, die mit $A$ identisch ist, verschwindet.

Wir betrachten nunmehr den Fall, dass in der Determinante $A$ der Curve $f(x, x)=0$ auch sämmtliche Unterdeterminanten $A_{i k}$ verschwinden. Es soll jedoch mindestens einer der drei Coefficienten $a_{11}, a_{22}, a_{33}$ von Null verschieden sein, denn für $a_{11}=a_{22}=a_{33}=0$ müsste wegen $A_{11}=A_{22}=A_{33}=0$ auch $a_{23}=a_{31}=a_{12}=0$ sein. Wir dürfen deshalb aunehmen, es sei etwa $a_{11} \gtrless 0$ und multipliciren nun die Gleichung (1) der Curve mit $a_{11}$; unter Rücksicht darauf, dass $a_{11} a_{22}=a_{12}{ }^{2}, a_{11} a_{33}=a_{13}{ }^{2}, a_{22} a_{93}=a_{23}{ }^{2}$, verwandelt sich dann (1) in

$$
\left(a_{11} x_{1}+a_{12} x_{2}+a_{13} x_{3}\right)^{2}=0,
$$

woraus folgt, dass der Kegelschuitt (1) im gegenwärtigen Fall eine doppelt zu zählende Gerade darstellt.

Ist umgekehrt $f(x, x)$ das Quadrat eines linearen Ausdrucks, so verschwinden alle Unterdeterminanten $A_{i k}$, denn für

$$
f(x x) \equiv\left(\alpha_{1} x_{1}+\alpha_{2} x_{2}+\alpha_{3} x_{3}\right)^{2}=0
$$

wird $a_{i k}=\alpha_{i} \alpha_{k}$, und eine beliebige Unterdeterminante $\left|\begin{array}{ll}a_{i l} & a_{i m} \\ a_{k l} & a_{k m}\end{array}\right|$ ererhält daher den Werth $\alpha_{i} \alpha_{k}\left|\begin{array}{cc}\alpha_{l} & \alpha_{m} \\ \alpha_{l} & \alpha_{m}\end{array}\right|$, welcher identisch Null ist.

Man kann somit sagen:

(42) Wenn sämmtliche Unterdeterminanten $A_{i k}$ der Determinante $A$ einer Curve zweiter Ordnung verschwinden, reducirt sich die Curve auf eine doppelt zu zählende Gerade (Doppelgerade), und umgekehrt.

Wir wollen jetzt die Beantwortung einer Frage wieder aufnehmen, die gelegentlich der Einführung des Mittelpunktes $z$ eines Kegelschnitts berührt wurde; es hatte sich in (25) gezeigt, dass auch bei Curven mit Mittelpunktslinie die Grösse $\frac{\mu}{p_{z}}(=x)$ einen ganz be- 
Endgiltige Bestimmung d. Grundconstante f. d. Transformation auf d. Mittelpunkt. 31 stimmten Werth besitzt, und es handelt sich nun darum, die Bestimmung dieses Werthes wirklich durchzuführen. Die Auflösung der Gleichungen

$$
\left\{\begin{array}{c}
\frac{1}{2} f^{\prime}\left(z_{t}\right)=\mu p_{1}, \quad \frac{1}{2} f^{\prime}\left(z_{2}\right)=\mu p_{2}, \quad \frac{1}{2} f^{\prime}\left(z_{3}\right)=\mu p_{3}, \\
p_{1} z_{1}+p_{2} z_{2}+p_{3} z_{3}=\frac{\mu}{x},
\end{array} .\right.
$$

denen die Coordinaten $z_{1}, z_{2}, z_{3}$ des Mittelpunktes genügen, ergibt

$$
A \cdot z_{i}=\mu\left(A_{i 1} p_{1}+A_{i 2} p_{2}+A_{i 3} p_{3} \dot{j}\right.
$$

woraus folgt

$$
\begin{aligned}
A p_{z} & =\mu\left\{A_{11} p_{1}{ }^{2}+2 A_{12} p_{1} p_{2}+\dot{A}_{22} p_{2}{ }^{2}+2 A_{13} p_{1} p_{3}+2 A_{23} p_{2} p_{3}+A_{33} p_{3}{ }^{2}\right\} \\
& =\mu \cdot F(p, p),
\end{aligned}
$$

daher

$$
\frac{\mu}{p_{z}}=\frac{A}{F(p, p)} .
$$

- Wenn nun $A$ und $F(p, p)$ verschwinden, ist von den drei Gleichungen (43) je eine die Folge der beiden anderen (vgl. 2), S. 25). Lassen wir daher die erste dieser Gleichungen ganz ausser Acht und ersetzen sie durch $u_{1} z_{1}+u_{2} z_{2}+u_{3} z_{3}=0$, wo $u_{1}, u_{2}, u_{3}$ beliebige Zahlen bedeuten, die jedoch den $p_{i}$ nicht proportional sein sollen, so erhält man in gleicher Weise wie oben

$$
\frac{\mu}{p_{z}}=\frac{A_{11} u_{1}+A_{12} u_{2}+A_{13} u_{3}}{\left|\begin{array}{llll}
a_{21} & a_{22} & a_{23} & p_{2} \\
a_{31} & a_{32} & a_{33} & p_{3} \\
p_{1} & p_{2} & p_{8} & 0 \\
u_{1} & u_{2} & u_{3} & 0
\end{array}\right|}
$$
wo für den Nenner auch $\frac{\partial F(p, \underline{p})}{\partial a_{11}} u_{1}+\frac{\partial F(p, p)}{\partial a_{12}} u_{2}+\frac{\partial F(p, \underline{p})}{\partial a_{13}} u_{3}$ ge-
setzt werden kann.

Zwei ähnliche Gleichungen wie (46) ergeben sich, wenn man in dem System (43) die zweite, resp. dritte Gleichung durch $u_{1} z_{1}+u_{2} z_{2}$ $+u_{3} z_{3}=0$ ersetzt, nämlich:

(46a)

$$
\left\{\begin{array}{c}
\frac{\mu}{p_{s}}=\frac{A_{91} u_{1}+A_{92} u_{2}+A_{23} u_{3}}{\frac{\partial F(p, p)}{\partial a_{31}} u_{1}+\frac{\partial F(p, p)}{\partial a_{22}} u_{2}+\frac{\partial F(p, p)}{\partial a_{23}} u_{3}} \\
\text { und } \left.\frac{\mu}{p_{s}}=\frac{A_{31} u_{1}+A_{32} u_{2}+A_{33} u_{3}}{\frac{\partial F(p, \underline{p})}{\partial a_{31}} u_{1}+\frac{\partial F(p, p)}{\partial a_{32}} u_{2}+\frac{\partial F(p, p)}{\partial a_{33}} u_{3}} \cdot{ }^{1}\right)
\end{array}\right.
$$

1) Setzt man von den willkürlichen $u_{i}$ je zwei gleich Null, das dritte gleich 1 , 80 entsteht $\mu: p_{z}=A_{11}: \frac{\partial F}{\partial a_{11}}=A_{12}: \frac{\partial F}{\partial a_{12}}=\cdots=A_{33}: \frac{\partial F}{\partial a_{33}}$. 
Endlich erhält man aus den drei Gleichungen (46) und (46a) unter Anwendung eines elementaren Satzes aus der Lehre von den Proportionen:

$$
\frac{\mu}{p_{z}}=\frac{A_{11} u_{1}^{2}+2 A_{12} u_{1} u_{2}+A_{22} u_{2}^{2}+2 A_{13} u_{1} u_{3}+2 A_{23} u_{2} u_{8}+A_{33} u_{3}^{2}}{\left|\begin{array}{lllll}
a_{11} & a_{12} & a_{13} & p_{1} & u_{1} \\
a_{21} & a_{22} & a_{23} & p_{2} & u_{2} \\
a_{31} & a_{32} & a_{33} & p_{3} & u_{3} \\
p_{1} & p_{2} & p_{3} & 0 & 0 \\
u_{1} & u_{2} & u_{3} & 0 & 0
\end{array}\right|}
$$

und hier ist $\frac{\mu}{p_{z}}$ für beliebige Werthe der $u_{i}$ bestimmt, auch wenn $A$ und $F(p, p)$ verschwinden.

Zwei Sehnen, die durch den Mittelpunkt des Kegelschnitts gehen und zu einander conjugirt sind (vgl. S. 26), bilden mit der unendlich fernen Geraden ein Dreieck, zu dessen wahrer projectiver Verallgemeinerung wir nun übergehen wollen.

Auf Grund des Satzes (16) liegen alle vierten harmonischen Punkte zu einem festen Punkte $y$ und zu den Schnittpunkten der durch $y$ gézogenen Strahlen mit der Curve zweiter Ordnung $f(x, x)=0$ auf einer Geraden, der Polare des Punktes $y$. Die Gleichung derselben war $f(x, y)=0$. Man nennt nun überhaupt zwei Punkte $x$ und $y$, die zu den Schnittpunkten ihrer Verbindungslinie mit einer Curve zweiter Ordnung harmonisch liegen, conjugirte oder harmonische Pole in Bezug auf die Curve; die Bedingung, dass $x$ und $y$ solche Pole seien, ist $f(x, y)=0$. Der eine Pol liegt immer auf der Polare des anderen. Offenbar gilt auch der Satz:

Die einzelnen Pare conjugirter Pole, die derselben Punktreihe angehören, bilden eine Involution; die Doppelpunkte der Involution sind die der Curve zweiter Ordnung angehörigen Punkte der Reihe.

Drei Punkte, von denen je zwei harmonische Pole in Bezug auf dieselbe Curve zweiter Ordnung sind, bestimmen ein Dreieck, das man als Poldreieck zu bezeichnen pflegt. Wir behaupten nun:

(49) Für eine gegebene Curve zweiter Ordnung gibt es dreifach unendlich viele Poldreiecke.

Denn zunächst ist eine Ecke dieses Dreiecks willkürlich gewählt, ihre Bestimmung hängt jedoch von zwei Constanten, nämlich von zwei Verhältnissen ihrer Coordinaten ab; durch diese erste Ecke ist die Gegenseite als Polare festgelegt, hingegen kann auf dieser Polare die zweite Ecke beliebig gewählt werden, wozu noch eine Constante erforderlich ist. Alsdann aber ist das Dreieck festgelegt, denn die Polare dieser zweiten Eicke schneidet auf der Polare der ersten die 
dritte Ecke aus; die Willkürlichkeit ist daher in der That eine dreifache.

Ferner gilt der Satz:

(50) Bezieht man eine Curve zweiter Ordnung auf ein Poldreieck als Coordinatendreieck, so kann ihre Gleichung nur noch die $Q$ uadrate der Variabeln enthalten.

Für die Polare eines beliebigen Punktes $y$ hat man nämlich die in (3) ausführlich angegebene Gleichung $f(x, y)=0$; lässt man $y$ etwa in die Ecke $x_{1}=x_{2}=0$ des Coordinatendreiecks rïcken, so wird die zugehörige Polare $a_{31} x_{1}+a_{32} x_{2}+a_{33} x_{3}=0$. Diese muss nun. mit der Gegenseite $x_{3}=0$ der Ecke $x_{1}=x_{2}=0$ zusammenfallen, falls das Dreieck ein Poldreieck sein soll, d. h. es müssen $a_{31}$ und $a_{32}$ wegfallen, wodurch man als Gleichung der Curve erhält

$$
a_{11} x_{1}{ }^{2}+2 a_{12} x_{1} x_{2}+a_{22} x_{2}{ }^{2}+a_{33} x_{3}{ }^{2}=0 .
$$

Verfährt man in solcker Weise auch bei den übrigen Ecken, so zeigt sich, dass überhaupt die Glieder mit $a_{12}, a_{31}, a_{23}$ wegfallen. Die Gleichung einer auf ein Poldreieck bezogenen Curve zweiter Ordnung lautet daher

$$
a_{11} x_{1}^{2}+a_{22} x_{2}^{2}+a_{33} x_{3}^{2}=0,
$$

sie enthält nur noch die Quadrate.

Offenbar gilt auch die Umkehrung des Satzes (50):

(52) Enthält die Gleichung einer Curve zweiter Ordung nur die Quadrate der Variabeln, so kann dieselbe als auf.ein Poldreieck bezogen angesehen werden.

Es möge zum Schlusse die Bestimmung der Coordinaten der Schnittpunkte einer Geraden mit einer Curve zweiter Ordnung in andrer Weise erfolgen als zu Anfang dieses Paragraphen.

Sei $f(x, x)=0$ die Gleichung der Curve, $u_{1} x_{1}+u_{2} x_{2}+u_{3} x_{3}=0$ oder, wie wir häufig kürzer setzen werden, $u_{x}=0$ diejenige der Geraden und sind $x_{1}, x_{2}, x_{3}$ die Coordinaten eines der gesuchten Schnittpunkte, also $x_{1} v_{1}+x_{2} v_{2}+x_{3} v_{3}=0$ dessen Gleichung in Liniencoordinaten $v_{i}$, so erhält man die Bedingung für die Coordinaten einer durch einen Schnittpunkt gehenden Geraden $v_{x}=0$, wenn es gelingt, aus den drei Gleichungen $f(x, x)=0, u_{x}=0, v_{x}=0$ eine Combination abzuleiten, welche die $x$ nicht mehr enthält. Diese Combination stellt das Product der beiden Schnittpunkte dar, wenn sie in den $v$ vom zweiten Grade wird.

- In Folge von $x_{1}: x_{2}: x_{3}=\left(u_{2} v_{3}-u_{3} v_{2}\right):\left(u_{3} v_{1}-u_{1} v_{3}\right):\left(u_{1} v_{2}-u_{2} v_{1}\right)$ erhält man sofort für das Product der beiden Schnittpunkte die Gleichung $\left.{ }^{1}\right)$

1) Die der Kürze halber in (53) nur durch Punkte angedeuteten Glieder GundeIfinger, Vorlesungen. 


$$
a_{11}\left(u_{2} v_{3}-u_{3} v_{2}\right)^{2}+\cdots+2 a_{23}\left(u_{3} v_{1}-u_{1} v_{3}\right)\left(u_{1} v_{2}-u_{2} v_{1}\right)+\cdots=0
$$

wofür man auch setzen kann

$$
\left.\left(\begin{array}{c}
u v \\
u v
\end{array}\right) \equiv\left|\begin{array}{lllll}
a_{11} & a_{12} & a_{13} & u_{1} & v_{1} \\
a_{21} & a_{22} & a_{23} & u_{2} & v_{2} \\
a_{31} & a_{32} & a_{33} & u_{3} & v_{3} \\
u_{1} & u_{2} & u_{3} & 0 & 0 \\
v_{1} & v_{2} & v_{3} & 0 & 0
\end{array}\right|=0^{1}\right)
$$

Falls der Kegelschnitt $f(x, x)=0$ von $u_{x}=0$ in zwei zusammenfallenden Punkten getroffen wird, stellt (53a) den betreffenden Punkt doppelt zählend dar. Sollte ferner (53a) für alle Werthe der $v_{i}$ $(i=1,2,3)$ befriedigt werden, so würde jede Gerade $v_{x}=0$ der Ebene durch einen Schnittpunkt von $f(x, x)=0$ und $u_{x}=0$ gehen, d. h. die Gerade $u_{x}=0$ würde einen Theil des alsdann ausgearteten Kegelschnitts bilden.

Die Gleichung der Schnittpunkte von $f(x, x)=0$ mit der Seite $x_{1}=0$ des Coordinatendreiecks erhält man aus (53a) durch die Substitution $u_{1}=1, u_{2}=u_{3}=0$ in der Gestalt

$$
a_{33} v_{2}^{2}-2 a_{23} v_{2} v_{3}+a_{22} v_{3}^{2}=0
$$

die Schnittpunkte mit den Seiten $x_{2}=0$ und $x_{3}=0$ sind analog gegeben durch

$$
a_{33} v_{1}^{2}-2 a_{31} v_{1} v_{3}+a_{11} v_{3}^{2}=0 \text { und } a_{22} v_{1}^{2}-2 a_{12} v_{1} v_{2}+a_{11} v_{2}^{2}=0
$$

Die beiden in (53a) enthaltenen Factoren lassen sich übrigens auch einzeln darstellen, und zwar in folgender Weise:

Sind. $s_{1}, s_{2}, s_{3}$ drei willkürlich gewählte Grössen, so besteht, wie in der Determinantentheorie gezeigt wird, die Relation

$$
\left(\begin{array}{l}
u s \\
u s
\end{array}\right)\left(\begin{array}{l}
u v \\
u v
\end{array}\right)=\left(\begin{array}{l}
u \\
u
\end{array}\right)\left(\begin{array}{l}
u s v \\
u s v
\end{array}\right)+\left(\begin{array}{l}
u s \\
u v
\end{array}\right)^{2}
$$

ergeben sich aus dem den Punkten vorausgehenden vollständig angegebenen Gliede durch cyklische Vertanschung der Indices.

1) Die durch „Rändern" mit zwei Reihen von Grössen $u_{i}$ resp. $v_{i}(i=1,2,3)$ aus $A$ hervorgehende Determinante werde kurz durch $\left(\begin{array}{ll}u & v \\ u & v\end{array}\right)$ bezeichnet; wird $A$ auf der einen Seite mit den $u$, auf der anderen mit den $v$ gerändert, so sei dies kurz durch $\left(\begin{array}{l}u \\ v\end{array}\right)$ bezeichnet, während $\left(\begin{array}{lll}u & v & w \\ u & v & w\end{array}\right)$ ausdrückt, dass die Determinante $A$ mit drei Reihen von Grössen $u_{i}, v_{i}, w_{i}(i=1,2,3)$ gerändert ist. Wird eine andere Determinante, z. B. eine aus Elementen $b_{i k}$ znsammengesetzte Determinante gerändert, so werde dies, um Verwechselung zu vermeiden, durch Zufügen des Index $b_{i k}$ bezeichnet; so bedentet z. B. $\left(\begin{array}{l}u \\ u\end{array}\right)_{b_{i k}}$, dass die Determinante der $b_{i k}$ mit je einer Reihe von Elementen $u_{1}, u_{2}, u_{3}$ gerändert ist. 
Schnittpanktepaar einer Curve zweiter Ordnung mit einer Geraden.

da $\operatorname{nun}\left(\begin{array}{l}u s v \\ u s v\end{array}\right)=-\sum \pm\left(u_{1} s_{2} v_{3}\right)^{2}$, so hat man

$$
\begin{aligned}
\left(\begin{array}{l}
u s \\
u s
\end{array}\right)\left(\begin{array}{l}
u \\
u \\
u
\end{array}\right) & =-\left(\begin{array}{l}
u \\
u
\end{array}\right) \sum \pm\left(u_{1} s_{2} v_{3}\right)^{2}+\left(\begin{array}{l}
u s \\
u v
\end{array}\right)^{2} \text { oder auch } \\
\left(\begin{array}{l}
u s \\
u s
\end{array}\right)\left(\begin{array}{l}
u v \\
u v v
\end{array}\right)= & \left\{\left(\begin{array}{l}
u s \\
u v
\end{array}\right)+\sqrt{\left(\begin{array}{l}
u \\
u
\end{array}\right)} \cdot \sum \pm\left(u_{1} s_{2} v_{3}\right)\right\} \\
& \left.\cdot\left\{\left(\begin{array}{l}
u s \\
u v
\end{array}\right)-\sqrt{\left(\begin{array}{l}
u \\
u
\end{array}\right)} \cdot \sum \pm\left(u_{1} s_{2} v_{3}\right)\right\}^{1}\right) .
\end{aligned}
$$

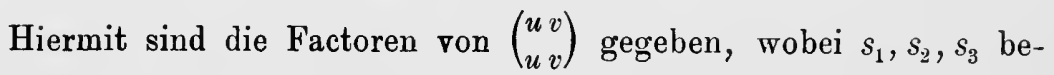
liebige Grössen bezeichnen, die nur den $u_{1}, u_{2}, u_{3}$ nicht proportional sein dürfen.

Eine andere Methode zur Bestimmung der Schnittpunkte einer Geraden und einer Curve zweiter Ordnung ist die folgende ${ }^{2}$ ). Wenn die Curve dargestellt ist durch $f(x, x)=0$ oder nach (6) durch $f_{1} x_{1}+f_{2} x_{2}+f_{3} x_{3}=0$, die Gerade durch $u_{1} x_{1}+u_{2} x_{2}+u_{3} x_{3}=0$, so folgen aus beiden Gleichungen zur Berechnung der Coordinaten $x_{1}, x_{2}, x_{3}$ der Schnittpunkte die drei in den $x$ linearen Gleichungen

$$
\rho x_{1}=f_{2} u_{3}-f_{3} u_{2}, \quad \rho x_{2}=f_{3} u_{1}-f_{1} u_{3}, \quad \rho x_{3}=f_{1} u_{2}-f_{2} u_{1},
$$

in denen $\rho$ einen noch $\mathrm{zu}$ bestimmenden Proportionalitätsfactor bedeutet. Um $\rho$ zu finden, substituiren wir die Werthe (57) in die Gleichung $x_{1} v_{1}+x_{2} v_{2}+x_{3} v_{3}=0$ eines der Schnittpunkte und erbalten so:

$$
\rho\left(x_{1} v_{1}+x_{2} v_{2}+x_{3} v_{3}\right)=\sum \pm\left(f_{1} u_{2} v_{3}\right) \text {. }
$$

Für das Quadrat $\rho^{2}\left(x_{1} v_{1}+x_{2} v_{2}+x_{3} v_{3}\right)^{2}$ folgt hieraus eine Darstellung in Form einer Determinante sechsten Grades

$$
\varrho^{2}\left(x_{1} v_{1}+x_{2} v_{2}+x_{3} v_{3}\right)^{2}=-\left|\begin{array}{llllll}
a_{11} & a_{12} & a_{13} & f_{1} & u_{1} & v_{1} \\
a_{21} & a_{22} & a_{23} & f_{2} & u_{2} & v_{2} \\
a_{31} & a_{32} & a_{33} & f_{3} & u_{3} & v_{3} \\
f_{1} & f_{2} & f_{3} & 0 & 0 & 0 \\
u_{1} & u_{2} & u_{3} & 0 & 0 & 0 \\
v_{1} & v_{2} & v_{3} & 0 & 0 & 0
\end{array}\right| .
$$

Wird hier die erste Verticalreihe mit $x_{1}$, die zweite mit $x_{2}$, die dritte mit $x_{3}$ multiplicirt und werden diese Reihen von der vierten

1) Vgl. Gundelfinger: „Intorno ad alcnne formole della teoria delle curve di secondo e terzo ordine". Annali di Matematica, Serie II, Bd. 5, S. 225. 1872.

2) Diese Methode hat Aronhold gegeben in einer Abhandlung über die Integration irrationaler Differentiale, Journal für die reine und angewandte Mathematik, Bd. 61, S. 103 f., 1862. 
subtrahirt, verfährt man hierauf in gleicher Weise mit den Horizontalreihen und beachtet, dass für die Coordinaten $x_{1}, x_{2}, x_{3}$ eines Schnittpunktes sowohl $f(x, x)$ als $u_{x}$ verschwinden, so wird

$\rho^{2}\left(x_{1} v_{1}+x_{2} v_{2}+x_{3} v_{3}\right)^{2}=-\left|\begin{array}{rrrrrr}a_{11} & a_{12} & a_{13} & 0 & u_{1} & v_{1} \\ a_{21} & a_{22} & a_{23} & 0 & u_{2} & v_{2} \\ a_{31} & a_{32} & a_{33} & 0 & u_{3} & v_{3} \\ 0 & 0 & 0 & 0 & 0 & -v_{x} \\ u_{1} & u_{2} & u_{3} & 0 & 0 & 0 \\ v_{1} & v_{2} & v_{3} & -v_{x} 0 & 0\end{array}\right|=\left(x_{1} v_{1}+x_{2} v_{2}+x_{3} v_{3}\right)^{2}\left(\begin{array}{l}u \\ u\end{array}\right)$

daher

$$
\begin{aligned}
\varrho^{2}=\left(\begin{array}{l}
u \\
u
\end{array}\right)=-\left(A_{11} u_{1}^{2}+2 A_{12} u_{1} u_{2}+A_{22} u_{2}^{2}\right. & +2 A_{13} u_{1} u_{3} \\
& \left.+2 A_{23} u_{2} u_{3}+A_{33} u_{3}^{2}\right)
\end{aligned}
$$

oder

$$
\varrho= \pm \sqrt{-F(u, u)}
$$

wenn zur Abkürzung gesetzt wird

$$
F(u, u) \equiv \sum_{1}^{3} \sum_{1}^{3} A_{i k} u_{i} u_{k} .
$$

Man hat nun die beiden Hauptfälle zu unterscheiden:
1) $\rho \gtrless 0$,
2) $\varrho=0$.

1) Im allgemeineren Falle $\varrho \gtrless 0$ erhält man, entsprechend den zwei verschiedenen Werthen von $\varrho$, mit Hilfe der Gleichungen (57) zwei verschiedene Punkte, die dem Kegelschnitt und der Geraden gemeinsam sind, und zwar sind dieselben reell oder imaginär, je nachdem $F(u, u)$ negativ oder positiv ist $\left.{ }^{1}\right)$.

2) Wenn $\varrho=0$, kann die Gerade $u_{x}=0$ mit der Curve

a) zwei zusammenfallende Punkte,

b) unendlich viele Punkte

gemeinsam haben, ist also entweder (a) eine Tangente der Curve (im eigentlichen oder uneigentlichen Sinne) oder (b), sie bildet einen Theil der Curve.

Die Gleichungen (57) verwandeln sich im Falle 2) in

$$
\frac{f_{1}}{u_{1}}=\frac{f_{2}}{u_{2}}=\frac{f_{3}}{u_{3}} \text {, wobei } f_{i}=\frac{1}{2} f^{\prime}\left(x_{i}\right) \text {, }
$$

1) Sind $u_{1}, u_{2}, u_{8}$ die Coordinaten der Verbindungslinie zweier Punkte $y$ und $z$, so kann man setzen $u_{1}=y_{2} z_{3}-y_{3} z_{2}, u_{2}=y_{3} z_{1}-y_{1} z_{3}, u_{3}=y_{1} z_{2}-y_{2} z_{1}$; nach (32) ist alsdann $f(y, y) \cdot f(z, z)-f^{2}(y, z)=F(u, u)$, daher $f(y, y) \cdot f(z, z)$ $=f^{2}(y, z)+F(u, u)$. Wenn nun die Gerade $u$ die Curve in imaginären Punkten trifft, ist $F(u, u)$, somit auch $f(y, y) \cdot f(z, z)$ positiv, es müssen daher in diesem Falle die Ausdrücke $f(y, y)$ und $f(z, z)$ gleiche Vorzeichen haben. 
und diese zwei Gleichungen ergeben entweder einen einzigen Schnittpunkt oder unendlich viele (die Gerade $u_{x}=0$ selbst).

Im Falle a) setze man $f_{i}=\sigma u_{i}(i=1,2,3)$, wo $\sigma$ einen Proportionalitätsfactor bedeutet, und für beliebige $y_{i}$ wird

$$
f_{1} y_{1}+f_{2} y_{2}+f_{3} y_{3}=\sigma\left(u_{1} y_{1}+u_{2} y_{2}+u_{3} y_{3}\right) \text {. }
$$

$\mathrm{Da}$ die $y_{i}$ beliebig sind, kann man für sie auch die Coordinaten irgend eines auf der Geraden $u_{x}=0$ gelegenen Punktes wählen, also bei völlig willkürlichen Werthen der $w_{i}$ setzen $y_{1}=u_{2} w_{3}-u_{3} v_{2}$, $y_{2}=u_{3} w_{1}-u_{1} w_{3}, y_{3}=u_{1} w_{2}-u_{2} w_{1}$. Die Coordinaten $x_{i}$ des gemeinschaftlichen Punktes der Curve $f(x, x)=0$ und der Geraden $u_{x}=0$ ergeben sich nun aus den zwei linearen Gleichungen:

$$
f^{\prime}\left(x_{1}\right) y_{1}+f^{\prime}\left(x_{2}\right) y_{2}+f^{\prime}\left(x_{3}\right) y_{3}=0 \text { und } u_{1} x_{1}+u_{2} x_{2}+u_{3} x_{3}=0 \text {, }
$$
oder unter Beibehaltung der völlig willkürlichen $w_{i}$ aus:

$$
\sum \pm\left(f^{\prime}\left(x_{1}\right) u_{2} w_{3}\right)=0 \text { und } u_{1} x_{1}+u_{2} x_{2}+u_{3} x_{3}=0
$$

Im Falle b) gehört jeder Punkt der Geraden $u_{x}=0$ dem Kegelschnitt an; da nun ein solcher Punkt Coordinaten von der Form besitzt $x_{1}=u_{2} w_{3}-u_{3} w_{2}, x_{2}=u_{3} w_{1}-u_{1} w_{3}, x_{3}=u_{1} w_{2}-u_{2} w_{1}$ bei völlig willkürlichen Werthen der $w_{i}$, so ergibt sich als nothwendige und ausreichende Bedingung für diesen Fall

$$
\left(\begin{array}{ll}
u & w \\
u & w
\end{array}\right) \equiv 0 \text {, }
$$

wie auch bereits S. 34 auf andere Weise gefunden wurde.

Umgekehrt sagt diese Bedingung aus, dass jeder Punkt von $\imath_{x}=0$ dem Kegelschnitt angebört, also die Gerade einen Theil desselben bildet.

Es werde noch bemerkt, dass man im Falle 2a) für die Coordinaten des gemeinsamen Pưktes aus (64) erhält

$\left.x_{1}: x_{2}: x_{3}=\left(f^{\prime}\left(y_{2}\right) u_{3}-f^{\prime}\left(y_{3}\right) u_{2}\right):\left(f^{\prime}\left(y_{3}\right) u_{1}-f^{\prime}\left(y_{1}\right) u_{3}\right):\left(f^{\prime}\left(y_{1}\right) u_{2}-f^{\prime}\left(y_{2}\right) u_{1}\right){ }^{1}\right)$ die Gleichung $x_{1} v_{1}+x_{2} v_{2}+x_{3} v_{3}=0$ dieses Punktes wird daher

oder auch

$$
\sum \pm\left(f^{\prime}\left(y_{1}\right) u_{2} v_{3}\right)=0
$$

$$
\left|\begin{array}{lllll}
a_{11} & a_{12} & a_{13} & u_{1} & v_{1} \\
a_{21} & a_{22} & a_{23} & u_{2} & v_{2} \\
a_{31} & a_{32} & a_{33} & u_{3} & v_{3} \\
u_{1} & u_{2} & u_{3} & 0 & 0 \\
w_{1} & w_{2} & w_{3} & 0 & 0
\end{array}\right|=0 \text {, d. h. }\left(\begin{array}{c}
u v \\
u w
\end{array}\right)=0
$$

bei völlig willkürlichen Werthen der $w_{i}$.

1) In der ersten Gleichung (64) wurden die $x_{i}$ und $y_{i}$ mit einander vertauscht. 
Sollte der Ausdruck (67) für alle Werthe der $y_{i}$ identisch verschwinden (also auch für solche Werthe, welche $u_{y}=0$ nicht erfüllen), so würde $f(x, x)=0$ mit der Doppelgeraden $u_{x} \cdot u_{x}=0$ identisch sein.

Wir wollen im Anschluss an (66) die wichtige Aufgabe lösen: Wenn $f(x, x)=0$ die Gleichung einer in ein Geradenpaar zerfallenen Curve zweiter Ordnung ist und $u_{x}=0$ die eine Gerade des Paares darstellt, die Gleichung der anderen Geraden zu bilden.

Jedenfalls geht jede Gerade $v_{x}=0$ der Ebene durch einen Schnittpunkt von $f(x, x)=0$ mit $u_{x}=0$ hindurch; die in (53a) abgeleitete Gleichung $\left(\begin{array}{l}u v \\ u v\end{array}\right)=0$ des Schnittpunktepaares wird nun für beliebige Werthe der $v$ erfüllt, und wir können daher setzen $v_{1}=x_{2} y_{3}-x_{3} y_{2}$, $v_{2}=x_{3} y_{1}-x_{1} y_{3}, v_{3}=x_{1} y_{2}-x_{2} y_{1}$, wobei die $y_{i}$ ganz willkürlich sind, jedoch $u_{y}=0$ nicht erfüllen dürfen. Es wird

$$
\begin{aligned}
& a_{11}\left(u_{2} v_{3}-u_{3} v_{2}\right)+a_{12}\left(u_{3} v_{1}-u_{1} v_{3}\right)+a_{13}\left(u_{1} v_{2}-u_{2} v_{1}\right) \\
& \quad=\left|\begin{array}{lll}
a_{11} & a_{12} & a_{13} \\
u_{1} & u_{2} & u_{3}
\end{array}\right|\left|\begin{array}{lll}
x_{1} & x_{2} & x_{3} \\
y_{1} & y_{2} & y_{3}
\end{array}\right|=\frac{1}{2} f^{\prime}\left(x_{1}\right) \cdot u_{y}-\frac{1}{2} f^{\prime}\left(y_{1}\right) \cdot u_{x},
\end{aligned}
$$

also

$$
\left(\begin{array}{l}
u v \\
u v
\end{array}\right)=f(x, x) \cdot u_{y}^{2}-2 f(x, y) \cdot u_{x} \cdot u_{y}+f(y, y) \cdot u_{x}^{2} \equiv 0 .
$$

Hieraus folgt

$$
f(x, x) \cdot u_{y}^{2} \equiv\left\{2 f(x, y) \cdot u_{y}-f(y, y) \cdot u_{x}\right\} u_{x}=0,
$$

womit als Gleichung der zweiten Geraden des Paares $f(x, x)=0$ gefunden ist

$$
2 f(x, y) \cdot u_{y}-f(y, y) \cdot u_{x}=0 .
$$

Besonders einfach wird die Rechnung natürlich, wenn man für die $y_{i}$ die Coordinaten einer Ecke des Coordinatendreiecks wählt; nur ist dabei zu beachten, dass man keine solche Ecke wählt, welche eventuell auf $u_{x}=0$ liegt.

$\S 5$.

\section{Die Curven zweiter Classe.}

Nachdem im vorhergehenden Paragraphen der geometrische Ort aller Punkte untersucht wurde, deren Coordinaten $x_{1}, x_{2}, x_{3}$ einer Gleichung zweiten Grades in $x_{1}, x_{2}, x_{3}$ genügen, wollen wir nun fragen, welche Eigenschaft alle geraden Linien besitzen, deren Coordinaten 
$u_{1}, u_{2}, u_{3}$ einer Gleichung zweiten Grades

$$
\begin{aligned}
\varphi(u, u) \equiv \alpha_{11} u_{1}^{2}+2 \alpha_{12} u_{1} u_{2}+\alpha_{22} u_{2}^{2} & +2 \alpha_{13} u_{1} u_{3} \\
& +2 \alpha_{23} u_{2} u_{3}+\alpha_{33} u_{3}{ }^{2}=0
\end{aligned}
$$

genügen.

Während man sich die Curve $f(x, x)=0$ entstanden denken kann durch Bewegung eines Punktes $x$, der die Curve beschreibt, stellt jedenfalls $\varphi(u, u)=0$ ein Gebilde dar, das umhüllt wird von allen Geraden, deren Coordinaten $u_{i}$ der Gleichung $\varphi(u, u)=0$ genügen. Man nennt dieses Gebilde eine Curve zweiter Classe.

Wird (1) der analogen algebraischen Beghandlung unterworfen wie $f(x, x)=0$ im vorhergehenden Paragraphen, so gelangt man zu Sätzen, die den früheren über Curven zweiter Ordnung entsprechen, es findet ein vollständiger Dualismus statt, indem jedem Satze über Curven zweiter Ordnung "dualistiscll" ein Satz über Curven zweiter Classe entspricht. Es ist demnach auch unnöthig diese dualistischen Sätze besonders abzuleiten, sie gehen aus den frïlieren hervor, indem man dort auftretende Punkte durch gerade Linien und umgekehrt gerade Linien durch Punkte ersetzt. Insbesondere ist die Verbindungslinie zweier Punkte nunmehr zu ersetzen durch den Schnittpunkt zweier Geraden, ein Punkt der Curve $f(x, x)=0$ durch eine Taugente der Curve $\varphi(u, u)=0$; an Stelle der Schnittpunkte einer Geraden mit einer Curve zweiter Ordnung treten die Tangenten, welche von einem Punkte an die Curve zweiter Classe gezogen werden können.

Wie bei Curven zweiter Ordnung sind auch jetzt zwei Hauptfälle zu unterscheiden, je nachdem nämlich die Discriminante

$$
\mathrm{A} \equiv \begin{array}{lll}
\alpha_{11} & \alpha_{12} & \alpha_{13} \\
\alpha_{21} & \alpha_{22} & \alpha_{23} \\
\alpha_{31} & \alpha_{32} & \alpha_{33}
\end{array} \mid, \alpha_{i k}=\alpha_{k i}
$$

von Null verschieden oder gleich Null ist.

Wir behaupten, dass im Falle $A \gtrless 0$ jede Gerade, deren Coordinaten $u_{i}$ die Gleichung $\varphi(u, u)=0$ befriedigen, Tangente des Kegelschnitts ist, dessen Gleichung in Punktcoordinaten lautet:

$$
\begin{gathered}
\Phi(x, x) \equiv \mathrm{A}_{11} x_{1}{ }^{2}+2 \mathrm{~A}_{12} x_{1} x_{2}+\mathrm{A}_{22} x_{2}{ }^{2}+2 \mathrm{~A}_{13} x_{1} x_{3} \\
\left.+2 \mathrm{~A}_{23} x_{2} x_{3}+\mathrm{A}_{33} x_{3}{ }^{2}=0^{1}\right) .
\end{gathered}
$$

Leitet man nämlich die Gleichung in Liniencoordinaten ab, welche

1) Es werde noch bemerkt, dass im Folgenden für das Functionszeichen und die Coefficienten der Gleichung einer gegebenen Curve zweiter Ordnung meistens kleine lateinische Buchstaben, für die Coefficienten der zugehörigen Gleichung in Liniencoordinaten grosse lateinische Buchstaben gewählt werden sollen. Ferner 
der Curve (3) zugehört, so ergibt sich nach (30) in $\S 4$ und mit Benutzung bekannter Relationen aus der Determinantentheorie, wie z. $B$.

$$
A_{22} A_{33}-A_{23}{ }^{2}=A \alpha_{11}, \quad A_{13} A_{23}-A_{12} A_{33}=A \alpha_{12},
$$

u. s. w., das Resultat

$$
\mathrm{A}\left(\alpha_{11} u_{1}^{2}+2 \alpha_{12} u_{1} u_{2}+\alpha_{22} u_{2}^{2}+2 \alpha_{13} u_{1} u_{3}+2 \alpha_{23} u_{2} u_{3}+\alpha_{33} u_{3}^{2}\right)=0
$$

oder $\varphi(u, u)=0$, da $\mathrm{A} \gtrless 0$ vorausgesetzt wurde.

Auf Grund dieser Thatsache und des analogen im vorhergehenden Paragraphen erhaltenen Resultates kann man sagen:

(5) Eine nicht ausartende Curve zweiter Classe kann immer auch betrachtet werden als eine Curve zweiter Ordnung, die nicht ausartet, und umgekehrt.

Im Falle $A=0$ ist uns aus $\S 4$ bekannt, dass der Ausdruck (1) in zwei lineare Factoren zerfallen muss, die von einander verschieden oder einander gleich sind, je nachdem auch nur eine der drei „Hauptunterdeterminanten" $A_{11}, A_{22}, A_{33}$ von Null verschieden ist, oder diese drei gleich Null sind ${ }^{1}$ ). Also

(6) Sobald die Determinante A der Curve zweiter Classe $\varphi(u, u)=0$ verschwindet, zerfällt die Curve in ein Punktepaar, und umgekehrt ${ }^{2}$ ).

(7) Wenn sämmtliche Unterdeterminanten $A_{i k}$ der Determinante $A$ einer Curve zweiter Classe verschwinden, reducirt sich die Curve auf einen doppelt zu zählenden Punkt (Doppelpunkt), und umgekehrt.

Auf Grund des Resultates (5) gelten alle in $\S 4$ für nicht ausartende Curven zweiter Ordnung gefundenen Sätze auch für Curven zweiter Classe.

Weitere Theoreme ergeben sich durch dualistische Uebertragung aus denen von $\S 4$, z. B.:

(8) Eine Curve zweiter Classe ist im allgemeinen durch fünf Tangenten bestimmt; gehen drei derselben durch einen und denselben Punkt, so zerfällt die Curve in ein Punktepaar; der eine Punkt dieses Paares wird unbestimmt, falls vier der Tangenten einem und demselben Büschel angehören.

mögen im allgemeinen kleine griechische Buchstaben bei der Gleichnng einer gegebenen Curve zweiter Classe benutzt werden, grosse griechische Buchstaben bei der zugehörigen Gleichnng in Punktcoordinaten.

1) Das Verschwinden dieser drei hat ja im Falle $A=0$ das Verschwinden aller $A_{i k}$ zur Folge.

2) In dem zu vorliegendem Paragraphen gehörigen Theil des Anhangs sind die beiden Punkte einzeln dargestellt. 
Dem Satze (9) in $\S 4$ entspricht:

(9) An jede Curve zweiter Classe lassen sich von einem beliebigen Punkte der Ebene im allgemeinen zwei Tangenten legen ${ }^{1}$ ).

Ist dieser Punkt der Schnitt zweier beliebigen Geraden mit den Coordinaten $u_{i}$ resp. $v_{i}(i=1,2,3)$, so sind die beiden Tangenten reell, wenn $\varphi^{2}(u, v)-\varphi(u, u) \cdot \varphi(v, v)>0$, sie sind imaginär, weln $\varphi^{2}(u, v)-\varphi(u, u) \cdot \varphi(v, v)<0$; sie fallen zusammen, wenn

$$
\varphi^{2}(u, v)-\varphi(u, u) \cdot \boldsymbol{\varphi}(v, v)=0 \text {. }
$$

Dabei ist $\varphi(u, v)$ definirt durch

$$
\begin{aligned}
\varphi(u, v) \equiv\left(\alpha_{11} u_{1}+\alpha_{12} u_{2}+\alpha_{13} u_{3}\right) v_{1}+\left(\alpha_{21} u_{1}+\alpha_{22} u_{2}+\alpha_{23} u_{3}\right) v_{2} \\
\quad+\left(\alpha_{31} u_{1}+\alpha_{32} u_{2}+\alpha_{33} u_{3}\right) v_{3} \\
\equiv \frac{1}{2}\left(v_{1} \varphi^{\prime}\left(u_{1}\right)+v_{2} \varphi^{\prime}\left(u_{2}\right)+v_{3} \varphi^{\prime}\left(u_{3}\right)\right) \\
\equiv \frac{1}{2}\left(u_{1} \varphi^{\prime}\left(v_{1}\right)+u_{2} \varphi^{\prime}\left(v_{2}\right)+u_{3} \varphi^{\prime}\left(v_{3}\right)\right)
\end{aligned}
$$

da $\alpha_{i k}=\alpha_{k i}$.

Nehmen wir an, die Gerade $v$ liege fest, während $u$ variabel ist, so stellt

$$
\varphi^{2}(u, v)-\varphi(u, u) \cdot \varphi(v, v)=0
$$

die Bedingung dar, der die $u_{i}$ genügen müssen, damit die Gerade $u_{x}=0$ durch einen der Geraden $v$ und der Curve $\varphi(u, u)=0$ gemeinsamen Punkt gehe, d. h. (11) ist die Gleichung des Schnittpunktepaars von $u_{x}=0$ mit der Curve und zwar eines Paares, weil (4) in den $u$ vom zweiten Grade ist. Da $v_{x}=0$ eine beliebig gewählte Gerade ist, kann man auch sagen: es stellt (11) die Bedingung dar, dass $u_{x}=0$ und $v_{x}=0$ sich in einem Punkte der Curve (1) schneiden. Sind

$$
x_{1}=u_{2} v_{3}-u_{3} v_{2}, x_{2}=u_{3} v_{1}-u_{1} v_{3}, x_{3}=u_{1} v_{2}-u_{2} v_{1}
$$

die Coordinaten des Punktes, so müssen sich diese in (11) einführen lassen, und kann alsdann (11) von der Gleichung der Curve $\varphi(u, u)=0$ in Punktcoordinaten nur um einen Zahlenfactor verschieden sein. In der That findet man

$$
\begin{gathered}
\varphi(u, u) \cdot \varphi(v, v)-\varphi^{2}(u, v) \\
\equiv \mathrm{A}_{11} x_{1}{ }^{2}+2 \mathrm{~A}_{12} x_{1} x_{2}+\mathrm{A}_{22} x_{2}{ }^{2}+2 \mathrm{~A}_{13} x_{1} x_{3}+2 \mathrm{~A}_{23} x_{2} x_{3}+\mathrm{A}_{33} x_{3}{ }^{2} \\
=-\left|\begin{array}{llll}
\alpha_{11} & \alpha_{12} & \alpha_{13} & x_{1} \\
\alpha_{21} & \alpha_{22} & \alpha_{23} & x_{2} \\
\alpha_{31} & \alpha_{32} & \alpha_{33} & x_{3} \\
x_{1} & x_{2} & x_{3} & 0
\end{array}\right|
\end{gathered}
$$

wobei die $x_{i}$ durch (12) definirt sind.

1) Der Fussnote 2) zu S. 20 entsprechend wäre zu erwähnen, dass man an eine 
Den Sätzen (16)-(19) in $\S 4$ entsprechen dualistisch die folgenden $(14)-(17)$ :

(14) Nimmt man auf einer Geraden $v$ beliebig viele Punkte an und construirt man den durch irgend einen dieser. Punkte gehenden vierten harmonischen Strahl zu $v$ und zu den beiden Tangenten, die vom Punkte an eine Curve zweiter Classe gezogen werden können, so gehen alle diese vierten harmonischen Strahlen durch einen und denselben Punkt, den Pol der Geraden $v$. Seine Gleichung ist $\varphi(u, v)=0$.

(15) Dreht sich eine Gerade um einen auf ihr gelegenen Punkt, beschreibt sie also ein Strahlenbüschel, so durchläuft der in Bezug auf eine Curve zweiter Classe genommene Pol der Geraden eine zum Strahlenbüschel projective Punktreihe ${ }^{1}$ ).

(16) Der Pol einer Geraden $v$ liegt auf den beiden Tangenten, welche in den der Geraden und der Curve zweiter Classe gemeinsamen Punkten gezogen werden können, er ist also der Schnittpunkt dieser beiden Tangenten.

(17) Der Pol einer Tangente $v$ der Curve $\varphi(u, u)=0$ fällt mit dem Berührungspunkt dieser Tangente zusammen, seine Gleichung ist $\varphi(u, v)=0$.

Den Formeln (26) in $\S 4$ für die Coordinaten der Polare des Punktes $y$ entsprechen folgende Formeln für die Coordinaten $x_{i}$ des Pols einer Geraden $v$ in Bezug auf $\varphi(u, u)=0$ :

$$
\left\{\begin{array}{l}
\varrho x_{1}=\alpha_{11} v_{1}+\alpha_{12} v_{2}+\alpha_{13} v_{3}=\frac{1}{2} \varphi^{\prime}\left(v_{1}\right) \\
\varrho x_{2}=\alpha_{21} v_{1}+\alpha_{22} v_{2}+\alpha_{23} v_{3}=\frac{1}{2} \varphi^{\prime}\left(v_{2}\right) \\
\varrho x_{3}=\alpha_{31} v_{1}+\alpha_{32} v_{2}+\alpha_{33} v_{3}=\frac{1}{2} \varphi^{\prime}\left(v_{3}\right) .
\end{array}\right.
$$

Hiernach gehört zwar zu jeder Geraden $v$ bei Curven zweiter Classe ein ganz bestimmter Pol $x$, dagegen zu jedem Punkte $x$ nur dann eine bestimmte Polare $v$, wenn sich die Gleichungen (18) ein-

Curve, die durch eine Gleichung $n^{\text {ten }}$ Grades in $u_{1}, u_{2}, u_{3}$ gegeben ist, an eine sogenannte Curve $n^{\text {ter }}$ Classe, im allgemeinen von einem Punkte aus $n$ Tangenten ziehen kann. Deshalb wird sie eben von der $n^{\text {ten }}$ Classe genannt.

1) Die Fassung dieses Satzes muss etwas modificirt werden, wenn der Kegelschnitt ein Punktepaar ist, auf dessen Träger das Centrum des Strablenbüschels liegt; sämmtliche Punkte der Punktreibe fallen dann offenbar zusammen mit dem vierten harmonischen Punkte zu dem gegebenen Paare und dem Centrum des Strahlenbüschels. Dieser vierte harmonische Punkt ist jetzt der Pol eines jeden Strahles im Büschel. 
deutig nach den $v_{i}$ auflösen lassen, also wenn $\mathrm{A} \gtrless 0$. Bei nicht ausartenden Kegelschnitten ist das Verhältniss zwischen Pol und Polare überhaupt ein völlig eindeutiges. Daraus und aus den Bemerkungen S. 25 über den Mittelpunkt $z$ einer Curve zweiter Ordnung geht hervor, dass derselbe auch angesehen werden kann als der Pol ${ }^{1}$ ) der unendlich fernen Geraden in Bezug auf den Kegelschnitt, und es möge dies auch für zerfallende Curven zweiter Classe gelten, da bei diesen jeder Geraden der Ebene eindeutig ein Pol zugehört.

Die Coordinaten der unendlich fernen Geraden sind nun die durch (7) und (5) in $\S 1$ definirten Grössen $p_{i}=\frac{e_{i}}{h_{i}}(i=1,2,3)$; daher findet man durch Auflösung von (26) in $\S 4$, wenn daselbst $u_{i}$ durch $p_{i}, y_{i}$ durch $z_{i}$ ersetzt wird, für den Mittelpunkt $z$ einer nicht ausartenden Curve zweiter Ordnung $f(x, x) \equiv \sum_{1}^{3} \sum_{1}^{3} a_{i k} x_{i} x_{k}=0 \quad$ die Coordinaten

$$
\left\{\begin{array}{l}
\sigma z_{1}=A_{11} p_{1}+A_{12} p_{2}+A_{13} p_{3}=\frac{1}{2} F^{\prime}\left(p_{1}\right) \\
\sigma z_{2}=A_{21} p_{1}+A_{22} p_{2}+A_{23} p_{3}=\frac{1}{2} F^{\prime}\left(p_{2}\right) \\
\sigma z_{3}=A_{31} p_{1}+A_{32} p_{2}+A_{33} p_{3}=\frac{1}{2} F^{\prime}\left(p_{3}\right)
\end{array}\right.
$$

wobei $\sigma$ ein Proportionalitätsfactor, während sich für die Coordinaten des Mittelpunktes einer beliebigen Curve zweiter Classe

$$
\varphi(u, u) \equiv \sum_{1}^{3} \sum_{1}^{3} \alpha_{i k} u_{i} u_{k}=0
$$

aus (18) in vorliegendem Paragraphen direct die Werthe ergeben

$$
\left\{\begin{array}{l}
\varrho z_{1}=\alpha_{11} p_{1}+\alpha_{12} p_{2}+\alpha_{13} p_{3}=\frac{1}{2} \varphi^{\prime}\left(p_{1}\right) \\
\varrho z_{2}=\alpha_{21} p_{1}+\alpha_{22} p_{2}+\alpha_{23} p_{3}=\frac{1}{2} \varphi^{\prime}\left(p_{2}\right) \\
\varrho z_{3}=\alpha_{31} p_{1}+\alpha_{32} p_{2}+\alpha_{33} p_{3}=\frac{1}{2} \varphi^{\prime}\left(p_{3}\right) .
\end{array}\right.
$$

Wir wollen nun untersuchen, was für eine Bedeutung die Gleichung in Liniencoordinaten $F(u, u) \equiv \sum_{i}^{3} \sum_{1}^{3} A_{i k} u_{i} u_{k}=0$ einer Curve zweiter Ordnung $f(x, x)=0$ besitzt, falls die Determinante $A$ der Curve verschwindet. Nach (34) in $\S 4$ stehen in diesem Falle die

1) Vgl. für den Fall einer Mittelpunktslinie die Fussnote zu S. 23. 
$A_{i k}$ in einfachem Zusammenhange mit den Coordinaten $\eta_{i}$ der Spitze des Geradenpaars, das von $f(x, x)=0$ dargestellt wird, der Art dass $A_{i k}$ proportional ist zu $\eta_{i} \eta_{k}$. Die Substitution dieser Werthe der $A_{i k}$ in $F(u, u)=0$ liefert

also:

$$
\left(\eta_{1} u_{1}+\eta_{2} u_{2}+\eta_{3} u_{3}\right)^{2}=0
$$

(21) Bildet man für einen in ein Geradenpaar ausgearteten Kegelschnitt die Gleichung in Liniencoordinaten, so repräsentirt dieselbe doppelt zählend die Spitze des Geradenpars. - Dualistisch folgt:

(22) Bildet man für einen in ein Punktepaar ausgearteten Kegelschnitt die Gleichung in Punktcoordinaten, so repräsentirt dieselbe doppelt zählend den Träger des Punktepaars.

Wenn ferner $f(x, x)=0$ eine doppelt zu zählende Gerade darstellt, kann natürlich von einer zugehörigen Gleichung in Liniencoordinaten $F(u, u)=0$ überhaupt nicht mehr die Rede sein, da alle $A_{i k}$ in diesem Falle gleich Null sind; analoges gilt, wenn $\varphi(u, u)=0$ einen Doppelpunkt darstellt, es gibt alsdann keine zugehörige Gleichung in Punktcoordinaten.

Den conjugirten Polen bei Curven zweiter Ordnung entsprechen dualistisch die conjugirten oder harmonischen Polaren bei Curven zweiter Classe: zwei gerade Linien, die harmonisch liegen zu den von ihrem Schnittpunkt an die Curve gezogenen Tangenten. Die Bedingung, dass zwei gerade Linien mit den Coordinaten $u_{i}$ resp. $v_{i}$ $(i=1,2,3)$ conjugirte Polaren seien in Bezug auf die Curve $\varphi(u, u)=0$, wird offenbar $\varphi(u, v)=0$.

Drei Geraden, von denen je zwei harmonische Polaren in Bezug auf dieselbe Curve zweiter Classe sind, bestimmen ein Dreiseit, das man als Poldreiseit bezeichnet.

Für conjugirte Polaren gelten. Sätze, die den früheren (48)-(50) und (52) in § 4 über conjugirte Pole dualistisch entsprechen. Es werde nur der folgende besonders hervorgehoben:

(23) Die einzelnen Paare conjugirter Polaren, die demselben Strahlenbüschel angehören, bilden eine Involution; die Doppelstrahlen der Involution bestehen aus denjenigen Geraden des Büschels, welche zugleich Tangenten der Curve zweiter Classe sind.

Ein wichtiges Beispiel bilden die bereits im vorhergehenden Paragraphen erwähnten Paare conjugirter Durchmesser; jedes Paar derselben liegt harmonisch zu den beiden Tangenten, die man vom Mittelpunkt der Curve ziehen kann und deren Berührungspunkte auf der 
Polare des Mittelpunktes, d.h. auf der unendlich fernen Geraden liegen. Man bezeichnet diese zwei Tangenten speciell als Asymptoten der Curre. Für sie gilt daher der Satz:

(24) Jedes Paar conjugirter Durchmesser eines Kegelschnitts liegt harmonisch zum Asymptotenpar.

Es möge nun noch die Gleichung dieses Asymptotenpaares für eine Curve zweiter Ordnung $f(x, x)=0$ abgeleitet werden. Zuvor werde die allgemeinere Aufgabe gelöst:

Die Gleichung des Tangentenpares aufustellen, das in den Schnittpunkten der Curve zweiter Ordnung $f(x, x)=0$ und der Geraden $u_{x}=0$ gezogen werden kann.

Die Coordinaten $y_{i}$ des Pols dieser Geraden sind nach Früherem proportional zu $\frac{1}{2} F^{\prime}\left(u_{i}\right)$, und diese Werthe wären nun an Stelle von $y_{i}$ einzusetzen in $f(x, x) \cdot f(y, y)-f^{2}(x, y)=0$. Hierdurch verwandelt sich $f(x, y)$ lediglich in $A\left(u_{1} x_{1}+u_{2} x_{2}+u_{3} x_{3}\right)$, indem alle übrigen Glieder von $f(x, y)$ wegfallen auf Grund des bekannten Determinantensatzes, dass die Summe der Producte aus den Elementen einer Reihe und den Unterdeterminanten der Elemente einer zweiten Reihe verschwindet oder gleich der Determinante selbst ist, je nachdem die zweite Reihe von der ersten verschieden ist oder nicht. Ferner wird $f(y, y)=A F(u, u)$, daher verwandelt sich $f(x, x) \cdot f(y, y)-f^{2}(x, y)=0$ in $A\left\{F(u, u) \cdot f(x, x)-A \cdot u_{x}^{2}\right\}=0$. Da bei nicht zerfallenden Kegelschnitten $A \gtrless 0$, bleibt

$$
F(u, u) \cdot f(x, x)-A \cdot u_{x}^{2}=0
$$

als Gleichung des Tangentenpaares.

Tritt speciell an die Stelle der Geraden $u_{x}=0$ die unendlich ferne Gerade $p_{x}=0$, so erhält man die Gleichung. des Asymptotenpaares in der Form:

$$
F(p, p) \cdot f(x, x)-A \cdot p_{x}^{2}=0 .
$$

Zum Schlusse werde noch bemerkt, dass dualistisch zu (53a) in § 4 nunmehr

$$
\left(\begin{array}{l}
y x \\
y x
\end{array}\right)_{\alpha_{i k}}=0
$$

das Tangentenpaar darstellt, welches vom Punkte $y$ an die Curve $\varphi(u, u) \equiv \sum_{i}^{3} \sum_{i}^{3} k \alpha_{i k} u_{i} u_{k}=0$ gezogen werden kann. 


\section{$\S 6$. \\ Classification der Kegelschnitte.}

Aus den Betrachtungen der beiden vorhergehenden Paragraphen geht hervor, dass man sowohl bei Curven zweiter Ordnung als auch bei solchen der zweiten Classe zwei Hauptarten zu unterscheiden hat: die eigentlichen, bei denen die Discriminante von Null verschieden ist, und die zerfallenden mit verschwindender Discriminante. Man theilt die Kegelschnitte noch weiter ein nach ihren Schnittpunkten mit der unendlich fernen Geraden, je nachdem nämlich diese Schnittpunkte von einander verschieden sind oder zusammenfallen; auch die Realität der Schnittpunkte ist zu berücksichtigen. Die Kriterien für die einzelnen Fälle ergeben sich leicht im Anschluss an die in $\S 4$ ausführlich behandelte Methode zur Bestimmung der Schnittpunkte einer Geraden und eines Kegelschnitts.

Es werde begonnen mit

\section{A) Curven zweiter Ordnung.}

Zunächst werde vorausgesetzt, dass die Curve $f(x, x)=0$ nicht zerfalle, mithin $A \geqslant 0$ sei. Wir schneiden die Curve mit der unendlich fernen Geraden

$$
p_{1} x_{1}+p_{2} x_{2}+p_{3} x_{3}=0,
$$

deren Coordinaten $p_{i}$ nach (5) in $\S 1$ die Werthe haben

$$
p_{1}=\frac{e_{1}}{h_{1}}, \quad p_{2}=\frac{e_{2}}{h_{2}}, \quad p_{3}=\frac{e_{3}}{h_{3}} \text {. }
$$

Zur Bestimmung der Coordinaten der Schnittpunkte dieser Geraden mit der Curve

(3) $f(x, x) \equiv a_{11} x_{1}{ }^{2}+2 a_{12} x_{1} x_{2}+a_{22} x_{2}{ }^{2}+2 a_{13} x_{1} x_{3}+2 a_{23} x_{2} x_{3}+a_{33} x_{3}{ }^{2}=0$ ist dann auf Grund der Resultate von $\S 4$ die Grösse

$$
\varrho= \pm \sqrt{-F(p, p)}
$$

zu substituiren in die Gleichungen

(5) $\quad \varrho x_{1}=f_{2} p_{3}-f_{3} p_{2}, \quad \varrho x_{2}=f_{3} p_{1}-f_{1} p_{3}, \quad \varrho x_{3}=f_{1} p_{2}-f_{2} p_{1}$.

Die Schnittpunkte werden daher

$$
\begin{gathered}
\text { reell, wenn } F(p, p)<0 \text { (Hyperbel), } \\
\text { imaginär, wenn } F(p, p)>0 \text { (Ellipse), }
\end{gathered}
$$

sie fallen zusammen, weun $F(p, p)=0$ (Parabel).

Die drei diesen Fällen entsprechenden Kegelschnitte bezeichnet 
man resp. als Hyperbel, Ellipse, Parabel; letztere wird von der unendlich fernen Geraden berührt.

Was den Fall $F(p, p)>0$ betrifft, so kann es hier vorkommen, dass nicht nur die unendlich ferne Gerade, sondern überhaupt jede Gerade der Ebene den Kegelschnitt in imaginären Punkten trifft, die Curve ist alsdann imaginär, und es wäre nun zu untersuchen, unter welcher Bedingung für die Coefficienten der Gleichung $f(x, x)=0$ dies eintritt.

Zu dem Zweck wählen wir auf der unendlich fernen Geraden einen beliebigen Punkt $y$; seine Polare $f(y, x)=0$ ist nach $\S 4$ ein Durchmesser des Kegelschnitts, und wenn die Curve auch imaginär sein sollte, ist die Polare jedenfalls eine Gerade, die sich um den zu $y$ conjugirten Pol (Mittelpunkt) dreht, also die ganze Ebene überstreicht, wenn $y$ die unendlich ferne Gerade durchläuft. Schneidet keine dieser unendlich vielen Polaren die Curve in reellen Punkten, so hat daher die Curve überhaupt keine reellen Punkte; die Bedingung hierfür ist nach $(60)$ in $\S 4$, dass $F(u, u)>0$ sei, wo nun

$$
u_{i}=a_{i 1} y_{1}+a_{i 2} y_{2}+a_{i 3} y_{3} \quad(i=1,2,3)
$$

die Coordinaten der Polare bedeuten. Aus dem in Form der Determinante - $\left(\begin{array}{l}u \\ u\end{array}\right)$ in $\S 4$ angegebenen Werthe von $F(u, u)$ ist nun ersichtlich, dass sich diese Grösse bei Einführung der $u_{i}$ verwandelt in $A f(y, y)$, man braucht nur die drei ersten Verticalreihen jener Determinante zu multipliciren resp. mit $y_{1}, y_{2}, y_{3}$ und von der vierten Reihe zu subtrahiren. Als Bedingung dafür, dass unsere Curve imaginär (eine imaginäre Ellipse) sei, tritt demnach zu $F(p, p)>0$ noch hinzu

$$
A f(y, y)>0
$$

wo $y_{i}(i=1,2,3)$ die Coordinaten eines im Unendlichen gelegenen Punktes bezeichnen. Wenn diese Ungleichung für irgend einen bestimmten Punkt $y$ der unendlich fernen Geraden $p_{x}=0$ erfüllt wird, findet sie auch für jeden anderen Punkt $z$ dieser Geraden statt, denn $f(y, y)$ und $f(z, z)$ haben zufolge der Fussnote zu S. 36 gleiches Vorzeichen, wenn die Gerade $y z$ die Curve in imaginären Punkten trifft. Zugleich erkennt man, dass $A f(y, y)<0$ in Verbindung mit $F(p, p)>0$ das Kriterium für eine reelle Ellipse abgibt. Uebrigens ist aus der soeben durchgeführten Betrachtung ersichtlich, dass im Falle der imaginären Curve das Product $A f(y, y)$ für jeden beliebigen Punkt $y$ der Ebene positiv wird. Doch ist es bei Untersuchung der Realität einer durch $f(x, x)=0$ gegebenen Curve nöthig, einen unendlich 
fernen Punkt $y$ zu wählen, weil bei Annahme eines ganz beliebigen Punktes $y$ der Ausdruck $A f(y, y)$ im Fall der reellen Ellipse verschiedene Vorzeichen hat, je nachdem der Punkt $y$ im Innern der Curve oder ausserhalb derselben liegt; denn beim Ueberschreiten der Curve muss ein Wechsel des Vorzeichens stattfinden.

Der Ausdruck $A \cdot f(y, y)$ kann noch in eine andere wichtige Gestalt gebracht werden; die Coordinaten eines auf der unendlich fernen Geraden gelegenen Punktes sind nämlich von der Form

$$
\sigma y_{1}=p_{2} v_{3}-p_{3} v_{2}, \quad \sigma y_{2}=p_{3} v_{1}-p_{1} v_{3}, \quad \sigma y_{3}=p_{1} v_{2}-p_{2} v_{1},
$$

wobei die $v_{i}$ völlig willkürliche Zahlenwerthe besitzen, nur nicht den $p_{i}$ proportional sein dürfen. Daher kann das Kriterium $A f(y, y) \gtrless 0$ auch ersetzt werden durch $\sigma^{2} A\left(\begin{array}{l}v p \\ v p\end{array}\right) \gtrless 0$, was hier gleichbedeutend ist mit

$$
A\left(\begin{array}{l}
v p \\
v p
\end{array}\right) \gtrless 0 \text {. }
$$

Man kann beispielsweise $v_{1}=v_{3}=0, v_{2}=1$ setzen, wodurch sich $A\left(\begin{array}{l}v p \\ v p\end{array}\right)$ verwandeln würde in $A\left(a_{11} p_{3}{ }^{2}+a_{33} p_{1}{ }^{2}-2 a_{13} p_{1} p_{3}\right)$, und eine weitere Vereinfachung würde eintreten, wenn statt der eigentlichen Dreieckscoordinaten homogene Parallelcoordinaten zu Grunde liegen. Alsdann wären etwa, wie am Schlusse von $\S 1$ gezeigt wurde, die Coordinaten $p_{1}$ und $p_{2}$ der unendlich fernen Geraden gleich Null, $p_{3}=1$, und der obige Ausdruck würde sich reduciren auf $A \cdot a_{11}$. Bei allgemeinen Dreieckscoordinaten dürfte die Rechnung am kürzesten sein bei Benutzung von $A f(y, y)$, wobei $y_{1}, y_{2}, y_{3}$ Zahlen bedeuten, die lediglich der Bedingung $p_{1} y_{1}+p_{2} y_{2}+p_{3} y_{3}=0$ genügen müssen, im übrigen aber völlig willkürlich gewählt sind.

Man kann noch die Frage aufwerfen, ob z. B. in der als Hyperbel bezeichneten Curvengattung nicht noch andere Kegelschnitte enthalten sind, die zwar gleichfalls die unendlich ferue Gerade in zwei verschiedenen reellen Punkten treffen, aber von einander wesentlich verschiedene Eigenschaften und Gestalten besitzen; und die analoge Frage wäre aufzuwerfen bei den als Ellipse und Parabel bezeichneten Curvengattungen.

Zur Entscheidung dieser Frage führen wir ein specielles Coordinatensystem ein.

Bei der Hyperbel ist das Tangentenpaar $T . T_{1}$ der Curve in ihren Schnittpunkten mit der unendlich fernen Geraden $p_{x}=0$ (das sogenannte Asymptotenpaar) reell und hat nach (26) in $\S 5$ die Gleichung 


$$
F(p, p) \cdot f(x, x)-A \cdot p_{x}^{2} \equiv T \cdot T_{1}=0 ;
$$

hieraus folgt, dass $f(x, x)=0$ in die Form gebracht werden kann T. $T_{1}-c \cdot p_{x}^{2}=0$, wo $c$ eine Constante darstellt, auf die es im Folgenden nicht weiter ankommt. Durch die Substitution: $T: p_{x}=x$, $T: p_{x}=y^{1}$ ) führen wir nun ein System schiefwinkliger Parallelcoordinaten ein und erhalten dann für die Hyperbel die Gleichung

$$
x \cdot y=\text { const., }=c,
$$

deren geometrische Deutung aussagt:

(10) Zieht man durch einen beliebigen Punkt der Hyperbel Parallelen zu den zwei Asymptoten, so hat das so entstehende Parallelogramm denselben Inhalt, wo der Punkt auch auf der Hyperbel liegen mag.

Durch diese Eigenschaft $y=c: x$ wird also eine ganz bestimmte Curvengestalt (und nicht mehrere) charakterisirt.

In analoger Weise zeigt man, dass auch die als Ellipse bezeichnete Curvengattung nur eine Form enthält. Das Tangentenpaar in den unendlich fernen Punkten der Curve ist jetzt imaginär, es wird daher $T T_{1}$ von der Form $X_{1}{ }^{2}+X_{2}{ }^{2}$, und zwar kann $T T_{1}$ auf unendlich viele verschiedene Arten in diese Form gebracht werden, denn auch wenn $X_{1}$ und $X_{2}$ ersetzt werden durch $X_{1} \cos \alpha-X_{2} \sin \alpha$, resp. $X_{1} \sin \alpha+X_{2} \cos \alpha$, wird die Summe der Quadrate dieser beiden Grössen gleich $X_{1}{ }^{2}+X_{2}{ }^{2} \cdot{ }^{2}$ ) Wenn bei Herstellung der Normalform (vgl. S. 10) die Gleichungen der Geraden $X_{1}=0$ und $X_{2}=0$ übergehen in $a X_{1}=0$, resp. $b X_{2}=0$ und man setzt $\frac{a X_{1}}{p_{x}}=x \sin w$, $\frac{b X_{2}}{p_{x}}=y \sin w$, so tritt an Stelle von $f(x, x) \equiv X_{1}{ }^{2}+X_{2}{ }^{2}-c p_{x}{ }^{2}=0$ eine Gleichung von der Form (vgl. auch (51) und (52) in $\S 4$, wemn $\left.p_{x}=X_{3}\right)$ : oder

$$
x^{2}+\frac{y^{2}}{a^{2}}=1
$$

$$
\frac{x^{2}}{a^{2}}+\frac{y^{2}}{b^{2}}=-1
$$

wenn man die noch auftretende Constante $c$ in den Nenner der linken Seite eingehen lässt. Sehen wir von dem Fall der sogenannten ima-

1) Wir denken uns die etwa hinzuzufügenden constanten Factoren in $T$ und $T_{1}$ eingegangen.

2) Es sei noch darauf hingewiesen, dass man $\operatorname{tg} \alpha$ so bestimmen kann, dass die zwei Geraden $X_{1} \cos \alpha-X_{2} \sin \alpha=0$ und $X_{1} \sin \alpha+X_{2} \cos \alpha=0$ zu einander normal sind. 
ginären Ellipse (11a) ab, so kann man durch passende Bestimmung von $\operatorname{tg} \alpha$ bewirken, dass (11) sogar in die Form gebracht werden kann $x^{2}+y^{2}=C^{2}$, worin offenbar $C$ die gemeinsame Länge der sogenannten gleichen conjugirten Durchmesser bedeutet. Indęm man also diese letzteren als Coordinatenaxen zu Grunde legt, zeigt sich auch die Ellipse (wie die Hyperbel) nur von der einen Constante $C$ abhängig und charakterisirt sich (vgl. § 11) durch Auflösung nach y als ein in sich geschlossenes Oval, mit dem Coordinatenanfang als Mittelpunkt.

Im Falle der als Parabel bezeichneten Curvengattung fixiren wir auf der Curve einen beliebigen Punkt und ziehen in ihm die Tangente $T=0$, welche die unendlich ferne Gerade in einem Punkte $y$ schneiden möge, so dass das von $y$ nach der Curve gezogene Tangentenpaar aus $T=0$ und aus $p_{x}=0$ besteht. Es gibt demnach zufolge $(10)$ in $\S 4$ eine Relation von der Form $f^{2}(x, y)-f(x, x) \cdot f(y, y) \equiv T \cdot p_{x}$, d. h. $f(x, x)=0$ wird identisch mit $f^{2}(x, y)-T \cdot p_{x}=0$. Führt man auch hier schiefwinklige Parallelcoordinaten ein durch die Substitution $\frac{f(y, x)}{p_{x}}=y, \frac{T}{p_{x}}=c \cdot x$, so erhält man

$$
y^{2}-c x=0,
$$

also wieder nur eine bestimmte Curvengestalt ${ }^{1}$ ).

Betrachten wir nun die zerfallenden Curven zweiter Ordnung $(A=0)$.

Zunächst werde der Fall berücksichtigt, dass nicht alle Unterdeterminanten $A_{i k}$ verschwinden, dass also zwei verschiedene Geraden vorliegen, und zwar möge vorläufig noch vorausgesetzt werden, dass keine derselben mit der unendlich fernen Geraden zusammenfällt. Ferner kann die Forderung, dąss nicht alle $A_{i k}$ verschwinden sollen, auf Grund der Fussnote zu S. 27 durch die einfachere ersetzt werden, dass nicht alle Hauptunterdeterminanten $A_{11}, A_{22}, A_{33}$ verschwinden; auch werde daran erinnert, dass im Falle $A=0$ die nicht verschwindenden Hauptunterdeterminanten gleiches Vorzeichen besitzen.

Man hat alsdann wieder die drei Möglichkeiten

$$
\boldsymbol{F}(p, p)<0, \quad \boldsymbol{F}(p, p)>0, \quad \boldsymbol{F}(p, p)=0,
$$

denen resp. entsprechen:

ein reelles Geradenpaar, ein imaginäres Geradenpaar

(jedesmal mit einem im Endlichen gelegenen Schnittpunkt), zwei Parallelen.

1) Die nähere Discussion der drei Curvenspecies: Hyperbel,- Ellipse und Parabel erfolgt in $\S 11$. 
Um zu entscheiden; ob die Parallelen reell oder imaginär sind, schneidet man dieselben mit einer beliebigen im Endlichen gelegenen Geraden $u_{x}=0$. Für $F(u, u)>0$ hat man alsdann zwei imaginäre, für $F(u, u)<0$ zwei reelle Parallelen, während $F(u, u)=0$ noch keine Entscheidung liefert. Man nimmt, wenn $F(u, u)=0$, noch die Seiten des Coordinatendreiecks zu Hilfe, die man überhaupt praktischer Weise am besten als schneidende Geraden $u_{x}=0$ wähIt. Schneidet man mit $x_{i}=0$, so reducirt sich $F(u, u)$ auf $A_{i i} u_{i}{ }^{2}$, es liefert daher jede nicht verschwindende Hauptunterdeterminante die Entscheidung. Das Parallelenpaar ist demnach reell oder imaginär, je nachdem irgend eine der drei Hauptunterdeterminanten $A_{i i}$ negativ oder positiv ist ${ }^{1}$ ).

Verschwinden $A$ und alle Hauptunterdeterminanten $A_{i i}$, so stellt $f(x, x)=0$ eine Doppelgerade dar.

Bei den bisherigen Betrachtungen war angenommen, dass die unendlich ferne Gerade keinen Theil des Kegelschnitts $f(x, x)=0$ bilde. Sollte eine Gerade des Geradenpaares $f(x, x)=0$ mit der unendlich fernen Geraden zusammenfallen, so besteht zufolge der Betrachtungen am Schlusse von $\S 4$ bei völlig willkürlichen Werthen der $w_{i}(i=1,2,3)$ die Relation

$$
\left(\begin{array}{l}
p w \\
p w
\end{array}\right) \equiv 0
$$

B) Curven zweiter Classe.

Auch bei Curven zweiter Classe hat man zunächst zwei Hauptarten zu unterscheiden: die eigentlichen, für welche die Determinante der Curvengleichung nicht verschwindet, und die in ein Punktepaar ausartenden mit verschwindender Determinante. Es sei

$$
\begin{aligned}
\varphi(u, u) \equiv \alpha_{11} u_{1}^{2}+2 \alpha_{12} u_{1} u_{2}+\alpha_{22} u_{2}^{2} & +2 \alpha_{13} u_{1} u_{3} \\
& +2 \alpha_{23} u_{2} u_{3}+\alpha_{33} u_{3}{ }^{2}=0
\end{aligned}
$$

die Gleichung einer Curve zweiter Classe, deren Determinante

$$
\mathrm{A} \equiv\left|\begin{array}{lll}
\alpha_{11} & \alpha_{13} & \alpha_{13} \\
\alpha_{21} & \alpha_{22} & \alpha_{23} \\
\alpha_{31} & \alpha_{32} & \alpha_{33}
\end{array}\right|
$$

vorläufig als von Null verschieden vórausgesetzt werden möge.

2) Bei gewöhnlichen homogenen Coordinaten fällt die eine Seite des Coordinatendreiecks (etwa $x_{3}=0$ ) mit der unendlich fernen Geraden zusammen 
Wir erhalten dann die Kriterien für die einzelnen nicht ausartenden Curvenarten dadurch, dass wir die Gleichung der Curve (14) in Punktcoordinaten aufstellen und auf sie die oben abgeleiteten Kriterien für Curven zweiter Ordnung anwenden; diese Gleichung ist:

$$
\begin{aligned}
\Phi(x, x) \equiv \mathrm{A}_{11} x_{1}{ }^{2}+2 \mathrm{~A}_{12} x_{1} x_{2}+\mathrm{A}_{22} x_{2}{ }^{2} & +2 \mathrm{~A}_{13} x_{1} x_{3}+2 \mathrm{~A}_{23} x_{2} x_{3} \\
& +\mathrm{A}_{33} x_{3}{ }^{2}=0 .
\end{aligned}
$$

Die Function $F(p, p)$, gebildet für (16), wird hier einfach $\mathrm{A} \varphi(p, p)$, wie man unter Berücksichtigung der bekannten Relationen wie

$$
A_{22} A_{33}-A_{23}{ }^{2}=A \alpha_{11}, \quad A_{12} A_{13}-A_{11} A_{23}=A \alpha_{23},
$$

u. s. w. leicht findet.

Für $A \varphi(p, p)>0$ stellt (14) eine Ellipse dar,

für $\mathrm{A} \varphi(p, p)<0$ eine Hyperbel,

für $\varphi(p, p)=0$ eine Parabel.

Besondere Beachtung verdient wieder der Fall, dass die Ellipse imaginär ist. Offenbar tritt dies ein, wenn sich aus einem beliebigen im Unendlichen gelegenen Punkte $y$ keine reellen Tangenten an die Curve ziehen lassen; überhaupt wird im Falle $A \varphi(p, p)>0$ die Gleichung $\varphi(u, u)=0$ eine reelle oder imaginäre Ellipse darstellen, je nachdem die aus einem unendlich fernen Punkte $y$ an die Curve gezogenen Tangenten reell oder imaginär sind. Wie nun bei einer Curve zweiter Ordnung $f(x, x)=0$ die Bedingung für die Realität der Schnittpunkte mit einer Geraden $u_{x}=0$ ausgedrückt war durch $F(u, u)<0$, so ist jetzt dualistisch die Bedingung für die Realität der von dem unendlich fernen Punkte $y$ an die Curve $\varphi(u, u)=0$ gezogenen Tangenten gegeben durch $\Phi(y, y)<0$, während im Falle $\Phi(y, y)>0$ diese Tangenten und damit die Curve $\varphi(u, u)=0$ überhaupt imaginär werden. Auch hier ist ähnlich wie oben (S. 47) zu bemerken, dass die Ungleichung $\Phi(y, y)>0$ für jeden anderen Punkt der unendlich fernen Geraden erfüllt wird, sobald sie nur für einen Punkt $y$ dieser Geraden erfüllt ist.

Dass die zerfallende Curve zweiter Classe $(A=0)$ ein Punktepaar darstellt, ist bereits in $\S 5$ erwähnt worden. In dualistisch analoger Weise, wie oben die Kriterien für ein reelles oder imaginäres Parallelenpaar gefunden wurden, lassen sich auch die Kriterien für ein reelles oder imaginäres Punktepaar entwickeln. Man hat zu

$\left(p_{1}=p_{2}=0, p_{3}=1\right)$; ausserdem ist alsdann im Falle der Parallelen stets $A_{83}=0$ (wegen $F(p, p)=0$ ); die Entscheidung der Realität hängt daher bei solchen Coordinaten von $\boldsymbol{A}_{11}$ oder $\boldsymbol{A}_{22}$ ab. 
prüfen, ob die Tangenten, welche von beliebigen Punkten $x$ der Ebene, am zweckmässigsten von den Ecken des Coordinatendreiecks, nach dem Punktepaar gezogen werden können, reell oder imaginär sind; wählt man etwa die Ecke $u_{i}=0$, so reducirt sich $\Phi(x, x)$ auf $A_{i i} x_{i}{ }^{2}$, und da die drei Hauptunterdeterminanten $A_{i i}$ im Falle $A=0$ gleiches Vorzeichen haben, auch nicht gleichzeitig Null sind, falls $\varphi(u, u)=0$ zwei verschiedene Punkte darstellt, ist das Punktepaar reell oder imaginär, je nachdem irgend eine der drei Hauptunterdeterminanten $A_{i i}$ negativ oder positiv ist.

Liegt einer der beiden Punkte im Unendlichen, so ist $\varphi(p, p)=0$; liegen jedoch beide Punkte im Unendlichen, so stellt die Gleichung (16), wie wir früher sahen, doppelt zählend die Verbindungslinie der Punkte des Paares dar und (16) muss daher durch die Coordinaten

$$
y_{1}: y_{2}: y_{3}=\left(p_{2} v_{3}-p_{3} v_{2}\right):\left(p_{3} v_{1}-p_{1} v_{3}\right):\left(p_{1} v_{2}-p_{2} v_{1}\right)
$$

eines beliebigen unendlich weiten Punktes erfüllt werden, $d . h$. es muss der Ausdruck $\Phi(y, y)$ verschwinden für völlig willkürliche Werthe der $v$. Es ist aber $\Phi(y, y) \equiv 0$ gleichbedeutend mit

$$
\varphi(p, p) \cdot \varphi(v, v)-\varphi^{2}(p, v) \equiv 0,
$$

wofür auch $\varphi(p, v)=0$ gesetzt werden kann, da

$$
\varphi(p, p)=0 .
$$

Die willkürliche Wahl der $v$ hat dann zur Folge, dass im gegenwärtigen Falle

$$
\left.\varphi^{\prime}\left(p_{1}\right)=0, \quad \varphi^{\prime}\left(p_{2}\right)=0, \quad \varphi^{\prime}\left(p_{3}\right)=0 .{ }^{1}\right)
$$

Es bleibt endlich noch der Fall zu erledigen, dass (14) einen einzigen doppelt zu zählenden Punkt darstellt. In $\$ 5$ wurde gezeigt, dass alsdann alle Unterdeterminanten $A_{i k}$ verschwinden; tritt hierzu noch $\varphi(p, p)=0$, so liegt der Doppelpunkt auf der unendlich fernen Geraden.

Wir wollen nun die erhaltenen Resultate über die Classification der Curven zweiter Ordnung und zweiter Classe übersichtlich zusammenstellen.

1) In $\S 2$ (Fussnote zu S. 14) wurde bereits von einer durch (21) in $\S 1$ definirten Function $\omega(u, u)$ bewiesen, dass sie den Gleichungen (17) genügt; diese Function stellt also, gleich Null gesetzt, ein im Unendlichen liegendes Punktepaar dar, welches überdies imaginär ist; denn die Hauptunterdeterminanten $\Omega_{11}, \Omega_{22}, \Omega_{33}$ sind nach (6) in $\S 2$ positiv. Im nächsten Paragraphen wird dieses Punktepaar eingebender behandelt werden. 
I. Abschnitt. $\$ 6$.

\section{Curven zweiter Ordnung.}

Es sei im Folgenden

$f(x, x) \equiv a_{11} x_{1}{ }^{2}+2 a_{12} x_{1} x_{2}+a_{22} x_{2}{ }^{8}+2 a_{13} x_{1} x_{3}+2 a_{23} \dot{x}_{2} x_{3}+a_{33} x_{3}{ }^{2}=0$ die Gleichung der Curve, $A \equiv \sum \pm\left(a_{11} a_{22} a_{33}\right)$ ihre Determinante; ferner sei

$F(u, u) \equiv A_{11} \ddot{u}_{1}{ }^{2}+2 A_{12} u_{1} u_{2}+A_{22} u_{2}{ }^{2}+2 A_{13} u_{1} u_{3}+2 A_{23} u_{2} u_{3}+A_{33} u_{3}{ }^{2}=0$ die Gleichung der Curve $f(x, x)=0$ in Liniencoordinaten, und $y_{1}, y_{2}, y_{3}$ seien die Coordinaten eines beliebig fixirten Punktes auf der unendlich fernen Geraden $p_{1} x_{1}+p_{2} x_{2}+p_{3} x_{3}=0$.

I) $A \gtrless 0$ : nicht ausartender Kegelschnitt.

1) $F(p, p)<0$, Ellipse.
a) imaginär, wenn $\left.A f(y, y)>0,{ }^{1}\right)$.
b) reell, wenn $\left.A f(y, y)<0 .{ }^{1}\right)$

2) $F(p, p)<0$, Hyperbel.

3) $F(p, p)=0$, Parabel.

II) $A=0$, nicht aber alle $A_{i i}$ gleich Null: zwei versehiedene Geraden.

1) $F(p, p)>0$, imaginäres Geradenpaar mit reeller Spitze im Endlichen.

2) $F(p, p)<0$, reelles Geradenpaar mit reeller Spitze im Endlichen.

3) $F(p, p)=0$, Parallelenpaar, und zwar

a) imaginär, wenn unter den drei Grössen $A_{i i}$ eine willkürlich ausgewählte positiv ist ${ }^{2}$ ),

b) reell, wenn unter den drei Grössen $A_{i i}$ eine willkürlich ausgewählte negativ ist $^{3}$ ).

III) $A=0$ und alle $A_{i i}$ gleich Null ${ }^{4}$ ): eine Doppelgerade.

Bildet die unendlich ferne Gerade einen Theil des Kegelschnitts, so ist für völlig willkürliche Werthe $w_{1}, w_{2}, w_{3}:\left(\begin{array}{l}p w \\ p w\end{array}\right) \equiv 0$.

1) Wenn diese Ungleichnng für irgend einen bestimmten Punkt $y$ auf $p_{x}=0$ stattfindet, findet sie anch für jeden anderen Punkt von $p_{x}=0$ statt.

2) Es ist dann für jedes Werthsystem $u_{1}: u_{2}: u_{3}$, welches $F(u, u)=0$ nicht erfüllt, $\boldsymbol{F}(u, u)>0$.

3) Es ist dann für jedes Werthsystem $u_{1}: u_{2}: u_{3}$, welches $F(u, u)=0$ nicht erfüllt, $F(u, u)<0$.

4) Man könnte auch sagen $F(u, u) \equiv 0$. 


\section{Curven zweiter Classe.}

Es sei im Folgenden

$\varphi(u, u) \equiv \alpha_{11} u_{1}{ }^{2}+2 \alpha_{12} u_{1} u_{2}+\alpha_{22} u_{2}{ }^{2}+2 \alpha_{13} u_{1} u_{3}+2 \alpha_{23} u_{2} u_{3}^{\circ}+\alpha_{33} u_{3}{ }^{2}=0$ die Greichung der Curve, $A \equiv \sum \pm\left(\alpha_{11} \alpha_{22} \alpha_{33}\right)$ ihre Determinante; ferner sei

$\Phi(x, x) \equiv \mathrm{A}_{11} x_{1}{ }^{2}+2 \mathrm{~A}_{12} x_{1} x_{2}+\mathrm{A}_{22} x_{2}{ }^{2}+2 \mathrm{~A}_{13} x_{1} x_{3}+2 \mathrm{~A}_{23} x_{2} x_{3}+\mathrm{A}_{33} x_{3}{ }^{2}=0$ die Gleichung der Curve $\varphi(u, u)=0$ in Punktcoordinaten, und $y_{1}, y_{2}, y_{3}$ seien die Coordinaten eires beliebig fixirten Punktes auf der unendlich fernen Geraden $p_{1} x_{1}+p_{2} x_{2}+p_{3} x_{3}=0$.

I) $\mathrm{A} \gtrless 0$ : nicht ausartender Kegelschnitt.

1) $\mathrm{A} \varphi(p, p)>0$, Ellipse.
a) imaginär, wenn $\left.\Phi(y, y)>0,{ }^{1}\right)$
b) reell, wenn $\left.\Phi(y, y)<0 .{ }_{0}^{1}\right)$

2) $\mathrm{A} \varphi(p, p)<0$, Hyperbel.

3) $\varphi(p, p)=0$, Parabel.

II) $\mathrm{A}=0$, nicht aber alle $\mathrm{A}_{i i}$ gleich Null: zwei verschiedene Punkte.

1) $\varphi(p, p) \gtrless 0$, beide Punkte liegen im.Endlichen, und zwar sind dieselben

a) imaginär, wenn unter den drei Grössen $A_{i i}$ eille willkürlich ausgewählte positiv ist $\left.{ }^{2}\right)$,

b) reell, wenn unter den drei Grössen $A_{i i}$ eine willkürlich ausgewählte negativ ist ${ }^{3}$ ).

2) $\varphi(p, p)=0$, nicht aber alle $\varphi^{\prime}\left(p_{i}\right)$ gleich Null: ein Punkt des Punktepaares im Endlichen, der andere im Unendlichen:

3) $\varphi(p, p)=0$ und alle $\varphi^{\prime}\left(p_{i}\right)=0$ : beide Punkte im Unendlichen, und zwar sind dieselben

a) imaginär $\}$ unter gleichen Bedingungen. wie bei 1 ).

III) $A=0$ und alle $A_{i}$ igleich Null ${ }^{4}$ ): ein Doppelpunkt.

1) $\varphi(p, p) \gtrless 0$, der Doppelpunkt liegt im Endlichen.

2) $\varphi(p, p)=0$, der Doppelpunkt liegt im Unendlichen.

1) Wenn diese Ungleichung für irgend einen bestimmten Punkt $y$ auf $p_{x}=0$ stattfindet, findet sie auch für jeden anderen Punkt von $p_{x}=0$ statt.

2) Es ist dann für jedes Werthsystem $x_{1}: x_{2}: x_{3}$; welches $\Phi(x, x)=0$ nicht erfüllt, $\Phi(x, x)>0$.

3) Es ist dann für jedes Werthsystem $x_{1}: x_{2}: x_{3}$, welches $\Phi(x, x)=0$ nicht erfüllt, $\Phi(x, x)<0$.

4) Man könnte auch sagen $\Phi(x, x) \equiv 0$. 
Liegen insbesondere schiefwinklige Parallelcoordinaten zu Grunde, so werden die Kriterien für Curven zweiter Ordnung und Classe äusserst einfach; man hat nur zu setzen $p_{1}=p_{2}=0, p_{3}=1$.

Die Gleichung $f(x, x)=0$ stellt daher im Falle $A \gtrless 0$ eine Ellipse, Hyperbel oder Parabel dar, je nachdem $A_{33}>0,<0,=0$, und zwar ist die Ellipse imaginär, wenn $A a_{11}$ oder $A a_{22}$ positiv, reell, wenn $A a_{11}$ oder $A a_{22}$ negativ $^{1}$ ). Ferner wird im Falle $A=0$ das sich im Endlichen schneidende Geradenpaar imaginär oder reell, je nachdem $A_{33}>0$ oder $<0$, man erhält ein Parallelenpaar für $A_{33}=0$, und zwar ein imaginäres, wenn $A_{11}$ oder $A_{22}$ positiv ist, ein reelles, wenn $A_{11}$ oder $A_{22}$ negativ ist.

Die Gleichung $\varphi(\imath, u)=0$ einer Curve zweiter Classe stellt bei schiefwinkligeu Parallelcoordinaten $\left(p_{1}=p_{2}=0, p_{3}=1\right)$ im Falle $A \gtrless 0$ eine Ellipse oder Hyperbel dar, je nachdem $A \alpha_{33}>0$, oder $<0$, eine Parabel, wenn $\alpha_{33}=0$, und zwar ist die Ellipse imaginär, wenn $A_{11}$ oder $A_{22}$ positiv, reell, wenn $A_{11}$ oder $A_{22}$ negativ ${ }^{2}$ ). Ferner liegt im Falle $A=0$ das Punktepaar im Endlichen, wenn $\alpha_{33} \gtrless 0$; ein Punkt des Paares liegt im Unendlichen, wenn $\alpha_{33}=0$, ohne dass noch $\alpha_{13}$ und $\alpha_{23}$ gleichzeitig verschwinden; beide Punkte liegen in Unendlichen, wenn $\alpha_{13}=\alpha_{23}=\alpha_{33}=0$.

\section{$\S 7$.}

Der Kreis, die imaginären Kreispunkte und ihre Beziehung zu dem Winkel zweier Geraden.

Die in $\S 2$ abgeleitete Formel

$$
\begin{aligned}
q= & \frac{u_{1} y_{1}+u_{2} y_{2}+u_{3} y_{3}}{ \pm \sqrt{\omega(u, u)} \cdot \sum_{1}^{3} p_{i} y_{i}}
\end{aligned}
$$

für den Abstand eines Punktes $y$ von einer Geraden $u$ enthält bei constanten $q$ und $y_{1}, y_{2}, y_{3}$ die Bedingung, der die Coordinaten $u_{i}$ von geraden Linien genügen müssen, wenn diese Geraden von einem festen Punkte $y$ gleichen Abstand haben, d. h. einen Kreis umhüllen sollen. Bezeichnet man diesen Abstand, also die Länge des Kreisradius, mit $r$, so ergibt sich

1) Da $A_{83}=a_{11} a_{22}-a_{12}{ }^{2}>0$, ist das Product $a_{11} a_{22}$ positiv, d. h. $a_{11}$ und $a_{22}$ haben im Fall der Ellipse gleiche Vorzeichen.

2) Da $A \alpha_{38}=A_{11} A_{22}-A_{12}{ }^{2}>0$, ist das Product $A_{11} A_{22}$ positiv, d. h. $A_{11}$ und $A_{22}$ haben im Fall der Ellipse gleiche Vorzeichen. 
(2) $r^{2}\left(p_{1} y_{1}+p_{2} y_{2}+p_{3} y_{3}\right)^{2} \omega(u, u)-\left(u_{1} y_{1}+u_{2} y_{2}+u_{3} y_{3}\right)^{2}=0$

als Gleichung in Liniencoordinaten des Kreises mit dem Mittelpunkte $y$ und dem Radius $r$.

Hierbei ist nach $(21)$ in $\S 1$ :

$$
\begin{aligned}
\omega(u, u) \equiv \frac{u_{1}^{2}}{e_{1}{ }^{2}}+\frac{u_{2}^{2}}{e_{2}^{2}}+\frac{u_{3}^{2}}{e_{3}{ }^{2}} & +\frac{2 u_{1} u_{2}}{e_{1} e_{2}} \cos \left(a_{1}, a_{2}\right)+\frac{2 u_{1} u_{3}}{e_{1} e_{3}} \cos \left(a_{1}, a_{3}\right) \\
& +\frac{2 u_{2} u_{3}}{e_{2} e_{3}} \cos \left(a_{2}, a_{3}\right) .
\end{aligned}
$$

Speciell für $r=0$ reducirt sich der Kreis auf den doppelt zu zählenden Mittelpunkt $y$; für $r=\infty$ erhält man $\omega(u, u)=0$, eine Curve zweiter Classe, die in ein Punktepaar zerfällt, da die Determinante dieses Ausdrucks nach $\S 2$ verschwindet. Unter Anwendung der in $\S 6$ abgeleiteten Kriterien $^{1}$ ) erkennt man, dass $\omega(u, u)=0$ ein im Unendlichen gelegenes imaginäres Punktepaar darstellt, das sogenannte Paar der „imaginären Kreispunkte“; bevor wir dasselbe näher betrachten, möge noch die Gleichung des Kreises in Punktcoordinaten abgeleitet werden. Man erhält dieselbe leicht aus (2) mit Hilfe des in $\S 2$ definirten Normalencentrums einer Geraden.

Ein Punkt $x_{1}, x_{2}, x_{3}$ des Kreises liegt zugleich auf einer Tangente der Curve, erfüllt also die Gleichung $u_{1} x_{1}+u_{2} x_{2}+u_{3} x_{3}=0$, wobei die $u_{i}$ Werthe sind, welche die Gleichung (2) befriedigen. Verbindet man den Punkt $x$ mit dem Mittelpunkte $y$ des Kreises, so steht diese Verbindungslinie bekanntlich auf der Tangente senkrecht, geht daher auch durch deren Normalencentrum, so dass dieses mit dem Mittelpunkte $y$ und dem Punkte $x$ der Kreisperipherie auf einer und derselben Geraden liegt. Hieraus folgt, dass für die Coordinaten des Punktes $x$ die Gleichungen bestehen

$$
\varrho x_{i}=y_{i}+\frac{\lambda}{2} \omega^{\prime}\left(u_{i}\right) \quad(i=1,2,3),
$$

in denen $\varrho$ und $\lambda$ irgend welche Zahlenfactoren bedeuten. Uebrigens erweist es sich als zweckmässig, die Gleichungen (4) durch $\lambda \mathrm{zu}$ dividiren, so dass man erhält

$$
\sigma x_{i}=\frac{1}{\lambda} y_{i}+\frac{1}{2} \omega^{\prime}\left(u_{i}\right),
$$

wobei $\sigma=\frac{\rho}{\lambda}$. Hierfür kann man, da $\frac{1}{2} \omega^{\prime}\left(u_{i}\right)=\omega_{i 1} u_{1}+\omega_{i 2} u_{2}+\omega_{i 3} u_{3}$, auch setzen:

$$
\sigma x_{i}=\frac{1}{\lambda} y_{i}+\omega_{i 1} u_{1}+\omega_{i 2} u_{2}+\omega_{i 3} u_{3} \quad(i=1,2,3) .
$$

1) Vgl. auch die Fussnote 1) zu S. 53. 
Durch Multiplication dieser drei Gleichungen mit $u_{1}, u_{2}$, resp. $u_{3}$ und darauf folgende Addition folgt ferner

$$
\sigma u_{x}=\frac{1}{2} u_{y}+\omega(u, u),
$$

und da die $x_{i}$ die Gleichung $u_{x}=0$ erfüllen, hat man

$$
\frac{1}{\lambda} u_{y}+\omega(u, u)=0 \text {. }
$$

Der hieraus hervorgehende Werth von $\omega(\imath, u)$ möge in die Gleichung (2) des Kreises in Liniencoordinaten substituirt werden, die sich alsdann nach Ausscheidung des Factors $u_{y}$ verwandelt in

$$
\frac{1}{\lambda}\left(p_{1} y_{1}+p_{2} y_{2}+p_{3} y_{3}\right)^{2} r^{2}+u_{1} y_{1}+u_{2} y_{2}+u_{3} y_{3}=0
$$

Für die fünf Grössen $u_{1}, u_{2}, u_{3}, \frac{1}{\lambda}, \sigma$ haben wir nun fünf homogene lineare Gleichungen, nämlich die drei Gleichungen (5), ferner (7) und $u_{x}=0$; das Resultat der Elimination dieser Grössen stellt sich als folgende Determinante dar:

$$
\left|\begin{array}{lllll}
\omega_{11} & \omega_{12} & \omega_{13} & y_{1} & x_{1} \\
\omega_{21} & \omega_{22} & \omega_{23} & y_{2} & x_{2} \\
\omega_{31} & \omega_{32} & \omega_{33} & y_{3} & x_{3} \\
y_{1} & y_{2} & y_{3} & r^{2} p_{y}{ }^{2} & 0 \\
x_{1} & x_{2} & x_{3} & 0 & 0
\end{array}\right|=0 .
$$

Durch Auflösung nach $r^{2}$ erhält man

$$
r^{2} p_{y}{ }^{2}\left(\Omega_{11} x_{1}{ }^{2}+\cdots+2 \Omega_{23} x_{2} x_{3}+\cdots\right)=[\omega],
$$

wobei $[\omega]$ aus $\omega(u, u)$ dadurch hervorgeht, dass man $u_{1}, u_{2}, u_{3}$ resp. ersetzt durch $x_{2} y_{3}-x_{3} y_{2}, x_{3} y_{1}-x_{1} y_{3}, x_{1} y_{2}-x_{2} y_{1}$. Hier ist nun $\Omega_{11} x_{1}{ }^{2}+\cdots+2 \Omega_{23} x_{2} x_{3}+\cdots$ nach (22) in $\S 5$ proportional dem Quadrat des Ausdrucks für die unendlich ferne Gerade, etwa gleich $\tau p_{x}{ }^{2}$, wobei mit Hilfe der Formeln (6) in $\S 2$ nebst zugehöriger Fussnote für $\tau$ gefunden wird $\left.{ }^{1}\right) \frac{16 \Delta^{4}}{\left(e_{1} e_{2} e_{3}\right)^{2}\left(a_{1} a_{2} a_{3}\right)^{2}} ;$ daher ergibt sich

$$
r^{2}=\frac{\left(e_{1} e_{2} e_{3}\right)^{2}\left(a_{1} a_{2} a_{8}\right)^{2} \cdot[\omega]}{16 \Delta^{4} p_{x}^{2} \cdot p_{y}^{2}},
$$

und wenn man statt $[\omega]$ unter Anwendung einer in der Fussnote zu S. 34 erklärten Schreibweise einsetzt $\left(\begin{array}{l}y x \\ y x\end{array}\right)_{\omega_{i k}}$, folgt

1) Weiter unten (Gl. (17)-(24)) werden noch andere Ausdrücke für die Constante $\tau$ abgeleitet. 


$$
r^{2}=\frac{\left(\begin{array}{l}
y x \\
y x
\end{array}\right)_{\omega_{i k}}}{\tau \cdot p_{x}{ }^{2} p_{y}{ }^{2}}
$$

oder

(10a)

$$
\left(\begin{array}{l}
y x \\
y x
\end{array}\right)_{\omega_{i k}}-r^{2} \tau p_{x}^{2} p_{y}^{2}=0 .
$$

Diese Gleichung stellt in Punktcoordinaten dar den Kreis mit dem Mittelpunkte $y$ und mit dem Radius $r$. Zugleich ist (10) die Formel für das Quadrat der Entfernung zweier Punkte, die durch ihre Coordinaten $x_{i}$, resp. $y_{i}(i=1,2,3)$ gegeben sind.

Speciell für $r=\infty$ reducirt sich der Kreis auf die doppelt zu zählende unendlich ferne Gerade; für $r=0$ erhält man

$$
\left(\begin{array}{l}
y x \\
y x
\end{array}\right)_{\omega_{i k}}=0
$$

und diese Gleichung repräsentirt zufolge einer Bemerkung am Schlusse von $\S 5$ das Tangentenpaar, welches vom Mittelpunkte $y$ an die Curve zweiter Classe $\omega(u, u)=0$ gezogen werden kann, d. h. (11) ist die Gleichung des imaginären Tangentenpaares, das vom Mittelpunkte y der für variabele $r$ in $(10 \mathrm{a})$ enthaltenen concentrischen Kreise an dic imaginären Kreispunkte gelegt ist $\left.{ }^{1}\right)$.

Wir wollen nun das Paar der imaginären Kreispunkte näher untersuchen. Von ihm gilt der Satz:

(12) Das imaginäre Kreispunktepaar $\omega(u, u)=0$ gehört allen Kreisen der Ebene an.

Haben nämlich die beiden Punkte des Paares die Coordinaten $\alpha_{1}, \alpha_{2}, \alpha_{3}$, resp. $\beta_{1}, \beta_{2}, \beta_{3}$, so ist:

$$
\boldsymbol{\omega}(u, u) \equiv\left(\alpha_{1} u_{1}+\alpha_{2} u_{2}+\alpha_{3} u_{3}\right)\left(\beta_{1} u_{1}+\beta_{2} u_{2}+\beta_{3} u_{3}\right),
$$

und aus der Gleichung eines Kreises in Liniencoordinaten

$$
\psi(u, u) \equiv r^{2} \cdot p_{y}{ }^{2} \cdot \omega(u, u)-\left(u_{1} y_{1}+u_{2} y_{2}+u_{3} y_{3}\right)^{2}=0
$$

erkennt man, dass die Gerade, welche etwa durch den Punkt $\alpha$ und den Mittelpunkt $y$ des Kreises (14) geht, eine Tangente von (14) ist, denn ihre Coordinaten $v_{1}, v_{2}, v_{3}$ bringen die beiden linearen Ausdrücke $\alpha_{1} u_{1}+\alpha_{2} u_{2}+\alpha_{3} u_{3}$ und $y_{1} u_{1}+y_{2} u_{2}+y_{3} u_{3}$ zum Verschwinden.

1) Es gibt noch eine andere Ausartung des Kreises, die nicht in der Form (10a) dargestellt werden kann und einen nicht im Endlichen gelegenen Mittelpunkt besitzt. Aus der elementaren Planimetrie ist nämlich bekannt, dass in einem System von Kreisen, die sämmtlich durch dieselben zwei (reellen oder imaginären) Punkte gehen, sich stets ein ausartender Kreis befindet, bestehend aus der Potenzlinie und der unendlich fernen Geraden. 
Gleiches gilt von der Verbindungslinie des Punktes $\beta$ mit dem Mittelpunkte $y$.

Aber $\alpha$ und $\beta$ sind auch geradezu die Berührungspunkte der vom Mittelpunkte $y$ aus an den Kreis gelegten (natürlich imaginären) Tangenten. Für $v_{1}, v_{2}, v_{3}$ als Coordinaten derjenigen Tangente der Curve (14) $\psi(u, u)=0$, welche etwa die Punkte $y$ und $\alpha$ verbindet, hat nämlich der Berührungspunkt nach (17) in $\S 5$ die Gleichung $\psi(u, v)=0$, und hierfür würde man aus (14) finden:

$$
\begin{aligned}
r^{2} \cdot p_{y}{ }^{2}\left\{\left(\alpha_{1} u_{1}\right.\right. & \left.+\alpha_{2} u_{2}+\alpha_{3} u_{3}\right)\left(\beta_{1} v_{1}+\beta_{2} v_{2}+\beta_{3} v_{3}\right) \\
& \left.+\left(\alpha_{1} v_{1}+\alpha_{2} v_{2}+\alpha_{3} v_{3}\right)\left(\beta_{1} u_{1}+\beta_{2} u_{2}+\beta_{3} u_{3}\right)\right\} \\
& -2\left(y_{1} u_{1}+y_{2} u_{2}+y_{3} u_{3}\right)\left(y_{1} v_{1}+y_{2} v_{2}+y_{3} v_{3}\right)=0 .
\end{aligned}
$$

Da nun unsere Tangente sowohl durch den Punkt $y$, als auch durch $\alpha$ geht, verschwinden $y_{1} v_{1}+y_{2} v_{2}+y_{3} v_{3}$ und $\alpha_{1} v_{1}+\alpha_{2} v_{2}+\alpha_{3} v_{3}$, es reducirt sich also die Gleichung des Berührungspunktes auf

$$
r^{2} \cdot p_{y}{ }^{2}\left(\dot{\alpha}_{1} u_{1}+\alpha_{2} u_{2}+\alpha_{3} u_{3}\right)\left(\beta_{1} v_{1}+\beta_{2} v_{2}+\beta_{3} v_{3}\right)=0,
$$

und dieser Ausdruck ist gleichbedeutend mit $\alpha_{1} u_{1}+\alpha_{2} u_{2}+\alpha_{3} u_{3}=0$, d. h. $\alpha$ ist der Berührungspunkt. Analoges gilt von $\beta$.

Hiermit ist zugleich bewiesen, dass das Punktepaar $\omega(u, u)=0$ allen Kreisen der Ebene angehört, denn die Function $\omega(u, u)$ ist unabhängig vom Mittelpunkt und Radius des Kreises, und auf Grund dieser Eigenschaft hat man den zwei Punkten $\omega(u, u)=0$ den Namen der „imaginären Kreispunkte“ gegeben.

Auch erkennt man nunmehr, weshalb ein Kreis schon durch drei Punkte vollständig bestimmt ist, während bei einem beliebigen Kegelschnitt im allgemeinen fünf Punkte zur eindeutigen Bestimmung erforderlich sind: es treten eben zu jenen drei Punkten noch die beiden, allen Kreisen der Ebene gemeinsamen imaginären Kreispunkte.

In § 5 war gezeigt worden, dass die Verbindungslinie der beiden Punkte einer in ein Punktepaar ausgearteten Curve zweiter Classe doppelt zählend durch die Gleichung dieser Curve in Punktcoordinaten dargestellt wird. Unter Anwendung dieses Satzes auf das Paar der imaginären Kreispunkte $\omega(u, u)=0$ muss daher

$$
\begin{aligned}
\Omega(x, x) \equiv \Omega_{11} x_{1}{ }^{2}+2 \Omega_{12} x_{1} x_{2} & +\Omega_{22} x_{2}{ }^{2}+2 \Omega_{13} x_{1} x_{3} \\
& +2 \Omega_{23} x_{2} x_{3}+\Omega_{33} x_{3}{ }^{2}=0
\end{aligned}
$$

die zugehörige Verbindungslinie, also die unendlich ferne Gerade, doppelt zählend darstellen, oder es muss seịn

$$
\Omega(x, x)=\tau\left(p_{1} x_{1}+p_{2} x_{2}+p_{3} x_{3}\right)^{2}, \quad \text { d. h. } \Omega_{i k}=\tau p_{i} p_{k},
$$

wobei $\tau$ einen Proportionalitätsfactor bedeutet. 
Wir wollen nui untersuchen, welchen Werth diese Constante $\tau$ besitzt. Durch Vergleichen der Coefficienten von $x_{1}{ }^{2}, x_{2}{ }^{2}, x_{3}{ }^{2}$ ergibt sich, wenn man für die $\Omega_{i i}$ und $p_{i}$ aus (6) in $\S 2$ und (5) in $\S 1$ die Werthe einsetzt:

$$
\frac{\tau e_{1}^{2}}{h_{1}{ }^{2}}=\frac{\sin ^{2}\left(a_{2}, a_{8}\right)}{e_{2}^{2} e_{3}{ }^{2}}, \frac{\tau e_{2}^{2}}{h_{2}{ }^{2}}=\frac{\sin ^{2}\left(a_{3}, a_{1}\right)}{e_{3} e_{1}^{2}}, \quad \frac{\tau e_{3}^{2}}{h_{3}{ }^{2}}=\frac{\sin ^{2}\left(a_{1}, a_{2}\right)}{e_{1}^{2} e_{2}{ }^{2}} .
$$

Der Inhalt des Coordinatendreiecks ist ferner gegeben durch $\Delta=\frac{a_{3} h_{3}}{2}$, und bezeichnet man mit $r$ den Radius des dem Dreieck umschriebenen Kreises, so ist $a_{3}=2 r \sin \left(a_{1}, a_{2}\right)$, daher $\Delta=r h_{3} \sin \left(a_{1}, a_{2}\right)$ und $h_{3} \sin \left(a_{1}, a_{2}\right)=\frac{\Delta}{r}$. Durch Substitution dieses Werthes in die Gleichung $\tau=\frac{h_{3}{ }^{2} \sin ^{2}\left(a_{1}, a_{2}\right)}{\left(e_{1} e_{2} e_{3}\right)^{2}}$ ergibt sich für die Proportionalitätsconstante $\tau$ der Ausdruck

$$
\tau=\frac{\Delta^{2}}{r^{2}\left(e_{1} e_{2} e_{3}\right)^{2}} .
$$

Unter Anwendung der bekannten Formeln

$$
\Delta=2 r^{2} \sin \left(a_{2}, a_{3}\right) \cdot \sin \left(a_{3}, a_{1}\right) \cdot \sin \left(a_{1}, a_{2}\right) \text { und } r=\frac{a_{1} a_{2} a_{3}}{4 \Delta}
$$

kann man $r$ aus (18) eliminiren und erhält dann

$$
\tau=\frac{2 \Delta \cdot \sin \left(a_{2}, a_{3}\right) \cdot \sin \left(a_{3}, a_{1}\right) \cdot \sin \left(a_{1}, a_{2}\right)}{\left(e_{1} e_{2} e_{3}\right)^{2}},
$$

resp.

$$
\tau=\frac{16 \Delta^{4}}{\left(e_{1} e_{2} e_{3}\right)^{2}\left(a_{1} a_{2} a_{3}\right)^{2}},
$$

eine Grösse, die auch bereits in der Gleichung (9) eines Kreises in Punktcoordinaten auftrat.

Durch Elimination von $\Delta$ mit Hilfe der Relation $r=\frac{2 \Delta^{2}}{h_{1} h_{2} h_{3}}$ ergibt sich aus (18):

oder auch

$$
\tau=\frac{h_{1} h_{2} h_{3} \cdot}{2 r\left(e_{1} e_{3} e_{3}\right)^{2}}
$$

$$
\tau=\frac{1}{2 r e_{1} e_{2} e_{3} p_{1} p_{2} p_{3}} .
$$

Eine weitere Bestimmung von $\tau$ besteht ebenfalls in der Vergleichung der Coefficienten von $\tau\left(\frac{e_{1} x_{1}}{h_{1}}+\frac{e_{2} x_{2}}{h_{2}}+\frac{e_{3} x_{3}}{h_{3}}\right)^{2}$ und $\Omega(x, x)$ und Addition der so entstehenden sechs Gleichungen. Man erhält nämlich zunächst:

$$
\frac{\tau e_{i}^{2}}{h_{i}^{2}}=\Omega_{i i}, \quad \frac{\tau e_{i} e_{k}}{h_{i} h_{k}}=\Omega_{i k}
$$


und hieraus

$$
\tau\left(\frac{e_{1}}{h_{1}}+\frac{e_{2}}{h_{2}}+\frac{e_{3}}{h_{3}}\right)^{2}=\Omega_{11}+\Omega_{22}+\Omega_{33}+2 \Omega_{12}+2 \Omega_{13}+2 \Omega_{23} ;
$$

nach (3) in $\S 1$ ist aber $\frac{e_{1}}{h_{1}}+\frac{e_{2}}{h_{2}}+\frac{e_{3}}{h_{3}}=1$, folglich

$$
\tau=\Omega_{11}+\Omega_{22}+\Omega_{33}+2 \Omega_{12}+2 \Omega_{13}+2 \Omega_{23} .
$$

Man kann die Function $\omega(u, u)$ auch als Summe zweier Quadrate darstellen, denn es ist beispielsweise

$$
\begin{aligned}
\omega(u, u) & =\left\{\frac{u_{1}}{e_{1}}+\frac{\cos \left(a_{1}, a_{2}\right)}{e_{2}} u_{2}+\frac{\cos \left(a_{1}, a_{3}\right)}{e_{3}} u_{3}\right\}^{2} \\
& +\left\{\frac{\sin \left(a_{1}, a_{2}\right)}{e_{2}} u_{2}-\frac{\sin \left(a_{1}, a_{8}\right)}{e_{3}} u_{3}\right\}^{2}
\end{aligned}
$$

woraus folgt:

(26) Die Function $\omega(u, u)$ kann für reelle Werthe der Variabeln $u_{1}, u_{2}, u_{3}$ nur Zahlenwerthe von gleichem Vorzeichen annehmen, es ist $\omega(u, u)$ eine sogenannte definite Form ${ }^{1}$ ).

Nach $(12 b)$ in $\S 2$ ist der Winkel zweier Geraden mit den Coordinaten $u_{i}$, resp. $v_{i}(i=1,2,3)$ gegeben durch

$$
\cos (u, v)=\frac{\omega(u, v)}{\sqrt{\omega(u, u)} \sqrt{\omega(v, v)}} .
$$

Für $\omega(u, v)=0$ sind die beiden Geraden zu einander normal; andrerseits ist nach S. $44 \omega(u, v)=0$ die Bedingung dafür, dass zwei gerade Linien $u$ und $v$ harmonische Polaren seien in Bezug auf das Paar der imaginären Kreispunkte, d. h.:

$\mathrm{Zwei}$ Geraden, die zu einander normal sind, können stets betrachtet werden als harmonische Polaren in Bezug auf das Paar der imaginären Kreispunkte, und umgekehrt: Wenn zwei gerade Linien harmonische Polaren sind in Bezug auf das Punktepar $\omega(u, u)=0$, sind sie zu einander normal.

Aus (27) folgt noch

$$
\sin (u, v)=\frac{ \pm \sqrt{\omega(u, u) \cdot \omega(v, v)-\omega^{2}(u, v)}}{\sqrt{\omega(u, u)} \cdot \sqrt{\omega(v, v)}}
$$

andrerseits ist nach (13) in $\S 5 \omega(u, u) \cdot \omega(v, v)-\omega^{2}(u, v)=\Omega(x, x)$, wenn

$$
x_{1}=u_{2} v_{3}-u_{3} v_{2}, \quad x_{2}=u_{3} v_{1}-u_{1} v_{3}, \quad x_{3}=u_{1} v_{2}-u_{2} v_{1}
$$

gesetzt wird,.daher nach (16) in vorliegendem Paragraphen

1) Näheres über solche Formen folgt in $\S 8$. 
oder:

$$
\omega(u, u) \cdot \omega(v, v)-\omega^{2}(u, v)=\tau \cdot p_{x}{ }^{2}=\tau \sum \pm\left(p_{1} u_{2} v_{3}\right)^{2}
$$

$$
\sin (u, v)=\frac{ \pm \sum \pm\left(p_{1} u_{2} v_{3}\right) \cdot \sqrt{\tau}}{\sqrt{\omega(u, u)} \sqrt{\omega(v, v)}}
$$

In noch ganz anderer Weise ist das Paar der imaginären Kreispunkte von Wichtigkeit bei Bestimmung der Grösse des Winkels zweier Geraden.

Das Doppelverhältuiss $\alpha$ zwischen zwei Punkten $x$ und $y$ und dem Schnittpunktepaar des Kegelschnitts $f(x, x)=0$ mit der Verbindungslinie der beiden Punkte ist nämlich nach (13) in $\S 4$ gegeben durch

dualistisch stellt

$$
\alpha=\frac{f(x, y)+\sqrt{f^{2}(x, y)-f(x, x) \cdot f(y, y)}}{f(x, y)-\sqrt{f^{2}(x, y)-f(x, x) \cdot f(y, y)}}
$$

$$
\alpha^{\prime}=\frac{\varphi(u, v)+\sqrt{\varphi^{2}(u, v)-\varphi(u, u) \cdot \varphi(v, v)}}{\varphi(u, v)-\sqrt{\varphi^{2}(u, v)-\varphi(u, u) \cdot \varphi(v, v)}}
$$

das Doppelverhältniss zweier Geraden mit den Coordinaten $\imath_{i}$, resp. $v_{i}$ zu dem von ibrem Schnittpunkte an irgend eine Curve zweiter Classe $\varphi(u, u)=0$ gelegten Tangentenpaare dar.

Der Winkel $\vartheta_{0}$ zweier Geraden $u$ und $v$ ist nun nach (27) gegeben durch

$$
\cos \vartheta_{0}=\frac{\omega(u, v)}{\sqrt{\omega(u, u)} \cdot \sqrt{\omega(v, v)}} .
$$

Nehmen wir im Nenner der rechten Seite nur den absoluten Werth $|\sqrt{\omega(u, u)} \sqrt{\omega(v, v)}|$, so hängt das Vorzeichen von $\cos \vartheta_{0}$ ab vou $\omega(u, v)$; je nachdem $\mid \frac{\omega(u, v)}{|\sqrt{\omega(u, u) \cdot \omega(v, v)}|}$ positiv oder negativ ist, liegt $\vartheta_{0}$ zwischen 0 und $\frac{\pi}{2}$ oder zwischen $\frac{\pi}{2}$ und $\pi$. Zugleich folgt

$$
\vartheta_{0}=\arccos \frac{\omega(u, v)}{|\sqrt{\omega(u, u) \cdot \omega(v, v)}|} .
$$

Es handle sich nun darum einen zwischen 0 und $\pi$ gelegenen Werth $\vartheta_{0}$ zu bestimmen der Art, dass $e^{\vartheta_{0} i}=z+i \sqrt{1-z^{2}}$; hierzu ist nöthig $\cos \vartheta_{0}=z, \sin \vartheta_{0}=+\sqrt{1-z^{2}}$, und hieraus ergibt sich, dass der Bogen $\vartheta_{0}=\arccos z$ im vorliegenden Falle zwischen 0 und $\pi$ liegt. Hätte man noch einen anderen Werth $\vartheta$ gefunden, für den $\cos \vartheta_{0}=\cos \vartheta$, so müsste $\vartheta \pm \vartheta_{0}= \pm 2 x \pi$ sein, wo $x$ irgend eine ganze Zahl bedeutet, denn alle Werthe $w$, für die $e^{i w}=1$, sind 
von der Form $w= \pm 2 \varkappa \pi$. Man hat daher $\vartheta_{0} i=\ln \left(z+i \sqrt{1-z^{2}}\right)$, wobei nun $\vartheta_{0}$ identisch ist mit dem zwischen 0 und $\pi$ gelegenen Werthe von $\operatorname{arc} \cos z$. Ferner ist auch $\vartheta_{0} i=\ln \frac{1}{z-i \sqrt{1-z^{2}}}$, mithin

$$
2 \vartheta_{0}=\frac{1}{i} \ln \frac{z+i \sqrt{1-z^{2}}}{z-i \sqrt{1-z^{2}}} \text { oder } \vartheta_{0}=\frac{1}{2 i} \ln \frac{z+\sqrt{z^{2}-1}}{z-\sqrt{z^{2}-1}}
$$

Durch Substitution des Werthes $z=\cos \vartheta_{0}=\frac{\omega(u, v)}{|\sqrt{\omega(u, u) \cdot \omega(v, v)}|}$ in diese Gleichung erhält man:

$$
\left.\vartheta_{0}=\frac{1}{2 i} \ln \frac{\omega(u, v)+\left|\sqrt{\omega^{2}(u, v)-\omega(u, u) \cdot \omega(v, v)}\right|}{\omega(u, v)-\left|\sqrt{\omega^{2}(u, v)-\omega(u, u) \cdot \omega(v, v)}\right|},{ }^{1}\right)
$$

und hieraus folgt mit Rücksicht auf (32):

(35) Der Winkel zweier Geraden ist gleich dem durch $2 i$ dividirten natürlichen Logarithmus des Doppelverhältnisses, welches die beiden Geraden mit dem von ihrem Schnittpunkte nach dem imaginären Kreispunktepaar gezogenen Geradenpare bilden.

Wir wollen bei dieser Gelegenheit noch den Winkel $\alpha$ bestimmen, welchen zwei durch $f(x, x)=0$ gegebene Geraden mit einander bilden.

Wären $u_{x}=0$ und $v_{x}=0$ die Gleichungen der beiden Geraden, daher $\sum_{1}^{3} \sum_{1}^{3} a_{i k} x_{i} x_{k} \equiv u_{x} v_{x}$, so hätte man nach (27) und (29):

$$
\begin{gathered}
\cos \alpha=\frac{\omega(u, v)}{\sqrt{\omega(u, u)} \sqrt{\omega(v, v)}}, \\
\sin \alpha=\frac{\sqrt{\omega(u, u) \cdot \omega(v, v)}-\omega^{2}(u, v)}{\sqrt{\omega(u, u)} \sqrt{\omega(v, v)}}=\frac{p_{x} \sqrt{\tau}}{\sqrt{\omega(u, u)} \sqrt{\omega(v, v)}},
\end{gathered}
$$

wobei $x_{1}=u_{2} v_{3}-u_{3} v_{2}, x_{2}=u_{3} v_{1}-u_{1} v_{3}, x_{3}=u_{1} v_{2}-u_{2} v_{1}$ die Coordinaten des Schnittpunktes der zwei Geraden bedeuten; andrerseits ist aber nach (34) in $\S 4$ das Product $x_{i} x_{k}$ proportional zu $A_{i k}$, und zwar findet man leicht $x_{i} x_{k}=-4 A_{i k}$, daher $\tau p_{x}{ }^{2}=-4 \tau F(p, p)$. Aus $2 a_{i k}=u_{i} v_{k}+v_{i} u_{k}$ folgt

$$
\begin{aligned}
\omega(u, v) & =\omega_{11} u_{1} v_{1}+\omega_{12}\left(u_{1} v_{2}+u_{2} v_{1}\right)+\cdots+\omega_{33} u_{3}{ }^{2} \\
& =a_{11} \omega_{11}+2 a_{12} \omega_{12}+a_{22} \omega_{22}+2 a_{13} \omega_{13}+2 a_{23} \omega_{23}+a_{33} \omega_{33},
\end{aligned}
$$

1) Die Vorzeichen der Wurzeln in (34) sind zu vertauschen, wenn $\boldsymbol{\vartheta}_{0}$ zwischen 0 und $-\pi$ liegt. Für beide Fälle hat man in (34) bei dem Logarithmus den Hauptwerth zu setzen, d. h. denjenigen, dessen imaginärer Theil zwischen $+i \pi$ und $-i \pi$ liegt. 
wofür $[a, \omega]$ gesetzt werde; endlich ist

$$
\omega(u, u) \cdot \omega(v, v)=\omega^{2}(u, v)-4 \tau F(p, p)=[a, \omega]^{2}-4 \tau F(p, p),
$$

es ergibt sich also

$$
\begin{gathered}
\cos ^{2} \alpha=\frac{[a, \omega]^{2}}{[a, \omega]^{2}-4 \tau F(p, p)}, \quad \sin ^{2} \alpha=\frac{-4 \tau F(p, p)}{[a, \omega]^{2}-4 \tau F(p, p)}, \\
\operatorname{tg}^{2} \alpha=-\frac{4 \tau F(p, p)}{[a, \omega]^{2}} .
\end{gathered}
$$

$\S 8$.

Ueber einen fundamentalen Satz aus der Theorie der definiten quadratischen Formen und über zwei Gattungen von Gleichungen mit nur reellen Wurzeln.

Im vorhergehenden Paragraphen wurde gezeigt, dass $\boldsymbol{\omega}(u, u)$ eine sogenannte definite Form, d. h. eine ganze homogene Function ist, welche für reelle Werthe der Variabeln nur Zahlenwertlie von gleichem Vorzeichen anzunehmen vermag.

Man bezeichnet überhaupt als "Form" eine ganze homogene Function mehrerer Variabeln, um eine Ausdrucksweise für die Gesammtheit aller Glieder zu haben, welche, in den Variabeln sämmtlich von gleicher Dimension, die Function zusammensetzen. Nach ihrer Dimension in den Variabeln heisst die Form von der 1., 2., 3., ...n $n^{\text {ten }}$ Ordnung, oder auch linear, quadratisch, kubisch, ...; nach der Anzahl der Variabeln heisst sie, von 2 beginnend, binär, ternär, ... So ist z. B. $a_{11} x_{1}{ }^{2}+2 a_{12} x_{1} x_{2}+a_{22} x_{2}{ }^{2}+2 a_{13} x_{1} x_{3}+2 a_{23} x_{2} x_{3}+a_{33} x_{3}{ }^{2}$ eine ternäre quadratische Form.

Ueber definite quadratische Formen gibt es nun einen für spätere Untersuchungen wichtigen Satz, den wir allgemein für den Fall vou $n$ Variabeln beweisen wollen unter Anwendung eines von Kronecker bei ähnlicher Gelegenheit benutzten Raisonnements ${ }^{1}$ ). Dieser Satz lautet:

(1) Ist $\varphi\left(u_{1}, u_{2}, \ldots u_{n}\right) \equiv \sum_{1}^{n} \sum_{1}^{n} \varphi_{i k} u_{i} u_{k}$ eine quadratische Form mit $n$ Variabeln $u_{1}, u_{2}, \ldots u_{n}$, welche für beliebige reelle Werthe der $u$ nur Zahlenwerthe mit demselben Vorzeichen annimmt (definit ist), und verschwindet diese Form für die Werthe $p_{1}, p_{2}, \ldots p_{n}$ der Variabeln, unter denen mindestens einer von Null verschieden ist, so verschwinden auch immer die Ausdrïcke $\varphi^{\prime}\left(p_{1}\right), \varphi^{\prime}\left(p_{2}\right), \ldots \varphi^{\prime}\left(p_{n}\right)$.

1) Vgl. Kronecker in den Monatsberichten der Berliner Akademie der Wissenschaften, Jahrgang 1868, S. $339 \mathrm{f}$. 
Zum Beweis dieses Satzes setze man:

$$
u_{1}=v_{1}+\lambda p_{1}, u_{2}=v_{2}+\lambda p_{2}, \cdots u_{n-1}=v_{n-1}+\lambda p_{n-1}, u_{n}=0+\lambda p_{n},
$$

wobei etwa $p_{n} \gtrless 0$ sei. Alsdann wird

$$
\begin{aligned}
\varphi\left(u_{1}, u_{2}, \cdots\right. & \left.u_{n-1}, u_{n}\right)=\varphi\left(v_{1}, v_{2}, \cdots v_{n-1}, 0\right) \\
& +\lambda\left\{\varphi^{\prime}\left(p_{1}\right) \cdot v_{1}+\varphi^{\prime}\left(p_{2}\right) \cdot v_{2}+\cdots+\varphi^{\prime}\left(p_{n-1}\right) \cdot v_{n-1}\right\} \\
& +\lambda^{2} \varphi\left(p_{1}, p_{2}, \cdots p_{n-1}, p_{n}\right)
\end{aligned}
$$

doch hat hierin der Factor von $\lambda^{2}$ nach Voraussetzung den Werth Null.

Um nun zu zeigen, dass die Ausdrücke $\varphi^{\prime}\left(p_{1}\right), \varphi^{\prime}\left(p_{2}\right), \ldots \varphi^{\prime}\left(p_{n}\right)$ verschwinden, nehme man an, es sei etwa $\varphi^{\prime}\left(p_{k}\right)$ von Null verschieden und setze $v_{1}=v_{2}=\cdots=v_{k-1}=v_{k+1}=\cdots=v_{n}=0, \quad v_{k}=1$; hierdurch verwandelt $\operatorname{sich} \varphi\left(u_{1}, u_{2}, \ldots u_{n}\right)$ in

$$
\varphi(0,0, \ldots, 1,0, \ldots, 0)+\lambda \varphi^{\prime}\left(p_{k}\right),
$$

und da hier $\lambda$ jeden beliebigen Werth annehmen kann, so ist dies auch, so lange $\varphi^{\prime}\left(p_{k}\right) \gtrless 0$, mit $\varphi\left(u_{1}, u_{2}, \ldots u_{n}\right)$ der Fall, was aber der über das Wesen der Function gemachten Voraussetzung definit zu sein widerspricht. Mithin muss $\varphi^{\prime}\left(p_{k}\right)$ verschwinden, und zwar zunächst für $k=1,2, \cdots(n-1)$, weil nur $\varphi^{\prime}\left(p_{1}\right), \varphi^{\prime}\left(p_{2}\right), \ldots \varphi\left(p_{n-1}\right)$ in dem Factor von $\lambda$ in (3) auftreten. Da aber

$\varphi\left(p_{1}, p_{2}, \cdots p_{n}\right)=p_{1} \varphi^{\prime}\left(p_{1}\right)+p_{2} \varphi^{\prime}\left(p_{2}\right)+\cdots+p_{n-1} \varphi^{\prime}\left(p_{n-1}\right)+p_{n} \varphi^{\prime}\left(p_{n}\right)=0$ so muss auch noch $\varphi^{\prime}\left(p_{n}\right)$ verschwinden; denn $p_{n}$ wurde als von Null verschieden angenommen.

Für den Fall der durch $\omega(u, u)$ definirten und im vorhergehenden Paragraphen näher betrachteten Form, welche gleich Null gesetzt die zwei imaginären Kreispunkte darstellt, sind die Coordinaten der unendlich fernen Geraden $p_{i}=\frac{e_{i}}{h_{i}}(i=1,2,3)$ solche Werthe, für welche $\omega(u, u)$ verschwindet; denn diese Gerade ist Trägerin des Punktepaars. Es ist dann zufolge des Satzes (1) stets $\omega^{\prime}\left(p_{i}\right)=0,(i=1,2,3)$, wie übrigens auch schon in $\S 2$ erwähnt wurde.

Um den Nutzen des Fundamentaltheorems (1) darzulegen, wollen wir einige Sätze über zwei Gattungen von Gleichungen beweisen, die im Folgenden, wie überhaupt in vielen Theilen der reinen und angewandten Mathematik, eine wichtige Rolle spielen. Der erste Satz lautet:

(4) Bezeichnet $\varphi\left(u_{1}, u_{2}, \cdots u_{n}\right) \equiv \sum_{1}^{n} \sum_{1}^{n} \alpha_{i k} u_{i} u_{k}$ eine beliebige quadratische Form der $n$ Variabeln $u_{1}, u_{2}, \cdots u_{n}$, ferner 
System quadratischer Formen, dessen Determinante nur reelle Factoren hat. 67

$$
\omega\left(u_{1}, u_{2}, \cdots u_{n}\right) \equiv \sum_{1}^{n} \sum_{1}^{n} \omega_{i k} u_{i} u_{k}
$$

eine definite quadratische Form derselben Veränderlichen und setzt man $-\lambda \alpha_{i k}+\omega_{i k}=\gamma_{i k}$, so besitzt die Gleichung $n^{\text {ten }}$ Grades in $\lambda$ :

$$
\Gamma \equiv \sum \pm\left(\gamma_{11} \gamma_{22} \cdots \gamma_{n n}\right)=0, \quad\left(\gamma_{i k}=\gamma_{k i}\right)
$$

stets nur reelle Wurzeln.

Ist nämlich $\lambda$ eine von Null verschiedene Wurzel dieser Gleichnng, so gibt es zufolge (4a) stets Werthe $c_{1}, c_{2}, \ldots c_{n}$, die nicht sämmtlich Null sind und die $n$ Gleichungen befriedigen:

$$
-\lambda \varphi^{\prime}\left(c_{i}\right)+\omega^{\prime}\left(c_{i}\right)=0(i=1,2, \cdots n) .
$$

Ist allgemein $c_{i}=c_{i}{ }^{0}+c_{i}{ }^{\prime} \sqrt{-1}$, so folgt durch Multiplication der $n$ Gleichungen (5) mit $c_{i}^{0}-c_{i}^{\prime} \sqrt{-1}$ und Addition derselben:

$$
\text { (6) } \begin{aligned}
-\lambda\left\{\varphi\left(c_{1}{ }^{0}, c_{2}{ }^{0} \cdots c_{n}{ }^{0}\right)+\varphi\left(c_{1}{ }^{\prime}, c_{2}{ }^{\prime}, \cdots c_{n}{ }^{\prime}\right)\right\} & +\omega\left(c_{1}{ }^{0}, c_{2}{ }^{0}, \cdots c_{n}{ }^{0}\right) \\
& +\omega\left(c_{1}{ }^{\prime}, c_{2}{ }^{\prime}, \cdots c_{n}{ }^{\prime}\right)=0 .
\end{aligned}
$$

Hier kann nun der Coefficient von $\lambda$ nicht verschwinden, denn sonst würde auch die Summe der beiden $\omega$ verschwinden müssen, d. h. die $c_{i}{ }^{0}$ und $c_{i}^{\prime}$ wären nach (1) solche Werthe, für die $\omega^{\prime}\left(c_{i}{ }^{0}\right)=0$ und $\boldsymbol{\omega}^{\prime}\left(c_{i}^{\prime}\right)=0$; dann wäre nach $(5)$ neben $\omega^{\prime}\left(c_{i}\right)=0$ auch noch $\varphi^{\prime}\left(c_{i}\right)=0$ $(i=1,2, \ldots n)$, d. h. die Gleichung (5) wäre für beliebige Werthe von $\lambda$ giltig, somit die Determinante $\sum \pm\left(\gamma_{11} \gamma_{22} \cdots \gamma_{n n}\right)$ identisch Null, und dieser Fall war bisher eo ipso ausgeschlossen. Der Coefficient von $\lambda$ in (6) ist demnach von Null verschieden, und in Folge dessen $\lambda$ gleich dem Quotienten zweier reellen Grössen, also in der That reell.

Für die Gleichung (4a) bestehen noch zwei weitere Sätze, die später von Wichtigkeit sind und daher hier bewiesen werden sollen ${ }^{1}$ ), nämlich:

A) Jeder $l$-fache Factor $\left(\lambda-\lambda_{1}\right)$ von $\Gamma=0$, wobei $\lambda_{1} \gtrless 0$, ist mindestens $(l-1) \mathrm{mal}$ in der Unterdeterminante $\Gamma_{i k}$ eines beliebigen Elementes $\gamma_{i k}$ der Determinante $\Gamma$ als Factor enthalten.

1) Das Princip der hier angewandten Beweismethode ist eine Umformung derjenigen, welche Herr Weierstrass gegeben hat in den Monatsberichten der Akademie der Wissenschaften zu Berlin, Jahrgang 1858, S. 207-220. 
B) Jede $l$-fache verschwindende Wurzel $\lambda=0$ der Gleichung $\Gamma=0$ ist mindestens $(l-2)$ fache Wurzel von $\Gamma_{i k}=0$.

Zum Beweise des Satzes A) gehen wir aus von dem Quotienten

oder

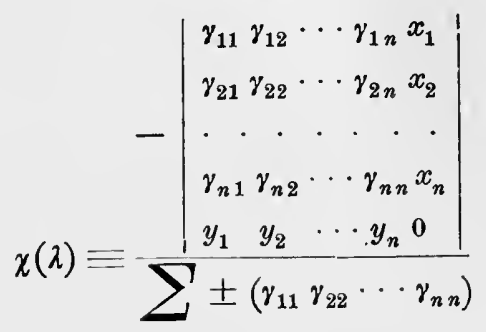

$$
\chi(\lambda)=\frac{\Gamma_{11} x_{1} y_{1}+\Gamma_{12}\left(x_{1} y_{2}+x_{2} y_{1}\right)+\cdots}{\sum \pm\left(\gamma_{11} \gamma_{22} \cdots \gamma_{n n}\right)},
$$

in welchem die $y_{i}$ willkürlich fixirte Constanten, die $x_{i}$ irgend welche variabele Grössen bedeuten. Die Anwendung einer Partialbruchzerlegung auf (9) möge alsdann, soweit sich die Brüche auf die Wurzel $\lambda=\lambda_{1}$ von $\Gamma=0$ beziehen, eine Summe liefern von der Form:

$$
\chi(\lambda)=\frac{\sum_{1}^{n} c_{i} x_{i}}{\left(\lambda-\lambda_{1}\right)^{e}}+\frac{\sum_{1}^{n} c_{i}^{\prime} x_{i}}{\left(\lambda-\lambda_{1}\right)^{e-1}}+\frac{\sum_{1}^{n} c_{i}^{\prime \prime} x_{i}}{\left(\lambda-\lambda_{1}\right)^{e-2}}+\cdots,
$$

wobei die $c, c^{\prime}, c^{\prime \prime}, \ldots$ abbängen von den willkürlich fixirten $y$ und von den $\gamma_{i k}$, und wir behaupten nun, dass hier, so lange $\lambda_{1} \geqslant 0$, der Exponent $e$ nicht grösser sein kann als 1. Da nämlich vorausgesetzt ist, dass $\Gamma$ nicht identisch verschwindet, kann man in (9) und (10) setzen

$$
x_{i}=-\frac{1}{2} \lambda \varphi^{\prime}\left(u_{i}\right)+\frac{1}{2} \omega^{\prime}\left(u_{i}\right),
$$

wodurch sich der Zähler von (9) verwandelt in $\left(y_{1} u_{1}+y_{2} u_{2}+\cdots+y_{n} u_{n}\right) \Gamma$, also $\chi(\lambda)$ nach Wegheben von $\Gamma$ in $y_{1} u_{1}+y_{2} u_{2}+\cdots+y_{n} u_{n}$. Für $\lambda=\lambda_{1}+h$ erhält man alsdann nach Multiplication mit $h^{e}$ an Stelle von (10) die Gleichung

$$
\begin{aligned}
u_{y} \cdot h^{e}= & \left\{-\left(\lambda_{1}+h\right) \varphi(c, u)+\omega(c, u)\right\} \\
& +h\left\{-\left(\lambda_{1}+h\right) \varphi\left(c^{\prime}, u\right)+\omega\left(c^{\prime}, u\right)\right\} \\
& +h^{2}\left\{-\left(\lambda_{1}+h\right) \varphi\left(c^{\prime \prime}, u\right)+\omega\left(c^{\prime \prime}, u\right)\right\}+\cdots
\end{aligned}
$$

Für $e>1$ mïissten mindestens die Coefficienten von $h^{0}$ und $h^{1}$ der rechten Seite gleich Null seiu, es wäre daher 


$$
\omega(c, u)-\lambda_{1} \varphi(c, u)=0
$$

und

$$
\left.\omega\left(c^{\prime}, u\right)-\lambda_{1} \varphi\left(c^{\prime}, u\right)-\varphi(c, u)=0^{1}\right) .
$$

Setzt man nun $u_{i}=c_{i}^{\prime}$ in (13), dagegen $u_{i}=c_{i}$ in (14) und subtrahirt man beide Gleichungen von einander, so folgt $\varphi(c, c)=0$, somit nach (13) auch $\omega(c, c)=0$; nach dem Fundamentaltheoreme (1) ist dann auch $\omega(c, u)=0$, denn $\omega(u, u)$ ist eine definite Form, und nach (13) nunmehr $\varphi(c, u)=0$, da $\lambda_{1} \gtrless 0$, d. h. für jeden Werth von $\lambda$ hätte man $\boldsymbol{\omega}(c, u)-\lambda \varphi(c, u)=0$, die Determinante $\Gamma$ würde identisch verschwinden gegen die Voraussetzung. So lange $\lambda \gtrless 0 \mathrm{kanu}$ folglich $e$ in (10) nicht grösser als 1 sein, d. h. in dem Quotienten $\chi(\lambda)$ muss sich die $l$-fache Wurzel $\lambda_{1}=\lambda$ von $\Gamma=0$ mindestens $(l-1)$ mal in Zähler und Nenner wegheben lassen, oder es muss wegen der Willkürlichkeit der $x_{i}$ und $y_{i}$ in $\chi(\lambda)$ der Factor $\lambda-\lambda_{1}$ in jeder Unterdeterminante $\Gamma_{i k}$ von $\Gamma$ mindestens $(l-1)$ mal enthalten sein.

Zum Beweis des Satzes B) in (8) verfahren wir zunächst gerade so wie bei A) und gelangen hierdurch zur Gleichung (12), in der jedoch nunmehr $\lambda_{1}=0$ zu setzen ist. Alsdann behaupten wir, dass für $\lambda_{1}=0$ der Exponent $e$ in (12) nicht grösser sein kann als 2. Für $e>2$ müsste nämlich neben den Coefficienten von $h^{0}$ und $h^{1}$ mindestens auch derjenige von $h^{2}$ verschwinden; in Folge von $\lambda_{1}=0$ gehen aber die Gleichungen (13) und (14) über in

$$
\omega(c, u)=0
$$

während das Verschwinden des Coefficienten von $h^{2}$ für $\lambda_{1}=0$ liefert:

$$
\omega\left(c^{\prime \prime}, u\right)-\varphi\left(c^{\prime}, u\right)=0 .
$$

Setzt man nun $u_{i}=c_{i}$ in (17), dagegen $u_{i}=c_{i}^{\prime \prime}$ in (15) und subtrahirt man beide Gleichungen von einander, so folgt $\varphi\left(c^{\prime}, c\right)=0$,

1) Es lassen sich die $y_{i}$ stets so fixiren, dass nicht alle $c_{i}(i=1,2, \cdots n)$ Null sind, denn sonst wäre für beliebige $x_{i}$ und $y_{i}$ der Factor $\lambda-\lambda_{1}$ auch $l$-fach in der Determinante $\left(\begin{array}{l}x \\ y\end{array}\right)_{\gamma_{i k}}$ enthalten, d. h. $l$-fach in jeder Unterdeterminante $\Gamma_{i k}$. Dies ist aber unmöglich, da alsdann auf Grund von

$$
\Gamma^{\prime}(\lambda)=\sum_{1}^{n} \sum_{1}^{n} \frac{\partial \Gamma}{\partial \gamma_{i k}} \frac{\partial \gamma_{i k}}{\partial \lambda}=-\sum_{1}^{n} \sum_{1}^{n} \Gamma_{i k} \alpha_{i k}=0
$$

auch $\Gamma^{\prime}(\lambda)$ den Factor $\left(\lambda-\lambda_{1}\right)^{l}$ enthalten würde. 
daher ist alsdann für $\iota_{i}=c_{i}^{\prime}$ in $(16) \omega\left(c^{\prime}, c^{\prime}\right)=0$ und nach dem Fundamentaltheorem $(1) \omega\left(c^{\prime}, u\right)=0$; hierdurch liefert $(16) \cdot \varphi(c, u)=0$, es wäre also mit Rücksicht auf (15) für jeden Werth von $\lambda \omega(c, u)$ $-\lambda \varphi(c, u)=0, \mathrm{~d}$. h. die Determinante $\Gamma$ würde identisch verschwinden, was ausgeschlossen war. Für $\lambda_{1}=0$ kann folglich $e$ in (10) nicht grösser als 2 sein; d. h. in dem Quotienten $\chi(\lambda)$ muss sich die etwa auftretende $l$-fache Wurzel $\lambda=0$ von $\Gamma=0$ mindestens $(l-2)$ mal in Zähler und Nenner wegheben lassen, oder es muss in jeder Unterdeterminante $\Gamma_{i k}$ von $\Gamma$ der Factor $\lambda$ mindestens $(l-2)$ mal enthalten sein.

Wir wollen nun noch eine zweite Gattung von Gleichungen betrachten, die ähnliche Eigenschaften haben wie die Gleichungen von der Form (4a).

Es sei nämlich $f \equiv \sum_{1}^{n} \sum_{1}^{n} a_{i k} x_{i} x_{k}$ eine beliebige quadratische Form der $n$ Variabeln $x_{1}, x_{2}, \cdots x_{n}, \omega \equiv \sum_{1}^{n} \sum_{1}^{n} \omega_{i k} u_{i} u_{k}$ eine quadratische Form der Variabeln $u_{1}, u_{2}, \ldots u_{n}$, welche für reelle Werthe der $u$ nur Zahlenwerthe von demselben Vorzeichen annimmt ${ }^{1}$ ). Alsdann bilde man den Ausdruck

$$
\begin{aligned}
\Psi(u, x) \equiv \frac{1}{4} \sum_{1}^{n} f^{\prime}\left(x_{i}\right) \cdot \omega^{\prime}\left(u_{i}\right) & \equiv \frac{1}{4} \sum_{1}^{n} \sum_{1}^{n} a_{i k} \omega^{\prime}\left(u_{i}\right) \cdot x_{k} \\
& \equiv \frac{1}{4} \sum_{1}^{n} \sum_{1}^{n} \alpha_{i k} u_{i} x_{k},
\end{aligned}
$$

wobei zur Abkürzung gesetzt ist

$$
\alpha_{i k}=\omega_{1 i} a_{1 k}+\omega_{2 i} a_{2 k}+\cdots+\omega_{n i} a_{n k} .
$$

und im allgemeinen $\alpha_{i k}$ nicht gleich $\alpha_{k i}$; ferner setze man

$$
u_{x} \equiv u_{1} x_{1}+u_{2} x_{2}+\cdots+u_{n} x_{n}
$$

und definire Grössen $\beta_{i k}$ durch

$$
\Psi(u, x)-\lambda \cdot u_{x} \equiv \sum_{1}^{n} \sum_{1}^{n} \beta_{i k} u_{i} x_{k} .
$$

Es besteht alsdann der Satz:

(19) Unter den soeben gemachten Voraussetzungen hat die Gleichung $n^{\text {ten }}$ Grades in $\lambda$ :

1) Es ist nicht nöthig, dass die Determinante von $\omega(u, u)$ verschwinde. Andererseits ist vorausgesetzt $a_{i k}=a_{k i}$ und $\omega_{i k}=\omega_{k i}$. 
oder auch

$$
\sum \pm\left(\beta_{11} \beta_{22} \cdots \beta_{n n}\right)=0
$$

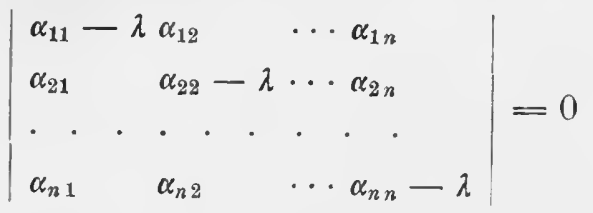

stets nur relle $W$ urzeln.

Zum Beweis dieses Satzes werde zunächst bemerkt, dass es zufolge Verschwindens der Determinante (19a) Werthe $t_{1}, t_{2}, \ldots t_{n}$ gibt, für welche

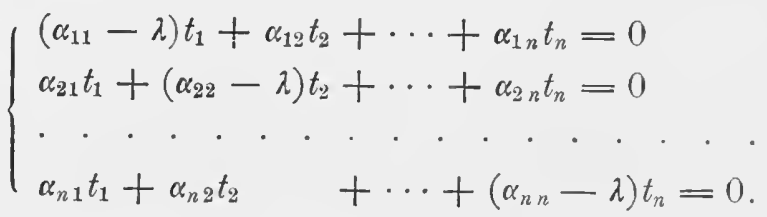

Zwei conjugirt complexen Wurzeln $\lambda$ von (19a) würden danı zwei conjugirt complexe Werthsysteme der $t$ zugehören, sie seien etwa

$$
t_{i}=v_{i}+\sqrt{-1} w_{i} \text { und } \overline{t_{i}}=v_{i}-\sqrt{-1} w_{i}(i=1,2, \cdots n) .
$$

Wir multipliciren nun die Gleichungen (20), nachdem in ihnen die mit dem Factor $\lambda$ behafteten Glieder auf die rechte Seite gebracht sind, resp. mit $\frac{\mathbf{1}}{\mathbf{2}} \boldsymbol{\varpi}^{\prime}\left(\bar{t}_{i}\right)$ und summiren über $i$, wodurch man erhält:

$$
\frac{1}{4} \sum_{1}^{n} \sum_{1}^{n} a_{k i} \omega^{\prime}\left(t_{k}\right) \cdot \omega^{\prime}\left(\bar{t}_{i}\right)=\lambda \cdot \sum_{1}^{n} \frac{1}{2} \omega^{\prime}\left(\bar{t}_{i}\right) \cdot t_{i} ;
$$

bei Substitution der für $t_{i}$ und $\bar{t}_{i}$ angenommenen conjugirt imaginären

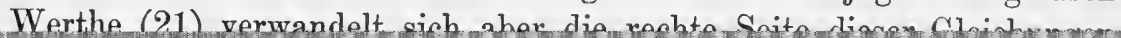


sprechende Werth von $\lambda$ derselbe sein, wie der den $\bar{t}_{i}$ entsprechende, d. h. der imaginäre Theil von $\lambda$ muss Null, $\lambda$ muss reell sein, w. z. b. w. Auch für die Gleichung (19a) gelten die analogen Sätze wie (7) und (8) bei der Gleichung (4a), nämlich:
A) Jeder $l$-fache Factor $\left(\lambda-\lambda_{1}\right)$, wobei $\lambda_{1} \gtrless 0$, von $\mathrm{B} \equiv \sum \pm\left(\beta_{11} \beta_{22} \cdots \beta_{n n}\right)=0$ ist mindestens $(l-1) \mathrm{mal}$ in der Unterdeterminante $B_{i k}$ eines beliebigen Elementes $\beta_{i k}$ der Determinante B als Factor enthalten.

(24) B) Jede $l$-fache verschwindende Wurzel $\lambda=0$ der Gleichung $B=0$ ist mindestens $(l-2)$ fache Wurzel von $\mathrm{B}_{i k}=0$.

Zum Beweis dieser Sätze gehen wir aus von dem Quotienten

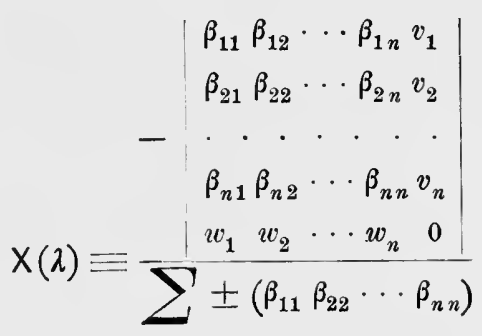

oder

$$
X(\lambda)=\frac{\mathrm{B}_{11} v_{1} w_{1}+\mathrm{B}_{12} v_{1} w_{2}+\mathrm{B}_{21} v_{2} w_{1}+\cdots}{\sum \pm\left(\beta_{11} \beta_{22} \cdots \beta_{n n}\right)},
$$

in welchem die $w_{i}$ willkürlich fixirte Constanten, die $v_{i}$ irgend welche variabele Grössen bedeuten. Die Anwendung einer Partialbruchzerlegung möge alsdann, soweit sich die Brüche auf die Wurzel $\lambda=\lambda_{1}$ von $B=0$ beziehen, eine Summe liefern von der Form

$$
X(\lambda)=\frac{\sum_{1}^{n} c_{i} v_{i}}{\left(\lambda-\lambda_{1}\right)^{e}}+\frac{\sum_{1}^{n} c_{i}^{\prime} v_{i}}{\left(\lambda-\lambda_{1}\right)^{e-1}}+\frac{\sum_{1}^{n} c_{i}^{\prime \prime} v_{i}}{\left(\lambda-\lambda_{1}\right)^{e-2}}+\cdots,
$$

wobei die $c, c^{\prime}, c^{\prime \prime}, \ldots$ abhängen von den willkürlich fixirten $w_{i}$ und von den $\beta_{i k}$, und wir behaupten nun, dass hier, so lange $\lambda_{1} \gtrless 0$, der Exponent $e$ nicht grösser sein kann als 1 . Denn setzt man

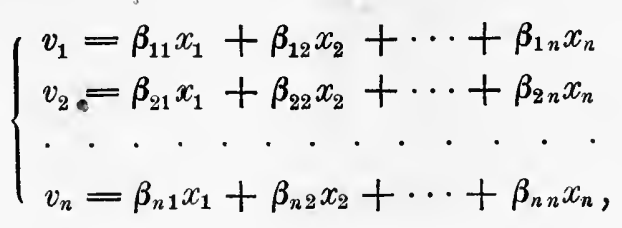


so verwandelt sich $\mathrm{X}(\lambda)$ in $\left.w_{1} x_{1}+w_{2} x_{2}+\cdots+w_{n} x_{n}{ }^{1}\right)$, und für $\lambda=\lambda_{1}+h$ erhält man nach Multiplication mit $h^{e}$ an Stelle von (26) die Gleichung

$$
\begin{aligned}
& w_{x} \cdot h^{e}=\left\{\Psi(c, x)-\left(\lambda_{1}+h\right) c_{x}\right\} \\
& \quad+h\left\{\Psi\left(c^{\prime}, x\right)-\left(\lambda_{1}+h\right) c_{x}^{\prime}\right\}+h^{2}\left\{\Psi\left(c^{\prime \prime}, x\right)-\left(\lambda_{1}+h\right) c_{x}^{\prime \prime}\right\}+\cdots
\end{aligned}
$$

Für $e>1$ müssten mindestens die Coefficienten von $h^{0}$ und $h^{1}$ der rechten Seite gleich Null sein, man hätte daher und

$$
\begin{gathered}
\Psi(c, x)-\lambda_{1} c_{x}=0 \\
\psi\left(c^{\prime}, x\right)-\lambda_{1} c_{x}^{\prime}-c_{x}=0 .
\end{gathered}
$$

Ist nun $p_{1}, p_{2}, \ldots p_{n}$ ein Werthsystem, für welches $\omega(p, p)=0$, daher nach (1) auch $\omega^{\prime}\left(p_{i}\right)=0$, so können jedenfalls die $c_{i}$ nicht proportional sein den $p_{i}$, denn sonst wäre auch

$$
\psi(c, x) \equiv \frac{1}{4} \sum_{i}^{n} f^{\prime}\left(x_{i}\right) \cdot \omega^{\prime}\left(c_{i}\right)
$$

identisch Null, daher nach (29) $c_{x} \equiv 0$, was nicht denkbar $\left.{ }^{2}\right)$. Man setze jetzt in (29) $x_{i}=\frac{1}{2} \omega^{\prime}\left(c_{i}^{\prime}\right)$, dagegen in (30) $x_{i}=\frac{1}{2} \omega^{\prime}\left(c_{i}\right)$; alsdann werden $\Psi(c, x)$ und $\Psi\left(c^{\prime}, x\right)$ identisch mit $\frac{1}{4} \sum_{1}^{n} \sum_{1}^{n} a_{i k} \omega^{\prime}\left(c_{i}\right) \cdot \omega^{\prime}\left(c_{k}^{\prime}\right)$ und wegen $a_{i k}=a_{k i}$ einander gleich. Zieht man daher nach diesen Substitutionen (29) und (30) vou einander ab, so bleibt übrig $\omega(c, c)=0$, was nicht möglich ist, wie soeben erwiesen. Im Falle $\lambda_{1} \gtrless 0$ kann folglich $e$ nicht grösser als 1 sein, u. s. w. (wie oben beim Beweis des Satzes (7)).

Zum Beweis des Satzes (24) verfahren wir zunächst gerade so wie bei (23) und gelangen hierdurch zur Gleichung (28), in der jedoch $\lambda_{1}=0 \mathrm{zu}$ setzen ist. Alsdann behaupten wir, dass für $\lambda_{1}=0$ der Exponent $e$ in (28) und (26) nicht grösser sein kann als 2. Für $e>2$ müsste nämlich neben den Coefficienten von $h^{0}$ und $h^{1}$ in (28) mindestens auch derjenige von $h^{2}$ verschwinden; in Folge von $\lambda_{1}=0$ gehen aber die Gleichungen (29) und (30) über in

$$
\Psi(c, x)=0
$$

und

(32)

$$
\Psi\left(c^{\prime}, x\right)-c_{x}=0,
$$

1) Man hat wie Seite 68, Zeile 8-9 von unten, die letzte Verticalreihe des Zählers in (25) vermöge der vorausgehenden Reihen zu transformiren.

2) Vgl. die Fussnote zu S. 69. 
während das Verschwinden des Coefficienten von $h^{2}$ für $\lambda_{1}=0$ liefert:

$$
\Psi\left(c^{\prime \prime}, x\right)-c_{x}^{\prime}=0 \text {. }
$$

Jetzt sind die $c_{i}$ in (31) proportional den $p_{i}$, denn setzt man in (31) $x_{i}=\frac{1}{2} \omega^{\prime}\left(c_{i}^{\prime}\right)$, in (32) $x_{i}=\frac{1}{2} \omega^{\prime}\left(c_{i}\right)$ und zieht beide Gleichungen von einander ab, so bleibt $\omega(c, c)=0$. Dagegen sind die $c_{i}^{\prime}$ in (32) nicht den $p_{i}$ proportional, sonst wäre auch $\Psi\left(c^{\prime}, x\right)=0$, somit wegen (32) $p_{x} \equiv 0$, was nicht denkbar. Wird nun in (32) gesetzt $x_{i}=\frac{1}{2} \omega^{\prime}\left(c_{i}^{\prime \prime}\right)$, in (33) $x_{i}=\frac{1}{2} \omega^{\prime}\left(c_{i}^{\prime}\right)$, so erhält man durch Subtraction $\omega\left(c^{\prime}, c^{\prime}\right)=0$, was nicht statthaben kann, denn die $c_{i}^{\prime}$ sind den $p_{i}$ nicht proportional.

Für $\lambda_{1}=0$ kann folglich $e$ in (26) nicht grösser sein als 2, u. s. w. (wie beim Beweis des Satzes (8)).

Wir wollen noch bemerken, dass die Sätze (4), (7) und (8) auch dann bestehen bleiben, wenn an Stelle der Formen $\varphi\left(u_{1}, u_{2}, \ldots u_{n}\right)$ und $\omega\left(u_{1}, u_{2}, \ldots u_{n}\right)$ zwei bilineare Formen treten

$$
\varphi(z, \xi) \equiv \sum_{1}^{n} \sum_{1}^{n} \alpha_{i k} z_{i} \xi_{k} \quad \text { und } \omega(z, \xi) \equiv \sum_{1}^{n} \sum_{1}^{n} \omega_{i k} z_{i} \xi_{k},
$$

welche folgende Bedingungen erfolgen: $\alpha_{i k}$ muss complex conjugirt sein zu $\alpha_{k i}$, analog $\omega_{i k} \mathrm{zu} \omega_{k i}$, so dass also $\alpha_{i i}$ und $\omega_{i i}$ reell sind; ferner müssen $z_{z}$ und $\xi_{k}$ complex conjugirte Werthe haben, z. B.

$$
z_{k}=x_{k}+y_{k} \sqrt{-1}, \quad \xi_{k}=x_{k}-y_{k} \sqrt{-1}
$$

endlich darf die Form $\omega(z, \xi)$ (eventuell auch mit verschwindender Determinante) nur Zahlenwerthe von gleichem Vorzeichen annehmen. Der Beweis obiger Behauptung beruht auch hier auf dem Satze, dass, wenn die Form $\boldsymbol{\omega}(z, \zeta)$ für zwei bestimmte Werthsysteme

$$
p_{k}=p_{k}^{0}+p_{k}^{\prime} \sqrt{-1} \text { und } \pi_{k}=p_{k}^{0}-p_{k}^{\prime} \sqrt{-1}
$$

der Variabeln $z_{k}$ und $\xi_{k}(k=1,2, \ldots n)$ verschwindet, immer auch $\omega(p, \xi)$ und $\omega(z, \pi)$ identisch verschwinden $\left.{ }^{1}\right)$.

Es möge noch besonders der Fall hervorgehoben werden, dass in der Form $\varphi(z, \xi)$ die Coefficienten $\alpha_{11}, \alpha_{22}, \ldots \alpha_{n n}$ sämmtlich Null, dagegen $\alpha_{i k}(i \gtrless k)$ rein imaginär sind, so dass $\alpha_{i k}=i \beta_{i k}, \alpha_{k i}=-i \beta_{i k}$

1) Für den Fall, dass $\sum \pm\left(\omega_{11} \omega_{22} \ldots \omega_{n n}\right) \gtrless 0$ hat Herr Christoffel einen bindenden algebraischen Beweis der verallgemeinerten Sätze (4) und (7) gegeben: „Verallgemeinerung einiger Theoreme des Herrn Weierstrass", Journal für die reine und angewandte Mathematik, Bd. 63, S. 255-272, 1864. 
und $\varphi(z, \xi)$ eine "alternirende bilineare Form" darstellt. In der nun analog zu (4a) gebildeten Determinante sind $\omega_{11}, \omega_{22}, \ldots \omega_{n n}$ die Diagonalelemente, die übrigen Elemente sind von der Form $-\lambda i \beta_{i k}+\omega_{i k}$, bezw. $+\lambda i \beta_{i k}+\omega_{k i}$. Es folgt daher der Satz: Wenn die oben definirte Form $\omega(z, \xi)$ definit ist und die bilineare Form $\sum_{1}^{n} \sum_{1}^{n} \beta_{i k} z_{i} \xi_{k}$ reelle Coefficienten hat der Art, dass $\beta_{i i}=0, \beta_{i k}=-\beta_{k i}$ ist, so ergibt die Determinante von $\omega(z, \xi)-\mu \sum_{i}^{n} \sum_{i}^{n} \beta_{i k} z_{i} \xi_{k}$ gleich Null gesetzt eine Gleichung, welche rein imaginäre Wurzeln $\mu=\lambda i$ hat und für welche die weiteren in (7) und (8) ausgedrückten Eigenschaften bestehen ${ }^{1}$.

Zum Schluss dieses der Algebra gewidmeten Paragraphen sei noch ein Satz erwähnt, der zwar mit den vorhergehenden Theoremen in keinem Zusammenhang steht, aber hier Stelle finden soll, weil er bei späteren Betrachtungen ein wichtiges algebraisches Hilfsmittel bildet. Derselbe lautet:

(34) Wenn zwei ganze homogene Functionen $7^{\text {ten }}$ Grades der $n$ Variabeln $x_{1}, x_{2}, \ldots x_{n}$ für alle Werthe der $x$ identisch sind, so sind die Coefficienten gleicher Potenzen der Variabeln einander gleich.

Zum Beweis dieses Satzes denkt man sich etwa $x_{2}, x_{3}, \ldots x_{n}$ beliebig fixirt, $x_{1}$ veränderlich; dann müssen nach einem bekannten Fundamentaltheorem die Coefficienten der gleichen Potenzen von $x_{1}$ identisch sein; alsdann fixirt man nur $x_{3}, x_{4}^{0}, \ldots x_{n}$ und lässt $x_{2}$ veränderlich u. s. w.

Ist z. B. $\sum_{1}^{3} \sum_{1}^{3} a_{i k} x_{i} x_{k}$ für alle Werthe der $x$ gleich

$$
\sum_{1}^{3} \sum_{1}^{3} b_{i k} x_{i} x_{k},
$$

so muss $a_{i k}=b_{i k}(i, k=1,2,3)$ sein. Daraus folgt nach Multiplication der gleichen Coefficienten $a_{i k}$ und $b_{i k}$ mit beliebigen Zahlen

1) Vergleiche Weierstrass: „Ueber ein die homogenen Functionen zweiten Grades betreffendes Theorem", Monatsberichte der Akademie der Wissenschaften zu Berlin, Jahrgang 1879, S. 430-439, sowie Lipschitz: „Beiträge zu der Theorie der gleichzeitigen Transformation von zwei quadratischen oder bilinearen Formen", Sitzungsberichte der Akademie der Wissenschaften zu Berlin, 1. Halbband des Jahrgangs 1890, S. $509 \mathrm{f}$. 
$\alpha_{i k}$ die Gleichung

$$
\begin{aligned}
& a_{11} \alpha_{11}+2 a_{12} \alpha_{12}+a_{22} \alpha_{22}+2 a_{13} \alpha_{13}+\cdots \\
= & b_{11} \alpha_{11}+2 b_{12} \alpha_{12}+b_{22} \alpha_{22}+2 b_{13} \alpha_{13}+\cdots
\end{aligned}
$$

Dieses specielle Verfahren zeigt, dass man den Satz (34) allgemein auch in folgender Form aussprechen kann:

(35) Wenn eine homogene Gleichung $k^{\text {ten }}$ Grades zwischen den $n$ Variabeln $x_{1}, x_{2}, \ldots x_{n}$ für alle Werthe der $x$ besteht, so bleibt sie auch noch richtig, wenn man die Producte der Variabeln durch die Coefficienten einer beliebigen Form $k^{\text {ten }}$ Grades von $n$ Veränderlichen ersetzt.

\section{$\S 9$.}

\section{Ein Excurs über Invarianten.}

Um eine Erklärung des Begriffs der Invariante zu geben, knüpft man am besten an das Multiplikationstheorem der Determinanten an. Wenn $n$ lineare Ausdrücke

$$
u_{i} \equiv a_{1 i} x_{1}+a_{2 i} x_{2}+\cdots+a_{n i} x_{n}(i=1,2, \ldots n),
$$

wobei im allgemeinen $a_{i k} \gtreqless a_{k i}$ sei, durch die Substitution

$$
x_{i}=\alpha_{i} X_{1}+\beta_{i} X_{2}+\cdots+\pi_{i} X_{n}(i=1,2, \ldots n) .
$$

ïbergehen in

$$
\mathfrak{u}_{i}=\mathfrak{a}_{1 i} X_{1}+\mathfrak{a}_{2 i} X_{2}+\cdots+\mathfrak{a}_{n i} X_{n}(i=1,2, \ldots n),
$$

so ist nach dem Multipliçationstheorem der Determinanten wobei

$$
\sum \pm\left(\mathfrak{a}_{\mathfrak{2 1}} \mathfrak{a}_{22} \cdots \mathfrak{a}_{n n}\right)=r \cdot \sum \pm\left(a_{11} a_{22} \cdots a_{n n}\right)
$$

die Determinante der Substitutionen (2) bezeichnet.

Wir bilden nun mit Hilfe eines neuen Systems von Veränderlichen $y_{1}, y_{2}, \ldots y_{n}$ den linearen Ausdruck

$$
\mathfrak{u}_{1} y_{1}+\mathfrak{u}_{2} y_{2}+\cdots+\mathfrak{H}_{n} y_{n}
$$

und transformiren denselben durch die Substitution

$$
y_{k}=\alpha_{k}{ }^{\prime} Y_{1}+\beta_{k}{ }^{\prime} Y_{2}+\cdots+\pi_{k}{ }^{\prime} Y_{n}(k=1,2, \ldots n),
$$

so dass sich (6) verwandelt in

$$
\sum_{1}^{n} \sum_{1}^{n} A_{i k} X_{i} Y_{k}
$$


wobei

$$
\left\{\begin{array}{l}
A_{i 1}=\mathfrak{a}_{i 1} \alpha_{1}^{\prime}+\mathfrak{a}_{i 2} \alpha_{2}{ }^{\prime}+\cdots+\mathfrak{a}_{i n} \boldsymbol{\alpha}_{n}{ }^{\prime} \\
A_{i 2}=\mathfrak{a}_{i 1} \beta_{1}{ }^{\prime}+\mathfrak{a}_{i 2} \beta_{2}{ }^{\prime}+\cdots+\mathfrak{a}_{i n} \beta_{n}{ }^{\prime} \\
\cdot \cdot \cdot \cdot \cdot \cdot \cdot \cdot \cdot \cdot \cdot \cdot \cdot \\
A_{i n}=\mathfrak{a}_{i 1} \pi_{1}^{\prime}+\mathfrak{a}_{i 2} \pi_{2}^{\prime}+\cdots+\mathfrak{a}_{i n} \pi_{n}{ }^{\prime} .
\end{array}\right.
$$

Alsdann ist nach (4) mit den entsprechenden Vertauschungen, resp. direct:

$$
\begin{aligned}
\sum \pm\left(A_{11} A_{22} \cdots A_{n n}\right) & =r^{\prime} \sum \pm\left(\mathfrak{a}_{11} \mathfrak{a}_{22} \cdots \mathfrak{a}_{n n}\right) \\
& =r r^{\prime} \sum \pm\left(a_{11} a_{22} \cdots a_{n n}\right),
\end{aligned}
$$

wobei

$$
r^{\prime}=\sum \pm\left(\alpha_{1}^{\prime} \beta_{2}{ }^{\prime} \cdots \pi_{n}{ }^{\prime}\right)
$$

die Determinante der Substitutionen (7) bezeichnet.

Hierbei lassen sich die $A_{i k}$ auch dadurch definiren, dass man sagt, durch die Substitutionen (2) und (7) sei der Ausdruck

$$
u_{1} y_{1}+u_{2} y_{2}+\cdots+u_{n} y_{n} \quad \text { oder } \sum_{1}^{n} \sum_{1}^{n} a_{i k} x_{i} y_{k}
$$

übergeführt worden in

$$
\sum_{i}^{n} \sum_{i}^{n} A_{i k} X_{i} Y_{k}
$$

Die Determinante

$$
\sum \pm\left(a_{11} a_{22} \cdots a_{n n}\right)
$$

ist nach dem Vorausgehenden eine derartige Verbindung der Coefficienten der gegebenen $n$ Formen (1), dass bei Bildung des entsprechenden Ausdrucks mit den Coefficienten der linear transformirten Formen (3) sich (14) zufolge (4) reproducirt bis auf die erste Potenz der Substitutionsdeterminante (5).

Allgemein nennt man nun Invariante eine solche Function der Coefficienten einer oder mehrerer Formen, welche, gebildet für die Coefficienten der durch eine und dieselbe Substitution linear transformirten Formen, sich bis auf eine Potenz der Substitutionsdeterminante reproducirt. Eine Invariante ist daher eine Function $\varphi$ jener Coefficienten $a_{i k} \cdot$ resp. $\mathfrak{a}_{i k}$, welche die Gleichung erfüllt

$$
\varphi\left(\mathfrak{a}_{i k}\right)=r^{\lambda} \cdot \varphi\left(a_{i k}\right) .
$$

Den Exponenten $\lambda$ der Substitutionsdeterminante nennt man das Gewicht des betreffenden invarianten Ausdrucks. 
Als absolute Invariante bezeichnet man eine solche Function der Coefficienten, .welche bei linearer Transformation völlig ungeändert bleibt, also das Gewicht $\lambda=0$ besitzt.

Enthält eine Verbindung von der Form (15) ausser den Coefficienten auch noch die Variabeln, so wird sie als Covariante bezeichnet; letztere würde daher eine Gleichung erfüllen von der Gestalt

$$
\varphi\left(\mathfrak{a}_{i k} ; X_{1}, X_{2}, \cdots X_{n}\right)=r^{\lambda} \cdot \varphi\left(a_{i k} ; x_{1}, x_{2} \cdots x_{n}\right) \text {. }
$$

Setzt man in (12) speciell $a_{i k}=a_{k i}$, sowie $x_{i}=y_{i}$ und $X_{i}=Y_{i}$ $(i=1,2, \ldots n)$, so verwandelt sich $(12)$ in eine quadratische Form von $n$ Variabeln $x_{1}, x_{2}, \ldots x_{n}$ :

$$
f(x, x) \equiv \sum_{1}^{n} \sum_{1}^{n} a_{i k} x_{i} x_{k},
$$

welche durch die Substitutionen (2) übergeführt wird in

$$
F(X, X) \equiv \sum_{i}^{n} \sum_{i}^{n} A_{i k} X_{i} X_{k}
$$

die beiden Transformationen (2) und (7). werden dann einander gleich und daher $r=r^{\prime}$.

Die Gleichung $f(x, x)=F(X, X)$ gilt nun, welche Werthe die $x$ und in Folge davon auch die $X$ haben mögen, sie gilt daher noch, wenn man $x_{i}$ ersetzt durch $x_{i}+\lambda y_{i}$ und $X_{i}$ durch $X_{i}+\lambda Y_{i}$, es ist

$$
\begin{aligned}
f\left(x_{1}+\lambda y_{1}, x_{2}+\lambda y_{2}, \cdots x_{n}+\lambda y_{n}\right) \\
\quad=F\left(X_{1}+\lambda Y_{1}, X_{2}+\lambda Y_{2}, \cdots X_{n}+\lambda Y_{n}\right)
\end{aligned}
$$

und da diese Gleichung für ganz beliebige Werthe des Parameters $\lambda$ stattfindet, müssen die Coefficienten gleicher Potenzen von $\lambda$ links und rechts einander gleich sein. Berücksichtigen wir nur die erste Potenz, so ist demnach

$$
\frac{\partial f}{\partial x_{1}} y_{1}+\frac{\partial f}{\partial x_{2}} y_{2}+\cdots+\frac{\partial f}{\partial x_{n}} y_{n}=\frac{\partial F}{\partial X_{1}} Y_{1}+\frac{\partial F}{\partial X_{2}} Y_{2}+\cdots+\frac{\partial F}{\partial X_{n}} Y_{n}
$$

durch Anwendung der Formel (10) folgt alsdann

$$
\sum \pm\left(A_{11} A_{22} \cdots A_{n n}\right)=r^{2} \cdot \sum \pm\left(a_{11} a_{22} \cdots a_{n n}\right) \text {. }
$$

Die hier auftretenden Determinanten $\operatorname{der} A_{i k}$ resp. $a_{i k}$ nennt man die Discriminante der Formen $(18)$, resp. $\left.(17) ;^{1}\right)$ wie aus (21) er-

1) Wir sind dieser Discriminante im Falle dreier Variabeln bereits in $\S 4$ begegnet; es wurde dort gezeigt, dass ihr Verschwinden die Ausartung des Kegelschnitts zur Folge hat. 
sichtlich, ist eine solche Discriminante zugleich eine Invariante, und zwar vom Gewicht 2.

Man kann auch, statt von einer einzigen Form $f(x, x)$ auszugehen, die lineare Combination $\lambda g(x, x)-f(x, x)$ oder ausführlicher

$$
\lambda \sum_{i}^{n} \sum_{i}^{n} b_{i k} x_{i} x_{k}-\sum_{i}^{n} \sum_{1}^{n} a_{i k} x_{i} x_{k}
$$

zu Grund legen; dieselbe werde kurz durch

$$
h(x, x) \equiv \sum_{1}^{n} \sum_{1}^{n} c_{i k} x_{i} x_{k}
$$

bezeichnet, wobei $c_{i k}=\lambda b_{i k}-a_{i k}$. Würde jetzt $h(x, x)$ durch die Substitution (2) transformirt in

so wäre nach (21):

$$
H(X, X)=\sum_{1}^{n} \sum_{i}^{n} C_{i k} X_{i} X_{k}
$$

$$
\sum \pm\left(C_{11} C_{22} \cdots C_{n n}\right)=r^{2} \cdot \sum \pm\left(c_{11} c_{22} \cdots c_{n n}\right)
$$

Die zwei hier auftretenden Discriminanten enthalten nun die $\lambda$ bis zum $n^{\text {ten }}$ Grade, und da (22) gilt, welche Werthe man auch dem Parameter $\lambda$ ertheilen mag, müssen die Coefficienten gleicher Potenzen von $\lambda$ links und rechts einander gleich sein. Die auf solche Weise einander entsprechenden Coefficienten sind überdies beiderseits gleich gebaut, nur besitzen die rechts stehenden noch den Factor $r^{2}$, die genannten Coefficienten müssen also Invarianten sein; sie heissen simultane Invarianten des Systems der beiden Formen $f(x, x)$ und $g(x, x)$.

In dem für das später Folgende wichtigen Falle dreier Variabeln $x_{1}, x_{2}, x_{3}$ erhält man

$$
\sum \pm\left(c_{11} c_{22} c_{33}\right)=\lambda^{3} B-3 \lambda^{2} \Theta+3 \lambda \mathrm{H}-A
$$

wo $A$ und $B$ die Discriminanten von $f(x, x)$ resp. $g(x, x)$ bedeuten, während $\Theta$ und $H$, wie die Ausrechnung zeigt, bestimmt sind durch

$$
\left\{\begin{array}{l}
3 \Theta=a_{11} B_{11}+a_{22} B_{22}+a_{33} B_{33}+2 a_{12} B_{12}+2 a_{13} B_{13}+2 a_{23} B_{23} \\
3 \mathrm{H}=b_{11} A_{11}+b_{22} A_{22}+b_{33} A_{33}+2 b_{12} A_{12}+2 b_{13} A_{13}+2 b_{23} A_{23}
\end{array} .\right.
$$

Hierbei sind die $A_{i k}$ und $B_{i k}$ die Unterdeterminanten der Elemente $a_{i k}$ und $b_{i k}$ in den Determinanten $A$ resp. $B$.

Die Ausdrücke (24) geben somit Beispiele ab für simultane In- 
varianten der beiden Formen $f(x, x)$ und $g(x, x)$; das Gewicht ist jedesmal gleich 2 .

Auch für Covarianten möge noch ein Beispiel gegeben werden, das überhaupt zeigt, wie man eine ganze Reihe solcher Functionen erhalten kann. Statt der quadratischen Formen (17) und (18) seien Formen beliebiger Ordnung gegeben; auf gleichem Wege wie oben gelangt man zu (19), und hieraus durch Vergleich der Coefficienten von $\lambda^{2} \mathrm{zu}$

$$
\frac{\partial^{2} f}{\partial x_{1}{ }^{2}} y_{1}{ }^{2}+2 \frac{\partial^{2} f}{\partial x_{1} \cdot \partial x_{2}} y_{1} y_{2}+\cdots=\frac{\partial^{2} F}{\partial X_{1}{ }^{2}} Y_{1}^{2}+2 \frac{\partial^{2} F}{\partial X_{1} \cdot \partial X_{2}} Y_{1} Y_{2}+\cdots
$$

Denkt man sich hier den $x_{1}, x_{2}, \ldots x_{n}$ irgend welche bestimmte Werthe ertheilt und $\frac{\partial^{2} f}{\partial x_{i} \partial x_{k}}=a_{i k}$ gesetzt, so würde (25) aussagen, dass eine quadratische Form $\sum_{1}^{n} \sum_{1}^{n} a_{i k} y_{i} y_{k}$ durch die Substitutionen (2) ${ }^{1}$ ) übergeführt sei in $\sum_{1}^{n} \sum_{1}^{n} A_{i k} Y_{i} Y_{k}$; dann ist auch

$$
r^{2} \sum \pm\left(a_{11} a_{22} \cdots a_{n n}\right)=\sum \pm\left(A_{11} A_{22} \cdots A_{n n}\right)
$$

nach Wiedereinführung der zweiten Differentialquotienten verwandelt sich aber diese Relation in

$$
r^{2} \cdot \sum \pm\left(\frac{\partial^{2} f}{\partial x_{1}{ }^{2}} \frac{\partial^{2} f}{\partial x_{2}{ }^{2}} \cdots \frac{\partial^{2} f}{\partial x_{n}{ }^{2}}\right)=\sum \pm\left(\frac{\partial^{2} F}{\partial X_{1}{ }^{2}} \frac{\partial^{2} F}{\partial X_{2}{ }^{2}} \cdots \frac{\partial^{2} F}{\partial X_{n}{ }^{2}}\right)
$$

Die hierdurch definirte Covariante der Form $n^{\text {ter }}$ Ordnung $f\left(x_{1}, x_{2} \ldots x_{n}\right)$ ist also die aus den zweiten partiellen Differentialquotienten von $f$ gebildete Determinante und wird die Hesse'sche Covariante oder Determinante von $f$ genannt; sie wird zur Discriminante, wenn $f$ von der zweiten Ordnung ist.

Es möge noch erwähnt werden ein Beispiel für eine simultane Invariante einer quadratischen und beliebig vieler linearer Formen von $n$ Variabeln, um zu zeigen, wie man noch zu einer anderen Art von invarianten Bildungen, zu den sogenannten Contravarianten gelangt. Der Einfachheit halber seien ausser der quadratischen Form (17) nur zwei lineare Formen gegeben, nämlich

$$
\left\{\begin{aligned}
v_{x} & \equiv v_{1} x_{1}+v_{2} x_{2}+\cdots+v_{n} x_{n} \\
\text { und } w_{x} & \equiv w_{1} x_{1}+w_{2} x_{2}+\cdots+w_{n} x_{n} .
\end{aligned}\right.
$$

1) Es ist in (2) jedoch zu ersetzen $x_{i}$ durch $y_{i}, X_{i}$ durch $Y_{i}(i=1,2, \ldots n)$. 
Simultane Invariante einer quadratischen and mehrerer linearer Formen.

Es wird sich dann zeigen, dass die Determinante

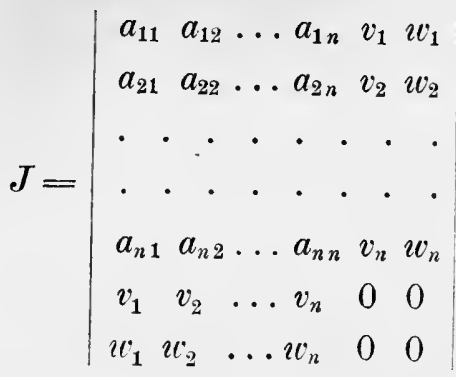

eine simultane Invariante der Formen $f(x, x), v_{x}$ und $w_{x}$ ist.

Zum Beweis führen wir mit Hilfe der beiden weiteren Variabeln $x_{n+1}$ und $x_{n+2}$ die Form ein:

$$
\varphi(x, x)=f(x, x)+2 x_{n+1} v_{x}+2 x_{n+2} v_{x}
$$

und transformiren dieselbe durch

$$
\left\{\begin{array}{l}
x_{i}=\alpha_{i} X_{1}+\beta_{i} X_{2}+\cdots+\pi_{i} X_{n}(i=1,2, \ldots n) \\
x_{n+1}=X_{n+1} \\
x_{n+2}=X_{n+2}
\end{array}\right.
$$

wodurch (29) übergehen möge in

$$
\begin{aligned}
& \Phi(X, X)= \\
& \sum_{1}^{n} \sum_{1}^{n} A_{i k} X_{i} X_{k}+2 X_{n+1}\left(V_{1} X_{1}+V_{2} X_{2}+\cdots+V_{n} X_{n}\right) \\
& +2 X_{n+2}\left(W_{1} X_{1}+W_{2} X_{2}+\cdots+W_{n} X_{n}\right) .
\end{aligned}
$$

Die Determinante der $n+2$ Substitutionen (30) ist hierbei, wie man leicht sieht, keine andere, als die früher mit $r$ bezeichnete Determinante der $n$ Substitutionen

$$
x_{i}=\alpha_{i} X_{1}+\beta_{i} X_{2}+\cdots+\pi_{i} X_{n}
$$

allein; ferner erkennt man, dass die Discriminante von (29) identisch ist mit dem durch (28) defiuirten Ausdruck $J$, während die Discriminante von (31) gegeben ist durch

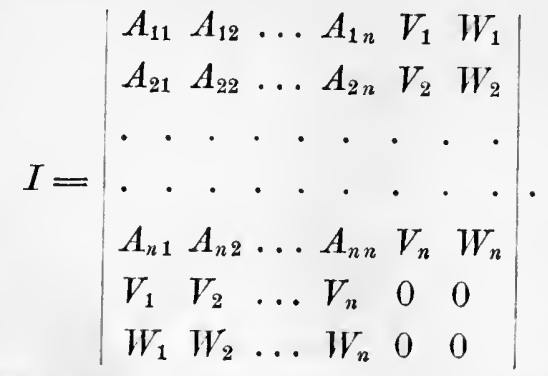

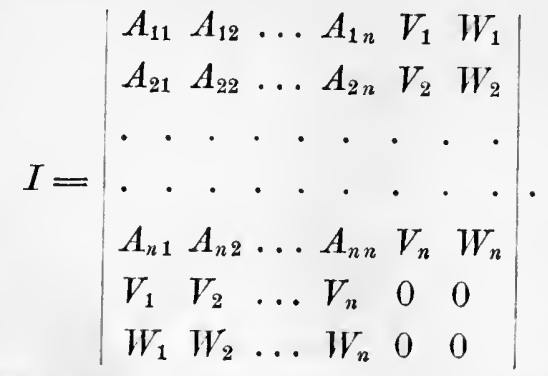


In Analogie zu (21) besteht aber die Relation

$$
I=r^{2} J
$$

es ist daher $J$ eine Invariante der Form (29) allein, oder auch der Formen $f(x, x), v_{x}$ und $w_{x}$, wenn wir die nur hilfsweise eingeführten Variabeln $x_{n+1}$ und $x_{n+2}$ wieder bei Seite lassen.

Fügt man statt nur zweier linearer Formen $v_{x}$ und $w_{x}$ zu $f(x, x)$ beliebig viele hinzu, so erkennt man aus dem Vorhergehenden nicht nur, in welcher Weise auch dann die zu (28) analoge Determinante angeordnet ist, sondern man sieht auch, dass diese Determinante eine simultane Invariante der Form $f(x, x)$ und der beliebig vielen hinzugefügten linearen Formen ist.

Beschränken wir uns auf den Fall einer quadratischen Form $f(x, x)$ und einer linearen Form von je drei Variabeln $x_{1}, x_{2}, x_{3}$, so ist die Determinante

$$
\left|\begin{array}{llll}
a_{11} & a_{12} & a_{13} & u_{1} \\
a_{21} & a_{22} & a_{23} & u_{2} \\
a_{31} & a_{32} & a_{33} & u_{3} \\
u_{1} & u_{2} & u_{3} & 0
\end{array}\right|
$$

jedenfalls eine Invariante vom Gewicht 2 ; dabei ist es ganz gleichgiltig, welche Werthe oder was für eine Bedeutung die $u_{1}, u_{2}, u_{3}$ haben. Fassen wir nun die gleich Null gesetzte Form $f(x, x)$ als Gleichung einer Curve zweiter Ordnung auf, $u_{x}=0$ als Gleichung einer Geraden, so sind die $u_{i}$ variabele Liniencoordinaten, und (34) stellt gleich Null gesetzt nach $\S 4$ die Gleichung des Kegelschnitts $f(x, x)=0$ in Liniencoordinaten dar.

Die Invariante (34) ist ein Beispiel aus einer grossen Gattung von Invarianten, zu denen man in folgender Weise gelangt: Es seien mehrere Formen beliebiger Ordnung und mit beliebig vielen Veränderlichen $x_{1}, x_{2}, \ldots x_{n}$ gegeben, darunter mindestens eine lineare Form $u_{1} x_{1}+u_{2} x_{2}+\cdots+u_{n} x_{n}$; sie werden sämmtlich der Transformation (2) unterworfen, wodurch speciell $u_{x}$ übergehe in

$$
U_{1} X_{1}+U_{2} X_{2}+\cdots+U_{n} X_{n}
$$

wobei alsdann

$$
\left\{\begin{array}{l}
U_{1}=\alpha_{1} u_{1}+\alpha_{2} u_{2}+\cdots+\alpha_{n} u_{n} \\
U_{2}=\beta_{1} u_{1}+\beta_{2} u_{2}+\cdots+\beta_{n} u_{n} \\
\cdot \cdot \cdot \cdot \cdot \cdot \cdot \cdot \cdot \cdot \cdot \cdot \\
U_{n}=\pi_{1} u_{1}+\pi_{2} u_{2}+\cdots+\pi_{n} u_{n}
\end{array}\right.
$$


Irgend eine Invariante des gegebenen Formensystems erfüllt nach (15) eine Gleichung von der Gestalt

$$
r^{2} \cdot J\left(a_{i k} ; u_{1}, u_{2}, \ldots u_{n}\right)=J\left(\mathfrak{a}_{i k} ; U_{1}, U_{2}, \ldots U_{n}\right),
$$

und man nennt eine Function der Coefficienten $a_{i k}$ einer oder mehrerer Formen $f(x, x)$, u. s. w., sowie eines Systems von Veränderlichen $u_{1}, u_{2}, \ldots u_{n}$ (für $n=3$ : Liniencoordinaten), welche durch die sogenannte "transponirte Substitution" (35) mit den neuen Veränderlichen $U_{1}, U_{2} \ldots U_{n}$ zusammenhängen, eine Contravariante der Formen mit den Coefficienten $a_{i k}$, wenn jene Function einer Gleichung wie (36) genügt.

$\mathrm{Z}$ wischenformen nennt man mit Invarianteneigenschaft versehene Functionen der Coefficienten $a_{i k}, \ldots$ und der Veränderlichen $x_{1}, x_{2}, \ldots x_{n}$ melhrerer simultaner Formen, unter denen wieder eine identisch ist mit $u_{1} x_{1}+u_{2} x_{2}+\cdots+u_{n} x_{n}$; demnach sind die Zwischenformen auch Functionen der $u_{1}, u_{2}, \ldots u_{n}$. Geometrisch würde dies im Falle dreier Variabeln bedeuten, dass. eine Function $J$ der Coefficienten einer oder mehrerer Formen vorläge, welche überdies die Coordinaten eines veränderlichen Punktes and einer veränderlichen Geraden enthält und gleich Null gesetzt eine rom Coordinatendreieck unabhängige Eigenschaft der einem bestimmten Punkte $x$, resp. einer bestimmten Geraden $u$ zugehörigen Curve ausdrückt.

Zum Schluss dieses Paragraphen wollen wir noch als eine Anwendung der Gleichung (21) zeigen, dass und auf welche Weise die Gleichung eines Kegelschnitts in Punktcoordinaten

$$
f(x, x) \equiv \sum_{1}^{3} \sum_{1}^{3} a_{i k} x_{i} x_{k}=0 \quad\left(a_{i k}=a_{k i}\right)
$$

in die Form gebracht werden kann (vgl. (10), § 4 und (25), §5):

$$
X_{1} X_{3}-c \cdot X_{2}^{2}=0 \text {, }
$$

in welcher $c$ ein Zahlenfactor ist und $X_{1}=0, X_{2}=0, X_{3}=0$ die Gleichungen dreier Geraden bedeuten, von denen $X_{1}=0$ und $X_{3}=0$ die Curve je in zwei zusammenfallenden Punkten schneiden, die auf der Geraden $X_{2}=0$ gelegen sind, d. h. $X_{1}=0$ und $X_{3}=0$ sind Tangenten des Kegelschnitts, $X_{2}=0$ die zugehörige Berührungssehne. Dass eine Transformation der Gleichung $f(x, x)=0$ in die Form $X_{1} X_{3}-X_{2}{ }^{2}=0$ möglich ist, unterliegt nach dieser Bemerkung keinem Zweifel mehr: man hat nur die beiden Tangenten und die Berührungssehne als Seiten des neuen Coordinatendreiecks zu Grunde zu legen. Es bleibt daher nur noch zu zeigen, in welcher Weise die Transformation durchzuführen ist, wenn die Coordinaten der beiden 
Berührungspunkte, die offenbar auch zur Fixirung des neuen Coordinatendreiecks ausreichen, gegeben sind.

Es seien $y_{1}, y_{2}, y_{3}$ und $z_{1}, z_{2}, z_{3}$ die Coordinaten dieser beiden Punkte, $t_{1}, t_{2}, t_{3}$ drei zunächst ganz willkürliche Grössen; alsdann denken wir uns die Gleichung $f(x, x)=0$ der Transformation unterworfen

$$
\left\{\begin{array}{l}
x_{1}=t_{1} \xi_{1}+y_{1} \xi_{2}+z_{1} \xi_{3} \\
x_{2}=t_{2} \xi_{1}+y_{2} \xi_{2}+z_{2} \xi_{3} \\
x_{3}=t_{3} \xi_{1}+y_{3} \xi_{2}+z_{3} \xi_{3}
\end{array}\right.
$$

wodurch sich $f(x, x)$ verwandelt in:

$$
\begin{aligned}
f(t, t) \cdot \xi_{1}{ }^{2} & +2 f(t, y) \cdot \xi_{1} \xi_{2}+f(y, y) \cdot \xi_{2}{ }^{2}+2 f(t, z) \cdot \xi_{1} \xi_{3} \\
& +2 f(y, z) \cdot \xi_{2} \xi_{3}+f(z, z) \cdot \xi_{3}{ }^{2}=0 .
\end{aligned}
$$

Hier fallen nun sofort die Glieder $f(y, y) \cdot \xi_{2}{ }^{2}$ und $f(z, z) \cdot \xi_{3}{ }^{2}$ weg, da die Punkte $y$ und $z$ auf $f(x, x)=0$ gelegen sind. Ferner besteht zufolge (21) dis Relation

$$
A \cdot \sum \pm\left(t_{1} y_{2} z_{3}\right)^{2}=\left|\begin{array}{lll}
f(t, t) & f(t, y) & f(t, z) \\
f(t, y) & 0 & f(y, z) \\
f(t, z) & f(y, z) & 0
\end{array}\right|
$$

wobei $A=\sum \pm\left(a_{11} a_{22} a_{33}\right)$, und aus dieser Gleichung folgt

$$
f^{2}(y, z) \cdot f(t, t)=2 f(y, z) \cdot f(z, t) \cdot f(t, y)-A \cdot \sum \pm\left(t_{1} y_{2} z_{3}\right)^{2},
$$

und wenn wir statt der willkürlichen Grössen $t_{i}$ überall $x_{i}$ setzen, so hat man

$$
f^{2}(y, z) \cdot f(x, x)=2 f(y, z) \cdot f(x, y) \cdot f(x, z)-A \cdot \sum \pm\left(x_{1} y_{2} z_{3}\right)^{2}
$$

Hier ist nun $f(y, z)$ ein reiner Zahlenfactor, $f(x, y)=0$ und $f(x, z)=0$ stellen nach $\S 4$ die Tangenten des Kegelschnitts in den Punkten $y$ und $z$ dar, $\sum \pm\left(x_{1} y_{2} z_{3}\right)=0$ die Berührungssehne. Setzen wir demnach

$$
f(x, y)=X_{1}, \quad f(x, z)=X_{3}, \quad \sum \pm\left(x_{1} y_{2} z_{3}\right)=X_{2},
$$

so verwandelt $\operatorname{sich} f(x, x)=0$ in

$$
2 f(y, z) \cdot X_{1} X_{3}-A X_{2}^{2}=0,
$$

und dieser Ausdruck ist in der That von derselben Form wie (38).

Analog folgt, dass die Gleichung einer Curve zweiter Classe $\varphi(u, u)=0$ in die Form gebracht werden kann

$$
2 \varphi(v, w) \cdot U_{1} U_{3}-\mathrm{A} U_{2}^{2}=0,
$$

wobei $U_{1} \equiv \varphi(u, v)=0$ und $U_{3} \equiv \varphi(u, w)=0$ die Berührungspunkte 
zweier Tangenten darstellen, deren Liniencoordinaten $v_{i} \operatorname{resp.} w_{i}(i=1,2,3)$ gegeben sind, während $U_{2} \equiv \sum \pm\left(u_{1} v_{2} w_{3}\right)=0$ die Gleichung des Schnittpunktes dieser beiden Tangenten (des Pols der Berührungssehne) ist.

\section{$\S 10$.}

Transformation der Curven zweiter Ordnung auf die Hauptaxen.

Als Hauptaxe einer Curve zweiter Ordnung

$$
\begin{aligned}
f(x, x) \equiv a_{11} x_{1}^{2}+2 a_{12} x_{1} x_{2}+a_{22} x_{2}^{2} & +2 a_{13} x_{1} x_{3} \\
& +2 a_{23} x_{2} x_{3}+a_{33} x_{33}^{2}=0
\end{aligned}
$$

möge eine im Endlichen gelegene Gerade bezeichnet werden, die mit der Polare ihres Normalencentrums zusammenfällt. Es sei

$$
u_{1} x_{1}+u_{2} x_{2}+u_{3} x_{3}=0
$$

die Gleichung einer Hauptaxe; die Coordinaten ihres Normalencentrums sind dann nach $\S 2: \frac{1}{2} \omega^{\prime}\left(u_{1}\right), \frac{1}{2} \omega^{\prime}\left(u_{2}\right), \frac{1}{2} \omega^{\prime}\left(u_{3}\right)$, und die Gleichung

$$
\frac{1}{4}\left\{\omega^{\prime}\left(u_{1}\right) f^{\prime}\left(x_{1}\right)+\omega^{\prime}\left(u_{2}\right) f^{\prime}\left(x_{2}\right)+\omega^{\prime}\left(u_{3}\right) f^{\prime}\left(x_{3}\right)\right\}=0
$$

oder

$$
\frac{1}{4} \sum_{1}^{3} \omega^{\prime}\left(u_{i}\right) \cdot f^{\prime}\left(x_{i}\right)=0
$$

stellt die Polare des Normalencentrums dar. Soll diese mit (2) zulsammenfallen, so muss die Identität stattfinden

$$
\begin{aligned}
{ }_{4}^{1}\left\{\omega^{\prime}\left(u_{1}\right) \cdot f^{\prime}\left(x_{1}\right)+\omega^{\prime}\left(u_{2}\right) \cdot f^{\prime}\left(x_{2}\right)\right. & \left.+\omega^{\prime}\left(u_{3}\right) \cdot f^{\prime \prime}\left(x_{3}\right)\right\} \\
& =\lambda\left(u_{1} x_{1}+u_{2} x_{2}+u_{3} x_{3}\right),
\end{aligned}
$$

wobei $\lambda$ ein passend zu bestimmender Proportionalitätsfactor ist ${ }^{1}$ ).

Durch Vergleichen der Coefficienten von $x_{1}, x_{2}, x_{3}$ erhält man:

$$
\sum_{1}^{3} \frac{1}{2} \omega^{\prime}\left(u_{i}\right) \cdot a_{i k}=\lambda u_{k} \quad(k=1,2,3),
$$

wofür mit Hilfe der abkürzenden Bezeichnung

$$
\alpha_{i k}=\omega_{1 i} a_{1 k}+\omega_{2 i} a_{2 k}+\omega_{3 i} a_{3 k}
$$

1) Die Zweckmässigkeit der Behandlung des Hanptaxenproblems mit Hilfe des Normalencentrums ist von Herrn Gundelfinger auch betont worden in seiner Abhandlung „Note sur un article de M. Brisse“, Nouvelles Annales de Mathématiques, Bd. 3 (3. Serie), S. 18, 1882. 
auch gesetzt werdeu kann

$$
\left.\alpha_{1 k} u_{1}+\alpha_{2 k} u_{2}+\alpha_{3 k} u_{3}=\lambda u_{k}{ }^{1}\right) \quad(k=1,2,3) .
$$

Dabei ist im allgemeinen $\alpha_{i k}$ von $\alpha_{k i}$ verschieden, wie (6) zeigt. Aus den drei Gleichungen (7) folgt durch Elimination der $u_{i}$ :

$$
\left|\begin{array}{lll}
\alpha_{11}-\lambda & \alpha_{21} & \alpha_{31} \\
\alpha_{12} & \alpha_{22}-\lambda & \alpha_{32} \\
\alpha_{13} & \alpha_{23} & \alpha_{33}-\lambda
\end{array}\right|=0
$$

eine Determinante, deren Elemente mitunter auch durch $\beta_{i k}$ bezeichnet werden sollen, so dass (8) gléichbedeutend ist mit:

(8a) $\quad \mathrm{B}(\lambda)=-\left|\begin{array}{lll}\beta_{11} & \beta_{21} & \beta_{31} \\ \beta_{12} & \beta_{22} & \beta_{32} \\ \beta_{13} & \beta_{23} & \beta_{33}\end{array}\right|=\left|\begin{array}{lll}\alpha_{11}-\lambda & \alpha_{21} & \alpha_{31} \\ \alpha_{12} & \alpha_{22}-\lambda & \alpha_{32} \\ \alpha_{13} & \alpha_{23} & \alpha_{33}-\lambda\end{array}\right|==0$.

Die Gleichung $B(\lambda)=0$ ist in den $\lambda$ vom dritten Grad, reducirt sich aber sofort auf eine solche vom zweiten Grad, da sie eine Wurzel $\lambda^{\prime \prime \prime}=0$ besitzt; ihre beiden anderen Wurzeln seien $\lambda^{\prime}$ und $\lambda^{\prime \prime}$. Das absolute Glied in (8) besitzt nämlich den Werth $\sum \pm\left(\alpha_{11} \alpha_{22} \alpha_{33}\right)$, der nach dem Multiplicationstheorem der Determinanten in das Product

$$
\sum \pm\left(\omega_{11} \omega_{22} \omega_{33}\right) \cdot \sum \pm\left(a_{11} a_{22} a_{33}\right)
$$

zerfällt, dessen erster Factor nach $(5)$ in $\S 2$ verschwindet. In Folge hiervon ist $B(\lambda)=0$ gleichbedeutend mit der Gleichung zweiten Grades:

$$
\begin{array}{r}
\lambda^{2}-\left(\alpha_{11}+\alpha_{22}+\alpha_{33}\right) \lambda+\left(\alpha_{11} \alpha_{22}-\alpha_{12} \alpha_{21}+\alpha_{22} \alpha_{33}-\alpha_{23} \alpha_{32}\right. \\
\left.+\alpha_{33} \alpha_{11}-\alpha_{31} \alpha_{13}\right)=0 .
\end{array}
$$

Mit Anwendung eines Satzes von $\mathrm{Hesse}^{2}$ ), demzufolge z. B. der Theil $\alpha_{11} \alpha_{22}-\alpha_{12} \alpha_{21}=\mathrm{A}_{33}$ des absoluten Gliedes in dieser Gleichung

1) Beiläufig werde bemerkt, dass man durch Vergleichen der Coefficienten von $u_{1}, u_{2}, u_{3}$ erbält: $\alpha_{i 1} x_{1}+\alpha_{i 2} x_{2}+\alpha_{i 3} x_{3}=\lambda x_{i}$.

2) Vgl. Hesse „Ueber Determinanten und ihre Anwendung in der Geometrie, insbesondere anf Curven vierter Ordnung". Crelle's Journal, Bd. 49, S. $243 \mathrm{f}$. (1853), oder auch die Brochüre: „Die Determinanten“, 2. Aufl., S. 47 (1872), oder auch Hesse's „Vorlesungen über analytische Geometrie des Raumes", revidirt und mit Zusätzen versehen von Gundelfinger, 3. Aufl., S. 98 f. (1876). - Nach diesem Satze von Hesse ist überhaupt die Unterdeterminante $\mathrm{A}_{i k}$ des Elementes $\alpha_{i k}$ der Determinante $\sum \pm\left(\alpha_{11} \alpha_{22} \alpha_{33}\right)$ gleich $\Omega_{1 i} A_{1 k}+\Omega_{2 i} A_{2 k}+\Omega_{3 i} A_{3 k}$, oder nach (16) in $\S 7$ gleich

$$
\tau p_{i}\left(A_{1 k} p_{1}+A_{2 k} p_{2}+A_{3 k} p_{3}\right)=\tau p_{i} \cdot \frac{1}{2} F^{\prime}\left(p_{k}\right) \text {. }
$$


gleich $\Omega_{13} A_{13}+\Omega_{23} A_{23}+\Omega_{33} A_{33}$ ist, erhält das absolute Glied die Gestalt

$$
\Omega_{11} A_{11}+2 \Omega_{12} A_{12}+\Omega_{22} A_{22}+2 \Omega_{13} A_{13}+2 \Omega_{23} A_{23}+\Omega_{33} A_{33}
$$

oder nach $(16)$ in $\S 7$ :

$$
\tau\left(A_{11} p_{1}{ }^{2}+2 A_{12} p_{1} p_{2}+A_{22} p_{2}{ }^{2}+2 A_{13} p_{1} p_{3}+2 A_{23} p_{22} p_{3}+A_{33} p_{3}{ }^{2}\right),
$$

d. h. $\tau F(p, p)$, wenn $F(u, u)=0$ die Gleichung der Curve (1) in Liniencoordinaten ist. Ferner findet man $\alpha_{11}+\alpha_{22}+\alpha_{33}$ mach (6) gleich

$$
a_{11} \omega_{11}+2 a_{12} \omega_{12}+a_{22} \omega_{22}+2 a_{13} \omega_{13}+2 a_{23} \omega_{23}+a_{33} \omega_{33},
$$

wofür wie in (36), § 7 gesetyt werden möge $[a, \omega]$, so dass sich $(10)$ verwandelt in

$$
\lambda^{2}-[a, \omega] \lambda+\tau F(p, p)=0 .
$$

Diese Gleichung lat stets reelle Wurzeln, denn sie ist mit (S) gleichbedentend; von (S) wurde aber die Realität der Wurzeln in $\$$ S nachgewiesen.

Der Fall zweier gleichen Wurzeln von (s), also auch der Fall, dass eine weitere Wurzel $\lambda=0$ von (8) auftritt, werde rorerst ausgeschlossen, und es seien $u_{1}^{\prime}, u_{2}^{\prime}, u_{3}^{\prime}$ resp. $u_{1}{ }^{\prime \prime}, u_{2}{ }^{\prime \prime}, u_{3}{ }^{\prime \prime}$ die Werthe der $u$, welche den zwei verschiedenen nicht verschwindenden Wurzeln $\lambda^{\prime}$ und $\lambda^{\prime \prime}$ von (8a) oder (11) nach (7) entsprechen. Der Wurzel $\lambda^{\prime \prime \prime}=0$ von $(8 \mathrm{a})$ würde entsprechen $\iota_{i}=p_{i}(i=1,2,3)$, denn nach $(5)$ wäre

$$
\sum_{1}^{3} \frac{1}{2} \omega^{\prime}\left(u_{i}\right) \cdot a_{i k}=0 \quad(k=1,2,3)
$$

und diese drei Gleichungen werden erfüllt durch das Werthsystem $u_{i}=p_{i}(i=1,2,3)$. Andrerseits können die $u_{i}^{\prime}$ und $u_{i}^{\prime \prime}$, so lange $\lambda^{\prime}$ und $\lambda^{\prime \prime}$ von Null verschieden sind, den $p_{i}$ nicht proportional sein, sonst wïre nach (5) $\sum_{i}^{3} \frac{1}{2} \omega^{\prime}\left(p_{i}\right) \cdot a_{i k}=\lambda p_{k} ;$ nun ist aber $\omega^{\prime}\left(p_{i}\right)=0$, mithin müsste auch $\lambda=0$ sein und (8a) hätte ausser $\lambda^{\prime \prime \prime}$ eine weitere verschwindende Wurzel.

Die Geraden

$$
u_{1}^{\prime} x_{1}+u_{2}^{\prime} x_{2}+u_{3}^{\prime} x_{3}=0 \text { und } u_{1}^{\prime \prime} x_{1}+u_{2}^{\prime \prime} x_{2}+u_{3}^{\prime \prime} x_{3}=\dot{0}
$$

sind hiernach die Hauptaxen der Curve (1), ${ }^{1}$ ) während die unendlich ferne Gerade $p_{1} x_{1}+p_{2} x_{2}+p_{3} x_{3}=0$ als eine uneigentliche Hauptaxe

1) Unter den obigen Voraussetzungen gibt es auch nur je ein Werthsystem der $u_{i}^{\prime}$ und eines der $u_{i}^{\prime \prime}$, denn wären zwei der Gleichungen (7) eine Folge der 
(der Wurzel $\lambda^{\prime \prime \prime}=0$ entsprechend) angesehen werden kann, da sie keine bestimmte Stellung in der Ebene hat.

Ersetzt man in (5) $\lambda$ durch $\lambda^{\prime}$, die $u_{i}$ durch $u_{i}^{\prime}$, multiplicirt man ferner (5) mit $\frac{1}{2} \omega^{\prime}\left(u_{k}^{\prime \prime}\right)$ und summirt hierauf über $k$, so entsteht

$$
\lambda^{\prime} \sum_{1}^{3} k u_{k}^{\prime} \cdot \frac{1}{2} \omega^{\prime}\left(u_{k}^{\prime \prime}\right)=\sum_{1}^{3} \sum_{1}^{3} \frac{1}{2} \omega^{\prime}\left(u_{i}^{\prime}\right) \cdot a_{i k} \cdot \frac{1}{2} \omega^{\prime}\left(u_{k}^{\prime \prime}\right),
$$

und auf analoge Weise erhält man auch

$$
\lambda^{\prime \prime} \sum_{1}^{3} u_{k}^{\prime \prime} \cdot{ }_{2}^{1} \omega^{\prime}\left(u_{k}^{\prime}\right)=\sum_{1}^{3} \sum_{1}^{3} \frac{1}{2} \omega^{\prime}\left(u_{k}^{\prime}\right) \cdot a_{k i} \cdot \frac{1}{2} \omega^{\prime}\left(u_{i}^{\prime \prime}\right) .
$$

Zufolge $a_{i k}=a_{k i}$ sind aber die rechten Seiten dieser beiden Gleichungen identisch und durch Subtraction folgt daher

$$
\left(\lambda^{\prime}-\lambda^{\prime \prime}\right) \sum_{1}^{3} u_{k}^{\prime} \cdot \frac{1}{2} \omega^{\prime}\left(u_{k}^{\prime \prime}\right)=0,
$$

oder, da $\lambda^{\prime} \gtrless \lambda^{\prime \prime}$, bleibt lediglich $\omega\left(u^{\prime}, u^{\prime \prime}\right)=0$, d. h. die Geraden $u_{x}^{\prime}$ und $u_{x}^{\prime \prime}$ sind zu einander normal (nach (28) in s 7).

Aus dem Vorhergehenden folgt:

(13) Bei jedem Kegelschnitt, für den die Gleichung (8a) nur verschiedene Wurzeln hat, gibt es zwei Hauptaxen, die überdies zu einander normal sind.

Wir wollen nun als Seiten eines neuen Coordinatendreiecks die beiden Hauptaxen und die unendlich ferne Gerade einführen; sind $X_{1}=0, X_{2}=0, X_{3}=0$ die Gleichungen der Seiten dieses Dreiecks, so ist daher

$$
\left\{\begin{array}{l}
X_{1} \equiv u_{1}^{\prime} x_{1}+u_{2}^{\prime} x_{2}+u_{3}^{\prime} x_{3} \\
X_{2} \equiv u_{1}^{\prime \prime} x_{1}+u_{2}^{\prime \prime} x_{2}+u_{3}^{\prime \prime} x_{3} \\
X_{3} \equiv p_{1} x_{1}+p_{2} x_{2}+p_{3} x_{3}
\end{array}\right.
$$

wobei die Verhältnisse der $u$ so bestimmt seien, dass

$$
\omega\left(u_{1}^{\prime}, u_{2}^{\prime}, u_{3}{ }^{\prime}\right)=1 \text { und } \omega\left(u_{1}^{\prime \prime}, u_{2}^{\prime \prime}, u_{3}^{\prime \prime}\right)=1 \text {, }
$$

also $X_{1}=0$ und $X_{2}=0$ Gleichungen gerader Linien in der Normalform rlarstellen. Vgl. S. 10.

Für $u_{1}, u_{2}, u_{3}$, resp. $U_{1}, U_{2}, U_{3}$ als Coordinaten einer Geraden, dritten, so müssten alle Unterdeterminanten $B_{i k}$ von (8a) verschwinden. $\mathbf{E s}$ ist aber $\mathrm{B}^{\prime}(\lambda)=\sum_{1}^{3} \sum_{1}^{3} \frac{\partial \mathrm{B}}{\partial \beta_{i k}} \frac{d \beta_{i k}}{d \lambda}=-\left(\mathrm{B}_{11}+\mathrm{B}_{22}+\mathrm{B}_{33}\right)$, daher würde sich ergeben $B^{\prime}(\lambda)=0$, was unmöglich ist, da (8a) keine Doppelwurzel besitzen sollte. 
Bestimmung der unendlich fernen Punkte auf den beiden Hauptaxen.

bezogen auf das ursprüngliche, resp. neue Dreieck, gilt nun die Relation (16)

$$
u_{1} x_{1}+u_{2} x_{2}+u_{3} x_{3}=U_{1} X_{1}+U_{2} X_{2}+U_{3} X_{3},
$$

und wenn man für die $X_{i}(i=1,2,3)$ ihre Werthe aus (14) einsetzt, entsteht beim Vergleichen der Coefficienten von $x_{1}, x_{2}, x_{3}$ links und rechts das System von Gleichungen:

$$
\left\{\begin{array}{l}
u_{1}=u_{1}^{\prime} U_{1}+u_{1}^{\prime \prime} U_{2}^{T}+p_{1} U_{3} \\
u_{2}=u_{2}^{\prime} U_{1}+u_{2}^{\prime \prime} U_{2}+p_{2} U_{3} \\
u_{3}=u_{3}^{\prime} U_{1}+u_{3}^{\prime \prime} U_{2}+p_{3} U_{3} .
\end{array}\right.
$$

Un dieses System nach $U_{1}$, resp. $U_{2}$ aufzulösen, addiren wir die drei Gleichungen, nachdem sie der Reihe nach mit $\frac{1}{2} \omega^{\prime}\left(u_{1}^{\prime}\right), \frac{1}{2} \omega^{\prime}\left(u_{2}^{\prime}\right), \frac{1}{2} \omega^{\prime}\left(u_{3}^{\prime}\right), \operatorname{resp} \cdot \frac{1}{2} \omega^{\prime}\left(u_{1}^{\prime \prime}\right), \frac{1}{2} \omega^{\prime}\left(u_{2}^{\prime \prime}\right), \frac{1}{2} \omega^{\prime}\left(u_{3}^{\prime \prime}\right)$ multiplicirt sind. Unter Berücksichtigung von (15) und (12), sowie des Umstandes, dass die $p_{i}$ die Coordinaten der unendlich fernen Geraden sind, erhält man auf solche Weise

$$
\left\{\begin{array}{l}
U_{1}=\frac{1}{2} \omega^{\prime}\left(u_{1}^{\prime}\right) u_{1}+\frac{1}{2} \omega^{\prime}\left(u_{2}^{\prime}\right) u_{2}+\frac{1}{2} \omega^{\prime}\left(u_{3}^{\prime}\right) u_{3} \\
U_{2}=\frac{1}{2} \omega^{\prime}\left(u_{1}^{\prime \prime}\right) u_{1}+\frac{1}{2} \omega^{\prime}\left(u_{2}^{\prime \prime}\right) u_{2}+\frac{1}{2} \omega^{\prime}\left(u_{3}^{\prime \prime}\right) u_{3} .
\end{array}\right.
$$

Die Auflösung des Systems (17) nach $U_{3}$ wird weiter unten (Gl. (31)) erfolgen.

Aus den drei Gleichungen (17) entstehen, indem man sie resp. mit $\omega_{i 1}, \omega_{i 2}, \omega_{i 3}$ multiplicirt und daun addirt, drei andere entsprechend den Werthen $i=1,2,3$ bei dieser Operation, nämlich:

$$
\left\{\begin{array}{l}
\frac{1}{2} \omega^{\prime}\left(u_{1}\right)=\frac{1}{2} \omega^{\prime}\left(u_{1}^{\prime}\right) U_{1}+\frac{1}{2} \omega^{\prime}\left(u_{1}^{\prime \prime}\right) U_{2} \\
\frac{1}{2} \omega^{\prime}\left(u_{2}\right)=\frac{1}{2} \omega^{\prime}\left(u_{2}^{\prime}\right) U_{1}+\frac{1}{2} \omega^{\prime}\left(u_{2}^{\prime \prime}\right) U_{2} \\
\frac{1}{2} \omega^{\prime}\left(u_{3}\right)=\frac{1}{2} \omega^{\prime}\left(u_{3}^{\prime}\right) U_{1}+\frac{1}{2} \omega^{\prime}\left(u_{3}^{\prime \prime}\right) U_{2},
\end{array}\right.
$$

und wenn man diese Gleichungen resp. mit $\frac{1}{2} f^{\prime}\left(x_{1}\right), \frac{1}{2} f^{\prime}\left(x_{2}\right), \frac{1}{2} f^{\prime}\left(x_{3}\right)$ multiplicirt und addirt, folgt unter Rücksicht auf (4) und (14):

$$
\left.\frac{1}{4} \sum_{1}^{3} i \omega^{\prime}\left(u_{i}\right) \cdot f^{\prime}\left(x_{i}\right)=\lambda^{\prime} U_{1} X_{1}+\lambda^{\prime \prime} U_{2} X_{2} \cdot{ }^{1}\right)
$$

1) Die hier gegebene Methode dürfte anch in synthetischer Uebertragung die zweckmässigste Bestimmung der Hauptaxen einer Curve oder Fläche zweiter Ordnung gewähren. Man vergleiche übrigens für den algebraischen Theil die Abhandlung von Clebseh und Gordan „Ueber biternäre Formen mit contragredienten Variabeln", Math. Annalen, Bd. 1, S. 385 ff., 1868. 
Ferner erhält man durch Multiplication des Systems (19) mit $u_{1}, u_{2}, u_{3}$ und Addition unter Berücksichtigung von (18):

$$
\omega\left(u_{1}, u_{2}, u_{3}\right)=U_{1}^{2}+U_{2}^{2} \text {. }
$$

Es waren nun die Ausdrücke $\frac{1}{2} \omega^{\prime}\left(u_{i}\right)(i=1,2,3)$ die Coordinaten des Normalencentrums der Geraden $u_{x}$, also die Coordinaten eines umendlich weit gelegenen Punktes; wir können für dieselben Werthe einführen von der Form $x_{i}+\varrho y_{i}$, wobei $\varrho$ so zu bestimmen ist, dass diese Werthe der Gleichung der unendlich fernen Geraden genügen, dass also $p_{x}+\varrho p_{y}=0$, und hieraus folgt $\varrho=-\frac{p_{x}}{p_{y}} ;$ man kann demnach stets setzen

$$
\frac{1}{2} \omega^{\prime}\left(u_{i}\right)=x_{i}-\frac{p_{x}}{p_{y}} y_{i} \quad(i=1,2,3),
$$

wo für beliebige $u_{i}$ die $x_{i}$ und $y_{i}$ ganz beliebige Zahlen sein dürfen, nur sei $p_{y} \geq 0$. Diese Ausdruicke (22) führen wir in (20) ein und erhalten hierdurch mit Rücksicht auf (18), sowie auf (14) die Relation:

$$
f(x, x)-f(x, y) \cdot \frac{p_{x}}{p_{y}}=\lambda^{\prime} X_{1}^{2}+\lambda^{\prime \prime} X_{2}^{2}-\frac{p_{x}}{p_{y}}\left(\lambda^{\prime} X_{1} Y_{1}+\lambda^{\prime \prime} X_{2} Y_{2}\right)
$$

wobei die $Y_{i}$ aus den $X_{i}$ hervorgehen, indem man in (14) die Coordinaten $x_{i}$ durch die $y_{i}$ ersetzt.

Gäbe es nun einen Punkt $y$, dessen Coordinaten die Ausdrücke $Y_{1}$ und $Y_{2}$ zum Verschwinden bringen, ohne dass $p_{y}$ gleich Null ist, so würde sich (23) verwandeln in

$$
f(x, x)-f(x, y) \frac{p_{x}}{p_{y}}=\lambda^{\prime} X_{1}^{2}+\lambda^{\prime \prime} X_{2}^{2} .
$$

Zufolge (20) wäre für einen solchen Punkt jedenfalls

$$
\frac{1}{4} \sum_{1}^{3} \omega^{\prime}\left(u_{i}\right) \cdot f^{\prime}\left(y_{i}\right)=0,
$$

woraus sich durch Einführung der Werthe (22) die für beliebige $x_{i}$ giltige Gleichung ergeben würde

oder

$$
\sum_{1}^{3} \frac{1}{2} f^{\prime}\left(y_{i}\right) \cdot x_{i}-\sum_{1}^{3} \frac{1}{2} f^{\prime}\left(y_{i}\right) \cdot y_{i} \cdot \frac{p_{x}}{p_{y}}=0
$$

$$
\sum_{1}^{3} \frac{1}{2} f^{\prime}\left(y_{i}\right) x_{t}=\frac{p_{x}}{p_{y}} f(y, y) \text {. }
$$


Durch Gleichsetzen der beiderseitigen Coefficienten von $x_{i}$ erhält man hieraus

$$
\frac{1}{2} f^{\prime}\left(y_{1}\right): p_{1}=\frac{1}{2} f^{\prime}\left(y_{2}\right): p_{2}=\frac{1}{2} f^{\prime}\left(y_{3}\right): p_{3}=f(y, y): p_{y} .
$$

Nun stimmen diese Gleichungen (26) genau überein mit den Gleichungen (22) und (23) in $\$ 4$, denen die Coordinaten des Mittelpunktes einer Curve zweiter Ordnung genügen. Man hat nur in (26) die Quotienten gleich $\mu$ zu setzen. Demnach sind die Coordinaten des Mittelpunktes solche Zahlenwerthe, welche die Gleichungen $Y_{1}=0$ und $Y_{2}=0$ befriedigen. Ausserdem folgt aus (26)

$$
f(x, y)=\mu\left(p_{1} x_{1}+p_{2} x_{2}+p_{3} x_{3}\right),
$$

und wenn man diesen Ausdruck in (24) einführt, entsteht schliesslich

$$
f(x, x)=\lambda^{\prime} X_{1}^{2}+\lambda^{\prime \prime} X_{2}{ }^{2}+\frac{\mu \cdot p_{x}{ }^{2}}{p_{y}} .
$$

Die Constante $\frac{\mu}{p_{y}}$ ist dabei durch (45) in $\S 4$ bestimmt; der Kürze halber werde gesetzt

$$
\frac{\mu}{p_{y}}=\varkappa,
$$

ferner ist $p_{x}$ nach (14) identisch mit $X_{3}$, so dass sich (27) verwandelt in:

$$
f(x, x)=\lambda^{\prime} X_{1}^{2}+\lambda^{\prime \prime} X_{2}{ }^{2}+x X_{3}{ }^{2} .
$$

Hiermit wäre die Transformation der allgemeinen Gleichung $f(x, x)=0$ einer Curve zweiter Ordnung auf die Hauptaxen durchgeführt, unter der Voraussetzung allerdings, dass die Gleichung (8a) nur verschiedene Wurzeln besitzt.

Es möge nuu noch die Auflösung des Systems (17) nach $U_{3}$ vollzogen werden. Jedenfalls stellt $U_{3}=0$ den Schnittpunkt der beiden Seiten $X_{1}=0, X_{2}=0$ des Coordinatendreiecks dar und, wie aus dem Vorhergehenden folgt, ist dieser Punkt zugleich der Mittelpunkt unseres Kegelschnitts. Seine Coordinaten genügen nach $(20)$ in $\S 5$, sowie nach (44) in $\S 4$ den Relationen

$$
y_{1}: y_{2}: y_{3}=F^{\prime}\left(p_{1}\right): F^{\prime}\left(p_{2}\right): F^{\prime}\left(p_{3}\right),
$$

wenn $F(u, u)=0$ die Gleichung der Curve $f(x, x)=0$ in Liniencoordinaten bedeutet. Multiplicirt man nun die Gleichungen (17) resp. mit $\frac{1}{2} F^{\prime}\left(p_{1}\right), \frac{1}{2} F^{\prime}\left(p_{2}\right), \frac{1}{2} F^{\prime}\left(p_{3}\right)$ und addirt dieselben, so verschwinden rechts in der Summe die Coefficienten von $U_{1}$ und $U_{2}$, denn die Coordinaten $F^{\prime}\left(p_{i}\right)$ oder $y_{i}$ bringen die mit jenen Coefficienten identischen Ausdrücke $Y_{1}$ und $Y_{2}$ zum Verschwinden, und es bleibt nur 


$$
\begin{aligned}
& \frac{1}{2} F^{\prime}\left(p_{1}\right) \cdot u_{1}+\frac{1}{2} F^{\prime}\left(p_{2}\right) \cdot u_{2}+\frac{1}{2} F^{\prime}\left(p_{3}\right) \cdot u_{3} \\
& =U_{3}\left\{\frac{1}{2} F^{\prime}\left(p_{1}\right) \cdot p_{1}+\frac{1}{2} F^{\prime}\left(p_{2}\right) \cdot p_{2}+\frac{1}{2} F^{\prime}\left(p_{3}\right) \cdot p_{3}\right\} \\
& =U_{3} \cdot F(p, p)
\end{aligned}
$$

man hat demnach

$$
U_{3}=\left\{\frac{1}{2} F^{\prime}\left(p_{1}\right) \cdot u_{1}+\frac{1}{2} F^{\prime}\left(p_{2}\right) \cdot u_{2}+\frac{1}{2} F^{\prime}\left(p_{3}\right) \cdot u_{3}\right\}: F(p, p) .
$$

Auch die Auflösung der Gleichungen (14) nach $x_{1}, x_{2}, x_{3}$ ist nun in einfachster Weise möglich. Wir machen zu dem Zweck lediglich Gebrauch von der Identitiit (16)

$$
u_{1} x_{1}+u_{2} x_{2}+u_{3} x_{3}=U_{1} X_{1}+U_{2} X_{2}+U_{3} X_{3},
$$

sowie von den Gleichungen (18) und (31), indem wir, um z. B. $x_{1}$ zu finden, in (16) setzen $u_{1}=1, u_{2}=u_{3}=0$ und die Werthe, welche $U_{1}, U_{2}, U_{3}$ für diesen Fall erhalten, aus (18) und (31) entnehmen. Man findet auf solche Weise

$$
\left\{\begin{array}{l}
x_{1}=\frac{1}{2} \omega^{\prime}\left(u_{1}^{\prime}\right) X_{1}+\frac{1}{2} \omega^{\prime}\left(u_{1}^{\prime \prime}\right) X_{2}+\frac{1}{2} \frac{F^{\prime}\left(p_{1}\right)}{F^{\prime}(p)} X_{3}, \text { und analog: } \\
x_{2}=\frac{1}{2} \omega^{\prime}\left(u_{2}^{\prime}\right) X_{1}+\frac{1}{2} \omega^{\prime}\left(u_{2}^{\prime \prime}\right) X_{2}+\frac{1}{2} \frac{F^{\prime}\left(p_{2}\right)}{F^{\prime}(p, p)} X_{3} \\
x_{3}=\frac{1}{2} \omega^{\prime}\left(u_{3}^{\prime}\right) X_{1}+\frac{1}{2} \omega^{\prime}\left(u_{3}^{\prime \prime}\right) X_{2}+\frac{1}{2} \frac{F^{\prime}\left(p_{3}\right)}{F^{\prime}(p, p)} X_{3} .
\end{array}\right.
$$

Die Bestimmung der Determinante

$$
r=\sum \pm\left(u_{1}^{\prime} u_{2}^{\prime \prime} p_{3}\right)
$$

der Transformation (14) ist folgendermassen zu erreichen. Gerade diese Determinante tritt auf bei den Gleichungen (17), vermöge deren, wic (21) zeigt, $\omega\left(u_{1}, u_{2}, u_{3}\right)$ übergeführt wird in $U_{1}{ }^{2}+U_{2}^{2}$. Nach § 9 (dualistisch zu (34) daselbst) besteht alsdann die Relation

$$
\left|\begin{array}{llll}
\omega_{11} & \omega_{12} & \omega_{13} & x_{1} \\
\omega_{21} & \omega_{22} & \omega_{23} & x_{2} \\
\omega_{31} & \omega_{32} & \omega_{33} & x_{3} \\
x_{1} & x_{2} & x_{3} & 0
\end{array}\right| r^{2}=\left|\begin{array}{llll}
1 & 0 & 0 & X_{1} \\
0 & 1 & 0 & X_{2} \\
0 & 0 & 0 & X_{3} \\
X_{1} & X_{2} & X_{3} & 0
\end{array}\right|=-X_{3}{ }^{2} ;
$$

andrerseits ist der Factor von $r^{2}$ nach (16) in $\S 7$ gleich $-\tau p_{x}^{2}$, daher $-\tau p_{x}^{2} \cdot r^{2}=-X_{3}^{2}$ oder

$$
r^{2}=\frac{1}{\tau},
$$

denn es ist $p_{x}=X_{3}$. Auch die Determinante der Transformation (32) lässt sich nun leicht berechnen. Denkt man sich nämlich das 
Gleichungen für die Hauptaxen und ihre unendlich fernen Punkte.

System (14) nach $x_{1}, x_{2}, x_{3}$ aufgelöst und setzt man etwa

$$
x_{i}=\alpha_{i} X_{1}+\beta_{i} X_{2}+\gamma_{i} X_{3} \text {, }
$$

so wird $\sum \pm\left(\alpha_{1} \beta_{2} \gamma_{3}\right)$ gleich der aus den Unterdeterminanten vou $r$ gebildeten Determinante, dividirt durch $r^{3}$, also gleich

$$
\frac{r^{\cdot 2}}{r^{3}}=\frac{1}{r}=\sqrt{\tau} \text {. }
$$

Für die Transformation (32) ist daher die Determinante gleich $\frac{1}{v}$ oder gleich $\sqrt{\tau}$.

Auch die Gleichungen für die beiden Hauptaxen, sowie für deren Schnittpunkte mit der unendlich fernen Geraden lassen sich leicht aufstellen.

Nach (20) ist

durch Subtraction der Identität

$$
\lambda^{\prime} U_{1} X_{1}+\lambda^{\prime \prime} U_{2} X_{2}=\frac{1}{4} \sum_{1}^{3} \omega^{\prime}\left(u_{i}\right) \cdot f^{\prime}\left(x_{i}\right) ;
$$

$$
\lambda^{\prime \prime}\left(U_{1} X_{1}+U_{2} X_{2}+U_{3} X_{3}\right)=\lambda^{\prime \prime}\left(u_{1} x_{1}+u_{2} x_{2}+u_{3} x_{3}\right)
$$

folgt hieraus

$$
\begin{aligned}
& \begin{array}{l}
\left(\lambda^{\prime}-\lambda^{\prime \prime}\right) U_{1} X_{1}=\frac{1}{4} \sum_{1}^{3} i \omega^{\prime}\left(u_{i}\right) \cdot f^{\prime}\left(x_{i}\right)+\lambda^{\prime \prime} U_{3} X_{3} \\
- \\
-\lambda^{\prime \prime}\left(u_{1} x_{1}+u_{2} x_{2}+u_{3} x_{3}\right),
\end{array}
\end{aligned}
$$

und in ganz analoger Weise

$$
\begin{aligned}
\left(\lambda^{\prime \prime}-\lambda^{\prime}\right) U_{2} X_{2}=\frac{1}{4} \sum_{i}^{3} \omega^{\prime}\left(u_{i}\right) \cdot f^{\prime}\left(x_{i}\right)+\lambda^{\prime} U_{3} X_{3} \\
\\
-\lambda^{\prime}\left(u_{1} x_{1}+u_{2} x_{2}+u_{3} x_{3}\right) .
\end{aligned}
$$
setzen

Dabei ist auf der rechten Seite auf Grund von (14) und (31) zu

$$
\begin{gathered}
X_{3}=p_{1} x_{1}+p_{2} x_{2}+p_{3} x_{3}, \\
U_{3}=\frac{1}{2}\left\{F^{\prime}\left(p_{1}\right) u_{1}+F^{\prime}\left(p_{2}\right) u_{2}+F^{\prime}\left(p_{3}\right) u_{3}\right\}: F(p, p) .
\end{gathered}
$$

$\mathrm{Da}$ ferner die rechten Seiten in (35) von der Form sein müssen

$$
\left(\alpha_{1} u_{1}+\alpha_{2} u_{2}+\alpha_{3} u_{3}\right)\left(\beta_{1} x_{1}+\beta_{2} x_{2}+\beta_{3} x_{3}\right),
$$

so erhält man die Ausdrücke für die beiden Axen in einfachster Weise dadurch, dass man in den zwei Gleichungen (35) rechts irgend zwei der Grössen $u_{1}, u_{2}, u_{3}$ gleich 0 , die dritte gleich 1 setzt oder, was auf dasselbe hinauskommt, man erhält die Gleichungen der Axen dadurch, dass man in (35) rechts den Factor irgend einer der drei Grössen $u_{1}, u_{2}, u_{3}$ gleich Null setzt. In entsprechender Weise wäre 
mit den $x$ zu verfahren, um die Gleichungen der unendlich fernen Punkte der beiden Axen zu erhalten.

Während bisher vorausgesetzt wurde, dass die beiden nicht verschwindenden Wurzeln $\lambda^{\prime}$ und $\lambda^{\prime \prime}$ der Gleichung (8a) von einander verschieden seien, werde nun angenommen, dass $\lambda^{\prime}=\lambda^{\prime \prime}$ sei, aber von $\lambda^{\prime \prime \prime}=0$ verschieden. Alsdann verschwinden nach (23) in $\S 8$ auch sämmtliche Unterdeterminanten $B_{i k}$, wenn man in ihnen für $\lambda$ den Werth der Doppelwurzel einsetzt; diese Wurzel findet man nach (11) gleich $\frac{1}{2}[a, \omega]$, da die Discriminante von (11) jetzt verschwindet. Von Wichtigkeit ist hier zunächst die aus (4) gebildete Form

$$
\frac{1}{4} \sum_{i}^{3} \omega^{\prime}\left(u_{i}\right) \cdot f^{\prime}\left(x_{i}\right)-\lambda^{\prime}\left(u_{1} x_{1}+u_{2} x_{2}+u_{3} x_{3}\right) \text {, }
$$

welche nach (7) und zufolge der Definition $\operatorname{der} \beta_{i k}$ identisch ist mit

$$
\sum_{1}^{3} \sum_{1}^{3} \beta_{i k} x_{i} u_{k} \text {. }
$$

Wir behaupten, dass diese in den $x_{i}$ und $u_{i}$ lineare und deshalb als „bilinear" bezeichnete Form im gegenwärtigen Falle in ein Product zweier Factoren zerfüllt, von denen der eine die $x_{i}$, der andere die $u_{i}$ separat enthält. In Folge von $\mathrm{B}_{i k}=0$ ist nämlich

$$
\beta_{11}: \beta_{12}: \beta_{13}=\beta_{21}: \beta_{22}: \beta_{23}=\beta_{31}: \beta_{32}: \beta_{33} \text {, }
$$

daher besteht u. a. die Proportion

$$
\begin{aligned}
\left(\beta_{11} u_{1}+\beta_{12} u_{2}+\beta_{13} u_{3}\right) & :\left(\beta_{21} u_{1}+\beta_{22} u_{2}+\beta_{23} u_{3}\right) \\
: & \left(\beta_{31} u_{1}+\beta_{32} u_{2}+\beta_{33} u_{3}\right)=\beta_{11}: \beta_{21}: \beta_{31} .
\end{aligned}
$$

Hieraus geht hervor, dass die Coefficienten von $x_{1}, x_{2}, x_{3}$ zu drei von den $u_{i}$ unabhängigen Grössen proportional sind, daher muss der Ausdruck (36) in zwei Factoren zerfallen, von denen der eine nur die $u_{i}$, der andere nur die $x_{i}$ enthält, d. h. man kann setzen

$$
\frac{1}{4} \sum_{1}^{3} \omega^{\prime}\left(u_{i}\right) \cdot f^{\prime}\left(x_{i}\right)-\lambda^{\prime}\left(u_{1} x_{1}+u_{2} x_{2}+u_{3} x_{3}\right)=-\lambda^{\prime} U_{3} X_{3} .
$$

Diese Gleichung gilt für alle Werthe der $u_{i}$ und $x_{i}$; setzt man $u_{i}=p_{i}$, ferner $x_{i}=\frac{1}{2} F^{\prime}\left(p_{i}\right): F(p, p)(i=1,2,3)$, so verschwindet jedesmal $\frac{1}{4} \sum_{1}^{3} \omega^{\prime}\left(u_{i}\right) \cdot f^{\prime}\left(x_{i}\right)$, und man erkennt, dass $U_{3}$ proportional ist $\mathrm{zu}$

$$
\frac{1}{2}\left(F^{\prime}\left(p_{1}\right) u_{1}+F^{\prime}\left(p_{2}\right) u_{2}+F^{\prime}\left(p_{3}\right) u_{3}\right): F(p, p),
$$


$X_{3}$ proportional zu $p_{1} x_{1}+p_{2} x_{2}+p_{3} x_{3} ;$ man kann die Proportionalitätsconstante auch $U_{3}$ zuschieben und $X_{3}=p_{1} x_{1}+p_{2} x_{2}+p_{3} x_{3}$ setzen. Demnach ist wieder $U_{3}=0$ die Gleichung des Mittelpunkts der Curve, $X_{3}=0$ diejenige der unendlich fernen Geraden. Es seien nun $U_{1}, U_{2}$ lineare Ausdrücke in Liniencoordinaten, $X_{1}, X_{2}$ solche in Punktcoordinaten, die mit $U_{3}$ und $X_{3}$ der Relation genügen

$$
u_{1} x_{1}+u_{2} x_{2}+u_{3} x_{3}=U_{1} X_{1}+U_{2} X_{2}+U_{3} X_{3}
$$

und wobei $X_{1}, X_{2}, X_{3}$ in der Normalform vorliegen; ausserdem seien $U_{1}=0$ und $U_{2}=0$ die Gleichungen von irgend zwei beliebigen harmonischen Polen des imaginären Kreispunktepaars $\omega(u, u)=0$, so dass $X_{1}=0$ und $X_{2}=0$ zwei zu einander normale Geraden darstellen. Man kann alsdann setzen

$$
\begin{gathered}
\left\{\begin{array}{l}
U_{1}=\frac{1}{2} \omega^{\prime}\left(u_{1}^{\prime}\right) u_{1}+\frac{1}{2} \omega^{\prime}\left(u_{2}^{\prime}\right) u_{2}+\frac{1}{2} \omega^{\prime}\left(u_{3}^{\prime}\right) u_{3} \\
U_{2}=\frac{1}{2} \omega^{\prime}\left(u_{1}^{\prime \prime}\right) u_{1}+\frac{1}{2} \omega^{\prime}\left(u_{2}^{\prime \prime}\right) u_{2}+\frac{1}{2} \omega^{\prime}\left(u_{3}^{\prime \prime}\right) u_{3}
\end{array}\right. \\
\left\{\begin{array}{l}
X_{1}=u_{1}^{\prime} x_{1}+u_{2}^{\prime} x_{2}+u_{3}^{\prime} x_{3} \\
X_{2}=u_{1}^{\prime \prime} x_{1}+u_{2}^{\prime \prime} x_{2}+u_{3}^{\prime \prime} x_{3},
\end{array}\right.
\end{gathered}
$$

wobei $\omega\left(u^{\prime}, u^{\prime \prime}\right)=0$. Durch Addition von (38) zu der mit $\lambda^{\prime}$ multiplicirten Relation (39) folgt

$$
\frac{1}{4} \sum_{1}^{3} \omega^{\prime}\left(u_{i}\right) \cdot f^{\prime}\left(x_{i}\right)=\lambda^{\prime}\left(U_{1} X_{1}+U_{2} X_{2}\right),
$$

und nun lassen sich dieselben Operationen anwenden, wie bei dcm oben ausführlich behandelten Falle zweier verschiedener Wurzeln $\lambda^{\prime}$ und $\lambda^{\prime \prime}$. Man gelangt hierdurch ähnlich wie bei (29) zu dem Resultat

$$
f(x, x)=\lambda^{\prime}\left(X_{1}{ }^{2}+X_{2}{ }^{2}\right)+x X_{3}{ }^{2} .
$$

Für diese Curve gibt es unendlich viele zu einander normale Hauptaxen, denn die Axen $X_{1}=0$ und $X_{2}=0$ haben lediglich der Forderung zu genügen, dass sie zu einander normal sind.

Die Bedingung, dass die zwei Wurzeln $\lambda^{\prime}$ und $\lambda^{\prime \prime}$ von (11) einander gleich seien, wird dargestellt durch das Verschwinden der Discriminante von (11), also durch

$$
[a, \omega]^{2}-4 \tau F(p, p)=0 .
$$

In Folge des.Umstandes, dass es immer zu einander normale Axen $X_{1}=0, X_{2}=0$ gibt (im Falle $\lambda^{\prime}=\lambda^{\prime \prime}$ sogar unendlich viele), können wir auch ein ganzes System von Bedingungen ableiten, welche 
die Coefficienten von $f(x, x)=0$ erfüllen müssen, damit diese Curve einen Kreis darstelle.

Der Kürze halber werde gesetzt

$$
\frac{1}{4} \sum_{1}^{3} \omega^{\prime}\left(u_{i}\right) f^{\prime}\left(x_{i}\right) \equiv \Psi(u, x) ;
$$

alsdann ist nach (20)

$$
\psi(\imath, x)=\frac{\partial \psi}{\partial x_{1}} x_{1}+\frac{\partial \psi}{\partial x_{2}} x_{2}+\frac{\partial \psi}{\partial x_{3}} x_{3}=\lambda^{\prime} U_{1} X_{1}+\lambda^{\prime \prime} U_{2} X_{2}
$$

ferner bestehen die Gleichungen

$$
\begin{aligned}
& u_{1} x_{1}+u_{2} x_{2}+u_{3} x_{3}=U_{1} X_{1}+U_{2} X_{2}+U_{3} X_{3}, \\
& p_{1} x_{1}+p_{2} x_{2}+p_{3} x_{3}=X_{3} .
\end{aligned}
$$

Betrachtet man hier $X_{1}, X_{2}, X_{3}$ als die ursprünglichen Veränderlichen, welche durch die Gleichungen (14) transformirt wurden, so besteht nach (4) in $\S 9$ die Beziehung

$$
\begin{array}{lll}
\frac{\partial \psi}{\partial x_{1}} & \frac{\partial \psi}{\partial x_{2}} & \frac{\partial \psi}{\partial x_{3}} \\
u_{1} & u_{2} & u_{3} \\
p_{1} & p_{2} & p_{3}
\end{array}|=r| \begin{array}{lll}
\lambda^{\prime} U_{1} & \lambda^{\prime \prime} U_{2} & 0 \\
U_{1} & U_{2} & U_{3} \\
0 & 0 & 1
\end{array} \mid=r\left(\lambda^{\prime}-\lambda^{\prime \prime}\right) U_{1} U_{2} .
$$

Da die Curve $f(x, x)=0$ im Falle $\lambda^{\prime}=\lambda^{\prime \prime}$ einen Kreis darstellt, ist die Bedingung hierfür gleichbedeutend mit

$$
\left|\begin{array}{lll}
\frac{\partial \psi}{\partial x_{1}} & \frac{\partial \psi}{\partial x_{2}} & \frac{\partial \psi}{\partial x_{3}} \\
u_{1} & u_{2} & u_{3} \\
p_{1} & p_{2} & p_{3}
\end{array}\right|=0
$$

und zwar muss diese Gleichung erfüllt werden, welche Werthe auch die $u_{i}$ haben mögen, d. h. in (47) müssen die Coefficienten von $u_{1}{ }^{2}$, $u_{2}{ }^{2}, u_{3}{ }^{2}, u_{1} u_{2}, u_{1} u_{3}, u_{2} u_{3}$ einzeln verschwinden, womit das oben erwähnte System von Bedingungen nun gefunden ist.

Man erkennt übrigens auch aus (46), dass

$$
\sum \pm\left(\frac{\partial \Psi}{\partial x_{1}} u_{2} p_{3}\right)=0
$$

das Product der Gleichungen für die unendlich fernen Punkte der beiden Axen ist. In ähnlicher Weise kann man ein Prodnct bilden für die Axen selbst; aus

$$
\left\{\begin{aligned}
\frac{\partial \Psi}{\partial u_{1}} u_{1}+\frac{\partial \Psi}{\partial u_{2}} u_{2}+\frac{\partial \Psi}{\partial u_{3}} u_{3} & =\lambda^{\prime} X_{1} U_{1}+\lambda^{\prime \prime} X_{2} U_{2} \\
x_{1} u_{1}+x_{2} u_{2}+x_{3} u_{3} & =X_{1}^{*} U_{1}+X_{2} U_{2}+X_{3} U_{3} \\
\frac{1}{2} F^{\prime}\left(p_{1}\right) u_{1}+\frac{1}{2} F^{\prime}\left(p_{2}\right) u_{2}+\frac{1}{2} F^{\prime}\left(p_{3}\right) u_{3} & =F(p, p) \cdot U_{3}
\end{aligned}\right.
$$


Product der Gleichungen für die beiden Hauptaxen.

folgt nämlich

$$
\text { (49) } \begin{aligned}
\left|\begin{array}{lll}
\frac{\partial \psi}{\partial u_{1}} & \frac{\partial \psi}{\partial u_{2}} & \frac{\partial \psi}{\partial u_{3}} \\
x_{1} & x_{2} & x_{3} \\
\frac{1}{2} F^{\prime}\left(p_{1}\right) & \frac{1}{2} F^{\prime}\left(p_{2}\right) & \frac{1}{2} F^{\prime}\left(p_{3}\right)
\end{array}\right| & =\left|\begin{array}{lll}
\lambda^{\prime} X_{1} & \lambda^{\prime \prime} X_{2} & 0 \\
X_{1} & X_{2} & X_{3} \\
0 & 0 & F(p, p)
\end{array}\right| \\
& =F(p, p) \cdot\left(\lambda^{\prime}-\lambda^{\prime \prime}\right) X_{1} X_{2},
\end{aligned}
$$

wobei vorausgesetzt ist $F(p, p) \gtrless 0$.

Hierbei ist

$$
\frac{\partial \psi}{\partial u_{1}}=\frac{1}{4} \omega_{11} f^{\prime}\left(x_{1}\right)+\frac{1}{4} \omega_{12} f^{\prime}\left(x_{2}\right)+\frac{1}{4} \omega_{13} f^{\prime}\left(x_{3}\right)=\frac{1}{2} \omega^{\prime}\left(f_{1}\right),
$$

analog

$$
\frac{\partial \psi}{\partial u_{2}}=\frac{1}{2} \omega^{\prime}\left(f_{2}\right), \quad \frac{\partial \psi}{\partial u_{3}}=\frac{1}{2} \omega^{\prime}\left(f_{3}\right) .
$$

Der Umstand, dass sich für den Fall eines Kreises aus (46) (und analog aus (49)) mehrere Bedingungen ergeben, obgleich doch schon die eine $\lambda^{\prime}=\lambda^{\prime \prime} \gtrless 0$ ausreichen würde, beruht darin, dass nach (23) in $\S 8$ für die Doppelwurzel ein ganzes System von Werthen $B_{i k}$ verschwindet. Auf Grund eines Satzes von $K_{r}$ onecker ${ }^{1}$ ) müssen sich diese Bedingungen nach Elimination von $\lambda$ schliesslich aber auf zwei reduciren. Man kann dies folgendermassen einsehen: Bei nicht ausartenden Kegelschnitten $(A \gtrless 0)$ kann man nämlich die kubische Gleichung $\mathrm{B}(\lambda)$ in die Gestalt einer symmetrischen Determinante bringen, indem man (8a) mit

$$
\left|\begin{array}{lll}
A_{11} & A_{21} & A_{31} \\
A_{12} & A_{22} & A_{32} \\
A_{13} & A_{23} & A_{33}
\end{array}\right|
$$

multiplicirt; unter Benutzung von

folgt

$$
\alpha_{i k}=\omega_{1 i} a_{1 k}+\omega_{2 i} a_{2 k}+\omega_{3 i} a_{3 k}
$$

$$
\left|\begin{array}{ccc}
A \omega_{11}-\lambda A_{11} & A \omega_{12}-\lambda A_{12} & A \omega_{13}-\lambda A_{13} \\
A \omega_{21}-\lambda A_{21} & A \omega_{22}-\lambda A_{22} & A \omega_{23}-\lambda A_{23} \\
A \omega_{31}-\lambda A_{31} & A \omega_{32}-\lambda A_{32} & A \omega_{33}-\lambda A_{33}
\end{array}\right|=0
$$

wofür auch eine Determinante gesetzt werden könnte, deren Elemente von der Form sind $\omega_{i k}-\mu A_{i k}$, wenn $\mu=\frac{\lambda}{A}$ ist. Diese kubische Gleichung (50) ersetzt also bei Curven zweiter Ordnung die frühere

1) Vgl. Baltzer "Theorie und Anwendung der Determinanten“, 5. Aufl., Leipzig 1881, S. 58. 
kubische Gleichung (8) in allen Fällen, in welchen der Kegelschnitt nicht ausartet. Man vergleiche hierzu auch die analoge Behandlung der confocalen Curven zweiter Classe in $\S 18$. Nennen wir die Elemente von $(50) \gamma_{i k}$, ihre Unterdeterminanten $\Gamma_{i k}$, so kann man drei Grössen $\Gamma_{i k}$ der Art wählen, dass ihr Verschwinden auch das Verschwinden aller übrigen Unterdeterminanten zur Folge hat; solche drei Grössen wären z. B. $\Gamma_{11}, \Gamma_{22}, \Gamma_{12}$, falls $\gamma_{33} \geqslant 0$. Durch Elimination von $\lambda$ reduciren sich die drei Bedingungen schliesslich auf zwei.

Darauf beruht auch die Thatsache, dass die Discriminante der quadratischen Gleichung (11) als Summe zweier Quadrate dargestellt werden kann. Bei Anwendung schiefwinkliger Parallelcoordinaten mit dem Axenwinkel $w$ ergibt sich zufolge der Bemerkungen zu (2) in $§ 2$ an Stelle der quadratischen Gleichung (11) die folgende:

$$
\lambda^{2} \sin ^{2} w-\lambda\left(a_{11}+a_{22}-2 a_{12} \cos w\right)+\left(a_{11} a_{22}-a_{12}{ }^{2}\right)=0,
$$

deren Discriminante

$$
4\left(a_{11} a_{22}-a_{12}{ }^{2}\right) \sin ^{2} w-\left(a_{11}+a_{22}-2 a_{12} \cos w\right)^{2}
$$

in die Form gebracht werden kann:

$$
\left(a_{11}-a_{22}\right)^{2} \sin ^{2} w+\left[\left(a_{11}+a_{22}\right) \cos w-2 a_{12}\right]^{2}=0 .
$$

Um zu sehen, wie sich diese Bedingung im allgemeinen Falle gestaltet, wollen wir statt der Dreieckscoordinaten in $f(x, x)=0$

Fig. 2.

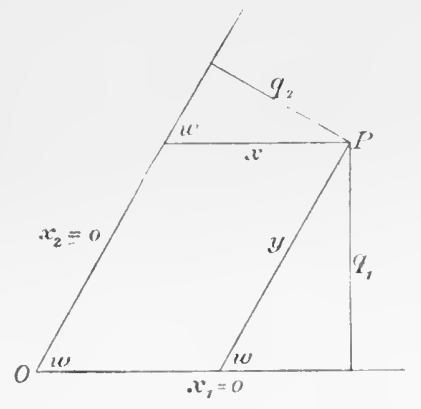
schiefwinklige Parallelcoordinaten einführen, bei welchen $x, y$ die schiefwinkligen Coordinaten eines Punktes $P, q_{1}$ und $q_{2}$ seine senkrechten Abstände von der $x$-Axe, resp. $y$-Axe bedeuten mögen. Diese Axen sollen mit den Seiten $x_{1}=0$, resp. $x_{2}=0$ des Coordinatendreiecks zusammenfallen. Alsdann hat man nach nebenstehender Figur und nach (1) in $\S 2$ die Gleichungen

$$
x=\frac{q_{2}}{\sin w}=\frac{x_{2}}{\sin w \cdot \sqrt{\omega_{22}} \cdot p_{x}}, \quad y=\frac{q_{1}}{\sin w}=\frac{x_{1}}{\sin w \cdot \sqrt{\omega_{11}} \cdot p_{x}} .
$$

Setzt man noch $\varrho p_{x}=1$, so folgt hieraus

$$
\begin{gathered}
\varrho x_{1}=y \sin w \cdot \sqrt{\omega_{11}}, \quad \varrho x_{2}=x \sin w \cdot \sqrt{\omega_{22}}, \\
\varrho x_{3}=\frac{1-\varrho\left(p_{1} x_{1}+p_{2} x_{2}\right)}{p_{3}}=\frac{1-\sin w\left(y \sqrt{\left.\omega_{11} p_{1}+x \sqrt{\omega_{22}} p_{2}\right)}\right.}{p_{3}} .
\end{gathered}
$$


Mit Hilfe dieser Transformationsformeln, welche natürlich $p_{3} \gtrless 0$ voranssetzen, wird nun die Gleichung der Curve zweiter Ordnung

$$
f(x, x) \equiv \sum_{1}^{3} \sum_{1}^{3} a_{i k} x_{i} x_{k}=0
$$

transformirt in eine Gleichung von der Form

$$
b_{11} x^{2}+2 b_{12} x y+b_{22} y^{2}+2 b_{13} x+2 b_{23} y+b_{33}=0,
$$

für welche sich die Bedingung des Kreises aus (52) ergibt durch Vertauschung von $a_{11}, a_{22}, a_{12}$ resp. mit $b_{11}, b_{22}, b_{12}$. Die Werthe dieser drei Grössen $b_{i k}$ sind leicht zu berechnen; durch Einführung derselben in (52) verwandelt sich diese Gleichung in:

$$
\begin{aligned}
& \left\{\omega_{22}\left(a_{22} p_{3}{ }^{2}+a_{33} p_{22}{ }^{2}-2 a_{23} p_{2} p_{3}\right)\right. \\
& \left.\quad-\omega_{11}\left(a_{11} p_{3}{ }^{2}+a_{33} p_{1}{ }^{2}-2 a_{13} p_{1} p_{3}\right)\right\}^{2} \sin ^{2} w \\
& +\left\{\left[\omega_{22}\left(a_{22} p_{3}{ }^{2}+a_{33} p_{2}{ }^{2}-2 a_{23} p_{2} p_{3}\right)\right.\right. \\
& \left.\quad+\omega_{11}\left(a_{11} p_{3}{ }^{2}+a_{33} p_{1}{ }^{2}-2 a_{13} p_{1} p_{3}\right)\right] \cos w \\
& \left.\quad-2 \sqrt{\omega_{11} \omega_{22}}\left(a_{12} p_{3}{ }^{2}+a_{33} p_{1} p_{2}-a_{13} p_{2} p_{3}-a_{23} p_{1} p_{3}\right)\right\}^{2}=0 .
\end{aligned}
$$

Ausser dieser Darstellung der Discriminante von (11) als Summe zweier Quadrate sind noch unendlich viele andere möglich; man darf nämlich in (54) nicht nur die Indices 1, 2, 3 cyklisch vertauschenf sondern kann auch von dem Umstande Gebrauch machen, dass die Summe zweier Quadrate $M^{2}+N^{2}$ wieder erhalten wird, wenn man $M$ ersetzt durch $M I \cos \alpha-N \sin \alpha, N$ durch $M \sin \alpha+N \cos \alpha$, wobei $\sin \alpha$ und $\cos \alpha$ Werthe sind, welche die Brüche

bei beliebigem $t$ annehmen.

$$
\sin \alpha=\frac{2 t}{1+t^{2}}, \quad \cos \alpha=\frac{1-t^{2}}{1+t^{2}}
$$

Wir wollen diese Untersuchungen über den Kreis nicht abschliessen, ohne noch einen Ausdruck für die Potenz eines Punktes in Bezug auf einen Kreis abzuleiten.

Nach (43) ist $f(x, x)$ für den Fall eines Kreises transformirbar in

$$
\lambda^{\prime}\left(X_{1}^{2}+X_{2}^{2}\right)+x X_{3}^{2}=0
$$

wobei $\lambda^{\prime}$ Doppelwurzel ist der quadratischen Gleichung

$$
\lambda^{2}-[a, \infty] \lambda+\tau F(p, p)=0,
$$

daher den Werth besitzt $\lambda^{\prime}=\frac{1}{2}[a, \omega]$. Setzen wir

$$
X_{3}=p_{x}, \quad \frac{X_{1}}{\bar{X}_{3}}=X, \quad \frac{X_{2}}{\bar{X}_{3}}=Y,
$$

so ist die Gleichung des Kreises bezogen auf rechtwinklige Coordinaten; 
dividirt man noch durch $\lambda^{\prime}$ und setzt $-\frac{x}{\lambda^{\prime}}$ gleich dem Quadrat des Radius $r^{2}$, so lautet die Gleichung des Kreises $X^{2}+Y^{2}-r^{2}=0$. Die Potenz $P$ eines Punktes mit den rechtwinkligen Coordinaten $x, y$ in Bezug auf diesen Kreis ist nun $x^{2}+y^{2}-r^{2}$, geht also aus

$$
\lambda^{\prime}\left(X_{1}^{2}+X_{2}^{2}\right)+x p_{x}^{2}
$$

hervor durch Division mit $\lambda^{\prime} p_{x}{ }^{2}$ oder mit $\frac{1}{2}[a, \omega] p_{x}{ }^{2}$ und Substitution der Coordinaten des betreffenden Punktes. Allgemein stellt daher

$$
P=\frac{2 f(y, y)}{[a, \omega] p_{y}{ }^{2}}=\frac{f(y, y)}{\lambda^{\prime} p_{y}{ }^{2}}
$$

die Potenz des Punktes $y_{1}, y_{2}, y_{3}$ dar in Bezug auf den Kreis $f(x, x)=0$.

Kehren wir nun wieder zur Untersuchung der kubischen Gleichung (8a) zurück!

Der Fall, dass diese Gleichung eine von Null verschiedene Doppelwurzel $\lambda^{\prime}=\lambda^{\prime \prime}$ besitzt, für welche nicht nur alle $\mathrm{B}_{i k}$, sondern auch

$$
\frac{1}{2} B^{\prime \prime}(\lambda)=\alpha_{11}-\lambda+\alpha_{22}-\lambda+\alpha_{33}-\lambda
$$

verschwindet, kann nicht eintreten. Denn es wäre nun $\lambda^{\prime}=\lambda^{\prime \prime}$ eine dreifache Wurzel der kubischen Gleichung (8a), also wegen $\lambda^{\prime \prime \prime}=0$ auch $\lambda^{\prime}=\lambda^{\prime \prime}=0$. Wie weiter unten gezeigt wird, besteht die Curve alsdann, so lange nicht auch alle $\alpha_{i k}$ verschwinden, aus einer im Endlichen und der im Unendlichen gelegenen Geraden (vgl. auch die Fussnote zu S. 59).

Es möge nunmehr angenommen werden, dass die kubische Gleichung (8a) als Doppelwurzel $\lambda^{\prime \prime}=\lambda^{\prime \prime \prime} \doteq 0$ besitzt, dagegen sei die Wurzel $\lambda^{\prime}$ von Null verschieden; hier ist $\lambda=0$ einfache Wurzel von (11), also nothwendig $F(p, p)=0$ und $\lambda^{\prime}=[a, \omega]$. Für $\lambda=0$ können jetzt nach (24) in $\$ 8$ die Unterdeterminanten $B_{i k}$ von Null verschieden sein, müssen es aber nicht; nehmen wir zuerst an die $B_{i k}$ oder, was nun wegen $\lambda^{\prime \prime}=\lambda^{\prime \prime \prime}=0$ auf dasselbe hinauskommt, die $A_{i k}$ seien nicht alle gleich Null.

In diesem Falle ist die Determinante $A$ der Curve $f(x, x)=0$ von Null verschieden. Wie in der Determinantentheorie gezeigt wird, besteht nämlich die Relation ${ }^{\text {) }}$

$$
A\left(\begin{array}{l}
u p \\
u p
\end{array}\right)=\left(\begin{array}{l}
u \\
u
\end{array}\right)\left(\begin{array}{l}
p \\
p
\end{array}\right)-\left(\begin{array}{l}
u \\
p
\end{array}\right)^{2}
$$

1) Vgl. über die Bezeichnungsweise die Fussnote zu S. 34. 
nach Voraussetzung ist aber $\left(\begin{array}{l}p \\ p\end{array}\right)=-F(p, p)=0$, für $A=0$ müsste daher auch $\left(\begin{array}{l}u \\ p\end{array}\right)$ verschwinden, d. h. es wäre

$$
F^{\prime}\left(p_{1}\right) u_{1}+F^{\prime}\left(p_{2}\right) u_{2}+F^{\prime}\left(p_{3}\right) u_{3}=0
$$

bei allen Werthen der $u_{i}$, folglich $F^{\prime}\left(p_{k}\right)=0(k=1,2,3)$, oder nach der Fussnote zu S. $86 \quad \mathrm{~A}_{i k}=0(i, k=1,2,3)$, was vorläufig ausgeschlossen wurde.

Der Kegelschnitt ist daher jedenfalls nicht ausartend und nach $\S 6$ eine Parabel. Hier wird durch passende Bestimmung linearer Ausdrücke $X_{1}, X_{2}, X_{3}$ die Gleichung der Curve $f(x, x)=0$ in die Form gebracht werden könuen wobei

$$
\begin{gathered}
f(x, x)=\lambda^{\prime} X_{1}^{2}+2 \varrho X_{2} X_{3}=0, \\
X_{3}=p_{1} x_{1}+p_{2} x_{2}+p_{3} x_{3}=0
\end{gathered}
$$

die unendlich ferne Gerade darstellt, während $X_{1}=0$ und $X_{2}=0$ die in der Normalform gegebenen Gleichungen zweier zu cinander senkrechten Geraden sind.

Man könnte diese Transformation analog wie bisher erledigen, indem man zwei Gleichungen herstellen würde von der Form

$$
\begin{aligned}
& \frac{1}{4} \sum_{1}^{3} \omega^{\prime}\left(u_{i}\right) \cdot f^{\prime \prime}\left(x_{i}\right)=\lambda^{\prime} U_{1} X_{1}+\varrho U_{2} X_{3}, \\
& u_{1} x_{1}+u_{2} x_{2}+u_{3} x_{3}=U_{1} X_{1}+U_{2} X_{2}+U_{3} X_{3} .
\end{aligned}
$$

Nimmt mau einen Punkt $y$ (den Scheitel der Curve $f(y, y)=0$ ) zu Hilfe, dessen Tangente als Normalencentrum den Berührungspunkt der Parabel mit der unendlich fernen Geraden hat, so wäre für diesen Punkt

$$
f^{\prime}\left(y_{1}\right) \cdot x_{1}+f^{\prime}\left(y_{2}\right) \cdot x_{2}+f^{\prime}\left(y_{3}\right) \cdot x_{3}=0
$$

oder kürzer $f_{1} x_{1}+f_{2} x_{2}+f_{3} x_{3}=0$ die Gleichung der Tangente, daher sind $\frac{1}{2} \omega^{\prime}\left(f_{1}\right), \frac{1}{2} \omega^{\prime}\left(f_{2}\right), \frac{1}{2} \omega^{\prime}\left(f_{3}^{\prime}\right)$ die Coordinaten des Normalencentrums der Tangente; andrerseits stellt nach (17) in $\S 5$

$$
\frac{1}{2} F^{\prime}\left(p_{1}\right) u_{1}+\frac{1}{2} F^{\prime}\left(p_{2}\right) u_{2}+\frac{1}{2} F^{\prime}\left(p_{3}\right) u_{3}=0
$$

den Berührungspunkt der Parabel mit der unendlich fernen Geraden dar, wenn $F(u, u)=0$ die Gleichung der Curve in Liniencoordinaten ist. Man hat daher die Gleichungen

$$
\frac{\omega^{\prime}\left(f_{1}\right)}{F^{\prime}\left(p_{1}\right)}=\frac{\omega^{\prime}\left(f_{2}\right)}{F^{\prime}\left(p_{2}\right)}=\frac{\omega^{\prime}\left(f_{3}\right)}{F^{\prime}\left(p_{3}\right)}
$$

und würde nun durch eine Transformation analog derjenigen auf den 
Mittelpunkt, wie sie oben für $f(x, x)=0$ durchgeführt wurde, die Form erhalten

$$
\lambda^{\prime} X_{1}^{2}+2 \varrho X_{2} X_{3}=0 \text {. }
$$

Diesen umständlichen und nur schwer zu den letzten Ergebnissen führenden Weg kann man auf folgende Art umgehen.

Die Gleichung des Berührungspunktes der Parabel $f(x, x)=0$ mit der unendlich fernen Geraden ist nach (53a) in $\S 4$ doppelt zählend gegeben durch

$$
\left(\begin{array}{ll}
p & u \\
p & u
\end{array}\right)=0 \text {. }
$$

Ist nun $U_{1}=0$ die Gleichung des Punktes, der auf der unendlich fernen Geraden zusammen mit dem unendlich fernen Berührungspunkt der Parabel ein zum imaginären Kreispunktepaar harmonisch gelegenes Punktepaar bildet, so wird ${U_{1}}^{2}$ von der Form sein

$$
U_{1}^{2} \equiv \omega(u, u) \cdot \lambda+\left(\begin{array}{l}
p u \\
p u
\end{array}\right)=0 .
$$

Hier ist $\lambda$ so zu bestimmen, dass die Coefficienten der zu (62) gehörigen Gleichung in Punktcoordinaten verschwinden, denn nur dann repräsentirt (62) nach (7) in $\S 5$ einen Doppelpunkt. Als Punktcoordinatengleichung für (62) findet man

$$
\left.\lambda^{2} \Omega(x, x)+\lambda[a, \omega] p_{x}^{2}=0,{ }^{1}\right)
$$

1) Dass der Coefficient von $\lambda^{2}$ in der Gleichung in Punktcoordinaten mit $\Omega(x, x)$ übereinstimmt, und dass der Coefficient von $\lambda^{0}$ identisch verschwindet, (denn $\left(\begin{array}{l}p u \\ p u\end{array}\right)$ ist ein vollständiges Quadrat) ist klar; es wäre also nur noch zu zeigen, dass der Coefficient von $\lambda^{1}$ gleich $[a, \omega] p_{x}{ }^{2}$ ist. $\mathrm{Zu}$ dem $\mathrm{Zweck}$ werde für den Augenblick gesetzt $\left(\begin{array}{l}p u \\ p u\end{array}\right)=\left(z_{1} u_{1}+z_{2} u_{2}+z_{3} u_{3}\right)^{2}$; stellt man nun für $\lambda \omega(u, u)+z_{u}{ }^{2}=0$ die Gleichung in Punktcoordinaten auf, etwa in Gestalt einer Determinante wie (13) in $\S 5$, so erkennt man, dass der Coefficient von $\lambda^{1}$ identisch ist mit $\left(\begin{array}{l}z x \\ z x\end{array}\right)_{\omega_{i k}}$. Dies gilt, welche Werthe die $\omega_{i k}$ haben mögen, daher auch noch, wenn man die Grössen $\omega_{i k}$ ersetzt durch Producte $y_{i} y_{k}$, wodurch $\operatorname{sich}\left(\begin{array}{l}z x \\ z x\end{array}\right)_{\omega_{i k}}$ verwandelt in $\sum \pm\left(y_{1} z_{2} x_{3}\right)^{2}$, und dieser Ausdruck geht aus $z_{u}{ }^{2}$ oder aus $\left(\begin{array}{l}p u \\ p u\end{array}\right)_{a_{i k}}$ hervor durch die.Substitution

$$
u_{1}=y_{2} x_{3}-y_{3} x_{2}, \quad u_{2}=y_{3} x_{1}-y_{1} x_{3}, \quad u_{3}=y_{1} x_{2}-y_{2} x_{1} .
$$

Andrerseits erhält hierdurch, wie ans einer am Schlusse von $\S 4$ durchgeführten Rechnung hervorgeht, $\left(\begin{array}{l}p u \\ p u\end{array}\right)_{a_{i k}}$ den Werth

$$
f(x, x) \cdot p_{y}{ }^{2}-2 f(x, y) \cdot p_{x} \cdot p_{y}+f(y, y) \cdot p_{x}^{2}
$$


es muss demnach, da $\Omega(x, x)=\tau p_{x}{ }^{2}$, die Gleichung erfüllt werden

$$
p_{x}^{2}\left(\lambda^{2} \tau+\lambda[a, \omega]\right)=0,
$$

und dies ist der Fall für $\lambda=-\frac{[a, \omega]}{\tau}$. In Folge von $\lambda^{\prime}=[a, \omega]$ kann man auch setzen $\lambda=-\frac{\lambda^{\prime}}{\tau}$, daher wird ${U_{1}}^{2}=0$ gleichbedeutend mit $-\lambda^{\prime} \omega\left(u_{1}, u_{2}, u_{3}\right)+\tau\left(\begin{array}{l}p u \\ p u\end{array}\right)$; es sei

$$
-\lambda^{\prime}{U_{1}}^{2}=-\lambda^{\prime} \cdot \omega\left(u_{1}, u_{2}, u_{3}\right)+\tau\left(\begin{array}{ll}
p & u \\
p & u
\end{array}\right) \text {. }
$$

Als Punkt $U_{2}=0$ wählen wir den Berührungspunkt der Parabel mit der unendlich fernen Geraden, und zwar sei (vgl. (61))

$$
\lambda^{\prime} U_{2}^{2}=\tau \cdot\left(\begin{array}{ll}
p & u \\
p & u
\end{array}\right)
$$

so dass in Folge der beiden letzten Gleichungen:

$$
\omega(u, u)=U_{1}^{2}+U_{2}{ }^{2} \text {. }
$$

Die Axe der Parabel ist die Polare des Punktes $U_{1}$; für $y_{1}, y_{2}, y_{3}$ als Coordinaten dieses Punktes sei

$$
-\lambda^{\prime} U_{1}^{2}=\left(y_{1} u_{1}+y_{2} u_{2}+y_{3} u_{3}\right)^{2}
$$

die Gleichung der Polare wird

$$
y_{1} \cdot f^{\prime}\left(x_{1}\right)+y_{2} \cdot f^{\prime}\left(x_{2}\right)+y_{3} \cdot f^{\prime \prime}\left(x_{3}\right)=0,
$$

oder auch für beliebige Werthe der $u_{i}$ :

$$
\left(y_{1} \cdot f^{\prime}\left(x_{1}\right)+y_{2} \cdot f^{\prime}\left(x_{2}\right)+y_{3} \cdot f^{\prime}\left(x_{3}\right)\right)\left(u_{1} y_{1}+u_{2} y_{2}+u_{3} y_{3}\right)=0 .
$$

Dies ist aber derselbe Ausdruck, den man erhält, wenn in (63) auf der linken Seite an Stelle von $u_{i}$ gesetzt wird $u_{i}+\lambda \cdot \frac{1}{2} f^{\prime \prime}\left(x_{i}\right)$ und nun der Coefficient von $\lambda^{1}$ berechnet wird; auf der rechten Seite von (63) ergibt dagegen der Coefficient von $\lambda^{1}$ nach dieser Substitution diè Formel:

$$
-\frac{1}{4} \lambda^{\prime} \sum_{1}^{3} \omega^{\prime}\left(u_{i}\right) \cdot f^{\prime}\left(x_{i}\right)+\tau\left(\begin{array}{l}
p u \\
p f
\end{array}\right) \equiv-\lambda^{\prime 2} X_{1} U_{1}\left(\text { vgl. 58); }{ }^{1}\right)
$$

dies ist richtig, welche Werthe auch die Producte $y_{i} y_{k}$ haben mögen, gilt daher nach (35) in $\S 8$ auch dann noch, wenn man wieder rückwärts $y_{i} y_{k}$ ersetzt durch $\omega_{i k}$. Alsdann verschwindet aber $p_{y}$, während $f(y, y)$ übergeht in $[a, \omega]$, es bleibt daher statt des Coefficienten $\left(\begin{array}{ll}z & x \\ z & x\end{array}\right)_{\omega_{i k}}$ von $\lambda^{1}$ lediglich $[a, \omega] p_{x}{ }^{2}$.

1) Der Factor $-\lambda^{\prime 2}$ ist hinzugefügt worden, damit $X_{1}=u_{1}^{\prime} x_{1}+u_{2}^{\prime} x_{2}+u_{8}^{\prime} x_{3}$ in der Normalform dargestellt werde: durch die Substitution $u_{i}=u_{i}^{\prime}$ in (67) folgt

$$
\frac{1}{4} \sum_{4}^{3} \omega^{\prime}\left(u_{i}^{\prime}\right) \cdot f^{\prime}\left(x_{i}\right)=\lambda^{\prime} X_{1} \sqrt{\omega\left(u_{1}^{\prime}, u_{2}^{\prime}, u_{3}^{\prime}\right)},
$$


wo $f_{i}$ zur Abkürzung gesetzt ist für $\frac{1}{2} f^{\prime}\left(x_{i}\right)$. Hiermit ist die Axe der Parabel bestimmt, wir wählen sje als Seite $X_{1}=0$ des Coordinatendreiecks. Doppelt zählend ergibt sich die Axe, wenn in (63) $u_{i}$ ersetzt wird durch $\frac{1}{2} f^{\prime}\left(x_{i}\right)$; der Ausdruck, welcher hierdurch aus $U_{1}$ hervorgeht, ist nach (4) gleich $\lambda^{\prime} X_{1}$, man hat daher nach (63):

$$
-\lambda^{\prime} \cdot \omega\left(f_{1} f_{2} f_{3}\right)+\tau\left(\begin{array}{l}
p f \\
p f
\end{array}\right)=-\lambda^{\prime 3} X_{1}{ }^{2} .
$$

Durch Multiplication der drei ersten Verticalreihen der Determinante $\left(\begin{array}{l}p f \\ p f\end{array}\right)$ mit $x_{1}, x_{2}$ resp. $x_{3}$ und Subtraction von der fünften Reihe und durch analoges Verfahren bei den Horizontalreihen verwandelt sich nun $\left(\begin{array}{l}p f \\ p f\end{array}\right)$ in $f(x, x) \cdot F(p, p)-A p_{x}{ }^{2}$ oder in $-A p_{x}{ }^{2}$, da $F(p, p)=0$, so dass an Stelle von (68) die Gleichung tritt

$$
\lambda^{\prime} \omega\left(f_{1}^{\prime}, f_{2}, f_{3}\right)+\tau A p_{x}{ }^{2}=\lambda^{\prime 3} X_{1}{ }^{2} .
$$

Das Tangenteupaar, welches rom Punkte $U_{1}=0$ mit den Coordinaten $y_{1}, y_{2}, y_{3}$ an die Parabel gezogen werden kann, besteht aus der unendlich fernen Geraden und der Scheiteltangente; die Gleichung dieses Geradenpaares ist

$$
f(y, y) \cdot f(x, x)-f^{2}(x, y)=0 .
$$

Nun geht $f(y, y)$ aus der linken Seite $-\lambda^{\prime} U_{1}^{2}$ von (63) hervor, wenn man die Producte $u_{i} u_{k}$ ersetzt durch $a_{i k}$, es wird daher

$$
f(y, y)=-\lambda^{\prime}[a, \omega]+2 \tau F(p, p)=-\lambda^{\prime}[a, \omega],
$$

da $F(p, p)=0$; ferner geht $f^{2}(x, y)$ oder

$$
\left\{y_{1} \cdot \frac{1}{2} f^{\prime}\left(x_{1}\right)+y_{2} \cdot \frac{1}{2} f^{\prime}\left(x_{2}\right)+y_{3} \cdot \frac{1}{2} f^{\prime}\left(x_{3}\right)\right\}^{2}
$$

aus (63) hervor, wenn man $u_{i}$ ersetzt durch $\frac{1}{2} f^{\prime}\left(x_{i}\right)$, wodurch sich, wie oben gezeigt wurde, der Ausdruck (68a) ergibt. An Stelle der Gleichung des Tangentenpaares $f(y, y) \cdot f(x, x)-f^{2}(x, y)=0$ erhält man somit:

$$
-\lambda^{\prime}[a, \omega] f(x, x)+\lambda^{\prime} \omega\left(f_{1}, f_{2}, f_{3}\right)+\tau A p_{x}{ }^{2}=0,
$$

mit Rücksicht auf (63), und durch die Bedingung $\sqrt{\omega\left(u_{1}^{\prime}, u_{2}^{\prime}, u_{3}{ }^{\prime}\right)}=1$ ist in der That $\lambda^{\prime}$ der richtige Factor von $X_{1}$, in Uebereinstimmung mit der Fundamentalgleichung (4). Aus (67) folgt für $x_{i}=\frac{1}{2} \omega^{\prime}\left(u_{i}^{\prime}\right)$, wieder mit Rüicksicht auf (4),

$$
U_{1}=\frac{1}{2} \omega^{\prime}\left(u_{1}^{\prime}\right) u_{1}+\frac{1}{2} \omega^{\prime}\left(u_{2}^{\prime}\right) u_{2}+\frac{1}{2} \omega^{\prime}\left(u_{3}^{\prime}\right) u_{3} .
$$


oder auch in Folge von $\lambda^{\prime}=[a, \omega]$ :

$$
[a, \omega]^{2} f(x, x)-[a, \omega] \cdot \omega\left(f_{1}, f_{2}, f_{3}\right)-\tau A p_{x}{ }^{2}=0 .
$$

Diese Gleichung stellt das Product aus der Scheiteltangente in die unendlich ferne Gerade dar; will man die Scheiteltangente einzcln haben, so ist nach der am Schlusse von $\S 4$ gegebenen Methode zu verfahren. Jedenfalls kann man setzen:

$$
[a, \omega]^{2} f(x, x)-[a, \omega] \cdot \omega\left(f_{1}, f_{2}, f_{3}\right)-\tau A p_{x}^{2}=2 \varrho \lambda^{\prime 2} X_{2} X_{3},
$$

wobei der Factor $\varrho$ so bestimmt sei, dass $X_{2}=0$ in der Normalform vorliegt; $X_{3}$ sei identisch mit $p_{1} x_{1}+p_{2} x_{3}+p_{3} x_{3}$. Aus $(70)$ folgt

$$
[a, \omega]^{2} f(x, x)=[a, \omega] \cdot \omega\left(f_{1}, f_{2}, f_{3}\right)+\tau A p_{x}{ }^{2}+2 \varrho \lambda^{\prime 2} X_{2} X_{3},
$$

daher mit Benutzung vou (68a)

$$
[a, \omega]^{2} f(x, x)=\lambda^{\prime 3} X_{1}^{2}+2 \varrho \lambda^{\prime 2} X_{2} X_{3}
$$

und nach Wegfall des gemeinsamen Factors $\lambda^{\prime}=[a, \omega]$ :

$$
f(x, x)=\lambda^{\prime} X_{1}^{2}+2 \varrho X_{2} X_{3} .
$$

Für $X_{1}, X_{2}, X_{3}, U_{1}$ und $U_{2}$ sind im Vorhergehenden Ausdrücke angegeben; $U_{3}$ ist vorlüufig nur insofern bestimmt, als $U_{3}=0$ den Schnittpunkt von $X_{1}=0$ und $X_{2}=0$ reprïsentirt; der in $U_{3}$ enthaltene Factor werde so fixirt, dass

$$
\left.u_{1} x_{1}+u_{2} x_{2}+u_{3} x_{3}=U_{1} X_{1}+U_{2} X_{2}+U_{3} X_{3} \cdot{ }^{1}\right)
$$

Für die Determinante $r$ der analogen Transformation wie in dem oben behandelten Falle zweier nicht verschwindenden Wurzeln $\lambda^{\prime}$ und $\lambda^{\prime \prime}$ findet man auch hier analog der Ableitung, von (34) den Werth

$$
r^{2}=\frac{1}{\tau} \text {. }
$$

Ferner ist $A=-\varrho^{2} \lambda^{\prime} r^{2}$ oder

$$
\rho^{2}=-\frac{A \tau}{\lambda^{\prime}}
$$

wenn über das Vorzeichen von $\rho$ verfügt ist, so ist damit auch über dasjenige von $X_{2}$ verfügt, weil $\varrho X_{2} X_{3}$ ein rationaler Ausdruck ist.

1) Dass in dieser Gleichung der Factor von $U_{1} X_{1}$ wirklich gleich 1 ist, erweist die Sabstitution $x_{i}=\frac{1}{2}-\omega^{\prime}\left(u_{i}^{\prime}\right), u_{i}=u_{i}^{\prime}$; man erhält durch sie

$$
\omega\left(u_{1}^{\prime}, u_{2}^{\prime}, u_{3}^{\prime}\right)=\sqrt{\omega\left(u_{1}^{\prime}, u_{2}^{\prime}, u_{3}^{\prime}\right)} \cdot \omega\left(u_{1}^{\prime}, u_{2}^{\prime}, u_{3}^{\prime}\right)
$$

(mit Hilfe von (63) oder (65)), also in Uebereinstimmung mit der obigen Forderung $\omega\left(u_{1}^{\prime}, u_{2}^{\prime}, u_{3}{ }^{\prime}\right)=1$. Analog erhält man durch die Substitution $x_{i}=\frac{1}{2} \omega^{\prime}\left(u_{i}^{\prime \prime}\right)$, $u_{i}=u_{i}{ }^{\prime \prime}$ links $\omega\left(u_{1}^{\prime \prime}, u_{2}{ }^{\prime \prime}, u_{8}{ }^{\prime \prime}\right)$, rechts nach $(65) \sqrt{\omega\left(u_{1}{ }^{\prime \prime}, u_{2}{ }^{\prime \prime}, u_{3}{ }^{\prime \prime}\right)}$. 
Es sei noch erwähnt, dass die Grösse $2 p=2\left|\frac{\varrho}{\lambda^{\prime}}\right|$ Parameter der Parabel genannt wird; näheres hierzu in $\S 11$.

Uebrigens lässt sich auch noch das Product aus dem Ausdruck für den Scheitel $U_{3}=0$ und demjenigen für den unendlich fernen Punkt der Axe elegant berechnen. Für die Gleichung der Parabel in Liniencoordinaten findet man nämlich nach (34), S. 82:

$$
F(u, u)=-\left(\varrho^{2} U_{1}^{2}+2 \lambda^{\prime} \varrho U_{2} U_{3}\right) r^{2}
$$

oder also

$$
\tau F(u, u)+\varrho^{2} U_{1}^{2}=-2 \lambda^{\prime} \varrho U_{2} U_{3}
$$

and mit Benutzung von (74):

$$
\tau F(u, u)-\frac{A \tau}{\lambda^{\prime}} U_{1}^{2}=-2 \lambda^{\prime} \varrho U_{2} U_{3} ;
$$

durch Substitution des für $U_{1}^{2}$ in (63) gefundenen Werthes folgt

$$
\lambda^{\prime 2} F(u, u)+A\left[\tau\left(\begin{array}{ll}
p & u \\
p & u
\end{array}\right)-\lambda^{\prime} \omega(u, u)\right]=-\frac{2 \lambda^{\prime 3} \rho}{\tau} U_{2} U_{3},
$$

so dass hiernach

$$
\lambda^{\prime 2} F(u, u)+A\left[\tau\left(\begin{array}{l}
p u \\
p u
\end{array}\right)-\lambda^{\prime} \cdot \omega(u, u)\right]=0, \quad \text { wobei } \lambda^{\prime}=[a, \omega],
$$

das oben verlangte Product darstellt. Da der Berührungspunkt $U_{2}=0$ der Parabel mit der unendlich fernen Geraden bekannt ist, kann mit Hilfe von (78) die Gleichung des Scheitels $U_{3}=0$ gefunden werden; man hat nur die am Schlusse von $\S 4$ gegebene Methode anzuwenden.

Während bisher vorausgesetzt wurde, dass für die Doppelwurzel $\lambda^{\prime \prime}=\lambda^{\prime \prime \prime}=0$ der kubischen Gleichung (8a) die Unterdeterminanten $\mathrm{B}_{i k}$ nicht alle verschwiinden, wollen wir nun untersuchen, was eintritt, wenn alle $B_{i k}$ und daher auch die Grössen $A_{i k}$, nicht aber $\alpha_{11}+\alpha_{22}+\alpha_{33}$ verschwinden. Aus (20) folgt für diesen Fall

$$
\frac{1}{4} \sum_{1}^{3} \omega^{\prime}\left(u_{i}\right) \cdot f^{\prime}\left(x_{i}\right)=\lambda^{\prime} U_{1} X_{1},
$$

für jeden Punkt $y$ der Geraden $X_{1}=0$ ist daher

$$
\frac{1}{4} \sum_{j}^{3} \omega^{\prime}\left(u_{i}\right) \cdot f^{\prime}\left(y_{i}\right)=0,
$$

d. h. es muss $f^{\prime}\left(y_{i}\right)$ proportional sein zu $p_{i}$, welchen Punkt $y$ von $X_{1}=0$ man auch wählen mag; es gibt zufolge $\$ 4$ nicht einen einzigen Mittelpunkt, sondern eine Mittelpunktslinie $X_{1}=0$. Die Curve $f(x, x)=0$ stellt in diesem Falle zwei parallele Geraden dar. Dass die Determinante $A$ von $f(x, x)$ verschwindet, geht auch aus dem gleichzeitigen Bestehen der Gleichungen $A_{i k}=0$ hervor, welche 
nach der Fussnote zu S. $86 \frac{1}{2} F^{\prime}\left(p_{k}\right)=0 \quad(k=1,2,3)$ zur Folge haben. Diese bestehen nur dann neben einander, wenn ihre Determinante, d. i. $A^{2}$, verschwindet. Da ausserdem $F(p, p)=0$, liegt in der That auch zufolge der in $\S 6$ abgeleiteten Kriterien ein Parallelenpaar vor. Durch Coordinatentransformation kann jetzt $f(x, x)$ übergeführt werden in

$$
f(x, x)=\lambda^{\prime} X_{1}{ }^{2}+x X_{3}{ }^{2},
$$

wo $x=\frac{\mu}{p_{y}}$ durch eine der Gleichungen (46) oder (47) in $\S 4$ bestimmt ist.

Der Fall endlich, dass für $\lambda^{\prime \prime}=\lambda^{\prime \prime \prime}=0$ auch $\beta_{11}+\beta_{22}+\beta_{33}$ verschwindet, also auch $\alpha_{11}+\alpha_{22}+\alpha_{33}$ Null ist, hat zur Folge, wie übrigens bereits S. 100 bemerkt wurde, dass ïberhaupt eine dreifache Wurzel $\lambda=0$ der Gleichung (8a) vorliegt. Jetzt verschwindet nach (11) neben $F(p, p)$ auch $[a, \omega]$, und dass $A=0$ ist, wurde soeben gezeigt. Jedenfalls liegt also ein Geradenpaar vor, etwa

$$
f(x, x)=u_{x} \cdot v_{x}
$$

zufolge $F(p, p)=0$ ist auch $\sum \pm\left(u_{1} v_{2} p_{3}\right)=0$. Ferner besteht nach (13) in $\$ 5$ die Relation

$$
\boldsymbol{\omega}(u, u) \cdot \omega(v, v)-\omega^{2}(u, v)=\tau \sum \pm\left(u_{1} v_{2} p_{3}\right)^{2} ;
$$

da die rechte Seite dieser Gleichung verschwindet und $\omega(u, v)$ mit $[a, \infty]$ identisch ist, mithin gleichfalls den Wertl Null hat, bleibt $\omega(u, u) \cdot \omega(v, v)=0, \mathrm{~d}$. h. für eine der beiden Geraden, etwa für $u_{x}=0$, muss sein $\omega^{\prime}\left(u_{i}\right)=0$, die $u_{i}$ müssen den $p_{i}$ proportional sein: eine Gerade des Pares $f(x, x)=0$ liegt im Unendlichen.

Wären schliesslich alle Grössen $\alpha_{i k}$ Null, so hätte man für irgend einen bestimmten, aber willkürlich gewählten Index $k(k=1,2,3)$ die drei Gleichungen:

$$
\omega_{1 i} a_{1 k}+\omega_{2 i} a_{2 k}+\omega_{3 i} a_{3 k}=0 \quad(i=1,2,3),
$$

aus denen folgt

$$
a_{1 k}: a_{2 k}: a_{3 k}=p_{1}: p_{2}: p_{3}
$$

mithin wäre jetzt $f(x, x)=0$ gleichbedeutend mit $p_{x}{ }^{2}=0$, es würde $f(x, x)=0$ deppelt zählend die unendlich ferne Gerade darstellen.

Wir wollen nun untersuchen, welche speciellen Fälle in der Gleichung (29) enthalten sind; wir schreiben (29) zu dem Zweck in der Form: 


$$
\frac{X^{2}}{-\frac{x}{\lambda^{\prime}}}+\frac{Y^{2}}{-\frac{x}{\lambda^{\prime \prime}}}-1=0,
$$

wobei $X=\frac{X_{1}}{X_{3}}, Y=\frac{X_{9}}{X_{3}}$ gesetzt wurde. Zufolge (11) bestehen hierbei für $\lambda^{\prime}$ und $\lambda^{\prime \prime}$ die Relationen

$$
\lambda^{\prime}+\lambda^{\prime \prime}=[a, \omega], \quad \lambda^{\prime} \lambda^{\prime \prime}=\tau F(p, p) ;
$$

ferner ist $x=\frac{A}{F(p, p)}$, und wenn $A=F(p, p)=0$, ist $x$ durch eine der Gleichungen (46) oder (47) in $\S 4$ bestimmt.

Es sei nun zunächst $F(p, p) \gtrless 0$ und I) $A \gtrless 0$ :

Im Falle 1) $F(p, p)<0$ haben $\lambda^{\prime}$ und $\lambda^{\prime \prime}$ nach (84) verschiedene Vorzeichen, und umgekehrt ist bei ungleichen Vorzeichen von $\lambda$ der Ausdruck $F(p, p)$ negativ, der Kegelschnitt eine Hyperbel.

Im Falle 2) $F(p, p)>0$ haben $\lambda^{\prime}$ und $\lambda^{\prime \prime}$ gleiche Vorzeichen und dasselbe Vorzeichen hat alsdann auch $\lambda^{\prime}+\lambda^{\prime \prime}=[a, \omega]$; die Curve (83) ist daher reell, wenn dieses Zeichen mit demjenigen von $-x$ übereinstimmt, d. h. für $-x\left(\lambda^{\prime}+\lambda^{\prime \prime}\right)>0$, oder auch für $F(p, p)>0$ und $A[a, \omega]<0$ ist die Curve (83) eine reelle Ellipse, während $F(p, p)>0$ und $A[a, \omega]>0$ eine imaginäre Curve (imaginäre Ellipse) ergibt ${ }^{1}$.

Es sei

II) $A=0$.

Da immer noch $F(p, p) \gtrless 0$ vorausgesetzt werden möge, ist hier $x=0$ und (29) verwandelt sich in

$$
\lambda^{\prime} X^{2}+\lambda^{\prime \prime} Y^{2}=0 \text {. }
$$

Diese Gleichung ist in zwei lineare Factoren zerlegbar, stellt also ein imaginäres oder reelles Geradenpaar dar, je nachdem $\lambda^{\prime}$ und $\lambda^{\prime \prime}$ gleiche oder verschiedene Vorzeichen haben, $d . h$. je nachdem $F(p, p)>0$ oder $<0$ ist. Die Spitze des Geradenpares liegt im Endlichen, weil nur im Falle $F(p, p)=0$ die unendlich ferne Gerade zwei zusammenfallende Punkte mit $f(x, x)=0$ gemeinsam hat.

1) Um nachzuweisen, dass diese Kriterien für die reelle und imaginäre Ellipse mit den in $\S 6$ aufgestellten Kriterien übereinstimmen, wäre zu zeigen, dass $[a, w]$ und $f(y, y)$ so lange $F(p, p)>0$ stets gleiche Vorzeichen haben, wobei $y_{1}, y_{2}, y_{3}$ die Coordinaten irgend eines auf der unendlich fernen Geraden gelegenen Punktes darstellen. Dieser Nachweis wird in dem zu vorliegendem Paragraphen gehörigen Theile des Anhangs geliefert; auch wird gezeigt, dass die eben aufgestellte Behauptung noch im Falle $F(p, p)=0$ richtig ist: 
Nahere Bestimmung der Dimension und Gestalt von Ellipse und Hyperbel. 109

Während bisher stets $F(p, p) \gtrless 0$ angenommen wurde, sei nun $F(p, p)=0$ und

I) $A \gtrless 0$. Jetzt stellt $f(x, x)=0$, wie wir sahen, eine Parabel dar, $x$ wird unendlich gross und in Folge dessen musste $f(x, x)$ auf eine von (29) verschiedene Form transformirt werden. Wenn hingegen

II) $\boldsymbol{A}=0$, so ist $x$ durch einen der Werthe (46) oder (47) in $\S 4$ bestimmt; eine Wurzel der Gleichung (11) ist Null (etwa $\lambda^{\prime \prime}$ ), so dass an Stelle von (29) die Gleichung tritt

$$
\lambda^{\prime} X^{2}+x=0,
$$

welche ein Parallelenpaar darstellt. Dasselbe ist imaginär oder reell, je nachdem $\lambda$ und $x$ gleiche oder verschiedene Vorzeichen besitzen, d. h. je nachdem das Product $\lambda^{\prime} x$, welches nach (47) in $\$ 4$ durch den Ausdruck

$$
\frac{[a, \omega] F(u, u)}{\left(\begin{array}{ll}
p & u \\
p & u
\end{array}\right)}
$$

ersetzt werden kann, positiv oder negativ ist; hierbei sind $u_{1}, u_{2}, u_{3}$ irgend welche Zahlen, die nur den $p_{1}, p_{2}, p_{3}$ nicht proportional sein sollen. Durch Einfülırung der Coordinaten

$$
y_{1}=p_{2} u_{3}-p_{3} u_{2}, \quad y_{2}=p_{3} u_{1}-p_{1} u_{3}, \quad y_{3}=p_{1} u_{2}-p_{2} u_{1}
$$

eines unendlich fernen Punktes verwandelt sich $\left(\begin{array}{l}p u \\ p u\end{array}\right)$ in $f(y, y)$; ferner haben nach der Fussnote zu S. 108 nunmehr $[a, \omega]$ und $f(y, y)$ gleiche Vorzeichen, so dass bei (87) nur noch das Vorzeichen von $F(u, u)$ in Betracht kommt. Hier darf etwa $u_{1}=1, u_{2}=u_{3}=0$ angenommen werden, wonach nur $A_{11}$ übrig bleibt; zufolge des Umstandes, dass im Falle $A=0$ die Hauptunterdeterminanten $A_{11}, A_{22}, A_{33}$ gleiche Vorzeichen besitzen und nicht gleichzeitig verschwinden können ${ }^{1}$ ), so lange $f(x, x)=0$ zwei verschiedene Geraden darstellt, erhält man in Uebereinstimmung mit $\S 6$ das Resultat, dass die beiden Parallelen imaginär oder reell sind, je nachdem unter den drei Grössen $A_{i i}$ $(i=1,2,3)$ eine willkürlich ausgewählte nicht verschwindende positiv, oder negativ ist.

\section{$\S 11$.}

Nähere Untersuchung der nicht ausartenden Kegelschnitte. Gleichung

Wie im vorhergehenden Paragraphen gezeigt wurde, stellt die (1)

$$
\lambda^{\prime} X^{2}+\lambda^{\prime \prime} Y^{2}+x=0
$$

1) Vgl. die Fussnote zu S. 27. 
eine reelle Ellipse dar, wenn $\lambda^{\prime}$ und $\lambda^{\prime \prime}$ dasselbe Vorzeichen haben wie $-x$; man kann in diesem Falle setzen

$$
-\frac{x}{\lambda^{\prime}}=a^{2},-\frac{x}{\lambda^{\prime \prime}}=b^{2},
$$

wo $a^{2}$ und $b^{2}$ positiv sind, und erhält hierdurch an Stelle von (1) die Gleichung

$$
\frac{X^{2}}{a^{2}}+\frac{Y^{2}}{b^{2}}-1=0
$$

Hierbei fallen die rechtwinkligen Coordinatenaxen ${ }^{1}$ ) mit den beiden Hauptaxen der Curve zusammen, der Coordinatenanfang mit dem Mittelpunkt der Ellipse; die Stücke von der Länge $a$, resp. $b$, welche durch die Curve auf den Coordinatenaxen abgeschnitten werden, bezeichnet man als die halben Axen der Ellipse, und zwar je nach ihrer Grösse als halbe grosse und halbe kleine Axe. Die Curve liegt überhaupt symmetrisch zur $X$ - und $Y$-Axe, wie nicht nur aus der Gleichung (3) hervorgeht, in der die Glieder mit ungeraden Exponenten der Variabeln fehlen, sondern auch aus der Definition der Hauptaxen als zweier zu einander senkrechten conjugirten Durchmesser folgt. Bei Untersuchung der Gestalt kann man sich daher auf den Verlauf der Curve in einem, etwa im ersten Qnadranten beschränken; alsdann folgt aus (3) $Y=+\frac{b}{a} \sqrt{a^{2}}-X^{\overline{2}}$; für $X=0$ wird $Y=+b$, mit wachsendem Werthe von $X$ nimmt $Y$ ab und verschwindet für $\mathrm{X}=a$. Die Ellipse hat demnach eine Gestalt, wie sie etwa in Fig. 3, S. 117 zum Ausdruck kommt. Für $a=b$ geht die Ellipse in einen Kreis über.

Die Gleichung (1) repräsentirt eine Hyperbel, wenn $\lambda^{\prime}$ und $\lambda^{\prime \prime}$ ungleiche Vorzeichen haben; es sei nun etwa $-\frac{x}{\lambda^{\prime}}$ positiv, gleich $a^{2}$, so wird $-\frac{x}{\lambda^{\prime \prime}}$ negativ, gleich $-b^{2}$, und (1) verwandelt sich nach Einführung dieser Grössen in

$$
\frac{X^{2}}{a^{2}}-\frac{Y^{2}}{b^{2}}-1=0 \text {. }
$$

Man erkenut aus dieser Gleichung, dass nur die eine der beiden Coordinatenaxen (in (4) die $X$-Axe) die Curve in reellen Punkten trifft; die Länge dieser Axenabschnitte (in (4) von der Grösse a) bezeichnet man als Länge der halben reellen Axe. Die andere Coordinatenaxe (in (4) die $\boldsymbol{Y}$-Axe) schneidet die Curve nicht reell, sie wird die Nebenaxe genannt. Die Grösse $b$ in (4) ist für die zu (4)

1) Vgl. auch S. $49 \mathrm{f}$. 
"conjugirte Hyperbel" $\frac{Y^{2}}{b^{2}}-\frac{X^{2}}{a^{2}}-1=0$ die analoge Grösse (halbe reelle Axe) wie $a$ bei (3).

Zur Discussion der Gleichung (4) wollen wir Polarcoordinaten einführen durch die Substitution $X=\varrho \cos \vartheta, Y=\varrho \sin \vartheta ;$ alsdann ergibt sich

$$
\varrho=\frac{a b}{\sqrt{b^{2} \cos ^{2} \vartheta-a^{2} \sin ^{2} \vartheta}} \text { oder } \varrho=\frac{a b}{\sqrt{b^{2}-\left(a^{2}+b^{2}\right) \sin ^{2} \vartheta} .}
$$

Auch hier genügt es den Verlauf der Curve im ersten Quadranten zu untersuchen; die Winkel $\vartheta$ werden von der positiven $X$-Axe aus gerechnet und nehmen zu in dem der Bewegung eines Uhrzeigers entgegengesetzten Sinne. Für $\vartheta=0$ wird $\varrho=a$, mit wachsendem Werthe von $\vartheta$ wächst auch $\varrho$ und erreicht einen unendlich grossen Werth für $\operatorname{tg} \vartheta=\frac{b}{a} ;$ dieser unendlich grosse Radiusvector trifft die Curve in zwei zusammenfallenden Punkten im Unendlichen, er ist eine As y mptote der Curve (die zweite Asymptote, welche den zweiten und vierten Quadranten durchschneidet, entspricht einem Winkel $\vartheta$, für den tg $\vartheta$ $\left.=-\frac{b}{a}\right) \cdot{ }^{1}$ ) Wächst der Winkel $\vartheta$ noch weiter, so dass $\operatorname{tg} \vartheta>\frac{b}{a}$, so sind die Schnittpunkte des zugehörigen Radiusvectors imaginär. Die Hyperbel hat demnach eine Gestalt, wie sie etwa in Fig. 4, S. 118 zum Ausdruck kommt. Im Falle $a=b$ heisst die Curve gleichseitige Hyperbel; bei ihr ist $\vartheta=45^{\circ}$, bezw. $135^{\circ}$, die Asymptoten sind als dann zu einander normal und halbiren die Winkel der Hauptaxen, wie überhaupt zweier conjugirten Durchmesser. Das letztere folgt daraus, dass nach (24) in $\S 5$ bei einem beliebigen Kegelschnitt jedes Paar solcher Durchmesser harmonisch liegt zu dem Asymptotenpaar; stehen die Asymptoten speciell auf einander senkrecht, so halbiren sie daher die Winkel zweier conjugirten Durchmesser.

Das Asymptotenpaar eines beliebigen Kegelschnitts $f(x, x)=0$ ist nach (26) in $\S 5$ gegeben durch $F(p, p) \cdot f(x, x)-A p_{x}{ }^{2}=0$; wendet man auf dieses, im Fall der Ellipse natürlich imaginäre, bei der Hyperbel reelle Geradenpaar eine der Formeln (36) in § 7 für den Winkel $\alpha$ zweier Geraden an, so verschwinden zufolge der Relationen $\omega^{\prime}\left(p_{i}\right)=0(i=1,2,3)$ die durch $A p_{x}{ }^{2}$ auftretenden Glieder, man hat daher z. B.

$$
\operatorname{tg}^{2} \alpha=-\frac{4 \tau F(p, p)}{[a, \omega]}=-\frac{4 \lambda^{\prime} \lambda^{\prime \prime}}{\left(\lambda^{\prime}+\lambda^{\prime \prime}\right)^{2}},
$$

woraus hervorgeht, dass dieser Winkel nur abhängig ist von dem Ver-

1) Vgl. auch die Bemerkungen über Asymptoten in § 5. 
hältniss $\lambda^{\prime}: \lambda^{\prime \prime}$, oder was dasselbe aussagt, nur abhängig ist von dem Verhältniss der Axen $b: a$.

Im Falle der Hyperbel $\frac{X^{2}}{a^{2}}-\frac{Y^{2}}{b^{2}}-1=0$ ist $-\frac{\lambda^{\prime}}{\lambda^{\prime \prime}}=+\frac{b^{2}}{a^{2}}$; setzt man diesen Quotienten gleich $\mu$, so wird

$$
\operatorname{tg}^{2} \alpha=\frac{4 \mu}{(1-\mu)^{2}}, \quad \operatorname{tg} \alpha=\frac{ \pm 2 \sqrt{\mu}}{1-\mu},
$$

und unter Rücksicht auf die Formel $\operatorname{tg} \alpha=\frac{2 \operatorname{tg} \frac{\alpha}{2}}{1-\operatorname{tg}^{2} \frac{\alpha}{2}}$ erkennt man sofort, dass $\sqrt{\mu}= \pm \frac{b}{a}$ gleich ist der Tangente des halben Asymptotenwinkels in Uebereinstimmung mit der oben gefundenen Formel

$$
\operatorname{tg} \vartheta= \pm \frac{b}{a}
$$

Man nennt übrigens solche Kegelschnitte, für welche das Verhältniss $\lambda^{\prime}: \lambda^{\prime \prime}$ oder auch der Winkel der beiden Asymptoten gleich gross ist, ähnliche Kegelschnitte.

Betrachten wir nun noch die Parabel. Die Gleichung dieser Curve wurde in die Form gebracht

$$
\lambda^{\prime} X_{1}^{2}+2 \varrho X_{2} X_{3}=0,
$$

wofür nach Einführung von $\frac{X_{1}}{X_{3}}=Y, \frac{X_{2}}{X_{3}}=X$ und nach Einführung des Parameters $2 p=-\frac{2 \rho}{\lambda^{\prime}}$ gesetzt werden kann

$$
Y^{2}-2 p X=0 \text {. }
$$

Man erkennt aus dieser Gleichung sofort, dass die Curve zur $X$-Axe symmetrisch liegt und dass bei positivem $p$ nur zu positiven Abscissen $X$ reelle Ordinaten $Y$ gehören, während bei negativem $p$ nur zu negativen Abscissen reelle Ordinaten gehören, d. h. die Curve liegt nur auf einer Seite der $Y$-Axe; letztere ist zugleich Tangente im Coordinatenanfang (Scheitel). Aus $Y=\sqrt{2 p X}$ folgt noch, dass die absoluten Längen der Ordinaten $Y$ mit wachsenden $X$ gleichfalls zunehmen, doch ist das Wachsthum von $Y$, wie (8) zeigt, im allgemeinen geringer als dasjenige von $X$. Dass die Parabel die unendlich ferue Gerade zur Tangente hat, ist bereits früher erwähnt worden.

Gehen wir nun wieder zurück zu den durch die Gleichung

$$
\lambda^{\prime} X_{1}^{2}+\lambda^{\prime \prime} X_{2}^{2}+x X_{3}^{2}=0
$$

dargestellten Kegelschnitten, und zwar werde vorläufig stets angenommen, dass die Curve nicht ausarte, also eine Ellipse oder Hyperbel 
vorliege. Die Gleichung in Liniencoordinaten wird alsdann

$$
F(u, u) \equiv\left(\lambda^{\prime \prime} x U_{1}^{2}+\lambda^{\prime} x U_{2}^{2}+\lambda^{\prime} \lambda^{\prime \prime} U_{3}^{2}\right) r^{2},
$$

wo $r$ die im vorhergehenden Paragraphen definirte Transformationsdeterminante bedeutet; ferner ist

$$
A=\lambda^{\prime} \lambda^{\prime \prime} x r^{2},
$$

so dass man mit Benutzung von (11) aus (10) erbält:

$$
\frac{F(u, u)}{A} \equiv \frac{U_{1}^{2}}{\lambda^{\prime}}+\frac{U_{2}^{2}}{\lambda^{\prime \prime}}+\frac{U_{3}^{2}}{x}=0 .
$$

Diese Curve wollen wir nun mit dem imaginären Kreispunktepaar

$$
\omega(u, u) \equiv U_{1}^{2}+U_{2}{ }^{2}=0
$$

zusammenstellen zu dem Ausdruck

$$
\frac{\mu F(u, u)}{A}-\omega(u, u) \equiv \mu\left\{\frac{U_{1}{ }^{2}}{\lambda^{\prime}}+\frac{U_{2}^{2}}{\lambda^{\prime \prime}}+\frac{U_{3}{ }^{2}}{x}\right\}-\left(U_{1}^{2}+U_{2}^{2}\right)=0,
$$

welcher für alle möglichen Werthe des Parameters $\mu$ ein ganzes System von Curven zweiter Classe darstellt. In diesem System sind gewisse ausartende Curven enthalten, die für das Folgende grosse Bedeutung haben ${ }^{1}$ ); den ihnen zugehörigen Parameterwerth erhält man dadurch, dass die Determinante von (13) gleich Null gesetzt wird, woraus sich ergibt $\left(\frac{\mu}{\lambda^{\prime}}-1\right)\left(\frac{\mu}{\lambda^{\prime \prime}}-1\right) \cdot \frac{\mu}{x}=0$. Diese Gleichung hat die drei Wurzeln

$$
\mu=0, \quad \mu=\lambda^{\prime}, \quad \mu=\lambda^{\prime \prime},
$$

deren erste das Kreispunktepaar liefert, während den Werthen $\mu=\lambda^{\prime}$ und $\mu=\lambda^{\prime \prime}$ die zwei Punktepaare entsprechen:

$$
\begin{aligned}
& \left\{\frac{\lambda^{\prime} F(u, u)}{A}-\omega(u, u) \equiv \frac{1}{\lambda^{\prime \prime}}\left\{\left(\lambda^{\prime}-\lambda^{\prime \prime}\right) U_{2}^{2}+\frac{\lambda^{\prime} \lambda^{\prime \prime}}{x} U_{3}^{2}\right\}\right. \\
& \equiv \frac{1}{\lambda^{\prime \prime}}\left\{U_{2} \sqrt{\lambda^{\prime}-\overline{\lambda^{\prime \prime}}}+U_{3} \sqrt{\frac{\overline{\lambda^{\prime} \lambda^{\prime \prime}}}{x}}\right\} \\
& \left\{U_{2} V \lambda^{\prime}-\lambda^{\prime \prime}-U_{3} V^{\frac{\lambda^{\prime}}{x} \lambda^{\prime \prime}}\right\}=0 \text { und } \\
& \left\{\frac{\lambda^{\prime \prime} F(u, u)}{A}-\omega(u, u) \equiv \frac{1}{\lambda^{\prime}}\left\{\left(\lambda^{\prime \prime}-\lambda^{\prime}\right) U_{1}^{2}+\frac{\lambda^{\prime} \lambda^{\prime \prime}}{x} U_{3}^{2}\right\}\right. \\
& \equiv \frac{1}{\lambda^{\prime}}\left\{U_{1} \sqrt{\lambda^{\prime \prime}-\lambda^{\prime}}+U_{3} \sqrt{\frac{\lambda^{\prime} \lambda^{\prime \prime}}{x}}\right\} \\
& \left\{U_{1} \sqrt{\lambda^{\prime \prime}-\lambda^{\prime}}-U_{3} \sqrt{\frac{\lambda^{\prime} \lambda^{\prime \prime}}{x}}\right\}=0 \text {. }
\end{aligned}
$$

Hierbei ist $U_{3}$ aus (31), S. 92 zu entnehmen, während alsdann

1) Der wahre Grund hiervon erbellt aus späteren Betrachtungen über sogenannte confocale Kegelschnitte ( $\$ 18$ und 19). 
$U_{1}^{2}$ und $U_{2}^{2}$ aus (12) und (12a) berechnet werden können (vgl. auch den Anhang).

Das eine dieser beiden Punktepaare ist immer reell, das andere imaginär, denn an Stelle von $\left(\lambda^{\prime}-\lambda^{\prime \prime}\right) U_{2}^{2}$ in der einen Gleichung steht das mit entgegengesetztem Vorzeichen versehene Glied $\left(\lambda^{\prime \prime}-\lambda^{\prime}\right) U_{1}^{2}$ in der anderen, während $\frac{\lambda^{\prime} \lambda^{\prime \prime}}{x} U_{3}^{2}$ jedesmal auftritt. Man nennt diese Punktepaare das reelle und das imaginäre Brennpunktepaar.

Ihre Gleichungen zeigen ferner, dass sie auf den zwei Axen der Curve liegen, und zwar harmonisch zum Mittelpunkt $U_{3}=0$ und zum unendlich fernen Punkt $\left(U_{2}=0\right.$ oder $\left.U_{1}=0\right)$ der einen oder anderen Hauptaxe, d. h.:

(15) Der Mittelpunkt einer Ellipse oder Hyperbel liegt sowohl zwischen den beiden reellen, als.auch zwischen den imagiuären Brennpunkten in der Mitte.

Werden die Gleichungen der Brennpunktepaare in der Form geschrieben

$$
\frac{\left(\lambda^{\prime}-\lambda^{\prime \prime}\right)}{x} U_{2}^{2}+\frac{\lambda^{\prime} \lambda^{\prime \prime}}{x^{2}} U_{3}^{2}=0, \text { resp. } \frac{\left(\lambda^{\prime \prime}-\lambda^{\prime}\right)}{x} U_{1}^{2}+\frac{\lambda^{\prime} \lambda^{\prime \prime}}{x^{2}} U_{3}^{2}=0
$$

und beachtet man im Falle einer Ellipse die Relationen (2), so folgt

$$
-\left(\frac{1}{a^{2}}-\frac{1}{b^{2}}\right) U_{2}^{2}+\frac{1}{a^{2} b^{2}} U_{3}^{2}=0, \text { resp. }\left(\frac{1}{a^{2}}-\frac{1}{b^{2}}\right) U_{1}^{2}+\frac{1}{a^{2} b^{2}} U_{3}^{2}=0
$$

woraus hervorgeht, dass für $a>b$ das reelle Brennpunktepaar auf $X_{2}=0$, für $b>a$ auf $X_{1}=0$, in jedem Falle also auf der grösseren der beiden Axen liegt. Man hat daher den Satz:

Bei einer Ellipse liegt das reelle Brennpunktepaar immer auf der grossen, das imaginäre Brennpunktepaar auf der kleinen Axe.

Wenn die Ellipse in einen Kreis übergeht, rücken alle Brennpunkte, wie die Ausdrücke (14) im Falle $\lambda^{\prime}=\lambda^{\prime \prime}$ ergeben, in den Mittelpunkt.

Auf analoge Weise wie bei (17) beweist man den Satz:

(18) Bei einer Hyperbel liegt das reelle Brennpunktepar immer auf der reellen Axe, das imaginäre Brennpunktepaar auf der Nebenaxe.

Durch Anwendung genau der entsprechenden Operationen, welche bei Ellipse und Hyperbel zu den Gleichungen (14) führten, gelangt man im Falle der Parabel, ausgehend von der im vorhergehenden Paragraphen gegebenen Darstellung

$$
f(x, x)=\lambda^{\prime} X_{1}^{2}+2 \varrho X_{2} X_{3}=0,
$$


Constantes Verhältniss d. Entfernungen eines Curvenp. v. Brennp. u. Directrix. 115 zu dem Brennpunktepaar der Parabel:

$$
\frac{\lambda^{\prime} F(u, u)}{A}-\omega(u, u)=\left(\frac{2 \lambda^{\prime}}{\varrho} U_{3}-U_{2}\right) U_{2}=0 .
$$

Hieraus folgt, da $X_{1}=0$ die Axe der Parabel ist:

(21) Bei einer Parabel gibt es nur ein reelles, kein imaginäres Brennpunktepaar; der eine Punkt des Paares liegt auf der Axe der Parabel im Endlichen, der andere auf derselben Geraden im Unendlichen.

Aus dem Vorhergehenden folgt, dass das reelle Brenupunktepaar eines Kegelschnitts $f(x, x)=0$ gegeben ist durch eine Gleichung von der Form

$$
\frac{\lambda^{\prime} F(u, u)}{A}-\omega(u, u) \equiv\left(y_{1} u_{1}+y_{2} u_{2}+y_{3} u_{3}\right)\left(z_{1} u_{1}+z_{2} u_{2}+z_{3} u_{3}\right)=0,
$$

wenn $y_{i}$ und $z_{i}(i=1,2,3)$ die Coordinaten der zwei Brennpunkte bezeichnew.

Wir führen nun in diese Gleichung für die $u_{i}$ speciell die Coordinaten der Verbindungslinie eines reellen Brennpunktes, etwa $y$, mit einem beliebigen Punkte $x$ der Ebene ein, setzen also

$$
u_{1}=y_{2} x_{3}-y_{3} x_{2}, \quad u_{2}=y_{3} x_{1}-y_{1} x_{3}, \quad u_{3}=y_{1} x_{2}-y_{2} x_{1} ;
$$

nach (31) und (32) in $\S 4$ verwandelt sich hierdurch $F(u, u)$ in

$$
f(x, x) \cdot f(y, y)-f^{2}(x, y)
$$

so dass an Stelle von (22) die Gleichung tritt

$$
\frac{\lambda^{\prime}}{A}\left\{f(x, x) \cdot f(y, y)-f^{2}(x, y)\right\}-\left(\begin{array}{l}
x y \\
x y
\end{array}\right)_{\omega_{i k}}=0,
$$

oder auch

$$
\frac{\lambda^{\prime}}{A} f(x, x) \cdot f(y, y) \equiv \frac{\lambda^{\prime}}{A} f^{2}(x, y)+\left(\begin{array}{l}
x y \\
x y
\end{array}\right)_{\omega_{i k}} .
$$

Für einen auf dem Kegelschnitt gelegenen Punkt $x$ verschwindet $f(x, x)$ und man hat alsdann

$$
\frac{\lambda^{\prime}}{A} f^{2}(x, y)=-\left(\begin{array}{l}
x y \\
x y
\end{array}\right)_{\omega_{i k}}
$$

Diese Gleichung gestattet eine ebenso einfache als wichtige geometrische Deutung. Mit Rücksicht auf die Formel (1) in $\S 2$ für den Abstand eines Punktes $x$ von einer Geraden und auf die Formel (10) in $\S 7$ für das Quadrat der Entfernung zweier Punkte folgt nämlich aus (25) der Satz:

(26) Für jeden auf einem Kegelschnitt gelegenen Punkt ist das Verhältniss seiner Abstände von einem Brennpunkt und dessen zugehöriger Polare (der sogenannten Directrix dieses Brennpunktes) constant. 
Aus der symmetrischen Gestalt der Kegelschnitte und der symmetrischen Lage der Brennpunkte folgt, dass dieses Verluältniss dasselbe ist, welchen der beiden Brennpunkte man auch wählen mag.

Ausserdem gilt der Satz:

(27) Jede Directrix steht senkrecht auf der Verbindungslinie der beiden Brennpunkte.

Für $v_{1}, v_{2}, v_{3}$ als Coordinaten der einen Directrix hat man nämlich für den zugehörigen Pol die Gleichung $F(v, u)=0$; andrerseits folgt aus (22):

$$
\frac{2 \lambda^{\prime} F^{\prime}(v, u)}{A} \equiv y_{u} z_{v}+y_{v} z_{u}+2 \omega(v, u) .
$$

Für die Coordinaten $u_{i}=w_{i}(i=1,2,3)$ der Verbindungslinie der beiden Brennpunkte verschwindet aber nicht nur $y_{w}$ und $z_{w}$, sondern auch $F(v, w)$, denn die Gerade $w$ geht durch den Pol von $v$, oder mit anderen Worten: die Geraden $v$ und $w$ sind conjugirte Polaren in Bezug auf den Kegelschnitt, ihre Coordinaten erfüllen daher nach $\S 5$ die Relation $F(v, v)=0$. Hierdurch reducirt sich $(28)$ auf $\omega(v, w)=0$, eine Bedingung, die bekanntlich aussagt, dass die Geraden $v$ und $w$ zu einander normal sind.

Aus der Gleichung (24) geht hervor, dass die Directrix $f(x, y)=0$ des Brennpunktes $y$ den Kegelschnitt im allgemeinen nicht in reellen Punkten trifft; denn für einen solchen Schnittpunkt wäre $f(x, x)=0$ und $f(x, y)=0,(24)$ würde sich verwandeln in $\left(\begin{array}{l}x y \\ x y\end{array}\right)_{\omega_{i k}}=0$, und dieser Ausdruck verschwindet nur, wenn

$$
\left(x_{2} y_{3}-x_{3} y_{2}\right):\left(x_{3} y_{1}-x_{1} y_{3}\right):\left(x_{1} y_{2}-x_{2} y_{1}\right)=p_{1}: p_{2}: p_{3} \text {, }
$$

d. h. wenn der Brennpunkt $y$ auf der unendlich fernen Geraden liegt, was nur bei dem im Unendlichen gelegenen Brennpunkt der Parabel der Fall ist. Im allgemeinen können daher von einem Brennpunkt keine reellen Tangenten an den Kegelschnitt gelegt werden ${ }^{1}$ ), nur bei dem unendlich fernen Brennpunkt der Parabel fällt diese Tangente mit der unendlich fernen Geraden, die zugleich Directrix und Parabeltangente ist, zusammen.

- Für die nähere Untersuchung der Lage von Directrix und Brennpunkt ist vor Allem von Wichtigkeit die Bemerkung, dass die zwei Schnittpunkte des Kegelschnitts mit der die Brennpunkte verbindenden Axe harmonisch liegen zu einem Brennpunkt und dem Schnittpunkt

1) Es folgt daraus die S. $110 \mathrm{ff}$. stillschweigend angenommene Thatsache, dass jeder Kegelschnitt den Brennpunkten die concave Seite zukehrt. 
der zugehörigen Directrix mit der Axe, denn die Directrix ist die Polare des Brennpunktes ${ }^{1}$ ).

Hieraus folgt:

(29) Die Entfernung des Scheitels der Parabel vom Brennpunkt ist so gross wie die Entfernung des Scheitels von der Directrix.

Wie S. 106 erwähnt, bezeichnet man bei der Parabel

$$
\lambda^{\prime} X_{1}^{2}+2 \varrho X_{2} X_{3}=0
$$

die Grösse $2\left|\frac{\rho}{\lambda^{\prime}}\right|$ als Parameter. Wählt man das Vorzeichen der Quadratwurzel $\varrho$ demjenigen von $\lambda^{\prime}$ entgegengesetzt, wodurch $\frac{\varrho}{\lambda^{\prime}}$ negativ, etwa gleich $-p$, wird, so verwandelt sich (19) in $X_{1}{ }^{2}-2 p X_{2} X_{3}=0$, oder unter Anwendung der gleichen Bezeichnungsweise wie bei (8) verwandelt sich (19) in $Y^{2}-2 p X=0$. Die Gleichung (20) zeigt ferner, dass der im Endlichen gelegene Brennpunkt der Parabel die Coordinaten hat $X=\frac{p}{2}, Y=0$; andrerseits hat die Ordinate des der Abscisse $X=\frac{p}{2}$ zugehörigen Curvenpunktes den Wertlı $Y= \pm p$, wie aus $Y^{2}-2 p X=0$ folgt; der Parameter $2 p$ ist demnach so gross, wie die Länge derjenigen Sehne der Parabel, welche durch den Brennpunkt senkrecht zur Axe gezogen ist.

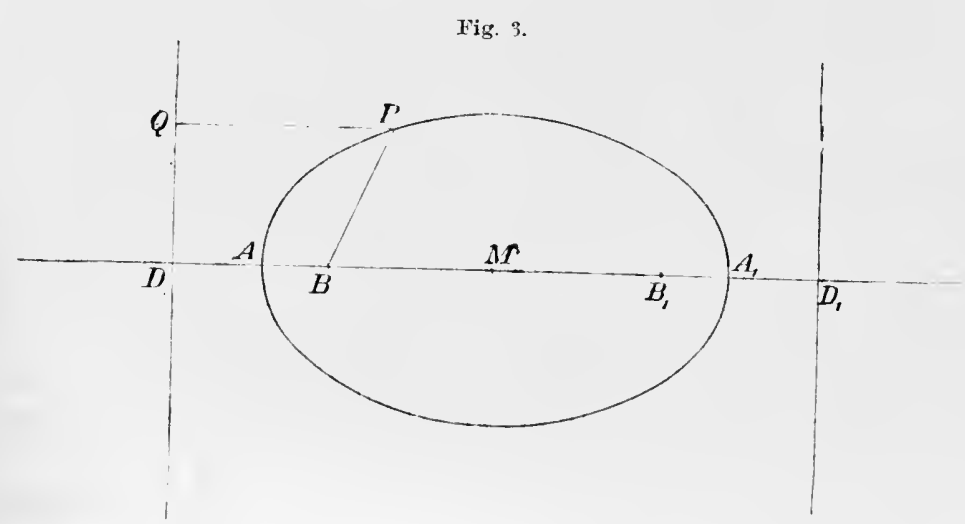

Ueberhaupt nennt man bei jedem Kegelschnitt die lialbe Länge der durch eimen Brennpunkt normal zur Axe gezogenen Sehne den Semiparameter. Wird das constante Verhältniss, von welchem in (26) die Rede ist, durch $e$ bezeichnet, so hat man für den Semipara-

1) Wenn im Folgenden von Brennpunkt und Directrix die Rede ist, meinen wir immer die dem betreffenden Brennpunkt als Polare zugehörige Directrix. 
meter den Werth $p=e \cdot B D$, wobei $B D$ den Abstand des Brennpunktes von . seiner Directrix bedeutet. Es folgt dies sofort mit Hilfe von (26), wenn man den betreffenden Punkt des Kegelschnitts in den Endpunkt einer Sehne der eben genannten Art verlegt.

Bezeichnen wir bei Ellipse und Hyjperbel mit $c$ den absoluten Werth des Abstandes $M B=M B_{1}$ der beiden Brennpunkte $B$ und $B_{1}$ vom Mittelpunkt (die sogenannte lineare Excentricität), mit $a$ die halbe Länge $M A=M A_{1}$ der die Brennpunkte verbindenden Axe $A A_{1}$, so ist bei der Ellipse jedenfalls $a>c$, bei der Hyperbel $a<c$.

Denn für eine Ellipse (3), bei welcher $a>b$, ist das reelle Brennpunktepaar nach $(16)$ gegeben durch $\left(a^{2}-b^{2}\right) \frac{U_{1}^{2}}{U_{s}^{2}}-1=0$, d. h. die reellen Brennpunkte haben die Coordinaten

$$
X= \pm \sqrt{a^{2}-b^{2}}= \pm c, \quad Y=0 .
$$

Für die Hyperbel (4) erhält man hingegen $\left(a^{2}+b^{2}\right) \frac{U_{1}{ }^{2}}{U_{3}^{2}}-1=0$, die Brennpunkte dieser Curve haben somit die Coordinaten

$$
X= \pm \sqrt{a^{2}+b^{2}}= \pm c, \quad Y=0 .
$$

Fig. 4.

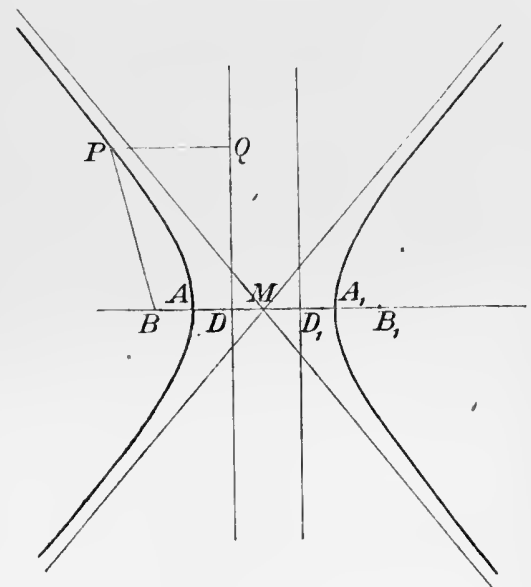

Nennen wir ausserdem $d$ den absolut genommenen Abstand $D M$ $=D_{1} M$ des Mittelpunktes von der Directrix, so folgt aus der oben erwähnten harmonischen Lage sowohl für die Ellipse als für die Hyperbel die Proportion

$$
\begin{gathered}
\frac{d+a}{d-a}=\frac{a+c}{a-c} \\
\text { oder } d: a=a: c
\end{gathered}
$$

d. h.:

(31) Die halbe Axe ist die mittlereProportionalezwischen der linearen Excentricität und dem Abstandedes Mittelpunktes von der Directrix.

Zufolge des Satzes (26) ist für jeden Punkt eines Kegelschnitts das Verhältniss seiner Abstände $P B$, resp. $P Q$ von einem Brennpunkt, resp. der zugehörigen Directrix constant. Für den Fall der Parabel ist die Bestimmung dieser Constanten bereits durch (29) erledigt, sie hat den Werth 1, man hat daher den Satz:

(32) Die Parabel ist geometrischer Ort aller Punkte, für 
welche die Abstande von einem festen Punkte (Brennpunkt) und einer festen Geraden (Directrix) einander gleich sind. Um das zuvor erwähnte constante Verhältniss $e=P B: P Q$ auch bei Ellipse und Hyperbel zu bestimmen, wählen wir als Punkt $P$ den Scheitel der Brennpunktaxe und finden sofort für beide Curvenarten $e=\frac{a-c}{d-a}$, woraus mit Hilfe der Relation $d c=a^{2}$ in (30) folgt

$$
e=\frac{c}{a} \text {. }
$$

Dieses Verhältniss wird als numerische Excentricität bezeichnet und ist $<1 \mathrm{im}$ Fall der Ellipse, $>1$ im Fall der Hyperbel, gleich 1 im Fall der Parabel. Man hat somit für die nicht ausartenden Kegelschnitte die folgeude gemeinsame Definition gefunden:

(34) Ein Kegelschnitt ist geometrischer Ort aller Punkte, für welche das Verhältniss der Abstände von einem festen Punkte (Brennpunkte) und einer festen Geraden (Directrix) constant ist; diese Constante ist $<1,>1,=1$, je nachden der Kegelschnitt eine Ellipse, Hyperbel oder Parabel darstellt.

(35) Umgekehrt, wenn ein Punkt $B$ und eine Gerade $G$ in der Ebene fest gegeben sind, so ist der geometrisclie Ort aller Punkte, für die das Verhältniss der Abstäude von dem festen Punkt und der festen Geraden einen gegebenen Werth $e=\frac{P B}{P Q}$ (vgl. z. B. Fig. 3) besitzt, ein Kegelschnitt, und zwar eine Ellipse, Hyperbel oder Parabel, je nachdem $e<1,>1$, $=1$ ist. Der gegebene Punkt ist ein Brenupunkt des Kegelschnitts, die Gerade die zugehörige Directrix.

Ist $v_{x}=0$ die Gleichung der Geraden, $y$ der feste Punkt, so ist nämlich nach (1) in $\S 2$ und (10) in $\S 7$ die Curve, welche die in (35) gestellten Bedingungen erfüllt, gegeben durch

$$
f(x, x) \equiv \omega(v, v) \cdot\left(\begin{array}{l}
y x \\
y x
\end{array}\right)_{\omega_{i k}}-\tau e^{2} \cdot p_{y}{ }^{2} \cdot v_{x}{ }^{2}=0 ;
$$

um die zugehörige Gleichung in Liniencoordinaten abzuleiten, setzen wir in (36) zunächst $-\tau e^{2} \boldsymbol{p}_{y}{ }^{2}: \boldsymbol{\omega}(v, v)=\lambda$ und erhalten hierdurch $\left(\begin{array}{l}y x \\ y x\end{array}\right)_{\omega_{i k}}+\lambda v^{2}{ }_{x}=0$. Der Coefficient von $\lambda^{2}$ in der gesuchten Gleichung verschwindet nun identisch, denn $v_{x}^{2}$ ist das Quadrat eines linearen Ausdrucks; hinsichtlich des Coefficienten von $\lambda^{1}$ findet man, ähnlich wie in der Fussnote zu S. 102 angedeutet, dass derselbe aus $\left(\begin{array}{l}y x \\ y x\end{array}\right)_{\omega_{i k}}$ hervorgeht, wenn man setzt 


$$
x_{1}=v_{2} u_{3}-v_{3} u_{2}, \quad x_{2}=v_{3} u_{1}-v_{1} u_{3}, \quad x_{3}=v_{1} u_{2}-v_{2} u_{1} .
$$

Man erhält hierdurch den Ausdruck

$$
\dot{\omega}(v, v) \cdot u_{y}{ }^{2}+v_{y}{ }^{2} \cdot \omega(u, u)-2 v_{y} u_{y} \omega(v, u) \text {. }
$$

Der Coefficient von $\lambda^{0}$ stimmt überein mit dem Ausdruck in Liniencoordinaten für $\left(\begin{array}{l}y x \\ y x\end{array}\right)_{\omega_{i k}}$; zur Ableitung desselben benutzen wir die Relation (32) in $\$ 4$, d. h. wir bilden $\left(\begin{array}{l}y x \\ y x\end{array}\right)_{\omega_{i k}} \cdot\left(\begin{array}{l}y z \\ y z\end{array}\right)_{\omega_{i k}}-\left(\begin{array}{l}y x \\ y z\end{array}\right)_{\omega_{i k}}^{2}$ und setzen darin an Stelle von $x_{2} z_{3}-x_{3} z_{2}, x_{3} z_{1}-x_{1} z_{3}, x_{1} z_{2}-x_{2} z_{1}$ resp. $u_{1}, u_{2}, u_{3}$. Die hier angegebene Differenz ist aber nach (55) in $\S 4$ auch gleich $\left(\begin{array}{l}y \\ y\end{array}\right)_{\omega_{i k}} \cdot\left(\begin{array}{l}y x z \\ y x z\end{array}\right)_{\omega_{i k}}$ oder also gleich $\tau p_{y}{ }^{2} \cdot u_{y}{ }^{2}$, daher erhält man als Gleichung von (36) in Liniencoordinaten die folgende:

(37) $F(u, u) \equiv \lambda\left\{\omega(v, v) \cdot u_{y}{ }^{2}+v_{y}{ }^{2} \cdot \omega(u, u)-2 v_{y} u_{y} \omega(v, u)\right\}+\tau p_{y}{ }^{2} u_{y}{ }^{2}=0$, und nach Einführung des Werthes von $\lambda$ ergibt sich nach Wegheben von $\tau p_{y}{ }^{2}$ :

$$
\left.e^{2} v_{y}{ }^{2} \omega(u, u)+u_{y}\left\{\left(e^{2}-1\right) \omega(v, v) u_{y}-2 c^{2} v_{y} \omega(v, u)\right\}=0 .{ }^{1}\right)
$$

Dieser Ausdruck ist von derselben Form, wie aus (22) für die Gleichung eines Kegelschnitts in Liniencoordinaten folgen würde, und man erkenut daraus, dass der festgegebene Punkt $y$ den einen Brennpunkt der Curve darstellt, während der andere Brennpunkt gegeben ist durch

$$
\left(e^{2}-1\right) \cdot \omega(v, v) \cdot u_{y}-2 e^{2} v_{y} \cdot \omega(v, u)=0 .
$$

Für den Fall der Parabel $(e=1)$ erhält man einfach $\omega(v, u)=0$, also die Gleichung des Normalencentrums der Geraden $v_{x}=0$, wie zu erwarten war, denn der zwcite Brennpunkt der Parabel liegt auf der Axe im Unendlichen.

Nachdem wir nunmehr wissen, dass der in (35) fest gegebene Punkt $y$ ein Brennpunkt des Kegelschnitts (36) ist, folgt aus (34) weiter, dass die gegebene Gerade $G$ die zugehörige Directrix sein muss, womit nun (35) vollständig bewiesen ist. Dass die Gerade $v_{x}=0$ Polare von $y$ ist, folgt übrigens auch aus (36); da nämlich $\left(\begin{array}{l}y x \\ y x\end{array}\right)_{\omega_{i k}}=0$ ein vom Punkte $y$ aus gezogenes imaginäres Geradenpaar $T T_{1}$ darstellt, ist die Gleichung (36) von der Form $T T_{1}-c \cdot v_{x}{ }^{2}=0$, wobei $c$ eine Constaute bedeuten möge. Man erkennt ferner, dass $T=0$ und $T_{1}=0$ Tangenten sind, welche die Curve in ihren imaginären Schnitt-

1) Vgl. für eine allgemeinere Aufgabe dieser Art die Transformation auf die Hauptaxen im Anhange zu vorliegendem Paragraphen. 
punkten mit $v_{x}=0$ berühren, d. h. $v_{x}=0$ ist die Polare des Punktes $y$, von welchem aus die beiden Tangenten gezogen sind.

Ist $P$ ein beliebiger Punkt eines Kegelschnitts, $P B$ der Abstand des Punktes rom einen Brennpunkt $B, P Q$ der Abstand von der zugehörigen Directrix (vgl. Fig. 3 und 4), so besteht nach (34) die Relation

$$
\frac{P B}{P Q}=\frac{c}{a} ;
$$

führt man noch den zweiten Brennpunkt $B_{1}$ ein, sowie den Abstand $P Q_{1}$ des Punktes $P$ von der zu $B_{1}$ gehörigen Directrix, so ist auch

$$
\frac{P B_{1}}{P Q_{1}}=\frac{c}{a}, \text { daher } \frac{P B \pm P B_{1}}{P Q \pm P Q_{1}}=\frac{c}{a} .
$$

Wenn wir uns nunmehr auf Ellipse und Hyperbel beschränken, kann nach (30) für $\frac{c}{a}$ gesetzt werden $\frac{a}{d}$ oder $\frac{2 a}{2 d}$, somit folgt

$$
P B \pm P B_{1}=\frac{2 a}{P Q \pm P Q_{1}}=
$$

Bei der Ellipse ist $P Q+P Q_{1}$ gleich dem Abstand $2 d$ der beiden Directricen, bei der Hyperbel gilt dasselbe von $P Q-P Q_{1}$, woraus hervorgeht, dass im Falle der Ellipse $P B+P B_{1}$ constant, nämlich gleich $2 a$ ist, während für die Hyperbel die Differenz $P B-P B_{1}$ den Werth $2 a$ hat. Wir können daher sagen:

(43) Für jeden Punkt einer Ellipse ist die Summe seiner Entfernungen von den beiden Brennpunkten (Summe der Brennstrahlen) coustant, und zwar gleich der grossen $\mathrm{Axe}^{\mathrm{l}}$ ).

Ferner:

(44) Für jeden Punkt einer Hyperbel ist die Differenz seiner Entfernungen von den beiden Brempunkten (Differenz der Brennstrahlen) constant, und zwar gleich der reellen $\mathrm{Axe}^{1}$ ).

\section{$\S 12$.}

Erzeugung der Kegelschnitte durch projective Strahlenbüschel und Punktreihen. Sätze von Pascal und Brianchon.

Bereits in $\S 4$ wurde erwähnt, dass die Gleichung eines Kegelschnitts, der durch fünf gegebene Punkte geht, in Form einer Determinante sechsten Grades dargestellt werden kann; sind $a_{i}, b_{i}, c_{i}, d_{i}, e_{i}$ $(i=1,2,3)$ die Coordinaten der gegebenen Punkte, wobei voraus-

1) Die wahre Quelle der zwei Sätze (43) und (44) wird erst in $\S 19$ angegeben werden. 
gesetzt werden möge, dass höchstens drei der Punkte in einer Geraden liegen, so ist diese Determinante die folgende:

$$
\left|\begin{array}{llllll}
a_{1}{ }^{2} & a_{2}{ }^{2} & a_{3}{ }^{2} & a_{1} a_{2} & a_{1} a_{3} & a_{2} a_{3} \\
b_{1}{ }^{2} & b_{2}{ }^{2} & b_{3}{ }^{2} & b_{1} b_{2} & b_{1} b_{3} & b_{2} b_{3} \\
c_{1}{ }^{2} & c_{2}{ }^{2} & c_{3}{ }^{2} & c_{1} c_{2} & c_{1} c_{3} & c_{2} c_{3} \\
d_{1}{ }^{2} & d_{2}{ }^{2} & d_{3}{ }^{2} & d_{1} d_{2} & d_{1} d_{3} & d_{2} d_{3} \\
e_{1}{ }^{2} & e_{2}{ }^{2} & e_{3}{ }^{2} & e_{1} e_{2} & e_{1} e_{3} & e_{2} e_{3} \\
x_{1}{ }^{2} & x_{2}{ }^{2} & x_{3}{ }^{2} & x_{1} x_{2} & x_{1} x_{3} & x_{2} x_{3}
\end{array}\right|=0 .
$$

Man kann die Gleichung desselben Kegelschnitts auch noch in anderer Form erhalten. Stellt nämlich

$$
(a b x) \equiv\left|\begin{array}{lll}
a_{1} & a_{2} & a_{3} \\
b_{1} & b_{2} & b_{3} \\
x_{1} & x_{2} & x_{3}
\end{array}\right|=0
$$

die Verbindungslinie der beiden Punkte $a$ und $b$ dar, analog $(b c x)=0$ diejenige von $b$ und $c$, u. s. w., so ist

$$
\lambda(a b x)(c d x)-(a d x)(b c x)=0
$$

für beliebige Werthe des Parameters $\lambda$ die Gleichung eines Kegelschnitts, der durch die vier Punkte $a, b, c, d$ hindurchgeht, denn (3) wird erfüllt, wenn man für $x_{1}, x_{2}, x_{3}$ die Coordinaten eines der vier genannten Punkte einsetzt. Damit (3) auch noch. durch die Coordinaten des fünften Punktes $e$ erfüllt wird, ist $\lambda$ entsprechend zu bestimmen; man findet sofort $\lambda=\frac{(a d e)(b c e)}{(a b e)(c d e)}$, wo beispielsweise

$$
(b c e) \equiv\left|\begin{array}{lll}
b_{1} & b_{2} & b_{3} \\
c_{1} & c_{2} & c_{3} \\
e_{1} & e_{2} & c_{3}
\end{array}\right|,
$$

und erhält somit als Gleichung des durch die fünf Punkte $a, b, c, d, c$ bestimmten Kegelschnitts:

$$
(a d e)(b c e)(a b x)(c d x)-(a b e)(c d e)(a d x)(b c x)=0 .
$$

Dieser Ausdruck ist in den Coordinaten der fünf Punkte $a, b, c, d, c$ und in den $x$ vom zweiten Grad; gleiches gilt von der oben erwähnten Determinante sechsten Grades, die demnach von (5) nur um einen Zahlenfactor verschieden sein kann, und man findet in der That diesen Factor gleich $1 .^{1}$ )

1) Die Gleichung (1) zeigt, dass die linke Seite von (5) bis auf das Vorzeichen ungeändert bleibt, wenn man irgend eine Permutation der fünf Buchstaben $a, b, c, d, e$ vornimmt. Anch ist leicht einzusehen, dass es für den betreffenden Kegelschnitt im ganzen $5 \cdot 3=15$ verschiedene Darstellungen gibt, 
Dualistisch folgt aus (3), dass für $\alpha_{i}, \beta_{i}, \gamma_{i}, \delta_{i}(i=1,2,3)$ als Coordinaten von vier Geraden die Gleichung

$$
\lambda(\alpha \beta u)(\gamma \delta u)-(\alpha \delta u)(\beta \gamma u)=0
$$

eine Curve zweiter Classe darstellt, welche die vier Geraden zu Tangenten hat; man kann dies auch in folgender Weise ausdrïcken: Sind $P=0$ und $Q=0, P_{1}=0$ und $Q_{1}=0$ die Gleichungen der Gegenecken irgend eines der Curve zweiter Classe umschriebenen Vierseits, so ist die Gleichung der Curve von der Form

$$
\lambda P Q-P_{1} Q_{1}=0 \text {. }
$$

Die Gleichung (5) kann man auch durch Elimination von $\mu$ ans

$$
\left\{\begin{array}{l}
(a d e)(c d x)-\mu(c d c)(b c x)=0 \quad \text { und } \\
(a b c)(a d x)-\mu(b c e)(a b x)=0
\end{array}\right.
$$

erhalten, woraus folgt:

(9) Die Punkte einer Curve zweiter Ordnung können betrachtet werden als die Schnittpunkte $z$ weier entsprechender Strahlen in den beiden Büscheln $\left.(8)^{1}\right)$; auch die Mittelpunkte dieser Büschel gehören der Curve an.

Setzt man

$$
\begin{aligned}
& (a d c)(c d x) \equiv U, \quad(c d e)(b c x) \equiv V, \\
& (a b e)(a d x) \equiv U_{1}, \quad(b c e)(a b x) \equiv V_{1},
\end{aligned}
$$

so verwandelt sich (8) in

$$
U-\mu V=0, \quad U_{1}-\mu V_{1}=0,
$$

und die Gleichung des Kegelschnitts wird

$$
\left|\begin{array}{ll}
U & V \\
U_{1} & V_{1}
\end{array}\right|=0
$$

Durch Multiplication dieser Determinante mit $\left|\begin{array}{ll}1 & -\mu_{0} \\ 1 & -\mu_{1}\end{array}\right|$, wobei $\mu_{0}$ und $\mu_{1}$ zwei beliebige verschiedene Zahlen bedeuten, erhält man

die sämmtlich von ähnlicher Form sind wie (5). Vgl. auch Hunyady im Journal für die reine und angewandte Mathematik, Bd. 83, S. 79, 1876. Durch (5) wird die in $\$ 4$ ausgesprochene Behauptung bestätigt, dass die Gleichnng des Kegelschnitts identisch verschwindet, wenn vier seiner Punkte, z. B. $b, c, d, e$, in einer Geraden liegen. Vgl. auch Pasch: „Ueber gewisse Determinanten, welche in der Lehre von den Kegelschnitten vorkommen", Journal für die reine und angewandte Mathematik, Bd. 89, S. 247-251, 1879.

1) Irgend vier Strahlen des einen Büschels haben bekanntlich dasselbe Doppelverhältniss wie die vier entsprechenden Strahlen des anderen. Man nennt zwei Strahlenbüschel von der Form (8) einander projectiv zugeordnet. 


$$
\left|\begin{array}{ll}
U-\mu_{0} V & U_{1}-\mu_{0} V_{1} \\
U-\mu_{1} V & U_{1}-\mu_{1} V_{1}
\end{array}\right|=0
$$

eine Gleichung, die sich auch durch Elimination des variabelen Parameters $\lambda$ ergibt aus

$$
\left\{\begin{array}{l}
U-\mu_{0} V-\lambda\left(U_{1}-\mu_{0} V_{1}\right)=0 \\
U-\mu_{1} V-\lambda\left(U_{1}-\mu_{1} V_{1}\right)=0
\end{array}\right.
$$

es sind also nun irgend zwei andere Strahlenbüschel, deren Centren der Gleichung der Curve (11) genügen, der Betrachtung zu Grunde gelegt. Irgend vier den Werthen $\lambda_{1}, \lambda_{2}, \lambda_{3}, \lambda_{4}$ des Parameters $\lambda$ entsprechende Strahlen schneiden sich auf der Curve, und da diese beiden Strahlenquadrupel nach (13) dasselbe Doppelverhältniss besitzen, so folgt der Satz:

(14) Verbindet man vier beliebige Punkte einer Curve zweiter Ordnung mit irgend einem fünften Curvenpunkt, so ist das Doppelverhältniss dieser vier Strahlen constant, wo auch der fünfte Punkt auf dem Kegelschnitte liegen mag.

Auch die Coordinaten des Schnittpunktes zweier entsprechenden Strahlen der Strahlenbüschel (10) lassen sich leicht berechnen. Ist nämlich

$$
\begin{aligned}
& U \equiv A x_{1}+B x_{2}+C x_{3}=0, \quad U_{1} \equiv A_{1} x_{1}+B_{1} x_{2}+C_{1} x_{3}=0 \\
& V \equiv \mathrm{A} x_{1}+\mathrm{B} x_{2}+\Gamma x_{3}=0, \quad V_{1} \equiv \mathrm{A}_{1} x_{1}+\mathrm{B}_{1} x_{2}+\Gamma_{1} x_{3}=0
\end{aligned}
$$

so treten an Stelle von (10) die Ausdrücke

$$
\begin{aligned}
& A x_{1}+B x_{2}+C x_{3}-\mu\left(\mathrm{A} x_{1}+\mathrm{B} x_{2}+\Gamma x_{3}\right)=0 \quad \text { und } \\
& A_{1} x_{1}+B_{1} x_{2}+C_{1} x_{3}-\mu\left(\mathrm{A}_{1} x_{1}+\mathrm{B}_{1} x_{2}+\Gamma_{1} x_{3}\right)=0,
\end{aligned}
$$

aus denen als Gleichung des gesuchten Schnittpunktes folgt:

$$
\left|\begin{array}{ccc}
A-\mu \mathrm{A} & B-\mu \mathrm{B} & C-\mu \Gamma \\
A_{1}-\mu \mathrm{A}_{1} & B_{1}-\mu \mathrm{B}_{1} & C_{1}-\mu \Gamma_{1} \\
u_{1} & u_{2} & u_{3}
\end{array}\right|=0 .
$$

Natürlich könnte man auch diejenigen Betrachtungen anstellen, die den bisherigen dualistisch entsprechen; wir wollen dies hier unterlassen und nur den dem Satze (9) entsprechenden hervorheben:

(18) Die Tangenten einer Curve zweiter Classe können betrachtet werden als die Verbindungslinien entsprechender Punkte zweier bestimmten „projectiven“ Punktreihen; auch die Träger dieser Punktreihen sind Tangenten der Curve.

$\mathrm{Um}$ zu fünf gegebenen Punkten eines Kegelschnitts einen beliebigen sechsten zú construiren, hat man nach (9) zwei von den fünf Punkten mit den drei übrigen zu verbinden; hierdurch sind zwei projective 
Strahlenbüschel festgelegt. Construirt man alsdann zu einem beliebigen Strahl des einẹn Büschels den entsprechenden des zweiten, so schneiden sich beide Strahlen in einem sechsten Punkte des Kegelschnitts. Analog ist nach (18) zu verfahren bei Construction irgend einer sechsten Tangente zu fünf gegebenen. Jedenfalls erfordern aber diese zwei Constructionen resp. die Lösung der Aufgaben: Wenn bei zwei projectiven Strahlenbüscheln je drei entsprechende Strahlen gegeben sind, zu einem beliebigen vierten Strahl des einen Büschels den entsprechenden des anderen zu construiren, bezw.: Wenn bei zwei projectiven Punktreihen je drei entsprechende Punkte gegeben sind, zu einem beliebigen vierten Punkte der einen Punktreihe den entsprechenden der anderen zu construiren. Diese zwei Aufgaben werden resp. mit Hilfe der folgenden zwei Sätze gelöst:

(19) Sind $s_{0}, s_{1}, s_{2}$ irgend drei Strahlen des einen Büschels, $\sigma_{0}, \sigma_{1}, \sigma_{2}$ die entsprechenden des zweiten und verbindet man den Schnittpunkt von $s_{0}$ und $\sigma_{1}$ mit demjenigen von $s_{1}$ und $\sigma_{0}$, den Schnittpunkt von $s_{1}$ und $\sigma_{2}$ mit demjenigen von $s_{2}$ und $\sigma_{1}$ (analog, wenu noch mehr entsprechende Strahlen gegeben wären), so gehen alle diese Verbindungslinien durch einen und denselben Punkt.

(20) Sind $p_{0}, p_{1}, p_{2}$ irgend drei Punkte einer Punktreihe, $\pi_{0}, \pi_{1}, \pi_{2}$ die entsprechenden einer zur ersten projectiven Punktreihe und verbindet man „kreuzweise" $p_{0}$ mit $\pi_{1}, p_{1}$ mit $\pi_{0}$, ferner $p_{0}$ mit $\pi_{2}, p_{2}$ mit $\pi_{0}, p_{1}$ mit $\dot{\pi}_{2}, p_{2}$ mit $\pi_{1}$ (analog, wenn noch mehr entsprechende Punkte gegeben wären), so liegen die Schnittpunte je zweier kreuzweise gezogenen Verbindungslinien in einer und derselben Geraden.

Wir beschränken uns hier auf den Beweis des Satzes (20), da der Beweis von (19) dualistisch entsprechend $z u$ führen ist.

Es seien $Y_{0}-\lambda Y_{1}=0$ und $T_{0}-\lambda T_{1}=0$ für variabele Werthe des Parameters $\lambda$ die Gleichungen der beiden Punktreihen $p$ und $\pi$. Der Schnittpunkt $V$ der beiden Träger hat dann als Punkt der einen Reihe eine Gleichung von der Form $V \equiv Y_{0}-\rho Y_{1}=0$, als Punkt der zweiten Reihe $V \equiv T_{0}-\sigma T_{1}=0$. Betrachtet man $V$ als Punkt der Reihe $p$, so entspricht ihm auf der Reihe $\pi: T \equiv T_{0}-\rho T_{1}=0$; wird $V$ als Punkt der Reihe $\pi$ angesehen, so ist sein entsprechender auf der Reihe $. p: Y \equiv Y_{0}-\sigma Y_{1}=0$. Aus dieser Gleichung, sowie aus $V \equiv Y_{0}-\varrho Y_{1}$ lassen sich nun $Y_{0}$ und $Y_{1}$ ausdrücken durch $Y$ und $V ;$ an Stelle von $Y_{0}-\lambda Y_{1}=0$ tritt alsdann die Gleichung $V-\frac{\rho-\lambda}{\sigma-\lambda} Y=0$, analog lässt sich $T_{0}-\lambda T_{1}=0$ ersetzen durch 
$V-\frac{\sigma-\lambda}{\varrho-\lambda} T=0$. Zwei Punkte $p_{i}$ und $p_{k}$ der einen Reihe sind nun gegeben durch

$$
V-\frac{\rho-\lambda_{i}}{\sigma-\lambda_{i}} Y=0, \quad \text { resp. } \quad V-\frac{\rho-\lambda_{k}}{\sigma-\lambda_{k}} Y=0,
$$

die entsprechenden $\pi_{i}$ und $\pi_{k}$ der anderen Reihe durch

$$
V-\frac{\sigma-\lambda_{i}}{\varrho-\lambda_{i}} T=0, \quad \text { resp. } \quad V-\frac{\sigma-\lambda_{k}}{\varrho-\lambda_{k}} T=0 ;
$$

hieraus folgt für einen beliebigen auf $p_{i} \pi_{k}$ gelegenen Punkt die Gleichung

$$
\left(V-\frac{\varrho-\lambda_{i}}{\sigma-\lambda_{i}} Y\right)-\mu\left(V-\frac{\sigma-\lambda_{k}}{\varrho-\lambda_{k}} T\right)=0,
$$

für einen auf $p_{k} \pi_{i}$ gelegenen Punkt:

$$
\left(V-\frac{\varrho-\lambda_{k}}{\sigma-\lambda_{k}} Y\right)-v\left(V-\frac{\sigma-\lambda_{i}}{\varrho-\lambda_{i}} T\right)=0 .
$$

Für den Schnittpunkt von $p_{i} \pi_{k}$ mit $p_{k} \pi_{i}$ müssen beide Gleichungen übereinstimmen, d. h. es muss sein $\mu=\nu=1$, wodurch $V$ herausfällt und wur $-\left(\varrho-\lambda_{i}\right)\left(\varrho-\lambda_{k}\right) Y+\left(\sigma-\lambda_{i}\right)^{\circ}\left(\sigma-\lambda_{k}\right) T=0$ übrig bleibt. Dieser Ausdruck ist linear aus $Y$ und $T$ zusammengesetzt, die Schnittpunkte aller kreuzweise gezogenen Verbindungslinien $p_{i} \pi_{k}$ und $p_{k} \pi_{i}$ liegen daher auf einer und derselben Geraden, und zwar auf derjenigen, welche die oben definirten Punkte $Y$ und $T$ verbindet; hiermit ist Satz (20) bewiesen.

Es ist wohl unnöthig zu zeigen, in welcher Weise dieser Satz benutzt wird, um bei zwei projectiven Punktreihen, von denen je drei entsprechende Punkte gegeben sind, zu einem beliebigen vierten Punkt der einen Reihe den zugehörigen der anderen zu construiren und hierdurch mit Hilfe von (18) eine Tangente der durch die beiden Punktreihen bestimmten Curve zweiter Classe zu erhalten. Mit Hilfe der dualistisch entsprechenden Construction kann man nach.(19) und (9) zu fünf Punkten einer Curve zweiter Ordnung weitere Punkte erhalten, wie übrigens schon oben erwälnt wurde.

Eine andere Constructionsmethode eines beliebigen sechsten Punktes liefert der Pascal'sche Satz. Derselbe lautet:

Bei jedem einer Curve zweiter Ordnung einbeschriebenen Sechseck schneiden sich die gegenüberliegenden Seiten in drei Punkten einer Geraden, der sogenannten Pascal'schen Geraden.

Zum Beweis dieses Satzes führen wir ein die Coordinaten $a_{i}, b_{i}$, $c_{i}, d_{i}, e_{i}, f_{i}(i=1,2,3)$ der sechs auf den Kegelschnitt gelegenen Ecken und nennen $u_{a b}, v_{a b}, w_{a b}$ die (sonst mit $u_{1}, u_{2}, u_{3}$ ) bezeichneten 
Liniencoordinaten der Verbindungslinie $a b$; analog $u_{c f}, v_{c f}, w_{c f}$ die Liniencoordinaten der Geraden of u. s. w., so dass z. B.:

$$
\left\{\begin{array}{l}
u_{a b} \doteq a_{2} b_{3}-a_{3} b_{2}, v_{a b}=a_{3} b_{1}-a_{1} b_{3}, w_{a b}=a_{1} b_{2}-a_{2} b_{1} \\
u_{c f}=c_{2} f_{3}-c_{3} f_{2}, v_{c f}=c_{3} f_{1}-c_{1} f_{3}, w_{c f}=c_{1} f_{2}-c_{2} f_{1} \text { u.s.w. }
\end{array}\right.
$$

Gegenüberliegende Seiten des Sechsecks sind nun z. B. $a b$ und $d c$, $b c$ und $c f, c d$ und $f a$; die Coordinaten des Schnittpunktes von $a b$ mit de seien $\gamma_{1}, \gamma_{2}, \gamma_{3}, b c$ und of mögen sich schneiden im Punkte $\alpha_{1}, \alpha_{2}, \alpha_{3}, c d$ und $f a$ in $\beta_{1}, \beta_{2}, \beta_{3}$. Kann man alsdann nachweisen, dass $\sum \pm\left(\alpha_{1} \beta_{2} \gamma_{3}\right)$ verschwindet, falls die sechs Punkte $a, b, c, d, e, f$ auf einem Kegelschnitt liegen, so ist damit der Pascal'sche Satz bewiesen.

Nun ist

$$
\sum \pm\left(\alpha_{1} \beta_{2} \gamma_{3}\right)=\left|\begin{array}{lll}
u_{b c} & v_{b c} & w_{b c} \\
u_{e f} & v_{e f} & w_{e f}
\end{array}\right| \cdot\left|\begin{array}{lll}
\beta_{1} & \beta_{2} & \beta_{3} \\
\gamma_{1} & \gamma_{z} & \gamma_{3}
\end{array}\right|=\left|\begin{array}{ll}
(b c \beta) & (b c \gamma) \\
(e f \beta) & (e f \gamma)
\end{array}\right|
$$

wemn man mit $(b c \beta)$ die Determinante $\sum \pm\left(b_{1} c_{z} \beta_{3}\right)$ bezeichnet und $(b c \gamma)$ u. s. w. analoge Bedeutung baben. Ferner ist

$$
(b c \beta)=\left|\begin{array}{lll}
b_{1} & b_{2} & b_{3} \\
c_{1} & c_{2} & c_{3}
\end{array}\right|\left|\begin{array}{ccc}
u_{c d} & v_{c d} & w_{c d} \\
u_{f a} & v_{f a} & w_{f a}
\end{array}\right|=\left|\begin{array}{ll}
(b c d) & (b f a) \\
(c c d) & (c f a)
\end{array}\right|=(b c d) \cdot(c f a),
$$

und analog findet man:

$$
(b c \gamma)=-(c a b)(b d e),(e f \beta)=-(c f a)(f c d),(e f \gamma)=(e a b)(f d e) .
$$

Durch Substitution dieser Werthe in (23) verwandelt sich $\sum \pm\left(\alpha_{1} \beta_{2} \gamma_{3}\right)$ in:

$$
(b c d)(c f a)(e a b)(f d e)-(c a b)(b c l e)(c f a)(f c d),
$$

und dieser Ausdruck geht aus (5) oder (1) hervor, wenn man daselbst die Buchstaben $a, b, c, d, e, x$ ersetzt resp. durch $c, a, e, d, b, f$. Da aber (5) die Bedingung darstellt, unter der die Punkte $a, b, c, d, \dot{e}, x$ auf einem und demselben Kegrelschnitt liegen, und da (1) bei irgend einer Permutation der Buchstaben $a, b, c, d, e, x$ nur sein Vorzeichen ändert, so ist auch (23) gleich Null gesetzt, die Bedingung dafür, dass die Punkte $a, b, c, d, e, f$ auf einem und demselben Kegelschnitt liegen. Es existirt daher eine Pascal'sche Gerade, in welcher Reihenfolge man auch die sechs Punkte $a, b, c, d, e, f$ zu einem Sechseck verbunden hätte.

Es sei bemerkt, dass man sechs Punkte $a, b, c, d, e, f$ eines Kegelschnitts auf 60 verschiedene Arten zu Sechsecken verbinden kann. Von den 6! Permutationen der Buchstaben $a, b, c, d, e, f$ liefern nämlich je 12 dasselbe Sechseck, denn es ist z. B. das Sechseck abcdef identisch mit jedem anderen, das durch cyklische 
Vertauschung der sechs Buchstaben (z. B. bcdefa), oder auch durch Umkehrung dieser Vertauschungen (z. B. $a f e d c b$ ) bestimmt ist. Die Anzahl der wirklich verschiedenen Sechsecke beträgt daher $6 !: 12=60$, und zu jedem dieser Sechsecke gehört eine Pascal'sche Gerade.

Dem Pascal'schen Satze entspricht dualistisch der Brianchon'sche Satz:

(25) Bei jedem einer Curve zweiter Classe umschriebenen Sechsseit gehen die drei Verbindungslinien der gegenüberliegenden Ecken durch einen Punkt.

Auch der Pascal'sche und der Brianchon'sche Satz lassen sich verwenden, un zu fünf gegebenen Punkten, resp. fünf gegebenen Tangenten eines Kegelschnitts beliebig viele weitere Punkte, resp. Tangenten zu finden. Sind z. B. die fünf Punkte $a, b, c, d, e$ eines Kegelschnitts gegeben und will man denjenigen Punkt $x$ finden, in welchem eine durch $a$ beliebig gezogene Gerade $G$ den Kegelschnitt nochmals schneidet, so wäre folgendermassen zu verfahren: Man sucht den Schnittpunkt $\gamma$ der zwei Geraden $a b$ und $d e$, ebenso denjenigen von $G$ mit $c d$, er sei $\beta$; die Gerade $b c$ treffe die Pascal'sche Gerade $\beta \gamma$ in $\alpha$, alsdann ist der Schnittpunkt von $G$ mit $\alpha e$ der gesuchte Punkt $x$. 


\section{Abschnitt.}

Kegelschnittbiischel nnd Kegelschnittnetze, sowic die dnalistisch entsprechenden Gebilde.

$\S 13$.

\section{Einleitende Bemerkungen.}

Bevor wir zur eigentlichen 'Theorie der Kegelschnittbüschel übergehen, wollen wir noch einige Bemerkungen über abkürzende Bezeichnungen vorausschicken, die im Folgenden angewandt werden.

Es seien $f=0$ und $g=0$ die Gleichungen zweier Curven zweiter Ordnung, und zwar

$$
f \equiv \sum_{1}^{3} \sum_{1}^{3} k a_{i k} x_{i} x_{k}=0, \quad g \equiv \sum_{1}^{3} \sum_{1}^{3} k b_{i k} x_{i} x_{k}=0 ;
$$

die Gleichung $\lambda g-f=0$ stellt alsdann entsprechend den unendlich vielen Werthen des Parameters $\lambda$ eine einfach unendliche Anzahl von Kegelschnitten dar, deren Gesammtheit man das durch $f=0$ und $g=0$ bestimmte Kegelschnittbüschel nennt. Der Kürze halber werde gesetzt:

(2) $\lambda g-f \equiv c_{11} x_{1}^{2}+2 c_{12} x_{1} x_{2}+c_{22} x_{2}{ }^{2}+2 c_{13} x_{1} x_{3}+2 c_{23} x_{2} x_{3}+c_{33} x_{3}{ }^{2}=0$,

$$
\text { wo } c_{i k}=\lambda b_{i k}-a_{i k} \text {. }
$$

Es sei ferner

$$
\begin{aligned}
\Psi_{0}(\lambda) & \equiv \sum \pm\left(c_{11} c_{22} c_{33}\right) \equiv\left|\begin{array}{lll}
c_{11} & c_{12} & c_{13} \\
c_{21} & c_{22} & c_{23} \\
c_{31} & c_{32} & c_{33}
\end{array}\right| \equiv C, \\
& -\Psi_{1}(\lambda) \equiv\left|\begin{array}{llll}
c_{11} & c_{12} & c_{13} & u_{1} \\
c_{21} & c_{22} & c_{23} & u_{2} \\
c_{31} & c_{32} & c_{33} & u_{3} \\
u_{1} & u_{2} & u_{3} & 0
\end{array}\right| \equiv\left(\begin{array}{l}
u \\
u
\end{array}\right)_{c_{i k}},
\end{aligned}
$$




$$
\Psi_{2}(\lambda) \equiv\left|\begin{array}{lllll}
c_{11} & c_{12} & c_{13} & u_{1} & v_{1} \\
c_{21} & c_{22} & c_{23} & u_{2} & v_{2} \\
c_{31} & c_{32} & c_{33} & u_{3} & v_{3} \\
u_{1} & u_{2} & u_{3} & 0 & 0 \\
v_{1} & v_{2} & v_{3} & 0 & 0
\end{array}\right| \equiv\left(\begin{array}{l}
u v \\
u v
\end{array}\right)_{c_{i k}} .
$$

Führt man noch den Ausdruck ein

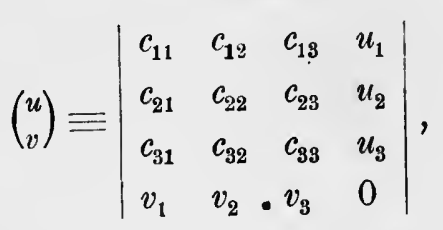

so besteht, wie in der Determinantentheorie gezeigt wird, die Relation

$$
C\left(\begin{array}{ll}
u & v \\
u & v
\end{array}\right)=\left(\begin{array}{l}
u \\
u
\end{array}\right)\left(\begin{array}{l}
v \\
v
\end{array}\right)-\left(\begin{array}{l}
u \\
v
\end{array}\right)^{2} .
$$

Die Entwicklung von $\Psi_{0}^{\prime}(\lambda)$ und $\Psi_{1}(\lambda)$ nach Potenzen von $\lambda$ ergibt Ausdrücke von der Form

$$
\begin{aligned}
& \Psi_{0}(\lambda) \equiv \lambda^{3} B-3 \lambda^{2} \Theta+3 \lambda H-A, \\
& \Psi_{1}(\lambda) \equiv F-2 \lambda H+\lambda^{2} G .
\end{aligned}
$$

Hierbei sind $A$ und $B$ die Determinanten der beiden Kegelschnitte $f$ und $g$, während $F=0$ und $G=0$ ihre Gleichungen in Liniencoordinaten bedeuten:

$$
\left\{\begin{array}{l}
F \equiv A_{11} u_{1}{ }^{2}+2 A_{12} u_{1} u_{2}+A_{22} u_{2}{ }^{2}+2 A_{13} u_{1} u_{3}+2 A_{23} u_{2} u_{3}+A_{33} u_{3}{ }^{2} \\
G \equiv B_{11} u_{1}{ }^{2}+2 B_{12} u_{1} u_{2}+B_{22} u_{2}{ }^{2}+2 B_{19} u_{1} u_{3}+2 B_{23} u_{2} u_{3}+B_{33} u_{3}^{2}
\end{array}\right.
$$

Ferner ist

$$
\begin{gathered}
\left\{\begin{array}{c}
3 \Theta \equiv a_{11} B_{11}+a_{22} B_{22}+a_{33} B_{33}+2 a_{23} B_{23}+2 a_{31} B_{31}+2 a_{12} B_{12} \\
3 \mathrm{H} \equiv b_{11} A_{11}+b_{22} A_{22}+b_{33} A_{33}+2 b_{23} A_{23}+2 b_{31} A_{31}+2 b_{12} A_{12}
\end{array}\right. \\
2 H \equiv(a, b)_{11} u_{1}{ }^{2}+2(a, b)_{12} u_{1} u_{2}+(a, b)_{22} u_{2}{ }^{2}+2(a, b)_{13} u_{1} u_{3} \\
+2(a, b)_{23} u_{2} u_{3}+(a, b)_{33} u_{3}{ }^{2}
\end{gathered}
$$

wobei zur Abkürzung gesetzt ist

$$
\left\{\begin{array}{l}
(a, b)_{11}=a_{22} b_{33}+a_{33} b_{22}-2 a_{23} b_{23} \\
(a, b)_{12}=a_{31} b_{32}+a_{32} b_{31}-a_{33} b_{12}-a_{12} b_{33}
\end{array}\right.
$$

während die übrigen $(a, b)_{i k}$ aus den hier angegebenen durch cyklische Vertauschung der Indices hervorgehen.

Es möge noch bemerkt werden, dass zufolge (3) die Gleichung besteht 


$$
\frac{d \Psi_{0}(\lambda)}{d \lambda}=\sum_{1}^{3} \sum_{1}^{3} \frac{\partial C}{\partial c_{i k}} \frac{\partial c_{i k}}{\partial \lambda}=\sum_{1}^{3} \sum_{1}^{3}{ }_{i}^{k} C_{i k} b_{i k},
$$

denn es ist $\frac{\partial c_{i k}}{\partial \lambda}$ nach (2) gleich $b_{i k}$, während $\frac{\partial C}{\partial c_{i k}}$ die Unterdeterminante $C_{i k}$ von $c_{i k}$ in $C$ darstellt; zufolge (8) hat man ausserden

$$
\frac{d \psi_{0}(\lambda)}{d \lambda}=3\left(\lambda^{2} B-2 \lambda \Theta+H\right) \text {. }
$$

Aus (8) folgt für den zweiten Differentialquotienten

$$
\frac{d^{2} \Psi_{0}(\lambda)}{d \lambda^{2}}=6 \lambda B-6 \Theta
$$

oder zufolge der Definition von $B$ und $\Theta$ :

$$
\begin{aligned}
\frac{d^{2} \Psi_{0}(\lambda)}{d \lambda^{2}} & =2 \lambda \sum_{1}^{3} \sum_{1}^{3} B_{i k} b_{i k}-2 \sum_{1}^{3} \sum_{i}^{3} B_{i k} a_{i k} \\
& =2\left\{B_{11} c_{11}+B_{22} c_{22}+B_{33} c_{33}+2 B_{23} c_{23}+2 B_{31} c_{31}+2 B_{12} c_{12}\right\}
\end{aligned}
$$

$\S 14$.

Bestimmung der Schnittpunkte und gemeinsamen Tangenten zwoier Kegelschnitte.

Es seien gegeben die zwei Kegelschnitte

$$
f \equiv \sum_{1}^{3} \sum_{1}^{3} a_{i k} x_{i} x_{k}=0 \quad \text { und } g \equiv \sum_{1}^{3} \sum_{1}^{3} b_{i k} x_{i} x_{k}=0 .
$$

Ist die Gleichung eines dritten Kegelschnitts von der Form

$$
\lambda g-f \equiv \sum_{1}^{3} \sum_{1}^{3} c_{i k} x_{i} x_{k}=0, \text { wo } c_{i k}=\lambda b_{i k}-a_{i k},
$$

so geht derselbe, welche Werthe auch der willkürliche Parameter $\lambda$ haben möge, stets durch die Schnittpunkte von $f$ und $g$ hindurch, denn für jeden dieser Schnittpunkte wird sowohl $f=0$, als $g=0$ erfüllt, daher auch $\lambda g-f=0$. Wir können daher sagen:

(3) Sind $f=0$ und $g=0$.die Gleichungen zweier Kegelschnitte, so stellt $\lambda g-f=0$ für alle möglichen Werthe des willkürlichen Parameters $\lambda$ ein ganzes System von Kegelschnitten dar, die sämmtlich durch die Schnittpunkte von $f=0$ und $g=0$ hindurchgehen.

Man bezeichnet dieses System als Kegelschnittbüschel und die gemeinsamen Schnittpunkte als Grundpunkte des Büschels.

Es liegt nun umgekehrt die Frage nahe, ob die Gleichung eines jeden Kegelschnitts, der durch alle Schnittpunkte von $f=0$ und $g=0$ 
geht, in die Form gebracht werden kann $\lambda g-f=0$. Um dies zu untersuchen, wenden wir uns zu der Bestimmung der Schnittpunkte von $f=0$ und $g=0$ und bemerken zunächst, dass man zu diesem Zweck irgend zwei Curven des Büschels (2) wählen kann. Denn jeder auf $\lambda g-f=0$ und auf $\mu g-f=0$ gelegene Punkt liegt auch auf $\lambda g-f-(\mu g-f)=0$, d. h. auf $g=0$, somit erfüllen seine Coordinaten auch $f=0$.

Fragen wir nun, wie viele zerfallende Kegelschnitte, also Geradenpaare in dem Büschel enthalten sind. Dabei werde angenommen, dass die Determinante $C$ nicht identisch verschwindet und, da es sich im Folgenden zunächst nur um die Schnittpunkte von $f=0$ und $g=0$ lrandelt, dass mindestens der eine der beiden Kegelschnitte, etwa $g=0$, nicht in ein Geradenpaar ausarte, dass also stets $B \gtrless 0$ sei.

Die Bedingung für das Zerfallen des Kegelschnitts $\lambda g-f=0$ ist nach $\S 4$ gegeben durch $\sum \pm\left(c_{11} c_{22} c_{33}\right)=0$, und da diese Gleichung in $\lambda$ vom dritten Grade, also von der Form ist

$$
\left.\Psi_{0}(\lambda) \equiv \lambda^{3} B-3 \lambda^{2} \Theta+3 \lambda H-A=0,{ }^{1}\right)
$$

hat man den Satz:

(5) In einem Kegelschnittbüschel sind im allgemeinen drei Geradenpaare vorhanden.

Sind $\lambda_{1}, \lambda_{2}, \lambda_{3}$ die den Geradenpaaren entsprechenden Werthe des Parameters $\lambda$, so erhält man die Gleichungen der Spitzen dieser Paare nach $\S 5$ doppelt zählend, wenn man in $\lambda_{i} g-f=0 \quad(i=1,2,3)$ Linieucoordinaten einführt. Nun ist aber die Gleichung von $\lambda g-f=0$ in Liniencoordinaten von der Form

$$
-\Psi_{1}(\lambda) \equiv\left|\begin{array}{llll}
c_{11} & c_{12} & c_{13} & u_{1} \\
c_{21} & c_{22} & c_{23} & u_{2} \\
c_{31} & c_{32} & c_{33} & u_{3} \\
u_{1} & u_{2} & u_{3} & 0
\end{array}\right|=0,
$$

und wenn dieser Ausdruck nach Potenzen von $\lambda$ entwickelt wird, ergibt sich

$$
\left.\Psi_{1}(\lambda) \equiv F-2 \lambda H+\lambda^{2} G=0 .^{2}\right)
$$

Sind daher $t_{1}{ }^{(i)}, t_{2}{ }^{(i)}, t_{3}{ }^{(i)}(i=1,2,3)$ die Coordinaten der Spitzen der drei Geradenpaare $\lambda_{i} g-f=0$, also

1) Ueber die Bildung der Ausdrücke $\Theta$ und $H$ vgl. (11) in $\S 13 ; A$ und $B$ sind die Determinanten von $f=0$, resp. $g=0$.

2) $F=0$ und $G=0$ sind die Gleichungen von $f=0$ und $g=0$ in Liniencoordinaten; über die Bildung des Ausdrucks $H$ vgl. (12) in § 13. 


$$
t_{1}^{(i)} u_{1}+t_{2}^{(i)} u_{2}+t_{3}^{(i)} u_{3}=0 \quad(i=1,2,3)
$$

die Gleichungen dieser Spitzen, so bestehen Identitäten von der Form

$$
\Psi_{1}\left(\lambda_{i}\right) \equiv F-2 \lambda_{i} H+\lambda_{i}{ }^{2} G \equiv\left\{t_{1}^{(i)} u_{1}+t_{2}{ }^{(i)} u_{2}+t_{3}{ }^{(i)} u_{3}\right\}^{2} .
$$

Hinsichtlich der Lage der drei Geradenpaare sind nun entsprechend der Verschiedenheit oder Gleichheit der Wurzeln $\lambda_{1}, \lambda_{2}, \lambda_{3}$ von (4) drei Hauptfälle zu unterscheiden, so lange die übrigen Curven des Büschels eigentliche, d. h. nicht ausartende Kegelschnitte repräsentiren.

I) Die drei Wurzeln $\lambda_{1} ; \lambda_{2}, \lambda_{3}$ sind von einander verschieden, daher $\psi_{0}^{\prime}\left(\lambda_{i}\right) \gtrless 0$, weun $\lambda_{i}$ eine dieser Wurzeln ist und $\Psi_{0}^{\prime}(\lambda)$ den Differentialquotienten von $\Psi_{a}(\lambda)$ bezeichnet. In diesern Falle kann nie die Spitze eines Geradenpaares, z. B. des dem Parameter $\lambda_{1}$ entsprechenden, auf einer Geraden eines anderen Paares liegen; denn wenn dies doch eintreten würde, so wäre diese Spitze ein allen Kegelschnitten des Büschels gemeinsamer Punkt, sie läge auch auf der Curve $g=0$, so dass $g\left(t_{1}{ }^{(1)}, t_{2}{ }^{(1)}, t_{3}{ }^{(1)}\right)=0$. Zwischen den $t^{(1)}$ und den Unterdeterminanten $C_{i k}$ eines Elementes $c_{i k}$ der Determinante $C \equiv \sum \pm\left(c_{11} c_{22} c_{33}\right)$ besteht aber nach (9) die Beziehung, dass jedes Product $t_{i} t_{k}$ zu $C_{i k}$ proportional ist, und hieraus geht hervor, dass $g\left(t_{1}{ }^{(1)}, t_{2}{ }^{(1)}, t_{3}{ }^{(1)}\right)$ den Werth erhält $\sum_{1}^{3} \sum_{1}^{3} C_{i k} b_{i k}$, der nach ${ }^{*}(14)$ in $\S 13$ mit $\Psi_{0}^{\prime}\left(\lambda_{1}\right)$ identisch ist. Mithin wäre $\Psi_{0}^{\prime}\left(\lambda_{1}\right)=0$, und dies war ausgeschlossen. Da die Schnittpunkte von irgend zwei Kegelschnitten des Büschels dieselben sind wie diejenigen von zwei Geradenpaaren des Büschels, letztere aber bei ihrer gegenwärtig nicht specialisirten Lage vier gemeinsame Punkte haben, so schneiden sicl im Falle I) die Curven des Büschels in vier verschiedenen Punkten.

II) Die zwei Wurzeln $\lambda_{1}$ und $\lambda_{2}$ von (4) seien einander gleich, dagegen $\lambda_{3} \gtrless \lambda_{1}$. Nun ist

$$
\Psi_{0}^{\prime}\left(\lambda_{1}\right)=0, \quad \Psi_{0}^{\prime \prime}\left(\lambda_{1}\right) \gtrless 0, \quad \Psi_{0}^{\prime}\left(\lambda_{3}\right) \gtrless 0 .
$$

Aus den soeben angestellten Betrachtungen geht hervor, dass die Spitze des dem Parameter $\lambda_{1}=\lambda_{2}$ entsprechenden Geradenpaares auch auf $g=0$ liegt, daher einer der Grundpunkte des Kegelschnittbüschels ist. Jedoch kann diese Spitze $t^{(1)}$ nicht mit derjenigen des Geradenpaares $\lambda_{3} g-f=0$ zusammenfallen. Nach $\S 4$ bestehen nämlich für die Coordinaten $t_{i}^{(1)}(i=1,2,3)$ die Gleichungen

$$
\lambda_{1} g^{\prime}\left(t_{1}^{(1)}\right)=f^{\prime}\left(t_{1}^{(1)}\right), \quad \lambda_{1} g^{\prime}\left(t_{2}^{(1)}\right)=f^{\prime}\left(t_{2}^{(1)}\right), \quad \lambda_{1} g^{\prime}\left(t_{3}^{(1)}\right)=f^{\prime}\left(t_{3}^{(1)}\right),
$$


daher ist auch

$$
\lambda g\left(t^{(1)}, x\right)-f\left(t^{(1)}, x\right)=\left(\lambda-\lambda_{1}\right) g\left(t^{(1)}, x\right),
$$

für beliebige Werthe $x_{i}$; fiele die Spitze $t^{(1)}$ mit $t^{(3)}$ zusammen, so wäre hiernach $\left(\lambda_{3}-\lambda_{1}\right) g\left(t^{(1)}, x\right)=0$, d. h. $\lambda_{1}=\lambda_{3}$, denn $g\left(t^{(1)}, x\right)$ kann für beliebige Werthe der $x$ nicht verschwinden, weil die Determinante $B$ als von Null verschieden vorausgesetzt war; die Gleichung $\lambda_{1}=\lambda_{3}$ war aber ausgeschlossen.

Es sind nun im Falle II) zwei Möglichkeiten vorhanden:

a) $\lambda_{1} g-f=0$ stellt zwei verschiedene Geraden dar, etwa $r_{x} \cdot s_{x}=0$, es ist also $F-2 \lambda_{1} H+\lambda_{1}{ }^{2} G$ nicht identisch Null,

b) $\lambda_{1} g-f=0$ ist eine Doppelgerade $r_{x}^{2}=0$, also

$$
F-2 \lambda_{1} H+\lambda_{1}{ }^{2} G \equiv 0 .{ }^{1} \text { ) }
$$

Jedenfalls berühren sich im Falle a) auf Grund der Gleichungen (10) und (11) die Kegelschnitte $f=0$ und $g=0$, daher sämmtliche Kegelschnitte des Büschels, im Punkte $t^{(1)}$; die gemeinsame Tangente muss somit auch Tangente an das Geradenpaar $\lambda_{3} g-f=0$ sein, d. h. mit einer Geraden dieses Paares zusammenfallen. Die Kegelschnitte des Büschels berühren sich in einem und demselben Punkte (der Spitze des Geradenpaares mit dem Parameter $\left(\lambda=\lambda_{1}=\lambda_{2}\right)$, und die gemeinsame Tangente ist eine Gerade des Paares $\lambda_{3} g-f=0$.

Im Falle b) rücken die Grundpunkte des Büschels paarweise in die zwei Schnittpunkte der Doppelgeraden $r_{x}{ }^{2}=0$ mit dem Geradenpaare $\lambda_{3} g-f$, und aus denselben Gründen wie bei a) ersieht man, dass sich alle Curven des Büschels in diesen zwei Punkten berühren und die Geraden des dem Parameter $\lambda_{3}$ entsprechenden Paares zu Tangenten in den zwei Berührungspunkten haben; es findet doppelte Berührung statt ${ }^{2}$ ).

Auch können diese Berührungspunkte nicht zusammenfallen, sonst würde $r_{x}=0$ Tangente aller Curven des Büschels sein, also auch des

1) Das zweite Geradenpaar $\lambda_{3} g-f=0$ kann nicht in eine Doppelgerade übergehen, sonst würden für die Wurzel $\lambda_{3}$ alle $C_{i k}$ verschwinden; nach (14) in $\S 13$ wäre also $\frac{d \Psi_{0}\left(\lambda_{s}\right)}{d \lambda}=0$, d: h. die kubische Gleichung (4) hätte noch eine zweite Doppelwurzel, was unmöglich ist.

2) Wie wir oben bemerkten, ist nun $\Psi_{1}\left(\lambda_{1}\right) \equiv F-2 \lambda_{1} H+\lambda_{1}{ }^{2} G \equiv 0$; nach einem allgemeinen Satze von Kronecker genügt aber bei einer symmetrischen Determinante das Verschwinden ron drei passend gewählten Unterdeterminanten, am das Verschwinden aller übrigen zu bewirken. Nach Elimiwation von $\lambda_{1}$ reduciren sich die drei ausgewählten Gleichungen auf zwei; der Fall der doppelten Berührung erfordert demnach die Erfüllung von zwei Bedingungen. Vgl. übrigens auch die Fussnote zu S. 97. 
Kegelschnitts $g=0$; aus

$$
\lambda_{1} g-f \equiv r_{x}^{2} \equiv \sum_{1}^{3} \sum_{1}^{3} k c_{i k} x_{i} x_{k} \text { (für } \lambda=\lambda_{1} \text { ) }
$$

folgt aber $r_{i} r_{k}=c_{i k}$, und wenn $r_{x}=0$ Tangente ist von $g=0$, so hätte man hiernach

$$
B_{11} c_{11}+2 B_{18} c_{12}+B_{22} c_{22}+2 B_{13} c_{13}+2 B_{23} c_{23}+B_{33} c_{33}=0
$$

oder nach (16) in $\S 13 \Psi_{0}^{\prime \prime}\left(\lambda_{1}\right)=0$, was ausgeschlossen war.

III) Die kubische Gleichung (4) hat eine dreifache Wurzel $\lambda_{1}$, es ist also neben $\Psi_{0}\left(\lambda_{1}\right)=0$ auch $\Psi_{0}^{\prime}\left(\lambda_{1}\right)=0$ und $\Psi_{0}^{\prime \prime}\left(\lambda_{1}\right)=0$; wir unterscheiden auch hier wieder:

a) $\lambda_{1} g-f=0$ stellt zwei verschiedene Geraden dar, etwa $r_{x} s_{x}=0$

b) $\lambda_{1} g-f=0$ ist eine Doppelgerade $r_{x}{ }^{2}=0$, also

$$
F-2 \lambda_{1} H+\lambda_{1}^{2} G \equiv 0 \text {. }
$$

Im Falle a) gehen wir aus von der Relation

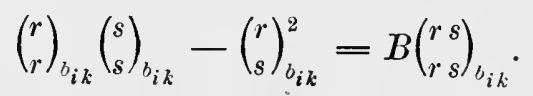

Da die Spitze des Geradenpaars wie im Falle II) allen Kegelschnitten des Büschels, somit auch $g=0$, angehört, ist jedenfalls $\left(\begin{array}{c}r s \\ r s\end{array}\right)_{b_{i k}}=0 ;$ ausserdem hat man

$$
\left(\begin{array}{l}
r \\
s
\end{array}\right)_{b_{i k}}=B_{11} r_{1} s_{1}+B_{12}\left(r_{1} s_{2}+r_{2} s_{1}\right)+\cdots=B_{11} c_{11}+2 B_{12} c_{12}+\cdots
$$

(für $\lambda=\lambda_{1}$ ),

und dieser Ausdruck ist nach (16) in $\S 13$ identisch mit $\frac{1}{2} \psi_{0}^{\prime \prime}\left(\lambda_{1}\right)$, verschwindet daher. In Folge von $\left(\begin{array}{l}r s \\ r s\end{array}\right)_{b_{i k}}=0$ und $\left(\begin{array}{l}r \\ s\end{array}\right)_{b_{i k}}=0$ reducirt sich nun (12) auf $\left(\begin{array}{l}r \\ r\end{array}\right)_{b_{i k}}\left(\begin{array}{l}s \\ s\end{array}\right)_{b_{i k}}=0$, d. h. eine der beiden Geraden, etwa $r_{x}=0$, muss den Kegelschnitt $g=0$, somit alle Curven des Büschels berühren, und zwar muss der Berührungspunkt die Spitze des Paares $\lambda_{1} g-f=0$ sein, da alle Kegelschnitte des Büschels durch diese Spitze hindurchgehen. Berührungspunkt und Spitze sind auch auf Grund von (10) nicht getrennt. Ferner können nicht beide Geraden $r_{x}=0$ und $s_{x}=0$ die Curven des Büschels berühren, sonst wären $r_{x}$ und $s_{x}$ identisch, was erst im Falle III b) angenommen wird.' Im gegenwärtigen Falle werden alle Kegelschnitte des Büschels von der einen Geraden des Paares $\lambda_{1} g-f=0$ berührt, von der anderen in zwei Punkten geschnitten, von denen der eine mit dem eben genannten 
Berührungspunkte identisch ist. Sämmtliche Kegelschnitte haben demnach drei mit der Spitze des Geradenpaares zusammenfallende Punkte gemeinsam, sie "osculiren" sich und schneiden sich überdies noch in einem vierten Punkte ${ }^{1}$ ).

Im Falle IIIb) rückt auch dieser vierte Punkt in den Berührungspunkt der Doppelgeraden mit den Kegelschnitten des Büschels, d. h. die einzelnen Curven haben vier zusammenfallende Punkte gemeinsam, und die gemeinsame Tangente ist die Doppelgerade $r_{x}^{2}=0$. Für die dreifache Wurzel verschwinden alle Unterdeterminanten $C_{i k}$, doch kann in ihnen der Factor $\lambda-\lambda_{1}$ nur einfach enthalten sein. Wäre nämlich dieser Factor doppelt in allen $C_{i k}$ enthalten, so würde derselbe in der linken Seite der Gleichung

$$
\left(\begin{array}{l}
u \\
u
\end{array}\right)\left(\begin{array}{l}
v \\
v
\end{array}\right)-\left(\begin{array}{l}
u \\
v
\end{array}\right)^{2}=C\left(\begin{array}{ll}
u & v \\
u & v
\end{array}\right)
$$

für beliebige Werthe $u_{i}, v_{i}$ vierfach enthalten sein, er wäre also in $\left(\begin{array}{ll}u & v \\ u & v\end{array}\right)$ einfach enthalten, d. h. es wäre für $\lambda=\lambda_{1} c_{i k}=0\left(i, l_{i}=1,2,3\right)$, die $a_{i k}$ wären den $b_{i k}$ proportional, daher die Kegelschnitte $f=0$ und $y=0$ identisch.

Es bleibt nun noch der Fall zu untersuchen, dass die Determinante $C$ identisch verscliwindet. Wenn dies eintritt, ist jeder Kegelschnitt des Büschels ein Geradenpaar, also auch $f=0$ und $g=0$. Es würden alsdann auftreten die Gleichungen $B_{11} a_{11}+2 B_{12} a_{12}+\cdots=0$ und $A_{11} b_{11}+2 A_{12} b_{12}+\cdots=0$, deren erste aussagt, dass die Spitze von $g=0$ auf $f=0$ liegt, während zufolge der zweiten die Spitze von $f=0$ auf $g=0$ liegt. Uebrigens sind wieder zwei Unterfälle möglich:

a) Die Spitzen von $f=0$ und $g=0$ sind verschieden, die Kegelschnitte des Büschels bilden Geradenpare, die eine Gerade gemeinsam haben; es ist also $f(x, x)$ von der Form $r_{x}, v_{x}, g(x, x)$ von der Form $r_{x} \cdot w_{x}$.

b) Die Spitzen von $f=0$ und $g=0$ fallen zusammen. Sind $c_{1}, c_{2}, c_{3}$ die Coordinaten der gemeinsamen Spitze und setzt man $x_{1}=y_{1}+\lambda c_{1}, x_{2}=y_{2}+\lambda c_{2}, x_{3}=\lambda c_{3}$, wobei $c_{3}$ von Null verschieden sei, so verwandelt sich $f\left(x_{1}, x_{2}, x_{3}\right)$ in $f\left(y_{1}, y_{2}, 0\right), g\left(x_{1}, x_{2}, x_{3}\right)$ in $g\left(y_{1}, y_{2} 0\right)$, denn $f(c, y)$ und $g(c, y)$ verschwinden identisch zufolge des

1) Durch Substitution der dreifachen Wurzel $\lambda_{1}$, welche zufolge $\Psi_{0}^{\prime \prime}\left(\lambda_{1}\right)=0$ den Werth besitzt $\lambda_{1}=\frac{\Theta}{B}$, in (14a) und (8) in $\S 13$ erbält man die Relationen

$$
\lambda_{1}=\frac{\Theta}{B}=\frac{H}{\Theta}=\frac{A}{H} .
$$


Umstandes, dass $c_{1}, c_{2}, c_{3}$ die Coordinaten der gemeinsamen Spitze sind. Es werden nunmehr $f$ und $g$ binäre Formen, und zwar stellen sie, sowie alle Kegelschnitte des Büschels, Geradenpaare dar, die von demselben Centrum ansgehen. Infolge der Identität der Spitzen sind natürlich die Grössen $A_{i k}$ den $B_{i k}$ proportional.

Kehren wir nunmehr zurück zu der S. 131 aufgeworfenen Frage, ob die Gleichnng eines jeden Kegelschnitts $K$, der durch die vier Schnittpunkte $\alpha, \beta, \gamma, \delta$ von $f=0$ und $g=0$ geht, in die Form gebracht werden kann $\lambda g-f=0$. Um dies nachzuweisen, fixiren wir auf $K$ einen fünften Punkt $\varepsilon_{1}, \varepsilon_{2}, \varepsilon_{3}$ und bestiumen in der Gleichung $\lambda g(x, x)-f(x, x)=0$ den Parameter $\lambda$ der Art, dass

$$
\lambda g(\varepsilon, \varepsilon)-f(\varepsilon, \varepsilon)=0
$$

alsdann hat der Kegelschnitt $f(\varepsilon, \varepsilon) \cdot g(x, x)-g(\varepsilon, \varepsilon) \cdot f(x, x)=0$ mit der Corve $K$ die vier Grundpunkte $\alpha, \beta, \gamma, \delta$ und den fünften Punkt $\varepsilon$ gemeinsam, fällt also mit $K$ zusammen. Sollten die liegelschnitte $f$ und $g$ eine der im Vorausgehenden unter II) und III) beschriebenen speciellen Lagen haben, so stände in allen Fällen der Kegelschnitt $K$ zu den Geradenpaaren, resp. zu $g=0$ in derselben Bezjehung, wie im Vorausgehenden der Kegelschnitt $f=0$ selbst, er würde also jedesmal durch die vier gemeinsamen Punkte von $f$ und $g$ gehen oder, was dasselbe aussagt, die Coefficienten seiner Gleichung würden vier in diesen Coefficienten lineare Gleichungen befriedigen.

Zum Schlusse dieser Betrachtungen wollen wir noch bemerken, dass man sehr wohl die Frage aufwerfen kann, ob nicht die vier Schnittpunkte zweier beliebig gelegenen Kegelschnitte immer eine gewisse specielle Lage $\mathrm{zu}$ einander haben ${ }^{1}$ ). Diese Frage ist zu verneinen, denn man kann stets Kegelschnitte angeben, die durch vier ganz beliebig fixirte Punkte der Ebene gehen. Legt man nämlich durch die vier Punkte zwei Geradenpaare, was bei der allgemeinen Lage der Punkte immer geschehen kann, und repräsentiren $g_{1}=0$, $g_{2}=0$ die Geraden des einen Paares, $g_{3}=0, g_{4}=0$ diejenigen des anderen, so sind $g_{1} g_{2}-\mu g_{3} g_{4}=0$ und $g_{1} g_{2}-\nu g_{3} g_{4}=0$ zwei Kegelschnitte, die durch jene vier beliebig fixirten Punkte gehen.

Nachdem im Vorhergehenden die Frage nach der Anzahl der

1) Bei gleichseitigen Hyperbeln z. B. tritt eine solche specielle Lage auf, indem alle gleichseitigen Hyperbeln, welche durch dieselben drei Punkte gehen, als vierten gemeinsamen Punkt den Höhenschnittpunkt des durch die drei übrigen bestimmten Dreiecks haben (vgl. den zn vorliegendem Paragraphen gehörigen Theil des Anhangs). 
Schnittpunkte erledigt ist, wollen wir nun sehen, wie viele gemeinsame Tangenten zwei Kegelschnitte besitzen, und wie man die Coordinaten oder die Gleichungen der Schnittpunkte und gemeinsamen Tangenten findet.

Wenn die Wurzeln der kubischen Gleichung (4) von einander verschieden sind, bestehen nach (9) für die Coordinaten $t_{1}{ }^{(i)}, t_{2}{ }^{(i)}, t_{3}{ }^{(i)}$ $(i=1,2,3)$ der Spitzen der drei in dem Kegelschnittbüschel enthaltenen Geradenpaare' die Identitäten

$$
\begin{gathered}
\Psi_{1}\left(\lambda_{i}\right) \equiv F-2 \lambda_{i} H+\lambda_{i}{ }^{2} G \equiv\left\{t_{1}{ }^{(i)} u_{1}+t_{2}{ }^{(i)} u_{2}+t_{3}{ }^{(i)} u_{3}\right\}^{2} \\
(i=1,2,3) .
\end{gathered}
$$

Vermittelst dieser Identitäten kann $\operatorname{man} F, H$ und $G$ oder allgemeiner $\Psi_{1}(\lambda)$ durch die in den drei Gleichungen (13) rechts stehenden Quadrate ausdrücken, und zwar entweder mit Hilfe directer Auflösung oder kürzer durch Partialbruchzerlegung:

$$
\frac{\psi_{1}(\lambda)}{\psi_{0}(\lambda)}=\left\{\begin{array}{l}
\Psi_{1}\left(\lambda_{1}\right) \\
\psi_{0}^{\prime}\left(\lambda_{1}\right) \lambda-\lambda_{1}
\end{array}+\frac{\psi_{1}\left(\lambda_{2}\right)}{\psi_{0}^{\prime}\left(\lambda_{2}\right)} \frac{1}{\lambda-\lambda_{2}}+\frac{\psi_{1}\left(\lambda_{3}\right)}{\psi_{0}^{\prime}\left(\lambda_{3}\right)} \frac{1}{\lambda-\lambda_{3}}\right\}
$$

Setzt man nämlich

so wird

$$
\frac{t_{1}^{(i)} u_{1}+t_{2}^{(i)} u_{2}+t_{3}^{(i)} u_{3}}{V \Psi_{0}^{\prime}\left(\lambda_{i}\right)} \equiv U_{i} \quad(i=1,2,3),
$$

und hiermit ergiebt sich der Satz:

(17) Die Spitzen der drei in dem Kegelschnittbüschel $\lambda g-f=0$ enthaltenen Geradenpaare bilden ein Poldreieck für jede andere Curve dieses Büschels.

Enthält nämlich die Gleichung eines Kegelschnitts nur die Quadrate der Veränderlichen, so ist derselbe, wie in $\S 4$ und 5 gezeigt wurde, auf ein Poldreieck als Coordinatendreieck bezogen.

Setzt man in der Gleichung

$$
\Psi_{1}(\lambda)=\Psi_{0}(\lambda)\left\{\frac{U_{1}^{2}}{\lambda-\lambda_{1}}+\frac{U_{2}^{2}}{\lambda-\lambda_{2}}+\frac{U_{3}^{2}}{\lambda-\lambda_{3}}\right\}
$$

den Parameter $\lambda$ gleich Null und beachtet, dass

$$
\Psi_{0}(\lambda)=B\left(\lambda-\lambda_{1}\right)\left(\lambda-\lambda_{2}\right)\left(\lambda-\lambda_{3}\right),
$$

so verwandelt sich $\Psi_{1}(\lambda)$ in $F$, und man erhält

$$
F=B\left(\lambda_{2} \lambda_{3} U_{1}^{2}+\lambda_{3} \lambda_{1} U_{2}^{2}+\lambda_{1} \lambda_{2} U_{3}^{2}\right),
$$

wofür man, wenn die Determinante $A$ des Kegelschnitts $f=0$ von Null verschieden ist, auf Grund der Relation $B \lambda_{1} \lambda_{2} \lambda_{3}=A$ auch setzen darf

$$
\frac{F}{A}=\frac{U_{1}^{2}}{\lambda_{1}}+\frac{U_{2}^{2}}{\lambda_{2}}+\frac{U_{3}^{2}}{\lambda_{3}}
$$


Für $\lambda=\infty$ folgt aus (18):

$$
\frac{G}{B}=U_{1}^{2}+U_{2}^{2}+U_{3}^{2} .
$$

Die Gleichungen (21) und (22) führen nun sofort zur Bestimmung der gemeinsamen Tangenten von $F=0$ und $G=0$, denn für die Coordinaten dieser Tangenten bestehen gleichzeitig die Gleichungen

$$
\frac{U_{1}^{2}}{\lambda_{1}}+\frac{U_{2}^{2}}{\lambda_{2}}+\frac{U_{3}^{2}}{\lambda_{3}}=0 \text { und } U_{1}^{2}+U_{2}^{2}+U_{3}^{2}=0,
$$

aus denen folgt

$$
U_{1}: U_{2}: U_{3}=\sqrt{\frac{1}{\lambda_{3}}-\frac{1}{\lambda_{3}}}: \pm \sqrt{\frac{1}{\lambda_{3}}-\frac{1}{\lambda_{1}}}: \pm \sqrt{\frac{1}{\lambda_{1}}-\frac{1}{\lambda_{9}}}
$$

wobei für die $U_{i}$ die Ausdrücke (15) einzusetzen sind. Es treten hierbei nur vier verschiedene Werthsysteme der $U_{i}$ auf, da von den acht an und für sich möglichen Combinationen der Vorzeichen in (23) je zwei für $U_{1}: U_{2}: U_{3}$ Werthe liefern, die sich nur un den Factor -1 unterscheiden. Wir können daher sagen:

(24) Es gibt im allgemeinen vier Tangenten, die zwei Kegelschnitten gemeinsam sind.

Die Erwägung, dass die Spitzen der drei in dem Kegelschnittbüschel vorhaudenen Geradenpaare ein allen Kegelschnitten des Biisehels gemeinsames Poldreieck bestimmen, führt darauf hin, in (16) an Stelle der willkürlichen Grössen $u_{1}, u_{2}, u_{3}$ die Differentialquotienten

$$
\frac{1}{2} g^{\prime}\left(x_{i}\right)=g_{i} \quad(i=1,2,3)
$$

einzuführen. Die Polare des Punktes $t^{(i)}$ in Bezug auf den Kegelschnitt $g(x, x)=0$ hat nämlich die Gleichung

$$
\begin{gathered}
\frac{1}{2} g^{\prime}\left(x_{1}\right) t_{1}{ }^{(i)}+\frac{1}{2} g^{\prime}\left(x_{2}\right) t_{2}^{(i)}+\frac{1}{2} g^{\prime}\left(x_{3}\right) t_{3}^{(i)}=0 \text { oder } \\
t_{1}^{(i)} g_{1}+t_{2}^{(i)} g_{2}+t_{3}{ }^{(i)} g_{3}=0 .
\end{gathered}
$$

Setzt man daher

$$
\frac{t_{1}^{(i)} g_{1}+t_{2}^{(i)} g_{2}+t_{3}^{(i)} g_{3}}{\sqrt{\Psi_{0}^{\prime}}\left(\overline{(}_{i}\right)}=X_{i} \quad(i=1,2,3),
$$

so ergibt sich sofort

$$
\begin{aligned}
-\left(\begin{array}{l}
g \\
g
\end{array}\right)_{c_{i k}}: \Psi_{0}(\lambda) & =\frac{X_{1}^{2}}{\lambda-\lambda_{1}}+\frac{X_{2}^{2}}{\lambda-\lambda_{2}}+\frac{X_{3}^{2}}{\lambda-\lambda_{3}} \\
& =\frac{1}{\lambda}\left\{\frac{X_{1}^{2}}{1-\frac{\lambda_{1}}{\lambda}}+\frac{X_{2}{ }^{2}}{1-\frac{\lambda_{2}}{\lambda}}+\frac{X_{3}{ }^{2}}{1-\frac{\lambda_{3}}{\lambda}}\right\}
\end{aligned}
$$

Ersetzt man andrerseits in (16) die Grössen, $u_{i}$ durch $\frac{1}{2} f^{\prime}\left(x_{i}\right)=f_{i}$ und berücksichtigt, dass 
so erhält man:

$$
\begin{aligned}
t_{1}^{(i)} f^{\prime}\left(x_{1}\right) & +t_{2}{ }^{(i)} f^{\prime}\left(x_{2}\right)+t_{3}^{(i)} f^{\prime}\left(x_{3}\right) \\
& =\lambda_{i}\left\{t_{1}^{(i)} g^{\prime}\left(x_{1}\right)+t_{2}{ }^{(i)} g^{\prime}\left(x_{2}\right)+t_{3}^{(i)} g^{\prime}\left(x_{9}\right)\right\},
\end{aligned}
$$

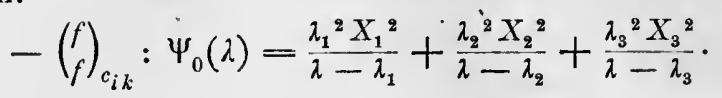

Aus (26) und (28) ergeben sich leicht die Gleichungen der auf das gemeinsame Poldreieck als Coordinatendreieck bezogenen Kegelschnitte $g=0$ und $f=0$, indem sich (26) durch die Substitution $\lambda=\infty$ verwandelt in

$$
g(x, x)=X_{1}{ }^{2}+X_{2}{ }^{2}+X_{3}{ }^{2},
$$

während (28) für $\lambda=0$ übergeht in

$$
f(x, x)=\lambda_{1} X_{1}{ }^{2}+\lambda_{2} X_{2}{ }^{2}+\lambda_{3} X_{3}{ }^{2} \text {. }
$$

Bildet man aus (29) und (30) die drei Differenzen $\lambda_{i} g-f=0$, so ergeben sich die Gleichungen der drei Geradenpaare, deren Spitzen nach der Definition der Ausdrücke $X_{i}$ in (25) mit den Punkten $U_{1}=0$, $U_{2}=0, U_{3}=0$, also mit den Ecken des Poldreiecks zusammenfallen. Man findet

$$
\left\{\begin{array}{l}
\left(\lambda_{1}-\lambda_{2}\right) X_{2}{ }^{2}+\left(\lambda_{1}-\lambda_{3}\right) X_{3}{ }^{2}=0 \\
\left(\lambda_{2}-\lambda_{3}\right) X_{3}{ }^{2}+\left(\lambda_{2}-\lambda_{1}\right) X_{1}{ }^{2}=0 \\
\left(\lambda_{3}-\lambda_{1}\right) X_{1}{ }^{2}+\left(\lambda_{3}-\lambda_{2}\right) X_{2}{ }^{2}=0
\end{array}\right.
$$

Die Ausdrücke für $g$ und $f$ in (29) und (30) führen überdies dazu, in eleganter Weise die Coordinaten der vier Punkte zu bestimmen, die diesen zwei Kegelschnitten gemeinsam sind, denn aus (31) folgt

$$
X_{1}: X_{2}: X_{3}=\sqrt{\lambda_{2}-\lambda_{3}}: \pm \sqrt{\lambda_{3}-\lambda_{1}}: \pm \sqrt{\lambda_{1}-\lambda_{2}} .
$$

Um aus (32) und (23) die Verhältnisse der $x_{i}$, resp. $u_{i}(i=1,2,3)$ $\mathrm{zu}$ berechnen, leiten wir in etwas allgemeinerer Weise eine Relation $\mathrm{ab}$, welche die $x_{i}$ durch $X_{1}, X_{2}, X_{3}$ und gleichzeitig die $u_{i}$ durch $U_{1}, U_{2}, U_{3}$ ausdrücken lehrt.

Wir substituiren zu diesem Zweck in (16) an Stelle der willkürlichen Veränderlichen $u_{i}$ die Grössen $u_{i}+\mu g_{i}$ und vergleichen auf beiden Seiten die Coefficienten von $\mu^{1}$. Alsdann entsteht die Formel

$$
-\left(\begin{array}{l}
g \\
u
\end{array}\right)_{c_{i k}}: \psi_{0}(\lambda)=\frac{U_{1} X_{1}}{\lambda-\lambda_{1}}+\frac{U_{2} X_{2}}{\lambda-\lambda_{2}}+\frac{U_{3} X_{3}}{\lambda-\lambda_{3}} .
$$

Für $\lambda=\infty$ erhält man hieraus nach einer leichten Transformation die gesuchte Relation:

$$
u_{1} x_{1}+u_{2} x_{2}+u_{3} x_{3}=U_{1} X_{1}+U_{2} X_{2}+U_{3} X_{3} .
$$

Für bestiumte Werthe der $x_{i}$ und variabele $u_{i}$ stellt die linke 
Seite dieser Relation gleich Null gesetzt einen Punkt dar. Wir denken uns nun für die $x_{i}$ Werthe eingesetzt, welche bewirken, dass die $X_{i}$ die Gleichungen (32) befriedigen; alsdann erhält man

$$
\sqrt{\lambda_{2}-\lambda_{3}} U_{1} \pm \sqrt{\lambda_{3}-\lambda_{1}} U_{2} \pm \sqrt{\lambda_{1}-\lambda_{2}} U_{3}=0 \text {, }
$$

und diese Gleichung ergibt, wenn man für die $U_{i}$ die Werthe (15) einsetzt, entsprechend den verschiedenen Combinationen der positiven und negativen Vorzeichen, die vier Schnittpunkte der Kegelschnitte $f(x, x)=0$ und $g(x, x)=0 . \quad$ Da zufolge (15) und (13)

$$
U_{i}=\frac{\sqrt{\left(F-2 \lambda_{i} H+\lambda_{i}^{2} \bar{G}\right)}}{\sqrt{\Psi_{0}^{\prime}\left(\lambda_{i}\right)}},
$$

so kann mit Hilfe von $\Psi_{0}^{\prime}\left(\lambda_{1}\right)=B\left(\lambda_{1}-\lambda_{2}\right)\left(\lambda_{1}-\lambda_{3}\right)$ und der analogen Werthe von $\Psi_{0}^{\prime}\left(\lambda_{2}\right)$ und $\psi_{0}^{\prime}\left(\lambda_{3}\right)$ die Gleichung (35) auch in die Form gebracht werden

$$
\begin{aligned}
& \left(\lambda_{2}-\lambda_{3}\right) \sqrt{\left(F-2 \lambda_{1} H+\lambda_{1}{ }^{2} G\right)} \pm\left(\lambda_{3}-\lambda_{1}\right) \sqrt{\left(F-2 \lambda_{2} H+\lambda_{2}{ }^{2} G\right)} \\
& \pm\left(\lambda_{1}-\lambda_{2}\right) \sqrt{\left(F-2 \lambda_{3} H+\lambda_{3}{ }^{2} G\right)=0 .}
\end{aligned}
$$

Für bestimmte Werthe der $u_{i}$ und variabele $x_{i}$ stellt die linke Seite von (34) eine Gerade dar. Setzt man für die $u_{i}$ Werthe ein, welche bewirken, dass die $U_{i}$ die Gleichungen (23) befriedigen, so erhält man die Gleichungen der vier gemeinsamen Tangenten von $f(x, x)=0$ und $g(x, x)=0$ in der Gestalt:

$$
\sqrt{\frac{1}{\lambda_{2}}-\frac{1}{\lambda_{3}}} X_{1} \pm \sqrt{\frac{1}{\lambda_{3}}-\frac{1}{\lambda_{1}}} X_{2} \pm \sqrt{\frac{1}{\lambda_{1}}-\frac{1}{\lambda_{2}}} X_{3}=0 \text {, }
$$

wobei die $X_{i}$ definirt sind durch $(25)$.

\section{$\S 15$.}

\section{Allgemeine Eigenschaften des Kegelschnittbüschels;}

$$
\text { die Kegelschnittschaar. }
$$

Zunächst möge die Frage beantwortet werden, welchen Werth der Parameter $\lambda$ annehmen muss, damit eine Curve des Büschels

eine gegebene Gerade

$$
\lambda g(x, x)-f(x, x)=0
$$

$$
u_{x} \equiv u_{1} x_{1}+u_{2} x_{2}+u_{3} x_{3}=0
$$

berühre. Nach $\S 4$ ist dies offenbar dann und nur dann der Fall, wenn

$$
\Psi_{1}(\lambda) \equiv-\left|\begin{array}{llll}
c_{11} & c_{12} & c_{13} & u_{1} \\
c_{21} & c_{22} & c_{23} & u_{2} \\
c_{31} & c_{32} & c_{33} & u_{3} \\
u_{1} & u_{2} & u_{3} & 0
\end{array}\right|=0
$$


d. h. wenn

$$
\Psi_{1}(\lambda) \equiv F-2 \lambda H+\lambda^{2} G=0 .
$$

Da diese Gleichung vom zweiten Grade in $\lambda$ ist, folgt:

(5) In dem Büschel $\lambda g-f=0$ gibt es im allgemeinen zwei Kegelschnitte, die eine gegebene Gerade berühren.

Um auch die Gleichung $H=0$ geometrisch zu deuten, gehen wir davon aus, dass nach (53a) in $\S 4$ die Gleichung des Schnittpunktepaares der Geraden (2) mit der Curve (1) in variabelen Liniencoordinaten $v_{1}, v_{2}, v_{3}$ dargestellt ist durch

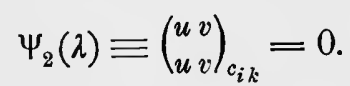

Diese Gleichung ist in $\lambda$ vom ersten Grad; bezeichnet man die Coefficienten von $\lambda^{0}$ und $\lambda^{1}$ in ihr mit $P$ und $-Q$, so entsteht

$$
\Psi_{2}(\lambda) \equiv P-\lambda Q=0,
$$

und es sind dann $P=0, Q=0$ die Gleichungen der zwei Schnittpunktepaare der Geraden (2) mit den Kegelschnitten $f=0$, resp. $g=0$. Denn zu dem Werthe $\lambda=0$ des Parameters, für welchen $P=0$, gehört die Curve $f=0$; zu dem Werthe $\lambda=\infty$, für welchen $Q=0$, die Curve $g=0$.

Speciell für eine der beiden Wurzeln $\lambda_{1}$ und $\lambda_{2}$ der quadratischen Gleichung (4) geht das Schnittpunktepaar über in den doppelt zu nehmenden Berührungspunkt, so dass man setzen kann:

$$
\left\{\begin{array}{l}
P-\lambda_{1} Q=\left(y_{1} v_{1}+y_{2} v_{2}+y_{3} v_{3}\right)^{2}=V_{1}{ }^{2} \\
P-\lambda_{2} Q=\left(z_{1} v_{1}+z_{2} v_{2}+z_{3} v_{3}\right)^{2}=\nabla_{2}{ }^{2}
\end{array}\right.
$$

Vermöge dieser beiden Relationen lassen sich nun $P$ und $Q$ ausdrücken durch $V_{1}^{2}$ und $V_{2}^{2}$. Zerlegt man nämlich den Ausdruck

$$
\frac{P-\lambda Q}{\left(\lambda-\lambda_{1}\right)\left(\lambda-\lambda_{2}\right)}
$$

in zwei Partialbrüche, so entsteht

$$
\begin{gathered}
\frac{P-\lambda Q}{\left(\lambda-\lambda_{1}\right)\left(\lambda-\lambda_{2}\right)}=\frac{P-\lambda_{1} Q}{\left(\lambda_{1}-\lambda_{2}\right)\left(\lambda-\lambda_{1}\right)}+\frac{P-\lambda_{2} Q}{\left(\lambda_{2}-\lambda_{1}\right)\left(\lambda-\lambda_{2}\right)}=0 \text { oder } \\
\frac{1}{\lambda_{1}-\lambda_{2}}\left\{\frac{V_{1}^{2}}{\lambda-\lambda_{1}}-\frac{V_{2}^{2}}{\lambda-\lambda_{2}}\right\}=0
\end{gathered}
$$

und die Form dieser Gleichung liefert unmittelbar den zuerst von Desargues ${ }^{1}$ ), später auch von Ch. Sturm gefundenen Satz, den wir den Desargues-Sturm'schen Satz nennen wollen:

1) Desargues: „Brouillon project d'une atteinte aux événemens des rencontres d'un cone avec un plan", Paris 1639, veröffentlicht in: "Oenvres de Desargues réunies et analysées par M. Poudra", Bd. I, S. 186 ff., Paris 1864. 
(11) Das Punktepaar, in welchem eine beliebige Curve des Büschels (1) eine gegebene Gerade trifft, ist harmonisch zu den Berührungspunkten derjenigen beiden Curven, welche die gegebene Gerade berühren.

Man kann diesen Satz auch in folgender Form aussprechen:

(12) Die Kegelschnitte eines Büschels treffen eine beliebige Gerade in Punkteparen einer Involution; die Doppelpunkte dieser Involution sind die Berührungspunkte derjenigen zwei Curven des Büschels, welche die gegebene Gerade zur Tangente haben.

Für $\lambda=0$ und $\lambda=\infty$ ergeben sich aus (10) die Punktepaare, in denen die Curven $f=0$ und $g=0$ die Gerade (2) treffen, nämlich

$$
\begin{gathered}
\left(V_{1}+\sqrt{\frac{\lambda_{1}}{\lambda_{2}}} V_{2}\right)\left(V_{1}-\sqrt{\frac{\lambda_{1}}{\lambda_{2}}} V_{2}\right)=0 \text { für die Curve } f=0, \\
\left(V_{1}+V_{2}\right)\left(V_{1}-V_{2}\right) \text { für } g=0 .
\end{gathered}
$$
ist nun

Das Doppelverhältniss $\alpha$ des ersten Punktepaares zum zweiten

$$
\alpha=\frac{\sqrt{\frac{\lambda_{1}}{\lambda_{2}}}-1}{\sqrt{\frac{\lambda_{1}}{\lambda_{2}}+1}}: \frac{\sqrt{\frac{\lambda_{1}}{\lambda_{2}}}+1}{\sqrt{\frac{\lambda_{1}}{\lambda_{2}}}-1},
$$

woraus folgt $\frac{\alpha-1}{\alpha+1}=-\frac{2 \sqrt{\lambda_{1} \lambda_{2}}}{\lambda_{1}+\lambda_{2}} ;$ erhebt man diesen Ausdruck ins Quadrat und führt man für das Product und die Summe der Wurzeln $\lambda_{1}, \lambda_{2}$ der quadratischen Gleichung (4) die Coefficienten ein, so ergibt sich die Beziehung:

$$
(\alpha-1)^{2} H^{2}-(\alpha+1)^{2} H G=0 .
$$

Diese Gleichung repräsentirt, wenn $\alpha$ eine gegebene Zahl ist, die Bedingung, der die Coordinaten $u_{i}$ einer geraden Linie genügen müssen, wenn die Gerade die Kegelschnitte $f$ und $g$ so schneiden soll, dass das Punktepaar $P$ mit dem Punktepaar $Q$ das gegebene Doppelverhältniss $\alpha$ bildet. Diese Bedingung ist für die $u_{i}$ vom vierten Grad, daher hat man den Satz:

(14) Alle geraden Linien, welche die zwei Kegelschnitte $f=0$ und $g=0$ so schneiden, dass die b'eiden Schnittpunktepaare ein gegebenes Doppelverhältniss $\alpha$ bilden, umhüllen eine Curve vierter Classe.

Für $\alpha=-1$ hat $\operatorname{man} \cdot H^{2}=0$. Das Verschwinden von $H$ sagt also aus, dass die Gerade (2) von den beiden Kegelschnitten $f=0$ und $g=0$ in zwei harmonischen Punktepaaren getroffen wird. Da 
$H$ in den $u_{i}$ vom zweiten Grad ist, stellt $H=0$ einen Kegelschnitt dar, und man kann somit sagen:

(15) Alle Geraden, welche zwei Kegelschnitte $f=0$ und $g=0$ in zwei harmonischen Punktepaaren treffen, umhüllen einen dritten Kegelschnitt $H=0$, den wir die harmonische Curve zweiter Classe nennen wollen.

Dieser Kegelschnitt wird auch von denjenigen acht Geraden berührt, welche in den vier Schnittpunkten von $f=0$ und $g=0$ als Tangenten dieser beiden Curven gezogen werden können. Construirt man nämlich in irgend einem der Schnittpunkte die Tangente, etwa von $f=0$, so schneidet dieselbe den Kegelschnitt $f=0$ in zwei zusammenfallenden Punkten; sollen nun die Schnittpunkte derselben Tangente mit $g=0$ harmonisch liegen zu diesem zusammengerückten Punktepaar, so muss einer der Schnittpunkte mit $g=0$ in dieses Punktepaar hereinfallen. Dies ist nun wirklich der Fall, jene Tangente trifft daher beide Kegelschnitte in der That harmonisch. Dasselbe würde gelten, wenn jene Tangente an den Kegelschnitt $g=0$ in einem der Schnittpunkte von $f=0$ mit $g=0$ gezogen worden wäre.

Man kann ferner fragen, was eintritt, wenn das Doppelverhältniss $\alpha=0$ ist, oder den reciproken Werth hiervon, $\infty$, annimmt. Bezeichnen wir die Schnittpunkte der Geraden $u_{x}=0$ mit dem Kegelschnitt $f=0$ durch 0 und 1 , ihre Schnittpunkte mit $g=0$ durch 2 und 3 , so hat das Doppelverhältniss $\alpha$ bekanntlich den Werth $\alpha=\frac{(20)}{(21)}: \frac{(30)}{(31)}=\frac{(20)(31)}{(21)(30)}$, wo (20) den Abstand des Punktes 0 rom Punkte 2 bedeutet. Dieses Doppelverbältniss ist nur dann Null oder unendlich gross, wenn einer der Abstände (20), (31), (21), (30) sich auf Null reducirt, d. h. wenn die Gerade die Kegelschnitte so trifft, dass ein auf $f=0$ gelegener Schnittpunkt mit einem auf $g=0$ gelegenen zusammenfüllt, wenn sie also durch einen der vier Schnittpunkte beider Kegelschnitte geht. Und so oft auch umgekehrt die Gerade durch einen der vier Schnittpunkte geht, wird das Doppelverhältniss gleich Null oder unendlich gross. Für $\alpha=0$ und $\alpha=\infty$ verwandelt sich aber (13) in:

$$
H^{2}-F G=0,
$$

und diese Gleichung wird daher erfüllt für jede Gerade, die durch einen der vier Schnittpunkte geht, d. h.:

(16a) Die vier Schnittpunkte der Kegelschnitte $f=0$ und $g=0$ sind gegeben durch die Gleichung $H^{2}-F G=0$.

$\mathrm{Zu}$ diesem Resultate gelangt man auch in folgender Weise. 
Die vorstehenden Betrachtungen hatten zur Voraussetzung, dass die beiden Wurzeln $\lambda_{1}$ und $\lambda_{2}$ der Gleichung (4) verschieden seien. Um auf den Fall $\lambda_{1}=\lambda_{2}$ näher einzugehen, ersetzen wir in der für ein beliebiges $\lambda$ und für alle Werthe der $\dot{v}_{i}$ bestehenden Identität

$$
\Psi_{2}(\lambda)=\frac{\partial \Psi_{1}(\lambda)}{\partial c_{11}} v_{1}{ }^{2}+2 \frac{\partial \Psi_{1}(\lambda)}{\partial c_{12}} v_{1} v_{2}+\cdots+\frac{\partial \Psi_{1}(\lambda)}{\partial c_{33}} v_{3}{ }^{2}
$$

die Grösse $\lambda$ durch die Doppelwurzel $\lambda_{1}$ und die Quadrate und Producte $v_{1}{ }^{2}, v_{1} v_{2}, \ldots v_{3}{ }^{2}$ durch $b_{11}, b_{12}, \ldots b_{33}$, was nach (35) in $\S 8$ geschehen darf. Alsdann wird die rechte Seite der letzten Identität gleich dem zufolge. Vorhandenseins der Doppelwurzel verschwindenden Differentialquotienten $\left.\frac{d \psi_{1}\left(\lambda_{1}\right)}{d \lambda_{1}}\right)$, während die linke Seite nach $(7)$ und (8) übergeht in $y_{1}{ }^{2} b_{11}+2 y_{1} y_{2} b_{12}+\cdots+y_{3}{ }^{2} b_{33}$, also in $g(y, y)$. Der auf der Curve $\lambda_{1} g-f=0$ befindliche Berührungspunkt $y$ liegt demnach gleichzeitig auch auf den Curven $g=0$ und $f=0$. Nun ist aber die Bedingung für die Gleichheit der Wurzeln $\lambda_{1}$ und $\lambda_{2}$ von $F-2 \lambda H+\lambda^{2} G=0$ ausgedrückt durch $H^{2}-F G=0$; die Erfüllung dieser Bedingung sagt daher aus, dass die Gerade $u_{x}=0$ durch einen der vier Schnittpunkte von $g=0$ und $f=0$ hindurchgeht.

Dem Kegelschnittbüschel entspricht dualistisch die Kegels chnittschaar: die Gesammtheit aller Curven zweiter Classe, welche vier gegebene Geraden zu Tangenten haben. Sind diese die gemeinsamen Tangenten der beiden Curven $\varphi(u, u)=0$ und $\psi(u, u)=0$, so stellt die Gleichung $\lambda \psi-\varphi=0$ für alle möglichen Werthe des Parameters $\lambda$ alle in der Schaar enthaltenen Curven dar.

Den bisher abgeleiteten Sätzen über Kegelschnittbüschel entsprechen solche über Schaaren; sie mögen hier ohne Beweis erwähnt werden, da die Beweisführung die analoge ist wie bei den Büscheln. (18) In einer Kegelschnittschaar sind im allgemeinen drei Punktepare vorhanden.

(19) Jede Gerade, welche nicht zugleich eine der vier gemeinsamen Tangenten der Kegelschnitte $\varphi(u, u)=0$ und $\psi(u, u)=0$ ist, wird von einem und nur einem der Schaar $\lambda \psi-\varphi=0$ angehörigen Kegedschnitte berührt.

(20) Die drei Träger der in einer Kegelschnittschaar enthaltenen Punktepare bilden ein Poldreiseit für jede Curve der Schaar.

(21) In einer Kegelschnittschaar gibt es im allgemeinen

1) Denn aus $c_{i k}=\lambda b_{i k}-a_{i k}$ folgt $b_{i k}=\frac{\partial c_{i k}}{\partial \lambda}$. 
zwei Curven, die durch einen gegebenen Punkt hindurchgehen.

Die Tangentenpaare, welche man von irgend einem Punkte an die Kegelschnitte einer Schaar legen kann, bilden eine Involution, deren Doppelstrablen aus den Tangenten derjenigen zwei Curven der Schaar bestehen, welche sich in dem gegebenen Punkte schneiden.

(23) Alle Punkte, von welchen an zwei gegebene Curven zweiter Classe harmonische Tangentenpare gezogen werden können, liegen auf einem dritten Kegelschnitt; derselbe werde die harmonische Curve zweiter Ordnung genannt.

Von besonderer Wichtigkeit ist diejenige Kegelschnittschaar, bei welcher eine der zwei Curven $\varphi=0, \psi=0$ aus dem imaginären Kreispunktepaar besteht; man spricht in diesem (in $\S 18$ ausführlicher behandelten) Falle von einer Schaar confocaler Kegelschnitte. Die zwei Curven einer solchen Schaar, die nach (21) durch einen beliebig gegebenen Punkt $(y)$ hindurchgehen, schneiden sich in demselben rechtwinklig, denn nach (22) liegen die Tangenten, welche in dem betreffenden Schnittpunkte $y$ an die beiden Curven gezogen werden können, harmonisch zu allen Tangentenpaaren, die von $y$ an die Kegelschnitte der Schaar gelegt sind, sie sind also larmonische Polaren des der Schaar angehörigen Kreispunktepaars, stehen daher nach (28) in \$ 7 auf einander senkrecht. Auch kann man zeigen, dass die zwei durch einen beliebigen Punkt $y$ der Ebene gehenden Kegelschnitte der confocalen Schaar immer reell sind. Die Gleichung der confocalen Schaar hat nämlich die Form:

$$
\lambda \varphi(u, u)-\omega(u, u) \doteq 0
$$

ist hier $\varphi(u, u) \equiv \sum_{1}^{3} \sum_{1}^{3} \alpha_{i k} u_{i} u_{k}$ und setzt man $\lambda \alpha_{i k}-\omega_{i k}=\gamma_{i k}$, so ist (24) gleichbedeutend mit $\sum_{1}^{3} \sum_{1}^{3} \gamma_{i k} u_{i} \imath_{k}=0$. Für die Parameter derjenigen zwei Curven der Schaar, welche durch den gegebenen Punkt $y$ gehen, hat man dualistisch zu (3) die Gleichung

$$
\left(\begin{array}{l}
y \\
y
\end{array}\right)_{\gamma_{i k}}=0 \text {, }
$$

und diese hat, wie wir behaupten, stets zwei reelle Wurzeln. Es folgt dies aus Satz (4) in $\S 8$, indem man in der Determinante (4a) in $\S 8$, auf welche sich der Satz bezieht, die Form $\sum_{1}^{n} \sum_{1}^{n} \alpha_{i k} u_{i} u_{k}$ ersetzt 
durch $\sum_{1}^{3} \sum_{1}^{3} \alpha_{i k} u_{i} u_{k}+2\left(y_{1} u_{1}+y_{2} u_{2}+y_{3} u_{3}\right) u_{4} ;$ wan erhält alsdann sofort die Determinante (25). Dass die zwei den Wurzeln von (25) entsprechenden Curven zweiter Classe nicht imaginär sind, folgt danm daraus, dass $y$ ein reeller Punkt dieser Curven ist. Wir können also sagen:

(26)

Durch einen beliebigen Punkt der Ebene gehen stets zwei reelle Kegelschnitte einer confocalen Schaar, und zwar schneiden sich dieselben in dem betreffenden Punkte rechtwinklig.

Es wird in $\S 18$ gezeigt werden, dass der eiue der beiden Kegelschnitte (so lange wenigstens die Schaar keine specielle Schaar ist) stets aus einer Ellipse, der andere aus einer Hyperbel besteht.

Für eine nicht ausartende Curve zweiter Classe $\varphi(u, u)=0$ bestehen ferner die drei Punktepaare der Schaar $\lambda \varphi(u, u)-\omega(u, u)=0$ aus $\omega(\imath, u)=0$ und den beiden Bremupunktepaaren (vgl. S. $113 \mathrm{f}$.).

Hieraus und aus (26) folgt in Verbindung mit (21) der Satz:

(27) Die Winkel derjenigen Tangentenpare, welche man von irgend einem Punkte $P$ an die Kegelschuitte eiuer confocalen Schaar, speciell auch an das.reelle Brennpuktepaar, legen kann, haben sämmtlich dieselbe Halbirungslinie; dieselbe besteht in der eiuen oder anderen Tangente der beiden durch $P$ gehenden Kegelschnitte der Schaar.

Nach (23) liegen die Punkte $y$, von welchen an zwei gegebene Curven zweiter Classe harmonische Tangentenpaare gezogen werden können, auf einem Kegelschnitt. Auch dieser Satz liefert besonders interessante Resultate, wenn man als eine der beiden Curven zweiter Classe das imaginäre Kreispunktepaar wählt, wälırend die andere gegeben sei durch

$$
\varphi(u, u) \equiv \sum_{1}^{3} \sum_{1}^{3} k \alpha_{i k} u_{i} u_{k}=0 .
$$

Es müssen nämlich nunmehr die von einem Punkte $y$ an $\varphi(\imath, u)=0$ gezogenen Tangenten harmonische Polaren sein von $\omega(u, u)=0$, also auf einander senkrecht stehen, so dass daher alle Punkte $y$, von welchen an eine Curve zweiter Classe zu einander rechtwinklige Tangenten gezogen werden könuen, auf einem Kegelschnitt liegen. Die Gleichung $\chi(x, x)=0$ desselben wird erhalten, indem man den dualistischen Ausdruck zu $H=0$ bildet und beachtet, dass die eine Curve zweiter Classe durch $\omega(u, u)=0$ gegeben ist; daher ergibt sich: 


$$
\begin{aligned}
2 \chi(x, x) & \equiv\left(\alpha_{22} \omega_{33}+\alpha_{33} \omega_{22}-2 \alpha_{23} \omega_{23}\right) x_{1}{ }^{2}+\cdots \\
& +2\left(\alpha_{31} \omega_{32}+\alpha_{32} \omega_{31}-\alpha_{33} \omega_{12}-\alpha_{12} \omega_{33}\right) x_{1} x_{2}+\cdots=0 .
\end{aligned}
$$

Man kann die Gleichung dieser Curve auch noch auf anderem Wege erhalten, wobei sich überdies sofort zeigt, dass dieselbe einen Kreis repräsentirt. Aus (22) in $\S 11$ folgt nämlich, dass die Gleichung einer Curve zweiter Classe in die Form gebracht werden kann

$$
\varphi(u, u) \equiv c \cdot \omega(u, u)+B \cdot B_{1}=0,
$$

wobei $B=0$ und $B_{1}=0$ die reellen Brennpunkte darstellen, $c$ eine Constante bedeutet. Der Ausdruck $\chi(x, x)$, gebildet für (30), besteht nun aus zwei Theilen, indem man $\chi(x, x)$ zu bilden hat für $c \cdot \omega(u, u)$, sowie für $B B_{1}$. Der erste Theil wird, wie man sofort sieht, gleich $c \cdot \tau p_{x}{ }^{2}$; der zweite ist identisch mit dem Ausdruck für den geometrischen Ort aller Punkte, von denen nach den Brennpunkten $B=0$ und $B_{1}=0$ zwei zu einander senkrechte Geraden gezogen werden können, und dieser Ort ist, wie man aus der elementaren Planimetrie weiss, ein Kreis mit der Verbindungslinie der zwei Brennpunkte als Durchmesser, etwa $K=0$. Infolge dessen wird $\chi$, gebildet für (30), von der Form $c \cdot \tau p_{x}{ }^{2}+K=0$, und diese Gleichung stellt, da sie sich von $K=0$ nur um das Glied $c \cdot \tau p_{x}^{2}$ unterscheidet, gleichfalls einen Kreis dar, welcher überdies, wie die Gleichung des Kreises (43) in $\S 10$ zeigt, mit $K=0$ und somit auch mit $f(u, u)=0$ concentrisch

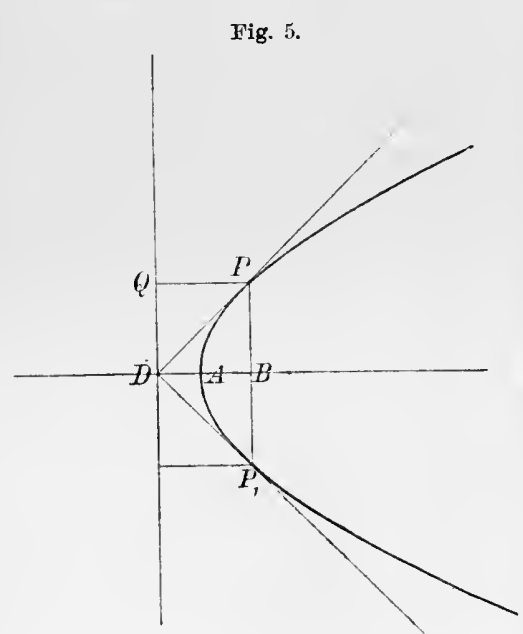

ist. Man nennt diesen Kreis $\chi(x, x)=0$ den Directorkreis des gegebenen Kegelschnitts und kann somit den Satz aussprechen:

(31) Der geometrische Ort aller Punkte, von denen an einen gegebenen Kegelschnitt Tangenten gezogen werden können, die zu einander rechtwinklig sind, ist ein mit dem Kegelschnitt concentrischer Kreis.

Besondere Beachtung verdient der Fall der Parabel, indem sich zeigen wird, dass hier der Directorkreis zerfällt in die unendlich ferne Gerade und in die Directrix der Parabel. Unter Anwendung derselben Betrachtungsweise wie zuvor auf die Gleichung (30), in welcher jedoch nunmehr $B_{1}=0$ den unendlich fernen Brennpunkt der Parabel darstellen möge, erkennt man, dass 
in diesem Falle $\chi(x, x)=0$ in die unendlich ferne Gerade, sowie in eine Parallele zu derjenigen Geraden zerfällt, welche durch den im Endlichen gelegenen Brennunkt senkrecht zur Axe der Parabel gezogen wird. Dass diese Parallele die Directrix ist, ergibt sich aus dem Umstande, dass die zwei aus dem Schnittpunkt $D$ von Axe und Directrix an die Curve gezogenen Tangenten zu einander normal sind. Eine Sehne, die in $B$ normal zur Axe steht und in $P, P_{1}$ die Parabel schneiden möge, ist offenbar die Polare von $D$; denn der unendlich ferne Punkt dieser Sehne (als Pol der Axe) und $B$ (als Pol der Directrix) sind beide conjugirt zu $D^{1}$ ). Andrerseits folgt aus der Definition der Parabel $P B=P Q=B D$, d. l. das Viereck $D B P Q$ ist ein Quadrat, somit $\Varangle P D B=\frac{1}{2} R$ und $P D P_{1}=R$. Es gilt demnach der Satz:

(32) Der geometrische Ort aller Punkte, von denen an eine Parabel zwei zu einander rechtwinklige Tangenten gezogen werden können, besteht aus der Directrix der Parabel und der unendlich fernen Geraden.

\section{$\S 16$.}

Einführung zweckmässiger Coordinatendreiecke in den versehiedenen speciellen Fällen, welche bei einem Kegelschnittbüschel auftreten können.

In dem allgemeinen Falle, in welchem die kubische Gleichung $\Psi_{0}(\lambda)=0$ nur verschiedene Wurzeln besitzt, hatte sich nach $\S 14$ als zweckmässigstes Coordinatendreieck das durch die Spitzen der drei in dem Kegelschnittbïschel vorhandenen Geradenpaare bestimmte Dreieck erwiesen; dasselbe war zugleich ein Poldreieck für alle Curven des Büschels. Bei Einführung desselben erhielten die Gleichungen der Curven $f=0$ und $g=0$ des Büschels $\lambda g-f=0$ die einfache Gestalt ${ }^{2}$ ):

$$
\left\{\begin{array}{l}
f=\lambda_{1} X_{1}{ }^{2}+\lambda_{2} X_{2}{ }^{2}+\lambda_{3} X_{2}{ }^{2}=0 \\
g=X_{1}{ }^{2}+X_{2}{ }^{2}+X_{3}{ }^{2}=0
\end{array}\right.
$$

Sobald nun aber die Gleichung $\Psi_{0}(\lambda)=0$ eine doppelte oder dreifache Wurzel besitzt, fallen zwei Ecken des zu Grunde gelegten Poldreiecks zusammen, und es verlieren überhaupt viele Entwickelungen in $\S 14$ ihre Giltigkeit. Zur näheren Untersuchung dieses Falles ist es vortheilhaft, die zur vielfachen Wurzel gehörigen Partialbrüche

1) Dies gilt übrigens für jeden Kegelschnitt, nicht nur für die Parabel.

2) Vgl. (30) und (29) in $\$ 14$. 
von $\frac{\Psi_{1}(\lambda)}{\Psi_{0}(\lambda)}$ zu bestimmen. Es besteht nun nach $(7)$ in $\S 13$ die Relation

$$
C\left(\begin{array}{l}
u v \\
u v
\end{array}\right)=\left(\begin{array}{l}
u \\
u
\end{array}\right)\left(\begin{array}{l}
v \\
v
\end{array}\right)-\left(\begin{array}{l}
u \\
v
\end{array}\right)^{2}
$$

wobei die $v_{i}(i=1,2,3)$ ganz beliebige Grössen bezeichnen und $\Psi_{0}(\lambda) \equiv \sum \pm\left(c_{11} c_{22} c_{33}\right)$ ersetzt ist durch $C$. Aus (2) folgt, da $\left(\begin{array}{l}u \\ u\end{array}\right)=-\Psi_{1}(\lambda)$, sofort:

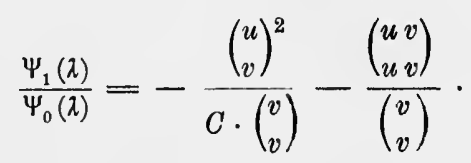

So lange wir annehmen, dass nicht alle Unterdeterminanten $C_{i k}$ in $C$ verschwinden, können wir uns zunächst die $v_{i}$ so bestimmt denken, dass für eine $e$-fache Wurzel $\lambda_{1}(e=2$ oder 3$)$ der Ausdruck $\left(\begin{array}{l}v \\ v\end{array}\right)$ bei völliger Willkürlichkeit der $v_{i}$ nicht verschwindet, mit anderen Worten, dass $\left(\begin{array}{l}v \\ v\end{array}\right)$ keinen Factor $\lambda-\lambda_{1}$ besitzt $^{1}$ ). In Folge dessen wird nur der Term $\frac{\left(\begin{array}{l}u \\ v\end{array}\right)^{2}}{c\left(\begin{array}{l}v \\ v\end{array}\right)}$ Partialbrüche veranlassen, die zur Wurzel $\lambda_{1}$ gehören. Bekanntlich ist die Gesammtheit derselben, wofern

$$
\frac{\left(\lambda-\lambda_{1}\right)^{e}\left(\begin{array}{l}
u \\
v
\end{array}\right)^{2}}{-C \cdot\left(\begin{array}{l}
v \\
v
\end{array}\right)}=\left\{\frac{\left(\lambda-\lambda_{1}\right)^{\frac{e}{2}} \cdot\left(\begin{array}{l}
u \\
v
\end{array}\right)}{\sqrt{-C \cdot\left(\begin{array}{l}
v \\
v
\end{array}\right)}}\right\}^{2}=U^{2}
$$

gesetzt wird, repräsentirt durch:

$$
\frac{\left[U^{2}\right]_{\lambda=\lambda_{1}}}{\left(\lambda-\lambda_{1}\right)^{e}}+\frac{\left[\frac{d \cdot U^{2}}{d \lambda}\right]_{\lambda=\lambda_{1}}}{\left(\lambda-\lambda_{1}\right)^{e-1}}+\cdots+\frac{\left[\frac{d^{e-1} \cdot U^{2}}{d \lambda}\right]_{\lambda=\lambda_{1}}}{(e-1) !\left(\lambda-\lambda_{1}\right)} .
$$

Führt man daher die Bezeichnungen ein:

$$
[U]_{\lambda=\lambda_{1}}=U^{(1)},\left[\frac{d U}{d \lambda}\right]_{\lambda=\lambda_{1}}=U^{(2)}, \frac{1}{2}\left[\frac{d^{2} U}{d \lambda^{2}}\right]_{\lambda=\lambda_{1}}=U^{(3)}
$$

1) Man kann beispielsweise einer der Grössen $v$ den Werth 1, den beiden anderen den Werth Null ertheilen, und zwar kann dies auf drei Arten geschehen, je nachdem $v_{1}$ oder $v_{2}$ oder $v_{3}$ gleich 1 gesetzt wird. Aber eine dieser drei Möglichkeiten führt stets zum Ziel, denn wenn dies nicht der Fall wäre, würden für jene Doppelwurzel $\lambda_{1}$ die Unterdeterminanten $C_{11}, C_{22}$ und $C_{33}$ verschwinden, und da für $\lambda=\lambda_{1}$ auch $C=0$, so würden alle Unterdeterminanten $C_{i k}$ verschwinden, was ausgeschlossen war: 
Coordinatendreieck bei speciellen Lagen der Kegelschnitte eines Büschels. 151 so erhält man für $\frac{\Psi_{1}(\lambda)}{\Psi_{0}(\lambda)}$, je nachdem $e=2$ oder $e=3$, die Darstellung:

$$
\frac{\Psi_{1}(\lambda)}{\Psi_{0}(\lambda)}=\frac{U^{(1)} U^{(1)}}{\left(\lambda-\lambda_{1}\right)^{2}}+2 \frac{U^{(1)} U^{(2)}}{\lambda-\lambda_{1}}+\frac{U_{3}^{2}}{\lambda-\lambda_{3}}
$$

oder diese andere:

$$
\frac{\Psi_{1}(\lambda)}{\Psi_{0}(\lambda)}=\frac{U^{(1)} U^{(1)}}{\left(\lambda-\lambda_{1}\right)^{3}}+2 \frac{U^{(1)} U^{(2)}}{\left(\lambda-\lambda_{1}\right)^{2}}+\frac{U^{(2)} U^{(2)}+2 U^{(1)} U^{(3)}}{\lambda-\lambda_{1}} .
$$

Darin sind $U^{(1)}, U^{(2)}, U^{(3)}$ lineare homogene Functionen der $u_{i}$, die durch (4) und (6) definirt sind, während $U_{3}$ mit dem bereits früher aufgetretenen und ähnlich bezeichneten Ausdrucke (15) in $\S 14$ übereinstimmt.

Ist die Grösse $\lambda_{1}$ eine Doppelwurzel von $\Psi_{0}(\lambda)=0$ und gleichzeitig für beliebige Werthe der $v_{i}$ eine einfache Wurzel von $\left(\begin{array}{l}v \\ v\end{array}\right)=0$, so liefern die beiden Terme in (3) Partialbrüche mit dem Nenner $\lambda-\lambda_{1}$; ferner sieht man aús (2), dass in diesem Falle $\lambda_{1}$ auch einfache Wurzel von $\left(\begin{array}{l}u \\ v\end{array}\right)=0$ ist. In Folge dessen lässt sich der Factor $\lambda-\lambda_{1}$ in Zähler und Nenner von $\frac{\left(\begin{array}{l}u \\ v\end{array}\right)^{2}}{C \cdot\left(\begin{array}{l}v \\ v\end{array}\right)}$ zweimal wegheben, so dass alsdann dieses Glied, ebenso wie $\frac{\left(\begin{array}{l}u v \\ u v\end{array}\right)}{\left(\begin{array}{l}v \\ v\end{array}\right)}$, im Nenner den Factor $\lambda-\lambda_{1}$ nur noch einmal besitzt. Durch Partialbruchzerlegung ergibt sich daher jetzt eine Gleichung von der Gestalt:

wobei

$$
\text { (10) } U^{(1)}=\left\{\frac{\left(\lambda-\lambda_{1}\right)^{\frac{1}{2}}\left(\begin{array}{l}
u \\
v
\end{array}\right)}{\sqrt{-C \cdot\left(\begin{array}{l}
v \\
v
\end{array}\right)}}\right\}_{\lambda=\lambda_{1}}, U^{(2)}=\left\{\frac{\left(\lambda-\lambda_{1}\right)^{\frac{1}{2}} \sqrt{\left(\begin{array}{l}
u v \\
u v
\end{array}\right)}}{\sqrt{-\left(\begin{array}{l}
v \\
v
\end{array}\right)}}\right\}_{\lambda=\lambda_{1}} \text {. }
$$

Der Fall, dass eine Doppelwurzel $\lambda_{1}$ von $\Psi_{0}(\lambda)=0$ auch Doppelwurzel von $\left(\begin{array}{l}v \\ v\end{array}\right)=0$ ist, kommt nicht in Betracht, da alsdann der Factor $\lambda-\lambda_{1}$ in der rechten Seite von (2) vierfach, somit in $\left(\begin{array}{ll}u & v \\ u & v\end{array}\right)$

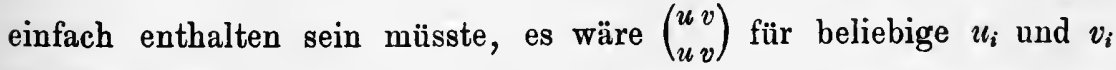
theilbar durch $\lambda-\lambda_{1}$, d. h. die Coefficienten $a_{i k}$ des Kegelschnitts $f(x, x)=0$ wären proportional den Coefficienten $b_{i k}$ von $g(x, x)=0$, 
beide Kegelschnitte somit identisch und überhaupt kein Büschel vorhanden.

Der letzte mögliche Specialfall wäre endlich derjenige, bei welchem der Factor $\lambda-\lambda_{1}$ in $\Psi_{0}(\lambda)$ zur dritten Potenz erhoben vorkommt und überdies in $\left(\begin{array}{l}v \\ v\end{array}\right)$, also auch (wie oben) in $\left(\begin{array}{l}u \\ v\end{array}\right)$, einfach enthalten ist. Nun lässt sich der Factor $\lambda-\lambda_{1}$ in Zähler und Nenner von $\frac{\left(\begin{array}{l}u \\ v\end{array}\right)^{2}}{c\left(\begin{array}{l}v \\ v\end{array}\right)}$ zweimal wegheben, so dass alsdann dieses Glied im Nenner noch den Factor $\left(\lambda-\lambda_{1}\right)^{2}$ besitzt. Setzt man

$$
\begin{gathered}
U=\frac{\left(\lambda-\lambda_{1}\right)\left(\begin{array}{l}
u \\
v
\end{array}\right)}{\sqrt{-C \cdot\left(\begin{array}{l}
v \\
v
\end{array}\right)},} \quad U^{(1)}=[U]_{\lambda=\lambda_{1}}, \quad U^{(2)}=\left[\frac{d}{d \lambda}\right]_{\lambda=\lambda_{1}}, \\
U^{(3)}=\left\{\frac{\left(\lambda-\lambda_{1}\right)^{\frac{1}{2}} \sqrt{\left(\begin{array}{l}
u \\
u
\end{array}\right)}}{\sqrt{-\left(\begin{array}{l}
v \\
v
\end{array}\right)}}\right\}_{\lambda=\lambda_{1}},
\end{gathered}
$$

so erhält man für $\frac{\Psi_{1}(\lambda)}{\psi_{0}(\lambda)}$ die Darstellung:

$$
\frac{\Psi_{1}(\lambda)}{\Psi_{0}(\lambda)}=\frac{U^{(1)} U^{(1)}}{\left(\lambda-\lambda_{1}\right)^{2}}+\frac{2 U^{(1)} U^{(2)}}{\lambda-\lambda_{1}}+\frac{U^{(3)} U^{(3)}}{\lambda-\lambda_{1}} .
$$

Der Fall, dass eine dreifache Wurzel von $\Psi_{0}(\lambda)=0$ zugleich Doppelwurzel von $\left(\begin{array}{l}v \\ v\end{array}\right)=0$ wäre, kommt nicht in Betracht, da bereits der Fall einer in $\left(\begin{array}{l}v \\ v\end{array}\right)=0$ zweifach enthaltenen Doppelwurzel von $\Psi_{0}(\lambda)=0$ auszuschliessen war.

Abgesehen von dem allgemeinen Falle, in welchem die Wurzeln von $\Psi_{0}(\lambda)$ von einander verschieden sind, haben wir also vier specielle Fälle zu unterscheiden, die wir nochmals kurz zusammenstellen und mit den in $\S 14$ mit IIa, IIb, IIIa, IIIb bezeichneten Fällen vergleichen, auch mit denselben Nummern bezeichnen wollen.

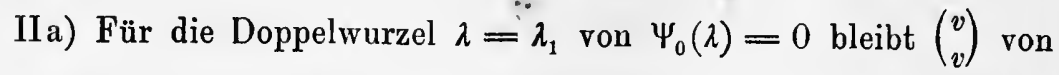
Null verschieden, es liegt daher der Fall. IIa) in $\S 14$ vor, die Kegelschnitte des Büschels berühren sich in einem und demselben Punkt, und die gemeinsame Tangente ist eine Gerade des Paares $\lambda_{3} g-f=0$. Man hat nach (7):

$$
\frac{\Psi_{1}(\lambda)}{\Psi_{0}(\lambda)}=\frac{U^{(1)} U^{(1)}}{\left(\lambda-\lambda_{1}\right)^{2}}+\frac{2 U^{(1)} U^{(2)}}{\left(\lambda-\lambda_{1}\right)}+\frac{U_{3}^{2}}{\lambda-\lambda_{3}} .
$$


IIIa) Für die dreifache Wurzel $\lambda=\lambda_{1}$ von $\Psi_{0}(\lambda)=0$ bleibt $\left(\begin{array}{l}v \\ v\end{array}\right)$ von Null verschieden, es liegt daher der Fall IIIa) in $\S 14$ vor, die Kegelschnitte des Büschels osculiren sich und schneiden sich noch in einem vierten Punkte. Man hat nach (8):

$$
\frac{\Psi_{1}(\lambda)}{\Psi_{0}(\lambda)}=\frac{U^{(1)} U^{(1)}}{\left(\lambda-\lambda_{1}\right)^{3}}+2 \frac{U^{(1)} U^{(2)}}{\left(\lambda-\lambda_{1}\right)^{2}}+\frac{U^{(2)} U^{(2)}+2 U^{(1)} U^{(3)}}{\lambda-\lambda_{1}} .
$$

IIb) Der Factor $\lambda-\lambda_{1}$ ist in $\Psi_{0}(\lambda)$ zweifach enthalten, in $\left(\begin{array}{l}v \\ v\end{array}\right)$ einfach, und zwar bei willkürlichen Werthen der Grössen $v_{i}$; es ist daher

$$
F-2 \lambda_{1} H+\lambda_{1}{ }^{2} G \equiv\left(\begin{array}{l}
v \\
v
\end{array}\right)_{\lambda_{2=\lambda_{1}}} \equiv 0,
$$

mithin liegt der Fall IIb) in $\S 14$ vor, zwischen den Kegelschnitten des Büschels findet doppelte Berührung statt. Man hat nach (9):

$$
\frac{\Psi_{1}(\lambda)}{\Psi_{0}(\lambda)}=\frac{U^{(1)} U^{(1)}}{\lambda-\lambda_{1}}+\frac{U^{(2)} C^{(2)}}{\lambda-\lambda_{1}}+\frac{U_{3}{ }^{2}}{\lambda-\lambda_{3}} .
$$

III b) Der Factor $\lambda-\lambda_{1}$ ist in $\Psi_{0}(\lambda)$ dreifach enthalten, in $\left(\begin{array}{l}v \\ v\end{array}\right)$ einfach, und zwar bei willkürlichen Werthen der Grössen $v_{i}$; es ist daher

$$
F-2 \lambda_{1} H+\lambda_{1}{ }^{2} G \equiv\left(\begin{array}{l}
v \\
v
\end{array}\right)_{\lambda=\lambda_{1}} \equiv 0,
$$

mithin liegt der Fall IIIb) in $\S 14$ vor, die Curven des Büschels haben vier zusammenfallende Punkte gemeinsam. Man hat nach (12):

$$
\frac{\Psi_{1}(\lambda)}{\Psi_{0}(\lambda)}=\frac{U^{(1)} U^{(1)}}{\left(\lambda-\lambda_{1}\right)^{2}}+2 \frac{U^{(1)} U^{(2)}}{\lambda-\lambda_{1}}+\frac{U^{(3)} U^{(3)}}{\lambda-\lambda_{1}} .
$$

Wir haben nun noch die den eben angegebenen speciellen Fällen entsprechenden Darstellungen der Kegelschnitte $f=0$ und $g=0$ in Punktcoordinaten zu untersuchen.

Zuvor wollen wir noch eine Formel ableiten, die im Folgenden mehrfach zur Anwendung kommt und sich auf die Determinante $\left(\begin{array}{l}g \\ g\end{array}\right)_{c_{i k}}$ bezieht, welche aus $\left(\begin{array}{l}u \\ u\end{array}\right)_{c_{i k}}$ dadurch hervorgeht, dass man $u_{i}$ ersetzt durch $g_{i}=\frac{1}{2} g^{\prime}\left(x_{i}\right)$. Multiplicirt man nämlich die drei ersten Verticalreihen von $\left(\begin{array}{l}g \\ g\end{array}\right)$ resp. mit $\frac{x_{1}}{\lambda}, \frac{x_{2}}{\lambda}, \frac{x_{3}}{\lambda}$ und subtrahirt sie von der vierten, und verfährt man hierauf in gleicher Weise mit den Horizontalreihen, so ergibt sich die Identität

$$
-\lambda^{2}\left(\begin{array}{l}
g \\
g
\end{array}\right)=(f+\lambda g) \cdot \Psi_{0}(\lambda)-\left(\begin{array}{l}
f \\
f
\end{array}\right) .
$$


Betrachten wir nun den Fall (IIa) (einfache Berührung).

Analog dem Verfahren, welches beim allgemeinen Falle benutzt wurde ${ }^{1}$ ), ersetzen wir in (IIa) die Veränderlichen $u_{i}$ durch die Differentialquotienten $\frac{1}{2} g^{\prime}\left(x_{i}\right)=g_{i}(i=1,2,3)$ und erhalten, wenn die vermöge dieser Substitution aus $U^{(1)}, U^{(2)}, U_{3}$ hervorgehenden Ausdrücke resp. durch $X_{2}, X_{1}, X_{3}$ bezeichnet werden:

$$
-\left(\begin{array}{l}
g \\
g
\end{array}\right): \Psi_{0}(\lambda)=\frac{X_{2}{ }^{2}}{\left(\lambda-\lambda_{1}\right)^{2}}+2 \frac{X_{2} X_{1}}{\lambda-\lambda_{1}}+\frac{X_{3}{ }^{2}}{\lambda-\lambda_{3}} .
$$

Der Grund, weshalb die aus $U^{(1)}$ und $U^{(2)}$ hervorgehenden Ausdrücke gerade mit $X_{2}$ und $X_{1}$ bezeichnet wurden, liegt darin, dass die Relation

$$
u_{1} x_{1}+u_{2} x_{2}+u_{3} x_{3}=U^{(1)} X_{1}+U^{(2)} X_{2}+U_{3} X_{3}
$$

erfüllt werden sollte. Dass diese nun erfüllt ist, zeigt man ähnlich wie bei (34) in $\S 14$ auf folgende Weise: Man ersetzt in (IIa) $u_{i}$ durch $u_{i}+\mu g_{i}$ und vergleicht beiderseits die Coefficienten von $\mu^{1}$, wodurch sich ergibt

$-2\left(\begin{array}{l}g \\ u\end{array}\right)_{c_{i k}}: B\left(\lambda-\lambda_{1}\right)^{2}\left(\lambda-\lambda_{3}\right)=\frac{2 U^{(1)} X_{2}}{\left(\lambda-\lambda_{1}\right)^{2}}+\frac{2\left(U^{(2)} X_{2}+U^{(1)} X_{1}\right)}{\lambda-\lambda_{1}}+\frac{2 U_{3}}{\lambda-X_{3}} ;$ hierbei ist für $\Psi_{0}(\lambda)$ der Werth $B\left(\lambda-\lambda_{1}\right)^{2}\left(\lambda-\lambda_{3}\right)$ substituirt, den dieser Ausdruck im gegenwärtigen Falle annimmt. Für $\lambda=\infty$ erhält man alsdann die Relation

$$
-\left(\begin{array}{l}
g \\
u
\end{array}\right)_{b_{i k}}: B=U^{(1)} X_{1}+U^{(3)} X_{2}+U_{3} X_{3}
$$

deren linke Seite mit $u_{1} x_{1}+u_{2} x_{2}+u_{3} x_{3}$ identisch ist, wie leicht einzusehen; hiermit ist die Beziehung (15) erwiesen.

Entwickelt man auf der rechten Seite von (14) vermöge der Formeln

$$
\frac{1}{\left(\lambda-\lambda_{i}\right)^{2}}=\frac{1}{\lambda^{2}}+\frac{2 \lambda_{i}}{\lambda^{3}}+\frac{3 \lambda_{i}{ }^{2}}{\lambda^{4}}+\cdots \text { und } \frac{1}{\lambda-\lambda_{i}}=\frac{1}{\lambda}+\frac{\lambda_{i}}{\lambda^{2}}+\frac{\lambda_{i}{ }^{2}}{\lambda^{3}}+\cdots
$$

nach absteigenden Potenzen von $\lambda$, so ergibt sich

$$
\begin{aligned}
-\lambda^{2}\left(\begin{array}{l}
g \\
g
\end{array}\right)=\Psi_{0}(\lambda)\left\{X_{2}{ }^{2}\left(1+\frac{2 \lambda_{1}}{\lambda}+\cdots\right)+\right. & 2 X_{2} X_{1}\left(\lambda+\lambda_{1}+\frac{\lambda_{1}{ }^{2}}{\lambda}+\cdots\right) \\
& \left.+X_{3}{ }^{2}\left(\lambda+\lambda_{3}+\frac{\lambda_{3}{ }^{2}}{\lambda}+\cdots\right)\right\},
\end{aligned}
$$

und hieraus durch Vergleichung der Coefficienten von $\lambda^{4}$ und $\lambda^{3}$ mit Rücksicht auf (13):

$$
\left\{\begin{array}{l}
g=2 X_{2} X_{1}+X_{3}^{2} \\
f=X_{2}^{2}+2 \lambda_{1} X_{1} X_{2}+\lambda_{3} X_{3}^{2}
\end{array}\right.
$$

1) Vgl. S. 139. 
Die Gleichungen dieser beiden Kegelschnitte in Liniencoordinaten erhält man aus (IIa) durch die Substitution $\lambda=0$, resp. $\lambda=\infty$ in der Form

$$
\left\{\begin{array}{l}
\frac{G}{B}=2 U^{(1)} U^{(2)}+U_{3}{ }^{2}, \\
F=B\left\{-\lambda_{3} U^{(1)} U^{(1)}+2 \lambda_{1} \lambda_{3} U^{(1)} U^{(2)}+\lambda_{1}{ }^{2} U_{3}{ }^{2}\right\},
\end{array}\right.
$$

wobei sich auf Grund der Relation $B \lambda_{1}{ }^{2} \lambda_{3}=A$ die zweite Gleichung auch ersetzen lässt durch

$$
\frac{F}{A}=-\frac{U^{(1)} U^{(1)}}{\lambda_{1}{ }^{2}}+\frac{2 U^{(1)} U^{(2)}}{\lambda_{1}}+\frac{U_{3}^{2}}{\lambda_{3}} .
$$

Ferner erhält man

$$
\left\{\begin{array}{l}
\lambda_{1} g-f=\left(\lambda_{1}-\lambda_{3}\right) X_{3}{ }^{2}-X_{2}{ }^{2} \\
\lambda_{3} g-f=2\left(\lambda_{3}-\lambda_{1}\right) X_{1} X_{2}-X_{2}{ }^{2}
\end{array}\right.
$$

woraus hervorgeht, dass $X_{2}=0, X_{3}=0$ die Coordinaten des gemeinsamen Berührungspunktes aller Kegelschnitte des Büchels sind, während für die Coordinaten der zwei übrigen Grundpunkte des Büschels die Proportionenreihe Geltung hat:

$$
X_{1}: X_{2}: X_{3}=1: 2\left(\lambda_{3}-\lambda_{1}\right): \pm 2 \sqrt{\lambda_{1}-\lambda_{3}} .
$$

Aus (17) und (18) ersieht man auch, dass $X_{2}=0$ die Gleichunng der Tangente des gemeinschaftlichen Berührungspunktes ist, sowie dass die Gerade $X_{3}=0$ harmonisch liegt zu $X_{2}=0$ und zu dem Geradenpaare $\lambda_{1} g-f=0$, welch letzteres die Verbindungslinien des Berührungspunktes mit den beiden anderen Grundpunkten des Büschels darstellt. Die Gerade $X_{3}=0$ schneidet

Fig. 6 .

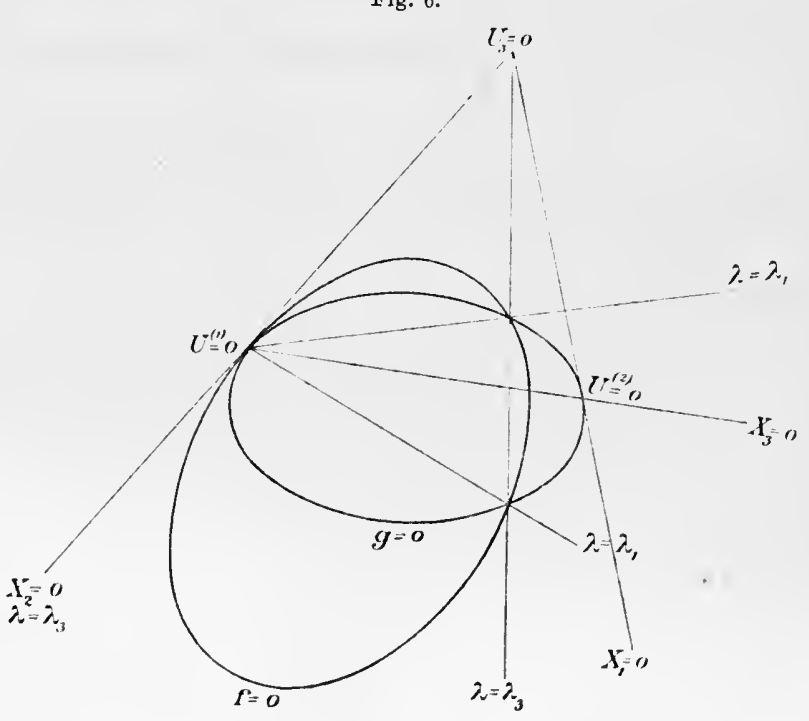

den Kegelschnitt $g=0$ in dessen zwei Berührungspunkten mit den Seiten $X_{1}=0$ und $X_{2}=0$ des Coordinatendreiecks (vgl. Fig. 6). 
Im Falle (IIIa) (Osculation) ersetzt man die durch die Substitution $u_{i}=\frac{1}{2} g^{\prime}\left(x_{i}\right)$ aus $U^{(1)}, U^{(2)}, U^{(3)}$ hervorgehenden Ausdrücke resp. durch $X_{3}, X_{2}, X_{1}$ und erhält hierdurch

$$
-\left(\begin{array}{l}
g \\
g
\end{array}\right): \Psi_{0}(\lambda)=\frac{X_{3}{ }^{2}}{\left(\lambda-\lambda_{1}\right)^{3}}+\frac{2 X_{2} X_{3}}{\left(\lambda-\lambda_{1}\right)^{2}}+\frac{X_{2}{ }^{2}+2 X_{1} X_{3}}{\lambda-\lambda_{1}} .
$$

Auch hier liegt der Grund dieser Bezeichnungsweise darin, dass die Relation

$$
u_{1} x_{1}+u_{2} x_{2}+u_{3} x_{3}=U^{(1)} X_{1}+U^{(2)} X_{2}+U^{(3)} X_{3}
$$

erfüllt werden soll, deren Bestehen in gleicher Weise wie im zuvor betrachteten Falle nachgewiesen wird.

Man könnte auch jetzt wieder die rechte Seite von (21) nach absteigenden Potenzen von $\lambda$ entwickeln und würde nach Multiplication mit $\lambda^{2}$ durch Vergleichen der Coefficienten von $\lambda^{4}$ und $\lambda^{3}$ mit Rücksicht auf (13) die Gleichungen der Kegelschnitte $f$ und $g$, bezogen auf das Dreieck $X_{1}, X_{2}, X_{3}$, erhalten. Eine andere Methode hierfür, die auch in allen Fällen zum Ziele führt, ist folgende. Aus (13) und (21) ergibt sich für den jetzt betrachteten Fall

$$
\begin{aligned}
-\lambda^{2}\left(\begin{array}{l}
g \\
g
\end{array}\right) & =(f+\lambda g) B\left(\lambda-\lambda_{1}\right)^{3}-\left(\begin{array}{l}
f \\
f
\end{array}\right) \\
& =\lambda^{2} B\left\{X_{3}{ }^{2}+2 X_{2} X_{3}\left(\lambda-\lambda_{1}\right)+\left(X_{2}{ }^{2}+2 X_{1} X_{3}\right)\left(\lambda-\lambda_{1}\right)^{2}\right\},
\end{aligned}
$$

woraus man durch Vergleichen der Coefficienten von $\lambda^{4}$ und $\lambda^{3}$ und nach Division durch $B$ erhält:

$$
\left\{\begin{aligned}
g & =X_{2}^{2}+2 X_{1} X_{3} \\
f-3 \lambda_{1} g & =2 X_{2} X_{3}-2 \lambda_{1} X_{2}^{2}-4 \lambda_{1} X_{1} X_{3}, \text { also } \\
f & =\lambda_{1} X_{2}^{2}+2 \lambda_{1} X_{1} X_{3}+2 X_{2} X_{3}
\end{aligned}\right.
$$

Ferner ist

$$
\lambda_{1} g-f=-2 X_{2} X_{3}
$$

Die Gleichungen in Liniencoordinaten werden:

$$
\left\{\begin{array}{l}
\frac{G}{B}=U^{(2)} U^{(2)}+2 U^{(1)} U^{(3)} \\
\frac{F}{A}=\frac{U^{(1)} U^{(1)}}{\lambda_{1}{ }^{3}}-\frac{2 U^{(1)} U^{(2)}}{\lambda_{1}{ }^{2}}+\frac{U^{(2)} U^{(2)}+2 U^{(1)} U^{(3)}}{\lambda_{1}} \\
\text { oder auch } \\
\frac{F}{B}=U^{(1)} U^{(1)}-2 \lambda_{1} U^{(1)} U^{(2)}+\lambda_{1}{ }^{2}\left(U^{(2)} U^{(2)}+2 U^{(1)} U^{(3)}\right)
\end{array}\right.
$$


Hier ist $U^{(1)}=0$ die Gleichung des allen Kegelschnitten des Büschels gemeinsamen Osculationspunktes, $X_{3}=0$ die gemeinsame Tangente; $U^{(3)}=0$ ist die Gleichung des vierten Schnittpunktes, $X_{1}=0$ stellt die in demselben an $g=0$ gezogene Tangente dar; $X_{2}=0$ ist die Gleichung der gemeinsamen Berührungssehne (vgl. Fig. 7).

Nachdem die Fälle (IIa) und (IIIa) im Vorhergehenden ausführlich behandelt sind, geben wir bei (IIb) und (IIIb) nur die Resultate an.

Sind im Falle (IIb) (doppelte Berührung) $X_{1}, X_{2}, X_{3}$ die Ausdrücke, welche aus $U^{(1)}$, $U^{(2)}, U_{3}$ vermöge der Substi-

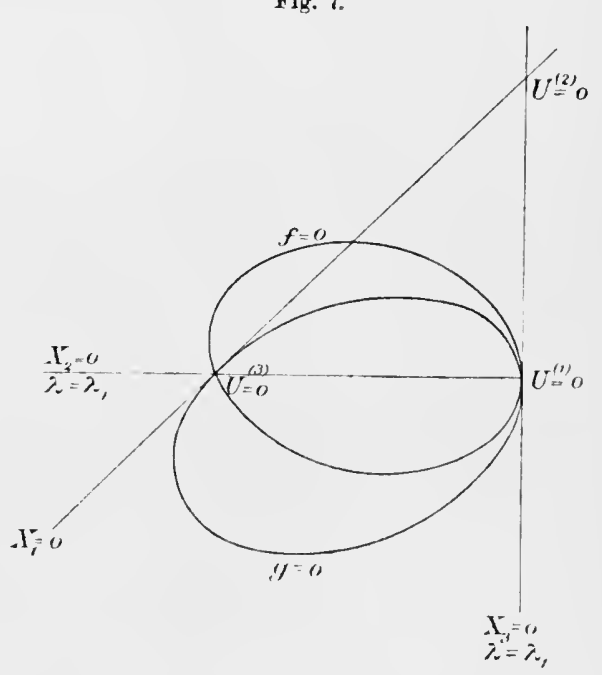
tution $u_{i}{ }^{\prime}=\frac{1}{2} g^{\prime}\left(x_{i}\right)$ hervorgehen, so erhält man die Gleichnugen der Kegelschnitte $f=0$ und $g=0$ in der Gestalt

ferner wird

$$
\left\{\begin{array}{l}
f=\lambda_{1}\left(X_{1}^{2}+X_{2}^{2}\right)+\lambda_{3} X_{3}^{2} \\
g=X_{1}^{2}+X_{2}^{2}+X_{3}^{2}
\end{array}\right.
$$

$$
\left\{\begin{array}{l}
\lambda_{1} g-f=\left(\lambda_{1}-\lambda_{3}\right) X_{3}^{2} \\
\lambda_{3} g-f=\left(\lambda_{3}-\lambda_{1}\right)\left(X_{1}^{2}+X_{2}^{2}\right)
\end{array}\right.
$$

und

$$
\left\{\begin{array}{l}
\frac{F}{A}=\frac{U^{(1)} U^{(1)}+U^{(2)} U^{(2)}}{\lambda_{1}}+\frac{U_{3}{ }^{2}}{\lambda_{3}}, \\
\frac{G}{B}=U^{(1)} U^{(1)}+U^{(2)} U^{(2)}+U_{3}{ }^{2} .
\end{array}\right.
$$

Hier stellt $X_{3}=0$ die Verbindungslinie der zwei gemeinsamen Berührungspunkte dar, während die Coordinaten dieser Punkte zu bestimmen sind aus $X_{1}: X_{2}= \pm \sqrt{-1}, X_{3}=0$. Die Auflösung dieser Gleichungen erfolgt wieder am einfachsten vermittelst der Identität

so dass

$$
u_{1} x_{1}+u_{2} x_{2}+u_{3} x_{3}=U^{(1)} X_{1}+U^{(2)} X_{2}+U_{3} X_{3},
$$

$$
U^{(1)}+\sqrt{-1} U^{(2)}=0, \quad U^{(1)}-\sqrt{-1} U^{(2)}=0
$$

die analytischen Darstellungen der Berührungspunkte werden. 
Im Falle (IIIb) (vier zusammenfallende Punkte gemeinsam) ersetzt man die durch die Substitution $u_{i}=\frac{1}{2} g^{\prime}\left(x_{i}\right)$ aus $U^{(1)}, U^{(2)}, U^{(3)}$ hervorgehenden Ausdrücke resp. durch $X_{2}, X_{1}, X_{3}$. Alsdann findet man

$$
\begin{gathered}
\left\{\begin{array}{l}
f=X_{2}{ }^{2}+2 \lambda_{1} X_{1} X_{2}+\lambda_{1} X_{3}{ }^{2} \\
g=2 X_{1} X_{2}+X_{3}{ }^{2},
\end{array}\right. \\
\lambda_{1} g-f=-X_{2}{ }^{2}, \\
\left\{\begin{array}{l}
\frac{F}{A}=-\frac{U^{(1)} U^{(1)}}{\lambda_{1}{ }^{2}}+\frac{2 U^{(1)} U^{(2)}+U^{(3)} U^{(3)}}{\lambda_{1}} \\
\frac{G}{B}=2 U^{(1)} U^{(2)}+U^{(3)} U^{(3)} \\
\frac{F}{B}=-\lambda_{1} U^{(1)} U^{(1)}+\lambda_{1}{ }^{2}\left(2 U^{(1)} U^{(2)}+U^{(3)} U^{(3)}\right) .
\end{array}\right.
\end{gathered}
$$

Hier ist $X_{2}=0$ die Gleichung der Tangente des gemeinsamen Berührungspunktes $U^{(1)}=0, X_{3}=0$ irgend eine durch diesen Punkt gehende Gerade, $X_{1}=0$ die Tangente des Kegelschnitts $y=0$ in dessen zweitem Schnittpunkte mit $X_{3}=0$.

\section{$\S 17$.}

Geometrische Deutung einiger algebraischer Formen.

In den vorhergehenden Paragraphen dieses Abschnitts wurde hauptsächlich untersucht, welche Eigenthümlichkeiten in der Lage der beiden Kegelschnitte $f=0, g=0$ gegen einander auftreten, wenn die Gleichung $\Psi_{0}(\lambda)=0$ mehrfache Wurzeln besitzt. Die folgenden Betrachtungen sollen zeigen, welche geometrische Bedeutung es hat, wenn die Coefficienten $\Theta$ und $H$ dieser Gleichung und einige andere damit zusammenhängende algebraische Formen verschwinden ${ }^{1}$ ).

Wir gehen aus von den Gleichungen in Liniencoordinaten

$$
\left\{\begin{aligned}
F \equiv A_{11} u_{1}{ }^{2}+2 A_{12} u_{1} u_{2}+A_{22} u_{2}{ }^{2} & +2 A_{13} u_{1} u_{3} \\
& +2 A_{23} u_{2} u_{3}+A_{33} u_{3}{ }^{2}=0 \\
G \equiv B_{11} u_{1}^{2}+2 B_{12} u_{1} u_{2}+B_{22} u_{2}{ }^{2} & +2 B_{13} u_{1} u_{3} \\
& +2 B_{23} u_{2} u_{3}+B_{33} u_{3}{ }^{2}=0
\end{aligned}\right.
$$

für die Kegelschnitte $f(x, x)=0$ und $g(x, x)=0$. Es ist alsdann die Determinante $\mathrm{A}$ von $F=0$ gleich $A^{2}$, wenn $A$ die aus den $a_{i k}$

1) Man vergleiche auch die von Herrn Gundelfinger gegebene Darstellung in $\S 3$ des dritten Supplementes zur dritten Auflage von Hesse's „Vorlesungen über analytische Geometrie des Raumes", 1876. 
gebildete Determinante bezeichnet, und jede Unterdeterminante $A_{i k}$ des Elementes $A_{i k}$ in A ist gleich $A a_{i k}$. Analoges gilt für den Kegelschnitt $G=0$.' Setzt man

(2) -

$$
\begin{array}{cccc}
-\mu A_{11}+\nu B_{11} & -\mu A_{12}+\nu B_{12} & -\mu A_{13}+\nu B_{13} & x_{1} \\
-\mu A_{21}+\nu B_{21} & -\mu A_{22}+\nu B_{22} & -\mu A_{23}+\nu B_{23} & x_{2} \\
-\mu A_{31}+\nu B_{31} & -\mu A_{32}+\nu B_{32} & -\mu A_{33}+\nu B_{33} & x_{3} \\
x_{1} & x_{2} & x_{3} & 0
\end{array}
$$

und vergleicht man diese Determinante mit dem analogen Ausdruck in (4), resp. (9) in $\S 13$, so erkennt man unter Rücksicht auf die eben erwähnten Determinantenrelationen, dass

$$
\gamma_{0}=A f, \quad \gamma_{2}=B y,
$$

dass ferner $\gamma_{1}$ gegeben ist durch

wo

$$
\begin{aligned}
2 \gamma_{1}=(\mathfrak{a}, \mathfrak{b})_{11} x_{1}{ }^{2} & +2(\mathfrak{a}, \mathfrak{b})_{12} x_{1} x_{2}+(\mathfrak{a}, \mathfrak{b})_{22} x_{2}{ }^{2}+2(\mathfrak{a}, \mathfrak{b})_{13} x_{1} x_{3} \\
& +2(\mathfrak{a}, \mathfrak{b})_{23} x_{2} x_{3}+(\mathfrak{a}, \mathfrak{b})_{33} x_{3}{ }^{2}
\end{aligned}
$$

$$
\begin{aligned}
& (\mathfrak{a}, \mathfrak{b})_{11}=A_{22} B_{33}+A_{33} B_{22}-2 A_{23} B_{2 ;} ; \\
& (\mathfrak{a}, \mathfrak{b})_{12}=A_{31} B_{32}+A_{32} B_{31}-A_{33} B_{12}-A_{12} B_{33},
\end{aligned}
$$

während die übrigen $(\mathfrak{a}, \mathfrak{b})_{i k}$ Ausdrücke bedeuten, die aus den hier angegebenen durch cyklische Vertauschung der Indices hervorgehen.

Der Kegelschnitt $\gamma_{1}=0$ entspricht daher dualistisch $H=0$ in $\S 13$ und $\S 15$ und ist nach (23) in $\S 15$ der geometrische Ort aller Punkte, von welchen an $F=0$ und $G=0$ zwei Paare zu einander harmonischer Tangenten gelegt werden können.

Wir wollen nun der Determinante (2) eine etwas andere Gestalt geben. $\mathrm{Zu}$ dem $\mathrm{Z}$ weck stellen wir $A$ und $B$ als Determinanten vierten Grades $\sum \pm\left(a_{11} a_{22} a_{33} a_{44}\right) \cdot \sum \pm\left(b_{11} b_{22} b_{33} b_{44}\right)$ dar, indem wir

$$
a_{44}=b_{44}=1 \text { und } a_{\gamma \delta}=b_{\gamma \delta}=0
$$

setzen, sobald einer der beiden Indices $\gamma$ und $\delta(\gamma \gtrless \delta)$ gleich vier wird. Alsdann erhält man durch Multiplication der Gleichung (2) mit der Identität

$$
\sum \pm\left(a_{11} a_{22} a_{33} a_{44}\right) \cdot \sum \pm\left(b_{11} b_{22} b_{33} b_{44}\right)=A B
$$

nach zweimaliger Anwendung des Multiplicationstheorems der Determinanten das Ergebniss, dass der Ausdruck 


$$
\begin{aligned}
& -\left|\begin{array}{cccc}
x a_{11}+\lambda b_{11} & x a_{12}+\lambda b_{12} & x a_{13}+\lambda b_{13} & f_{1} \\
x a_{21}+\lambda b_{21} & x a_{22}+\lambda b_{22} & x a_{23}+\lambda b_{23} & f_{2} \\
x a_{31}+\lambda b_{31} & x a_{32}+\lambda b_{32} & x a_{33}+\lambda b_{33} & f_{3} \\
g_{1} & g_{2} & g_{3} & 0
\end{array}\right| \\
& =x^{2} A g+2 x \lambda \chi+\lambda^{2} B f
\end{aligned}
$$

durch die Substitution $x=\nu B, \lambda=\mu A$ übergeht in

$$
\left(\mu^{2} \gamma_{0}+2 \mu \nu \gamma_{1}+\nu^{2} \gamma_{2}\right) A B
$$

und dass daher das System von Gleichungen besteht

$$
\gamma_{0}=A f, \quad \gamma_{1}=\chi, \quad \gamma_{2}=B g
$$

(vgl. übrigens (3) und (4)).

Den Functionen $\gamma_{1}$, sowie $A g$ und $B f$ lässt sich eine Gestalt geben, aus welcher man ohne Mühe die besonderen geometrischen Beziehungen ableiten kann, die zwischen $f=0$ und $g=0$ eintreten, weun einzelne Coefficienten der Potenzen von $\lambda$ in $\sum \pm\left(c_{11} c_{22} c_{33}\right)$ verschwinden. Wir setzen, um hierauf näher einzugehen, in der Determinante (5) den variabelen Parameter $x=-1$ und addiren die resp. mit $x_{1}, x_{2}, x_{3}$ multiplicirten drei ersten Verticalreihen sämmtlich zur letzten Verticalreihe, so dass sich mit Anwendung der bereits früher definirten Bezeichnung ergibt:

$$
\begin{aligned}
A g-2 \lambda \chi+\lambda^{2} B f & =-\lambda\left(\begin{array}{l}
g \\
g
\end{array}\right)-g \cdot \sum \pm\left(c_{11} c_{22} c_{33}\right) \\
& =-\lambda\left(\begin{array}{l}
g \\
g
\end{array}\right)-g\left\{\lambda^{3} B-3 \lambda^{2} \Theta+3 \lambda H-A\right\} .
\end{aligned}
$$

Nennt man $F\left(g_{1}, g_{2}, g_{3}\right), H\left(g_{1}, g_{2}, g_{3}\right)$ etc. die Ausdrücke, welche aus $F, H$ etc. vermöge der Substitution $u_{i}=\frac{1}{2} g^{\prime}\left(x_{i}\right)=g_{i}$ hervorgehen, so entsteht durch Vergleichung entsprechender Glieder auf beiden Seiten der letzten Identität:

$$
\begin{aligned}
& B f=-2 H\left(g_{1}, g_{2}, g_{3}\right)+3 \Theta g, \\
& 2 \chi=-F\left(g_{1}, g_{2}, g_{3}\right)+3 \mathrm{H} g,
\end{aligned}
$$

und hieraus durch Vertauschung der $a_{i k}$ mit den $b_{i k}$ :

$$
\begin{aligned}
& A g=-2 H\left(f_{1}, f_{2}, f_{3}\right)+3 H f, \\
& 2 \chi=-G\left(f_{1}, f_{2}, f_{3}\right)+3 \Theta f .
\end{aligned}
$$

Es sind aber $g_{1}, g_{2}, g_{3}$ die Coordinaten der Polare eines Punktes $x$ in Bezug auf den Kegelschnitt $g=0$, und da $F(u, u)=0$ die Gleichung von $f=0$ in Liniencoordinaten bedeutet, so stellt die 
Deutung der einfachsten simultanen Invarianten zweier Kegelschnitte. 161

Gleichung $F\left(g_{1}, g_{2}, g_{3}\right)=0$, welche die $x$ als Variabeln enthält, den geometrischen Ort derjenigen Punkte dar, deren Polaren in Bezug auf $g=0$ zugleich Tangenten von $F=0$ sind. Analoge Bedeutung hat $G\left(f_{1}, f_{2}, f_{3}\right)=0$.

Ebenso folgt, dass $H\left(g_{1}, g_{2}, g_{3}\right)=0$, resp. $H\left(f_{1}, f_{2}, f_{3}\right)=0$ der geometrische Ort derjenigen Punkte ist, deren Polaren in Bezug auf $g=0$, resp. $f=0$ zugleich Tangenten von $H$ sind, d. h. deren Polaren in Bezug auf $g=0$, resp. $f=0$ die beiden Kegelschnitte $f$ und $g$ in harmonischen Punkteparen treffen ${ }^{1}$ ).

Unter der vorläufigen Annahme, dass die Determinanten $A$ und $B$ nicht verschwinden, wollen wir nun die geometrische Bedeutung von $\Theta=0$ untersuchen.

Aus $(8 \mathrm{~b})$ folgt, dass für $\Theta=0$ die Kegelschnitte $G\left(f_{1}, f_{2}, f_{3}\right)=0$ und $\chi=0$ identisch werden, d. h. von jedem Punkte $x$, dessen Polare $m$ in Bezug auf $f=0$ die Curve $g=0$ berührt, lassen siclı alsdann gleichzeitig an $f$ und $g$ zwei Paare zu einander harmonischer Tangenten legen ${ }^{2}$. Ein jedes solches Tangentenpaar, das an $g=0$ gelegt ist, bildet nun zusammen mit der Polare $m$ ein dem Kegelschnitt $g=0$ umschriebenes Poldreiseit von $f=0$. Man hat also den Satz: (9) Wenn $\Theta$ verschwindet, können dem Kegelschuitt $g=0$ unendlich viele Poldreiseite von $f=0$ umschrieben werden. Die Ecken aller dieser Poldreiseite liegen auf dem Kegelschnitt $G\left(f_{1}, f_{2}, f_{3}\right)=0$.

(10) Und umgekehrt: Ist die Curve $g=0$ irgend einem bebestimmten Poldreiseit von $f=0$ eingesclirieben, so wird $\Theta=0$.

Die Coordinaten $x_{i}$ einer beliebigen Ecke eines solchen Poldreiseits erfüllen nämlich die Gleichungen $\chi=0$ und $G\left(f_{1}, f_{2}, f_{3}\right)=0$, somit wegen $(8 b)$ auch $\Theta f=0$; da aber die Polare des Punktes $x$ in Bezug auf $f=0$ nicht durch diesen Puukt hindurchgehen soll, können seine Coordinaten nicht $f=0$ erfüllen, sondern muss $\Theta$ verschwinden.

Eine zweite geometrische Bedeutung von $\Theta=0$ folgt aus (7a). Diese Gleichung zeigt, dass für $\Theta=0$ jeder Punkt $p$ von $f=0$ gleichzeitig dem Kegelschnitt $H\left(g_{1}, g_{2}, g_{3}\right)=0$ angehört, d. h. dass $p$ ein Punkt ist, dessen Polare in Bezug auf $g=0$ die beiden Kegelschnitte $f$ und $g$ in zwei harmonischen Punktepaaren $p_{1}, p_{2}$ und $\pi_{1}, \pi_{2}$ schneidet.

1) Zufolge der in $\S 15$ abgeleiteten Bedeutung des Kegelschnitts $I=0$.

2) Mit Berücksichtigung der geometrischen Bedeutung der Kegelschnitte $\gamma_{1} \equiv \chi=0$ und $G\left(f_{1}, f_{2}, f_{3}\right)=0$.

Gundelfinger, Vorlesungen. 
Offenbar bilden die drei auf dem Kegelschnitt $f$ gelegenen Punkte $p_{1}$, $p_{2}, p_{3}$ ein Poldreieck von $g=0$, denn die Polare des Punktes $p_{1}$ in Bezug auf $g$ muss sowohl durch den Pol $p$ der Geraden $p_{1} p_{2}$ hindurchgehen, als auch durch den vierten harmonischen Punkt zu $p_{1}$, $\pi_{1}, \pi_{2}$, d. i. $p_{2}$, und analoges gilt von der Polare des Punktes $p_{2}$, sie fällt mit $p p_{1}$ zusammen. Man kann daher den Satz aussprechen:

(11) Wenn $\Theta$ verschwindet, geht der Kegelschnitt $f(x, x)=0$ durch unendlich viele Poldreiecke von $G(u, u)=0$. Die Seiten dieser Poldreiecke berühren sämmtlich den Kegelschnitt $H=0$.

Umgekehrt kann man auch zeigen:

(12) Wenn von zwei Kegelschnitten $f=0$ und $G=0$ der eine $(f=0)$ durch irgend ein Poldreieck des anderen geht, verschwindet $\Theta$.

Die Coordinaten $x_{i}$ irgend einer Ecke $p$ des Dreiecks $p p_{1} p_{2}$ genügen nämlich nach der Voraussetzung den zwei Relationen

$$
f=0, H\left(g_{1}, g_{2}, g_{3}\right)=0,
$$

und somit wegen (7a) der Gleichung $\Theta g=0$. Da die Gerade $p_{1} p_{2}$ nicht durch $p$ gehen soll, kann der Punkt $p$ nicht auf $g=0$ liegen, sondern muss $\Theta$ verschwinden.

Wir wollen unter Benutzung einer von Herrn Rosanes eingeführten Bezeichnung ${ }^{1}$ ) sagen, dass im Falle $\Theta=0$ die Kegelschnitte $f=0$ und $G=0$ conjugirt liegen ${ }^{2}$ ). Diese Bezeichnungsweise ist äusserst zweckmässig, zumal die Relation $\Theta=0$ in dem besonderen Falle, dass die $B_{i k}$ die Coefficienten einer ein Paar von Punkten $y, z$ darstellenden Curve zweiter Classe sind, übergeht in die Relation $f(y, z)=0$, welche nach $\S 4$ aussagt, dass $y$ und $z$ conjugirte Pole sind von $f=0$.

Hält man die Theoreme (9) und (10) zusammen, so folgt:

1) Vgl. Rosanes: „Ueber Systeme von Kegelschnitten“, Math. Annalen, Bd. 6, S. 268, 1872.

2) Allgemeiner nennt man eine Curve zweiter Ordnung

$$
f(x, x) \equiv \sum_{1}^{3} \sum_{1}^{3} a_{i k} x_{i} x_{k}=0
$$

conjugirt zu einer (eventuell ausartenden) Curve zweiter Classe

$$
\varphi(u, u) \equiv \sum_{1}^{3} \sum_{1}^{3} \alpha_{i k} u_{i} u_{k}=0,
$$

wenn $a_{11} \alpha_{11}+a_{22} \alpha_{22}+a_{33} \alpha_{33}+2 a_{23} \alpha_{23}+2 a_{31} \alpha_{31}+2 a_{12} \alpha_{12}=0$. 
Deutung der einfachsten simultanen Invarianten zweier Kegelschnitte. 163

(13) Wenn ein Kegelschnitt die Seiten irgend eines Poldreiseits einer Curve.zweiter Ordnung berührt, so berührt er stets auch noch unendlich-viele Poldreiseite der Curve.

Analog liefern (11) und (12) den Satz:

(14) Wenn ein Kegelschnitt durch irgend ein Poldreieck einer Curve zweiter Ordnung geht, so geht er stets auch noch durch unendlich viele Poldreiecke der Curve.

Ausserdem folgt aus den Sätzen (9)-(12):

(15) Wenn die Curve $g=0$ Poldreiseiten von $f=0$ eingeschrieben ist, so lässt sich gleichzeitig die Curve $f=0$ Poldreiecken von $g=0$ umschreiben, und umgekehrt.

Vertauscht man in den Theoremen (9)-(12) die Cofficienten $a_{i k}$ mit den $b_{i k}$, also $f$ mit $g, F$ mit $G$ (oder benutzt man die Relationen (7b) und (8a)), so ergibt sich die geometrische Bedeutung der Beziehung $\mathrm{H}=0$. Man erhält alsdann die Sätze (16)-(19):

(16) Wenn $\mathrm{H}$ verschwindet, können dem Kegelschnitt $f=0$ unendlich viele Poldreiseite von $g=0$ umschrieben werden. Die Ecken aller dieser Poldreiseite liegen auf dem Kegelschnitt $F\left(g_{1}, g_{2}, g_{3}\right)=0$.

(17) Ist die Curve $f=0$ irgend einem bestimmten Poldreiseit von $g=0$ eingeschrieben, so wird $\mathrm{H}=0$.

(18) Wenn $\mathrm{H}$ verschwindet, geht der Kegelschnitt $g(x, x)=0$ durch unendlich viele Poldreiecke von $F(u, u)=0$. Die Seiten dieser Poldreiecke berühren sämmtlich den Kegelschuitt $H=0$.

(19) Wenn von zwei Kegelschnitten $F=0$ und $g=0$ der eine $(g=0)$ durch irgend ein Poldreieck des anderen geht, verschwindet $H$.

Es möge nun angenommen werden, dass der Kegelschnitt $f==0$ in ein Geradenpaar zerfällt, also $A=0$ ist. Man hat dann eine Identität von der Form:

$$
F=\left(t_{1} u_{1}+t_{2} u_{2}+t_{3} u_{3}\right)^{2}
$$

in der die $t_{i}$ die Coordinaten der Spitze des Geradenpaares bedeuten, und in der nach (35) in $\$ 8$ die Quadrate und Producte $u_{i} u_{k}$ durch die $b_{i k}$ ersetzt werden dürfen. Die vermöge dieser Substitution entstehende Gleichung

$$
3 \mathrm{H}=g\left(t_{1}, t_{2}, t_{3}\right)
$$

lehrt:

(21) Die Spitze eines durch $f=0$ dargestellten Geradenpaares liegt auf dem Kegelschnitt $g=0$, weun der Ausdruck 
$H$ verschwindet, und umgekehrt hat das Verschwinden von $H$ zur Folge, dass die Spitze jenes Geradenpares auf $g=0$ liegt.

Wären dagegen die beiden Relationen $A=0$ und $\Theta=0$ gleichzeitig befriedigt, so hat, wie wir oben sahen, jeder Punkt des Geradenpaares $f=0$, insbesondere also auch der Scheitel desselben, die Eigenschaft, dass seine Polare in Bezug auf $g=0$ die zwei Kegelschnitte $f$ und $g$ in harmonischen Punktepaaren schneidet, oder mit anderen Worten, dass sich von jenem Scheitel an die Curve $g=0$ ein Tangentenpaar legen lässt, das harmonisch ist zu dem Geradenpaare selbst. Also:

(22) Ein Geradenpaar $f=0$ bildet ein harmonisches Polarenpaar des Kegelschnitts $g=0$, sobald $\Theta$ verschwindet.

Wenn von vier harmonischen Strahlen zwei in eine Gerade fallen, wird bekanntlich gleichzeitig noch ein dritter Strahl mit dieser Geraden identisch. Durch Verbindung der Theoreme (21) und (22) ergibt sich daher:

(23) Bestehen die drei Relationen $A=0, \mathrm{H}=0, \Theta=0$ neben einander, so wird die Curve $g=0$ von der einen Geraden des Geradenpaares $f=0$ in dessen Scheitel berührt.

Das Tangentenpaar, das man im Falle $A=0$ von der Spitze $t$ des Geradenpaares $f=0$ an den Kegelschnitt $g=0$ legen kann, ist gegeben durch

$$
g(x, x) \cdot g(t, t)-g^{2}(x, t)=0,
$$

und da nun $t_{1}, t_{2}, t_{3}$ die Coordinaten der Spitze des Geradenpaares bedeuten, sind die Quadrate und Producte der $t$ proportional zu den Unterdeterminanten $A_{i k}$, so dass sich die Gleichung des Tangentenpaares bei Einführung dieser Grössen verwandelt in

$$
3 \mathrm{H} g-F\left(g_{1}, g_{2}, g_{3}\right)=0,
$$

und dieser Ausdruck ist nach $(7 \mathrm{~b})$ äquivalent mit $\chi=0$. Man hat daher folgenden Satz:

(24) Wenn der Kegelschnitt $f=0$ in ein Geradenpaar zerfällt, so repräsentirt $\chi=0$ das Tangentenpaar, das von der Spitze des Geradenpaares an den Kegelschnitt $g=0$ gelegt werden kann.

Im Falle $\mathrm{H}=0$ liegt nach (21) die Spitze von $f=0$ auf dem Kegelschnitt $g=0$; die beiden Tangenten, die für $A=0, \mathrm{H} \gtrless 0$ von der Spitze des Geradenpaares $f=0$ an die Curve $g=0$ gelegt werden können und nach (24) durch $\chi=0$ dargestellt sind, fallen daher; wenn $H=0$, zusammen, und wir können in Ergänzung zu (21) sagen: 
(25) Wenn die Relationen $A=0, \mathrm{H}=0$ neben einander bestehen, liegt die Spitze des Geradenpaares $f=0$ auf $g=0$. Die Tangente der Curve in diesem Punkte wird doppelt zählend dargestellt durch $\chi=0$.

Verschwindet auch noch $\Theta$, so gelangen wir zum Satze (23). Ausserdem folgt:

(26) Wenn $A=0, \mathrm{H}=0, \Theta=0, B=0$, so zerfallen beide Kegelschnitte in Geradenpare, und zwar der Art, dass beiden Paaren eine Gerade gemeinsam ist. Die Verbindungs. linie der Spitzen wird doppelt zählend dargestellt dureh $\chi=0$.

Dabei ist vorausgesetzt, dass nicht auch noch die Unterdeterminanten $A_{i k}$ den $B_{i k}$ proportional sind, denn im Falle einer solchen Proportionalität würden nach S. $137 f^{\prime}=0$ und $g=0$ für $A=B$ $=\mathrm{H}=\mathrm{O}=0$ zwei Geradenpaare mit gemeinsamer Spitze darstellen.

\section{$\S 18$.}

\section{Confocale Kegelschnitte.}

Sind $\varphi(u, u)=0$ und $\psi(u, \imath)=0$ die Gleichungen irgend zweier Kegelschnitte in Liniencoordinaten, so stellt

$$
\mu \varphi(u, u)-\psi(u, u)=0
$$

für variirende Werthe des Parameters $\mu$ nach $\S 15$ eine Schaar von Kegelschnitten dar: die Gesammtheit aller Curven zweiter Classe, welche die vier gemeinsamen Tangenten von $\varphi=0$ und $\psi=0$ berühren. In dieser Schaar sind nach (18) in $\S 15$ insbesondere drei Punktepaare enthalten, nämlich die Paare der Schnittpunkte der vier gemeinsamen Tangenten oder der sechs Ecken des durch diese vier Geraden gebildeten vollständigen Vierseits. Dabei sind diese Punkte der Art zu Paaren zusammenzufassen, dass immer zwei Gegenecken des Vierseits zusammengehören.

Von besonderer Wichtigkeit ist der Fall, dass die eine der zwei Grundcurven der Schaar, etwa $\psi(u, u)=0$, nicht eine beliebige Curve zweiter Classe, sondern speciell das Paar der imaginären Kreispunkte $\omega(u, u)=0$ ist; man bezeichnet alsdann das System

$$
\mu \varphi(u, u)-\omega(u, u)=0,
$$

wie bereits S. 146 erwähnt, als eine confocale Schaar, von Kegelschnitten. Während die Determinante von $\omega$ gleich $\mathrm{Null}$ ist, wollen wir diejenige von $\varphi$ als nicht verschwindend voraussetzen. Es sei ferner 
und

$$
\varphi(u, u) \equiv \sum_{1}^{3} \sum_{1}^{3} \alpha_{i k} u_{i} u_{k}
$$

$$
\begin{gathered}
\mu \varphi(u, u)-\omega(u, u) \equiv \gamma_{11} u_{1}^{2}+2 \gamma_{12} u_{1} u_{2}+\gamma_{22} u_{2}^{2}+2 \gamma_{13} u_{1} u_{3} \\
+2 \gamma_{23} u_{2} u_{3}+\gamma_{33} u_{3}^{2} \\
\text { wobei } \gamma_{i k}=\mu \alpha_{i k}-\omega_{i k} .
\end{gathered}
$$

Die Determinante $\Gamma(\mu) \equiv \sum \pm\left(\gamma_{11} \gamma_{22} \gamma_{33}\right)$ repräsentirt gleich Null gesetzt eine kubische Gleichung in $\mu$, welche nach (4) in $\S 8$ nur reelle Wurzeln besitzt. Man findet (dualistisch zu (8) in $\S 13$ ):

$$
\Gamma(\mu) \equiv \sum \pm\left(\gamma_{11} \gamma_{22} \gamma_{33}\right)=\mu^{3} \mathrm{~A}-\mu^{2} \mathrm{~A}_{1}+\mu \mathrm{A}_{2}
$$

wobei $A, A_{1}, A_{2}$ die Werthe besitzen:

$$
\left\{\begin{array}{l}
A \equiv \sum \pm\left(\alpha_{11} \alpha_{22} \alpha_{33}\right) \\
A_{1}=A_{11} \omega_{11}+A_{22} \omega_{22}+A_{33} \omega_{33}+2 A_{12} \omega_{12}+2 A_{13} \omega_{13}+2 A_{23} \omega_{23} \\
A_{2} \equiv \alpha_{11} \Omega_{11}+\alpha_{22} \Omega_{22}+\alpha_{33} \Omega_{33}+2 \alpha_{12} \Omega_{12}+2 \alpha_{13} \Omega_{13}+2 \alpha_{23} \Omega_{23} \\
\quad \equiv \tau \varphi(p, p) \quad(\operatorname{vgl} .(16) \text { in } \S 7) .
\end{array}\right.
$$

Dabei ist $A_{i k}$ die Unterdeterminante des Elementes $\alpha_{i k}$ in A. Das absolute Glied in (4) fehlt, weil die Determinante von $\omega(\imath, u)$ gleich Null ist. Uebrigens werden wir weiterhin von der Relation Gebranch machen:

$$
\Gamma(\mu) \equiv A \mu\left(\mu-\mu_{1}\right)\left(\mu-\mu_{2}\right)
$$

in welcher $\mu_{1}$ und $\mu_{2}$ die zwei von Null verschiedenen Wurzeln der Gleichung

$$
\Gamma(\mu) \equiv \mu^{3} \mathrm{~A}-\mu^{2} \mathrm{~A}_{1}+\mu \mathrm{A}_{2}=0
$$

bezeichnen; es ist daun offenbar

$$
\mathrm{A}_{1}=\mathrm{A}\left(\mu_{1}+\mu_{2}\right), \quad \mathrm{A}_{2} \equiv \tau \varphi(p, p)=\mathrm{A} \mu_{1} \mu_{2} \text {. }
$$

Setzt man noch

$(9) \quad \mathrm{X}(\mu) \equiv-\left|\begin{array}{cccc}\mu \alpha_{11}-\omega_{11} & \mu \alpha_{12}-\omega_{12} & \mu \alpha_{13}-\omega_{13} & x_{1} \\ \mu \alpha_{21}-\omega_{21} & \mu \alpha_{22}-\omega_{22} & \mu \alpha_{23}-\omega_{23} & x_{2} \\ \mu \alpha_{31}-\omega_{31} & \mu \alpha_{32}-\omega_{32} & \mu \alpha_{33}-\omega_{33} & x_{3} \\ x_{1} & x_{2} & x_{3} & 0\end{array}\right| \equiv-\left(\begin{array}{l}x \\ x\end{array}\right)_{\gamma_{i k}}$,

so folgt durch Partialbruchzerlegung:

$$
\frac{X(\mu)}{\Gamma(\mu)}=\frac{X\left(\mu_{1}\right)}{\Gamma^{\prime}\left(\mu_{1}\right)} \frac{1}{\mu-\mu_{1}}+\frac{X\left(\mu_{2}\right)}{\Gamma^{\prime}\left(\mu_{2}\right)} \frac{1}{\mu-\mu_{2}}+\frac{X(0)}{\Gamma^{\prime}(0)} \cdot \frac{1}{\mu} .
$$


Transformation confocaler Ellipsen und Hyperbeln auf die Hauptaxen. 167

Wie aus (9) zu ersehen, ist hier

$$
\mathrm{X}(0) \equiv \sum_{1}^{3} \sum_{1}^{3} \Omega_{i k} x_{i} x_{k}=\tau\left(p_{1} x_{1}+p_{2} x_{2}+p_{3} x_{3}\right)^{2},
$$

unter $p_{1}, p_{2}, p_{3}$ die Coordinaten der unendlich fernen Geraden verstanden; ferner ist nach (7) und (8):

$$
\Gamma^{\prime}(0)=\mathrm{A}_{2}=\tau \varphi(p, p) \text {. }
$$

Für eine Wurzel $\mu_{i}$ der Gleichung (7) wird X $(\mu)$ ein vollstïndiges Quadrat, da in diesem Falle der zugehörige Kegelschnitt der Schaar in ein Punktepaar ausartet, dessen Träger doppelt zählend durelı $X(\mu)=0$ dargestellt wird. Man kann daher setzen:

$$
\frac{\mathrm{X}\left(\mu_{i}\right)}{\Gamma^{\prime}\left(u_{i}\right)}=\left[u_{1}{ }^{(i)} x_{1}+u_{2}{ }^{(i)} x_{2}+u_{3}{ }^{(i)} x_{3}\right]^{2}=\mu_{i} X_{i}{ }^{2} \quad(i=1,2),
$$

und erhält, wenn man für $p_{1} x_{1}+p_{2} x_{2}+p_{3} x_{3}$ noch $X_{3}$ einführt:

$$
\begin{aligned}
& \mathrm{X}(\mu) \\
& \Gamma(\mu)
\end{aligned}=\frac{\mu_{1} X_{1}{ }^{2}}{\mu-\mu_{1}}+\frac{\mu_{2} X_{2}{ }^{2}}{\mu-\mu_{2}}+\frac{X_{3}{ }^{2}}{\mu \cdot \varphi(p, p)} .
$$

Speciell die Werthe $\mu=\infty$ und $\mu=0$ liefern hieraus die Gleichungen der Kegelschnitte $\varphi(u, u)=0$ und $\omega(u, u)=0$ in Punktcoordinaten

$$
\left\{\begin{array}{l}
\frac{\Phi(x, x)}{\mathrm{A}}=\mu_{1} X_{1}{ }^{2}+\mu_{2} X_{2}{ }^{2}+\frac{X_{3}{ }^{2}}{\varphi(p, p)}, \text { wobei } \\
\Phi(x, x) \equiv \sum_{1}^{3} \sum_{1}^{3} \mathrm{~A}_{i k} x_{i} x_{k} \\
\Omega(x, x)=\tau X_{3}{ }^{2} .
\end{array}\right.
$$

Wir ersetzen nun in der Determinante (9) die $x_{i}$ durch $\frac{1}{2} \varphi^{\prime}\left(u_{i}\right)=\varphi_{i}$ und nemnen $\frac{U_{1}}{\mu_{\mathrm{t}}}, \frac{U_{2}}{\mu_{2}}, \varphi(p, p) \cdot U_{3}$ die hierdurch aus $X_{1}, X_{2}, X_{3}$ in (13) hervorgehenden Ausdrücke, so dass also

$$
\begin{aligned}
\frac{U_{i}}{\mu_{i}} & =\frac{u_{1}{ }^{(i)} \varphi_{1}+u_{2}{ }^{(i)} \varphi_{2}+u_{3}{ }^{(i)} \varphi_{3}}{\sqrt{\mu_{i}}} \quad(i=1,2), \\
U_{3} & =\frac{p_{1} \varphi_{1}+p_{2} \varphi_{2}+p_{3} \varphi_{3}}{\varphi(p, p)}=\frac{\varphi(p, u)}{\varphi(p, p)}
\end{aligned}
$$

alsdann folgt aus (14):

$$
-\left(\begin{array}{l}
\varphi \\
\varphi
\end{array}\right)_{\gamma_{i k}}: \Gamma(\mu)=\frac{U_{1}^{2}}{\mu_{1}\left(\mu-\mu_{1}\right)}+\frac{U_{2}^{2}}{\mu_{2}\left(\mu-\mu_{2}\right)}+\frac{\varphi(p, p) U_{3}^{2}}{\mu} .
$$

Analog $\mathrm{zu}(13)$ in $\S 16$ ist nun:

$$
-\frac{\left(\begin{array}{c}
\varphi \\
\varphi
\end{array}\right) \mu^{2}}{\Gamma(\mu)}=\omega+\mu \varphi-\frac{\left(\begin{array}{c}
\omega \\
\omega
\end{array}\right)}{\Gamma(\mu)}
$$


und wenn man in (17) auf der rechten Seite nach absteigenden Potenzen von $\mu$ entwickelt, erhält man

$-\frac{\left(\begin{array}{l}\varphi \\ \varphi\end{array}\right)}{\Gamma(\mu)} \mu^{2}=\mu^{2}\left\{\frac{U_{1}{ }^{2}}{\mu_{1}}\left(\frac{1}{\mu}+\frac{\mu_{1}}{\mu^{2}}+\cdot \cdot\right)+\frac{U_{8}{ }^{2}}{\mu_{2}}\left(\frac{1}{\mu}+\frac{\mu_{2}}{\mu^{2}}+\cdot \cdot\right)+\frac{\varphi(p, p) \cdot U_{3}{ }^{2}}{\mu}\right\}$,

so dass sich durch Vergleichung der Coefficienten von $\mu^{1}$ und $\mu^{0}$ in den beiden letzten Relationen ergibt:

$$
\left\{\begin{array}{l}
\varphi(u, u)=\frac{U_{1}^{2}}{\mu_{1}}+\frac{U_{2}^{2}}{\mu_{2}}+\varphi(p, p) \cdot U_{3}{ }^{2}, \\
\omega(u, u)=U_{1}^{2}+U_{2}^{2} .
\end{array}\right.
$$

Hierbei geht aus $\omega(u, u)=U_{1}{ }^{2}+{U_{2}}^{2}$ hervor, dass die Ausdrücke $U_{1}^{2}$ und $U_{2}^{2}$ positiv sind, denn $\omega(u, u)$ ist nach $\S 7$ eine definite, positive Form und als Summe zweier reellen Quadrate darstellbar. Aus der Realität von $U_{1}$ und $U_{2}$ folgt alsdann auch diejenige von $X_{1}$ und $X_{2}$; die Ausdrücke $U_{3} \equiv \frac{\varphi(p, u)}{\varphi(p, p)}$ und $X_{3} \equiv p_{1} x_{1}+p_{2} x_{2}+p_{3} x_{3}$ sind selbstverständlich reell. Es werde bei dieser Gelegenheit noch bemerkt, dass die $X_{i}$ und $U_{i}$ in (13) und (16) wesentlich mit Rücksicht auf die Gleichungen (18) und (19) gewählt wurden.

Dass die Coordinaten $U_{1}, U_{2}, U_{3}, X_{1}, X_{2}, X_{3}$ auch die Relation

$$
u_{1} x_{1}+u_{2} x_{2}+u_{3} x_{3}=U_{1} X_{1}+U_{2} X_{2}+U_{3} X_{3}
$$

erfüllen, zeigt man auf analoge Weise, wie bei (34) in $\S 14$ : man ersetzt in (14) die $x_{i}$ durch $x_{i}+\varrho \varphi_{i}$, vergleicht beiderseits die Coefficienten von $\varrho^{1}$ und setzt alsdann $\mu=\infty$.

Die drei in der Kegelschnittschaar (2) enthaltenen Punktepaare entsprechen den Wurzeln $\mu=0, \mu=\mu_{1}, \mu=\mu_{2}$ der kubischen Gleichung $\Gamma(\mu)=0$ und sind dargestellt resp. durch das imaginäre Kreispunktepaar $\omega(u, u)=0$, sowie durch $\mu_{1} \varphi-\omega=0$ und $\mu_{2} \varphi-\omega=0$. Man findet für die beiden letzteren, ausgedrückt in den Coordinaten $U_{i}$, die Gleichungen

$$
\left\{\begin{array}{l}
\mu_{1} \varphi-\omega=\frac{\left(\mu_{1}-\mu_{2}\right)}{\mu_{2}} U_{2}^{2}+\mu_{1} \varphi(p, p) U_{3}^{2}=0, \\
\mu_{2} \varphi-\omega=\frac{\left(\mu_{2}-\mu_{1}\right)}{\mu_{1}} U_{1}^{2}+\mu_{2} \varphi(p, p) U_{3}^{2}=0 .
\end{array}\right.
$$

Wie schon in $\S 11$ erwähnt wurde, repräsentiren diese zwei, in der Schaar $\mu \varphi-\omega=0$ enthaltenen Punktepaare die zwei Brennpunktepaare des Kegelschnitts $\varphi(u, u)=0$, und da dieselben (abgesehen von $\omega(u, u)=0$ ) die einzigen Punktepaare sind, die der Schaar angehören, so folgt, dass alle Curven der Schaar $\mu \varphi-\omega=0$ dieselben Brennpunkte (Foci) besitzen. Aus diesem Grunde werden 
die Kegelschnitte dieser Schaar als confocal bezeichnet; dass dieselben auch concentrisch sind, folgt aus (15) in $\S 11$.

Die Quadrate der halben Axen des Kegelschnitts $\Phi(x, x)=0$ besitzen, wie (15) zeigt, die Werthe

$$
a^{2}=-\frac{1}{\mu_{1} \varphi(p, p)}, \quad b^{2}=-\frac{1}{\mu_{2} \varphi(p, p)},
$$

falls z. B. (15) eine Ellipse darstellt. Durch Einführung dieser Ausdrücke in (14), sowie durch die Substitution $\frac{1}{\mu \varphi(p, p)}=\lambda$ geht die in (14) gegebene Gleichung einer confocalen Schaar in Punkteoordinaten über in die gewöhnlich gebräuchliche Form

$$
\left.\frac{X_{1}{ }^{2}}{a^{2}+\lambda}+\frac{X_{2}{ }^{2}}{b^{2}+\lambda}-X_{3}{ }^{2}=0 .{ }^{1}\right)
$$

Die Gleichungen (20) der beiden Brennpunktepaare lassen sich noch in eine andere einfache Gestalt bringen durch Einführung der Werthe von $U_{1}, U_{2}, U_{3}$ aus $(16)$; beachtet man hierbei, dass nacl

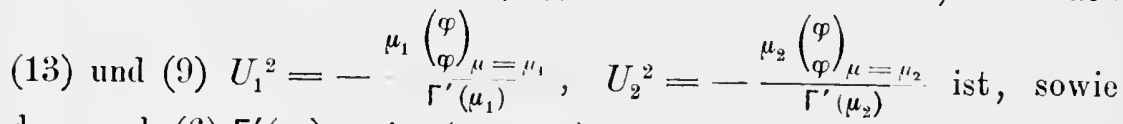
dass nach $(6) \Gamma^{\prime}\left(\mu_{1}\right)=\mathrm{A} \mu_{1}\left(\mu_{1}-\mu_{2}\right), \Gamma^{\prime}\left(\mu_{2}\right)=\mathrm{A} \mu_{2}\left(\mu_{2}-\mu_{1}\right)$, so folgt

$$
\begin{aligned}
& \mu_{1} \varphi-\omega=\frac{\left(\begin{array}{l}
\varphi \\
\varphi
\end{array}\right)_{\mu=\mu_{2}}}{\mathrm{~A} \mu_{2}}+\frac{\mu_{1} \cdot \varphi^{2}(p, u)}{\varphi(p, p)}, \\
& \mu_{2} \varphi-\omega=\frac{\left(\begin{array}{l}
\varphi \\
\varphi
\end{array}\right)_{\mu=\mu_{1}}}{\mathrm{~A} \mu_{1}}+\frac{\mu_{2} \cdot \varphi^{2}(p, u)}{\varphi(p, p)},
\end{aligned}
$$

und wenn man diese Gleichungen mit $A \mu_{2}$ resp. $A \mu_{1}$ multiplicirt, ergeben sich mit Rücksicht auf $\mathrm{A} \mu_{1} \mu_{2}=\tau \varphi(p, p)$ für die zwei Brcunpunktepaare die Gleichungen

$$
\left\{\begin{array}{l}
\mathrm{A} \mu_{2}\left(\mu_{1} \varphi-\omega\right)=\left(\begin{array}{l}
\varphi \\
\varphi
\end{array}\right)_{\mu=\mu_{2}}+\tau \cdot \varphi^{2}(p, u)=0, \\
\mathrm{~A} \mu_{1}\left(\mu_{2} \varphi-\omega\right)=\left(\begin{array}{l}
\varphi \\
\varphi
\end{array}\right)_{\mu=\mu_{1}}+\tau \cdot \varphi^{2}(p, u)=0 .
\end{array}\right.
$$
folgende:

Aus diesen zwei Relationen folgt übrigens durch Subtraction die

$$
\mathrm{A}\left(\mu_{1}-\mu_{2}\right) \omega \equiv\left(\begin{array}{l}
\varphi \\
\varphi
\end{array}\right)_{\mu=\mu_{2}}-\left(\begin{array}{l}
\varphi \\
\varphi
\end{array}\right)_{\mu=\mu_{1}},
$$

1) Man beachte, dass $a^{2}+\lambda-\left(b^{2}+\lambda\right)=a^{2}-b^{2}=\frac{1}{\varphi(p, p)}\left(\frac{1}{\mu_{2}}-\frac{1}{\mu_{1}}\right)$ unabhängig von $\lambda$ ist, in Uebereinstimmung mit der Definition der confocalen Kegelschnitte. 
und wenn man hier für $\left(\begin{array}{l}\varphi \\ \varphi\end{array}\right)$ seinen Werth einführt, oder auch in der zweiten Gleichung (18) die Werthe $U_{1}, U_{2}$ aus (16) substituirt, ergibt sich die Relation

$$
\omega=\mu_{1}\left(u_{1}{ }^{(1)} \varphi_{1}+u_{2}^{(1)} \varphi_{2}+u_{3}^{(1)} \varphi_{3}\right)^{2}-\mu_{2}\left(u_{1}{ }^{(2)} \varphi_{1}+u_{2}{ }^{(2)} \varphi_{2}+u_{3}^{(2)} \varphi_{3}\right)^{2},
$$

vermöge deren ebenso wie vermöge (22) sofort die Gleichungen der beiden imaginären Kreispunkte einzeln erhalten werden könnten. Dabei sind die $u_{1}^{\left({ }^{(i)}\right.} \varphi_{1}+u_{2}^{(i)} \varphi_{2}+u_{3}^{(i)} \varphi_{3}(i=1,2)$ zu entnehmen aus $(16)$, mit Rücksicht auf (13).

In $\S 15$ wurde gezeigt, dass durch jeden Punkt $P$ der Ebene zwei reelle Kegelschnitte der Schaar $\mu \varphi-\omega=0$ hindurchgehen und sich in $P$ rechtwinklig schneiden; wir wollen nun nachweisen, dass im allgemeinen Falle, d. h. so lange die Gleichung (7) keine Doppelwurzel besitzt, der eine dieser Kegelschnitte eine Ellipse, der andere eine Hyperbel ist.

Es seien $y_{1}, y_{2}, y_{3}$ die Coordinaten des Punktes $P$; alsdann ist $\left(\begin{array}{l}y \\ y\end{array}\right)_{\gamma_{i k}}=0$ nach (9) die Bedingung dafür, dass ein Kegelschnitt der Schaar durch $P$ hindurchgehe. Nennen wir $Y_{i}$ das Resultat der Substitution der Coordinaten $y_{1}, y_{2}, y_{3}$ in die Ausdrücke $X_{i}$, so lässt sich die Bedingung $\left(\begin{array}{l}y \\ y\end{array}\right)=0$ nach (14) auch ersetzen durch

$$
-\left(\begin{array}{l}
y \\
y
\end{array}\right)_{\gamma_{i k}}: \Gamma(\mu)=\frac{\mu_{1} Y_{1}{ }^{2}}{\mu-\mu_{1}}+\frac{\mu_{2} Y_{2}{ }^{2}}{\mu-\mu_{2}}+\frac{Y_{3}{ }^{2}}{\mu \cdot \varphi(p, p)}=0
$$

oder durch

$$
\begin{aligned}
\Psi(\mu) \equiv & \mu \mu_{1}\left(\mu-\mu_{2}\right) \cdot \varphi(p, p) Y_{1}^{2}+\mu \mu_{2}\left(\mu-\mu_{1}\right) \varphi(p, p) Y_{2}^{2} \\
& +\left(\mu-\mu_{1}\right)\left(\mu-\mu_{2}\right) Y_{3}^{2}=0
\end{aligned}
$$

Die Ergebnisse der Substitution von $\mu=\mu_{1}$ und $\mu=\mu_{2}$ in $\Psi(\mu)$ sind nun stets entgegengesetzten Vorzeichens, während die Substitution einer hinreichend grossen, positiven oder negativen, Zahl (dieselbe werde symbolisch durch $\pm \infty$ bezeichnet) in den Ausdruck (24a) jedesmal ein Ergebniss von gleichem Vorzeichen liefert, mag die Zahl positiv oder negativ sein.

Ist nun $\mu_{1}>\mu_{2}$ und etwa $\psi\left(\mu_{1}\right)$ von entgegengesetztem Vorzeichen mit $\Psi(+\infty)$, so liegt eine Wurzel $\mu^{\prime}$ der quadratischen Gleichung (24a) zwischen $+\infty$ und $\mu_{1}$, die andere $\mu^{\prime \prime}$ zwischen $\mu_{1}$ und $\mu_{2}$.

Hat dagegen der Ausdruck $\psi\left(\mu_{2}\right)$ für $\mu_{1}>\mu_{2}$ das entgegengesetzte Vorzeichen von $\Psi(+\infty)$ und daher auch von $\Psi(-\infty)$, so liegt eine Wurzel $\mu^{\prime}$ von (24a) zwischen $\mu_{2}$ und $-\infty$, die andere $\mu^{\prime \prime}$ zwischen $\mu_{1}$ und $\mu_{2}$. 
Jedesmal ergibt die Substitution der zwischen $\mu_{1}$ und $\mu_{2}$ gelegenen Wurzel $\mu=\mu^{\prime \prime}$ in (24) eine Hyperbel bei glejchem Vorzeichen von $\mu_{1}$ und $\mu_{2}$, eine Ellipse bei ungleichem Vorzeichen. Die Substitution der anderen Wurzel $\mu^{\prime}$ ergibt hingegen bei gleichem Vorzeichen von $\mu_{1}$ und $\mu_{2}$ eine Ellipse, bei ungleichem eine Hyperbel. Dasselbe Resultat würde man erhalten, wenn nicht, wie soeben vorausgesetzt war, $\mu_{1}>\mu_{2}$, sondern umgekehrt $\mu_{2}>\mu_{1}$ wäre.

In allen Fällen liefert die eine Wurzel der quadratischen Gleichung (24a) eine Ellipse, die andere eine Hyperbel, und somit ist der Satz bewiesen:

(25) So lange die Gleichung (7) $\Gamma(\mu)=0$ keine Doppelwurzel besitzt, gehen dureh jedell Punkt der Ebene zwei sich rechtwinklig schneidende Curven der confocalen Schar $\mu \varphi(u, u)-\omega(u, u)=0$; die eine dieser Curven ist eine Ellipse, die andere eine Hyperbel.

Auch folgt hieraus, dass die in der confocalen Schaar enthaltenen Kegelschnitte derselben Art sich nicht in reellen Punkten schneiden. Etwas Aehnliches findet statt, wenn die Kegelschnitte der confocalen Schaar nur aus Parabeln bestehen (vgl. weiter unten).

Wir wollen nun sehen, was eintritt, wenn die Gleichung $\Gamma(\mu)=0$ mehrfache Wurzeln besitzt; die Determinante A werde dabei noeh als von Null verschieden vorausgesetzt. Hier ist zunächst zu beachten, dass der Fall einer dreifachen Wurzel von $\Gamma(\mu)=0$ überhaupt ausgeschlossen ist, denn eine solche müsste nach (7) gleich Null sein, also zufolge des Satzes (8) in $\S 8$ in alleu Unterdeterminanten $\Gamma_{i k}$ der die Determinante $\sum \pm\left(\gamma_{11} \gamma_{22} \gamma_{33}\right)$ bildenden Elemente $\gamma_{i k}$ einfach enthalten sein. Für die Wurzel $\mu=0$ reduciren sich aber diese Unterdeterminanten auf $\Omega_{i k}=\tau p_{i} p_{k}$, mithin auf Ausdrücke, die nicht alle verschwinden können.

Es sind also nur die Fälle zu untersuchen, in denen $\Gamma(\mu)=0$ eine Doppelwurzel besitzt, und zwar werde zuerst angenommen diese Doppelwurzel sei die nicht verschwindende Wurzel $\mu=\mu_{1}$, so dass $\Gamma(\mu)=A \mu\left(\mu-\mu_{1}\right)^{2}$.

Wir verfahren alsdann ähnlich wie in $\S 16$ bei Behandlung der speciellen Fälle, die bei dem Kegelschnittbüschel auftreten können, indem wir ausgehen von der Relation:

$$
\frac{\mathrm{X}(\mu)}{\Gamma(\mu)}=-\frac{\left(\begin{array}{l}
x \\
y
\end{array}\right)^{2}}{\Gamma(\mu) \cdot\left(\begin{array}{l}
y \\
y
\end{array}\right)}-\frac{\left(\begin{array}{l}
x y \\
x y
\end{array}\right)}{\left(\begin{array}{l}
y \\
y
\end{array}\right)} .
$$

Nach (7) in $\S 8$ werden für die Doppelwurzel $\mu_{1}$ alle Unter- 
determinanten $\Gamma_{i k}$ zu Null, d. h. $\mu_{1}$ ist für beliebige Werthe der $y_{i}$ eine einfache Wurzel von $\left(\begin{array}{l}y \\ y\end{array}\right)=0$ oder auch von $\left(\begin{array}{l}x \\ y\end{array}\right)=0$, es liefern daher die beiden Terme in (26) Partialbrüche mit dem Nenner $\mu-\mu_{1} \cdot{ }^{1}$ ) Ferner lässt sich der Factor $\mu-\mu_{1}$ in Zähler und Nenner von

$$
\frac{\left(\begin{array}{l}
x \\
y
\end{array}\right)^{2}}{\Gamma(\mu) \cdot\left(\begin{array}{l}
y \\
y
\end{array}\right)}
$$

zweimal wegheben, so dass alsdann dieses Glied, ebenso wie $\left(\begin{array}{l}x y \\ x y\end{array}\right):\left(\begin{array}{l}y \\ y\end{array}\right)$, im Nenner den Factor $\mu-\mu_{1}$ nur noch einmal besitzt und wegen $\left(\begin{array}{l}y \\ y\end{array}\right)=0$, nach der zu (2) in $\S 16$ analogen Formel, in Bezug auf die $x_{i}$ ein vollstïndiges Quadrat ist. Durch Partialbruchzerlegung ergibt sich daher jetzt eine Gleichung von der Gestalt

$$
\frac{X(\mu)}{\Gamma(\mu)}=\frac{\mu_{1} X^{(1)} X^{(1)}}{\mu-\mu_{1}}+\frac{\mu_{1} X^{(2)} X^{(2)}}{\mu-\mu_{1}}+\frac{X_{3}{ }^{2}}{\mu \cdot \varphi(p, p)},
$$

wobei

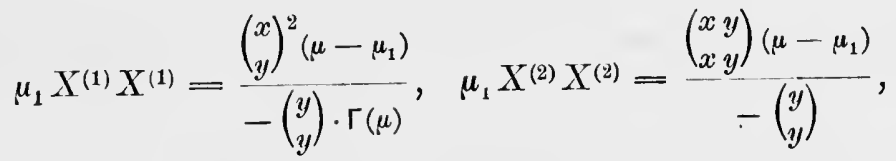

während $X_{3}$ mit dem bereits früher aufgetretenen und ebenso bezeichneten Ausdrucke in (14) übereinstimmt.

In derselben Weise wie in dem oben ausführlich behandelten allgemeinen Falle findet man

$$
\begin{aligned}
& \left\{\begin{array}{l}
\frac{\Phi(x, x)}{\mathrm{A}}=\mu_{1}\left(X^{(1)} X^{(1)}+X^{(2)} X^{(2)}\right)+\frac{X_{3}{ }^{2}}{\varphi(p, p)}, \\
\Omega(x, x)=\tau X_{3}{ }^{2}
\end{array}\right. \\
& \left\{\begin{array}{l}
\varphi(u, u)=\frac{U_{1}{ }^{2}+U_{2}{ }^{2}}{\mu_{1}}+\varphi(p, p) \cdot U_{3}{ }^{2}, \\
\omega(u, u)=U_{1}{ }^{2}+U_{2}{ }^{2},
\end{array}\right.
\end{aligned}
$$

wobei $\frac{U_{1}}{\mu_{1}}, \frac{U_{2}}{\mu_{1}}, \varphi(p, p) U_{3}$ die durch die Substitution $x_{i}=\frac{1}{2} \varphi^{\prime}\left(u_{i}\right)$ aus $X^{(1)}, X^{(2)}, X_{3}$ hervorgehenden Ausdrücke bedeuten.

Ausserdem findet man

$$
\mu_{1} \varphi-\omega=\mu_{1} \varphi(p, p) \cdot U_{3}{ }^{2} .
$$

Die Gleichungen (29) und (30) lassen erkennen, dass nunmehr die confocale Schaar $\mu \varphi-\omega=0$ aus concentrischen Kreisen

1) Vgl. die Behandlung des analogen Falles in $\S 16$, welche daselbst zu der Gleichung (9) in $\S 16$ führt. 
besteht, und zwar gehört der Mittelpunkt $U_{3}=0$ doppelt zählend dieser Schaar an; die zwei im allgemeinen Falle vorhandenen Brennpunktepaare sind nun zusammengefallen und in diesen Mittelpunkt gerückt. Man erkennt auch, dass eine confocale Schaar nur aus Kreisen besteht, sobald auch nur eine Curve derselben ein Kreis ist. Durch jeden Punkt $y$ der Ebene geht ein Kreis der Schaar, denn durch diesen Punkt ist die Curve, da ihr Mittelpunkt gleiclffalls gegeben, vollständig bestimmt; algebraisch erhellt diese Thatsache aus dem gleich Null gesetzten Ausdrucke (27), denn die so entstehende Gleichung ist in $\mu$ linear.

Bei dieser Gelegenheit wollen wir untersuchen, welche Bedingungen erfüllt werden müssen, damit überhaupt die Gleichung einer Curve zweiter Classe $\varphi(u, u)=0$ einen Kreis darstelle. Wir behaupten, dass diese Bedingungen in dem Verschwinden der Coefficienten einer gewissen quadratischen Form der $u_{i}(i=1,2,3)$ bestehen, und zwar ist diese Form gegeben durch die Determinante

$$
\mathrm{K} \equiv\left|\begin{array}{lll}
\frac{1}{2} \varphi^{\prime}\left(u_{1}\right) & \frac{1}{2} \varphi^{\prime}\left(u_{2}\right) & \frac{1}{2} \varphi^{\prime}\left(u_{3}\right) \\
\frac{1}{2} \omega^{\prime}\left(u_{1}\right) & \frac{1}{2} \omega^{\prime}\left(u_{2}\right) & \frac{1}{2} \omega^{\prime}\left(u_{3}\right) \\
\frac{1}{2} \varphi^{\prime}\left(p_{1}\right) & \frac{1}{2} \varphi^{\prime}\left(p_{2}\right) & \frac{1}{2} \varphi^{\prime}\left(p_{3}\right)
\end{array}\right|=0,
$$

welche eine simultane Contravariante von $\varphi(u, u), \omega(u, u)$ nnd $p_{x}$ repräsentirt. Es hängen nämlich die $X_{i}$ mit den $x_{i}$ zusammen durch die Gleichungen (vgl. (13)):

$$
\begin{aligned}
& X_{i}=\frac{1}{\sqrt{\mu_{i}}}\left(u_{1}{ }^{(i)} x_{1}+u_{2}{ }^{(i)} x_{2}+u_{3}{ }^{(i)} x_{3}\right) \quad(i=1,2), \\
& X_{3}=p_{1} x_{1}+p_{2} x_{2}+p_{3} x_{3},
\end{aligned}
$$

daher die $u_{i}$ mit den $U_{i}$ durch die transponirte Substitution

$$
u_{i}=\frac{u_{i}^{(1)}}{\sqrt{\mu_{1}}} U_{1}+\frac{u_{i}^{(2)}}{\sqrt{\mu_{2}}} U_{2}+p_{i} U_{3} \quad(i=1,2,3),
$$

und es sei $r=\sum \pm\left(\frac{u_{1}^{(1)}}{\sqrt{\mu_{1}}} \frac{u_{2}^{(2)}}{\sqrt{\mu_{2}}} p_{3}\right)$ die Determinante dieser Substitution. Mit Rücksicht auf (18) und auf $p_{x}=X_{3}$ besteht folglich die Relation:

$$
r \cdot \mathrm{K}=\left|\begin{array}{ccc}
\frac{U_{1}}{\mu_{1}} & \frac{U_{2}}{\mu_{2}} & \varphi(p, p) U_{3} \\
U_{1} & U_{2} & 0 \\
0 & 0 & \varphi(p, p)
\end{array}\right|=\varphi(p, p)\left(\frac{1}{\mu_{1}}-\frac{1}{\mu_{2}}\right) U_{1} U_{2}
$$


Dabei ist die Determinante der Transformation $r$ von Null verschieden, denn die drei Punkte $U_{1}=0, U_{2}=0, U_{3}=0$ liegen nicht in einer Geraden.

Die Gleichung (33) zeigt, dass das Verschwinden von (32), so lange $\varphi(p, p) \gtrless 0$, die Beziehung $\mu_{1}=\mu_{2}$ zur Folge hat, es gibt also $\mathrm{K}=0$ bei beliebigen $u_{i}$ in der That die Bedingungen dafür, dass $\varphi(u, u)=0$ einen Kreis darstelle.

Uebrigens verschwinden für die Doppelwurzel $\mu_{1}=\mu_{2}$, wie bereits erwähnt, auch die oben mit $\Gamma_{i k}$ bezeichneten sechs Unterdeterminanten, und es gilt hier wieder dieselbe Bemerkung, wie S. 97 bei Aufstellung der Bedingungen dafür, dass eine Gleichung $f(x, x)=0$ in Punktcoordinaten einen Kreis darstelle.

Wir gehen nun weiter in der Behandlung der speciellen Fälle, welche für die Wurzeln der kubischen Gleichung $\Gamma(\mu)=0$ bei confocalen Kegelschnitten eintreten können.

Der Fall, dass die Doppelwurzel $\mu_{1}=\mu_{2}$ auch Doppelwurzel ist von $\left(\begin{array}{l}y \\ y\end{array}\right)=0$, muss aus demselben Grunde ausgeschlossen werden, aus dem dies schon bei Kegelschnittbüscheln (vgl. S. 151) geschah.

Es möge jetzt angenommen werden, die Gleichung $\Gamma(\mu)=0$ habe die Doppelwurzel $\mu=0$ und die von Null verschiedene Wurzel $\mu=\mu_{1}$; alsdann ist nach (7) $\mathrm{A}_{2}=\tau \varphi(p, p)=0$ und $\Gamma(\mu)=\mathrm{A} \mu^{2}\left(\mu-\mu_{1}\right)$. Jedenfalls können für $\mu=0$ nicht alle Unterdeterminanten $\Gamma_{i k}$ verschwinden, demn diese gehen durch die Annahme $\mu=0$ in $\Omega_{i k}=\tau p_{i} p_{k}$ über $\left.^{1}\right)$. Alsdann veranlasst in (26) nur der Term $\frac{\left(\begin{array}{l}x \\ y\end{array}\right)^{2}}{-\left(\begin{array}{l}y \\ y\end{array}\right) \cdot \Gamma(\mu)}$ Partialbrïche, die zur Wurzel $\mu=0$ gehören. Setzen wir

$$
\frac{\mu^{2}\left(\begin{array}{l}
x \\
y
\end{array}\right)^{2}}{-\left(\begin{array}{l}
y \\
y
\end{array}\right) \Gamma(\mu)}=X \quad \text { und } \quad \frac{X\left(\mu_{1}\right)}{\Gamma^{\prime}\left(\mu_{1}\right)}=\mu_{1} X_{1}^{2}
$$

so $\left.\operatorname{wird}^{2}\right)$

$$
\frac{X(\mu)}{\Gamma(\mu)}=\frac{\left[X^{2}\right]_{\mu=0}}{\mu^{2}}+\frac{\left[\frac{d \cdot X^{2}}{d \mu}\right]_{\mu=0}}{\mu}+\frac{\mu_{1} X_{1}^{2}}{\mu-\mu_{1}}
$$

mit Einführung der Bezeichnungsweise:

$$
[X]_{\mu=0}=v_{3} X_{3}, \quad\left[\frac{d X}{d \mu}\right]_{\mu=0}=X_{2}, \quad \text { wobei } v_{3}^{2}=-\frac{\tau}{\mathrm{A} \mu_{1}},
$$
verwandelt sich (34) in:

1) Vgl. auch S. 171.

2) Vgl. S. 150. 


$$
\left.\frac{\mathrm{X}(\mu)}{\Gamma(\mu)}=\frac{-\tau}{\mathrm{A} \mu_{1}} \frac{X_{3}{ }^{2}}{\mu^{2}}+2 \sqrt{\frac{-\tau}{\mathrm{A} \mu_{1}}} \frac{X_{2} X_{3}}{\mu}+\frac{\mu_{1} X_{1}{ }^{2}}{\mu-\mu_{1}}, 1\right)
$$

und hieraus erhält man für $\mu=\infty$, resp. $\mu=0$ die Gleichungen von $\varphi(u, u)=0$ und $\omega(u, u)=0$ in Punktcoordinaten:

$$
\left\{\begin{array}{l}
\frac{\Phi(x, x)}{\mathrm{A}}=2 \sqrt{\frac{-\tau}{\overline{\mathrm{A} \mu}}} X_{2} X_{3}+\mu_{1} X_{1}{ }^{2}, \\
\Omega(x, x)=\tau X_{3}{ }^{2} .
\end{array}\right.
$$

Wir ersetzen nun in $\mathrm{X}(\mu)$ die $x_{i}$ durch $\frac{1}{2} \varphi^{\prime}\left(u_{i}\right)=\varphi_{i}$ und bezeichnen die vermöge dieser Substitution aus $X_{3}, X_{2}, X_{1}$ hervorgehenden Ausdrücke resp. durch $\left.\sqrt{\frac{\bar{A} \mu_{1}}{-\tau}} U_{2}, \sqrt{\frac{\bar{A} \mu_{1}}{-\tau}} U_{3},{ }_{\mu_{1}}^{U_{1}}{ }^{1}\right)$ alsdann folgt aus (34):

$$
-\left(\begin{array}{l}
\varphi \\
\varphi
\end{array}\right)_{\gamma_{i k}}: \Gamma(\mu)=\frac{U_{2}{ }^{2}}{\mu^{2}}+2 \sqrt{\frac{\mathrm{A} \mu_{1}}{-\tau}} \frac{U_{2} U_{3}}{\mu}+\frac{U_{1}{ }^{2}}{\mu_{1}\left(\mu-\overline{\mu_{1}}\right)},
$$

und diese Gleichung liefert nach einer der in den oben behandelten speciellen Fällen angewandten Methoden:

$$
\begin{gathered}
\left\{\begin{array}{l}
\varphi(u, u)=2 \sqrt{\frac{\bar{A} \mu_{1}}{-\tau}} U_{2} U_{3}+U_{1}{ }^{2}, \\
\omega(u, u)=U_{1}^{2}+U_{2}^{2}
\end{array}\right. \\
\mu_{1} \varphi-\omega=\left\{2 \mu_{1} \sqrt{\frac{A \mu_{1}}{-\tau}} U_{3}-U_{2}\right\} U_{2} .
\end{gathered}
$$

Die Gleichungen (35), (37) und (38) lassen erkennen, dass nunmehr die confocale Schaar $\mu \varphi-\omega=0$ nur aus Parabeln besteht, welche sämmtlich die Gerade $X_{1}=0$ zur Axe haben; der gemeinsame im Endlichen gelegene Brennpunkt hat die Gleichung

$$
2 \mu_{1} \sqrt{\frac{\overline{\mathrm{A} \mu_{1}}}{-\tau}} U_{3}-U_{2}=0,
$$

der unendlich ferne ist $U_{2}=0$. Die Gerade $X_{2}=0$ ist die Scheitcltangente der Parabel $\varphi(u, u)=0,2 p=\frac{2}{\mu_{1}} \sqrt{\overline{\overline{\mathrm{A}} \mu_{1}}}$ der Parameter $\left.{ }^{2}\right)$. Ferner erkennt man, dass eine confocale Schaar nur aus Parabeln besteht, sobald auch nur eine Parabel der Schaar angehört.

1) Der Grund dieser Bezeichnungsweise liegt darin, dass die Relationen

sowie

$$
\Omega(x, x)=\tau X_{3}{ }^{2}, \quad \omega(u, u)=U_{1}{ }^{2}+U_{2}{ }^{2},
$$

erfüllt werden sollten.

$$
u_{1} x_{1}+u_{2} x_{2}+u_{3} x_{3}=U_{1} X_{1}+U_{2} X_{2}+U_{3} X_{3}
$$

2) Sobald man hier über das Vorzeichen der Wurzel verfügt hat, ist anch die Richtnng festgelegt, nach der sich die Parabel erstreckt. 
Uebrigens folgt wieder in derselben Weise wie bei (26) in $\$ 15$, dass durch jeden Punkt der Ebene zwei reelle der Schaar angehörige Parabeln hindurchgehen, und zwar schneiden sie sich in dem betreffenden Punkte rechtwinklig. Aus (35) geht ausserdem hervor, dass man eigentlich zwei Systeme von Parabeln in einer confocalen Schaar unterscheiden kann, indem sich je nach dem Vorzeichen ihres Parameters die Curven nach entgegengesetzten Seiten öffnen. Man kann auch zeigen, dass die zwei confocalen Parabeln, welche sich in irgend einem Punkte der Ebene schneiden, nicht demselben System angehören. Zu diesem Zweck wollen wir die Gleichung.(34) der confocalen Parabeln in Punktcoordinaten zunächst dadurch umformen, dass wir an Stelle des variabelen Parameters $\mu$ einführen $\lambda=\frac{\mu_{1}}{\mu}$ und $\frac{1}{\mu_{1}} \sqrt{\overline{\frac{-\tau}{A \mu_{1}}}}$ setzen gleich $\left.-p,{ }^{1}\right)$ wodurch sich (34) nach Multiplication mit $\frac{\mu}{\mu_{1}}$ verwandelt in:

$$
\begin{gathered}
\lambda p^{2} X_{3}{ }^{2}-2 p X_{2} X_{3}+\frac{X_{1}{ }^{2}}{1-\lambda}=0 \text { oder in } \\
\frac{X_{1}{ }^{2}}{1-\lambda}-2 p\left(X_{2}-\frac{p}{2} \lambda X_{3}\right) X_{3}=0 .
\end{gathered}
$$

Durch die Transformation $X_{1}=\xi_{1}, X_{2}=\xi_{2}+\frac{p}{2} \xi_{3}, X_{3}=\xi_{3}$ wird die Gerade $X_{2}=0$ parallel zu sich selbst verschoben, so dass sie durch den gemeinsamen Brennpunkt hindurchgeht; an Stelle von (39a) tritt alsdann

$$
\frac{\xi_{1}{ }^{2}}{1-\lambda}-2 p\left\{\xi_{2}+\frac{p}{2}(1-\lambda) \xi_{3}\right\} \xi_{3}=0,
$$

und wenn man $p(1-\lambda)$ ersetzt durch die mit $\lambda$ veränderliche Grösse $q$, welche für $\lambda=0$ den halben Parameter $p$ der Parabel $\varphi(u, u)=0$ gibt, so folgt

$$
\xi_{1}^{2}-2 q\left(\xi_{2}+\frac{q}{2} \xi_{3}\right) \xi_{3}=0 .
$$

Um die Grössen $q$ für diejenigen zwei Parabeln zu bestimmen, welche durch irgend einen Punkt $\eta$ der Ebene gehen, sind die Coordinaten $\eta_{1}, \eta_{2}, \eta_{3}$ dieses Punktes in (41) zu substituiren; nach Potenzen von $q$ geordnet erhält man

$$
\Psi(q) \equiv-q^{2} \eta_{3}{ }^{2}-2 q \eta_{2} \eta_{3}+\eta_{1}{ }^{2}=0 .
$$

Für $q= \pm \infty$ wird $\Psi(q)$ negativ, für $q=0$ positiv, mithin hat diese quadratische Gleichung stets eine positive und eine negative

1) Durch diese letzte Substitution erhält $\Phi(x, x)=0$ die gewöhnlich gebrïuchliche Gestalt $X_{1}^{2}-2 p X_{2} X_{3}=0$. 
Wurzel, d. h. die Parameter zweier durch einen Punkt $\eta$ der Ebene gehenden Parabeln einer confocalen Schaar sind von entgegengesetztem Vorzeichen. Wie (41) zeigt, gehören diese Curven also in der That den zwei entgegengesetzt gerichteten Systemen an ${ }^{1}$ ). Die beiden Parameter werden nach (42) entgegengesetzt gleich, wenn $\eta_{2}=0$, d. h. wenn der Punkt $\eta$ der Senkrechten angehört, welche im gemeinsamen Brennpunkt auf der Axe errichtet ist; nur dann sind die zwei zugehörigen Parabeln congruent, natürlich aber entgegengesetzt gerichtet.

Wie bei confocalen Ellipsen und Hyperbeln sich Curven derselben Art nicht in reellen Punkten schneiden, folgt auch jetzt wieder, dass Parabeln desselben Systems sich nicht in reellen Punkten schneideı.

Hiermit ist die Untersuchung des Falles einer verschwindenden Doppelwurzel von $\Gamma(\mu)=0$ erledigt; die Unterdeterminanten $\Gamma_{i k}$ konnten, wie wir sahen, für dieselbe nicht alle verschwinden, und es wäre nur noch die Frage zu beantworten, ob nicht vielleicht die $\Gamma_{i k}$ bei Substitution der von Null verschiedenen Wurzel $\mu_{1}$ verschwinden. Auch dieser Fall ist ausgeschlossen, denn, wie wir schon in der Fussnote zu S. 134 bei ähnlicher Gelegenheit zeigten, würde mit allen $\Gamma_{i k}$ auch $\frac{d \Gamma\left(\mu_{1}\right)}{d \mu}$ verschwinden, d. h. die kubische Gleichung $\Gamma(\mu)=0$ hätte noch eine zweite Doppelwurzel $\mu=\mu_{1}$, was unmöglich ist.

Bisher war vorausgesetzt worden, dass die Determinante $A$ voll $\varphi(u, u)=0$ nicht verschwinde; es kamen dann ausser dem allgemeinen Fall, in welchem die kubische Gleichung $\Gamma(\mu)=0$ verschiedene Wurzeln besitzt, nur zwei specielle Fälle in Betracht: die nicht verschwindende Doppelwurzel $\mu=\mu_{1}$, für welche alle Unterdeterminanten $\Gamma_{i k}$ zu Null werden und die Doppelwurzel $\mu=0$. Im allgemeinen Falle bestehen die Curven der confocalen Schaar aus Ellipsen und Hyperbeln, ausserdem sind drei Punktepaare in der Schaar enthalten: die zwei Brennpunktepaare und das imaginäre Kreispunktepaar. Im Falle der Doppelwurzel $\mu=\mu_{1}$ hat man eine Schaar concentrischer Kreise, ferner den gemeinsamen Mittelpunkt doppelt zählend, sowie das imaginäre Kreispunktepaar. Im Falle der Doppelwurzel $\mu=0$ besteht die Schaar aus Parabeln; ausserdem gehören hinzu das Brennpunktepaar (ein im Endlichen und ein im Unendlichen gelegener Brennpunkt) und das imaginäre Kreispunktepaar.

Es fragt sich nun, wie man diese Punktepaare findet, wenn die Determinante A von $\varphi(u, u)$ verschwindet, wenn also $\varphi(u, u)=0$

1) Vgl. zu dieser Darstellung Hesse: „Sieben Vorlesungen aus der analytischen Geometrie der Kegelschnitte“, Leipzig 1874, S. 52, oder auch Zeitschrift für Mathematik und Physik, hrsgg. von Schlömilch, Bd. 19, S. 52. 
selbst eine zerfallende Curve der Schaar ist. Man kann sich alsdann die confocale Schaar $\mu \varphi-\omega=0$ gegeben denken in der Form

$$
\begin{aligned}
\mu \varphi-\omega & \equiv \mu(\varphi-\nu \omega)+(\mu \nu-1) \omega \\
& \equiv(1-\mu \nu)\left\{\frac{\mu}{1-\mu \nu}(\varphi-\nu \omega)-\omega\right\}=0,
\end{aligned}
$$

wobei $\nu$ ein Werth sei, für den die Determinante von $\varphi-v \omega$ nicht verschwindet. Setzt $\operatorname{man} \frac{\mu}{1-\mu \nu}=\lambda$, so tritt in den bisherigen Formeln $\varphi-\nu \omega$ an Stelle von $\varphi$ und $\lambda$ an Stelle von $\mu$; die den Werthen $\lambda$ entsprechenden $\mu$ findet man aus $\mu=\frac{\lambda}{\lambda \nu+1}$.

Wenn es sich nur darum handelt, das Punktepaar zu finden, welches neben $\varphi(u, u)=0$ und $\omega(u, u)=0$ der confocalen Schaar angehört, kann man daher so verfahren, dass man $\varphi(u, u)$ ersetzt durch

$$
\dot{\varphi}(u, u)-v \omega(u, u) \text {, }
$$

wobei $v$ ein beliebiger Parameter ist, der nur so gewählt sein muss, dass die Determinante von $\varphi-\nu \omega$ nicht verschwindet. Die oben entwickelten Formeln wendet man alsdann an auf die.Schaar

$$
\lambda(\varphi-v \omega)-\boldsymbol{\omega}=0,
$$

d. h. man ersetzt in diesen Formeln den Ausdruck $\varphi$ durch $\varphi-\nu \omega$, oder die Coefficienten $\alpha_{i k}$ der Gleichung $\varphi=0$ durch $\alpha_{i k}-\nu \omega_{i k}$.

Besteht die confocale Schaar aus Ellipsen und Hyperbeln, so ist nach (18)

$$
\left\{\begin{aligned}
\varphi-\nu \omega & =\frac{U_{1}{ }^{2}}{\lambda_{1}}+\frac{U_{2}{ }^{2}}{\lambda_{2}}+\varphi(p, p) U_{3}{ }^{2} \\
\omega & =U_{1}{ }^{2}+U_{2}{ }^{2} .
\end{aligned}\right.
$$

Nimmt man an, dass $\operatorname{sich}(43 a)$ etwa für $\lambda_{1}=-\frac{1}{\nu}$ auf $\varphi=0$ reducire, so wird

$$
\varphi=\left(\frac{1}{\lambda_{2}}+\nu\right) U_{2}^{2}+\varphi(p, p) U_{3}^{2} .
$$

Wenn die confocale Schaar aus concentrischen Kreisen besteht, findet man nach (30):

daher

$$
\left\{\begin{aligned}
\varphi-\nu \omega & =\frac{U_{1}^{2}+U_{2}^{2}}{\lambda_{1}}+\varphi(p, p) U_{3}^{2} \\
\omega & =U_{1}^{2}+U_{2}^{2}
\end{aligned}\right.
$$

so dass für $-\nu=\frac{1}{\lambda_{1}}$ die Gleichung $\varphi=0$ den Mittelpunkt der Schaar darstellt. 
Besteht endlich die confocale Schaar aus Parabeln, so ist nach (37)

$$
\left\{\begin{aligned}
\varphi-\nu \omega & =2 \sqrt{\frac{\bar{A} \lambda_{1}}{-\tau}} U_{2} U_{3}+\frac{U_{1}^{2}}{\lambda_{1}} \\
\omega & =U_{1}^{2}+U_{2}^{2},
\end{aligned}\right.
$$

daher für $\nu=-\frac{1}{\lambda_{1}}$

$$
\varphi=2 \sqrt{\frac{A \lambda_{1}}{-\tau}} U_{2} U_{3}+\nu U_{2}^{2}
$$

dabei sind, wie erwähnt, in den rechts stehenden Ausdrücken die Grössen $\alpha_{i k}$ stets zu ersetzen durch $\alpha_{i k}-v \omega_{i k}$.

\section{$\S 19$.}

Weitere Untersuchungen über die Brennpunkte: Doppeltberührende Kreise, Sätze über Brennstrahlen.

Im vorhergehenden Paragraphen wurde u. A. gezeigt, dass die Brennpunktepaare der Curve $\varphi(u, \imath)=0$, welche eine Ellipse oder Hyperbel darstellen möge, gegeben sind durch die zwei Gleichungen

$$
\left\{\begin{array}{l}
\mu_{1} \varphi-\omega \equiv \frac{\mu_{1}-\mu_{2}}{\mu_{2}} U_{2}^{2}+\mu_{1} \varphi(p, p) \cdot U_{3}^{2}=0, \\
\mu_{2} \varphi-\omega \equiv \frac{\mu_{2}-\mu_{1}}{\mu_{1}} U_{1}^{2}+\mu_{2} \varphi(p, p) \cdot U_{3}^{2}=0 .
\end{array}\right.
$$

Ganz ähnliche Ausdrücke hat man für die Brennpunkte eines durch seine Gleichung in Punktcoordinaten gegebenen Kegelschnitts $f(x, x)=0$; dieselben finden sich in $\S 11$, Gl. (14) und speciell für die Ellipse in Gl. (16).

Um die Vorstellung zu fixiren, wollen wir noch annehmen, das reelle Brennpunktepaar sei

$$
\mu_{2} \varphi-\omega \equiv \frac{\mu_{2}-\mu_{1}}{\mu_{1}} U_{1}^{2}+\mu_{2} \varphi(p, p) \cdot U_{3}^{2}=0 ;
$$

die Coefficienten von $U_{1}{ }^{2}$ und $U_{2}{ }^{2}$ müssen alsdann entgegengesetzte Vorzeichen haben. Sind $B=0, B_{1}=0$ die Gleichungen der zwei reellen Brennpunkte, so ist demnach jedenfalls

$$
\left\{\begin{aligned}
\mu_{2} \varphi-\omega=B B_{1} & = \pm\left(\sigma_{1}{ }^{2} U_{1}^{2}-\sigma_{3}{ }^{2} U_{3}{ }^{2}\right), \\
\sigma_{1}{ }^{2}: \sigma_{3}{ }^{2} & =\left(\frac{1}{\mu_{2}}-\frac{1}{\mu_{1}}\right) \frac{1}{\varphi(p, p)} .
\end{aligned}\right.
$$

Hier gestattet die Identität $\mu_{2} \varphi-\omega \equiv B B_{1}$ eine einfache geometrische Deutung. Für jede Gerade, deren Coordinaten $u_{i}$ die Gleichnng $\varphi(u, u)=0$ erfüllen, also für jede Tangente des Kegelschnitts $\varphi$, folgt nämlich mit Rücksicht auf (1) in $\S 2$ sofort der Satz:

(3) Bei jedem Kegelschnitt ist das Product aus den Ab- 
ständen der beiden Brennpunkte von einer beliebigen Tangente constant, und zwar gleich dem Quadrat der halben kleinen Axe im Falle einer Ellipse, gleich dem Quadrat der halben Nebenaxe im Falle einer Hyperbel.

Der soeben angegebene Werth der Constante ergibt sich, wenn man die Tangente im einen Endpunkte der die reellen Brennpunkte verbindenden Axe zieht, bei der Ellipse noch einfacher mit Hilfe der Tangente in einem Scheitel der kleinen Axe.

Aus (2) folgt mit Hilfe eines beliebigen Multiplicators $\nu$ :

$$
\begin{gathered}
4 \nu\left(\mu_{2} \varphi-\omega\right)=\left[\nu B+B_{1}\right]^{2}-\left[\nu B-B_{1}\right]^{2}, \quad \text { oder } \\
4 \nu \omega-\left(\nu B-B_{1}\right)^{2} \equiv 4 \nu \mu_{2} \varphi-\left(\nu B+B_{1}\right)^{2}=0 .
\end{gathered}
$$

Diese Gleichung repräsentirt nach $(2)$ in $\$ 7$ ein System von Kreisen: reell für die positiven Werthe von $\nu$, imaginär für negative $\nu$. Uebrigens lassen sich beide Arten auch getrennt darstellen, wobei $\nu$ jedesmal als positiv angenommen werden darf; das reelle System ist nämlich alsdann durch (4a) gegeben, das imaginäre durch die aus (4) folgende Gleichung

$$
4 \nu \omega+\left(\nu B+B_{1}\right)^{2} \equiv 4 v \mu_{2} \varphi+\left(\nu B-B_{1}\right)^{2}=0 .
$$

Betrachten wir speciell das bei positivem $\nu$ reelle System von Kreisen (4a).

Aus (4a) (und ähnlich aus (5)) folgt alsdann, mit Rücksicht auf (2) in $\S 7$, dass diese Kreise den Mittelpunkt $\nu B-B_{1}=0$ haben und den Kegelschnitt $\varphi=0$ doppelt berühren, nämlich in den Berührungspunkten der vom Punkte $\nu B+B_{1}=0$ an die Curve gelegten Tangenten. Ausserdem sind $B=0, B_{1}=0, \nu B-B_{1}=0$ und $v B+B_{1}=0$ vier harmonisch gelegene Punkte. Genau dieselbe Betrachtung liesse sich, wie (38) in $\S 18$ zeigt, anstellen, wenn die Curve $\varphi(u, u)=0$ eine Parabel ist; man hat daher den Satz:

(6) Es gibt bei jedem Kegelschnitt ein System doppelt berührender Kreise, für welche der Mittelpunkt und der zugehörige Pol der Berührungssehne harmonisch liegen zu den zwei reellen Brennpunkten.

Um die Lage dieser Kreise im einzelnen zu untersuchen, sind die Fälle der Ellipse, Hyperbel und Parabel gesondert zu betrachten. Indem wir wegen des Näheren auf die Abhandlung von Steiner: „Elementare Lösung einer geometrischen Aufgabe, und über einige damit in Beziehung stehende Eigenschaften der Kegelschnitte" ${ }^{\text {(1) }}$ ver-

1) Crelle's Journal, Bd. 37, S. 174 ff., 1847, oder auch „Gesammelte Werke", Bd. II, S. $404 \mathrm{ff}$. 
weisen, wollen wir nur den Fall der Ellipse noch kurz berühren. Die Gleichung für die Mittelpunkte der einzelnen Kreise wird $\nu B-B_{1}=0$, oder nach (2) $v\left(\sigma_{1} U_{1}+\sigma_{3} U_{3}\right)-\left(\sigma_{1} U_{1}-\sigma_{3} U_{3}\right)=0$, d. h.

$$
(\nu-1) \sigma_{1} U_{1}+(\nu+1) \sigma_{3} U_{3}=0 ;
$$

der Mittelpunkt des dem Parameter $v$ entsprechenden Kreises hat daher die homogenen rechtwinkligen Coordinaten $\frac{(\nu-1) \sigma_{1}}{(\nu+1) \sigma_{3}}: 0: 1$. Der Radius $r$ ergibt sich nach (2) in $\S 7$ aus $r^{2}=\frac{4 \nu}{(\nu+1)^{2} \sigma_{3}^{2}}$. Wie man mit Hilfe der in der Differentialrechnung gebräuchlichen Methode findet, wird der Radius ein Maximum für $v=1$; ein Minimum $v=-1$ kommt nicht in Betracht, da ihm kein reeller Kreis entspricht. Der grösste Radius hat demnach die Länge

$$
\frac{1}{\sigma_{3}}=\sqrt{-\frac{1}{\mu_{2} \varphi(p, p)}}
$$

oder er ist gleich der halben kleinen Axe der Ellipse, denn wie aus (15) in $\S 18$ hervorgeht, sind $-\mu_{1} \varphi(p, p)$ und $-\mu_{2} \varphi(p, p)$ die reciproken Werthe $\frac{1}{a^{2}}$ und $\frac{1}{b^{2}}$ der Quadrate der halben Axen $a$ und $l$ der Ellipse. Für $v=0$ und $v=\infty$ erhält man Kreise vom Radius Null, nämlich die beiden Brennpunkte $B_{1}$ und $B$.

Ein zweites System von Kreisen erhält man, wenn dieselben Betrachtungen wie zuvor angestellt werden mit Bezug auf das imaginäre Brennpunktepaar des Kegelschnitts $\varphi(u, u)=0$. Bevor wir hierzu übergehen, möge die Aufgabe gelöst werden, zu dem imaginären Brennpunktepaare beliebig viele harmonische Polenpaare zu construiren.

Es sei wieder

$$
\mu_{2} \varphi-\omega \equiv B B_{1} \equiv \pm\left(\sigma_{1}{ }^{2} U_{1}{ }^{2}-\sigma_{3}{ }^{2} U_{3}{ }^{2}\right)=0
$$

die Gleichung für die zwei reellen Brennpunkte,

$$
\mu_{1} \varphi-\omega \equiv \nu_{2} U_{2}^{2}+\nu_{3} U_{3}^{2}=0
$$

diejenige 'für die imaginären. Es müssen dann natürlich die beiden reellen Grössen $\nu_{2}$ und $\nu_{3}$ gleiches Vorzeichen haben, denn andernfalls wäre $\nu_{2}{U_{2}}^{2}+v_{3} \dot{U}_{3}{ }^{2}$ in zwei reelle lineare Factoren zerlegbar. Ausserdem müssen die Grössen $v$ negativ sein, denn bei positiven $v$ wäre nach (8) der Kegelschnitt $\varphi(u, u)=0$, den wir als reell voraussetzen, gleich der Summe der drei Quadrate $U_{1}^{2}+\left(\nu_{2}+1\right) U_{2}^{2}+\nu_{3} U_{3}^{2}=0$, da $\omega=U_{1}{ }^{2}+U_{2}^{2}$. Man kann demnach die Gleichung (8) ersetzen durch

(9) $\mu_{1} \varphi-\omega \equiv-\left(T_{2}{ }^{2}+T_{3}{ }^{2}\right)=0$, wo $T_{2}{ }^{2}=-\nu_{2} U_{2}{ }^{2}, T_{3}{ }^{2}=-\nu_{3} U_{3}{ }^{2}$, oder auch durch 


$$
\mu_{1} \varphi-\omega \equiv-\left(T_{2} \cos t-T_{3} \sin t\right)^{2}-\left(T_{2} \sin t+T_{3} \cos t\right)^{2},
$$

wobei $t$ einen völlig willkürlichen Winkel bezeichnet. Führẹn wir zur Abkürzung ein $V \equiv T_{2} \cos t-T_{3} \sin t, W=T_{2} \sin t+T_{3} \cos t$, so verwandelt sich $(10)$ in

$$
\mu_{1} \varphi-\omega \equiv-\left(V^{2}+W^{2}\right) .
$$

Indem man diese Gleichung in Verbindung bringt mit (7), ergibt sich durch Elimination von $\varphi$ die Relation

oder auch

$$
\left(\mu_{1}-\mu_{2}\right) \omega=-\mu_{2}\left(V^{2}+W^{2}\right)-\mu_{1} B B_{1}
$$

$$
\left(\mu_{1}-\mu_{2}\right) \omega+\mu_{2} V^{2}=-\mu_{2} W^{2}-\mu_{1} B B_{1} .
$$

Bevor wir diese Gleichung geometrisch deuten, werde bemerkt, dass die Punkte $V=0$ und $W=0$, welchen Werth auch der Parameter $t$ haben mag, harmonisch liegen zu

$$
V+i W=0 \text { und } V-i W=0
$$

oder, was dasselbe bedeutet, zu $T_{2}+i T_{3}=0$ und $T_{2}-i T_{3}=0$, also zu dem imaginären Brennpunktepaar.

Die linke Seite von (12) stellt nun, gleich Null gesetzt, einen Kreis dar mit dem Mittelpunkt $V$, während die rechte Seite von (12) aussagt, dass auf diesem Kreis auch das reelle Brennpunktepaar liegt und dass die in den Brennpunkten gezogenen Tangenten sich in $W$ schneiden $^{1}$ ). Ist also z. B. der reelle Punkt $V$ gegeben und will man zu ihm und zu dem imaginären Brennpunktepaar, welches bei unseren obigen Annahmen zugleich mit $V$ auf der Geraden $X_{1}=0$ liegt, den vierten harmonischen Punkt $W$ construiren, so ziehe man um $V$ als Mittelpunkt den durch die reellen Brennpunkte $B, B_{1}$ gehenden Kreis; die Tangenten desselben in $B$ und $B_{1}$ schneiden sich in dem auf $X_{1}=0$ gelegenen Punkte $W$.

Dass dieser Kreis reell ist, geht nicht nur aus der Realität seines Mittelpunktes und der zwei Punkte $B, B_{1}$ hervor, sondern kann auch aus seiner Gleichung $\left(\mu_{1}-\mu_{2}\right) \omega+\mu_{2} V^{2}=0$ oder

$$
\left(\frac{\mu_{1}}{\mu_{2}}-1\right) \omega+V^{2}=0
$$

geschlossen werden. Die oben mit $\nu_{2}$ bezeichnete Grösse muss nämlich, wie wir sahen, bei unseren Annahmen negativ sein, andrerseits besitzt dieselbe nach (1) den Werth $\frac{\mu_{1}}{\mu_{2}}-1$, mithin ist in

$$
\left(\frac{\mu_{1}}{\mu_{2}}-1\right) \omega+V^{2}=0
$$

1) Vgl. hierzu den Schluss von $\$ 9$. 
die positive definite Form $\infty$ multiplicirt mit einer negativen Grösse, so dass diese Gleichung in der That eine reelle Curve repräsentirt.

Wenden wir uns uun zu dem oben erwähuten zweiten System von Kreisen!

Aus der Identität (11) folgt:

$$
V^{2}-\omega \equiv-\left(\mu_{1} \varphi+W^{2}\right)
$$

ein Ausdruck, der gleich Null gesetzt bei variabelem $t$ ein System von Kreisen darstellt, deren Mittelpunkte ${ }^{1}$ ) $V$ auf der Verbindungslinie der imaginären Brennpunkte liegen; es kann also von diesem Kreissystem eigentlich nur bei Ellipse und Hyperbel die Rede sein, da bei einer Parabel das imaginäre Brennpunktepaar im Endlichen fehlt. Indem man die Gleichung (13) in analoger Weise geometrisch deutet, wie es oben bei (4a) geschehen war, folgt der Satz:

(14) Bei jeder Ellipse und Hyperbel gibt es ein System doppelt berührender Kreise, für welche der Mittelpunkt und der zugehörige Pol der Berührungssehne harmonisch liegen zu dem imaginären Brenupunktepaar ${ }^{2}$.

Vermöge der Identität $V \equiv T_{2} \cos t-T_{3} \sin t$ kann jeder auf der Nebenaxe $X_{1}=0$ gelegene Punkt als Centrum eines den Kegelschnitt $\varphi(u, u)=0$ doppelt berührenden Kreises angesehen werden. (15) Das System von Kreisen (14) hat noeh die weitere interessante Eigenschaft, dass jeder solche Kreis der Ort der Fusspunkte der geraden Linien ist, welche von den Brennpunkten des gegebenen Kegelschnitts $\varphi(u, u)=0$ nach allen Tangenten von $\varphi$ unter demselben bestimmten Winkel $t$ gezogen werden ${ }^{3}$ ).

Wir wollen hier der Kürze halber nur eingehen auf den Fall $t=90^{\circ}$ und eine directe Behandlung des Problems geben. Es werde dabei wieder angenommen, dass das reelle Brennpunktepaar $B, B_{1}$ gegeben sei durch $\mu_{2} \varphi-\omega=0$. Soll eine Gerade mit den Coordinaten $u_{1}, u_{2}, u_{3}$ Tangente sein des gegebenen Kegelschnitts, so müssen die $u_{i}$ die Gleichung befriedigen $\varphi(u, u)=0$; andrerseits befriedigt eine durch einen reellen Brennpunkt gehende Gerade $v$ die Gleichung

1) Für den Radius $r$ findet man leicht nach Einsetzen des Werthes von $V$ mit Rücksicht auf (2) in § 7 die Formel $r=\frac{1}{\sin t} \sqrt{\frac{-1}{\mu_{1} \varphi(p, p)}}$, oder bei gleicher Bezeichnungsweise wie S. 181 wird $r=\frac{a}{\sin t}$.

2) Näheres über dieses System von Kreisen bei Steiner a. a. 0 .

3) Aucb hierzu vgl. Steiner a. a. 0. 


$$
\mu_{2} \varphi(v, v)=\omega(v, v) \text {. }
$$

Die Coordinaten $x_{1}, x_{2}, x_{3}$ des Schnittpunktes einer Tangente $u$ mit einer durch $B$ oder $B_{1}$ gehenden Geraden $v$ sind

$$
x_{1}=u_{2} v_{3}-u_{3} v_{2}, \quad x_{2}=u_{3} v_{1}-u_{1} v_{3}, \quad x_{3}=u_{1} v_{2}-u_{2} v_{1},
$$

und wir fragen nun nach dem geometrischen Ort aller Punkte $x$ von solcher Beschaffenheit, dass die von $x$ nach der Curve $\varphi(u, u)=0$ gezogenen Tangenten normal sind zu dem einen, resp. anderen der zwei von $x$ nach dem Brennpunktepaar gezogenen Strahlen. Der Ort der Punkte $x$ muss alsdann der oben gewünschte Kreis sein.

Um die Gleichung dieses Ortes aufzustellen, gehen wir aus von einer Identität, die für die Gleichung

$$
\begin{aligned}
2 \chi(x, x) & =\left(\alpha_{22} \omega_{33}+\alpha_{33} \omega_{22}-2 \alpha_{23} \omega_{23}\right) x_{1}{ }^{2}+\cdots \\
& +2\left(\alpha_{31} \omega_{32}+\alpha_{32} \omega_{31}-\alpha_{33} \omega_{12}-\alpha_{12} \omega_{33}\right) x_{1} x_{2}+\cdots=0
\end{aligned}
$$

des Directorkreises ${ }^{1}$ ) der Curve zweiter Classe

$$
\varphi(u, u) \equiv \sum_{i}^{3} \sum_{i}^{3} \alpha_{i k} u_{i} u_{k}=0
$$

besteht; diese Identität lautet ${ }^{2}$ ):

1) Vgl. (29) und (31) in § 15.

2) Zur Ableitung dieser Identität werde Folgendes bemerkt: Der Directorkreis ist nach $\S 15$ der geometrische Ort aller Punkte $x$, von denen zu einander rechtwinklige Tangenten an den Kegelschnitt $\varphi(u, u)=0$ gezogen werden können; diese Tangenten müssen somit harmonische Polarenpaare des Kreispunktepaars $\omega(u, u)=0$ sein. Ist nun ein beliebiger Punkt $x$ der Ebene der Schnittpunkt irgend zweier Geraden $u$ und $v$, so besitzt das Doppelverhältniss dieses Geradenpaares zu dem von $x$ an den Kegelschnitt $\varphi(u, u)=0$ gezogenen T'angentenpaare den Werth $\frac{x_{1}}{x_{2}}$, wobei $x_{1}$ und $x_{2}$ die Wurzeln sind der quadratischen Gleichung

$$
\varphi(v, v)+2 x \varphi(v, u)+x^{2} \varphi(u, u)=0 ;
$$

andrerseits besitzt das Doppelverhältniss des Geradenpaares $u, v$ zu den von $x$ aus nach den imaginären Kreispunkten gezogenen Geraden den Werth $\frac{\lambda_{1}}{\lambda_{2}}$, wobei $\lambda_{1}$ und $\lambda_{2}$ die Wurzeln sind der quadratischen Gleichung

$$
\omega(v, v)+2 \lambda \omega(v, u)+\lambda^{2} \omega(u, u)=0 .
$$

Es ergibt sich dies sofort aus den dualistisch entsprechenden Betrachtungen zu denen von S. $20 \mathrm{f}$. Sollen nun die vom Punkte $x$ an $\varphi(u, u)=0$ und an $\omega(u, u)=0$ gezogenen Tangentenpaare zu einander harmonisch sein, so muss, wie wir als bekannt voraussetzen, die Relation erfüllt sein:

$$
x_{1} x_{2}-\frac{1}{2}\left(x_{1}+x_{2}\right)\left(\lambda_{1}+\lambda_{2}\right)+\lambda_{1} \lambda_{2}=0,
$$

und wenn man hier für die Prodncte nnd Summen der Wurzeln der zwei in $x$ 


$$
2 \chi(x, x) \equiv \varphi(u, u) \cdot \omega(v, v)-2 \varphi(u, v) \cdot \omega(u, v)+\varphi(v, v) \cdot \omega(u, u),
$$

wobei für die $x_{i}, u_{i}$ und $v_{i}(i=1,2,3)$ die Relationen (16) gelten. In unserem Falle sollte die Gerade $u$ eine Tangente des gegebenen Kegelschnitts sein und mit der zugehörigen Geraden $v$ einen rechten Winkel bilden, daher ist $\varphi(u, u)=0$ und $\omega(u, v)=0$; ausserdem erfüllen, wie oben bereits erwähnt, die Coordinaten $v_{i}$ die Relation

$$
\mu_{2} \varphi(v, v)=\omega(v, v) \text {. }
$$

Die Gleichung (18) kann man somit im vorliegenden Falle ersetzen durch

$$
2 \chi(x, x)-\frac{\omega(u, u) \cdot \omega(v, v)}{\mu_{2}}=0 .
$$

Nun ist nach (13) in $\S 5$

$$
\omega(u, u) \cdot \omega(v, v)-\omega^{2}(u, v)=\Omega(x, x) \equiv \tau p_{.}{ }^{2} ;
$$

da aber $\omega(u, v)=0$, so lässt sich jetzt bereits an Stelle von $\omega(u, u) \cdot \omega(v, v)$ substituiren $\tau p_{x}{ }^{2}$, wodurch sich (19) verwandelt in

$$
2 \mu_{2} \chi(x, x)-\tau\left(p_{1} x_{1}+p_{2} x_{2}+p_{3} x_{3}\right)^{2}=0 .
$$

Dies ist die gewünschte Gleichung des Kreises; dass dieselbe wirklich einen Kreis darstellt, geht daraus hervor, dass sie sich von der Gleichung des Directorkreises $\chi(x, x)=0$ nur um das Glied $\tau p_{x}{ }^{2}$ unterscheidet. Ausserdem ist der Kreis (20) mit $\chi(x, x)=0$ concentrisch, daher nach $\S 15$ auch mit dem gegebenen Kegelschnitt $\varphi(u, u)=0$.

Rein geometrisch folgt auch, dass die Fusspunkte der Normalen, welche von den Brennpunkten auf die Scheiteltangenten der die Brennpunkte verbindenden Axe gefällt werden, mit den Scheiteln dieser Axe zusammenfallen; letztere ist daher ein Durchmesser des Kreises (20). Die Gleichung von (20) in Liniencoordinaten ergibt sich also aus (13), wenn man in $V$ setzt $t=90^{\circ} ;$ man erhält alsiann $T_{3}^{2}-\omega$, oder nach (9)

$$
-v_{3} U_{3}^{2}-\left(U_{1}^{2}+U_{2}^{2}\right)=0
$$

wofür mit Rücksicht auf (8) in diesem und (20) im vorhergehenden Paragraphen gesetzt werden kann

und $\lambda$ quadratischen Gleichungen die Coefficienten dieser Gleichung einführt, folgt nach Wegbringen des Nenners

$$
\varphi(u, u) \cdot \omega(v, v)-2 \varphi(u, v) \cdot \omega(u, v)+\varphi(v, v) \cdot \omega(u, u)=0 .
$$

Dieser Ausdruck kann sich also von $2 \chi(x, x)$ in (17) nur um einen Zahlenfactor unterscheiden; man findet denselben leicht gleich 1 . Wäre $\omega(u, u)=0$ eine beliebige Carve zweiter Classe, so würde übrigens dieselbe Identität stattfinden; nur würde dann $\chi(x, x)=0$ den bei (23) in $\S 15$ als harmonische Curve zweiter Ordnung bezeichneten Kegelschnitt darstellen. 


$$
U_{1}^{2}+U_{2}^{2}+\mu_{1} \cdot \varphi(p, p) U_{3}^{2}=0 .
$$

Ausserdem zeigt die Gleichung (15) in $\S 18$, dass $\mu_{1} \varphi(p, p)$ gleich $-\frac{1}{a^{2}}$ ist, wenn wir mit $a$ die halbe Länge derjenigen Axe des. Kegelschnitts $\varphi(u, u)=0$ bezeichnen, welche die reellen Brennpunkte verbindet ${ }^{1}$ ). Der Kreis (21a) oder (20) hat also (auch in Uebereinstimmung mit der Fussnote zu S. 183) den Radius $a$, falls der gegebene Kegelschnitt eine Ellipse oder Hyperbel ist.

Sollte $\varphi(u, u)=0$ eine Parabel darstellen, so würde $\chi(x, x)=0$ zufolge der Bemerkungen am Schlusse von $\S 15$ aus der Directrix und der unendlich fernen Geraden bestehen; die Curve (20) würde daher in eine Parallele zur Directrix und in die unendlich ferne Gerade zerfallen. Wie man geometrisch sofort einsieht und auch algebraisch leicht gezeigt werden kann, ist diese Parallele die Scheiteltangente. Auf Grund des Vorhergehenden kann man die zwei Sätze aussprechen:

(22) Die Fusspunkte der Normalen, welche von einem Brennpunkte einer Ellipse oder Hyperbel auf die Tangenten dieser Curve gefällt werden, liegen auf einem mit dem gegebenen Kegelschnitt concentrischen Kreis, der diejenige Hauptaxe des Kegelschnitts zum Durchmesser hat, welche die reellen Brennpunkte verbindet.

(23) Die Fusspunkte der Normalen, welche vom Brennpunkte einer Parabel auf die Tangenten dieser Curve gefällt werden, liegen auf der Scheiteltangente der Parabel.

Man kann auch zeigen, dass die zwei Systeme (6) und (14) die einzigen Systeme von Kreisen sind, welche den Kegelschnitt $\varphi(u, u)=0$ doppelt berühren. Die Gleichung irgend eines Kreises in Liniencoordinaten ist nämlich nach $\S 7$ von der Form $\omega-a C^{2}=0$, wo $a$ eine bestimmte Constante, $C=0$ die Gleichung des Mittelpunktes bedeutet. Soll dieser Kreis den Kegelschnitt $\varphi(u, u)=0$ doppelt berühren, so muss es einen Parameterwerth $\mu$ geben, für welchen $\mu \varphi-\left(\omega-a C^{2}\right)$ ein vollständiges Quadrat $P^{2}$ wird, wobei $P=0$ den Pol der Berührungssehne darstellt. Aus $\mu \varphi-\left(\omega-a C^{2}\right)=P^{2}$ folgt aber $\mu \varphi-\omega=P^{2}-a C^{2}$, und hier ist die rechte Seite dieser Gleichung in zwei lineare Factoren zerlegbar; lassen wir den Werth . $\mu=0$, dem das jetzt nicht in Betracht kommende imaginäre Kreispunktepaar entspricht, unberücksichtigt, so gibt es, wie wir in $\S 18$ sahen, nur noch zwei Werthe des Parameters $\mu$, für welche $\mu \varphi-\omega$

1) Vgl. hierzu auch die Bemerkungen zu (16) in $\$ 11$. 
zerfällt, und diese Werthe lieferten im allgemeinen Falle das reelle und imaginäre Brennpunktepaar. Beide Punktepaare wurden aber bei Ableitung der Sätze (6), resp. (14) zu Grunde gelegt.

Zur Ableitung weiterer wichtiger Sätze werde ausgegangen von der Gleichung (7):

$$
\mu_{2} \varphi-\omega \equiv B B_{1}=0 \text {, }
$$

die wieder das reelle Brennpunktepaar darstellen möge. Andrerseits wurde am Schlusse von $\S 9$ gezeigt, dass die Gleichung einer beliebigen nicht ausartenden Curve zweiter Classe $\varphi(u, u)=0$ in die Form gebracht werden kann:

$$
\varphi(u, u) \equiv a V_{1} V_{3}-b V_{2}{ }^{2}=0 .
$$

Hierbei sind $V_{1}=0, V_{3}=0$ die Gleichungen irgend zweier Punkte $P$ und $Q$ der Curve, $V_{g}=0$ repräsentirt den Pol der Verbindungssehne $P Q$, während $a$ und $b$ Coefficienten bezeichnen, auf deren nähere Bedeutung es jetzt nicht ankommt.

Aus (7) und (24) folgt

oder auch:

$$
\mu_{2}\left(a V_{1} V_{3}-b V_{2}^{2}\right)-\omega=B B_{1},
$$

$$
\mu_{2} a V_{1} V_{3}-B T_{1}=\mu_{2} b V_{2}^{2}+\omega .
$$

Die rechte Seite dieser Gleichungr liefert nun, grleich Null gesetzt, einen Kreis mit dem Mittelpunkte $V_{2}=0$; andrerseits zeigt die linke Seite von (25), dass die Verbindungslinien der Punkte $V_{1}=0$ und $B=0, V_{1}=0$ und $B_{1}=0, V_{3}=0$ und $B=0, V_{3}=0$ und $B_{1}=0$ Tangenten dieses Kreises sind. Es folgt also der Satz:

(26) Die zwei Strahlenpaare, welche irgend zwei Punkte $(P$ und $Q$ ) einer Curve zweiter Classe $K$ mit deren Brenupunkten verbinden, sind Tangenten eines Kreises, dessen Mittelpunkt der Pol der Verbindungslinie $P Q$ in Bezug auf $K$ ist.

Bei der Lage der Sehne $P Q$ sind drei verschiedene Fälle zu unterscheiden: 1) sie geht weder durch den Mittelpunkt noch durch einen Brennpunkt des gegebenen Kegelschnitts $\varphi(u, u)=0$; 2) die Sehne geht durch einen der beiden Brennpunkte; 3) sie ist ein Durchmesser der Curve $\varphi=0$.

Die wichtigste Anwendung des Satzes (26) im Falle 1) besteht in der Ableitung der Thatsache, dass bei einer Ellipse die Summe der Brennstrahlen eines Curvenpunktes, bei einer Hyperbel die Differenz der Brennstrahlen constant ist. Damit ist alsdann dieses für die mechanische Erzeugung der genannten Curven so wichtige Theorem 
von den Brennstrahlen hergeleitet ganz unabhängig von der Gleichung der Curve in Punktcoordinaten, sondern nur unter Anwendung der Liniencoordinaten.

Im ersten Falle ist nämlich das Vierseit, welches durch die von $P$ und $Q$ nach den Brennpunkten $B$ und $B_{1}$ des Kegelschnitts gezogenen Tangenten gebildet wird, einem Kreise umschrieben und daher nach einem elementaren Satze der Geometrie entweder die Summe oder die Differenz zweier Seiten dieses Vierseits resp. gleich der Summe oder Differenz der beiden anderen Seiten ${ }^{1}$ ).

Hat der Punkt $P$ von den beiden Brennpunkten die Entfernungen $r$ resp. $r_{1}, Q$ die Entfernungen $\varrho$ resp. $\varrho_{1}$, so ist hiernach entweder

$$
\left\{\begin{array}{l}
r+r_{1}=\varrho+\varrho_{1} \text { oder } \\
r-r_{1}=\varrho-\varrho_{1} \text { oder } \\
r-r_{1}=\varrho_{1}-\varrho .
\end{array}\right.
$$

Es sei nun $2 a$ der absolute Werth dieser drei Ausdrücke, $2 c$ die Entfernung der beiden Brennpunkte von einander; alsdann kann die Gleichung $r+r_{1}=2 a$ nur stattfinden, wenn $a>c$, denn in jedem Dreieck ist die Summe zweier Seiten grösser als die dritte Seite; andrerseits kann die Gleichung $r-r_{1}=2 a$ nur bestehen, wenn $a<c$, da in jedem Dreieck die Differenz zweier Seiten kleiner als die dritte Seite ist.

Für alle Punkte eines Kegelschnitts ist demnach entweder die Summe oder die Differenz der Brennstrahlen constant. So lange die zwei Brennpunkte im Endlichen liegen und die Summe der Brennstrahlen keinen unendlich grossen constanten Werth besitzt, schneidet die Verbindungslinie der Brennpunkte $B, B_{1}$ die Curve jedenfalls in zwei Punkten $A$ und $A_{1}$, welche auf den Verlängerungen der Strecke $B B_{1}$ über die zwei Brennpunkte hinaus gelegen sind, denn es muss $2 a>2 c$ sein, die Brennpunkte liegen daher zwischen den Scheiteln der Axe $A A_{1}$, der Kegelschnitt ist eine Ellipse. Wenn die Differenz der Brennstrahlen constant ist, trifft die Gerade $B B_{1}$ die Curve in zwei zwischen $B$ und $B_{1}$ gelegenen Punkten $A$ und $A_{1}$, denn es muss

1) Der Ausdruck „Vierseit" ist hier im allgemeinsten Sinne aufzufassen das Vierseit kann convex, concav und überschlagen sein. Die Lehrbücher der elementaren Geometrie enthalten den Satz vom "Tangentenvierseit" meist nur mit Bezug auf convexe Figuren und beschränken sich auf die Angabe, dass in den Tangentenvierseiten die Summe je zweier gegenüberliegenden Seiten dieselbe sei. Es war wohl Steiner, der zuerst auf diesen Mangel hinwies in seiner Note: „Ueber das dem Kreise umschriebene Viereck", Crelle's Journal, Bd. 32, S. 305-310, 1846, oder auch „Gesammelte Werke" Bd. II, S. 381-388. 
$2 a<2 c$ sein, die Brennpunkte liegen daher auf den Verlängerungen der Strecke $A A_{1}$, der Kegelschnitt ist eine Hyperbel ${ }^{1}$ ). Der Fall $a=c=\infty$, in welchem also einer der beiden Brennpunkte im Unendlichen liegt, liefert die Parabel.

In untenstehender Figur 8, welche den Fall erläutern soll, dass die gegebene Curve eine Ellipse ist, sind $G$ und $H$ die Berührungspunkte der Brennstrahlen $P B$, bezw. $P B_{1}$ mit dem durch (26) definirten Kreis, $M$ der Mittelpunkt dieses Kreises, $I$ und $K$ sind die Berührungspunkte der Brennstrahlen $Q B$, bezw. $Q B_{1}$. Da nun die Tangenten, welche von irgend einem Punkte der Ebene nach der Peripherie eines Kreises gezogen werden, bekanntlich gleiche Lünge haben, so ist $G B=I B$ und $H B_{1}=K B_{1}$, daher

$$
G B+H B_{1}=I B+K B_{1}
$$

hierfür kaun aber gesetzt werden

d. h.

$$
P B+P H+H B_{1}=I B+Q I+Q B_{1},
$$

$$
P B+P B_{1}=Q B+Q B_{1} \text { oder } r+r_{1}=\varrho+\varrho_{1} \text {. }
$$

Dass diese constante Summe gleich der grossen Axe der Ellipse ist, folgt sofort, wenn man den beliebig gew ̈̈̈llten Punkt $P$ in einen

Fig. 8.

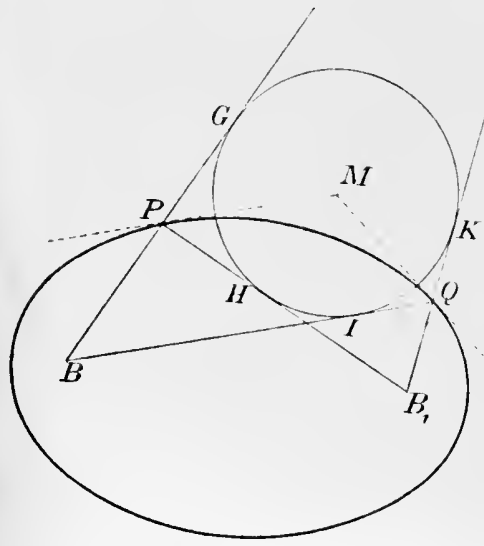

Fig. 9.

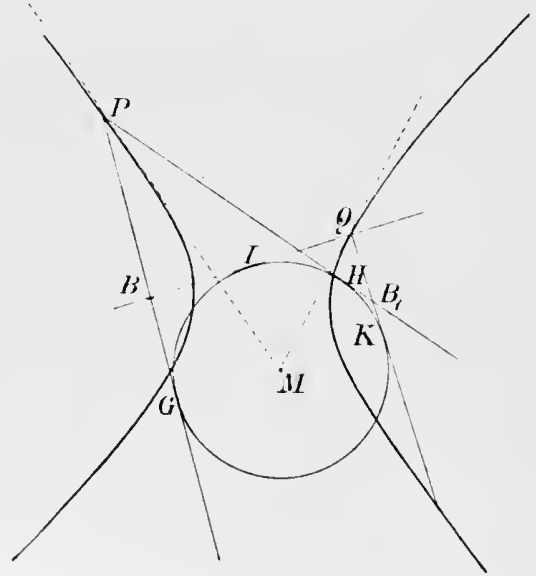

Scheitel dieser Axe legt. In Fig. 9, welche den Fall der Hyperbel erläutern soll, ist die Bezeichnung der in Betracht konmenden Punkte die entsprechende wie bei Fig. 8. Man hat alsdann die Gleichungen

$$
P H=P B+I B, \quad Q B_{1}+H B_{1}=Q I,
$$

1) Vgl. hierzu die andere Ableitung dieser Sätze am Schlnsse von $\S 11$. 
woraus durch Addition folgt

d. h.

$$
P H+H B_{1}+Q B_{1}=P B+I B+Q I
$$

$$
P B_{1}+Q B_{1}=P B+Q B \text { oder } r_{1}+\varrho_{1}=r+\varrho \text {, }
$$

also $r-r_{1}=\varrho_{1}-\varrho$. Hieraus geht auch hervor, dass die constante

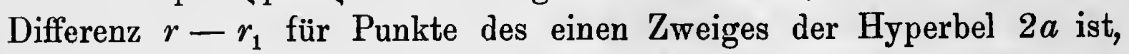
für Punkte des anderen Zweiges wird hingegen $\varrho-\varrho_{1}=-2 a$.

Da der Mittelpunkt $M$ des in (26) definirten Kreises der Pol der Sehne $P Q$, daher Schnittpunkt der in $P$ und $Q$ an den gegebenen Kegelschnitt gezogenen Tangenten ist, und da die Gerade PM nach einem Satze der elementaren Geometrie die Halbirungslinie des Winkels ist, den die zwei von $P$ an den Kreis gezogenen Tangenten mit einander bilden, so folgt (vgl. auch (27) in $\S 15$ ):

(28) Die Tangente in einem beliebigen Punkte $P$ eines Kegelschnitts bildet gleiche Winkel mit den von $P$ nach den Brennpunkten gezogenen Strahlen. Speciell im Falle einer Parabel bildet also die Tangente in $P$ gleiche Winkel mit zwei Strahlen, deren einer den Punkt $P$ mit dem im End-. lichen gelegenen Brennpunkt verbindet, während der andere durch $P$ parallel zur Axe der Parabel gezogen ist.

Will man näher untersuchen, welchen Winkel der zwei von $P$ nach den Brennpunkten gezogenen Strahlen die Tangente halbirt, so sind die drei Kegelschnitte Ellipse, Hyperbel und Parabel für sich zu betrachten.

Es sei zunächst die Curve eine Ellipse, jedoch seien nur der Curvenpunkt $P$ und die zwei Brennpunkte $B, B_{1}$ gegeben. Wir behaupten, dass in diesem Falle die Tangente den Nebenwinkel von

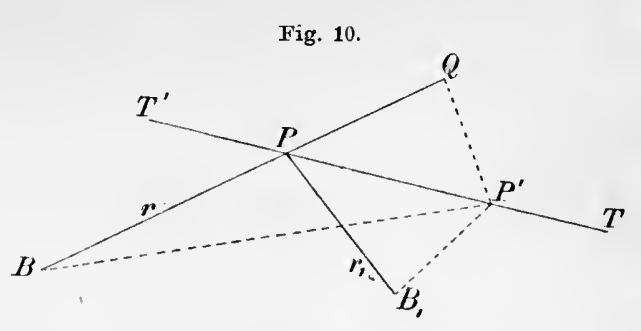
$\Varangle B P B_{1}$ halbirt (Fig. 10). Es lässt sich nämlich nachweisen, dass für jeden von $P$ verschiedenen Punkt $P^{\prime}$ der Halbirungslinie $P T$ die Summe der Brennstrahlen grösser ist als $r+r_{1}$. Um dies $\mathrm{zu}$ zeigen, verlängere man den einen Brennstrahl, z. B. $r$, um die Länge des anderen $r_{1}$; es sei $Q$ der Endpunkt dieser Verlängerung, also $B Q=r+r_{1}$. Setzt man noch $P^{\prime} B=\varrho, P^{\prime} B_{1}=\varrho_{1}$, so ist alsdann

$$
\varrho+\varrho_{1}=P^{\prime} B+P^{\prime} B_{1}=P^{\prime} B+P^{\prime} Q ;
$$

in Folge der Ungleichung $P^{\prime} B+P^{\prime} Q>B Q$ ist aber $\varrho+\varrho_{1}>r+r_{1}$, 
d. h. der auf $P T$ willkürlich gewählte Punkt $P^{\prime}$ kann nicht auf der Ellipse liegen; in ganz analoger Weise zeigt man, dass auch auf der Verlängerung der Geraden $T P$ in der Richtung nach $T^{\prime}$ kein Punkt der Ellipse liegen kann; die Gerade $T P$ hat folglich mit der Ellipse nur den Punkt $\boldsymbol{P}$ gemeinsam, sie ist eine Tangente der Curve.

Aehnlich zeigt man, dass im Falle einer Hyperbel die Tangente des Punktes $P$ denjenigen Winkelraum halbirt, welchen die Halbstrahlen $P B$ und $P B_{1}$ bilden (Fig. 11). Um dies zu zeigen, trage man den einen Brennstrahl, z. B. $r_{1}$, auf den anderen $r$ von $P$ aus ab, so dass $P Q=r_{1}$, daher

$$
P B-P Q=r-r_{1} \text {. }
$$

Für jeden von $P$ verschiedenen Punkt $P^{\prime}$ der Geraden $P T$ ist alsdann die Differenz der Bremnstrahlen $\varrho-\varrho_{1}$ gleicl $P^{\prime} B-P^{\prime} B_{1}=P^{\prime} B-P^{\prime} Q$. In Folge der für das Dreieck $B Q P^{\prime}$ bestehenden

Fig. 11.

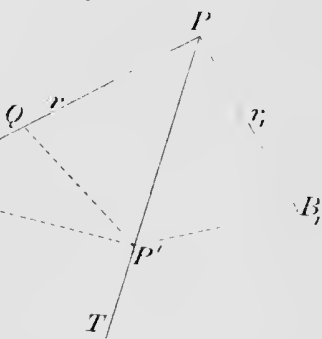
Ungleichung $P^{\prime} B-P^{\prime} Q<Q B$ ist aber $\varrho-\varrho_{1}<r-r_{1}$, d. h. der auf $P T$ willkürlich gewählte Punkt $P^{\prime}$ kann nicht auf der Hyperbel liegen; die Gerade $P T$ hat also nur den Punkt $P$ mit der Curve gemeinsam, sie ist eine Tangente der Curve.

Aus diesen für die Construction der Tangente bei Ellipse und Hýperbel gegebenen Beweisführungen folgt die Thatsache, dass es zu zwei gegebenen Punkten $B, B_{1}$ und zu einer festen Geraden $g$ stets einen Kegelschnitt gibt, welcher $B$ und $B_{1}$ zu Brenupunkten, $g$ zur Tangente hat, und zwar ist derselbe eine Ellipse oder Hyperbel, jc nachdem die Gerade die Verbindungslinie $B B_{1}$ ausserhalb der Strecke $B B_{1}$ oder zwischen den Punkten $B$ und $B_{1}$ trifft. Im ersten Falle hat der Berührungspunkt $P$ der Tangente die Eigenschaft, dass für ihn die Summe der Entfernungen von $B$ und $B_{1}$ kleiner ist, als für jeden anderen Punkt von $g$; im zweiten Falle ist für $P$ die Differenz der Entfernungen von $B$ und $B_{1}$ grösser als für jeden anderen Punkt von $g$.

Im Falle der Parabel sei $B$ der im Endlichen gelegene, $B_{1}$ der uuendlich ferne Brennpunkt; die Tangente $P T$ des Punktes $P$ halbirt alsdann den Nebenwinkel von $\Varangle B P B_{1}$ (Fig. 12), also den Winkel $B P Q$, welchen der Brennstrahl $P B$ mit dem auf die Directrix gefällten Lothe $P Q$ bildet. Es geht dies wieder daraus hervor, dass die Halbirungslinie $P T$ nur den Punkt $P$ mit der Parabel gemeinsam hat; für jeden von $P$ verschiedenen Punkt $P^{\prime}$ auf $P T$ ist nämlich 
$P^{\prime} Q=P^{\prime} B$, und diese Strecke $P^{\prime} B$ müsste auch gleich dem von $P^{\prime}$ auf die Directrix gefällten Lothe $P^{\prime} Q^{\prime}$ sein, es wäre mithin $P^{\prime} Q=P^{\prime} Q^{\prime}$, was unmöglich ist.

Aus dem Satze (26) lassen sich leicht noch weitere Folgerungen ziehen. Da der Pol der Sehne $P Q$ mit dem Mittelpunkte $M$ des in

Fig. 12.

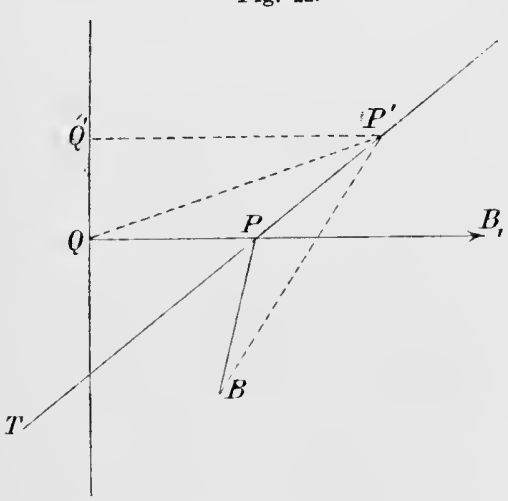

(26) definirten Kreises zusammenfällt und in Folge dessen der Winkel $G B Q$ durch $B M, \Varangle P B_{1} K$ durch $B_{1} M$ (Fig. 8 und 9) halbirt wird, ergibt sich:

(29) Die Verbindungslinie eines Brennpunktes mit dem Pol einer Sehne halbirt den Winkel des Geradenpaares, das den Brennpunkt mit den Endpunkten der Sehne verbindet $\left.{ }^{1}\right)$. Wir hatten oben (S. 187) für die Lage des durch (26) definirten Kreises drei verschiedene Fälle angenommen; nachdem wir bisher den ersten Fall betrachtet, möge nun untersucht werden, was eintritt, wenn die Sehne $P Q$ durch einen der Brennpunkte hindurchgeht (eine Focalsehne ist). Es wird sich das Resultat ergeben:

(30) Jede durch einen einzigen Brennpunkt $B$ des Kegelschnitts gezogene Sehne $P Q$ berührt in $B$ einen Kreis, dessen Mittelpunkt $M$ der Pol der Sehne $P Q$ ist; dieser Kreis hat auch die beiden Geraden zu Tangenten, welche die Endpunkte $P, Q$ der Sehne mit dem anderen Brennpunkte $B_{1}$ verbinden (Fig. 13).

Zum Beweis dieses Satzes gehen wir wieder aus von der Reiation (25) $\mu_{2} a V_{1} V_{3}-B B_{1}=\mu_{2} b V_{2}^{2}+\omega$, wobei $V_{1}=0, V_{3}=0$ Gleichungen waren für die Punkte $P, Q$, während $V_{2}=0$ den Pol der Sehne $P Q$ darstellte. Da im jetzigen Falle $P, Q$ und $B$ in einer Geraden liegen sollen, muss zwischen den Ausdrücken $V_{1}, V_{3}$ und $B$ eine lineare Identität stattfinden, d. h. mit $V_{1}=0$ und $V_{3}=0$ wird auch $B=0$ erfüllt; wie (25) zeigt, ist also die Gerade $P Q$ jedenfalls Tangente des Kreises mit dem Mittelpunkte $V_{2}=0$. Der Berührungspunkt ist Pol der Tangente in Bezug auf den Kreis. Es seien nun $W_{1}, W_{3}, \mathrm{~B}, \mathrm{~B}_{1}$ die Ausdrücke, in welche sich $V_{1}, V_{3}, B, B_{1}$ verwandelu

1) Weitere Folgerungen finden sich in dem zu vorliegendem Paragraphen gehörigen Theil des Anbanges. 
bei Substitution der Coordinaten der Geraden $P Q$ an Stelle der variabelen Liniencoordinaten. Die Gleichung des Pols von $P Q$ in Bezug auf den Kreis ist alsdann:

$$
W_{3} V_{1}+W_{1} V_{3}+\mathrm{B}_{1} B+\mathrm{B} B_{1}=0 ;
$$

da aber $W_{3}=0, W_{1}=0$ und $\mathrm{B}=0$ durch die Coordinaten der Geraden $P Q$ erfüllt werden, bleibt lediglich $\mathrm{B}_{1} B=0$ oder $B=0$ als Gleichung des Berührungspunktes, und hiermit ist (30) bewiesen.

Der Mittelpunkt $M$ des Kreises liegt als Pol einer Focalsehne natürlich auf der Polare des Brennpunktes $B, \mathrm{~d}$. h. auf der Directrix von $B$; da ferner beim Kreis der nach dem Berührungspunkt einer Tangente gezogene Radius zur Tangente rechtwinklig ist, folgt sofort:

(31) Die Polare eines a uf der Directrix gelegenen Punktes $M$ steht in dem der Di-

Fig. 13.

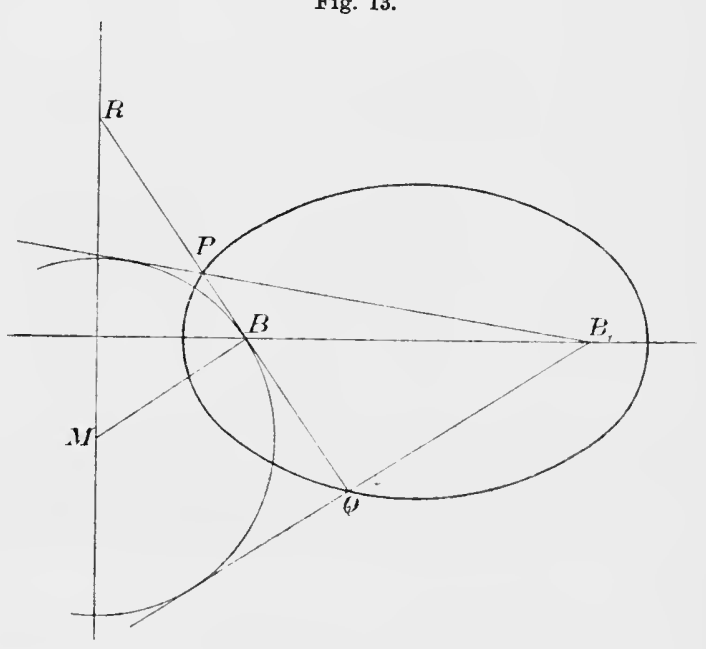
rectrix zugehörigen Brennpunkte $B$ senkrecht auf der Verbindungslinie $M B$, oder auch:

(32) Die Verbindungslinie des Pols einer Focalsehne mit dem zugehörigen Brennpunkt ist zur Focalsehne rechtwinklig.

Schneidet die Focalsehne $P Q$ die Directrix in $R$ (Fig. 13), so ist $R B M$ ein Poldreiseit des Kegelschnitts, denn die Gerade $R Q$ ist Polare von $M$, die Directrix $R M$ Polare des Brennpunktes $B$, daher auch $M B$ Polare von $R$. In Folge dessen sind $B M$ und $B R$ conjugirte Polaren in Bezug auf den Kegelschnitt und mit Rücksicht auf (32) erhält man den Satz:

(33) Zwei conjugirte Polaren eines Kegelschnitts, die sich in einem Brennpunkte der Curve schneiden, sind zu einander normal ${ }^{1}$ ).

Umgekehrt gilt auch der Satz:

1) Ein anderer Beweis dieses Satzes findet sich in dem zu vorliegendem Paragraphen gehörigen Theil des Anhanges. 
(34) Zwei in einem Brennpunkt sich rechtwinklig schneidende Geraden sind harmonische Polaren des Kegelschnitts.

Der leichte Beweis dieses Satzes möge dem Leser überlassen werden.

Da $M P$ die Curve zweiter Classe in $P$ berührt, folgt:

Das zwischen dem Berührungspunkt und einer Directrix gelegene Stück einer Tangente wird rom zugehörigen Brennpunkt aus unter rechtem Winkel gesehen.

Eigenartig ist der dritte Fall, welcher für die Lage der Sehne $P Q$ bei (26) in Betracht kommt, nämlich der Fall, dass $P Q$ ein Durchmesser der Curve $\varphi(u, u)=0$ ist. Hier rückt der Mittelpunkt $V_{2}=0$ des in (26) definirten Kreises als Pol der Geraden $P Q$ ins Unendliche, und der Kreis geht über in ein auf der unendlich fernen Geraden gelegenes Punktepaar. Setzt man nämlich den Ausdruck in (25) $\mu_{2} b V_{2}^{2}+\omega(u, u)$ gleich $\psi(u, u)$, so verschwindet, wenn $V_{2}=0$ einen unendlich fernen Punkt darstellt, nicht nur $\psi(p, p)$, sondern es verschwinden auch die drei Ausdrücke $\psi^{\prime}\left(p_{i}\right) \quad(i=1,2,3)$, wobei $p_{1}, p_{2}, p_{3}$ die Coordinaten der unendlich fernen Geraden bedeuten; nach den in $\$ 6$ aufgestellten Kriterien der Curven zweiter Classe ist dann in der That $\psi(u, u)=0$ die Gleichung eines im Unendlichen gelegenen Punktepaares.

Die zwei von den Endpuukten des Durchmessers $P Q$ nach dem einen Brennpunkte gezogenen Strahlen sind infolge der symmetrischen Gestalt. des Kegelschnitts zu den nach dem anderen Brennpunkte gezogenen parallel; diese zwei Paare paralleler Strahlen bestimmen daher die zwei Richtungen, welche nach den Punkten des oben erwähnten unendlich fernen Punktepaares verlaufen.

Wir hatten den wichtigen Satz (26) abgeleitet mit Hilfe der Relationen $\mu_{2} \varphi=B B_{1}+\omega$ und $\varphi=a V_{1} V_{3}-b V_{2}^{2}$; ersetzt man den letzteren Ausdruck von $\varphi(u, u)$ durch $U_{1} U_{2}-U_{3} U_{4}$, wobei $U_{1}=0$ und $U_{2}=0, U_{3}=0$ und $U_{4}=0$ die Gleichungen zweier Paare Gegenecken irgend eines dem Kegelschnitt $\varphi(u, u)=0$ umschriebenen Vierseits sind $^{1}$ ), so erhält man die Relation:

$$
\mu_{2}\left(U_{1} U_{2}-U_{3} U_{4}\right)=B B_{1}+\omega
$$

woraus folgt

$$
\mu_{2} U_{1} U_{2}-B B_{1}=\mu_{2} U_{3} U_{4}+\omega .
$$

Die rechte Seite dieser Gleichung repräsentirt gleich Null gesetzt einen Kegelschnitt, der die Punkte $U_{3}$ und $U_{4}$ zu Brennpunkten hat,

1) Vgl. (7) in § 12. 
während die linke Seite sofort ein diesem Kegelschnitt umschriebenes Vierseit erkennen lässt. Man hat daher den Satz:

(38) Wird einem Kegelschnitt $\varphi(u, u)=0$ ein beliebiges Vierseit umschrieben, so gibt es stets einen zweiten Kegelschnit, der irgend ein Paar von Gegenecken des Vierseits als Brennpunktepaar besitzt und gleichzeitig die beiden Strahlenpaare berührt, welche die Brennpunkte der Originalcurve $\varphi$ mit einem zweiten Paar von Gegenecken verbinden.

In dem speciellen Falle, dass das umschriebene Vierseit ein $\mathrm{Pa}$ ralleltrapez wird, rückt ein Punkt des Paares $U_{3}, U_{4}$ ins Unendliche; die Gleichung $\mu_{2} U_{3} U_{4}+\omega=0$ repräsentirt alsdann eine Parabel, und man kann daher im Anschluss an (37) und (38) den Satz aussprechen: (39) Wird einem Kegelschnitt $\varphi(u, u)=0$ ein beliebiges Paralleltrapez umschrieben, so gibt es stets eine Parabel, die den Schnittpunkt der nicht parallelen Seiten des Trapezes zum Brennpunkt hat und gleichzeitig die beiden Strahlenpaare berührt, welche die Brennpunkte des gergebenen Kegelschnitts mit einem der beiden übrigen Paare von Gegenecken verbinden.

\section{$\$ 20$.}

Ueber einige Curven, welche in invarianter Beziehung stehen zu einem Kegelschnittbüschel, resp. einer Kegelschnittschaar.

Es seien

$$
f(x, x) \equiv \sum_{1}^{3} \sum_{1}^{3} a_{i k} x_{i} x_{k}=0 \text { und } g(x, x) \equiv \sum_{1}^{3} \sum_{1}^{3} b_{i k} x_{i} x_{k}=0
$$

die Gleichungen zweier Curven zweiter Ordnung, $y_{1}, y_{2}, y_{3}$ die Coordinaten eines festen Punktes; alsdann gilt der Satz:

(1) Die Polaren eines festen Punktes $y$ in Bezug auf alle Kegelschnitte des Büschels $\lambda g(x, x)-f(x, x)=0$ schneiden sich in einem zweiten festen Punkte.

Die Polare des Punktes $y$ in Bezug auf irgend einen Kegelschnitt des Büschels hat nämlich die Gleichung:

(2) $\left\{\begin{array}{l}\left(\lambda g_{1}-f_{1}\right) y_{1}+\left(\lambda g_{2}-f_{2}\right) y_{2}+\left(\lambda g_{3}-f_{3}\right) y_{3}=0 \text { oder } \\ \lambda g(x, y)-f(x, y) \equiv \lambda\left(g_{1} y_{1}+g_{2} y_{2}+g_{3} y_{3}\right)-\left(f_{1} y_{1}+f_{2} y_{2}+f_{3} y_{3}\right)=0\end{array}\right.$

wobei

$$
\begin{aligned}
g_{i} & \equiv \frac{1}{2} g^{\prime}\left(x_{i}\right)=b_{1 i} x_{1}+b_{2 i} x_{2}+b_{3 i} x_{3} \\
f_{i} & \equiv \frac{1}{2} f^{\prime}\left(x_{i}\right)=a_{1 i} x_{1}+a_{2 i} x_{2}+a_{3 i} x_{3}
\end{aligned}
$$


und hier stellt (2) für alle beliebige Werthe des Parameters $\lambda$ gerade Linien dar, die sich in einem und demselben Punkte schneiden. Bezeichnen wir diesen Punkt, der als Schnittpunkt von $g(x, y)=0$ und $f(x, y)=0$ leicht zu ermitteln wäre, durch $x$, denken wir uns hingegen nun $y$ variabel, so sieht man sofort, dass auch umgekehrt die in Bezug auf alle Kegelschnitte des Büschels genommenen Polaren von $x$ durch den Punkt $y$ gehen. Infolge dieses gegenseitigen Entsprechens nennt man die beiden Punkte $x$ und $y$ conjugirt hinsichtlich der Kegelschnitte des Büschels.

An den Satz (1) reiht sich unmittelbar die Frage nach dem geometrischen Ort derjenigen zu Punkten $y$ in Bezug auf das Büschel $\lambda g-f=0$ conjugirten Punkte $x$, welche man erhält, wenn die $y$ auf einer und derselben Geraden mit den Coordinaten $u_{1}, u_{2}, u_{3}$ liegen. Dem Satze (1) zufolge genügt es, irgend zwei Kegelschnitte des Büschels, etwa $f=0$ und $g=0$, der Betrachtung zu Grunde zu legen. Es müssen dann zusammen bestehen die drei Gleichungen:

$$
\left\{\begin{array}{l}
f_{1} y_{1}+f_{2} y_{2}+f_{3} y_{3}=0 \\
g_{1} y_{1}+g_{2} y_{2}+g_{3} y_{3}=0 \\
u_{1} y_{1}+u_{2} y_{2}+u_{3} y_{3}=0
\end{array}\right.
$$

aus denen man durch Elimination von $y_{1}, y_{2}, y_{3}$ erhält:

$$
N \equiv\left|\begin{array}{lll}
f_{1} & f_{2} & f_{3} \\
g_{1} & g_{2} & g_{3} \\
u_{1} & u_{2} & u_{3}
\end{array}\right|=0 .
$$

Diese Gleichung stellt, da sie in den $x$ vom zweiten Grade ist, einen Kegelschnitt dar, und zu demselben Kegelschnitt gelangt man auch bei der Frage nach dem geometrischen Ort der in Bezug auf alle Kegelschnitte eines Büschels genommenen Pole einer und derselben Geraden mit den Coordinaten $u_{1}, u_{2}, u_{3}$. Zwischen den $u_{i}$ und den Coordinaten $x_{i}$ des zugehörigen Pols bestehen nämlich, wenn eine bestimmte Curve $\lambda g-f=0$ des Büschels zu Grunde gelegt wird, die Relationen

$$
\varrho u_{1}=\lambda g_{1}-f_{1}, \quad \varrho u_{2}=\lambda g_{2}-f_{2}, \quad \varrho u_{3}=\lambda g_{3}-f_{3},
$$

wo $\varrho$ einen Proportionalitätsfactor bedeutet. Durch Elimination von $\varrho$ und $\lambda$ erhält man hieraus die Gleichung für den Ort der Pole jener Geraden $u$ in Bezug auf alle Curven des Büschels in Gestalt einer Determinante, die sich von (4) nur durch Vertauschung der Horizontalund Verticalreihen unterscheidet, somit dieselbe Curve liefert wie (4). Wir können daher sagen: 
(6) Die in Bezug auf sämmtliche Kegelschnitte eines Büschels genommenen Pole einer gegebenen Geraden liegen auf einem und demselben Kegelschnitt $N$. Dieser ist zugleich der Ort aller derjenigen Punkte, welche den Punkten der Geraden im Sinne des Satzes (1) conjugirt sind.

Wir wollen den Kegelschnitt $N$ den Polkegelschnitt der gegebenen Geraden $u$ nennen. Es lassen sich leicht mehrere Punkte angeben, die ihm angehören. Solche Punkte sind z. B. die Spitzen der drei in dem Kegelschnittbüschel enthaltenen Geradenpaare, also die Ecken des dem Viereck der Grundpunkte zugehörigen Diagonaldreiecks oder (nach (17) in $\S 14$ ) die Ecken des gemeinsamen Pol. dreiecks aller Curven des Büschels. Man erhält nämlich, wie wir sahen, die Gleichung eines solchen Geradenpaares, wemn man in

$$
\lambda g(x, x)-f(x, x)=0
$$

für $\lambda$ eine Wurzel $\lambda^{\prime}$ einer gewissen kubischen Gleichung einsetzt; die Coordinaten der Spitze findet man hierauf aus den drei Gleichungen

$$
\lambda^{\prime} g_{i}-f_{i}=0 \quad(i=1,2,3) .
$$

Zufolge dieser Gleichungen werden aber die Ausdrücke $g_{i}$ den entsprechenden $f_{i}$ proportional, wenn man in diese Ausdrücke die Coordinaten der Spitze einführt; in (4) werden demnach zwei Reihen einander proportional, die Determinante (4) verschwindet, d. h. die Spitzen jener drei Geradenpaare liegen in der That auf dem Polkegelschnitt $N$ der Geraden $u$.

Sechs weitere Punkte von (4) sind die vierten harmonischen Punkte zu dem Schnittpunkte der Geraden $u$ mit irgend einer von den sechs Geraden des Büschels und zu den zwei auf der betreffenden Geraden liegenden Grundpunkten. Dies ergibt sich in folgender Weise.

Es seien $A, B, C, D$ die vier Grundpunkte des Büschels und $E$ der Schnittpunkt der Geraden $u$ mit der einem ausartenden Kegelschnitt des Büschels angehörigen Geraden $A D$. Die Polare des Punktes $E$ in Bezug auf das Geradenpaar $A B, D C$, das sich in $L$ schneiden möge, ist alsdann die Verbindungslinie von $L$ mit dem vierten harmonischen Punkte $E^{\prime}$ zu $E$ und zu dem Punktepaare $A, D$. Durch $E^{\prime}$ geht aber auch die Polare von $E$ in Bezug auf das Geralenpaar $A C, B D$, dessen Schnittpunkt $M$ sei. Baher ist $E^{\prime}$ ïberhaupt der Schnittpunkt aller Polaren des Punktes $E$ der Geraden $u$ in Bezug auf alle Kegelschnitte des Büschels, und zufolge (6) ist somit $E^{\prime}$ ein Punkt des Kegelschnitts $N$.

Analog ist es bei den übrigen Punkten, die zu den jeweiligen 
Schnittpunkten von $u$ mit den Geradenpaaren des Büschels in der angegebenen Weise harmonisch zugeordnet sind.

Da man zufolge des Vorausgehenden neun Punkte des Kegelschnitts $N$ leicht sofort construiren kann, bezeichnet man ihn bisweilen als "Kegelschnitt der neun Punkte".

Uebrigens lassen sich noch zwei weitere Punkte desselben angeben: die Doppelpunkte der Involution, welche nach (12) in $\S 15$ auf der Geraden $u$ von ihren Schnittpunkten mit den Curven des Büschels gebildet wird. Diese Doppelpunkte sind, wie wir in $\S 15$ sahen, die Berührungspunkte derjenigen zwei Curven des Büschels, welche die Gerade $u$ zur Tangente haben; sie sind also die Pole von $u$ in Bezug auf diese zwei berührenden Curven.

Ueberhaupt lassen sich selbstverständlich unendlich viele Punkte des Kegelschnitts $N$ dadurch finden, dass man zu einem beliebigen Punkte $P$ der gegebenen Geraden $u$ den im Sinne des Satzes (1) conjugirten Punkt $Q$ sucht. Da $Q$ Schnittpunkt der Polaren von $P$ in Bezug auf die Kegelschnitte des Büschels $\lambda g-f=0$ ist, wird man $Q$ am einfachsten dadurch erhalten, dass man die Polaren von $P$ in Bezug auf zwei dem Büschel angehörige Geradenpaare construirt. Man braucht dann nur $P$ mit den Spitzen dieser Geradenpaare zu verbinden und zu diesen Verbindungslinien und dem zugehörigen Geradenpaare jedesmal die vierte harmonische Gerade zu construiren; der Schnittpunkt dieser beiden vierten Harmonischen ist $Q$.

Eine andere Art der Construction von Punkten der Curve $N$ wäre endlich die, dass man in den Schnittpunkten der gegebenen Geraden mit irgend einem Kegelschnitt des Büschels die Tangenten zieht; dieselben schneiden sich in dem Pol der Geraden in Bezug auf den betreffenden Kegelschnitt, und dieser Pol ist nach (6) ein Punkt der Curve $N$.

Nach dieser allgemeinen Betrachtung des Kegelschnitts $N$ wollen wir als Gerade $u$ insbesondere die unendlich ferne Gerade wählen; die ihr zugehörigen Pole sind alsdann die Mittelpunkte der Kegelschnitte des Büschels, und so erhält man den Satz:

(7) Die Mittelpunkte der Curven zweiter Ordnung des Büschels $\lambda g(x, x)-f(x, x)=0$ liegen auf einem Kegelschnitt $M$; die Gleichung desselben ist:

$$
M \equiv\left|\begin{array}{lll}
f_{1} & f_{2} & f_{3} \\
g_{1} & g_{2} & g_{3} \\
p_{1} & p_{2} & p_{3}
\end{array}\right|=0,
$$


wobei $p_{1}, p_{2}, p_{3}$ die Coordinaten der unendlich fernen Geraden bedeuten.

Dieser "Mittelpunktskegelschnitt" geht, wie aus den vorhergehenden allgemeinen Betrachtungen folgt, durch die Spitzen der in den Büschel enthaltenen Geradenpaare, sowie durch die Mitten der sechs Seiten des durch die Grundpunkte des Büschels gebildeten vollständigen Vierecks. Da nun irgend vier Punkte der Ebene als Schnittpunkte zweier Kegelschnitte, daher als Grundpunkte eines Büschels betrachtet werden können, kann man den Satz aussprechen:

(8) Die Mittelpunkte der sechs Seiten eines vollständigen Vierecks liegen zugleich mit den Ecken des zugehörigen Diagonaldreiecks auf einem und demselben Kegelschnitt.

Da ferner nach (5) in $\S 15$ jede Gerade der Ebene, somit auch die unendlich ferne Gerade, von zwei Kegelschnitten des Büschels berührt wird, befinden sich in demselben zwei Parabeln, die freilich auch in eine einzige zusammenfallen oder imaginär werden können. Mit Rücksicht darauf, dass der Mittelpunktskegelschnitt $I I$ durch die Berührungspunkte dieser beiden Parabeln mit der unendlich fernen Geraden geht, erhält man den Satz:

(9) Die Asymptoten des Mittelpunktskegelschnitts sind parallel zu den Axen der zwei in dem Büschel vorhandenen Parabeln.

Von der Realität dieser Parabeln hängt somit die Realität der Asymptoten des Kegelschnitts $M$ ab, es folgt also weiter:

(10) Der Mittelpunktskegelschnitt ist eine Hyperbel, $\mathrm{Pa}$ rabel oder Ellipse, je nachdem sich dnrch die Grundpunkte des Büschels zwei verschiedene reelle Parabeln legen lassen, oder diese Parabeln in eine einzige zusammenfallen, oder imaginär sind.

Zu den Schnittpunkten des Kegelschnitts $M$ mit der unendlich fernen Geraden liegen nun nach (24) in $\$ 5$ nicht nur die unendlich fernen Punkte irgend zweier conjugirten Durchmesser von $M$ harmonisch, sondern nach (11) in $\S 15$ auch alle Punktepaare, in denen die unendlich ferne Gerade von Kegelschnitten des Büschels getroffen wird. Hieraus folgt:

(11) Die Asymptoten eines jeden dem Büschel angehörigen Kegelschnitts sind parallel zu einem Pare conjugirter Durchmesser des Mittelpunktskegelschnitts, sowie mit Berücksichtigung von (9):

(12) Bei einem jeden Kegelschnitt des Büschels ist ein 
bestimmtes Paar conjugirter Durchmesser parallel zu den Asymptoten des Mittelpunktskegelschnitts.

Wir wollen nun diejenigen Sätze aufstellen, die sich ergeben, wenn man die dualistisch entsprechenden Betrachtungen zu denen am Anfang des vorliegenden Paragraphen anstellt bei einer Kegelschnittschaar $\lambda \psi(u, u)-\varphi(u, u)=0$. Es sind alsdann Punkte zu ersetzen durch gerade Linien, u. s. w.

Dem Satze (1) entspricht der folgende:

(13) Die Pole einer festen Geraden $v$ in Bezug auf die Kegelschnitte einer Schaar liegen auf einer zweiten Geraden $u$, und umgekehrt liegen die Pole von $u$ auf der Geraden $v$.

Zwei sich in solcher Weise entsprechende Geraden bezeichnet man als conjugirt hinsichtlich der Kegelschnittschaar. Man sieht auch, dass für $\varphi(u, u)=0$ und $\psi(u, u)=0$ als Gleichungen zweier Curven der Schaar die Gerade $u$, welche den Ort der Pole der Geraden $v$ darstellt, die Gleichung hat

$$
\left|\begin{array}{lll}
\frac{1}{2} \varphi^{\prime}\left(v_{1}\right) & \frac{1}{2} \varphi^{\prime}\left(v_{2}\right) & \frac{1}{2} \varphi^{\prime}\left(v_{3}\right) \\
\frac{1}{2} \psi^{\prime}\left(v_{1}\right) & \frac{1}{2} \psi^{\prime}\left(v_{2}\right) & \frac{1}{2} \psi^{\prime}\left(v_{3}\right) \\
x_{1} & x_{2} & x_{3}
\end{array}\right|=0 .
$$

Wählt man als Gerade $v$ die unendlich ferne Gerade mit den Coordinaten $p_{1}, p_{2}, p_{3}$, so sind die zugehörigen Pole die Mittelpunkte der Kegelschnitte der Schaar; man hat somit den Satz:

Die Mittelpunkte aller Kegelschnitte der Schaar

$$
\lambda \psi(u, u)-\varphi(u, u)=0
$$

liegen auf einer Geraden; die Gleichung derselben ist

$$
\sum \pm\left(\frac{1}{2} \varphi^{\prime}\left(p_{1}\right) \quad \frac{1}{2} \psi^{\prime}\left(p_{2}\right) \quad x_{3}\right)=0
$$

Dem Satze (6) entspricht:

(16) Die in Bezug auf sämmtliche Kegelschnitte einer Schaar genommenen Polaren eines gegebenen Punktes y umhüllen einen und denselben Kegelschnitt N. Dieser ist zu. gleich die Enveloppe aller derjenigen Geraden, welche zu den durch den gegebenen Punkt gehenden Geraden im.Sinne des Satzes (13) conjugirt sind.

Die Gleichung des Kegelschnitts $N$ ist 


$$
\mathrm{N} \equiv\left|\begin{array}{lll}
\frac{1}{2} \varphi^{\prime}\left(u_{1}\right) & \frac{1}{2} \varphi^{\prime}\left(u_{2}\right) & \frac{1}{2} \varphi^{\prime}\left(u_{3}\right) \\
\frac{1}{2} \psi^{\prime}\left(u_{1}\right) & \frac{1}{2} \psi^{\prime}\left(u_{2}\right) & \frac{1}{2} \psi^{\prime}\left(u_{3}\right) \\
y_{1} & y_{2} & y_{3}
\end{array}\right|=0 .
$$

Aehnlich wie oben bei dem dualistisch entsprechenden Kegelschnitte $N$ lassen sich auch hier sofort mehrere Tangenten von $\mathrm{N}=0$ angeben. Es sind dies z. B. 1) die Träger der drei in der Kegelschnittschaar enthaltenen Punktepaare (oder die Seiten des dem Vierseit der gemeinsamen Tangenten aller Curven der Schaar zugehörigen Diagonaldreiseits); 2) sechs andere Tangenten erhält man folgendermassen: man verbindet den gegebenen Punkt $y$ mit den drei Punktepaaren der Kegelschnittschaar, also mit den sechs Ecken des der Schaar zu Grunde gelegten vollständigen Vierseits und zieht die vierte harmonische Gerade zu einer jeden dieser Verbindungslinien und zu den zwei Seiten des Vierseits, die sich in der betreffenden Ecke schneiden. Diese sechs vierten harmonischen Geraden sind alsdann Tangenten von N. 3) Die durch den gegebenen Punkt gezogenen Tangenten derjenigen zwei Kegelschnitte der Schaar, welche sich nach (21) in $\S 15$ in diesem Punkte schneiden, gehören gleichfalls der Curve zweiter Classe $\mathrm{N}$ an.

Unendlich viele Tangenten von $\mathrm{N}$ kann man natürlich durch die dualistische Construction zu jener erhalten, welche oben für beliebig viele Punkte des Kegelschnitts $N$ angegeben wurde. Es dürfte jedoch unnöthig sein, dies noch weiter auszuführen.

Wir wollen nun den Fall besonders betrachten, in welchem die zu Grunde liegende Kegelschnittschaar eine confocale Schaar

$$
\lambda \varphi(u, u)-\omega(u, u)=0
$$

ist. Alsdann ist der Kegelschnitt $N$ eine Parabel; denn er berührt die Träger der in der Schaar enthaltenen Punktepaare, und zu diesen Trägern gehört auch die unendlich ferne Gerade. Auch aus der Gleichung

$$
\left|\begin{array}{ccc}
\frac{1}{2} \varphi^{\prime}\left(u_{1}\right) & \frac{1}{2} \varphi^{\prime}\left(u_{2}\right) & \frac{1}{2} \varphi^{\prime}\left(u_{3}\right) \\
\frac{1}{2} \omega^{\prime}\left(u_{1}\right) & \frac{1}{2} \omega^{\prime}\left(u_{2}\right) & \frac{1}{2} \omega^{\prime}\left(u_{3}\right) \\
y_{1} & y_{2} & y_{3}
\end{array}\right|=0
$$

geht hervor, dass diese Curve von der unendlich fernen Geraden berührt wird; denn für $u_{i}=p_{i}(i=1,2,3)$ wird (18) erfüllt. Weitere Tangenten der Parabel (18) sind die Axen des confocalen Systems 
(als Verbindungslinien der reellen, resp. imaginären Brennpunkte), sowie die in dem gegebenen Punkte $y$ gezogenen Tangenten derjenigen zwei Kegelschnitte der Schaar, welche sich in $y$ schneiden. Da dieser Durchschnitt nach (26) in $\S 15$ unter rechtem Winkel stattfindet, kann man auch sagen, dass jene Parabel die in $y$ gezogenen zu einander senkrechten Normalen ${ }^{1}$ ) der beiden Kegelschnitte berührt, welche sich in $y$ schneiden. Wir wollen die Curve (18) als Steiner'sche Parabel des Punktes $y$ in Bezug auf den Kegelschnitt $\varphi(u, u)=0$ bezeichnen, da Steiner zuerst ihre Bedeutung für die Theorie der Kegelschnitte, insbesondere für die Construction des sogenannten Krümmungsradius, erkannte ${ }^{2}$ ). Bevor wir hierauf eingehen, sei noch erwähnt, dass die Gleichung (18) ungeändert bleibt, wenn man in ihr $\varphi^{\prime}\left(u_{i}\right)(i=1,2,3)$ ersetzt durch $\lambda \varphi^{\prime}\left(u_{i}\right)-\omega^{\prime}\left(u_{i}\right)$, wobei $\lambda$ ein beliebiger Parameter ist; es gibt demnach zu einem gegebenen Punkte $y$ in Bezug auf alle Kegelschnitte einer confocalen Schaar nur eine einzige Steiner'sche Parabel.

Aus dem Umstand, dass irgend eine Tangente $u$ der Steiner'schen Parabel das Normalencentrum $\omega^{\prime}\left(u_{i}\right)(i=1,2,3)$ hat, folgt sofort mit Rücksicht auf (18) das Theorem:

(18a) Die von einem Punkte $y$ auf irgend eine Tangente der Steiner'schen Parabel gefällte Normale geht durch den Pol dieser Tangente in Bezug auf den Kegelschnitt $\varphi(u, u)=0$. Insbesondere folgt hieraus:

(18b) Die Steiner'sche Parabel berührt auch die beiden Normalen in den Berührungspunkten der von $y$ an den Kegelschnitt $\varphi(u, u)=0$ gelegten Tangenten.

Für eine gemeinsame Tangente von $\varphi(u, u)=0$ und (18) hat man daher den Satz:

(18c) Die'vier gemeinsamen Tangenten der Steiner'schen Parabel und des gegebenen Kegelschnitts $\varphi(u, u)=0$ berïhren letzteren in den Fusspunkten der vier Normalen, die vom Punkte $y$ nach $\varphi(u, u)=0$ gezogen werden können.

Rückt speciell der Punkt $y$ auf den Kegelschnitt $\varphi(u, u)=0$, so fallen die in (18b) erwähnten beiden Normalen zusammen, und es ergibt sich der Satz:

(18d) Die Steiner'sche Parabel berührt die Normale des Punktes $y$ in dem zu $y$ gehörigen „Krümmungsmittelpunkte“,

1) Als Normale des Punktes $y$ einer Curve bezeichnet man die Gerade, welche in $y$ die Tangente dieses Punktes rechtwinklig schneidet.

2) Vgl. Steiner: „Ueber algebraische Curven und Flächen“, Crelle's Journal, Bd. 49, S. 339, 1854, oder auch "Gesammelte Werke“, Bd. II, S. 629. 
d. h. im Mittelpunkte desjenigen Kreises, der in y drei aufeinander folgende Punkte mit dem Kegelschnitte $\varphi(u, u)=0$ gemeinsam hat.

Dieser Mittelpunkt kann nämlich aufgefasst werden als der Schnittpunkt zweier jener auf einander folgenden Normalen.

Wir wollen nun annehmen, es sei $F(u, u)=0$ die Gleichung in Liniencoordinaten des Kegelschnitts $f(x, x)=0$; alsdann erhält man die Coordinaten des Pols $x$ irgend einer Tangente $u$ der Steiner'schen Parabel in Bezug auf $f(x, x)=0$ durch Auflösung der Gleichungen $\left.\sigma u_{i}=\frac{1}{2} f^{\prime}\left(x_{i}\right)^{1}\right)(i=1,2,3)$ nach $x_{1}, x_{2}, x_{3}$. Die Pole $x$ sämmtlicher Tangenten der Steiner'schen Parabel

$$
\frac{1}{4} \sum \pm\left(F^{\prime}\left(u_{1}\right) \omega^{\prime}\left(u_{2}\right) y_{3}\right)=0
$$

genügen daher einer Gleichung, die man erhält, wemn in (19) die $u_{i}$ ersetzt werden durch $\frac{1}{2} f^{\prime}\left(x_{i}\right)=f_{i}$; hierdurch verwandelt sich $F^{\prime \prime}\left(u_{i}\right)$ in $A x_{i}, \omega^{\prime}\left(u_{i}\right)$ in $\omega^{\prime}\left(f_{i}\right)$, und es ergibt sich daher, so lange man $A$ als von Null verschieden voraussetzt, als Ort der Pole die Curve zweiter Ordnung

$$
\sum \pm\left(\omega^{\prime}\left(f_{1}\right) x_{2} y_{3}\right)=0,
$$

welche der Steiner'schen Parabel reciprok entspricht und eine gleichseitige Hyperbel darstellt. Da der Punkt $y$ ganz willkürlich angenommen ist, folgt nämlich aus (20) die Proportion

$$
\omega^{\prime}\left(f_{1}\right): \omega^{\prime}\left(f_{2}\right): \omega^{\prime}\left(f_{3}\right)=x_{1}: x_{2}: x_{3},
$$

und unter Auwendung des Proportionalitätsfactors $\lambda$ hat man

$$
\lambda x_{i}=\frac{1}{2} \omega^{\prime}\left(f_{i}\right) \quad(i=1,2,3),
$$

oder wenn (ganz ebenso wie bei der Lösung des Hauptaxenproblems in $\S 10)$ gesetzt wird $\alpha_{i k}=\omega_{1 i} a_{1 k}+\omega_{2 i} a_{2 k}+\omega_{3 i} a_{3 k}$, so folgt

$$
\lambda x_{i}=\alpha_{i 1} x_{1}+\alpha_{i 2} x_{2}+\alpha_{i 3} x_{3} \quad(i=1,2,3) .
$$

Diese drei Gleichungen führen nach Elimination der $x_{i}$ zu derjenigen Gleichung dritten Grades in $\lambda$, welche bereits in $\S 10$ auftrat und nur reelle Wurzeln besitzt, darunter eine gleich Null. Der Wurzel $\lambda^{\prime \prime \prime}=0$ entspricht ein Punkt mit den Coordinaten

$$
x_{1}: x_{2}: x_{3}=\mathrm{A}_{i 1}: \mathrm{A}_{i 2}: \mathrm{A}_{i 3},
$$

wofür zufolge der Fussnote $S .86$ auch gesetzt werden kann

1) $\sigma$ bedeutet eiven Proportionalititsfactor. 


$$
\frac{1}{2} F^{\prime}\left(p_{1}\right): \frac{1}{2} F^{\prime}\left(p_{2}\right): \frac{1}{2} F^{\prime}\left(p_{3}\right),
$$

und dies sind die Coordinaten des Mittelpunktes der Curve $f(x, x)=0$. Ebenso folgt aus den Betrachtungen zu Anfang von $\S 10$, dass den zwei im allgemeinen von Null verschiedenen Wurzeln jener kubischen Gleichung die unendlich fernen Punkte der beiden Hauptaxen des Kegelschnitts $f(x, x)=0$ entsprechen; durch diese zwei Punkte geht daher die Curve (20) hindurch, d. h. ihre Asymptoten sind parallel zu den Hauptaxen von $f(x, x)=0$, sind somit zu eịnander normal, oder die Curve (20) ist eine gleichseitige Hyperbel. Dieselbe hat die wichtige Eigenschaft, dass sie durch die Fusspunkte der Normalen hindurchgeht, welche man von einem beliebigen Punkte $y$ der Ebene nach dem Kegelschnitt $f(x, x)=0$ ziehen kann. Um dies zu beweisen, wollen wir allgemeiner zeigen, dass von einem beliebigen Punkte $y$ in der Ebene $n^{2}$ Normalen nach einer Curve $n^{\text {ter }}$ Ordnung gezogen werden können und dass die $n^{2}$ Fusspunkte derselben zugleich mit $y$ auf einer Curve $n^{\text {ter }}$ Ordnung liegen. Beim Beweis dieses Satzes lässt sich wieder in sehr einfacher Weise der Begriff des Normalencentrums einer Geraden verwerthen. Sind nämlich $y_{1}, y_{2}, y_{3}$ die Coordinaten des gegebenen Punktes, $x_{1}, x_{2}, x_{3}$ diejenigen des Fusspunktes irgend einer von $y$ aus gezogenen Normalen, so ist dieser Fusspunkt $x$ zugleich Berührungspunkt einer Tangente mit den Coordinaten $f_{i}=\frac{1}{n} f^{\prime}\left(x_{i}\right),(i=1,2,3)$. Das Normalencentrum dieser Tangente, ihr Berührungspunkt $x$ und der gegebene Punkt $y$ liegen nun in einer Geraden, es ist also

$$
\left|\begin{array}{ccc}
\frac{1}{2} \omega^{\prime}\left(f_{1}\right) & \frac{1}{2} \omega^{\prime}\left(f_{2}\right) & \frac{1}{2} \omega^{\prime}\left(f_{3}\right) \\
x_{1} & x_{2} & x_{3} \\
y_{1} & y_{2} & y_{3}
\end{array}\right|=0,
$$

denn die Grössen $\omega^{\prime}\left(f_{i}\right)(i=1,2,3)$ sind nach $\S 2$ die Coordinaten des genannten Normalencentrums. Die Gleichung (22) ist in den $x_{i}$ vom $n^{\text {ten }}$ Grade; in Verbindung mit der Curve $n^{\text {ter }}$ Ordnung $f=0$ ergibt sie $n^{2}$ Werthsysteme $x_{1}: x_{2}: x_{3}$ für die Coordinaten von Fusspunkten der durch den Punkt $y$ gezogenen Normalen. Man erkennt auch sofort, dass $y$ auf der Curve (22) gelegen ist. Wir können das Resultat dieser Betrachtungen für den Fall, dass ein Kegelschnitt $f(x, x)=0 \mathrm{zu}$ Grunde gelegt ist, zusammenfassen in dem Satze:

(23) Von einem beliebigen Punkte $y$ der Ebene kann man nach einem Kegelschnitte $f(x, x)=0$ im allgemeinen vier Normalen ziehen. Die Fusspunkte derselben liegen a uf der 
gleichseitigen Hyperbel (22), welche durch den Punkt $y$ und den Mittelpunkt des gegebenen Kegelschnitts hindurchgeht, und deren Asymptoten zu den Hauptaxen dieses Kegelschnitts parallel sind.

Allgemeiner folgt unmittelbar aus (22) für irgend einen Punkt $x$ der gleichseitigen Hyperbel die Definition:

(23a) Die gleichseitige Hyperbel (22) ist der geometrische Ort aller Punkte $x$, deren (gerade) Polare in Bezug auf die Curve $f(x, x)=0$ normal ist zur Verbindungslinie von $x$ mit dem gegebenen Punkte $y$.

Wenn man in dem Ausdruck für den Polkegelschnitt $N$ in (4) die Curven $f(x, x)=0$ und $g(x, x)=0$ ersetzt durch irgend zwei andere Curven $x_{1} f+\lambda_{1} g=0$, resp. $x_{2} f+\lambda_{2} g=0$ des Büschels $\lambda g(x, x)-f(x, x)=0$, so reproducirt sich $N$, indem nur der Factor $x_{1} \lambda_{2}-x_{2} \lambda_{1}$ vortritt; Analoges gilt natürlich von $N$ in (17). Gebilde von dieser Beschaffenheit bezeichnet man allgemein als Combinanten. Es ist zu erwarten, dass zu ihnen auch derjenige Ausdruck gehört, welcher gleich Null gesetzt die vier Schnittpunkte der Kegelschnitte des Büschels $\lambda g(x, x)-f(x, x)=0$ darstellt, denn es muss gleichgiltig sein, welche zwei Curven des Büschels man für die Bestimmung der Schnittpunkte auswählt. Zum Zweck der Ableitung des betreffenden Ausdrucks denken wir uns den Kegelschnitt

$$
N=\frac{1}{4} \sum \pm\left(f^{\prime}\left(x_{1}\right) g^{\prime}\left(x_{2}\right) u_{3}\right)=0
$$

in die Form gebracht

$N_{11} x_{1}{ }^{2}+2 N_{12} x_{1} x_{2}+N_{22} x_{2}{ }^{2}+2 N_{13} x_{1} x_{3}+2 N_{23} x_{2} x_{3}+N_{33} x_{3}{ }^{2}=0$,

wobei die Coefficienten $N_{i k}$ in den $a_{i k}, b_{i k}$ und $u_{i}$ vom ersten Grad sind. Wir sahen S. 198, dass der Polkegelschnitt $N$ die Gerade $u$ in den Doppelpunkten der Involution trifft, welche auf $u$ von den Schnittpunkten mit den Curven des Büschels gebildet wird. Wenn diese zwei Doppelpunkte in einen zusammenrücken, berührt die Gerade $u$ ihren eigenen Polkegelschnitt; aus den Betrachtungen S. 144 folgt, dass dieses Zusammenrücken der Doppelpunkte nur dann eintreten kann, wenn die Gerade $u$ durch einen der vier Schnittpunkte geht. Die Bedingung dafür, dass die Gerade $u$ den ihr zugehörigen Kegelschnitt berührt, ist aber identisch mit der Gleichung der Curve

$$
\sum_{i}^{3} \sum_{1}^{3} N_{i k} x_{i} x_{k}=0
$$

in Liniencoordinaten; dieselbe ist in den $N_{i k}$ vom zweiten, also in 
den $u_{i}$ vom vierten Grad und muss daher gleichbedeutend sein mit dem Product der Ausdrücke für die vier Schnittpunkte der Kegelschnitte des Büschels.

Dualistisch entsprechend ist $\mathrm{zu}$ verfahren, wenn man aus

$$
\mathrm{N}=\frac{1}{4} \sum \pm\left(\varphi^{\prime}\left(u_{1}\right) \psi^{\prime}\left(u_{2}\right) y_{3}\right)=0
$$

die Gleichung für die vier Tangenten herleiten will, welche den Curven der Schaar $\lambda \psi(u, u)-\varphi(u, u)=0$ gemeinsam sind.

Der Polkegelschnitt $N$ ist auch von Wichtigkeit bei Ableitung einer Gleichung für die Seiten des gemeinsamen Poldreiecks der Curven des Büschels $\lambda g(x, x)-f(x, x)=0$. Nach S. 197 geht nämlich $N$ durch die Ecken dieses Dreiecks hindurch, gleichgiltig welche Werthe die Coordinaten der zugehörigen Geraden $u$ haben mögen; da aber $N$ nach (4) von der Form ist $N_{1} u_{1}+N_{2} u_{2}+N_{3} u_{3}=0$, wobei $\quad N_{1}=f_{2} g_{3}-f_{3} g_{2}, \quad N_{2}=f_{3} g_{1}-f_{1} g_{3}, \quad N_{3}=f_{1} g_{2}-f_{2} g_{1}$, so müssen in Folge der Willkürlichkeit der $u$ die drei Kegelschnitte $N_{i}=0(i=1,2,3)$ gleichfalls durch die Ecken des Poldreiecks gehen. Fixirt man nun auf irgend einer Seite dieses Dreiecks einen Punkt $x$ und construirt man den vierten harmonischen Punkt $y$ zu $x$ und zu den zwei auf der betreffenden Seite liegenden Ecken des Poldreiecks, so sind $x$ und $y$ harmonische Pole in Bezug auf jede der drei Curven zweiter Ordnung $N_{i}=0$, d. h. die Coordinaten $x_{i}$ und $y_{i}$ dieser Punkte erfüllen die drei Gleichungen

$$
y_{1} \frac{\partial N_{i}}{\partial x_{1}}+y_{2} \frac{\partial N_{i}}{\partial x_{2}}+y_{3} \frac{\partial N_{i}}{\partial x_{3}}=0 \quad(i=1,2,3)
$$

aus welchen durch Elimination der $y$ folgt:

$$
\sum \pm\left(\frac{\partial N_{1}}{\partial x_{1}} \frac{\partial N_{2}}{\partial x_{2}} \frac{\partial N_{3}}{\partial x_{3}}\right)=0
$$

Diese Gleichung wird, wie aus der Art und Weise ihrer Ableitung hervorgeht, stets von den Coordinaten $x_{i}$ solcher Punkte erfüllt, welche auf den Seiten des Poldreiecks liegen; da sie in den $x_{i}$ vom dritten Grad ist, muss sie daher mit dem Product der Ausdrücke für die Seiten des gemeinsamen Poldreiecks der Kegelschnitte des Büschels $\lambda g(x, x)-f(x, x)=0$ identisch sein.

Es seien nun $x_{1}, x_{2}, x_{3}$ die Coordinaten irgend eines der drei Schnittpunkte von $N_{1}=0, N_{2}=0, N_{3}=0$, also die Coordinaten einer Ecke des Poldreiecks; jede Gerade $u$, die durch einen der drei gemeinsamen Schnittpunkte geht, genügt alsdann einer Gleichung $u_{1} x_{1}+u_{2} x_{2}+u_{3} x_{3}=0$ oder $u_{x}=0$, mithin auch den drei Gleichungen $x_{1} u_{x}=0, x_{2} u_{x}=0, x_{3} u_{x}=0$. Diese repräsentiren zusammen mit 
$N_{i}=0(i=1,2,3)$ sechs Gleichungen, welche $x_{1}{ }^{2}, x_{2}{ }^{2}, x_{3}{ }^{2}, x_{1} x_{2}$, $x_{2} x_{3}, x_{3} x_{1}$ linear enthalten. Durch Elimination dieser sechs Grösseıı erhält man eine Determinante sechsten Grades, welche $u_{1}, u_{2}, u_{3}$ im dritten Grade enthält. Da dieselbe für jede Gerade $u$ verschwindet, welche durch irgend eine der Ecken des Poldreiecks geht, ist sie mit dem Product der Ausdrücke für diese Ecken identisch ${ }^{1}$ ).

Dualistisch entsprechend könnte man mit Hilfe des durch (17) definirten Kegelschnitts N Gleichungen ableiten für die Ecken und Seiten des gemeinsamen Poldreiecks aller Curven der Kegelschnittschaar $\lambda \psi(u, u)-\varphi(u, u)=0$.

Wählt man insbesondere eine confocale Schaar

$$
\lambda \varphi(u, u)-\omega(u, u)=0,
$$

so besteht das gemeinsame Poldreiseit nach $(20)$ in $\S 15$ aus den Hauptaxen des Kegelschnitts $\varphi(u, \imath)=0$ und aus der unendlich fernen Geraden; das Product der Ansdrücke für die Ecken dieses Poldreiseits (die unendlich feruen Punkte der Hauptaxen und den Mittelpunkt von $\varphi(u, u)=0$ ) ist also dualistisch zu (24) gegeben durch $\sum \pm\left(\frac{\partial \Pi_{1}}{\partial u_{1}} \frac{\partial \Pi_{2}}{\partial u_{2}} \frac{\partial \Pi_{3}}{\partial u_{3}}\right)=0$, wenn wir mit $\Pi_{1}, \Pi_{2}, \Pi_{3}$ die Coefficienten von $y_{1}, y_{2}, y_{3}$ in der Gleichung (18) der Steiner'schen Parabel bezeichnen. Das Product der Ausdrücke für die Hauptaxen von $\varphi(u, u)=0$ und für die unendlich ferne Gerade würde dualistisch zu vorausgehenden Betrachtungen durch eine Determinante sechsten Grades gegeben sein.

$\mathrm{Zu}$ derjenigen Gattung von Formen, die wir oben als Combinanten bezeichneten, gehört nach S. 205 auch die folgende:

$$
\psi(x, x) \equiv \frac{3}{2} \sum_{1}^{3} \sum_{i}^{3} \frac{\partial^{2} N}{\partial x_{i} \partial u_{k}} \frac{\partial^{2} N}{\partial x_{k} \partial u_{i}},
$$

wobei $N=\frac{1}{4} \sum \pm\left(\frac{\partial f}{\partial x_{1}} \frac{\partial g}{\partial x_{2}} u_{3}\right)$. Legt man insbesondere das gemeinsame Poldreieck des Büschels $\lambda g(x, x)-f(x, x)=0$ zu Grunde, so müssen $f(x, x)=0$ und $g(x, x)=0$ nach $(50)$ in $\S 4$ von der Form sein:

$$
\begin{aligned}
& f(x, x) \equiv \mu_{1} x_{1}{ }^{2}+\mu_{2} x_{2}{ }^{2}+\mu_{3} x_{3}{ }^{2}=0 \\
& g(x, x) \equiv \nu_{1} x_{1}{ }^{2}+\nu_{2} x_{2}{ }^{2}+\nu_{3} x_{3}{ }^{2}=0
\end{aligned}
$$

1) Auf Grund von Entwickelungen in $\$ 23$ kann man sagen, dass die Cayley'sche Curve des durch $N_{1}=0, N_{2}=0$ und $N_{3}=0$ bestimmten Kegelschnittnetzes in das Product der Ausdrücke für die Ecken des obigen Poldreiecks ausartet; auch wird dort eine einfache Methode zur Berechnung der Determinante sechsten Grades angegeben werden. 
für die Combinante $\psi$ findet man alsdann den Ausdruck

$$
\begin{gathered}
\frac{1}{3} \psi \equiv\left(\mu_{1} v_{2}-\mu_{2} \nu_{1}\right)\left(\mu_{3} v_{1}-\mu_{1} v_{3}\right) x_{1}{ }^{2}+\left(\mu_{2} v_{3}-\mu_{3} v_{2}\right)\left(\mu_{1} v_{2}-\mu_{2} v_{1}\right) x_{2}{ }^{2} \\
+\left(\mu_{3} \nu_{1}-\mu_{1} v_{3}\right)\left(\mu_{2} v_{3}-\mu_{3} v_{2}\right) x_{3}{ }^{2}=0
\end{gathered}
$$

wofür wir kürzer setzen wollen $A x_{1}{ }^{2}+B x_{2}{ }^{2}+C x_{3}{ }^{2}=0$. Diese Gleichung zeigt, dass der Kegelschnitt $\psi=0$ das Geradenpaar $A x_{1}{ }^{2}+B x_{2}{ }^{2}=0$ oder

$$
\left(\mu_{1} \nu_{2}-\mu_{2} \nu_{1}\right)\left\{\left(\mu_{3} \nu_{1}-\mu_{1} \nu_{3}\right) x_{1}{ }^{2}+\left(\mu_{2} \nu_{3}-\mu_{3} \nu_{2}\right) x_{2}{ }^{2}\right\}=0
$$

doppelt berührt in dessen Schnittpunkten mit $x_{3}=0$. Durch die Ecke $x_{1}=x_{2}=0$ des Poldreiecks geht nun ausser den zwei Seiten $x_{1}=0$ und $x_{2}=0$ noch ein dem Kegelschnittbüschel angehöriges Geradenpaar; die drei Geradenpaare des Büschels erhält man nämlich aus $\lambda g(x, x)-f(x, x)=0$ für die Werthe $\mu_{i}: \nu_{i}(i=1,2,3)$ des Parameters $\lambda$, und speciell $\lambda=\mu_{3}: \nu_{3}$ liefert

$$
\left(\mu_{3} \nu_{1}-\mu_{1} v_{3}\right) x_{1}{ }^{2}+\left(\mu_{3} \nu_{2}-\mu_{2} \nu_{3}\right) x_{2}{ }^{2}=0,
$$

also ein Geradenpaar, das zu $A x_{1}{ }^{2}+B x_{2}{ }^{2}=0$ harmonisch liegt. Andrerseits sind auch die Seiten $x_{1}=0$ und $x_{2}=0$ des Poldreiecks harmonisch zu $A x_{1}{ }^{2}+B x_{2}{ }^{2}=0$, und da dieses Dreieck zugleich Diagonaldreieck des durch die Grundpunkte des Kegelschnittbüschels bestimmten Vierecks ist, so folgt:

(28) Durch jeden Scheitel des Diagonaldreiecks gehen zwei Diagonalen und ein Geradenpaar des Kegelschnittbüschels. Diese zwei Strahlenpaare bestimmen eine Involution, deren Doppelstrahlen den Kegelschnitt $\psi(x, x)=0$ in dessen Schnittpunkten mit der dritten Diagonale berühren ${ }^{1}$ ).

\section{$\S 21$.}

\section{Krümmungskreis und Evolute.}

Bereits im vorhergehenden Paragraphen wurden wir gelegentlich der Untersuchung der Steiner'schen Parabel zu dem Krümmungskreis irgend eines auf dem Kegelschnitt gelegenen Punktes geführt. Wir wollen nun zeigen, wie man die Coordinaten des Mittelpunktes und

1) Weitere Eigenschaften des Kegelschnitts $\psi=0$ findet man in Herru Gundelfinger's Note „Zur Theorie des Kegelschnittbüschels", Zeitschrift für Mathematik und Physik, hrsgg. von Schlömilch, 20. Jahrgang, S. 153-159, 1874; vgl. auch „Ueber das Schliessungsproblem bei zwei Kegelschnitten", Crelle's Journal, Bd. 83, S. 172f., 1877. Ueber die Bedeutung der Form $\psi(x, x)$ bei Ableitung der Realitätskriterien für die Schnittpunkte zweier Kegelschnitte gibt der Anhang zu diesen Vorlesungen nähere Auskunft. 
die Länge des Radius dieses Kreises findet, und zwar möge hierbei ganz allgemein eine Curve $k^{\text {ter }}$ Classe $\varphi\left(u_{1}, u_{2}, u_{3}\right)=0$ zu Grunde gelegt werden. Unter der Voraussetzung, dass $u_{1}, u_{2}, u_{3}$ die Coordinaten einer Tangente dieser Curve, mithin $\varphi^{\prime}\left(u_{1}\right), \varphi^{\prime}\left(u_{2}\right), \varphi^{\prime}\left(u_{3}\right)$ die Coordinaten des Berührungspunktes $P$ sind, besteht also unsere nächste Aufgabe darin, den Mittelpunkt des dem Berührungspunkte $P$ der Tangente $u$ zugehörigen Krümmungskreises zu bestimmen. Jedenfalls liegt dieser Mittelpunkt zugleich mit $P$ und mit dem Normalencentrum der Tangente $u$ auf einer und derselben Geraden; sind $y_{1}$, $y_{2}, y_{3}$ die Coordinaten des Krümmungsmittelpunktes, so hat man demnach die drei Gleichungen

$$
\varrho y_{i}=\frac{1}{k} \varphi^{\prime}\left(u_{i}\right)+\frac{\lambda}{2} \omega^{\prime}\left(u_{i}\right) \quad(i=1,2,3),
$$

wo $\varrho$ einen Proportionalitätsfactor, $\lambda$ eine noch näher zu bestimmende Grösse bedeutet.

Für den Berührungspunkt der zunächst benachbarten Tangente mit den Coordinaten $u_{i}+d u_{i}(i=1,2,3)$ werden die Coordinatell des Krümmungsmittelpunktes dieselben wie in (1), also mit Anwendung des Proportionalitätsfactors $\mu$ :

$$
\begin{gathered}
\varrho y_{i}=\mu\left\{\frac{1}{k} \varphi^{\prime}\left(u_{i}\right)+\frac{1}{k} d \varphi^{\prime}\left(u_{i}\right)+\frac{(\lambda+d \lambda)}{2}\left(\omega^{\prime}\left(u_{i}\right)+d \omega^{\prime}\left(u_{i}\right)\right)\right\} \\
(i=1,2,3),
\end{gathered}
$$

so dass man nach Subtraction der Gleichungen (1) und (2) und mit Vernachlässigung der unendlich kleinen Grössen zweiter Ordnung $d \lambda \cdot d \omega^{\prime}\left(u_{i}\right)$ erhält

$$
\begin{aligned}
(3)\left[\frac{1}{k} \varphi^{\prime}\left(u_{i}\right)+\frac{\lambda}{2} \omega^{\prime}\left(u_{i}\right)\right](1-\mu) & =\mu\left[\begin{array}{l}
1 \\
k
\end{array} d \varphi^{\prime}\left(u_{i}\right)+\frac{d \lambda}{2} \omega^{\prime}\left(u_{i}\right)+\frac{\lambda}{2} d \omega^{\prime}\left(u_{i}\right)\right] \\
(i & =1,2,3) .
\end{aligned}
$$

Man kann nun leicht zeigen, dass der Factor $\mu$ gleich der Einheit sein muss. Zu dem Zweck multipliciren wir die drei Gleichungen (3) resp. mit $p_{i}$, wobei $p_{i}(i=1,2,3)$ die Coordinaten der unendlich fernen Geraden bedeuten, und erhalten unter Berücksichtigung von $\boldsymbol{\omega}^{\prime}\left(p_{i}\right)=0$ die Relation

oder auch

$$
\begin{aligned}
& {\left[\varphi^{\prime}\left(p_{1}\right) u_{1}+\varphi^{\prime}\left(p_{2}\right) u_{2}+\varphi^{\prime}\left(p_{3}\right) u_{3}\right](1-\mu)} \\
& \quad=\mu\left[\varphi^{\prime}\left(p_{1}\right)^{\circ} \cdot d u_{1}+\varphi^{\prime}\left(p_{2}\right) \cdot d u_{2}+\varphi^{\prime}\left(p_{3}\right) \cdot d u_{3}\right],
\end{aligned}
$$


Unter der Annahme, dass der Berührungspunkt der Tangente $u$ in Endlichen liege, ist $\left.\varphi(p, u) \gtrless 0^{1}\right)$; daher können die Glieder mit $d u_{i}$ als Factor vernachlässigt werden, es ergibt sich also in der That $\mu=1$. In Folge dessen sind die Gleichungen (3) zu ersetzen durch

$$
\frac{1}{k} d \varphi^{\prime}\left(u_{i}\right)+\frac{\lambda}{2} d \omega^{\prime}\left(u_{i}\right)+\frac{d \lambda}{2} \omega^{\prime}\left(u_{i}\right)=0 \quad(i=1,2,3) .
$$

Nun ist $d \varphi^{\prime}\left(u_{i}\right)=\frac{\partial^{2} \varphi}{\partial u_{i} \partial u_{1}} d u_{1}+\frac{\partial^{2} \varphi}{\partial u_{i} \partial u_{2}} d u_{2}+\frac{\partial^{2} \varphi}{\partial u_{i} \partial u_{3}} d u_{3}$, und wemn man die Bezeichnungsweise einführt

$$
\varphi_{i l}=\frac{1}{k(k-1)} \frac{\partial^{2} \varphi}{\partial u_{i} \partial u_{l}}, \quad \omega_{i l}=\frac{1}{2} \frac{\partial^{2} \omega}{\partial u_{i} \partial u_{l}},
$$

erhält man

$$
\begin{aligned}
& \frac{1}{k} d \varphi^{\prime}\left(u_{i}\right)=\left(l_{i}-1\right)\left\{\varphi_{i 1} d u_{1}+\varphi_{i 2} d u_{2}+\varphi_{i 3} d u_{3}\right\} \\
& \frac{1}{2} d \omega^{\prime}\left(u_{i}\right)=\omega_{i 1} d u_{1}+\omega_{i 2} d u_{2}+\omega_{i 3} d u_{3},
\end{aligned}
$$

es geht also (5) über in

$$
\begin{aligned}
\left\lceil(k-1) \varphi_{i 1}\right. & \left.+\lambda \omega_{i 1}\right] d u_{1}+\left[(k-1) \varphi_{i 2}+\lambda \omega_{i 2}\right] d u_{2} \\
& +\left[(k-1) \varphi_{i 3}+\lambda \omega_{i 3}\right] d u_{3}+\frac{d \lambda}{2} \omega^{\prime}\left(u_{i}^{3}\right)=0 .
\end{aligned}
$$

Da auch die Gerade mit den Coordinaten $u_{i}+d u_{i}$ Taugente der Curve $\varphi(u, u)=0$ sein soll, tritt zu diesen Gleichungen noch

$$
\varphi_{1} d u_{1}+\varphi_{2} d u_{2}+\varphi_{3} d u_{3}=0, \text { wobei } \varphi_{i}=\frac{1}{k} \frac{\partial \varphi}{\partial u_{i}},
$$

so dass man durch Elimination von $d u_{1}, d u_{2}, d u_{3}$ und $d \lambda$ aus (7) und (8) erhält:

$$
\left|\begin{array}{cccc}
(k-1) \varphi_{11}+\lambda \omega_{11} & (k-1) \varphi_{12}+\lambda \omega_{12} & (k-1) \varphi_{13}+\lambda \omega_{13} & \omega_{1} \\
(k-1) \varphi_{21}+\lambda \omega_{21} & (k-1) \varphi_{22}+\lambda \omega_{22} & (k-1) \varphi_{23}+\lambda \omega_{23} & \omega_{2} \\
(k-1) \varphi_{31}+\lambda \omega_{31} & (k-1) \varphi_{32}+\lambda \omega_{32} & (k-1) \varphi_{33}+\lambda \omega_{33} & \omega_{3} \\
\varphi_{1} & \varphi_{2} & \varphi_{3} & 0
\end{array}\right|=0 .
$$

Diese Determinante hat nun gleiche Gestalt wie (5) in $\S 17$; bei Entwickelung nach Potenzen von $\lambda$ enthält daher der Coefficient von $\lambda^{2}$ die Determinante von $\omega(u, u)$ als Factor; da dieselbe jedoch verschwindet, bleibt nur

$$
(k-1)^{2} \sum \pm\left(\varphi_{11} \varphi_{22} \varphi_{33}\right) \cdot \omega(u, u)+2(k-1) \lambda \chi=0
$$

1) Infolge des Umstandes, dass auch die beiden imaginären Kreispunkte im Unendlichen einem jeden Kreis angehören, ist die Beschränkung auf einen im Endlichen gelegenen Berührungspunkt geboten. 
wobei $2 \chi$ nach $(8 \mathrm{~b})$ in $\S 17$ den Werth besitzt

$$
\left(\varphi_{11} \Omega_{11}+2 \varphi_{12} \Omega_{12}+\cdots\right) \varphi(u, u)-\Omega\left(\varphi_{1}, \varphi_{2}, \varphi_{3}\right) .
$$

Da die Gerade $u$ Tangente der gegebenen Curve ist, erfüllen ihre Coordinaten die Gleichung $\varphi(u, u)=0$, und $2 \chi$ reducirt sich auf

$$
-\Omega\left(\varphi_{1}, \varphi_{2}, \varphi_{3}\right)=-\tau\left(\varphi_{1} p_{1}+\varphi_{2} p_{2}+\varphi_{3} p_{3}\right)^{2}=-\tau \varphi^{2}(p, \imath) .
$$

Demnach ist im gegenwärtigen Falle (9) gleichbedeutend mit:

(10) $(k-1)^{2} \sum \pm\left(\varphi_{11} \varphi_{22} \varphi_{33}\right) \cdot \omega(u, u)-(k-1) \lambda \cdot \tau \varphi^{2}(p, u)==0$;

für die Grösse $\lambda$ findet man also den Werth

$$
\lambda=\frac{(k-1) \sum \pm\left(\varphi_{11} \varphi_{22} \varphi_{33}\right) \cdot \omega(u, u)}{\tau \cdot \varphi^{2}(p, u)},
$$

so dass sich mit Rücksicht auf (1) als Gleichung des Krümmungsmittelpunktes in variabelen Liniencoordinaten $v_{1}, v_{2}, v_{3}$ ergibt:

$$
\varphi(u, v)+\frac{(k-1) \sum \pm\left(\varphi_{11} \varphi_{22} \varphi_{33}\right) \cdot \omega(u, u)}{\tau \cdot \varphi^{2}(p, u)} \omega(u, v)=0 .
$$

Auch ein Ausdruck für die Länge des Krümmungsradius lässt sich nun leicht ableiten.

Die Coordinaten des Berührungspunktes der Tangente $u$ der Curve $\varphi(u, u)=0$ sind, wie bereits bemerkt wurde, $x_{i}={ }_{l}^{1} \varphi^{\prime}\left(u_{i}\right)(i=1,2,3)$; nach (1) kann daher die Gleichung des Krümmungsmittelpunktes auch gegeben werden in der Form $v_{x}+\lambda \omega(\imath, v)=0$, wobei $\lambda$ durch (11) definirt ist. Vermöge $\boldsymbol{\nu}=\lambda \sqrt{\omega(u, u)}$ kann man diese Gleichung ersetzen durch

$$
v_{x}+\frac{v \cdot \omega(u, v)}{\sqrt{\omega(u, u)}}=0
$$

woraus $v=-\frac{v_{x} \sqrt{\omega(u, u)}}{\omega(u, v)}$ folgt. Mit Hilfe von $(12 \mathrm{~b})$ in $\S 2$ wollen wir nun einführen den Cosinus des Winkels zwischen der Tangente $u$ und der willkürlich durch den Krümmungsmittelpunkt gelegten Geraden $v$; alsdann ist

$$
\nu=\frac{-v_{x}}{\cos (u, v) \cdot \sqrt{\omega(v, v)}}, \quad \text { daher } \quad \frac{\nu}{p_{x}}=\frac{-v_{x}}{\cos (u, v) \cdot p_{x} \sqrt{\omega(v, v)}} .
$$

Auf der rechten Seite dieser Gleichung bedeutet $\frac{v_{x}}{p_{x} \sqrt{\omega(v, v)}}$ nach (1) in $\S 2$ den Abstand $q$ des Currenpunktes $x$ von der Geraden $v$, mithin wird $\frac{\nu}{p_{x}}=\frac{-q}{\cos (u, v)}$; aus einer einfachen Figur erkennt man aber 
sofort, dass $\frac{q}{\cos (u, v)}$ gleich dem mit positivem oder negativem Vorzeichen versehenen Abstand des Curvenpunktes $x$ vom Krümmungsmittelpunkte $y$, also absolut genommen gleich der Länge $r$ des Krümmungsradius ist. In Folge dessen ist $\frac{\nu}{p_{x}}=r^{1}$ ), und wenn man die Identität $p_{x} \equiv \frac{1}{k}\left(p_{1} \varphi^{\prime}\left(u_{1}\right)+p_{2} \varphi^{\prime}\left(u_{2}\right)+p_{3} \varphi^{\prime}\left(u_{3}\right)\right)=\varphi(p, u)$ beachtet, wird $r=\frac{\lambda \sqrt{\omega(u, u)}}{\varphi(p, u)} ;$ mit Rücksicht auf (11) ergibt sich demnach für die Länge des zum Berührungspunkt der Tangente $u$ gehörigen Krümmungsradius der $\left(\operatorname{erth}^{2}\right)$ :

$$
r=\frac{(k-1) \sum \pm\left(\varphi_{11} \varphi_{22} \varphi_{33}\right) \cdot \omega^{\frac{3}{2}}(u, u)}{\tau \cdot \varphi^{3}(p, u)} .
$$

Ist die gegebene Curve $\varphi(u, u)=0$ insbesondere die Curve zweiter Classe $\varphi(u, u) \equiv \sum_{1}^{3} \sum_{i}^{3} \alpha_{i k} u_{i} u_{k}=0$, so verwandelt sich $\sum \pm\left(\varphi_{11} \varphi_{22} \varphi_{33}\right)$ in die Determinante A, und man erhält:

$$
r=\frac{\mathrm{A} \cdot \omega^{\frac{3}{2}}(u, u)}{\tau \cdot \varphi^{3}(p, u)}
$$

die Gleichung des Krümmungsmittelpunktes wird

$$
\varphi(u, v)+\frac{\mathrm{A} \cdot \omega(u, u)}{\tau \cdot \varphi^{2}(p, u)} \omega(u, v)=0 .
$$

Wenn man die Coordinaten des zu einem Punkte $x$ einer Curve zweiter Ordnung $f(x, x)=0$ gehörigen Krümmungsmittelpunktes und die Länge des Krümmungsradius bestimmen will, denkt man sich zu $f(x, x) \equiv \sum_{1}^{3} \sum_{1}^{3} a_{i k} x_{i} x_{k}=0$ die Gleichung in Liniencoordinaten

$$
F(u, u) \equiv \sum_{1}^{3} \sum_{1}^{3} A_{i k} u_{i} u_{k}=0
$$

1) Allgemein folgt hieraus, dass für einen Punkt $y$ mit den Coordinaten

$$
x_{i}+\frac{\nu \cdot \omega^{\prime}\left(u_{i}\right)}{2 \sqrt{\omega(u, u)}}, \quad(i=1,2,3),
$$

der also auf der Verbindungslinie von $x$ mit dem Normalencentrum einer gegebenen Geraden $u$ gelegen ist, die Grösse $v$ die einfache Bedeutung hat $\frac{\nu}{p_{x}}=r$, wobei $r$ die Entfernung der beiden Punkte $x$ und $y$ bezeichnet.

2) Vgl. auch Hesse: „Ueber Curven dritter Classe und Curven dritter Ordnung', Crelle's Journal, Bd. 38, S. 244, 1847. 
Krümmungsmittelpunkt und Krümmungsradius einer Curve $n^{\text {ter }}$ Ordnung. 213 gebildet und kann nun die bisher entwickelten Formeln anwenden. Es ist dabei zu beachten, dass zwischen den Coordinaten des Punktes $x$ und denjenigen seiner Tangente der Zusammenhang besteht

oder umgekehrt

$$
\varrho u_{i}=\frac{1}{2} f^{\prime}\left(x_{i}\right)=f_{i},
$$

$$
A x_{i}=\varrho\left(A_{i 1} u_{1}+A_{i 2} u_{2}+A_{i 3} u_{3}\right)=\frac{\varrho}{2} F^{\prime}\left(u_{i}\right),
$$

wobei $\varrho$ Proportionalitätsfactor ist. In Folge dessen verwandelt sich die Grösse $\varphi(p, u)$ oder $F(p, u)$ nunmehr in $\frac{A}{\varrho}\left(p_{1} x_{1}+p_{2} x_{2}+p_{3} x_{3}\right)$, an Stelle von $\omega(u, u)$ tritt $\frac{1}{\rho^{2}} \omega\left(f_{1}, f_{2}, f_{3}\right)$, A wird gleich $A^{2}$. Bei Substitution dieser Ausdrücke in (15) hebt sich der Proportionalitätsfactor $\varrho$ weg und man erhält für den Radius $r$ des zum Punkte $x$ gehörigen Krümmungskreises:

$$
r=\begin{gathered}
\omega^{\frac{3}{2}}\left(f_{1}, f_{2}, f_{3}\right) \\
t \cdot A \cdot p_{x}^{3}
\end{gathered}
$$

aus (16) ergibt sich als Gleichung des Krümmungsmittelpunktes:

$$
x_{1} v_{1}+x_{2} v_{2}+x_{3} v_{3}+\frac{\omega\left(f_{1}, f_{2}, f_{3}\right) \cdot \omega(f, v)}{\tau \cdot A \cdot p_{x}^{2}}=0,
$$

die Coordinaten desselben sind demnach

$$
\sigma y_{i}=x_{i}+\frac{\omega\left(f_{1}, f_{2}, f_{3}\right)}{t \cdot A_{p_{x}}{ }^{2}}\left(\omega_{i 1} f_{1}+\omega_{i 2} f_{2}+\omega_{i 3} f_{3}\right) \quad(i=1,2, \dot{3}),
$$

wobei $\sigma$ einen Proportionalitätsfactor bezeichnet.

Auf demselben Wege, der eingeschlagen wurde zur Bestimmung des einem beliebigen Punkte einer Curve $7_{i}^{\text {ter }}$ Classe zugehörigen Krümmungskreises, kann man auch vorgehen, wenn eine Curve $n^{\text {ter }}$ Ordnung $f(x, x)=0$ gegeben ist. Man würde für die Länge des Krümmungsradius finden

wobei

$$
r=\frac{\omega^{\frac{3}{2}}\left(f_{1}, f_{2}, f_{3}\right)}{(n-1) \tau \cdot p_{x}^{3} \cdot \sum \pm\left(f_{11} f_{22} f_{33}\right)}
$$

$$
f_{i}=\frac{1}{n} \frac{\partial f}{\partial x_{i}}, \quad f_{i k}=\frac{1}{n(n-1)} \frac{\partial^{2} f}{\partial x_{i} \partial x_{k}}
$$

die Gleichung des dem Punkte $x$ zugehörigen Krümmungsmittelpunktes wird

$$
x_{1} v_{1}+x_{2} v_{2}+x_{3} v_{3}+\frac{\omega\left(f_{1}, f_{2}, f_{3}\right) \cdot \omega(f, v)}{(n-1) \tau \cdot p_{x}^{2} \cdot \sum \pm\left(f_{11} f_{22} f_{33}\right)}=0
$$


seine Coordinaten sind daher

$$
\varrho y_{i}=x_{i}+\frac{\omega\left(f_{1}, f_{2}, f_{8}\right)\left(\omega_{i 1} f_{1}+\omega_{i 2} f_{2}+\omega_{i 3} f_{3}\right)}{(n-1) \tau \cdot p_{x}{ }^{2} \cdot \sum \pm\left(f_{11} f_{22} f_{33}\right)} .
$$

Construirt man in jedem Punkte einer gegebenen Curve die Normale, so umhüllen alle Normalen eine neue Curve, die sogenannte Evolute der gegebenen; zwei auf einander folgende Normalen schneiden sich in einem Punkt der Evolute, und da dieser Schnittpunkt zugleich Centrum eines Krümmungskreises ist, so repräsentirt die Evolute auch den Ort der Krümmungsmittelpunkte, welche den einzelnen Punkten der gegebenen Curve zugehören.

Wir wollen nun die Gleichung der Evolute ableiten, falls die gegebene Curve von der zweiten Classe ist und die Gleichung hat

$$
\varphi(u, u) \equiv \sum_{i}^{3} \sum_{i}^{3} \alpha_{i k} u_{i} u_{k}=0 .
$$

Es sei $u_{x}=0$ eine Tangente dieses Kegelschnitts, $v_{x}=0$ die zugehörige Normale; beide schliessen einen rechten Winkel ein, und es ist daher nach (13) in $\S 2$

$$
\omega(u, v) \equiv \omega^{\prime}\left(v_{1}\right) u_{1}+\omega^{\prime}\left(v_{2}\right) u_{2}+\omega^{\prime}\left(v_{3}\right) u_{3}=0 .
$$

Ferner besteht noch die Gleichung

$$
\varphi^{\prime}\left(u_{1}\right) v_{1}+\varphi^{\prime}\left(u_{2}\right) v_{2}+\varphi^{\prime}\left(u_{3}\right) v_{3}=0,
$$

denn der Berührungspunkt der Tangente $u$ liegt auch auf der Normale $v$; übrigens können wir im gegenwärtigen Falle auch $u$ und $v$ vertauschen und erhalten alsdann $\varphi^{\prime}\left(v_{1}\right) u_{1}+\varphi^{\prime}\left(v_{2}\right) u_{2}+\varphi^{\prime}\left(v_{3}\right) u_{3}=0$. Aus dieser Gleichung und aus $\omega(u, v)=0$ folgt

(25) $u_{1}: u_{2}: u_{3}=\left[\varphi^{\prime}\left(v_{2}\right) \omega^{\prime}\left(v_{3}\right)-\varphi^{\prime}\left(v_{3}\right) \omega^{\prime}\left(v_{2}\right)\right]:\left[\varphi^{\prime}\left(v_{3}\right) \omega^{\prime}\left(v_{1}\right)-\varphi^{\prime}\left(v_{1}\right) \omega^{\prime}\left(v_{3}\right)\right]$

$$
:\left[\varphi^{\prime}\left(v_{1}\right) \omega^{\prime}\left(v_{2}\right)-\varphi^{\prime}\left(v_{2}\right) \omega^{\prime}\left(v_{1}\right)\right] \text {, }
$$

so dass man nach Einführung dieser Werthe der $u$ in (24) für die Evolute unsrer Curve zweiter Classe die Gleichung in Liniencoordinaten $v$ erhält:

$$
\begin{array}{ccccc}
\alpha_{11} & \alpha_{12} & \alpha_{13} & \frac{1}{2} \varphi^{\prime}\left(v_{1}\right) & \frac{1}{2} \omega^{\prime}\left(v_{1}\right) \\
\alpha_{21} & \alpha_{22} & \alpha_{23} & \frac{1}{2} \varphi^{\prime}\left(v_{2}\right) & \frac{1}{2} \omega^{\prime}\left(v_{2}\right) \\
\alpha_{31} & \alpha_{32} & \alpha_{33} & \frac{1}{2} \varphi^{\prime}\left(v_{3}\right) & \frac{1}{2} \omega^{\prime}\left(v_{3}\right) \\
\frac{1}{2} \varphi^{\prime}\left(v_{1}\right) & \frac{1}{2} \varphi^{\prime}\left(v_{2}\right) & \frac{1}{2} \varphi^{\prime}\left(v_{3}\right) & 0 & 0 \\
\frac{1}{2} \omega^{\prime}\left(v_{1}\right) & \frac{1}{2} \omega^{\prime}\left(v_{2}\right) & \frac{1}{2} \omega^{\prime}\left(v_{3}\right) & 0 & 0
\end{array} \mid=0 .
$$


Multiplicirt man die drei ersten Horizontalreihen dieser Determinante resp. mit $v_{1}, v_{2}, v_{3}$ und subtrahirt sie von der vierten Reihe und verfährt man in gleicher Weise mit den Verticalreihen, so sieht man sofort, dass die Determinante sich zerlegen lässt in:

$$
A \omega^{2}(v, v)-\varphi(v, v)\left\{A_{11} \omega_{1}{ }^{2}+\cdots+2 A_{23} \omega_{2} \omega_{3}+A_{33} \omega_{3}{ }^{2}\right\}=0,
$$

wobei $\omega_{i}=\frac{1}{2} \omega^{\prime}\left(v_{i}\right)$ und $\mathrm{A}_{i k}$ die Unterdeterminante von $\alpha_{i k}$ in

$$
\mathrm{A}=\left|\begin{array}{lll:}
\alpha_{11} & \alpha_{12} & \alpha_{13} \\
\alpha_{21} & \alpha_{22} & \alpha_{23} \\
\alpha_{31} & \alpha_{32} & \alpha_{33}
\end{array}\right| \text { ist. }
$$

Die Gleichung (27) enthält die variabelen Liniencoordinaten $v$ im vierten Grade, demnach ist die Evolute eines Kegelschnitts im allgemeinen von der vierten Classe. Es stimmt dies auch damit überein, dass man nach (23) in $\S 20$ von einem beliebigen Punkte der Ebene nach einem Kegelschnitt im allgemeinen vier Normalen ziehen kann; dieselben sind zufolge der Definition der Evolute Tangenten dieser Curve.

Es liegt nun die Frage nahe, welche geometrische Bedeutung der Factor von $\varphi(v, v)$ in dieser Gleichung der Evolute besitzt. Zunächst bemerkt man, dass $\omega_{i}(i=1,2,3)$ nach $\S 2$ die Coordinaten eines unendlich fernen Punktes sind, und zwar des Normalencentrums der Geraden $v$; ausserdem liegt dieser Punkt, wenn

$$
\chi \equiv A_{11} \omega_{1}{ }^{2}+2 A_{12} \omega_{1} \omega_{2}+A_{22} \omega_{2}{ }^{2}+2 A_{13} \omega_{1} \omega_{3}+2 A_{23} \omega_{2} \omega_{3}+A_{33} \omega_{3}{ }^{2}=0,
$$

auf der Curve $\varphi(u, u)=0$, denn die $\omega_{i}$ erfüllen im Falle $\chi=0$ die Gleichung des gegebenen Kegelschnitts in Punktcoordinaten. Hieraus folgt, dass $\chi=0$ die Bedingung ist, dass das Normalencentrum einer Geraden mit den Coordinaten $v$ ein Asymptotenpunkt des Kegelschnitts sei, oder mit anderen Worten, dass die Gerade $v$ durch das Normalencentrum einer der beiden Asymptoten gehe.

Es möge nun die Formel (27) auf deu Fall angewandt werden, dass rechtwinklige homogene Coordinaten zu Grunde liegen; ferner sei der gegebene Kegelschnitt eine Ellipse oder Hyperbel, welche auf die Hauptaxen als Coordinatenaxen bezogen ist. Man hat alsdamn nach $\S 10$ :

$$
\left\{\begin{array}{l}
\omega(U, U) \equiv U_{1}^{2}+U_{2}^{2}=0 \\
\varphi(U, U) \equiv \frac{U_{1}{ }^{2}}{\lambda^{\prime}}+\frac{U_{2}^{2}}{\lambda^{\prime \prime}}+\frac{U_{3}{ }^{2}}{x}=0
\end{array}\right.
$$


so dass an Stelle von (27) die Gleichung tritt

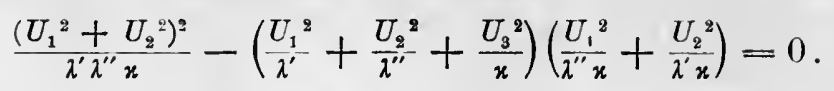

Wenn dagegen vorgelegt ist die Parabel

$$
\varphi(u, u) \equiv \varrho U_{1}^{2}+2 \lambda^{\prime} U_{2} U_{3}=0,
$$

ergibt sich an Stelle von (27) der Ausdruck

oder auch:

$$
-\varrho \lambda^{\prime 2}\left(U_{1}^{2}+U_{2}^{2}\right)^{2}-\left(\varrho U_{1}^{2}+2 \lambda^{\prime} U_{2} U_{3}\right)\left(-\lambda^{\prime 2} U_{1}^{2}\right)=0,
$$

$$
U_{2}\left\{\varrho U_{2}^{3}+2 \varrho U_{1}^{2} U_{2}-2 \lambda^{\prime} U_{1}^{2} U_{3}\right\}=0 \text {; }
$$

es scheidet sich demnach im Falle der Parabel der Factor $U_{2}=0$ aus, welcher nach $\S 10$ den Berührungspunkt der Parabel mit der unendlich fernen Geraden repräsentirt; der übrig bleibende Ausdruck ist eine Curve dritter Classe.

Es möge endlich noch gezeigt werden, wie man die Gleichung in Punktcoordinaten für die Evolute einer gegebenen Curve zweiter Ordnung

findet.

$$
f(x, x) \equiv \sum_{1}^{3} \sum_{1}^{3} a_{i k} x_{i} x_{k}=0
$$

Nach (23) in $\S 20$ können von einem beliebigen Punkte $y$ der Ebene im allgemeinen vier Normalen nach einem Kegelschnitt gezogen werden, und zwar werden deren Fusspunkte aus (32) ausgeschnitten durch die gleichseitige Hyperbel:

$$
g(x, x) \equiv\left|\begin{array}{ccc}
\frac{1}{2} \omega^{\prime}\left(f_{1}\right) & \frac{1}{2} \omega^{\prime}\left(f_{2}\right) & \frac{1}{2} \omega^{\prime}\left(f_{3}\right) \\
x_{1} & x_{2} & x_{3} \\
y_{1} & y_{2} & y_{3}
\end{array}\right|=0 .
$$

Aus der oben gegebenen Definition der Evolute folgt, dass der Punkt $y$ der Evolute angehört, wenn von den vier Normalen zwei zusammenfallen, oder mit anderen Worten: wenn die gleichseitige Hyperbel (33) den gegebenen Kegelschnitt (32) berührt. Die Bedingung hierfür ist nach $\S 14$, dass die kubische Gleichung

$$
\left.\lambda^{3} B-3 \lambda^{2} \Theta+3 \lambda H-A=0^{1}\right)
$$

1) Ueber die Bildung der Ausdrücke $\Theta$ und $H$ vgl. (11) in § 13; dabei ist $g(x, x)$ in (33) gesetzt gleich $\sum_{1}^{3} \sum_{1}^{3} b_{i k} x_{i} x_{k} . A$ und $B$ sind die Determinanten von (32), resp. (33). 
zwei gleiche Wurzeln besitzt; in dieser Gleichung muss jedoch für den vorliegenden Fall $H$ nach (19) in $\S 17$ verschwinden, denn die Curve $g=0$ geht durch ein Poldreieck von $f=0$ hindurch, nämlich wie aus (23) in $\S 20$ hervorgeht, durch dasjenige Dreieck, welches den Mittelpunkt und die unendlich fernen Punkte der Hauptaxen von $f=0$ zu Ecken hat. Die Bedingung aber, dass die kubische Gleichung $\lambda^{3} B-3 \lambda^{2} \Theta-A=0$ zwei gleiche Wurzeln besitzt, ist bekanntlich

$$
4 \Theta^{3}+A B^{2}=0
$$

Diese Gleichung ist in den Coefficienten von $g=0$, also auch in den $y_{1}, y_{2}, y_{3}$, vom sechsten Grad, die Evolute eines Kegelschnitts ist daher im allgemeinen von der sechsten Ordnung.

Wir wollen nun wieder die Formel (35) auf den Fall anwenden, dass rechtwinklige homogene Coordinaten zu Grunde liegen; der gegebene Kegelschnitt sei zunächst eine auf die Hauptaxen als Coordinatenaxen bezogene Ellipse oder Hyperbel. Man hat alsdann nach $\$ 10$ :

$$
\left\{\begin{array}{l}
\omega(U, U)=U_{1}^{2}+U_{2}^{2} \\
f(X, X)=\lambda^{\prime} X_{1}^{2}+\lambda^{\prime \prime} X_{2}^{2}+x X_{3}^{2}
\end{array}\right.
$$

daher nach (33):

$$
\begin{aligned}
j(x, x) & \equiv\left|\begin{array}{ccc}
\lambda^{\prime} X_{1} & \lambda^{\prime \prime} X_{2} & 0 \\
X_{1} & X_{2} & X_{3} \\
Y_{1} & Y_{2} & Y_{3}
\end{array}\right| \\
& =Y_{1} \lambda^{\prime \prime} X_{2} X_{3}-Y_{2} \lambda^{\prime} X_{3} X_{1}+Y_{3}\left(\lambda^{\prime}-\lambda^{\prime \prime}\right) X_{1} X_{2}=0 .
\end{aligned}
$$

Ferner findet man:

$$
\begin{aligned}
A & =\lambda^{\prime} \lambda^{\prime \prime} x, \quad B=-\frac{Y_{1} Y_{2} Y_{3} \lambda^{\prime} \lambda^{\prime \prime}\left(\lambda^{\prime}-\lambda^{\prime \prime}\right)}{4}, \\
3 \Theta & =-\frac{1}{4}\left\{\lambda^{\prime} \lambda^{\prime \prime}\left(\lambda^{\prime \prime} Y_{1}^{2}+\lambda^{\prime} Y_{2}^{2}\right)+x\left(\lambda^{\prime}-\lambda^{\prime \prime}\right)^{2} Y_{3}{ }^{2}\right\}
\end{aligned}
$$

so dass an Stelle von (35) die Gleichung tritt:

$$
\begin{aligned}
& -\frac{1}{27}\left\{\lambda^{\prime} \lambda^{\prime \prime}\left(\lambda^{\prime \prime} Y_{1}{ }^{2}+\lambda^{\prime} Y_{2}{ }^{2}\right)+\varkappa\left(\lambda^{\prime}-\lambda^{\prime \prime}\right)^{2} Y_{3}{ }^{2}\right\}^{3} \\
& +\lambda^{\prime} \lambda^{\prime \prime} x \cdot Y_{1}{ }^{2} Y_{2}{ }^{2} Y_{3}{ }^{2} \cdot \lambda^{\prime 2} \lambda^{\prime \prime 2}\left(\lambda^{\prime}-\lambda^{\prime \prime}\right)^{2}=0
\end{aligned}
$$

oder kürzer:

(39a) $\left\{\lambda^{\prime \prime} Y_{1}^{2}+\lambda^{\prime} Y_{2}^{2}+\frac{x}{\lambda^{\prime} \lambda^{\prime \prime}}\left(\lambda^{\prime}-\lambda^{\prime \prime}\right)^{2} Y_{3}^{2}\right\}^{3}-27 x\left(\lambda^{\prime}-\lambda^{\prime \prime}\right)^{2} Y_{1}^{2} Y_{2}^{2} Y_{3}^{2}=0$.

Ist gegeben die Parabel:

$$
f(x, x) \equiv \lambda^{\prime} X_{1}^{2}+2 \varrho X_{2} X_{3}=0,
$$


so findet man:

$$
\begin{aligned}
g(x, x) & \equiv\left|\begin{array}{ccc}
\lambda^{\prime} X_{1} & \varrho X_{3} & 0 \\
X_{1} & X_{2} & X_{3} \\
Y_{1} & Y_{2} & Y_{3}
\end{array}\right| \\
& =Y_{1} \varrho X_{3}{ }^{2}-\left(Y_{2} \lambda^{\prime}+Y_{3} \varrho\right) X_{3} X_{1}+Y_{3} \lambda^{\prime} X_{1} X_{2}=0
\end{aligned}
$$

ferner wird:

(42) $A=-\lambda^{\prime} \varrho^{2}, B=-\frac{1}{4} \lambda^{\prime 2} \varrho Y_{1} Y_{3}^{2}, 3 \Theta=-\frac{1}{2} \lambda^{\prime} \varrho\left(\lambda^{\prime} Y_{2}+\varrho Y_{3}\right) Y_{3}$,

so dass an Stelle von (35) die Gleichung tritt:

$$
-\frac{4}{8 \cdot 27} \lambda^{\prime 3} \varrho^{3}\left(\lambda^{\prime} Y_{2}+\varrho Y_{3}\right)^{3} Y_{3}^{3}-\frac{\lambda^{\prime} 5 \varrho^{4}}{16} Y_{1}^{2} Y_{3}^{4}=0
$$

oder kïrzer:

$$
8\left(\lambda^{\prime} Y_{2}+\varrho Y_{3}\right)^{3}+27 \lambda^{\prime 2} \varrho Y_{1}^{2} Y_{3}=0 ;
$$

es scheidet sich dennach im Falle der Parabel der Factor $Y_{\mathbf{3}}{ }^{3}=0$ aus, welcher dreifach zählend die unendlich ferne Gerade repräsentirt; der ïbrig bleibende Ausdruck ist eine Curve dritter Ordnung.

$$
\S 22-25 .
$$

Kegelschnittnetz und Kegelschnittgewebe.

$\S 22$.

\section{Die Hessiane eines Kegelschnittnetzés.}

Es seien gegeben drei Curven zweiter Ordnung:

$$
\varphi(x, x)=0, \quad \psi(x, x)=0, \quad \chi(x, x)=0,
$$

die nicht einem und demselben Büschel angehören niögen. Man hat alsdann folgenden Satz:

$\left.I^{1}{ }^{1}\right)$ Jeder Punkt $y$, dessen Polaren in Bezug auf drei gegebene Curven zweiter Ordnung (1) sich in einem Punkte $x$ schneiden, liegt zugleich mit diesem Punkte auf der Curve dritter Ordnung

$$
\begin{gathered}
\sum \pm\left(\varphi_{1} \dot{\psi}_{2} \chi_{3}\right)=0, \text { wobei } \varphi_{i}=\frac{1}{2} \frac{\partial \varphi}{\partial x_{i}}, \psi_{i}=\frac{1}{2} \frac{\partial \psi}{\partial x_{i}}, \chi_{i}=\frac{1}{2} \frac{\partial \chi}{\partial x_{i}} \\
(i=1,2,3) .
\end{gathered}
$$

1) Die Sätze dieses und des folgenden Paragraphen werden durch fortlaufende römische Ziffern bezeichnet, um alsdann in $\$ 24$ die dualistisch entsprechenden Sätze mit denselben Ziffern versehen zu können. 
Die Polaren eines Punktes $y$ in Bezug auf die drei Kegelschnitte (1) haben nämlich die Gleichungen

$$
\begin{gathered}
\varphi_{1} y_{1}+\varphi_{2} y_{2}+\varphi_{3} y_{3}=0, \quad \psi_{1} y_{1}+\psi_{2} y_{2}+\psi_{3} y_{3}=0 \\
\chi_{1} y_{1}+\chi_{2} y_{2}+\chi_{3} y_{3}=0,
\end{gathered}
$$

in denen auch, wie wir in $\S 4$ sahen, die $x_{i}$ mit den $y_{i}$ vertauscht werden können. Die durch (3) repräsentirten Geraden schneiden sich aber in einem Punkte, sobald die Determinante der Coefficienten verschwindet, und diese Determinante ist eben mit (2) identisch, stellt also, da sie in den Variabeln vom dritten Grade ist, eine Curve dritter Ordnung dar. Man bezeichnet dieselbe nach dem Mathematiker Otto Hesse ${ }^{1}$ ) als die Hesse'sche Curve oder Hessiane der drei gegebenen Kegelschnitte (1).

Auch die Umkehrung von I ist giltig und lautet:

Ia. Jeder Punkt $x$ der Curve dritter Ordnung (2) kann angesehen werden als ein Punkt, dessen Polaren in Bezug auf die drei gegebenen Kegelschnitte (1) sich in einem Punkte schneiden.

Es folgt dies daraus, dass man beim Bestehen von (2) stets drei Grössen $y_{1}, y_{2}, y_{3}$ finden kann, welche die Gleichungen (3) erfüllen.

Zwei auf die in I und Ia angegebene Art zusammengehörige Punkte $x$ und $y$ heissen conjugirte oder harmonische Pole jener Curve dritter Ordnung (3). Multiplicirt man die drei Gleichungen (3) resp. mit den Parametern $x, \lambda, \mu$ und addirt, so sieht man, dass die Punkte $x$ und $y$ harmonische Pole sind für jeden Kegelschnitt, dessen Gleichung von der Form ist

$$
\varkappa \varphi(x, x)+\lambda \psi(x, x)+\mu \chi(x, x)=0 .
$$

Die Gesammtheit aller Kegelschnitte, die man erhält, wenn in (4) den Parametern alle möglichen Werthe ertheilt werden, heisst ein Kegelschnittnetz. Dasselbe fasst unendlich viele Kegelschnittbïschel in sich. Sind z. B.

$$
x_{1} \varphi+\lambda_{1} \psi+\mu_{1} \chi=0 \text { und } x_{2} \varphi+\lambda_{2} \psi+\mu_{2} \chi=0
$$

irgend zwei bestimmte Curven des Netzes, so repräsentirt

$$
x_{1} \varphi+\lambda_{1} \psi+\mu_{1} \chi+\varrho\left(x_{2} \varphi+\lambda_{2} \psi+\mu_{2} \chi\right)=0
$$

bei veränderlichem Parameter $\varrho$ ein Kegelschnittbüschel; ein zweites Büschel werde dargestellt durch

$$
x_{3} \varphi+\lambda_{y} \psi+\mu_{3} \chi+\sigma\left(x_{4} \varphi+\lambda_{4} \psi+\mu_{4} \chi\right)=0,
$$

1) Vgl. Hesse: „Ueber die Wendepunkte der Curven dritter Ordnung", Journal für die reine und angewandte Mathematik, Bd. 28, S. 105, 1844. 
wobei $\sigma$ einen veränderlichen Parameter bedeutet. Man kann nun $\varrho$ und $\sigma$ immer so bestimmen, dass die beiden Gleichungen (5) und (6) einen und denselben Kegelschnitt repräsentiren; es muss dann sein

$$
\left\{\begin{array}{c}
x_{1}+\varrho x_{2}=\tau\left(x_{3}+\sigma x_{4}\right), \quad \lambda_{1}+\varrho \lambda_{2}=\tau\left(\lambda_{3}+\sigma \lambda_{4}\right), \\
\mu_{1}+\varrho \mu_{2}=\tau\left(\mu_{3}+\sigma \mu_{4}\right) .
\end{array}\right.
$$

Aus diesen Gleichungen lassen sich die drei Unbekannten $\varrho, \tau, \sigma \tau$ stets berechnen, vorausgesetzt, dass die Determinante $\sum \pm\left(x_{2} \lambda_{3} \mu_{4}\right)$ von Null verschieden ist, also vorausgesetzt, dass der durch den Index 2 charakterisirte Kegelschnitt nicht dem Büschel angehört, das durch die Kegelschnitte mit den Indices 3 und 4 in (6) bestimmt ist. Man hat somit den wichtigen Satz:

II. Irgend $z$ wei in dem Kegelschnittnetze enthaltenen Büschel haben stets einen Kegelschnitt gemeinsam.

Ein weiterer Satz lautet:

III. Die Spitzen aller in einem Kegelschnittnetze enthaltenen Geradenpare liegen auf der Hessiane, und umgekehrt: Jeder Punkt der Hessiane ist der Schnittpunkt eines solchen Geradenpaares.

Man kann dies auch in folgender Form aussprechen:

IIIa. Die Hessiane ist der geometrische Ort der Scheitel aller Poldreiecke, die irgend welchen Kegelschnitten des Netzes gemeinsam sind.

Die Form III dieses Satzes ergibt sich daraus, dass die Coordinaten der Spitze irgend eines in dem Netze enthaltenen Geradenpaares die Gleichungen erfüllen:

(8) $x \varphi_{1}+\lambda \psi_{1}+\mu \chi_{1}=0, x \varphi_{2}+\lambda \psi_{2}+\mu \chi_{2}=0, x \varphi_{3}+\lambda \psi_{3}+\mu \chi_{3}=0$, aus denen durch Elimination von $x, \lambda, \mu$ wieder (2) folgt. Auf Grund des Satzes (17) in $\S 14$, demzufolge die drei Spitzen der in einem Kegelschnittbüschel enthaltenen Geradenpaare ein Poldreieck für jede Curve des Büschels bilden, lässt sich der Satz III auch in der Form III a aussprechen.

Für die conjugirten Pole der Hessiane gilt der fundamentale Satz:

IV. Wenn zwei Punktepaare eines vollständigen Vierseits conjugirte Polepare der Hessiane sind, so sind auch die Punkte des dritten Pares conjugirte Pole.

Der Beweis dieses Satzes fällt zusammen mit dem Beweis des Satzes von $\mathrm{Hesse}^{1}$ ):

1) Hesse: „De curvis et superficiebus secundi ordinis“, Journal für die reine und angewandte Mathematik, Bd. 20, S. 301, 1840. Vgl. auch die Abhaudlung 
Wenn zwei Punktepaare eines vollständigen Vierseits harmonische Pole eines Kegelschnitts sind, so sind auch die Punkte des dritten Paares harmonische Pole des Kegelschnitts („Polvierseit").

Dieser Satz ergibt sich folgendermassen: Der gegebene Kegelschnitt sei dargestellt durch

$$
f(x, x) \equiv \sum_{1}^{3} \sum_{1}^{3} a_{i k} x_{i} x_{k}=0,
$$

die beiden Punktepaare seien

und

$$
\alpha(u, u) \equiv \sum_{1}^{3} \sum_{1}^{3} k \alpha_{i k} u_{i} u_{k}=0
$$

$$
\beta(u, u) \equiv \sum_{1}^{3} \sum_{1}^{3} k \beta_{i k} u_{i} u_{k}=0,
$$

wobei nun die Determinanten A und B dieser beiden Functionen verschwinden. Die Bedingungen, dass die Punktepaare $\alpha$ und $\beta$ in Bezug auf $f=0$ conjugirt seien, sind dann, wie dualistisch aus (22) in $\S 17$ folgt, ausgedrückt durch

$$
\left\{\begin{array}{l}
\Theta_{\alpha} \equiv \alpha_{11} a_{11}+2 \alpha_{12} a_{12}+\alpha_{22} a_{22}+2 \alpha_{13} a_{13}+2 \alpha_{23} a_{23}+\alpha_{33} a_{33}=0 \text { und } \\
\Theta_{\beta} \equiv \beta_{11} a_{11}+2 \beta_{12} a_{12}+\beta_{22} a_{22}+2 \beta_{13} a_{13}+2 \beta_{23} a_{23}+\beta_{33} a_{33}=0 .
\end{array}\right.
$$

Da die Gleichung des dritten Punktepaares von der Form ist ${ }^{1}$ )

$$
\alpha(u, u)+\varrho \beta(u, u)=0,
$$

hat man als Bedingung dafür, dass auch dieses Punktepaar zu $f(x, x)=0$ conjugirt ist, die Relation $\Theta_{\alpha}+\varrho \Theta_{\beta}=0$, und diese wird in der That erfüllt, da $\Theta_{\alpha}$ und $\Theta_{\beta}$ einzeln verschwinden. Hiermit ist der obige Hesse'sche Satz bewiesen, daher auch Satz IV, wenn man als Kegelschnitt $f(x, x)=0$ irgend eine Curve des Netzes (4) wählt.

Man kann den Satz IV noch in der oft zwecknässigeren Form aussprechen:

IVa. Verbindet man irgend einen Punkt $z$ der Hessiane mit zwei conjugirten Polen $x$ und $\xi$, so schneiden die Verbindungslinien $z x$ und $z \xi$ die Hessiane noch in je einem weiteren conjugirten Pole $y$, resp. $\eta$, und die Geraden $y \xi$ und $x \eta$ treffen sich in dem zu $z$ conjugirten Pole $\xi$ der Hessiane.

"Ueber Curven dritter Ordnung und die Kegelschnitte, welche diese Curven in drei verschiedenen Punkten berühren", in demselben Journal, Bd. 36, S. 146 f., 1847.

1) Folgt dualistisch aus den Betrachtungen S. 137. 
Denu dass der dritte Schnittpunkt $\eta$ der Geraden $z \xi$ mit der Curve der conjugirte Pol zu $y$ ist, ergibt sich folgendermassen: Bezeichnen wir die zu $z, x, y$ conjugirten Pole resp. durch $\xi^{\prime}, \xi, \eta^{\prime}$, so müssen dieselben ein Dreieck bilden, bei dem z. B. die Seite $\xi \eta^{\prime}$ durch $z$ hindurchgeht, d. h. aber $\eta^{\prime}$ muss auf der Geraden $z \xi$ liegen, und da $\eta^{\prime}$ auch der Curve angehört, muss dieser Punkt mit dem dritten Schnittpunkte $\eta$ der Geraden $z \xi$ und der Curve zusammenfallen. Ebenso ergibt sich, dass $\xi^{\prime}$ mit $\xi$ identisch ist.

Aus IV a folgt ein weiteres interessantes Theorem:

V. Die Tangenten in zwei conjugirten Polen $p$ und $\pi$ der Hessiane schneiden sich in einem Punkte dieser Curve, und zwar ist derselbe conjugirt zu dem dritten Schnittpunkte der Geraden $p \pi$ mit der Curve.

Nennen wir nämlich $q$ den dritten Schnittpunkt der Geraden $p \pi$ mit der Hessiane und $x$ den zu $q$ conjugirten Pol, so sind nach IVa die dritten Schnittpunkte, welche die beiden von $\pi$ nach den zugeordneten Polen $q$ und $x$ gezogenen Strahlen mit der Curve gemein haben, conjugirt. Einer dieser dritten Schnittpunkte ist $p$, und da dessen zugeordneter $\mathrm{Pol} \pi$ ist, so fällt der dritte Schnittpunkt der Geraden $\pi x$ und der Hessiane mit $\pi$ zusammen, d. h. $x$ liegt auf der in $\pi$ gezogenen Tangente; aus analogem Grunde liegt $x$ auch auf der in $p$ gezogenen Tangente, womit alsdann gezeigt ist, dass sich diese beiden Tangenten in dem zu $q$ conjugirteu Curveupunkte $x$ schneiden.

\section{$\S 23$.}

\section{Die Cayley'sche Curve eines Kegelschnittnetzes.}

Diese Curve ist definirt durch folgenden Satz:

VI. Die Geradenpare, die sich in einem Kegelschnittnetze befinden, umhüllen eine Curve dritter Classe, die sogenannte Cayley'sche Curve des Netzes ${ }^{1}$ ).

Zum Beweis dieses Satzes seien

1) Man nennt diese Curve anch vielfach die Hermite'sche Curve des Netzes mit Rücksicht anf die von Herrn Hermite im 57. Bande 'von Crelle's Journal, S. 371-375, 1860, gegebene Darstellung. In dem speciellen Falle, dass die Ausdrücke $\varphi(x, x), \psi(x, x), \chi(x, x)$ in (1) die partiellen Differentialquotienten einer ternären kubischen Form bedeuten, geht die Hermite'sche Curve in die sogenannte Cayley'sche Curve der Curve dritter Ordnung über. Mit Rücksicht hierauf haben wir die Bezeichnung Cayley'sche Curve gewählt. 


$$
\left\{\begin{array}{l}
\varphi(x, x) \equiv \sum_{1}^{3} \sum_{1}^{3} a_{i k} x_{i} x_{k}=0 \\
\psi(x, x) \equiv \sum_{1}^{3} \sum_{1}^{3} b_{i k} x_{i} x_{k}=0 \\
\chi(x, x) \equiv \sum_{1}^{3} \sum_{1}^{3} c_{i k} x_{i} x_{k}=0
\end{array}\right.
$$

drei Curven des Netzes, die nicht einem und demselben Büschel angehören; eine Curve dieses Netzes

$$
\varkappa \varphi(x, x)+\lambda \psi(x, x)+\mu \chi(x, x)=0
$$

zerfüllt alsdann in ein Linienpaar

$$
\left(u_{1} x_{1}+u_{2} x_{2}+u_{3} x_{3}\right)\left(v_{1} x_{1}+v_{2} x_{2}+v_{3} x_{3}\right)=0,
$$

wenn die Bedingungen erfüllt werden:

$$
\left\{\begin{aligned}
\varkappa a_{11}+\lambda b_{11}+\mu c_{11} & =u_{1} v_{1}, \\
x a_{22}+\lambda b_{22}+\mu c_{22} & =u_{2} v_{2}, \\
x a_{33}+\lambda b_{33}+\mu c_{33} & =u_{3} v_{3}, \\
2\left(x a_{23}+\lambda b_{23}+\mu c_{23}\right) & =u_{2} v_{3}+u_{3} v_{2}, \\
2\left(x a_{31}+\lambda b_{31}+\mu c_{31}\right) & =u_{3} v_{1}+u_{1} v_{33}, \\
2\left(x a_{12}+\lambda b_{12}+\mu c_{12}\right) & =u_{1} v_{2}+u_{2} v_{1} .
\end{aligned}\right.
$$

Durch Elimination von $x, \lambda, \mu, v_{1}, v_{2}, v_{3}$ erhält man hieraus die Gleichung der Cayley'schen Curve in der Gestalt

$$
\left|\begin{array}{rrrrrr}
a_{11} & b_{11} & c_{11} & u_{1} & 0 & 0 \\
a_{22} & b_{22} & c_{22} & 0 & u_{2} & 0 \\
a_{33} & b_{33} & c_{33} & 0 & 0 & u_{3} \\
2 a_{23} & 2 b_{23} & 2 c_{23} & 0 & u_{3} & u_{2} \\
2 a_{31} & 2 b_{31} & 2 c_{31} & u_{3} & 0 & u_{1} \\
2 a_{12} & 2 b_{12} & 2 c_{12} & u_{2} & u_{1} & 0
\end{array}\right|=0
$$

und diese Determinante repräsentirt in der That eine Curve dritter Classe.

Umgekehrt gilt auch der Satz:

VII. Jede Tangente der Cayley'schen Curve gehört einem Geradenpaare des zugehörigen Kegelschnittnetzes an.

Dies folgt daraus, dass sich auf Grund der Gleichung (4) stets sechs Grössen $x, \lambda, \mu, v_{1}, v_{2}, v_{3}$ bestimmen lassen, welche die Relationen (3) erfüllen. Hierbei können die $v_{i}$ nicht sämmtlich verschwinden, da sonst $x a_{i k}+\lambda b_{i k}+\mu c_{i k}=0$ wäre, die Kegelschnitte 
$\varphi, \psi, \chi$ also einem und demselben Büschel angehören würden, was gegen die Voraussetzung ist.

Ein weiterer Satz lautet:

VIII. Jede Gerade, welche zwei conjugirte Pole $x$ und $y$ der Hessiane verbindet, ist Tangente der Cayley'schen Curve.

Zunächst müssen nämlich, da $x$ und $y$ conjugirte Pole sind, die Gleichungen bestehen

$$
\varphi(x, y)=0, \quad \psi(x, y)=0, \quad \chi(x, y)=0,
$$

wobei

$$
\begin{aligned}
\varphi(x, y) \equiv\left(a_{11} x_{1}+a_{12} x_{2}+a_{13} x_{3}\right) y_{1} & +\left(a_{21} x_{1}+a_{22} x_{2}+a_{23} x_{3}\right) y_{2} \\
& +\left(a_{31} x_{1}+a_{32} x_{2}+a_{33} x_{3}\right) y_{3},
\end{aligned}
$$

während $\psi(x, y)$ und $\chi(x, y)$ analog gebildet sind. Die Coordinaten $u_{1}, u_{2}, u_{3}$ der Verbindungslinie der beiden Pole genügen nun der Gleichung

$$
f\left(u_{1}, u_{2}, u_{3}\right) \equiv\left(u_{1} x_{1}+u_{2} x_{2}+u_{3} x_{3}\right)\left(u_{1} y_{1}+u_{2} y_{2}+u_{3} y_{3}\right)=0
$$

suchen wir die Enveloppe dieser Verbindungslinie, so müssen noch erfüllt werden die drei Gleichungen

$$
\frac{\partial f}{\partial u_{i}} \equiv u_{x} y_{i}+u_{y} x_{i}=0 \quad(i=1,2,3),
$$

und die Gleichung der Enveloppe ergibt sich schliesslich durch Elimination der sechs Grössen $x_{1} y_{1}, x_{2} y_{2}, x_{3} y_{3}, x_{1} y_{2}+x_{2} y_{1}, x_{2} y_{3}+x_{3} y_{2}$, $x_{3} y_{1}+x_{1} y_{3}$ aus den sechs Gleichungen (5) und (7) in Form einer Determinante, die sich von (4) nur dadurch unterscheidet, dass die Horizontal- und Verticalreihen vertauscht sind und jede der drei ersten Verticalreihen mit dem Factor $\frac{1}{2}$ multiplicirt erscheint.

Das Theorem VIII lässt sich auch in folgender Form aussprechen:

VIIIa. Jede Gerade, welche die Kegelschnitte des Netzes in Punktepaaren einer Involution schneidet, berührt die Cayley'sche Curve. Die Doppelpunkte der Involution auf dieser Geraden sind die conjugirten Pole der Hessiane.

Es geht dies daraus hervor, dass die Verbindungslinie zweier conjugirten Pole von allen Kegelschnitten des Netzes harmonisch getheilt wird und dass auf solche Weise eine Involution entsteht, welche diese conjugirten Pole zu Doppelpunkten hat.

Umgekehrt gilt auch folgender Satz:

VIIIb. Jede Tangente der Cayley'schen Curve trifft die Kegelschnitte des Netzes in Punktepaaren einer Involution.

Sind nämlich $u_{1}, u_{2}, u_{3}$ die Coordinaten einer Tangente der Cayley'schen Curve, so besteht nach VII eine Identität von der Form 


$$
\begin{aligned}
\left(u_{1} x_{1}+u_{2} x_{2}+u_{3} x_{3}\right) & \left(u_{1} x_{1}+v_{2} x_{2}+v_{3} x_{3}\right) \\
& \equiv \varkappa \varphi(x, x)+\lambda \psi(x, x)+\mu \chi(x, x),
\end{aligned}
$$

und wenn $\xi_{i}$ und $\eta_{i}(i=1,2,3)$ die Coordinaten zweier ganz willkürlichen Punkte bezeichnen, hat man auch

$$
u_{\xi} v_{\eta}+u_{\eta} v_{\xi}=2\{\varkappa \varphi(\xi, \eta)+\lambda \psi(\xi, \eta)+\mu \chi(\xi, \eta)\} .
$$

Da $\varkappa, \lambda, \mu$ nicht gleichzeitig verschwinden können, sei etwa $\mu$ von Null verschieden. Sind dann insbesondere $\xi$ und $\eta$ die Punkte jener Tangente, welche harmonisch liegen sowohl zu den Sclmittpunkten der Tangente mit $\varphi=0$, als auch $\mathrm{zu}$ denen mit $\psi=0$, so hat man die Gleichungen:

$$
u_{\xi}=0, \quad u_{\eta}=0, \quad \varphi(\xi, \eta)=0, \quad \psi(\xi, \eta)=0 ;
$$

aus (9) ergibt sich daher $\chi(\xi, \eta)=0$, d. h. das Punktepaar $\xi, \eta$ liegt auch harmonisch zu den Schnittpunkten jener Tangente mit $\chi=0$, man hat folglich auf der Tangente eine Involution, deren Doppelpunkte durch $\xi$ und $\eta$ gebildet werden.

Der obige Satz VIIIa, dass die Cayley'sche Curve von allen Geraden umhüllt wird, welche die Kegelschnitte des Netzes in Punktepaaren einer Involution schneiden, gestattet die Gleichung dieser Curve in einer Gestalt abzuleiten, die für viele Fälle zweckmässiger ist als die Berechnung der Determinante (4). Einer Geraden $u_{x}=0$ entspricht nämlich nach (6) in $\S 20$ als Ort ihrer Pole in Bezug auf die Kegelschnitte $\varkappa \varphi+\lambda \psi=0$ des Netzes $x \varphi+\lambda \psi+\mu \chi=0$ ein Kegelschnitt

$$
N_{\varphi, \psi} \equiv \frac{1}{4} \sum \pm\left(\varphi^{\prime}\left(x_{1}\right) \psi^{\prime}\left(x_{2}\right) u_{3}\right)=0
$$

und dieser trifft, wie gleichfalls in $\S 20$ gezeigt wurde, die Gerade $u_{x}=0$ in den Doppelpunkten $p, q$ der Involution, welche auf $u_{x}=0$ von den Kegelschnitten des Büschels ausgeschnitten wird. Bildet man nun nach (15) in $\S 15$ die Bedingung, dass die Gerade $u_{x}=0$ die Kegelschnitte $N_{\varphi, \psi}$ und $\chi=0$ in zwei harmonischen Punktepaaren trifft, so hat man hiermit die Gleichung der Cayley'schen Curve in Liniencoordinaten, denn das auf der Geraden gelegene Punktepaar $p, q$ liegt alsdann harmonisch zu den Schnittpunktepaaren der Geraden und der Kegelschnitte $\varphi=0, \psi=0, \chi=0$, d. h. die Gerade trifft die Kegelschnitte des Netzes in Punktepaaren einer Involution, ist somit Tangente der Cayley'schen Curve. 


\section{$\S 24$.}

\section{Das Kegelschnittgewebe.}

Es seien gegeben drei Curven zweiter Classe:

$$
\Phi(u, u)=0, \quad \Psi(u, u)=0, \quad \mathrm{X}(u, u)=0,
$$

die nicht einer und derselben Schaar angehören mögen. Es lassen sich alsdann Sätze aufstellen, die zu den in $\S 22$ und $\S 23$ bewiesenen dualistisch sind.

Zunächst werde bemẹrkt, dass eine Gleichung von der Form

$$
\varkappa \Phi(u, u)+\lambda \Psi(u, u)+\mu \mathrm{X}(u, u)=0
$$

entsprechend den zweifach unendlich vielen Werthen der Verhältnisse der Parameter $x, \lambda, \mu$ zweifach unendlich viele Curven zweiter Classe darstellt, deren Gesammtheit man als das durch $\Phi=0, \psi=0, X=0$ bestimmte Kegelschnittgewebe bezeichnet.

Dem Satze $I$ in $\S 22$ entspricht dualistisch ${ }^{1}$ ):

I. Jede Gerade $v$, deren Pole in Bezug auf drei gegebene Curven zweiter Classe (1) auf derselben Geraden u liegen, berïhrt zugleich mit dieser Geraden die Curve dritter Classe

$$
\sum \pm\left(\frac{\partial \Phi}{\partial u_{1}} \frac{\partial \Psi}{\partial u_{2}} \frac{\partial X}{\partial u_{3}}\right)=0
$$

die sogenannte Hesse'sche Curve oder Hessiane des Kegelschnittgewebes (2).

Zwei auf die soeben angegebene Art zusammengehörige Geraden heissen conjugirte Tangenten oder harmonische Polaren der Hessiane (3).

Ia. Umgekehrt kann auch jede Tangente der Curve dritter Classe (3) angesehen werden als eine Gerade, deren Pole in Bezug auf die drei gegebenen Kegelschnitte (1) in einer Geraden liegen.

II. Irgend zwei in dem Kegelschnittgewebe (2) enthaltenen Schaaren haben stets einen Kegelschnitt gemeinsam.

III. Die Träger aller in einem Kegelschnittgewebe enthaltenen Punktepaare berühren die Hessiane, und umgekehrt: Jede Tangente der Hessiane ist der Träger eines solchen Punktepaares.

1) Die Beweise der den Sätzen I-VIIIb in $\S 22$ und $\S 23$ dualistisch zugehörigen und durch dieselben Ziffern bezeichneten Sätze wurden in allen Fällen unterlassen, in denen sie den früher gegebenen Beweisen der entsprechenden Sätze mutatis mutandis gleich und besonders einfach waren. 
IIIa. Die Hessiane ist die Einhüllende der Seiten aller Poldreiecke, die irgend welchen Kegelschnitten des Gewebes gemeinsam sind.

IV. Wenn zwei Seitenpaare eines vollständigen Vierecks conjugirte Tangenten der Hessiane sind, so sind auch die Geraden des dritten Paares conjugirte Tangenten der gleichen Curve.

IVa. Eine Tangente $u$ der Hessiane des Gewebes (2) schneidet $z w e i$ conjugirte Tangenten $s$ und $\sigma$ derselben Curve in zwei Punkten $(u, s)^{1}$ ) und $(u, \sigma)$, von welchen sich noch zwei weitere conjugirte Tangenten $t$ und $\tau$ der Hessiane legen lassen; die Verbindungslinie der Punkte $(t, \sigma)$ und $(\tau, s)$ ist überdies die zu $u$ conjugirte Tangente $v$.

Dass nämlich die dritte Tangente $\tau$, die man von $(u, \sigma)$ an die Curve legen kann, in der That die zu $t$ conjugirte Tangente ist, ergibt sich folgendermassen: Bezeichnen wir die zu $\imath, s, t$ conjugirten Tangenten resp. durch $v^{\prime}, \sigma, \tau^{\prime}$, so müssen dieselben ein Dreiseit bilden, bei dem z. B. die Ecke $\left(\sigma \tau^{\prime}\right)$ auf $u$ liegt, d. h. aber $\tau^{\prime}$ geht durch $(u, \sigma)$ hindurch, und da $\tau^{\prime}$ Tangente der Curve ist, muss dieselbe mit der dritten von $(u, \sigma)$ an dic Curve gelegten Tangente $\tau$ zusammenfallen. Ebenso ergibt sich, dass auch $v^{\prime}$ mit $v$ identisch ist.

Aus IV a folgt weiter:

V. Die Gerade, welche die Berührungspunkte zweier conjugirten Tangenten $p$ und $\pi$ der Hessiane verbindet, ist selbst Tangente an diese Curve, und zwar conjugirt zu der dritten Tangente, welche vom Punkte $(p, \pi)$ an die Hessiane gelegt werden kann.

Nennen wir nämlich $q$ die dritte Tangente, welche sich vom Punkte $(p, \pi)$ an die Hessiane legen lässt und $x$ die zu $q$ conjugirte Tangente, so sind nach IV a auch die dritten Tangenten, welche sich von den Punkten $(\pi, q)$ und $(\pi, x)$ an die Curve legen lassen, conjugirt. Eine derselben ist $p$, und da ihre zugehörige $\pi$ ist, so fällt die dritte Tangente, die man von $(\pi, x)$ an die Curve legen kann, mit $\pi$ zusammen, d. h. aber $(\pi, x)$ ist der Berührungspunkt der Tangente $\pi$, und aus analogem Grunde ist $(p, x)$ der Berührungspunkt der Tangente $p$, so dass also in der That die Verbindungslinie $x$ dieser beiden Punkte die zu jener dritten Tangente $q$ conjugirte ist.

Der Cayley'schen Curve eines Kegelschnittnetzes entspricht eine analoge Curve des Gewebes auf Grund des folgenden Satzes:

1) $(p, q)$ bezeichnet allgemein den Schnittpunkt der Geraden $p$ und $q$. 
VI. Die Punktepare, die sich in einem Kegelschnittgewebe befinden, liegen auf einer Curve dritter Ordnung, der sogenannten Cayley'schen Curve des Gewebes.

Umgekehrt:

VII. Jeder Punkt dieser Cayley'schen Curve gehört einem Punktepaare des zugehörigen Kegelschnittgewebes an.

VIII. Der Schnittpunkt zweier conjugirten Tangenten der Hessiane ist ein Punkt der Cayley'schen Curve.

VIIIa. Alle Punkte, von welchen an die Kegelschnitte des Gewebes Tangentenpaare einer Involution gelegt werden können, liegen auf der Cayley'schen Curve. Die Doppelstrahlen dieser Involution sind die conjugirten Polaren der Hessiane des Gewebes.

Umgekehrt:

VIIIb. Von jedem Punkte der Cayley'schen Curve lassen sich an die Kegelschnitte des Gewebes Tangentenpare einer Involution legen.

\section{$\S 25$.}

Ueber einen merkwürdigen Dualismus, der zwischen der Hesse'schen und Cayley'schen Curve besteht.

Wir sind nunmehr in der Lage, ein wichtiges Uebertragungsprincip aufzustellen, welches gestattet, jedem Satz für die Hessiane ${ }^{1}$ ) sofort einen entsprechenden für die Cayley'sche Curve an die Seite zu stellen. Es ist zu diesem Zweck eine Curve dritter Classe einzuführen:

$$
P \equiv p_{111} u_{1}^{3}+3 p_{112} u_{1}^{2} u_{2}+3 p_{122} u_{1} u_{2}^{2}+\cdots+6 p_{123} u_{1} u_{2} u_{3}=0,
$$

wobei die Coefficienten $p_{x \lambda \mu}$ den im allgemeinen stets erfüllbaren neun Gleichungen zu genügen haben:

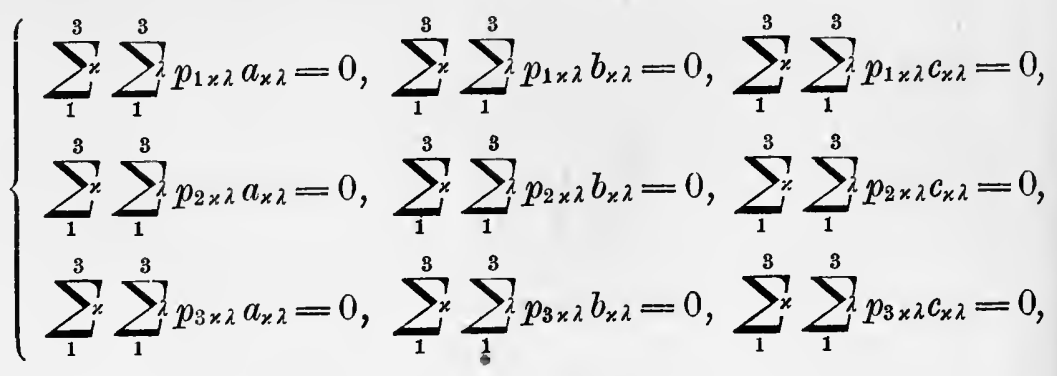

1) Wir werden in diesem Paragraphen unter der Hessiane, wenn nicht das Gegentheil bemerkt ist, stets die Hessiane des in $\S 22$ und $\S 23$ betrachteten Kegelschnittnetzes verstehen. 
in welchen $a_{x \lambda}, b_{x \lambda}, c_{x \lambda}$ die Coefficienten der Gleichungen dreier Curven zweiter Ordnung

$$
\varphi(x, x)=0, \quad \psi(x, x)=0, \quad \chi(x, x)=0
$$

darstellen, die nicht einem und demselben Büschel angehören.

Als erste Polare einer Geraden $v$ in Bezug auf die Curve dritter Classe $P=0$ bezeichnet man nun eine Curve, deren Gleicliung lautet ${ }^{1}$ )

$$
v_{1} \frac{\partial P}{\partial u_{1}}+v_{2} \frac{\partial P}{\partial u_{2}}+v_{3} \frac{\partial P}{\partial u_{3}}=0 .
$$

Diese Gleichung ordnet jeder Geraden $v$ der Ebene eine Curve zweiter Classe zu; den zweifach unendlich vielen Geraden der Ebene entsprechend repräsentirt daher (4) ein Kegelschnittgewebe. Für dasselbe gilt der wichtige Satz:

IX. Die Punktepare des Gewebes (4) sind harmonische Pole der Hessiane des Netzes

$$
\varkappa \varphi(x, x)+\lambda \psi(x, x)+\mu \chi(x, x)=0 .
$$

Soll nämlich eine Curve zweiter Classe (4) ein Punktepaar $x, y$ darstellen, so mïssen die Relationen bestehen

(6) $2\left(v_{1} p_{1 \times \lambda}+v_{2} p_{2 \varkappa \lambda}+v_{3} p_{3 \varkappa \lambda}\right)=x_{\star} y_{\lambda}+x_{\lambda} y_{*} \quad(x, \lambda=1,2,3)$.

Multiplicirt man nun (6) mit $a_{k \lambda}$ und summirt man sowohl über $x$ als über $\lambda$ von 1 bis 3 , so folgt mit Rücksicht auf das System (2):

$$
0=\sum_{1}^{3} \sum_{1}^{3} a_{* \lambda} x_{k} y_{\lambda},
$$

und ebenso würde man analog erhalten

$$
0=\sum_{1}^{3} \sum_{1}^{3} b_{x \lambda} x_{x} y_{\lambda} \quad \text { und } 0=\sum_{1}^{3} \sum_{1}^{3} c_{x \lambda} x_{x} y_{\lambda},
$$

womit gezeigt ist, dass die Punkte $x$ und $y$ harmonische Pole der Hessiane des Netzes (5) sind.

Dem soeben bewiesenen Theoreme steht reciprok das folgende gegenüber:

$X$. Die Geradenpare des Netzes (5) sind harmonische Polaren der Hessiane des Gewebes (4).

Die Coordinaten $u_{i}$ und $v_{i}$ eines jeden im Netze (5) enthaltenen Geradenpaares genügen nämlich dem System von Gleichungen

$$
2\left(\varkappa a_{\varrho \sigma}+\lambda b_{\rho \sigma}+\mu c_{\rho \sigma}\right)=u_{\varrho} v_{\sigma}+u_{\sigma} v_{\varrho} \quad(\varrho, \sigma=1,2,3) .
$$

1) Man vergleiche hierzu die auf die dualistisch entsprechenden Gebilde bei Curven $n^{\text {ter }}$ Ordnung sich beziehende Fussnote zu S. 22. 
Multiplicirt man (8) mit $p_{\tau \varrho \sigma}$ und summirt man über $\varrho$ und $\sigma$ von 1 bis 3 , so ergeben sich wegen (2) die drei Gleichungen

$$
\sum_{1}^{3} \sum_{1}^{3} p_{\varrho \sigma \tau} u_{\rho} v_{\sigma}=0 \quad(\tau=1,2,3),
$$

aus denen hervorgeht, dass die Geraden $u$ und $v$ harmonische Polaren der Hessiane des Gewebes (4) sind.

Da nun ferner nach III in $\S 22$ jeder Punkt der Hessiane eines Netzes zugleich der Schnittpunkt zweier dem Curvennetze angehörigen Geraden ist und diese zugleich nach dem soeben bewiesenen Satze harmonische Polaren der Hessiane des Gewebes (4) sind, liegt jeder Punkt der Hessiane des Netzes (5) nach VIII in $\S 24$ auch auf der Cayley'schen Curve des Gewebes (3).

Und da dualistisch nach III in $\$ 24$ jede Tangente der Hessiane eines Gewebes (4) zugleich Träger eines dem Gewebe angehörigen Punktepaares ist und die Punkte eines solchen Paares nach IX harmonische Pole der Hessiane des Netzes (5) sind, so ist jede Tangente der Hessiane des Gewebes (4) nach VIII in $\S 23$ zugleich auch Tangente der Cayley'schen Curve des Netzes (5).

Es lässt sich somit die Hessiane des Netzes (5) auffassen als die Cayley'sche Curve des Gewebes (4), und umgekehrt die Cayley'sche Curve des Netzes (5) als die Hessiane des (Gewebes $(4)^{1}$ ).

Die Eigenschaften dieser beiden Curven lassen sich daher unnittelbar auf einander übertragen.

Die Theoreme II, IV, IV a, V, VIIIa und VIIIb ị § 24 liefern z. B. für die Hesse'sche und Cayley'sche Curve des Gewebes erster Polaren

$$
v_{1} \frac{\partial P}{\partial u_{1}}+v_{2} \frac{\partial P}{\partial u_{2}}+v_{3} \frac{\partial P}{\partial u_{3}}=0
$$

d. h. für die Cayley'sche und Hesse'sche Curve des Netzes

$$
\varkappa \varphi(x, x)+\lambda \psi(x, x)+\mu \chi(x, x)=0
$$

sofort die folgenden Sätze XI, XII, XIIa, XIII, XIV:

XI. Die acht Seiten zweier vollständigen Vierseite, deren Paare von Gegenecken conjugirte Pole der Hessiane des Netzes (11) sind, berühren einen Kegelschnitt.

1) Der hier gegebene Beweis ist im Grunde auf den Fall beschränkt, dass (lie drei Kegelschnitte (3) keinen Punkt gemeinsam haben; diese Beschränkung wird in $\$ 26$ durch den Beweis der beiden Theoreme (38) und (39) vollständig aufgehoben werden. 
Denn die Hessiane dieses Netzes lässt sich auch auffassen als Cayley'sche Curve eines Gewebes; nach VII in $§ 24$ gehört aber jeder Punkt dieser Curve einem Punktepaare des Gewebes an, so dass jedes der beiden Vierseite durch seine Seiten eine in dem betreffenden Gewebe enthaltene Kegelschnittschaar bestimmt. Nach II in $\$ 24$ haben aber die zwei so entstehenden Kegelschnittschaaren eine Curve zweiter Classe gemeinsam, d. h. jene acht Seiten berühren einen Kegelschnitt.

XII. Wenn zwei Seitenpaare eines vollständigen Vierecks conjugirte Tangenten der Cayley'schen Curve eines Kegelschnittnetzes sind, so bilden auch die Geraden des dritten Paares conjugirte Tangenten der gleichen Curve.

XIIa. Eine Tangente $u$ der Cayley'schen Curve eines Netzes schneidet zwei conjugirte Tangenten $s$ und $\sigma$ derselben Curve in zwei Punkten, von welchen sich noch zwei weitere conjugirte Tangenten $t$ und $\tau$ der Curve legen lassen. Die Verbindungslinie der Punkte $(t, \sigma)$ und $(s, \tau)$ ist überdies die zu $u$ conjugirte Tangente $v$.

XIII. Die Gerade, welche die Berïhrungspunkte жweier conjugirten Tangenten $u$ und $v$ der Cayley'schen Curve verbindet, ist selbst Tangente an diese Curve, und zwar conjugirt zu der dritten Tangente, welche vom Punkte $(u, v)$ an die Cayley'sche Curve gelegt werden kann.

XIV. Die Geraden, welche irgend einen Punkt $p$ der Hessiane des Netzes (5) mit zwei conjugirten Polen verbinden, bilden eine Involution. Die Doppelstrahlen dieser Involution bestehen aus dem durch den Punkt gehendell Paare conjugirter Tangenten der Cayley'schen Curve.

Wendet man diesen Satz XIV nach dem bekannten Principe der Dualität an auf die Hessiane des Gewebes (10), d. h. auf die Cayley'sche Curve des Netzes (11), so folgt:

XV. Die Punkte, in denen irgend eine Tangente der Cayley'schen Curve des Netzes (11) von zwei conjugirten Tangenten dieser Curve getroffen wird, bilden eine Involution. Die Doppelstrahlen derselben bestehen aus dem auf der Tangente liegenden Paare conjugirter Pole der Hessiane.

Lässt man die Spitze des in Satz XIV erwähnten Strahlensystems in einen von zwei conjugirten Polen $p$ und $\pi$ der Hessiane rücken, so ergibt sich:

XVI. Die Tangente eines Punktes $p$ der Hessiane und die Verbindungslinie dieses Punktes mit seinem conjugirten Pole x liegen harmonisch zu den beiden conjugirten Tangenten 
der Cayley'schen Curve, welche ausser $p \pi$ noch an die Cayley'sche Curve gezogen werden können.

Lässt man analog den Träger des Punktsystems in XV mit einer von zwei conjugirten Tangenten der Cayley'schen Curve zusammenfallen, so folgt der Satz:

XVII. Jede Tangente der Cayley'schen Curve des Netzes (11) trifft die Hessiane in einem Paar conjugirter Pole, das harmonisch liegt zum dritten Schnittpunkt mit der Hessiane und zum Berührungspunkt mit der Cayley'schen Curve.

\section{$\S 26$.}

\section{Ueber conjugirte, lineare Kegelschnittsystem ${ }^{1}$ ).}

Der Satz, dass ein Kegelschnitt durch fünf Tangenten im allgemeinen vollständig und eindeutig bestimmt ist, bildet nur einen speciellen Fall des folgenden Satzes:

(1) Es gibt nur eine einzige Curve zweiter Classe, die conjugirt liegt $\left.{ }^{2}\right)$ zu den fünf gegebenen Curven zweiter Ordnung $f_{i}(x, x)=0(i=1,2,3,4,5)$, vorausgesetzt, dass nicht zwischen den fünf Ausdrücken $f_{i}(x, x)$ eine lineare Identität stattfindet.

Es ist also vorausgesetzt, dass keine Relation stattfindet von der Form

$$
\lambda_{1} f_{1}(x, x)+\lambda_{2} f_{2}(x, x)+\lambda_{3} f_{3}(x, x)+\lambda_{4} f_{4}(x, x)+\lambda_{5} f_{5}(x, x) \equiv 0,
$$

oder dass nicht gleichzeitig die sechs Gleichungen erfüllt werden:

$$
\lambda_{1} a_{i k}+\lambda_{2} b_{i k}+\lambda_{3} c_{i k}+\lambda_{4} d_{i k}+\lambda_{5} e_{i k}=0 \quad(i, k=1,2,3),
$$

in welchen $a_{i k}, b_{i k}, \ldots$ die Coefficienten in den Gleichungen der fünf gegebenen Curven zweiter Ordnung bedeuten.

Soll nun die Curve zweiter Classe

$$
\varphi(u, u) \equiv \sum_{1}^{3} \sum_{1}^{3} \alpha_{i k} u_{i} u_{k}=0
$$

1) Die Grundlage zu den hier behandelten Anfgaben findet sich der Hauptsache nach schon bei Hesse in der Abhandlung: „De curvis et superficiebus secundi ordinis‘c, Journal für die reine und angewandte Mathematik, Bd. 20, S. 293, $\S 8,1840$. Ueber die Deutung der simultanen Invariante $\Theta=0$ vergleiche man auch Hesse's Abhandlung: ,Ueber die geometrische Bedeutung der linearen Bedingungsgleichung zwischen den Coefficienten einer Gleichung zweiten Grades", in demselben Journal, Bd. 45, S. 82-90, 1852.

2) Vgl. zu dieser Bezeichnungsweise S. 162. 
conjugirt liegen zu den fünf Curven $f_{i}(x, x)=0$, so müssen neben (4) nach S. 162 die fünf Gleichungen bestehen

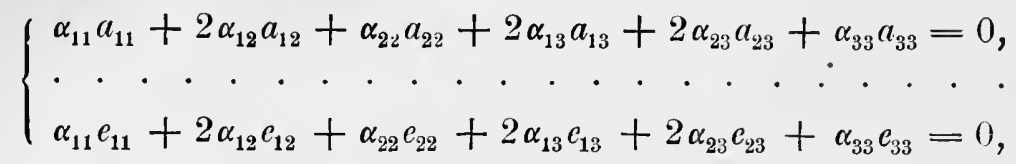

d. h. es muss die Determinante verschwinden:

$$
\left|\begin{array}{llllll}
a_{11} & a_{12} & a_{22} & a_{13} & a_{23} & a_{33} \\
b_{11} & b_{12} & b_{22} & b_{13} & b_{23} & b_{33} \\
c_{11} & c_{12} & c_{22} & c_{13} & c_{23} & c_{33} \\
d_{11} & d_{12} & d_{22} & d_{13} & d_{23} & d_{33} \\
e_{11} & c_{12} & c_{22} & e_{13} & e_{23} & c_{33} \\
u_{1}{ }^{2} & u_{1} u_{2} & u_{2}{ }^{2} & u_{1} u_{3} & u_{2} u_{3} & u_{3}^{2}
\end{array}\right|=0 .
$$

Auch können hier die Coefficienten der Quadrate und Producte $u_{i} u_{k}$ nicht sämmtlich Null sein, weil auf Grund der Voraussetzungen iiber die Ausdrücke (3) mindestens eine der sechs Unterdeterminanten der in der letzten Horizontalreihe von (6) stehenden Elemente von Null verschieden ist; es stellt mithin (6) die Gleichung der gesuchten Curve zweiter Classe $\varphi(u, u)=0$ dar.

Man erkennt auch sofort aus (5), dass diese Curve conjugirt liegt zu jedem Kegelschnitt des Systems

$$
\mu_{1} f_{1}+\mu_{2} f_{2}+\mu_{3} f_{3}+\mu_{4} f_{1}+\mu_{5} f_{5}=0 .
$$

Es gilt nun ferner der fundamentale Satz:

(7) Jede Curve zweiter Ordnung $f_{6}(x, x)=0$, welche conjugirt liegt zu (4), hat eine Gleichung von der Form

$$
f_{6} \equiv \mu_{1} f_{1}+\mu_{2} f_{2}+\mu_{3} f_{3}+\mu_{4} f_{4}+\mu_{5} f_{5}=0 \text {. }
$$

Sind nämlich $f_{i k}$ die Coefficienten von $f_{6}(x, x)=0$, so tritt zu den Relationen (5) noch hinzu

$$
\alpha_{11} f_{11}+2 \alpha_{12} f_{12}+\alpha_{22} f_{22}+2 \alpha_{13} f_{13}+2 \alpha_{23} f_{23}+\alpha_{33} f_{33}=0
$$

es verschwindet also eine Determinante, die aus (6) dadurch hervorgeht, dass man in der letzten Horizontalreihe $u_{i} u_{k}$ ersetzt durch $f_{i k}$. Nach einem fundamentalen Satze der Determinantentheorie gibt es also sechs Factoren $\lambda_{1}, \ldots \lambda_{6}$, für welche die sechs Gleichungen bestehen

$$
\lambda_{1} a_{i k}+\lambda_{2} b_{i k}+\lambda_{3} c_{i k}+\lambda_{4} d_{i k}+\lambda_{5} e_{i k}+\lambda_{6} f_{i k}=0 ;
$$

dabei muss $\lambda_{6}$ von Null verschieden sein, weil sonst gegen Voraussetzung die Relationen (3) erfüllt würden. Aus (9) folgt daher 
weln

$$
\begin{aligned}
f_{i k} & =-\frac{\lambda_{1} a_{i k}+\lambda_{2} b_{i k}+\lambda_{3} c_{i k}+\lambda_{4} d_{i k}+\lambda_{5} e_{i k}}{\lambda_{6}} \\
& =\mu_{1} a_{i k}+\mu_{2} b_{i k}+\mu_{3} c_{i k}+\mu_{4} d_{i k}+\mu_{5} e_{i k},
\end{aligned}
$$

$$
\mu_{i}=-\frac{\lambda_{i}}{\lambda_{6}} \quad(i=1,2,3,4,5)
$$

gesetzt wird; hiermit ist (7) bewiesen.

Sind die fünf Kegelschnitte $f_{i}(x, x)=0$ Doppelgeraden, sind also ihre Gleichungen von der Form $u_{x}^{\left(i^{2}\right.}=0$, so ist die Curve zweiter Classe $\varphi(u, u)=0$ der Kegelschnitt, der die fünf Geraden zu Tangenten hat. Für eine sechste Tangente $f_{6} \equiv u_{x}{ }^{(6)^{2}}=0$ würde alsdann eine ähnliche Relation wie (8) bestehen, man hat also den Satz:

(10) Die Ausdrücke für irgend sechs Tangenten $u_{x}^{(i)}=0$ $(i=1,2, \ldots 6)$ eines Kegelschnitts genügen einer Gleichung von der Form

$$
\lambda_{1} u_{x}^{(1)^{2}}+\lambda_{2} u_{x}^{(2)^{2}}+\lambda_{3} u_{x}^{(3)^{2}}+\lambda_{4} u_{x}^{(4)^{2}}+\lambda_{5} u_{x}^{(5)^{2}}+\lambda_{6} u_{x}^{(6)^{2}}=0,
$$

und umgekehrt.

Wir wollen nun dieselben Betrachtungen wie bisher anstellen für den Fall, dass nur vier Curven zweiter Ordnung gegeben sind. Es gilt hier der Satz:

(11) Sind vier Curven zweiter Ordnung $f_{i}(x, x)=0(i=1,2,3,4)$ gegeben, zwischen deren Gleichungen keine lineare Identität stattfindet, so gibt es unendlich viele, einer Schaar angehörige Curven zweiter Classe, welche zu den vier gegebenen Kegelschnitten conjugirt liegen.

Hier ist vorausgesetzt, dass nicht gleichzeitig die sechs Relationen erfüllt werden :

$$
\lambda_{1} a_{i k}+\lambda_{2} b_{i k}+\lambda_{3} c_{i k}+\lambda_{4} d_{i k}=0(i, k=1,2,3),
$$

in welchen $a_{i k}, b_{i k}, c_{i k}, d_{i k}$ die Coefficienten in den Gleichungen der vier gegebenen Curven zweiter Ordnung bedeuten.

Soll nun die Curve zweiter Classe (4) conjugirt liegen zu den vier Kegelschnitten $f_{i}(x, x)=0$, so müssen die vier Relationen bestehen

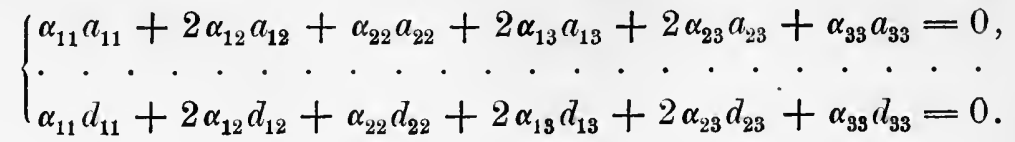

Auf Grund der gemachten Voraussetzungen muss nun mindestens eine aus Coefficienten $a, b, c, d$ gebildete Determinante vierten Grades der Matrix 


$$
\| \begin{array}{llllll}
a_{11} & a_{12} & a_{22} & a_{13} & a_{23} & a_{33} \\
b_{11} & b_{12} & b_{22} & b_{13} & b_{23} & b_{33} \\
c_{11} & c_{12} & c_{22} & c_{13} & c_{23} & c_{33} \\
d_{11} & d_{12} & d_{22} & d_{13} & d_{23} & d_{33}
\end{array} \mid
$$

von Null verschieden sein; es sei etwa, um die Vorstellung zu fixiren:

$$
d \equiv\left|\begin{array}{llll}
a_{11} & a_{12} & a_{22} & a_{13} \\
b_{11} & b_{12} & b_{22} & b_{13} \\
c_{11} & c_{12} & c_{22} & c_{13} \\
d_{11} & d_{12} & d_{22} & d_{13}
\end{array}\right| \geqslant 0 .
$$

Alsdaun stellen wir zusammen die fünf Gileichungen:

$$
\left\{\begin{array}{l}
\alpha_{11} a_{11}+2 \alpha_{12} a_{12}+\alpha_{22} a_{22}+2 \alpha_{13} a_{13}=-\left(2 \alpha_{23} a_{23}+\alpha_{33} a_{33}\right), \\
\alpha_{11} b_{11}+2 \alpha_{12} b_{12}+\alpha_{22} b_{22}+2 \alpha_{13} b_{13}=-\left(2 \alpha_{23} b_{23}+\alpha_{33} b_{33}\right), \\
\alpha_{11} c_{11}+2 \alpha_{12} c_{12}+\alpha_{22} c_{22}+2 \alpha_{13} c_{13}=-\left(2 \alpha_{23} c_{23}+\alpha_{33} c_{33}\right), \\
\alpha_{11} d_{11}+2 \alpha_{12} d_{12}+\alpha_{22} d_{22}+2 \alpha_{13} d_{13}=-\left(2 \alpha_{23} d_{23}+\alpha_{33} d_{33}\right), \\
\alpha_{11} u_{1}^{2}+2 \alpha_{12} u_{1} u_{2}+\alpha_{22} u_{2}{ }^{2}+2 \alpha_{13} u_{1} u_{3}+\left(2 \alpha_{23} u_{2} u_{3}+\alpha_{33} u_{3}{ }^{2}\right)=0 .
\end{array}\right.
$$

Mit Hilfe der vier ersten dieser Gleichungen kann man $\alpha_{11}, \alpha_{12}$, $\alpha_{22}, \alpha_{13}$ eindeutig und linear durch $\alpha_{23}$ und $\alpha_{33}$ ausdriicken, welch letztere Grössen vollständig beliebig bleiben; die so berechneten Werthe von $\alpha_{11}, \alpha_{12}, \alpha_{22}, \alpha_{13}$ setzt man nun in die letzte Gleichung (16) ein und multiplicirt dieselbe mit der alsdann links im Nenner auftretenden Determinante $d$. Es ist noch zu beachten, dass nur für $\alpha_{23}=\alpha_{33}=0$ in der letzten Gleichung (16) der links stehende Ausdruck für alle Werthe der $u$ verschwinden kann. Fände das Gegentheil statt, so müsste der Coefficient von $u_{2} u_{3}$ und derjenige von $u_{3}{ }^{2}$ gleichfalls verschwinden. Diese beiden Coefficienten sind aber $\alpha_{23} d$ und $\alpha_{33} d$, es müssten also $\alpha_{23}$ und $\alpha_{33}$ verschwinden.

Es gibt demnach unendlich viele Curven zweiter Classe der oben verlangten Art, und zwar sind ihre Gleichungen von der Form

$$
\alpha_{23} \psi(u, u)+\alpha_{33} \chi(u, u)=0,
$$

wobei $\alpha_{23}$ und $\alpha_{33}$ willkürliche Parameter bedeuten, oder, wie wir allgemeiner sagen wollen, ihre Gleichungen sind

$$
\alpha \psi(u, u)+\beta \chi(u, u)=0,
$$

die einzelnen Curven gehören somit sämmtlich derselben Schaar an. Auch erkennt man wieder, dass auf Grund von (16) jede Curve dieser Schaar conjugirt ist zu jedem Kegelschnitt des Systems

$$
\mu_{1} f_{1}+\mu_{2} f_{2}+\mu_{3} f_{3}+\mu_{4} f_{4}=0 .
$$


Umgekehrt gilt der Satz:

(19) Wenn eine Curve zweiter Ordnung $f_{5}(x, x)=0$ conjugirt liegt zu jeder Curve der Schaar (17), so ist ihre Gleichung von der Form (18).

Es sei $\varphi(u, u) \equiv \sum_{1}^{3} \sum_{1}^{3} \alpha_{i k} u_{i} u_{k}=0$ irgend ein Kegelschnitt der Schaar (17); alsdann tritt zu den vier Gleichungen (13) noch hinzu $\alpha_{11} e_{11}+2 \alpha_{12} e_{12}+\alpha_{22} e_{22}+2 \alpha_{13} e_{13}+2 \alpha_{23} e_{23}+\alpha_{33} e_{33}=0$, wenn wir mit $e_{i k}$ die Coefficienten von $f_{5}(x, x)=0$ bezeichnen. Werden diese fünf Gleichungen resp. mit $\lambda_{1}, \lambda_{2}, \lambda_{3}, \lambda_{4}, \lambda_{5}$ multiplicirt und hierauf addirt, und setzt man zur Abkürzung

$$
\left[i, l_{0}\right] \equiv \lambda_{1} a_{i k}+\lambda_{2} b_{i k}+\lambda_{3} c_{i k}+\lambda_{4} d_{i k}+\lambda_{5} e_{i k},
$$

so folgt

$$
\begin{gathered}
\alpha_{11}[1,1]+2 \alpha_{12}[1,2]+\alpha_{22}[2,2]+2 \alpha_{13}[1,3] \\
+2 \alpha_{23}[2,3]+\alpha_{33}[3,3]=0 .
\end{gathered}
$$

Dem Umstande zufolge, dass $\varphi(u, u)=0$ der Schaar (17) angehört, ist die Gleichung dieser Curve von der Form

$$
\alpha \cdot \sum_{1}^{3} \sum_{1}^{3} k \gamma_{i k} u_{i} u_{k}+\beta \cdot \sum_{1}^{3} \sum_{1}^{3} \delta_{i k} u_{i} u_{k}=0,
$$

wenn wir mit $\gamma_{i k}$ und $\delta_{i k}$ die Coefficienten von $\psi$ und $\chi$ in (17) bezeichnen. Hier ist mindestens eine aus diesen Coefficienten gebildete Determinante der Gestalt $\left|\begin{array}{ll}\gamma_{i k} & \gamma_{l m} \\ \delta_{i k} & \delta_{l m}\end{array}\right|$ von Null verschieden; nehmen wir, um die Vorstellung zu fixiren, etwa an, es sei

$$
\gamma_{23} \delta_{33}-\gamma_{33} \delta_{23} \gtrless 0 \text {. }
$$

Wir denken uns dann $\lambda_{1}, \lambda_{2}, \lambda_{3}, \lambda_{4}, \lambda_{5}$ so bestimmt, dass alle Grössen $[i, k]$ verschwinden mit Ausnahme von $[2,3]$ und $[3,3]$; hierdurch reducirt sich (21) auf

$$
2 \alpha_{23}[2,3]+\alpha_{33}[3,3]=0,
$$

wobei nochmals darauf hingewiesen werden möge, dass $\alpha_{i k}=\alpha \gamma_{i k}+\beta \delta_{i k}$ und $\alpha, \beta$ ganz willkürlich sind. Setzt man nun $\alpha=1, \beta=0$, bezw. $\alpha=0, \beta=1$, so treten an Stelle von (23) die zwei Gleichungen

$$
2 \gamma_{23}[2,3]+\gamma_{33}[3,3]=0,2 \delta_{23}[2,3]+\delta_{33}[3,3]=0,
$$

aus welchen auf Grund von (22) folgt $[2,3]=0,[3,3]=0$. Hiermit ist aber nun gezeigt, dass alle Grössen $[i, k](i, k=1,2,3)$ verschwinden, dass also in der That $e_{i k}=-\frac{\lambda_{1} a_{i k}+\cdots+\lambda_{4} d_{i k}}{\lambda_{5}}$; auch 
muss $\lambda_{5}$ in den Relationen $[i, k]=0$ von Null verschieden sein, sonst würden gegen die Voraussetzung die Gleichungen (12) bestehen.

Wenn man speciell als Curve $f_{5}(x, x)=0$ irgend eine doppelt zu zählende gemeinsame Tangente $u_{x}^{(i)^{2}}=0$ der Kegelschnitte $\psi(u, u)=0$ und $\chi(u, u)=0$ wählt, erhält man, da es im allgemeinen vier verschiedene solcher Tangenten gibt, den Satz:

Sind $f_{i}(x, x)=0(i=1,2,3,4)$ die Gleichungen von vier Curven zweiter Ordnung, so lassen sich im allgemeinen auf vier verschiedene Arten Factoren $\lambda_{1}, \lambda_{2}, \lambda_{3}, \lambda_{4}$ so bestimmen, dass $\lambda_{1} f_{1}+\lambda_{2} f_{2}+\lambda_{3} f_{3}+\lambda_{4} f_{4}$ das Quadrat eines linearen Ausdrucks darstellt.

Uebrigens folgt hieraus auch umgekehrt, dass jede von vier gegebenen, linear unabhängigen, tern̈̈ren quadratischen Formen als lineare homogene Function derselben vier Quadrate $\left(u x^{\left.(i)^{2}\right)}\right.$ dargestellt werden kann, so lange die Curven der Schaar $\alpha \psi(u, u)+\beta \chi(u, u)=0$ vier verschiedene gemeinsame Tangenten haben.

Wenn nur drei Kegelschnitte gegeben sind, tritt an Stelle von (11) der folgende Satz:

(26) Sind drei Curven zweiter Ordnung $f_{i}(x, x)=0(i=1,2,3)$ gegeben, $z$ wischen deren Gleichungen keine lineare Identität stattfindet, so gibt es unendlich viele, ein Gewebe bildende Curven zweiter Classe, welche zu den drei gegebenen Kegelschnitten conjugirt liegen.

Jede Curve $\varphi(u, u) \equiv \sum_{1}^{3} \sum_{1}^{3} \alpha_{i k} u_{i} u_{k}=0$ des Gewebes muss sich also durch drei solcher Curven ausdrücken lassen in der Form:

$$
\varphi(u, u) \equiv \gamma \psi(u, u)+\delta \chi(u, u)+\varepsilon \eta(u, u) .
$$

Nach Voraussetzung liönnen nämlich nicht gleichzeitig die sechs Relationen erfüllt werden

$$
\lambda_{1} a_{i k}+\lambda_{2} b_{i k}+\lambda_{3} c_{i k}=0 \quad(i, k=1,2,3),
$$

in welchen $a_{i k}, b_{i k}, c_{i k}$ die Coefficienten in den Gleichungen der drei gegebenen Curven zweiter Ordnung bedeuten; es muss also mindestens eine Determinante dritten Grades der Matrix

$$
\mid \begin{array}{llllll}
a_{11} & a_{12} & a_{22} & a_{13} & a_{23} & a_{33} \\
b_{11} & b_{12} & b_{22} & b_{13} & b_{23} & b_{33} \\
c_{11} & c_{12} & c_{22} & c_{13} & c_{23} & c_{33}
\end{array} \|
$$

von Null verschieden sein; es sei etwa, um die Vorstellung \%u fixiren: 


$$
d \equiv\left|\begin{array}{lll}
a_{11} & a_{12} & a_{22} \\
b_{11} & b_{12} & b_{22} \\
c_{11} & c_{12} & c_{22}
\end{array}\right| \gtrless 0 .
$$

Alsdann müssen analog zu (13), bezw. (16) die Gleichungen bestehen:

$$
\left\{\begin{array}{l}
\alpha_{11} a_{11}+2 \alpha_{12} a_{12}+\alpha_{22} a_{22}=-\left(2 \alpha_{13} a_{13}+2 \alpha_{23} a_{23}+\alpha_{33} a_{33}\right) \\
\alpha_{11} b_{11}+2 \alpha_{12} b_{12}+\alpha_{22} b_{22}=-\left(2 \alpha_{13} b_{13}+2 \alpha_{23} b_{23}+\alpha_{33} b_{33}\right) \\
\alpha_{11} c_{11}+2 \alpha_{12} c_{12}+\alpha_{22} c_{22}=-\left(2 \alpha_{13} c_{13}+2 \alpha_{23} c_{23}+\alpha_{33} c_{33}\right) \\
\alpha_{11} u_{1}^{2}+2 \alpha_{12} u_{1} u_{2}+\alpha_{22} u_{2}{ }^{2}+\left(2 \alpha_{13} u_{1} u_{3}+2 \alpha_{23} u_{2} u_{3}+\alpha_{33} u_{3}^{2}\right)=0
\end{array}\right.
$$

Mit Hilfe der drei ersten dieser Gleichungen kann man nun wieder $\alpha_{11}, \alpha_{12}, \alpha_{22}$ eindeutig und linear ausdrücken durch $\alpha_{13}, \alpha_{23}, \alpha_{33}$, welch letztere Grössen ganz beliebig bleiben. Durch Substitution der Werthe von $\alpha_{11}, \alpha_{12}, \alpha_{22}$ in die letzte Gleichung (31) erhält man einen Ausdruck zweiten Grades in $u$ mit $\alpha_{13}, \alpha_{23}, \alpha_{33}$ als willkürlichen Parametern. Dabei sind die Ausdrücke zweiten Grades der $u_{i}$, die mit $\alpha_{13}, \alpha_{23}, \alpha_{33}$ multiplicirt sind, linear unabhängig, d. h. nur für $\alpha_{13}=\alpha_{23}$ $=\alpha_{33}=0$ kann der in der letzten Gleichung (31) links stehende Ausdruck für alle Werthe der $u$ verschwinden. Fände das Gegentheil statt, so müssten die Coefficienten von $u_{1} u_{3}, u_{2} u_{3}, u_{3}{ }^{2}$ verschwinden; diese sind aber $\alpha_{13} d, \alpha_{23} d, \alpha_{33} d$, es müssten also $\alpha_{13}, \alpha_{23}$ und $\alpha_{33}$ verschwinden.

Es gibt demnach zweifach unendlich viele Curven der oben verlangten Art, und zwar sind ihre Gleichungen, wie wir nun allgemeiner sagen können, von der Form (27), sie gehören somit sämmtlich demselben Gewebe an.

Auch erkennt man wieder, dass auf Grund von (31) jede Curve des Gewebes (27) conjugirt ist zu jedem Kegelschnitt des Netzes

$$
\mu_{1} f_{1}+\mu_{2} f_{2}+\mu_{3} f_{3}=0 \text {. }
$$

Umgekehrt gilt der Satz:

(32a) Wenn eine Curve zweiter Ordaung $f_{4}(x, x)=0$ conjugirt liegt zu jeder Curve des Gewebes (27), so ist ihre Gleichung von der Form (32).

Zum Beweis dieses Satzes sei $\varphi(u, u) \equiv \sum_{i}^{3} \sum_{i}^{3} \alpha_{i k} u_{i} u_{k}=0$ irgend ein Kegelschnitt des Gewebes (27); alsdann tritt zu den drei ersten Gleichungen von (31) noch hinzu

$$
\alpha_{11} d_{11}+2 \alpha_{12} d_{12}+\alpha_{22} d_{22}+2 \alpha_{13} d_{13}+2 \alpha_{23} d_{23}+\alpha_{33} d_{33}=0,
$$

wenn wir mit $d_{i k}$ die Coefficienten von $f_{4}(x, x)=0$ bezeichnen. Durch 
Multiplication dieser vier Gleichungen mit $\lambda_{1}, \lambda_{2}, \lambda_{3}, \lambda_{4}$ und Addition erhält man

$$
\begin{gathered}
\alpha_{11}(1,1)+2 \alpha_{12}(1,2)+\alpha_{22}(2,2)+2 \alpha_{13}(1,3) \\
+2 \alpha_{23}(2,3)+\alpha_{33}(3,3)=0
\end{gathered}
$$

wobei zur Abkürzung gesetzt ist

$$
(i, k) \equiv \lambda_{1} a_{i k}+\lambda_{2} b_{i k}+\lambda_{3} c_{i k}+\lambda_{4} d_{i k} .
$$

Dem Umstande zufolge, dass $\varphi(u, u)=0$ dem Gewebe (27) angehört, ist die Gleichung dieser Curve von der Form

$$
\gamma \sum_{1}^{3} \sum_{1}^{3} k \gamma_{i k} u_{i} u_{k}+\delta \sum_{1}^{3} i \sum_{1}^{3} \delta_{i k} u_{i} u_{k}+\varepsilon \sum_{1}^{3} \sum_{1}^{3} \varepsilon_{i k} u u_{i} u_{k}=0,
$$

wenn wir mit $\gamma_{i k}, \delta_{i k}, \varepsilon_{i k}$ die Coefficienten von $\psi, \chi$ und $\eta$ in (27) bezeichnen. Es ist nun mindestens eine aus diesen Coefficienten gebildete Determinante von Null verschieden; nehmen wir etwa an, es sei

$$
\left|\begin{array}{lll}
\gamma_{13} & \gamma_{23} & \gamma_{33} \\
\delta_{13} & \delta_{23} & \delta_{33} \\
\varepsilon_{13} & \varepsilon_{23} & \varepsilon_{33}
\end{array}\right| \gtrless 0
$$

Wir denken uns alsdanu die Verhältnisse $\lambda_{1}: \lambda_{2}: \lambda_{3}: \lambda_{4}$ so bestimmt, dass die drei Grössen $(1,1),(1,2)$ und $(2,2)$ verschwinden, wodurch sich (33) reducirt auf

$$
2 \alpha_{13}(1,3)+2 \alpha_{23}(2,3)+\alpha_{33}(3,3)=0,
$$

und hier sind in $\alpha_{i k}=\gamma \cdot \gamma_{i k}+\delta \cdot \delta_{i k}+\varepsilon \cdot \varepsilon_{i k}$ die Parameter $\gamma, \delta$, $\varepsilon$ ganz willkürlich. Setzt man nun einmal $\gamma=1, \delta=\varepsilon=0$, ferner $\delta=1, \gamma=\varepsilon=0$, endlich $\varepsilon=1, \gamma=\delta=0$, so treten an Stelle von (36) die drei Gleichungen

$$
\left\{\begin{array}{l}
2 \gamma_{13}(1,3)+2 \gamma_{23}(2,3)+\gamma_{33}(3,3)=0 \\
2 \delta_{13}(1,3)+2 \delta_{23}(2,3)+\delta_{33}(3,3)=0 \\
2 \varepsilon_{13}(1,3)+2 \varepsilon_{23}(2,3)+\varepsilon_{33}(3,3)=0
\end{array}\right.
$$

aus welchen auf Grund von (35) folgt $(1,3)=(2,3)=(3,3)=0$. Mithin verschwinden sämmtliche Grössen $(i, k)$, nach (34) ist also in der That $d_{i k}=-\frac{\lambda_{1} a_{i k}+\lambda_{2} b_{i k}+\lambda_{3} c_{i k}}{\lambda_{4}}$; auch muss $\lambda_{4}$ von Null verschieden sein, sonst würden gegen die Voraussetzung die Gleichungen (28) bestehen.

Das Theorem (32a) ist, wenn man von einem directen Beweis absehen will, im Grunde schon durch (26) erwiesen. Denn alle ternäre quadratische Formen, die zu drei gegebenen, linear unabhängigen 
quadratischen Formen von contragredienten Veränderlichen conjugirt sind, lassen sich nach (26) als lineare Functionen dreier linear unabhängigen quadratischen Formen ausdrücken. Da nun in (32a) die drei gegebenen (linear unabhängigen) Formen $f_{1}, f_{2}, f_{3}$ conjugirt sind zu den drei Formen $\psi, \chi, \eta$ in (27), so muss jeder Kegelschnitt $f_{4}$, der zu jeder Curve des Gewebes (27) conjugirt sein soll, eine Gleichung haben von der Form

$$
f_{4} \equiv \mu_{1} f_{1}+\mu_{2} f_{2}+\mu_{3} f_{3} .
$$

Mittels der bisherigen Betrachtungen lassen sich sofort die zwei fundamentalen Theoreme beweisen:

(38) Die Cayley'sche Curve des Gewebes (27) ist identisch mit der Hessiane des Netzes (32), und:

(39) Die Hessiane des Gewebes (27) ist identisch mit der Cayley'schen Curve des Netzes (32).

Nach VI und VII in $\S 24$ besteht nämlich die Cayley'sche Curve des Gewebes aus den Punktepaaren des Gewebes; diese sind aber conjugirte Polenpaare des Netzes. Umgekehrt wird nach $I$ in $\S 24$ die Hesse'sche Curve des Gewebes eingehüllt von den harmonischen Polarenpaaren des Gewebes; diese sind aber identisch mit den Geradenpaaren des Netzes.

Der Vollständigkeit halber sei noch darauf aufmerksan gemacht, dass selbstverständlich die zu vorstehenden Sätzen dualistisch entsprechenden in analoger Art zu beweisen sind. Auch gilt das Beweisverfahren noch bei beliebig vielen Formen mit einer willkürlichen Anzahl von Veränderlichen.

Ein eleganter Beweis der in vorliegendem Paragraphen abgeleiteten Sätze kann allgemein auch mit Hilfe eines von Herrn Brill gefundenen fundamentalen Theorems ${ }^{1}$ ) gegeben werden.

1) Brill: „Ueber zwei Berührungsprobleme“, Math. Annalen Bd. 4, S. 530, 1871; vgl. auch Pasch: „Zur Theorie der linearen Complexe", Journal für die reine und angewandte Mathematik, Bd. 75, S. 108, 1872. 


\section{Anhang.}

Ergänzungen zur voranstehenden Theorie und Lösung von Aufgaben.

\section{Anwendung von \& 1-3.}

1. Das Product $h_{1} h_{2} h_{3}$ hat positiven Werth, wenn der Einheitspunkt trigonal gelegen ist, negativen Werth bei tetragonaler Lage ${ }^{1}$ ).

Liegt der Einheitspunkt $E$ im Inneren des Dreiecks, so ist $h_{1} h_{2} h_{3}$ sicher positiv; beim Uebergang von $E$ in ein tetragonales Feld ändert eine der Grössen $h_{i}$ das Vorzeichen, das Product wird also negativ; beim Uebergang aus dem Inneren des Dreiecks in ein trigonales Feld ändern zwei der Grössen $h_{i}$ das Vorzeichen, das Product bleibt also positiv.

2. Bei beliebiger Lage des Einheitspunktes ist das Product $\frac{q_{1} q_{2} q_{3}}{h_{1} h_{2} h_{3}}$ positiv, wenn der zugehörige Punkt $P$ trigonal gelegen ist, negativ bei tetragonaler Lage.

Aus $\left.(1)^{2}\right)$ und aus einer Bemerkung S. 2, Zeile $13-9$ v. u. geht hervor, dass bei trigonaler Lage des Punktes $P$ das Product der $q_{i}$ stets gleiches Vorzeichen hat mit dem Product der $h_{i}$ (positives oder negatives Vorzeichen, je nachdem $E$ trigonal oder tetragonal gelegen ist). Ebenso folgt, dass bei tetragonaler Lage des Punktes $P$ das Product der $q_{i}$ stets das dem Product der $h_{i}$ entgegengesetzte Vorzeichen hat, und zwar ist das Product der $q_{i}$ positiv oder negativ, je nachdem der Einheitspunkt tetragonal oder trigonal gelegen ist. Bei tetragonaler Lage des Punktes $P$ ist also der Quotient $\frac{q_{1} q_{2} q_{3}}{h_{1} h_{2} h_{3}}$ in der That negativ.

1) Wir sagen im Folgenden ein Punkt liege trigonal, wenn er im Inneren oder in einem der drei trigonalen Felder des Coordinatendreiecks gelegen ist; hingegen liegt er tetragonal, wenn er sich in einem der drei tetragonalen Felder befindet.

2) Die in Klammern eingeschlossenen Zahlen, denen keine Seiten- oder Paragraphenzahl beigefügt ist, bezeichnen die Nummern der Aufgaben des Anhanges. 
3. Ein Punkt mit den Coordinaten $x_{1}: x_{2}: x_{3}$ liegt trigonal oder tetragonal, je nachdem der Ausdruck

$$
\frac{x_{1} x_{2} x_{3}}{p_{1} p_{2} p_{3}}\left(p_{1} x_{1}+p_{2} x_{2}+p_{3} x_{3}\right)
$$

positiv oder negativ ist; die Lage des Einheitspunktes ist dabei ganz willkürlich.

Führt man in das Product $\frac{q_{1} q_{2} q_{3}}{h_{1} h_{2} h_{3}}$ für die $q_{i}$ aus (6), S. 3 die Werthe ein, so verwandelt sich dasselbe in $\frac{e_{1} e_{2} e_{3} x_{1} x_{2} x_{3}}{p_{x}^{3} \cdot h_{1} h_{2} h_{3}}$ oder in $\frac{p_{1} p_{2} p_{3} x_{1} x_{2} x_{3}}{\left(p_{1} x_{1}+p_{2} x_{2}+p_{3} x_{3}\right)^{3}} \cdot$ Nach Multiplication mit dem positiven Factor $p_{x}^{4}$ erhält man den Ausdruck $x_{1} x_{2} x_{3} p_{1} p_{2} p_{3}\left(p_{1} x_{1}+p_{2} x_{2}+p_{3} x_{3}\right)$, der mit $\frac{x_{1} x_{2} x_{3}}{p_{1} p_{2} p_{3}}\left(p_{1} x_{1}+p_{2} x_{2}+p_{3} x_{3}\right)$ und mit $\frac{q_{1} q_{2} q_{3}}{h_{1} h_{2} h_{3}}$ gleiches Vorzeichen hat und bei Anwendung von (2) obigen Satz liefert. Das Vorzeichen des Ausdrucks ändert sich übrigens nicht, wenn man die $p_{i}$ und $x_{i}(i=1,2,3)$ durch Werthe ersetzt, die ihnen proportional sind.

4. Gegeben sind drei Geraden $u_{x}=0, v_{x}=0, w_{x}=0$ und die unendlich ferne Gerade $p_{x}=0$, bezogen auf ein Coordinatendreieck mit deu Seiten $x_{1}=0, x_{2}=0, x_{3}=0$. Wann liegt ein Punkt mit den Coordinaten $y_{1}: y_{2}: y_{3}$ trigoual in Bezug auf das durch $u_{x}=0$, $v_{x}=0, w_{x}=0$ gebildete Dreieck, wann liegt er tetragonal?

Um dies zu untersuchen, setze man $\varrho u_{x}=X_{1}, \varrho v_{x}=X_{2}$, $\varrho w_{x}=X_{3}$, wobei $\varrho$ einen Proportionalitätsfactor bedeutet. In Folge der Relation

oder

$$
\left|\begin{array}{llll}
u_{1} & u_{2} & u_{3} & u_{x} \\
v_{1} & v_{2} & v_{3} & v_{x} \\
w_{1} & w_{2} & w_{3} & w_{x} \\
p_{1} & p_{2} & p_{3} & p_{x}
\end{array}\right| \equiv 0
$$

hat man

$$
\left.(u v w) p_{x}=(p v w) u_{x}+(p w u) v_{x}+(p u v) w_{x}{ }^{1}\right)
$$

$$
\varrho(u v w) p_{x}=(p v w) X_{1}+(p w u) X_{2}+(p u v) X_{3},
$$

so dass also die Gleichung der unendlich fernen Geraden im Coordinatensystem der $X_{i}$ die Gestalt erhält

$$
(p v w) X_{1}+(p w u) X_{2}+(p u \dot{v}) X_{3} \equiv P_{1} X_{1}+P_{2} X_{2}+P_{3} X_{3}=0 \text {. }
$$

1) $(u v w)$ ist zur Abkürzung gesetzt für $\Sigma \pm\left(u_{1} v_{2} w_{3}\right)$, analoges gilt von $(p v w)$ u. s. w. 
Die Coordinaten des Punktes $y$ im Coordinatensystem der $X_{i}$ seien $Y_{1}: Y_{2}: Y_{3} ;$ nach (3) liegt alsdann der Punkt $Y$ trigonal oder tetragonal in Bezug auf das durch $u_{x}=0, v_{x}=0, w_{x}=0$ gebildete Dreieck, je nachdem

$$
\frac{Y_{1} Y_{2} Y_{3}}{P_{1} P_{2} P_{3}}\left(P_{1} Y_{1}+P_{2} Y_{2}+P_{3} Y_{3}\right)>0 \text { oder }<0
$$

d. h. je nachdem

$\frac{u_{y} v_{y} w_{y} \cdot\left[(p v w) u_{y}+(p w u) v_{y}+(p u v) w_{y}\right]}{(p v w)(p w u)(p u v)}=\frac{u_{y} v_{y} w_{y} p_{y}(u v w)}{(p v w)(p w u)(p u v)}>0$ oder $<0$.

Der jedenfalls positive Factor $\varrho^{4}$ wurde hierbei ausser Acht gelassen.

5. Die Gleichung einer Geraden, die durch den Schnittpunkt der Geraden $u_{x}=0$ und $v_{x}=0$, sowie durch einen Punkt $y$ hindurchgeht, ist $v_{y} u_{x}-v_{y} v_{x}=0$.

In $u_{x}-\lambda v_{x}=0$ ist $\lambda$ so zu bestimmen, dass diese Gleichung durch $y$ erfüllt wird. Ersetzt man $v_{x}=0$ durch $p_{x}=0$, so folgt:

6. Die Gleichung einer durch den Punkt $y$ parallel zu $u_{x}=0$ gezogenen Geraden ist $p_{y} u_{x}-u_{y} p_{x}=0$.

7. Die "Schwerlinie“, welche die Ecke $x_{2}=x_{3}=0$ des Coordinatendreiecks mit der Mitte der Gegenseite verbindet, hat die Gleichung $p_{2} x_{2}-p_{3} x_{3}=0$.

Die genannte Verbindungslinie ist die vierte Harmonische zu $x_{2}=0, x_{3}=0$ und zu der durch $x_{2}=x_{3}=0$ gezogenen Parallelen der Gegenseite; letztere ist aber nach (6) gegeben durch $p_{2} x_{2}+p_{3} x_{3}=0$.

8. Die Gleichung der Halbirungslinie des von den beiden Geraden $a_{x}=0$ und $b_{x}=0$ gebildeten Winkels ist $\frac{a_{x}}{\sqrt{\omega(a, a)}}-\frac{b_{x}}{\sqrt{\omega(b, b)}}=0$.

Jeder Punkt der Halbirungslinie hat von beiden Geraden gleichen Abstand; mit Anwendung von (1) in $\S 2$ findet man die Gleichung. Aus den Bemerkungen zu (21) in $\S 1$ folgt, dass die Gleichung die Halbirungslinie desjenigen Winkels der beiden Geraden darstellt, in welchem der Einheitspunkt liegt, wenn man das Vorzeichen von $\sqrt{\omega(a, a)}$ und $\sqrt{\omega(b, b)}$ mit demjenigen von $a_{1}+a_{2}+a_{3}$, bezw. $b_{1}+b_{2}+b_{3}$ übereinstimmen lüsst. Gibt man nur einer dieser Wurzeln ein anderes Vorzeichen, so erhält man die Halbirungslinie des Winkels, in welchem der Einheitspunkt nicht liegt.

Beide Halbirungslinien zusammen sind dargestellt durch

$$
\omega(b, b) \cdot a_{x}^{2}-\omega(a, a) \cdot b_{x}^{2}=0 .
$$


Insbesondere folgt:

9. Die Gleichung $\frac{x_{2}}{\sqrt{\omega_{2.2}}}-\frac{x_{8}}{\sqrt{\omega_{33}}}=0$ repräsentirt die Halbirungslinie desjenigen Winkels der beiden Seiten $x_{2}=0, x_{3}=0$ des Coordinatendreiecks, in welchem der Einheitspunkt liegt, $\frac{x_{2}}{\sqrt{\omega_{22}}}+\frac{x_{3}}{\sqrt{\omega_{33}}}=0$ die Halbirungslinie des Nebenwinkels.

10. Die Gerade, welche durch den Punkt $y$ normal zu $u_{x}=0$ gezogen wird, hat die Gleichung

$$
\left|\begin{array}{lll}
x_{1} & x_{2} & x_{3} \\
\omega^{\prime}\left(u_{1}\right) & \omega^{\prime}\left(u_{2}\right) & \omega^{\prime}\left(u_{3}\right) \\
y_{1} & y_{2} & y_{3}
\end{array}\right|=0 .
$$

Denn sie geht durch $y$ und das Normalencentrum von $u_{x}=0$. Insbesondere folgt:

11. Die Gleichungen der Höhen des Coordinatendreiecks sind: $\omega_{31} x_{2}-\omega_{12} x_{3}=0, \quad \omega_{12} x_{3}-\omega_{23} x_{1}=0, \quad \omega_{23} x_{1}-\omega_{31} x_{2}=0$.

12. Die Coordinaten der Mitte der Verbindungslinie zweier Punkte $y$ und $z$ sind $y_{i} p_{z}+z_{i} p_{y},(i=1,2,3)$.

Irgend ein Punkt der Verbindungslinie hat Coordinaten

$$
y_{i}+\lambda z_{i},(i=1,2,3) \text {; }
$$

der unendlich ferne muss erfüllen $p_{y}+\lambda p_{z}=0$, für ihn wird

$$
\lambda=-p_{y}: p_{z}
$$

dem Mittelpunkt der Strecke $(y z)$ entspricht als viertem harmonischem Punkte zu $y, z$ und zu dem unendlich fernen Punkte der Werth

$$
\lambda=+p_{y}: p_{z} \text {. }
$$

Die Gleichung des Mittelpunktes ist $p_{z} u_{y}+p_{y} u_{z}=0$.

13. Die Gleichung des Mittellothes der Verbindungslinie zweier Punkte $y$ und $z$ ist $p_{z}\left(\begin{array}{ll}y & x \\ y & z\end{array}\right)_{\omega_{i k}}-p_{y}\left(\begin{array}{ll}z & x \\ z & y\end{array}\right)_{\omega_{i k}}=0$.

Sind

$$
v_{1}=y_{2} z_{3}-y_{3} z_{2}, \quad v_{2}=y_{3} z_{1}-y_{1} z_{3}, \quad v_{3}=y_{1} z_{2}-y_{2} z_{1}
$$

die Coordinaten der Verbindungslinie $(y z)$, so ist die Gleichung des Mittellothes nach (10) und (12):

$$
\left|\begin{array}{lll}
\omega^{\prime}\left(v_{1}\right) & y_{1} p_{z}+z_{1} p_{y} & x_{1} \\
\omega^{\prime}\left(v_{2}\right) & y_{2} p_{z}+z_{2} p_{y} & x_{2} \\
\omega^{\prime}\left(v_{3}\right) & y_{3} p_{z}+z_{3} p_{y} & x_{3}
\end{array}\right|=0
$$


die nach Einführung der $v_{i}$ in die Gestalt gebracht werden kann

$$
\left|\begin{array}{lllll}
\omega_{11} & \omega_{12} & \omega_{13} & y_{1} p_{z}+z_{1} p_{y} & x_{1} \\
\omega_{21} & \omega_{22} & \omega_{23} & y_{2} p_{z}+z_{2} p_{y} & x_{2} \\
\omega_{31} & \omega_{32} & \omega_{33} & y_{3} p_{z}+z_{3} p_{y} & x_{3} \\
y_{1} & y_{2} & y_{3} & 0 & 0 \\
z_{1} & z_{2} & z_{3} & 0 & 0
\end{array}\right|=0 \text { oder } p_{z}\left(\begin{array}{ll}
y & x \\
y & z
\end{array}\right)_{\omega_{i k}}-p_{y}\left(\begin{array}{ll}
z & x \\
z & y
\end{array}\right)_{\omega_{i k}}=0 \text {. }
$$

Insbesondere folgt:

14. Die Mittellothe der Seiten $x_{1}=0, x_{2}=0, x_{3}=0$ des Coordinatendreiecks haben bezw. die Gleichungen

$$
\begin{aligned}
& \left(\omega_{12} p_{2}-\omega_{13} p_{3}\right) x_{1}-\omega_{11}\left(p_{2} x_{2}-p_{3} x_{3}\right)=0, \\
& \left(\omega_{23} p_{3}-\omega_{21} p_{1}\right) x_{2}-\omega_{22}\left(p_{3} x_{3}-p_{1} x_{1}\right)=0, \\
& \left(\omega_{31} p_{1}-\omega_{32} p_{2}\right) x_{3}-\omega_{33}\left(p_{1} x_{1}-p_{2} x_{2}\right)=0 .
\end{aligned}
$$

Zum Beweis, dass sich diese drei Geraden in einem und demselben Punkte schneiden, multiplicire man ihre Gleichungen resp. mit $p_{1}{ }^{2}, p_{2}{ }^{2}, p_{3}{ }^{2}$ und eliminire die Grössen $\omega_{i i} p_{i}$ vermittelst der Relationen wie $\omega_{11} p_{1}=-\left(\omega_{12} p_{2}+\omega_{13} p_{3}\right)$, u. s. w. Werden hierauf die drei Gleichungen addirt, so ergibt sich eine identisch verschwindende Summe.

Die obigen Gleichungen der Mittellothe können noch in eine andere Gestalt gebracht werden, welche zeigt, dass diese Geraden durch die Ecken des dem Coordinatendreieck parallel eingeschriebenen Dreiecks hindurchgehen. Multiplicirt man nämlich z. B. die Gleichung des Mittellothes von $x_{1}=0$ mit $p_{1}$ und ersetzt $\omega_{11} p_{1}$ wieder durch $-\left(\omega_{12} p_{2}+\omega_{13} p_{3}\right)$, so erhält man:

$$
\omega_{12} p_{2}\left(p_{1} x_{1}+p_{2} x_{2}-p_{3} x_{3}\right)-\omega_{13} p_{3}\left(p_{1} x_{1}-p_{2} x_{2}+p_{3} x_{3}\right)=0 .
$$

Die Mittellothe von $x_{2}=0$ und $x_{3}=0$ werden:

$$
\begin{aligned}
& \omega_{23} p_{3}\left(-p_{1} x_{1}+p_{2} x_{2}+p_{3} x_{3}\right)-\omega_{21} p_{1}\left(p_{1} x_{1}+p_{2} x_{2}-p_{3} x_{3}\right)=0, \\
& \omega_{31} p_{1}\left(p_{1} x_{1}-p_{2} x_{2}+p_{3} x_{3}\right)-\omega_{32} p_{2}\left(-p_{1} x_{1}+p_{2} x_{2}+p_{3} x_{3}\right)=0 .
\end{aligned}
$$

Dass die in den $x_{i}$ linearen Klammerfactoren die Seiten des dem Coordinatendreieck parallel eingeschriebenen Dreiecks darstellen, wird in (22) gezeigt.

15. Der Schwerpunkt des Coordinatendreiecks hat die Coordinaten

Folgt sofort aus (7).

$$
x_{1}: x_{2}: x_{3}=\frac{1}{p_{1}}: \frac{1}{p_{2}}: \frac{1}{p_{3}} .
$$


16. Die Mittelpunkte des dem Coordinatendreieck eingeschriebenen und der drei angeschriebenen Kreise haben die Coordinaten

$$
x_{1}: x_{2}: x_{3}= \pm \sqrt{\omega_{11}}: \pm \sqrt{\omega_{22}}: \pm \sqrt{\omega_{33}},
$$

wobei gleiche Vorzeichen der drei Wurzeln dem eingeschriebenen Kreise entsprechen, falls der Einheitspunkt imerhalb des Dreiecks gelegen ist; die übrigen Combinationen der Vorzeichen liefern die Mittelpunkte der drei angeschriebenen Kreise.

Folgt sofort aus (9).

1\%. Der Höhenschnittpunkt des Coordinatendreiecks hat die Coordinaten

Folgt sofort aus (11).

$$
x_{1}: x_{2}: x_{3}=\frac{1}{\omega_{23}}: \frac{1}{\omega_{31}}: \frac{1}{\omega_{12}} .
$$

18. Der Mittelpunkt des dem Coordinatendreieck umschriebenen Kreises hat die Coordinaten

$$
x_{1}: x_{2}: x_{3}=\omega_{11} \omega_{23}: \omega_{22} \omega_{31}: \omega_{33} \omega_{12} \text {. }
$$

Folgt sofort durch Berechnung des Schnittpunktes der Mittellothe, deren Gleichungen in (14) gegeben sind.

19. Man bestimme für das Coordinatendreieck die Coordinaten des Mittelpunktes $N$ des eingeschriebenen Kreises, des Mittelpunktes $M$ des umschriebenen Kreises, des Schwerpunktes $S$, des Höhenschnittpunktes $H$, wenn der Einheitspunkt $E$ in einem dieser vier Punkte gelegen ist.

Das mit Hilfe von (15)-(18) durch Einführung der Stücke des Coordinatendreiecks leicht abzuleitende Resultat möge folgender Tabelle entnommen werden, in welcher $\alpha, \beta, \gamma$ die Winkel des Dreiecks bezeichnen.

\begin{tabular}{l|c|c|c|c}
\hline & Einheitspunkt in $N$ & Einheitspunkt in $M$ & Einheitspunkt in $S$ & Einheitspunkt in 1 \\
\hline Coord. von $N$ & $1: 1: 1$ & $\frac{1}{\cos \alpha}: \frac{1}{\cos \beta}: \frac{1}{\cos \gamma}$ & $\sin \alpha: \sin \beta: \sin \gamma$ & $\cos \alpha: \cos \beta: \cos$ \\
\hline Coord. von $M$ & $\cos \alpha: \cos \beta: \cos \gamma$ & $1: 1: 1$ & $\sin 2 \alpha: \sin 2 \beta: \sin 2 \gamma$ & $\cos ^{2} \alpha: \cos ^{2} \beta: \cos ^{2}$ \\
\hline Coord. von $S$ & $\frac{1}{\sin \alpha}: \frac{1}{\sin \beta}: \frac{1}{\sin \gamma}$ & $\frac{1}{\sin 2 \alpha}: \frac{1}{\sin 2 \beta}: \frac{1}{\sin 2 \gamma}$ & $1: 1: 1$ & $\cot \alpha: \cot \beta: \cot$ \\
\hline Coord. von $H$ & $\frac{1}{\cos \alpha}: \frac{1}{\cos \beta}: \frac{1}{\cos \gamma}$ & $\frac{1}{\cos ^{2} \alpha}: \frac{1}{\cos ^{2} \beta}: \frac{1}{\cos ^{2} \gamma}$ & $\operatorname{tg} \alpha: \operatorname{tg} \beta: \operatorname{tg} \gamma$ & $1: 1: 1$
\end{tabular}


20. Man bilde die Gleichung der unendlich fernen Geraden, wenn der Einheitspunkt eine der vier eben genannten Lagen hat.

Die Gleichung der unendlich fernen Geraden ist allgemein

$$
\frac{e_{1}}{h_{1}} x_{1}+\frac{e_{2}}{h_{2}} x_{2}+\frac{e_{3}}{h_{3}} x_{3}=0
$$

man findet leicht nachstehendes Resultat:

Einheitspunkt in $N: x_{1} \sin \alpha+x_{2} \sin \beta+x_{3} \sin \gamma=0$,

Einheitspunkt in $M: x_{1} \sin 2 \alpha+x_{2} \sin 2 \beta+x_{3} \sin 2 \gamma=0$,

Einheitspunkt in $S: x_{1}+x_{2}+x_{3}=0$,

Einheitspunkt in $H: x_{1} \operatorname{tg} \alpha+x_{2} \operatorname{tg} \beta+x_{3} \operatorname{tg} \gamma=0$.

21. Welche geometrische Bedeutung haben die drei Geraden

$$
-v_{1} x_{1}+v_{2} x_{2}+v_{3} x_{3}=0, \quad v_{1} x_{1}-v_{2} x_{2}+v_{3} x_{3}=0 \text {, }
$$

$$
v_{1} x_{1}+v_{2} x_{2}-v_{3} x_{3}=0 \text {, }
$$

wenn

$$
v_{1} x_{1}+v_{2} x_{2}+v_{3} x_{3}=0
$$

irgend eine fest gegebene Gerade darstellt?

Wir brauchen nur eine Gerade zu untersuchen, etwa

$$
-v_{1} x_{1}+v_{2} x_{2}+v_{3} x_{3}=0
$$

die Bedeutung der anderen ist dann analog. Diese Gerade ist offenbar die vierte Harmonische zu $v_{x}=0$ und $\mathrm{zu}$ dem Geradenpaare $x_{1}=0, v_{2} x_{2}+v_{3} x_{3}=0$, wobei letztere Gleichung die Verbindungrlinie des Schnittpunktes von $v_{x}=0$ und $x_{1}=0$ mit der Ecke $x_{2}=x_{3}=0$ des Coordinatendreiecks darstellt. Man kann also sagen: Verbindet man den Schnittpunkt $Q$ der Seite $A_{2} A_{3}$ oder $x_{1}=0$ des Coordinatendreiecks $A_{1} A_{2} A_{3}$ und einer Geraden $v_{x}=0$ mit der Gegenecke $A_{1}$ des Dreiecks, so repräsentirt $-v_{1} x_{1}+v_{2} x_{2}+v_{3} x_{3}=0$ die vierte harmonische Gerade zu dem durch $Q$ gehenden Geradenpaare $A_{2} A_{3}, Q A_{1}$ und zu $v_{x}=0$.

Die drei obigen Geraden schneiden also bezw. die Seiten $x_{1}=0$, $x_{2}=0, x_{3}=0$ des Coordinatendreiecks in Punkten, die auf

liegen.

$$
v_{1} x_{1}+v_{2} x_{2}+v_{3} x_{3}=0
$$

Wenn man als Gerade $v_{x}=0$ die unendlich ferne wählt $\left(v_{i}=p_{i}\right)$, ergibt sich:

22. Die Seiten des dem Coordinatendreieck parallel eingeschriebenen Dreiecks haben die Gleichungen:

$$
\begin{gathered}
-p_{1} x_{1}+p_{2} x_{2}+p_{3} x_{3}=0, \quad p_{1} x_{1}-p_{2} x_{2}+p_{3} x_{3}=0, \\
p_{1} x_{1}+p_{2} x_{2}-p_{3} x_{3}=0 .
\end{gathered}
$$


23. Man bestimme die Länge $l$ der Normalen, welche vom Punkte $y$ auf diejenige Seite des dem Coordinatendreieck parallel eingeschriebenen Dreiecks gefällt ist, welche zu $x_{1}=0$ parallel verläuft.

Die Gleichung dieser Parallelen ist nach (22):

$$
-p_{1} x_{1}+p_{2} x_{2}+p_{3} x_{3}=0
$$

für die Coordinaten $\imath_{i}$ dieser Geraden wird

$$
\omega(u, u)=-4 p_{1}\left(\omega_{12} p_{2}+\omega_{13} p_{3}\right)=4 \omega_{11} p_{1}{ }^{2},
$$

daher findet man nach $(1)$ in $\S 2$

$$
l=\frac{-p_{1} y_{1}+p_{2} y_{2}+p_{3} y_{3}}{2 p_{1} \sqrt{ } \omega_{11}\left(p_{1} y_{1}+p_{2} y_{2}+p_{3} y_{3}\right)} .
$$

Analoge Werthe ergeben sich für die Abstände des Punktes $y$ von den beiden anderen Seiten des parallel eingeschriebenen Dreiecks.

24. Die Geraden, welche die auf den Seiten $x_{1}=0, x_{2}=0$, $x_{3}=0$ des Coordinatendreiecks gelegenen Fusspunkte $A^{\prime}, B^{\prime}, C^{\prime}$ der Höhen dieses Dreiecks verbinden, haben die Gleichungen:

$$
\begin{aligned}
& \left.B^{\prime} C^{\prime}\right) \quad-\omega_{23} x_{1}+\omega_{31} x_{2}+\omega_{12} x_{3}=0, \\
& \left.C^{\prime} A^{\prime}\right) \quad \omega_{23} x_{1}-\omega_{31} x_{2}+\omega_{12} x_{3}=0, \\
& \left.A^{\prime} B^{\prime}\right) \quad \omega_{23} x_{1}+\omega_{31} x_{2}-\omega_{12} x_{3}=0 .
\end{aligned}
$$

Die Coordinaten der Fusspunkte $A^{\prime}, B^{\prime}, C^{\prime}$ sind nach (11): für $A^{\prime}$ ) $x_{1}=0, x_{2}: x_{3}=\omega_{12}: \omega_{13}$, für $\left.B^{\prime}\right) x_{2}=0, x_{1}: x_{3}=\omega_{21}: \omega_{23}$, für $\left.C^{\prime}\right) x_{3}=0, x_{1}: x_{2}=\omega_{31}: \omega_{32}$.

Hieraus folgen leicht die obigen Gleichungen.

25. Der Inhalt $J$ eines Dreiecks, dessen Ecken $P_{1}, P_{2}, P_{3}$ die Coordinaten haben $x_{1}, x_{2}, x_{3} ; y_{1}, y_{2}, y_{3} ; z_{1}, z_{2}, z_{3}$, ist gegeben durch die Formel

$$
\pm J=\frac{p_{1} p_{2} p_{3} \cdot \Delta \cdot \sum \pm\left(x_{1} y_{2} z_{3}\right)}{p_{x} p_{y} p_{z}},
$$

wobei das positive oder negative Vorzeichen zu stehen hat, je nachdem das Dreieck $P_{1} P_{2} P_{3}$ mit dem Coordinatendreieck 1-2-3 $(=\Delta)$ gleichstimmig liegt oder nicht, $d . h$. je nachdem man beim Unlauf des Dreiecks $P_{1} P_{2} P_{3}$ im Sinne $P_{1}-P_{2}-P_{3}$ die Fläche des Dreiecks auf derselben Seite hat wie beim Umlauf von $\Delta$ in Sinne $1-2-3$, oder nicht.

Bezogen auf irgend ein rechtwinkliges Hilfscoordinatensystem 
seien $x^{\prime}, y^{\prime} ; x^{\prime \prime}, y^{\prime \prime} ; x^{\prime \prime \prime}, y^{\prime \prime \prime}$ die Coordinaten der Ecken des Dreiecks $\boldsymbol{P}_{1} \boldsymbol{P}_{2} \boldsymbol{P}_{3} ;$ man hat alsdann für den Inhalt $J$ die Gleichung

$$
J= \pm \frac{1}{2}\left|\begin{array}{lll}
x^{\prime} & y^{\prime} & 1 \\
x^{\prime \prime} & y^{\prime \prime} & 1 \\
x^{\prime \prime \prime} & y^{\prime \prime \prime} & 1
\end{array}\right|
$$

wobei das positive oder negative Vorzeichen zu setzen ist, je nachdem die Seite $P_{1} P_{2}$ zur Seite $P_{1} P_{3}$ so liegt wie die positive $x$-Axe zur positiven $y$-Axe, oder umgekehrt ${ }^{1}$ ). Zufolge (9) in $\S 3$ bestehen nun zwischen den rechtwinkligen und Dreieckscoordinaten der Ecken $P_{1}, P_{2}, P_{3}$ Beziehungen von der Form:

$$
\begin{gathered}
\sigma^{\prime} x^{\prime}=A_{1} x_{1}+B_{1} x_{2}+C_{1} x_{3} \quad \sigma^{\prime \prime} x^{\prime \prime}=A_{1} y_{1}+B_{1} y_{2}+C_{1} y_{3} \\
\sigma^{\prime} y^{\prime}=A_{2} x_{1}+B_{2} x_{2}+C_{2} x_{3} \quad \sigma^{\prime \prime} y^{\prime \prime}=A_{2} y_{1}+B_{2} y_{2}+C_{2} y_{3} \\
\sigma^{\prime}=A_{3} x_{1}+B_{3} x_{2}+C_{3} x_{3} \quad \sigma^{\prime \prime}=A_{3} y_{1}+B_{3} y_{2}+C_{3} y_{3} \\
\sigma^{\prime \prime \prime} x^{\prime \prime \prime}=A_{1} z_{1}+B_{1} z_{2}+C_{1} z_{3} \\
\sigma^{\prime \prime \prime} y^{\prime \prime \prime}=A_{2} z_{1}+B_{2} z_{2}+C_{2} z_{3} \\
\sigma^{\prime \prime \prime}=A_{3} z_{1}+B_{3} z_{2}+C_{3} z_{3},
\end{gathered}
$$

wobei die Grössen $A_{3}, \mathcal{B}_{3}, C_{3}$ jedenfalls den Coordinaten $p_{1}, p_{2}, p_{3}$ der unendlich fernen Geraden proportional sind. Durch Einfïlırung der Dreieckscoordinaten in den Ausdruck für $J$ erluält maı zufolge des Multiplicationstheorems der Determinanten

$$
\begin{aligned}
J & = \pm \frac{1}{2} \sum \pm\left(A_{1} B_{2} C_{3}\right) \cdot \sum \pm\left(x_{1} y_{2} z_{3}\right): \sigma^{\prime} \sigma^{\prime \prime} \sigma^{\prime \prime \prime} \\
& = \pm \frac{1}{2} \frac{\sum \pm\left(A_{1} B_{2} C_{3}\right)}{A_{3} B_{3} C_{3}} \cdot \sum \pm\left(x_{1} y_{2} z_{3}\right): \frac{\sigma^{\prime} \sigma^{\prime \prime} \sigma^{\prime \prime \prime}}{A_{3} B_{3} C_{3}}
\end{aligned}
$$

ṇun sind aber

$$
\frac{A_{1}}{A_{3}}, \frac{A_{2}}{A_{3}} ; \frac{B_{1}}{B_{3}}, \frac{B_{2}}{B_{33}} ; \frac{C_{1}}{C_{3}}, \frac{C_{2}}{C_{3}}
$$

die rechtwinkligen Coordinaten der Ecken des Coordinatendreiecks $\triangle$, daher $\Delta= \pm \frac{1}{2} \sum \pm\left(A_{1} B_{2} C_{3}\right): A_{3} B_{3} C_{3}$, wo wieder das positive oder negative Vorzeichen gilt, je nachdem die Seiten $\overline{12}$ und 13 des

1) Wir setzen die (übrigens leicht abzuleitende) Formel für $J$ bei rechtwinkligen Coordinaten als bekaunt voraus und bemerken nur noch, dass eine ähnliche Vorzeichenregel wie die obige zuerst, allerdings bei anderer Gelegenheit, von Gauss gegeben sein dürfte in seinen „Disquisitiones generales circa superficies curvas", Commentationes soc. reg. scient. Gotting. rec., Bd. 6, S. 106, 1827, oder Gauss' Werke, hr'sgg. von der kgl. Ges. d. Wissensch. zu Göttingen, Bd.4, S. 225, oder in der deutschen, von Herrn W angerin besorgten Ausgabe S. 10 (Ostwald's Classiker der exacten Wissenschaften, Nr. 5). 
Dreiecks $\Delta$ so zu einander liegen wie die positive $x$-Axe zur positiven $y$-Axe, oder umgekehrt. Es wird folglich

$$
\pm J=\frac{\Delta \cdot \sum \pm\left(x_{1} y_{2} z_{3}\right) \cdot A_{3} B_{3} C_{3}}{\sigma^{\prime} \sigma^{\prime \prime} \sigma^{\prime \prime \prime}}
$$

und wir können nun sagen, dass hier das positive oder negative Vorzeichen zu stehen hat, je nachdem die Dreiecke $P_{1} P_{2} P_{3}$ und $\Delta$ gleichstimmig liegen oder nicht. Mit Hilfe von $A_{3}: B_{3}: C_{3}=p_{1}: p_{2}: p_{3}$ ergibt sich sofort die zu beweisende Formel. Man könnte an Stelle von $p_{1} p_{2} p_{3} \Delta$ auf Grund der Formeln S. 61 einsetzen $\frac{1}{2 \sqrt{\tau}}$ und hätte alsdaun $\pm 2 J=\frac{\sum \pm\left(x_{1} y_{2} z_{3}\right)}{p_{x} \cdot p_{y} \cdot p_{z} \sqrt{\tau}}$, und zwar wäre hier rechts $\sqrt{\tau}$ dasselbe Vorzeichen $\mathrm{zu}$ ertheilen, welches $p_{1} p_{2} p_{3}$ besitzt.

\section{Anwendung von $\S 4-6$.}

26. Die Gleichung einer dem Coordinatendreieck umschriebenen Curve zweiter Ordnung ist von der Form

$$
a_{1} x_{2} x_{3}+a_{2} x_{3} x_{1}+a_{3} x_{1} x_{2}=0 .
$$

Die quadratischen Glieder müssen fehlen, weil die Gleichung erfüllt werden muss sowohl durch $x_{2}=x_{3}=0$, als durch $x_{3}=x_{1}=0$, als durch $x_{1}=x_{2}=0$.

Dualistisch folgt:

27. Die Gleichung einer dem Coordinatendreiseit eingeschriebenen Curve zweiter Classe ist von der Form

$$
\alpha_{1} u_{2} u_{3}+\alpha_{2} u_{3} u_{1}+\alpha_{3} u_{1} u_{2}=0 \text {. }
$$

28. Wie lautet die Gleichung einer dem Coordinatendreieck umschriebenen Curve zweiter Ordnung, deren Mittelpunkt die Coordinaten $y_{1}, y_{2}, y_{3}$ besitzt?

In $f(x, x) \equiv 2\left(a_{1} x_{2} x_{3}+a_{2} x_{3} x_{1}+a_{3} x_{1} x_{2}\right)=0$ sind die $a$ auszudrücken durch die $y$; unter Berücksichtigung von

folgt

$$
y_{1}: y_{2}: y_{3}=\frac{1}{2} F^{\prime}\left(p_{1}\right): \frac{1}{2} F^{\prime}\left(p_{2}\right): \frac{1}{2} F^{\prime}\left(p_{3}\right)
$$

$$
\begin{gathered}
\varrho y_{1}=a_{1}\left(-a_{1} p_{1}+a_{2} p_{2}+a_{3} p_{3}\right), \quad \varrho y_{2}=a_{2}\left(a_{1} p_{1}-a_{2} p_{2}+a_{3} p_{3}\right) \\
\varrho y_{3}=a_{3}\left(a_{1} p_{1}+a_{2} p_{2}-a_{3} p_{3}\right)
\end{gathered}
$$


Mittelpunkt eines Kegelschnitts in spec. Lage zum Coordinatendreieck, 251 woraus man erhält

$$
\begin{gathered}
\sigma a_{1}=y_{1}\left(-p_{1} y_{1}+p_{2} y_{2}+p_{3} y_{3}\right), \quad \sigma a_{2}=y_{2}\left(p_{1} y_{1}-p_{2} y_{2}+p_{3} y_{3}\right), \\
\sigma a_{3}=y_{3}\left(p_{1} y_{1}+p_{2} y_{2}-p_{3} y_{3}\right),
\end{gathered}
$$

mit $\varrho$ und $\sigma$ als Proportionalitätsfactoren.

29. Wie lautet die Gleichung einer dem Coordinatendreiseit eingeschriebenen Curve zweiter Classe, deren Mittelpunkt die Coordinaten $y_{1}, y_{2}, y_{3}$ besitzt?

In $\varphi(u, u) \equiv 2\left(\alpha_{1} u_{2} u_{3}+\alpha_{2} u_{3} u_{1}+\alpha_{3} u_{1} u_{2}\right)=0$ sind die $\alpha$ auszudrücken durch die $y$; unter Berücksichtigung von

folgt

$$
y_{1}: y_{2}: y_{3}=\frac{1}{2} \varphi^{\prime}\left(p_{1}\right): \frac{1}{2} \varphi^{\prime}\left(p_{2}\right): \frac{1}{2} \varphi^{\prime}\left(p_{3}\right)
$$

$$
\varrho y_{1}=\alpha_{3} p_{2}+\alpha_{2} p_{3}, \quad \varrho y_{2}=\alpha_{3} p_{1}+\alpha_{1} p_{3}, \quad \varrho y_{3}=\alpha_{2} p_{1}+\alpha_{1} p_{2},
$$

woraus man erhält

$$
\begin{gathered}
\sigma \alpha_{1}=p_{1}\left(-p_{1} y_{1}+p_{2} y_{2}+p_{3} y_{3}\right), \quad \sigma \alpha_{2}=p_{2}\left(p_{1} y_{1}-p_{2} y_{2}+p_{3} y_{3}\right), \\
\sigma \alpha_{3}=p_{3}\left(p_{1} y_{1}+p_{2} y_{2}-p_{3} y_{3}\right),
\end{gathered}
$$

mit $\varrho$ und $\sigma$ als Proportionalitätsfactoren.

30. Die Gleichung derjenigen Curve zweiter Ordnung, welche das Coordinatendreieck zum Poldreieck hat und deren Mittelpunkt die Coordinaten $y_{1}, y_{2}, y_{3}$ besitzt, lautet

$$
p_{1} y_{2} y_{3} x_{1}^{2}+p_{2} y_{3} y_{1} x_{2}{ }^{2}+p_{3} y_{1} y_{2} x_{3}{ }^{2}=0 \text {. }
$$

Die Gleichung ist nach (50), S. 33 von der Form

$$
a_{11} x_{1}{ }^{2}+a_{23} x_{2}{ }^{2}+a_{33} x_{3}{ }^{2}=0 \text {; }
$$

andrerseits findet man nach (23), S. 25 für die Coordinaten des Mittelpunktes $p_{1}: p_{2}: p_{3}=a_{11} y_{1}: a_{22} y_{2}: a_{33} y_{3}$, daher

$$
a_{11}: a_{22}: a_{33}=p_{1} y_{2} y_{3}: p_{2} y_{3} y_{1}: p_{3} y_{1} y_{2} \text {. }
$$

31. Die Gleichung derjenigen Curve zweiter Classe, welche das Coordinatendreiseit zum Poldreiseit hat und deren Mittelpunkt die Coordinaten. $y_{1}^{\prime}, y_{2}, y_{3}$ besitzt, lautet

$$
p_{2} p_{3} y_{1} u_{1}^{2}+p_{3} p_{1} y_{2} u_{2}^{2}+p_{1} p_{2} y_{3} u_{3}^{2}=0 \text {. }
$$

Die Gleichung ist von der Form $\alpha_{1} u_{1}{ }^{2}+\alpha_{2} u_{2}{ }^{2}+\alpha_{3} u_{3}{ }^{2}=0$; andrerseits findet man nach (20), S. $43 y_{1}: y_{2}: y_{3}=\alpha_{1} p_{1}: \alpha_{2} p_{2}: \alpha_{3} p_{3}$, daher $\alpha_{1}: \alpha_{2}: \alpha_{3}=p_{2} p_{3} y_{1}: p_{3} p_{1} y_{2}: p_{1} p_{2} y_{3}$.

32. In welcher Weise wird die Gestalt aller durch drei Punkte 
$A, B, C$ der Ebene gehenden Curven zweiter Ordnung durch die Lage ihres Mittelpunktes bedingt? ${ }^{1}$ )

Die Gleichung des dem Coordinatendreieck umschriebenen Kegelschnitts mit dem Mittelpunkte $y$ ist nach (28)

$f(x, x) \equiv 2\left\{y_{1}\left(-p_{1} y_{1}+p_{2} y_{2}+p_{3} y_{3}\right) x_{2} x_{3}+y_{2}\left(p_{1} y_{1}-p_{2} y_{2}+p_{3} y_{3}\right) x_{3} x_{1}\right.$

oder

$$
\left.+y_{3}\left(p_{1} y_{1}+p_{2} y_{2}-p_{3} y_{3}\right) x_{1} x_{2}\right\}=0
$$

$$
2\left\{y_{1} r_{y} x_{2} x_{3}+y_{2} s_{y} x_{3} x_{1}+y_{3} t_{y} x_{1} x_{2}\right\}=0,
$$

wenn zur Abkürzung gesetzt wird

$$
\begin{aligned}
& r_{y} \equiv-p_{1} y_{1}+p_{2} y_{2}+p_{3} y_{3} \\
& s_{y} \equiv p_{1} y_{1}-p_{2} y_{2}+p_{3} y_{3} \\
& t_{y} \equiv p_{1} y_{1}+p_{2} y_{2}-p_{3} y_{3} .
\end{aligned}
$$

Man findet nun für die Determinante $A$ den Werth

ferner wird

$$
A=2 y_{1} y_{2} y_{3} r_{y} s_{y} t_{y}
$$

$$
\begin{aligned}
& F(p, p)= \\
& -\left(p_{1}{ }^{4} y_{1}{ }^{4}+p_{2}{ }^{4} y_{2}{ }^{4}+p_{3}{ }^{4} y_{3}{ }^{4}-2 p_{1}{ }^{2} p_{2}{ }^{2} y_{1}{ }^{2} y_{2}{ }^{2}-2 p_{1}{ }^{2} p_{3}{ }^{2} y_{1}{ }^{2} y_{3}{ }^{2}-2 p_{2}{ }^{2} p_{3}{ }^{2} y_{2}{ }^{2} y_{3}{ }^{2}\right) \\
& =p_{y} r_{y} s_{y} t_{y} .
\end{aligned}
$$

Die Curve zerfällt also, wenn eine der Coordinaten $y_{i}$ gleich Null ist, d. h. wenn der Mittelpunkt $y$ auf einer Seite des Dreiecks $A B C$ liegt; man sieht leicht, dass alsdann der Kegelschnitt aus dieser Seite und der Verbindungslinie von $y$ mit der gegenüberliegenden Ecke besteht. Rückt $y$ in eine der Ecken $A, B, C$, so zerfällt der Kegelschnitt in die zwei, in der betr. Ecke sich schneidenden Seiten von $A B C$. Die Discriminante verschwindet auch noch, wenn $r_{y}$ oder $s_{y}$ oder $t_{y}$ gleich Null ist, d. h. (zufolge (22)) wenn $y$ auf einer Seite des dem Dreieck $A B C$ parallel eingeschriebenen Dreiecks $A^{\prime} B^{\prime} C^{\prime}$ liegt; da alsdann auch $F(p, p)=0$, so zerfällt der Kegelschnitt in ein Parallelenpaar, und zwar besteht dasselbe, falls z. B. $y$ auf $B^{\prime} C^{\prime}$ liegt, aus der zu $B^{\prime} C^{\prime}$ parallelen Seite $B C$ des Dreiecks und einer durch die gegenüberliegende Ecke $A$ gezogenen Parallelen.

Im Falle $p_{1} y_{1}+p_{2} y_{2}+p_{3} y_{3}=0$ ist $F(p, p)=0, A \gtrless 0$, die Curve also eine Parabel.

Aus dem Vorhergehenden erkennt man; dass bei Aufstellung der

1) Vgl. Steiner: "Teoremi relativi alle coniche inscritte e circoscritte", Journal für die reine und angewandte Mathematik, Bd. 30, S.98, 1846, oder Giornale arcadico di Roma, Bd. 99, S. 147-161, oder „Gesammelte Werke", Bd. 2, S. $329 \mathrm{f}$. 
Kriterien das parallel eingeschriebene Dreieck $A^{\prime} B^{\prime} C^{\prime}$ eine wesentliche Rolle spielt.

Setzt man $r_{y}=p_{1} Y_{1}, s_{y}=p_{2} Y_{2}, t_{y}=p_{3} Y_{3}$, so wird

und

$$
p_{y}=p_{1} Y_{1}+p_{2} Y_{2}+p_{3} Y_{3}
$$

$$
F(p, p)=p_{1} p_{z} p_{3} Y_{1} Y_{2} Y_{3}\left(p_{1} Y_{1}+p_{2} Y_{2}+p_{3} Y_{3}\right) \text {. }
$$

Dieser Ausdruck ist nach (3) positiv oder negativ, je nachdem der Punkt $Y$ in Bezug auf das durch die Geraden $Y_{i}=0(i=1,2,3)$ gebildete, dem gegebenen Dreieck $A B C$ parallel eingeschriebene Dreieck $A^{\prime} B^{\prime} C^{\prime}$ trigonal oder tetragonal gelegen ist. Mit Rücksicht auf die in $\S 6$ gegebenen Kriterien der Kegelschnitte folgt also:

Der umschriebene Kegelschnitt ist eine Ellipse, wenn der Mittelpunkt in Bezug auf das dem gegebenen Dreieck parallel eingeschriebene Dreieck trigonal und im Endlichen liegt; bei tetragonaler Lage im Endlichen ist der Kegelschnitt eine Hyperbel.

33. Man behandele dieselbe Frage wie in (32) für Curven zweiter Classe, die einem Dreieck $A B C$ eingeschrieben sind ${ }^{1}$ ).

Die Gleichung dieser Curven wird nach (29) folgende:

$$
\begin{gathered}
\frac{1}{2} \varphi(u, u) \equiv p_{1}\left(-p_{1} y_{1}+p_{2} y_{2}+p_{3} y_{3}\right) u_{2} u_{3}+p_{2}\left(p_{1} y_{1}-p_{2} y_{2}+p_{3} y_{3}\right) u_{3} u_{1} \\
+p_{3}\left(p_{1} y_{1}+p_{2} y_{2}-p_{3} y_{3}\right) u_{1} u_{2}=0 .
\end{gathered}
$$

Bei analoger Behandlung dieser Gleichung wie zuvor gelangt man im wesentlichen zu denselben Kriterien wie bei (32), nur treten an Stelle der Parallelenpaare natürlich Punktepaare, und ein weiterer Unterschied ist dadurch veranlasst, dass bei dem Werthe der Discriminante der Factor $y_{1} y_{2} y_{3}$ fehlt, so dass statt der früheren sich inl Endlichen schneidenden Geradeupaare nummehr Hyperbeln auftreten, welche diejenige Seite des gegebenen Dreiecks $A B C$ zur Asymptote haben, auf der der Mittelpunkt $y$ liegt.

34. Man behandele dieselbe Frage wie in (32) und (33) für Curven zweiter Ordnung, die das Dreieck $A B C$ (Coordinatendreieck) zun Poldreieck haben.

Die Gleichung dieser Curven ist nach (30):

hier ist

$$
p_{1} y_{2} y_{3} x_{1}^{2}+p_{2} y_{3} y_{1} x_{2}^{2}+p_{3} y_{1} y_{2} x_{3}^{2}=0 \text {; }
$$

$$
A=p_{1} p_{2} p_{3} y_{1}^{2} y_{2}^{2} y_{3}^{2}, F(p, p)=p_{1} p_{2} p_{3} y_{1} y_{2} y_{3}\left(p_{1} y_{1}+p_{2} y_{2}+p_{3} y_{3}\right) \text {, }
$$
also folgt wieder mit Rücksicht auf (3): Sobald der Mittelpunkt $y$ in

1) Steiner a. a. O., im Journal für die reine und angewandte Mathematik Bd. 30, S. 97, in den "Gesammelten Werken“ Bd. 2, S. 329. 
eine Seite des Coordinatendreiecks rückt, repräsentirt die Gleichung der Curve diese Seite doppelt zählend; der Kegelschnitt ist eine Ellipse oder Hyperbel, je nachdem $y$ in Bezug auf das Dreieck $A B C$ trigonal oder tetragonal liegt. Hierbei ist jedoch zu bemerken, dass einem im Inneren des Dreiecks gelegenen Mittelpunkte überhaupt kein reeller Kegelschnitt zugehört. Denn nimmt man an, dass der Einheitspunkt z. B. im Inneren des Dreiecks liege, so sind die $y_{i}$ und $p_{i}$, mithin die Coefficienten von $x_{1}{ }^{2}, x_{2}{ }^{2}$ und $x_{3}{ }^{2}$ positiv, die Summe dreier Quadrate müsste also verschwinden. Die Curve ist eine Parabel, wenn $y$ im Unendlichen liegt, jedoch nicht im uneudlich fernen Punkte einer Seite des Dreiecks $A B C$.

35. Die Verbindungslinien der Ecken eines Dreiecks mit den auf den gegenüberliegenden Seiten gelegenen Berührungspunkten eines eingeschriebenen Kegelschnitts schneiden sich in einem und demselben Punkte.

Das Dreieck wird als Coordinatendreieck gewählt; die Gleichung des Kegelschnitts ist dann von der Form

Aus

$$
\varphi(u, u) \equiv \alpha_{1} u_{2} u_{3}+\alpha_{2} u_{3} u_{1}+\alpha_{3} u_{1} u_{2}=0 .
$$

$$
\alpha_{1} \varphi(u, u) \equiv\left(\alpha_{2} u_{1}+\alpha_{1} u_{2}\right)\left(\alpha_{3} u_{1}+\alpha_{1} u_{3}\right)-\alpha_{2} \alpha_{3} u_{1}{ }^{2},
$$

geht hervor, dass $\frac{u_{1}}{\alpha_{1}}+\frac{u_{2}}{\alpha_{2}}=0$ und $\frac{u_{1}}{\alpha_{1}}+\frac{u_{3}}{\alpha_{3}}=0$ die Berïhrungspunkte des Kegelschnitts mit den Seiten $x_{3}=0$, resp. $x_{2}=0$ darstellen; analog ist $\frac{u_{2}}{\alpha_{2}}+\frac{u_{3}}{\alpha_{3}}=0$ der Berührungspunkt mit $x_{1}=0$. Es repräsentirt nun $\frac{u_{1}}{\alpha_{1}}+\frac{u_{2}}{\alpha_{2}}+\frac{u_{3}}{\alpha_{3}}=0$ einen Punkt, welcher auf der Verbindungslinie irgend eines der drei eben genannten Berührungspunkte mit der gegenüberliegenden Ecke liegt, also gehen diese drei Geraden durch jenen Punkt.

Dualistisch folgt:

36. Die Schnittpunkte der Seiten eines Dreiecks mit den durch die gegenüberliegende Ecke gezogenen Tangenten eines umschriebenen Kegelschnitts liegen in einer und derselben Geraden.

Auch die Unkehrung von (35) ist giltig, nämlich:

37. Die Verbindungslinien irgend eines Punktes mit den Ecken eines Dreiecks schneiden die gegenüberliegenden Seiten in drei Punkten, welche die Berührungspunkte eines dem Dreieck eingeschriebenen Kegelschnitts bilden. 
Sind $y_{i}(i=1,2,3)$ die Coordinaten des gegebenen Punktes, so haben die auf den Seiten $x_{1}=0, x_{2}=0, x_{3}=0$ gelegenen eben erwähnten Schnittpunkte bezw. die Gleichungen

$$
y_{2} u_{2}+y_{3} u_{3}=0, \quad y_{3} u_{3}+y_{1} u_{1}=0, \quad y_{1} u_{1}+y_{2} u_{2}=0
$$

andrerseits berührt der dem Dreieck eingeschriebene Kegelschnitt $\alpha_{1} u_{2} u_{3}+\alpha_{2} u_{3} u_{1}+\alpha_{3} u_{1} u_{2}=0$ z. B. die Seite $x_{1}=0$ im Punkte $\frac{u_{2}}{\alpha_{2}}+\frac{u_{3}}{\alpha_{3}}=0$, welcher mit $y_{2} u_{2}+y_{3} u_{3}=0$ identisch wird, sobald man $\alpha_{2}: \alpha_{3}=\frac{1}{y_{2}}: \frac{1}{y_{3}}$ setzt, und analog ist es bei den Berührungspunkten mit $x_{2}=0$ und $x_{3}=0$. Der betreffende eingeschriebene Kegelschnitt hat daher die Gleichung $\frac{u_{2} u_{3}}{y_{1}}+\frac{u_{3} u_{1}}{y_{2}}+\frac{u_{1} u_{2}}{y_{3}}=0$.

Ebenso gilt die Umkehrung von (36):

38. Die Verbindungslinien der Ecken eines Dreiecks mit den auf den gegenüberliegenden Seiten gelegenen Schnittpunkten irgend einer Geraden sind Tangenten eines dem Dreieck umschriebenen Kegelschnitts.

Aus (37) folgt mit Rücksicht auf (15) sofort:

39. Die Gleichung desjenigen Kegelschnitts, der die Seiten eines Dreiecks in ihren Mittelpunkten berührt, ist

$$
p_{1} u_{2} u_{3}+p_{2} u_{3} u_{1}+p_{3} u_{1} u_{2}=0
$$

eine reelle Ellipse.

40. Verbindet man einen Punkt $P$ mit den Ecken eines Dreiecks und construirt man in jeder Ecke zu den zwei anliegenden Seiten und $\mathbf{z u}$ der eben genannten Verbindungslinie den vierten harmonischeu Strahl, so schneiden diese Strahlen die Gegenseiten des Dreiecks in drei Punkten, die auf einer und derselben Geraden, der sogenannten Harmonicale des Punktes $P$ in Bezug auf das gegebene Dreieck, liegen.

Für $y_{i}$ als Coordinaten des gegebenen Punktes $P$ sind die Gleichungen der vierten harmonischen Strahlen

$$
y_{3} x_{2}+y_{2} x_{3}=0, \quad y_{3} x_{1}+y_{1} x_{3}=0, \quad y_{2} x_{1}+y_{1} x_{2}=0,
$$

und diese schneiden die Gegenseiten in den Punkten $y_{2} u_{2}-y_{3} u_{3}=0$, $y_{1} u_{1}-y_{3} u_{3}=0, y_{1} u_{1}-y_{2} u_{2}=0$, welche, wie leicht ersichtlich, auf $\operatorname{der}$ Geraden $\frac{x_{1}}{y_{1}}+\frac{x_{2}}{y_{2}}+\frac{x_{3}}{y_{3}}=0$ liegen. Dieselbe heisst die lineare oder gerade Polare des Punktes $y$ in Bezug auf das als ausartende Curve 
dritter Ordnung betrachtete Dreieck $x_{1} x_{2} x_{3}=0^{1}$ ). Man versteht nämlich unter der geraden Polare eines Punktes $y$ in Bezug auf eine beliebige Curve $n^{\text {ter }}$ Ordnung $f\left(x_{1}, x_{2}, x_{3}\right)=0$ die Gerade mit der Gleichung $x_{1}\left(\frac{\partial f}{\partial x_{1}}\right)+x_{2}\left(\frac{\partial f}{\partial x_{2}}\right)+x_{3}\left(\frac{\partial f}{\partial x_{3}}\right)=0$, wobei in die Differentialquotienten $\left(\frac{\partial f}{\partial x_{i}}\right)$ an Stelle von $x_{i}$ die Coordinaten $y_{i}$ des gegebenen Punktes zu substituiren sind. Analog ist die konische Polare oder der Polarkegelschnitt von $y$ in Bezug auf eine Curve $f\left(x_{1}, x_{2}, x_{3}\right)=0$ gegeben durch die Gleichung $\sum_{1}^{3} \sum_{i}^{3}\left(\frac{\partial^{2} f}{\partial x_{i} \partial x_{k}}\right) x_{i} x_{k}=0$, wobei wieder in $\left(\frac{\partial^{2} f}{\partial x_{i} \partial x_{k}}\right)$ die $x_{i}$ durch die Coordinaten $y_{i}$ des gegebenen Punktes zu ersetzen sind ${ }^{2}$ ). In Aufg. (42) wird die konische Polare von $y$ in Bezug auf $x_{1} x_{2} x_{3}=0$ auftretell.

Es ist leicht zu sehen, wie der obigen geraden Polare

$$
\frac{x_{1}}{y_{1}}+\frac{x_{2}}{y_{2}}+\frac{x_{3}}{y_{3}}=0
$$

auch umgekehrt der Punkt $P$ entspricht, wie also allgemein zu jeder Geraden $v_{x}=0$ ein Punkt $\frac{u_{1}}{v_{1}}+\frac{u_{2}}{v_{2}}+\frac{u_{3}}{v_{3}}=0$ construirt werden kamn.

41. Wie lautet der dem Satze (40) dualistisch zugehörige?

42. Die Verbindungslinien eines Punktes $y$ mit den Ecken eines Dreiecks $A B C$ schneiden die Seiten des Dreiecks in drei Punkten $A_{1}, B_{1}, C_{1}$; man beweise, dass die Verbindungslinien $B_{1} C_{1}, C_{1} A_{1}, A_{1} B_{1}$ dieser Punkte die Seiten $B C, C A, A B$ in Punkten treffen, die auf der in (40) behandelten geraden Polare des Punktes $y$ liegen. Man zeige ferner, dass die Verbindungslinien der Ecken des Dreiecks mit den auf den Gegenseiten gelegenen Schnittpunkten der geraden Polare Tangenten der (dem Dreieck umschriebenen) konischen Polare des Punktes $y$ sind in Bezug auf das als ausartende Curve dritter Ordnung betrachtete Dreieck.

Für die Verbindungslinien $B_{1} C_{1}, C_{1} A_{1}, A_{1} B_{1}$ findet man leicht die Gleichungen

$$
-\frac{x_{1}}{y_{1}}+\frac{x_{2}}{y_{2}}+\frac{x_{3}}{y_{3}}=0, \quad \frac{x_{1}}{y_{1}}-\frac{x_{2}}{y_{2}}+\frac{x_{3}}{y_{3}}=0, \frac{x_{1}}{y_{1}}+\frac{x_{2}}{y_{2}}-\frac{x_{3}}{y_{3}}=0 ;
$$

diese Geraden schneiden die Seiten $B C, C A, A B$ des Dreiecks resp. in

1) Vgl. Cayley "Sur quelques théorèmes de la géométrie de position", Journal für die reine und augewandte Mathematik, Bd. 34, S. 275, 1847.

2) Vgl. auch die Fussnote zu S. 22. 
denselben Punkten $y_{2} u_{2}-y_{3} u_{3}=0, y_{1} u_{1}-y_{3} u_{3}=0, y_{1} u_{1}-y_{2} u_{2}=0$, welche schon in (40) vorkamen und auf der geraden Polare

$$
\frac{x_{1}}{y_{1}}+\frac{x_{2}}{y_{2}}+\frac{x_{3}}{y_{3}}=0
$$

liegen. Die Verbindungslinien dieser Punkte mit den gegenüberliegenden Ecken des Dreiecks sind nach (38) Tangenten eines dem Dreieck umschriebenen Kegelschnitts $y_{1} x_{2} x_{3}+y_{2} x_{3} x_{1}+y_{3} x_{1} x_{2}=0$, der die konische Polare von $y$ in Bezug auf $x_{1} x_{2} x_{3}=0$ darstellt $^{1}$ ).

43. Verbindet man die Punktepaare, in denen ein beliebiger Kegelschnitt die Seiten eines Dreiecks schneidet, mit den gegenüberliegenden Ecken, so sind diese drei Paare Verbindungslinien Tangenten eines zweiten Kegelschnitts ${ }^{2}$ ).

Für dasjenige Geradenpaar, welches die auf der Seite $x_{1}=0$ des Coordinatendreiecks gelegenen Punkte des gegebenen Kegelschnitts $\sum_{i}^{3} \sum_{1}^{3} a_{i k} x_{i} x_{k}=0$ mit der gegenüberliegenden Ecke verbindet, ergibt sich unmittelbar die Gleichung $a_{22} x_{2}{ }^{2}+2 a_{23} x_{2} x_{3}+a_{33} x_{3}{ }^{2}=0$; für die zwei anderen Paare analoger Verbindungslinien erhält man $a_{11} x_{1}^{2}+2 a_{13} x_{1} x_{3}+a_{33} x_{3}{ }^{2}=0$ und $a_{11} x_{1}{ }^{2}+2 a_{12} x_{1} x_{2}+a_{22} x_{2}{ }^{2}=0$. Diese drei Geradenpaare sollen Tangenten einer und derselben Curve zweiter Classe $\sum_{1}^{3} \sum_{1}^{3} b_{i k} u_{i} u_{k}=0$ sein. Das vom Punkte $u_{1}=0$ an diese Curve gezogene Tangentenpaar hat nun nach (27), S. 45 und dualistisch zu (54), S. 34 die Gleichung $b_{33} x_{2}{ }^{2}-2 b_{23} x_{2} x_{3}+b_{22} x_{3}{ }^{2}=0$, es müssten also die Relationen bestehen $b_{33}=\rho a_{22}, b_{23}=-\rho a_{23}$, $b_{22}=\varrho a_{33}$, in denen $\varrho$ einen Proportionalitätsfactor bedeutet. Die zwei anderen Tangentenpaare liefern ausserdem die Beziehungen $b_{33}=\sigma a_{11}, b_{13}=-\sigma a_{13}, b_{11}=\sigma a_{33}$ und $b_{11}=\tau a_{22}, b_{12}=-\tau a_{12}$, $b_{22}=\tau a_{11}$ ( $\sigma$ und $\tau$ Proportionalitätsfactoren), und es folgt nun sofort $\varrho: \sigma: \tau=a_{11}: a_{22}: a_{33}$, so dass man die Gleichung des gesuchten Kegelschnitts in der Gestalt erhält

$$
\begin{aligned}
a_{22} a_{33} u_{1}^{2}+a_{33} a_{11} u_{2}^{2}+a_{11} a_{22} u_{3}^{2}-2 a_{11} a_{23} u_{2} u_{3} & -2 a_{22} a_{31} u_{3} u_{1} \\
& -2 a_{33} a_{12} u_{1} u_{2}=0 .
\end{aligned}
$$

Ebenso gilt der dualistisch entsprechende Satz:

1) Vgl. auch hier die oben citirte Abhandlung von Cayley, S. 275, sowie Schröter: "Ueber perspectivisch liegende Dreiecke", Math. Annalen, Bd. 2, S. 562, 1870.

2) Vgl. Hesse „Satz ans der Lehre von den Kegelschnitten", Journal für die reine und angewandte Mathematik, Bd. 65, S. 384, 1865. 
44. Legt man von den Ecken eines Dreiecks die Tangenten an einen Kegelschnitt, so schneiden diese die Gegenseiten in drei Punktepaaren eines zweiten Kegelschnitts.

45. Man beweise nachstehenden von Steiner ohne Beweis mitgetheilten Satz $^{1}$ :

„Zieht man aus den Ecken $a, b, c$ eines gegebeneu Dreiecks durch einen in seiner Ebene liegenden unbestimmten Punkt $p$ Strahlen, welche die Gegenseiten beziehlich in den Punkten $a_{1}, b_{1}, c_{1}$ treffen, und verlangt, es soll das Product $a p \cdot b p \cdot c p=p a_{1} \cdot p b_{1} \cdot p c_{1}$ sein, so ist der Ort des Punktes $p$ diejenige dem Dreieck $a b c$ umschriebene Ellipse, welche den Schwerpunkt desselben zum Mittelpunkt hat."

In $\frac{a p}{p a_{1}} \cdot \frac{b p}{p b_{1}} \cdot \frac{c p}{p c_{1}}=1$ kann der Quotient $\frac{a p}{p a_{1}}$ aufgefasst werden als das Doppelverhältniss des Punktepaares $a, a_{1}$ zu dem aus $p$ und dem unendlich fernen Punkte $P_{\infty}$ der Geraden $a a_{1}$ bestehenden Punktepaar; dasselbe Doppelverhältniss besitzen die Strahlen $b a, b a_{1}, b p, b P_{\infty}$. Sind $y_{1}, y_{2}, y_{3}$ die Coordinaten des Punktes $p$, bezogen auf das vorgelegte Dreieck als Coordinatendreieck, so ist $y_{3} x_{2}-y_{2} x_{3}=0$ die Gleichung der Geraden $a a_{1}$, daher hat $b P_{\infty}$ als Parallele zu $a a_{1}$ eine Gleichung von der Form $y_{3} x_{2}-y_{2} x_{3}+\lambda\left(p_{1} x_{1}+p_{2} x_{2}+p_{3} x_{3}\right)=0$, wobei man $\lambda$ dadurch bestimmt, dass diese Gerado durch die Ecke $b$ des Dreiecks gehen soll. Man finclet $\lambda=-y_{3}: p_{2}$ und erhält so als Gleichung von $b P_{\infty}$ die folgende: $p_{1} y_{3} x_{1}+\left(p_{2} y_{2}+p_{3} y_{3}\right) x_{3}=0$; die Gleichungen von $b a, b a_{1}$ und $b p$ sind bezw. $x_{3}=0, x_{1}=0$ und $y_{3} x_{1}-y_{1} x_{3}=0$, so dass sich für das gesuchte Doppelverhältniss der Werth ergibt:

$$
\frac{a p}{p a_{1}}=-\frac{p_{2} y_{2}+p_{\mathrm{s}} y_{3}}{p_{1} y_{1}}=-\frac{p_{y}-p_{1} y_{1}}{p_{1} y_{1}} .
$$

Analoge Ausdrücke erhält man für $\frac{b p}{p b_{1}}$ und $\frac{c p}{p c_{1}}$, daher unterliegen die Coordinaten $y_{i}$ der Bedingung

$$
\left(p_{y}-p_{1} y_{1}\right)\left(p_{y}-p_{2} y_{2}\right)\left(p_{y}-p_{3} y_{3}\right)=-p_{1} y_{1} \cdot p_{2} y_{2} \cdot p_{3} y_{3}
$$

woraus bei Ausführung der Multiplication der Klammern folgt

$$
f(y, y) \equiv p_{2} p_{8} y_{2} y_{3}+p_{3} p_{1} y_{3} y_{1}+p_{1} p_{2} y_{1} y_{2}=0 \text {. }
$$

Offenbar stellt diese Gleichung eine dem Dreieck umschriebene Curve zweiter Ordnung dar, und zwar eine Ellipse, weil $F(p, p)$ positiv ist. Für ihren Mittelpunkt $\eta$ findet man die Coordinaten

1) „Lehrsätze“. Journal für die reine und angewandte Mathematik, Bd. 45, S. 177, 1852, oder anch "Gesammelte Werke", Bd. 2, S. 431. 


$$
\eta_{1}: \eta_{2}: \eta_{3}=\frac{1}{p_{1}}: \frac{1}{p_{2}}: \frac{1}{p_{3}},
$$

die nach (15) auch dem Schwerpunkt des Dreiecks angehören. Nach (42) ist diese Ellipse die konische Polare des Schwerpunktes in Bezug auf $x_{1} x_{2} x_{3}=0$.

46. Die Berührungspunkte der beiden Tangenten, die vom Punkte $y$ an den Kegelschnitt $f(x, x)=0$ gelegt werden köunen, sind gegeben durch die Gleichung $A\left(y_{1} v_{1}+y_{2} v_{2}+y_{3} v_{3}\right)^{2}-f(y, y) \cdot F(v, v)=0$.

Soll eine Gerade $v_{x}=0$ durch irgend einen von zwei Punkten des Kegelschnitts $f(x, x)=0$ gehen, deren Tangeuten sich in einem Punkte $y$ schneiden, so besteht zwischen den Coordinaten $v_{i}$ der Geraden und den Coordinaten $y_{i}$ des Punktes nach (25), S. 45 die Relation $A v_{y}{ }^{2}-F(v, v) f(y, y)=0$. Nimmt man die $v_{i}$ als gegeben an, so stellt diese Gleichung in variabelen Punktcoordinaten das Tangentenpaar dar, welches in den Schnittpunkten der Curve $f(x, x)=0$ und der Geraden $v_{x}=0$ gezogen werden kann. Nimmt man die $y_{i}$ als gegeben an, so stellt analog dieselbe Gleichung in variabelen Liniencoordinaten $v_{i}$ das Paar von Berührungspunkten der beiden Tangenten dar, die vom Punkte $y$ an den Kegelschnitt $f(x, x)=0$ gelegt werden könuen ${ }^{1}$ ).

Folgt auch so: Die Berührungspunkte sind die Schnittpunkte der Curve nit der Polare $f(x, y)=0$; daher hat man in der Gleichung $\left(\begin{array}{ll}u & v \\ u & v\end{array}\right)$ des Schnittpunktepaares von $u_{x}=0$ mit $f(x, x)=0$ die $u_{i}$ zu ersetzen durch $\frac{1}{2} f^{\prime}\left(y_{i}\right)$. Subtrahirt man in der Determinante $\left(\begin{array}{ll}u & v \\ u & v\end{array}\right)$ die mit $y_{1}, y_{2}, y_{3}$ multiplicirten ersten drei Reihen von den die $\frac{1}{2} f^{\prime}\left(y_{i}\right)$ enthaltenden Reihen, so erhält man sofort für die Determinante den Ausdruck $A y_{v}{ }^{2}+f(y, y) \cdot\left(\begin{array}{l}v \\ v\end{array}\right)=0$ oder also $A y_{v}{ }^{2}-f(y, y) \cdot F(v, v)=0$.

Dualistisch folgt:

47. Die beiden Tangenten, die in den Schnittpunkten einer Geraden $v$ und eines Kegelschnitts $\varphi(u, u)=0$ gezogen werden können, sind gegeben durch die Gleichung

$$
\mathrm{A}\left(v_{1} x_{1}+v_{2} x_{2}+v_{3} x_{3}\right)^{2}-\varphi(v, v) \cdot \Phi(x, x)=0 .
$$

1) Eine analoge Gleichung tritt auch bei Carven $n^{\text {ter }}$ Ordnung anf; vgl. speciell für Curven dritter Ordnung die Abhandlung von Herrn Gundelfinger: "Ueber geometrische Deutung algebraischer Formen, die in der Theorie der Curven dritter Ordnung auftreten". Math. Annalen, Bd. 8, S. 143, Zeile 11, 1874. 
48. Es sei $f(x, x)=0$ die Gleichung einer Curve zweiter Ordnung, $g(x, x) \equiv \sum_{1}^{3} \sum_{1}^{3} c_{i k} x_{i} x_{k}=0$ diejenige irgend eines Tangentenpaares der Curve; man leite die Gleichung der beiden Berührungspunkte ab.

Der Punkt $y$, von welchem aus die beiden Tangenten gezogen sind, hat nach S. $28 \mathrm{f}$. Coordinaten, die sich ergeben aus $\varrho y_{i} y_{k}=C_{i k}$, wobei $\varrho$ einen Proportionalitätsfactor und $C_{i k}$ die Unterdeterminante des Elementes $c_{i k}$ in der Determinante von $\psi(x, x)$ bezeichnet. Diese Werthe der $y_{i} y_{k}$ sind in die in (46) abgeleitete Gleichung des Paares der Berührungspunkte einzusetzen, wodurch man erhält

$$
A G(v, v)-[a, C] F(v, v)=0
$$

hierbei ist $G(v, v) \equiv \sum_{1}^{3} \sum_{1}^{3} C_{i k} v_{i} v_{k}$ und

$$
[a, C] \equiv a_{11} C_{11}+2 a_{12} C_{12}+a_{22} C_{22}+2 a_{13} C_{13}+2 a_{23} C_{23}+a_{33} C_{33} .
$$

Dualistisch folgt:

49. Ist $\varphi(u, u) \equiv \sum_{1}^{3} \sum_{1}^{3} \alpha_{i k} u_{i} u_{k}=0$ die Gleichung einer Curve zweiter Classe, $\psi(u, u) \equiv \sum_{1}^{3} \sum_{1}^{3} k \gamma_{i k} u_{i} \imath_{k}=0$ diejenige irgend eines Punktepaares auf derselben, so ist das Paar der Tangenten, das in diesen Punkten gezogen werden kann, gegeben durch

$$
\mathrm{A} \Psi(x, x)-[\alpha, \Gamma] \Phi(x, x)=0 .
$$

Die hier angewandte Bezeichnuugsweise bedarf wohl keiner näheren Erläuterung.

Aus (48) folgt leicht:

50. Ist $\varphi(u, u) \equiv \sum_{1}^{3} \sum_{1}^{3} \alpha_{i k} u_{i} u_{k}=0$ die Gleichung einer Curve zweiter Classe, $g(x, x) \equiv \sum_{1}^{3} \sum_{1}^{3} c_{i k} x_{i} x_{k}=0$ diejenige irgend eines Tangentenpaares derselben, so stellt $\mathrm{A} G(v, v)-[\mathrm{A}, C] \varphi(v, v)=0$ das Paar der Berührungspunkte dar.

Aus (49) folgt ebenso:

๖1. Ist $f(x, x)=0$ die Gleichung einer Curve zweiter Ordnung, $\psi(u, u) \equiv \sum_{1}^{3} \sum_{1}^{3} \gamma_{i k} u_{i} u_{k}=0$ diejenige irgend eines Punktepaares auf 
derselben, so stellt $A \Psi(x, x)-[A, \Gamma] f(x, x)=0$ das zugehörige Tangentenpaar dar.

52. Man stelle die Bedingung auf, unter der von dem Schnittpunkte $y$ zweier Geraden $u_{x}=0, v_{x}=0$ an den Kegelschnitt $f(x, x)=0$ reelle oder imaginäre Tangenten gezogen werden können.

Die Tangenten sind reell oder imaginär, je nachlem in dem Strahlenbüschel $v_{x}+\lambda u_{x}=0$ Strahlen vorhanden sind, die der Gleichung des Kegelschnitts in Liniencoordinaten genügen oder nicht. Es hängt also die Entscheidung ab von der Realität der Wurzeln der Gleichung $F\left(v_{1}+\lambda u_{1}, v_{2}+\lambda u_{2}, v_{3}+\lambda u_{3}\right)=0$ oder

$$
F(v, v)+2 \lambda F(v, u)+\lambda^{2} F(u, u)=0 ;
$$

dieselben sind reell oder imaginär, je nachdem

$$
F(u, u) F(v, v)-F^{2}(u, v)<0 \text { oder }>0 .
$$

Bei Einführung der Coordinaten $y_{i}$ des Schnittpunktes der Geraden $u_{x}=0$ und $v_{x}=0$ kann man die linke Seite dieser Ungleichung auch ersetzen durch $A f(y, y)$. Beide Tangenten fallen zusammen, wenn $f(y, y)=0$, d. h. der Punkt $y$ auf der Curve selbst liegt, oder wenn $A=0$, d. h. der Kegelschnitt aus einem Geradenpaare besteht.

53. Die Gleichung des Geradenpaares, das ron einem beliebigen Punkte $y$ nach den Schnittpunkten des Kegelschnitts $f(x, x)=0$ mit einer Geraden $u_{x}=0$ gezogen werden kann, lautet

$$
u_{y}{ }^{2} \cdot f(x, x)-2 u_{x} u_{y} f(x, y)+f(y, y) u_{x}{ }^{2}=0 .
$$

Man hat den Parameter $\lambda$ zu eliminiren aus den beiden Gleichungen $f(x, x)+2 \lambda f(x, y)+\lambda^{2} f(y, y)=0$ und $u_{x}+\lambda u_{y}=0$, wodurch sich obige Gleichung ergibt.

Liegt insbesondere der Punkt $y$ auf der Curve selbst, so hat man einfacher $u_{y} f(x, x)-2 u_{x} f(x, y)=0$.

54. Der zu der Richtung der Geraden $v_{x}=0$ conjugirte Durchmesser des Kegelschnitts $f(x, x)=0$ hat die Gleichung

$$
\sum \pm\left(v_{1} p_{2} \frac{1}{2} f^{\prime}\left(x_{3}\right)\right)=0
$$

Der gesuchte Durchmesser ist die Polare des unendlich feruen Punktes von $v_{x}=0$, hat demnach die Gleichung $\frac{1}{2} f^{\prime}\left(x_{1}\right)\left(v_{2} p_{3}-v_{3} p_{2}\right)+\frac{1}{2} f^{\prime}\left(x_{2}\right)\left(v_{3} p_{1}-v_{1} p_{3}\right)+\frac{1}{2} f^{\prime}\left(x_{3}\right)\left(v_{1} p_{2}-v_{2} p_{1}\right)=0$. 
55. Die Gleichung für den Mittelpunkt derjenigen Sehne, welche durch die Schnittpunkte des Kegelschnitts $f(x, x)=0$ mit der Geraden $v_{x}=0$ bestimmt ist, lautet $\left(\begin{array}{ll}v & u \\ v & p\end{array}\right)_{a_{i k}}=0$.

Der gesuchte Mittelpunkt ist der Schnittpunkt von $v_{x}=0$ mit dem zu der Richtung von $v_{x}=0$ conjugirten Durchmesser, der nach (54) gegeben ist durch $\sum \pm\left(v_{1} p_{2} \frac{1}{2} f^{\prime}\left(x_{3}\right)\right)=0$. Denkt man sich diese Gleichung geordnet nach $x_{1}, x_{2}, x_{3}$, so ist leicht zu sehen, dass dieser Schnittpunkt in den veränderlichen Liniencoordinaten $u_{i}$ die Gleichung hat $\left(\begin{array}{ll}v & u \\ v & p\end{array}\right)_{a_{i k}}=0$.

56. Die Gleichung des zu der Geraden $v_{x}=0$ parallelen Tangentenpaares des Kegelschnitts $f(x, x)=0$ aufzustellen.

Das Tangentenpaar ist vom unendlich fernen Punkt der Geraden $v_{x}=0$ an den Kegelschnitt gezogen; dieser Punkt hat die Coordinaten $y_{1}: y_{2}: y_{3}=\left(p_{2} v_{3}-p_{3} v_{2}\right):\left(p_{3} v_{1}-p_{1} v_{3}\right):\left(p_{1} v_{2}-p_{2} v_{1}\right)$, welche nach S. 21 in $f(x, x) \cdot f(y, y)-f^{2}(x, y)=0$ zu substituiren sind. Man findet:

$$
f(x, x) \cdot\left(\begin{array}{ll}
p & v \\
p & v
\end{array}\right)_{a_{i k}}-\frac{1}{4}\left[\sum \pm\left(f^{\prime}\left(x_{1}\right) p_{2} v_{3}\right)\right]^{2}=0 .
$$

Diese Gleichung kann noch in eine andere Gestalt gebracht werden durch Anwendung der Formel (32), S. 27:

$$
f(x, x) f(y, y)-f^{2}(x, y)=F(u, u),
$$

wenn $u_{1}=x_{2} y_{3}-x_{3} y_{2}$, etc. In unserem Falle wird $u_{1}=p_{1} v_{x}-v_{1} p_{x}$, so dass sich die Gleichung des Tangentenpaares verwandelt in

$$
F(p, p) v_{x}^{2}+F(v, v) p_{x}{ }^{2}-2 F(p, v) p_{x} v_{x}=0 .
$$

Aus dieser Formel erkennt man auch, dass im Falle der Parabel $(F(p, p)=0)$ die eine Tangente mit der unendlich fernen Geraden zusammenfällt, während die andere gegeben ist durch

$$
F(v, v) \cdot p_{x}-2 F(p, v) \cdot v_{x}=0 .
$$

57. Ist $f(x, x)=0$ die Gleichung eines Geradenpaares $(A=0)$, so sind die beiden Geraden einzeln dargestellt durch

$$
\frac{1}{4} \sum \pm\left(f^{\prime}\left(x_{1}\right) f^{\prime}\left(y_{2}\right) u_{3}\right) \pm f(y, x) \sqrt{-F(u, u)}=0,
$$

wobei die $y_{i}$ und $u_{i}$ völlig willkürliche Werthe besitzen, die nur nicht den Coordinaten eines auf dem Geradenpaare liegenden Punktes, bezw. einer durch die Spitze gehenden Geraden proportional sein dürfen. 
Bestimmung der Geraden resp. Punkte eines ausartenden Kegelschnitts. 263

Es ist $f(y, x)=0$ die Polare eines beliebigen Punktes $y$ in Bezug auf das Geradenpaar $f(x, x)=0$; auf dieser Polare wählen wir einen willkürlichen Punkt $z$, der aus der Polare ausgeschnitten werde durch eine Gerade $u_{x}=0$. Die Verbindungslinien der Spitze des Geradenpaares mit den beiden Punkten $y$ und $z$ sind alsdann bezw. $f(z, x)=0$ und $f(y, x)=0$, wobei statt $f(z, x)=0$ auch gesetzt werden kann $\frac{1}{4} \sum \pm\left(f^{\prime}\left(x_{1}\right) f^{\prime}\left(y_{2}\right) u_{3}\right)=0$, denu es ist $z_{1}=\frac{1}{2} f^{\prime}\left(y_{2}\right) u_{3}-\frac{1}{2} f^{\prime}\left(y_{3}\right) u_{3}$; $z_{2}$ und $z_{3}$ haben analoge Werthe. $\mathrm{Zu}$ diesen Verbindungslinien liegen die Geraden $f(x, x)=0$ harmonisch, sie müssen demnach einzeln Gleichungen haben von der Form

$$
\pm \lambda f(y, x)+\frac{1}{4} \sum \pm\left(f^{\prime}\left(x_{1}\right) f^{\prime}\left(y_{2}\right) u_{3}\right)=0
$$

wobei $\lambda$ so zu bestimmen ist, dass der Punkt mit den Coordinaten $\lambda y_{i}+z_{i},(i=1,2,3)$, auf $f(x, x)=0$ liegt, d. h. man hat $\lambda$ zu bestimmen aus $\lambda^{2} f(y, y)+2 \lambda f(y, z)+f(z, z)=0$. Nun ist $f(y, z) \equiv 0$, $f(z, z)$ wird $f(y, y) F(u, u)-A u_{y}{ }^{2}$, also gleich $f(y, y) \cdot F(u, u)$, da $A=0$; man hat daher $f(y, y)\left\{\lambda^{2}+F(u, u)\right\}=0$ oder $\lambda=\sqrt{-F(u, u)}$. Dualistisch folgt:

58. Ist $\varphi(u, u)=0$ die Gleichung eines Punktepaares, so sind die beiden Punkte einzeln dargestellt durch

$$
\frac{1}{4} \sum \pm\left(\varphi^{\prime}\left(u_{1}\right) \varphi^{\prime}\left(v_{2}\right) x_{3}\right) \pm \varphi(v, u) \sqrt{-\Phi(x, x)}=0,
$$

wobei die $v_{i}$ und $x_{i}$ analogen Einschränkungen unterliegen wie die $y_{i}$ und $\iota_{i}$ in (57), übrigens aber völlig willkürliche Zahlen bedeuten.

59. Das Product aus den senkrechten Entfernungen eines beliebigen Punktes eines Kegelschnitts von den beiden Asymptoten ist constant.

Für $g_{x} h_{x}=0$, als Gleichung der beiden Asymptoten, ist $f(x, x)=0$ nach (26), S. 45 von der Form $g_{x} h_{x}+\frac{A}{F(p, p)} p_{x}{ }^{2}=0$, daher

$$
\frac{g_{x} h_{x}}{\sqrt{\omega(g, g)} \sqrt{\omega(h, h)}} \cdot \frac{1}{p_{x}^{2}}=-\frac{A}{\sqrt{\omega(g, g)} \sqrt{\omega(h, h)} F(p, p)} .
$$

Die linke Seite dieser Gleichung ist nach (1), S. 9 das Product der Abstände des Curvenpunktes $x$ von den beiden Asymptoten, die rechte Seite eine Constante. - Der Satz kann auch mit Hilfe von (9) odler (10), S. 49 bewiesen werden, da aus $x y=$ const. noch

$x \sin w \cdot y \sin w=$ const. 
folgt, wobei $w$ den Winkel der beiden Asymptoten bezeichnet; $x \sin w$ und $y$ sin $w$ sind aber die oben erwähnten senkrechten Entfernungen ${ }^{1}$ ).

60. Zieht man durch einen beliebigen Punkt $P$ der Ebene eine. Parallele zu einer Asymptote der Hyperbel, so wird das Stück der Parallelen, welches zwischen ihrem Schnittpunkte $Q$ mit der Polare von $P$ und dem Punkte $P$ selbst liegt, von der Hyperbel halbirt.

Die Punkte $P, Q$ bilden ein Paar conjugirter Pole in Bezug auf die Curve; da der eine Schnittpunkt der Geraden $P Q$ und der Curve im Unendlichen liegt, muss der andere die Strecke $P Q$ halbiren.

Aus gleichem Grunde gilt bei der Parabel der Satz:

61. Zieht man durch einen beliebigen Punkt $P$ der Ebene eine Parallele zur Axe der Parabel $^{2}$ ), so wird das Stück dieser Geraden, welches zwischen ihrem Schnittpunkte mit der Polare von $P$ und dem Punkte $P$ selbst liegt, von der Parabel halbirt.

62. Die Polare eines Punktes $P$ in Bezug auf einen Kegelschnitt ist parallel zu dem conjugirten Durchmesser desjenigen Durchmessers $D$, auf welchem $P$ gelegen ist.

Denn wenn $P$ den Durchmesser $D$ durchläuft, dreht sich die zugehörige Polare um dessen Pol; derselbe liegt aber im Unendlichen, so dass also die einzelnen Polaren, zu denen auch der conjugirte Durchmesser gehört, einander parallel sind.

63. Zieht man von einem Punkte $P$ die zwei Tangenten an einen Kegelschnitt, so geht derjenige Durchmesser $D$, auf welchem $P$ liegt, durch den Halbirungspunkt $H$ der Berührungssehne $M N$.

Folgt mit Hilfe von (62), denn $M N$ gehört demjenigen Sehnensystem an, zu welchem $D$ conjugirt ist, d. h. der Durchmesser $D$ halbirt die Sehne $M N$.

Ebenso leicht folgt:

1) Es lässt sich leicht zeigen (unter Rücksicht auf S. 64), dass das constante Product den Werth besitzt $\frac{-A \sin v}{F(p, p) \sqrt{-4 \tau F(p, p)}}$, wobei $\sin ^{2} v=\frac{1}{1+\cot ^{2} w}$ auf Grund von (6), S. 111 berechnet werden kann. Bei Einführung der Längen $a$ und $b$ der halben Axen findet man im Falle einer Hyperbel die Constante gleich $\frac{a^{2} b^{2}}{a^{2}+b^{2}}$, wobei $a$ und $b$ die auf S. $110 \mathrm{f}$. angegebene Bedentung für die Hyperbel besitzen. Vgl. auch (112).

2) Zur Definition der Axe der Parabel vgl. S. 103. 
64. Zieht man von einem Punkte $\boldsymbol{P}$ die zwei Tangenten an einen Kegelschnitt, so geht die Verbindungslinie von $P$ mit dem Halbirungspunkte der Berührungssehne durch den Mittelpunkt der Curve.

Denn diese Verbindungslinie ist die Polare des auf der Berührungssehne unendlich weit gelegenen Punktes.

65. Bei jeder gleichseitigen Hyperbel $^{1}$ ) werden die Winkel zwischen zwei conjugirten Durchmessern durch die Asymptoten halbirt.

Nach (24), S. 45 liegen die einzelnen Paare conjugirter Durchmesser harmonisch zum Asymptotenpaar; da das letztere bei der gleichseitigen Hyperbel aus zwei zu einander normalen Geraden besteht, halbiren diese Geraden die Winkel zweier conjugirten Durchmesser.

66. Es seien zwei Kegelschnitte, die das Coordinatendreieck zum Poldreieck haben, gegeben durch ihre Gleichungen

$$
f \equiv a_{1} x_{1}{ }^{2}+a_{2} x_{2}{ }^{2}+a_{3} x_{3}{ }^{2}=0 \text { und } g \equiv b_{1} x_{1}{ }^{2}+b_{2} x_{2}{ }^{2}+b_{3} x_{3}{ }^{2}=0 \text {. }
$$

Unter welcher Bedingung wird der erste Kegelschnitt von denjenigen Polaren umhüllt, welche den Punlten des ersten in Bezug auf den zweiten Kegelschnitt zugehören?

Die Polare eines auf $f=0$ gelegenen Punktes $x_{1}, x_{2}, x_{3}$ hat mit Bezug auf $g=0$ die Gleichung $b_{1} x_{1} X_{1}+b_{2} x_{2} X_{2}+b_{3} x_{3} X_{3}=0$; sie hat daher die Coordinaten $u_{i}=b_{i} x_{i}(i=1,2,3)$. Hieraus folgt $x_{i}=u_{i}: b_{i}$ und durch Substitution in $f=0$ erhält man

$$
\frac{a_{1} u_{1}{ }^{2}}{b_{1}{ }^{2}}+\frac{a_{2} u_{2}{ }^{2}}{b_{2}{ }^{2}}+\frac{a_{3} u_{3}{ }^{2}}{b_{3}{ }^{2}}=0 .
$$

Diese Gleichung stellt in Liniencoordinaten den Kegelschnitt $f$ dar, falls $a_{i}: b_{i}{ }^{2}$ proportional ist zu $1: a_{i}$, d. h. wenn $a_{1}{ }^{2}: a_{2}{ }^{2}: a_{3}{ }^{2}=b_{1}{ }^{2}: b_{2}{ }^{2}: b_{3}{ }^{2}$ oder $b_{1}: b_{2}: b_{3}= \pm a_{1}: \pm a_{2}: \pm a_{3}$.

Jeder der vier Kegelschnitte

$$
\begin{array}{r}
a_{1} x_{1}{ }^{2}+a_{2} x_{2}{ }^{2}+a_{3} x_{3}{ }^{2}=0 \\
-a_{1} x_{1}{ }^{2}+a_{2} x_{2}{ }^{2}+a_{3} x_{3}{ }^{2}=0 \\
a_{1} x_{1}{ }^{2}-a_{2} x_{2}{ }^{2}+a_{3} x_{3}{ }^{2}=0 \\
a_{1} x_{1}{ }^{2}+a_{2} x_{2}{ }^{2}-a_{3} x_{3}{ }^{2}=0
\end{array}
$$

hat daher die Eigenschaft, dass er selbst wieder entsteht, wenn man seine „reciproke Polare" in Bezug auf einen der drei übrigen nimmt. Man erkennt leicht, dass einer der vier Kegelschnitte imaginär sein muss.

1) Zur Definition der gleichseitigen Hyperbel vgl. S. 111. 


\section{Anwendung von $\$ 7-9$.}

67. Jeder Kegelschnitt, der durch die imaginären Kreispunkte geht, ist ein Kreis.

Nach (43), S. 84 ist die Gleichung eines solchen Kegelschnitts in Liniencoordinaten jedenfalls von der Form

$$
\omega(u, u)-\lambda\left(y_{1} u_{1}+y_{2} u_{2}+y_{3} u_{3}\right)^{2}=0,
$$

wobei $\omega(u, u)=0$ die Gleichung des imaginären Kreispunktepaares, $y_{1} u_{1}+y_{2} u_{2}+y_{3} u_{3}=0$ diejenige des Pols der unendlich fernen Geraden, also des Mittelpunktes der Curve darstellt, während $\lambda$ irgend einen numerischen Coefficienten bezeichnet. Nach (2), S. 57 ist aber ein Ausdruck von der obigen Form die Gleichung eines Kreises.

Uebrigens kann man zum Beweise des Satzes ebenso gut ausgehen von der Gleichung eines Kegelschnitts in Punktcoordinaten.

68. Ist $K=0$ die Gleichung eines Kreises in Punktcoordinaten, so stellt auch $K^{\prime} \equiv x K-\lambda p_{x} q_{x}=0$ einen Kreis dar, wobei $x$ und $\lambda$ willkürliche Parameter sind, während $p_{x}=0$ die Gleichung der unendlich fernen, $q_{x}=0$ diejenige einer beliebigen Geraden ist.

Denn die Schnittpunkte der unendlich fernen Geraden mit $K^{\prime}=0$ sind dieselben wie diejenigen mit $K=0$, nämlich die imaginären Kreispunkte; nach (67) ist daher auch der Kegelschnitt $K^{\prime}=0$ ein Kreis. Für $x=0$ zerfällt derselbe in die Gerade $q_{x}=0$ und die unendlich ferne Gerade, wodurch die in der Fussnote zu S. 59 erwähnte Ausartung entsteht.

Uebrigens kann jeder Kreis bei willkürlicher Wahl von $K$ in der Form $K^{\prime}=0$ dargestellt werden, da das Verhältniss $x: \lambda$ und die Gerade $q$ so bestimmt werden können, dass der Kreis durch drei beliebig gegebene Punkte geht.

69. Sind $K_{1}=0$ und $K_{2}=0$ die Gleichungen zweier Kreise in Punktcoordinaten, so stellt auch $K_{3} \equiv \varkappa K_{1}-\lambda K_{2}=0$, wobei $x$ und $\lambda$ beliebige Parameter bedeuten, einen Kreis dar; derselbe geht offenbar durch die Schnittpunkte von $K_{1}=0$ und $K_{2}=0$, sein Mittelpunkt liegt daher auf der Centrale von $K_{1}$ und $K_{\mathrm{q}}$.

70. Die gemeinsame Sehne der beiden Kreise $K_{0} \equiv K-p_{x} q_{x}=0$ und $K_{1} \equiv K-p_{x} r_{x}=0$ ( $K=0$ Gleichung eines dritten Kreises) liat die Gleichung $q_{x}-r_{x}=0$.

Ein Punkt, dessen Coordinaten die Ausdrücke $K_{0}$ und $K_{1}$ zum Verschwinden bringen, gehört beiden Kreisen an, erfüllt aber auch die 
Gleichung $K_{0}-K_{1} \equiv p_{x}\left(r_{x}-q_{x}\right)=0$; ein solcher Punkt liegt demnach auch entweder auf der unendlich fernen Geraden (imag. Kreispunkt) oder auf $r_{x}-q_{x}=0, \mathrm{~d}$. h. diese Gleichung stellt die gemeinsame Sehne, die sogenannte Radicalaxe oder Potenzlinie der beiden Kreise dar.

71. Die Radicalaxen je zweier von drei Kreisen schneiden sich in einem und demselben Punkte, dem sogenannten Radicalcentrum oder Potenzmittelpunkt der drei Kreise.

Es seien

$$
K_{0} \equiv K-p_{x} q_{x}=0, \quad K_{1} \equiv K-p_{x} r_{x}=0, \quad K_{2} \equiv K-p_{x} s_{x}=0
$$

die Gleichungen der drei Kreise; alsdann sind die zugehörigen Radicalaxen nach (70) dargestellt durch $q_{x}-r_{x}=0, r_{x}-s_{x}=0, s_{x}-q_{x}=0$, und diese drei Geraden haben offenbar einen gemeinsamen Schnittpunkt.

72. Die beiden imaginären Kreispunlite sind einzeln dargestellt durch

$$
\frac{1}{4} \sum \pm\left(\omega^{\prime}\left(u_{1}\right) \omega^{\prime}\left(v_{2}\right) x_{3}\right) \pm i \omega(v, u) p_{x} \sqrt{\tau}=0,
$$

wobei die $v_{i}$ und $x_{i}$ völlig willkürliche Werthe besitzen, die nur nicht den Gleichungen $\omega(v, v)=0$, bezw. $p_{x}=0$ genügen dürfen.

Folgt aus (58), denn nun ist $\Phi(x, x) \equiv \tau p_{x}{ }^{2}$. - Man kann übrigens für $\omega^{\prime}\left(v_{i}\right),(i=1,2,3)$, auch die Coordinaten irgend eines Punktes auf der unendlich fernen Geraden eiusetzen, also Zahlenwerthe $z_{i}$, welche nur die Gleichung $p_{1} z_{1}+p_{2} z_{2}+p_{3} z_{3}=0$ erfüllen müssen.

73. Die Gleichung des dem Coordinatendreieck umschriebenen Kreises lautet $\omega_{11} p_{1} x_{2} x_{3}+\omega_{22} p_{2} x_{3} x_{1}+\omega_{33} p_{3} x_{1} x_{2}=0$.

Jedenfalls ist die Gleichung von der Form $a_{1} x_{2} x_{3}+a_{2} x_{3} x_{1}+a_{3} x_{1} x_{2}$; der Mittelpunkt des umschriebenen Kreises hat nach (18) die Coordinaten $\omega_{11} \omega_{23}: \omega_{22} \omega_{31}: \omega_{33} \omega_{12}$, daher wird $a_{1}$ nach (28) gleich

$$
\omega_{11} \omega_{28}\left(-\omega_{11} \omega_{23} p_{1}+\omega_{22} \omega_{31} p_{2}+\omega_{33} \omega_{12} p_{3}\right) \text {, }
$$

wobei der Klammerfactor auf Grund der Relationen $\frac{1}{2} \omega^{\prime}\left(p_{i}\right)=0$ identisch wird mit $-2 \omega_{31} \omega_{12} p_{1}$, daher $a_{1}$ proportional $\mathrm{zu} \omega_{11} p_{1}$.

74. Die Gleichung in Liniencoordinaten des dem Coordinatendreieck eingeschriebenen Kreises ist

$\left(\omega_{23}-\sqrt{\omega_{22} \omega_{33}}\right) u_{2} u_{3}+\left(\omega_{31}-\sqrt{\omega_{33} \omega_{11}}\right) u_{3} u_{1}+\left(\omega_{12}-\sqrt{\omega_{11} \omega_{22}}\right) u_{1} u_{2}=0$, oder auch 


$$
\begin{aligned}
& p_{1}\left(-p_{1} \sqrt{\omega_{11}}+p_{2} \sqrt{\omega_{22}}+p_{3} \sqrt{\omega_{33}}\right) u_{2} u_{3} \\
& \quad+p_{2}\left(p_{1} \sqrt{\omega_{11}}-p_{2} \sqrt{\omega_{22}}+p_{3} \sqrt{\omega_{33}}\right) u_{3} u_{1} \\
& \quad+p_{3}\left(p_{1} \sqrt{\omega_{11}}+p_{2} \sqrt{\omega_{22}}-p_{3} \sqrt{\omega_{33}}\right) u_{1} u_{2}=0 .
\end{aligned}
$$

Die Gleichung muss einerseits von der Form sein

$$
2\left(\alpha_{1} u_{2} u_{3}+\alpha_{2} u_{3} u_{1}+\alpha_{3} u_{1} u_{2}\right)=0
$$

andrerseits nach (2), S. 57 von der Form $r^{2} p_{y}{ }^{2} \boldsymbol{\omega}(u, u)-u_{y}{ }^{2}=0$; hieraus folgt durch Vergleichung der Coefficienten

$$
y_{1}: y_{2}: y_{3}=\sqrt{\omega_{11}}: \sqrt{\omega_{22}}: \sqrt{\omega_{33}} \text {, }
$$

wie bereits in (16) erhalten wurde, ferner $\alpha_{1}=r^{2} p_{y}{ }^{2} \omega_{23}-y_{2} y_{3}$, $r^{2} p_{y}{ }^{2}=y_{1}{ }^{2}: \omega_{11}$, daher $\alpha_{1}=\frac{y_{1}{ }^{2} \omega_{23}}{\omega_{11}}-y_{2} y_{3}$ und nach Einführung der den $y_{i}$ proportionalen $\sqrt{\omega_{i i}}$ erhält man $\alpha_{1}=\omega_{23}-\sqrt{\omega_{22} \omega_{33}}$. Mit Benutzung der Gleichung für die dem Coordinatendreieck eingeschriebene Curve zweiter Classe mit dem Mittelpunkte y (29) erhält man die zweite oben angegebene Form; beide sind selbstverständlich in einander überführbar. Gibt man den drei Grössen $\sqrt{\omega_{11}}, \sqrt{\omega_{22}}, \sqrt{\omega_{33}}$ nicht dieselben Vorzeichen, so gelangt man zu den Gleichungen der drei angeschriebenen Kreise.

75. Die Gleichung, sowie den Mittelpunkt und Radius desjenigen Kreises zu finden, für welchen das Coordinatendreieck ein Poldreieck ist.

Die Gleichung in Liniencoordinaten eines auf das Coordinatendreieck als Poldreieck bezogenen Kegelschnitts ist nach S. 33 und 44 von der Form $\alpha_{1} u_{1}{ }^{2}+\alpha_{2} u_{2}{ }^{2}+\alpha_{3} u_{3}{ }^{2}=0$; andrerseits muss sie als Gleichung eines Kreises von der Form sein $r^{2} p_{y}{ }^{2} \omega(u, u)-u_{y}{ }^{2}=0$. Durch Vergleichung der Coefficienten folgt hieraus

$$
y_{1}: y_{2}: y_{3}=\omega_{31} \omega_{12}: \omega_{12} \omega_{23}: \omega_{23} \omega_{31} \text {, }
$$

der Mittelpunkt fällt daher nach (17) in den Höhenschnittpunkt des Dreiecks.

Ferner wird $\alpha_{1}=r^{2} p_{y}{ }^{2} \omega_{11}-y_{1}{ }^{2}, r^{2} p_{y}{ }^{2}=y_{2} y_{3}: \omega_{23}$, daher

$$
\alpha_{1}=\frac{y_{2} y_{3} \omega_{11}}{\omega_{23}}-y_{1}{ }^{2}=\omega_{31} \omega_{12}\left(\omega_{11} \omega_{23}-\omega_{31} \omega_{12}\right)=-\omega_{31} \omega_{12} \Omega_{23} ;
$$

analoge Werthe besitzen $\alpha_{2}$ und $\alpha_{3}$, so dass

$$
\alpha_{1}: \alpha_{2}: \alpha_{3}=\omega_{31} \omega_{12} \Omega_{23}: \omega_{12} \omega_{23} \Omega_{31}: \omega_{23} \omega_{31} \Omega_{12},
$$

oder nach (16), S. 60 wird

$$
\alpha_{1}: \alpha_{2}: \alpha_{3}=\omega_{31} \omega_{12} p_{2} p_{3}: \omega_{12} \omega_{23} p_{3} p_{1}: \omega_{23} \omega_{31} p_{1} p_{2} .
$$


Die gewünschte Gleichung des Kreises in Liniencoordinaten wird daher

$$
\omega_{31} \omega_{12} p_{2} p_{3} u_{1}^{2}+\omega_{12} \omega_{23} p_{3} p_{1} u_{2}^{2}+\omega_{23} \omega_{31} p_{1} p_{2} u_{3}^{2}=0
$$

in Puuktcoordinaten erhält man

$$
\omega_{23} p_{1} x_{1}^{2}+\omega_{31} p_{2} x_{2}{ }^{2}+\omega_{12} p_{3} x_{3}{ }^{2}=0,
$$

was übrigens auch direct aus (30) folgt.

Der Radius des Kreises kann mit Hilfe von $r^{2} p_{y}{ }^{2}=y_{2} y_{3}: \omega_{23}$ berechnet werden. Bei Einführung der den $y_{i}$ proportionalen Werthe findet man $r^{2}\left(p_{1} \omega_{31} \omega_{12}+p_{2} \omega_{12} \omega_{23}+p_{3} \omega_{23} \omega_{31}\right)^{2}=\omega_{23} \omega_{31} \omega_{12} ;$ nun ist aber $p_{1} \omega_{31} \omega_{12}+p_{2} \omega_{12} \omega_{23}+p_{3} \omega_{23} \omega_{31}=\omega_{23} \omega_{31} p_{3}-\omega_{12} \omega_{33} p_{3}=\Omega_{12} p_{3}=\tau p_{1} p_{2} p_{3}$, daher $r^{2}=\frac{\omega_{23} \omega_{31} \omega_{12}}{\tau^{2} p_{1}^{2} p_{2}^{2} p_{3}^{2}}$, und wenn man die Formeln S. 61 benutzt, wird $r=\frac{2 \Delta}{\sqrt{\tau}} \sqrt{\omega_{23} \omega_{31} \omega_{12}}$. Durch Substitution der Werthe $\omega_{i k}$ und

$$
\sqrt{\tau}=\frac{\Delta}{r_{0} e_{1} e_{2} e_{3}},
$$

wobei $r_{0}$ den Radius des dem Dreieck umschriebenen Kreises bezeichnet, wird $r=2 \dot{r}_{0} \sqrt{-\cos A_{1} \cdot \cos A_{2} \cdot \cos A_{3}}$, woraus hervorgeht, dass der obige Kreis nur reell ist, wenn ein stumpfwinkliges Dreieck vorliegt.

76. Die Gleichung des Kreises in Punktcoordinaten $y_{i}$, der die Verbindungslinie zweier gegebenen Punkte $a$ und $b$ zum Durchmesser hat, ist $\left(\begin{array}{ll}a & y \\ b & y\end{array}\right)_{\omega_{i k}}=0$.

Es seien $a_{i}$ und $b_{i},(i=1,2,3)$, die Coordinaten der beiden Punkte; das Geradenpaar, welches einen beliebigen Punkt $y$ der Ebene mit $a$ und $b$ verbindet, hat alsdann die Gleichung

$$
\sum \pm\left(x_{1} y_{2} a_{3}\right) \cdot \sum \pm\left(x_{1} y_{2} b_{3}\right)=0
$$

und wenn der Winkel des Geradenpaares ein Rechter ist, liegt der Punkt $y$ auf der Peripherie des gesuchten Kreises. Die Bedingung hierfür erhält man zufolge der Formel für $\cos ^{2} \alpha$ in (36), S. 65, indem man in der Gleichung des Geradenpaares die Producte $x_{i} x_{k}$ ersetzt durch $\omega_{i k}$; die so entstehende Gleichung lässt sich kurz schreiben in der Form $\left(\begin{array}{ll}a & y \\ b & y\end{array}\right)_{\omega_{i k}}=0$. (Vgl. auch (225).)

Insbesondere hat der über der Seite $x_{1}=0$ des Coordinatendreiecks als Durchmesser beschriebene Kreis die Gleichung

$$
\omega_{23} x_{1}^{2}+\omega_{11} x_{2} x_{3}-\omega_{12} x_{3} x_{1}-\omega_{31} x_{1} x_{2}=0 .
$$


77. Man bilde die Gleichung in Liniencoordinaten des Kreises, der die Verbindungslinie zweier gegebenen Punkte $a$ und $b$ zum Durchmesser hat.

Ist $\varphi(u, u) \equiv a_{u} b_{u}=0$ die Gleichung des Punktepaares, so hat man für den gesuchten Kreis nach (2), S. 57 und (20), S. 43 einen Ausdruck von der Form

$$
\begin{aligned}
& r^{2}\left\{\frac{1}{2} \varphi^{\prime}\left(p_{1}\right) p_{1}+\frac{1}{2} \varphi^{\prime}\left(p_{2}\right) p_{2}+\frac{1}{2} \varphi^{\prime}\left(p_{3}\right) p_{3}\right\}^{2} \omega(u, u)- \\
& -\left\{\frac{1}{2} \varphi^{\prime}\left(p_{1}\right) u_{1}+\frac{1}{2} \varphi^{\prime}\left(p_{2}\right) u_{2}+\frac{1}{2} \varphi^{\prime}\left(p_{3}\right) u_{3}\right\}^{2}=0
\end{aligned}
$$

wo nun der Radius $r$ noch zu bestimmen ist. Derselbe ist gleich der halben Entfernung der beiden Punkte $a$ und $b$, daher nach (10), S. 59 $4 r^{2}=\left(\begin{array}{ll}a & b \\ a & b\end{array}\right)_{\omega_{i k}}: \tau p_{a}{ }^{2} p_{b}{ }^{2} ;$ es besteht nun, wie auch aus einer Bemerkung S. 64 hervorgeht, stets die Relation

$$
\begin{gathered}
\sum \pm\left(a_{1} b_{2} x_{3}\right)^{2} \\
=-4\left(\mathrm{~A}_{11} x_{1}{ }^{2}+2 \mathrm{~A}_{12} x_{1} x_{2}+\mathrm{A}_{22} x_{2}{ }^{2}+2 \mathrm{~A}_{13} x_{1} x_{3}+2 \mathrm{~A}_{23} x_{2} x_{3}+\mathrm{A}_{33} x_{3}{ }^{2}\right),
\end{gathered}
$$

in der die $A_{i k}$ die Unterdeterminanten in der Determinante von $\varphi(u, u)$ bezeichnen, und diese Relation ist nach (35), S. 76 noch giltig, wenn man die Producte $x_{i} x_{k}$ ersetat durch $\omega_{i k}$. Hierdurch verwandelt sich $\sum \pm\left(a_{1} b_{2} x_{3}\right)^{2}$ in $\left(\begin{array}{ll}a & b \\ a & b\end{array}\right)_{\omega_{i k}}$, und man erhält $\left(\begin{array}{ll}a & b \\ a & b\end{array}\right)_{\omega_{i k}}=-4[\mathrm{~A}, \omega]$; ferner ist $p_{a} p_{b}=\varphi(p, p)$, mithin $4 r^{2}=-\frac{4[\mathrm{~A}, \omega]}{\tau \varphi^{2}(p, p)}$, und die Gleichung des Kreises erhält also die Gestalt $[\mathrm{A}, \omega] \cdot \omega(u, u)+\tau \varphi^{2}(p, u)=0$.

78. Ein Kegelschnitt wird von einer Geraden in zwei Punkten geschnitten; man stelle die Gleichung des Kreises auf, der die Verbindungslinie der beiden Schnittpunkte zum Durchmesser hat.

Es sei $f(x, x)=0$ die Gleichung des Kegelschnitts, $v_{x}=0$ diejenige der Geraden; ein Punkt $y$ liegt auf der Peripherie des ge. nannten Kreises, wenn das von $y$ aus nach den Schnittpunkten von $f(x, x)=0$ und $v_{x}=0$ gezogene Geradenpaar eineu rechten Winkel einschliesst. Die Gleichung dieses Geradenpaares ist nach (53)

$$
v_{y}{ }^{2} f(x, x)-2 v_{x} v_{y} f(x, y)+f(y, y) v_{x}{ }^{2}=0
$$

die Bedingung für den rechten Winkel erhält man zufolge der Formel für $\cos ^{2} \alpha$ in (36), S. 65 dadurch, dass man in der Gleichung des Geradenpaares die $x_{i} x_{k}$ ersetzt durch $\omega_{i k}$. Alsdann entsteht

$$
\begin{aligned}
{[a, \omega] v_{y}{ }^{2}-\frac{1}{2}\left\{\omega^{\prime}\left(v_{1}\right) f^{\prime}\left(y_{1}\right)+\omega^{\prime}\left(v_{2}\right) f^{\prime}\left(y_{2}\right)\right.} & \left.+\omega^{\prime}\left(v_{3}\right) f^{\prime}\left(y_{3}\right)\right\} v_{y}+ \\
& +\omega(v, v) f(y, y)=0 .
\end{aligned}
$$


79. Welche geometrische Bedeutung hat diese Gleichung, wenn die Coordinaten $y_{i}$ als fest gegeben, dagegen die Liniencoordinaten $v_{i}$ als veränderlich betrachtet werden?

80. Man bestimme den Winkel, den die Tangente eines Kreises mit einer durch den Berührungspunkt gezogenen Sehne $u_{x}=0$ bildet.

Bekanntlich ist dieser Winkel (er sei $\alpha$ ) halb so gross wie der der Sehne zugehörige Centriwinkel; daher wird $\cos \alpha$ gleich dem durch die Länge $r$ des Kreisradius dividirten Abstand des Kreismittelpunktes $y$ von der Geraden $u_{x}=0$, d. h. es wird $\cos \alpha=\frac{u_{y}}{r p_{y}} \frac{\sqrt{\omega(u, u)}}{}$.

81. Man bestimme die Länge der Sehne, welche durch die Schnittpunkte des Kegelschnitts $f(x, x)=0$ mit der Geraden $\iota_{x}=0$ begrenzt ist.

Sind $y_{i}$ und $z_{i},(i=1,2,3)$, die Coordinaten der Schnittpunkte, so hat man für das Quadrat $r^{2}$ der Entfernung dieser beiden Punkte nach (10), S. 59 den Ausdruck $r^{2}=\left(\begin{array}{l}y \\ y \\ y\end{array}\right)_{(1) i k}: \tau p_{y}{ }^{2} p_{z}{ }^{2}$. In veränderlichen Liniencoordinaten $v_{i}$ ist das Schnittpunktepaar nach (53a), S. 34 gegeben durch $\psi(v, v) \equiv\left(\begin{array}{ll}u & v \\ u & v\end{array}\right)_{a_{i k}}=0$, man hat also $v_{y} \cdot v_{z} \equiv\left(\begin{array}{ll}u & v \\ u & v\end{array}\right)_{a_{i k}}$ und

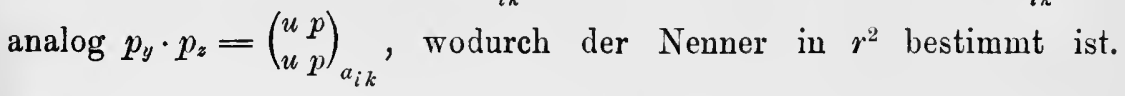
Ferner besteht nach einer Bemerking auf S. 64 die Relation

$$
\sum \pm\left(y_{1} z_{2} x_{3}\right)^{2}=-4\left(\Psi_{11} x_{1}{ }^{2}+\cdots+2 \Psi_{23} x_{2} x_{3}+\cdots\right)
$$

wobei die $\Psi_{i k}$ die Unterdeterminanten in der Determinante von $\psi(v, v)$ bezeichnen; da diese Relation für alle Werthe der $x$ besteht, so bleibt sie nach (35), S. 76 noch richtig, wenn man die Producte $x_{i} x_{k}$ ersetzt durch $\omega_{i k}$, wovon sofort Gebrauch gemacht werden wird. Andrerseits stellt $\Psi(x, x)=0$ das Quadrat des Trägers des Punktepaares $y, z$ dar, muss daher $u_{x}{ }^{2}$ als einen Factor enthalten, und da $\Psi(x, x)=0$ auch erfüllt wird, wenn $u_{x}$ eine Tangente der Curve $f(x, x)=0$ ist, so wird der andere Factor bis auf einen Zahlencoefficienten gleich $F(u, u)$; man findet in der That

$$
\Psi(x, x)=u_{x}^{2}\left(A_{11} u_{1}^{2}+\cdots+2 A_{23} u_{2} u_{3}+\cdots\right)=u_{x}^{2} \cdot F(u, u) .
$$

Durch Substitution dieses Ausdrucks in $\sum \pm\left(y_{1} z_{2} x_{3}\right)^{2}=-4 \Psi(x, x)$ und Vertauschung der $x_{i} x_{k}$ mit den $\omega_{i k}$ folgt $\left(\begin{array}{l}y \\ y \\ y\end{array}\right)_{\omega_{i k}}=-4 \omega(u, u) \cdot F(u, u)$. 
Mit Benutzung dieses und des oben für $p_{y} p_{z}$ gefundenen Werthes verwandelt sich die Formel für $r^{2}$ in

$$
r^{2}=-\frac{4 \omega(u, u) \cdot F(u, u)}{\tau_{\tau}\left(\begin{array}{ll}
p & u \\
p & u
\end{array}\right)_{a_{i k}}^{2}}
$$

Hieraus folgt sofort:

82. Alle in dem Kegelschnitt $f(x, x)=0$ gezogenen Sehnen von derselben Länge $r$ umhüllen die Curve vierter Classe

$$
4 \omega(u, u) \cdot F(u, u)+r^{2} \tau\left(\begin{array}{ll}
p & u \\
p & u
\end{array}\right)_{a_{i k}}^{2}=0 .
$$

83. Die Länge $r$ der Strecke, welche auf der Geraden $u_{x}=0$ durch zwei andere Geraden $v_{x}=0$ und $w_{x}=0$ abgeschnitten wird, ist gegeben durch

$$
r^{2}=\frac{\omega(u, u) \cdot \sum \pm\left(u_{1} v_{2} w_{3}\right)^{2}}{\tau \cdot \sum \pm\left(v_{1} u_{2} p_{3}\right)^{2} \sum \pm\left(w_{1} u_{2} p_{3}\right)^{2}} .
$$

Man hat bei der in (81) abgeleiteten Formel $f(x, x)$ zu ersetzen durch $v_{x} v_{x}$; alsdann ist (dualistisch zu einer Bemerkung auf S. 64) $F(u, u)=-\frac{1}{4} \sum \pm\left(u_{1} v_{2} w_{3}\right)^{2}$, während $\operatorname{sich}\left(\begin{array}{ll}u & p \\ u & p\end{array}\right)_{a_{i k}}$ verwandelt in $\sum \pm\left(v_{1} u_{2} p_{3}\right) \cdot \sum \pm\left(w_{1} u_{2} p_{3}\right)$

84. Ein Winkel von constanter Grösse, dessen Scheitel in einem beliebig aber fest gewählten Punkte $y$ eines Kegelschnitts liegt, dreht sich um seinen Scheitel. Was für eine Curve wird von der jeweiligen Verbindungslinie der beiden Schnittpunkte des Kegelschnitts mit den Schenkeln des Winkels umhüllt?

Die Gleichung der beiden Schenkel ist nach (53)

$$
g(x, x) \equiv \sum_{1}^{3} \sum_{i}^{3} b_{i k} x_{i} x_{k} \equiv 2 u_{x} f(x, y)-u_{y} f(x, x)=0
$$

wenn $u_{1}, u_{2}, u_{3}$ die Coordinaten der eben genannten Verbindungslinie bezeichnen. Auf diese Gleichung ist die Formel $\operatorname{tg}^{2} \alpha=-\frac{4 \tau G(p, p)}{[b, \omega]^{2}}$ $((36)$, S. 65) für den Winkel $\alpha$ des durch $g(x, x)=0$ dargestellten Geradenpaares anzuwenden. Der Zähler $G(p, p)$ muss proportional werden zu $p_{y}{ }^{2}$, denn $G(v, v)=0$ würde die Spitze $y$ des Geradenpaares doppelt darstellen. Auch wird $G(p, p)=0$ erfüllt, wenn $u_{x}=0$ eine Tangente der Curve $f(x, x)=0$ ist; daher wird der andere Factor von $G(p, p)$ bis auf einen Zahlencoefficienten gleich $F(u, u)$, und man findet 
in der That $G(p, p)=F(u, u) \cdot p_{y}{ }^{2}$. Die Grösse $[b, \omega]$ im Nenner geht aus $g(x, x)$ dadurch hervor, dass man die Producte $x_{i} x_{k}$ ersetzt durch $\omega_{i k}$; man erhält alsdann

$$
\frac{1}{2}\left\{f^{\prime}\left(y_{1}\right) \cdot \omega^{\prime}\left(u_{1}\right)+f^{\prime}\left(y_{2}\right) \cdot \omega^{\prime}\left(u_{2}\right)+f^{\prime}\left(y_{3}\right) \cdot \omega^{\prime}\left(u_{3}\right)\right\}-[a, \omega] u_{y} .
$$

Für die Umhüllungscurve folgt daher die Gleichung

$$
\begin{aligned}
\operatorname{tg}^{2} \alpha\left\{\frac { 1 } { 2 } \left\{f^{\prime}\left(y_{1}\right) \cdot \omega^{\prime}\left(u_{1}\right)+f^{\prime}\left(y_{2}\right) \cdot \omega^{\prime}\left(u_{2}\right)\right.\right. & \left.\left.+f^{\prime}\left(y_{3}\right) \cdot \omega^{\prime}\left(u u_{3}\right)\right\}-[a, \omega] u_{y}\right\}^{2}+ \\
& +4 \tau p_{y}{ }^{2} \cdot F(u, u)=0
\end{aligned}
$$

mithin eine Curve zweiter Classe.

In dem besonderen Falle, dass der rotirende Winkel ein Rechter ist, reducirt sich die Curve auf den doppelt zu zählenden Punkt

$$
\frac{1}{2}\left\{\left(f^{\prime}\left(y_{1}\right) \cdot \omega^{\prime}\left(u_{1}\right)+f^{\prime}\left(y_{2}\right) \cdot \omega^{\prime}\left(u_{2}\right)+f^{\prime}\left(y_{3}\right) \cdot \omega\left(u_{3}\right)\right\}-[a, \omega] u_{y}=0,\right.
$$

wie übrigens auch aus (79) für $f(y, y)=0$ folgen würde. Dieser Punkt liegt nothwendig auf der in $y$ senkrecht zur Tangente von $y$ gezogeuen Geraden (Normale von $y$ ), denn wenn der eine Schenkel des rotirenden rechten Winkels die Lage der Tangente des Kegelschnitts im Punkte $y$ einnimmt, fällt die obige variabele Verbindungslinie mit der Normale dieses Punktes zusammen. Mit Benutzung dieser Thatsache lässt sich lediglich durch Anwendung eines rechten Winkels in einem beliebigen Punkte eines Kegelschnitts die Normale construiren ${ }^{1}$ ).

Man erkennt übrigens aus der Gleichung der im allgemeinen Falle (bei beliebigem $\alpha$ ) entstehenden Umhüllungscurve zweiter Classe, dass diese Curve den gegebenen Kegelschnitt doppelt berührt und dass der im Falle $\alpha=90^{\circ}$ entstehende Punkt stets Pol der betreffenden Berührungssehne ist.

85. Welche Bedingung müssen die Coordinaten $w_{1}, w_{2}, w_{3}$ einer Geraden erfüllen, wenn dieselbe mit einer Geraden des Paares

$$
f(x, x) \equiv u_{x} v_{x}=0
$$

einen Winkel $\alpha$ bilden soll, für $\operatorname{den} \operatorname{tg} \alpha=t$ gegeben ist?

Für den Winkel, den die beiden Geraden $u$ und $w$ mit einander bilden, hat man nach S. 64 die Formel $\operatorname{tg} \alpha=t=\frac{\sqrt{\tau} \sum \pm\left(p_{1} u_{2} w_{3}\right)}{\omega(u, w)}$,

1) Dieses Theorem wurde zuerst von Frégier bewiesen und auch auf den Raum für Flächen 2. Ordnung erweitert in seiner Abhandlung "Théorèmes nouveaux sur les lignes et surfaces du second ordre", Annales de Mathématiques, herausgegeben von Gergonne, Bd. 6, S. $229 \mathrm{ff}$, 1816; zuvor war das Theorem von Frégier angekündigt worden in der Correspondance sur l'école polytechnique, brsgg. von Hachette, Bd. 3, S. 394, 1816. 
daher $\omega(u, w) \cdot t-\sqrt{\tau} \sum \pm\left(p_{1} u_{2} w_{3}\right)=0$. Da in der Aufgabe unentschieden ist, ob die Gerade $w$ mit $u_{x}=0$ oder mit $v_{x}=0$ den Winkel $\alpha$ bilden soll, so ist auch $\omega(v, w) t-\sqrt{\tau} \sum \pm\left(p_{1} v_{2} w_{3}\right)=0$ zu berücksichtigen; die Lösung der Aufgabe wird folglich durch Multiplication der beiden in $t$ linearen Gleichungen erhalten in der Gestalt

$$
\begin{gathered}
\omega(u, w) \omega(v, w) t^{2}- \\
-\sqrt{\tau}\left\{\omega(u, w) \sum \pm\left(p_{1} v_{2} w_{3}\right)+\omega(v, w) \sum \pm\left(p_{1} u_{2} w_{3}\right)\right\} t \\
+\tau \sum \pm\left(p_{1} u_{2} w_{3}\right) \cdot \sum \pm\left(p_{1} v_{2} w_{3}\right)=0
\end{gathered}
$$

Offeubar ist der Coefficient von $t^{2}$ in dieser Gleichung

$$
f\left(\frac{1}{2} \omega^{\prime}\left(w_{1}\right), \frac{1}{2} \omega^{\prime}\left(w_{2}\right), \quad \frac{1}{2} \omega^{\prime}\left(w_{3}\right)\right) \text {; }
$$

der Coefficient von $t^{1}$ ist mit Rücksicht auf $2 f(x, y)=u_{x} v_{y}+u_{y} v_{x}$ identiscl mit

$$
-\sqrt{\tau}\left\{\frac{1}{2} \omega^{\prime}\left(w_{1}\right) f^{\prime}\left(y_{1}\right)+\frac{1}{2} \omega^{\prime}\left(w_{2}\right) f^{\prime}\left(y_{2}\right)+\frac{1}{2} \omega^{\prime}\left(w_{3}\right) f^{\prime}\left(y_{3}\right)\right\},
$$

wobei $y_{1}=w_{2} p_{3}-w_{3} p_{2}, \quad y_{2}=w_{3} p_{1}-w_{1} p_{3}, \quad y_{3}=w_{1} p_{2}-w_{2} p_{1}$. Das absolute Glied wird $\tau\left(\begin{array}{cc}p & w \\ p & w\end{array}\right)_{i \dot{ }}$. Uebrigens stellt die Gleichung für variabele Liniencoordinaten $w_{i}$ ein Punktepaar im Unendlichen dar, das für $t=\infty$ mit den Normalencentra der zwei gegebenen Geraden zusammenfällt.

86. Der Winkel $\alpha$ der Tangenten, welche rom Punkte $y$ an die Curve zweiter Classe $\varphi(u, u) \equiv \sum_{1}^{3} \sum_{1}^{3} \alpha_{i k} u_{i} u_{k}=0$ gezogen werden können, ist gegeben durch die Formel

wobei

$$
\operatorname{tg} \alpha= \pm \frac{p_{y} \sqrt{-\tau \cdot \Phi(y, y)}}{x(y, y)}
$$

$$
\begin{aligned}
2 \chi(y, y) & \equiv\left(\alpha_{22} \omega_{33}+\alpha_{33} \omega_{22}-2 \alpha_{23} \omega_{23}\right) y_{1}^{2}+\cdots \\
& +2\left(\alpha_{31} \omega_{23}+\alpha_{23} \omega_{31}-\alpha_{33} \omega_{12}-\alpha_{12} \omega_{33}\right) y_{1} y_{2}+\cdots
\end{aligned}
$$

Die Gleicbung des Tangentenpaares ist nach (27), S. 45

$$
g(x, x) \equiv \sum_{1}^{3} \sum_{1}^{3} b_{i k} x_{i} x_{k} \equiv\left(\begin{array}{ll}
y & x \\
y & x
\end{array}\right)_{\alpha_{i k}}=0
$$

und nun hat man eine der Formeln (36), S. 65 für den Winkel eines durch 
$f(x, x)=0$ dargestellten Geradenpaares anzuwenden auf $g(x, x)=0$; es folgt alsdann $\operatorname{tg}^{2} \alpha=-\frac{4 \tau G(p, p)}{[b, \omega]^{2}}$. Es ist

$$
\begin{aligned}
{[b, \omega] } & =\alpha_{11}\left(y_{2}{ }^{2} \omega_{33}+y_{3}{ }^{2} \omega_{22}-2 y_{2} y_{3} \omega_{23}\right)+\cdot \\
& +2 \alpha_{12}\left(y_{3} y_{1} \omega_{23}+y_{2} y_{3} \omega_{31}-y_{3}{ }^{2} \omega_{12}-y_{1} y_{2} \omega_{33}\right)+\cdots= \\
& =\left(\alpha_{22} \omega_{33}+\alpha_{33} \omega_{22}-2 \alpha_{23} \omega_{23}\right) y_{1}{ }^{2}+\cdots+ \\
& +2\left(\alpha_{31} \omega_{23}+\alpha_{23} \omega_{31}-\alpha_{33} \omega_{12}-\alpha_{12} \omega_{33}\right) y_{1} y_{2}+\cdots,
\end{aligned}
$$

wofür $2 \chi(y, y)$ gesetzt werden möge $\left.{ }^{1}\right)$. Die Grösse $G(u, u)$ muss (ähnlich wie in (84)) proportional werden zu $u_{y}{ }^{2}$; denn $G(u, u)=0$ würde die Spitze $y$ des Tangentenpaares doppelt zählend darstellen. Ferner wird $G(u, u)=0$ erfüllt, wenn $y$ auf der Curve liegt, daher wird der andere Factor von $G(p, p)$ bis auf einen Zahlencoefficienten gleich $\Phi(y, y)$, und man findet in der That $G(u, u)=u_{y}{ }^{2} \cdot \Phi(y, y)$, mithin $G(p, p)=p_{y}{ }^{2} . \Phi(y, y)$. Durch Substitution der für $[b, \omega]$ und $G(p, p)$ gefundenen Werthe in die Formel für $\operatorname{tg}^{2} \alpha$ folgt das oben angegebene Resultat.

Diese Formel kann auch noch abgeleitet werden mit Benutzung der $\mathrm{zu}(13), \S 15$ dualistischen Relation

$$
(\alpha-1)^{2} \chi^{2}(y, y)-(\alpha+1)^{2} \Phi(y, y) \cdot \psi(y, y)=0
$$

für das Doppelverhältniss, welches die vom Punkte $y$ nach zwei Curven zweiter Classe $\varphi(u, u)=0$ und $\psi(u, u)=0$ gezogenen Tangentenpaare mit einander bilden. Man hat nur an Stelle von $\psi(u, u)=0$ das imaginäre Kreispunktepaar zu setzen und den in (35), S. 64 ausgesprochenen Satz anzuwenden, wonach der Winkel der von $y$ an $\varphi(u, u)=0$ gezogenen Tangenten aus dem eben genannten Doppelverhältniss $m$ durch Division mit $2 i$ hervorgeht. Bei Uebergang zu den trigonometrischen Functionen wäre dann Gebrauch zu machen von der Relation $\ln m=2 i \cdot \arccos \frac{m+1}{2 \sqrt{m}}$.

Denkt man sich $\operatorname{tg} \alpha$ fest gegeben, dagegen den Punkt $y$ variabel, so folgt sofort:

87. Der geometrische Ort aller Punkte $y$, von denen betrachtet eine Curve zweiter Classe $\varphi(u, u)=0$ unter einem gegebenen Winkel $\alpha$ oder dessen Nebenwinkel erscheint, ist die Curve vierter Ordnung

$$
\operatorname{tg}^{2} \alpha \cdot \chi^{2}(y, y)+\tau \Phi(y, y) \cdot p_{y}{ }^{2}=0 .
$$

1) Hinsichtlich dieser Bezeichnungsweise vergleiche man auch (29), S. 148. Für $\alpha=90^{\circ}$ erbält man den Satz (31), S. 148. 
Besteht insbesondere die gegebene Curve zweiter Classe $\varphi(u, u)=0$ aus einem Punktepaar $\left(\varphi(u, u) \equiv a_{u} b_{u}=0\right)$, so wird $\Phi(y, y)$ proportional dem Quadrat des Trägers, und zwar ist nach einer Bemerkung auf S. $64 \Phi(y, y)=-\frac{1}{4} \sum \pm\left(a_{1} b_{2} x_{3}\right)^{2}$; ferner verwandelt sich $2 \chi(y, y)$ in $\left(\begin{array}{ll}a & y \\ b & y\end{array}\right)_{\omega_{i k}}$, und man erhält das Resultat:

88. Der geometrische Ort aller Punkte $y$, von denen betrachtet die Strecke zwischen zwei Punkten $a, b$ unter einem gegebenen Winkel $\alpha$ oder dessen Nebenwinkel erscheint, besteht aus den zwei Kreisen

$$
\operatorname{tg} \alpha \cdot\left(\begin{array}{ll}
a & y \\
b & y
\end{array}\right)_{\omega_{i k}} \pm \sqrt{\tau} \cdot \sum \pm\left(a_{1} b_{2} y_{3}\right) \cdot p_{y}=0 .
$$

Dass der betreffende Ort in der That aus zwei Kreisen besteht, ist nicht nur aus der elementaren Geometrie bekannt, sondern folgt auch aus (76) mit Rücksicht auf (68); denn weil $\left(\begin{array}{ll}a & y \\ b & y\end{array}\right)_{\omega_{i k}}=0$ den über der Strecke $a b$ als Durchmesser errichteten Kreis darstellt, ist nach (68) auch jede der beiden sich im Vorzeichen von $\sqrt{\tau}$ unterscheidenden Curven ein Kreis.

89. Der Winkel $\alpha$ der Tangenten, welche vom Punkte $y$ an die Carve zweiter Ordnung $f(x, x) \equiv \sum_{1}^{3} \sum_{i}^{3} a_{i k} x_{i} x_{k}=0$ gezogen werden können, ist gegeben durch die Formel $\operatorname{tg} \alpha= \pm \frac{p_{y} \sqrt{-\tau A f(y, y)}}{H}$, wobei

$$
\begin{aligned}
2 H \equiv\left(A_{22} \omega_{33}\right. & \left.+A_{33} \omega_{22}-2 A_{23} \omega_{23}\right) y_{1}{ }^{2}+\cdots \\
& +2\left(A_{31} \omega_{23}+A_{23} \omega_{31}-A_{33} \omega_{12}-A_{12} \omega_{33}\right) y_{1} y_{2}+\cdots
\end{aligned}
$$

Folgt unmittelbar aus (86), wenn man sich zu $f(x, x)=0$ die Gleichung in Liniencoordinaten hergestellt denkt. - Für $\alpha=90^{\circ}$ erhält man den in (31), S. 148 ausgesprochenen Satz.

90. Man bestimme den Winkel zwischen dem Durchmesser $u_{x}=0$ des Kegelschnitts $f(x, x)=0$ und dem zugehörigen conjugirten Durchmesser.

Die Gleichung des letzteren ist nach (54)

$$
v_{x} \equiv \sum \pm\left(u_{1} p_{2} \frac{1}{2} f^{\prime}\left(x_{3}\right)\right)=0
$$

und nach $12 \mathrm{~b}$, S. 13 wird $\cos ^{2}(u, v)=\frac{1}{4} \frac{\left(v_{1} \omega^{\prime}\left(u_{1}\right)+v_{2} \omega^{\prime}\left(u_{2}\right)+v_{8} \omega^{\prime}\left(u_{3}\right)\right)^{2}}{\omega(u, u) \cdot \omega(v, v)}$, wo nun noch die $v_{i}$ eliminirt werden sollen. Es ist 
Winkel zweier conjugirten Durchmesser. Satz von Newton.

$$
\frac{1}{2}\left(v_{1} \omega^{\prime}\left(u_{1}\right)+v_{2} \omega^{\prime}\left(u_{2}\right)+v_{3} \omega^{\prime}\left(u_{3}\right)\right) \equiv \omega(u, v)=\sum \pm\left(\frac{\partial \psi}{\partial x_{1}} u_{2} p_{3}\right)
$$

wobei $\Psi$, wie in (45), S. 96, zur Abkürzung gesetzt ist für

$$
\frac{1}{4} \sum_{1}^{3} \omega^{\prime}\left(u u_{i}\right) f^{\prime}\left(x_{i}\right)
$$

ferner wird nach S. $63 \omega(u, u) \cdot \omega(v, v)=\omega^{2}(u, v)+\tau \sum \pm\left(p_{1} u_{2} v_{3}\right)^{2}$, und wenn man hier an Stelle der $v_{i}$ ihre eigentlichen Werthe einsetzt, erhält man $\omega(u, u) \cdot \omega(v, v)=\sum \pm\left(\frac{\partial \psi}{\partial x_{1}} u_{2} p_{3}\right)^{2}+\tau\left(\begin{array}{l}u p \\ u p\end{array}\right)_{a_{i k}}^{2}$, folglich wird

daher

$$
\cos ^{2}(u, v)=\frac{\sum \pm\left(\frac{\hat{\frac{\partial}{}}}{\hat{\partial} x_{1}} u_{z} p_{3}\right)^{2}}{\sum \pm\left(\frac{\partial \psi}{\partial x_{1}} u_{2} p_{3}\right)^{2}+\tau\left(\begin{array}{cc}
u & p \\
u & p
\end{array}\right)_{a_{i k}}^{2}},
$$

und

$$
\sin ^{2}(u, v)=\frac{\tau\left(\begin{array}{ll}
u & p \\
u & p
\end{array}\right)_{a_{i k}}^{2}}{\sum \pm\left(\frac{\partial \psi}{\partial x_{1}} u_{2} p_{3}\right)^{2}+\tau\left(\begin{array}{ll}
u & p \\
u & p
\end{array}\right)_{a_{i k}}^{2}}
$$

$$
\operatorname{tg}(u, v)=\left(\begin{array}{ll}
u & p \\
u & p
\end{array}\right) \underset{a_{i k}}{ } \sqrt{\tau}: \sum \pm\left(\frac{\partial \psi}{\partial x_{1}} u_{2} p_{3}\right)
$$

91. Durch die vier Punkte, in denen irgend ein Tangentenpaar eines Kegelschnitts von einem beliebigen zweiten Tangentenpaare getroffen wird, lässt sich ein Geradenpaar legen, das durch den Schnittpunkt der den beiden Tangentenparen zugehörigen zwei Berührungssehnen geht und ausserdem zu den letzteren harmonisch liegt ${ }^{1}$ ).

Nach (42), S. 84 kann die Gleichung eines Kegelschnitts in die Form gebracht werden $X_{1} X_{3}-X_{2}{ }^{2}=0$, wo $X_{1}=0, X_{3}=0$ irgend zwei Tangenten darstellen, während $X_{2}=0$ die Gleichung der zugehörigen Berührungssehne ist; mit Hilfe zweier anderen Tangenten desselben Kegelschnitts sei dessen Gleichung in die Form gebracht

1) Die Veranlassung, dass Plücker („System der analytischen Geometrie“, Berlin 1835, S. 117) diesen Satz al won Newton herrührend bezeichnet, dürfte wohl eine Untersuchung in dessen "Philosophias naturalis principia mathematica“ gegeben haben. Vgl. z. B. die von Cotes besorgte und im Jahre 1714 in Amsterdam erschienene Ausgabe, S. 86 f., sowie S. 84, Corol. 2, oder auch die deutsche von Wolfers besorgte Uebersetzung "Sir Isaac Newton's Mathematische Principien der Naturlehre", Berlin 1872, S. 109, sowie S. 107, Zusatz 2. 
$x_{1} x_{3}-x_{2}{ }^{2}=0$, so dass $X_{1} X_{3}-X_{2}{ }^{2}=x_{1} x_{3}-x_{2}{ }^{2}$. Hieraus folgt $X_{1} X_{3}-x_{1} x_{3}=\left(X_{2}+x_{2}\right)\left(X_{2}-x_{2}\right)$. Die geometrische Deutung dieser Gleichung liefert obigen Satz, der auch in folgender Form ausgesprochen werden kann:

91 a. Die Geraden, welche die Berührungspunkte jezweier Gegenseiten eines dem Kegelschnitt umschriebenen Vierseits verbinden, gehen durch den Schnittpunkt der beiden Diagonalen desselben, und zwar liegen sie harmonisch zu den zwei Diagonalen.

92. Die Berührungssehne irgend zweier Tangenten einer Hyperbel ist parallel zu den Verbindungslinien der Schnittpunkte des Tangentenpaares mit dem Asymptotenpaare und liegt in der Mitte zwischen diesen Verbindungslinien ${ }^{1}$ ).

Folgt aus (91), wenn man als eines der beiden Tangentenpaare das Asymptotenpaar wählt und beachtet, dass die vierte harmonische Gerade zu zwei Parallelen und zur unendlich fernen Geraden mit der Mittellinie der beiden Parallelen zusammenfällt.

Aus (92) folgt unmittelbar:

93. Der Berührungspunkt einer Tangente der Hyperbel liegt in der Mitte zwischen den Schnittpunkten dieser Tangente mit den zwei Asymptoten.

94. Alle Dreiecke, welche die beiden Asymptoten und eine beliebige Tangente einer und derselben Hyperbel zu Seiten haben, sind inhaltsgleich.

Fig. 14.

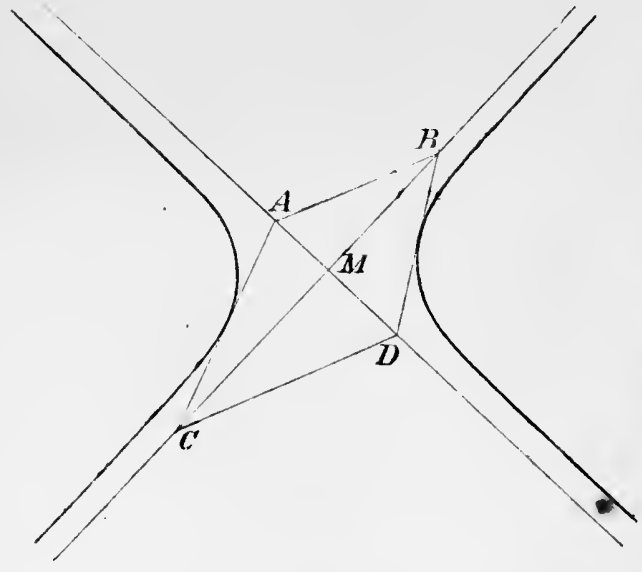

Nach (92) ist $A B$ parallel $C D$, daher $\triangle C D A=\triangle C D B$, woraus $\triangle A M C=\triangle B M D$ folgt (Fig. 14).

95. Jede Tangente einer Parabel halbirt im Berührungspunkte $B$ die Verbindungslinie des Mittelpunktes $M$ einer zur Tangente parallelen Sehne $R S$ mit dem Pol $P$ dieser Sehne.

Man fixire ein vom Punkte $P$ an die Curve gezogenes

1) Es bedarf wohl kaum der Erwähnung, dass natürlich diejenigen zwei Verbindungslinien gemeint sind, welche weder mit einer Asymptote noch mit einer der zwei anderen Tangenten zusammenfallen. 
Tangentenpaar mit den Berührungspunkten $R, S$, sowie die zur Sehne $R S$ parallel gezogene Tangente mit dem Berührungspunkte $B$, welche zusammen mit der unendlich fernen Geraden ein zweites Tangentenpaar der Parabel bildet. Die Berührungssehne desselben ist eine durch $B$ parallel zur $\mathrm{Axe}^{1}$ ) gezogene Gerade, welche die Sehne $R S$ in deren Halbirungspunkte $M$ trifft. Von den vier Schnittpunkten der beiden Tangentenpaare liegen nun zwei $(C$ und $D)$ im Endlichen, die beiden anderen unendlich weit; das durch die vier Punkte ausserdem noch mögliche Geradenpaar besteht daher hier aus zwei durch $C$ resp. $D$ gezogenen Parallelen zu den von $P$ an die Curve gelegten Tangenten. Da ferner nach (91) der Schnittpunkt dieses Geradenpaares mit dem Schnittpunkt $M$ der zwei Berülrungssehnen zusammenfüllt, so entsteht ein Parallelogramm $P C M I D$, dessen Diagonalen $P M$ und $C D$ sich im Punkte $B$ halbiren.

96. Die Diagonalen irgend eines dem Kegelschnitt umschriebenen Parallelogramms $A B C D$ bilden ein Paar conjugirter Durchmesser.

Die Verbindungslinien $E G$ und $F H$ der Berührungspunkte je zweier gegenüberliegenden Seiten des Parallelogramms (Fig. 15) sind als Polaren unendlich weit liegender Punkte jedenfalls Durchmesser. Durch den Schnittpunkt $M$ derselben gehen nach (91a) auch die Diagonalen des umschriebenen Parallelogramms, sie sind daher gleichfalls Durchmesser und liegen nach (91a) harmonisch zu den Diago-

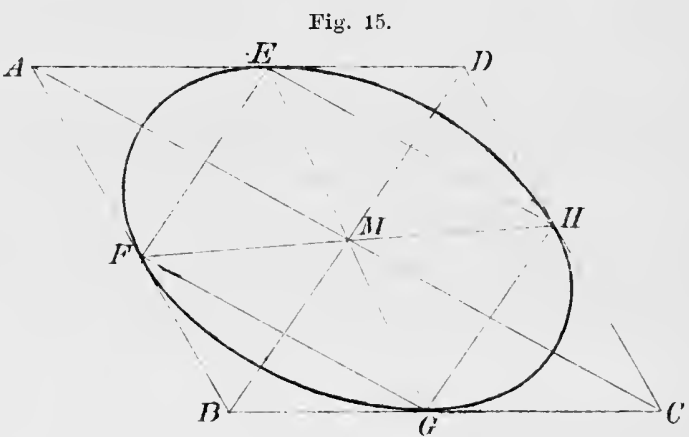
nalen des durch die Berührungssehnen gebildeten Vierecks EFGH, das gleichfalls ein Parallelogramm ist, denn je zwei seiner gegenüberliegenden Seiten, z. B. $E H$ und $F G$, sind als Polaren von Punkten $B$ und $D$ eines und desselben Durchmessers einander parallel. Da $M I A$ und $M D$ harmonisch liegen zu $M E$ und $M H$, und da ferner nach (63) $M D$ die Sehne $E H$ halbirt, so ist der Durchmesser $A C$ zur Sehne $E H$ parallel, gehört somit demjenigen Sehnensystem an, das von $B D$ halbirt wird.

1) Ueber die Definition der Axe der Parabel vgl. S. 103. 
Zugleich folgt:

97. Die Seiten irgend eines dem Kegelschnitt eingeschriebenen Parallelogramms sind zwei conjugirten Durchmessern parallel, oder in etwas anderer Form:

98. Die beiden Sehnen, welche die Endpunkte eines Durchmessers mit einem beliebigen Punkte des Kegelschnitts verbinden, sind parallel zu zwei conjugirten Durchmessern. Man nennt solche Sehnen Supplementarsehnen.

Umgekehrt gilt auch der Satz:

99. Zieht man durch die Endpunkte $F, H$ eines Durchmessers gerade Linien $F E$ und $H E$ parallel zu zwei conjugirten Durchmessern $M D$ und $M A$, so schneiden sich jene Geraden in einem Punkte des Kegelschnitts.

Würde nämlich die Gerade $H E$ den Kegelschnitt nicht in ihrem Schnittpunkte mit $F E$ treffen, sondern in einem anderen Punkte $E^{\prime}$, so sind $F E^{\prime}$ und $H E^{\prime}$ nach (98) zwei conjugirten Durchmessern parallel; nun ist $H E^{\prime}$ parallel $M A$, daher $F E^{\prime}$ parallel $M D$, d. h. $F E^{\prime}$ fällt mit $F E$ und $E^{\prime}$ mit $E$ zusammen.

100. Zieht man durch einen beliebigen Punkt $P$ eines Kegelschnitts zwei Sehnen (mit den Gleichungen $S_{2}=0$ und $S_{3}=0$ ) und in dem weiteren Schnittpunkt dieser Sehnen und der Curve die Tangenten (mit den Gleichungen $T_{3}=0$, bezw. $T_{2}=0$ ), so liegt die Verbindungslinie des Punktes $P$ mit dem Schnittpunkte dieser beiden Tangenten harmonisch zur Tangente in $P$ und zu dem Sehnenpaare.

Ist $T_{1}=0$ die Gleichung der Tangente in $P$, so kann der Kegelschnitt nach (42), S. 84 dargestellt werden sowohl durch $T_{1} T_{2}-S_{3}{ }^{2}=0$ als durch $T_{1} T_{3}-S_{2}{ }^{2}=0$. Hieraus folgt

$$
T_{1}\left(T_{2}-T_{3}\right) \equiv\left(S_{3}+S_{2}\right)\left(S_{3}-S_{2}\right),
$$

d. h. der eine der beiden Factoren $S_{3}+S_{2}=0$ und $S_{3}-S_{2}=0$ repräsentirt gleich Null gesetzt die Tangente $T_{1}=0$ des Punktes $P$, der andere die oben genannte Verbindungslinie. Die Gleichungen $S_{\mathbf{3}}=0$, $S_{2}=0, S_{3}+S_{2}=0$ und $S_{3}-S_{2}=0$ gehören aber vier harmonischen Strahlen eines Büschels an.

Dualistisch folgt:

101. Fixirt man auf irgend einer Tangente $T$ eines Kegelschnitts zwei beliebige Punkte $P_{2}, P_{3}$ und zieht man die zwei von $P_{2}, P_{3}$ noch möglichen Tangenten an die Curve, sowie die zugehörige Berührungs- 
sehne, so liegt das Punktepaar $P_{2}, P_{3}$ harmonisch zu dem Berührungspunkte der Tangente $T$ und zu dem Schnittpunkte dieser Tangente mit der eben genannten Berührungssehne.

Dieser Satz ist übrigens gleichbedeutend mit dem folgenden:

102. Die Verbindungslinie der Berührungspunkte zweier Seiten eines dem Kegelschnitt umschriebenen Dreiecks schneidet die dritte Seite in einem Punkte, welcher harmonisch liegt zum Berührungspunkte dieser Seite und zu den auf ihr gelegenen Ecken des Dreiecks.

103. Fixirt man bei einem Kegelschnitt irgend ein Tangentenpaar und die zugehörige Berührungssehne, so steht das Product aus den Abständen eines beliebigen Punktes der Curve von den beiden Tangenten in constantem Verhältuiss zu dem Quadrat des Abstandes dieses Punktes von der Berührungssehne.

Die Gleichung der Curve ist von der Form $X_{1} X_{3}-X_{2}{ }^{2}=0$, und hier werden durch $X_{1}=0, X_{3}=0$ die beiden Tangenten dargestellt, durch $X_{2}=0$ die Berührungssehne. Führt man in $X_{1} X_{3}-X_{2}{ }^{2}=0$ an Stelle von $X_{1}, X_{2}, X_{3}$ nach (1), S. 9 die Abstände eines Punktes von diesen Geraden ein, wobei die Coordinaten des Punktes der Gleichung des Kegelschnitts genügen müssen, so folgt für diese Abstände $q_{1}, q_{2}, q_{3}$ eine Relation von der Form $q_{1} q_{3}-c \cdot q_{2}{ }^{2}=0$, wo $c$ eine Constante bedeutet, deren Werth natürlich auch von den Coordinaten der Geraden $X_{1}, X_{2}, X_{3}$ abhängt.

104. Man beweise, dass für den Fall eines Kreises die Constante $c$ gleich der Einheit ist.

Dualistisch ergibt sich aus (103):

105. Fixirt man auf einem Kegelschnitt irgend ein Punktepaar $P, Q$ und den Schnittpunkt $S$ der beiden in diesen Punkten gezogenen Tangenten, so steht das Product aus den Abständen einer beliebigen Tangente der Curve von den beiden Punkten $P$ und $Q$ in constantem Verhältniss zu dem Quadrat des Abstandes dieser Tangente von dem Punkte $S$.

\section{Anwendung von \& 10-12.}

106. Alle Parallelogramme, welche dadurch entstehen, dass man in den Endpunkten zweier conjugirten Durchmesser die Tangenten an eine Ellipse zieht, haben denselben Flächeninhalt wie das Rechteck, dessen Seiten durch die vier Scheiteltangenten der Curve gebildet werden. 
Bezogen auf zwei conjugirte Durchmesser als Axen eines Systems homogener schiefwinkliger Parallelcoordinaten lautet die Gleichung des Kegelschnitts $a_{11} x_{1}{ }^{2}+a_{22} x_{2}{ }^{2}+x x_{3}{ }^{2}=0$. Die halben Längen $r_{1}$ und $r_{2}$ der zwei conjugirten Durchmesser folgen aus $r_{1}^{2}=-\frac{x}{a_{11}}$, $r_{2}^{2}=-\frac{x}{a_{22}}$; daher wird $r_{1} r_{2} \sin w=\sqrt{\frac{x^{2} \cdot \sin ^{2} v}{a_{11} a_{22}}}$, wobei $w$ den Coordinatenwinkel bedeutet. Die quadratische Gleichung (51), S. 98, auf uuseren Fall angewandt, liefert $\frac{a_{11} a_{22}}{\sin ^{2} w}=\lambda^{\prime} \lambda^{\prime \prime}$; andererseits ist aber nach (2), S. $110 \frac{x^{2}}{\lambda^{\prime} \lambda^{\prime \prime}}=a^{2} b^{2}$, wenn die halben Axen des Kegelschnitts mit $a$ und $b$ bezeichnet werden. Daher wird $r_{1} r_{2} \sin w=a b$, also coustant, und der Inhalt des ganzen Parallelogramms ist $4 a b$.

Aus $r_{1} r_{2} \sin w=a b$ folgt auch noch der Satz:

10\%. Alle Parallelogramme, welche dadurch entstehen, dass man die Endpunkte zweier conjugirten Durchmesser verbindet, haben gleichen Flächeninhalt. Derselbe ist halb so gross wie derjenige des Rechtecks, dessen Seiten die vier Scheiteltangenten der Ellipse sind.

108. Bei jeder Ellipse ist die Summe der Quadrate zweier conjugirten Durchmesser constant, bei jeder Hyperbel deren Differenz.

Wird in ähnlicher Weise bewiesen wie (106), nur sind dabei noch anzuwenden die Relationen $a^{2}=-\frac{x}{\lambda^{\prime}}, b^{2}=\mp \frac{x}{\lambda^{\prime \prime}}(-$ oder + , je nachdem Ellipse oder Hyperbel, vgl. S. 110), sowie die aus (51), S. 98 folgende Relation $\frac{a_{11}+a_{22}}{\sin ^{2}}=\lambda^{\prime}+\lambda^{\prime \prime}$. Auch hat man zu beachten, dass bei der Hyperbel zufolge $F(p, p)<0$ (was in (106) gleichbedeutend mit $\left.a_{11} a_{22}<0\right)$ die Grössen $\lambda^{\prime}$ und $\lambda^{\prime \prime}$, sowie $r_{1}{ }^{2}=-\frac{x}{a_{11}}$ und $-r_{2}{ }^{2}=-\frac{x}{a_{22}}$ ungleiche Vorzeichen haben; man findet $r_{1}{ }^{2} \pm r_{2}{ }^{2}=a^{2} \pm b^{2}$, wobei das positive oder negative Vorzeichen zu stehen hat, je nachdem der Kegelschnitt eine Ellipse oder Hyperbel ist.

109. Diejenigen zwei conjugirten Durchmesser einer Ellipse, welche den grössten Winkel einschliessen, sind einander gleich.

Nach (106) ist $r_{1} r_{2} \sin w=a b$, und hier wird der stumpfe Nebenwinkel $w$ ein Maximum, wenn $\sin w$ ein Minimum, d. l. wenn $r_{1} r_{2}$ oder auch $r_{1}{ }^{2} r_{2}{ }^{2}$ ein Maximum ist. Da nach (108) $r_{1}{ }^{2}+r_{2}{ }^{2}=a^{2}+b^{2}$, so tritt dies ein für $r_{1}^{2}=r_{2}^{2}$; ferner folgt alsdann bei der Ellipse $r_{1}{ }^{2}=\frac{a^{2}+b^{2}}{2}$ und $\sin w=\frac{2 a b}{a^{2}+b^{2}}$, daher $\operatorname{tg} \frac{w}{2}= \pm \frac{b}{a}$. Bei der Hyperbel können im allgemeinen keine gleichen conjugirten 
Durchmesser auftreten, da dort die Differenz $r_{1}{ }^{2}-r_{2}{ }^{2}$ gleich ist der Differenz der Quadrate der halben Axen; nur bei der gleichseitigen Hyperbel sind die conjugirten Durchmesser einander gleich.

110. Zieht man irgend zwei zu einander senkrechte Halbmesser eines Kegelschnitts, so ist bei der Ellipse die Summe, bei der Hyperbel die Differenz der Quadrate der reciproken Werthe für die Lüngen dieser Halbmesser constant.

Bezogen auf irgend ein rechtwinkliges Coordinatensystem, dessen Anfang in Mittelpunkte der Curve liege, ist die Gleichung der Curve von der Form $a_{11} x^{2}+2 a_{12} x y+a_{22} y^{2}+x=0$. Die Quadrate der Abschnitte auf den Coordinatenaxen sind $x^{2}=-\frac{x}{u_{11}}, y^{2}=-\frac{x}{a_{22}}$, daher $\frac{1}{x^{2}}+\frac{1}{y^{2}}=-\frac{a_{11}+a_{22}}{x}=-\frac{\lambda^{\prime}+\lambda^{\prime \prime}}{x}=\frac{1}{a^{2}} \pm \frac{1}{b^{2}}$ (nach (51), S. 98 und nach S. 110). Bezieht man dieselbe Curve auf ein anderes rechtwinkliges System, dessen Anfang wiederum in deren Mittelpunkte liegt, so wird ihre Gleichung etwa $b_{11} x^{2}+2 b_{12} x y+b_{22} y^{2}+x^{\prime}=0$. Während jetzt die Quadrate der Axenabschnitte natürlich andere Werthe haben wie zuvor, wird die Summe der reciproken Werthe gleich $-\frac{b_{11}+b_{28}}{x^{\prime}}$ oder (analog wie oben) gleich $\frac{1}{a^{2}} \pm \frac{1}{b^{2}}$, daher in der That constant.

111. Sind $d_{1}, d_{2}, d_{3}$ die Abstände des Mittelpunktes eines Kegelschnitts von den Seiten eines Poldreiecks mit den Höhen $h_{1}, h_{2}, h_{3}$, so besteht die Relation $\frac{1}{h_{1} d_{1}}+\frac{1}{h_{2} d_{9}}+\frac{1}{h_{3} d_{3}}=-\left(\frac{1}{a^{2}}+\frac{1}{b^{2}}\right)$.

Die Gleichung der Curve $a_{11} x_{1}{ }^{2}+a_{22} x_{2}{ }^{2}+a_{33} x_{3}{ }^{2}=0$ verwandelt sich bei Einführung der Coordinaten $y_{1}: y_{2}: y_{3}=\frac{p_{1}}{a_{11}}: \frac{p_{2}}{a_{28}}: \frac{p_{3}}{a_{33}}$ ihres Mittelpunktes in $\frac{p_{1}}{y_{1}} x_{1}{ }^{2}+\frac{p_{2}}{y_{2}} x_{2}{ }^{2}+\frac{p_{3}}{y_{3}} x_{3}{ }^{2}=0$. Für diese Curve wird nach (11), S. 87

$$
\lambda^{\prime}+\lambda^{\prime \prime}=\frac{p_{1} \omega_{11}}{y_{1}}+\frac{p_{2} \omega_{22}}{y_{2}}+\frac{p_{3} \omega_{33}}{y_{3}}=\frac{1}{e_{1} h_{1} y_{1}}+\frac{1}{e_{2} h_{2} y_{2}}+\frac{1}{e_{3} h_{3} y_{3}} ;
$$

ferner ist $x$ nach (28), S. 91 und (45), S. 31 gleich

so dass man erhält

$$
\frac{A}{F(p, p)}=\frac{1}{p_{1} y_{1}+p_{2} y_{2}+p_{3} y_{3}}
$$

$\frac{\lambda^{\prime}+\lambda^{\prime \prime}}{x}=-\left(\frac{1}{a^{2}}+\frac{1}{b^{2}}\right)=\left(p_{1} y_{1}+p_{2} y_{2}+p_{3} y_{3}\right)\left(\frac{1}{e_{1} h_{1} y_{1}}+\frac{1}{e_{2} h_{8} y_{2}}+\frac{1}{e_{3} h_{3} y_{3}}\right)$. Mit Hilfe von $d_{i}=\frac{e_{i} y_{i}}{p_{1} y_{1}+p_{2} y_{8}+p_{3} y_{3}}$ folgt hieraus die zu beweisende Formel. 
112. Nach (10), S. 49 hat das Parallelogramm, welches entsteht, wenn man durch einen beliebigen Punkt $P$ der Hyperbel Parallelen zu den zwei Asymptoten zieht, constanten Inhalt. Man bestimme den Werth dieser Constante.

Für $g_{x} h_{x}=0$ als Gleichung der beiden Asymptoten ist $f(x, x)=0$ nach (26), S. 45 von der Form $g_{x} h_{x}+\frac{A}{F(p, p)} p_{x}{ }^{2}$. Es seien nun $X_{1}$, $X_{2}$ die auf den Asymptoten $g_{x}=0, h_{x}=0$ durch die Parallelen abgeschnittenen Stücke, $g$ und $h$ die Entfernungen des Punktes $\boldsymbol{P}$ von den beiden Asymptoten. Alsdann ist $g=X_{2} \sin \alpha, h=X_{1} \sin \alpha$, wobei $\alpha$ den Winkel der Asymptoten bezeichnet, für welchen nach (6), S. $111 \sin ^{2} \alpha=\frac{-4 \tau F(p, p)}{[a, \omega]^{2}-4 \tau F(p, p)}$. Mit Hilfe der in (59) aufgestellten Gleichung ergibt sich nun

$$
X_{1} X_{2}=\frac{A\left\{[a, \omega]^{2}-4 \tau F(p, p)\right\}}{\sqrt{\omega(g, g)} \sqrt{\omega(h, h)} F(p, p) \cdot 4 \tau F(p, p)} .
$$

Ferner sind die für $f(x, x) \equiv g_{x} h_{x}+\frac{A}{F(p, p)} p_{x}{ }^{2}$ gebildeten Ausdrücke $[a, \omega]$ und $F(p, p)$, wie leicht zu erkennen, dieselben wie die für $g_{x} h_{x}$ gebildeten, und zwar findet man $[a, \omega]^{2}-4 \tau F(p, p)=\omega(g, g) \cdot \omega(h, h)$, so dass sich der Ausdruck für $X_{1} X_{2}$ verwandelt in

$$
X_{1} X_{2}=\frac{A \sqrt{[a, \omega]^{2}-4 \tau F(p, p)}}{4 \tau F^{2}(p, p)} .
$$

Es seien nun $\lambda^{\prime}$ und $\lambda^{\prime \prime}$ die Wurzeln der Gleichung

$$
\lambda^{2}-[a, \omega] \lambda+\tau F(p, p)=0
$$

((11), S. 87), daher $\sqrt{[a, \omega]^{2}-4 \tau F(p, p)}=\lambda^{\prime}-\lambda^{\prime \prime} ;$ nach (2) S. 110 ist alsdann $a^{2}=-\frac{x}{\lambda^{\prime}}, b^{2}=-\frac{x}{\lambda^{\prime \prime}}$, wo $x=\frac{A}{F(p, p)}$ und $a^{2}, b^{2}$ die Quadrate der halben Axen bezeichnen. Durch Einführung dieser Grössen erhält man $X_{1} X_{2}=\frac{x\left(\lambda^{\prime}-\lambda^{\prime \prime}\right)}{4 \lambda^{\prime} \lambda^{\prime \prime}}=\frac{a^{2}+b^{2}}{4}$, da speciell bei der Hyperbel $b^{2}$ zu ersetzen ist durch $-b^{2}$. Die oben erwähnten Parallelogramme haben demnach den gemeinsamen Inhalt $X_{1} X_{2} \sin \alpha=\frac{a^{2}+b^{2}}{4} \sin \alpha$.

113. Der Winkel $\alpha$ des Asymptotenpaares einer auf schiefwinklige Parallelcoordinaten mit dem Axenwinkel $w$ bezogenen Curve zweiter Ordnung $f(x, x)=0$ ist gegeben durch

$$
\operatorname{tg}^{2} \alpha=\frac{-4\left(a_{11} a_{92}-a_{12}^{2}\right) \sin ^{2} w}{\left(a_{11}+a_{92}-2 a_{12} \cos w\right)^{2}} .
$$


Asymptotenwinkel. Quadrate der Gleichungen für die Hauptaxen. 285

Folgt sofort aus (6), S. 111 mit Benutzung von (2) S. 10 und $p_{1}=p_{2}=0, p_{3}=1$.

114. Der Winkel $\alpha$ des Asymptotenpaares der Curve zweiter Classe $\varphi(u, u) \equiv \sum_{1}^{8} \sum_{1}^{8} k \alpha_{i k} u_{i} u_{k}=0$ ist allgemein gegeben durch die Formel

$$
\operatorname{tg}^{2} \alpha=-\frac{4 \tau \mathrm{A} \varphi(p, p)}{[\mathrm{A}, \omega]^{2}}
$$

speciell für schiefwinklige Coordinaten erhält man

$$
\operatorname{tg}^{2} \alpha=-\frac{4 \alpha_{33} \sin ^{2} w}{\left(\mathrm{~A}_{11}+\mathrm{A}_{22}-2 \mathrm{~A}_{12} \cos w\right)^{2}} .
$$

Folgt aus (6), S. 111, indem man sich die Gleichung $\varphi(u, u)=0$ zuvor in Punktcoordinaten übertragen denkt.

115. Die Hauptaxen der Curve zweiter Ordnung $f(x, x)=0$ mit einem im Endlichen gelegenen Mittelpunkte sind doppelt zählend gegegeben durch

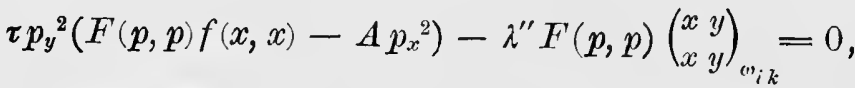

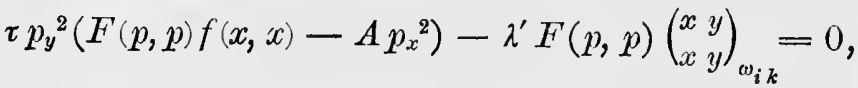

wobei durch $y_{1}: y_{2}: y_{3}=F^{\prime}\left(p_{1}\right): F^{\prime}\left(p_{2}\right): F^{\prime}\left(p_{3}\right)$ die Coordinaten des Mittelpunktes definirt sind und $\lambda^{\prime}, \lambda^{\prime \prime}$ die Wurzeln der Gleichung $\lambda^{2}-[a, \omega] \lambda+\tau F(p, p)=0$ bedeuten $\left.{ }^{1}\right)$.

Aus $\boldsymbol{\omega}(u, u)=U_{1}^{2}+U_{2}^{2}$ in (21), S. 90 folgt

$r^{2}\left|\begin{array}{ccccc}\omega_{11} & \omega_{12} & \omega_{13} & x_{1} & y_{1} \\ \omega_{21} & \omega_{22} & \omega_{23} & x_{2} & y_{2} \\ \omega_{31} & \omega_{32} & \omega_{33} & x_{3} & y_{3} \\ x_{1} & x_{2} & x_{3} & 0 & 0 \\ y_{1} & y_{2} & y_{3} & 0 & 0\end{array}\right|=\left|\begin{array}{ccccc}1 & 0 & 0 & X_{1} & 0 \\ 0 & 1 & 0 & X_{2} & 0 \\ 0 & 0 & 0 & X_{3} & Y_{3} \\ X_{1} & X_{2} & X_{3} & 0 & 0 \\ 0 & 0 & Y_{3} & 0 & 0\end{array}\right|=Y_{3}{ }^{2}\left(X_{1}{ }^{2}+X_{2}{ }^{2}\right)$

ferner ist nach (29), S. $91 f(x, x)-x X_{3}{ }^{2}=\lambda^{\prime} X_{1}{ }^{2}+\lambda^{\prime \prime} X_{2}{ }^{2}$. Mit Benutzung von $X_{3}=p_{x}, Y_{3}=p_{y}, x=A: F(p, p), r^{2}=1: \tau$ erhält man

$$
\begin{aligned}
& \tau F(p, p) p_{y}{ }^{2}\left(\lambda^{\prime}-\lambda^{\prime \prime}\right) X_{1}{ }^{2} \equiv \tau p_{y}{ }^{2}\left(F(p, p) f(x, x)-A p_{x}{ }^{2}\right) \\
& -\lambda^{\prime \prime} F(p, p)\left(\begin{array}{ll}
x & y \\
x & y
\end{array}\right)_{\omega_{i k}}=0 \text {, }
\end{aligned}
$$

1) Andere Gleichungen zur Bestimmung der Hauptaxen sind (35) und (35a), S. 93, sowie (49), S. 97. 


$$
\begin{aligned}
\tau F(p, p) p_{y}{ }^{2}\left(\lambda^{\prime \prime}-\lambda^{\prime}\right) X_{2}{ }^{2} & \equiv \tau p_{y}{ }^{2}\left(F(p, p) f(x, x)-A p_{x}{ }^{2}\right) \\
& -\lambda^{\prime} F(p, p)\left(\begin{array}{ll}
x & y \\
x & y
\end{array}\right)_{\omega_{i k}}=0 .
\end{aligned}
$$

Bei schiefwinkligen Parallelcoordinaten $\left(x_{1}: x_{2}: x_{3}=x: y: 1\right)$ verwandeln sich diese Gleichungen in

$$
\begin{gathered}
A_{33}\left\{A_{33} f(x, y, 1)-A\right\} \\
-\lambda\left\{\left(A_{32}-A_{33} y\right)^{2}+\left(A_{31}-A_{33} x\right)^{2}-2\left(A_{33} y-A_{32}\right)\left(A_{31}-A_{33} x\right) \cos w\right\}=0,
\end{gathered}
$$

wobei für $\lambda$ die eine oder andere Wurzel zu setzen ist der Gleichung

$$
\lambda^{2} \sin ^{2} w-\lambda\left(a_{11}+a_{22}-2 a_{12} \cos w\right)+a_{11} a_{22}-a_{12}{ }^{2}=0
$$

und $f(x, y, 1)=0$ die gegebene Curve darstellt.

116. Man bilde die Gleichungen für die Hauptaxen der auf rechtwinklige Coordinaten bezogenen Curve zweiter Ordnung

$$
11 x^{2}+14 y^{2}-4 x y-10 x-20 y+10=0 .
$$

Die quadratische Gleichung $\lambda^{2}-[a, \omega] \lambda+\tau F(p, p)=0$ wird hier $\lambda^{2}-25 \lambda+150=0$ und hat die Wurzeln $\lambda^{\prime}=15, \lambda^{\prime \prime}=10$; ferner findet $\operatorname{man} A=-150, A_{31}=90, A_{32}=120, A_{33}=150$. Die Wurzel $\lambda^{\prime}=15$ liefert alsdann nach (115) die Gleichung

$$
\begin{array}{r}
150\left\{150\left(11 x^{2}+14 y^{2}-4 x y-10 x-20 y+10\right)+150\right\} \\
-15\left\{(120-150 y)^{2}+(90-150 x)^{2}\right\}=0,
\end{array}
$$

welche gleichbedeutend ist mit $4 x^{2}+y^{2}+4 x y-8 x-4 y+4=0$ oder mit $(2 x+y-2)^{2}=0$; die Gleichung der einen Axe ist daher $2 x+y-2=0$. Die Wurzel $\lambda^{\prime}=10$ liefert eine ganz ähnliche Gleichung, welche gleichbedeutend ist mit

$$
x^{2}+4 y^{2}-4 x y+2 x-4 y+1=0
$$

oder mit $(x-2 y+1)^{2}=0$, die Gleichung der anderen Axe ist daher $x-2 y+1=0$.

11\%. Die Hauptaxen der Curve zweiter Classe $\varphi(u, u)=0$ mit einem im Endlichen gelegenen Mittelpunkte sind doppelt zählend gegeben durch

$$
\begin{gathered}
\tau p_{y}{ }^{2}\left(\varphi(p, p) . \Phi(x, x)-\mathrm{A} p_{x}^{2}\right)-\lambda^{\prime \prime} \varphi(p, p)\left(\begin{array}{ll}
x & y \\
x & y
\end{array}\right)_{\omega_{i k}}=0 \\
\tau p_{y}{ }^{2}\left(\varphi(p, p) . \Phi(x, x)-\mathrm{A} p_{x}^{2}\right)-\lambda^{\prime} \varphi(p, p)\left(\begin{array}{ll}
x & y \\
x & y
\end{array}\right)_{\omega_{i k}}=0
\end{gathered}
$$

wobei durch $y_{1}: y_{2}: y_{3}=\varphi^{\prime}\left(p_{1}\right): \varphi^{\prime}\left(p_{2}\right): \varphi^{\prime}\left(p_{3}\right)$ die Coordinaten des Mittelpunktes definirt sind und $\lambda^{\prime}, \lambda^{\prime \prime}$ die Wurzeln der Gleichung $\lambda^{2}-[\mathrm{A}, \omega] \lambda^{2}+\tau \mathrm{A} \varphi(p, p)=0$ bedeuten. 
Folgt aus (115), indem man sich die Gleichung der Curve zweiter Classe $\varphi(u, u)=0$ in die Gleichung in Punktcoordinaten $\Phi(x, x)=0$ übertragen denkt und dann die dort angegebenen Formeln anwendet.

Bei schiefwinkligen Parallelcoordinaten $\left(x_{1}: x_{2}: x_{3}=x: y: 1\right)$ verwandeln sich die obigen Gleichungen in:

$$
\alpha_{33}\left(\alpha_{33} \Phi(x, y, 1)-\mathrm{A}\right)
$$

$-\lambda\left\{\left(\alpha_{32}-\alpha_{33} y\right)^{2}+\left(\alpha_{31}-\alpha_{33} x\right)^{2}-2\left(\alpha_{33} y-\alpha_{32}\right)\left(\alpha_{31}-\alpha_{33} x\right) \cos w\right\}=0$, wobei für $\lambda$ die eine oder andere Wurzel zu setzen ist der Gleichung

$$
\lambda^{2} \sin ^{2} w-\lambda\left(\mathrm{A}_{11}+\mathrm{A}_{22}-2 \mathrm{~A}_{12} \cos v\right)+\mathrm{A} \alpha_{33}=0 .
$$

118. Das Product der Gleichungen für die beiden Hauptaxen der Curve zweiter Ordnung $f(x, x)=0$ mit einem im Endlichen gelegenen Mittelpunkte ist bei schiefwinkligen Parallelcoordinaten $\left(x_{1}: x_{2}: x_{3}=x: y: 1\right)$ gegeben durch:

$$
\left|\begin{array}{ccc}
a_{11} x+a_{12} y+a_{13}-\left(a_{21} x+a_{22} y+a_{23}\right) \cos v & x & A_{31} \\
a_{21} x+a_{22} y+a_{23}-\left(a_{11} x+a_{12} y+a_{13}\right) \cos v & y & A_{32} \\
0 & 1 & A_{33}
\end{array}\right|=0 .
$$

Folgt aus (49), S. 97.

Ebenso folgt:

119. Das Product der Gleichungen für die beiden Hauptaxen der Curve zweiter Classe $\varphi(u, u)=0$ mit einem im Endlichen gelegenen Mittelpunkte ist bei schiefwinkligen Parallelcoordinaten gegeben durch

$$
\left|\begin{array}{ccc}
\mathrm{A}_{11} x+\mathrm{A}_{12} y+\mathrm{A}_{13}-\left(\mathrm{A}_{21} x+\mathrm{A}_{22} y+\mathrm{A}_{23}\right) \cos w & x & \alpha_{31} \\
\mathrm{~A}_{21} x+\mathrm{A}_{22} y+\mathrm{A}_{23}-\left(\mathrm{A}_{11} x+\mathrm{A}_{12} y+\mathrm{A}_{13}\right) \cos w & y & \alpha_{32} \\
0 & 1 & \alpha_{33}
\end{array}\right|=0 .
$$

120. Die unendlich fernen Punkte der Hauptaxen des Kegelschnitts $f(x, x)=0$ sind doppelt zählend gegeben durch

$$
\tau\left(\begin{array}{ll}
p & u \\
p & u
\end{array}\right)_{a_{i k}}-\lambda^{\prime} \omega(u, u)=0 \text {, bezw. } \quad \tau\left(\begin{array}{ll}
p & u \\
p & u
\end{array}\right)_{a_{i k}}-\lambda^{\prime \prime} \omega(u, u)=0,
$$

wobei $\lambda^{\prime}$ und $\lambda^{\prime \prime}$ die Wurzeln sind der quadratischen Gleichung $\left.\lambda^{2}-[a, \omega] \lambda+\tau F(p, p)=0 .{ }^{1}\right)$

Nach (29) und (21) in $\S 10$ ist

$$
r^{2}\left(\lambda^{\prime \prime} x U_{1}^{2}+\lambda^{\prime} x U_{2}^{2}+\lambda^{\prime} \lambda^{\prime \prime} U_{3}^{2}\right)=F(u, u), \quad U_{1}{ }^{2}+U_{2}^{2}=\omega(u, u) ;
$$

1) Eine andere Methode zur Bestimmung der unendlich fernen Punkte de: Hauptaxen wurde S. 93 gegeben. 
mit Benutzung von $x=\frac{A}{\boldsymbol{F}(p, p)}, r^{2}=\frac{1}{\tau}, U_{3}=\frac{F(p, u)}{F(p, p)}, \lambda^{\prime} \lambda^{\prime \prime}=\tau F(p, p)$, sowie der Relation $\left(\begin{array}{l}p \\ p\end{array}\right)\left(\begin{array}{l}u \\ u\end{array}\right)-\left(\begin{array}{l}p \\ u\end{array}\right)^{2}=A\left(\begin{array}{ll}p & u \\ p & u\end{array}\right)$ folgt

$$
\begin{aligned}
& \left(\lambda^{\prime \prime}-\lambda^{\prime}\right) U_{1}^{2}=\tau\left(\begin{array}{ll}
p & u \\
p & u
\end{array}\right)_{a_{i k}}-\lambda^{\prime} \omega(u, u), \\
& \left(\lambda^{\prime}-\lambda^{\prime \prime}\right) U_{2}^{2}=\tau\left(\begin{array}{ll}
p & u \\
p & u
\end{array}\right)_{a_{i k}}-\lambda^{\prime \prime} \omega(u, u) .
\end{aligned}
$$

Speciell für schiefwinklige Parallelcoordinaten

erhält man

$$
u_{1}: u_{2}: u_{3}=u: v: 1
$$

$$
\begin{aligned}
& a_{11} v^{2}+a_{22} u^{2}-2 a_{12} u v-\lambda^{\prime}\left(u^{2}+v^{2}-2 u v \cos w\right)=0 \quad \text { und } \\
& a_{11} v^{2}+a_{22} u^{2}-2 a_{12} u v-\lambda^{\prime \prime}\left(u^{2}+v^{2}-2 u v \cos w\right)=0,
\end{aligned}
$$

wobei $\lambda^{\prime}$ und $\lambda^{\prime \prime}$ die Wurzeln sind der quadratischen Gleichung

$$
\lambda^{2} \sin ^{2} w-\lambda\left(a_{11}+a_{22}-2 a_{12} \cos w\right)+a_{11} a_{22}-a_{12}{ }^{2}=0 .
$$

Im Falle der Parabel ist nach S. $100 \mathrm{f}$. die eine Wurzel (etwa $\lambda^{\prime \prime}$ ) gleich Null, die andere gleich $[a, \omega]$; die eben abgeleiteten Gleichungen lauten alsdann $\tau\left(\begin{array}{ll}p & u \\ p & u\end{array}\right)_{a_{i k}}-[a, \omega] \omega(u, u)=0$, bezw. $\left(\begin{array}{ll}p & u \\ p & u\end{array}\right)_{a_{i k}}=0$ und repräsentiren nach (63) und (64), S. 103 den unendlich fernen Punkt der Scheiteltangente, bezw. der Axe.

Bei schiefwinkligen Parallelcoordinaten erhält man in Falle der Parabel für den umendlich fernen Punkt der Scheiteltangente (doppelt zählend) die Gleichung

$$
\begin{aligned}
\left(a_{11}+a_{22} \cos ^{2} w\right. & \left.-2 a_{12} \cos w\right) u^{2}+\left(a_{22}+a_{11} \cos ^{2} w-2 a_{18} \cos w\right) v^{2} \\
& -2\left(a_{11} \cos w+a_{22} \cos w-a_{12}-a_{12} \cos ^{2} w\right) u v=0
\end{aligned}
$$

für den unendlich fernen Punkt der Axe (doppelt zählend):

$$
a_{11} v^{2}+a_{22} u^{2}-2 a_{12} u v=0 \text {. }
$$

121. Das Product der Gleichungen für die unendlich fernen Punkte der Hauptaxen des Kegelschnitts $f(x, y, 1)=0$ mit einem im Endlichen gelegenen Mittelpunkte ist bei schiefwinkligen Parallelcoordinaten gegeben durch:

$$
\left(a_{12}-a_{22} \cos w\right) u^{2}+\left(a_{11} \cos w-a_{12}\right) v^{2}+\left(a_{22}-a_{11}\right) u v=0 .
$$

Folgt aus (46), S. 96. Es ist übrigens klar, dass mit den unendlich fernen Punkten auch die Richtungen der Hauptaxen gegeben sind, also die Winkel, unter denen diese Richtungen gegen die Coordinatenaxen geneigt sind. 
122. Die Axe der auf schiefwinklige Parallelcoordinaten

$$
\left(x_{1}: x_{2}: x_{3}=x: y: 1\right)
$$

mit dem Axenwinkel $w$ bezogenen Parabel $\left.{ }^{1}\right) f(x, y, 1)=0$ ist gegeben durch jede der folgenden zwei Gleichungen

$$
\begin{gathered}
\left(a_{11}-a_{12} \cos v\right) x+\left(a_{12}-a_{22} \cos w\right) y \\
+\frac{\left(a_{11}+a_{22}-2 a_{12} \cos w\right)\left(a_{13}-a_{23} \cos w\right)+A_{13} \sin ^{2} w}{a_{11}+a_{22}-2 a_{12} \cos w}=0, \\
+\frac{\left(a_{12}-a_{11} \cos w\right) x+\left(a_{22}-a_{12} \cos w\right) y}{\left.a_{22}-2 a_{12} \cos w\right)\left(a_{23}-a_{13} \cos w\right)+A_{23} \sin ^{2} w}=0 .
\end{gathered}
$$

Speciell für rechtwinklige Coordinaten erhält man

oder auch (falls z. B. $a_{11}=a_{12}=0$ ):

$$
a_{11} x+a_{12} y+\frac{a_{11} a_{13}+a_{12} a_{23}}{a_{11}+a_{22}}=0
$$

$$
a_{12} x+a_{22} y+\frac{a_{12} a_{13}+a_{22} a_{23}}{a_{11}+a_{22}}=0 .
$$

Die obigen Formeln folgen beide aus (67), S. 103 und $\mathrm{zwar}$ die erste durch die Substitution $u_{2}=u_{3}=0, u_{1}=1$, die zweite durch $u_{1}=u_{3}=0, u_{2}=1$; selbstverständlich hat man auch die S. 8 angegebenen Relationen bei schiefwinkligen Parallelcoordinaten zu benutzen. Man kann beiden Formeln auch folgende Gestalt geben:

$$
\begin{gathered}
\left(a_{11}-a_{12} \cos w\right) x+\left(a_{12}-a_{22} \cos w\right) y \\
+\frac{\left(a_{11}-a_{12} \cos w\right)\left(a_{13}-a_{23} \cos w\right)+\left(a_{12}-a_{22} \cos w\right)\left(a_{23}-a_{13} \cos w\right)}{a_{11}+a_{22}-2 a_{12} \cos w}=0, \\
\left.+\frac{\left(a_{12}-a_{11} \cos w\right) x+\left(a_{22}-a_{12} \cos w\right) y}{a_{11}+a_{22}-2 a_{12} \cos w} \cos w\right)\left(a_{13}-a_{23} \cos w\right)+\left(a_{22}-a_{12} \cos w\right)\left(a_{23}-a_{13} \cos w\right) \\
a_{11}=0 .
\end{gathered}
$$

Auf Grund der Relation $a_{11} a_{22}-a_{12}{ }^{2}=0$ lassen sich alle die hier angegebenen Gleichungen für die Axe in einander überführen.

123. Die Gleichung der Scheiteltangente der auf schiefwinklige Parallelcoordinaten $\left(x_{1}: x_{2}: x_{3}=x: y: 1\right)$ mit dem Axenwinkel $w$ bezogenen Parabel $\left.{ }^{1}\right) f(x, y, 1)=0$ lautet:

$$
\begin{gathered}
\left(A_{13}+A_{23} \cos v\right) x+\left(A_{23}+A_{13} \cos w\right) y \\
+\frac{A \sin ^{2} w-\left(a_{11}+a_{22}-2 a_{12} \cos w\right)\left(A_{11}+A_{22}+2 A_{12} \cos w\right)}{2\left(a_{11}+a_{22}-2 a_{12} \cos w\right)}=0 .
\end{gathered}
$$

Ergibt sich aus der Gleichung (69a), S. 105 für das Product aus der Scheiteltangente in die unendlich ferne Gerade, indem die Glieder

1) Die Gleichung der Directrix wird in (242)-(244) gegeben. 
mit $x_{1}^{2}, x_{2}^{2}, x_{1} x_{2}$ nunmehr wegfallen, so dass sich $x_{3}$ ausscheiden lässt. Speciell für rechtwinklige Coordinaten erhält man

$$
A_{13} x+A_{23} y+\frac{A-\left(a_{11}+a_{22}\right)\left(A_{11}+A_{22}\right)}{2\left(a_{11}+a_{22}\right)}=0
$$

124. Bei schiefwinkligen Parallelcoordinaten ist der Parameter $2 p$ der Parabel $f(x, y, 1)=0$ gegeben durch

$$
2 p=\frac{2 \sqrt{-A} \sin ^{2} w}{\sqrt{\left(a_{11}+a_{22}-2 a_{12} \cos w\right)^{3}}} .
$$

Folgt aus der in $\S 10$ und $\S 11$ erwähnten allgemeinen Formel $2 p=\frac{2 \varrho}{\lambda^{\prime}}, \varrho^{2}=-\frac{A \tau}{\lambda^{\prime}}$ für den Parameter der Parabel.

125. Der Parameter der Parabel in Liniencoordinaten $\varphi(u, u)=0$ ist allgemein gegeben durch $2 p=\frac{2 \mathrm{~A}}{[\mathrm{~A}, \omega]} \sqrt{\frac{-\tau}{[\mathrm{A}, \omega]}}$.

Ergibt sich aus der Formel für den Parameter der Parabel bei Punktcoordinaten, wenn man sich zuvor $\varphi(u, u)=0$ in Punktcoordinaten übertragen denkt.

Speciell für schiefwinklige Parallelcoordinaten findet man

$$
\frac{2 A \sin ^{2} w}{\sqrt{-\left(A_{11}+A_{22}-2 A_{12} \cos w\right)^{3}}} \text {. }
$$

126. Die zwei Brennpunktepaare der auf schiefwinklige Parallelcoordinaten bezogenen Curve zweiter Ordnung $f(x, y, 1)=0$ sind gegeben durch die Gleichungen:

$$
\begin{aligned}
& \sin ^{2} w \cdot \lambda^{\prime} F(u, v, 1)-A\left(u^{2}+v^{2}-2 u v \cdot \cos w\right)=0, \\
& \sin ^{2} w \cdot \lambda^{\prime \prime} F(u, v, 1)-A\left(u^{2}+v^{2}-2 u v \cdot \cos w\right)=0,
\end{aligned}
$$

wobei $\lambda^{\prime}, \lambda^{\prime \prime}$ die Wurzeln sind der quadratischen Gleichung

$$
\lambda^{2} \sin ^{2} w-\lambda\left(a_{11}+a_{22}-2 a_{12} \cos w\right)+a_{11} a_{22}-a_{12}^{2}=0 .
$$

Folgt sofort aus (14), S. 113.

Speciell im Falle der Parabel erhält man für das Product der Gleichungen des im Endlichen und des im Unendlichen gelegenen Brennpunktes den Ausdruck

$$
\left(a_{11}+a_{22}-2 a_{12} \cos w\right) F(u, v, 1)-A\left(u^{2}+v^{2}-2 u v \cdot \cos v\right)=0 \text {. }
$$

und

127. Die Bedingung dafür, dass zwei Kegelschnitte

$$
f(x, x) \equiv \sum_{1}^{3} \sum_{1}^{3} a_{i k} x_{i} x_{k}=0
$$




$$
g(x, x)=\sum_{1}^{3} \sum_{1}^{3} k b_{i k} x_{i} x_{k}=0
$$

einander ihnlich seien, ist $\frac{F(p, p)}{[a, \omega]^{2}}=\frac{G(p, p)}{[b, \omega]^{2}}$.

Nach S. 112 muss das Verhältniss $\lambda^{\prime}: \lambda^{\prime \prime}$ oder, was auf dasselbe hinauskommt, der Winkel der beiden Asymptoten derselbe sein; mit Anwendung von (6), S. 111 ergibt sich die obige Bedingung.

Speciell für schiefwinklige Parallelcoordinaten erhält man

$$
A_{33}\left(b_{11}+b_{22}-2 b_{12} \cos w\right)^{2}=B_{33}\left(a_{11}+c_{22}-2 a_{12} \cos w\right)^{2} \text {. }
$$

128. Aehnliche Kegelschnitte haben gleiche numerische Excentricität.

Folgt daraus, dass die Verhältnisse der Längen der Hauptaxen einander gleich sind.

129. Zwei Kegelschnitte sollen ähnlich liegend genannt werden, wenn ihre Hauptaxen gleiche Richtung haben und wenn die aus einem unendlich fernen Punkte an beide Curven gezogenen Tangenten gleichzeitig reell oder imaginär sind. Wann sind diese Bedingungen erfüllt?

Die nnendlich fermen Punkte der Hauptaxen müssen für die zwei Kegelschnitte $f(x, x)=0$ und $\fallingdotseq(x, x)=0$ dieselben sein; sie sind für $f(x, x)=0$ nach (46), S. 96 gegeben durch $\sum \pm\left(\frac{\partial \psi}{\partial x_{1}} p_{2} u_{3}\right)=0$, also durch eine Gleichung von der Form $\sum_{i}^{3} \sum_{i}^{3} \gamma_{i k} u_{i} u_{k}=0$, während man bei dem anderen Kegelschnitt einen analogen Ausdruck

$$
\sum_{1}^{3} \sum_{1}^{3} k \delta_{i k} u_{i} u_{k}=0
$$

erhält. Die unendlich ferven Punkte der Hauptaxen sind also dicselben, wenn $\frac{\gamma_{11}}{\delta_{11}}=\frac{\gamma_{12}}{\delta_{12}}=\frac{\gamma_{22}}{\delta_{22}}=\frac{\gamma_{13}}{\delta_{13}}=\frac{\gamma_{23}}{\delta_{23}}=\frac{\gamma_{33}}{\delta_{33}}$.

Die aus einem unendlich fernen Punkte $y$ an beide Curven gezogenen Tangenten sind nach (52) gleichzeitig reell oder imaginär, wenn $A f(y, y)$ und $B g(y, y)$ gleiche Vorzeichen haben; die $y_{i}$ haben die Gleichung $p_{y}=0$ zu erfüllen.

Speciell bei schiefwinkligen Parallelcoordinaten werden die Bedingungen:

1) $\quad \frac{a_{12}-a_{11} \cos w}{b_{12}-b_{11} \cos w}=\frac{a_{11}-a_{22}}{b_{11}-b_{22}}=\frac{a_{12}-a_{22} \cos w}{b_{12}-b_{22} \cos w}$,

2) $A\left(a_{11} y_{1}^{2}+2 a_{12} y_{1} y_{2}+a_{22} y_{2}^{2}\right)$ und $B\left(b_{11} y_{1}{ }^{2}+2 b_{12} y_{1} y_{2}+b_{22} y_{2}{ }^{2}\right)$ 
müssen gleiche Vorzeichen haben bei völlig willkürlichen Werthen von $y_{1}$ und $y_{2}$, also z. B. $A a_{11}$ und $B b_{11}$ oder auch $A a_{22}$ und $B b_{22}$ müssen je gleiche Vorzeichen haben.

130. Unter welchen Bedingungen sind zwei Kegelschnitte ähnlich und ähnlich liegend?

Vor Allem müssen beide nach (127) und (129) gleichen Asymptotenwinkel und gleichgerichtete Hauptaxen besitzen, d. h. ihre Asymptoten müssen einander parallel sein, oder die Schnittpunkte der beiden Kegelschnitte mit der unendlich fernen Geraden müssen dieselben sein. Ferner ist nach (129) erforderlich, dass die von einem unendlich fernen Punkte an beide Curven gezogenen Tangenten gleichzeitig reell oder imaginär sind.

1) Die Schnittpunkte der unendlich fernen Geraden mit $f(x, x)=0$ und mit $g(x, x)=0$ sind nach (53a), S. 34 dieselben, wenn

$$
\left(\begin{array}{ll}
p & u \\
p & u
\end{array}\right)_{a_{i k}}:\left(\begin{array}{ll}
p & u \\
p & u
\end{array}\right)_{b_{i k}}=\text { const., }
$$

welche Werthe auch die $u_{i}$ haben mögen. 2) Als weitere Bedingung erhält man aus (129): $A f(y, y)$ und $B g(y, y)$ müssen für $y_{i}$ als Coordinaten eines unendlich fernen Punktes gleiche Vorzeichen haben.

Setzt man

$$
\left(\begin{array}{ll}
p & u \\
p & u
\end{array}\right)_{a_{i k}} \equiv \sum_{1}^{3} \sum_{i}^{3} k \mu_{i k} u_{i} u_{k}, \quad\left(\begin{array}{ll}
p & u \\
p & u
\end{array}\right)_{b_{i k}} \equiv \sum_{1}^{3} \sum_{1}^{3} \nu_{i k} u_{i} u_{k},
$$

so wird die Bedingung 1): $\frac{\mu_{11}}{\nu_{11}}=\frac{\mu_{12}}{\nu_{12}}=\frac{\mu_{29}}{\nu_{22}}=\frac{\mu_{13}}{\nu_{13}}=\frac{\mu_{23}}{\nu_{23}}=\frac{\mu_{33}}{v_{33}}$.

Die Bedingung 1) liesse sich auch in folgender Form aussprechen: Ist $f(x, x)=0$ die Gleichung des einen Kegelschnitts, so muss diejenige des anderen von der (für Beweise geometrischer Sätze wichtigen) Form sein $\lambda f(x, x)+p_{x} q_{x}=0$, wo $p_{x}=0$ die unendlich ferne Gerade, $q_{x}=0$ eine zweite Gerade darstellt und $\lambda$ einen von Null verschiedenen Parameter bezeichnet.

Speciell für schiefwinklige Parallelcoordinaten werden die Bedingungen 1): $\frac{a_{11}}{b_{11}}=\frac{a_{12}}{b_{12}}=\frac{a_{92}}{b_{29}}$.

131. Das Product $a^{2} b^{2}$ aus den Quadraten der halben Axen der Ellipse oder Hyperbel $f(x, x)=0$ ist gegeben durch die Formel

$$
a^{2} b^{2}=\frac{A^{2}}{\tau F^{3}(p, p)}
$$


Folgt aus (2), S. 110 bei 'Einführung von $x=A: F(p, p)$, $\lambda^{\prime} \lambda^{\prime \prime}=\tau F(p, p)$. Speciell bei schiefwinkligen Parallelcoordinaten ist $a^{2} b^{2}=\frac{A^{2} \sin ^{2} w}{\left(a_{11} a_{22}-a_{12}^{2}\right)^{3}}$.

Da der Flächeninhalt $E$ einer Ellipse, wie in der Integralrechnung gezeigt wird, gleich $a b \pi$ ist, ergibt sich:

132. Der Inhalt der Ellipse $f(x, x)=0,(F(p, p)>0)$, ist gegeben durch die Formel $E=\frac{A \cdot \pi}{\boldsymbol{F}^{\prime}(p, p) \sqrt{\tau F(p, p)}}$; bei schiefwinkligen Parallelcoordinaten ist $E=\frac{A \pi \sin w}{\sqrt{\left.a_{11} a_{22}-a_{1} 2^{3}\right)^{3}}}$.

133. Für das Product $a^{2} b^{2}$ aus den Quadraten der halbcn Axen der Ellipse oder Hyperbel $\varphi(u, u)=0$ hat man die Relation

$$
a^{2} b^{2}=\frac{\mathrm{A}}{\tau \cdot q^{3}(p, p)}
$$

speciell bei schiefwinkligen Parallelcoordinaten ist $a^{2} b^{2}=\frac{A \sin ^{2} w}{\alpha_{33}{ }^{3}}$.

Folgt aus (131), indem man sich die Gleichung $\varphi(u, u)=0$ in Punktcoordinaten übertragen denkt.

Hieraus ergibt sich wie bei (132):

134. Der Flächeninhalt der Ellipse $\varphi(u, u)=0,(A \varphi(p, p)>0)$, ist gegeben durch die Formel $E=\frac{\sqrt{A} \pi}{\varphi(p, p) \sqrt{\tau \varphi(p, p)}}$; bei schiefwinkligen Parallelcoordinaten ist $E=\frac{\sqrt{A} \pi \sin w}{\alpha_{33} \sqrt{\alpha_{33}}}$.

135. Die Summe, bezw. Differenz der Quadrate der halben Axen der Ellipse, bezw. Hyperbel $f(x, x)=0$ ist:

$$
a^{2} \pm b^{2}=-\frac{A[a, \omega]}{\tau F^{2}(p, p)} \text {. }
$$

Nach S. 110 ist $a^{2} \pm b^{2}=-x\left(\frac{1}{\lambda^{\prime}}+\frac{1}{\lambda^{\prime \prime}}\right)=-\frac{x\left(\lambda^{\prime}+\lambda^{\prime \prime}\right)}{\lambda^{\prime} \lambda^{\prime \prime}} ;$ mit Hilfe von $x=A: F(p, p), \lambda^{\prime}+\lambda^{\prime \prime}=[a, \omega], \lambda^{\prime} \lambda^{\prime \prime}=\tau F(p, p)$ erhält man die gewünschte Formel.

136. Die Summe, bezw. Differenz der Quadrate der halben Axen der Ellipse, bezw. Hyperbel $\varphi(u, u) \equiv \sum_{1}^{3} \sum_{1}^{3} \alpha_{i k} u_{i} u_{k}=0$ ist:

$$
a^{2} \pm b^{2}=-\frac{[\mathrm{A}, \infty]}{\tau \varphi^{2}(p, p)} \text {. }
$$


Folgt aus (135), indem man sich die Gleichung $\varphi(u, u)=0$ in Punktcoordinaten übertragen denkt.

137. Ist $f(x, x)=0$ die Gleichung eines Kreises, so ist der Radius $r$ desselben gegeben durch $r^{2}=\frac{-2 A}{[a, \omega] \cdot F(p, p)}$.

Ergibt sich aus (135), indem man $a=b=r$ setzt und von der nach (44), S. 95 im Falle eines Kreises bestehenden Relation

$$
[a, \omega]^{2}-4 \tau F(p, p)=0
$$

Gebrauch macht. Vgl. auch (55), S. 100.

138. Ist $\varphi(u, u)=0$ die Gleichung eines Kreises, so ist der Radius $r$ desselben gegeben durch $r^{2}=\frac{-2 \mathrm{~A}}{[\mathrm{~A}, \omega] \cdot \varphi(p, p)}$, oder auch durch $r^{2}=\frac{-[\mathrm{A}, \omega]}{2 \tau \varphi^{2}(p, p)}$.

Kann aus (137) abgeleitet werden, indem $\operatorname{man}^{\prime} \varphi(u, u)=0$ in eine Gleichung in Punktcoordinaten $\Phi(x, x)=0$ verwandelt; folgt aber auch aus (136). Die zwei angegebenen Werthe von $r^{2}$ können vermöge der im Falle eines Kreises bestehenden Relation

$$
[A, \omega]^{2}-4 A \tau \varphi(p, p)=0
$$

in einander übergeführt werden.

139. Man bilde die Invarianten $A, F(p, p)$ und $[a, \omega]$ für die Gleichung des dem Coordinatendreieck umschriebenen Kegelschnitts mit dem Mittelpunkte $y$.

Unter Anwendung der Abkürzung $r_{y} \equiv-p_{1} y_{1}+p_{2} y_{2}+p_{3} y_{3}$, $s_{y} \equiv p_{1} y_{1}-p_{2} y_{2}+p_{3} y_{3}, \quad t_{y} \equiv p_{1} y_{1}+p_{2} y_{2}-p_{3} y_{3}$ ist die Gleichung der Curve nach (28): $f(x, x)=2\left(y_{1} r_{y} x_{2} x_{3}+y_{2} s_{y} x_{3} x_{1}+y_{3} t_{y} x_{1} x_{2}\right)=0$, und man findet alsdann

$$
\begin{aligned}
& A=2 y_{1} y_{2} y_{3} r_{y} s_{y} t_{y}, \quad \cdot F(p, p)=p_{y} r_{y} s_{y} t_{y}, \\
& {[a, \omega]=2\left(y_{1} r_{y} \omega_{23}+y_{2} s_{y} \omega_{31}+y_{3} t_{y} \omega_{12}\right) .}
\end{aligned}
$$

140. Die analoge Aufgabe für den dem Coordinatendreiseit eingescliriebenen Kegelschnitt mit dem Mittelpunkte $y$.

Die Gleichung dieses Kegelschnitts ist nach (29)

$$
\varphi(u, u) \equiv 2\left(p_{1} r_{y} u_{2} u_{3}+p_{2} s_{y} u_{3} u_{1}+p_{3} t_{y} u_{1} u_{2}\right)=0 ;
$$

alsdann findet man

$$
\begin{gathered}
\mathrm{A}=2 p_{1} p_{2} p_{3} r_{y} s_{y} t_{y}, \quad \varphi(p, p)=2 p_{1} p_{2} p_{3} p_{y} \\
{[\mathrm{~A}, \omega]=-p_{1}{ }^{2} \omega_{11} r_{y}{ }^{2}-p_{2}{ }^{2} \omega_{22} s_{y}{ }^{2}-p_{3}{ }^{2} \omega_{33} t_{y}{ }^{2}+2 p_{1} p_{2} \omega_{12} r_{y} s_{y}} \\
+2 p_{1} p_{3} \omega_{13} r_{y} t_{y}+2 p_{2} p_{3} \omega_{23} s_{y} t_{y}
\end{gathered}
$$


oder auch $4 p_{1} p_{2} p_{3}\left(p_{1} \omega_{23} y_{1}^{2}+p_{2} \omega_{31} y_{2}^{2}+p_{3} \omega_{12} y_{3}^{2}\right)$, wenn die Grössen $\omega_{11}, \omega_{22}, \omega_{33}$ mit Hilfe der Relationen $\dot{\omega}^{\prime}\left(p_{i}\right)=0$ eliminirt werden.

141. Die analoge Aufgabe für diejenige Curve zweiter Ordnung, die das Coordinatendreieck zum Poldreieck hat.

Die Gleichung des Kegelschnitts ist nach (30)

$$
p_{1} y_{2} y_{3} x_{1}^{2}+p_{2} y_{3} y_{1} x_{2}{ }^{2}+p_{3} y_{1} y_{2} x_{3}{ }^{2}=0
$$

alsdiann wird $A=p_{1} p_{2} p_{3} y_{1}{ }^{2} y_{2}{ }^{2} y_{3}{ }^{2}, F(p, p)=p_{1} p_{2} p_{3} y_{1} y_{2} y_{3} p_{y}$,

$$
[a, \omega]=\omega_{11} p_{1} y_{2} y_{3}+\omega_{22} p_{2} y_{3} y_{1}+\omega_{33} p_{3} y_{1} y_{2} \text {. }
$$

142. Die analoge Aufgabe für diejenige Curve zweiter Classe, die das Coordinatendreiseit zum Poldreiseit hat.

Die Gleichung des Kegelschnitts ist nach (31)

$$
p_{2} p_{3} y_{1} u_{1}{ }^{2}+p_{3} p_{1} y_{2} u_{2}{ }^{2}+p_{1} p_{2} y_{3} u_{3}{ }^{2}=0 ;
$$

daher findet man $\mathrm{A}=p_{1}{ }^{2} p_{2}{ }^{2} p_{3}{ }^{2} y_{1} y_{2} y_{3}, \varphi(p, p)=p_{1} p_{2} p_{3} p_{y}$,

$$
[\mathrm{A}, \omega]=p_{1} p_{2} p_{3}\left(\omega_{11} p_{1} y_{2} y_{3}+\omega_{22} p_{2} y_{3} y_{1}+\omega_{33} p_{3} y_{1} y_{2}\right)
$$

Man beweise nachstehenden Satz von Steiner ${ }^{1}$ ):

143. Die Mittelpunkte aller einem Dreieck umschriebenen Kegelschnitte, für welche das Product ihrer Halbaxen constant ist, liegen auf einer Curve sechster Ordnung.

Das Product $U$ der halben Axen der Curve $f(x, x)=0$ ist nach (131) gleich $\frac{A}{\sqrt{\tau F^{3}(p, p)}}$; durch Substitution der in (139) gefundenen Werthe von $A$ und $F(p, p)$ erhält man für den Ort der Mittelpunkte die Curve sechster Ordnung

$$
U^{2} \tau p_{y}{ }^{3} r_{y} s_{y} t_{y}-4 y_{1}{ }^{2}{y_{2}}^{2}{y_{3}}^{2}=0 \text {. }
$$

Die Discussion dieser Gleichung möge dem Leser überlassen sein; uur werde bemerkt, dass Steiner in seiner Abhandlung verschiedene Skizzen von dem Verlauf der Curve gibt.

Von Steiner ruhrt auch der folgende Satz her:

1) "Teoremi relativi alle coniche inscritte e circoscritte", Journal für die reine und angewandte Mathematik, Bd. 30, S. 99, 1846, oder Giornale arcadico di Roma, Bd. 99, S. 147-161, oder „Gesammelte Werke“, Bd. 2, S. 331.

Ein Beweis dieses und mehrerer der folgenden Sïtze findet sich auch bei anderen Autoren, besonders aber in einer Abhandlung von Gino Loria: "Studi sulla teoria delle coordinate triangolari e sulla geometria analitica di un piano nello spazio", Giornale di Matematiche, hrsgg. von G. Battaglini, Bd. 24, S. 164 $-241,1885$. 
144. Die Mittelpunkte aller einem Dreiseit eingeschriebenen Kegelschnitte, für welche das Product $J$ ihrer Halbaxen constant ist, liegen auf einer Curve dritter Ordnung ${ }^{1}$ ).

Bei gleicher Behandlung dieser Aufgabe wie im vorhergehenden Beispiel erbält man als Gleichung der Curve

$$
4 J^{2} \tau p_{1}^{2} p_{z}{ }^{2} p_{3}{ }^{2} p_{y}{ }^{3}-r_{y} s_{y} t_{y}=0 .
$$

145. Die Mittelpunkte aller Curven zweiter Ordnung, die ein gegebenes Dreieck zum Poldreieck haben und bei denen das Product der Halbaxen constant ist, liegen auf einer Curve dritter Ordnung.

Wählt man das betr. Dreieck zum Coordinatendreieck, so ist die Gleichung des Kegelschnitts nach (30):

$$
p_{1} y_{2} y_{3} x_{1}{ }^{2}+p_{2} y_{3} y_{1} x_{2}{ }^{2}+p_{3} y_{1} y_{2} x_{3}{ }^{2}=0 \text {. }
$$

Für das Product $P=a b$ der Halbaxen besteht nach (131) die Relation $P^{2}=\frac{A^{2}}{\tau F^{3}(p, p)}$; andrerseits ist nunmehr

$$
A=p_{1} p_{2} p_{3} y_{1}{ }^{2} y_{2}{ }^{2} y_{3}{ }^{2}, F(p, p)=p_{1} p_{2} p_{3} y_{1} y_{2} y_{3}\left(p_{1} y_{1}+p_{2} y_{2}+p_{3} y_{3}\right) \text {, }
$$

daher erhält man als Gleichung der Curve

$$
P^{2} \tau p_{1} p_{2} p_{3}\left(p_{1} y_{1}+p_{2} y_{2}+p_{3} y_{3}\right)^{3}-y_{1} y_{2} y_{3}=0 \text {. }
$$

Aus den drei vorhergehenden Aufgaben ergeben sich sofort die drei folgenden Sätze:

146. Der Inhalt der dem Coordinatendreieck umschriebenen Ellipse mit dem Mittelpunkte $y$ ist $E=\frac{2 y_{1} y_{2} y_{3} \pi}{p_{y} \sqrt{\tau p_{y} r_{y} s_{y} t_{y}}}$.

Denn der Inhalt einer Ellipse ist bekanntlich gleich dem mit $\pi$ multiplicirten Producte der Halbaxen. Vgl. (157).

Ebenso folgt:

147. Der Inhalt der dem Coordinatendreieck eingeschriebenen Ellipse mit dem Mittelpunkte $y$ ist gleich $\frac{\sqrt{r_{y} s_{y} t_{y}}}{2 p_{1} p_{2} p_{3} p_{y} \sqrt{\tau p_{y}}} \pi$.

Endlich folgt:

148. Der Inhalt derjenigen Ellipse, welche das Coordinatendreieck zum Poldreieck und den Punkt $y$ zum Mittelpunkt hat, ist gleich $\frac{\sqrt{y_{1} y_{2} y_{3}}}{p_{y} \sqrt{\tau p_{1} p_{2} p_{3} p_{y}}} \pi$.

1) Steiner a. a. 0 . 
In genau derselben Weise wie bei (143), (144), (145) erhält man unter Anwendung der Relationen (135) und (136) die folgenden drei Sätze:

149. Die Mittelpnnkte allor einem Dreieck (Coordinatendreieck) umschriebenen Kegelschnitte, für welche die Summe oder Differenz (je nachdem die Curve Ellipse oder Hyperbel) der Quadrate der Halbaxen $a^{2} \pm b^{2}$ constant ist, liegen auf der Curve fünfter Ordnung ${ }^{1}$ )

$$
\tau Q p_{y}{ }^{2} r_{y} s_{y} t_{y}+4 y_{1} y_{2} y_{3}\left(y_{1} r_{y} \omega_{23}+y_{2} s_{y} \omega_{31}+y_{3} t_{y} \omega_{12}\right)=0 \text {, }
$$

wobei $Q=a^{2} \pm b^{2}$.

150. Die Mittelpunkte aller dem Coordinatendreieck eingeschriebenen Kegelschnitte, für welche $a^{2} \pm b^{2}=Q$ constant ist, liegen auf dem Kreis $\left.\tau Q p_{1} p_{2} p_{3} p_{y}{ }^{2}+\left(p_{1} \omega_{23} y_{1}{ }^{2}+p_{2} \omega_{31} y_{2}{ }^{2}+p_{3} \omega_{12} y_{3}{ }^{2}\right)=0 .{ }^{2}\right)$

Derselbe ist mit demjenigen Kreis concentrisch, der das Coordinatendreieck zum Poldreieck hat (75); denu die Gleichungen beider unterscheiden sich nur um ein Glied mit $p_{y}{ }^{2}$.

151. Die Mittelpunkte aller Kegelschnitte, die ein gegebenes Dreieck zum Poldreieck haben und bei denen $a^{2} \pm l^{2}=Q$ constant ist, liegen auf dem Kreise

$$
\tau Q p_{1} p_{2} p_{3} p_{y}{ }^{2}+\left(\omega_{11} p_{1} y_{2} y_{3}+\omega_{22} p_{2} y_{3} y_{1}+\omega_{33} p_{3} y_{1} y_{2}\right)=0 .
$$

Derselbe ist mit dem umschriebenen Kreise des Dreiecks (73) concentrisch, denn die Gleichungen beider unterscheiden sich nur um ein Glied mit $p_{y}{ }^{2} \cdot{ }^{3}$ )

152. Man beweise nachstehenden Satz von Steine $\left.\mathrm{r}^{4}\right)$ :

„Unter der unendlichen Menge von Kegelschnitten, welche einem gegebenen Dreieck sich einschreiben lassen, sind nur immer je 6 und 6 einander gleich (congruent); die Mittelpunkte von je 6 gleichen Kegelschnitten liegen in einem Kreise, und alle diese Kreise haben einen ausgezeichneten Punkt des Dreiecks zum gemeinsamen Mittelpunkte."

1) Das Aggregat $y_{1} r_{y} \omega_{23}+y_{2} s_{y} \omega_{31}+y_{3} t_{y} \omega_{12}=0$ in der Gleichung der Curve 5. Ordnung stellt nach (165) den sogenannten Feuerbach'schen Kreis des Dreiecks dar.

2) Vgl. Paul Serret "Géométrie de Direction", Paris 1869, S. $77 \mathrm{f}$. und S. 145, corollaire III.

3) Paul Serret, a. a. O., S. 156, corollaire III.

4) „Lehrsätze und Aufgaben". Journal für die reine und angewandte Mathematik, Bd. 30, S. 273, 1845, oder auch „Gesammelte Werke“, Bd. 2, S. 345. 
Die Mittelpunkte aller eingeschriebenen Kegelschnitte, für welche das Product $a^{2} b^{2}$ aus den Quadraten der Halbaxen constant ist, liegen nümlich nach (144) auf einer Curve dritter Ordnung; die Mittelpunkte der eingeschriebenen Kegelschnitte, für welche $a^{2} \pm b^{2}=$ const., liegen nach (150) auf einem Kreise, der mit demjenigen concentrisch ist, für welchen das Dreieck ein Poldreieck ist. Der Kreis und die Curve dritter Ordnung schneiden sich in 6 Punkten; dieselben sind also Mittelpunkte eingeschriebener Kegelschnitte, für welche $a^{2} b^{2}$, andrerseits $a^{2} \pm b^{2}$ denselben Werth hat; diese Kegelschnitte sind daher congruent. Aus (75) folgt noch, dass der oben genannte „ausgezeichnete Punkt des Dreiecks" der Höhenschnittpunkt ist.

153. Unter der unendlich grossen Anzahl von Kegelschnitten, die sich einem gegebenen Dreieck umschreiben lassen, sind je 6 cinander gleich.

Wir haben hier, ähnlich wie bei der vorhergehenden Aufgabe, auszugehen von den Gleichungen

$$
a^{2} b^{2}=\frac{A^{2}}{\tau F^{3}(p, p)} \text { und } a^{2} \pm b^{2}=-\frac{A \cdot[a, \omega]}{\tau F^{2}(p, p)}
$$

(vgl. (131) und (135)); aus ihnen folgt

$$
\left(a^{2} \pm b^{2}\right) A+a^{2} b^{2} \cdot[a, \omega] F(p, p)=0
$$

sowie $\left(a^{2} \pm b^{2}\right) \tau F(p, p)-a^{2} b^{2} \cdot[a, \omega]^{2}=0$. Die eine dieser beiden abgeleiteten Gleichungen ist in den Coefficienten des dem Coordinatendreieck umschriebenen Kegelschnitts

$$
f(x, x) \equiv 2\left(a_{23} x_{2} x_{3}+a_{31} x_{3} x_{1}+a_{12} x_{1} x_{2}\right)=0
$$

vom dritten, die andere vom zweiten Grade. Aus beiden würde man also für die Verhältnisse der Coefficienten sechs Werthe erhalten.

* 154. Man beweise nachstehenden Steiner'schen Satz ïber Kegelschnitte, von denen der eine einem Dreieck (etwa dem Coordinatendreieck) umschrieben, der andere eingeschrieben ist $^{1}$ ):

„Sollen die beiden Kegelschnitte gleichen Inhalt haben, oder sollen die Producte ihrer Halbaxen gleich sein, so besteht der Ort ihres gemeinsamen Mittelpunktes $p$ aus zwei verschiedenen Curven dritten Grades $P^{3}$ und $P_{1}^{3}$."

„Die eine dieser Curven, $P^{3}$, ist in der Art speciell, dass ihre drei Asymptoten sich in einem Punkte und zwar im Schwerpunkt des Dreiecks schneiden, und dass dieselben zugleich Wendetangenten

1) „Vermischte Sätze und Aufgaben“, Journal für die reine und angewandte Mathematik, Bd. 55, S. 362 f., 1858, oder "Gesammelte Werke“, Bd. 2, S. 669. 
(Wendeasymptoten) und zudem den Seiten des Dreiecks parallel sind. Die drei hyperbelartigen $\mathrm{Z}$ weige der Curve liegen in den drei Räumen über den Seiten des Dreiecks und berühren die respectiven Seiten in ihren Mitten. Für jeden Punkt $p$ in dieser Curve sind die zugehörigen Kegelschnitte Hyperbeln."

"Die andere Curve, $P_{1}{ }^{3}$, besteht aus zwei getrennten Theilen, der eine ist ein sogenanntes Oval und der andere hat drei hyperbelartige Zweige; das Oval liegt innerhalb des Dreiecks und berïhrt dessen Seiten in ihren Mitten; der andere Theil hat die Seiten des dem gegebenen Dreieck parallel umschriebenen Dreiecks zu Asymptoten und seine drei Zweige liegen in den Scheitelwinkeln dieses Dreiecks. Für jeden Punkt $p$ in diesem dreizweigigen Theil sind die Kegelschnitte Ellipsen, dagegen für jeden Punkt des Ovals sind dieselben Hyperbeln."

Man hat die Gleichungen (143) und (144) nach $U^{2}$ und $J^{2}$ aufzulösen; durch Gleichsetzen der beiden Werthe erhält man eine Gleichung, die in das Product der beiden nachstehenden durch das Vorzeichen des Klammerausdrucks sich unterscheidenden Gleichungen zerfällt:

$$
4 p_{1} p_{2} p_{3} y_{1} y_{z} y_{3} \pm r_{y} s_{y} t_{y}=0 \text {. }
$$

Die nähere Discussion dieser Curven sei dem Leser übcrlassen. Nur mögen zur Erleichterung des Beweises der oben angeführten Behauptungen Steiner's einige Andeutungen gegeben werden.

A) Die dem negativen Vorzeichen entsprechende Curve ist die von Steiner mit $P^{3}$ bezeichnete. Die Schnittpunkte der unendlich fernen Geraden mit den Seiten des Coordinatendreiecks sind Wendepunkte der Curve, und zwar haben die drei "Wendeasymptoten" bei Anwendung barycentrischer Coordinaten $\left(p_{1}=p_{2}=p_{3}\right)$ die Gleichungen $-2 y_{1}+y_{2}+y_{3}=0, y_{1}-2 y_{2}+y_{3}=0, y_{1}+y_{2}-2 y_{3}=0$. Diese drei Geraden sind den Seiten des Dreiecks parallel und schneiden sich im Schwerpunkte $y_{1}=y_{2}=y_{3}$; die Mitten der Seiten werden von der Curve berührt. Endlich zeigt die Einführung der Abstände $q_{1}, q_{2}, q_{3}$ des Punktes $y$ von den drei Seiten des Coordinatendreiecks $A_{1} A_{2} A_{3}$, sowie der Abstände $q_{1}^{\prime}, q_{2}^{\prime}, q_{3}^{\prime}$ von den Seiten des parallel eingeschriebenen Dreiecks $A_{1}{ }^{\prime} A_{2}{ }^{\prime} A_{3}{ }^{\prime}$ (vgl. auch (32)), dass die Curve nur in den tetragonalen Feldern verlaufen kann, und zwar nur in dem Winkelraum zwischen je zwei sich in das betr. Feld erstreckenden Wendeasymptoten. Die Gleichung der Curve erhält nämlich bei Einführung der $q$ und $q^{\prime}$ die Gestalt $q_{1} q_{2} q_{3}=2 q_{1}^{\prime} q_{2}^{\prime} q_{3}{ }^{\prime}$; untersucht man diese Gleichung mit Berücksichtigung der Vorzeichen der $q$ und $q^{\prime}$ in den verschiedenen Theilen der Ebene und beachtet hierbei, dass die Punkte $A_{1}{ }^{\prime}, A_{2}{ }^{\prime}, A_{3}{ }^{\prime}$ Berührungspunkte sind, sowie dass die oben er- 
wähnten durch den Schwerpunkt gezogenen Parallelen zu den Seiten Wendeasymptoten darstellen, so zeigt sich, dass die Curve aus drei im Unendlichen zusammenhängenden hyperbolischen $\mathrm{Z}_{\text {weigen }}$ besteht, die in ihrer Gesammtheit als ein einziger Zug aufzufassen sind. Nach (32) und (33) sind die zugehörigen Kegelschnitte Hyperbeln.

B) Auch die dem positiven Vorzeichen entsprechende Curve

$$
4 y_{1} y_{2} y_{3}+r_{y} s_{y} t_{y}=0
$$

hat drei unendlich ferne Wendepunkte; hier haben jedoch die Wendeasymptoten bei Anwendung barycentrischer Coordinaten die Gleichungen $y_{2}+y_{3}=0, y_{3}+y_{1}=0, y_{1}+y_{2}=0$, sind also die durch die Ecken des Coordinatendreiecks parallel zu den Gegenseiten gezogenen Geraden. Die Seiten des Dreiecks $A_{1} A_{2} A_{3}$ werden in ihren Mitten von der Curve berührt, aber nicht wie zuvor durch drei hyperbolische Zweige, sondern durch ein innerhalb $A_{1} A_{2} A_{3}$ liegendes und $A_{1}{ }^{\prime} A_{2}{ }^{\prime} A_{3}{ }^{\prime}$ umschliessendes Oval. Es folgt dies aus der nunmehr giltigen Gleichung $q_{1} q_{2} q_{3}=-2 q_{1}^{\prime} q_{2}^{\prime} q_{3}^{\prime}$. Bei Berücksichtigung der Vorzeichen $\operatorname{der} q$ und $q^{\prime}$, sowie des Umstandes, dass die Curve die Seiten des zu $A_{1} A_{2} A_{3}$ gehörigen parallel umschriebenen Dreiecks zu Wendeasymptoten hat, folgt, dass die Curve aus dem eben genannten Oval besteht und überdies noch aus drei hyperbolischen $\mathrm{Z}$ weigen, die in den äusseren trigonalen Feldern des parallel umschriebenen Dreiecks verlaufen und als ein im Unendlichen zusammenhängender Zug aufzufassen sind. Nach (32) und (33) sind die dem Oval zugehörigen Kegelschnitte

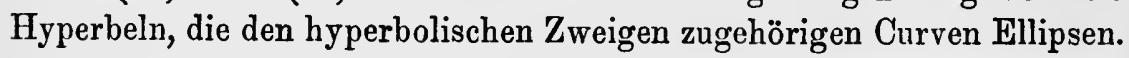

155. Aehnlich wie zuvor kann auch ein Satz von Steiner über gewisse Paare von Kegelschnitten bewiesen werden ${ }^{1}$ ). Man denke sich in einer Ebene irgend zwei Dreiecke $A B C$ und $\mathfrak{A} \mathfrak{B C}$ gegeben, „so ist jeder Punkt $p$ der Ebene zugleich der Mittelpunkt von zwei Kegelschnitten $P^{2}$ und $P_{1}{ }^{2}$, die dem ersten, und von zwei Kegelschnitten $\mathfrak{P}^{2}$ und $\mathfrak{P}_{1}^{2}$, die dem anderen Dreieck beziehlich um- und eingeschrieben sind“. Steiner behauptet nun:

„Sollen entweder die beiden Kegelschnitte

$P^{2}$ und $\mathfrak{F}^{2}$, oder $P_{1}^{2}$ und $\mathfrak{P}_{1}^{2}$, oder $P^{2}$ und $\mathfrak{P}_{1}{ }^{2}$

gleichen Inhalt oder gleiches Axenproduct haben, so ist der Ort des Punktes $p$ beziehlich eine Curve neunten, dritten, sechsten Grades."

Zum Beweis dieses Satzes hat man nur nöthig die in (143) und (144) abgeleiteten Gleichungen für den Punkt $p$ zweimal aufzustellen:

1) A. a. O., im Journal für die reine und angewandte Mathematik Bd. 55, S. 372, in den "Gesammelten Werken" Bd. 2, S. 678. 
Concentrische Kegelschnitte mit Nebenbedingungen; Ort der Mittelpunkte. 301

einmal bezogen auf das Dreieck $A B C$ mit dem Inhalt $\triangle$, dann auf das Dreieck $\mathfrak{A} \mathfrak{B} \mathfrak{C}$ mit dem Inhalt $\mathfrak{D}$, in welch letzterem Falle die Coordinaten von $p$ durch $\mathfrak{y}_{1}, \mathfrak{y}_{2}, \mathfrak{y}_{3}$ bezeichnet werden mögen. Diese Gleichungen sind nun nach $U^{2}$, bezw. $J^{2}$ aufzulösen und die erhaltenen Lösungen so gleichzusetzen, wie es in dem Steiner'schen Satze verlangt wird. Führt man ferner statt der den Dreiecken $\Delta$ und $\mathfrak{D}$ zugehörigen Constanten $\tau$ und $\tau^{\prime}$ die Inhalte ein vermöge der Formeln $\tau=\frac{3^{6}}{4 \Delta^{2}}, \quad \tau^{\prime}=\frac{3^{6}}{4 D^{2}}, \quad$ so erhält man, den obigen drei Fällen entsprechend, bei Benutzung barycentrischer Coordinaten die drei Gleichungen:

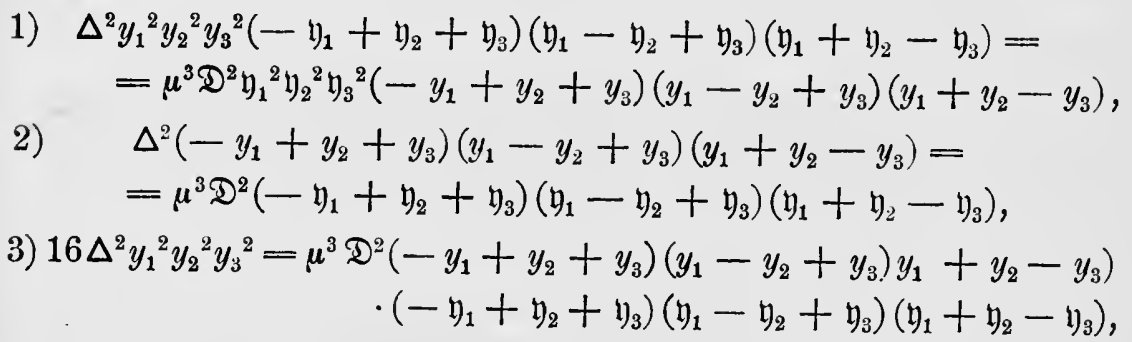

wobei die $\mathfrak{y}_{i}$ lineare Functionen von $y_{1}, y_{2}, y_{3}$ sind, deren Coeffcienten durch die Lage des Dreiecks $\mathfrak{A} B \mathbb{C}$ im Vergleich zu $A B C$ bestimmt sind; es ist etwa (ähnlich wie bei (4), S. 15)

$$
\begin{aligned}
& \mu \mathfrak{y}_{1}=a_{1} y_{1}+a_{2} y_{2}+a_{3} y_{3} \\
& \mu \mathfrak{y}_{2}=b_{1} y_{1}+b_{2} y_{2}+b_{3} y_{3} \\
& \mu \mathfrak{y}_{3}=c_{1} y_{1}+c_{2} y_{2}+c_{3} y_{3}
\end{aligned}
$$

und jedenfalls, da wir barycentrische Coordinaten zu Grunde legten, $\mu\left(\mathfrak{y}_{1}+\mathfrak{y}_{2}+\mathfrak{y}_{3}\right)=y_{1}+y_{2}+y_{3}$, vorausgesetzt, dass $\mu$ und die $a, b, c$ analog definirt sind wie S. 15 .

156. Es sei $J=a b$ das Product der Halbaxen eines dem Dreieck $A B C$ eingeschriebenen Kegelschnitts, ferner seien $A^{\prime}, B^{\prime}, C^{\prime}$ die Mitten der Seiten dieses Dreiecks und $r$ der Radius des ihm umschriebenen Kreises; sind alsdann $q_{1}^{\prime}, q_{2}^{\prime}, q_{3}^{\prime}$ die Abstände des Mittelpunktes $y$ jenes Kegelschnitts von den Seiten des Dreiecks $A^{\prime} B^{\prime} C^{\prime}$, so gilt stets die Relation $J^{2}=4 r q_{1}^{\prime} q_{2}^{\prime} q_{3}{ }^{\prime}{ }^{1}$ )

Wir wählen das Dreieck $A B C$ zum Coordinatendreieck und nennen $q_{1}, q_{2}, q_{3}$ die Abstände des Mittelpunktes $y$ des Kegelschnitts von den

1) Vgl. Steiner: „Teoremi relativi alle coniche inscritte e circoscritte“, Journal für die reine und angewandte Mathematik, Bd. 30, S. 97, 1846, oder Giornale arcadico di Roma, Bd. 99, S. 147-161, oder "Gesammelte Werke", Bd. 2, S. 329. 
Seiten; alsdanu ist nach (6), S. $3 y_{i}=\frac{q_{i} p_{y}}{e_{i}}$ oder bei Anwendung barycentrischer Coordinaten $y_{i}=\frac{q_{i}\left(y_{1}+y_{2}+y_{3}\right)}{h_{i}}$. Durch Einführung dieser Werthe in die bei (144) aufgestellte Gleichung erhält man die Relation $4 J^{2} \tau=3^{6}\left(-\frac{q_{1}}{h_{1}}+\frac{q_{2}}{h_{2}}+\frac{q_{3}}{h_{3}}\right)\left(\frac{q_{1}}{h_{1}}-\frac{q_{2}}{h_{2}}+\frac{q_{3}}{h_{3}}\right)\left(\frac{q_{1}}{h_{1}}+\frac{q_{2}}{h_{2}}-\frac{q_{3}}{h_{3}}\right)$, und unter Benutzung von $\frac{q_{1}}{h_{1}}+\frac{q_{2}}{h_{2}}+\frac{q_{3}}{h_{3}}=1$, sowie von $\frac{h_{i}}{2}-q_{i}=q_{i}^{\prime}$, ergibt sich $4 J^{2} \tau-\frac{3^{6} \cdot 8}{h_{1} h_{2} h_{3}} q_{1}^{\prime} q_{2}^{\prime} q_{3}^{\prime}=0 \quad$ oder $\quad J^{2}=\frac{2.3^{6} q_{1}^{\prime} q_{2}^{\prime} q_{8}^{\prime}}{h_{1} h_{2} h_{3} \cdot \tau}$. Nach (21), S. 61 ist aber nunmehr $\tau=\frac{3^{6}}{2 r h_{1} h_{2} h_{3}}$, daher $J^{2}=4 r q_{1}^{\prime} q_{2}^{\prime} q_{3}^{\prime}$.

Im Fall einer Ellipse folgt für deren Inhalt $E$ sofort die Relation $E^{2}=4 \pi^{2} r q_{1}^{\prime} q_{2}^{\prime} q_{3}^{\prime}$.

15\%. Es sei $U$ das Product der Halbaxen eines dem Dreieck $A B C$ umschriebenen Kegelschnitts, $r$ der Radius des umschriebenen Kreises; ferner mögen $q_{i}$ und $q_{i}^{\prime},(i=1,2,3)$, für den betr. Kegelschnitt die analoge Bedeutung haben wie in (156) für den ein-

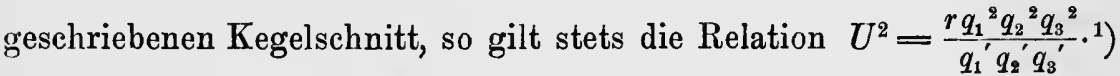

Folgt aus (143) in gleicher Weise wie (156) aus (144).

Im Fall einer Ellipse erhält man für ihren Inhalt $E$ die Relation

$$
E^{2}=\frac{\pi^{2} r q_{1}^{2} q_{2}^{2} q_{3}^{2}}{q_{1}^{\prime} q_{2}^{\prime} q_{3}^{\prime}}
$$

Diese Formel folgt auch aus (146), wenu man (1), S. 9, ferner (22), S. 61 und die Relationen wie $\omega\left(-p_{1}, p_{2}, p_{3}\right)=4 \omega_{11} p_{1}^{2}$ benutzt.

158. Für zwei concentrische Kegelschnitte, deren einer dem Dreieck umschrieben, der andere eingeschrieben ist, folgen aus (156) und (157) die Relationen $J U= \pm 2 r q_{1} q_{2} q_{3}$ und $\left.\frac{2 U}{J}= \pm \frac{q_{1} q_{2} q_{3}}{q_{1}^{\prime} q_{2}^{\prime} q_{3}{ }^{\prime}}{ }^{2}\right)$

159. Sind $J^{\prime}$ und $U^{\prime}$ die analogen Ausdrücke für das parallel eingeschriebene Dreieck $A^{\prime} B^{\prime} C^{\prime}$, wie $J$ und $U$ für das Dreieck $A B C$, so gilt die Relation $4 J^{\prime} U^{\prime}= \pm J^{2} \cdot{ }^{3}$ )

1) Steiner, a. a. O., im Journal für die reine und angewandte Mathematik Bd. 30, S. 98, oder "Gesammelte Werke“ Bd. 2, S. 330.

2) Vgl. Steiner „Vermischte Sätze und Aufgaben“, Journal für die reine und angewandte Mathematik, Bd. 55, S. 364, 1858, oder "Gesammelte Werke", Bd. 2, S. 670.

3) Siehe die Fussnote 1). 
Denn der Radius des dem Dreieck $A^{\prime} B^{\prime} C^{\prime}$ umschriebenen Kreises ist halb so gross als der Radius des umschriebenen Kreises von $A B C$, also $\frac{r}{2}$; daher ist nach (158) $J^{\prime} U^{\prime}= \pm r q_{1}{ }^{\prime} q_{2}^{\prime} q_{3}^{\prime}$, wofür nach (156) aueh $\frac{1}{4} J^{2}$ gesetzt werden kann.

160. Es sei $P$ das Product der Halbaxen derjenigen Curve zweiter Ordnung mit dem Mittelpunkte $y$, für die das Coordinatendreieck Poldreieck ist; bei gleicher Bezeichnungsweise wie in (156) gilt alsdann die Relation $P^{2}=2 r q_{1} q_{2} q_{3}$.

Nach (145) ist $P^{2}=\frac{y_{1} y_{2} y_{3}}{\tau p_{1} p_{2} p_{3}\left(p_{1} y_{1}+p_{2} y_{2}+p_{3} y_{3}\right)^{3}}$; mit Benutzung der Formeln $\frac{y_{i}}{p_{y}}=\frac{q_{i}}{e_{i}}$ (vgl. (6), S. 3), sowie der Relation $\tau=\frac{1}{2 r e_{1} e_{2} e_{3} p_{1} p_{2} p_{3}}$ (vgl. (22), S. 61) folgt die gewünschte Gleichung.

161. Hat man drei concentrische Kegelschnitte, von denen der eine ein gegebenes Dreieck zum Poldreieck hat, der zweite ihm eingeschrieben, der dritte umschrieben ist, so besteht zwischen den Producten $P, J, U$ der Halbaxen dieser Kegelschnitte die einfache Relation $P^{2}= \pm J U$.

Folgt sofort aus (158) und (160). Uebrigens können, wie die Resultate in (32), (33) und (34) zeigen, die drei Curven niemals gleichzeitig Ellipsen sein.

162. Die Bedingung dafür, dass die Curve zweiter Ordnung $f(x, x)=0$ eine gleichseitige Hyperbel repräsentirt, ist $[a, \omega]=0$.

Folgt aus (6), S. 111, denn die Asymptoten müssen zu einander normal sein.

Speciell für schiefwinklige Parallelcoordinaten mit dem Axenwinkel $\omega$ erhält man (vgl. (51), S. 98) $a_{11}+a_{22}-2 a_{12} \cos \omega=0$.

163. Die Bedingung dafür, dass die Curve zweiter Classe

$$
\varphi(u, u) \equiv \sum_{1}^{3} \sum_{1}^{3} \alpha_{i k} u_{i} u_{k}=0
$$

eine gleichseitige Hyperbel repräsentirt, ist

$$
[A, \omega] \equiv A_{11} \omega_{11}+\cdots+2 A_{12} \omega_{12}+\cdots=0 .
$$

Folgt aus (114). 
164. Der geometrische Ort der Mittelpunkte aller einem Dreieck eingeschriebenen gleichseitigen Hyperbeln ist derjenige Kreis, für welchen das Dreieck ein Poldreieck ist.

Der dem Coordinatendreieck eingeschriebene Kegelschnitt

$$
\alpha_{1} u_{2} u_{3}+\alpha_{2} u_{3} u_{1}+\alpha_{3} u_{1} u_{2}=0
$$

repräsentirt nach (163) eine gleichseitige Hyperbel, wenn

$$
\alpha_{1}{ }^{2} \omega_{11}+\alpha_{2}{ }^{2} \omega_{22}+\alpha_{3}{ }^{2} \omega_{33}-2 \alpha_{1} \alpha_{2} \omega_{12}-2 \alpha_{1} \alpha_{3} \omega_{13}-2 \alpha_{2} \alpha_{3} \omega_{23}=0 .
$$

Bei Einführung der Coordinaten des Mittelpunktes verwandelt sich diese Bedingung nach (140) in $p_{1} \omega_{23} y_{1}{ }^{2}+p_{2} \omega_{31} y_{2}{ }^{2}+p_{3} \omega_{12} y_{3}{ }^{2}=0$, also nach (75) in die Gleichung des Kreises, für den das Coordinatendreieck ein Poldreieck ist. Dieser Kreis ist jedoch nur bei stumpfwinkligen Dreiecken reell, also können nur stumpfwinkligen Dreiecken gleichseitige Hyperbeln eingeschrieben werden. Vgl. auch (324).

165. Der geometrische Ort der Mittelpunkte aller einem Dreieck umschriebenen gleichseitigen Hyperbeln ist ein Kreis, der durch die Fusspunkte der Höhen und Mitten der Seiten des Dreiecks hindurchgeht (Feuerbach'scher Kreis).

Die Gleichung eines dem Coordinatendreieck umschriebenen Kegelschnitts ist von der Form $a_{1} x_{2} x_{3}+a_{2} x_{3} x_{1}+a_{3} x_{1} x_{2}=0$. Sind nun $y_{i}$ $(i=1,2,3)$ die Coordinaten des Mittelpunktes, so hat man nach (23), S. 25

$$
\begin{aligned}
& \varrho p_{1}=\quad a_{2} y_{3}+a_{3} y_{2} \\
& \varrho p_{2}=a_{1} y_{3}+a_{3} y_{1} \\
& \varrho p_{3}=a_{1} y_{2}+a_{2} y_{1} ;
\end{aligned}
$$

$\mathrm{zu}$ diesen drei Gleichungen tritt noch die Bedingung der gleichseitigen Hyperbel $a_{1} \omega_{23}+a_{2} \omega_{31}+a_{3} \omega_{12}=0$, worauf man die Gleichung des gesuchten Ortes durch Elimination von $\varrho, a_{1}, a_{2}, a_{3}$ in der Gestalt erhält:

$$
\left|\begin{array}{cccc}
p_{1} & 0 & y_{3} & y_{2} \\
p_{2} & y_{3} & 0 & y_{1} \\
p_{3} & y_{2} & y_{1} & 0 \\
0 & \omega_{23} & \omega_{31} & \omega_{12}
\end{array}\right|=0
$$

d. h. $\quad \omega_{23} p_{1} y_{1}^{2}+\omega_{31} p_{2} y_{2}^{2}+\omega_{12} p_{3} y_{3}^{2}-\left(\omega_{31} p_{1}+\omega_{32} p_{2}\right) y_{1} y_{2}$

$$
-\left(\omega_{21} p_{1}+\omega_{23} p_{3}\right) y_{1} y_{3}-\left(\omega_{12} p_{2}+\omega_{13} p_{3}\right) y_{2} y_{3}=0 \text {. }
$$

Diese Gleichung lässt sich auch in die Form bringen

$$
\begin{aligned}
2\left(\omega_{23} p_{1} y_{1}^{2}\right. & \left.+\omega_{31} p_{2} y_{2}^{2}+\omega_{12} p_{3} y_{3}^{2}\right) \\
& -\left(p_{1} y_{1}+p_{2} y_{2}+p_{3} y_{3}\right)\left(\omega_{23} y_{1}+\omega_{31} y_{2}+\omega_{12} y_{3}\right)=0,
\end{aligned}
$$


woraus nach (68) und (75) folgt, dass sie einen Kreis darstellt. Für die Coordinaten der Schnittpunkte des Kreises mit der Seite $y_{1}=0$ des Coordinatendreiecks erbält man $\left(\omega_{31} y_{2}-\omega_{12} y_{3}\right)\left(p_{2} y_{2}-p_{3} y_{3}\right)=0$, diese Punkte sind daher nach (11) und (7) der Fusspunkt der zur Seite $y_{1}=0$ gehörigen Höhe und die Mitte dieser Seite. Der obige Kreis geht also überhaupt durch die Mittelpunkte der Seiten und Fusspunkte der Höhen des Dreiecks; man bezeichnet ihn als den Feuerbach'schen Kreis ${ }^{1}$ ).

Eine andere Form der Gleichung dieses Kreises ergibt sich aus der obigen unter Anwendung von $\omega^{\prime}\left(p_{i}\right)=0$ und lautet

$$
\begin{aligned}
\omega_{23} p_{1} y_{1}{ }^{2}+\omega_{31} p_{2} y_{2}{ }^{2}+\omega_{12} p_{3} y_{3}{ }^{2}+\omega_{11} p_{1} y_{2} y_{3} & +\omega_{22} p_{2} y_{3} y_{1} \\
& +\omega_{33} p_{3} y_{1} y_{3}=0 .
\end{aligned}
$$

Eine weitere Form der Gleichung, welche direct aus der Bedingung $[a, \omega]=$ folgt $(\operatorname{vgl} .(139))$, ist

$$
\begin{aligned}
\omega_{23} y_{1}\left(-p_{1} y_{1}+p_{2} y_{2}+p_{3} y_{3}\right) & +\omega_{31} y_{2}\left(p_{1} y_{1}-p_{2} y_{2}+p_{3} y_{3}\right) \\
& +\omega_{12} y_{3}\left(p_{1} y_{1}+p_{2} y_{2}-p_{3} y_{3}\right)=0,
\end{aligned}
$$

wobei die Klammerfactoren gleich Null gesetzt, die Seiten des dem Coordinatendreieck parallel eingeschriebenen Dreiecks darstellen.

Die oben angegebene Bediugung der gleichseitigen Hyperbel ist übrigens, wie man mit Hilfe der Coordinaten des Höhenschnittpunktes (17) sofort erkennt, identisch mit der, dass der umschriebene Kegelschnitt durch diesen Punkt hindurchgehe. Hieraus folgt:

166. Jede gleichseitige Hyperbel, die durch die Ecken eines Dreiecks geht, geht auch durch den Höhenschnittpunkt des Dreiecks ${ }^{2}$ ).

Ferner folgt umgekehrt:

167. Jeder Kegelschnitt, der durch die Ecken und den Höhenschnittpunkt eines Dreiecks geht, ist eine gleichseitige Hyperbel.

168. Verbindet man drei Punkte einer gleichseitigen Hyperbel durch Sehnen und legt man durch die Mitten dieser Selmen einen Kreis, so geht derselbe durch den Mittelpunkt der Curve.

Folgt aus (165), denn der betreffende Kreis ist der Feuerbach'sche Kreis des Sehnendreiecks.

1) Feuerbach: „Eigenschaften einiger merkwürdigen Punkte des geradlinigen Dreiecks und mehrerer durch sie bestimmten Linien und Figuren". Nürnberg 1822. Vgl. daselbst besonders die Abschnitte II, IV (\$ 56), V und VI (9. Satz).

2) Diese gleichseitigen Hyperbeln bilden daher nach § 14 ein Büschel; aus (8), S. 199 folgt alsdann, dass auch die Mitten der drei oberen Höhenabschnitte auf dem Fenerbach'schen Kreise liegen. 
169. Der geometrische Ort der Mittelpunkte aller gleichseitigen Hyperbeln, die ein gegebenes Dreieck zum Poldreieck haben, ist der dem Dreieck umschriebene Kreis.

Die Gleichung der Curve zweiter Ordnung mit dem Mittelpunkte $y$, die das Coordinatendreieck zum Poldreieck hat, ist nach (30)

$$
p_{1} y_{2} y_{3} x_{1}{ }^{2}+p_{2} y_{3} y_{1} x_{2}{ }^{2}+p_{3} y_{1} y_{2} x_{3}{ }^{2}=0 ;
$$

dieselbe ist eine gleichseitige Hyperbel, wemu

$$
p_{1} y_{2} y_{3} \omega_{11}+p_{2} y_{3} y_{1} \omega_{22}+p_{3} y_{1} y_{2} \omega_{33}=0
$$

d. h. nach (73) der Mittelpunkt $y$ muss auf dem umschriebenen Kreis des Dreiecks liegen. (Vgl. auch (326) und (327).)

170. Alle gleichseitigen Hyperbeln, die ein gegebenes Dreieck zum Poldreieck haben, gehen durch die Mittelpunkte derjenigen vier Kreise hindurch, welche die drei Seiten des Dreiecks berühren.

Die Bedingung $a_{11} \omega_{11}+a_{22} \omega_{22}+a_{33} \omega_{33}=0$ dafür, dass der Kegelschnitt $a_{11} x_{1}{ }^{2}+a_{22} x_{2}{ }^{2}+a_{33} x_{3}{ }^{2}=0$ eine gleichseitige Hyperbel sei, ist nach (16) identisch mit der, dass die oben genannten vier Mittelpunkte auf der Curve liegen.

Ebenso folgt:

171. Jeder Kegelschnitt, der ein gegebenes Dreieck zum Poldreieck hat und durch den Mittelpunkt irgend eines der vier Kreise geht, die die Seiten des Dreiecks berühren, geht auch durch die Mittelpunkte der drei übrigen Kreise hindurch und ist eine gleichseitige Hyperbel.

* 172. Man beweise nachstehenden Satz von Steiner $\left.{ }^{1}\right)$ :

„Die Mittelpunkte jeder Schaar unter sich ähnlicher und dem gegebenen Dreieck umschriebener Kegelschnitte liegen in einer Curve vierten Grades, welche die Mitten der Dreiecksseiten zu Doppelpuniten hat."

Nach (127) sind Curven zweiter Ordnung einander ähnlich, wenn die Grösse $\frac{F(p, p)}{[a, \omega]^{2}}$, gebildet für diese Curven, denselben constanten Werth $c$ besitzt. Wendet man dies an auf die Gleichung eines dem Coordinatenàreieck umschriebenen Kegelschnitts mit dem Mittelpunkte $y$ (vgl. (139)), so folgt als Gleichung des Ortes:

$$
p_{y} r_{y} s_{y} t_{y}-4 c\left(y_{1} r_{y} \omega_{23}+y_{2} s_{y} \omega_{31}+y_{3} t_{y} \omega_{12}\right)^{2}=0 \text {, }
$$

1) „Vermischte Sätze und Anfgaben“, Journal für die reine und angewandte Mathematik, Bd. 55, S. 369, 1858, oder auch "Gesammelte Werke", Bd. 2, S. 675. 
Ort d. Mittelpunkte u. Enveloppe von ähnlichen, umschrieb. Kegelschnitten. 307

wobei $c$ nach (6), S. 111 definirt ist durch $-\frac{\operatorname{tg}^{2} \alpha}{4 \tau}$, wenn $\alpha$ den Winkel der beiden Asymptoten bezeichnet. Bei Einführung des parallel eingeschriebenen Dreiecks als Coordinatendíeieck vermöge der Formeln $\mu p_{1} z_{1}=r_{y}, \mu p_{2} z_{2}=s_{y}, \mu p_{3} z_{3}=t_{y}$ (vgl. (22)), erhält man mit Benutzung der Relationen $\omega^{\prime}\left(p_{i}\right)=0$ die Gleichung der Curve in der Form

$$
\begin{aligned}
p_{1} p_{2} p_{3}\left(p_{1} z_{1}+p_{2} z_{2}\right. & \left.+p_{3} z_{3}\right) z_{1} z_{2} z_{3} \\
& -c\left(\omega_{11} p_{1} z_{2} z_{3}+\omega_{22} p_{2} z_{3} z_{1}+\omega_{33} p_{3} z_{1} z_{2}\right)^{2}=0 .
\end{aligned}
$$

Diese Gleichung stellt offenbar eine Curve vierter Ordnung dar, welche die Ecken des neuen Coordinatendreiecks, also die Seitenmitten des ursprünglichen Dreiecks zu Doppelpunkten hat.

Im Anschluss an die oben benutzte Relation

$$
\psi \equiv 4 \tau F(p, p)+[a, \omega]^{2} \operatorname{tg}^{2} \alpha=0
$$

lässt sich die Frage erledigen nach der sogenannten Enveloppe der Schaar umschriebener Kegrelschnitte. Wird wieder das Coordinatendreieck zu Grunde gelegt, so muss neben $\psi=0$ die Gleichung bestehen $f(x, x) \equiv 2\left(a_{23} x_{2} x_{3}+a_{31} x_{3} x_{1}+a_{12} x_{1} x_{2}\right)=0$, welche sich mittels der Substitution $x_{2} x_{3}: x_{3} x_{1}: x_{1} x_{2}=z_{1}: z_{2}: z_{3}$ verwandelt in $a_{23} z_{1}+a_{31} z_{2}+a_{12} z_{3}=0$. Deutet man nun $a_{23}, a_{31}, a_{12}$ als Liniencoordinaten $\left(u_{1}, u_{2}, u_{3}\right), z_{1}, z_{2}, z_{3}$ als Punktcoordinaten, so entspricht jeder Tangente der Curve zweiter Classe $\psi=0$ eine Gerade

oder ein Kegelschnitt

$$
a_{23} z_{1}+a_{31} z_{2}+a_{12} z_{3}=0
$$

$$
a_{23} x_{2} x_{3}+a_{31} x_{3} x_{1}+a_{12} x_{1} x_{2}=0,
$$

und den Schnittpunkten der auf einander folgenden Tangenten entsprechen die Schnittpunkte der auf einander folgenden Kegelschnitte der Schaar; die Gesammtheit dieser Schnittpunkte bildet die sogenannte Enveloppe der Schaar. Die Bedingung, dass zwei von dem Punkte z an die Curve $\psi\left(a_{23}, a_{31}, a_{12}\right)=0$ gezogene Tangenten zusammenfallen, wird (als Gleichung der Curve $\psi=0$ in Punktcoordinaten $z_{i}$ ) in den $z_{i}$ vom zweiten Grade, in den $x_{i}$ also rom vierten; die Enveloppe ist daher eine Curve vierter Ordnung, welche die Ecken des Coordinatendreiecks zu Doppelpunkten hat, wie aus $z_{1}: z_{2}: z_{3}=x_{2} x_{3}: x_{3} x_{1}: x_{1} x_{2}$ lervorgeht. Legt man den Einheitspunkt in den Mittelpunkt des eingeschriebenen Kreises, setzt man also $e_{1}=e_{2}=e_{3}=\rho$, so stellt $F(p, p)=0$, wenn $a_{23}, a_{31}, a_{12}$ als Liniencoordinaten $u_{1}, u_{2}, u_{3}$ aufgefasst werden, nach (73) den umschriebenen Kreis des Dreiecks dar, $[a, \omega]^{2}=0$ seinen Mittelpunkt doppelt zählend; mit Rücksicht auf (2), S. 57 folgt alsdann, dass $\psi\left(a_{23}, a_{31}, a_{12}\right)=0$ die Gleichung in Linien- 
coordinaten eines mit dem umschriebenen Kreis concentrischen Kreises ist. Den Tangenten des letzteren entsprechen nach dem Vorausgehenden umschriebene ähnliche Kegelschnitte, dem Kreis selbst die Enloppe der Kegelschnitte ${ }^{1}$ ).

Es werde noch bemerkt, dass diese Verwandlung eines mit dem umschriebenen Kreis concentrischen Kreises in eine Curve vierter Ordnung vermöge der Gleichungen $z_{1}: z_{2}: z_{3}=x_{2} x_{3}: x_{3} x_{1}: x_{1} x_{2}$ eine Steiner'sche Transformation genannt wird ${ }^{2}$ ).

Eine andere Auffassung von $\psi=0$ ist folgende: Deutet man in

$$
f(x, x) \equiv 2\left(a_{23} x_{2} x_{3}+a_{31} x_{3} x_{1}+a_{12} x_{1} x_{2}\right)=0,
$$

sowie in $\psi\left(a_{23}, a_{31}, a_{12}\right)=0$ die Grössen $a_{23}, a_{31}, a_{12}$ als Coordinaten eines Punktes $a$, so stellt $f(x, x)=0$ nach (42) die konische Polare von $a$ in Bezug auf die ausartende Curve dritter Ordnung $x_{1} x_{2} x_{3}=0$ dar. Für alle konischen Polaren von gegebenem Asymptotenwinkel $\alpha$ muss der Punkt $a$ auf dem Kegelschnitt $4 \tau F(p, p)+[a, \omega]^{2} \operatorname{tg}^{2} \alpha=0$ $\left(a_{23}, a_{31}, a_{12}\right.$ variabele Punktcoordinaten) liegen, der die Ellipse $F(p, p)=0$ in ihren Schnittpunkten mit der Geraden $a_{23} \omega_{23}+a_{31} \omega_{31}+a_{12} \omega_{12}=0$ doppelt berührt. Diese Gerade ist nach (40) und (17) die Harmonicale $H$ des Höhenschnittpunktes, während $F(p, p)=0$ nach (39) die Seiten des Dreiecks in ihren Mittelpunkten berührt und eine Ellipse ist. Sollen die Polaren $f(x, x)=0$ Parabeln sein, so muss $a$ auf $F(p, p)=0$ liegen; sollen dieselben gleichseitige Hyperbeln sein, so ist der Ort von $a$ die Gerade $H$. Die Polare wird ein Kreis nur wenn $a$ der Pol von $H$ in Bezug auf $F(p, p)=0$ ist. Diese Theoreme decken sich vollständig mit denen, wielche Steiner erwähnt in einem Nachtrag zu seiver Abhandlung über algebraische Curven, welche einen Mittelpunkt haben ${ }^{3}$ ); man hat nur als Coordinatendreieck dasjenige zu wählen, welches von den drei Asymptoten der bei Steiner als "Basis" auftretenden Curve dritter Ordnung gebildet wird.

1) Die hier gegebene Beantwortung der oben gestellten Frage wurde vor mehr als siebzehn Jahren von Herrn Gundelfinger an Herrn Fiedler mitgetheilt. Vgl. übrigens noch Steiner a. a. O., oder auch "Systematische Entwickelung der Abhängigkeit geometrischer Gestalten von einander", Anhang
Nr. 39, 1832.

2) Vgl. Steiner's Abhandlung: „Développement d'une série de théorèmes relatifs aux sections coniques", Annales de Mathématiques, Bd. 19, S. 37-64, 1828, oder auch „Gesammelte Werke", Bu. 1, S. 189-210. Ferner vgl. § 59 der soeben citirten systematischen Entwickelung.

3) Journal für die reine und angewandte Mathematik, Bd. 47, S. 107, 1854, oder ,Gesammelte Werke“, Bd. 2, S. 600. 
173. „Jede Schaar unter sich ähnlicher und einem gegebenen Dreieck $A B C$ eingeschriebener Kegelschnitte hat ihre Mittelpunkte in irgend einer Curve vierten Grades"1).

Bei analogem Verfahren wie in (172) erhält man die Gleichung der Curve bezogen auf das gegebene Dreieck als Coordinatendreieck in der Gestalt

$$
p_{y} r_{y} s_{y} t_{y}-4 c\left(p_{1} \omega_{23} y_{1}^{2}+p_{2} \omega_{31} y_{2}^{2}+p_{3} \omega_{12} y_{3}^{2}\right)^{2}=0 .
$$

174. Ueber die Schaar unter sich ähnlicher und einem gegebenen Dreieck eingeschriebener Kegelschnitte stellt Steiner noch folgende Sätze ohne Beweis auf ${ }^{2}$ ):

"Die Glieder solcher Schaar Kegelschnitte sind zu vier und vier ähnlich liegend, d. h. es gibt im allgemeinen je vier dem gegebenen Dreieck eingeschriebene Kegelschnitte, welche irgend einem gegebenen Kegelschnitte ähnlich und mit ihm ähnlichliegend sind.

Sind die vier Kegelschnitte Ellipsen, so sind ihre Mittelpunkte allemal die Ecken eines vollständigen Vierecks, dessen drei Paar Gegenseiten sich in den Ecken des gegebenen Dreiecks schneiden." -

„Das Product der Halbaxen solcher vier Ellipsen, die dem gegebenen Dreieck eingeschrieben und ähnlich und ähnlichliegend sind, ist constant und zwar der vierten Potenz der Dreieckstäche gleich. Oder sind $\mathfrak{r}, \mathfrak{r}_{1}, \mathfrak{r}_{2}, \mathfrak{r}_{3}$ die Radien derjenigen vier Kreise, welche mit den Ellipsen gleichen Inhalt haben, so ist $\mathfrak{r r}_{1} \mathfrak{r}_{2} \mathfrak{r}_{3}=\Delta^{*}$."

„Die vorstehenden Sätze, die einfachleitshalber nur fïr die Ellipsen ausgesprochen sind, gelten analoger Weise auch für Hyperbeln."

Die Gleichung eines dem Coordinatendreieck eingeschriebenen Kegelschnitts mit dem Mittelpunkte $y$ ist nach (29)

$$
\frac{1}{2} \varphi(u, u) \equiv \alpha_{1} u_{2} u_{3}+\alpha_{2} u_{3} u_{1}+\alpha_{3} u_{1} u_{2}=0,
$$

wobei $\sigma \alpha_{1}=p_{1} r_{y}, \sigma \alpha_{2}=p_{2} s_{y}, \sigma \alpha_{3}=p_{3} t_{y}$, wenn zur Abkürzung gesetzt wird $r_{y} \equiv-p_{1} y_{1}+p_{2} y_{2}+p_{3} y_{3}, \quad s_{y} \equiv p_{1} y_{1}-p_{2} y_{2}+p_{3} y_{3}$, $t_{y} \equiv p_{1} y_{1}+p_{2} y_{2}-p_{3} y_{3}$. Soll der Kegelschnitt mit einem gegebenen ähnlich und ähnlich liegend sein, so ist nach (130) erforderlich, dass beide von der unendlich fernen Geraden in denselben Punkten getroffen werden. (Von der weiteren Bedingung, dass die aus einem unendlich

1) Steiner: "Lebrsätze und Aufgaben", Journal für die reine und angewandte Mathematik, Bd. 30, S. 273, 1845, oder "Gesammelte Werke“, Bd. 2, S. 346. Ferner: "Vermischte Sätze und Aufgaben", Bd. 55 des eben genannten Journals, S. 369, 1858, oder "Gesammelte Werke", Bd. 2, S. 675.

2) Journal für die reine und angewandte Mathematik, Bd. 55, S. 369 f., oder "Gesammelte Werke", Bd. 2, S. $675 \mathrm{f}$. 
fernen Punkte an beide Curven gezogenen Tangenten gleichzeitig reel oder imaginär seien, wird nach Steiner abgesehen.) Ist

$$
\psi(u, u) \equiv \beta_{u} \gamma_{u}=0
$$

die Gleichung des Schnittpunktepaars mit der unendlich fernen Geraden, so ist nach (43), S. 84 die Gleichung der Curve auch von der Form $\psi(u, u)-\varrho \cdot u_{y}{ }^{2}=0$, und da die Glieder mit $u_{i}{ }^{2}$ fehlen müssen, folgt sofort für die Coordinaten $y_{i}$ des Mittelpunktes

$$
y_{1}= \pm \sqrt{\beta_{1} \gamma_{1}}, \quad y_{2}= \pm \sqrt{\beta_{2} \gamma_{2}}, \quad y_{3}= \pm \sqrt{\beta_{3} \gamma_{3}} \text {. }
$$

Den verschiedenen Combinationen der Vorzeichen entsprechend gibt es also in der That vier eingeschriebene Kegelschnitte, die dem gegebenen Kegelschnitte ähnlich sind. Aus den Werthen der Coordinaten der vier Mittelpunkte folgt auch leicht, dass diese vier Punkte die Ecken eines vollständigen Vierecks bilden, dessen drei Paar Gegenseiten sich in den Ecken des Coordinatendreiecks schneiden.

Das Product aus den Quadraten der Halbaxen der Curve $\varphi(u, u)=0$ ist nach (133) gegeben durch $a^{2} b^{2}=\frac{A}{\tau \varphi^{3}(p, p)}$; für

wird nun

$$
\varphi(u, u) \equiv 2\left(\alpha_{1} u_{2} u_{3}+\alpha_{2} u_{3} u_{1}+\alpha_{3} u_{1} u_{2}\right)=0
$$

$$
\mathrm{A}=2 \alpha_{1} \alpha_{2} \alpha_{3}=\frac{2 p_{1} p_{2} p_{3} r_{y} s_{y} t_{y}}{\sigma^{3}},
$$

$\varphi\left(p, p^{\prime}\right)=2\left(\alpha_{1} p_{2} p_{3}+\alpha_{2} p_{3} p_{1}+\alpha_{3} p_{1} p_{2}\right)=2\left(\frac{\alpha_{1}}{p_{1}}+\frac{\alpha_{2}}{p_{2}}+\frac{\alpha_{3}}{p_{3}}\right) p_{1} p_{2} p_{3}$, wofür auch gesetzt werden kann

$$
\frac{2 p_{1} p_{2} p_{3}}{\sigma}\left(r_{y}+s_{y}+t_{y}\right)=\frac{2 p_{1} p_{2} p_{3} p_{y}}{\sigma} .
$$

Man erhält demnach $a^{2} b^{2}=\frac{2 p_{1} p_{2} p_{3} r_{y} s_{y} t_{y}}{\tau .8 p_{1}^{3} p_{2}^{3} p_{3}^{3} p_{y}^{3}}=\frac{r_{y} s_{y} t_{y}}{4 \tau p_{1}^{2} p_{2}^{2} p_{3}^{2} p_{y}^{3}}$ als Ausdruck für das Product aus den Quadraten der Halbaxen der Curve mit dem Mittelpunkte $+y_{1},+y_{2},+y_{3} ;$ bei dem Kegelschnitt mit dem Mittelpunkte $-y_{1},+y_{2},+y_{3}$ ergibt sich also $a_{1}{ }^{2} b_{1}{ }^{2}=\frac{p_{y} t_{y} s_{y}}{4 \tau p_{1}^{2} p_{2}^{2} p_{3}^{2} \cdot r_{y}^{3}}$. Hieraus ersieht man, dass bei Berücksichtigung von vier ähulich liegenden Curven der Schaar sich in dem Product $a^{2} b^{2} \cdot a_{1}{ }^{2} b_{1}{ }^{2} \cdot a_{2}{ }^{2} b_{2}{ }^{2} \cdot a_{3}{ }^{2} b_{3}{ }^{2}$ sïmmtliche Factoren $p_{y} r_{y} s_{y} t_{y}$ in Zähler und Nenner wegheben, so dass $a b \cdot a_{1} b_{1} \cdot a_{2} b_{2} \cdot a_{3} b_{3}=\frac{1}{16 \tau^{2} p_{1}{ }^{4} p_{2}{ }^{4} p_{3}{ }^{4}} \cdot$ Aus (20), S. 61 folgt, wenn man $a_{i}=\frac{2 \Delta}{h_{i}}$ benutzt und $\frac{e_{i}}{h_{i}}$ ersetzt durch $p_{i}$, die Relation $\tau=\frac{1}{4 p_{1}{ }^{2} p_{2}{ }^{2} p_{3}{ }^{2} \Delta^{2}}$, es ist also in der That das Product aus den Halbaxen von vier Kegelschnitten der oben genannten Art gleich $\Delta^{4}$. 
Sind die vier Kegelschnitte einander ähnliche Ellipsen, so ist (33) und S. 252, Zeile $16 \mathrm{ff}$. anzuwenden, und es zeigen alsdann die nur in den Vorzeichen verschiedenen Coordinaten der Mittelpuukte, dass einer derselben im Innern des dem gegebenen parallel eingeschriebenen Dreiecks, die drei anderen in den trigonalen Feldern liegen. Dem entsprechend besteht die Curve vierter Ordnung, welche nach (173) den Ort für die Mittelpunkte aller ähnlicheu, dem gegebenen Dreieck eingeschriebenen Ellipsen bildet, aus vier getrennten Ovalen. Aucl ist die Curve noch dadurch interessant, dass sie 28 reelle Doppeltangenten besitzt $^{1}$ ). -

Wir hatten oben der Gleichung des Kegelschnitts die Form gegeben $\psi(u, u)-\varrho u_{y}{ }^{2}=0$; stellt uun allgemeiner $\psi(u, u)=0$ nicht ein Punktepaar, sondern irgend eine Curve zweiter Classe dar, so wird dieselbe offenbar von $\chi(u, u) \equiv \psi(u, u)-\varrho u_{y}{ }^{2}=0$ doppelt berührt, und zwar ist $y$ der Pol der Berührungssehne. Man kann nun verlangen, dass $\chi(u, u)=0$ dem Coordinatendreiseit eingeschrieben, also von der Form sei $2\left(\alpha_{1} u_{2} u_{3}+\alpha_{2} u_{3} u_{1}+\alpha_{3} u_{1} u_{2}\right)=0$ und würde alsdann für $y_{1}: y_{2}: y_{3}$ wieder vier Werthsysteme erhalten von ähnlicher Beschaffenheit wie die obigen. Man erhält auf solche Weise den Satz:

175. Es gibt vier Kegelschnitte, die einem gegebenen Dreiseit eingeschrieben sind und einen gegebenen Kegelschnitt doppelt berïhren. Die Pole der vier Berührungssehnen bilden die Ecken eines vollständigen Vierecks, dessen drei Paar Gegenseiten sich in den Ecken des Dreiseits schneiden.

176. Welche Curve wird von der Schaar ähnlicher, einem gegebenen Dreieck eingeschriebener Kegelschnitte umhüllt? $\left.{ }^{2}\right)$

Ausser der Gleichung $2\left(\alpha_{1} u_{2} u_{3}+\alpha_{2} u_{3} u_{1}+\alpha_{3} u_{1} u_{2}\right)=0$ eines dem Coordinatendreieck eingeschriebenen Kegelschnitts muss, wie sich uus (127) oder aus (114) ergibt, die Bedingung

$$
[\mathrm{A}, \omega]^{2} \operatorname{tg}^{2} \alpha+4 \tau \mathrm{A} \varphi(p, p)=0
$$

erfüllt sein; dieselbe lautet im gegenwärtigen Falle

$$
\begin{gathered}
\psi\left(\alpha_{1}, \alpha_{2}, \alpha_{3}\right) \\
\equiv\left(\alpha_{1}{ }^{2} \omega_{11}+\alpha_{2}{ }^{2} \omega_{22}+\alpha_{3}{ }^{2} \omega_{33}-2 \alpha_{2} \alpha_{3} \omega_{23}-2 \alpha_{3} \alpha_{1} \omega_{31}-2 \alpha_{1} \alpha_{2} \omega_{12}\right)^{2} \operatorname{tg}^{2} \alpha \\
+16 \tau \alpha_{1} \alpha_{2} \alpha_{3} p_{1} p_{2} p_{3}\left(\frac{\alpha_{1}}{p_{1}}+\frac{\alpha_{2}}{p_{2}}+\frac{\alpha_{3}}{p_{3}}\right)=0 .
\end{gathered}
$$

1) Näheres in der Dissertation von A eschlimann: "Zur Theorie der ebenen Curven vierter Ordnung", Zürich 1880.

2) Auch diese Frage wird von Steiner a. a. 0 . aufgeworfen. 
Aehnlich wie in (172) setze man nun $u_{2} u_{3}=v_{1}, u_{3} u_{1}=v_{2}, u_{1} u_{2}=v_{3}$, wodurch die Gleichung des Kegelschnitts übergeht in

$$
\alpha_{1} v_{1}+\alpha_{2} v_{2}+\alpha_{3} v_{3}=0
$$

ferner deute man die $\alpha_{i}$ als variabele Punktcoordinaten, die $v_{i}$ als Liniencoordinaten. Bildet man die Bedingung, dass die Gerade

$$
v_{1} \alpha_{1}+v_{2} \alpha_{2}+v_{3} \alpha_{3}=0
$$

die Curve vierter Ordnung $\psi\left(\alpha_{1}, \alpha_{2}, \alpha_{3}\right)=0$ berühre, so erhält man die „Enveloppe“ der Schaar ähnlicher Kegelschnitte; an Stelle von $v_{1}: v_{2}: v_{3}$ ist natürlich einzusetzen $u_{2} u_{3}: u_{3} u_{1}: u_{1} u_{2}$. Die weitere Ausführung sei dem Leser überlassen.

17\%. Die Mittelpunkte aller unter sich ähnlicher Kegelschnitte, die ein gegebenes Dreieck zum Poldreieck haben, liegen in einer Curve vierter Ordnung, welche die Ecken des Dreiecks zu Doppelpunkten hat.

In analoger Weise wie bei (172) und (173) erhält man mit Rücksicht auf (34) als Gleichung der Curve:

$$
p_{1} p_{2} p_{3} y_{1} y_{2} y_{3}\left(p_{1} y_{1}+p_{2} y_{2}+p_{3} y_{3}\right)
$$

wobei $c=-\frac{\operatorname{tg}^{2} \alpha}{4 \tau}$.

$$
-c\left(\omega_{11} p_{1} y_{2} y_{3}+\omega_{22} p_{2} y_{3} y_{1}+\omega_{33} p_{3} y_{1} y_{2}\right)^{2}=0,
$$

Diese Curve hat in Bezug auf das Coordinatendreieck genau dieselbe Lage wie die in (172) vorkommende Curve in Bezug auf das parallel eingeschriebene Dreieck.

178. Man suche den geometrischen Ort für die Mittelpunkte je zweier concentrischer Kegelschnitte, die unter sich ähnlich sind und von denen der eine dem Coordinatendreieck umschrieben, der andere ihm eingeschrieben ist ${ }^{1}$ ).

Der Einfachheit halber mögen wieder barycentrische Coordinaten zu Grunde gelegt werden. Die Gleichung des umschriebenen Kegelschnitts mit dem Mittelpunkte $y$ ist dann nach (32)

$$
\frac{1}{2} f(x, x) \equiv y_{1} r_{y} x_{2} x_{3}+y_{2} s_{y} x_{3} x_{1}+y_{3} t_{y} x_{1} x_{2}=0
$$

1) Auch an diesen Ort denkt Steiner, indem er (Journal für die reine und angewandte Mathematik, Bd. 55, S. 363, 1858, oder „Gesammelte Werke“, Bd. 2, S. 670) die Frage aufwirft: „Besteht der Ort aus vier Geraden und einer Curve vierten Grades?" Steiner's Vermuthung ist richtig, die Curve zerfällt allerdings in zwei Kreise, während die vier Geraden sich aus $F(p, p)=0$ oder $G(p, p)=0$ ergeben und demnach aus der unendlich fernen Geraden und den Seiten des parallel eingeschriebenen Dreiecks bestehen. 
Auftreten des umschriebenen Kreises bei gewissen concentr. Kegelschnitten. 313 die des eingeschriebenen nach (33) $g(x, x) \equiv r_{y}{ }^{2} x_{1}{ }^{2}+s_{y}{ }^{2} x_{2}{ }^{2}+t_{y}{ }^{2} x_{3}{ }^{2}-2 r_{y} s_{y} x_{1} x_{2}-2 r_{y} t_{y} x_{1} x_{3}-2 s_{y} t_{y} x_{2} x_{3}=0$. Man findet hiernach $F(p, p)=\frac{1}{9}\left(y_{1}+y_{2}+y_{3}\right) r_{y} s_{y} t_{y}$,

$$
\begin{gathered}
{[a, \omega]=-2\left(\omega_{23} y_{1}^{2}+\omega_{31} y_{2}^{2}+\omega_{12} y_{3}^{2}+\omega_{11} y_{2} y_{3}+\omega_{22} y_{3} y_{1}+\omega_{33} y_{1} y_{2}\right),} \\
G(p, p)=\frac{4}{9}\left(y_{1}+y_{2}+y_{3}\right) r_{y} s_{y} t_{y}, \\
{[b, \omega]=-4\left(\omega_{23} y_{1}^{2}+\omega_{31} y_{2}^{2}+\omega_{12} y_{3}^{2}\right) .}
\end{gathered}
$$

Bildet man nun nach (127) die Gleichung $\frac{F(p, p)}{[n, \omega]^{2}}-\frac{G(p, p)}{[b, \omega]^{2}}=0$, so erhält man

$$
\begin{aligned}
\left(\omega_{23} y_{1}^{2}+\omega_{31} y_{2}^{2}+\omega_{12} y_{3}^{2}+\omega_{11} y_{2} y_{3}+\omega_{22} y_{3} y_{1}+\omega_{33} y_{1} y_{2}\right)^{2} \\
-\left(\omega_{23} y_{1}{ }^{2}+\omega_{31} y_{2}{ }^{2}+\omega_{12} y_{3}{ }^{2}\right)^{2}=0
\end{aligned}
$$

Diese Differenz zerfällt sofort in die zwei Factoren

und

$$
\omega_{11} y_{2} y_{3}+\omega_{22} y_{3} y_{1}+\omega_{33} y_{1} y_{2}=0
$$

$$
2\left(\omega_{23} y_{1}{ }^{2}+\omega_{31} y_{2}{ }^{2}+\omega_{12} y_{3}{ }^{2}\right)+\omega_{11} y_{2} y_{3}+\omega_{22} y_{3} y_{1}+\omega_{33} y_{1} y_{2}=0,
$$

von denen der erste nach (73) den umschriebenen Kreis des Dreiecks darstellt, der zweite einen Kreis, welcher dem System (Büschel) von Kreisen

$$
\lambda\left(\omega_{23} y_{1}{ }^{2}+\omega_{31} y_{2}{ }^{2}+\omega_{12} y_{3}{ }^{2}\right)+\omega_{11} y_{2} y_{3}+\omega_{22} y_{3} y_{1}+\omega_{33} y_{1} y_{2}=0
$$

angehört. In diesem Büschel sind ïberhaupt einige besonders wichtige Curven enthalten: $\lambda=0$ ergibt den umschriebenen Kreis des Dreiecks, $\lambda=1$ den Feuerbach'schen Kreis, $\lambda=\infty$ denjenigen Kreis, für welchen das Dreieck ein Poldreieck ist.

179. Man beweise nachstehenden von Steiner ohne Beweis mitgetheilten Satz $\left.{ }^{1}\right)$ :

"Werden durch irgend einen Punkt $p$ in der Ebene eines gegebenen Dreiecks $A B C$ diejenigen drei Geraden $r r_{1}, s s_{1}, t t_{1}$ gezogen, welche beziehlich von den Seiten $A$ und $B, B$ und $C, C$ und $A$ begrenzt und durch den Punkt $p$ gehälftet werden, so liegen ihre drei Paar Endpunkte $r, r_{1} ; s, s_{1} ; t, t_{1}$ allemal in irgend einem Kegelschnitte $C^{2}$, welcher nothwendigerweise den Punkt $p$ zum Mittelpunkt hat."

1) „Lehrsätze“. Journal für die reine und angewandte Mathematik, Bd. 45, S. 177, 1852, oder auch "Gesammelte Werke", Bd. 2, S. 431. 
Bevor wir auf den Beweis dieses speciellen Satzes eingehen, möge ein allgemeinerer Satz von Steiner bewiesen werden, welcher lautet ${ }^{1}$ ): „Durch jeden Punkt $P$ in der Ebene einer gegebenen Curve $C^{m}$ gehen im allgemeinen $\frac{1}{2} m(m-1)$ Sehnen $S$, und ihre $m(m-1)$ Endpunkte $\left(a\right.$ und $a_{1}$ ) liegen allemal in einer um einen Grad niedrigeren Curve $J^{m-1}$, welche nothwendigerweise den Pol $P$ zum Mittelpunkt hat." Dabei bezeichnet Steiner als "Sehnen" solche durch $P$ gezogene Strahlen, für welche irgend zwei ihrer Schnittpunkte mit $C^{m}$ gleichweit von $P$ abstehen und auf entgegengesetzten Seiten von $P$ liegen. Den Ort $J^{m-1}$ nennt Steiner die ,innere Polare des Pols $P$ in Bezug auf die Basis $C^{m \text { ". }}$

Zum Beweis dieses Satzes sei $f\left(x_{1}, x_{2}, x_{3}\right)=0$ die Gleichung der gegebenen Curve $C^{m}$, der Pol $P$ habe die Coordinaten $y_{1}, y_{2}, y_{3}$, und $u_{x}=0$ sei eine vorläufig beliebig, aber fest gewählte Gerade. Wir fixiren nuu auf $C^{m}$ irgend einen Punkt $z$ und fragen, welcher Bedingung die Coordinaten desselben ausser $f\left(z_{1}, z_{2}, z_{3}\right)=0$ noch geniigen müssen, damit ein weiterer Schnittpunkt $z^{\prime}$ der Geraden $y z$ und der Curve zusammen mit $z$ harmonisch liege zu $y$ und zu dem Schnittpunkte der Geraden $y z$ mit $u_{x}=0$. Der letztere hat jedenfalls die Coordinaten $z_{i} u_{y}-y_{i} u_{z}(i=1,2,3)$, oder auch $z_{i}-\lambda_{1} y_{i}$, wenn man $u_{z}: u_{y}=\lambda_{1}$ setzt; der Punkt $z^{\prime}$ möge die Coordinaten haben $z_{i}-\lambda y_{i}$. Wir haben dann auf der Geraden $y z$ ein Punktepaar mit den Coordinaten $z_{i}-\lambda_{1} y_{i}$, resp. $y_{i}, \quad(i=1,2,3)$, und ein solches wit den Coordinaten

und

$$
z_{i}=\left(z_{i}-\lambda_{1} y_{i}\right)+\lambda_{1} y_{i}
$$

$$
z_{i}-\lambda y_{i}=\left(z_{i}-\lambda_{1} y_{i}\right)+\left(\lambda_{1}-\lambda\right) y_{i}, \quad(i=1,2,3) .
$$

Die Bedingung, dass beide zu einander harmonisch liegen, ist

$$
\frac{\lambda_{1}}{\lambda_{1}-\lambda}=-1
$$

woraus $\lambda=2 \lambda_{1}$ folgt; der Punkt $z^{\prime}$ hat demnach die Coordinaten $z_{i}-2 \lambda_{1} y_{i}$, welche nun noch der Gleichung $f\left(z_{1}{ }^{\prime}, z_{2}{ }^{\prime}, z_{3}{ }^{\prime}\right)=0$ genügen sollen. Man erhält hierdurch $f\left(z_{1}-2 \lambda_{1} y_{1}, z_{2}-2 \lambda_{1} y_{2}, z_{3}-2 \lambda_{1} y_{3}\right)=0$,

1) „Ueber solche algebraische Curven, welche einen Mittelpunkt haben, und über darauf bezügliche Eigenschaften allgemeiner Curven, sowie über geradlinige Transversalen der letzteren." Journal für die reine und angewandte Mathematik, Bd. 47, S. 32 f., 1851, oder auch "Gesammelte Werke“t, Bd. 2, S. 527f. Vgl. auch die Abhandlung von Herrn Güssfeldt: „Ueber Curven, welche einen harmonischen Pol und eine harmonische Gerade besitzen, und darauf bezügliche Eigenschaften allgemeiner algebraischer Curven, mit besonderer Berücksichtigung der Curven dritter Ordnung." Math. Annalen, Bd. 2, S. 83, 1868. 
und dies ist die Gleichung einer Curve $(m-1)^{\text {ter }}$ Ordnung, denn in der Entwickelung dieser Function fällt das erste Glied $f\left(z_{1}, z_{2}, z_{3}\right)$ weg, weil der Punkt $z$ auf der Curve $f\left(z_{1}, z_{2}, z_{3}\right)=0$ liegt. Der übrig bleibende Ausdruck stellt die oben geforderte Bedingung dar.

Es ist nun klar, dass man zum Beweis des oben an erster Stelle ausgesprochenen Steiner'schen Satzes nur nöthig hat, die Gerade $u_{x}=0$ mit der unendlich fernen $p_{x}=0$ zusammenfallen zu lassen und als gegebene Curve $m^{\text {ter }}$ Ordnung die aus den drei Seiten des Coordinatendreiecks bestehende ausgeartete Curve dritter Ordnung $z_{1} z_{2} z_{3}=0$ zu nehmen. Die Punktepaare $r, r_{1} ; s, s_{1} ; t, t_{1}$ liegen dann auf einem Kegelschnitt $C^{2}$, dessen Gleichung sich aus

$$
\left(z_{1}-2 \lambda_{1} y_{1}\right)\left(z_{2}-2 \lambda_{1} y_{2}\right)\left(z_{3}-2 \lambda_{1} y_{3}\right)=0
$$

ergibt, weun man hier das Product $z_{1} z_{2} z_{3}$ weglässt und $\lambda_{1}=p_{i}: p_{y}$ setzt; die Gleichung lautet:

$$
\begin{aligned}
4 y_{1} y_{2} y_{3} p_{z}{ }^{2}-2\left(y_{2} y_{3} z_{1}\right. & \left.+y_{3} y_{1} z_{2}+y_{1} y_{2} z_{3}\right) p_{y} p_{z} \\
& +p_{y}{ }^{2}\left(y_{1} z_{2} z_{3}+y_{2} z_{3} z_{1}+y_{3} z_{1} z_{2}\right)=0 .
\end{aligned}
$$

Steiner stellt a. a. O. noch einen weiteren Satz auf hinsichtlich eines Kegelschuitts, der zu dem soeben erhaltenen und zu dem Pole $p$ in einer einfachen Beziehung steht, nämlich:

„Zieht man ferner aus demselben Punkte $p$ Strahlen $\alpha, \beta, \gamma$ nach den Ecken $a, b, c$ des Dreiseits und coustruirt in jeder Ecke zu den zwei anliegenden Seiten und dem jedesmaligen Strahle den vierten, dem letzteren zugeordneten, harmonischen Strahl, beziehlich $\alpha_{1}, \beta_{1}$ und $\boldsymbol{\gamma}_{1}$, so werden diese drei neuen Strahlen in den respectiven Ecken des Dreiecks allemal von einem solchen Kegelschnitte $C_{1}^{2}$ berührt, welcher jenem Kegelschnitte $C^{2}$ ähnlich ist und mit ihm ähnlich liegt, so dass die sich entsprechenden Axen beider Kegelschnitte parallel sind, ebenso ihre Asymptoten, falls sie Hyperbeln sind."

Die eben genannten vierten harmonischen Strahlen haben, wie man leicht sieht, die Gleichungen: $y_{3} x_{2}+y_{2} x_{3}=0, y_{3} x_{1}+y_{1} x_{3}=0$, $y_{2} x_{1}+y_{1} x_{2}=0$, wenn wieder $y_{i}(i=1,2,3)$ die Coordinaten des Punktes $p$ bedeuten; da der Kegelschnitt $C_{1}{ }^{2}$ durch die Ecken des Dreiecks gehen soll, hat er jedenfalls eine Gleichung von der Form $a_{1} x_{2} x_{3}+a_{2} x_{3} x_{1}+a_{3} x_{1} x_{2}=0$, und die Tangenten desselben in den Ecken des Dreiecks

$$
a_{3} x_{2}+a_{2} x_{3}=0, \quad a_{3} x_{1}+a_{1} x_{3}=0, \quad a_{2} x_{1}+a_{1} x_{2}=0
$$

sind mit den obigen vierten harmonischen Strahlen identisch, wenn man setzt $a_{1}=y_{1}, a_{2}=y_{2}, a_{3}=y_{3}$. Die Gleichung des Kegelschnitts $C_{1}{ }^{2}$ ist demnach $y_{1} x_{2} x_{3}+y_{2} x_{3} x_{1}+y_{3} x_{1} x_{2}=0$, woraus nach (42) 
hervorgeht, dass diese Curve mit der konischen Polare des Punktes $y$ in Bezug auf $x_{1} x_{2} x_{3}=0$ identisch ist. Sie ist zu der S. 315 erhaltenen Curve $C^{2}$ ähnlich und ähnlich gelegen, denn beide Curven werden von der unendlich fernen Geraden in denselben Punkteu getroffen.

Auch alle übrigen von Steiner a. a. O. aufgeführten Sätze über die gegenseitige Beziehung der Curven $C^{2}$ und $C_{1}{ }^{2}$ folgen nun leicht, so z. B. dass durch jeden dem Dreieck umschriebenen Kegelschnitt $C_{1}{ }^{2}$ der Punkt $p$, sowie der ihm zugehörige Kegelschnitt $C^{2}$ bestimmt ist. Denn die Coordinaten $y_{i}$ des Punktes $p$ sind gleich den Coefficienten $a_{i}$ in der Gleichung des umschriebenen Kegelschnitts. Ferner ist nunmehr bewiesen: „Es gibt nur einen Pol $p$, für welchen der zugehörige Kegelschnitt $C^{2}$ ein Kreis wird, oder bei welchem die drei Geraden $r r_{1}, s s_{1}, t t_{1}$ einander gleich werden; derselbe wird durch den dem Dreieck $a b c$ umschriebenen Kreis bestimmt.“

Die beiden Kegelschnitte sind gleichseitige Hyperbeln, wenn der Pol $p$ auf der Geraden $\omega_{23} y_{1}+\omega_{31} y_{2}+\omega_{12} y_{3}=0$ liegt, denn auf diese Gleichung reduciren sich die Ausdrücke für die Curven $C^{2}$ und $C_{1}{ }^{2}$ bei Anwendung der Bedingung $[a, \omega]=0$ für die gleichseitige Hyperbel. Die so erhaltene Gerade ist nach (24) und (21) diejenige, auf welcher die drei Schnittpunkte der Seiten des Dreiecks mit den Verbindungslinien der gegenüberliegenden Höhenfusspunkte gelegen sind.

Soll der Kegelschnitt $C_{1}{ }^{2}$ eine Parabel sein, so zerfällt $C^{2}$ in ein Paar ron Parallelen, die von $p$ gleichweit abstehen und mit der Axe der Parabel gleiche Richtung haben. Denn wenn $C_{1}{ }^{2}$ eine Parabel darstellt, haben beide Kegelschnitte mit der unendlich fernen Geraden dieselben zwei unendlich nahen Punkte gemeinsam, doch kann $C^{2}$ keine Parabel sein, da der Mittelpunkt $p$ von $\dot{C}^{2}$ im Endlichen liegt, es muss demnach $C^{2}$ in ein Parallelenpaar zerfallen, dessen Mittelpunktslinie durch $p$ geht. Der Kegelschnitt $C_{1}{ }^{2}$ ist eine Parabel, wenn der Punkt $y$ anf der Curve liegt

$$
p_{1}^{2} y_{1}^{2}+p_{2}{ }^{2} y_{2}^{2}+p_{3}{ }^{2} y_{3}{ }^{2}-2 p_{2} p_{3} y_{2} y_{3}-2 p_{3} p_{1} y_{3} y_{1}-2 p_{1} p_{2} y_{1} y_{2}=0
$$

ihre Gleichung in Liniencoordinaten ist

$$
p_{1} u_{2} u_{3}+p_{2} u_{3} u_{1}+p_{3} u_{1} u_{2}=0 \text {, }
$$

und man zeigt mit deren Hilfe leicht, dass diese Curve den Scliwerpunkt des Dreiecks zum Mittelpunkt hat und die Seiten des Dreiecks in ihren Mitten berührt. (Vgl. auch (39).)

Alle diese Sätze werden von Steiner a. a. O. ohne Beweis aufgestellt. 
180. Man bestimme die Potenz eines Punktes $y$ in Bezug anf einen Kreis, dessen Radius gleich $r$ ist und dessen Mittelpunkt die Coordinaten $c_{1}, c_{2}, c_{3}$ hat.

Die Gleichung des Kreises ist nach (10a), S. 59 :

$$
K \equiv\left(\begin{array}{ll}
c & x \\
c & x
\end{array}\right)_{\omega_{i k}}-r^{2} \tau p_{c}^{2} p_{x}^{2}=0
$$

andrerseits ist nacl S. 100 die Potenz eines Punktes $y$ in Bezug auf den Kreis $f(x, x)=0$ gegeben durch $\frac{2 f(y, y)}{[a, \omega] \cdot p_{y}{ }^{2}}$. Im gegenwärtigen Falle wird $[a, \omega]$ gleich $-2\left(\begin{array}{l}c \\ c\end{array}\right)_{\omega_{i k}}=2 \tau p_{c}^{2}$, so dass man für die Potenz $P$ den Ausdruck erhält $P=\frac{[K]}{\tau^{\prime}{ }_{c}^{2} p_{y}{ }^{2}}$, wobei $[K]$ die Substitution der Coordinaten des Punktes $y$ in die linke Seite der obigen Kreisgleichung $K=0$ andeutet.

181. Man bilde die Gleichung für den geometrischen Ort aller Punkte, von denen aus zwei Krreise gleich gross erscheinen. Es seien

$K_{1} \equiv\left(\begin{array}{ll}a & x \\ a & x\end{array}\right)_{\omega_{i k}}-r_{1}{ }^{2} \tau p_{a}^{2} p_{x}{ }^{2}=0 \quad$ und $\quad K_{2}=\left(\begin{array}{ll}b & x \\ b & x\end{array}\right)_{\omega_{i k}}-r_{2}{ }^{2} \tau p_{b}{ }^{2} p_{x}{ }^{2}=0$

die Gleichungen der beiden Kreise mit den Mittelpunkten $a, b$ und den Radien $r_{1}, r_{2}$. Die trigonometrische Tangente des halben Winkels, unter dem vom Punkte $y$ aus der Kreis $K_{1}$ erscheint, ist gleich dem Quotienten aus dem Radius $r_{1}$ und der Länge der von $y$ an den Kreis gezogenen Tangente, welch letztere bekanntlich mit der Quadratwurzel aus der Potenz übereinstimmt. Man erhält daher nach (180) als Gleichung des betreffenden Ortes: $K_{3} \equiv r_{2}^{2} p_{b}^{2} K_{1}-r_{1}^{2} p_{a}^{2} K_{2}=0$ oder $r_{2}{ }^{2} p_{b}{ }^{2}\left(\begin{array}{ll}a & x \\ a & x\end{array}\right)_{\omega_{i k}}-r_{1}{ }^{2} p_{a}{ }^{2}\left(\begin{array}{ll}b & x \\ b & x\end{array}\right)_{\omega_{i k}}=0$. Diese Gleichung stellt nach (69) einen Kreis dar, dessen Mittelpunkt auf der Centrale von $K_{1}$ und $K_{2}$ liegt. Die Punkte der Centrale, von denen aus die zwei gegebenen Kreise gleich gross erscheinen, sind der äussere und der innere Aehnlichkeitspunkt (Schnittpunkt der zwei äusseren, bezw. der zwei inneren gemeinschaftlichen Tangenten); der Kreis $K_{3}=0$ hat also die Verbindungslinie dieser beiden Punkte zum Durchmesser (Aehnlichkeitskreis).

182. Die Gleichung der Linie gleicher Potenzen (Potenzlinie, Radicalaxe) zweier durch

$f(x, x) \equiv \sum_{1}^{3} i \sum_{1}^{3} k a_{i k} x_{i} x_{k}=0 \quad$ und $\quad g(x, x) \equiv \sum_{1}^{3} \sum_{1}^{3} b_{i k} x_{i} x_{k}=0$

gegebenen Kreise zu bilden. 
Ist $y$ ein Punkt der Potenzlinie, so muss nach (55), S. 100 die Relation stattfinden $\frac{2 f(y, y)}{[a, \omega] p_{y}{ }^{2}}=\frac{2 g(y, y)}{[b, \omega] p_{y}{ }^{2}}$, oder auch

$$
[b, \omega] f(y, y)-[a, \omega] g(y, y)=0 .
$$

Diese Gleichung muss $p_{y}$ als Factor enthalten (vgl. dic Fussnote zu S. 59); der andere, nach der auf S. 38 angegebenen Methode zu bestimmende Factor stellt die Potenzlinie dar.

183. Die Bedingung dafür, dass sich die zwei Kreise $f(x, x)=0$ und $\varphi(u, u)=0$ rechtwinklig schneiden, ist

oder auch

$$
4 \tau \varphi(p, p) \cdot[\alpha, a]-[\mathrm{A}, \omega] \cdot[a, \omega]=0
$$

$$
4[\alpha, a] \cdot[\alpha, \Omega]-[\mathrm{A}, \omega] \cdot[a, \omega]=0 .
$$

Nach Multiplication mit einem gewissen Factor $\mu$ geht $\varphi(u, u)=0$ in die Normalform $\varrho^{2} p_{y}{ }^{2} \omega(u, u)-u_{y}{ }^{2}=0$ (vgl. (2), S. 57) über, so dass also $\mu . \varphi(u, u)=\varrho^{2} p_{y}{ }^{2} \omega(u, u)-u{ }_{y}{ }^{2}$, wobei $\varrho$ den Radius, $y$ den Mittelpunkt des Kreises $\varphi(u, u)=0$ bezeichnet. Zur Bestimmung des Factors $\mu$ setzen wir $u_{i}=p_{i}(i=1,2,3)$ und finden sofort $\mu=-p_{y}{ }^{2}: \varphi(p, p)$, also $u_{y}{ }^{2}=\rho^{2} p_{y}{ }^{2} \omega(u, u)+\frac{\varphi(u, u) \cdot p_{y}{ }^{2}}{\varphi(p, p)}$. Die zwei Kreise schneiden sich rechtwinklig, wenn die Potenz des Mittelpunktes $y$ von $\varphi(u, u)=0$ in Bezug auf deu Kreis $f(x, x)=0$ gleich $\varrho^{2}$ wird, d. h. $\operatorname{nach}(55)$, S. 100, wenn $\frac{2 f(y, y)}{[a, \omega] p_{y}{ }^{2}}=\rho^{2}$ oder $f(y, y)=\frac{\rho^{2} \cdot[a, \omega] \cdot p_{y}{ }^{2}}{2}$. Andrerseits muss die soeben abgeleitete Gleichung für $u_{y}{ }^{2}$ gelten, welche Werthe auch die $u_{i}$ haben mögen, sie gilt daher nach (35), S. 76 auch noch, wenn man die Producte $u_{i} u_{k}$ ersetzt durch $a_{i k}$; man erhält alsdann $f(y, y)=\varrho^{2} p_{y}{ }^{2}[a, \omega]+\frac{[\alpha, a] p_{y}{ }^{2}}{\varphi(p, p)}$. Aus den zwei für $f(y, y)$ angegebenen Ausdrücken folgt durch Gleichsetzen $\frac{\varrho^{2} \cdot[a, \omega]}{2}+\frac{[\alpha, a]}{\varphi(p, p)}=0$, und wenn man für $\varrho^{2}$ seinen in (138) angegebenen Werth $\frac{-[A, \omega]}{2 \tau \varphi^{2}(p, p)}$ einsetzt, ergibt sich $\frac{-[A, \omega] \cdot[a, \omega]}{4 \tau \varphi(p, p)}+[\alpha, a]=0$ oder

$$
4 \tau \varphi(p, p) \cdot[\alpha, a]-[\mathrm{A}, \omega] \cdot[a, \omega]=0,
$$

wofür auch zufolge $\tau p_{i} p_{k}=\Omega_{i k}$ gesetzt werden kann

$$
4[\alpha, \Omega] \cdot[\alpha, a]-[\mathrm{A}, \omega] \cdot[a, \omega]=0 . \quad \text { Vgl. (224.) }
$$

184. Man berechne die Potenz eines Punktes $y$ in Bezug auf den dem Coordinatendreieck umschriebenen Kreis. 
Die Gleichung dieses Kreises ist nach (73)

daher

$$
\omega_{11} p_{1} x_{2} x_{3}+\omega_{22} p_{2} x_{3} x_{1}+\omega_{33} p_{3} x_{1} x_{2}=0,
$$

$$
\begin{gathered}
{[a, \omega]=\omega_{11} p_{1} \omega_{23}+\omega_{22} p_{2} \omega_{31}+\omega_{33} p_{3} \omega_{12}=} \\
-2\left(\omega_{12} \omega_{13} p_{1}+\omega_{21} \omega_{23} p_{2}+\omega_{31} \omega_{32} p_{3}\right)=-2 \tau p_{1} p_{2} p_{3} .
\end{gathered}
$$

Für die Potenz fiudet man somit nach (55), S. 100 den Wertlı

$$
P_{U}=\frac{\omega_{11} p_{1} y_{2} y_{3}+\omega_{22} p_{2} y_{3} y_{1}+\omega_{33} p_{3} y_{1} y_{9}}{-\tau p_{1} p_{2} p_{3}\left(p_{1} y_{1}+p_{2} y_{2}+p_{3} y_{3}\right)^{2}}
$$

185. Dieselbe Aufgabe in Bezug auf denjenigen Kreis, für welchen das Coordinatendreieck Poldreieck ist.

Die Gleichung dieses Kreises ist nach (75)

$$
\begin{gathered}
\omega_{23} p_{1} x_{1}{ }^{2}+\omega_{31} p_{2} x_{2}{ }^{2}+\omega_{12} p_{3} x_{3}{ }^{2}=0, \\
\text { daher }[a, \omega]=\omega_{23} p_{1} \omega_{11}+\omega_{31} p_{2} \omega_{22}+\omega_{12} p_{3} \omega_{33}=-2 \tau p_{1} p_{2} p_{3} \text { (wie } \\
\text { zuvor) und } P_{P}=\frac{\omega_{23} p_{1} y_{1}{ }^{2}+\omega_{31} p_{2} y_{2}{ }^{2}+\omega_{12} p_{3} y_{3}{ }^{2}}{-\tau p_{1} p_{2} p_{3}\left(p_{1} y_{1}+p_{2} y_{2}+p_{3} y_{3}\right)^{2}}
\end{gathered}
$$

186. Dieselbe Aufgabe für den Feuerbach'schen Kreis.

Die Gleichung desselben ist nach (165)

$$
f(x, x) \equiv 2\left(\omega_{23} x_{1} r_{x}+\omega_{31} x_{2} s_{x}+\omega_{12} x_{3} t_{x}\right)=0,
$$

daher $[a, \omega]=-4\left(\omega_{23} p_{1} \omega_{11}+\omega_{31} p_{2} \omega_{22}+\omega_{12} p_{3} \omega_{33}\right)=8 \tau p_{1} p_{2} p_{3}$ (wie bei (184)) und $P_{F}=\ldots f(y, y)$

$$
4 \tau p_{1} p_{2} p_{3}\left(p_{1} y_{1}+p_{2} y_{2}+p_{3} y_{3}\right)^{2}
$$

187. Man berechne die Grösse $a^{2} \pm l^{2}$ für den dem Coordinatendreieck umschriebenen Kegelschnitt mit dem Mittelpunkte $y$; dabei gilt in $a^{2} \pm b^{2}$ das positive oder negative Vorzeichen, je naclidem der Kegelschnitt eine Ellipse oder Hyperbel ist.

Für die Curve $f(x, x)=0$ hat man nach (135) die Relation $a^{2} \pm b^{2}=-\frac{A[a, \omega]}{\tau F^{2}(p, p)} ;$ durch Substitution der in (139) gefundenen Werthe von $A$ und $F(p, p)$ ergibt siclı auf ähnliche Weise und bei gleicher Bezeichnung wie in (156):

$$
a^{2} \pm b^{2}=\frac{-[a, \omega] q_{1} q_{2} q_{3}}{p_{1} p_{2} p_{3} q_{1}^{\prime} q_{2}^{\prime} q_{3}{ }^{\prime} \cdot 4 \tau p_{y}{ }^{2}}
$$

wobei $[a, \omega]=2\left(y_{1} r_{y} \omega_{23}+y_{2} s_{y} \omega_{31}+y_{3} t_{y} \omega_{12}\right)$, so dass $[a, \omega]=0$ nach (165) die Gleichung des Feuerbach'schen Kreises wäre. Mit Hilfe der in (186) abgeleiteten Potenz $\mathcal{P}_{F}$ eines Punktes $y$ in Bezug auf diesen Kreis findet man $a^{2} \pm b^{2}=-\frac{q_{1} q_{2} q_{3}}{q_{1}^{\prime} q_{2}^{\prime} q_{3}{ }^{\prime}} P_{F}$.

188. Dieselbe Aufgabe für den dem Coordinatendreieck eingeschriebenen Kegelschnitt mit dem Mittelpunkte $y$. 
Für die Curve $\varphi(u, u)=0$ lat man nach (136) die Relation $a^{2} \pm b^{2}=-\frac{[\mathrm{A}, \infty]}{\tau \varphi^{2}(p, p)} ;$ durch Substitution der in (140) angegebenen Werthe von $[\mathrm{A}, \omega]$ und $\varphi(p, p)$ erhält man

$$
a^{2} \pm b^{2}=-\frac{\left(\omega_{23} p_{1} y_{1}{ }^{2}+\omega_{31} p_{2} y_{2}{ }^{2}+\omega_{12} p_{3} y_{3}{ }^{2}\right)}{\tau p_{1} p_{2} p_{3}\left(p_{1} y_{1}+p_{2} y_{2}+p_{3} y_{3}\right)^{2}}
$$

Mit Rücksicht auf das in (185) erhaltene Resultat erkennt man sofort, dass $a^{2} \pm b^{2}$ gleich der Potenz des Mittelpunktes $y$ in Bezug auf denjenigen Kreis ist, der das Coordinatendreieck zum Poldreieck hat.

189. Dieselbe Aufgabe für diejenige Curve zweiter Ordnung mit dem Mittelpunkte $y$, die das Coordinatendreieck zun Poldreieck hat. Mit Hilfe der in (141) angegebenen Werthe von $A,[a, \omega]$ und $F(p, p)$ findet $\operatorname{man} a^{2} \pm b^{2}=-\frac{\left(\omega_{11} p_{1} y_{2} y_{3}+\omega_{22} p_{2} y_{3} y_{1}+\omega_{33} p_{3} y_{1} y_{2}\right)}{\tau p_{1} p_{2} p_{3}\left(p_{1} y_{1}+p_{2} y_{2}+p_{3} y_{3}\right)^{2}}$ es ist also $a^{2} \pm b^{2}$ nach (184) gleich der Potenz des Mittelpunktes $y$ in Bezug auf den dem Coordinatendreieck umschriebenen Kreis.

190. Ist $\alpha$ der Asymptotenwinkel des einem Dreieck umschriebenen Kegelschnitts mit dem Mittelpunkte $y$, so besteht die einfache Relation $\operatorname{tg}^{2} \alpha=-\frac{4 q_{1}{ }^{\prime} q_{2}{ }^{\prime} q_{3}{ }^{\prime} \cdot r}{P_{F^{\prime}}{ }^{2}}$, worin $r$ den Radius des dem Dreieck umschriebenen Kreises bezeichnet und die Grössen $q^{\prime}$, sowie $\boldsymbol{P}_{\boldsymbol{F}}$ dieselbe Bedeutung haben wie in (187).

Führt man in die Formel $\operatorname{tg}^{2} \alpha=-\frac{4 \tau F(p, p)}{[a, \omega]^{2}}$ (vgl. (6), S. 111) des Asymptotenwinkels die Halbaxen $a$ und $b$ des Kegelschnitts ein, so wird $\operatorname{tg}^{2} \alpha=\frac{\mp 4 a^{2} b^{2}}{\left(a^{2} \pm b^{2}\right)^{2}}$, wobei das obere oder untere Vorzeichen zu stehen hat, je nachdem die Curve eine Ellipse oder Hyperbel ist. Mit Hilfe von (157) und (187) folgt alsdanu die obige Relation.

Auf gleiche Weise fiudet man die folgenden zwei Sätze:

191. Ist $\alpha$ der Asymptotenwinkel des einem Dreieck eingeschriebenen Kegelschnitts mit dem Mittelpunkte $y$, so besteht die Relation $\operatorname{tg}^{2} \alpha=-\frac{16 q_{1}{ }^{\prime} q_{2}^{\prime} q_{3}{ }^{\prime} r}{P_{P}{ }^{2}}$, worin $P_{P}$ die Potenz des Punktes $y$ in Bezug auf denjenigen Kreis bezeichnet, für welchen das betr. Dreieck Poldreieck ist.

192. Ist $\alpha$ der Asymptotenwinkel desjenigen Kegelschnitts, welcher ein gegebenes Dreicck zum Poldreieck und den Punkt $y$ zum Mittelpunkt hat, so besteht die Relation $\operatorname{tg}^{2} \alpha=\frac{-8 q_{1} q_{2} q_{3} \cdot v^{*}}{P_{U}{ }^{2}}$, worin $P_{U}$ die 
Potenz des Punktes $y$ in Bezug auf den dem betr. Dreieck umschriebenen Kreis bezeichnet.

193. Welches ist der geometrische Ort aller Punkte $x$, für die das Verhältniss des Quadrats ihres Abstandes von einer festen Geraden $v_{x}=0 \mathrm{zu}$ der Potenz in Bezug auf einen Kreis vom Radius $v$ und mit dem Mittelpunkte $c_{1}, c_{2}, c_{3}$ gleich einer Constanten $1: \lambda^{2}$ ist?

Mit Hilfe der Formel (1), S. 9 für den Abstand eines Punktes von einer Geraden und der in (180) abgeleiteten Formel für die Potenz in Bezug auf einen durch Mittelpunkt und Radius gegebenen Kreis findet man als Gleichung des Ortes:

oder

$$
\lambda^{2} \tau p_{c}^{2} v_{x}^{2}=\omega(v, v)\left[\left(\begin{array}{cc}
c & x \\
c & x
\end{array}\right)_{\omega_{i k}}-r^{2} \tau p_{c}^{2} \gamma^{2} x^{2}\right]
$$

$$
f(x, x) \equiv\left(\begin{array}{l}
c x \\
c x
\end{array}\right)_{\omega_{i k}}-r^{2} \tau p_{c}{ }^{2} p_{x}{ }^{2}-\frac{\lambda^{2} \tau p_{c}{ }^{2} v_{x}{ }^{2}}{\omega(v, v)}=0 .
$$

Diese Gleichung repräsentirt einen Kegelschnitt, und zwar von gleicher Art wie $\left(\begin{array}{ll}c & x \\ c & x\end{array}\right)_{\omega_{i k}}-\frac{\lambda^{2} \tau p_{c}{ }^{2} v_{x}{ }^{2}}{\omega(v, v)}=0$, denn beide werden von $p_{x}{ }^{2}=0$ in denselben Punkten getroffen; nach S. 119, wo die letztgenannte Curve bereits auftrat, ist daher der Kegelschnitt eine Ellipse, Hyperbel oder Parabel, je nachdem die positive Constante $\lambda<1,>1,=1$.

Es möge nun noch die Gleichung in Liniencoordinaten für obigen Kegelschnitt abgeleitet werden; in Punktcoordinaten hat man einen Ausdruck von der Form $\left(\begin{array}{ll}c & x \\ c & x\end{array}\right)_{()_{i k}}+m p_{x}{ }^{2}+n v_{x}{ }^{2}=0$, wenn zur Abkürzung gesetzt ist $m=-r^{2} \tau p_{c}{ }^{2}, n=-\lambda^{2} \tau p_{c}{ }^{2}: \omega(v, v)$. Die Gleichung in Liniencoordinaten von $g(x, x) \equiv\left(\begin{array}{ll}c & x \\ c & x\end{array}\right)_{\omega_{i k}}+n v_{x}{ }^{2}=0$ lautet nach S. $119 \mathrm{f}$ :

$$
G(u, u) \equiv u\left\{\omega(v, v) u_{c}{ }^{2}+v_{c}{ }^{2} \omega(u, u)-2 v_{c} u_{c} \omega(v, u)\right\}+\tau p_{c}{ }^{2} u_{c}{ }^{2}=0
$$

nun ist aber die Gleichung in Liniencoordinaten zu bilden für

$$
g(x, x)+m p_{x}^{2}=0 .
$$

Der Coefficient von $m^{2}$ in derselben verschwindet identisch ${ }^{1}$ ), derjenige von $m^{1}$ geht aus $g(x, x)$ dadurch hervor, dass man $x_{1}$ ersetzt durch $p_{2} u_{3}-p_{3} u_{2}$ und analog bei $x_{2}, x_{3}$ verfährt; der Coefficient von $m^{0}$ wird $G(u, u)$. Es ergibt sich also

$$
\begin{aligned}
G(u, u)+m\left\{\omega(p, p) u_{c}{ }^{2}\right. & \left.+p_{c}{ }^{2} \omega(u, u)-2 p_{c} u_{c} \omega(p, u)\right\} \\
& +m n \sum \pm\left(p_{1} v_{2} u_{3}\right)^{2}=0,
\end{aligned}
$$

1) Vgl. hier und im Folgenden die Bemerkungen $\mathrm{S} .119 \mathrm{f}$. 
und wenn man für $m$ und $n$ ihre Werthe einsetzt, sowie die Relationen berücksichtigt

$\omega(p, p)=0, \omega(p, u)=0, \tau \sum \pm\left(p_{1} v_{2} u_{3}\right)^{2}=\omega(u, u) \omega(v, v)-\omega^{2}(v, u)$,

folgt schliesslich als Gleichung der Curve in Liniencoordinaten:

$\varphi(u, u) \equiv\left\{\left(1-\lambda^{2}\right) r^{2} p_{c}{ }^{2} \omega(v, v)+\lambda^{2} v_{c}{ }^{2}\right\} \omega(u, u)+\left(\lambda^{2}-1\right) \omega(v, v) u_{c}{ }^{2}$ $-2 \lambda^{2} v_{c} u_{c} \omega(v, u)+\lambda^{2} r^{2} p_{c}^{2} \omega^{2}(v, u)=0$.

Für $\lambda==1$ erhält man einfach

$$
v_{c}^{2} \omega(u, u)-2 v_{c} u_{c} \omega(v, u)+r^{2} p_{c}^{2} \omega^{2}(v, u)=0,
$$

woraus (ähnlich wie S. 120) hervorgeht, dass das Brennpunktepaar in diesem Falle gegeben ist durch $\omega(v, u)\left\{r^{2} p_{c}^{2} \omega(v, u)-2 v_{c} u_{c}\right\}=0$, der im Unendlichen gelegene Brennpunkt liegt also im Normalencentrum der Geraden $v_{x}=0$.

Im Falle $\lambda \gtrless 1$ hat man für das eine Brennpunktepaar die Gleichung $\left(\lambda^{2}-1\right) \omega(v, v) u_{c}^{2}-2 \lambda^{2} v_{c} u_{c} \omega(v, u)+\lambda^{2} r^{2} p_{c}^{2} \omega^{2}(v, u)=0$, welche nach Multiplication mit $\left(\lambda^{2}-1\right) \omega(v, v)$ in zwei sich nur im Vorzeichen der Quadratwurzel unterscheidende Factoren zerfällt, nämlich:

$$
\begin{gathered}
\left(\lambda^{2}-1\right) \omega(v, v) u_{c}-\lambda^{2} v_{c} \omega(v, u) \\
\pm \lambda V \lambda^{2} v_{c}^{2}-r^{2} p_{c}^{2}\left(\lambda^{2}-1\right) \omega(v, v) \omega(v, u)=0 .
\end{gathered}
$$

Diese beiden Brennpunkte sind stets reell, wenn die positive Constante $\lambda<1$, und für $\lambda>1$ nur dann, wenn der Ausdruck unter der Wurzel positiv ist. Sie liegen, wie ihre Gleichungen zeigen, auf der vom Mittelpunkte $c$ des gegebenen Kreises auf die Gerade $v_{x}=0$ gefällten Normale. Für den Mittelpunkt $\mu_{u}=0$ des Kegelschnitts $\varphi(u, u)=0$ fiudet man sofort die Gleichung

$$
\mu_{u} \equiv \frac{\varphi(p, u)}{p_{c}}=\left(\lambda^{2}-1\right) \omega(v, v) u_{c}-\lambda^{2} v_{c} \omega(v, u)=0 .
$$

Die Verbindungslinie dieses Punktes mit dem unendlich fernen Punkte der Geraden $v_{x}=0$, also die Axe, welche zur Verbindungslinie der beiden eben erwähnten Brennpunkte normal ist, hat die Gleichung $v_{c} p_{x}+\left(\lambda^{2}-1\right) p_{c} v_{x}=0$.

Nach Multiplication mit $\left(\lambda^{2}-1\right) \omega(v, v)$ ergibt sich für die Gleichung der Curve bei Benutzung des Mittelpunktes die Relation $\left(\lambda^{2}-1\right) \omega(v, v) \cdot \varphi(u, u) \equiv\left[\left(\lambda^{2}-1\right) r^{2} p_{c}{ }^{2} \omega(v, v)-\lambda^{2} v_{c}{ }^{2}\right] \omega(v, v) \cdot \omega(u, u)$
$+\mu_{u}{ }^{2}-\left[r^{2} p_{c}{ }^{2}\left(\lambda^{2}-1\right) \omega(v, v)-\lambda^{2} v_{c}{ }^{2}\right] \lambda^{2} \tau \cdot \sum \pm\left(p_{1} v_{2} u_{3}\right)^{2}=0$,

aus der man für das zweite Brennpunktepaar die Gleichung erhält

$$
\mu_{u} \pm \lambda \sqrt{\tau} \sqrt{r^{2} p_{c}{ }^{2}\left(\lambda^{2}-1\right) \omega(v, v)-\lambda^{2} v_{c}{ }^{2}} \sum \pm\left(p_{1} v_{2} u_{3}\right)=0 \text {. }
$$


Fortsetzung. Constante Summe oder Differenz d. Tang. an zwei Kreise. 323

Setzt man zur Abkürzung $r^{2}{p_{c}}^{2}\left(\lambda^{2}-1\right) \omega(v, v)-\lambda^{2} v_{c}{ }^{2}=k$ und wendet die Identität an $\omega(v, v) \omega(u, u) \equiv \omega^{2}(v, u)+\tau \sum \pm\left(p_{1} v_{2} u_{3}\right)^{2}$, so wird

$$
\begin{gathered}
\left(\lambda^{2}-1\right) \omega(v, v) \varphi(u, u) \\
\equiv k \omega^{2}(v, u)+\mu_{u}^{2}+k\left(1-\lambda^{2}\right) \tau \sum \pm\left(p_{1} v_{2} u_{3}\right)^{2}=0 .
\end{gathered}
$$

Für die Transformation dieses Kegelschnitts auf die beiden Hauptaxen ist vou Wichtigkeit die Bemerkung, dass die Gleichungen $\mu_{u}=0$, $\omega(v, u)=0, \sum \pm\left(p_{1} v_{2} u_{3}\right)=0$ den Mittelpunkt des Kegelschnitts und die unendlich fernen Punkte der beiden Hauptaxen darstellen.

Bei Anwendung von $\omega(u, u)=U_{1}^{2}+U_{2}^{2}$ und $U_{3}=\frac{\varphi(p, u)}{\varphi(p, p)}$ (vgl. (21) und (31) in $\S 10$ ) folgen unter Rücksicht auf die Identität $\omega(v, v) \cdot \boldsymbol{\omega}(u, u) \equiv \boldsymbol{\omega}^{2}(v, u)+\tau \sum\left(p_{1} v_{2} u_{3}\right)^{2}$ die Relationen

$$
U_{1}=\frac{\omega(v, u)}{\sqrt{\omega(v, v)}}, \quad U_{2}=\frac{\sqrt{\tau} \sum^{ \pm} \pm\left(p_{1} v_{2} u_{3}\right)}{\sqrt{\omega(v, v)}}, \quad U_{3}=\frac{\mu^{\prime \prime}}{\left(\lambda^{2}-1\right) \omega(v, v) p_{c}},
$$

mit Hilfe deren sich $\varphi(u, u)$ verwandelt in

$$
\varphi(u, u) \equiv \frac{k U_{1}{ }^{2}}{\lambda^{2}-1}-k U_{2}^{2}+\left(\lambda^{2}-1\right) \omega(v, v) \cdot p_{c}^{2} U_{3}^{2}=0 .
$$

Wird an Stelle von $k$ der Abstand $d$ des Kreismittelpunktes $c$ von der festen Geraden $v$ eingeführt, wobei $d=\frac{v_{c}}{\sqrt{\omega(v, v)} p_{c}}$, so erhält man vermöge der Beziehung $k=\frac{v_{c}\left\{r^{2} \lambda^{2}-r^{2}-\lambda^{2} d^{2}\right\}}{d^{2}}$ die Gleichung

$$
\frac{\varphi(u, u)}{\left(1-\lambda^{2}\right) \omega(v, v) p_{c}^{2}}=\frac{r^{2}\left(1-\lambda^{2}\right)+\lambda^{2} d^{2}}{\left(1-\lambda^{2}\right)^{2}} U^{2}+\frac{r^{2}\left(1-\lambda^{2}\right)+\lambda^{2} d^{2}}{1-\lambda^{2}} V^{2}-1=0 ;
$$

hierbei wurde noch $U_{1}: U_{2}: U_{3}$ ersetzt durch $U: V: 1$. Die Gleichung in Punktcoordinaten des auf die Hauptaxen als rechtwinklige Coordinatenaxen bezogenell Kegelschnitts $\varphi(u, u)=0$ lautet daher

$$
\frac{\left(1-\lambda^{2}\right)^{2}}{r^{2}\left(1-\lambda^{2}\right)+\lambda^{2} d^{2}} X^{2}+\frac{1-\lambda^{2}}{r^{2}\left(1-\lambda^{2}\right)+\lambda^{2} d^{2}} Y^{2}-1=0 .
$$

194. Man beweise nachstehenden Satz von Steiner $\left.{ }^{1}\right)$ :

„Sind in einer Ebene irgend zwei Kreise $A^{2}, B^{2}$ gegeben, und zieht man aus einem willkürlichen Punkte $X_{0}$ an jeden Kreis eine

1) „Ueber einige neue Bestimmungs-Arten der Curven zweiter Ordnung nebst daraus folgenden neuen Eigenschaften derselben Curven". Journal für die reine und angewandte Mathematik, Bd.45, S. 189, 1852, oder auch "Gesanmelte Werke", Bd. 2, S. 447. 
Tangente $\alpha, \beta$ und verlangt, es soll entweder die Summe, $(\alpha+\beta)$, oder der Unterschied, $(\alpha-\beta)$ oder $(\beta-\alpha)$, dieser Tangenten einer gegebenen Länge $l$ gleich sein, so ist der Ort des Punktes $X_{0}$ allemal irgend ein Kegelschnitt $C^{2}$, welcher jeden der beiden Kreise doppelt berührt (reell oder imaginär), und von dessen Axen immer die eine oder andere auf der Mittelpunktslinie $A B$ der Kreise liegt."

Sind $f(x, x)=0, g(x, x)=0$ die Gleichungen der beiden Kreise $A^{2}, B^{2}$, so ist nach (55), S. 100 und den Bedingungen der Aufgabe entsprechend:

daher

$$
\sqrt{\frac{2 f(x, x)}{[a, \omega] p_{x}^{2}}} \pm \sqrt{\frac{2 g(x, x)}{[b, \omega] p_{x}^{2}}}=l
$$

oder auch

$$
\left[\frac{2 f(x, x)}{[a, \omega]}-\frac{2 g(x, x)}{[b, \omega]}-l^{2} p_{x}\right]^{2}=\frac{8 l^{2} \cdot g(x, x) p_{x}^{2}}{[b, \omega]}
$$

$$
\left[\frac{2 g(x, x)}{[b, \omega]}-\frac{2 f(x, x)}{[a, \omega]}-l^{2} p_{x}\right]^{2}=\frac{8 l^{2} f(x, x) p_{x}{ }^{2}}{[a, \omega]} .
$$

Nun ist $\frac{2 f(x, x)}{[a, \omega]}-\frac{2 g(x, x)}{[b, \omega]}$ nach (182) gleich dem Product der Ausdrïcke für die unendlich ferne Gerade und die Radicalaxe $R_{x}$ der zwei gegebenen Kreise, also von der Form $R_{x} p_{x}$; die eine der beiden vorstehenden Gleichungen verwandelt sich also in $\left(R_{x}-l^{2} p_{x}\right)^{2}=\frac{8 l^{2} g(x, x)}{[b, \omega]}$. Hieraus folgt, dass der Ort des Punktes $X_{0}$ in der That ein Kegelschnitt $C^{2}$ ist, der z. B. den Kreis $g(x, x)=0$ in seinen Schnittpunkten mit der zur Radicalaxe parallelen Geraden $R_{x}-l^{2} p_{x}=0$ doppelt berührt; analoges findet man für $f(x, x)=0$ bei Benutzung der zweiten obigen Gleichung von $C^{2}$. Tiefere Einsicht gewährt folgender Weg.

Es ist $\frac{2 f(x, x)}{[a, \omega] p_{x}^{2}}-\frac{2 g(x, x)}{[b, \omega] p_{x}^{2}}$ gleich der Differenz aus den ins Quadrat erhobenen Längen der Tangenten, welche vom Punkte $x$ an die Kreise $A^{2}, B^{2}$ gezogen werden können. Andrerseits ist diese Differenz, wie mit Hilfe einer einfachen Figur gezeigt werden kann, gleich dem Rechteck aus dem Abstand des Punktes $x$ von der Radicalaxe und aus der doppelten Entfernung $4 c$ der zwei Kreiscentren. Hieraus folgt, dass der Kegelschnitt $C^{2}$ der geometrische Ort aller Punkte $x$ ist, für welche das Verhältnis des Quadrats ihres Abstandes von einer Geraden $4 c R_{x}{ }^{\prime}-l^{2} p_{x}=0$ zu der Potenz in Bezug auf den Kreis $g(x, x)=0$ gleich der Constanten $\frac{4 l^{2}}{(4 c)^{2}}$; dabei ist $R_{x}^{\prime}$ der Ausdruck für die Normalform von $R_{x}$ und die Gerade $4 c R_{x}^{\prime}-l^{2} p_{x}=0$ repräsentirt eine Parallele zur Radicalaxe im Abstande $\frac{l^{2}}{4 c}$. 
Man sieht nun sofort, dass die ganze Aufgabe zurückgeführt ist auf die vorhergehende und man kann somit z. B. unmittelbar ablesen, dass für $l=2 c$ der Ort der Punkte $X_{0}$ eine Parabel ist, wir verweisen daher bezüglich der weiteren Einzelheiten auf (193) und die oben citirte Abhandlung von Steiner.

195. Die Gleichung in Liniencoordinaten derjenigen Parabel, welche einen gegebenen Punkt $y$ zum Brennpunkt, eine gegebene Gerade $v$ zur Directrix hat, lautet:

$$
\varphi(u, u) \equiv v_{y} \cdot \omega(u, u)-2 u_{y} \cdot \omega(v, u)=0 .
$$

Es sind $u_{y}=0$, bezw. $\omega(v, u)=0$ die Gleichungen des im Endlichen, bezw. im Unendlichen gelegenen Brennpunktes; die Parabel wird daher nach (22), S. 115 dargestellt durch einen Ausdruck von der Form $\varphi(u, u) \equiv \omega(u, u)+\lambda u_{y} \cdot \omega(v, u)=0$. Nun muss der Pol der Geraden $v$ identisch sein mit dem Punkte $y$, es muss

$$
2 \omega(v, u)+\lambda\left[u_{y} \cdot \omega(v, v)+v_{y} \cdot \omega(v, u)\right]=0
$$

gleichbedeutend sein mit $u_{y}=0$, d. h. man hat

$$
2 \omega(v, u)+\lambda v_{y} \cdot \omega(v, u)=0 \text {, }
$$

woraus $\lambda=-2: v_{y}$.

Folgt auch aus (38) in $\S 11$ für $e=1$, sowie ans (193) für $r=0, \lambda=1$.

196. Fixirt man irgend zwei Tangenten $\left(T\right.$ und $\left.T_{1}\right)$ eines Kegelschnitts (mit der Berührungssehne $S$ ) und den einen Brennpunkt $B$ (mit der Directrix $D$ ), so gibt es stets einen zweiten Kegelschnitt, welcher gleichfalls die beiden Geraden $T$ und $T_{1}$ berührt (mit $D$ als Berührungssehne) und welcher den Punkt $B$ als Bremnpunkt mit $S$ als zugehöriger Directrix hat.

Die Gleichung einer Curve zweiter Ordnung kann nach (42), S. 84 in die Form gebracht werden $X_{1} X_{3}-X_{2}{ }^{2}=0$, wobei $X_{1}=0$ und $X_{3}=0$ die Gleichungen zweier Tangenten bedeuten mit der Berührungssehne $X_{2}=0$. Andrerseits kann die Gleichung der Curve nach S. 115 und 119 auch in die Form gebracht werden $\left(\begin{array}{ll}y & x \\ y & x\end{array}\right)_{\omega_{i k}}-d_{x}{ }^{2}=0$, wobei $y_{1}, y_{2}, y_{3}$ die Coordinaten des einen Brennpunktes sind und $d_{x}=0$ dessen Directrix repräsentirt. Es gilt demnach eine Relation von der Form $X_{1} X_{3}-X_{2}^{2}=\left(\begin{array}{ll}y & x \\ y & x\end{array}\right)_{\omega_{i k}}-d_{x}^{2}$, aus welcher folgt

$$
X_{1} X_{3}+d_{x}^{2}=\left(\begin{array}{ll}
y & x \\
y & x
\end{array}\right)_{\omega_{i k}}+X_{2}^{2} .
$$

Die geometrische Deutung dieser Relation liefert sofort den obigen Satz. 
197. Haben drei Curven zweiter Ordnung eine gemeinsame Sehne, so schueiden sich die drei übrigen Sehnen, welche je zweien der Curven gemeinsam sind, in einem und demselben Punkte.

Die Gleichungen der drei Kegelschnitte sind von der Form

$$
f(x, x)+x u_{x} r_{x}=0, f(x, x)+\lambda u_{x} s_{x}=0, f(x, x)+\mu u_{x} t_{x}=0 ;
$$

$u_{x}=0$ ist die den drei Curven gemeinsame Sehne, die Gleichungen der drei übrigen sind $x r_{x}-\lambda s_{x}=0, \lambda s_{x}-\mu t_{x}=0, \mu t_{x}-x r_{x}=0$, und diese gehen durch einen und denselben Punkt.

In diesem Satze ist (71) als specieller Fall enthalten.

Dualistisch folgt:

198. Haben drei Curven zweiter Classe zwei gemeinsame Tangenten, so liegen die Spitzen der übrigen drei Paare von Tangenten, die je zweien der Curven gemeinsam sind, auf einer Geraden.

Insbesondere ergibt sich hieraus der in (20), S. 125 bewiesene Satz, wenn man als Curven zweiter Classe drei Punktepaare nimmt, von denen je ein Punkt auf der einen, der zweite auf der andereı Geraden eines Geradenpaares liegt.

199. Sind $y_{1}, y_{2}, y_{3}$ die Coordinaten eines Punktes der unendlich fernen Geraden, so ist das Product $[a, \omega] f(y, y)$ positiv, so lange $F(p, p) \geq 0$. Vgl. die Fussnote zu S. 108 .

Nach (32) und (31), S. 27 besteht für alle Werthe der $x_{i}$ und $y_{i}$ die Identität

$$
\begin{aligned}
A_{11}\left(x_{2} y_{3}-x_{3} y_{2}\right)^{2}+\cdots & +2 A_{23}\left(x_{3} y_{1}-x_{1} y_{3}\right)\left(x_{1} y_{2}-x_{2} y_{1}\right)+\cdots \\
& =f(x, x) f(y, y)-f^{2}(x, y)
\end{aligned}
$$

man kann also nach (35), S. 76 die Producte $x_{i} x_{k}$ ersetzen durch $\omega_{i k}$, wodurch sich die Relation ergibt

$$
\begin{aligned}
2 H \equiv & \left(A_{22} \omega_{33}+A_{33} \omega_{22}-2 A_{23} \omega_{23}\right) y_{1}{ }^{2}+\cdots \\
& \quad+2\left(A_{31} \omega_{23}+A_{23} \omega_{31}-A_{33} \omega_{12}-A_{12} \omega_{33}\right) y_{1} y_{2}+\cdots \\
= & {[a, \omega] f(y, y)-\omega\left(f_{1}, f_{2}, f_{3}\right), }
\end{aligned}
$$

oder auch

$$
[a, \omega] f(y, y)=\omega\left(f_{1}, f_{2}, f_{3}\right)+2 H .
$$

Werden nun für die $y_{i}$ die Coordinaten eines im Unendlichen gelegenen Punktes eingeführt durch $y_{1}=p_{2} v_{3}-p_{3} v_{2}, y_{2}=p_{3} v_{1}-p_{1} v_{3}$, $y_{3}=p_{1} v_{2}-p_{2} v_{1}$, so verwandelt sich $2 H$ in $F(p, p) \cdot \omega(v, v)$, und 
man erhält $[a, \omega] f(y, y)=\omega\left(f_{1}, f_{2}, f_{3}\right)+F(p, p) \cdot \omega(v, v)$. Hiermit ist der oben verlangte Nachweis geführt, denn $\omega(u, u)$ ist eine positive definite Form.

\section{Anwendung von $\S 13-17$.}

200. Alle Curven zweiter Ordnung, die ein gegebenes Dreieck zum Poldreieck haben und durch einen gegebenen Punkt $y$ hindurchgehen, bilden ein Büschel; die Grundpunkte, desselben sind der gegebene Punkt mit den Coordinaten $+y_{1},+y_{2},+y_{3}$ und die drei Punkte mit den Coordinaten $-y_{1},+y_{2},+y_{3} ;+y_{1},-y_{2},+y_{3}$ und $+y_{1},+y_{2},-y_{3}$.

Die Gleichung einer Curve des Systems ist von der Form

$$
a_{1} x_{1}{ }^{2}+a_{2} x_{2}{ }^{2}+a_{3} x_{3}{ }^{2}=0
$$

hierzu tritt noch die Bedingungsgleichung $a_{1} y_{1}{ }^{2}+a_{2} y_{2}{ }^{2}+a_{3} y_{3}{ }^{2}=0$. Wird diese von dem Werthsystem $+y_{1},+y_{2},+y_{3}$ erfüllt, so genïgen ihr anch die Coordinaten der drei übrigen oben genamuten Punkte.

Analog folgt:

201. Alle Curven zweiter Classe, die ein und dasselbe Dreiseit zum Poldreiseit haben und eine gegebene Gerade $v$ berühren, bilden eine Schaar; die gemeinsamen Tangenten derselben sind die gegebene Gerade $r_{1} x_{1}+v_{2} x_{2}+v_{3} x_{3}=0$ und die Geraden

und

$$
-v_{1} x_{1}+v_{2} x_{2}+v_{3} x_{3}=0, \quad v_{1} x_{1}-v_{2} x_{2}+v_{3} x_{3}=0
$$

$$
v_{1} x_{1}+v_{2} x_{2} \cdots v_{3} x_{3}=0 .
$$

Für $v_{i}=p_{i}$ folgt mit Rücksicht auf $(22)$ :

202. Alle Parabeln, für welche ein gegebenes Dreiseit Poldreiseit ist, berühren die Verbindungslinien der Seitenmitten dieses Dreiseits.

203. Wenn von den vier reellen Schnittpunkten zweier Kegelschnitte der eine in Bezug auf das, dem gemeinsamen Poldreieck parallel eingeschriebene Dreiseit trigonal gelegen ist, so ist dies auch bei den drei übrigen der Fall, und zwar liegt alsdann im Inneren des parallel eingeschriebenen Dreiseits und in den drei trigonalen Feldern je ein Schnittpunkt.

Folgt aus den Entwicklungen S. 252, Zeile $16 \mathrm{ff}$. und daraus, dass sich nach (32), S. 140 die auf das gemeinsame Poldreieck bezogenen Coordinaten der Schnittpunkte nur durch die vier verschiedenen hier 
in Betracht kommenden Vorzeichencombinationen von $\pm y_{1}: \pm y_{2}: \pm y_{3}$ unterscheiden ${ }^{1}$ ).

204. Welche Gebilde sind als ausartende, einem ganz im Endlichen liegenden Dreieck umschriebene Parabeln zu betrachten?

Legt man das Coordinatendreieck zu Grunde, so ist ein umschriebener Kegelschnitt gegeben durch $2\left(a_{29} x_{2} x_{3}+a_{31} x_{3} x_{1}+a_{12} x_{1} x_{2}\right)=0$; hierzu tritt die Bedingung der Parabel $F(p, p)=0$. Soll die Curve ausarten, so muss $A=2 a_{23} a_{31} a_{12}$ verschwinden; es sei etwa $a_{23}=0$, so dass $F(p, p)=a_{31}{ }^{2} p_{2}{ }^{2}+a_{12}{ }^{2} p_{3}{ }^{2}-2 a_{31} a_{12} p_{2} p_{3}=0$ oder

$$
\left(a_{31} p_{2}-a_{12} p_{3}\right)^{2}=0 \text {, d. h. } a_{31}: a_{12}=p_{3}: p_{2} \text {. }
$$

Die Gleichung des betr. Gebildes wird daher $x_{1}\left(p_{3} x_{3}+p_{2} x_{2}\right)=0$, d. l.: Unter den einem gegebenen Dreieck umschriebenen Parabeln gibt es drei ausartende, bestehend aus je einer Seite des Dreiecks und der Parallelen durch die gegenüberliegende Ecke.

In ähnlicher Weise oder auch geometrisch findet man die folgenden Sätze über ausartende Parabeln:

205. Unter den einem Dreiseit eingeschriebenen Parabeln befinden sich drei ausartende, bestehend aus je einer Ecke des Dreiseits und dem unendlich fernen Punkte der Gegenseite.

206. Unter den Parabeln, die ein. gegebenes Dreieck zum Poldreieck haben, befinden sich drei ausartende, bestehend aus je einer doppelt zu zählenden Seite des Dreiecks.

Man hat hier nämlich die Gleichungen $a_{1} x_{1}{ }^{2}+a_{2} x_{2}{ }^{2}+a_{3} x_{3}{ }^{2}=0$, $a_{2} a_{3} p_{1}{ }^{2}+a_{3} a_{1} p_{2}{ }^{2}+a_{1} a_{2} p_{3}{ }^{2}=0$ und $a_{1} a_{2} a_{3}=0 ;$ aus $a_{1}=0$ folgt aber $a_{2} a_{3} p_{1}{ }^{2}=0$, also entweder $a_{2}=0$ oder $a_{3}=0$.

20\%. Unter den Parabeln, die ein gegebenes Dreiseit zum Poldreiseit haben, befinden sich drei ausartende, bestehend aus dem Mittelpunkte und dem unendlich fernen Punkte je einer Seite des Dreiseits.

208. Welche Gebilde sind als ausartende, einem Dreieck umschriebene gleichseitige Hyperbeln zu betrachten?

Man hat hier die Gleichungen $2\left(a_{23} x_{2} x_{3}+a_{31} x_{3} x_{1}+a_{12} x_{1} x_{2}\right)=0$, $[a, \omega]=a_{29} \omega_{23}+a_{31} \omega_{31}+a_{12} \omega_{12}=0$ und $A=2 a_{23} a_{31} a_{12}=0$. Setzt man etwa $a_{23}=0$, so wird $a_{31}: a_{12}=\omega_{12}:-\omega_{31}$, die Gleichung der Curve wird also $x_{1}\left(\omega_{31} x_{2}-\omega_{12} x_{3}\right)=0$, d. h. (vgl. (11)): Unter den

1) Vgl. hierzu einen Satz von Steiner in der mehrfach citirten Arbeit im Journal für die reine und angewandte Mathematik, Bd. 55, S. 369-370, 1858, oder "Gesammelte Werke", Bd. 2, S. 676. 
Ausartende Parabeln (gleichseitige Hyperbeln) in einer Schaar (Büschel). 329

einem gegebenen Dreieck umschriebenen gleichseitigen Hyperbeln gibt es drei ausartende, bestehend aus je einer Seite des Dreiecks und der zugehörigen Höhe.

209. Welche Gebilde sind als ausartende, einem Dreiseit eingeschriebene gleichseitige Hyperbeln zu betrachten?

Hier sind die Gleichungen zu erfüllen

$$
\begin{gathered}
2\left(\alpha_{1} u_{2} u_{3}+\alpha_{2} u_{3} u_{1}+\alpha_{3} u_{1} u_{2}\right)=0, \\
\alpha_{1}{ }^{2} \omega_{11}+\alpha_{2}{ }^{2} \omega_{22}+\alpha_{3}{ }^{2} \omega_{33}-2 \alpha_{1} \alpha_{2} \omega_{12}-2 \alpha_{1} \alpha_{3} \omega_{13}-2 \alpha_{2} \alpha_{3} \omega_{2: 3}=0, \\
\mathrm{~A}=2 \alpha_{1} \alpha_{2} \alpha_{3}=0 .
\end{gathered}
$$

Setzt man etwa $\alpha_{1}=0$, so bleibt als Gleichung der Curve

$$
u_{1}\left(\alpha_{2} u_{3}+\alpha_{3} u_{2}\right)=0 \text {, }
$$

ein Punktepaar; bei Einführung von Punktcoordinaten erhält man $\left(\alpha_{2} x_{2}-\alpha_{3} x_{3}\right)^{2}=0$, also den durch die Ecke $u_{1}=0$ gehenden Träger des Punktepaares doppelt zählend. Die Bedingung der gleichseitigen Hyperbel verwandelt sich bei Substitution der Coordinaten

$$
u_{1}: u_{2}: u_{3}=0: \alpha_{2}:\left(-\alpha_{3}\right)
$$

des Trägers in $\omega(u, u)=0$, worin jedoch $u_{1}=0$, d. h. der Träger muss durch einen der beiden Kreispunkte gehen, sowie natürlich durch die Ecke $u_{1}=0$. Das nach den imaginären Krireispunkten gehende Geradenpaar möge als circulares Geradenpaar bezeichnet werden ${ }^{1}$ ), so dass wir sagen können: Als eingeschriebene gleichseitige Hyperbel ist jede doppelt gezählte Gerade zu betrachten, die einem der drei circularen Geradenpaare angehört, welche von einer Ecke des Dreiseits nach einem der beiden imaginären Kreispunkte gezogen werden können; es gibt daher sechs solche ausartende gleichseitige Hyperbeln.

In ähnlicher Weise findet man die folgenden zwei Sätze:

210. Unter den gleichseitigen Hyperbeln, die ein gegebenes Dreieck zum Poldreieck haben, befinden sich drei ausartende, bestehend aus den drei Paaren von Winkelhalbirenden der Winkel des Dreiecks.

211. Unter den gleichseitigen Hyperbeln, die ein gegebenes Dreiseit zum Poldreiseit haben, befinden sich drei ausartende, bestehend aus je einer doppelt zu zählenden Ecke des Dreiseits.

1) Die Verbindungslinien irgend eines Punktes $\boldsymbol{P}$ der Ebene mit den beiden Kreispunkten bilden also das durch $P$ gezogene circulare Geradenpaar. 
Mit Rücksicht darauf, dass nach (17), S. 138 die Spitzen der drei in einem Kegelschnittbüschel enthaltenen Geradenpaare das gemeinsame Poldreieck aller Curren des Büschels bilden, sowie mit Rücksicht auf die dualistisch entsprechende Thatsache, dass die Träger der drei in einer Kegelschnittschaar enthaltenen Punktepaare das gemeinsame Poldreiseit aller Curven der Schaar bilden, folgen aus (208) und (205) sofort die Sätze:

212. Das Büschel gleichseitiger Hyperbeln, die einem gegebenen Dreieck umschrieben sind, hat die Fusspunkte der Höhen des Dreiecks zum gemeinsamen Poldreieck.

213. Die Schaar von Parabeln, die einem gegebenen Dreiseit eingeschrieben sind, hat das dem gegebenen parallel umschriebene Dreiseit zum gemeinsamen Poldreiseit, oder mit anderen Worten:

214. Bei jeder einem gegebenen Dreiseit eingeschriebenen Parabel gehen die Berührungssehnen durch die Ecken des dem gegebenen parallel umschriebenen Dreiseits ${ }^{1}$ ). Vgl. (202).

215. In jedem Kegelschnittbüschel ist im allgemeinen eine gleichseitige Hyperbel enthalten; befinden sich insbesondere zwei solcher Curven in dem Büschel, so besteht es überhaupt nur aus gleichseitigen Hyperbeln.

$$
f(x, x) \equiv \sum_{1}^{3} \sum_{1}^{3} a_{i k} x_{i} x_{k}=0 \quad \text { und } g(x, x) \equiv \sum_{i}^{3} \sum_{1}^{3} b_{i k} x_{i} x_{k}=0
$$

die Gleichungen der Grundcurven des Büschels $\lambda g-f=0$, so erhält man für eine gleichseitige Hyperbel nach (162) die Bedingungsgleichung $\lambda[b, \omega]-[a, \omega]=0$, welche in $\lambda$ linear ist. - Wenn zwei gleichseitige Hyperbeln im Büschel enthalten sind, müssen $[b, \omega]$ und $[a, \omega]$ gleichzeitig verschwinden; die angegebene Bedingungsgleichung ist dann für alle Werthe von $\lambda$ erfüllt $\left.^{2}\right)$. Dass im letzteren

1) Vgl. Schröter: „Die Theorie der Kegelschnitte, gestützt auf projectivische Eigenschaften" (zweiter "Theil der von Geiser und Schröter herausgegebenen Vorlesungen Steiner's über synthetische Geometrie), 2. Aufl, Leipzig 1876, S. 277.

2) Aus zwei Gleichungen von der Form

$$
\lambda_{1}[b, \omega]-[a, \omega]=0 \text { und } \lambda_{2}[b, \omega]-[a, \omega]=0 \text {, }
$$

wobei $\lambda_{1} \gtrless \lambda_{2}$, folgt durch Subtraction $[l, \omega]=0$, daher auch $[a, \omega]=0$. 
Falle ein Grundpunkt des Büschels der Höhenschnittpunkt des durch die drei anderen gebildeten Dreiecks ist, folgt aus (166).

216. Ist in einem Kegelschnittbüschel ein Kreis enthalten, so sind die Axen der beiden Parabeln, welche dem Büschel angehören, zu einander normal.

Dass in dem Büschcl zwei Parabeln enthalten sind, folgt aus (5), S. 142, denn hiernach gibt es in einem Kegelschnittbüschel zwei Curven, die eine gegebene Gerade, also auch z. B. die unendlich ferne, berühren ${ }^{1}$ ). Die übrigen Kegelschnitte des Büschels treffen diese Gerade nach dem Desargues-Sturm'schen Satze (vgl. (12), S. 143) in Punktepaaren einer Involution, deren Doppelpunkte die Berührungspunkte der zwei Parabeln mit der unendlich fernen Geraden sind; sie liegen zu allen übrigen Schnittpunktepaaren harmonisch, im vorliegenden Falle also auch zum imaginären Kreispunktepaar, d. h. die Axen der zwei in dem Büschel enthaltenen Parabeln sind zu einander normal.

21\%. Umgekehrt gilt auch der Satz: Die Schnittpunkte zweier Parabeln, deren Axen sich rechtwinklig schneiden, liegen auf einem Kreise.

Denn unter den Punktepaaren, welche auf der unendlich fernen Geraden zu dem von den Berührungspunkten der beiden Parabeln gebildeten Paare der Doppelpunkte der Involution harmonisch liegen, befindet sich im gegenwärtigen Falle auch das imaginäre Kreispunktepaar, da die Axen der zwei Parabeln sich rechtwinklig schneiden. Es ist daher in dem durch die Schnittpunlite der Parabeln definirten Kegelschnittbüschel auch ein Kreis enthalten.

218. Es seien $f(x, x)=0$ und $g(x, u)=0$ die Gleichungen zweier Kegelschnitte, ferner sei durch $\lambda_{1} g-f=0$ eines der drei in dem Büschel $\lambda g-f=0$ enthaltenen Geradenpaare dargestellt; welche Bedeutung hat der Kegelschnitt $\lambda_{1} g+f=0$ ?

Jedenfalls gehört er dem Büschel an; ist $y$ irgend einer der vier Grundpunkte, so haben die in ihm an $f=0, g=0, \lambda_{1} g+f=0$ gezogenen Tangenten resp. die Gleichungen

$$
f(y, x)=0, \quad g(y, x)=0, \quad \lambda_{1} g(y, x)+f(y, x)=0 ;
$$

diese letzte Tangente liegt aber harmonisch zu $\lambda_{1} g(y, x)-f^{\prime}(y, x)=0$, also zu der durch $y$ gezogenen Geraden des Paares $\lambda_{1} g-f=0$. Hiermit ist der fragliche Kegelschnitt bestimmt.

1) Vgl. auch S. 199. 
219. Werden einem vollständigen Vierseit zwei Kegelschnitte eingeschrieben, so liegen die acht Punkte, in denen sie die Seiten berühren, auf einem dritten Kegelschnitte.

Auf S. 144 wurde gezeigt, dass die in den vier Schnittpunkten zweier Kegelschnitte gezogenen Tangenten, deren Anzahl also zusammen acht beträgt, einen dritten Kegelschnitt berühren. Hieraus folgt dualistisch der obige Satz.

220. Legt man durch einen Punkt $y$ des Kegelschnitts $f(x, x)=0$ Parallelen zu irgend einem Paar conjugirter Durchmesser eines anderen Kegelschnitts $g(x, x)=0$, so schneidet jedes solche Parallelenpaar die Curve $f(x, x)=0$ in zwei weiteren Punkten, deren jeweilige Verbindungslinien durch einen bestimmten Punkt $P$ hindurchgehen ${ }^{1}$ ).

Das Geradenpaar, welches den auf $f(x, x)=0$ gelegenen Punkt $y$ mit den Schnittpunkten der Curve $f(x, x)=0$ und der Geraden $v_{x}=0$ rerbindet, hat nach (53) die Gleichung

$$
\psi(x, x) \equiv v_{y} f(x, x)-2 v_{x} f(y, x)=0,
$$

und dieses Geradenpaar ist zu zwei conjugirten Durchmessern des Kegelschnitts $g(x, x)=0$ parallel, wenn die Schnittpunkte der unendlich fernen Geraden mit $\psi(x, x)=0$ und $g(x, x)=0$ zwei harmonische Punktepaare sind. Die Bedingung hierfür geht nach (15), S. 144 aus der Gleichung der harmonischen Curve zweiter Classe $H=0$ von $g(x, x)=0$ und $\psi(x, x)=0$ dadurch hervor, dass man in ihr die variabelen Liniencoordinaten $u_{1}, u_{2}, u_{3}$ ersetzt durch $p_{1}, p_{2}, p_{3}$. Da die Gleichung $H=0$ die Coefficienten von $g(x, x)$ und $\psi(x, x)$, daher auch die $v_{i}$, linear enthält, stellt sie in der That (in variabelen Liniencoordinaten $v_{1}, v_{2}, v_{3}$ ) einen Punkt $P$ dar.

Ist die Curve $g(x, x)=0$ ein Kreis, so erhält man den bereits in (84) für den Fall eines um seinen Scheitel rotirenden rechten Winkels abgeleiteten Satz von Frégier.

Ersetzt man $g(x, x)$ durch ein Kegelschnittbüschel

$$
g(x, x)+\lambda k(x, x)=0
$$

1) Für den speciellen Fall, dass $y$ der Mittelpunkt des Kegelschnitts $g(x, x)=0$ ist, wurde dieser Satz zuerst von Frégier ausgesprochen in seiner Note: "Théorèmes nouveaux sur les lignes et surfaces du second ordre", Annales de Mathématiques, Bd. 7, S. 95 f., 1816 und 1817; vgl, auch einen Artikel von Frégier unter gleichem Titel in Bd. 6, S. $321 \mathrm{ff}$., 1816, in derselben Zeitschrift.

Das dem allgemeinen Satze bei Oberflächen zweiter Ordnung analoge Theorem hat zuerst Hesse ausgesprochen in seiner Abhandlung "Ueber Oberflächen zweiter Ordnung", Journal für die reine und angewandte Mathematik, Bd. 18, S. 110, 1837. 
so enthält der Ausdruck $H\left(p_{1}, p_{2}, p_{3}\right)$, gebildet für $g(x, x)+\lambda k(x, x)$, den Parameter $\lambda$ wieder linear, die Punkte $P$ erfüllen also jetzt eine Punktreihe ${ }^{1}$ ).

Ersetzt man ferner $g(x, x)$ durch die Gesammtheit der einem gegebenen Vierseit eingeschriebenen Kegelschnitte, also durch die Curven einer Schaar, welche in. Liniencoordinaten etwa durch

$$
G(u, u)+\lambda K(u, u)=0 \text {, }
$$

in Punktcoordinaten durch eine Gleichung von der Form

$$
g_{0}(x, x)+2 \lambda g_{1}(x, x)+\lambda^{2} g_{2}(x, x)=0
$$

dargestellt ist, so wird der Ausdruck $H(p, p)$, gebildet für diese Gleichung, von der Form $V_{0}+2 \lambda V_{1}+\lambda^{2} V_{2}=0$, wobei die $V$ in den Liniencoordinaten $v_{i}$ linear sind. Den unendlich vielen Werthen des Parameters $\lambda$ entsprechen unendlich viele Punkte $P$, welche eine Curve erfülleu. Bei Bildung ihrer Gleichung beachte man, dass für eine beliebig fixirte Gerade $v$ die in $\lambda$ quadratische Gleichung

$$
V_{0}+2 \lambda V_{1}+\lambda^{2} V_{2}=0
$$

im allgemeinen zwei verschiedene Werthe gibt. Soll die Gerade eine Tangente sein, so müssen diese zwei Wurzeln zusammenfallen, und man erhält alsdann $V_{0} V_{2}-V_{1}^{2}=0$, also eine Curve zweiter Classe, die von allen Punkten $P$ erfüllt wird ${ }^{2}$ ).

221. Man beweise nachstehenden Satz von Steine ${ }^{3}$ ):

"Zieht man in einem gegebenen Kegelschnitte $K_{1}$ ein System paralleler Sehnen $G G_{1}$ nach beliebiger Richtung $R$, so liegen ihre Mitten $P$ in einem Durchmesser $F F_{1}=2 f$ desselben; und beschreibt man über den Sehnen, als Durchmesser, Kreise $P$, so haben diese irgend einen bestimmten anderen Kegelschnitt $K$ zur Enveloppe, und zwar berühren sie ihn doppelt, jeder in zwei Punkten $C$."

Um die Gleichung der Kreise $P$ zu erhalten, ist ähnlich zu verfahren wie in $(78) ;^{4}$ ) nur tritt an Stelle der Geraden $v_{x}=0$ jetzt ein Büschel paralleler Geraden, d. h. es ist $v_{x}$ zu ersetzen durch

1) Vgl. Schröter: „Die Theorie der Kegelschnitte, gestützt auf projectivische Eigenschaften" (zweiter Theil der von Geiser und Schröter herausgegebenen Vorlesungen Steiner's über synthetische Geometrie), 2. Aufl, Leipzig 1876, S. 266.

2) Vgl. Schröter, a. a. O. S. 290.

3) „Elementare Lösung einer geometrischen Aufgabe, und über einige damit in Beziehung stehende Eigenschaften der Kegelschnitte", Journal für die reine und angewandte Mathematik, Bd. 37, S. 179, 1847, oder auch ,Gesammelte Werke", Bd. 2; S. 408.

4) Diese Gleichung stellt im Grunde den Directorkreis dar, welcher dem dnrch die Endpunkte einer Sehne gebildeten Punktepaare zugehört. 
$v_{x}+\lambda p_{x}=0$. Nehmen wir zunächst ein beliebiges Strahlenbüschel $v_{x}+\lambda w_{x}=0$, so würde man für das System von Kreisen in variabelen Punktcoordinaten $y_{i}$ die Gleichung erhalten:

$$
\begin{aligned}
\left(v_{y}+\lambda w_{y}\right)^{2} \cdot[a, \omega] & -\frac{1}{2}\left(v_{y}+\lambda w_{y}\right)\left\{f^{\prime}\left(y_{1}\right)\left[\omega^{\prime}\left(v_{1}\right)+\lambda \omega^{\prime}\left(w_{1}\right)\right]+\cdots\right. \\
& \left.+f^{\prime}\left(y_{3}\right)\left[\omega^{\prime}\left(v_{3}\right)+\lambda \omega^{\prime}\left(w_{3}\right)\right]\right\} \\
& +\left\{\omega(v, v)+2 \lambda \omega(v, w)+\lambda^{2} \omega(w, w)\right\} f(y, y)=0,
\end{aligned}
$$

dabei ist $f(x, x)=0$ die Gleichung des gegebenen Kegelschnitts $K_{1}$. Wenn man nach Potenzen des Parameters $\lambda$ ordnet, verwandelt sich die Gleichung des Systems von Kreisen in einen Ausdruck von der Form $\mathrm{K}_{r}+2 \lambda \mathrm{X}_{v, w}+\lambda^{2} \mathrm{~K}_{w}=0$, wobei

$$
\begin{aligned}
\mathrm{K}_{v} \equiv[a, \omega] v_{y}{ }^{2}-\frac{1}{2}\left\{\omega^{\prime}\left(v_{1}\right) f^{\prime}\left(y_{1}\right)+\cdots\right. & \left.+\omega^{\prime}\left(v_{3}\right) f^{\prime}\left(y_{3}\right)\right\} \imath, \\
& +\omega(v, v) f(y, y)=0
\end{aligned}
$$

denjenigen Kreis darstellt, der über der Sehne $v_{x}=0$ als Durchmesser errichtet ist, und $K_{w}$ die gleiche Bedeutung besitzt mit Bezug auf die Sehne $w_{x}=0$. Ferner ist

$$
\begin{aligned}
\mathrm{X}_{v, w} & \equiv[a, \omega] v_{y} w_{y}-\frac{1}{4}\left[\omega^{\prime}\left(v_{1}\right) f^{\prime}\left(y_{1}\right)+\cdots+\omega^{\prime}\left(v_{3}\right) f^{\prime}\left(y_{3}\right)\right] w_{y} \\
& -\frac{1}{4}\left[\omega^{\prime}\left(w_{1}\right) f^{\prime}\left(y_{1}\right)+\cdots+\omega^{\prime}\left(w_{3}\right) f^{\prime}\left(y_{3}\right)\right] v_{y}+\omega(v, w) f(y, y) .
\end{aligned}
$$

Nimmt man ein Büschel paralleler Strahlen $\left(v_{i}=p_{i}\right)$, so bleibt der Ausdruck $\mathrm{K}_{w}$ unverändert, $\mathrm{K}_{v}$ geht dagegen über in $\mathrm{K}_{p} \equiv[a, \omega] p_{y}{ }^{2}$, $\mathrm{X}_{v, w}$ in $\mathrm{X}_{p, w} \equiv[a, \omega] p_{y} w_{y}-\Psi_{w, y} \cdot p_{y}$, wobei $\Psi(w, y)$ gerade so wie in (45), S. 96 definirt ist durch $\frac{1}{4} \sum_{i}^{3} i \omega^{\prime}\left(w_{i}\right) f^{\prime}\left(y_{i}\right)$, so dass $\Psi(w, y)=0$ nach (20a), S. 24 die Gleichung desjenigen Durchmessers vou $f(x, x)=0$ repräsentirt, welcher zur Richtung des Normalencentrums der Geraden $w$ conjugirt ist.

Die Enveloppe aller Kreise des Systems $\mathrm{K}_{p}+2 \lambda \mathrm{X}_{p, w}+\lambda^{2} \mathrm{~K}_{w}=0$ wird (vgl. (220)) $K_{p} K_{w}-X_{p, w}^{2}=0$, und diese Gleichung stellt nach Ausscheidung des Factors $p_{y}{ }^{2}$ einen Kegelschnitt dar; werden die oben angegebenen Werthe eingesetzt, so findet man als Gleichung dieses Kegelschnitts $K=[a, \omega] \omega(w, w) f(y, y)-\psi^{2}(w, y)=0$. Dass derselbe von allen Kreisen des Systems doppelt berührt wird, geht daraus hervor, dass man für den Ausdruck $\mathrm{K}_{p} \mathrm{~K}_{w}-\mathrm{X}_{p, w}^{2}$ nach Multiplication mit $(\lambda-\mu)^{2}$ die Identität hat

$$
\begin{gathered}
\left(\mathrm{K}_{p}+2 \lambda \mathrm{X}_{p, w}+\lambda^{2} \mathrm{~K}_{w}\right)\left(\mathrm{K}_{p}+2 \mu \mathrm{X}_{p, w}+\mu^{2} \mathrm{~K}_{w}\right) \\
-\left[\left(\mathrm{K}_{p}+\lambda \mathrm{X}_{p, w}\right)+\left(\mathrm{X}_{p, w}+\lambda \mathrm{K}_{w}\right) \mu\right]^{2} \equiv\left(\mathrm{K}_{p} \mathrm{~K}_{w}-\mathrm{X}_{p, w}^{2}\right)(\lambda-\mu)^{2},
\end{gathered}
$$


wobei $\lambda$ und $\mu$ ganz willkürliche Parameter bezeichnen. Die Form der Gleichung $K=0$ zeigt ferner, dass der gegebene Kegelschnitt $K_{1}$ von $K$ in den Endpunkten eines diesen beiden Curven gemeinsamen Durchmessers berührt wird. Auch die meisten übrigen von Steiner a. a. O. aufgestellten Sätze lassen sich auf Grund der vorstehenden Entwickelungen ableiten.

222. Wird irgend ein Punkt $y$ eines Kegelschnitts verbunden mit den Punktepaaren, die auf der Curve durch die Strahlen eines beliebigen Strahlenbüschels ausgeschnitten werden, so bilden diese Paare von Verbindungslinien eine Involution. Die Doppelstrahlen dieser Involution werden durch die Geraden gebildet, welche vom Punkte $y$ nach den Berührungspunkten der zwei in dem Strahlenbüschel enthaltenen Kegelschnittstangenten gezogen werden können.

Das Geradenpaar, welches vom Punkte $y$ der Curve $f(x, x)=0$ nach deren Schnittpunkten mit $u_{x}=0$ gezogen werden kann, ist nach (53) gegeben durch $u_{y} f(x, x)-2 u_{x} \cdot f(x, y)=0$. Ersetzt man die Gerade $u_{x}=0$ durch die Strahlen eines Büschels $v_{x}+\lambda w_{x}=0$, so erhält manl: $v_{y} f(x, x)-2 v_{x} f(x, y)+\lambda\left\{w_{y} f(x, x)-2 w_{x} f(x, y)\right\}=0$, ein Ausdruck, der in $\lambda$ linear ist. Alle diese Geradenpaare, welche man für varïrende Werthe $\lambda$ erhält, treffen nach (12), S. 143 eine beliebige Gerade in Punktepaaren einer Involution. Die Geradenpaare bilden daher selbst eine Involution; offenbar gehen ihre Doppelstrahlen von $y$ nach den Berührungspunkten der beiden Tangenten, die sich von dem Centrum des Strahlenbüschels $v_{x}+\lambda w_{x}=0$, dem sog. Involutionscentrum ${ }^{1}$ ), an den Kegelschnitt ziehen lassen.

Hieraus folgt eine einfache Construction der Doppelstrahlen einer Involution, die durch zwei Strahlenpaare bestimmt ist: Man legt durch das gemeinsame Centrum der Strahlenpaare einen beliebigen Kegelschnitt, am einfachsten einen Kreis; derselbe möge von dem einen Strahlenpaare noch getroffen werden in den Punkten $p$ und $p^{\prime}$, von dem anderen in $q$ und $q^{\prime}$. Alsdann zieht man $p p^{\prime}$, sowie $q q^{\prime}$, Schnittpunkt sei $z$, und construirt die Polare von $z$ in Bezug auf den oben erwähnten Hilfskegelschnitt. Ihre Schnittpunkte mit demselben ergeben durch Verbindung mit dem Centrum der Strahlenpaare die gesuchten Doppelstrahlen.

223. Wie lautet der dem obigen Satz dualistisch entsprechende?

1) Vgl. v. Staudt, „Beiträge zur Geometrie der Lage“. Erstes Heft, Nürnberg 1856 , S. 47. 
224. Man untersuche das gemeinsame Poldreieck eines Büschels von Kreisen.

Sind $K_{0}=0$ und $K_{1}=0$ die Gleichungen zweier Kreise, so enthält nach (182) das Büschel $K_{0}-\lambda K_{1}=0$ einen •in ein Geradenpaar ausartenden Kreis, bestehend aus der Potenzlinie und der unendlich fernen Geraden (vgl. S. 59). ${ }^{1}$ ) Die Spitze dieses Geradenpaares, also nach (17), S. 138 eine Ecke des den Kreisen des Büschels gemeinsamen Poldreiecks, ist der unendlich ferne Punkt der Potenzlinie, und die Polare dieses Punktes kann nur die gemeinsame Centrale sein. Die auf ihr gelegenen Ecken des Poldreiecks müssen harmonisch liegen zu dem Schnittpunktepaar der Centrale sowohl mit $K_{0}$ als mit $K_{1}$, sie müssen daher die Doppelpunkte der Involution sein, welche auf der Centrale durch ihre Schnittpunktepaare mit den einzelnen Kreisen des Büschels gebildet wird; man nennt diese Doppelpunkte die Grenzpunkte des Büschels. Sie sind zugleich die Spitzen der zwei in dem Büschel enthaltenen circularen Geradenpaare. Das gemeinsame Poldreieck besteht somit aus den zwei Grenzpunkten und dem unendlich fernen Punkte der Potenzlinie. Zugleich folgt, dass die nach dem letztgenannten Punkte gehenden zwei Seiten des Dreiecks zur Centrale normal sind, denn jede derselben liegt zugleich mit der Centrale harmonisch zu dem durch die betreffende Ecke gehenden circularen Geradenpaare. Die Grenzpunkte sind als Doppelpunkte der oben genannten Involution bekanntlich imaginär oder reell, je nachdem das auf der Centrale von $K_{0}$ ausgeschnittene Punktepaar durch das von $K_{1}$ ausgeschnittene getrennt oder nicht getrennt wird, d. h. je nachdem die Kreise des Büschels eine reelle oder imaginäre gemeinsame Schnittsehne besitzen ${ }^{2}$ ).

Jeder Punkt der Potenzlinie hat bekanntlich gleiche Potenz in Bezug auf alle Kreise des Büschels. Zieht man also von irgend 'einem Punkte der Potenzlinie Tangenten an diese Kreise, so liegen die Berührungspunkte auf einem Kreis, der durch die Grenzpunkte geht und

1) Unter Anwendung der Bezeichnung in (182) ist $\lambda=\frac{[a, \infty]}{[b, \omega]}$; übrigens könnte die Wurzel $\lambda$ auch aus einer der sechs Gleichungen entnommen werden, die in der auf $K_{0}-\lambda K_{1}=0$ angewandten Identität $\left(\begin{array}{ll}p & w \\ p & w\end{array}\right) \equiv 0$ enthalten sind (vgl. (13), S. 51). Die Bedingungen in (183) liessen sich hierdurch mannigfach umformen.

2) Vgl. Steiner: „Ueber einige neue Bestimmungsarten der Curven zweiter Ordnung nebst daraus folgenden neuen Eigenschaften derselben Curven". Journal für die reine und angewandte Mathematik, Bd. 45, S. $206 \mathrm{f}$, 1852, oder „Gesammelte Werke", Bd. 2, S. 463. 
die einzelnen Kreise rechtwinklig schneidet. Hiermit ist auch eine Construction der zwei Grenzpunkte gegeben. Zugleich entsteht durch alle, die eben genannten rechtwinklig schneidenden Kreise ein zweites Büschel, das die Grenzpunkte des ersten zu Grundpunkten hat.

225. Die Gleichung des Directorkreises der Curve zweiter Ordnung $f(x, x)=0$ lautet

$$
\begin{aligned}
2 H & \equiv\left(A_{22} \omega_{33}+A_{33} \omega_{22}-2 A_{23} \omega_{23}\right) x_{1}^{2}+\cdots \\
& +2\left(A_{31} \omega_{23}+A_{23} \omega_{31}-A_{33} \omega_{12}-A_{12} \omega_{33}\right) x_{1} x_{2}+\cdots=0 .
\end{aligned}
$$

Folgt sowohl aus der in (29), S. 148 gegebenen Gleichung des Directorkreises einer Curve zweiter Classe, als auch aus der in (89) abgeleiteten Formel für den Winkel $\alpha$ der Tangenten, welche von irgend einem Punkte an die Curve $f(x, x)=0$ gezogen werden können; man hat in letzterem Falle nur $\alpha=90^{\circ}$ zu setzen.

Uebrigens sei der Vollständigkeit habber noch daran erinnert, dass nach (199) der Ausdruck $2 H$ auch in die Form gebracht werden kann $2 H \equiv[a, \omega] f(x, x)-\omega\left(f_{1}, f_{2}, f_{3}\right)$, wobei $f_{i}=\frac{1}{2} f^{\prime}\left(x_{i}\right)$, welche dadurch wichtig ist, dass in ihr ein Glied auftritt mit $f(x, x)$ als Factor.

226. Die Gleichung des Directorkreises der auf schiefwinklige Parallelcoordinaten $\left(x_{1}: x_{2}: x_{3}=x: y: 1\right)$ bezogenen Curve zweiter Ordnung $f(x, y, 1)=0$ lautet:

$$
\begin{aligned}
& \left(a_{11}+a_{22}-2 a_{12} \cos w\right) f(x, y, 1)-\left(a_{11} x+a_{12} y+a_{13}\right)^{2} \\
& -\left(a_{21} x+a_{22} y+a_{23}\right)^{2}+2\left(a_{11} x+a_{12} y+a_{13}\right)\left(a_{21} x+a_{22} y+a_{23}\right) \cos w=0 .
\end{aligned}
$$

Folgt sofort aus der zuvor erwähnten Form

$$
[a, \omega] f(x, x)-\omega\left(f_{1}, f_{2}, f_{3}\right)=0
$$

für die Gleichung des Directorkreises.

227. Man beweise, dass die Gleichung in Liniencoordinaten des Directorkreises der Curve zweiter Ordnung $f(x, x)=0$ die Gestalt besitzt

$$
A \cdot[a, \omega] \cdot \omega(u, u)+\tau F^{2}(p, u)=0 .
$$

Kann leicht vermöge der kanonischen Form

$$
\lambda^{\prime} X_{1}^{2}+\lambda^{\prime \prime} X_{2}^{2}+x X_{3}^{2}=0
$$

der Curvengleichung $f(x, x)=0$ (vgl. (29), S. 91) verificirt werden.

Ein anderer Beweis folgt aus (228) mit Benutzung von (10a), S. 59. 
228. Ist $\varphi(u, u)=0$ die Gleichung einer Curre zweiter Classe, für welche $\varphi(p, p) \gtrless 0,2 \chi \equiv \sum_{1}^{3} \sum_{1}^{3}(\alpha, \omega)_{i k} x_{i} x_{k}=0$ die Gleichung des zugehörigen Directorkreises (vgl. (29), S. 148), so besteht die Relation $2 \varphi(p, p) \cdot \chi(x, x) \equiv\left(\begin{array}{l}y x \\ y x\end{array}\right)_{\omega_{i k}}+[\mathrm{A}, \boldsymbol{\omega}] \cdot p_{x}{ }^{2}$, in welcher

$$
y_{i}=\frac{1}{2} \varphi^{\prime}\left(p_{i}\right), \quad(i=1,2,3),
$$

die Coordinaten des Mittelpunktes von $\varphi(u, u)=0$ bedeuten.

Man bilde die Determinante aus den Elementen $\omega_{i k}+\lambda \alpha_{i k}$ und rändere dieselbe sowohl bei den Horizontal- als bei den Verticalreihen mit den zwei Reihen $\frac{1}{2} \varphi^{\prime}\left(p_{1}\right), \frac{1}{2} \varphi^{\prime}\left(p_{2}\right), \frac{1}{2} \varphi^{\prime}\left(p_{3}\right)$ und $x_{1}, x_{2}, x_{3}$; die vier übrig bleibenden Stellen in der so entstehenden Determinante 5. Grades fülle man durch Nullen aus. Nach Multiplication der drei ersten Verticalreihen mit $\frac{p_{1}}{\lambda}, \frac{p_{2}}{\lambda}, \frac{p_{3}}{\lambda}$ und Subtraction derselben von der vierten, sowie nach gleichem Verfahren bei den Horizontalreihen lässt sich die Determinante unter Berücksichtigung von $\omega^{\prime}\left(p_{i}\right)=0$ zerlegen in

$-\frac{1}{\lambda} \varphi(p, p)\left(\begin{array}{l}x \\ x\end{array}\right)_{\omega_{i k}+\lambda \alpha_{i k}}-\frac{p_{x}^{2}}{\lambda^{\ddagger}} \sum \pm\left(\omega_{11}+\lambda \alpha_{11}, \omega_{22}+\lambda \alpha_{22}, \omega_{33}+\lambda \alpha_{33}\right)=0$.

Diese Umformung der ursprünglichen Determinante gilt, welchen Werth auch der Parameter $\lambda$ haben mag, es müssen daher die Coefficienten gleicher Potenzen von $\lambda$ dieselben sein. Durch Vergleichen der Coefficienten von $\lambda^{0}$ folgt $\left(\begin{array}{ll}y & x \\ y & x\end{array}\right)_{\omega_{i k}}=\varphi(p, p) \cdot 2 \chi(x, x)-[\mathrm{A}, \omega] p_{x}{ }^{2}$, woraus sofort die verlangte Relation hervorgeht.

229. Der Radius $r$ des Directorkreises eines Kegelschnitts ist gegeben durch die Formel $r=\sqrt{a^{2} \pm b^{2}}$, wo $a$ und $b$ die Längen der Halbaxen bedeuten und in $a^{2} \pm b^{2}$ das positive oder negative Vorzeichen zu stehen hat, je nachdem die Curve eine Ellipse oder Hyperbel ist.

Aus der in (228) abgeleiteten Gleichung des Directorkreises $\left(\begin{array}{ll}y & x \\ y & x\end{array}\right)_{\omega_{i k}}+[\mathrm{A}, \omega] p_{x}{ }^{2}=0$ der Curve zweiter Classe $\varphi(u, u)=0$ folgt mit Rücksicht auf (10a), S. 59 die Relation $r^{2}=-\frac{[\mathrm{A}, \omega]}{\tau \varphi^{2}(p, p)}$, und dieser Ausdruck ist nach (136) gleich $a^{2} \pm b^{2}$.

230. Für eine gleichseitige Hyperbel artet der Director- 
Ort der Punkte, von welchen ans 2 wei Strecken gleich gross erscheinen. 339

kreis in das circulare Geradenpaar aus, dessen Spitze der Mittelpunkt der Curve ist. Vgl. die Fussnote zu S. 329.

Folgt wegen $a^{2}-b^{2}=0$ aus (229), mit Rücksicht auf S. 148 .

231. Man beweise nachstehenden Satz von Steine ${ }^{1}$ ):

"Sind in einer Ebene zwei begrenzte Geraden $A B$ und $C D$ in beliebiger fester Lage gegeben, so besteht der Ort desjenigen Punktes, aus welchem dieselben unter gleichen Winkeln (oder auch unter Winkeln, die zwei Rechte betragen) gesehen werden, aus zwei Curven dritten Grades. Beide Curven gehen durch die vier Endpunkte der gegebenen Geraden, sowie durch ibren gegenseitigen Schnittpunkt. Ferner haben die Curven diejenigen zwei Punkte gemein, aus welchen beide Geraden unter rechten Winkeln erscheinen. Die zwei übrigen gemeinschaftlichen Punkte der Curven sind imaginär und liegen auf der unendlich entfernten Geraden."

Es seien $\varphi(u, u) \equiv \alpha_{u} \cdot \beta_{u}=0$ und $\psi(u, u) \equiv \gamma_{u} \cdot \delta_{u}=0$ die Gleichungen der beiden Punktepaare $A, B$ und $C, D$,

$$
m_{x} \equiv \sum \pm\left(\alpha_{1} \beta_{2} x_{3}\right)=0 \text { und } n_{x} \equiv \sum \pm\left(\gamma_{1} \delta_{2} x_{3}\right)=0
$$

diejenigen ihrer Träger. Sind nun $y_{i}(i=1,2,3)$ die Coordinaten irgend eines auf dem gesuchten Orte gelegenen Punktes, so müssen die Winkel, unter welchen die beiden Punktepaare von $y$ aus erscheinen, einander gleich sein oder sich zu zwei Rechten ergänzen, d. h. man hat nach (88):

$$
\operatorname{tg} \alpha= \pm \frac{m_{y} p_{y} \sqrt{\tau}}{H}= \pm \frac{n_{y} p_{y} \sqrt{\tau}}{K}
$$

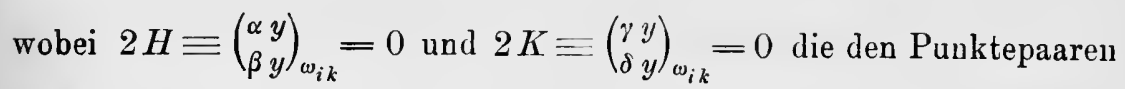
$\alpha, \beta$, bezw. $\gamma, \delta$ zugehörigen Directorkreise darstellen. Als Ort des Punktes $y$ erhält man die zwei Curven dritter Ordnung $K m_{y}-H n_{y}=0$ und $K m_{y}+H n_{y}=0$, aus deren Gleichungen man leicht die oben angeführten Behauptungen von Steiner ablesen kann. Die zwei im Unendlichen gelegenen Schnittpunkte der beiden Curven sind natürlich die imaginären Kreispunkte.

232. Wann artet der Directorkreis einer Curve zweiter Classe $\varphi(\imath, u)=0$ in ein Geradenpaar aus?

Nach S. 148 f. tritt dies ein im Falle der Parabel $(\varphi(p, p)=0)$, ferner nach $(228)$, wenn $[A, \omega]=0$, d. b. im Falle der gleichseitigen

1) „Aufgaben und Lehrsätze". Journal für die reine und angewandte Mathematik, Bd. 45, S. 375, 1852, oder auch "Gesammelte Werke", Bd. 2, S. 487. 
Hyperbel. Das Product dieser beiden Bedingungen ist in den $\alpha_{i k}$ vom dritten Grade, muss daher mit der Discriminante der (in den $\alpha_{i k}$ gleichfalls linearen) Gleichung des Directorkreises bis auf einen Zahlenfactor identisch sein.

233. Die Directorkreise einer Kegelschnittschaar bilden ein Büschel.

Folgt sofort daraus, dass die Gleichung des Directorkreises der Curve $\lambda \psi(u, u)-\varphi(u, u)=0$ in den Coefficienten von $\psi$ und $\varphi$, also auch in dem Parameter $\lambda$ linear ist.

Besteht die Schaar insbesondere aus confocalen Kegelschnitten, so sind die zugehörigen Directorkreise natürlich alle concentrisch.

234. Es gibt zwei feste Punkte in der Ebene, von denen an alle Kegelschnitte einer Schaar zu einander rechtwinklige Tangenten gezogen werden können.

Da die Directorkreise der Schaar nach (233) ein Büschel bilden, schneiden sie sich in einem und demselben im Endlichen gelegenen Punktepaare. Zur Construction desselben zeichnet man am einfachsten die Directorkreise, welche den in der Schaar enthaltenen Punktepaaren zugehören, d. h. diejenigen Kreise, welche je eine der Diagonalen des gemeinsamen Tangentenvierseits als Durchmesser haben. Es folgt also der Satz:

235. Die drei Kreise, welche man über den Diagonalen eines vollständigen Vierseits als Durchmesser beschreiben kann, schneiden sich in einem und demselben Punktepaare.

236. Durch fünf Geraden sind fünf Vierseite bestimmt; jedem derselben lässt sich eine Schaar von Kegelschnitten einschreiben, für welche die zugehörigen Directorkreise sich nach (233) in demselben im Endlichen gelegenen Puuktepaare schneiden. So entstehen im ganzen fünf Punktepaare, von denen gezeigt werden soll, dass sie auf einem und demselben Kreise liegen.

Jenen fünf Kegelschnittschaaren gehört gemeinschaftlich derjenige Kegelschnitt an, welcher die fünf gegebenen Geraden berührt; sein Directorkreis muss demnach durch die oben genannten fünf Punktepaare hindurchgehen.

23\%. Die Directricen einer Schaar von Parabeln bilden ein Strahlenbüschel.

Folgt daraus, dass die Directorkreise einer beliebigen Kegelschnitt- 
schaar nach (233) ein Büschel von Kreisen bilden; im Falle der Parabel zerfällt nach S. $148 \mathrm{f}$. jeder Kreis dieses Büschels in die Directrix der Parabel and die unendlich ferne Gerade; die Gleichung der Directrix bleibt aber in dem Parameter $\lambda$ der Schaar

linear. Vgl. auch (244).

$$
\lambda \psi(u, u)-\varphi(u, u)=0
$$

238. Die Directricen aller einem und demselben Dreiseit eingeschriebenen Parabeln schneiden sich im Höhenschnittpunkte des Dreiseits ${ }^{1}$ ).

Die Directricen bilden nach (237) jedenfalls ein Strahlenbüschel. Andrerseits sind in der Parabelschaar nach (205) drei ausartende Curven enthalten, gebildet durch je eine Ecke des Dreiseits und den unendlich fernen Punkt der Gegenseite. Die Directricen dieser Punktepaare bestehen aber je aus der von der betreffenden Ecke auf die Gegenseite gefällten Normale, d. h. aus den Höhen des Dreiseits.

239. Die Höhenschnittpunkte derjenigen vier Dreiseite, die durch vier beliebige Geraden bestimmt sind, liegen auf einer und derselben Geraden, und zwar auf der Directrix derjenigen Parabel, welche jene vier Geraden berührt ${ }^{2}$ ).

Nach (238) gehen die Directricen aller einem Dreiseit eingeschriebenen Parabeln durch dessen Höhenschnittpunkt; dasselbe muss also auch von der Directrix derjenigen Parabel gelten, welche die Seiten der vier Dreiseite berührt, sie muss folglich durch die vier Höhenschnittpunkte gehen.

Offenbar gilt auch der Satz:

240. Die Höhenschnittpunkte aller Dreiseite, die einer Parabel umschrieben sind, liegen auf der Directrix der Curve $^{3}$ ).

241. Die Directricen aller Parabeln, für welche ein gegebenes Dreiseit Poldreiseit ist, schneiden sich im Mittelpunkt des umschriebenen Kreises ${ }^{4}$ ).

1) Vgl. Steiner: "Geometrische Lehrsätze". Journal für die reine und angewandte Mathematik, Bd. 2, S. 191, 1827, oder auch "Gesammelte Werke", Bd. 1, S. 134.

2) Vgl. Steiner: „Développement d'une série de théorèmes relatifs aux sections coniques". Annales de Matbématiques, hregg. von Gergonne, Bd. 19, S. 59, oder auch "Gesammelte Werke", Bd. 1, S. 207.

3) Ibid.

4) Vgl. Schröter: „Die Theorie der Kegelschnitte, gestützt anf projectivische Eigenschaften" (zweiter Theil der von Geiser und Schröter herausgegebenen Vorlesungen Steiner's über synthetische Geometrie), 2. Aufl., Leipzig 1876, S. 407. 
Die Parabeln bilden nach (201) einc Schaar, ihre Directricen nach (237) ein Strahlenbüschel. In der Parabelschaar sind nach (207) drei ausartende Curven enthalten, bestehend aus dem Mittelpunkte und dem unendlich fernen Punkte je einer Seite des Dreiseits; die zugehörigen Directricen sind aber die in dem Mittelpunkte auf der jeweiligen Seite errichteten Normalen, die sich nach (237) in einem und demselben Punkte schneiden bekanntlich im Centrum des dem Dreiseit umschriebenen Kreises.

Folgt auch aus (202) mit Benutzung von (238), denn der Höhenschnittpunkt des parallel eingeschriebenen Dreiseits ist zugleich Centrum des dem gegebenen Dreiseit umschriebenen Kreises.

242. Die Directrix der Parabel $f(x, x)=0$ hat die Gleichung

$$
\begin{aligned}
\{[a, \omega] f(y, x) & \left.-\frac{1}{4} \sum_{1}^{3} i \omega^{\prime}\left(f_{i}\right) \cdot f^{\prime}\left(x_{i}\right)\right\} 2 p_{y} \\
& -\left\{[a, \omega] f(y, y)-\omega\left(f_{1}, f_{2}, f_{3}\right)\right\} p_{x}=0 ;
\end{aligned}
$$

dabei sind $y_{1}, y_{2}, y_{3}$ ganz willkürliche Zahlen (z. B. $y_{1}=y_{2}=0, y_{3}=1$, falls $\left.p_{3} \gtrless 0\right)$, die jedoch nicht die Gleichung $p_{1} y_{1}+p_{2} y_{2}+p_{3} y_{3}=0$ erfüllen dürfen; $f_{i}$ ist zur Abkürzung gesetzt für $\frac{1}{2} f^{\prime}\left(y_{i}\right)$.

Wir gehen aus von der Gleichung des Directorkreises

$$
2 H \equiv[a, \omega] f(x, x)-\omega\left(f_{1}, f_{2}, f_{3}\right)=0
$$

(vgl. (199) und (225)); dieselbe muss im gegenwärtigen Falle nach S. $148 \mathrm{f}$. sich in zwei lineare Factoren zerspalten lassen, deren einer die unendlich ferne Gerade $p_{x}=0$, der andere die Directrix repräsentirt. Wendet man die S. 38 gegebene Methode zur Bestimmung dieses zweiten Factors an, so folgt die obige Gleichung.

243. Die Directrix der auf schiefwinklige Parallelcoordinaten $\left(x_{1}: x_{2}: x_{3}=x: y: 1\right)$ bezogenen Parabel $f(x, y, 1)=0$ hat die Gleichung

$$
\begin{aligned}
\left(A_{13}+A_{23} \cos w\right) x & +\left(A_{23}+A_{13} \cos w\right) y \\
& -\frac{1}{2}\left(A_{11}+A_{22}+2 A_{12} \cos w\right)=0 .
\end{aligned}
$$

Folgt aus (242) durch die Substitution $p_{1}=p_{2}=0, p_{3}=1$, $y_{1}=y_{2}=0, \quad y_{3}=1, \quad \omega_{11}=\omega_{22}=\frac{1}{\sin ^{2} w}, \quad \omega_{12}=-\frac{\cos w}{\sin ^{2} w}$, $\omega_{13}=\omega_{23}=\omega_{33}=0$ (vgl. S. 8).

244. D ectrix der auf schiefwinklige Parallelcoordinaten $\left(u_{1}: u_{2}: u_{3}=u: v: 1\right)$ bezogenen Parabel $\varphi(u, v, 1)=0$ hat die Gleichung 
$\left(\alpha_{13}+\alpha_{23} \cos w\right) x+\left(\alpha_{23}+\alpha_{13} \cos w\right) y-\frac{1}{2}\left(\alpha_{11}+\alpha_{22}+2 \alpha_{12} \cos w\right)=0$.

Folgt aus (243).

245. Man bilde die Gleichungen der Axe, Scheiteltangente und Directrix der auf rechtwinklige Coordinaten bezogenen Parabel

$$
9 x^{2}+16 y^{2}-24 x y-4 x-28 y-16=0 \text {. }
$$

Für die Axe findet man nach (122) die Gleichung

$$
9 x-12 y+\frac{-18+168}{25}=0 \text { oder } 3 x-4 y+2=0 \text {. }
$$

Mit Hilfe der Werthe $A_{13}=200, A_{23}=150, A=-2500$, $A_{11}=-452, A_{22}=-148$ wird die Gleichung der Scheiteltangente nach (123)

$$
200 x+150 y+\frac{-2500+25 \cdot 600}{2 \cdot 25}=0 \text { oder } 4 x+3 y+5=0 .
$$

Für die Directrix ergibt sich nach (243):

$$
200 x+150 y+\frac{600}{2}=0 \text { oder } 4 x+3 y+6=0 .
$$

Der Parameter $2 p$ der Parabel wird nach (124)

$$
2 p=\frac{2 \sqrt{2500}}{\sqrt{ } 25^{3}}= \pm \frac{4}{5} \text {. }
$$

246. Der Directorkreis eines Kegelschnitts schneidet alle diejenigen Kreise senkrecht, welche durch Poldreiecke des Kegelschnitts hindurchgehen ${ }^{1}$ ).

Zum Beweise dieses Satzes fragen wir zunächst, welche Beziehung zwischen den Coordinaten des Mittelpunktes und der Länge des Radius eines Kreises bestehen muss, wenn der Kreis durch Poldreiecke der Curve zweiter Classe $\varphi(u, u)=0$ hindurchgehen, also zu dieser Curve conjugirt sein soll. Die Gleichung eines Kreises mit dem Mittelpunkte $y$ und dem Radius $r$ lautet nun nach (10a), S. 59

$$
\left(\begin{array}{l}
y x \\
y x
\end{array}\right)_{\omega_{i k}}-r^{2} \tau p_{y}^{2} p_{x}^{2}=0 \text {. }
$$

Will man die verlangte Bedingung haben, so sind zufolge (12), S. 162 die Producte $x_{i} x_{k}$ zu ersetzen durch die Coefficienten $\alpha_{i k}$ von $\varphi(u, u)=0$. Hierdurch verwandelt sich $\left(\begin{array}{l}y x \\ y x\end{array}\right)_{\omega_{i k}}$ in $2 \chi(y, y) \equiv \sum_{1}^{3} \sum_{1}^{3}(\alpha, \omega)_{i k} y_{i} y_{k}$, $p_{x}{ }^{2}$ in $\varphi(p, p)$, und man erbält $2 \chi(y, y)-r^{2} \tau \varphi(p, p) \cdot p_{y}{ }^{2}=0$. Ist nun der Mittelpunkt $y$ gegeben, so folgt für das Quadrat des Radius

1) Vgl. Schröter a. a. O. S. 185. Dieser Satz wurde zuerst von Faure anfestellt (Nouvelles Annales de Mathématiques, Bd. 19 der 1. Serie). 
$r^{2}=\frac{2 \chi}{\tau \varphi(p, p) \cdot p_{y}{ }^{2}}$, und dieser Ausdruck ist nach (55), S. 100 gleich der Potenz des Punktes $y$ in Bezug auf den Directorkreis von $\varphi(u, u)=0$, woraus der oben aufgestellte Satz folgt, den man also in nachstehender Form aussprechen kann:

24\%. Die Potenz des Mittelpunktes eines Kegelschnitts in Bezug auf den einem beliebigen Poldreieck unschriebenen Kreis ist gleich dem Quadrate des Radius des Directorkreises $\left.{ }^{x}\right)$.

Im Falle der Parabel ist $\varphi(p, p)=0$, und die Coordinaten $y_{i}$ der Mittelpunkte aller Kreise, welche Poldreiecken umschrieben sind, erfüllen alsdann die Gleichung des Directorkreises $2 \chi(x, x)=0$; da dieser aber für die Parabel in die unendlich ferne Gerade und die Directrix zerfällt, so folgt:

248. Die Mittelpunkte aller Kreise, welche Poldreiecken einer Parabel umschrieben sind, liegen anf der Directrix der Parabel ${ }^{1}$ ).

249. Wenn eine gleichseitige Hyperbel conjugirt liegt zu einer Parabel, d. b. also wenn die Hyperbel durch die Ecken unendlich vieler Poldreiecke der Parabel hindurchgeht, oder wenn die Parabel unendlich viele Poldreiseite der Hyperbel berührt, so trifft die Axe der Parabel die gleichseitige Hyperbel in einem Punktepaare, dessen Mittelpunkt der im Endlichen gelegene Brennpunkt der Parabel ist.

Die Gleichung der gleichseitigen Hyperbel sei $f(x, x)=0$, wobei natürlich $[a, \omega]=0$; die Parabel sei durch eine Gleichung in Liniencoordinaten gegeben in der Form

$$
\omega(u, u)+\left(y_{1} u_{1}+y_{2} u_{2}+y_{3} u_{3}\right)\left(z_{1} u_{1}+z_{2} u_{2}+z_{3} u_{3}\right)=0
$$

(vgl. S. 120), wobei $y$ der im Endlichen gelegene, $z$ der unendlich ferme Brennpunkt sein möge. Die Bedingung, dass beide Curven conjugirt liegen, ist nach S. $162[a, \omega]+f(y, z)=0$ oder $f(y, z)=0$ da $[a, \omega]=0$; daher sind $y$ und $z$ harmonische Pole in Bezug auf $f(x, x)=0$, und zwar liegt $z$ im Unendlichen, also $y$ in der Mitte zwischen den Schnittpunkten der Parabelaxe $y z$ und der gleichseitigen Hyperbel.

250. Welche geometrische Bedeutung hat die Grösse $\frac{f(y, y)}{[a, \omega] p_{y}{ }^{2}}$, wenn $y_{1}, y_{2}, y_{3}$ die Coordinaten eines nicht auf dem Kegelschnitt $f(x, x)=0$ gelegenen Punktes bezeichnen?

Die Gleichung in Liniencoordinaten eines Kreises vom Radius $r$ und mit dem Mittelpunkte $y$ ist nach (2), S. 57: $r^{2} p_{y}{ }^{2} \omega(u, u)-u_{y}{ }^{2}=0$.

1) Vgl. die Fussnote zu S. 343. 
Die Bedingung dafür, dass dieser Kreis conjugirt liege zu dem Kegelschnitt $f(x, x)=0$, wird nach S. 162 dadurch erhalten, dass man die $u_{i} u_{k}$ ersetzt durch $a_{i k}$. Alsdann entsteht $r^{2} \cdot[a, \omega] \cdot p_{y}{ }^{2}-f(y, y)=0$, woraus folgt, dass $\frac{f(y, y)}{[a, \omega] p_{y}{ }^{2}}$ gleich ist dem Quadrat des Radius desjenigen Kreises, welcher den Punkt $y$ zum Mittelpunkt hat und zu $f(x, x)=0$ conjugirt liegt, also einem Poldreiseit von $f(x, x)=0$ eingeschrieben ist. Man köunte in Analogie zu (55), S. 100 den Ausdruck $\frac{2 f(y, y)}{[a, \omega] p_{y}{ }^{2}}$ nennen die Potenz des Punktes $y$ in Bezug auf den Kegelschnitt $f(x, x)=0$; sie wäre offenbar gleich dem Quadrat des Radius des Directorkreises für jenen Kreis, welcher $y$ zum Mittelpunkt hat und einem Poldreiseit von $f(x, x)=0$ eingeschrieben ist.

Ausserdem folgt sofort:

251. Soll ein Kreis irgend ein Poldreiseit des Kegelschuitts $f(x, x)=0$ berühren, oder der Kegelschnitt irgend einem Poldreieck des Kreises umschrieben sein, so muss bei gegebenem Radius $r$ der Mittelpunkt $y$ des Kreises auf dem Kegelschnitt

$$
f(y, y)-r^{2} \cdot[a, \omega] p_{y}{ }^{2}=0
$$

liegen, der nach (130) mit $f(x, x)=0$ concentrisch, ähnlich und ähnlich gelegen ist.

Der Mittelpunkt $y$ ist auch der Höhensclnnittpunkt $H$ des betreffenden Dreiecks; bezeichnet man ferner mit $A, B, C$ dessen Ecken, mit $A_{1}, B_{1}, C_{1}$ die Fusspunkte der zugehörigen Höhen, mit $r$ den Radius desjenigen Kreises, welcher das Dreieck zum Poldreieck hat, so ist, wie die elementare Planimetrie lehrt ${ }^{1}$ ),

$$
r^{2}=H A \cdot H A_{1}=H B \cdot H B_{1}=H C \cdot H C_{1} .
$$

In Folge dessen kann man auch sagen ${ }^{2}$ ):

252. Soll das Product aus den Abschnitten der Höhen eines dem Kegelschnitt $f(x, x)=0$ eingeschriebenen Dreiecks constant gleich $r^{2}$ sein, so ist der Ort des Höhenschnittpunktes der Kegelschnitt

$$
f(y, y)-r^{2} \cdot[a, \omega] p_{y}^{2}=0 .
$$

1) Das betr. Theorem wird sich übrigens in (322) bei allgemeineren Betrachtungen über Kreisnetze aus der Definition der Potenz ergeben.

2) Vgl. zu (252)-(254) Salmon: „Analytische Geometrie der Kegelschnitte", frei bearbeitet von W. Fiedler, 5. Aufl., 2. Theil, S. 641. 1888. Daselbst ist auch die betreffende Litteratur citirt. 
253. Soll das Product aus den Abschnitten der Höhen eines den Kegelschnitt $\varphi(u, u)=0$ umschriebenen Dreiecks constant gleich $r_{1}{ }^{2}$ sein, so ist der Ort des Höhenschnittpunktes der mit dem Kegelschnitt concentrische Kreis $2 \chi(y, y)-r_{1}{ }^{2} \tau \varphi(p, p) \cdot p_{y}{ }^{2}=0$.

Die Bedingung dafür, dass der Kegelschnitt $\varphi(u, u)=0$ irgend einem Poldreiseit des Kreises $\left(\begin{array}{l}y x \\ y x\end{array}\right)_{\omega_{i k}}-r_{1}{ }^{2} \tau p_{y}{ }^{2} p_{x}{ }^{2}=0$ eingeschrieben sei, wird nämlich nach (17), S. 163 dadurch gebildet, dass man in der Gleichung des Kreises die $x_{i} x_{k}$ ersetzt durch $\alpha_{i k}$. Hierdurch verwandelt sich $\left(\begin{array}{l}y x \\ y x\end{array}\right)_{\omega_{i k}}$ in $2 \chi(y, y)$ (vgl. (29), S. 148), $p_{x}^{2}$ in $\varphi(p, p)$, und man erhält $2 \chi(y, y)-r_{1}{ }^{2} \tau \varphi(p, p) \cdot p_{y}{ }^{2}=0$. Bei gegebenem Werthe von $r^{2}$ ergibt sich mit Rücksicht auf die Bemerkungen in (251) der obige Satz; $2 \chi(y, y)$ ist natürlich der Ausdruck für den Directorkreis von $\varphi(u, u)=0$.

Werden die Ausdrücke für $r^{2}$ und $r_{1}{ }^{2}$ in (251) und (253) einander gleichgesetzt, so erhält man nach Wegheben von $p_{y}{ }^{2}$ die Gleichung $\tau \varphi(p, p) f(y, y)-2[a, \omega] \chi(y, y)=0$, woraus folgt:

254. Soll ein Dreieck dem Kegelschnitt $f(x, x)=0$ eingeschrieben, dem Kegelschnitt $\varphi(u, u)=0$ umschrieben sein, so ist der Ort seines Höhenschnittpunktes die Curve zweiter Ordnung

$$
\tau \boldsymbol{\varphi}(p, p) f(y, y)-2[a, \omega] \chi(y, y)=0 .
$$

255. Wann ist die eine Asymptote einer Curve zweiter Ordnung $f(x, x)=0$ parallel zu einer Asymptote der Curve $g(x, x)=0$ ?

Die unendlich ferne Gerade muss durch einen der vier Schnittpunkte von $f=0$ und $g=0$ gehen; zufolge (16), S. 144 erhält man also die Bedingung $F(p, p) G(p, p)-H^{2}(p, p)=0$; speciell für schiefwinklige Parallelcoordinaten wird dieselbe

$$
4 A_{33} B_{33}-\left(a_{11} b_{22}+a_{22} b_{11}-2 a_{12} b_{12}\right)^{2}=0 .
$$

\section{Anwendung von $\$ 18-19$.}

256. Die beiden Brennpunktepaare einer auf schiefwinklige Parallelcoordinaten $\left(u_{1}: u_{2}: u_{3}=u: v: 1\right)$ bezogenen Curve zweiter Classe $\varphi(u, v, 1)=0$ sind gegeben durch

$$
\mu \varphi(u, v, 1)-\frac{u^{2}+v^{2}-2 u v \cos v}{\sin ^{2} w}=0,
$$


wobei $\mu$ die eine oder andere Wurzel ist der quadratischen Gleichung

$$
\mathrm{A} \cdot \sin ^{2} w \cdot \mu^{2}-\left(\mathrm{A}_{11}+\mathrm{A}_{22}-2 \mathrm{~A}_{12} \cos v\right) \mu+\alpha_{33}=0 \text {. }
$$

Folgt aus (20), S. 168 mit Rücksicht auf (4) und (5), S. 166 bei Anwendung vou Parallelcoordinaten.

25\%. Wenn die eine Reihe der Brennpunkte aller Kegelschnitte, welche die Seiten eines Dreiecks berühren, auf einem dem Dreieck umschriebenen Kegelschnitte liegt, so erfüllt die andere Reihe eine gerade Linie, und umgekehrt.

Es sei $\varphi(u, u) \equiv 2\left(\alpha_{1} u_{2} u_{3}+\alpha_{2} u_{3} u_{1}+\alpha_{3} u_{1} u_{2}\right)=0$ die Gleichung eines dem Coordinatendreieck eingeschriebenen Kegelschnitts;

$$
y_{i} \text { und } z_{i},(i=1,2,3) \text {, }
$$

seien die Coordinaten der zwei reellen Brennpunkte. Alsdann besteht wach S. 168 eine Relation von der Form

$$
\begin{aligned}
2 \mu\left(\alpha_{1} u_{2} u_{3}\right. & \left.+\alpha_{2} u_{3} u_{1}+\alpha_{3} u_{1} u_{2}\right)+\omega(u, u) \\
& =\left(y_{1} u_{1}+y_{2} u_{2}+y_{3} u_{3}\right)\left(z_{1} u_{1}+z_{2} u_{2}+z_{3} u_{3}\right),
\end{aligned}
$$

wobei $\mu$ einen gewissen Factor darstellt, der für das Folgende gleichgiltig ist. Aus dieser Relation ergeben sich durch Coefficientenvergleichung für die Coordinaten der beiden Brennpunkte die einfachen Beziehungen $\omega_{11}=y_{1} z_{1}, \omega_{22}=y_{2} z_{2}, \omega_{33}=y_{3} z_{3}$ oder

$$
y_{i}=\frac{\omega_{i i}}{z_{i}},(i=1,2,3) \text {. }
$$

Beschreibt also der eine Brennpunkt, etwa $y$, einen dem Coordinatendreieck umschriebenen Kegelschnitt $m_{1} y_{2} y_{3}+m_{2} y_{3} y_{1}+m_{3} y_{1} y_{2}=0$, so durchläuft der andere die Gerade $\frac{m_{1} z_{1}}{\omega_{11}}+\frac{m_{2} z_{2}}{\omega_{22}}+\frac{m_{3} z_{3}}{\omega_{33}}=0$, und offenbar gilt auch die Umkehrung. Durchläuft ferner der eine Brennpunkt die unendlich ferne Gerade $p_{1} z_{1}+p_{2} z_{2}+p_{3} z_{3}=0$, so sind alle Kegelschnitte $\varphi(u, u)=0$ Parabeln, welche die Seiten des Dreiecks berühren, und die im Endlichen gelegenen Brennpunkte dieser Parabeln erfüllen alsdann die Curve $\omega_{11} p_{1} y_{2} y_{3}+\omega_{22} p_{2} y_{3} y_{1}+\omega_{33} p_{3} y_{1} y_{2}=0$, die nach (73) den umschriebenen Kreis des Dreiecks darstellt.

Hiermit ist auch der Satz bewiesen:

258. Die Schnittpunkte dreier Tangenten einer Parabel liegen mit dem Brennpunkte auf einem und demselben Kreis.

259. Geht die Halbirungslinie des Winkels, den zwei Tangenten eines Kegelschnitts mit einander bilden, durch den einen Brennpunkt 
der Curve, so geht sie auch durch den anderen Brennpunkt hindurch, d. h. sie ist eine Hauptaxe.

Die beiden Tangenten wählt man als Seiten eines Coordinatendreiseits, eine beliebige weitere Tangente als dritte Seite; alsdann kann man die in (257) abgeleiteten Relationen benutzeu. Die Halbirungslinie des von zwei Tangenten $x_{2}=0$ und $x_{3}=0$ gebildeten Winkels hat nun nach (9) die Gleichung $\frac{x_{2}}{\sqrt{\omega_{22}}}-\frac{x_{3}}{\sqrt{\omega_{33}}}=0$, und da diese Gleichung durch die Coordinaten des einen Brennpunktes $y$ erfüllt werden soll, hat man $\frac{y_{2}}{\sqrt{\omega_{22}}}-\frac{y_{3}}{\sqrt{\omega_{33}}}=0$, woraus mit Rücksicht auf die Relationen $y_{i}=\frac{\omega_{i i}}{z_{i}}$ folgt $\frac{z_{9}}{\sqrt{\omega_{22}}}-\frac{z_{3}}{\sqrt{\omega_{33}}}=0$, d. h. der andere Brennpunkt liegt gleichfalls auf der Winkelhalbirenden.

260. Liegt der eine Brennpunkt eines Kegelschnitts · im Höhensclinittpunkt eines Tangentendreiecks der Curve, so liegt der andere im Mittelpunkt des dem Dreieck umschriebenen Kreises, und umgekehrt.

Die Coordinaten des Höhenschnittpunktes sind nach (17)

$$
y_{1}: y_{2}: y_{3}=\frac{1}{\omega_{23}}: \frac{1}{\omega_{31}}: \frac{1}{\omega_{12}}
$$

mit Hilfe der in (257) abgeleiteten Beziehungen zwischen den Coordinaten der beiden Brennpunkte folgt für den anderen Brennpunkt

$$
z_{1}: z_{2}: z_{3}=\omega_{11} \omega_{23}: \omega_{22} \omega_{31}: \omega_{33} \omega_{12} \text {, }
$$

d. h. der letztere fällt nach (18) mit dem Mittelpunkte des dem Tangentendreieck umschriebenen Kreises zusammen. Offenbar gilt auch die Umkehrung.

261. Die Fusspunkte der Normalen, welche von einem beliebigen Punkte des einem Dreieck umschriebenen Kreises auf die Seiten des Dreiecks gefällt werden, liegen in einer Geraden.

Der Punkt des umschriebenen Kreises kann nach (257) angesehen werden als Brempunkt einer dem Dreieck eingeschriebenen Parabel. Nach (23), S. 186 liegen aber die Fusspunkte der Normalen, welche vom Brennpunkte einer Parabel auf die Tangenten dieser Curve gefällt werden, auf der Scheiteltangente der Parabel, also in einer Geraden.

262. Wenn die eine Reihe der Brennpunkte aller Kegelschnitte, für welche ein gegebenes Dreiseit Poldreiseit ist, auf einer Geraden liegt, so erfüllt die andere Reihe einen Kegelschnitt. 
Die Gleichung eines Kegelschnitts, der das Coordinatendreiseit zum Poldreiseit hat, ist $\varphi(u, u) \equiv \alpha_{1} u_{1}^{2}+\alpha_{2} u_{2}{ }^{2}+\alpha_{3} u_{3}{ }^{2}=0$. Aehnlich wie in (257) besteht nun eine Relation von der Form

$$
\begin{aligned}
\mu\left(\alpha_{1} u_{1}^{2}\right. & \left.+\alpha_{2} u_{2}^{2}+\alpha_{3} u_{3}^{2}\right)+\omega(u, u) \\
& =\left(y_{1} u_{1}+y_{2} u_{2}+y_{3} u_{3}\right)\left(z_{1} u_{1}+z_{2} u_{2}+z_{3} u_{3}\right),
\end{aligned}
$$

wenn $y_{i}$ und $z_{i},(i=1,2,3)$, die Coordinaten der Brennpunkte von $\varphi(u, u)=0$ bedeuten. Aus dieser Relation folgt

$$
2 \omega_{23}=y_{2} z_{3}+y_{3} z_{2}, \quad 2 \omega_{31}=y_{3} z_{1}+y_{1} z_{3}, \quad 2 \omega_{12}=y_{1} z_{2}+y_{2} z_{1},
$$

daher

$$
\begin{aligned}
z_{1}: z_{2}: z_{3}=\left(-\omega_{23} y_{1}+\omega_{31} y_{2}+\omega_{12} y_{3}\right) y_{1}: & \left(\omega_{23} y_{1}-\omega_{31} y_{2}+\omega_{12} y_{3}\right) y_{2} \\
: & \left(\omega_{23} y_{1}+\omega_{31} y_{2}-\omega_{12} y_{3}\right) y_{3} .
\end{aligned}
$$

Beschreibt nun der eine Brennpunkt $z$ die Gerade

$$
m_{1} z_{1}+m_{2} z_{2}+m_{3} z_{3}=0 \text {, }
$$

so durchläuft der andere den Kegelschnitt

$$
\begin{gathered}
\omega_{23} m_{1} y_{1}{ }^{2}+\omega_{31} m_{2} y_{2}{ }^{2}+\omega_{12} m_{3} y_{3}{ }^{2} \\
-\left(\omega_{12} m_{2}+\omega_{13} m_{3}\right) y_{2} y_{3}-\left(\omega_{23} m_{3}+\omega_{21} m_{1}\right) y_{3} y_{1}-\left(\omega_{31} m_{1}+\omega_{32} m_{2}\right) y_{1} y_{2}=0 .
\end{gathered}
$$

Beschreibt insbesondere der eine Brennpunkt die unendlich ferne Gerade $p_{z}=0$, so sind alle Kegelschnitte $\varphi(u, u)=0$ Parabeln, die das Dreiseit zum Poldreiseit haben, und die im Endlichen gelegenen Brennpunkte erfüllen alsdann den Feuerbach'schen Kreis, denn obige Gleichung stellt nach (165) für $m_{i}=p_{i}$ diesen Kreis dar ${ }^{1}$ ).

263. „Jeder beliebige Punkt in der Ebene eines gegebenen geradlinigen Dreiecks kann einer der Brennpunkte eines Kegelschnitts sein, der alle drei Seiten des Dreiecks berührt. Man soll nun untersuchen, welche Lage der Punkt in Beziehung auf das Dreieck haben müsse, damit der Kegelschnitt entweder Parabel, oder Ellipse, oder Hyperbel sei." 2 )

1) Vgl. Schröter: „Die Theorie der Kegelschnitte, gestützt auf projectivische Eigenschaften" (zweiter Theil der von Geiser und Schröter herausgegebenen Vorlesungen Steiner's über synthetische Geometrie), 2. Aufl., Leipzig 1876, S. 407.

2) Steiner stellt diese Aufgabe im Journal für die reine und angewandte Mathematik, Bd. 2, S. 96, 1827 („Gesammelte Werke“, Bd. 1, S. 128) und beantwortet sie in einem anderen Artikel in demselben Journal, Bd. 2, S. 191, 1827 (Ges. Werke, Bd. 1, S. 134). Vgl. auch Steiner's Abhandlung „Développement d'une série de théorèmes relatifs aux sections coniques", Annales de Mathématiques, Bd. 19, S. 47, 1828, oder "Gesammelte Werke", Bd. 1, S. 198. Ferner ver- 
Man wählt das Dreieck als Coordinatendreieck und hat dann für den Kegelschnitt die Gleichung

$$
\varphi(u, u) \equiv 2\left(\alpha_{1} u_{2} u_{3}+\alpha_{2} u_{3} u_{1}+\alpha_{3} u_{1} u_{2}\right)=0,
$$

welche nach S. 55 eine Ellipse, Hyperbel oder Parabel repräsentirt, je nachdem $A \varphi(p, p)$, d. h. $2 \alpha_{1} \alpha_{2} \alpha_{3}\left(\alpha_{1} p_{2} p_{3}+\alpha_{2} p_{3} p_{1}+\alpha_{3} p_{1} p_{2}\right)>0$, $<0,=0$ ist. Sind $y_{i}$ und $z_{i},(i=1,2,3)$, die Coordinaten der Brennpunkte des Kegelschnitts, so besteht wie in (257) die Relation

$$
\begin{aligned}
2 \mu\left(\alpha_{1} u_{2} u_{3}\right. & \left.+\alpha_{2} u_{3} u_{1}+\alpha_{3} u_{1} u_{2}\right)+\omega(u, u) \\
& =\left(y_{1} u_{1}+y_{2} u_{2}+y_{3} u_{3}\right)\left(z_{1} u_{1}+z_{2} u_{2}+z_{3} u_{3}\right) ;
\end{aligned}
$$

aus ihr folgen, wenn man mit Hilfe von $y_{i} z_{i}=\omega_{i i}$ die $z$ eliminirt und die Coefficienten von $v_{2} u_{3}, u_{3} u_{1}, u_{1} u_{2}$ beiderseits vergleicht, für die Grössen $\alpha_{i}$ bei gegebenem Brennpunkt $y$ die Werthe

ferner wird

$$
\begin{aligned}
& 2 \mu \alpha_{1}=\left(y_{2}{ }^{2} \omega_{33}+y_{3}{ }^{2} \omega_{22}-2 y_{2} y_{3} \omega_{23}\right): y_{2} y_{3} \\
& 2 \mu \alpha_{2}=\left(y_{3}{ }^{2} \omega_{11}+y_{1}{ }^{2} \omega_{33}-2 y_{3} y_{1} \omega_{31}\right): y_{3} y_{1} \\
& 2 \mu \alpha_{3}=\left(y_{1}{ }^{2} \omega_{22}+y_{2}{ }^{2} \omega_{11}-2 y_{1} y_{2} \omega_{12}\right): y_{1} y_{2}
\end{aligned}
$$

$2 \mu\left(\alpha_{1} p_{2} p_{3}+\alpha_{2} p_{3} p_{1}+\alpha_{3} p_{1} p_{2}\right)=p_{y} \cdot p_{z}=p_{y}\left(\frac{\omega_{11} p_{1}}{y_{1}}+\frac{\omega_{22} p_{2}}{y_{2}}+\frac{\omega_{33} p_{3}}{y_{3}}\right)$, so dass nun $\mathrm{A} \varphi(p, p)$ gleichbedeutend wird mit

$$
\begin{aligned}
& \left(y_{2}{ }^{2} \omega_{33}+y_{3}{ }^{2} \omega_{22}-2 y_{2} y_{3} \omega_{23}\right)\left(y_{3}{ }^{2} \omega_{11}+y_{1}{ }^{2} \omega_{33}-2 y_{3} y_{1} \omega_{31}\right) \\
& \cdot\left(y_{1}{ }^{2} \omega_{22}+y_{2}{ }^{2} \omega_{11}-2 y_{1} y_{2} \omega_{12}\right) p_{y}\left(\frac{\omega_{11} p_{1}}{y_{1}}+\frac{\omega_{22} p_{2}}{y_{2}}+\frac{\omega_{33} p_{8}}{y_{3}}\right) .
\end{aligned}
$$

Dabei ist der eigentlich noch auftretende Divisor $16 \mu^{4} y_{1}{ }^{2} y_{2}{ }^{2} y_{3}{ }^{2}$ weggelassen worden, da es nur auf das Vorzeichen von $\mathrm{A} \varphi(p, p)$ ankommt. Die drei ersten Factoren, z. B. $y_{2}{ }^{2} \omega_{33}+y_{3}{ }^{2} \omega_{22}-2 y_{2} y_{3} \omega_{23}$, sind nun, wie man aus der Bedeutung der Grössen $\omega_{i k}$ erkennt, sicher positiv, also ohne Einfluss auf das Vorzeichen; nach Multiplication mit dem positiven Product $y_{1}{ }^{2} y_{2}{ }^{2} y_{3}{ }^{2}$ wird daher $\mathrm{A} \varphi(p, p)$ gleichbedeutend mit

$$
K \equiv \frac{y_{1} y_{2} y_{3}}{p_{1} p_{2} p_{3}} p_{y} \cdot p_{1} p_{2} p_{3}\left(\omega_{11} p_{1} y_{2} y_{3}+\omega_{22} p_{2} y_{3} y_{1}+\omega_{33} p_{3} y_{1} y_{2}\right)
$$

Der Factor $\frac{y_{1} y_{2} y_{3}}{p_{1} p_{2} p_{3}} p_{y}$ ist nun nach (3) positiv oder negativ, je nachdem der Punkt $y$ in Bezug auf das Dreieck trigonal oder tetragonal gelegen ist. Der Klammerfactor stellt, gleich Null gesetzt, nach (73) den dem Dreieck umschriebenen Kreis dar; an ihn gehen, wie mit Benutzung des in (52) gegebenen Kriteriums folgt, von einem Punkte $y$

gleiche man Geiser: "Die Theorie der Kegelschnitte in elementarer Darstellung" (erster Theil der von Geiser und Schröter herausgegebenen Vorlesungen Steiner's über synthetische Geometrie), 2. Aufl., Leipzig 1875, S. 156 f. 
aus reelle oder imaginäre Tangenten, $d . h$. der Punkt liegt innerhalb oder ausserhalb des Kreises, je nachdem

$$
\omega_{11} \omega_{22} \omega_{33} p_{1} p_{2} p_{3}\left(\omega_{11} p_{1} y_{2} y_{3}+\omega_{22} p_{2} y_{3} y_{1}+\omega_{33} p_{3} y_{1} y_{2}\right)
$$

$<0$ oder $>0$ ist. Hierbei ist der stets positive Factor $\omega_{11} \omega_{22} \omega_{33}$ ohne Einfluss auf das Vorzeichen. Man erkennt hiernach, dass $K$ positiv, der Kegelschnitt also eine Ellipse ist, entweder wenu der Brennpunkt $y$ innerhalb des Dreiecks liegt, oder wenn der Brennpunkt ausserhalb des umschriebenen Kreises und in Bezug auf das Dreieck tetragonal gelegen ist. Der Kegelschnitt ist dagegen eine Hyperbel, entweder wenn der Brennpunkt $y$ innerhalb des umschriebenen Kreises tetragonal liegt, oder wenn $y$ ausserhalb dieses Kreises trigonal gelegen ist. Ferner sieht man, dass der Kegelschnitt eine Parabel ist, wenn $y$ auf der unendlich fernen Geraden oder auf dem umschriebenen Kreis des Dreiecks liegt (vgl. (257)).

264. Der geometrische Ort für die Brennpunkte der Kegelschnitte einer Schaar jst eine Curve dritter Ordnung.

Durch die zwei Gleichungen

und

$$
\varphi(u, u) \equiv 2\left(\alpha_{1} u_{2} u_{3}+\alpha_{2} u_{3} u_{1}+\alpha_{3} u_{1} u_{2}\right)=0
$$

$$
\varphi(v, v) \equiv 2\left(\alpha_{1} v_{2} v_{3}+\alpha_{2} v_{3} v_{1}+\alpha_{3} v_{1} v_{2}\right)=0
$$

wird eine Schaar von Kegelschnitten bestimmt, welche die Seiten des Coordinatendreiseits und die Gerade $v$ berühren.

Nach Substitution der in (263) angegebenen, durch die Cooràinaten des einen Brennpunktes $y$ ausgedrückten Werthe von $\alpha_{1}, \alpha_{2}, \alpha_{3}$ in die Gleichung $\varphi(v, v)=0$ erhält man die Curve dritter Ordnung

$$
\begin{gathered}
v_{2} v_{3} y_{1}\left(\omega_{22} y_{3}{ }^{2}+\omega_{33} y_{2}{ }^{2}-2 \omega_{23} y_{2} y_{3}\right)+v_{3} v_{1} y_{2}\left(\omega_{33} y_{1}{ }^{2}+\omega_{11} y_{3}{ }^{2}-2 \omega_{31} y_{3} y_{1}\right) \\
+v_{1} v_{2} y_{3}\left(\omega_{11} y_{2}{ }^{2}+\omega_{.22} y_{1}{ }^{2}-2 \omega_{12} y_{1} y_{2}\right)=0 .
\end{gathered}
$$

Nach Addition und Subtraction von $\left(\omega_{11} v_{1}{ }^{2}+\omega_{22} v_{2}{ }^{2}+\omega_{33} v_{3}{ }^{2}\right) y_{1} y_{2} y_{3}$ kann man diese Gleichung in die Form setzen

$$
\begin{gathered}
\left(\omega_{11} v_{1} y_{2} y_{3}+\omega_{22} v_{2} y_{3} y_{1}+\omega_{33} v_{3} y_{1} y_{2}\right)\left(v_{1} y_{1}+v_{2} y_{2}+v_{3} y_{3}\right) \\
-\omega(v, v) y_{1} y_{2} y_{3}=0 .
\end{gathered}
$$

Bei constanten $y$ und variabelen $v$ stellt sie diejenige Curve zweiter Classe dar, welche die Seiten des Coordinatendreiecks berührt und einen bestimmten Punkt $y$ zum Brennpunkt hat.

Ist speciell $v_{x}=0$ die unendlich ferme Gerade $\left(v_{i}=p_{i}\right)$, so besteht die Kegelschnittschaar aus Parabeln, $\omega(v, v)$ ist alsdann gleich Null, und der Ort der Brennpunkte zerfällt in Uebereinstimmung mit (257) 
in den umschriebenen Kreis des Dreiecks und die unendlich ferne Gerade.

265. Man bilde die Gleichung der Curve dritter Ordnung, auf welcher die Brennpunkte aller Kegelschnitte liegen, die ein gegebenes Dreiseit zum Poldreiseit haben und eine feste Gerade berühren.

Dass die Brennpunkte eine Curve dritter Ordnung erfüllen, folgt aus (264), denn die Kegelschnitte bilden eine Schaar. Für

$$
v_{1} x_{1}+v_{2} x_{2}+v_{3} x_{3}=0
$$

als Gleichung der gegebenen Geraden ist das System von Kegelschnitten $\varphi(u, u) \equiv \alpha_{1} u_{1}{ }^{2}+\alpha_{2} u_{2}{ }^{2}+\alpha_{3} u_{3}{ }^{2}=0$ der Bedingung unterworfen $\alpha_{1} v_{1}{ }^{2}+\alpha_{2} v_{2}{ }^{2}+\alpha_{3} v_{3}{ }^{2}=0$; ausserdem besteht eine Relation von der Form

$$
\begin{gathered}
\mu\left(\alpha_{1} u_{1}^{2}+\alpha_{2} u_{2}^{2}+\alpha_{3} u_{3}^{2}\right)+\omega(u, u) \\
=\left(y_{1} u_{1}+y_{2} u_{2}+y_{3} u_{3}\right)\left(z_{1} u_{1}+z_{2} u_{2}+z_{3} u_{3}\right) .
\end{gathered}
$$

Eliminirt man aus dieser Gleichung die Grössen $z_{i}$ mit Hilfe der in (262) gefundenen Beziehungen zwischen den $y_{i}, z_{i}$ und $\omega_{i k}$, und vergleicht man beiderseits die Coefficienten von $u_{1}{ }^{2}, u_{2}{ }^{2}, u_{3}{ }^{2}$, so folgt

$$
\mu \alpha_{1}=\left(-\omega_{23} y_{1}+\omega_{31} y_{2}+\omega_{12} y_{3}\right) y_{1}^{2}-\omega_{11} y_{1} y_{2} y_{3}
$$

einen analogen Werth haben $\mu \alpha_{2}$ und $\mu \alpha_{3}$. Durch Substitution in $\alpha_{1} v_{1}{ }^{2}+\alpha_{2} v_{2}{ }^{2}+\alpha_{3} v_{3}{ }^{2}=0$ erhält man die Curve dritter Ordnung $\begin{aligned} & v_{1}{ }^{2} y_{1}{ }^{2}\left(-\omega_{z 3} y_{1}+\omega_{31} y_{2}+\omega_{12} y_{3}\right)+v_{2}^{2} y_{2}{ }^{2}\left(\omega_{23} y_{1}-\omega_{31} y_{2}+\omega_{12} y_{3}\right) \\ + & v_{3}^{2} y_{3}{ }^{2}\left(\omega_{23} y_{1}+\omega_{31} y_{2}-\omega_{12} y_{3}\right)-\left(\omega_{11} v_{1}^{2}+\omega_{22} v_{2}{ }^{2}+\omega_{33} v_{3}^{2}\right) y_{1} y_{2} y_{3}=0\end{aligned}$ wofür auch gesetzt werden $\mathrm{kann}$

$$
\begin{gathered}
{\left[-\omega_{23} v_{1} y_{1}^{2}-\omega_{31} v_{2} y_{2}^{2}-\omega_{12} v_{3} y_{3}^{2}+\left(\omega_{12} v_{2}+\omega_{13} v_{3}\right) y_{2} y_{3}\right.} \\
\left.+\left(\omega_{21} v_{1}+\omega_{23} v_{3}\right) y_{1} y_{3}+\left(\omega_{31} v_{1}+\omega_{32} v_{2}\right) y_{1} y_{2}\right]\left(v_{1} y_{1}+v_{2} y_{2}+v_{3} y_{3}\right) \\
-\omega(v, v) \cdot y_{1} y_{2} y_{3}=0 .
\end{gathered}
$$

Zugleich ist klar, dass diese Gleichung bei constanten $y$ und variabelen $v$ die Curve zweiter Classe darstellt, für welche das Coordinatendreiseit ein Poldreiseit und ein bestimmter Punkt $y$ der eine Brempunkt ist.

Ist speciell $v_{x}=0$ die unendlich ferne Gerade $\left(v_{i}=p_{i}\right)$, so besteht die Kegelschnittschaar aus Parabeln, $\omega(v, v)$ verschwindet, die Curve dritter Ordnung zerfällt alsdann in Uebereinstimmung mit (262) in den Feuerbach'schen Kreis und die unendlich ferne Gerade ${ }^{1}$ ).

1) Für Nr. 264 und 265 vgl. auch den Anhang zu $\$ 22-26$. 
266. Die Verbindungslinien irgend eines Punktes $P$ eines Kegelschnitts mit den beiden Brennpuukten bilden mit der Tangente und Normale des Punktes $P$ vier harmonische Strahlen.

Folgt daraus, dass die Tangente und Normale von $P$ nach (28), S. 190 die Winkel der beiden Breunstrahlen halbiren.

Hieraus folgt weiter:

267. Die Tangente und Normale in irgend einem Punkte des Kegelschnitts treffen die Hauptaxe der Curve in zwei Punkten, die zu den Brempunkten harmonisch liegen.

Speciell für die Parabel folgt:

268. Die Tangente und Normale in irgend einem Punkte einer Parabel treffen die Axe in zwei Punkten, welche vom Brennpunkt gleiche Entfernung haben und auf verschiedenen Seiten desselben liegen.

269. Verbindet man einen Brennpunkt $B$ eines Kegelschnitts mit den Berührungspunkten $P, Q$ und mit dem Schnittpunkte $M$ zweier Tangenten, so halbirt die letztgenannte Verbindungslinie den Winkel $P B Q$ der beiden von $B$ aus nach $P$ und $Q$ gezogenen Strahlen (Fig. 8 und 9, S. 189).

Zufolge des Satzes (26), S. $187^{1}$ ) ist $M$ der Mittelpunkt eines Kreises, der die Verbindungslinien der Brennpunkte wit den zwei Berührungspunkten $P, Q$ zu Tangenten hat. Die Gerade $M B$ ist demnach eine durch $B$ gehende Centrale des Kreises und halbirt als solche bekanntlich den Winkel der beiden von $B$ an den Kreis gelegten Tangenten, die mit $B P$ und $B Q$ zusammenfallen.

270. Die Enveloppe aller Geraden, für welche das Product ihrer Entfernungen von zwei festen Punkten constant ist, ist eine Curve zweiter Classe, die die zwei gegebenen Punkte zu Brennpunkten hat.

Es seien $y_{i}$ und $z_{i},(i=1,2,3)$, die Coordinaten der festen Punkte, $u_{1}, u_{2}, u_{3}$ die Coordinaten einer Geraden. Mit Hilfe von (1), S. 9 erhält man für das obige Product

$$
\frac{\left(y_{1} u_{1}+y_{z} u_{2}+y_{3} u_{3}\right)\left(z_{1} u_{1}+z_{2} u_{2}+z_{3} u_{3}\right)}{\omega(u, u) p_{y} p_{z}}=\text { const. }
$$

und diese Gleichung stellt offenbar eine Curve zweiter Classe dar,

1) Weitere Anwendungen des 'Theorems (26), S.187 hat Herr Lucian Gottscho in der Lösung einer Preisaufgabe gegeben, die anschliessend an dieses Theorem Herr Gundelfinger im Jahre 1893 den Studirenden der mathematisch-naturwissenschaftlichen Abtheilung der Technischen Hochschule zu Darmstadt gestelit hatte.

Gundelfinger, Vorlesungen. 
welche nach (20), S. 168 die zwei gegebenen Punkte $y$ und $z$ zu Brennpunkten hat. Ertheilt man der Constanten alle möglichen Werthe, so entsteht eine confocale Schaar von Kegelschnitten.

Uebrigens kann die Gleichung der Curve auch geschrieben werden in der Form $\frac{u_{y} \cdot u_{z}}{p_{y} \cdot p_{z} \cdot \omega(u, u)}=$ const.; aus den Bemerkungen S. 191 und S. 9 folgt alsdann, dass sie eine Ellipse oder Hyperbel darstellt, je nachdem der Constanten ein positiver oder negativer Werth ertheilt wird.

Der obige Satz bildet übrigens die Umkehrung von (3), S. 179.

271. Zwei conjugirte Polaren eines Kegelschnitts, die sich in einem Brennpunkte der Curve schneiden, sind zu einander normal.

Für $y_{i}$ und $z_{i}$ als Coordinaten der Brennpunkte des Kegelschnitts besteht nach (20), S. 168 eine Relation von der Form

$$
\lambda_{1} \varphi(u, u)-\omega(u, u)=y_{u} z_{u} .
$$

Sind nun $u$ und $v$ conjugirte Polaren in Bezug auf $\varphi(u, u)=0$, so ist $\varphi(u, v)=0$ oder $y_{u} z_{v}+y_{v} z_{u}+2 \omega(u, v)=0$, und da die Polaren durch den einen Brennpunkt, etwa $y$, gehen sollen, verschwinden sowohl $y_{u}$ als $y_{v}$, es bleibt nur $\omega(u, v)=0$, d. h. die beiden Geraden $u$ und $v$ sind nach S. 13 zu einander normal. (Vgl. übrigens (33), S. 193.)

Aehnlich beweist man die Umkehrung, dass zwei in einem Brennpunkt sich rechtwinklig schneidende Geraden conjugirte Polaren des Kegelschnitts sind.

\section{Anwendung von \& 20-21.}

272. Man bilde die Gleichung für den geometrischen Ort der Mittelpunkte des Büschels von Kegelschnitten, die dem Coordinatendreieck umschrieben sind und durch einen und denselben Punkt $z$ gehen.

Für den Mittelpunkt $x$ einer Curve zweiter Ordnung bestehen nach (22), S. 25 die Relationen $\frac{1}{2} f^{\prime}\left(x_{i}\right)=\varrho p_{i},(i=1,2,3)$, wobei $\varrho$ einen Proportionalitätsfactor bedeutet. Ausgehend von

$$
2\left(a_{1} x_{2} x_{3}+a_{2} x_{3} x_{1}+a_{3} x_{1} x_{2}\right)=0
$$

als Gleichung einer dem Coordinatendreieck umschriebenen Curve erhält man also die drei Gleichungen

$$
\begin{aligned}
& \varrho p_{1}=\quad a_{2} x_{3}+a_{3} x_{2} \\
& \varrho p_{2}=a_{1} x_{3}+a_{3} x_{1} \\
& \varrho p_{3}=a_{1} x_{2}+a_{2} x_{1}
\end{aligned}
$$


hierzu tritt noch $a_{1} z_{2} z_{3}+a_{2} z_{3} z_{1}+a_{3} z_{1} z_{2}=0$, so dass man durch Elimination von $a_{1}, a_{2}, a_{3}$ und $\varrho$ als Ort des Mittelpunktes $x$ erhält:

oder

$$
\left|\begin{array}{llll}
p_{1} & 0 & x_{3} & x_{2} \\
p_{2} & x_{3} & 0 & x_{1} \\
p_{3} & x_{2} & x_{1} & 0 \\
0 & z_{2} z_{3} & z_{3} z_{1} & z_{1} z_{2}
\end{array}\right|=0
$$

$$
p_{1} z_{2} z_{3} x_{1}^{2}+\cdots-\left(p_{2} z_{2}+p_{3} z_{3}\right) z_{1} x_{2} x_{3}-\cdots=0,
$$

wobei die zu dem vorhergehenden symmetrischen Glieder durch Punkte angedeutet sind. Für $z_{i}=\frac{1}{\omega_{23}}$ vgl. (165).

273. Man bilde die Gleichung für den geometrischen Ort der Mittelpunkte aller dem Coordinatendreieck eingeschriebenen und eine und dieselbe Gerade $v$ berührenden Kegelschnitte.

Es sei $2\left(\alpha_{1} u_{2} u_{3}+\alpha_{2} u_{3} u_{1}+\alpha_{3} u_{1} u_{2}\right)=0$ die Gleichung eines dem Coordinatendreieck eingeschriebenen Kegelschnitts. Zu den drei Gleichungen

$$
\begin{aligned}
& \varrho x_{1}=\quad \alpha_{2} p_{3}+\alpha_{3} p_{2} \\
& \varrho x_{2}=\alpha_{1} p_{3}+\alpha_{3} p_{1} \\
& \varrho x_{3}=\alpha_{1} p_{2}+\alpha_{2} p_{1}
\end{aligned}
$$

für die Coordinaten $x_{i}$ des Mittelpunktes tritt noch als vierte hinzu $\alpha_{1} v_{2} v_{3}+\alpha_{2} v_{3} v_{1}+\alpha_{3} v_{1} v_{2}=0$. Durch Elimination von $\varrho, \alpha_{1}, \alpha_{2}, \alpha_{3}$ folgt

oder

$\begin{array}{llll}x_{1} & 0 & p_{3} & p_{2} \\ x_{2} & p_{3} & 0 & p_{1} \\ x_{3} & p_{2} & p_{1} & 0 \\ 0 & v_{2} v_{3} & v_{3} v_{1} & v_{1} v_{2}\end{array}=0$

$$
\begin{gathered}
\left(-p_{1} v_{2} v_{3}+p_{2} v_{3} v_{1}+p_{3} v_{1} v_{2}\right) p_{1} x_{1}+\left(p_{1} v_{2} v_{3}-p_{2} v_{3} v_{1}+p_{3} v_{1} v_{2}\right) p_{2} x_{2} \\
+\left(p_{1} v_{2} v_{3}+p_{2} v_{3} v_{1}-p_{3} v_{1} v_{2}\right) p_{3} x_{3}=0
\end{gathered}
$$

also die Gleichung einer Geraden, in Uebereinstimmung mit (15), S. 200.

274. Man bilde die Gleichung für den geometrischen Ort der Mittelpunkte aller Kegelschnitte, die das Coordinatendreieck zum Poldreieck haben und durch einen und denselben Punkt $z$ gehen.

Ausgehend von $a_{1} x_{1}{ }^{2}+a_{2} x_{2}{ }^{2}+a_{3} x_{3}{ }^{2}=0$ erhält man in derselben Weise wie bei (272) für den Ort des Mittelpunktes in variabelen Punktcoordinaten $x_{i}$ die Gleichung: 
oder

$$
\left|\begin{array}{cccc}
p_{1} & x_{1} & 0 & 0 \\
p_{2} & 0 & x_{2} & 0 \\
p_{3} & 0 & 0 & x_{3} \\
0 & z_{1}^{2} & z_{2}^{2} & z_{3}^{2}
\end{array}\right|=0
$$

$$
p_{1} z_{1}^{2} x_{2} x_{3}+p_{2} z_{2}^{2} x_{3} x_{1}+p_{3} z_{3}^{2} x_{1} x_{2}=0 \text {. }
$$

Für $z_{i}= \pm \sqrt{\omega_{i i}}$ erhält man den umschriebenen Kreis des Dreiecks. Vgl. (73), (74), (169) und (171).

275. Man bilde die Gleichung für den geometrischen Ort der Mittelpunkte aller Kegelschuitte, die das Coordinatendreiseit zum Poldreiseit haben und eine und dieselbe Gerade $v$ berühren.

Ausgehend von $\alpha_{1} u_{1}{ }^{2}+\alpha_{2} u_{2}{ }^{2}+\alpha_{3} u_{3}{ }^{2}=0$ erhält man auf gleiche Weise wie bei (273) für den Ort des Mittelpunktes $x$ die Gleichung

oder

$$
\left|\begin{array}{cccc}
x_{1} & p_{1} & 0 & 0 \\
x_{2} & 0 & p_{2} & 0 \\
x_{3} & 0 & 0 & p_{3} \\
0 & {v_{1}}^{2} & v_{2}{ }^{2} & v_{3}{ }^{2}
\end{array}\right|=0
$$

$$
v_{1}^{2} p_{2} p_{3} x_{1}+v_{2}^{2} p_{3} p_{1} x_{2}+v_{3}^{2} p_{1} p_{2} x_{3}=0 .
$$

Eine andere Ableitung von (274) und (275) ergibt sich mit Hilfe von $(30)$ und (31).

276. Mau untersuche, auf welche Weise die Art der Kegelschnitte eines Büschels, bezw. des zugehörigen Mittelpunktskegelschnitts, durch die Gestalt des als reell vorausgesetzten Vierecks der Grundpunkte bedingt ist ${ }^{1}$ ).

Was zunächst den Mittelpunktskegelschnitt $M$ betrifft, so ist derselbe nach (10), S. 199 eine Hyperbel, Parabel oder Ellipse, je nachdem sich durch die vier Grundpunkte des Büschels zwei verschiedene reelle Parabeln legen lassen, oder diese Parabeln in eine eiuzige zusammenfallen, oder inaginär sind. Die Grundpunkte des Büschels mögen nun bestehen aus den Ecken des Coordinatendreiecks und einem Punkte $z$; nach (272) lautet alsdann die Gleichung des Kegelschnitts $M$ :

$$
\begin{aligned}
p_{1} z_{2} z_{3} x_{1}{ }^{2} & +p_{2} z_{3} z_{1} x_{2}^{2}+p_{3} z_{1} z_{2} x_{3}^{2}-\left(p_{2} z_{2}+p_{3} z_{3}\right) z_{1} x_{2} x_{3} \\
& -\left(p_{3} z_{3}+p_{1} z_{1}\right) z_{2} x_{3} x_{1}-\left(p_{1} z_{1}+p_{2} z_{2}\right) z_{3} x_{1} x_{2}=0 .
\end{aligned}
$$

1) Vgl. Möbius: „Der barycentrische Calcul“, Leipzig 1827, § 253 und § 254 (Bd. 1 der gesammelten Werke, S. 325-327); ferner vergleiche man Steiner: "Vermischte Sätze und Aufgaben", Journal für die reine und angewandte Mathematik, Bd. 55, S. 372 f., 1858, oder auch "Gesammelte Werke", Bd, 2, S. $678 \mathrm{f}$. 
Die Gattung dieser Curve $M$ ist dieselbe wie die einer anderen Curve, deren Gleichung aus derjenigen von $\boldsymbol{M}$ durch Addition von

$$
\left(p_{1} x_{1}+p_{2} x_{2}+p_{3} x_{3}\right)\left(x_{1} z_{2} z_{3}+x_{2} z_{3} z_{1}+x_{3} z_{1} z_{2}\right)
$$

hervorgeht, denn beide Curven werden von $p_{x}=0$ in denselben Punkten getroffen. Man erhält hierdurch die Gleichung

$$
\left.p_{1} z_{2} z_{3} x_{1}{ }^{2}+p_{2} z_{3} z_{1} x_{2}{ }^{2}+p_{3} z_{1} z_{2} x_{3}{ }^{2}=0,{ }^{1}\right)
$$

welche eine Ellipse oder Hyperbel repräsentirt, je nachdem

$$
p_{1} p_{2} p_{3} z_{1} z_{2} z_{3}\left(p_{1} z_{1}+p_{2} z_{2}+p_{3} z_{3}\right)
$$

$>0$ oder $<0$ ist, d. h. zufolge (3) je nachdem der Punkt $z$ in Bezug auf das Coordinatendreieck trigonal oder tetragonal gelegen ist; im Falle $p_{s}=0$, also wenn $z$ im Unendlichen liegt, ist der Kegelschnitt $M$ eine Parabel. Mit Rücksicht auf die Betrachtungen S. 199 erhält man aus dem Vorstehenden das Resultat:

„Haben vier Punkte in einer Ebene eine solche Lage gegen einander, dass jeder derselben ausserhalb des Dreiecks, welches die drei anderen bilden, befindlich ist, so lassen sich durch sie zwei verschiedene Parabeln beschreiben. Liegt dagegen einer der vier Punkte innerhalb des von den drei anderen gebildeten Dreiecks, so kann durch sie keine Parabel beschhrieben werden". ${ }^{2}$ ) Im ersten Falle ist der Mittelpunktskegelschnitt eine Hyperbel, im anderen Falle eine Ellipse.

Es mögen nun noch die übrigen Kegelschnitte betrachtet werden, welche man durch die vier Grundpunkte legen kann. Wir behaupten, dass hier der Satz gilt:

"Wenn von vier Punkten in einer Ebene jeder derselben ausserhalb des von den drei anderen gebildeten Dreiecks liegt, so lassen sich durch sie sowohl Ellipsen als Hyperbeln und zwei verschiedene Parabeln beschreiben. Liegt aber der eine innerhalb des von den drei anderen gebildeten Dreiecks, so können durch sie weder Ellipsen noch Parabeln, sondern bloss Hyperbeln geführt werden". ${ }^{3}$ )

Zum Beweis dieses Satzes führen wir in den Ausdruck

$$
\begin{aligned}
F(p, p)= & -a_{1}{ }^{2} p_{1}{ }^{2}-a_{2}{ }^{2} p_{2}{ }^{2}-a_{3}{ }^{2} p_{3}{ }^{2} \\
& +2 a_{2} a_{3} p_{2} p_{3}+2 a_{3} a_{1} p_{3} p_{1}+2 a_{1} a_{2} p_{1} p_{2},
\end{aligned}
$$

dessen Vorzeichen über die Gattung der Curve

1) Derjenige Kegelschnitt, welcher das Coordinatendreieck zum Poldreieck, den Punkt $z$ zum Mittelpunkt hat, besitzt nach (30) dieselbe Gleichung. Mit Rücksicht auf (75) und (17) erkennt man auch, dass sie einen Kreis nur dann darstellt, wenn $z$ der Höhenschnittpuukt des Coordinatendreiecks ist.

2) Vgl. Möbius a. a. $0 . \S 253$.

3) Möbins ibid. § 254. 


$$
f(x, x) \equiv 2\left(a_{1} x_{2} x_{3}+a_{2} x_{3} x_{1}+a_{3} x_{1} x_{2}\right)=0
$$

Auskunft gibt, an Stelle der Coefficienten $a$ willkürliche Parameter $v$ ein, indem wir mit Rücksicht auf $\frac{a_{1}}{z_{1}}+\frac{a_{2}}{z_{2}}+\frac{a_{3}}{z_{3}}=0$ setzen

$$
a_{1}=\frac{v_{3}}{z_{2}}-\frac{v_{9}}{z_{3}}, \quad a_{2}=\frac{v_{1}}{z_{3}}-\frac{v_{3}}{z_{1}}, \quad a_{3}=\frac{v_{9}}{z_{1}}-\frac{v_{1}}{z_{2}},
$$

oder wenn $\frac{1}{z_{i}}$ ersetzt wird durch $u_{i}$, so folgt $a_{1} u_{1}+a_{2} u_{2}+a_{3} u_{3}=0$ und $a_{1}=u_{2} v_{3}-u_{3} v_{2}, a_{2}=u_{3} v_{1}-u_{1} v_{3}, a_{3}=u_{1} v_{2}-u_{2} v_{1}$. Hierdurch verwandelt sich $F(p, p)$ in

$$
\begin{aligned}
-\left(u_{2} v_{3}-u_{3} v_{2}\right)^{2} p_{1}{ }^{2} & -\left(u_{3} v_{1}-u_{1} v_{3}\right)^{2}{p_{2}}^{2}-\left(u_{1} v_{2}-u_{2} v_{1}\right)^{2} p_{3}{ }^{2} \\
& +2\left(u_{3} v_{1}-u_{1} v_{3}\right)\left(u_{1} v_{2}-u_{2} v_{1}\right) p_{2} p_{3}+\cdots,
\end{aligned}
$$

also in einen Ausdruck, der gleich Null gesetzt zufolge (53), S. 34 in variabelen Liniencoordinaten $v$ das Schnittpunktepaar der Geraden $u_{x}=0$ nit dem Kegelschnitt (vgl. (39) und S. 216)

$$
\begin{aligned}
E(x, x) \equiv p_{1}{ }^{2} x_{1}{ }^{2}+p_{2}{ }^{2} x_{2}{ }^{2}+p_{3}{ }^{2} x_{3}{ }^{2}-2 p_{2} p_{3} x_{2} x_{3}-2 p_{3} p_{1} x_{3} x_{1} \\
-2 p_{1} p_{2} x_{1} x_{2}=0
\end{aligned}
$$

darstellen wïrde. Ist dieses Punktepaar imaginär, so behält der letzte Ausdruck von $F(p, p)$ für alle Werthe der $v$ sein Vorzeichen, und zwar das negative, es bleibt also $F(p, p)$ auch für alle Werthe der $a$ negativ, $d . h$. durch die Grundpunkte des Büschels können nur Hyperbelu gelegt werden. Der Kegelschnitt $E(x, x)=0$ wird aber nach S. 36 von der Geraden $u_{x}=0$ in imagiuären oder reellen Punkten getroffen, je nachdem

$$
p_{1} p_{2} p_{3}\left(p_{1} u_{2} u_{3}+p_{2} u_{3} u_{1}+p_{3} u_{1} u_{2}\right) \equiv \frac{p_{1} p_{2} p_{3}}{z_{1} z_{2} z_{3}}\left(p_{1} z_{1}+p_{2} z_{2}+p_{3} z_{3}\right)
$$

$>0$ oder $<0$ ist, d. h. je nachdem der Punkt $z$ in Bezug auf das Coordinatendreieck trigonal oder tetragonal gelegen ist. Im ersten Falle bestehen die Curven des Büschels nur aus Ellipsen. Das Schnittpunktepaar von $E(x, x)=0$ mit $\left.u_{x}=0^{1}\right)$ ist dagegen reell, d. h. der Ausdruck $F(p, p)$ kann für beliebige Werthe der $v_{i}$, also auch der $a_{i}$, sein Vorzeichen wechseln, wenn der Punkt $z$ tetragonal liegt; wegen des Vorzeichenwechsels von $F(p, p)$ gehen also in diesem Falle durch die Grundpunkte des Büschels sowohl Hyperbeln als Ellipsen und Parabelı, für welch letztere nach S. 199 die Anzahl gleich 2 ist.

Eine andere noch tiefer eindringende Behandlung ist folgende ${ }^{2}$ ). Auf ein gemeinsames Poldreieck bezogen seien

1) Man beachte, dass die Gerade $u_{x}=0$ die Harmonicale des Punktes $z$ (vgl. (40)) in Bezug auf das Dreieck $x_{1} x_{2} x_{3}$ darstellt.

2) Diese Methode kann auch zur Behandlung des Falles von vier imaginären 
$f(x, x) \equiv \lambda_{1} x_{1}{ }^{2}+\lambda_{2} x_{2}{ }^{2}+\lambda_{3} x_{3}{ }^{2}=0$ und $g(x, x) \equiv x_{1}{ }^{2}+x_{2}{ }^{2}+x_{3}{ }^{2}=0$ die Gleichungen zweier Kegelschnitte, die ein Büschel $\lambda g-f=0$ mit vier reellen Grundpunkten bestimmen; dabei müssen die $x_{i}{ }^{2}$ theilweise negativ sein. In Liniencoordinaten erhält man für dieses Büschel die Gleichung $\frac{u_{1}{ }^{2}}{\lambda-\lambda_{1}}+\frac{u_{2}{ }^{2}}{\lambda-\lambda_{2}}+\frac{u_{3}{ }^{2}}{\lambda-\lambda_{3}}=0$, woraus folgt, dass die Coordinaten des Mittelpunktes der dem Parameter $\lambda$ entsprechenden Curve die Werthe besitzen $\varrho x_{i}=\frac{p_{i}}{\lambda-\lambda_{i}},(i=1,2,3)$, mit $\varrho$ als Proportionalitätsfactor $\left.{ }^{1}\right)$. Die Parameter der zwei in dem Büschel enthaltenen Parabeln sind durch die mit $p_{1} x_{1}+p_{2} x_{2}+p_{3} x_{3}=0$ gleichbedeutende quadratische Gleichung bestimmt

$$
\frac{p_{1}^{2}}{\lambda-\lambda_{1}}+\frac{p_{2}^{2}}{\lambda-\lambda_{2}}+\frac{p_{3}{ }^{2}}{\lambda-\lambda_{3}}=0
$$

besitzt dieselbe zwei complexe Wurzeln, so ist der Mittelpunktskegelschnitt $M$ eine Ellipse und, wie wir num wissen, sind alle Curven des Büschels Hyperbeln. Hat jedoch die quadratische Gleichung reelle Wurzeln $\lambda^{\prime}$ und $\lambda^{\prime \prime}$, so ist $M$ eine Hyperbel, das Büschel besteht aus Ellipsen, Hyperbeln und zwei Parabeln. Den zwischen $\lambda^{\prime}$ und $\lambda^{\prime \prime}$ gelegenen Werthen des Parameters $\lambda$ entspricht eine stetige Reihe von Mittelpunkten, also ein Zweig der Hyperbel $M$; allen ausserhalb des Intervalles von $\lambda^{\prime}$ bis $\lambda^{\prime \prime}$ gelegenen Werthen des Parameters $\lambda$, wozu auch $\lambda= \pm \infty$ gehört, entspricht wieder eine stetige Reihe von Mittelpunkten: der andere Zweig der Hyperbel $M$; für $\lambda=\lambda^{\prime}$ und $\lambda=\lambda^{\prime \prime}$ erhält man hingegen die unendlich fernen Punkte von $M$, denen als Curven des Büschels, wie erwähnt, die zwei Parabeln zugehören. Man erkennt ferner, dass aus Gründen der Stetigkeit die Mittelpunkte der in dem Büschel enthaltenen Ellipsen einen Zweig des Kegelschnitts $M$, die Mittelpunkte der Hyperbeln den anderen Zweig von $M$ erfüllen ${ }^{2}$ ).

277. Man untersuche, auf welche Weise die einem Vierseit eingeschriebenen Kegelschnitte nach der Lage ihrer Mittelpunkte angeordnet $\operatorname{sind}^{3}$ ). .

Schnittpunkten verwandt werden. Vgl. den Artikel über die Kriterien für die Realität der Schnittpnnkte $z$ weier Kegelschnitte im vorliegenden Theile des Anhanges.

1) Natürlich sind die $p_{i}$ theilweise imaginär.

2) Vgl. Steiner a. a. 0 .

3) Vgl. Steiner a. a. O., in Journal für die reine und angewandte Mathematik, Bd. 55, S. 374, in den "Gesammelten Werken", Bd. 2, S. 680. 
Auf das gemeinsame Poldreiseit bezogen seien

und

$$
F(u, u) \equiv \lambda_{1} u_{1}^{2}+\lambda_{2} u_{2}^{2}+\lambda_{3} u_{3}^{2}=0
$$

$$
G(u, u) \equiv u_{1}^{2}+u_{2}^{2}+u_{3}^{2}=0
$$

die Gleichungen zweier Kegelschnitte, durch welche eine Schaar

$$
\lambda G-F \equiv\left(\lambda-\lambda_{1}\right) u_{1}{ }^{2}+\left(\lambda-\lambda_{2}\right) u_{2}{ }^{2}+\left(\lambda-\lambda_{3}\right) u_{3}{ }^{2}=0
$$

mit vier reellen gemeinsamen Tangenten bestimmt sei. Die dem Parameter $\lambda$ entsprechende Curve der Schaar ist nach S. 55 eine Ellipse, Hyperbel oder Parabel, je nachdem

$$
\begin{gathered}
K \equiv\left(\lambda-\lambda_{1}\right)\left(\lambda-\lambda_{2}\right)\left(\lambda-\lambda_{3}\right)\left\{\left(\lambda-\lambda_{1}\right) p_{1}{ }^{2}+\left(\lambda-\lambda_{2}\right) p_{2}{ }^{2}\right. \\
\left.\left.+\left(\lambda-\lambda_{3}\right) p_{3}{ }^{2}\right)\right\}
\end{gathered}
$$

$>0,<0,=0$ ist. Führt man in diesen Ausdruck die Coordinaten

$$
\varrho y_{i}=\left(\lambda-\lambda_{i}\right) p_{i},(i=1,2,3) \text {, }
$$

des Mittelpunktes ein, so verwandelt er sich in

$$
\left.\frac{y_{1} y_{2} y_{3}}{p_{1} p_{2} p_{3}}\left(p_{1} y_{1}+p_{2} y_{2}+p_{3} y_{3}\right),{ }^{1}\right)
$$

wobei der jedenfalls positive Proportionalitätsfactor $\rho^{4}$ im Zähler weggelassen wurde. Hieraus folgt mit Rücksicht auf (4), dass der Kegelschnitt eine Ellipse oder Hyjperbel ist, je. nachdem sein Mittelpunkt in Bezug auf das der Kegelschnittschaar gemeinsame Poldreiseit trigonal oder tetragonal liegt. Ausserdem liegen nach (15), S. 200 die Mittelpunkte aller Curven der. Schaar auf einer Geraden $\mathfrak{M}$, welche durch die Mitten $\alpha, \beta, \gamma$ der drei Diagonalen des Vierseits geht, denn die zwei Endpunkte jeder Diagonale bilden ein der Schaar angehöriges Punktepaar; die Diagonalen selbst sind nach (20), S. 145 die Seiten des gemeinsamen Poldreiseits. Dem unendlich fernen Punkte $\delta$ der Mittelpunktsgeraden $\mathfrak{M}$ entspricht natürlich eine Parabel (die einzige nicht zerfallende, die im allgemeinen in der Schaar enthalten); passirt man beim Durchlaufen der Geraden $\mathfrak{M}$ die Punkte $\left.\alpha, \beta, \gamma,{ }^{2}\right) \delta$ in ihrer alphabetischen Reihenfolge und ist etwa die Strecke $\delta \alpha$ in Bezug auf das Poldreiseit tetragonal gelegen, daher $\alpha \beta$ trigonal, $\beta \gamma$ tetragonal, $\gamma \delta$ wieder trigonal gelegen, so enthalten nach dem Vorausgehenden die Strecken $\alpha \beta$ und $\gamma \delta$ die Mittelpunkte zweier Gruppen Ellipsen, während die Mittelpunkte zweier Gruppen Hyperbeln auf den Strecken $\beta \gamma$ und $\delta \alpha$ liegen.

1) Bei der Transformation von $f(x, x)=0$ und $g(x, x)=0$ in eine Summe von je drei Quadraten können zwar die $y_{i}$ bezw. $p_{i}$ rein imaginär werden, der obige Ausdruck würde aber stets reell bleiben.

2) Man beachte die in (207) erwähnten ausartenden Parabeln. 
Aehnliche Kegelschnitte einer Schaar. Maximum des Asymptotenwinkels. 361

Zu demselben Resultat wäre man gelangt bei directer Benutzung des oben mit $K$ bezeichneten Ausdrucks; führt man in $K$ den der Parabel entsprechenden Parameter

$$
\lambda^{\prime}=\left(\lambda_{1} p_{1}{ }^{2}+\lambda_{2}{p_{2}}^{2}+\lambda_{3} p_{3}^{2}\right):\left(p_{1}^{2}+{p_{2}}^{2}+p_{3}{ }^{2}\right)
$$

ein, so wird $K=\left(\lambda-\lambda_{1}\right)\left(\lambda-\lambda_{2}\right)\left(\lambda-\lambda_{3}\right)\left(\lambda-\lambda^{\prime}\right)\left(p_{1}{ }^{2}+p_{2}{ }^{2}+p_{3}{ }^{2}\right)$. Es wäre jetzt nur zu beachten, dass $\lambda^{\prime}$ grösser als die kleinste und kleiner als die grösste der drei Grössen $\lambda_{1}, \lambda_{2}, \lambda_{3}$ sein kann; immer lassen sich die Grössen $\lambda$ so ordnen, dass z. B. die Folge $\lambda_{1}, \lambda^{\prime}, \lambda_{2}, \lambda_{3}$ entweder eine wachsende oder abnehmende Zahlenreihe bildet.

Zufolge (177) erfüllen die Mittelpunkte aller unter sich ähnlicher Kegelschnitte, welche das Coordinatendreieck zum Poldreieck haben, die Curve vierter Ordnung

$$
\begin{aligned}
4 \tau p_{1} p_{2} p_{3} y_{1} y_{2} y_{3}\left(p_{1} y_{1}\right. & \left.+p_{2} y_{2}+p_{3} y_{3}\right) \\
& +\operatorname{tg}^{2} \alpha\left(\omega_{11} p_{1} y_{2} y_{3}+\omega_{22} p_{2} y_{3} y_{1}+\omega_{33} p_{3} y_{1} y_{2}\right)^{2}=0,
\end{aligned}
$$

die in den Ecken des Coordinatendreiecks je einen Doppelpunkt hat. $\mathrm{Da}$ diese Curve von der Geraden $\mathfrak{M}$ in vier Punkten geschnitten wird, gibt es unter den einem Vierseit eingeschriebenen Kegelschnitten im allgemeinen je vier einander ähnliche ${ }^{1}$ ). Jedoch gibt es nur zwei eingeschriebene gleichseitige Hyperbeln, und zwar sind ihre Mittelpunkte die Schnittpunkte des dem Poldreiseit umschriebenen Kreises $\omega_{11} p_{1} y_{2} y_{3}+\omega_{22} p_{2} y_{3} y_{1}+\omega_{33} p_{3} y_{1} y_{2}=0$ mit der Geraden $\mathfrak{M}$.

Man kann an das Vorausgehende die Aufgabe knüpfen, die Mittelpunkte derjenigen dem Vierseit eingeschriebenen Kegelschnitte zu bestimmen, für welche $\operatorname{tg}^{2} \alpha$ ein Maximum oder Minimum wird. Nach (192) ist $\operatorname{tg}^{2} \alpha=-\frac{8 q_{1} q_{2} q_{3} r}{P_{V}^{2}}$, worin $q_{i}$ die Abstände des Mittelpunktes $y$ von den Seiten des der Schaar zugehörigen Poldreiseits bezeichnen, während $r$ den Radius des umschriebenen Kreises, $P_{U}$ die Potenz von $y$ in Bezug auf diesen Kreis bedeutet. Da nun die Mittelpunkte $y$ auf der Geraden $\mathfrak{M}$ liegen und die Lothe $q_{i}$, abgesehen von den Sinus der Winkel, unter denen $\mathfrak{M}$ gegen die Seiten des Poldreiseits geneigt ist, gleich sind aen Strecken $y \alpha, y \beta, y \gamma$, da ferner $P_{U}$ gleich dem Product $y \alpha^{\prime} . y \beta^{\prime}$ ist, wenn $\alpha^{\prime}, \beta^{\prime}$ die Schnittpunkte von $\mathfrak{M}$ mit dem umschriebenen Kreis bedeuten, so wird die eben genannte Aufgabe

1) Die geometrische Behandlung der ähnlichen Kegelschnitte, die einem Büschel, bezw. einer Schaar angehören, lässt sich sehr übersichtlich auch nnter Beihilfe des Satzes (222) vermöge der zwei in (220) berührten Theoreme erledigen, auf welche sich die dort angeführten und für diese Behandlungsweise zu berücksichtigenden Citate des Werkes von Schröter beziehen. 
identisch damit, auf $\mathfrak{M}$ einen Punkt $y$ der Art zu bestimmen, dass $\frac{y \alpha \cdot y \beta \cdot y \gamma}{y \alpha^{2} \cdot y \beta^{\prime 2}}$ ein Maximum oder Minimum wird.

Bei Einführung irgend eines Anfangspunktes auf der Mittelpunktslinie möge $y$ die Abscisse $\eta$ haben, ferner seien $\alpha, \beta, \gamma, \alpha^{\prime}, \beta^{\prime}$ die Abscissen der durch die gleichen Buchstaben bezeichneten Punkte; alsdann verwandelt sich der letztangegebene Ausdruck in

$$
\frac{(\eta-\alpha)(\eta-\beta)(\eta-\gamma)}{\left(\eta-\alpha^{\prime}\right)^{2}\left(\eta-\beta^{\prime}\right)^{2}},
$$

und durch logarithmische Differentiation folgt

$$
\frac{1}{\eta-\alpha}+\frac{1}{\eta-\beta}+\frac{1}{\eta-\gamma}=2\left(\frac{1}{\eta-\alpha^{\prime}}+\frac{1}{\eta-\beta^{\prime}}\right)
$$

als Bedingung für ein Maximum oder Minimum.

Uebrigens ist die ganze Discussion auch ohne Differentiation durchführbar, lediglich durch nähere Betrachtung der Function

$$
\left.\frac{(\eta-\alpha)(\eta-\beta)(\eta-\gamma)}{\left(\eta-\alpha^{\prime}\right)^{2}\left(\eta-\beta^{\prime}\right)^{2}} \cdot{ }^{1}\right)
$$

278. Liegen die vier Grundpunkte eines Kegelschnittbüschels auf einem Kreise, so ist der Mittelpunktskegelschnitt des Büschels eine gleichseitige Hyperbel und die Axen aller. Kegelschnitte des Büschels sind parallel zu den zwei auf einander normal stehenden Asymptoten dieser Hyperbel ${ }^{2}$ ).

Nach (216) sind im vorliegenden Falle die Axen der zwei in dem Büschel enthaltenen Parabeln zu einander normal; die Asymptoten des Mittelpunktskegelschnitts $M$ sind aber wach (9), S. 199 zu diesen Axen parallel, daher ist $I I$ eine gleichseitige Hyperbel. Mit Hilfe von (12), S. $199 \mathrm{f}$. ergibt sich, dass die Hauptaxen aller Kegelschnitte des Büschels zu den Asymptoten dieser Hyperbel parallel sind.

Hieraus folgt weiter:

279. Wird ein Kegelschnitt von einem Kreise in vier Punkten geschnitten, so sind die sechs Halbirungslinien der Winkel zwischen den drei Paar Gegenseiten des Vierecks der Schnittpunkte zu je drei den Hauptaxen des Kegelschnitts parallel ${ }^{3}$ ).

1) Vgl. hierzu Plücker: „Analytisch-geometrische Entwicklungen“, 2. Bd., Essen 1831, S $222 \mathrm{ff}$.

2) Vgl. Schröter: „Die Theorie der Kegelschnitte, gestütat auf projectivische Eigenschaften" (zweiter Theil der von Geiser und Schröter herausgegebenen Vorlesungen Steiner's über synthetische Geometrie), 2. Auf., Leipzig 1876, S. 311.

3) Vgl. Steiner: "Vermischte Sütze und Aufgaben", Journal für die reine und angewandte Mathematik, Bd. 55, S. 359, 1858, oder auch "Gesammelte Werke", Bd. 2, S. $665 \mathrm{f}$. 
Denn die Halbirungslinien derjenigen zwei Winkel, welche von den Geraden eines Paares der Gegenseiten gebildet werden, können als die Axen dieses Geradenpaares angesehen werden.

Dieser Satz lässt sich auch in folgender Weise aussprechen:

280. „Halbirt man in einem Viereck im Kreise sowohl die Winkel zwischen den Diagonalen als auch die Winkel, welche die gegenüberliegenden Seiten einschliessen, so sind von den sechs Geraden, welche diese Winkel halbiren, drei und drei parallel", und selbstverständlich sind die drei ersten zu den drei anderen normal ${ }^{1}$ ).

281. Die Pole einer Geraden $v$ in Bezug auf die Kegelschnitte einer confocalen Schaar liegen auf einer zu $v$ normalen Geraden.

Für $\lambda \varphi(u, u)-\omega(u, u)=0$ als Gleichung der confocalen Schaar ist nach (14), S. 200 der Ort der Pole von $v$ die Gerade

$$
g_{x} \equiv \sum \pm\left(\varphi^{\prime}\left(v_{1}\right) \omega^{\prime}\left(v_{2}\right) x_{3}\right)=0 .
$$

Da bier $\omega(u, u)=0$ das imaginäre Kreispunlitepaar darstellt, sind $\boldsymbol{\omega}^{\prime}\left(v_{i}\right),(i=1,2,3)$, die Coordinaten des Normalencentrums der Geraden $v$; dieselben genügen aber, wie die Determinante für $g_{x}$ zeigt, der Gleichung $g_{x}=0$, d. h. $g_{x}=0$ ist zu der Geraden $v_{x}=0$ normal. Ebenso erkennt man ans der Determinante, dass $g_{x}=0$ Normale desjenigen bestimmten Kegelschnittes der Schaar $\lambda \varphi(u, u)-\omega(u, u)=0$ ist, welcher die gegebene Gerade $v$ berührt, denn die Coordinaten des Berührungspunktes $\lambda \varphi^{\prime}\left(v_{i}\right)-\omega^{\prime}\left(v_{i}\right),(i=1,2,3)$, genügen ebenfalls der Gleichung $g_{x}=0$. Vgl. (18), S. 201 bei gegebenen $x_{i}$ und veränderlichen $v_{i}$.

* 282. Excurs über binäre kubische Formen.

Es sei gegeben eine binäre kubische Form:

$$
f(x, y) \equiv a x^{3}+3 b x^{2} y+3 c x y^{2}+d y^{3}
$$

ihre Hesse'sche Covariante ist alsdann

$$
h(x, y)=\frac{1}{36}\left[\frac{\partial^{2} f}{\partial x^{2}} \frac{\partial^{2} f}{\partial y^{2}}-\left(\frac{\hat{c}^{2} f}{\partial x \cdot \partial y}\right)^{2}\right]
$$

und hat das Gewicht 2. Eine andere Covariante ist

$$
q(x, y) \equiv \frac{1}{3}\left(\frac{\partial f}{\partial x} \frac{\partial h}{\partial y}-\frac{\partial f}{\partial y} \frac{\partial h}{\partial x}\right)
$$

1) Steiner: „Aufgaben und Lehrsätze, erstere aufzulösen, letztere zu beweisen", Journal für die reine und angewandte Mathematik, Bd. 2, S. 97, 1827, oder anch "Gesammelte Werke", Bd. 1, S. 128. 
sie hat das Gewicht 3. Die Discriminante $r$ der kubischen Gleichung $f(x, y)=0$ ist

$$
r=4\left(a c-b^{2}\right)\left(b d-c^{2}\right)-(a d-b c)^{2}
$$

und stimmt überein mit der Discriminante der Hesse'schen Covariante

$$
h(x, y)=\left(a c-b^{2}\right) x^{2}+(a d-b c) x y+\left(b d-c^{2}\right) y^{2} .
$$

Für $q(x, y)$ findet man

$$
\begin{aligned}
q(x, y) & =\left(a^{2} d-3 a b c+2 b^{3}\right) x^{3}+3\left(a b d+b^{2} c-2 a c^{2}\right) x^{2} y \\
& +3\left(2 b^{2} d-a c d-b c^{2}\right) x y^{2}+\left(3 b c d-a d^{2}-2 c^{3}\right) y^{3} .
\end{aligned}
$$

Wir transformiren nun $f$ mit Hilfe der Substitution

$$
x=\alpha X-\beta Y, \quad y=\alpha^{\prime} X-\beta^{\prime} Y,
$$

deren Umkehrung gegeben ist durch

$$
-\Delta X=\beta^{\prime} x-\beta y, \quad-\Delta Y=\alpha^{\prime} x-\alpha y,
$$

wobei

$$
\Delta \equiv \alpha^{\prime} \beta-\alpha \beta^{\prime}
$$

die Determinante der Transformation bedentet.

Durch $(3)$ möge $f$ übergehen in

$$
F\left(X, I^{\prime}\right)=A X^{3}+3 B X^{2} Y+3 C X Y^{2}+D Y^{3} \text {. }
$$

Alsdann besteht die Relation

$$
\begin{gathered}
\Delta^{2} h(x, y)=H(X, Y) \\
=\left(A C-B^{2}\right) X^{2}+(A D-B C) X Y+\left(B D-C^{2}\right) Y^{2},
\end{gathered}
$$

und wenn man auch hier die Discriminante bildet, folgt

$$
\Delta^{2} \cdot \Delta^{4} \cdot r=4\left(A C-B^{2}\right)\left(B D-C^{2}\right)-(A D-B C)^{2} .
$$

Ist die Discriminante $r$ von Null verschieden und sind insbesondere $\alpha^{\prime}: \alpha$ und $\beta^{\prime}: \beta$ in $(3 a)$ die Wurzeln der quadratischen Gleichung $h(x, y)=0$, so müssen in $F(X, Y)$ die Coefficienten $B$ und $C$ gleich Null sein, denn die Form (6) muss nun proportional sein zu $X Y$, d. h. man hat $A C-B^{2}=0, B D-C^{2}=0, A D-B C \gtrless 0$. Es kann aber $A$ nicht verschwinden, sonst wäre auch $B=0$ und $F(X, Y)$ hätte den Factor $Y^{2}$; ebenso kann $D$ nicht verschwinden, sonst wäre auch $C=0$ und $F(X, Y)$ hätte den Factor $X^{2}$. Andrerseits folgen aus $A C-B^{2}=0$ und aus $B D-C^{2}=0$ die Gleichungen $\left(A C-B^{2}\right) C+\left(B D-C^{2}\right) A=0$ und $\left(A C-B^{2}\right) D+\left(B D-C^{2}\right) B=0$ oder

$$
(A D-B C) B=0 \quad \text { und } \quad(A D-B C) C=0,
$$

daher ist nothwendig $B=C=0$.

Es erhält also unter den gegenwärtigen Annahmen $F$ die einfache Gestalt: 


$$
F(X, Y)=A X^{3}+D Y^{3}
$$

für die Covariante $Q$ findet man

$$
\left.\Delta^{3} q=Q=A D\left(A X^{3}-D Y^{3}\right) \cdot{ }^{1}\right)
$$

Nach Zerlegung in Factoren sei nun

$$
f=\left(x-\lambda_{1} y\right)\left(x-\lambda_{2} y\right)\left(x-\lambda_{3} y\right) \text {. }
$$

Man kann alsdann $x-\lambda_{i} y=0,(i=1,2,3)$, geometrisch als drei Strahlen betrachten, die bezogen sind auf zwei Grundstrahlen $x=0$ und $y=0$; oder wenn man $X=0$ und $Y=0$ als Grundstrahlen einführt, so zeigt sich, dass die drei durch $F=0$ gegebenen Strahlen die Parameter haben $\lambda_{1}=-\sqrt[3]{\frac{D}{A}}, \lambda_{2}=-\varepsilon \sqrt[3]{\frac{D}{A}}, \lambda_{3}=-\varepsilon^{2} \sqrt[3]{\frac{D}{A}}$, wobei $\varepsilon$ und $\varepsilon^{2}$ die complex conjugirten dritten Wurzeln der positiven Einheit bedeuten. Das Doppelverhältniss je zweier dieser Strahlen zu $X=0$ und $Y=0$ besitat entweder den Werth $\varepsilon$ oder $\varepsilon^{2}$. Die Darstellung von $Q$ in (9) ergibt den Satz, dass die drei durch $Q=0$ dargestellten Strahlen erhalten werden, indem man zu je einem Strahlenpaare von $F=0$ und zu dem übrig bleibenden dritten Strahle von $F=0$ je $\cdot$ den viertelı harmonischen bestimmt. Aus dieser Deutung geht hervor, dass $q$ von der Form ist ${ }^{2}$ )

$$
\begin{aligned}
-27 q= & \left\{\left(2 \lambda_{1}-\lambda_{2}-\lambda_{3}\right) x+\left(2 \lambda_{2} \lambda_{3}-\lambda_{1} \lambda_{2}-\lambda_{1} \lambda_{3}\right) y\right\} \\
& \left\{\left(2 \lambda_{2}-\lambda_{3}-\lambda_{1}\right) x+\left(2 \lambda_{3} \lambda_{1}-\lambda_{2} \lambda_{3}-\lambda_{2} \lambda_{1}\right) y\right\} \\
& \left\{\left(2 \lambda_{3}-\lambda_{1}-\lambda_{2}\right) x+\left(2 \lambda_{1} \lambda_{2}-\lambda_{3} \lambda_{1}-\lambda_{3} \lambda_{2}\right) y\right\}
\end{aligned}
$$

denn man kann in der That die Gleichung, die sich ergibt, wenn man den ersten dieser drei Factoren gleich Null setzt, in die Form bringen

$$
\frac{\lambda_{1}-\lambda_{2}}{\lambda_{1}-\lambda_{3}}: \frac{x-\lambda_{2} y}{x-\lambda_{3} y}=-1
$$

Die Hesse'sche Covariante $h$ wird $^{3}$ )

1) Zwischen den Grössen $Q, R, F$ und $H$ besteht die einfache Relation:

$$
Q^{2}+R F^{2}+4 H^{3}=0 \text {. }
$$

Nach Division durch $\Delta^{6}$ folgt $q^{2}+r f^{2}+4 h^{3}=0$, und zwar gilt diese Relation für alle Werthe der Coefficienten von $F$, die $R$ nicht zu Null machen; sie besteht daher auch für alle Werthe der Coefficienten von $f$, die $r$ nicht zu Null machen, d. h. die Relation gilt identisch, mithin auch im Falle $r=0$.

2) Vgl. Salmon: "Lessons introductory to the Modern Higher Algebra", 4. Aufl, Dublin 1885, S. 184.

3) Ibid. S. 185. Vgl. auch Hesse: „Transformation einer beliebigen homogenen Function dritten Grades von zwei Variabeln durch lineäre Sabstitution neuer Variabeln in eine Form, welche nur die dritten Potenzen der neuen Variabeln enthält." Journal für die reine und angewandte Matbematik, Bd. 38, S. 265. 1847. 


$$
\begin{aligned}
-9 h= & \left\{\left(\lambda_{1}+\varepsilon \lambda_{2}+\varepsilon^{2} \lambda_{3}\right) x+\left(\lambda_{2} \lambda_{3}+\varepsilon \lambda_{3} \lambda_{1}+\varepsilon^{2} \lambda_{1} \lambda_{2}\right) y\right\} \\
& \left\{\left(\lambda_{1}+\varepsilon^{2} \lambda_{2}+\varepsilon \lambda_{3}\right) x+\left(\lambda_{2} \lambda_{3}+\varepsilon^{2} \lambda_{3} \lambda_{1}+\varepsilon \lambda_{1} \lambda_{2}\right) y\right\} .
\end{aligned}
$$

Das Doppelverhältniss eines Strahlenpaares von $F=0$ zu dem noch übrigen dritten Strahle von $F=0$ und zu einem Strahle von $H=0$ besitzt nämlich den Werth $-\varepsilon$ oder $-\varepsilon^{2}$; andrerseits kanu die Gleichung, die sich ergibt, wenn man den ersten Factor von (13) gleich Null setzt, in die Form gebracht werden

$$
\frac{\lambda_{1}-\lambda_{3}}{\lambda_{2}-\lambda_{3}}: \frac{x-\lambda_{1} y}{x-\lambda_{2} y}=-\varepsilon \text {. }
$$

Man nennt vier Stralılen, deren Doppelverhältniss gleich einer imaginären dritten Wurzel aus -1 ist, äquianharmonisch gelegen.

* 283. Man bestimme diejenigen (sechs) Kegelschnitte des Büschels $\lambda g-f=0$, für welche die vier Grundpunkte ein gegebenes Doppelverhältniss bilden ${ }^{1}$ ).

Zieht man von irgend einem Punkte $y$ einer bestimmten Curve $\lambda_{0} g-f=0$ des durch $f=0$ und $g=0$ erzeugten Kegelschnittbüschels nach den vier Grundpunkten Strahlen, so bilden diese letzteren ein gewisses Doppelverhältniss, das sogenannte Doppelverhältniss der vier Grundpunkte für den Kegelschnitt $\lambda_{0} g-f=0$. Dasselbe hat nach (14), S. 124 stets gleichen Werth, wo auch der Punkt $y$ auf $\lambda_{0} g-f=0$ angenommen werden möge. Es wird nun gefragt, welche Gleichung der Parameter $\lambda$ erfüllen muss, damit das Doppelverhältniss einer gegebenen Zahl $s$ gleich werde.

Lässt man den auf $\lambda_{0} g-f=0$ gelegenen Punkt $y$ mit einem der vier Grundpunkte zusammenfallen, so bestehen die vier zu betrachtenden Strahlen aus der Tangente $\lambda_{0} g(y, x)-f(y, x)=0$ in diesem Grundpunkte und aus den drei durch ihn gehenden Geraden $\lambda_{i} g(y, x)-f(y, x)=0,(i=1,2,3)$, des Kegelschnittbüschels. Hierbei ist $\lambda_{i}$ eine Wurzel der durch $\mu$ homogen gemachten kubischen Gleichung $\Psi_{0}(\lambda, \mu) \equiv \lambda^{3} B-3 \lambda^{2} \mu \Theta+3 \lambda \mu^{2} H-\mu^{3} A=0$, so dass das Doppelverhältniss $s$ identisch wird mit dem aus $\lambda: \mu=\lambda_{0}$ und aus den Wurzeln von $\Psi_{0}(\lambda, \mu)=0$ gebildeten. Da dieses Doppelverhältniss durch eine lineare Transformation nicht geändert wird, kann man an Stelle von $\lambda$ und $\mu$ neue Variabeln einführen durch

$$
\lambda=\alpha \lambda^{\prime}-\beta \mu^{\prime}, \quad \mu=\gamma \lambda^{\prime}-\delta \mu^{\prime},
$$

1) Vgl. Gundelfinger: "Zur Theorie des Kegelschnittbüschels", Zeitschrift für Mathematik und Physik, 20. Jahrgang, S. 153-159, 1874. 
wobei jetzt $\lambda^{\prime}, \mu^{\prime}$ nicht wie früher, S. 364, die Factoren der Hesseschen Covariante $h(\lambda, \mu)$ von $\Psi_{0}(\lambda, \mu)$ sein sollen, sondern irgend zwei Factoren von $\Psi_{0}(\lambda, \mu)$ selbst. Ueberhaupt möge sich die Form $\Psi_{0}(\lambda, \mu)$ durch diese Transformation verwandeln in $3 \lambda^{\prime} \mu^{\prime}\left(\lambda^{\prime}-\mu^{\prime}\right)$, und $\lambda-\lambda_{0} \mu$ möge übergehen in $\lambda^{\prime}-\sigma \mu^{\prime}$. Hier bedeutet $\sigma=\lambda^{\prime}: \mu^{\prime}$ das Doppel. verhältniss von $\lambda^{\prime}=0, \mu^{\prime}=0, \lambda^{\prime}-\mu^{\prime}=0$ und $\lambda^{\prime}-\sigma \mu^{\prime}=0$, oder auch das oben mit $s$ bezeichuete Doppelverhältniss der vier Wurzeln von $\Psi_{0}(\lambda, \mu)\left\{\lambda-\lambda_{0} \mu_{3}\right\}=0$.

Wir führen nun die Covarianten $h$ und $q$ der Form $\Psi_{0}(\lambda, \mu)$ ein. Nach (6), bezw. (13) und (2a), bezw. (11) in (282) ist in unserem Falle

$$
\left\{\begin{aligned}
\Delta^{2} h(\lambda, \mu) & =-\lambda^{\prime 2}+\lambda^{\prime} \mu^{\prime}-\mu^{\prime 2}=-\left(\lambda^{\prime}+\varepsilon \mu^{\prime}\right)\left(\lambda^{\prime}+\varepsilon^{2} \mu^{\prime}\right), \\
\Delta^{3} q(\lambda, \mu) & =2 \lambda^{\prime 3}-3 \lambda^{\prime 2} \mu^{\prime}-3 \lambda^{\prime} \mu^{\prime 2}+2 \mu^{\prime 3} \\
& =\left(\lambda^{\prime}+\mu^{\prime}\right)\left(2 \lambda^{\prime}-\mu^{\prime}\right)\left(\lambda^{\prime}-2 \mu^{\prime}\right), \text { wobei } \Delta=\beta \gamma-\alpha \delta
\end{aligned}\right.
$$

daher

$$
-\frac{h^{3}(\lambda, \mu)}{q^{2}(\lambda, \mu)}=\frac{\left(\lambda^{\prime 2}-\lambda^{\prime} \mu^{\prime}+\mu^{\prime 2}\right)^{3}}{\left(\lambda^{\prime}+\mu^{\prime}\right)^{2}\left(2 \lambda^{\prime}-\mu^{\prime}\right)^{2}\left(\lambda^{\prime}-2 \mu^{\prime}\right)^{2}},
$$

und wenn man für $\lambda^{\prime}: \mu^{\prime}$ das Doppelverhältniss $s$ einführt, folgt

$$
-\frac{h^{3}(\lambda, \mu)}{q^{2}(\lambda, \mu)}=\frac{\left(1-s+s^{2}\right)^{3}}{(1+s)^{2}(2 s-1)^{2}(s-2)^{2}}=\frac{(s+\varepsilon)^{3}\left(s+\varepsilon^{2}\right)^{3}}{(1+s)^{2}(2 s-1)^{2}(s-2)^{2}} .
$$

Diese Gleichung sechsten Grades in $\lambda: \mu$ liefert die Parameter derjenigen sechs Kegelschnitte des Büschels $\lambda g-\mu f=0$, für welche die vier Grundpunkte das gegebene Doppelverhältniss $s$ bilden.

Die vorstehenden Betrachtungen lassen sich leicht auf die Kegelschnittschaar $v G(u, u)-F(u, u)=0$ übertragen. Die Parameter $\nu=\nu_{1}: \nu_{2}$ der drei in dieser Schaar enthaltenen Punktepaare werden erhalten durch Auflösung der kubischen Gleichung

$$
\Phi\left(\nu_{1}, \nu_{2}\right) \equiv \nu_{1}{ }^{3} B^{2}-3 \nu_{1}{ }^{2} \nu_{2} B \mathrm{H}+3 v_{1} \nu_{2}{ }^{2} A \Theta-\nu_{2}{ }^{3} A^{2}=0 .
$$

Da vermöge der linearen Substitution $\lambda=A \nu_{2}, \mu=B \nu_{1}$ die binäre Form $\Psi_{0}(\lambda, \mu)$ übergeht in $\Psi_{0}\left(A v_{2}, B v_{1}\right)=-A B \cdot \Phi\left(v_{1}, v_{2}\right)$, so ist

$$
\left\{\begin{aligned}
h(\lambda, \mu) & =\frac{1}{36}\left[\frac{\partial^{2} \Phi}{\partial \nu_{1}{ }^{2}} \frac{\partial^{2} \Phi}{\partial \nu_{2}{ }^{2}}-\left(\frac{\partial^{2} \Phi}{\partial \nu_{1} \cdot \partial \nu_{2}}\right)^{2}\right] \\
-q(\lambda, \mu) & =\frac{1}{3}\left[\frac{\partial \Phi}{\partial \nu_{1}} \frac{\partial h}{\partial \nu_{2}}-\frac{\partial \Phi}{\partial \nu_{2}} \frac{\partial h}{\partial \nu_{1}}\right]
\end{aligned}\right.
$$

hierbei war zu beachten, dass die Substitutionsdeterminante den Werth - $A B$ besitzt. Mit Rücksicht auf (4) erhält man also:

$$
-\frac{h^{3}\left(A v_{2}, B v_{1}\right)}{q^{2}\left(A v_{2}, B v_{1}\right)}=\frac{\left(1-s+s^{2}\right)^{3}}{(1+s)^{2}(2 s-1)^{2}(s-2)^{2}} .
$$

Diese Gleichung ist in $s$ vom sechsten Grade, woraus folgt: 
*284. In der Schaar $\nu_{1} G(u, u)-v_{2} F(u, u)=0$ gibt es sechs Kegelschuitte, für welche die vier gemeinsamen Grundtangenten ein gegebenes Doppelverhältniss bilden ${ }^{1}$ ).

Ferner gilt der Satz:

* 285. Das Doppelverhältniss der vier Grundpunkte des Büschels $\lambda g-f=0$ für die Curve zweiter Ordnung $f=0$, bezw. $g=0$ ist lasselbe wie dasjenige der vier gemeinsamen Tangenten von $f=0$ und $g=0$ für die Curve $g=0$, bèzw. $f=0^{2}$ ).

Deun für $\lambda=0$ verwandelt sich (4) in dieselbe Gleichung wie (7) für $\nu_{2}=0$; ebenso liefert die Substitution $\mu=0$ in (4) dieselbe Gleichung wie $\nu_{1}=0$ in $(7)$.

* Zusammenlang zwischen der Combinante $\psi$, den äquianharmonischen und harmonischen Kegelschnitten, sowie dem Poldreiseit des Büschels.

*286. Für die Kegelschnitte

$$
f \equiv \lambda_{1} x_{1}{ }^{2}+\lambda_{2} x_{2}{ }^{2}+\lambda_{3} x_{3}{ }^{2}=0, \quad g \equiv x_{1}{ }^{2}+\dot{x}{ }^{2}+x_{3}{ }^{2}=0
$$

hat die Combinante $\psi$ nach (27), S. 208 den Werth:

$$
-\frac{1}{3} \psi \equiv \mathrm{A} x_{1}{ }^{2}+\mathrm{B}{x_{2}}^{2}+\Gamma x_{3}{ }^{2}=0,
$$

wobei

$$
\left\{\begin{array}{l}
\mathrm{A}=\left(\lambda_{1}-\lambda_{2}\right)\left(\lambda_{1}-\lambda_{3}\right) \\
\mathrm{B}=\left(\lambda_{2}-\lambda_{3}\right)\left(\lambda_{2}-\lambda_{1}\right) \\
\Gamma=\left(\lambda_{3}-\lambda_{1}\right)\left(\lambda_{3}-\lambda_{2}\right) .
\end{array}\right.
$$

Auch besteht noch die Relation ${ }^{3}$ )

$$
-\frac{1}{9} \psi \equiv(\Theta f+\mathrm{H} g-2 \chi) \text {, }
$$

in welcher $2 \chi$ durch (6) und (4) in $\S 17$ definirt ist. Ferner werde gesetzt $\mathrm{A} x_{1}{ }^{2}=A, \mathrm{~B} x_{2}{ }^{2}=B, \Gamma x_{3}{ }^{2}=C$, so dass

$$
-\frac{1}{3} \psi=A+B+C \text {. }
$$

Alsdann stellen auch

$$
A+\varepsilon B+\varepsilon^{2} C=0 \text { und } A+\varepsilon^{2} B+\varepsilon C=0
$$

zwei Kegelschnitte des Büschels $\lambda g-f=0$ dar, und zwar werden, wie sogleich gezeigt werden soll, dieselben in jedem der vier Grund-

1) Vgl. Gundelfinger a. a. O. S. 158.

2) Ibid. (Fussnote).

3) Diese Relation bleibt anch noch giltig, wenn zwei der Grössen $\lambda_{i}$ einander gleich werden; vgl. das entsprechende Raisonnement in der Fussnote 1) zu S. 365 . 
Aequianharmonische und harmonische Kegelschnitte. Poldreiseit. 369 punkte je von einem der beiden Strahlen berührt; deren jeder zu den nach den drei übrigen Grundpunkten gezogenen-Strahlen äquianharmonisch gelegen ist. Die Curven ( 6$)$ heissen daher die äquianharmonischen Kegelschnitte des Büschels.

Ebenso sind

(7) $-2 A+B+C=0, A-2 B+C=0$ und $A+B-2 C=0$ diejenigen Kegelschnitte des Büschels, für welche das analog wie bei (6) betrachtete Doppelverhältniss gleich -1 ist.

Beweisen wir zunächst die Behauptung iiber $(G)$. Die kubische Gleichung zur Berechnung der in dem Büschel $\lambda g-f=0$ enthaltenen Geradenpaare ist $\left(\lambda-\lambda_{1}\right)\left(\lambda-\lambda_{2}\right)\left(\lambda-\lambda_{3}\right)=0$; ein Factor ihrer Hesse'schen Covariante hat daher nach (13), S. 366 die Gestalt

$$
\lambda\left(\lambda_{1}+\varepsilon \lambda_{2}+\varepsilon^{2} \lambda_{3}\right)+\left(\lambda_{2} \lambda_{3}+\varepsilon \lambda_{3} \lambda_{1}+\varepsilon^{2} \lambda_{1} \lambda_{2}\right)
$$

und ergibt in Verbindung mit $\lambda g-f=0$, d. h. $\lambda=f: g$, die Gleichung

$$
\begin{aligned}
\left(\lambda_{1}+\varepsilon \lambda_{2}\right. & \left.+\varepsilon^{2} \lambda_{3}\right)\left(\lambda_{1} x_{1}{ }^{2}+\lambda_{2} x_{2}{ }^{2}+\lambda_{3} x_{3}{ }^{2}\right) \\
& +\left(\lambda_{2} \lambda_{3}+\varepsilon \lambda_{3} \lambda_{1}+\varepsilon^{2} \lambda_{1} \lambda_{2}\right)\left(x_{1}{ }^{2}+x_{2}{ }^{2}+x_{3}{ }^{2}\right)=0
\end{aligned}
$$

oder

$$
A+\varepsilon B+\varepsilon^{2} C=0 \text {. }
$$

Mit Hilfe des zweiten Factors der Hesse'schen Covariante erliält man

$$
A+\varepsilon^{2} B+\varepsilon C=0 \text {. }
$$

Die oben für die Kegelschnitte ( 7 ) ausgesprochene Behauptung beweist man ganz analog unter Benutzung der kubischen Covariante $q$. Der Factor $\lambda\left(2 \lambda_{1}-\lambda_{2}-\lambda_{3}\right)+\left(2 \lambda_{2} \lambda_{3}-\lambda_{1} \lambda_{2}-\lambda_{1} \lambda_{3}\right)$ derselben ergibt in Verbindung mit $\lambda g-f=0$ die Gleichung

oder

$$
\begin{aligned}
& \left(2 \lambda_{1}-\lambda_{2}-\lambda_{3}\right)\left(\lambda_{1} x_{1}{ }^{2}+\lambda_{2} x_{2}{ }^{2}+\lambda_{3} x_{3}{ }^{2}\right) \\
& +\left(2 \lambda_{2} \lambda_{3}-\lambda_{1} \lambda_{2}-\lambda_{1} \lambda_{3}\right)\left(x_{1}{ }^{2}+x_{2}{ }^{2}+x_{3}{ }^{2}\right)=0
\end{aligned}
$$

$$
2 A-B-C=0 \text {. }
$$

Auf Grund der vorangehenden Bemerkungen zu $(6)$ und (7) folgt, indem in (13), S. 366 und (11), S. $365 x=f, y=g$ gesetzt wird:

$$
\begin{aligned}
& -9 h(f, g)=\left(A+\varepsilon B+\varepsilon^{2} C\right)\left(A+\varepsilon^{2} B+\varepsilon C\right), \\
& -27 q(f, g) \\
=(2 A & -B-C)(-A+2 B-C)(-A-B+2 C) .
\end{aligned}
$$

Als weitere Covariante möge noch eingeführt werden

$$
\Omega \equiv \frac{1}{8} \sum \pm\left(\frac{\partial f}{\partial x_{1}} \frac{\partial g}{\partial x_{2}} \frac{\partial \psi}{\partial x_{3}}\right)
$$

für (1) berechnet findet man daher

$$
\Omega=9\left(\lambda_{2}-\lambda_{3}\right)\left(\lambda_{3}-\lambda_{1}\right)\left(\lambda_{1}-\lambda_{2}\right) x_{1} x_{2} x_{3},
$$




$$
\bar{\Omega}^{2}=-81 A B C \text {. }
$$

Da nun für willkürliche Werthe $A, B, C$ stets die Identität stattfindet $27 A B C=(A+B+C)^{3}-3(A+B+C)\left(A+\varepsilon B+\varepsilon^{2} C\right)\left(A+\varepsilon^{2} B+\varepsilon C\right)$

$$
+(2 A-B-C)(-A+2 B-C)(-A-B+2 C) \text {, }
$$

so ergibt sich mit Hilfe von $(10),(5)$ und $(8)$ die Relation

$$
\Omega^{2}=\frac{1}{9} \psi^{3}+27 \psi \cdot h(f, g)+81 q(f, g) .
$$

Die simultanen Invarianten $\Theta$ und $H$, gebildet für die zwei äquianharmonischen Kegelschnitte, verschwinden, denn sie werden, wie man leicht sieht, gleichbedeutend mit $1+\varepsilon+\varepsilon^{2} \equiv 0$.

Bildet man ferner für die zwei äquianharmonischen Kegelschnitte die Gleichung der harmonischen Curve zweiter Classe $H$, so wird dieselbe

$$
2 H \equiv \mathrm{B} \Gamma\left(\varepsilon^{2}+\varepsilon^{4}\right) u_{1}^{2}+\Gamma \mathrm{A}\left(\varepsilon+\varepsilon^{2}\right) u_{2}^{2}+\mathrm{AB}\left(\varepsilon+\varepsilon^{2}\right) u_{3}^{2}=0,
$$

also gleichbedeutend mit der Gleichung von $\psi=0$ in Liniencoordinaten. Dasselbe Resultat ergibt sich, wenn man die Gleichung der harmonischen Curve zweiter Ordnung $\chi=0$ aufstellt. Wir können daher sagen:

*287. "Die Combinante $\psi$ wird eingehüllt von-allen Geraden, welche die beiden äquianharmonischen Kegelschnitte nach harmonischen Punkteparen schneiden, und ist gleichzeitig der geometrische Ort aller Punkte, von welchen aus sich an dieselben beiden Kegelschnitte harmonische Tangentenpaare legen lassen."

Ein weiterer Satz lautet:

*288. Die zwei äquianharmonischen Curven und $\psi$ bilden ein System dreier Kegelschnitte, von welchen je zwei einander in Bezug auf den dritten "als Directrix polar entsprechen", d. h. die in Bezug auf eine der drei Curven genommenen Polaren von Punkten einer anderen Curve des Systems umhüllen die dritte Curve ${ }^{2}$ ).

Man zeigt dies nach ähnlichem Verfahren wie bei (66).

Für die drei harmonischen Kegelschnitte gilt der Satz:

*289. „Jeder der drei harmonischen Kegelschnitte hat mit $\psi$ eine doppelte Berührung, wobei die Berührungssehnen

1) Gundelfinger a. a. O. S. 157.

2) Ibid. 
mit den Seiten des gemeinsamen Polardreiecks zusammenfallen."1)

Folgt sofort daraus, dass z. B. die Gleichung des harmonischen Kegelschnitts $2 \mathrm{~A} x_{1}{ }^{2}-\mathrm{B} x_{2}{ }^{2}-\Gamma x_{3}{ }^{2}=0$ auch geschrieben werden kann in der Form $3 \mathrm{~A} x_{1}{ }^{2}-\psi(x, x)=0$.

*290. Jeder der drei harmonischen Kegelschnitte geht durch unendlich viele Poldreiecke von $\psi(x, x)=0$.

Die Gleichung ron $\psi=0$ in Liniencoordinaten ist nämlich $\frac{u_{1}^{2}}{\mathrm{~A}}+\frac{u_{2}^{2}}{\mathrm{~B}}+\frac{u_{3}^{2}}{\Gamma}=0 ;$ um zu zeigen, dass z. B. der harmonische Kegelschnitt $2 \mathrm{~A} x_{1}{ }^{2}-\mathrm{B}{x_{2}}^{2}-\Gamma x_{3}{ }^{2}=0$ durch unendlich viele Poldreiecke von $\psi$ geht, wäre nach (11), S. 162 zu beweisen, dass die Invariante $\Theta$, gebildet für beide Curven, verschwindet. Man findet aber in der That für diese Invariante den Werth $\frac{2 A}{A}-\frac{B}{B}-\frac{r}{r} \equiv 0$. Offenbar sind die in (289) und (290) angegebenen Beziehungen der harmonischen Kegelschnitte zu $\psi=0$ für diese Curven charakteristisch.

*291. Kriterien der Realität für die Schnittpunk te zweier Curven zweiter Ordnung.

Die Gleichungen der vier Schnittpunkte zweier Kegelschnitte $f(x, x)=0$ und $g(x, x)=0$ sind nach (37), S. 141:

(1) $\left(\lambda_{2}-\lambda_{3}\right) \sqrt{F-2} \bar{\lambda}_{1} H+\lambda_{1}{ }^{2} G \pm\left(\lambda_{3}-\lambda_{1}\right) \sqrt{F-2} \lambda_{2} H+\lambda_{2}{ }^{2} G$

$$
\pm\left(\lambda_{1}-\lambda_{2}\right) \sqrt{F-2 \lambda_{3} H+\lambda_{3}{ }^{2} G=0,}
$$

wobei $\lambda_{1}, \lambda_{2}, \lambda_{3}$ die Wurzeln sind der kubischen Gleichung

$$
\Psi_{0}(\lambda) \equiv \lambda^{3} B-3 \lambda^{2} \Theta+3 \lambda H-A=0,
$$

also auch $\Psi_{0}(\lambda)=B\left(\lambda-\lambda_{1}\right)\left(\lambda-\lambda_{2}\right)\left(\lambda-\lambda_{3}\right)$.

Ist die Discriminante

$$
\begin{aligned}
R & \equiv 4\left(B H-\Theta^{2}\right)\left(A \Theta-H^{2}\right)-(\Theta H-A B)^{2} \\
& =\frac{B^{4}}{27}\left(\lambda_{1}-\lambda_{2}\right)^{2}\left(\lambda_{2}-\lambda_{3}\right)^{2}\left(\lambda_{3}-\lambda_{1}\right)^{2}
\end{aligned}
$$

dieser kubischen Gleichung negativ, so hat die Gleichung bekanntlich eine reelle und zwei complex conjugirte Wurzeln. Hält man in (1) das Vorzeichen des ersten Gliedes fest (es sei positiv gewählt), und ist $\lambda_{1}$ die reelle Wurzel, so sind alsdann die beiden folgenden Glieder complex conjugirt, und (1) ergibt entsprechend den verschiedenen Vor-

1) Gundelfinger a. a. 0 . 
zeichen der Wurzeln jedenfalls zwei reelle und zwei imaginäre Schnittpunkte.

Ist die Discriminante (3) positiv, so hat (2) drei reelle Wurzeln, es werden daher auch die drei Ausdrücke $F-2 \lambda_{i} H+\lambda_{i}{ }^{2} G$ reell. Haben dieselben gleiches Vorzeichen, so sind die vier Schnittpunkte nach (1) reell; das Vorzeichen kann alsdann, da die Geradenpaare $\lambda_{i} g-f=0(i=1,2,3)$ in diesem Falle reell sind, nach S. 36 nur negativ sein. Haben zwei der Grössen $F-2 \lambda_{i} H+\lambda_{i}{ }^{2} G$ ein anderes Vorzeichen als die dritte, so sind die vier Schnittpunkte imaginär.

Bezieht man die beiden Kegelschnitte

$f(x, x) \equiv \sum_{1}^{3} \sum_{1}^{3} a_{i k} x_{i} x_{k}=0$, und $g(x, x) \equiv \sum_{1}^{3} \sum_{1}^{3} b_{i k} x_{i} x_{k}=0$

auf das gemeinsame Poldreieck, so werden ihre Gleichungen nach (30) und (29) in $\S 14$ :

$$
\left\{\begin{array}{l}
f(x, x)=\lambda_{1} X_{1}^{2}+\lambda_{2} X_{2}^{2}+\lambda_{3} X_{3}^{2}=0 \\
g(x, x)=X_{1}^{2}+X_{2}^{2}+X_{3}^{2}=0
\end{array}\right.
$$

wo z. B. $X_{1}$ nach (25), (15) und (13) in $\S 14$ definirt ist durch

$$
X_{1}=\left[\frac{\sqrt{F-2 \lambda_{1} H+\lambda_{1}{ }^{2} G}}{\sqrt{B\left(\lambda_{1}-\lambda_{2}\right)\left(\lambda_{1}-\lambda_{3}\right)}}\right]_{u_{i}=\frac{1}{2} g^{\prime}\left(x_{i}\right)},
$$

analog $X_{2}$ und $X_{3}$.

Hierbei wird vorausgesetzt, dass die Determinante $B$ von $g(x, x)=0$ nicht verschwindet; diese Annahme ist erlaubt, da es im Folgenden nur auf die Bestimmung der Schnittpunkte, bezw. die Realität derselben ankommt.

Ist $r$ die Determinante der Substitution, durch welche die ursprünglichen Gleichungen (4) in die Gestalt (5) transformirt werden, so ist

$$
r^{2} B=1
$$

und nach (27) in $\S 20$ :

(8) $\begin{aligned}-\frac{1}{3} r^{2} \psi=\left(\lambda_{1}-\lambda_{2}\right)\left(\lambda_{1}-\lambda_{3}\right) X_{1}^{2} & +\left(\lambda_{2}-\lambda_{1}\right)\left(\lambda_{2}-\lambda_{3}\right) X_{2}^{2} \\ & +\left(\lambda_{3}-\lambda_{1}\right)\left(\lambda_{3}-\lambda_{2}\right) X_{3}^{2} .\end{aligned}$

Mit Hilfe von (6) und (7) kann für (8) gesetzt werden

worin

$$
\begin{gathered}
-\frac{1}{3} \psi=\left(F-2 \lambda_{1} H+\lambda_{1}{ }^{2} G\right)+\left(F-2 \lambda_{2} H+\lambda_{2}{ }^{2} G\right) \\
+\left(F-2 \lambda_{3} H+\lambda_{3}{ }^{2} G\right), \\
u_{i}=\frac{1}{2} g^{\prime}\left(x_{i}\right) .
\end{gathered}
$$

Diese Formel, die wir soeben für den Fall ungleicher Wurzeln von (2) mit Hilfe der kanonischen Formen (5) abgeleitet haben, gilt 
ganz allgemein. Schreibt man nämlich die rechte Seite von $(8 a)$ in der Form $3 F-2\left(\lambda_{1}+\lambda_{2}+\lambda_{3}\right) H+\left(\lambda_{1}{ }^{2}+\lambda_{2}{ }^{2}+\lambda_{3}{ }^{2}\right) G$ und drückt man nun die Summen $\lambda_{1}+\lambda_{2}+\lambda_{3}$ und $\lambda_{1}{ }^{2}+\lambda_{2}{ }^{2}+\lambda_{3}{ }^{2}$ durch die Coefficienten von (2) aus, so folgt

$$
-\frac{1}{9} B^{2} \psi=B^{2} F+\Theta(3 \Theta G-2 B H)-2 B H G,
$$

wobei rechts $u_{i}$ durch $\frac{1}{2} g^{\prime}\left(x_{i}\right)$ zu ersetzen ist. Alsdann verwandelt sich die rechte Seite nach (7a) und (7b) in $\S 17$ in $B^{2}(\mathrm{H} g-2 \chi+\Theta f)$, es folgt also $-\frac{1}{9} \psi=\Theta f+\mathrm{H} g-2 \chi$ in Uebereinstimmung mit (4), S. 368 .

Die nothwendige und ausreichende Bedingung dafür, dass die Ausdrücke $F-2 \lambda_{i} H+\lambda_{i}{ }^{2} G(i=1,2,3)$ für beliebige Werthe der $u_{k}=\frac{1}{2} g^{\prime}\left(x_{k}\right)$ negativ seien, fällt also auf Grund von $(\delta a)$ damit zusammen, dass die Combinante $\psi$ eine positive definite Form ist.

Bevor wir diese Untersuchung über Realität der Schnittpunkte weiter fortsetzen, wollen wir für $\psi(x, x)=0$ noch die Gleichung in Liniencoordinaten ableiten. Aus ( 8 ) findet man

$$
\begin{aligned}
& \quad \frac{1}{9} r^{6} \cdot \psi(u, u)=-\left(\lambda_{2}-\lambda_{3}\right)^{2}\left(\lambda_{1}-\lambda_{2}\right)\left(\lambda_{1}-\lambda_{3}\right) U_{1}^{2} \\
& -\left(\lambda_{3}-\lambda_{1}\right)^{2}\left(\lambda_{2}-\lambda_{3}\right)\left(\lambda_{2}-\lambda_{1}\right) U_{2}{ }^{2}-\left(\lambda_{1}-\lambda_{2}\right)^{2}\left(\lambda_{3}-\lambda_{1}\right)\left(\lambda_{3}-\lambda_{2}\right) U_{3}{ }^{2}
\end{aligned}
$$

und erhält nach Einfiihrung der Werthe der $U_{i}$ aus (36), S. 141 mit Rücksicht auf die Relation $r^{2} B=1$ die Gleichung:

$$
-\frac{1}{9} r^{4} \Psi(u, u)=\left(\lambda_{2}-\lambda_{3}\right)^{2}\left(F-2 \lambda_{1} H+\lambda_{1}{ }^{2} G\right)
$$

$$
+\left(\lambda_{3}-\lambda_{1}\right)^{2}\left(F-2 \lambda_{2} H+\lambda_{2}{ }^{2} G\right)+\left(\lambda_{1}-\lambda_{2}\right)^{2}\left(F-2 \lambda_{3} H+\lambda_{3}{ }^{2} G\right) \text {. }
$$

Wir wollen nun die kubische Gleichung (2) durch eine Variabele $\mu$ homogen machen. Alsdann ist die Hesse'sche Covariante $h$ von (2) nach (13), S. 366 gegeben durch

$$
\begin{aligned}
-18 h(\lambda, \mu) & =B^{2}\left\{\left(\lambda_{2}-\lambda_{3}\right)^{2}\left(\lambda \dot{-} \lambda_{1} \mu\right)^{2}+\left(\lambda_{3}-\lambda_{1}\right)^{2}\left(\lambda-\lambda_{2} \mu\right)^{2}\right. \\
& \left.+\left(\lambda_{1}-\lambda_{2}\right)^{2}\left(\lambda-\lambda_{3} \mu\right)^{2}\right\}
\end{aligned}
$$

wofür wegen $r^{2} B=1$ auch gesetzt werden kann

$$
\begin{aligned}
-18 r^{4} h(\lambda, \mu) & =\left(\lambda_{2}-\lambda_{3}\right)^{2}\left(\lambda-\lambda_{1} \mu\right)^{2}+\left(\lambda_{3}-\lambda_{1}\right)^{2}\left(\lambda-\lambda_{2} \mu\right)^{2} \\
& +\left(\lambda_{1}-\lambda_{2}\right)^{2}\left(\lambda-\lambda_{3} \mu\right)^{2} .
\end{aligned}
$$

Es geht also (9) aus (10) dadurch hervor, dass man setzt $\lambda^{2}=F$, $\lambda \mu=H, \mu^{2}=G$. Andrerseits hat man für die Hesse'sche Covariante von (2) den Ausdruck

$$
h(\lambda, \mu)=\left(B \mathrm{H}-\Theta^{2}\right) \lambda^{2}+(\Theta H-A B) \lambda \mu+\left(A \Theta-H^{2}\right) \mu^{2},
$$


daher wird

(11) $\frac{1}{162} \Psi(u, u)=\left(B H-\Theta^{2}\right) F+(\Theta H-A B) H+\left(A \Theta-H^{2}\right) G$.

Nach (8) ist

$$
r^{8} \sum \pm\left(\psi_{11} \psi_{22} \psi_{33}\right)=27\left(\lambda_{\overline{2}}-\lambda_{3}\right)^{2}\left(\lambda_{3}-\lambda_{1}\right)^{2}\left(\lambda_{1}-\lambda_{2}\right)^{2}
$$

andrerseits ist $B^{4}\left(\lambda_{2}-\lambda_{3}\right)^{2}\left(\lambda_{3}-\lambda_{1}\right)^{2}\left(\lambda_{1}-\lambda_{2}\right)^{2}=27 R$, in Folge von $r^{2} B=1$ wird daher

$$
\sum \pm\left(\psi_{11} \psi_{22} \psi_{33}\right)=729 R
$$

Denkt man sich nun eine Transformation wie (35), S. 28 angewandt auf

$\psi(x, x)=\psi_{11} x_{1}{ }^{2}+2 \psi_{12} x_{1} x_{2}+\psi_{22} x_{2}{ }^{2}+2 \psi_{13} x_{1} x_{3}+2 \psi_{23} x_{2} x_{3}+\psi_{33} x_{3}{ }^{2}$, so zeigt (36), S. 29 , dass die Relation stattfindet ${ }^{1}$ )

$$
\begin{aligned}
\psi_{11} \cdot \psi(x, x) & =\left(\psi_{11} x_{1}^{\prime}+\psi_{12} x_{2}\right)^{2}+\left(\psi_{11} \psi_{22}-\psi_{12}{ }^{2}\right) x_{2}{ }^{\prime 2} \\
& +\frac{\psi_{11} \cdot \sum_{\psi_{11}} \pm\left(\psi_{11} \psi_{22} \psi_{33}\right)}{\psi_{22}{ }^{2}} x_{3}{ }^{2} .
\end{aligned}
$$

Hieraus geht hervor, dass $\psi$ stets dann und nur dann definit ist, wenn $\psi_{11} \cdot\left(\sum \pm \psi_{11} \psi_{22} \psi_{33}\right)$ und $\psi_{11} \psi_{22}-\psi_{12}{ }^{2}$ gleiche Vorzeichen haben. Da wir nun an und für sich den Fall $R>0$ betrachten, muss also $\sum \pm\left(\psi_{11} \psi_{23} \psi_{33}\right)$ positiv sein, ebenso $\psi_{11}$, denn $\psi(x, x)$ sollte nach $(8 a)$ und S. 373, Zeile 13 eine positive definite Form darstellen; hieraus folgt, dass auch

$$
\psi_{33}=\psi_{11} \psi_{22}-{\psi_{12}}^{2}>0
$$

sein muss. Die Bedingung, dass sich die Kegelschnitte (4) in vier reellen Punkten schneiden, ist daher

$$
R>0, \quad \psi_{11}>0, \quad \Psi_{33} \equiv \psi_{11} \psi_{22}-{\psi_{12}}^{2}>0 \text {. }
$$

Es ist klar, dass man allgemeiner hierfür setzen könnte:

$$
R>0, \quad \psi_{i i}>0, \quad \Psi_{k k}>0, \quad \text { wobei } i \gtrless k .
$$

Im Falle der einfachen Berührung der beiden Kegelschnitte hat die Gleichung (2) zwei gleiche Wurzeln, etwa $\lambda_{1}=\lambda_{2} ; R$ verschwindet. Für die Realität der beiden übrigen Schnittpunkte ist nothwendig, dass das Geradenpaar, welches der Doppelwurzel entspricht und nach S. 133 und 155 den stets reellen Berührungspunkt mit den zwei übrigen

1) $\mathrm{Da} \sum \pm\left(\psi_{11} \psi_{22} \psi_{33}\right)$ nach (12) $\gtreqless 0$, so muss $\psi_{11}=\psi(1,0,0)$ nach (1), S. 65 von Null verschieden sein; gleiches gilt von $\psi_{11} \psi_{22}-\psi_{12}{ }^{2}$, da sonst nichtverschwindende Werthe $p_{1}, p_{2}$ existiren, für welche $\psi_{11} p_{1}+\psi_{12} p_{2}=0$ und $\psi_{21} p_{1}+\psi_{22} p_{2}=0$, d. h. für welche $\psi\left(p_{1}, p_{2}, 0\right)=0$ wäre. 
Schnittpunkten verbindet, reell sei; hierzu genügt $F-2 \lambda_{1} H+\lambda_{1}^{2} G<0$, oder nach (11) $\Psi(u, u)>0$ für die Coordinaten aller Geraden $u_{i}$, die nicht durch den Berührungspunkt gehen. Aehnlich wie S. 51 kann man auch sagen, dass irgend eine der drei Hauptunterdeterminanten $\Psi_{i i}>0$ sein muss. Im entgegengesetzten Falle sind die zwei übrigen Schnittpunkte imaginär. .

Im Falle der doppelten Berührung ist $\lambda_{1}=\lambda_{2}$ und

$$
F-2 \lambda_{1} H+\lambda_{1}^{2} G \equiv 0,
$$

daher auch $\Psi(u, \imath) \equiv 0$. Die Gleichung $\lambda_{1} g-f=0$ repräsentirt die stets reelle Berührungssehne doppelt zählend, $\lambda_{3} g-f=0$ die Tangenten in den zwei Berührungspunkten. Die beiden Berührungspunkte sind also reell oder imaginär, je nachdem das Geradenpaar $\lambda_{3} g-f=0$ reell oder imaginär ist, $\mathrm{d}$. $\mathrm{h}$. je nachdem

$$
F-2 \lambda_{3} H+\lambda_{3}{ }^{2} G<0 \text { oder }>0
$$

für die Coordinaten $u_{i}$ aller Geraden, die nicht durch einen der Berührungspunkte gehen. Man könnte zufolge $(s a)$ auch sagen, dass die zwei Berührungspunkte reell oder imaginär sind, je nachdem $\psi(x, x)>0$ oder $<0$ ist für jeden Punkt $x$, der nicht auf der Berülırungssehne liegt, d. h. kürzer je nachdem eine der Grössen $\psi_{i i}>0$ oder $<0$. Diese Sehne wird übrigens, wie $(s a)$ zeigt, im gegenwärtigen Falle doppelt zählend auch durch $\psi(x, x)=0$ dargestellt.

Setzt $\operatorname{man} h_{11}=B \mathrm{H}-\Theta^{2}, 2 h_{12}=\Theta \mathrm{H}-A B, h_{22}=A \Theta-\mathrm{H}^{2}$, so ist nach $(11) \frac{1}{162} \psi(u, u)=h_{11} F+2 h_{12} I I+h_{22} G$; man kann daher sagen, dass im Falle der doppelten Berührung sämmtliche Hauptunterdeterminanten $\Psi_{i i}$ verschwiuden müssen, dagegen können $h_{11}$ und $h_{22}$ nicht gleichzeitig Null sein, weil sonst in Folge von

$$
R=4\left(h_{11} h_{22}-h_{12}{ }^{2}\right)=0
$$

auch $h_{12}$ verschwinden und alsdann Osculation vorliegen würde. Die Berührungspunkte sind reell oder imaginär, je nachdem irgend eine der Grössen $\psi_{i i}$ positiv oder negativ ist.

Im Falle der Osculation verschwinden zufolge der Fussnote zu S. 136 die Ausdrücke $h_{11}, h_{12}$ und $h_{22}$.

Die Kegelschnitte des Büschels haben endlich vier zusammenfallende Punkte gemeinsam, wenn $\psi(x, x) \equiv 0$. Es geht dies aus den Betrachtungen S. 135 und 136 hervor, wenn man dabei die oben gefundene Relation ( $8 a$ ) beachtet.

Wir wollen nun die erhaltenen Resultate über die Realität der Schnittpunkte zweier Curven zweiter Ordnung $f(x, x)=0, g(x, x)=0$ übersichtlich zusammenstellen. 
Es sei im Folgenden

$$
\psi(x, x) \equiv \sum_{i}^{3} \sum_{1}^{3} \psi_{i k} x_{i} x_{k} \equiv \frac{3}{2} \sum_{1}^{3} \sum_{1}^{3} \frac{\partial^{2} N}{\partial x_{i} \partial u_{k}} \frac{\partial^{2} N}{\partial x_{k} \partial u_{i}},
$$

wobei

$$
N \equiv \frac{1}{4} \sum \pm\left(\frac{\partial f}{\partial x_{1}} \frac{\partial g}{\partial x_{2}} u_{3}\right)
$$

ferner sei $\quad R=\frac{1}{729} \sum \pm\left(\psi_{11} \psi_{22} \psi_{33}\right)=4\left(h_{11} h_{22}-h_{12}{ }^{2}\right)$,

$$
\Psi(u, u) \equiv \sum_{1}^{3} \sum_{1}^{3} \Psi_{i k} u_{i} u_{k}=162\left(h_{11} F+2 h_{12} H+h_{22} G\right),
$$

wobei $h_{11}=B \mathrm{H}-\Theta^{2}, 2 h_{12}=\Theta \mathrm{H}-A B, h_{22}=A \Theta-\mathrm{H}^{2}$.

I) $R \gtrless 0:$ vier verschiedene Schnittpunkte.

1) $R<0$ : zwei reelle und zwei imaginäre Schnittpunkte.

2) $R>0$ : vier reelle oder vier imaginäre Schnittpunkte.

a) $\psi_{i i}>0$ und $\psi_{k k}>0, i \gtrless k$, vier reelle Schnittpunkte ( $i$ und $k$ irgend zwei bestimmte, verschiedene Zahlen aus derReihe $1,2,3)^{1}$ ),

b) die Bedingungen a) sind nicht erfüllt: vier imaginäre Schnittpunkte.

II) $R=0$ : specielle Fälle der Berührung.

1) Nicht alle $\psi_{i i}$ gleich Null: einfache Berührung, zwei Schnittpunkte fallen zusammen; die beiden anderen sind:

a) reell, wenn unter den drei Grössen $\psi_{i i}$ eine willkürlich ausgewählte positiv ist ${ }^{2}$ ),

b) imaginär, wenn unter den drei Grössen $\Psi_{i i}$ eine willkürlich ausgewählte negativ ist ${ }^{3}$ ).

2) Alle $\Psi_{i i}$ gleich Null, nicht aber alle $\psi_{i i}:^{4}$ )

a) $h_{11}$ und $h_{22}$ nicht gleichzeitig Null: doppelte Berührung, und zwar

a) reell, wenn unter den drei Grössen $\psi_{i i}$ eine willkürlich ausgewählte positiv ist,

$\beta$ ) imaginär, wenn unter den drei Grössen $\psi_{i i}$ eine willkürlich ausgewählte negativ ist.

b) $h_{11}=h_{22}=h_{12}=0$ : Osculation.

c) Auch alle $\psi_{i i}$ gleich $N^{5}{ }^{5}$ ): die vier Schnittpunkte fallen zusammen.

1) Wenn diese Bedingungen neben $R>0$ für irgend ein bestimmtes $i$, bezw. $k$ erfüllt sind, so gelten sie anch für jedes $i$, bezw. $k$.

2) Es ist dann für jedes Werthsystem $u_{1}: u_{2}: u_{3}$, welches $\Psi(u, u)=0$ nicht erfüllt, $\Psi(u, u)>0$.

3) Es ist dann für jedes Werthsystem $u_{1}: u_{2}: u_{3}$, welches $\Psi(u, u)=0 \mathrm{nicht}$ erfüllt, $\Psi(u, u)<0$.

4) Man könnte auch sagen $\psi(u, u)$ identisch Null, nicht aber $\psi(x, x)$.

5) Man könnte auch sagen $\psi(x, x) \equiv 0$. 
*292. Um die Realität der vier gemeinsamen Tangenten von $f(x, x)=0$ und $g(x, x)=0$ zu untersuchen, hat man genau dieselben Betrachtungen wie zuvor durchzuführen, jedoch ausgehend von einer Combinante $\varphi(u, u)$, welche aus $F(u, u)$ und $G(u, u)$ in derselben Weise zu bilden ist wie $\psi(x, x)$ aus $f(x, x)$ und $g(x, x)$. Man fiudet

$$
\begin{aligned}
-\frac{1}{3} \varphi(\imath, u) & =\lambda_{2} \lambda_{3}\left(\lambda_{1}-\lambda_{2}\right)\left(\lambda_{1}-\lambda_{3}\right) u_{1}^{2}+\cdots \\
& +\lambda_{1} \lambda_{2}\left(\lambda_{3}-\lambda_{1}\right)\left(\lambda_{3}-\lambda_{2}\right) u_{3}{ }^{2}
\end{aligned}
$$

oder auch $\frac{1}{9} \varphi(u, u)=-(A \Theta G+B H F-2 A B H)$; an Stelle von $\Psi(u, u)$ tritt

$$
\Phi(x, x)=A B\left\{\left(A \Theta-\mathrm{H}^{2}\right) B f+(\Theta \mathrm{H}-A B) \chi+\left(B \mathrm{H}-\Theta^{2}\right) A g\right\} \cdot
$$

Andere, allerdings etwas complicirtere Kriterien sind zum ersten Male (auch für Schnittpunkte) von Herrn K. Kem mer ${ }^{1}$ ) gegeben worden.

* 293. Man bilde die in (292) erwähnte Combinante $\varphi(u, u)$ für die auf rechtwinklige Coordinaten bezogenen Kegelschnitte

$$
a^{2} u_{1}^{2}+b^{2} u_{2}{ }^{2}-u_{3}{ }^{2}=0 \text { und } u_{1}^{2}+u_{2}^{2}=0 \text {, }
$$

also für die Curven einer confocalen Schaar.

Man findet $-\frac{1}{3} \varphi(u, u)=\left(a^{2}-b^{2}\right) u_{1}^{2}-\left(a^{2}-b^{2}\right) u_{2}^{2}+1$, und dieser Ausdruck stellt gleich Null gesetzt die conjugirte gleichseitige Hyperbel zu derjenigen dar, welche die Verbindungslinie der zwei Brennpunkte der confocalen Schaar zur reellen Hauptaxe hat. Ueber die geometrische Bedeutung der letzteren vgl. die unten ${ }^{2}$ ) citirte Abhandlung von Steiner.

294. Wie viele Punkte gibt es auf der Curve zweiter Ordnung $f(x, x)=0$, in denen der Krümmungsradius $r$ einen gegebenen Werth besitzt?

Aus (17), S. 213 folgt $r^{2} \tau^{2} A^{2} p_{x}^{6}-\omega^{3}\left(f_{1}, f_{2}, f_{3}\right)=0$; nach (199) ist aber $\omega\left(f_{1}, f_{2}, f_{3}\right)=[a, \omega] f(x, x)-2 H$, wofür nun $-2 H$ gesetzt werden kann, da der Punkt $x$ auf $f(x, x)=0$ liegen soll. Wir erhalten also $r^{2} \tau^{2} \dot{A}^{2} p_{x}^{6}+8 H^{3}=0$ oder

1) „Kriterien der Realität für die Schnittpunkte von Linien zweiter Ordnung". Inaugaral-Dissertation. Giessen 1878.

2) „Elementare Lösung einer geometrischen Aufgabe, und über einige damit in Beziehnng stehende Eigenschaften der Kegelschnitte". Journal für die reine und angewandte Mathematik, Bd. 37, S. 185, 1847, oder "Gesammelte Werke", Bd. 2, S. 414. 


$$
\begin{aligned}
&\left(r^{\frac{2}{3}} \tau^{\frac{2}{3}} A^{\frac{2}{3}} p_{x}^{2}+2 H\right)\left(r^{\frac{2}{3}} \tau^{\frac{2}{3}} A^{\frac{2}{3}} p_{x}{ }^{2}+2 H \varepsilon\right) \\
&\left(r^{\frac{2}{3}} \tau^{\frac{2}{3}} A^{\frac{2}{3}} p_{x}{ }^{2}+2 H \varepsilon^{2}\right)=0,
\end{aligned}
$$

wobei $\varepsilon$ und $\varepsilon^{2}$ die complex conjugirten dritten Wurzeln der positiven Einheit bedeuten. Jeder der drei Klammerfactoren stellt eine Curve zweiter Ordnung dar, doch ist nur die erste reell; ihre Schnittpunkte mit $f(x, x)=0$ liefern diejenigen Punkte, in denen $r$ einen gegebenen Werth hat. Im allgemeinen gibt es also deren je 4, wenn wir von den 8 jedenfalls imaginären absehen, die bei Berücksichtigung der complexen Klammerfactoren in Betracht kommen. Die vier Punkte von gleichem Krümmungsradius $r$ liegen auf der Curve

$$
r^{\frac{2}{3}} \tau^{\frac{2}{3}} A^{\frac{2}{3}} p_{x}^{2}+2 H=0,
$$

welche einen mit dem Directorkreis $H=0$ und somit auch mit $f(x, x)=0$ concentrischen Kreis darstellt, was übrigens ans Gründen der Symmetrie klar ist. Bei der Parabel zerfällt nach S. $148 \mathrm{f}$. der Directorkreis in die Directrix und die unendlich ferne Gerade; daraus geht hervor, dass bei der Parabel die Punkte von gleichem Krümmungsradius auf Parallelen zur Directrix gelegen sind.

295. Wie viele Punkte gibt es im allgemeinen auf einer Curve $u^{\text {ter }}$ Ordnung, in denen der Krümmungsradius $r$ einen gegebenen Werth besitzt?

Aus (20), S. 213 folgt

$$
r^{2}(n-1)^{2} \tau^{2} p_{x}{ }^{6} \sum \pm\left(f_{11} f_{22} f_{33}\right)^{2}-\omega^{3}\left(f_{1}, f_{2}, f_{3}\right)=0
$$

d. h. alle Punkte von gleichem Krümmungsradius $r$ liegen auf einer Curve $6(n-1)^{\text {ter }}$ Ordnung; da sie auch auf der Curve $f\left(x_{1}, x_{2}, x_{3}\right)=0$ gelegen sind, gibt es im allgemeinen je $6 n(n-1)$ Punkte von der verlangten Beschaffenheit.

296. Es gibt im allgemeinen $6 k(k-1)$ Tangenten einer Curve $7^{\text {ter }}$ Classe, in deren Berührungspunkten der Krümmungsradius $r$ einen gegebenen Werth besitzt.

Folgt aus (14), S. 212 in gleicher Weise wie das Resultat von (295) aus (20), S. 213; speciell für Curven zweiter Classe findet man mit Hilfe von (15), S. 212 ein ähnliches Resultat wie bei (294): es können höchstens vier reelle Punkte von gleicher Krümmung vorhanden sein.

297. Man beweise, dass die Gleichung des Krümmungsmittelpunktes, der dem Berührungspunkte einer Tangente $u$ der Curve $k^{\text {ter }}$ Classe $\varphi\left(u_{1}, u_{2}, u_{3}\right)=0$ zugehört, die Form besitzt 


$$
\left|\begin{array}{cccc}
\frac{\partial D_{1}}{\partial u_{1}} & \frac{\partial D_{1}}{\partial u_{2}} & \frac{\partial D_{1}}{\partial u_{3}} & v_{1} \\
\frac{\partial D_{2}}{\partial u_{1}} & \frac{\partial D_{2}}{\partial u_{2}} & \frac{\partial D_{2}}{\partial u_{3}} & v_{2} \\
\frac{\partial D_{3}}{\partial u_{1}} & \frac{\partial D_{3}}{\partial u_{2}} & \frac{\partial D_{3}}{\partial u_{3}} & v_{3} \\
\varphi^{\prime}\left(u_{1}\right) & \varphi^{\prime}\left(u_{2}\right) & \varphi^{\prime}\left(u_{3}\right) & 0
\end{array}\right|=0,
$$

wobei $v_{1}, v_{2}, v_{3}$ variabele Liniencoordinaten bedeuten und die $D$ definirt sind durch

$$
\begin{gathered}
D_{1}=\varphi^{\prime}\left(u_{2}\right) \omega^{\prime}\left(u_{3}\right)-\varphi^{\prime}\left(u_{3}\right) \omega^{\prime}\left(u_{2}\right), \quad D_{2}=\varphi^{\prime}\left(u_{3}\right) \omega^{\prime}\left(u_{1}\right)-\varphi^{\prime}\left(u_{1}\right) \omega^{\prime}\left(u_{3}\right), \\
D_{3}=\varphi^{\prime}\left(u_{1}\right) \omega^{\prime}\left(u_{2}\right)-\varphi^{\prime}\left(u_{2}\right) \omega^{\prime}\left(u_{1}\right) .
\end{gathered}
$$

298. Man suche im Anschluss an (297) die Länge des zum Berührungspunkte der Tangente $u$ gehörigen Krümmungsradius der Curve $l_{i}^{\text {ter }}$ Classe $\varphi\left(u_{1}, u_{2}, u_{3}\right)=0$. (Vgi. die Fussnote 1) zu S. 212.)

299. Die Evolute einer Curve $\imath^{\text {ter }}$ Ordnung ist von der Ordnung $3 n(n-1)$.

Es ist beim Beweis dieses Satzes zu fragen, in wieviel Punliten eine Gerade $u_{y}=0$ die betr. Evolute schneidet; man hat also nach (23), S. 214 gleichzeitig zu betrachten die Gleichungen

$$
(n-1) \tau p_{x}{ }^{2} \cdot \sum \pm\left(f_{11} f_{22} f_{33}\right) u_{x}+\omega\left(f_{1}, f_{2}, f_{3}\right) \cdot \omega(f, u)=0
$$

und $f\left(x_{1}, x_{2}, x_{3}\right)=0$, von denen die erste in den $x$ vom Grade $3(n-1)$, die zweite vom Grade $n$ ist. Aus beiden folgen in allgemeinen $3 n(n-1)$ Werthsysteme von $x_{1}: x_{2}: x_{3}$, womit obige Behauptung bewiesen; natürlich ist vorausgesetzt, dass die gegebene Curve $n^{\text {ter }}$ Ordnung keine singulären Punkte enthält. Das Auftreten solcher Punkte würde diese Zahl $3 n(n-1)$ reduciren.

$\mathrm{Zu}$ derselben Zahl $3 n(n-1)$ gelangt man auch in folgender Weise.

Aus der Definition der Evolute folgt, dass ein Punkt $y$ dieser Curve angehört, wenn von den $n^{2}$ Normalen, die nach S. 204 von $y$ nach der Curve $n^{\text {ter }}$ Ordnung $f\left(x_{1}, x_{2}, x_{3}\right)=0$ gezogen werden können, zwei zusammenfallen, d. h. wenn $f\left(x_{1}, x_{2}, x_{3}\right)=0$ von der Curve $n^{\text {ter }}$ Ordnung (22), S. 204, auf der die Fusspunkte jener Normalen liegen, berührt wird (vgl. auch S. 216 unten); wir wollen die Gleichung dieser letzten Curve durch $g\left(x_{1}, x_{2}, x_{3}\right)=0$ bezeichnen, und haben nun zu untersuchen, von wie hohem Grade in den $y$ die Bedingung der Berührung wird. Es werde zuvor allgemeiner gefragt, wie hoch in den Coefficienten der Gleichung einer Curve $m^{\text {ter }}$ Ordnung $g\left(x_{1}, x_{2}, x_{3}\right)=0$ 
die Bedingung dafür ist, dass $g=0$ die Curve $n^{\text {ter }}$ Ordnung $f=0$ berührt ${ }^{1}$ ). Ersetzt man $g=0$ durch $g+\lambda h=0$, wo $h=0$ eine andere Curve $m^{\text {ter }}$ Ordnung darstellen möge, so hat man zunächst für die Berührungspunkte $x$ von $f=0$ mit $g+\lambda h=0$ die drei Gleichungen $\varrho f^{\prime}\left(x_{i}\right)=g^{\prime}\left(x_{i}\right)+\lambda h^{\prime}\left(x_{i}\right),(i=1,2,3)$, aus denen durch Elimination von $\varrho$ und $h$ folgt $\sum \pm\left(f^{\prime}\left(x_{1}\right) g^{\prime}\left(x_{2}\right) h^{\prime}\left(x_{3}\right)\right)=0$. Die Schnittpunkte dieser Curve von der Ordnung $n-1+2(m-1)=n+2 m-3$ mit $f=0$ sind diejenigen Punkte, in denen $f$ von einer Curve des Büschels $g+\lambda h=0$ berührt wird. Da die Anzahl dieser Schnittpunkte $(n+2 m-3) n$ beträgt, müssen die Coefficienten von $g+\lambda h=0$, also auch die von $g=0$ allein, in die Bedingung der Berührung im Grade $(n+2 m-3) n$ eingehen. In dem oben speciell vorliegenden Falle ist $g\left(x_{1}, x_{2}, x_{3}\right)=0$ von der $n^{\text {ten }}$ Ordnung, die Bedingung der Berührung von $g=0$ mit $f=0$ wird daher in den Coefficienten von $g$, somit auch in den $y_{i}$, vom Grade $(3 n-3) n$ oder $3 n(n-1)$, d. h. die Evolute einer Curve $n^{\text {ter }}$ Ordnung ist von der Ordnung $3 n(n-1)$.

*300. Man gebe einen Weg an zur Bildung der Gleichung für die Evolute einer Curve $l^{\text {ter }}$ Classe $\varphi\left(u_{1}, u_{2}, u_{3}\right)=0$.

Sind $u_{1}, u_{2}, u_{3}$ die Coordinaten der Tangente irgend eines Punktes der gegebenen Curve, $v_{1}, v_{2}, v_{3}$ die Coordinaten der zugehörigen Normale, so hat man $\omega^{\prime}\left(v_{1}\right) u_{1}+\omega^{\prime}\left(v_{2}\right) u_{2}+\omega^{\prime}\left(v_{3}\right) u_{3}=0$; ferner ist $\varphi^{\prime}\left(u_{1}\right) v_{1}+\varphi^{\prime}\left(u_{2}\right) v_{2}+\varphi^{\prime}\left(u_{3}\right) v_{3}=0$, da die Normale durch den Berührungspunkt der Tangente $u$ geht. Um die Gleichung der Evolute in Liniencoordinaten $\mathrm{zu}$ erhalten, sind die $u_{i} \mathrm{zu}$ eliminiren aus der Gleichung $k^{\text {ten }}$ Grades $\varphi\left(u_{1}, u_{2}, u_{3}\right)=0$, aus einer Gleichung ersten Grades und einer vom Grade $(k-1)$. Die beiden letzten Gleichungen sind in den $v_{i}$ linear, während $\varphi\left(u_{1}, u_{2}, u_{3}\right)=0$ die $v_{i}$ überhaupt nicht enthält. Die Resultante ist alsdann, wie in der Algebra gezeigt wird, in den $v$ vom Grade $k(k-1)+k \cdot 1=k^{2}$. Zugleich folgt, dass für eine Curve $k^{\text {ter }}$ Classe die Evolute von der Classe $k^{2}$ ist.

$\mathrm{Zu}$ demselben Resultat gelangt man in nachstehender Weise: Setzt man der Kürze halber $x_{i}=\omega^{\prime}\left(v_{i}\right)$, so liegen die Gleichungen vor:

$$
\begin{gathered}
\varphi\left(u_{1}, u_{2}, u_{3}\right)=0, \quad \varphi^{\prime}\left(u_{1}\right) v_{1}+\varphi^{\prime}\left(u_{2}\right) v_{2}+\varphi^{\prime}\left(u_{3}\right) v_{3}=0, \\
u_{1} x_{1}+u_{2} x_{2}+u_{3} x_{3}=0 .
\end{gathered}
$$

Die Elimination von $u_{1}, u_{2}, u_{3}$ liefert das Product der Gleichungen der $k(k-1)$ Tangenten, welche die gegebene Curve $\varphi=0$ mit

1) Vgl. hierzu „Analytische Geometrie der höheren ebenen Curven von Salmon", deutsch bearbeitet von W. Fiedler, 2. Aufl., S. 103. 


$$
\varphi^{\prime}\left(u_{1}\right) v_{1}+\varphi^{\prime}\left(u_{2}\right) v_{2}+\varphi^{\prime}\left(u_{3}\right) v_{3}=0
$$

gemeinsam hat; speciell erhält man wieder den Ausdruck für die Evolute in variabelen Liniencoordinaten $v_{i}$ durch die Substitution $x_{i}=\omega^{\prime}\left(v_{i}\right)$, so dass also im Eliminationsresultate die $v_{i}$ vermöge der $x_{i}$ eingehen im Grade $k(k-1)$ und vermöge der in

$$
\varphi^{\prime}\left(u_{1}\right) v_{1}+\varphi^{\prime}\left(u_{2}\right) v_{2}+\varphi^{\prime}\left(u_{3}\right) v_{3}=0
$$

explicit stehenden $v_{i}$ im Grade $k \cdot 1$, somit insgesammt im Grade $k(k-1)+k=k^{2}$.

301. Man bestimme den geometrischen Ort der Punkte $y$, von denen an die Curve zweiter Ordnung $f(x, x)=0$ vier Normalen vou gegebenem Doppelverhältniss gelegt werden können $\left.{ }^{1}\right)$.

Die Fusspunkte der Normalen werden nach (22), S. 204 aus $f(x, x)=0$ ausgeschnitten durch die gleichseitige Hyperbel

$$
g(x, x) \equiv \sum \pm\left(\frac{1}{2} \omega^{\prime}\left(f_{1}\right) x_{2} y_{3}\right)=0 \text {. }
$$

Nach (283) ist nun für diese Curve $g(x, x)=0$ und für $f(x, x)=0$ zu bilden die kubische Form

$$
\Psi_{0}(\lambda, \mu) \equiv \lambda^{3} B-3 \lambda^{2} \mu \Theta+3 \lambda \mu^{2} \mathrm{H}-\mu^{3} A,
$$

in welcher jedoch $H$ verschwindet, wie bereits S. 217 unter Angabe des Grundes erwähnt. Alsdann hat man, von dieser kubischen Form ausgehend, die Gleichung $-\frac{h^{3}(\lambda, \mu)}{q^{2}(\lambda, \mu)}=\frac{\left(1-s+s^{2}\right)^{3}}{(1+s)^{2}(2 s-1)^{2}(s-2)^{2}}$ zu bilden, in der $s$ das gegebene Doppelverhältniss bedeutet (vgl. (4), S. 367). Im allgemeinen gehen die $y_{i}$ in $h(\lambda, \mu)$ im vierten Grade ein, in $q(\lambda, \mu)$ im sechsten Grade; der Ort der Punkte $y$ ist also im allgemeinen eine Curve 12. Ordnung. Da jedoch speciell von einem Punkte $y$ des Kegelschnitts $g(x, x)=0$ des Büschels $\lambda g-\mu f=0$ die vier Normalen von gegebenem Doppelverhältniss gezogen werden sollen, so ist zu setzen $\lambda=1, \mu=0$, wodurch sich $h(\lambda, \mu)$ nach S. 364 reducirt auf - $\Theta^{2}, q(\lambda, \mu)$ auf $-A B^{2}-2 \Theta^{3}$. Diese Ausdrücke wären nun zu substituiren in $-\frac{h^{3}(\lambda, \mu)}{q^{2}(\lambda, \mu)}=k$, wobei zur Abkürzung gesetzt ist

$$
\frac{\left(1-s+s^{2}\right)^{3}}{(1+s)^{2}(2 s-1)^{2}(s-2)^{2}}=k
$$

man erhält alsdann $\Theta^{6}-k\left(A B^{2}+2 \Theta^{3}\right)^{2}=0$ oder

$$
\left\{\Theta^{3}+\sqrt{k}\left(A B^{2}+2 \Theta^{3}\right)\right\}\left\{\Theta^{3}-\sqrt{k}\left(A B^{2}+2 \Theta^{3}\right)\right\}=0,
$$

die Curve 12. Ordnung zerfällt also in zwei Curven sechster Ordnung.

1) Die hier gegebene Methode wurde im Jahre 1877 Herrn W. Fiedler von Herrn Gundelfinger mitgetheilt. 
Speciell für $s=0$ müssen zwei von den vier Normalen zusammenfallen, der Punkt $y$ also auf der Evolute liegen; wenn $s=0$, wird $k=\frac{1}{4}$ und man erhält in der That als Ort von $y$ die zwei Curven sechster Ordnung $4 \Theta^{3}+A B^{2}=0$ und $A B^{2}=0$, deren erste in Uebereinstimmung mit (35), S. 217 die Evolute darstellt, während die zweite nach (38), S. 217 das doppelt zu zählende Product aus den Hauptaxen von $f(x, x)=0$ und der unendlich fernen Geraden repräsentirt.

302. Man bilde eine Gleichung zur Bestimmung der Fusspunkte der Normalen, welche von einem beliebigen Punkte $y$ nach einem Kegelschnitt $f(x, x)=0$ gezogen werden.

Sind $y_{i}$ die Coordinaten des beliebig gegebenen Punktes, $x_{i}$ die Coordinaten eines Normalenfusspunktes, $\boldsymbol{\omega}^{\prime}\left(f_{i}\right)$ die Coordinaten des Normalencentrums der Tangente in $x$, so bestehen die Relationen $y_{i}=\lambda x_{i}+\omega^{\prime}\left(f_{i}\right),(i=1,2,3)$, denn die drei Punkte $y, x$ und das genannte Normalencentrum liegen in einer Geraden (vgl. auch (19), S. 213). Nach Einführung der Werthe $\omega^{\prime}\left(f_{i}\right)$ folgt unter Anwendung der in (6), S. 85 gebrauchten Bezeichnungsweise:

$$
\begin{array}{lll}
y_{1}=\left(\alpha_{11}+\lambda\right) x_{1} & +\alpha_{12} x_{2} & +\alpha_{13} x_{3} \\
y_{2}=\alpha_{21} x_{1} & +\left(\alpha_{22}+\lambda\right) x_{2}+\alpha_{23} x_{3} \\
y_{3}=\alpha_{31} x_{1} & +\alpha_{32} x_{2} & +\left(\alpha_{33}+\lambda\right) x_{3} .
\end{array}
$$

Es sei nun $\mathrm{B} \equiv \sum \pm\left(\beta_{11} \beta_{22} \beta_{33}\right)$ die aus den Coefficienten der $x$ in diesen drei Gleichungen gebildete Determinante, so dass also z. B. $\beta_{22}=\alpha_{22}+\lambda, \beta_{31}=\alpha_{31}$ u. s. f.; ferner sei $B_{i k}$ die Unterdeterminante von $\beta_{i k}$ in $\mathrm{B}$. Die Auflösung obiger Gleichungen nach $x_{i}$ ergibt alsdann $\mathrm{B} x_{i}=\mathrm{B}_{1 i} y_{1}+\mathrm{B}_{2 i} y_{2}+\mathrm{B}_{3 i} y_{3}$. Diese Werthe der $x$ substituirt man in $f(x, x)=0$ und erhält dadurch eine biquadratische Gleichung für $\lambda$, denn die $B_{i k}$ enthalten $\lambda$ im zweiten Grade. Durch Einsetzen der Wurzeln $\lambda$ in $\mathrm{B} x_{i}=\mathrm{B}_{1 i} y_{1}+\mathrm{B}_{2 i} y_{2}+\mathrm{B}_{3 i} y_{3}$ ergeben sich die Coordinaten $x_{i}$ jener Normalenfusspunkte.

\section{Anwendung von $\S 22-26$.}

303. Man beweise, dass die Hessiane, sowie die Cayleysche Curve eines Kegelschnittnetzes Combinanten sind.

Gehen wir aus von dem Netz $\varkappa \varphi+\lambda \psi+\mu \chi=0$, so ist zu zeigen, dass sich bei Substitution von

$$
x_{i} \varphi+\lambda_{i} \psi+\mu_{i} \chi=0,(i=1,2,3),
$$


an Stelle von $\varphi$, resp. $\psi$, resp. $\chi$ die Ausdrücke für die Hessiane und für die Cayley'sche Curve nur um eine Potenz der Determinante der Substitution $\sum \pm\left(x_{1} \lambda_{2} \mu_{3}\right)$ ändern.

Wenn man nun für $x_{i} \varphi+\lambda_{i} \psi+\mu_{i} \chi$ entsprechend den Werthen 1, 2, 3 des Index $i$ zur Abkürzung setzt $f(x, x), g(x, x), h(x, x)$, so wird die Gleichung der Hessiane des durch $f, g$ und $h$ bestimmten Netzes:

$\sum \pm\left(f^{\prime}\left(x_{1}\right), g^{\prime}\left(x_{2}\right), h^{\prime}\left(x_{3}\right)\right)=\sum \pm\left(x_{1} \lambda_{2} \mu_{3}\right) \cdot \sum \pm\left(\varphi^{\prime}\left(x_{1}\right) \psi^{\prime}\left(x_{2}\right) \chi^{\prime}\left(x_{3}\right)\right)$ womit für die Hessiane obige Behauptung erwiesen ist.

Die Gleichung der Cayley'schen Curve werde dadurch aufgestellt, dass man zuerst den Polkegelschnitt einer Geraden $u$ in Bezug auf $f(x, x)$ und $g(x, x)$ sucht. Derselbe wird

$N(x, x) \equiv\left(\lambda_{1} \mu_{2}-\lambda_{2} \mu_{1}\right) N_{1}+\left(\mu_{1} x_{2}-\mu_{2} x_{1}\right) N_{2}+\left(x_{1} \lambda_{2}-x_{2} \lambda_{1}\right) N_{3}^{r}=0$, wobei zur Abkürzung gesetzt ist

$$
\begin{gathered}
N_{1} \equiv \sum \pm\left(\psi^{\prime}\left(x_{1}\right) \chi^{\prime}\left(x_{2}\right) u_{3}\right), \quad N_{2} \equiv \sum \pm\left(\chi^{\prime}\left(x_{1}\right) \varphi^{\prime}\left(x_{2}\right) u_{3}\right), \\
N_{3} \equiv \sum \pm\left(\varphi^{\prime}\left(x_{1}\right) \psi^{\prime}\left(x_{2}\right) u_{3}\right) .
\end{gathered}
$$

Jetzt muss noch zu $N(x, x)$ und zu $h(x, x)$ die Gleichung des harmonischen Kegelschnitts gebildet werden. Die Curve $N_{1}=0$ wird nun von jeder Geraden $u$ in einem Punktepaare getroffen, das harmonisch liegt zu den Schnittpunkten dieser Geraden sowohl mit $\psi=0$, als mit $\chi=0$; daher ist

$$
\begin{aligned}
& \left(N_{1}, \psi\right)_{11} u_{1}^{2}+2\left(N_{1}, \psi\right)_{12} u_{1} u_{2}+\cdots \equiv 0 \quad \text { und } \\
& \left.\left(N_{1}, \chi\right)_{11} u_{1}^{2}+2\left(N_{1}, \chi\right)_{12} u_{1} u_{2}+\cdots \equiv 0 .{ }^{1}\right)
\end{aligned}
$$

Aehnliches gilt von $N_{2}$ und $N_{3}$. In Folge dessen bleiben bei Bildung der Gleichung des harmonischen Kegelschnitts von $N$ und $h$ nur übrig die Glieder:

$$
\begin{aligned}
& x_{3}\left(\lambda_{1} \mu_{2}-\lambda_{2} \mu_{1}\right)\left\{\left(N_{1}, \varphi\right)_{11} u_{1}^{2}+2\left(N_{1}, \varphi\right)_{12} u_{1} u_{2}+\cdots\right\}+ \\
+ & \lambda_{3}\left(\mu_{1} x_{2}-\mu_{2} x_{1}\right)\left\{\left(N_{2}, \psi\right)_{11} u_{1}^{2}+2\left(N_{2}, \psi\right)_{12} u_{1} u_{2}+\cdots\right\}+ \\
+ & \mu_{3}\left(x_{1} \lambda_{2}-x_{2} \lambda_{1}\right)\left\{\left(N_{3}, \chi\right)_{11} u_{1}^{2}+2\left(N_{3}, \chi\right)_{12} u_{1} u_{2}+\cdots\right\} .
\end{aligned}
$$

Jede dieser drei geschweiften Klammern enthält denselben Ausdruck für die dem Netz $\varkappa \varphi+\lambda \psi+\mu \chi=0$ zugehörige Cayley'sche Curve; von letzterer ist daher die Cayley'sche Curve des durch $f, g, h$ bestimmten Netzes in der That nur verschieden um den Factor $\sum \pm\left(x_{1} \lambda_{2} \mu_{3}\right)$.

1) Ueker die Bezeichnungsweise vgl. (12), S. 130. 
304. Man bilde die Gleichung der Hesse'schen Curve eines Netzes, dessen einzelne Curven sämmtlich einen und denselben Punkt gemeinsam haben.

Als Seiten des Coordinatendreiecks führen wir ein die Tangenten $x_{1}=0, x_{2}=0$ zweier Kegelschnitte $\varphi(x, x)=0$ und $\psi(x, x)=0$ des Netzes in dem allen Curven gemeinsamen Punkte $P$; die Tangente $x_{1}=0$ von $\varphi$ trifft $\psi$ in einem zweiten Punkte $Q$, die Tangente $x_{2}=0$ von $\psi$ trifft $\varphi$ in einem zweiten Punkte $R$; die Gerade $Q R$ sei die dritte Seite des Coordinatendreiecks. Wird eine weitere Curve des Netzes durch das Geradenpaar $x_{1} x_{2}=0$ gebildet, so erhält man als Gleichung des Netzes:

$$
\begin{aligned}
x\left(a_{22} x_{2}{ }^{2}+2 a_{12} x_{1} x_{2}+2 a_{13} x_{1} x_{3}\right)+\lambda\left(b_{11} x_{1}{ }^{2}\right. & \left.+2 b_{12} x_{1} x_{2}+2 b_{23} x_{2} x_{3}\right) \\
& +2 \mu x_{1} x_{2}=0,
\end{aligned}
$$

und hier kann noch (vgl. (303)) $a_{12}=b_{12}=0$ gesetzt werden. Für die Hessiame des Netzes folgt

$$
f\left(x_{1}, x_{2}, x_{3}\right) \equiv a_{13} b_{11} x_{1}{ }^{3}+a_{22} b_{23} x_{2}{ }^{3}-2 a_{13} b_{23} x_{1} x_{2} x_{3}=0,
$$

eine Curve dritter Ordnung, die in dem gemeinsamen Punkte der Kegelschnitte des Netzes einen Doppelpunkt hat mit den Geraden $x_{1}=0, x_{2}=0$ als Doppelpunktstangenten. (Auf der Geraden $x_{3}=0$ liegen die Wendepunkte dieser Curve dritter Ordnung, d. h. diejenigen Punkte, in denen der Krümmungsradius unendlich gross ist, also nach (20), S. 213 der Ausdruck $\sum \pm\left(\frac{\partial^{2} f}{\partial x_{1}{ }^{2}} \frac{\partial^{2} f}{\partial x_{9}{ }^{2}} \frac{\partial^{2} f}{\partial x_{3}{ }^{2}}\right)$ verschwindet.) Durch passende Wahl des Einheitspunktes kann die Hessiane in der.Form dargestellt werden $\left.X_{1}{ }^{3}+X_{2}{ }^{3}+6 k X_{1} X_{2} X_{3}{ }^{1}\right)=0$.

305. Für dasselbe Kegelschnittnetz die Gleichung der Cayleyschen Curve aufzustellen.

Nach der auf S. 225 angegebenen Methode bilden wir zuerst die Gleichung des Polkegelschnitts einer Geraden $u$ in Bezug auf

$$
\varphi \equiv a_{22} x_{2}{ }^{2}+2 a_{13} x_{1} x_{3}
$$

und $\chi \equiv x_{1} x_{2}$, alsdann die Gleichung der harmonischen Curve zweiter Classe zu dem Polkegelschnitt und zu $\psi \equiv b_{11} x_{1}{ }^{2}+2 b_{23} x_{2} x_{3}$. Man findet alsdann für die Cayley'sche Curve:

$$
u_{3}\left(a_{22} b_{1} u_{3}^{2}-4 a_{13} b_{23} u_{1} u_{2}\right)=0,
$$

sie zerfällt also in den allen Kegelschnitten des Netzes gemeinsamen Punkt und eine Curve zweiter Classe, welche in den Ecken $u_{1}=0$,

1) Vgl. Hesse: „Ueber Curven dritter Ordnung und die Kegelschnitte, welche diese Curven in drei verschiedenen Punkten berühren." Journal für die reine und angewandte Mathematik, Bd. 36, S. 168, 1847. 
resp. $u_{2}=0$ des Coordinatendreiecks von dessen Seiten $x_{2}=0$, resp. $x_{1}=0$ berührt wird, während $x_{3}=0$ die Berührungssehne, $u_{3}=0$ deren Pol darstellt.

306. Haben alle Kegelschnitte eines Netzes eine gemeinsame Sehne, so zerfällt die Hessiane in diese Sehne und einen Kegelschnitt.

Die drei Gleichungen

$$
f(x, x)=0, \quad f(x, x)+2 m_{x} q_{x}=0, \quad f(x, x)+2 n_{x} q_{x}=0
$$

stellen drei Kegelschnitte dar, welche durch die Schnittpunkte der Geraden $q_{x}=0$ mit $f(x, x)=0$ hindurchgehen. Die Gleichung des Kegelschnittnetzes ist daher im vorliegenden Falle

$$
x f(x, x)+2 \lambda m_{x} q_{x}+2 \mu n_{x} q_{x}=0 .
$$

Die Hessiane des Netzes wird

oder auch

$$
\left|\begin{array}{lll}
f_{1} & m_{x} q_{1}+q_{x} m_{1} & n_{x} q_{1}+q_{x} n_{1} \\
f_{2} & m_{x} q_{2}+q_{x} m_{2} & n_{x} q_{2}+q_{x} n_{2} \\
f_{3} & m_{x} q_{3}+q_{x} m_{3} & n_{x} q_{3}+q_{x} n_{3}
\end{array}\right|=0
$$

$q_{x}{ }^{2} \sum \pm\left(f_{1} m_{2} n_{3}\right)+q_{x} m_{x} \sum \pm\left(f_{1} q_{2} n_{3}\right)-q_{x} n_{x} \sum \pm\left(f_{1} q_{2} m_{3}\right)=0$

wobei sich in der That der Factor $q_{x}$ ausscheiden lässt. Mit Hilfe der Identität

folgt

$$
\begin{array}{llll}
f_{1} & f_{2} & f_{3} & f(x, x) \\
q_{1} & q_{2} & q_{3} & q_{x} \\
m_{1} & m_{2} & m_{3} & m_{x}
\end{array} m_{1} \equiv 0
$$$$
\begin{gathered}
f(x, x) \cdot \sum \pm\left(q_{1} m_{2} n_{3}\right)-q_{x} \sum \pm\left(f_{1} m_{2} n_{3}\right)+m_{x} \sum \pm\left(f_{1} q_{2} n_{3}\right) \\
-n_{x} \sum \pm\left(f_{1} q_{2} m_{3}\right)=0,
\end{gathered}
$$

so dass die Gleichung des in der Hessiane enthaltenen Kegelschnitts die Gestalt erlält

$$
2 q_{x} \sum \pm\left(f_{1} m_{2} n_{3}\right)-f(x, x) \sum \pm\left(q_{1} m_{2} n_{3}\right)=0 \text {. }
$$

Führt man die Coordinaten des Schnittpunktes $z$ der beiden Geraden $m$ und $n$ ein durch $z_{1}=m_{2} n_{3}-m_{3} n_{2}$, u. s. w., so entsteht

$$
2 q_{x} f(z, x)-q_{2} f(x, x)=0 .
$$

Auch diese Curve hat mit den Kegelschnitten des Netzes die Sehne $q_{x}=0$ gemeinsam; ausserdem trifft sie irgend eine Curve $g(x, x)=0$ 
des Netzes in deren Schnittpunkten mit der Polare $g(z, x)=0$ des Punktes $z$ in Bezug auf $g(x, x)=0$.

Von Wichtigkeit ist endlich noch die Bemerkung, dass die Polare des Punktes $z$ in Bezug auf den der Hessiane angehörigen Kegelschnitt $2 q_{x} f(z, x)-q_{s} f(x, x)=0$ mit der gemeinsamen Sehne der einzelnen Curven des Netzes zusammenfällt; der Punkt $z$ ist nach (197) zugleich derjenige, in welchem sich die übrigen Sehnen schneiden, die je zwei Curven des Netzes gemeinsam sind.

30\%. Haben alle Kegelschnitte eines Netzes zwei Punkte gemeinsam, so zerfällt die Cayley'sche Curve in drei Punkte, nämlich. in die zwei gemeinsamen und in einen dritten Punkt, der mit demjenigen identisch ist, in welchem sich nach (197) die übrigen gemeinsamen Sehnen von je zwei Curven des Netzes schneiden.

Wie in (306) wird ausgegangen von dem Netz

$$
x f(x, x)+2 \lambda q_{x} m_{x}+2 \mu q_{x} n_{x}=0 .
$$

Nach der Bemerkung am Schlusse von $\S 23$ kann man die Gleichung der Cayley'schen Curve bilden, indem man erst den Polkegelschnitt von $q_{x} m_{x}=0$ und $q_{x} n_{x}=0$ berechnet und alsdann zu ihm und $f(x, x)=0$ die harmonische Curve zweiter Classe. Die Gleichung des ersteren kann auf dieselbe Art abgeleitet werden, wie die der Hessiane in (306), nur sind die $f_{i}$ daselbst zu ersetzen durch die $u_{i}$; man findet $q_{x}\left\{2 \sum \pm\left(u_{1} m_{2} n_{3}\right) \cdot q_{x}-\sum \pm\left(q_{1} m_{2} n_{3}\right) \cdot u_{x}\right\}=0$, der Kegelschnitt zerfällt also in die gemeinsame Sehne $q_{x}=0$ und die Gerade

$$
r_{x} \equiv 2 \sum \pm\left(u_{1} m_{2} n_{3}\right) q_{x}-\sum \pm\left(q_{1} m_{2} n_{3}\right) u_{x}=0
$$

Die harmonische Curve zweiter Classe zu $f(x, x)=0$ und $q_{x} r_{x}=0$ ist $\left(\begin{array}{ll}q & u \\ r & u\end{array}\right)_{a_{i k}}=0$, oder auch $2 \sum \pm\left(u_{1} m_{2} n_{3}\right) \cdot\left(\begin{array}{ll}q & u \\ q & u\end{array}\right)_{a_{i k}}=0$, zufolge der obigen einfachen Relation, welche zwischen den drei Geraden $q_{x}=0$, $r_{x}=0, u_{x}=0$ besteht, vermöge deren dieselben durch einen und denselben Punkt gehen. Der Factor $\left(\begin{array}{ll}q & u \\ q & u\end{array}\right)_{a_{i k}}=0$ stellt aber nach (53a) in $\S 4$ das Schnittpunktepaar der Geraden $q_{x}=0$ mit $f(x, x)=0$ dar, während $\sum \pm\left(u_{1} m_{2} n_{3}\right)=0$ die Gleichung des in (306) durch $z$ bezeichneten und daselbst näher besprochenen Punktes ist.

308. Haben alle Kegelschnitte eines Netzes drei Punkte gemeinsam, so besteht die Hessiane aus den drei Verbindungslinien dieser Punkte, die Cayley'sche Curve aus den drei Punkten selbst.

Es genügt hier das Netz zu betrachten, welches durch alle dem 
Kegelschnittgewebe mit ein, zwé oder drei gemeinsamen Tangenten. 387.

Coordinatendreieck umschriebenen Kegelschnitte gebildet wird; mau • kommt dann sehr leicht zu obigem Resultat.

Dualistisch zu den voranstehenden Sätzen ergeben sich die folgenden:

309. Die Hessiane eines Kegelschnittgewebes, dessen einzelne Curven eine Tangente gemeinsam haben, ist eine Curve dritter Classe, welche die gemeinsame Tangente zur Doppeltangente hat.

Ausserdem lässt sich noch Folgendes über diese Curve sagen. Es seien $P$ und $Q$ die Berührungspunkte der Doppeltangente mit der Hessiane und $\varphi(u, u)=0$, bezw. $\psi(u, u)=0$ irgend zwei Kegelschnitte des Gewebes; von $P$ kann alsdann noch eine weitere Tangente an $\psi(u, u)=0$ gezogen werden, von $Q$ eine solche an $\varphi(u, u)=0$. Durch den Schnittpunkt $S$ dieser beiden 'Tangenten gehen die drei Rückkehrtangenten der Curve dritter Classe, d. l. die Tangenten in solchen Punkten, in denen die Länge des Krümmungsradius gleich Null ist. Nach (14), S. 212 genügen diese Tangenten der Gleichung $\sum \pm\left(\frac{\partial^{2} F}{\partial u_{1}{ }^{2}} \frac{\partial^{2} F}{\partial u_{2}{ }^{2}} \frac{\partial^{2} F}{\partial u_{3}{ }^{2}}\right)=0$, wenn durch $F\left(u_{1}, u_{2}, u_{3}\right)=0$ die Hessiane dargestellt wird. Vgl. (304).

310. Die Cayley'sche Curve eines Kegelschnittgewebes, dessen einzelne Curven eine Tangente gemeinsam haben, zerfüllt in diese Gerade und in einen Kegelschnitt. Bei gleicher Bezeichnung wie in (309) sind $P$ und $Q$ die Berührungspunkte der beiden 'langenten $P S$ und $Q S$, also $S$ der Pol der Berührungssehne. Vgl. (305).

311. Haben alle Kegelschnitte eines Gewebes zwei gemeinsame T'angenten, so zerfällt die Hessiane in den Schnittpunkt dieser Tangenten und in einen Kegelschnitt. Vgl. (306).

312. Die Cayley'sche Curve eines solchen Gewebes zerfällt in die beiden gemeinsamen Tangenten und in eine dritte Gerade. Auf ihr liegen die Spitzen der übrigen drei Paare von Tangenten, die je zwei Curven des Gewebes gemeinsam sind. Vgl. (307):

313. Haben alle Kegelschnitte eines Gewebes drei Tangenten gemeinsam, so besteht die Hessiane aus den drei Schnittpunkten dieser Tangenten, die Cayley'sche Curve aus den drei Tangenten selbst. Vgl. (308).

314. Man bilde die Gleichung der Hessiane eines Kegelschnittnetzes, in welchem sich eine Doppelgerade befindet.

Für $x \varphi(x, x)+\lambda \psi(x, x)+\mu w_{x}^{2}=0$ zerfällt die Hessiane in 
$w_{x}=0$ und in $\sum \pm\left(\frac{\partial \varphi}{\partial x_{1}} \frac{\partial \psi}{\partial x_{2}} w_{3}\right)=0$, den Polkegelschnitt der Geraden $w$ in Bezug auf das Büschel $x \varphi(x, x)+\lambda \psi(x, x)=0$.

Uebrigens ist die Doppelgerade eines Netzes für das conjugirte Gewebe eine gemeinsame Tangente, denn wenn eine Curve $\varphi(u, u)=0$ conjugirt sein soll zu der Doppelgeraden $w_{x}^{2}=0$, so heisst dies die Gerade $w$ soll die Curve berühren. Die Hessiane des Netzes ist aber nach (38), S. 240 identisch mit der Cayley'schen Curve des conjugirten Gewebes; nach (310) besteht sie also aus der Geraden $w_{x}=0$ und aus einem Kegelschnitt.

315. Die Cayley'sche Curve eines Kegelschnittnetzes, in welchem sich eine Doppelgerade befindet, ist eine Curve dritter Classe, welche diese Gerade zur Doppeltangente hat.

Die Cayley'sche Curve des Netzes ist nach (39), S. 240 identisch mit der Hessiane des conjugirten Gewebes, das zufolge (314) aus Kegelschnitten besteht, die eine Tangente gemeinsam haben.

Repräsentirt $x \varphi(x, x)+\lambda \psi(x, x)+\mu w_{x}{ }^{2}=0$ das Netz, so ergibt sich übrigens nach S. 225 die Gleichung der Cayley'schen Curve dadurch, dass man in der Gleichung $\sum \pm\left(\frac{\partial \varphi}{\partial x_{1}} \frac{\partial \psi}{\partial x_{2}} u_{3}\right)=0$ des Polkegelschnitts einer Geraden $u$ an Stelle von $x_{1}: x_{2}: x_{3}$ einführt

$$
\left(w_{2} u_{3}-w_{3} u_{2}\right):\left(w_{3} u_{1}-w_{1} u_{3}\right):\left(w_{1} u_{2}-w_{2} u_{1}\right) \text {. }
$$

Nach (309), bezw. (304) muss sich die Cayley'sche Curve schliesslich darstellen lassen durch einen Ausdruck von der Form

$$
a U_{1}^{3}+b U_{2}^{3}-6 k U_{1} U_{2} U_{3}=0 \text {. }
$$

316. Die Hessiane eines Kegelschnittnetzes, in welchem sich zwei Doppelgeraden befinden, besteht aus den beiden Geraden und aus der in Bezug auf irgend eine Curve des Netzes genommenen Polaren des Schnittpunktes der zwei Geraden.

Denn die Hessiane des Netzes $x \varphi(x, x)+\lambda v_{x}^{2}+\mu w_{x}{ }^{2}=0$ hat die Gleichung $v_{x} w_{x} \cdot \sum \pm\left(\varphi^{\prime}\left(x_{1}\right) v_{2} w_{3}\right)=0$.

317. Die Cayley'sche Curve eines Kegelschnittnetzes, in welchem sich zwei Doppelgeraden befinden, besteht aus dem Schnittpunkte der beiden Geraden und aus dem harmonischen Kegelschnitt zu diesem Geradenpaare und zu irgend einer Curve des Netzes.

Nach S. 225 erhält man die Gleichung der Cayley'schen Curve des Netzes $x \varphi(x, x)+\lambda v_{x}{ }^{2}+\mu w_{x}{ }^{2}=0$, indem man die Bedingungsgleichung dafür aufstellt, dass die Gerade $u_{x}=0$ die Curve $\varphi(x, x)=0$ 
und das Geradenpaar $v_{x} w_{x} \cdot \sum \pm\left(v_{1} w_{2} u_{3}\right)=0$ in zwei harmonischen Punktepaaren schneidet. Ist $\varphi(x, x)$ definirt durch

$$
\sum_{1}^{3} \sum_{1}^{3} a_{i k} x_{i} x_{k}=0,
$$

so würde man als Cayley'sche Curve den Punkt $\sum \pm\left(v_{1} w_{2} u_{3}\right)=0$ und die Curve zweiter Classe $\left(\begin{array}{ll}v & u \\ w & u\end{array}\right)_{a_{i k}}=0$ erlialten.

318. Die Gesammtheit der Kegelschnitte, welche ein gegebenes Dreieck zum Poldreieck haben, bildet ein Netz; die Hessiane desselben besteht aus den Seiten, die Cayley'sche Curve aus den Ecken des betreffenden Dreiecks.

Wählt man das gegebene Dreieck zum Coordinatendreieck, so ist die Gesammtheit der in Rede stehenden Kegelschnitte gegeben durch $x x_{1}{ }^{2}+\lambda x_{2}{ }^{2}+\mu x_{3}{ }^{2}=0$; man kommt alsdann leicht zu obigem Resultat.

Da dieses Netz drei Doppelgeraden enthält, so besteht das conjugirte Gewebe aus der Gesammtheit aller Kegelschnitte, welche diese Geraden berühren; die Hessiane des Netzes ist nach S. 240 identisch mit der Cayley'schen Curve des Gewebes, die Cayley'sche Curve des Netzes identisch mit der Hessiane des Gewebes. Mit Hilfe von (313) wäre man also gleichfalls zu obigem Resultat gelangt.

319. Die Cayley'sche Curve eines Netres von Kreisen besteht aus dem imaginären Kreispunktepare und dem gemeinsamen Potenzmittelpunkte.

Dass das imagginäre Kreispunktepaar der Cayley'schen Curve angehört, folgt sofort aus (307); dass der noch übrige Bestandtheil dieser Curve der Potenzmittelpunkt ist, zeigt (307) in Verbindung mit (71).

320. Die Hessiane eines Netzes von Kreisen besteht aus der unendlich fernen Geraden und einem Kreise, der alle Curven des Netzes rechtwinklig schneidet, dem sogenannten Orthogonalkreise des Netzes.

Dass die unendlich ferne Gerade dem Orte angehört, folgt sofort aus (306). Ein weiterer Bestandtheil der Hessiane ist nach (306) ein Kegelschnitt, der gleichfalls durch die zwei, allen Curven des Netzes gemeinsamen Punkte hindurchgeht, also in unserem Falle ein Kreis ist; er möge durch $O$ bezeichnet werden. Der in (306) durch $z$ bezeichnete Puukt ist jetzt der Potenzmittelpunkt aller Kreise des Netzes; 
seine Polare in Bezug auf den Kreis 0 fällt nach (306) mit der gemeinsamen Sehne aller Kreise des Netzes, also mit der unendlich fernen Geraden zusammen, woraus hervorgeht, dass der Potenzmittelpunkt $z$ aller Kreise des Netzes zugleich Mittelpunkt von $O$ ist. Jedenfalls ist also $O$ mit dem Orthogonalkreis concentrisch. Dass aber beide zusammenfallen, ergibt sich in folgender Weise: Die Hessiane ist nach III, S. 220 der geometrische Ort für die Spitzen aller in dem Netze enthaltenen Geradenpaare. Diese Spitzen rühren zum Theil von denjenigen ausartenden Kreisen her, welche durch die unendlich ferne Gerade und die Radicalaxe irgend zweier Kreise des Netzes gebildet werden (vgl. S. 59), und zwar erfüllen sie dann, wie bereits erwähnt, die unendlich ferne Gerade; zum Theil rühren sie her von den in dem Netze enthaltenen circularen Geradenpaaren (vgl. (224)) und erfüllen alsdann den Kreis. $O$, der somit ein Ort für die Grenzpunkte aller dem Netze angehörigen Kreisbüschel ist. Irgend ein Radius von $O$ ist daher, da der Mittelpunkt $z$ Potenzmittelpunkt ist, als Tangente an einen (allerdings ausartenden) Kreis des-Netzes zu betrachten. Mithin muss $O$ der Orthogonalkreis aller in dem Netze enthaltenen Kreise sein.

321.' Die Directorkreise aller Kegelschnitte eines Ge: webes bilden ein Netz.

Denn die Gleichung des Directorkreises irgend einer Curve

$$
\eta(u, u) \equiv \varkappa \varphi(u, u)+\lambda \psi(u, u)+\mu \chi(u, u)=0
$$

des Gewebes ist nach (29), S. 148 in den Parametern $x, \lambda, \mu$ linear.

322. Die Directorkreise aller einem Dreieck eingeschriebenen Kegelschnitte bilden ein Netz, dessen Hessiane aus der unendlich fernen Geraden und aus demjenigen Kreise besteht, für welchen das gegebene Dreieck Poldreieck ist. Nach (320) ist dieser Kreis zugleich Orthogonalkreis für alle Kreise des Netzes und nach (230) Ort: der Mittelpunkte aller eingeschriebenen gleichseitigen Hyperbeln.

Zu den dem Dreieck eingeschriebenen Kegelschnitten gehören als ausartende Gebilde die parweise genommenen Ecken des Dreiecks; die zugehörigen Directorkreise haben die Seiten des Dreiecks zu Durchmessern und schneiden sich zu je zweien in den Endpunkten einer Höhe. Die Höhen sind daher Radicalaxen für Kreise des Netzes, der Höhenschnittpunkt also der Mittelpunkt des Orthogonalkreises. Sind nun : $A, B, C$ die Ecken des gegebenen Dreiecks, $A_{1}, B_{1}, C_{1}$ die Fusspunkte der zugehörigen Höhen und ist $H$ der Höhenschnittpunkt, so 
bestehen nach der elementaren Definition der Potenz (vgl. auch (251)) die Relationen

$$
H A \cdot H A_{1}=H B \cdot H B_{1}=H C \cdot H C_{1} ;
$$

da ferner z. B. die Seite $B C$, auf welcher der Höhenfusspunkt $A_{1}$ gelegen ist, mit $H A_{1}$ einen rechten Winkel einschliesst, so sind $A$ und $A_{1}$, wie in der elementaren Planimetrie gezeigt wird, harmonische Pole in Bezug auf einen Kreis mit dem Mittelpunkte $H$ und dem Radius $\varrho=\sqrt{H A \cdot H A_{1}}$. Nun ist aber $H A \cdot H A_{1}$ auch gleich der Potenz des Punktes $H$ in Bezug auf diejenigen Kreise, welche $A A_{1}$ zur Schnittsehne haben und zu denen die ïber $A C$ und $A B$ als Durchmessern construirten Kreise gehören; daraus geht hervor, dass jener Kreis mit dem Mittelpunkte $I$ und dem Radius $\varrho=\sqrt{H A \cdot H} \overline{A_{1}}$ der Orthogonalkreis des Netzes der Directorkreise ist. Für ihn sind $A$ und $A_{1}$, analog $B$ und $B_{1}$, sowie $C$ und $C_{1}$ eonjugirte Pole, er hat daher in der That das gegebene Dreieck zum Poldreieck. Aus $\varrho^{2}=H A \cdot H A_{1}$ folgt wieder wie in (75), dass der Radius des Kreises, somit auch der Kreis selbst, nur dann reell ist, wenn $H A$ und $H A_{1}$ gleiche Richtung haben, d. h. wenn $H$ ausserhalb des Dreiccks $A B C$ liegt, wenn also $A B C$ ein stumpfwinkliges Dreieck ist.

Aus Vorstehendem folgt:

323. Die Tangenten, welche von dem Sehnittpunkte $H$ der Höhen eines Dreiecks an die über den Seiten als Durchmessern construirten Kreise gezogen werden können, sind von gleicher Länge, und zwar eben so lang wie die Tangenten, welche von $H$ an die Directorkreise der vier dem Dreieck eingeschriebenen Kreise gezogen werden könneı.

324. Die Entfernung $l$ des Scbnittpunlites der Höhen eines Dreiecks von dem Mittelpunkte irgend eines dem Dreieck eingeschriebenen Kegelschnitts ist gegeben durch $l^{2}=\varrho^{2}+\left(a^{2} \pm b^{2}\right)$, wobei $\varrho$ den Radius desjenigen Kreises bezeichnet, für welchen das gegebene Dreieck Poldreieck ist, während $a$ und $b$ die halben Hauptaxen des eingeschriebenen Kegelschnitts bedeuten. Dabei hat in $a^{2} \pm b^{2}$ das positive oder negative Vorzeichen zu stehen, je nachdem die Curve eine Ellipse oder Hyperbel ist.

Folgt aus (322) mit Rücksicht auf (229). Uebrigens erkennt man wieder sofort, dass die Mittelpunkte aller einem Dreieck eingeschriebenen gleichseitigen Hyperbeln $\left(a^{2}-b^{2}=0\right)$ auf demjenigen Kreise liegen, für welchen das Dreieck ein Poldreieck ist (vgl. 164).

325. Die Radicalaxe der Directorkreise von irgend zwei Kegel- 
schnitten, die einem Dreieck eingeschrieben sind, ist Directrix für eine dem Dreieck eingeschriebene Parabel.

Folgt aus (322) mit Rücksicht auf (237) und (238).

326. Die Directorkreise aller Kegelschnitte desjenigen Gewebes, dessen Curven ein gegebenes Dreiseit zum Poldreiseit haben, werden von dem umschriebenen Kreise des Dreiseits orthogonal geschnitten.

Wählt man als Dreiseit das Coordinatendreiseit, so ist

$$
x u_{1}^{2}+\lambda u_{2}^{2}+\mu u_{3}^{2}=0
$$

die Gleichung des Gewebes; zu den Curven des Gewebes gehören auch die doppelt zu zählenden Ecken des Dreiseits und die zugehörigen Directorkreise bestehen aus dem von der betreffenden Ecke aus gezogenen circularen Geradenpaare. Der Orthogonalkreis des Netzes der Directorkreise muss auch diese Geradenpaare rechtwinklig schneiden, daher nach den Ausführungen zu (320) durch deren Spitzen gehen, also durch die Ecken des oben erwähnten Poldreiseits.

32\%. Die Mittelpunkte aller gleichseitigen Hyperbeln, die sich in irgend einem Kegelschnittgewebe befinden, liegen auf einem Kreise. (Vgl. (169).)

Der Mittelpunkt jeder gleichseitigen Hyperbel kann nach (230) aufgefasst werden als der der Curve zugehörige Directorkreis (vgl. (11), S. 59); die Directorkreise des Gewebes bilden aber ein Netz, auf dessen Orthogonalkreis also die Mittelpunkte jener gleichseitigen Hyperbeln liegen müssen.

328. Die Directricen aller in irgend einem Kegelschnittgewebe enthaltenen Parabeln gehen durch den Mittelpunkt des Orthogonalkreises aller Directorkreise des Gewebes.

Jede Directrix ist, zusammen mit der unendlich fernen Geraden, als Directorkreis der zugehörigen Parabel anzusehen; alle Directorkreise bilden ein Kreisnetz, dessen Orthogonalkreis also auch die Directricen der Parabeln rechtwinklig schneidet, d. h. diese Directricen müssen durch den Mittelpunkt jenes Orthogonalkreises gehen. Vgl. auch (320) und den Schluss von (306).

329. Man nennt Polviereck ${ }^{1}$ ) einer Curve zweiter Classe ein solches, bei dem zwei Paare Gegenseiten conjugirte Polaren in Bezug auf die Curve sind. Nach einem Satze von Hesse ${ }^{2}$ ) besteht alsdann

1) Vgl. Reye: „Die Geometrie der Lage", 3. Aufl,, 1. Abth., 1886, S. 220.

2) Vgl. S. $220 \mathrm{f}$. 
auch das dritte Paar Gegenseiten aus coujugirten Polaren. Es gilt nun der weitere Satz ${ }^{1}$ ):

Die Verbindungslinien der Ecken $A, B, C$ eines Dreiecks mit den Polen $a, b, c$ der gegenüberliegenden Seiten gehen durch einen und denselben Punkt $D$, der mit $A, B$ und $C$ ein Polviereck bildet.

Sei nämlich zunächst $D$ der Schnittpunkt der Geraden $A a$ und $B b$, so ist $A B C D$ ein vollstïndiges Viereck, durch dessen Ecken die drei Geradenpaare gelegt sind: $B C$ und $A D, C A$ und $B D, A B$ und $C D$. Die Geraden des ersten Paares sind conjugirt, demn $A D$ geht nach Construction durch den Pol $a$ von $B C$; ebenso sind $C A$ und $B D$ conjugirt, denn $B D$ geht nach Construction durch den Pol $b$ von $C A$. Nach dem oben erwähnten Satze von Hesse sind alsdann auch die Geraden $A B$ und $C D$ des dritten Paares conjugirt, d. h. $C D$ geht durch den Pol $c$ von $A B$.

Man construirt demnach irgend ein Polviereck, indem man drei Ecken $A, B, C$ desselben willkürlich annimmt; durch sie ist die vierte Ecke $D$ bestimmt.

330. Wenn eine Curve zweiter Ordnung

$$
g \equiv \sum_{i}^{3} \sum_{1}^{3} y_{i k} x_{i} x_{k}=0,
$$

die zu einer Curve zweiter Classe $F=0$ conjugirt ist, durch drei Ecken $A, B, C$ eines Polvierecks $A B C D$ von $F=0$ hindurchgeht, so muss auch noch die vierte Ecke $D$ auf $g=0$ liegen $^{2}$ ).

Die Geradenpaare $B C$ und $A D$, sowie $C A$ und $B D$ bilden zwei Curven zweiter Ordnung, zu denen die vier doppelt zu zählenden Punkte $A=U_{\alpha}^{2}, B={U_{\beta}}^{2}, C=U_{\gamma}{ }^{2}, D=U_{\delta}^{2}$ conjugirt liegen. Nach $\S 26$ muss daher die Gleichung der Curve zweiter Classe $F=0$ von der Form sein $F(u, u)=a_{1} U_{\alpha}^{2}+a_{2} U_{\beta}^{2}+a_{3} U_{\gamma}{ }^{2}+a_{4} U_{\delta}{ }^{2}$, so dass die Bedingung der conjugirten Lage wird:

$$
[F, g] \equiv a_{1}\left[U_{\alpha}{ }^{2}, g\right]+a_{2}\left[U_{\beta}{ }^{2}, g\right]+a_{3}\left[U_{\gamma}{ }^{2}, g\right]+a_{4}\left[U_{\delta}{ }^{2}, g\right]=0,
$$

wobei $\left[U_{\alpha}^{2}, g\right]$ zur Abkürzung gesetzt ist für

$$
g_{11} \alpha_{1}^{2}+2 g_{12} \alpha_{1} \alpha_{2}+\cdots+g_{33} \alpha_{3}^{2}
$$

und analoges gilt von den übrigen $\left[U^{2}, g\right]$. Nun ist aber nach Voraussetzung $\left[U_{\alpha}^{2}, g\right]=\left[U_{\beta}{ }^{2}, g\right]=\left[U_{\gamma}^{2}, g\right]=0$, also muss, wenn nicht

1) Vgl. Reye a. a. O., S. 221, sowie die S. 220 citirte Arbeit von Hesse im Journal für die reine und angewandte Mathematik, Bd. 20, S. 302, Nr. 15. 1840.

2) Reye a. a. O. S. 225. 
$a_{4}$ bereits Null ist (d. h. wenn nicht die Ecken $A, B, C$ bereits ein Poldreieck von $F$ bilden) auch,

verschwinden.

$$
\left[U_{\delta}^{2}, g\right] \equiv g_{11} \delta_{1}{ }^{2}+2 g_{12} \delta_{1} \delta_{2}+\cdots+g_{33} \delta_{3}{ }^{2}
$$

\section{Beispiel eines Kegelschnittgewebes mit zwei Doppelpunkten.}

331. Man beweise nachstehenden Satz von Steiner ${ }^{1}$ ):

"Der Ort einer Geraden $G$, welche in zwei gegebenen festen Kreisen $A^{2}$ und $B^{2}$ solche Sehnen $s$ und $s_{1}$ bildet, deren Verhältniss irgend einen gegebenen Werth $k$ hat, so dass $s: s_{1}=k$, ist allemal irgend ein bestimmter Kegelschnitt $G^{2}$; und alle auf diese Weise bestimmten Kegelschnitte, wofern der Werth $k$ nach einander alle Grössen durchläuft, bilden eine Curvenschaar ${ }^{2}$ ), $B\left(G^{2}\right)$, mit vier (reellen oder imaginären) gemeinschaftlichen Tangenten $\left(R, R_{1}, S, S_{1}\right)$, and zwar gehören die gegebenen Kreise $A^{2}$ und $B^{2}$ selbst mit za diesem Büschel, nämlich sie entsprechen beziehlich den Werthen $k=0$ und $k=\infty$. Dem Werthe $k=0$ oder $s=s_{1}$ entspricht die Parabel $\mathfrak{P}^{2}$, welche den Mittelpunkt $M$ der Centrale der beiden Kreise $A^{2}, B^{2}$ zum Brennpunkt und die Radicalaxe $L$ zur Tangente im Scheitel hat. Dem Werthe $k=a: b^{3}$ ) entsprechen beide Aehnlichkeitspunkte $\mathfrak{x}$ und $\mathfrak{x}_{1}$, die zusammen eine specielle $G^{2}$ sind."

Der Mittelpunkt $y$ des Kreises $A^{2}$ hat von der Geraden $G\left(u_{x}=0\right)$ die Entfernung $\frac{u_{y}}{p_{y} \sqrt{\omega(u, u)}}$, daher ist $\left(\frac{s}{2}\right)^{2}=a^{2}-\frac{u_{y}{ }^{2}}{p_{y}{ }^{2} \cdot \omega(u, u)}$; für $z$ als Mittelpunkt des Kreises $B^{2}$ ist analog $\left(\frac{s_{1}}{2}\right)^{2}=b^{2}-\frac{u_{z}{ }^{2}}{p_{z}{ }^{2} \cdot \omega(u, u)}$. Setzt man $A^{2} \equiv a^{2} p_{y}{ }^{2} \omega(u, u)-u_{y}{ }^{2}, B^{2} \equiv b^{2} p_{z}{ }^{2} \omega(u, u)-u_{z}{ }^{2}$, so ist $p_{z}{ }^{2} A^{2}-k^{2} p_{y}{ }^{2} B^{2}=0$ die Gleichung des gesuchten Kegelschnitts. Für variabele Werthe von $k^{2}$ stellt dieselbe offenbar eine ganze Schaar von Kegelschnitten dar. Dem Werthe $k=a: b$ entspricht das Punktepaar $a^{2} p_{y}{ }^{2} u_{z}{ }^{2}-b^{2} p_{z}{ }^{2} u_{y}{ }^{2}=0$, welches aus den zwei Aehnlichkeitspunkten besteht, denn es liegt auf der Verbindungslinie der Mittelpunkte $y$ und $z$. Der Werth $z_{i}=1$ liefert den Kegelschnitt:

$$
\left(a^{2}-b^{2}\right) p_{y}{ }^{2} p_{z}{ }^{2} \omega(u, u)+p_{y}{ }^{2} u_{z}{ }^{2}-p_{z}{ }^{2} u_{y}{ }^{2}=0,
$$

1) „Ueber einige neue Bestimmungs-Arten der Curven zweiter Ordnung nebst daraus folgenden neuen Eigenschaften derselben Curven". Journal für die reine und angewandte Mathematik, Bd.45, S. 210, 1852, oder auch "Gesammelte Werke" Bd. 2, S. 467 .

2) Bei Steiner steht „einen Curvenbüschel".

3) $a$ und $b$ sind die Radien von $A^{2}$, bezw. $B^{2}$. 
dessen Brennpunktepaar $p_{y}{ }^{2} u_{z}{ }^{2}-p_{z}{ }^{2} u_{y}{ }^{2}=0$ nach (12) aus dem Mittelpunkt der Strecke $y z$ und aus dem unendlich fernen Punkt der Centrale besteht; dieser Kegelschnitt ist daher eine Parabel, deren Axe mit der Centrale zusammenfällt. Auch die Radicalaxe der beiden Kreise $A^{2}$ und $B^{2}$ ist eine Tangente der Parabel, denn für die Radicalaxe ist jedenfalls $s=s_{1}$, daher $k=1$; da diese Gerade auf der Centrale, also auf der Axe der Parabel normal steht, ist sie die Scheiteltangente. Bei beliebigem Werthe von $k$ hat der Mittelpunkt des Kegelschnitts die Gleichung $k^{2} p_{y} u_{z}-p_{z} u_{y}=0$; das eine Brennpunktepaar ist gegeben durch $k^{2} p_{y}{ }^{2} u_{z}{ }^{2}-p_{z}{ }^{2} u_{y}{ }^{2}=0$, liegt also harmonisch zu den Mittelpunkten der beiden Kreise. Allerdings stellt diese letzte Gleichung das reelle Brennpunktepaar nur dann dar, wenn $k^{2}$ positiv ist, $d$. h. wenn $s^{2}$ und $s_{1}{ }^{2}$ gleichzeitig positiv oder gleichzeitig negativ sind oder mit anderen Worten: wenn die Gerade, welche die Sehnen $s$ und $s_{1}$ bildet, die Kreise $A^{2}$ und $B^{2}$ beide reell oder beide imaginär trifft ${ }^{1}$ ).

Will man den Ort der Brennpunkte aller Curven der Schaar $p_{z}{ }^{2} A^{2}-l^{2} p_{y}{ }^{2} B^{2}=0$ haben, so ist diese Gleichung, ähnlich wie es bei einem einzigen Kegelschnitte schon S. 113 geschah, zusammenzustellen mit dem imaginären Kreispunktepaare $\omega(u, u)=0$. Man erhält hierdurch das Kegelschnittgewebe

$\varkappa\left\{a^{2} p_{y}{ }^{2} \omega(u, u)-u_{y}{ }^{2}\right\} p_{z}{ }^{2}-\lambda k^{2}\left\{b^{2} p_{z}{ }^{2} \omega(u, u)-u_{z}{ }^{2}\right\} p_{y}{ }^{2}+\mu \omega(u, u)=0$, das gleichbedeutend ist nit dem Gewebe $x u_{y}{ }^{2}+\lambda u_{z}{ }^{2}+\mu \omega(u, u)=0$. Die Punktepaare desselben, also die Brennpunlite der oben betrachteten Schaar, liegen nach VI, S. 228 auf der Cayley'schen Curve des Gewebes, welche nunmehr in die Verbindungslinie $y z$ und in denjenigen Kegelschnitt zerfällt, von welchem an das Punktepaar $y, z$ und an $\omega(\imath, u)=0$ harmonische Tangentenpaare gezogen werden können. (Dies folgt durch dualistische Uebertragung aus (317)). Die von einem Punkte des eben genannten Kegelschnitts nach $y$ und $z$ gezogenen Geraden mïssen also einen rechteu Winkel einschliessen, d. h. die Curve ist der über der Strecke $y z$ als Durchmesser errichtete Kreis.

Durch das Vorhergehende sind, mit Rücksicht auf die Gleichung $k^{2} p_{y} u_{z}-p_{z} u_{y}=0$ des Mittelpunktes von $G^{2}$, die nachstehenden Sätze ${ }^{2}$ ) bewiesen:

1) Die Grösse $k^{2}$ ist offenbar negativ, wenn der eine Kreis in reellen, der andere in imaginären Punkten getroffen wird. In ähnlicher Weise gilt die am Schlusise von (193) für den Fall eines positiven $\lambda^{2}$ abgeleitete Gleichung auch dann noch, wenn $\lambda^{2}$ negativ ist, $\mathrm{d}$. h. wenn die Potenz des Punktes $x$ negativ wird.

2) Steiner a. a. O. In dem angeführten Satze sind die Mittelpunkte der zwei gegebenen Kreise mit $A$ und $B$ bezeichnet; ihre Verbindungslinie mit $X$. 
„Die Mittelpunkte der Ortscurven, $B\left(G^{2}\right)$, liegen sämmtlich in der Axe $X$, auf welche zugleich auch je eine Axe von jeder Curve fällt. $O b$ die erste oder zweite Axe der Curve auf $X$ fällt, hängt davon ab, ob ihr Mittelpunkt jenseits der Strecke $A B$, oder ob er in dieser Strecke liegt. Dadurch scheiden sich die Curven in zwei $\mathrm{Ab}$ theilungen, etwa $\operatorname{Gr}\left(G_{1}{ }^{2}\right)$ und $\operatorname{Gr}\left(G_{2}{ }^{2}\right)$. In Hinsicht der Brennpunkte dieser beiden Gruppen hat es folgende Bewandtniss:

Die Brennpunkte der $\operatorname{Gr}\left(G_{1}{ }^{2}\right)$ liegen in der Axe $X$ und jedes Paar Brennpunkte $f$ und $f_{1}$ ist zu den Punkten $A$ und $B$ zugeordnet harmonisch. Dagegen liegen die Brennpunkte der $\operatorname{Gr}\left(G_{2}{ }^{2}\right)$ in dem Kreise $M_{0}^{2}$, welcher die Strecke $A B=2 c$ zum Durchmesser hat, so dass jedes Paar Brennpunkte zugleich die Endpunkte einer zu diesem Durchmesser senkrechten Sehne des Kreises sind."

332. „Die Tangenten jedes Kegelschnittes $G^{2}$, welcher mit zwei Kreisen $A^{2}$ und $B^{2}$ vier reelle oder imaginäre Tangenten gemeinsam hat, bilden in diesen Kreisen solche Sehnen $s$ und $s_{1}$, deren Verhältniss constant ist, $d . h$. für alle Tangenten denselben bestimmten Werth k hat". ${ }^{1}$ )

Folgt aus der Gleichung des Kegelschnittes $G^{2}$, die von der Form sein muss $A^{2}-\lambda B^{2}=0$; man hat nur in diese Gleichung auf Grund der schon in (331) benutzten Formeln die Grössen $s^{2}$ und $s_{1}^{2}$ einzuführen.

Ueber Kegelschnitte, die zwei andere doppelt berühren.

(Netze und Gewebe mit zwei Doppelgeraden, bezw. Doppelpunkten.)

Soll ein Kegelschnitt $h(x, x)=0$ zwei andere $f(x, x)=0$ und $g(x, x)=0$ doppelt berühren, so muss es gerade Linien $v_{x}=0$ und $w_{x}=0$ geben der Art, dass

$$
h(x, x) \equiv f(x, x)+v_{x}{ }^{2} \text { und } h(x, x) \equiv \lambda g(x, x)+w_{x}{ }^{2},
$$

d. h. man hätte $f-\lambda g \equiv w_{x}^{2}-v_{x}{ }^{2} \equiv\left(w_{x}+v_{x}\right)\left(w_{x}-v_{x}\right)$; es müssen somit die Geraden $v$ und $w$ durch die Spitzen eines der drei Geradenpaare gehen, welche in dem Büschel $f-\lambda g=0$ enthalten sind, und ausserdem müssen $v$ und $w$ zu dem betreffenden Geradenpaare harmonisch liegen. Den drei Wurzeln $\lambda_{1}, \lambda_{2}, \lambda_{3}$ der kubischen Gleichung (4), S. 132 entsprechend gibt es somit drei Systeme von Berührungscurven. Mit Rücksicht darauf, dass nach (17), S. 138 die Spitzen der

1) Steiner a. a. 0 . 
Drei Systeme Kegelschnitte, die zwei gegebene Kegelschn. doppelt berühren. 397

drei in $f-\lambda g=0$ enthaltenen Geradenpaare für jede Curve des Büschels ein Poldreieck bilden, folgt der Satz von Steiner ${ }^{1}$ ):

333. „Die gesammten Kegelschnitte $C^{2}$, welche beide gegebenen Ellipsen $\left.{ }^{2}\right) A^{2}$ und $B^{2}$ doppelt berühren, zerfallen vermöge ihrer Beziehung zu den drei Polen $x, y$ und $z$ in drei verschiedene Schaaren $S\left(C_{x}{ }^{2}\right), S\left(C_{y}{ }^{2}\right)$ und $S\left(C_{z}{ }^{2}\right)$, welche sich jedoch im allgemeinen gleich verhalten und gleiche Eigenschaften haben, so dass wir der Kürze halber uur von der einen Schaar, etwa von $S\left(C_{x}{ }^{2}\right)$ zu sprechen brauchen."

„Berührt eine Curve $C_{x}{ }^{2}$ die Ellipse $A^{2}$ in den Punkten $p$ und $p_{1}$ und die Ellipse $B^{2}$ in den Punkten $q$ und $q_{1}$, so gehen die Berührungssebnen $p p_{1}$ und $q q_{1}$ durch den Pol $x$ und sind allemal zu den Gegenseiten $\mathfrak{Z}$ und $\mathfrak{X}_{1}$ zugeordnet harmonisch."

Auch die Umkehrung dieses Satzes wird von Stein er aufgestellt:

334. „Zieht man durch den Pol $x$ irgend zwei zu den Seiten $\mathfrak{t}$ und $\mathfrak{X}_{1}$ zugeordnete harmonische Gerade, etwa $G$ und $H$, so schneiden sie die Ellipsen $A^{2}$ und $B^{2}$ beziehlich in solchen Punkten $p p_{1}$ und $q q_{1}$, in welchen dieselben von einer Curve $C_{x}{ }^{2}$ berührt werden; und fermer schneiden sie verwechselt, $H$ die $A^{2}$ und $G$ die $B^{2}$, in solchen Punkten $p^{0}, p_{\mathbf{1}}{ }^{0}$ und $q^{0}, q_{1}{ }^{0}$, in welchen $A^{2}$ und $B^{2}$ von einer anderen Curve $C_{x}{ }^{2}$ berührt werden."

Ist nämlich $f-\lambda_{1} g=\mathfrak{X} \cdot \mathfrak{X}_{1}$, so wird anch gleichzeitig für beliebige Werthe $\mu$ und $\nu$ :

oder man hat

$$
f-\lambda_{1} g=\frac{1}{4 \mu \nu}\left\{\left(\mu \mathfrak{X}+\nu \mathfrak{X}_{1}\right)^{2}-\left(\mu \mathfrak{X}-\nu \mathfrak{X}_{1}\right)^{2}\right\},
$$

und

$$
4 \mu \nu f+\left(\mu \mathfrak{X}-\nu \mathfrak{X}_{1}\right)^{2} \equiv 4 \mu \nu \lambda_{1} g+\left(\mu \mathfrak{X}+\nu \mathfrak{X}_{1}\right)^{2}
$$

$$
4 \mu \nu f-\left(\mu \mathfrak{X}+\nu \mathfrak{X}_{1}\right)^{2} \equiv 4 \mu \nu \lambda_{1} g-\left(\mu \mathfrak{X}-\nu \mathfrak{X}_{1}\right)^{2} .
$$

Hierbei sind $\mu \mathfrak{X}-\nu \mathfrak{X}_{1}=0$ und $\mu \mathfrak{X}+\nu \mathfrak{X}_{1}=0$ die oben mit $G$ und $H$

1) „Allgemeine Betrachtung über einander doppelt berührende Kegelschnitte“, Journal für die reine und angewandte Mathematik, Bd. 45, S. 213, 1852, oder "Gesammelte Werke", Bd. 2, S. 472. In dieser AbhandIung finden sich auch die übrigen Sätze, die im Folgenden zwischen Anführungszeichen gesetzt sind. Die wahre Quelle, aus der Steiner seine Sätze geschöpft hat, dürfte jedoch nicht die Theorie der Kegelschnittnetze sein, sondern, wie die Ausführungen des Herrn W. Fiedler treffend zeigen, die Methode der descriptiven Geometrie. Vergleiche W. Fiedler: „Ueber die Durchdringung gleichseitiger Rotationshyperboloide von parallelen Axen". Acta Mathematica, Bd. 5, S. 331-408, 1884.

2) In Bezug auf den hier genau citirten Wortlaut Steiner's möge bemerkt werden, dass nach der Einleitung zu dieser Nummer im Folgenden $((333)-(342))$ statt der Ellipsen $A^{2}$ and $B^{2}$ irgend zwei Kegelschnitte gesetzt werden können. 
bezeichneten Geraden. Es bedarf wohl keiner näheren Erläuterung, wie mit den beiden letzten Identitäten die Steiner'sche Behauptung er wiesen ist.

Ist $x$ die Ecke des gemeinsamen Poldreiecks der Kegelschnitte $f=0$ und $g=0$, in der sich $\mathfrak{X}$ und $\mathfrak{X}_{1}$ schneiden, und ist $X$ die Gegenseite dieser Ecke, so zeigt die Form $4 \mu \nu f+\left(\mu \mathfrak{X}-\nu \mathfrak{X}_{1}\right)^{2}=0$ der Gleichung irgend eines der doppelt berührenden Kegelschnitte die Richtigkeit des Satzes:

335. „Alle Curven $C_{x}{ }^{2}$ haben gemeinschaftlich $x$ und $X$ zu Pol und Polaren."

Auch kaun man zeigen:

336. „Von den gemeinschaftlichen Secanten je zweier Curven $C_{x}{ }^{2}$ geht immer ein Paar, etwa $G$ und $H$, durch den Pol $x$, und sie sind allemal zu $\mathfrak{X}$ und $\mathfrak{X}_{1}$ zugeordnet harmonisch."

Zum Beweise werde gesetzt

$$
\psi(\mu, \nu) \equiv 4 \mu \nu f+\left(\mu \mathfrak{X}-\nu \mathfrak{X}_{1}\right)^{2} \equiv 4 \mu \nu \lambda_{1} g+\left(\mu \mathfrak{X}+\nu \mathfrak{X}_{1}\right)^{2} ;
$$

sind $\mu_{1}, \nu_{1}$ willkürliche Parameter, so ist alsdann

$$
\mu_{1} \nu_{1} \cdot \psi(\mu, \nu)-\mu \nu \cdot \psi\left(\mu_{1}, \nu_{1}\right) \equiv\left(\mu \nu_{1}-\mu_{1} \nu\right)\left(\mu \mu_{1} \mathfrak{X}^{2}-\nu \nu_{1} \mathfrak{X}_{1}^{2}\right),
$$

und zufolge dieser Identität stellt jedenfalls $\mu \mu_{1} \mathfrak{t}^{2}-\nu \nu_{1} \mathfrak{X}_{1}{ }^{2}=0$ ein Paar gemeinschaftlicher Secanten der Kegelschnitte $\psi(\mu, v)=0$ und $\psi\left(\mu_{1}, v_{1}\right)=0$ dar, und zwar geht dieses Paar durch den Schnittpunkt $x$ von $\mathfrak{X}$ mit $\mathfrak{X}_{1}$ und liegt harmonisch zu dem Paare $\mathfrak{X}, \mathfrak{X}_{1}$.

337. „Die acht Berührungspunkte je zweier Curven $C_{x}{ }^{2}$ mit den Ellipsen $A^{2}$ und $B^{2}$ liegen jedesmal in irgend einem Kegelschnitt $D^{2}$."

Zunächst werde als Ablürzung eingeführt $\mathfrak{X}^{2}=A, \mathfrak{X}_{1}{ }^{2}=\Gamma$, $2 f-\mathfrak{X} \mathfrak{X}_{1} \equiv 2 \lambda_{1} g+\mathfrak{X} \mathfrak{X}_{1} \equiv \mathrm{B}$, wodurch man erhält

$$
\psi(\mu, \nu) \equiv \mu^{2} \mathrm{~A}+2 \mu \nu \mathrm{B}+\nu^{2} \Gamma \text {. }
$$

Ferner gilt nun die Identität

$$
\begin{gathered}
\left(\mathrm{A} \mu^{2}+2 \mathrm{~B} \mu \nu+\Gamma \nu^{2}\right)\left(\mathrm{A} \mu_{1}{ }^{2}+2 \mathrm{~B} \mu_{1} \nu_{1}+\Gamma \nu_{1}{ }^{2}\right) \\
-\left\{(\mathrm{A} \mu+\mathrm{B} \nu) \mu_{1}+(\mathrm{B} \mu+\Gamma \nu) \nu_{1}\right\}^{2} \equiv\left(\mathrm{A} \Gamma-\mathrm{B}^{2}\right)\left(\mu \nu_{1}-\mu_{1} \nu\right)^{2} \\
\equiv\left\{\left(f-\lambda_{1} g\right)^{2}-\left(f+\lambda_{1} g\right)^{2}\right\}\left(\mu \nu_{1}-\mu_{1} \nu\right)^{2} \equiv-4 \lambda_{1}\left(\mu \nu_{1}-\mu_{1} \nu\right)^{2} f g,
\end{gathered}
$$

daher

$$
\left\{(\mathrm{A} \mu+\mathrm{B} v) \mu_{1}+(\mathrm{B} \mu+\Gamma \nu) \nu_{1}\right\}^{2}
$$

$\equiv\left(\mathrm{A} \mu^{2}+2 \mathrm{~B} \mu \nu+\Gamma \nu^{2}\right)\left(\mathrm{A} \mu_{1}{ }^{2}+2 \mathrm{~B} \mu_{1} \nu_{1}+\Gamma \nu_{1}{ }^{2}\right)+4 \lambda_{1}\left(\mu \nu_{1}-\mu_{1} \nu\right)^{2} f g$.

Diese Identität zeigt nicht nur, dass die acht Berührungspunkte der Kegelschnitte $\psi(\mu, \nu)=0$ und $\psi\left(\mu_{1}, \nu_{1}\right)=0$ mit $f=0$ und $g=0$ 
auf dem Kegelschnitt $(\mathrm{A} \mu+\mathrm{B} \nu) \mu_{1}+(\mathrm{B} \mu+r \nu) \nu_{1}=0$ liegen, sondern sie liefert auch (bei gleicher Bezeichnung wie in (333)) die Umkehrung:

338. „Legt man durch die vier Berührungspunkte $p$ und $p_{1}$, $q$ und $q_{1}$ irgend einen willkürlichen Kegelschnitt $D^{2}$, so schneidet er die gegebenen Curven $A^{2}$ und $B^{2}$ in vier solchen neuen Punkten $p^{0}$ und $p_{1}{ }^{0}, q^{0}$ und $q_{1}{ }^{0}$, in welchen dieselben von einer anderen Curve $C_{x}{ }^{2}$ berührt werden." 1 )

Sind $r, s, t, u$ die vier Schnittpunkte der Kegelschnitte $f=0$ und $g=0$, so gilt ferner der Satz:

339. „Jede vier Berührungspunkte $p, p_{1}, q, q_{1}$ liegen einerseits mit den Ecken $r$ und $s$ in einem Kegelschnitte, etwa $M I^{2}$, und andererseits mit den Ecken $t$ und $u$ in einem Kegelschnitte $M M_{1}^{2}{ }^{2} "$

- Dieser Satz ergibt sich mit Hilfe der Identität

$$
\begin{aligned}
\left(\mu^{2} \mathrm{~A}+2 \mu \nu \mathrm{B}+v^{2} \Gamma\right) \nu_{1}{ }^{2} & -\left(\mu_{1}{ }^{2} \mathrm{~A}+2 \mu_{1} \nu_{1} \mathrm{~B}+\nu_{1}{ }^{2} \Gamma\right) \nu^{2} \\
& \equiv\left(\mu \nu_{1}-\mu_{1} \nu\right)\left\{\left(\mu \nu_{1}+\mu_{1} \nu\right) \mathrm{A}+2 \nu \nu_{1} \mathrm{~B}\right\}
\end{aligned}
$$

vermöge deren rechts $\Gamma$ eliminirt ist, und ganz analog könnte mau verfahren, um $\mathrm{A}$ zu eliminiren. Die Gleichung $\left(\mu \nu_{1}+\mu_{1} v\right) \mathrm{A}+2 v \nu_{1} \mathrm{~B}=0$ des Kegelschnitts $M^{2}$ zeigt nun, dass derselbe durch die Schnittpunkte von $\mathfrak{X}$ mit $\mathrm{B}=0$, also durch die Punkte $r$ und $s$ geht; andererseits liegen, wie die linke Seite der Identität zeigt, auf $M I^{2}$ auch die Schnittpunkte zweier Kegelschnitte $\psi(\mu, \nu)=0$ und $\psi\left(\mu_{1}, \nu_{1}\right)=0$, die $f$ und $g$ doppelt berühren.

Da überdies $\mathrm{A}=\mathfrak{X}^{2}, \mathrm{~B}=2 f-\mathfrak{X} \mathfrak{X}_{1}=f+\lambda_{1} g$, so berühren sich auch alle Curven $M^{2}$ in den Punkten $r$ und $s$, und zwar sind die gemeinschaftlichen Berührungstangenten $\Re$ und $\mathfrak{S}$ die Tangenten des Kegelschnitts $f+\lambda_{1} g=0$ in seinen Schnittpunkten mit $\mathfrak{X}=0 .{ }^{2}$ )

Man hat daher den Satz:

340. „Die gesammten Kegelschnitte $M^{2}$ berühren einander in den Punkten $r$ und $s$, so dass sie daselbst gemeinschaftliche Berührungstangenten, etwa $\Re$ und $\subseteq$ haben mit der gemeinschaftlichen Berührungssehue $r s=\mathfrak{X}$ und somit einen speciellen Curven-Büschel, $B\left(M^{2}\right)$, bilden." Analoges gilt natürlich von den Kegelschnitten $M_{1}^{2}$.

1).Die Willkürlichkeit des Kegelschnitts $D^{2}$ tritt in seiner Gleichung dadurch zu Tage, dass sie den Parameter $\mu_{1}: v_{1}$ enthält. vgl. (218).

2) Ueber die geometrische Bedeutung des Kegelschnitts $f+\lambda_{1} g=0$ 
Ein weiterer Satz benutzt das Vierseit, welches durch die vier gemeinsamen Tangenten $R, S, T, U$ der Kegelschnitte $f=0$ und $g=0$ gebildet wird; er lautet:

341. „Werden zwischen je zwei Paar zusammengehöriger Berührungspunkte $p$ und $p_{1}, q$ und $q_{1}$ die vier Wechselsehnen $p q, p q_{1}$, $p_{1} q$ und $p_{1} q_{1}$ gezogen, so berühren dieselben insgesammt einen bestimmten Kegelschnitt, etwa $X^{2}$, welcher dem Vierseit $R S T U$ eingeschrieben ist und auch die zwei Gegenseiten $\mathfrak{X}$ und $\mathfrak{X}_{1}$ berührt (indem die letzteren, sowie die Tangenten $R, S, T, U$ specielle Wechselsehnen sind)." Die Gleichung von $X^{2}$ ist $F(u, u)-\lambda_{1}{ }^{2} G(u, u)=0$.

Die Gleichungen der Kegelschnitte durch zwei Paare Berührungspunkte sind nach (337) von der Form $x A+\lambda B+\mu \Gamma=0$; diese Curven bilden daher ein specielles Netz, and die oben genannten Wechselsehnen sind Geraden, die dem Netze angehören. Nach VI, S. 222 umhüllen dieselben die Cayley'sche Curve des Netzes, welch letztere im vorliegenden Falle, da $A=\mathfrak{t}^{2}=0$ und $\Gamma=\mathfrak{X}_{1}{ }^{2}=0$ Doppelgeradell darstellen, wach (317) aus dem Schnittpunkte $x$ dieser beiden Geraden und aus dem harmonischen Kegelschnitte zu irgend einer Curve des Netzes, etwa $\mathrm{B}=f+\lambda_{1} g$, und zu $\mathfrak{X} \mathfrak{X}_{1}=f-\lambda_{1} g$ besteht. Die Gleichung des harmonischen Kegelschnitts wird

$$
F(u, u)-\lambda_{1}^{2} G(u, u)=0
$$

daher sind die Geraden des speciellen Netzes $x A+\lambda B+\mu \Gamma=0$ zum Theil Tangenten von $F-\lambda_{1}{ }^{2} G=0$, zum Theil gehen sie durch den Punkt $x$. Die Curve $F-\lambda_{1}{ }^{2} G=0$ berührt, wie ihre Gleichung zeigt, die vier gemeinsamen Tangenten $R, S, T, U$ von $f=0$ und $g=0$, und als harmonischer Kegelschnitt zu $f-\lambda_{1} g=0$ und $f+\lambda_{1} g=0$ berührt sie nach S. 144 auch die in deren Schnittpunkten, den Grundpunkten des Büschels, gezogenen Tangenten von $f+\lambda_{1} g=0$; ferner berührt sie das Geradenpaar $f-\lambda_{1} g=0$.

Die Hessiane des Netzes doppelt berührender Kegelschnitte $S\left(C_{x}{ }^{2}\right)$ besteht nach (316) aus dem Geradenpaare $\mathfrak{X}, \mathfrak{X}_{1}$ und aus der in Bezug auf irgend eine Curve des Netzes genommenen Polaren des Schnittpunktes $x$ von $\mathfrak{X}$ und $\mathfrak{X}_{1}$. Mit Hilfe von XVII, S. 232 und unter Riicksicht darauf, dass $F-\lambda_{1}{ }^{2} G=0$ von Wechselsehnen berührt wird und einen Bestandtheil der Cayley'schen Curve bildet, folgt:

342. Der Berührungspunkt jeder Wechselsehne mit dem Kegelschnitt $X^{2}$ liegt harmonisch zu den Schnittpunkten dieser Wechselsehne mit dem Geradenpaare $\mathfrak{X}, \mathfrak{X}_{1}$ und mit der Polaren des Punktes $x$ in Bezug auf irgend einen der doppelt berührenden Kegelschnitte $C_{x}{ }^{2}$. 
Nach diesem allgemeinen Satze berichtigen sich einige Angaben Steiner's, die bereits von Herrn Schur als unrichtig erkannt worden $\left.\operatorname{sind}^{1}\right)$.

Aus den Sätzen (333) bis (342) folgen leicht die entsprechenden für. diejenigen Kegelschnitte, welche speciell zwei Kreise doppelt berühren. Vor Allem ist hier aber zu bemerken, dass von den drei Systemen $S\left(C_{x}{ }^{2}\right), S\left(C_{y}{ }^{2}\right), S\left(C_{z}{ }^{2}\right)$ eines im Vergleich mit den beiden anderen besonders ausgezeichnet ist, indem eine Ecke $x$ des gemeinsamen Poldreiecks der beiden Kreise $f=0$ und $g=0$ nach (224) in Richtung der Radicalaxe im Unendlichen liegt, während die beiden anderen Ecken $y$ und $z$ zugleich die Grenzpunkte des Büschels von Kreisen $f-\lambda g=0$ sind. Wir wollen der Kürze halber auf die den früheren Sätzen entsprechenden jetzt nicht näher eingehen, sondern verweisen auf die betreffende Abhandlung von Steiner. ${ }^{2}$ ). Nur die nach (341) von den Wechselsehnen umhüllte Curve, sowie den Ort der Brennpunkte aller Kegelschnitte, welche die zwei Kreise doppelt berühren, wollen wir noch etwas näher betrachten, und zwar für dasjenige System doppelt berührender Kegelschnitte, dessen Berührungssehnen nach (333) durch den im Unendlichen gelegenen Pol $x$ gehen, also zur Radicalaxe parallel sind und von ihr gleich weit abstehen.

343. Es seien

$$
f \equiv\left(\begin{array}{ll}
y & x \\
y & x
\end{array}\right)_{\omega_{i k}}-r^{2} \tau p_{y}{ }^{2} p_{x}{ }^{2}=0 \quad \text { und } \quad g \equiv\left(\begin{array}{ll}
z & x \\
z & x
\end{array}\right)_{\omega_{i k}}-s^{2} \tau p_{z}{ }^{2} p_{x}{ }^{2}=0
$$

die Gleichungen der zwei Kreise mit den Mittelpunkten y, bezw. $z$ und den Radien $r$, bezw. $s$; alsdann ist nach (180) und (182)

$$
p_{z}^{2} f-p_{y}{ }^{2} g=0
$$

der Ausdruck für das Product aus der Radicalaxe und die unendlich ferne Gerade; die bisher mit $\lambda_{1}$ bezeichnete Grösse besitzt also den Werth $p_{y}{ }^{2}: p_{z}{ }^{2}$. Ferner wird

$F(u, u)=\tau p_{y}{ }^{2}\left[u_{y}{ }^{2}-r^{2} p_{y}{ }^{2} \omega(u, u)\right], G(u, u)=\tau p_{z}{ }^{2}\left[u_{z}{ }^{2}-s^{2} p_{z}{ }^{2} \omega(u, u)\right]$; für den nach (341) von den Wechselsehnen umhüllten Kegelschnitt $F-\lambda_{1}{ }^{2} G=0$ erhält man somit die Gleichung

$$
p_{z}{ }^{2} u_{y}{ }^{2}-p_{y}{ }^{2} u_{z}{ }^{2}-p_{y}{ }^{2} p_{z}{ }^{2}\left(r^{2}-s^{2}\right) \omega(u, u)=0 .
$$

1) Vgl. Steiner's gesammelte Werke, Bd. 2, S. 740.

2) „Ueber einige neue Bestimmungs-Arten der Curven zweiter Ordnung nebst daraus folgenden neuen Eigenschaften derselben Curven." Journal für die reine und angewandte Mathematik, Bd. 45, S. $196 \mathrm{ff}$., 1852, oder "Gesammelte Werke", Bd. 2, S. $454 \mathrm{ff}$. 
Man erkennt sofort, dass die Brennpunkte dieser Curve gegeben sind durch $p_{z} u_{y} \pm p_{y} u_{z}=0$, wobei $p_{z} u_{y}+p_{y} u_{z}=0$ nach (12) den Mittelpunkt der Strecke $y z$ darstellt, während $p_{z} u_{y}-p_{y} u_{z}=0$ auf der gemeinsamen Centrale der zwei gegebenen Kreise im Unendlichen liegt.

Hieraus geht hervor, dass der Ort der Wechselsehnen eine Parabel $X^{2}$ ist mit der Centrale der zwei Kreise als Axe und mit der Radicalaxe als Scheiteltangente; übrigens würde dies rein geometrisch auch daraus folgen, dass die Curve $F-\lambda_{1}{ }^{2} G=0$ von den Geraden $\mathfrak{X}$ und $\mathfrak{X}_{1}$ des Paares $f-\lambda_{1} g=0$ berührt wird.

Aus (342) ergibt sich ferner:

344. Der Schnittpunkt $\mathfrak{m}$ einer Wechselsehne $w$ mit der Radicalaxe $\mathfrak{X}$ liegt in der Mitte zwischen dem Berührungspunkte der Parabel $X^{2}$ mit dieser Wechselsehne und dem Schnittpunkte von $w$ mit der Centrale. Da die Radicalaxe zugleich Scheiteltangente der Parabel $X^{2}$ ist und der Mittelpunkt $M$ der Strecke $y z$ den Brennpunkt bildet, so folgt mit Rücksicht auf (23), S. 186: Die Normale, welche vom Punkte $M$ auf eine Tangente der Parabel $X^{2}$ gefällt wird, trifft dieselbe in dem mit $\mathfrak{n}$ bezeichneten Punkte; es ist klar, wie man hiernach umgekehrt beliebig viele Tangenten von $X^{2}$ construiren kann. Da der Kegelschnitt $X^{2}$ mit den zwei Kreisen $f=0$ und $g=0$ nach (341) vier Tangenten gemeinsam hat und eine Parabel ist, erhält man mit Hilfe von (332) und (331) den Satz:

345. „Jede Wechselsehne bildet in den gegebenen Kreisen gleiche Sehnen; d. h. schneidet z. B. die Gerade $p q$ die Kreise $A^{2}$ und $B^{2}$ zum zweiten Mal, etwa in den Punkten $p^{0}$ und $q^{0}$, so ist stets die Sehne $\left.p p^{0}=q q^{0 .} \cdot{ }^{* 1}\right)$

Betrachten wir nun noch die Brennpunkte derjenigen Kegelschnitte, welche die zwei Kreise $A^{2}, B^{2}$ doppelt berühren. Geht man von den Gleichungen der Kreise $F(u, u)=0, G(u, u)=0$ in Liniencoordinaten aus, so ist die Ableitung der Gleichung des die beiden Kreise doppelt berührenden Kegelschnittsystems genau analog zu dem früher (S. $398 \mathrm{ff}$.) betrachteten allgemeinen Falle bei Punktcoordinaten. An Stelle des Geradenpaares $f-\lambda_{1} g=0$ tritt jetzt ein Punktepaar $F-\lambda^{\prime} G=0$, das auf der Centrale $y z$ liegen soll. Aus den oben angegebenen Ausdrücken für $F$ und $G$ findet man leicht $\lambda^{\prime}=r^{2} p_{y}{ }^{4}: s^{2} p_{z}{ }^{4}$ so dass

$s^{2} p_{z}{ }^{4} F(u, u)-r^{2} p_{y}{ }^{4} G(u, u)=0 \quad$ oder $\quad \tau p_{y}{ }^{2} p_{z}{ }^{2}\left(s^{2} p_{z}{ }^{2} u_{y}{ }^{2}-r^{2} p_{y}{ }^{2} u_{z}{ }^{2}\right)=0$

1) Steiner a. a. 0 . 
dieses Punktepaar darstellt; es gehört der Schaar von Kreisen $F-\lambda G=0$ an und besteht aus dem Schnittpunkte der zwei äusseren, andrerseits dem der zwei inneren Tangenten der Kreise $F=0, G=0$, d. l. $F-\lambda^{\prime} G=0$ repräsentirt die zwei Aehnlichkeitspunkte von $A^{2}$ und $B^{2}$. Wir wollen für dieselben als Abkürzung einführen

$$
u_{m} \equiv\left(s p_{z} u_{y}+r p_{y} u_{z}\right)=0, \quad u_{n} \equiv\left(s p_{z} u_{y}-r p_{y} u_{z}\right)=0,
$$

so dass $F-\lambda^{\prime} G$ gleichbedeutend wird mit $\tau p_{y}{ }^{2} p_{z}{ }^{2} u_{m} \cdot u_{n}$.

Wir führen nun mit Steiner die Definitionen ein: „Die Berührungstangenten der Curve $C^{2}$ und der Kreise $A^{2}$ und $B^{2}$, d. h. diejenigen Tangenten, welche in den Punkten $p$ und $p_{1}, q$ und $q_{1}$ zugleich die Curve und die respectiven Kreise berühren, sollen $P$ und $P_{1}, Q$ und $Q_{1}$ beissen."

„Der Schnitt $P P_{1}$, d. h. von $P$ mit $P_{1}$, heisse $\mathfrak{p}$, und der Schnitt $Q Q_{1}$ heisse $\mathfrak{p}_{1}$; ferner mögen die Wechselschnitte $P Q$ und $P_{1} Q_{1}, P Q_{1}$ und $P_{1} Q$ beziehlich durch $q$ und $q_{1}, \mathfrak{r}$ und $\mathfrak{r}_{1}$ bezeichnet werden, so dass also $\mathfrak{p}$ und $\mathfrak{p}_{1}, \mathfrak{q}$ und $\mathfrak{q}_{1}, \mathfrak{r}$ und $\mathfrak{r}_{1}$ die Gegenecken des vollständigen Vierseits $P P_{1} Q Q_{1}$ sind."

Alsdann folgt dualistisch zu (341) der Ort für Wechselschnitte, indem man zu $F(u, u)$ und $G(u, u)$ die Ausdrücke in Punkteoordinaten $\mathfrak{f}(x, x)$ und $\mathfrak{g}(x, x)$ herstellt und alsdann $\mathfrak{f}-\lambda^{\prime 2} \mathfrak{g}=0$ bildet. Natürlich sind $f(x, x)$ und $g(x, x)$ den früheren $f(x, x)$ und $g(x, x)$ proportional, nämlich $\mathfrak{f}(x, x)=-\tau^{2} r^{2} p_{y}{ }^{6} f(x, x), g(x, x)=-\tau^{2} s^{2} p_{z}{ }^{6} g(x, x)$, daher wird $\overline{\mathrm{i}}-\lambda^{\prime 2} \mathrm{~g}=0$ gleichbedeutend mit

$$
\tau^{2} r^{2} s^{2} p_{y}{ }^{6} p_{z}{ }^{6}\left\{r^{2} p_{y}{ }^{2}\left(\begin{array}{ll}
z & x \\
z & x
\end{array}\right)_{\omega_{i k}}-s^{2} p_{z}{ }^{2}\left(\begin{array}{ll}
y & x \\
y & x
\end{array}\right)_{\omega_{i k}}\right\}=0,
$$

und diese Gleichung repräsentirt nach (181) den über der Verbindungslinie der beiden Aehnlichkeitspunkte als Durchmesser construirten „Aehnlichkeitskreis". Wie nach (341) die Wechselsehnen zum Theil Tangenten von $F-\lambda_{1}{ }^{2} G=0$ sind, zum Theil durch den Punkt $x$ gehen, so liegen jetzt die Wechselschuitte $\mathfrak{p}, p_{1}$ auf der Centrale $X$, die Wechselschuitte $\mathfrak{q}, \mathfrak{q}_{\mathfrak{l}}, \mathfrak{r}, \mathfrak{r}_{1}$ auf dem Aehnlichkeitskreise $\mathfrak{f}-\lambda^{\prime 2} \mathfrak{g}=0$.

Der zu $\psi(\mu, v) \equiv \mu^{2} \mathrm{~A}+2 \mu \nu \mathrm{B}+v^{2} \Gamma=0$ in (337) dualistische Ausdruck liefert nun das System doppelt berührender Kegelschnitte; um ihn zu erhalten ist an Stelle von $\mathrm{A}=\mathfrak{X}^{2}, \Gamma=\mathfrak{X}_{1}{ }^{2}, \mathrm{~B}=f+\lambda_{1} g$ in (337) bezw. zu setzen

$$
u_{m}{ }^{2}, \quad u_{n}{ }^{2}, \quad s^{2} p_{z}{ }^{2} u_{y}{ }^{2}+r^{2} p_{y}{ }^{2} u_{z}{ }^{2}-2 r^{2} s^{2} p_{y}{ }^{2} p_{z}{ }^{2} \omega(u, u),
$$

welch letzterer Ausdruck gleichbedeutend ist mit $F+\lambda^{\prime} G$. Werden an Stelle von $u_{y}$ und $u_{z}$ die Ausdrücke $u_{m}$ und $u_{n}$ eingeführt, so ergibt sich für das System doppelt berïhrender Kegelschnitte die Gleichung 


$$
\mu^{2} u_{m}^{2}+\nu^{2} u_{n}{ }^{2}+\mu \nu\left(u_{m}^{2}+u_{n}^{2}\right)-4 \mu \nu r^{2} s^{2} p_{y}{ }^{2} p_{z}{ }^{2} \omega(u, u)=0 .
$$

Diese Curven gehören also dem System an

$$
\varkappa^{\prime} u_{m}^{2}+\lambda^{\prime} u_{n}^{2}+\mu^{\prime} \omega(u, u)=0,
$$

d. h. einem speciellen Kegelschnittgewebe. Ein ähnliches Gewebe trat bereits in (331) auf; dort wurde auch gezeigt, dass die Cayley'sche Curve der Ort für die Brennpunkte der einzelnen Curven des Gewebes ist und aus der Verbindungslinie der zwei Punkte $u_{m}$ und $u_{n}$, sowie aus dem über $m n$ als Durchmesser construirten Kreise besteht:

346. Es liegen also die Brennpunkte der das obige Gewebe bildenden doppelt berïhrenden Kegelschnitte zum Theil auf der Centrale, zum Theil auf dem Aehnlichkeitskreise. Die Gleichung des Gewebes zeigt übrigens noch, dass die in ihm enthaltenen Punktepaare $x^{\prime} u_{m}{ }^{2}+\lambda^{\prime} u_{n}{ }^{2}=0$, d. h. die auf der Centrale $X$ befindlichen Wechselschnitte $\mathfrak{p}$ und $\mathfrak{p}_{1}$ zu den zwei Aehnlichkeitspunkten harmonisch gelegen sind.

Bezüglich weiterer Einzelheiten sei nochmals auf die oben citirte Abhandlung von Steiner verwiesen. Uebrigens ist durch die Ergebnisse der Nummern (333)-(346) in Zusammenhang mit (193) und (194) der allgemeine Theil der Steiner'schen Arbeit erledigt. Man hat nur zu beachten, dass in (334) mit Rücksicht auf S. 324, Zeile 14 zu setzen ist $4 \mu \nu=\frac{8 l^{2}}{[a, \omega]}, \nu=l^{2}\left(\right.$ daher $\left.\mu=\frac{2}{[a, \omega]}\right)$, ferner $\mathfrak{X}_{1}=p_{x}$, $\mu \mathfrak{X}=R_{x}$.

Brennpunktcurve für die einem Vierseit eingeschriebenen Kegelschnitte.

Es sei $x \varphi(u, u)+\lambda \psi(u, u)=0$ die Gleichung der einem Vierseit eingeschriebenen Kegelschnittschaar. Aehnlich wie in (331) folgt alsdann, dass die Cayley'sche Curve des Gewebes

$$
\varkappa \varphi(u, u)+\lambda \psi(u, u)+\mu \omega(u, u)=0
$$

der Ort der in dem Gewebe enthaltenen Punktepaare, also der Ort für die Brennpunkte der genannten Schaar ist.

347. Die Hessiane des Gewebes wird nach III, S. 226 von den Trägern der Punktepaare, d. h. von den Axen aller Kegelschnitte der Schaar umhüllt. Ferner gelten wieder alle aus $\S 22-\S 26$ folgenden Sätze über Kegelschnittgewebe, nur ist zu beachten, dass nunmehr das imaginäre Kreispunktepaar $\boldsymbol{\omega}(u, u)=0$ dem Gewebe angehört. Da nach (38), S. 240 die Cayley'sche Curve identisch ist mit der Hessiane des conjugirten Netzes, so lässt sich z. B. Satz V, S. 222 anwenden, 
und zwar würde alsdann folgen, dass die in dem Punktepaar $\boldsymbol{\omega}(u, u)=0$ gezogenen imaginären Asymptoten der Cayley'schen Curve dritter Ordnung sich in einem Punkte $q$ dieser Curve schneiden, der conjugirt ist zu dem reellen, dritten Schnittpunkte $x$ der unendlich fernen Geraden mit der Curve. Hieraus geht herror, dass $q$ den im Endlichen gelegenen Brennpunkt, $x$ den unendlich fernen Brennpunkt der einzigen Parabel darstellt, die in der Kegelschnittschaar enthalten ist $^{1}$ ).

Eine weitere Eigenschaft der Brennpunktscurve $C_{3}$ ergibt sich aus XIV, S. 231, wenn wir wieder die Cayley'sche Curve als Hessiane des conjugirten Netzes auffassen. Hiernach bilden die Strahlen, welche irgend einen Punkt von $C_{3}$ mit zwei conjugirten Polen verbinden, eine Involution, und zwar sind die Doppelstrahlen dieser Involution nunmehr zu einander rechtwinklig, da die imaginären Kreispunkte unter den conjugirten Polen enthalten sind; daraus folgt, dass alle Strahlenpaare der Involution dieselben Winkelhalbirenden besitzen. Man erhält somit nachstehenden Satz von Schröter ${ }^{2}$ ):

348. „Zieht man von irgend einem Punkte $a$ der allgemeinen Brennpunktscurve $C_{3}$ zwei Strahlen nach zwei beliebigen andern Punkten derselben $b$ und $c$ und nach den conjugirten $\beta$ und $\gamma$, so ist allemal $\Varangle b a c=\Varangle \beta a \gamma^{\prime \prime}$, oder diese beiden Winkel ergänzen sich zu zwei Rechten.

Der letzte Zusatz über die Ergänzung zu zwei Rechten findet sich allerdings bei Schröter nicht. Doch folgt aus dem eben erwähnten Satze, wie Schröter zeigt ${ }^{3}$ ), das Theorem von Steiner ${ }^{4}$ ):

349. "Sind $a$ und $\alpha, b$ und $\beta, c$ und $\gamma$ die Brennpunkte irgend dreier demselben Vierseit eingeschriebenen Kegelschnitte, so findet zwischen ihren gegenseitigen Abständen allemal die Relation statt, dass z. B.

ist."

$$
\frac{a c \cdot \alpha c}{b c \cdot \beta c}=\frac{a \gamma \cdot \alpha \gamma}{b \gamma \cdot \beta \gamma}
$$

Zufolge des Hesse'schen Satzes IV, S. 220 kann man sich nämlich leicht drei conjugirte Polepaare construiren; bilden die Punkte

1) Vgl. Schröter: „Ueber eine besondere Curve $3^{\text {ter }}$ Ordnung and eine einfache Erzeugungsart der allgemeinen Curve $3^{\text {ter }}$ Ordnung“. Math. Annalen, Bd. 5, S. 58, 1871.

2) „Ueber Curven dritter Ordnung“. Math. Annalen, Bd. 6, S. 87, 1872.

3) A. a. O. S. 88.

4) „Lehrsätze“. Journal für die reine und angewandte Mathematik, Bd. 45, S. 180, 1852, oder "Gesammelte Werke", Bd. 2, S. 434. 
$a, b, c$ ein Dreieck, so liegen alsdann die zugehörigen conjugirten Pole $\alpha, \beta, \gamma$ auf einer und derselben Geraden, und zwar ausserdem $\alpha$ auf der Seite $b c$ des Dreiecks, $\beta$ auf $c a, \gamma$ auf $a b$. Man hat alsdann

$$
\frac{a c}{b c}=\frac{\sin (a b c)}{\sin (b a c)}, \quad \frac{\alpha c}{\beta c}=\frac{\sin (\alpha \beta c)}{\sin (\beta \alpha c)}, \quad \frac{a \gamma}{\beta \gamma}=\frac{\sin (a \beta \gamma)}{\sin (\beta a \gamma)}, \quad \frac{\alpha \gamma}{b \gamma}=\frac{\sin (\alpha b \gamma)}{\sin (b \alpha \gamma)},
$$

woraus mit Rücksicht auf

$$
\begin{array}{ll}
\sin (a b c)=\sin (\alpha b \gamma), & \sin (\alpha \beta c)=\sin (a \beta \gamma), \\
\sin (b a c)=\sin (\beta a \gamma), & \sin (\beta \alpha c)=\sin (b \alpha \gamma)
\end{array}
$$

die Steiner'sche Relation folgt.

350. Zur Ableitung der Gleichung der Brennpunktscurve kann man noch folgenden Weg einschlagen.

Sind $q_{x}=0, r_{x}=0, s_{x}=0, t_{x}=0$ die vier gemeinsamen Tangenten aller Kegelschnitte der Schaar $x^{\prime} \varphi(u, u)+\lambda^{\prime} \psi(u, u)=0$, so repräsentirt die Gleichung

im Verein mit

$$
x q_{x}^{2}+\lambda r_{x}^{2}+\mu s_{x}^{2}+\nu t_{x}^{2}=0
$$

$$
x \omega(q, q)+\lambda \omega(r, r)+\mu \omega(s, s)+\nu \omega(t, t)=0
$$

das zu dem Gewebe $x^{\prime} \varphi(u, u)+\lambda^{\prime} \psi(u, u)+\mu^{\prime} \omega(u, u)=0$ conjugirte Kegelschnittnetz; denn jede Curve des Netzes liegt conjugirt zu $\varphi(u, u)=0, \mathrm{zu} \psi(u, u)=0$ und zu $\omega(u, u)=0$, also zu jedem Kegelschnitt des Gewebes. Die Hessiane dieses Netzes ist, wie bereits oben erwähnt, identisch mit der Cayley'schen Curve des Gewebes, d. h. mit der Brennpunktscurve für die dem Tangentenvierseit eingeschriebene Kegelschnittschaar. Bei Ableitung der Gleichung der Hessiane hat man zu beachten, dass diese Curve der Ort für die Spitzen aller Geradenpaare des Netzes ist; daher müssen die drei Gleichungen bestehen:

$$
x q_{x} q_{i}+\lambda r_{x} r_{i}+\mu s_{x} s_{i}+\nu t_{x} t_{i}=0, \quad(i=1,2,3),
$$

und hierzu tritt noch die Relation

$$
x \omega(q, q)+\lambda \omega(r, r)+\mu \omega(s, s)+\nu \omega(t, t)=0 .
$$

Bei Elimination von $x, \lambda, \mu, v$ erhält man für die Hessiane die Determinante vierten Grades

$\left|\begin{array}{llll}q_{x} q_{1} & r_{x} r_{1} & s_{x} s_{1} & t_{x} t_{1} \\ q_{x} q_{2} & r_{x} r_{2} & s_{x} s_{2} & t_{x} t_{2} \\ q_{x} q_{3} & r_{x} r_{3} & s_{x} s_{3} & t_{x} t_{3} \\ \omega(q, q) & \omega(r, r) & \omega(s, s) & \omega(t, t)\end{array}\right|=0$


Zwei Curven, von welchen aus zwei Strecken gleich gross erscheinen. 407

also

$$
\begin{aligned}
\omega(q, q) \cdot(r s t) r_{x} s_{x} t_{x}-\omega(r, r) \cdot(s t q) s_{x} t_{x} q_{x} & +\omega(s, s) \cdot(t q r) t_{x} q_{x} r_{x} \\
& -\omega(t, t) \cdot(q r s) q_{x} r_{x} s_{x}=0 .
\end{aligned}
$$

Wenn statt des beliebigen Vierseits insbesondere ein Dreiseit und die unendlich ferne Gerade gegeben sind, bestehen die Kegelschnitte der Schaar aus den dem Dreiseit eingeschriebenen Parabeln; wird der Einfachheit halber das Coordinatendreiseit zu Grunde gelegt, so erhält man als Gleichung der Brennpunktscurve

$$
p_{x} \mid \begin{array}{cccc}
p_{1} & x_{1} & 0 & 0 \\
p_{2} & 0 & x_{2} & 0 \\
p_{3} & 0 & 0 & x_{3} \\
0 & \omega_{11} & \omega_{22} & \omega_{33}
\end{array}=0
$$

welche nun in Uebereinstimmung mit (264) und (257) in die unendlich ferne Gerade und den umschriebenen Kreis des Dreiseits zerfällt.

351. Man bilde ähnlich wie in (350) die Gleichung der Brennpunktscurve für diejenigen Kegelschnitte, welche ein gegebenes Dreiseit zum Poldreiseit haben and eime feste Gerade berühren. Vgl. (262) und (265).

352. Es mögen nun die im Vorausgehenden gefundenen Resultate über die Brennpunktscurve einer Kegelschnittschaar angewandt werden auf den Fall, dass die zwei Gewebe vorliegen

$$
\begin{aligned}
& \text { 1) } x u_{a} \cdot u_{d}+\lambda u_{b} \cdot u_{c}+\mu \omega(u, u)=0, \\
& \text { 2) } x u_{a} \cdot u_{c}+\lambda u_{b} \cdot u_{d}+\mu \omega(u, u)=0 .
\end{aligned}
$$

Die Cayley'sche Curve des ersten Gewebes ist der Ort der Brennpunkte aller Kegelschnitte, die einem durch die Geraden $a b, a c, d b, d c$ gebildeten Vierseit eingeschrieben sind, während die Cayley'sche Curve des zweiten Gewebes der analoge Ort für diejenigen Kegelschnitte ist, die dem Vierseit der Geraden $a b, a d, c b, c d$ eingeschrieben sind. Ausserdem sind für 1) die Punktepaare $a$ und $d$, sowie $b$ und $c$ conjugirte Polenpaare, während von $a$ und $c$, sowie $b$ und $d$ gleiches gilt mit Bezug auf das Gewebe 2). Mit Rücksicht auf den Satz von Schröter in (348) folgt alsdann, dass von jedem Punkte einer der beiden Brennpunktscurven, die den Geweben 1) und 2) zugehören, die Strecken $a b$ und $c d$ unter gleich grossen Winkeln erscheinen oder unter solchen Winkeln, die sich zu zwei Rechten ergänzen ${ }^{1}$ ); und zwar gibt es auf jeder Curve einen Theil, von dessen Punkten aus

1) Vgl. (231). 
die Strecken gleich gross erscheinen und einen anderen Theil, für den die betreffenden Winkel Supplementwinkel sind. Die Nothwendigkeit dieser Erscheinung wird schon dadurch erklärt, dass auf Grund der Kriterien. in (277) jede der beiden in 1) und 2) für $\mu=0$ enthaltenen Kegelschnittschaaren im allgemeinen zwei Gruppen Ellipsen und zwei Gruppen Hyperbeln aufweist und dass von jedem Punkte der Cayleyschen Curve eines jeden der beiden Gewebe, also von dem Orte der Brennpunkte, die Strecken $a b$ und $c d$ unter gleichen oder supplementären Winkeln erscheinen können auf Grund elementarer Sätze ${ }^{1}$ ).

Auch zeigt sich dies bereits in dem einfacheren Falle, wo die Strecken $a b$ und $c d$ in einem Punkte zusammenstossen, so dass etwa $b$ mit $c$ zusammenfällt; die beiden obigen Gewebe sind alsdann

$$
\begin{aligned}
& \text { 3) } x u_{a} u_{d}+\lambda u_{b}{ }^{2}+\mu \omega(u, u)=0 \text {, } \\
& \text { 4) } x u_{a} u_{b}+\lambda u_{b} u_{t}+\mu \omega(u, u)=0 \text {. }
\end{aligned}
$$

Im Falle 3) folgt dualistisch zu (315), dass die Cayley'sche Curve des Gewebes eine Curve dritter Ordnung ist, die den Punkt $b$ zum Doppelpunkt hat. Bei Ableitung ihrer Gleichung verfahren wir ähnlich wie S. 225, bilden also zuerst den Ausdruck für den einem Punkte $x$ nach (16), S. 200 zugehörigen Polarkegelschnitt $N$; man findet

$$
\mathrm{N} \equiv u_{b} \cdot u_{a} \cdot \sum \pm\left(d_{1} b_{2} x_{3}\right)+u_{b} \cdot u_{d} \cdot \sum \pm\left(a_{1} b_{2} x_{3}\right)=0
$$

Alsdann ist die Bedingung dafür aufzustellen, dass von dem Punkte $x$ an $\mathrm{N}=0$ und $\omega(u, u)=0$ harmonische Tangentenpaare gelegt werden können. Man erhält hierdurch

$$
H_{a b} \cdot \sum \pm\left(d_{1} b_{2} x_{3}\right)+H_{b d} \cdot \sum \pm\left(a_{1} b_{2} x_{3}\right)=0 \text {, }
$$

wobei $H_{a b}$ und $H_{b d}$ die Ausdrücke für die den Punktepaaren $a, b$, bezw. $b, d$ zugehörigen Directorkreise bedeuten, also für diejenigen Kreise, welche die Strecken $a b$, bezw. $b d$ zum Durchmesser haben (vgl. (231)).

Eine einfache Construction der Brennpunktscurve ron 3$)^{2}$ ) ergibt sich folgendermassen. Der Ausdruck $\lambda u_{b}{ }^{2}+\mu \omega(u, u)$ in der Gleichung des Gewebes 3) repräsentirt nach (2), S. 57 gleich Null gesetzt für beliebige Werthe der Parameter $\lambda, \mu$ ein System concentrischer Kreise um den Mittelpunkt $b$. Aus

1) Vgl. Geiser: „Die Theorie der Kegelschnitte in elementarer Darstellung" (erster Theil der von Geiser und Schröter herausgegebenen Vorlesungen Steiner's über synthetische Geometrie), 2. Aufl., Leipzig 1876, S. 59 und 93. Diese „elementaren" Sätze ergeben sich übrigens sofort mit Hilfe der Figuren 8 und 9, S. 189.

2) Vgl. Schröter, a. a. O. Math. Annalen, Bd. 6, S. 86. 


$$
x u_{a} u_{d}+\lambda u_{b}^{2}+\mu \omega(u, u)=0
$$

folgt alsdann, dass die Punktepaare dieses Gewebes 3), also Punkte der Curve dritter Ordnung, erhalten werden, indem man von den Punkten $a$ und $d$ an die concentrischen Kreise um $b$ die Tangentenpaare legt. Die Schnittpunkte je zweier Tangentenpaare, die demselben Kreise angehören, sind auf der Curve dritter Ordnung gelegen; denn die Coordinaten dieser Punkte genügen der Gleichung des Gewebes 3). ${ }^{1}$ ) Auch erkennt man aus der Construction, dass in der That von irgend einem Punkte der Curve die Strecken $b a$ und $b d$ entweder gleich gross oder unter Supplementwinkeln erscheinen; die Curvenbogen, auf denen das eine oder andere stattfindet, werden durch die Punkte $a$ und $d$, welche gleichfalls der Curve angehören, begrenzt.

Einen eigenthümlichen Fall repräsentirt das Gewebe 4). Dualistisch zu (307) folgt, dass die Cayley'sche Curve nummehr zerfällt in das circulare Geradenpaar $\left(\begin{array}{ll}b & x \\ b & x\end{array}\right)_{\omega_{i k}}=0$, dessen Spitze in $b$ liegt, und in die Verbindungslinie der beiden Punkte $a$ und $d$. Von allen Punkten, die auf dieser Linie zwischen $a$ und $b$ gelegen sind, erscheinen offenbar die Strecken $b a$ und $b d$ unter Supplementwinkeln; von allen Punkten, die auf einer Verlängerung der Strecke ad gelegen sind, erscheinen $b a$ und $b d$ gleich gross.

Bei Anwendung der in (231) abgeleiteten Gleichungen zeigt sich, dass für $\alpha_{i}=a_{i}, \beta_{i}=\gamma_{i}=b_{i}, \delta_{i}=d_{i}$ die nicht zerfallende Curve dritter Ordnung erhalten wird, weun man in $K . m_{y} \mp H . n_{y}=0$ das Vorzeichen - wählt, während die ausartende Curve dem Vorzeichen + entspricht.

353. Die Axen aller Kegelschnitte, welche in den Geweben 3) und 4) enthalten sind, gehen zum Theil durch den Punkt $b$, zum Theil umhüllen sie je eine Parabel, welche speciell im Falle 3) aus der Steiner'schen Parabel des Punktes $b$ in Bezug auf das Punktepaar $a, d$ besteht.

Die Enveloppe der Axen ist die Hessiane der betr. Gewebe; im Falle 3) ergibt sich dualistisch zu (314) als Hessiane der Punkt $u_{b}=0$ und der Kegelschnitt $\sum \pm\left(\varphi^{\prime}\left(u_{1}\right) \boldsymbol{\omega}^{\prime}\left(u_{2}\right) b_{3}\right)=0$, wobei

$$
\varphi(u, u)=u_{a} u_{d},
$$

und dieser Kegelschnitt repräsentirt nach S. $201 \mathrm{f}$. die Steiner'sche

1) Vgl. VI, S. 228. 
Parabel von $b$ in Bezug auf $\left.\varphi(u, u)=0 .{ }^{1}\right)$ Im Falle 4) erbält man dualistisch zu (306) wiederum den Punkt $b$ und die Parabel

$$
2 u_{b} \cdot \sum \pm\left(\frac{1}{2} \omega^{\prime}\left(u_{1}\right) a_{2} d_{3}\right)-\omega(u, u) \cdot \sum \pm\left(b_{1} a_{2} d_{3}\right)=0
$$

Wir wollen die Enveloppe der Axen aller Kegelschnitte einer Schaar noch in einem anderen Falle betrachten, der uns zu der sogenannten Steiner'schen Curve dritter Classe führt.

\section{Die Steiner'sche Curve dritter Class $\left.\theta^{2}\right)$.}

354. Die Axen aller Parabeln, die ein gegebenes Dreiseit zum Poldreiseit haben, umhüllen eine Curve dritter Classe mit einer Doppeltangente und drei Rüickkehrpunkten.

Alle Parabelu, die das Coordinatendreiseit zum Poldreiseit haben, sind gegeben durch

(1) $\alpha_{1} u_{1}{ }^{2}+\alpha_{2} u_{2}{ }^{2}+\alpha_{3} u_{3}{ }^{2}=0$, wobei $\alpha_{1} p_{1}{ }^{2}+\alpha_{2} p_{2}{ }^{2}+\alpha_{3} p_{3}{ }^{2}=0$.

Wir stellen nun diese Schaar von Parabeln mit $\omega(\imath, \imath)=0$ zusammen zù der Gleichung

$$
\alpha_{1} u_{1}^{2}+\alpha_{2} u_{2}^{2}+\alpha_{3} u_{3}^{2}+\mu \omega(u, u)=0,
$$

welche ein Gewebe darstellt, da die Parameter $\alpha_{i}$ der Bedingung $\alpha_{1} p_{1}{ }^{2}+\alpha_{2} p_{2}{ }^{2}+\alpha_{3} p_{3}{ }^{2}=0$ unterworfen sind. Die Hessiane des Gewebes ist die von den Axen der Parabeln eingehüllte Curve; um ihre Gleichung abzuleiten, sind die nach $u_{i}(i=1,2,3)$ genommenen partiellen Differentialquotienten von (2) gleich Null zu setzen, d. h. man hat

$$
\begin{aligned}
& \alpha_{1} u_{1}+\frac{1}{2} \omega^{\prime}\left(u_{1}\right)=0 \\
& \alpha_{2} u_{2} \quad+\frac{1}{2} \omega^{\prime}\left(u_{2}\right)=0 \\
& \alpha_{3} u_{3}+\frac{1}{2} \omega^{\prime}\left(u_{3}\right)=0,
\end{aligned}
$$

und hierzu tritt noch

$$
\alpha_{1} p_{1}^{2}+\alpha_{2}{p_{2}}^{2}+\alpha_{3} p_{3}^{2}=0
$$

1) Näberes über diese Parabel findet man in der oben citirten Abhandlung von Schröter in Bd. 6 der Math. Annalen.

2) Steiner: „Ueber eine besondere Curve dritter Classe (und vierten Grades)", Journal für die reine und angewandte Mathematik, Bd.53, S. 231-237, 1856, oder „Gesammelte Werke", Bd. 2, S. 639-647. Vgl. anch Cremona: „Sur l'hypocycloïde à trois rebroussements", in demselben Journal, Bd. 64, S. 101-123, 1864. 
durch Elimination der $\alpha_{i}$ erhält man die Gleichung der Curve in Gestalt der Determinante

$$
\left|\begin{array}{cccc}
u_{1} & 0 & 0 & \frac{1}{2} \omega^{\prime}\left(u_{1}\right) \\
0 & u_{2} & 0 & \frac{1}{2} \omega^{\prime}\left(u_{2}\right) \\
0 & 0 & u_{3} & \frac{1}{2} \omega^{\prime}\left(u_{3}\right) \\
p_{1}{ }^{2} & p_{2}{ }^{2} & p_{3}{ }^{2} & 0
\end{array}\right|=0 .
$$

Die unendlich ferne Gerade $p_{x}=0$ ist für diese Curve eine Doppeltangente, denn ersetzt man $u_{i}$ durch $p_{i}+\lambda v_{i},(i=1,2,3)$, so verschwinden die Coefficienten von $\lambda^{0}$ und $\lambda^{1}$ auf Grund der Relationen $\omega^{\prime}\left(p_{i}\right)=0$, der Factor von $\lambda^{2}$ repräsentirt gleich Null gesetzt das Paar der Berührungspunkte der unendlich fernen Geraden mit der Curve. (Vgl. auch (309).) Für den synthetischen Nachweis der drei Rückkehrtangenten vergleiche man die wiederholt citirte Arbeit von Schröter ${ }^{1}$ ); für die analytische Bestimmung der Rückkehrtangenten gewährt (309) die nöthigen Hilfsmittel.

Die Steiner'sche Curve dritter Classe steht in einer einfachen Beziehung zu der Brenupunktscurve derjenigen Parabeln, welche das der Betrachtung zu Grunde liegende Dreiseit zum Poldreiseit haben, also nach (265), bezw. (351) zu dem Feuerbach'schen Kreise'des Dreiseits und zu der unendlich fernen Geraden. Diese Beziehung ist folgende:

355. Legt man von einem Punkte des Feuerbach'schen Kreises das Tangentenpaar an irgend eine der Parabeln, die das zugehörige Dreiseit zum Poldreiseit haben, so sind die Winkelhalbirenden dieses Geradenpaares Tangenten der Steiner'schen Curve dritter Classe und somit auch Axen zweier Parabeln, deren vier Schnittpunkte nach (217) auf einem Kreise liegen.

Nach VIIIb und VIIIa, S. 228 lassen sich nämlich von irgend einem Punkte der Brennpunktscurve an die Kegelschnitte des zugehörigen Gewebes Tangentenpaare einer Involution legen, deren Doppelstrahlen conjugirte Polaren der Hessiane, also Tangenten der Hessiane sind. Im vorliegenden Falle müssen diese Doppelstrahlen zu einander rechtwinklig sein, denn sie müssen harmonisch liegen zu dem nach den imaginären Kreispunkten gezogenen Geradenpaare, das ja gleichfalls dem betr. Gewebe angehört; daraus folgt aber, dass die Doppelstrahlen die Winkelbalbirenden sind aller übrigen Geradenpaare der Involution.

1) Math. Annalen, Bd. 6, S. 96. 
$\mathrm{Zu}$ genau derselben Steiner'schen Curve (3) in (354) gelangt man bei Lösung der Aufgabe:

356. Man bilde die Gleichung derjenigen Curve, welche von den Scheiteltangenten aller einem Dreiseit eingeschriebenen Parabeln umhüllt wird. Vgl. (264) und (261).

Sind $y_{i}$, bezw. $t_{i},(i=1,2,3)$, die Coordinaten des Scheitels, bezw. des unendlich fernen Punktes der Scheiteltangente der Parabel, sind ferner $z_{i},(i=1,2,3)$, die Coordinaten des Berührungspunktes der Curve mit der unendlich fernen Geraden, so besteht die Relation

$$
\alpha_{1} v_{2} v_{3}+\alpha_{2} v_{3} v_{1}+\alpha_{3} v_{1} v_{2} \equiv v_{y} v_{z}-v_{t}^{2} \text {, }
$$

falls die Parabel dem Coordinatendreiseit eingeschrieben ist; dabei ist (vgl. (62), S. 102) $\omega(v, v)=v_{z}{ }^{2}+v_{t}{ }^{2}$. Die Coordinaten $u_{i}$ der Scheiteltangente genügen nun den Gleichungen $u_{y}=0$ und $u_{t}=0$; da aber nach (1) $y_{i} z_{i}-t_{i}{ }^{2}=0$, so lässt sich $u_{y}=0$ ersetzen durch

oder durch

$$
\frac{u_{1} t_{1}^{2}}{z_{1}}+\frac{u_{2} t_{2}^{2}}{z_{2}}+\frac{u_{3} t_{3}^{2}}{z_{3}}=0
$$

$$
u_{1} z_{2} z_{3} t_{1}{ }^{2}+u_{2} z_{3} z_{1} t_{2}{ }^{2}+u_{3} z_{1} z_{2} t_{3}{ }^{2}=0 \text {. }
$$

Mit Rücksicht auf die, aus $\omega(v, v)=v_{z}{ }^{2}+v_{t}{ }^{2}$ folgenden Relationen $z_{i} z_{k}=\omega_{i k}-t_{i} t_{k}$ lässt sich die letzte Gleichung in die Form bringen

$$
u_{1} \omega_{23} t_{1}^{2}+u_{2} \omega_{31} t_{2}^{2}+u_{3} \omega_{12} t_{3}{ }^{2}-t_{1} t_{2} t_{3}\left(u_{1} t_{1}+u_{2} t_{2}+u_{3} t_{3}\right)=0,
$$

wofür auch gesetzt werden kann

(2) $\omega_{23} u_{1}\left(p_{2} u_{3}-p_{3} u_{2}\right)^{2}+\omega_{31} u_{2}\left(p_{3} u_{1}-p_{1} u_{3}\right)^{2}+\omega_{12} u_{3}\left(p_{1} u_{2}-p_{2} u_{1}\right)^{2}=0$;

denn $u_{t}$ ist gleich Null, da der Punkt $t$ auf der Scheiteltangente $u$ liegt, und aus $u_{t}=0, p_{t}=0$ folgt

$$
t_{1}: t_{2}: t_{3}=\left(p_{2} u_{3}-p_{3} u_{2}\right):\left(p_{3} u_{1}-p_{1} u_{3}\right):\left(p_{1} u_{2}-p_{2} u_{1}\right) \text {. }
$$

Die Curve (2) ist mit der in (354) erhaltenen identisch, wie man durch Vergleichung der Coefficienten sofort erkennt.

Eine dritte Erzeugungsweise genau derselben Steiner'schen Curve dritter Classe, die in (354) und (356) erbalten wurde, ist folgende:

35\%. Die Asymptoten aller einem Dreieck umschriebenen gleichseitigen Hyperbeln umhüllen die Steiner'sche Curve dritter Classe.

Die Hessiane des Gewebes (2) in (354) ist nämlich nach (39), S. 240 für das conjugirte Netz die Cayley'sche Curve, also die Enveloppe aller Geradenpaare des conjugirten Netzes; die Gleichung des letzteren ist

wobei

$$
\begin{gathered}
a_{1} x_{2} x_{3}+a_{2} x_{3} x_{1}+a_{3} x_{1} x_{2}+\mu p_{x}^{2}=0 \\
a_{1} \omega_{23}+a_{2} \omega_{31}+a_{3} \omega_{12}=0
\end{gathered}
$$


denn auf Grund der Bedingungsgleichung $a_{1} \omega_{23}+a_{2} \omega_{31}+a_{3} \omega_{12}=0$ und zufolge $\boldsymbol{\omega}(p, p)=0$ ist jede Curve dieses Netzes conjugirt zu jeder Parabel des Gewebes (2) in (354). Nach (162) stellt aber nunmehr $a_{1} x_{2} x_{3}+a_{2} x_{3} x_{1}+a_{3} x_{1} x_{2}=0$ die Gesammtheit des Büschels gleichseitiger Hyperbein dar, die dem Coordinatendreieck umschrieben sind (vgl. auch (166)), und die Geradenpaare des Netzes (1) sind (vgl. (26), S. 45) die Asymptotenpaare der gleichseitigen Hyperbeln.

358. Viertens kann man die Curve in (354) dadurch ableiten, dass man die Gleichung des zu dem Netz (1) in (357) conjugirten Gewebes aufstellt und dessen Hessiane bildet. Es möge diese Aufgabe ganz allgemein gelöst werden, indem wir ein beliebiges Dreieck zu Grunde legen, dessen Ecken gegeben seien durch $u_{\alpha}=0$, $u_{\beta}=0, u_{\gamma}=0$; ist alsdann $u_{\delta}=0$ die Gleichung des dem Dreieck zugehörigen Höhenschnittpunktes, so lautet die Gleichung des conjugirten Gewebes

$$
x u_{\alpha}^{2}+\lambda u_{\beta}^{2}+\mu u_{\gamma}^{2}+v u_{\delta}^{2}=0,
$$

wozu noch die Bedingungsgleichung tritt

$$
x p_{\alpha}^{2}+\lambda p_{\beta}^{2}+\mu p_{\gamma}^{2}+v p_{\delta}^{2}=0,
$$

damit jede Curve des Gewebes auch conjugirt sei zu dem Glied $p_{x}{ }^{2}$ des Netzes (1) in (357). Hieraus geht hervor, dass sämmtliche Curven des Gewebes Parabeln sind, und zwar enthält das Gewebe alle Parabeln, für welche die vier Punkte $\alpha, \beta, \gamma, \delta$ ein Polviereck bilden im Sinne von (329). Dabei ist $u_{\delta}$ gleichbedeutend mit

$$
\frac{u_{\alpha}}{\left(\begin{array}{cc}
\alpha & \beta \\
\alpha & \gamma
\end{array}\right)_{\omega_{i k}}}+\frac{u_{\beta}}{\left(\begin{array}{ll}
\beta & \gamma \\
\beta & \alpha
\end{array}\right)_{\omega_{i k}}}+\frac{u_{\gamma}}{\left(\begin{array}{ll}
\gamma & \alpha \\
\gamma & \beta
\end{array}\right)_{\omega_{i k}}}=0 .
$$

Für die Hessiane erhält man, dualistisch zu (350), die Gleichung

$$
\left|\begin{array}{cccc}
\alpha_{1} u_{\alpha} & \beta_{1} u_{\beta} & \gamma_{1} u_{\gamma} & \delta_{1} u_{\delta} \\
\alpha_{2} u_{\alpha} & \beta_{2} u_{\beta} & \gamma_{2} u_{\gamma} & \delta_{2} u_{\delta} \\
\alpha_{3} u_{\alpha} & \beta_{3} u_{\beta} & \gamma_{3} u_{\gamma} & \delta_{3} u_{\delta} \\
p_{\alpha}{ }^{2} & p_{\beta}{ }^{2} & p_{\gamma}{ }^{2} & p_{\delta}{ }^{2}
\end{array}\right|=0
$$

welche sich für

$$
\begin{gathered}
u_{\alpha} \equiv u_{1}=0, \quad u_{\beta} \equiv u_{2}=0, \quad u_{\gamma} \equiv u_{3}=0, \\
u_{\delta} \equiv \frac{1}{\omega_{23}} u_{1}+\frac{1}{\omega_{31}} u_{2}+\frac{1}{\omega_{12}} u_{3}=0,
\end{gathered}
$$

d. h. wenn das Coordinatendreiseit der Betrachtung zu Grunde gelegt wird, reducirt auf: 


$$
\left|\begin{array}{cccc}
u_{1} & 0 & 0 & \frac{1}{\omega_{23}}\left(\omega_{23} u_{1}+\omega_{31} u_{2}+\omega_{12} u_{3}\right) \\
0 & u_{2} & 0 & \frac{1}{\omega_{31}}\left(\omega_{23} u_{1}+\omega_{31} u_{2}+\omega_{12} u_{3}\right) \\
0 & 0 & u_{3} & \frac{1}{\omega_{12}}\left(\omega_{23} u_{1}+\omega_{31} u_{2}+\omega_{12} u_{3}\right) \\
p_{1}{ }^{2} & p_{2}{ }^{2} p_{3}{ }^{2} & \left(\frac{p_{1}}{\omega_{23}}+\frac{p_{2}}{\omega_{31}}+\frac{p_{3}}{\omega_{12}}\right)^{2}
\end{array}\right|=0 .
$$

Diese Gleichung ist mit (3) in (354) gleichbedeutend, indem sie aus (3) in (354) durch Multiplication mit $-\frac{1}{\omega_{23} \omega_{31} \omega_{12}}$ hervorgeht.

359. Welche Curve wird von den Axen aller einem Dreiseit eingeschriebenen Parabeln umhüllt? Vgl. (347).

Die Axen sind als Verbindungslinien der Brennpunkte die Träger aller Punktepaare, welche in dem durch

$$
\begin{gathered}
\alpha_{1} u_{2} u_{3}+\alpha_{2} u_{3} u_{1}+\alpha_{3} u_{1} u_{2}+\mu \omega(u, u)=0 \\
\alpha_{1} p_{2} p_{3}+\alpha_{2} p_{3} p_{1}+\alpha_{3} p_{1} p_{2}=0
\end{gathered}
$$

bestimmten Gewebe enthalten sind; für die Hessiane findet man die Curve dritter Classe

$$
\left|\begin{array}{cccc}
0 & u_{3} & u_{2} & \omega^{\prime}\left(u_{1}\right) \\
u_{3} & 0 & u_{1} & \omega^{\prime}\left(u_{2}\right) \\
u_{2} & u_{1} & 0 & \omega^{\prime}\left(u_{3}\right) \\
p_{2} p_{3} & p_{3} p_{1} & p_{1} p_{2} & 0
\end{array}\right|=0 .
$$

Da alle Kegelschnitte des Gewebes die unendlich ferne Gerade zur gemeinsamen Tangente haben, ist diese Gerade nach (309) eine Doppeltangente der Curve dritter Classe. Wie nun eine Curve dritter Ordnung mit Doppelpunkt drei Wendepunkte besitzt, so hat die Curve dritter Classe mit Doppeltangente drei Rückkehrpunkte. Wir sind also auch bei vorliegender Aufgabe wieder zu einer analogen Curve dritter Classe geführt worden wie bei (354), (356) und (357). ${ }^{1}$ )

Auf gleiche Weise wie in (355) erhält man mit Rücksicht auf den Schluss von (350) und (264) den Satz:

360. Legt man von einem Punkte des einem Dreieck umschriebenen Kreises das Tangentenpaar an eine der dem Dreieck eingeschriebenen Parabeln, so sind die Winkelhalbirenden dieses Geradenpaares

1) Vgl. Steiner: „Vermischte Sätze und Aufgaben", Journal für die reine und angewandte Mathematik, Bd. 55, S. 371, 1858, oder "Gesammelte Werke", Bd. 2, S. $677 \mathrm{f}$. 
Tangenten der in (359) erhaltenen Curve dritter Classe und somit auch Axen zweier eingeschriebenen Parabeln, deren vier Schnittpunkte nach (217) auf einem Kreise liegen.

361. Welche Curve wird von den Scheiteltangenten aller Parabeln umhüllt, die ein gegebenes Dreiseit zum Poldreiseit haben? Vgl. (265) und den Beweis zu (261).

Bei analoger Behandlung dieser Aufgabe wie in (356) gelangt man zu der Gleichung

$$
\begin{gathered}
\omega_{11} u_{1}\left(p_{3} u_{1}-p_{1} u_{3}\right)\left(p_{1} u_{2}-p_{2} u_{1}\right)+\omega_{22} u_{2}\left(p_{1} u_{2}-p_{2} u_{1}\right)\left(p_{2} u_{3}-p_{3} u_{2}\right) \\
+\omega_{33} u_{3}\left(p_{2} u_{3}-p_{3} u_{2}\right)\left(p_{3} u_{1}-p_{1} u_{3}\right)+\omega_{23} u_{1}\left(p_{2} u_{3}-p_{3} u_{2}\right)^{2} \\
+\omega_{31} u_{2}\left(p_{3} u_{1}-p_{1} u_{3}\right)^{2}+\omega_{12} u_{3}\left(p_{1} u_{2}-p_{2} u_{1}\right)^{2}=0 .
\end{gathered}
$$

Von dieser Curve dritter Classe gilt gleiches wie bei der in (360) erhaltenen: sie hat die unendlich ferne Gerade zur Doppeltangente und besitzt drei Rückkehrpunkte.

Zum Schlusse der voranstehenden Betrachtungen möge noch bemerkt werden, dass die meisten speciellen Sätze, welche in der S. 410 citirten Abhandlung Steiner's enthalten sind, sich aus den allgemeinen Theoremen in $\S \S 22-26$, aus dem Satze (249) und aus dem Umstande ergeben, dass die Cayley'sche Curve des in (359) betrachteten Gewebes nach (350) und (264) durch den dem betr. Dreieck umschriebenen Kreis und die unendlich ferne Gerade gebildet wird, die Cayley'sche Curve des Gewebes in (354) nach (351) und (265) in den Feuerbach'schen Kreis und die unendlich ferne Gerade zerfällt.

*Um die Vorzüge der analytisch-geometrischen Methode bei Behandlung verwickelter Probleme der Integralrechnung darzulegen, erschien es zweckmässig, im Folgenden (362) und (363) noch zwei Anwendungen dieser Art aufzunehmen.

* 362. Anwendung der Poldreiecke auf ein Integral von Aronhold.

Bezeichnet in beliebigen homogenen oder Dreieckscoordinaten

$$
f\left(x_{1}, x_{2}, x_{3}\right) \equiv \sum_{i}^{3} \sum_{i}^{3} a_{i k} x_{i} x_{k}=0, a_{i k}=a_{k i},
$$

die Gleichung eines Kegelschnitts, so ist nach den Ausführungen von Aronhold ${ }^{1}$ ) unter der Voraussetzung

1) Journal für die reine und angewandte Mathematik, Bd. 61, S. 96-103, 1862. 


$$
f(x, y, 1)=0
$$

das Integral

$$
J=\int \frac{d x}{\frac{1}{2} f^{\prime}(y)\left(u_{1} x+u_{2} y+u_{8}\right)},
$$

oder das für beliebige $\xi_{1}, \xi_{2}, \xi_{3}$ damit identische Integral $^{1}$ )

$$
\int \frac{\sum \pm\left(\xi_{1} x_{z} d x_{3}\right)}{\frac{1}{2}\left[\xi_{1} f^{\prime}\left(x_{1}\right)+\xi_{2} f^{\prime}\left(x_{2}\right)+\xi_{3} f^{\prime}\left(x_{3}\right)\right]\left(u_{1} x_{1}+u_{2} x_{2}+u_{3} x_{3}\right)}=J
$$

durch einen einzigen Logarithmus nach folgender Regel darstellbar:

Man bezeichne mit

$$
F(u, u) \equiv \sum_{1}^{3} \sum_{1}^{3} A_{i k} u_{i} u_{k}
$$

den Ausdruck in Liniencoordinaten für den Kegelschnitt (1) und berechne bei beliebig fixirtem Vorzeichen von $\sqrt{-F(u, u)}$ die Werthe $\xi_{1}: \xi_{2}: \xi_{3}$ aus dem System linearer Gleichungen ${ }^{2}$ )

$$
\begin{aligned}
& \sqrt{-F(u, u)} \xi_{1}=\frac{1}{2}\left(u_{2} f^{\prime}\left(\xi_{3}\right)-u_{3} f^{\prime}\left(\xi_{2}\right)\right) \\
& \sqrt{-F(u, u)} \xi_{2}=\frac{1}{2}\left(u_{3} f^{\prime}\left(\xi_{1}\right)-u_{1} f^{\prime}\left(\xi_{3}\right)\right) \\
& \sqrt{-\overline{F(u, u)} \xi_{3}}=\frac{1}{2}\left(u_{1}\left(f^{\prime}\left(\xi_{2}\right)-u_{2} f^{\prime}\left(\xi_{1}\right)\right),\right.
\end{aligned}
$$

so wird

$$
J=\frac{1}{\sqrt{-F(u, u)}} \log \frac{\xi_{1} f^{\prime}\left(x_{1}\right)+\xi_{2} f^{\prime}\left(x_{2}\right)+\xi_{3} f^{\prime}\left(x_{3}\right)}{x_{1} u_{1}+x_{2} u_{2}+x_{3} u_{3}}+\text { Const. }
$$

Dieses Ergebniss ist praktisch zunächst nur verwendbar, wenn $F(u, u)$ einen negativen Zahlenwerth besitzt. Da die Umformung des Logarithmus in eine cyklometrische Function nicht ohne mühsame Rechnung durchführbar, und da überdies die elegante Fassung des Endergebnisses nicht gerade auf der Hand liegt, möge im Folgenden das Integral (3) auf einem (von dem Aronhold'schen völlig verschiedenen) Wege abgeleitet werden, der sämmtliche Umformungen mit Leichtigkeit gibt und die Bedeutung einiger der wichtigsten Sätze von $\S 4$ für die Integralrechnung zur Anschauung bringt.

1) Ueber die Identität der Integrale (3) und (3a) vgl. Aronhold a. a. O., S. 97-98.

2) Zufolge der Entwicklungen S. $35 \mathrm{f}$. gibt die Auflösung dieses Systems entsprechend dem. Vorzeichen von $\sqrt{-F(u, u)}$ die Coordinaten des einen oder anderen Schnittpunktes der Geraden $u_{x}=0$ mit dem Kegelschnitt (1). 
$\mathrm{Zu}$ dem $\mathrm{Z}$ weck seien $y_{1}, y_{2}, y_{3}$ die Coordinaten eines Punktes, für welcben

$$
u_{1} y_{1}+u_{2} y_{2}+u_{3} y_{3}=0, \text { dagegen } f\left(y_{1}, y_{2}, y_{3}\right) \gtrless 0 ;
$$

ferner sei

$$
\left\{\begin{array}{l}
z_{1}=\frac{1}{2} f^{\prime}\left(y_{2}\right) u_{3}-\frac{1}{2} f^{\prime}\left(y_{3}\right) u_{2} \\
z_{2}=\frac{1}{2} f^{\prime}\left(y_{3}\right) u_{1}-\frac{1}{2} f^{\prime}\left(y_{1}\right) u_{3} \\
z_{3}=\frac{1}{2} f^{\prime}\left(y_{1}\right) u_{2}-\frac{1}{2} f^{\prime}\left(y_{2}\right) u_{1},
\end{array}\right.
$$

so dass also der Punkt $z$ den Schnittpunkt von $u_{x}=0$ mit der Polare des Punktes $y$ in Bezug auf den Kegelschnitt (1) darstellt.

Durch die Substitution

$$
x_{i}=y_{i} X_{1}+z_{i} X_{2}+\frac{1}{2} F^{\prime}\left(u_{i}\right) X_{3}, \quad(i=1,2,3),
$$

wird nun die Curve (1) auf ein Poldreieck bezogen, denn ihre Gleichung verwandelt sich in

$$
f(x, x) \equiv f(y, y) X_{1}{ }^{2}+f(z, z) X_{2}{ }^{2}+A F(u, u) X_{3}{ }^{2} .
$$

Zum Nachweis dieser Behauptung beachte man, dass aus $(8)$ durch Multiplication mit $\frac{1}{2} f^{\prime}\left(y_{i}\right)$, bezw. $\frac{1}{2} f^{\prime}\left(z_{i}\right)$, bezw. $u_{i}$ und jeweilige Summation in Bezug auf $i$ von 1 bis 3 mit Rücksicht auf $u_{y}=0$, $u_{z} \equiv 0, f(y, z) \equiv 0$ die Auflösungen hervorgehen

\section{Ferner wird}

$$
X_{1}=\frac{f(y, x)}{f(y, y)}, \quad X_{2}=\frac{f(z, x)}{f(z, z)}, \quad X_{3}=\frac{u_{x}}{F(u, u)} .
$$

$\frac{1}{2} f^{\prime}\left(x_{i}\right)=a_{i 1} x_{1}+a_{i 2} x_{2}+a_{i 3} x_{3}=\frac{1}{2} f^{\prime}\left(y_{i}\right) X_{1}+\frac{1}{2} f^{\prime}\left(z_{i}\right) X_{2}+A u_{i} X_{3}$, daher

$$
\left\{\begin{aligned}
f(x, x) & =f(y, x) X_{1}+f(z, x) X_{2}+A u_{x} X_{3} \\
& =f(y, y) X_{1}{ }^{2}+f(z, z) X_{2}{ }^{2}+A F(u, u) X_{3}{ }^{2} .
\end{aligned}\right.
$$

Wenn man noch die leicht abzuleitende Beziehung

$$
f(z, z)=f(y, y) \cdot F(u, u)-A u_{y}{ }^{2}=f(y, y) \cdot F(u, u)
$$

beachtet, so folgt durch Einführung der Werthe der $X_{i}$ aus $(8 a)$ in die Gleichung (9), dass $f(x, x)=0$ gleichbedeutend ist mit

$$
F(u, u) \cdot f^{2}(y, x)+f^{2}(z, x)+A f(y, y) \cdot u_{x}^{2}=0 .
$$

Wir wollen zunächst annehmen, dass $F(u, u)>0$ sei, d. h. dass die Gerade $u_{x}=0$ die Curve (1) nicht in reellen Punkten schneidet. Alsdann muss nach (10) jedenfalls $A f(y, y)<0$ sein, denn der Kegelschnitt soll reell vorausgesetzt werden. Es werde nun gesetzt 


$$
\frac{\sqrt{F(u, u)} f(y, x)}{\sqrt{-A f(y, y)} u_{x}}=\cos t, \frac{f(z, x)}{\sqrt{-A f(y, y)} u_{x}}=\sin t,
$$

woraus folgt

oder

$$
\frac{\sqrt{F(u, u)}}{\sqrt{-A f(y, y)}} d\left(\frac{f(y, x)}{u_{x}}\right)=-\sin t \cdot d t
$$

$$
\frac{u_{x} \sqrt{F(u, u)}}{f(z, x)} d\left(\frac{f(y, x)}{u_{x}}\right)=-d t .
$$

Es ist aber

$$
u_{x}^{2} d\left(\frac{f(y, x)}{u_{x}}\right)=u_{x} f(y, d x)-f(y, x)\left(u_{1} d x_{1}+u_{2} d x_{2}+u_{3} d x_{3}\right),
$$

andrerseits

$$
\begin{aligned}
\sum \pm\left(z_{1} x_{2} d x_{3}\right) & =\left|\begin{array}{ccc}
\frac{1}{2} f^{\prime}\left(y_{1}\right) & \frac{1}{2} f^{\prime}\left(y_{2}\right) & \frac{1}{2} f^{\prime}\left(y_{3}\right) \\
u_{1} & u_{2} & u_{3}
\end{array}\right| \cdot\left|\begin{array}{ccc}
x_{1} & x_{2} & x_{3} \\
d x_{1} & d x_{2} & d x_{3}
\end{array}\right| \\
& =f(y, x)\left(u_{1} d x_{1}+u_{2} d x_{2}+u_{3} d x_{3}\right)-u_{x} f(y, d x),
\end{aligned}
$$

daher

$$
u_{x}^{2} \cdot d\left(\frac{f(y, x)}{u_{x}}\right)=-\sum \pm\left(z_{1} x_{2} d x_{3}\right) \text {, }
$$

so dass sich (12) verwandelt in

$$
\frac{\sqrt{F(u, u)}}{f(z, x) \cdot u_{x}} \sum \pm\left(z_{1} x_{2} d x_{3}\right)=d t
$$

Hieraus folgt sofort

$$
\int \frac{\sum \pm\left(z_{1} x_{2} d x_{3}\right)}{f(z, x) \cdot u_{x}}=\frac{1}{\sqrt{F(u, u)}} t+\text { Const. }
$$

wobei natürlich links die $z_{i}$ durch beliebige andere Grössen $\xi_{i}$ ersetzt werden könnten (vgl. $3 a$ ).

Wenn $F(u, u)<0$, d. h. wenn die Gerade $u_{x}=0$ den Kegelschnitt (1) in reellen Punkten schneidet, schreiben wir (10) in der Form

$$
\begin{gathered}
(f(z, x)+\sqrt{-F(u, u)} f(y, x))(f(z, x)-\sqrt{-F(u, u)} f(y, x)) \\
=-A f(y, y) u_{x}^{2},
\end{gathered}
$$

wo die beiden Factoren der linken Seite die Tangenten des Kegelschnitts (1) in seinen Schnittpunkten mit $u_{x}=0$ darstellen. Der Gleichung (15) wird jedenfalls genügt durch die Substitution

$$
\left\{\begin{array}{l}
\frac{f(z, x)+\sqrt{-F(u, u)} f(y, x)}{u_{x}}=t \\
\frac{f(z, x)-\sqrt{-F(u, u)} f(y, x)}{u_{x}}=-\frac{A f(y, y)}{t},
\end{array}\right.
$$


aus welcher folgt

$$
\frac{2 f(z, x)}{u_{x}}=t-\frac{A f(y, y)}{t}, \quad \frac{2 \sqrt{-F(u, u)} f(y, x)}{u_{x}}=t+\frac{A f(y, y)}{t} ;
$$

ferner ist

daler

$$
2 \sqrt{-F(u, u)} d\left(\frac{f(y, x)}{u_{x}}\right)=\left(t-\frac{\Delta f(y, y)}{t}\right) \frac{d t}{t}
$$

$$
\frac{u_{x} \sqrt{-F(u, u)}}{f(z, x)} \cdot d\left(\frac{f(y, x)}{u_{x}}\right)=\frac{d t}{t}
$$

so dass man mit Hilfe von (13) erhält:

und

$$
\begin{gathered}
\sum \frac{\sum \pm\left(z_{1} x_{2} d x_{3}\right)}{f(z, x) \cdot u_{x}}=\frac{-1}{\sqrt{-F(u, u)}} \frac{d t}{t} \\
\int \frac{\sum \pm\left(z_{1} x_{9} d x_{3}\right)}{f(z, x) \cdot u_{x}}=\frac{-1}{\sqrt{-F(u, u)}} \log t \\
=\frac{-1}{\sqrt{-F(u, u)}} \log \frac{f(z, x)+\sqrt{-F(u, u)} f(y, x)}{u_{x}}+\text { Const. }
\end{gathered}
$$

(vgl. (6) und (7)).

Auch mit Hilfe der hyperbolischen Functionen lïsst sich im Falle $F(u, u)<0$ der Werth des Integrals $(17 a)$ ausdrücken. Wir wollen hierbei zwei Fälle unterscheiden, je nachdem $A f(y, y)$ positiv oder negativ ist; es sei zunächst $A f(y, y)>0$, etwa gleich $+\Delta^{2}$. Alsdann lässt sich (10) in die Form bringen

setzt man jetzt

$$
\left(\frac{\sqrt{-F(u, u)} f(y, x)}{\Delta \cdot u_{x}}\right)^{2}-\left(\frac{f(z, x)}{\Delta \cdot u_{x}}\right)^{2}=1 ;
$$

$$
\left\{\begin{aligned}
\frac{\sqrt{-F(u, u)} f(y, x)}{\Delta \cdot u_{x}} & =\cos \operatorname{hyp} \tau=\frac{1}{2}\left(e^{\tau}+e^{-\tau}\right), \\
\frac{f(z, x)}{\Delta \cdot u_{x}} & =\sin \text { hyp } \tau=\frac{1}{2}\left(e^{\tau}-e^{-\tau}\right),
\end{aligned}\right.
$$

so folgt in ähnlicher Weise wie oben

$$
\int \frac{\sum \pm\left(z_{1} x_{2} d x_{3}\right)}{f(z, x) u_{x}}=-\frac{1}{\sqrt{-F(u, u)}} \tau+\text { Const. }
$$

Wenn $A f(y, y)<0$, etwa gleich $-\Delta^{2}$ ist, schreibt man (10) in der Form

setzt

$$
\left(\frac{f(z, x)}{\Delta \cdot u_{x}}\right)^{2}-\left(\frac{\sqrt{-F(u, u)} f(y, x)}{\Delta \cdot u_{x}}\right)^{2}=1
$$


und findet wieder

$$
\frac{f(z, x)}{\Delta \cdot u_{x}}=\cos \text { hyp } \tau, \quad \frac{\sqrt{-F(u, u)} f(y, x)}{\Delta \cdot u_{x}}=\sin \text { hyp } \tau
$$

$$
\int \frac{\sum \pm\left(z_{1} x_{2} d x_{3}\right)}{f(z, x) \cdot u_{x}}=-\frac{1}{\sqrt{-\boldsymbol{F}(u, u)}} \tau+\text { Const. }
$$

$\mathrm{Um}$ weitere Theoreme zu erhalten, setzen wir

$$
y_{1}=u_{2} a_{3}-u_{3} a_{2}, \quad y_{2}=u_{3} a_{1}-u_{1} a_{3}, \quad y_{3}=u_{1} a_{2}-u_{2} a_{1} ;
$$

alsdann ist $f(z, x)$ nach $(7)$ zunächst gleich

$$
\sum \pm\left(\frac{1}{2} f^{\prime}\left(y_{1}\right) u_{2} \frac{1}{2} f^{\prime}\left(x_{3}\right)\right)
$$

und wenu man für $y_{i}$ die Werthe (19) einführt, wird

daher

$$
\frac{1}{2} f^{\prime}\left(y_{i}\right)=\sum \pm\left(a_{i 1} u_{2} a_{3}\right)
$$

$$
f(z, x)=\left|\begin{array}{ccccc}
a_{11} & a_{12} & a_{13} & u_{1} & a_{1} \\
a_{21} & a_{22} & a_{23} & u_{2} & a_{2} \\
a_{31} & a_{32} & a_{33} & u_{3} & a_{3} \\
u_{1} & u_{2} & u_{3} & 0 & 0 \\
\frac{1}{2} f^{\prime}\left(x_{1}\right) & \frac{1}{2} f^{\prime}\left(x_{2}\right) & \frac{1}{2} f^{\prime}\left(x_{3}\right) & 0 & 0
\end{array}\right|
$$

Hieraus folgt

$$
=F(u, u) a_{x}-F(u, a) u_{x} .
$$

$$
\frac{F(u, u) \cdot a_{x}}{u_{x}}=F(u, a)+\frac{f(z, x)}{u_{x}},
$$

und durch Multiplication dieser Gleichung mit (14) erhält man:

$$
\frac{F(u, u) \cdot a_{x} \cdot \sum \pm\left(z_{1} x_{2} d x_{3}\right)}{u_{x}^{2} \cdot f(z, x)}=\frac{F(u, a)}{\sqrt{\boldsymbol{F}^{2}(u, u)}} d t+\frac{\sqrt{-A f(y, y)}}{\sqrt{\boldsymbol{F}(u, u)}} \sin t d t
$$

wobei für $\frac{f(z, x)}{u_{x}}$ der aus der zweiten Gleichung (11) folgende Werth eingesetzt wurde. Die Integration von (22) ergibt sich sofort mit Benutzung der ersten Gleichung (11) in der Gestalt ${ }^{1}$ )

$$
\begin{array}{r}
F(u, u) \int \frac{a_{x} \cdot \sum_{u_{x}{ }^{2} \cdot f(z, x)} \pm\left(z_{1} x_{2} d x_{3}\right)}{u^{2}}=\frac{F(u, a)}{\sqrt{F^{\prime}(u, u)}} t-\frac{f(y, x)}{u_{x}} \\
=F(u, a) . \int \frac{\sum \pm\left(z_{1} x_{2} d x_{3}\right)}{f(z, x) \cdot u_{x}}-\frac{f(y, x)}{u_{x}},
\end{array}
$$

wobei $f(y, x)=\sum \pm\left(\frac{1}{2} f^{\prime}\left(x_{1}\right) u_{3} a_{3}\right)$.

1) Vgl. hierzu Aronhold a. a. 0. S. $105 \mathrm{f}$. 
Beiläufig folgt im Falle $F(u, u)=0$ :

$$
\int \frac{\sum \pm\left(z_{1} x_{2} d x_{3}\right)}{f(z, x) \cdot u_{x}}=\frac{1}{\boldsymbol{F}(u, a)} \cdot \frac{f(y, x)}{u_{x}}+\text { Const. }
$$

Wird die Gleichung (21) auf die $n^{\text {te }}$ Potenz erhoben und hierauf mit (14) multiplicirt, so erhält man allgemeiner ${ }^{1}$ )

$$
\begin{aligned}
& \frac{F^{n}(u, u) \cdot a_{x}^{n} \cdot \sum \pm\left(z_{1} x_{2} d x_{3}\right)}{u_{x}^{n+1} \cdot f(z, x)}= \\
& \quad=[F(u, a)+\sqrt{-A f(y, y)} \sin t]^{n} \cdot \frac{d t}{\sqrt{F(u, u)}} \cdot
\end{aligned}
$$

Bei der Integration hätte man hier so zu verfahren, dass nur Potenzen von $\sin t$ und $\cos t$ auftreten, für die alsdanu die Werthe (11) zu substituiren wären.

Mit dem Vorhergehenden ist gezeigt, wie das Integral

$$
\int \frac{a_{x}^{n} \cdot \sum_{u_{x}^{n+1} \cdot f(z, x)} \pm\left(z_{1} x_{2} d x_{3}\right)}{u_{x}}
$$

zurïckgeführt werden kann auf das einfachere

$$
\int \sum \frac{ \pm\left(z_{1} x_{2} d x_{3}\right)}{u_{x} \cdot f(z, x)}
$$

welches auf Grund von. (14a) und (17a) durch eine cyklometrische Function oder einen Logarithmus ausgedrückt wird, je nachdem $F(u, u)>0$ oder $<0$ ist.

Auch die Integration von

$$
\int \varphi(x, y) \cdot d x
$$

wobei $\varphi(x, y)$ eine beliebige rationale Function von $x$ und $y$ ist, während $x$ und $y$ durch die Gleichung zweiten Grades $f(x, y, 1)=0$ verbunden sind, ist vermöge der Formeln (s) zurückgeführt auf die Integration rationaler Functionen entweder von $\sin t$ und $\cos t$ (im Falle (11)) oder von $t$ (im Falle (16)). Zum Zweck eines eingehenderen Studiums dieses allgemeinen Falles vergleiche man die Entwickelungen von Aronhold zu Gleichung (6)-(9), S. $130 \mathrm{f}$. in der oben citirten Abhandlung.

1) Man vergleiche auch hier die Darstellung bei Aronbold a. a. 0. S. $109 \mathrm{ff}$. 
* 363. Ueber eine Verallgemeinerung der Weierstrassschen Methode, elliptische Integrale auf die Normalform zu reduciren ${ }^{\mathbf{1}}$.

Die Grundlage der soeben genannten Methode besteht darin, dass das Integral

$$
\int_{x_{0}}^{x} \frac{d x}{\sqrt{R(x)}}, R(x)=A x^{4}+4 B x^{3}+6 C x^{2}+4 B^{\prime} x+A^{\prime}
$$

vermöge der Substitution

$$
\left\{\begin{array}{c}
s=\frac{G_{1}\left(x_{0}, x\right)+\sqrt{R\left(x_{0}\right)} \sqrt{R(x)}}{2\left(x-x_{0}\right)^{2}} \\
G_{1}\left(x_{0}, x\right)=x_{0}{ }^{2}\left(A x^{2}+2 B x+C\right)+2 x_{0}\left(B x^{2}+2 C x+B^{\prime}\right) \\
+\left(C x^{2}+2 B^{\prime} x+A^{\prime}\right)
\end{array}\right.
$$

in die Normalform

$$
\int_{s}^{\infty} \frac{d s}{\sqrt{4 s^{3}-g_{2} s-g_{3}}}
$$

übergeführt wird. Bei der hohen Bedeutung, welche diese Substitution (2) namentlich bei Anwendungen der elliptischen Functionen besitzt, wollen wir im Folgenden eive analoge Formel für das allgemeinere Integral

$$
J=\int \frac{\sum \pm\left(c_{1} x_{2} d x_{3}\right)}{\left[\frac{1}{2} c_{1} g^{\prime}\left(x_{1}\right)+\frac{1}{2} c_{2} g^{\prime}\left(x_{2}\right)+\frac{1}{2} c_{3} g^{\prime}\left(x_{3}\right)\right] \sqrt{f(x, x)}}, g(x, x)=0
$$

gewinnen, in welchem $g(x, x)$ und $f(x, x)$ irgend zwei ternäre quadratische Formen

$$
\left\{\begin{array}{l}
g(x, x) \equiv \sum_{1}^{3} \sum_{1}^{3} b_{i k} x_{i} x_{k}, \quad b_{i k}=b_{k i}, \\
f(x, x) \equiv \sum_{1}^{3} \sum_{1}^{3} a_{i k} x_{i} x_{k}, \quad a_{i k}=a_{k i},
\end{array}\right.
$$

bedeuten, und welches beim Schliessungsproblem der dem Kegelschnitte $g(x, x)=0$ eingeschriebenen und dem Kegelschnitte $f(x, x)=0$ umschriebenen Polygone auftritt ${ }^{2}$ ). Abgesehen davon, dass die auf

1) Der Inhalt der bier folgenden Entwickelung wurde im Jabre 1886 von Herrn Gundelfinger dem der Wissenschaft leider so früh entrissenen Mathematiker Halphen mitgetheilt. Vgl. auch das Werk von Halphen: „Traité des fonctions elliptiques et de leurs applications", Bd. 2, Paris 1888, S. 411.

2) Vgl. Gundelfinger: „Ueber das Schliessungsproblem bei zwei Kegelschnitten", Journal für die reine und angewandte Mathematik, Bd. 83, S. 171-174, 1877 . 
$\int \frac{d x}{\sqrt{R(x)}}$ bezüglichen Formeln sofort aus dem Integrale (4) hervorgehen, wenn man

$$
\begin{aligned}
& g \equiv 4 x_{1} x_{3}-x_{2}{ }^{2}, \text { also } x_{1}: x_{2}: x_{3}=x^{2}: 2 x: 1, \\
& f \equiv A x_{1}{ }^{2}+2 B x_{1} x_{2}+C x_{2}{ }^{2}+2 C x_{1} x_{3}+2 B^{\prime} x_{2} x_{3}+A^{\prime} x_{3}{ }^{2}
\end{aligned}
$$

annimmt, sind in (4) noch eine Anzahl weiterer wichtiger und vielfach behandelter Integrale enthalten.

Hat z. B. $g(x, x)$ die Gestalt

$$
g(x, x) \equiv x_{1}{ }^{2}+x_{2}{ }^{2}-x_{3}{ }^{2}=0,
$$

oder, was auf dasselbe hinauskommt, setzt man

$$
x_{1}: x_{3}=\cos \varphi, \quad x_{2}: x_{3}=\sin \varphi,
$$

so gelangt man zu dem von Gauss in der "Determinatio attractionis etc." 1) und von Jacobi²) behandelten Integrale. Ebenso kann man das Pentagramma mirificum von $\mathrm{Gauss}^{3}$ ) äusserst elegant ohne Auflösung einer kubischen Gleichung wach den hier mitzutheilenden Formeln behandeln, wenn man $g=0$ als die Gleichung eines Kegels zweiter Ordnung in rechtwinkligen Raumcoordinaten $x_{1}, x_{2}, x_{3}$ und $f=0$ als die Gleichung des dazu gehörigen Reciprokalkegels auffasst.

Wir behaupten nun:

(6) Bedeuten $c_{1}, c_{2}, c_{3}$ die Coordinaten eines willkürlich, aber fest gewählten Punktes auf dem Kegelschnitt $g(x, x)=0$ und setzt man

$$
\begin{aligned}
G(\lambda) & =\sum \pm\left(\lambda b_{11}-a_{11} \lambda b_{22}-a_{22} \lambda b_{33}-\left(a_{33}\right)\right. \\
& =\lambda^{3} B-3 \lambda^{2} \Theta+3 \lambda H-A,
\end{aligned}
$$$$
\text { (8) }\left\{\begin{array}{r}
\psi(x, x) \equiv 3 \mathrm{H} g(x, x)-2 \chi(x, x), \\
\text { wobei } \left.\chi(x, x) \equiv \sum_{1}^{3} \sum_{1}^{3}(a, b)_{i k} f_{i} y_{k},{ }^{4}\right) f_{i}=\frac{1}{2} f^{\prime}\left(x_{i}\right), \\
y_{k}=\frac{1}{2} g^{\prime}\left(x_{k}\right),
\end{array}\right.
$$

1) Commentationes soc. reg. scient. Gotting. rec., Bd. 4, S. 21-48, 1818, oder Gauss' Werke, hrsgg. 'von der kgl. Ges. d. Wissensch. zu Göttingen, Bd. 3, S. $331-355$.

2) Journal für die reine und angewandte Mathematik, Bd. 8, S. 253 ff., 1832.

3) Gauss' Werke, Bd. 3, S. $481-490$.

4) Durch Vergleichung der Coefficienten von $\lambda^{1}$ in (5), S. 160 erkennt man, dass

$$
\sum_{1}^{3} \sum_{1}^{3}(a, b)_{i k} f_{i} g_{k}=\sum_{i}^{3} \sum_{1}^{3}(A, B)_{i k} x_{i} x_{k} .
$$


so geht vermöge der Transformation

$$
\lambda^{2} g(c, x)-2 \lambda f(c, x)-\frac{\psi(c, x)}{B}=0, \quad g(x, x)=0
$$

das Differential

$$
d J=\frac{\sum \pm\left(c_{1} x_{2} d x_{3}\right)}{g(c, x) \sqrt{f(x, x)}}, \quad g(x, x)=0
$$

über in:

$$
-\frac{d \lambda}{\sqrt{G(\lambda)}} .
$$

Zum Beweis dieser Behauptung werde zur Abkürzung eingeführt

$$
\Phi(x, x) \equiv \lambda^{2} g(x, x)-2 \lambda f(x, x)-\frac{\psi(x, x)}{B} ;
$$

alsdann kann man die Transformation (9) einfach ausdrücken durch

$$
\left\{\begin{array}{l}
\Phi(c, x) \equiv \frac{1}{2} \Phi^{\prime}\left(c_{1}\right) x_{1}+\frac{1}{2} \Phi^{\prime}\left(c_{2}\right) x_{2}+\frac{1}{2} \Phi^{\prime}\left(c_{3}\right) x_{3}=0 \\
g(x, x) \equiv \frac{1}{2} g^{\prime}\left(x_{1}\right) x_{1}+\frac{1}{2} g^{\prime}\left(x_{2}\right) x_{2}+\frac{1}{2} g^{\prime}\left(x_{3}\right) x_{3}=0
\end{array}\right.
$$

Hieraus folgt

$$
\varrho x_{1}=\frac{1}{4}\left\{\Phi^{\prime}\left(c_{2}\right) g^{\prime}\left(x_{3}\right)-\Phi^{\prime}\left(c_{3}\right) g^{\prime}\left(x_{2}\right)\right\},
$$

und analoge Werthe hat man für $\varrho x_{2}$ und $\varrho x_{3}$, wobei der Proportionalitätsfactor $\varrho$ ähnlich wie S. $35 \mathrm{f}$. gegeben ist durch

$$
\varrho^{2}=-\frac{1}{4}\left\{B_{11} \Phi^{\prime}\left(c_{1}\right)^{2}+2 B_{12} \Phi^{\prime}\left(c_{1}\right) \cdot \Phi^{\prime}\left(c_{2}\right)+\cdots\right\}
$$

oder auch durch

$$
\varrho^{2}=4\left(\lambda^{3} B-3 \lambda^{2} \Theta+3 \lambda H-A\right) f(c, c),
$$

denn es besteht die Identität

$$
\begin{aligned}
-\frac{1}{4}\left\{B_{11} \Phi^{\prime}\left(c_{1}\right)^{2}\right. & \left.+2 B_{12} \Phi^{\prime}\left(c_{1}\right) \cdot \Phi^{\prime}\left(c_{2}\right)+\cdots\right\} \\
= & 4\left(\lambda^{3} B-3 \lambda^{2} \Theta+3 \lambda H-A\right) f(c, c),
\end{aligned}
$$

welche mit Hilfe "kanonischer Formen" für $g(x, x)$ und $f(x, x)$ bestätigt werden kann $^{1}$ ). Durch Einführung der Werthe für $\varrho x_{i}$ in (10) erhält man

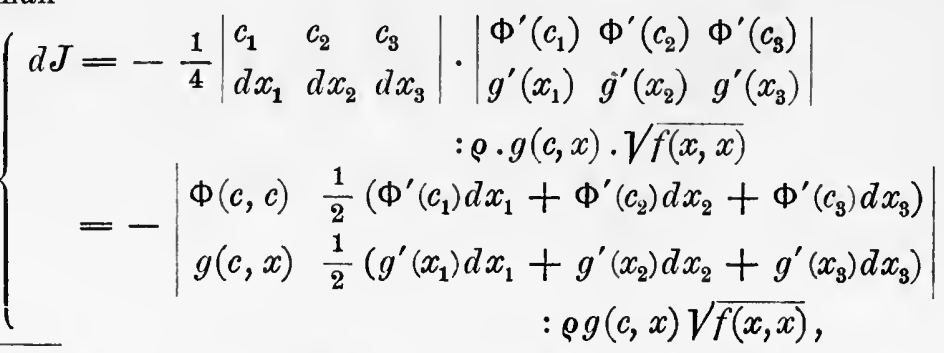

1) Vgl. auch die Bemerkung am Schluss dieser Betrachtungen, S. 426. 
woraus zufolge $g^{\prime}\left(x_{1}\right) d x_{1}+g^{\prime}\left(x_{2}\right) d x_{2}+g^{\prime}\left(x_{3}\right) d x_{3}=0$ hervorgeht:

$(17 a)$

$$
d J=\frac{\frac{1}{2}\left(\Phi^{\prime}\left(c_{1}\right) d x_{1}+\Phi^{\prime}\left(c_{2}\right) d x_{2}+\Phi^{\prime}\left(c_{3}\right) d x_{3}\right)}{\rho \sqrt{f(x, x)}} .
$$

Andrerseits folgt durch totale Differentiation der Substitution (9): (18) $\frac{1}{2}\left(\Phi^{\prime}\left(c_{1}\right) d x_{1}+\Phi^{\prime}\left(c_{2}\right) d x_{2}+\Phi^{\prime}\left(c_{3}\right) d x_{3}\right)+2 d \lambda(\lambda g(c, x)-f(c, x))=0$; daher wird

$$
d J=\frac{-2 d \lambda(\lambda g(c, x)-f(c, x))}{\varrho \sqrt{f(x, x)}},
$$

und hier kann für $\lambda g(c, x)-f(c, x)$ nach Auflösung der in $\lambda$ quadratischen Gleichung $(9)$ gesetzt werden

$$
\sqrt{\frac{g(c, x) \cdot \psi(c, x)}{B}+B f^{2} \overline{(c, x)}},
$$

so dass sich (19) verwandelt in

$$
d J=\frac{-2 d \lambda \sqrt{g(c, x) \cdot \psi(c, x)+B f^{2}(c, x)}}{\varrho \sqrt{\bar{B}}} \cdot \frac{\sqrt{f(x, x)}}{\sqrt{f(x,}} .
$$

Es besteht nun die Relation

$$
\left\{\begin{aligned}
& \left(f(c, c) f(x, x)-f^{2}(c, x)\right) B \\
= & g(c, x) \cdot \psi(c, x)-(3 \mathrm{H} g(c, c)-\chi(c, c)) g(x, x)+\chi(x, x) \cdot g(c, c),
\end{aligned}\right.
$$

welche mit Hilfe kanonischer Formen bewiesen werden kann und sich im gegenwärtigen Falle zufolge $g(x, x)=0$ und $g(c, c)=0$ reducirt auf (22)

$$
g(c, x) \cdot \psi(c, x)+B f^{2}(c, x)=B f(c, c) f(x, x) .
$$

Auf Grund dieser Gleichung erhält man aus (20):

$$
d J=-\frac{2 \sqrt{f(c, c)} d \lambda}{\varrho},
$$

folglich mit Rücksicht auf $(15 a)$ :

$$
d J=\frac{-d \lambda}{\sqrt{\lambda^{3} B-3 \lambda^{2} \Theta+3 \lambda H-A}}=\frac{-d \lambda}{\sqrt{G(\lambda)}} .
$$

Hier wäre noch zu setzen $\lambda B-\Theta=4 s$.

Bezüglich der Bestimmung des Vorzeichens sei endlich bemerkt, dass $\sqrt{f(x, x)}$ vorgelegt, also gegeben ist; bei $\sqrt{f(c, c)}$ kann das Vorzeichen willkürlich gewählt werden; doch ist alsdann das Vorzeichen von $\sqrt{G(\lambda)}$ in dem Ausdrucke $\varrho=2 \sqrt{f(c, c)} \sqrt{G(\lambda)}$ so zu wählen, dass in den Auflösungen (14) der Substitution (9) die $x_{i}$ demjenigen der beiden Schnittpunkte von $g(x, x)=0$ und $\Phi(c, x)=0$ angehören, welcher durch ein fest gewähltes Vorzeichen von $\varrho$ in (14) bestimmt wurde. 
Was die geometrische Bedeutung der Substitution (9) betrifft, so möge noch bemerkt werden, dass die Identität (21) im Falle $g(c, c)=0$, der ja bei unserer Untersuchung vorliegt, auch geschrieben werden kann in der Form

$$
B f(c, c) f(x, x)-\chi(c, c) g(x, x)=g(c, x) \psi(c, x)+B f^{2}(c, x),
$$

aus welcher mit Hilfe der Entwickelungen S. $83 \mathrm{f}$. hervorgeht, dass $B f(c, c) f(x, x)-\chi(c, c) g(x, x)=0$ derjenige Kegelschnitt des Büschels $f(x, x)-\lambda g(x, x)=0$ ist, welcher nach (5) in $\S 15$ die Tangente $g(c, x)=0$ von $g(x, x)=0$ gleichfalls berührt. Andrerseits zeigen die rechte Seite von (24) und die Substitution (9), dass derselbe Kegelschnitt die Enveloppe ${ }^{1}$ ) aller Geraden ist, die für veränderliche Werthe von $\lambda$ durch $\lambda^{2} g(c, x)-2 \lambda f(c, x)-\frac{\psi(c, x)}{B}=0$ dargestellt werden.

Will man speciell die übrigen gemeinschaftlichen Tangenten des letzterwähnten und des Kegelschnitts $g(x, x)=0$ haben, so ist nach S. 36 auf Grund von (13) und (15) zu setzen $\varrho=0$ oder

$$
B_{11} \Phi^{\prime 2}\left(c_{1}\right)+2 B_{12} \Phi^{\prime}\left(c_{1}\right) \Phi^{\prime}\left(c_{2}\right)+\cdots=0 .
$$

Es zeigt sich hier eine Bestätigung des Umstandes, dass nach (285) das Doppelverhältniss der vier gemeinsamen Tangenten von (24) gleich ist dem Doppelverhältniss der vier auf $g(x, x)=0$ gelegenen Grundpunkte des Büschels $\lambda g(x, x)-f(x, x)=0$.

Die allgemeine Auflösung der Substitution (9) oder (13) ist durch (14) und (15) gegeben.

1) Den unendlich vielen Werthen des Parameters $\lambda$ in (9) entsprechen unendlich viele Geraden, welche eine Curve, die sogenannte Enveloppe des Systems von Geraden, umhüllen. Wie bereits in (220) erwähnt wurde, erhält man die Gleichung der Enveloppe dadurch, dass man die Discriminante derjenigen Gleichung bildet, welche den Parameter als Veränderliche enthält. 


\section{Alphabetisches Sachregister.}

Die Zahlen beziehen sich auf die Seiten. Die Buchstabe K. ist als Abkürzung gebraucht für Kegelschnitt; die übrigen als Abkürzungen dienenden grossen Buchstaben bezeichnen das Wort, unter dem sie vorkommen. Die Worte eingeschrieben oder umschrieben beziehen sich, wenn nichts anderes bemerkt ist, auf ein Dreieck.

\begin{abstract}
Abstand eines Punktes von einer Geraden 3 f. 9.56 ; der Brennpunkte von einer Tangente $179 \mathrm{f}$. $353 \mathrm{f}$.; des Mittelpunktes von der Directrix 118; des Scheitels der Parabel vou Brennpunkt und Directrix 117. 119; eines Curvenpunktes vou Brennpunkt und Directrix 115. $118 \mathrm{f}$; ; eines Curvenpunktes von zwei Tangenten und von der Berührungssehne 281 ; eines Curvenpunktes von den Asymptoten 263; gerader Linien von zwei festen Punkten 353 f; zweier Punkte 59.
\end{abstract}

Aequianharmonische Kegelschnitte eines Büschels $368 \mathrm{ff}$; Strahlen 366 .

Aehnliche Kegelschnitte 112. $290 \mathrm{f}$; ; iu einem Büschel 361 , in einer Schair 361 ; umschriebene K. $306 \mathrm{ff}$. $312 \mathrm{f}$; eingeschriebene K. $309 \mathrm{ff} .312 \mathrm{f}$; ; bei gegebenem Poldreieck 312. 361; concentrische $K$. $312 \mathrm{f}$.

Aehnlich liegende Kegelschnitte 291. 309 ; zugleich ähnlich 292. 345 .

Aehnlichkeitskreis 317. $403 \mathrm{f}$.

Aehnlichkeitspunkte 317. $403 \mathrm{f}$.

Asymptoten 45. 49. 111. 278. 284; ibr Winkel 111f. 284. 320; Max. oder Min. des Winkels bei $\mathbf{K}$, einer Schaar $361 \mathrm{f}$; ibr Abstand von einem Curvenpunkt 263; Parallele zu einer A. 264; K. mit einer parallelen A. 346 ; Enveloppe der A. der umschr. gleichseitigen Hyperbeln 412.

Ausartende Kegelschnitte einem Dreieck umschrieben $328 f$, eingeschrieben $328 f$. 341 ; bei gegebenem Poldreiseit $328 \mathrm{f}$. 342; bei gegebenem Poldreieck 329; vgl. auch Geradenpaar und Punktepaar.

Axen der Kegelschnitte 85. $87 \mathrm{f}$, , der
Ellipse 110. 114. 169, der Hyperbel 110 f. 114 , der Parabel 103. 115. 289; Länge der A. 110; Product ihrer Längen 292f. 295 f. $298-303$. 309; gleiches Axenproduct bei umschriebenen K. 295, bei eingeschriebenen K. 296; gleiches Axenproduct bei K. mit gegebenem Poldreieck 296; Summe oderDifferenz der Axen 293. 297. $319 \mathrm{f}$; constante Summe oder Differenz der Axen bei umschr. K. 297; Gleichungen der Axen 93. $96 \mathrm{f}$. 207. $285 \mathrm{ff} .382$, ihrer unendlich fernen Punkte 93. 96. $287 \mathrm{f}$; Parallele zur Axe der Parabel 264; Enveloppe der A. der K. cines Gewebes 409, der Parabeln mit gegebenem Poldreiseit $410 \mathrm{f}$; Enveloppe der A. der eingeschr. Parabeln 414.

Berührung: Berïhrungspunkt der Tangente eines K. 42; Grad der Bedingungsgleichung für Berührung bei Curven $m^{\text {ter }}$ und $n^{\text {ter }}$ Ordnung $379 \mathrm{f}$; einfach berührende K. 134.152. $154 \mathrm{f}$; doppelt berührende Kegelschnitte 311 370. 396-404, speciell für zwei Kreise 401-404; doppelt berïhrende Kreise 180 f. $183.333 \mathrm{ff}$.

Brennpunkte: ibre Lage $114 \mathrm{ff}$, bei eingeschriebenen $K$. $347 \mathrm{f}$, , bei eingeschr. Parabeln 347 f. 351 f. 407; ihre Lage bei gegebenem Poldreiseit $348 \mathrm{f}$., bei gegeb. Poldreiseit und gemeinsamer Tangente 352. 407; ihr Ábstand von einer Tangente 179f. $353 \mathrm{f}$; F Fusspunkte der von $B$. auf Tangenten gefällten Lothe $183 \mathrm{f}$. 186. 348; zwei Brennstrahlenpaare als Tangenten eines K. 187. 192 ff. 353 ; zwei Brennstrahlenpaare durch ein Punktepaar 
im Unendlichen 194; Verbindungslinie von B. mit Pol einer Sehne 192, einer Focalsehne 193; Brennpunkte von Kegelschnitten auf einer Geraden gelegen $347 \mathrm{ff}$, auf einem $K$. gelegen $348 \mathrm{f}$.; Brennpunkte einer Schaar $351 \mathrm{f}$. 395f.; Gleichungen der Brennpunkte 113f. 290. $346 \mathrm{f}$,, bei der Parabel 115; Ort der B. für die einem Vierseit eingeschr. K. 404-409.

Brianchon'scher Satz 128.

Büschel von Kegelschnitten, seine Definition 129. 131; Grundpunkte des B. 131; Geradenpaare in einem $132 \mathrm{f}$. 138 f.; B. einfach berührender K. 134. 152. $154 \mathrm{f}$., doppelt berührender $K$. 134. 153. 157 ; osculirender $K$. $135 \mathrm{f}$. 153. $156 \mathrm{f}$., mit vier zusammenfallenden Grundpunkten 136. 153. 158; aus Geradenpaaren bestehend $136 \mathrm{f}$; aus gleichseitigen Hyperbeln bestehend 330; Parabeln im B. 199. 331. $357 \mathrm{ff}$. 362; Schnittpunkte der $K$. eines B. mit einer Geraden $142 \mathrm{f}$; Kegelschn. im B. bei gegebener Tangente $141 \mathrm{ff}$; Büschel von Kreisen 336, seine Grenzpunkte $336 \mathrm{f}$. 390.401.

Cayley'sche Curve eines Netzes 207. 222-225. 231. 240. 388; einfachste Bildung ihrer Gleichung 225; als Hessiane eines Gewebes 230.240. $388 \mathrm{f}$; eines Netzes von Kreisen 389; eines Gewebes 228-240. 387. 405-409; als Combinante $382 \mathrm{f}$.

Combinante 205. 382 f.; $\psi 207$ f. $368-$ 371. 372-377.

Concentrische Kegelschnitte mit Nebenbedingungen 298-303; Kreise $172 \mathrm{f}$.

Confocale Kegelschnitte, Definition 146. 165; ihre Schnittpunkte 146f. 170; ihre Arten 170f.; Parabeln $175 \mathrm{f}$; Winkel der Tangentenpaare an conf. K. 147.

Congruente Kegelschnitte eingeschrieben $297 \mathrm{f}$., umschrieben 298.

Conjugirte Hyperbel 111. 377; Polaren 44, in Bezug auf das Kreispunktepaar 62; durch einen Brennpunkt 193 f. 354; Geraden in Bezug aut eine Schaar 200; Tangenten der Hessiane eines Gewebes 226. 229; Durchmesser $24 \mathrm{ff} .111 .261 .264 .279$ f. 281 f. 332 , ihr Winkel $276 \mathrm{f}$. 282, Summe oder Differenz ihrer Quadrate 282; Durchmesser von gleicher Grösse 50. 282; Pole 32, in Bezug auf das Kreispunktepaar 95, in Bezug auf das imaginäre Brennpunktepaar 181; Pole in Bezug auf ein Büschel 196, der Hessiane eines Netzes $219 \mathrm{ff}$. 224.229-232;
Lage von Kegelschnitten 162. $344 \mathrm{f}$. 393, von Kegelschnittsystemen 232240; Lage von gleichseitiger Hyperbel und Parabel 344.

Constante $\tau 60 \mathrm{ff} .92 \mathrm{f}$.

Contravariante 80. 83.

Coordinaten eines Punktes 2, einer Geraden $5 \mathrm{f}$; ; barycentrische 7 , homogene 8 , schiefwinklige Parallelcoord. 8. 56.

Covariante 78. 80; Hesse'sche 80, vgl. auch kubische Form; geometrische Deutung von Cov. 161. $163 \mathrm{ff}$; Relation zwischen Cov. eines Büschels $159 \mathrm{f}$.

Curve zweiter Ordnung, bezw. Classe 18, bezw. 27. 39, näheres s. bei Kegelschnitt; $n^{\text {ter }}$ Ordnung $21 ; n^{\text {ter }}$ Classe 42.

Definite Form 65. 326 f. 373 f.; vgl. auch 49. Desargues - Sturm'scher Satz $142 \mathrm{f}$.

Determinante des K. s. Discriminante; Multiplicationstheorem der Det. 76.

Directorkreis 148. 337; sein Radius 338. 344 , bei d. gleichseitigen Hyperbel $338 \mathrm{f}$; Relation $\mathrm{zwischen}$ seiner Gleichung und der des K. 338; seine Ausartungen $339 \mathrm{f}$; ; seine Beziehungen $\mathrm{zu}$ den durch Poldreiecken eines $K$. gehenden Kreisen $343 \mathrm{f}$; Radicalaxe der D. für $z$ wei eingeschriebene $K$. $391 \mathrm{f}$; B B̈schel der D. einer Schaar 340; Netz der D. eines Gewebes $390 \mathrm{f}$; eines Gewebes von K. mit gemeinsamem Poldreiseit 392.

Directrix, Definition 115, ibre Lage 116; der Parabel $117 \mathrm{ff} .148 \mathrm{f}$., als Ort für d. Mittelpunkte von Kreisen, die Poldreiecken umschrieben $344 ; \mathrm{Be}$ ziehung zwischen $\mathrm{D}$. und Berïhrungssehne zweier Tangenten 325.

Discriminante des Kegelschnitts 26. 29. 39 f. 43 f. 50 ; als Invariante 78 ff. 81 .

Doppelgerade 30. 51. 54 .

Doppelpunkt 40. 53. 55; als Ausartung eines Kreises 57.

Doppelverhältniss der Schnittpunkte einer Geraden mit zwei K. $143 \mathrm{f}$; von vier Punkten eines K. 124; der vier Grundpunkte eines Büschels $366 \mathrm{ff}$. 426; der vier gemeinsamen Tangenten einer Schaar $367 \mathrm{f}$. 426; der Schnittpunkte von Gerade und Kegelschnitt zu zwei Punkten auf der Geraden 21. 63.

Dreieck, sein Inhalt $248 \mathrm{ff}$; gebildet durch eine Tangente und zwei Asymptoten 278.

Dreiseit, parallel umschrieben 330; parallel eingeschrieben $247 \mathrm{f}$.

Dualistisch entsprechende Sätze 39.

Durchmesser s. conjugirte Durchmesser. 
Einheitspunkt 1.

Ellipse 46 f. 49. 52. 54f. 108; imaginäre E. 49 f. 54 f. 108; ihre Gestalt 50.110 , ihre Axen 110. 114. 169, ihr Inhalt 293. 296; Definition mit Hilfe der Brennstrahlen 121; eingeschrieben mit Mittelpunkt im Schwerpunkt 255.316; nmschrieben mit Mittelpunkt im Schwerpunkt $258 \mathrm{f}$.

Entfernung s. Abstand.

Enveloppe gerader Linien, die einer Bedingung unterworfen $272 \mathrm{f}$.

Evolute einer Curve 2. Ordnung 216f., speciell Ellipse oder Hyperbel 217. 382, Parabel $217 \mathrm{f}$.; einer Curve 2. Classe $214 \mathrm{f}$, speciell Ellipse oder Hyperbel 215 f., Parabel 216; einer Curve $n^{\text {ter }}$ Ordnung $379 \mathrm{f}$; einer Curve $k^{\text {ter }}$ Classe $380 \mathrm{f}$.

Excentricität, lineare 118, numerische 119.

Feld, trigonales und tetragonales 2 . Vgl. auch Tetragonal und Trigonal.

Feuerbach'scher Kreis 304f. 313. 349. 352. 411; Potenz in Bezug auf ihn $319 \mathrm{f}$.

Form 65; bilineare F. 74f. 94; definite F. 65. 326 f. $373 \mathrm{f}$; vgl. auch kubische Form.

Frégier, Theorem von 273; verallgemeinert $332 \mathrm{f}$.

Function, Identität zweier ganzen homo. genen F. $75 \mathrm{f}$.

Gerade, ihre Gleichung 5; ibre Gl. in der Normalform 10; unendlich ferne 3. 51. 54. 107. 326, ihre Schnittpunkte mit einem Kegelschnitt $46 \mathrm{ff}$.; die unendlich ferne G. doppelt zäblend als Ausartung .eines Kreises $59 \mathrm{f}$.

Geradenpaar 29 f. 38. 44. 50. 54. 108. 262, im Kegelschnittbüschel $132 \mathrm{f}$. $138 \mathrm{f}$; ; kubische Gl. zur Bestimmung der G. im Kegelschnittbüschel 132136. 149-152; als harmonisches Po. larenpaar zu einem Kegelschnitt 164; circulares 329. 336. 339. 390. 392. 409; nach den Schnittpunkten eines Kegelschnitts und einer Secante gezogen 261; Spitze eines G. 29, 44, dieselbe auf einem Kegelschnitt gelegen $163 \mathrm{ff}$; Hessiane als Ort für die Spitzen der Geradenpaare in einem Netz 220.

Gewebe von Kegelschnitten, Definition 226; von Kegelschnitten mit einer, zwei oder drei gemeinsamen Tangenten 387; mit einem Doppelpunkt 408 f.; mit zwei Doppelpunkten 394. $403 \mathrm{f}$; ; Directorkreise der $K$. eines
Gewebes $390 \mathrm{ff}$; Ort für die Mittelpunkte der gleichseitigen Hyperbeln eines G. 392.

Gleichseitige Hyperbel vgl. Hyperbel.

Gleichungen mit nur reellen Wurzeln 67-72. 87; mit nur rein imaginäre Wurzeln 75; vgl. auch kubische Gleichungen.

Grenzpunkte eines Büschels von Kreisen 336 f. 390.401.

Grundpunkte eines Kegelschnittbüschels 131; ihr Doppelverhältniss $366 \mathrm{ff}$.

Halbmesser eines Kegelschnitts zu einander rechtwinklig 283.

Harmonicale eines Punktes in Bezug auf ein Dreieck 255. 358.

Harmonische Polaren, bezw. Pole vgl. conjugirte Polaren, bew. Pole.

Harmonische Lage von Geraden, bezw. Punkten bei Kegelschnitten $277 \mathrm{f}$. $280 \mathrm{f}$. 314 f. 344. 353. 392 f, vgl. anch conjugirte Polaren, bezw. Pole.

Harmonische Curve zweiter Ordnung 146. 159.370; $\mathrm{zw}$ weiter Classe 144.370. $388 \mathrm{f}$;; Kegelschnitte eines Büschels $369 \mathrm{ff}$.

Hauptaxen, Transformation eines K. auf die Hauptaxen 85. 91; vgl. auch Axe.

Hermite'sche Curve s. Cayley'sche Curve.

Hessiane als Combinante $382 \mathrm{f}$; eines Gewebes 226 f. 240.387; als Cayley'sche Curve eines Netzes 230. 240. $388 \mathrm{f}$.; conjugirte Tangenten der $\mathrm{H}$. eines Gewebes 226. 229; umhüllt von den Trägern der Punktepaare des Gewebes $226 \mathrm{f}$; eines Netzes 218-222. $230 \mathrm{f}$. 240. 384; als Cayley'sche Curve eines Gewebes 230. 240. 389. 405; conjugirte Pole der $H$. eines Netzes 219 ff. 224. $229-232$; als Ort für die Spitzen der Geradenpaare des Netzes 220; eines Netzes von Kreisen $389 \mathrm{f}$.

Höhen des Coordinatendreiecks 244. 330 .

Höhenfusspunkte im Dreieck 248. 330 .

Höhenschuittpunkt des Coordinatendreiecks 246. 341. 345. 390f. 413; gelegen auf den dem Dreieck umschriebenen gleichseitigen Hyperbeln 305 . $330 \mathrm{f}$; ; Tangenten vom $\mathrm{H}$. an die über den Seiten des Dreiecks als Durchmessern beschriebenen Kreise 391; Ort des $\mathrm{H}$. bei gewissen eingeschriebenen oder umschriebenen Kegelschnitten 345 f. 348.; Relation zwischen H. und Mittelpunkt eines eingeschriebenen K. 391.

Hyperbel 46 ff. 52.54 f. 108; ihre Gestalt 49. 111; ibre Axen 110. 114; Definition mit Hilfe der Brennstrablen 121; conjugirte H. 111. 377; gleichseitige 
H. 111. 265, Bedingung für dieselbe 303 , ihre conjugirten Durchmesser 265, ihr Directorkreis $338 \mathrm{f}$; gleichseitige $\mathrm{H}$. im Kegelschnittbüschel 330 , in einer Schaar 361 ; Schnittpunkte zweier gleichseitiger H. 137; Büschel gleichseitiger $\mathrm{H} .330$; umschriebene gleichseitige $H$. gehen durch den Höhenschnittpunkt 305. $330 \mathrm{f}$; ; ihr Poldreieck 330; Ort der Mittelpunkte umschriebener gleichs. H. $304 \mathrm{f}$, eingeschriebener gleichs. H. 304. 390f.; Ort der Mittelpunkte gleichs. $H$. mit gegebenem Poldreieck 306, der gleichs. H. eines Gewebes 392; gleichs. H. als Mittelpunktskegelschnitt 362 ; conjugirte Luge von gleichs. $\mathbf{H}$. und Parabel 344; Enveloppe der Asymptoten der umschr. gleichs. H. 412.

Integral, Anwendung von Poldreiecken bei gewissen I. 415-421, Transformation der elliptischen I. auf die Normalform $422-426$.

Invarianten 77; ibr Gewicht 77; absolute 78; sinultane 79. $81 \mathrm{f}$; geometrische Deutung von I. 161-165; Berechnung gewisser fundamentaler $I$. $294 \mathrm{f}$.

Involution conjugirter Pole 32 , conjugirter Polaren 44; Construction der Doppelstrahlen einer I. 335; Involutionscentrum 335 .

Kegelschnitt, seine Gleichung in Punktcoordinaten 19 , in Liniencoordinaten 27. 39 f. 51. 82. y1. 113. 120; definirt mit Hilfe von Brenupunkt und Directrix 115. 117. 119, mit Hilfe der Brennstrablen 121. $187 \mathrm{ff}$; bestimmt durch fünf Punkte $19 \mathrm{f}$. 121f. 137, durch fünf Tangenten 40.123 ; bestimmt durch das Brennpunktepaar und eine Tangente 191; specielle Formen der Gleichung eines K. $49 \mathrm{f}$. $83 \mathrm{ff}$.; umschriebener K. 250. 254 f. 256 f. 295. 297-304. 319. 345, bei gegebenem Mittelpunkt 250. 294. 320 ; congruente umschr. K. 298; eingeschriebener K. 250. 254 f. 296 . $297-304$. 319 f. 346 , bei gegebenem Mittelpunkt 251. 294. 320 ; congruente eingeschr. K. $297 \mathrm{f}$; mit gegebenem Poldreieck 33. $296 \mathrm{f}$. 306. 320. 327, bei gegebenem Mittelpunkt 251. 295. 303. 320; mit gegebenem Poldreiseit und Mittelpunkt 251. 295; ausartende umschr. K. $328 \mathrm{f}$., eingeschr. K. 328f. 341; ausartende $\mathrm{K}$. bei gegebenem Poldreieck 329, - Poldreiseit 328 f. 342 ; Kriterien für
K. $54 \mathrm{f}$, bei schiefwinkligen Parallelcoordinaten 56; Art des umschriebenen K. abhängig von der Lage des Mittelpunktes $251 \mathrm{ff}$; ; Art des eingeschr. $K$. abhängig von der Lage des Mittelpunktes 253 , ebenso bei $K$. mit gegebenem Poldreieck $253 \mathrm{f}$; A Art der K. eines Büschels 356-359, einer Schaar $359 \mathrm{ff}$; Art des K., der durch drei Tangenten und einen Brennpunkt gegeben $349 \mathrm{ff}$.

Kreis als Ort von Punkten $57 \mathrm{ff} .110$. $266 \mathrm{f}$; umbüllt von Geraden 57 ; ansartend in eine im Endlichen und die im Unendlichen gelegene Gerade 59, in die doppelt zu zählende unendlich ferne Gerade $59 \mathrm{f}$, in ein circulares Geradenpaar 59 ; in das imaginäre Kreispunktepaar 57; in einen doppelt zu zählenden Punkt 57; Bedingungen für einen Kreis bei Punktcoordinaten $96 \mathrm{ff}$, bei Liniencoordinaten 173 ; Bestimmung des Radius 294; umschriebener Kreis 267. 297. $301 \mathrm{ff}$. 306. 313. $347 \mathrm{f}$. 392, Potenz in Bezug auf ihn $318 \mathrm{ff}$, sein Mittelpunkt 246.341.348; der nmschr. Kress als Ort der Brennpunkte der eingeschr. Parabeln $347 \mathrm{f}$. 351 f. 407. 414; ein- und angeschriebene Kreise $267 \mathrm{f}$, ihre Mittelpunkte 246. 306; mit gegebenem Dreieck als Poldreieck $268 \mathrm{f}$. 297. 304. 313. 345. $390 \mathrm{f}$, Potenz in Bezug auf ihn $319 \mathrm{f}$; Feuerbach'scher Kreis 304 f. 313. 349. 352, Potenz in Bezug auf ihn $319 \mathrm{f}$; concentrische Kreise 172f.; im Kegelschnittbüschel 331. 362; Kreisbüschel 336, seine Grenzpunkte 336 f. 390.401; mit zwei Brenustrahlenpaaren als Tangenten $187 \mathrm{ff}$; mit Focalsehne als Tangente, Pol der Sehne als Mittelpunkt 192; über der Hauptaxe als Durchmesser $184 \mathrm{f}$; über gegebener Strecke als Durchmesser 269f.; Ort, von welchem $\mathrm{zwei}$ Kreise gleich gross erscheinen 317 .

Kreispunktepaar, imaginäres 57. 60.62. 113. 266 f. 326 f. 389 ; conjugirte Polaren in Bezug auf dasselbe 62; conjugirte Pole 95.

Kriterien für die Kegelschnitte $54 \mathrm{f}$; bei schiefwinkligen Parallelcoordinaten 56.

Krümmungskreis bei Curven $k^{\text {ter }}$ Classe $208 \mathrm{f}$.

Krümmungsmittelpunkt bei Kegelschnitten 202. $212 \mathrm{f}$; ; bei Curven $n^{\text {ter }}$ Ordnung 213 ; bei Curven $k^{\text {ter }}$ Classe $209 \mathrm{ff}$. $378 \mathrm{f}$.

Krümmungsradius bei Kegelschnitten $212 \mathrm{f}$, , bei Curven $n^{\text {ter }}$ Ordnung 213, 
bei Carven $k^{\text {ter }}$ Classe 212. 379; von gleicher Länge bei einem Kegelschnitt 377 f., bei Curve $n^{\text {ter }}$ Ordnung 378, bei Curve $k^{\text {ter }}$ Classe 378 .

Kubische Form, binäre 363-366; ihre Discriminante $364 \mathrm{f}$. 371. 374; ihre Hesse'sche Covariante 363-367. $369 \mathrm{f}$. 373 ; ihre kubische Covariante $363 \mathrm{ff}$. 367. $369 f$.

Kubische Gleichung bei der Transformation auf die Hauptaxen 86; zur Bestimmung der Geradenpaare eines Büschels 132-136. 149-152; zur Bestimmung der Punktepaare einer confocalen Schaar 166. 168.

Liniencoordinaten, Gleichung des Kegelschnitts in 27.39f. 51. 82.91. 113.120; Transformation der L. 17.

Mitte einer Strecke 244.

Mittelloth einer Strecke 244, der Seiten des Coordinatendreiecks 245.

Mittelpunkt des Kegelschnitts $25.31 \mathrm{f}$. 43. 91. 265; seine Lage 114; trigonale oder tetragonale Lage des $M$. bei umschr. K. $251 \mathrm{ff}$, bei eingeschr. $K$. 253; bei K. mit gegebenem Poldreieck $253 \mathrm{f}$; ; des umschr. Kreises 246. 341. 348 , der ein- und angeschr. Kreise 246. 306, des Kreises mit gegebenem Poldreieck 268; Ort der Mittelpunkte umschr. K. mit Nebenbedingungen 295. 297-300; Ort der M. eingeschr. K. mit Nebenbedingungen 296. $298 \mathrm{ff}$; Ort der M. von Kegelschnitten bei gegebenem Poldreieck and Nebenbedingungen 296. $355 \mathrm{f}$; Ort der concentr. Kegelschnitte mit Nebenbedingungen $300 \mathrm{f}$; Ort der M. umschr. gleichseitiger Hyperbeln $304 \mathrm{f}$., eingeschr. gleichs. Hyperbeln 304. $390 \mathrm{f}$; Kreis als Ort der M. der gleichseitigen Hyperbeln eines Gewebes 392; Directrix der Parabel als Ort für $\mathbf{M}$. von Kreisen, die Poldreiecken der Parabel umschrieben sind 344; Ort der M. einer Schaar 200. 355 f. 359362 ; Relation zwischen M. und Höhenschnittpunkt des Dreiecks, dem ein Kegelschnitt eingeschr. 391 .

Mittelpunktslinie, Kegelschnitte mit 25. $31 \mathrm{f}$.

Mittelpunktskegelschnitt eines Büschels 198 f. $354 \mathrm{ff}$; ; Art desselben 356-359; gleichseitige Hyperbel als Mittelpunktskegelschnitt 362 .

Netz ron Kegelschnitten, Definition 219; von $\mathrm{K}$. mit einem, zwei oder drei ge- meinsamen Punkten 384-387; mit einer Doppelgeraden $387 \mathrm{f}$; mit zwei Doppelgeraden $388 \mathrm{f}$. 396-400; mit gemeinsamen Poldreieck 389; gebildet durch die Directorkreise der Kegelschnitte eines Gewebes 390; gebildet durch die Directorkreise der einen Dreieck eingeschr. Kegelschnitte $390 \mathrm{t}$. Newton'scher Satz 277.

Normal, Bedingung, dass zwei Geraden zu einander normal 13. 62; vgl. auch orthogonal.

Normale zu einer Geraden 244. 248; eines Kegelschnitts 273. 353; gleichseitige Hyperbel durch die Fusspunkte der von einem Punkt nach einem $K$. gezogenen Normalen $203 \mathrm{ff} .381$; von einem Punkte nach einer Curve $n^{\text {ter }}$ Ordnang 204. 379, deren Fusspunkt 382; von einem Pankte des umschr. Kreises auf die Seiten eines Dreiecks gefällt 348; Ort der Punkte, aus denen sich bei Kegelschnitten vier $\mathrm{N}$. von gegebenem Doppelverhältniss ziehen lassen $381 \mathrm{f}$.

Normalencentrum einer Geraden 13. 24. 85. 90. 244; Polare des Normalencentrums 85 .

Normalform, Gleichung der Geraden in der $10 ; N$. der elliptischen Integrale $422-426$.

Orthogonal kreis eines Netzes von Kreisen $389-392$.

Orthogonalschneiden zweier Kreise 318. 337 ; vgl. auch normal.

Parabel 46. 50. 52. 54f. 109; ihre Gestalt 50.112; Axe derselben 103.115. 289 ; Scheitel 106. 117; Scheiteltangente 104f. 289; Parameter 106. 117. 290 ; Berührungspunkt mit der uuendlich fernen Geraden $101 \mathrm{ff} .106$; Brennpunkte 115; Directrix 117. 342 f;; Directrix als Ort der Schnittpunkte rechtwinkliger Tangenten $148 \mathrm{f}$; Directrix als Ort für die Mittelpunkte von Kreisen, die Poldreiecken der Parabel umschrieben 344; Scheiteltangente als Ort der Fusspunkte von Lothen, die rom Brennpunkt auf Tangenten gefällt 186; Definition mit Hilfe von Brennpunkt und Directrix $118 \mathrm{ff}$. 325; confocale P. $175 \mathrm{f}$.; Steinersche P. 201 f. 409; Parabeln im Kegelschnittbüschel 199 . 331 . $357 \mathrm{ff}$. 362 ; Axen zweier $P$. zn einander normal 331. 411. 415; Schaar von P. mit gegebenem Poldreiseit 327. 349; Directricen einer Schaar von Parabeln $340 \mathrm{f}$; 
Directricen der P. mit gegebenem Poldreiseit $341 \mathrm{f}$.; Schaar eingeschriebener P., ihr Poldreiseit 330, ihre Directricen 341. 392; Directricen der P. eines Gewebes 392; Dreieck einer P. umschrieben 347; Kreis durch Poldreiecke einer P. 344; umschriebener Kreis als Ort für die Brennpunkte eingeschr. Parabeln 347 f. 351 f. 407 ; Feuerbach'scher Kreis als Ort für die Brennpunkte von Parabeln mit gegebenem Poldreiseit 347. 352. 411; conjugirte Lage von $P$. und gleichseitiger Hyperbel 344 ; Parallele zur Axe der P. 264; Enveloppe der Axen der eingeschr. P. 414 ; Enveloppe der Axen von P. mit gegebenem Poldreiseit $410 \mathrm{f}$. 413; Enveloppe der Scheiteltangenten der eingeschr. P. 412, der P. mit gegebenem Poldreiseit 415.

Parallele zu einer Geraden 243; zur Axe der Parabel 264.

Parallelenpaar 50f. 54. 106 f. 109 ; bestebend aus Tangenten eines K. 262.

Parallelogramm, einem K. eingeschrieben 280. 282, umschrieben 279. $281 \mathrm{f}$.

Parameter der Parabel 106. 117. 290.

Pascal'sche Gerade und Satz von Pascal $126 \mathrm{ff}$.

Pascal'sches Sechseck 127.

Pol einer Geraden in Bezug auf einen Kegelschnitt 42; Pole einer Geraden in Bezug auf eine Schaar 200, eine confocale Schaar 363, in Bezug auf ein Büschel 196f.; Involution conjugirter Pole 32.

Polare eines Punktes in Bezug auf einen Kegelschnitt $23 \mathrm{f}$. 26. 264; Polaren eines Punktes in Bezug auf ein Büschel 195, in Bezug auf eine Schaar 200; Geradenpaar als harmonisches Polarenpaar zu einem K. 164; Involution conjugirter Polaren $44 ;(n-k)^{\text {te }}$ Polare 22 ; lineare $\mathrm{P}$. eines Punktes in Bezug auf ein Dreieck $255 \mathrm{f}$; ; konische P. eines Punktes in Bezug auf ein Dreieck 256f. 259. 316. 358; innere P. eines Punktes in Bezug auf eine Curve 314.

Poldreieck 32f. 265. 283. 327.330; beim Kegelschnittbüschel $138 \mathrm{ff}$. 206 f. 327 ; beim Kreisbüschel 336; Product d. Seiten d.gemeinsamen P. einesBüschels $369 \mathrm{f}$; einem $\mathrm{K}$. eingeschrieben $162 \mathrm{f}$. $343 \mathrm{ff}$. 371 ; bei umschriebenen gleichseitigen Hyperbeln 330 ; Ort der Mittelpunkte der gleichseitigen Hyperbeln mit gegebenem Poldreieck 306; Kreis mit gegebenem Dreieck als Poldreieck 268f. 297. 304. 313. 345. 390f; An- wendung der P. bei gewissen Integralen 415-421.

Poldreiseit 44.330; bei der Kegelschnittschaar 145. 207. 327; Schaar von Parabeln mit gegebenem P. 327.349; einem $\mathrm{K}$. umschrieben 161.163. $344 \mathrm{ff}$; Ort der Brennpunkte von $K$. bei gegebenem P. 348 f. 352.

Polkegelschnitt einer Geraden 197 f. 388; seine Anwendung bei Bildung der Gleichung der Cayley'schen Curve 225.

Polviereck einer Curve zweiter Classe $392 \mathrm{f}$;; Polviereck für Parabeln 413.

Polvierseit eines Kegelschnitts 221.

Potenz eines Punktes in Bezug auf einen Kreis $99 \mathrm{f}$. 317. 318-323; in Bezug auf einen Kegelschnitt 345; eines Punktes in Bezug auf den umschr. Kreis $318 \mathrm{ff}$; in Bezug auf den Feuerbach'schen Kreis $319 \mathrm{f}$; in Bezug auf den Kreis mit gegebenem Dreieck als Poldreieck $319 \mathrm{f}$; eines Kegelschnittcentrums in Bezug auf den einem Poldreieck umschr. Kreis 344; Quotient aus Potenz und Quadrat des Abstandes eines Punktes von einer Geraden $321 \mathrm{ff}$.

Potenzlinie zweier Kreise 59. 267. $317 \mathrm{f}$. 336. 390 ; der Directorkreise zweier eingeschr. Kegelschnitte $391 \mathrm{f}$.

Potenzmittelpunkt 267. 326. 389.

Projective Punktreibe vgl. Punktreihe.

Projective Strahlenbüschel vgl. Strahlenbüschel.

Punkt, seine Gleichung 5.

Punktcoordinaten 2, ihre Transformation 14 ff. 88. 92.

Punktepaar 40. 44. 52 f. 55. 263; Träger eines P. 44; kubische Gleichung zur Bestimmung der Punktepaare einer confocalen Schaar 166. 168.

Punktreihe, Definition der Kegelschnitte durch projective P. 124; Construction entsprechender Punkte zweier projectiver P. $125 \mathrm{f} .326$.

Radicalaxe, vgl. Potenzlinie.

Realität der Schnittpunkte bei Kegelschnitten, vgl. Schnittpunkte.

Schaar von Kegelschnitten, ihre Definition 145; Punktepaare in einer Schaar 145; Kegelschnitte einer Schaar durch einen gegebenen Punkt 145 f.; Mittelpunkte der $K$. einer Sch. 200. $355 \mathrm{f}$. 359-362.

Schnittpunkte von Gerade und Kegelschnitt 20 f. 33 ff. 41 ; der Seiten eines Dreiecks mit einem K. 34. 257; Doppelverhältniss der Schn. von Gerade und Kegelschnitt zu zwei Punkten auf 
der Geraden 21. 63; Schn. zweier Kegelschnitte 131. $140 \mathrm{f}$. $144 \mathrm{f}$. $205 \mathrm{f}$. 327; Lage derselben bei einfacher Berübrung 134. $374 \mathrm{ff}$, , bei doppelter Berührung 134. $375 \mathrm{f}$, , bei Osculation $135 \mathrm{f}$. $375 \mathrm{f}$; vier zusammenfallende Schn. 136.375f.; Realitätskriterien der Schn. zweier K. 371-376; Schn. zweier gleichseitiger Hyperbeln 137; von Kegelschnitt und Kreis 362; Verbindnngslinie eines Punktes mit Schn. zweier Geraden 243; Schn. zweier Tangentenpaare eines K. $277 \mathrm{f}$.

Scbwerlinien des Coordinatendreiecks 243.

Schwerpunkt des Coordinatendreiecks $245 \mathrm{f}$; ; als Mittelpunkt einer eingeschr. Ellipse 255. 316, einer umschr. Ellipse 258 f.

Sehne, Länge einer S. beim Kegelschnitt 271 f.; Mittelpunkt der S. 262; Kegelschnittsebnen von gleicher Lünge 272; drei K. mit gemeinsamer S.326. $385 \mathrm{f}$.; Netz von K. mit gemeinsamer S. 385 f.; constantes Verhältniss der durch eine Gerade ansgeschnittenen S. zweier Kreise $394 \mathrm{ff}$; Kreis über Kegelschnittsehne als Durchmesser 270.

Semiparameter $117 \mathrm{f}$;

Steiner'sche Curve dritter Classe $410-$ 415; Steiner'sche Parabel 201 f. 409.

Strahlenbüschel, Definition der Kegelschnitte durch projective S. 123 ; Construction entsprechender Strahlen zweier projectiver S. $125 \mathrm{f}$.

Strecke, Mitte einer S. 244; Mittelloth 244; Mittelloth der Seiten des Coordinatendreiecks 245; Ort der Punkte, von denen eine $S$. unter gegebenem Winkel erscheint 276; Ort der Punkte, von denen zwei S. unter gleichen oder supplementären Winkeln erscheinen 339 . $407 \mathrm{ff}$.

Sturm, Sturm-Desargues'scher Satz $142 \mathrm{f}$. Supplementarsehnen 280.

Tangente eines Kegelschnitts 24. 27. 42. 44. 280; T. und Normale in einem Punkte eines K. 353; Stück einer T. zwischen Berührungspunkt und Directrix gelegen 194; Tangentenpaar von einem Punkt an einen K. 21. 23. 41. 45. $264 \mathrm{f}$; d die zugehörigen Berührungspunkte $259 \mathrm{f}$; ;on der Spitze eines Geradenpaars an einen K. 164f.; T. von den Ecken eines Dreiecks an einen K. $257 \mathrm{f}$; ; parallele T. 262 ; Bedingnng für reelle oder imaginäre Tangenten von einem Punkte an einen K. 261 ; Tangentenpaar in den Schnitt-

Gundelfinger, Vorlesungen. punkten von Gerade und K. $45.259 \mathrm{ff}$. ; Winkel der von einem Punkte an einen K. gezogenen T. $274 \mathrm{ff}$.; Winkel der an confocale $K$. gezogenen Tangentenpaare 147 ; rechtwinklige $T$. an einen K. 148, speciell an eine Parabel 148f.; Schnittpunkte zweier Tangentenpaare eines K. $277 \mathrm{f}$; Relation $\mathrm{zwischen}$ sechs $\mathrm{T}$. eines $\mathrm{K}$. 234; T. an die Kegelschnitte einer Schaar 146; rechtwinklige $\mathrm{T}$. an die einzelnen $K$. einer Schaar 340; Doppelverhältniss der vier gemeinsamen $T$. einer Schaar $367 \mathrm{f}$; gemeinsame T. zweier K. 138 f. 141. 145. 206, Realitätskriterien derselben 377 ; constante Summe oder Differenz der an zwei Kreise gezogenen T. $323 \mathrm{ff}$; drei Kegelschnitte mit zwei gemeinsamen T. 326 .

Tetragonale oder trigonale Felder 2; Kriterium für tetrag. oder trig. Lage eines Punktes 242 f.; Product der Abstände eines Punktes von den Seiten des Coordinatendreiecks bei trig. oder tetrag. Lage des Einheitspunktes 241 ; Product der Höhen des Coordinatendreiecks bei trig. oder tetrag. Lage des Einbeitspunktes 241; Art der umschriebenen $\mathrm{K}$. bei trig. oder tetrag. Lage des Mittelpunktes $251 \mathrm{ff}$., dasselbe für die eingeschriebenen K. 253 , für die K. mit gegebenem Poldreieck $253 \mathrm{f}$.

Transformation der Punktcoordinaten $14 \mathrm{ff}$. 88. 92; der Liniencoordinaten 17. 89.92 ; eines K. auf die Hauptaxen 85. 91 .

Trapez, Schnittpunkt der zwei nicht parallelen Gegenseiten eines einem K. umschr. T. als Brennpunkt einer Parabel 195.

Trigonal, vgl. Tetragonal.

Unendlich ferne Gerade, vgl. Gerade.

Viereck, Mittelpunkte der sechs Seiten eines vollständigen V. 199; Halbirungslinien der Winkel zwischen den Gegenseiten eines vollstïndigen V. $362 \mathrm{f}$.

Vierseit, nmschriebenes 278. $332 \mathrm{f}$; Kreise über den Diagonalen eines vollständigen V. 340; zwei Gegenecken eines umschr. V. als Brennpunkte eines anderen Kegelschnitts 195; Ort der Brennpunkte für die einem V. eingeschr. K. 404-409.

Wechselschnitte $403 \mathrm{f}$.

Wechselselınen $400 \mathrm{ff}$. 
Winkel zweier Geraden $11 \mathrm{ff} .63 \mathrm{ff} .273 \mathrm{f}$., als Doppelverhältniss $63 \mathrm{f}$.; Halbirungslinie des W. zweier Geraden 243f.; W. zweier conjugirter Durchmesser 276 f. 282; W. der von einem Punkt an einen $K$. gezogenen Tangenten $274 \mathrm{ff}$.; $W$. der an, confocale $K$. gezogenen Tangentenpaare 147; W. $\mathrm{zwischen}$ Tangente und Brennstrahlen des Berührungspunktes 190; Ort der Punkte, von denen eine Strecke unter gegebenem W. erscheint 276; Ort der Punkte, von denen zwei Strecken unter gleichen oder supplementären W. erscheinen $\mathbf{3 3 9}$. $407 \mathrm{ff}$.

Zwischenform 83. 



\section{DAY USE}

\section{RETURN TO DESK FROM WYHCH BORROWED}

ASTRON.MATH-STAT.

This book is due LIBRARY

is due on the last date stamped below, or on the date to which renewed.

Renewed books are subject to immediate recall.

\begin{tabular}{|c|c|}
\hline $00 \frac{1}{2}$ & \\
\hline NOY 1.01998 & MAY 142001 \\
\hline Livin 201000 & JUL 042001 \\
\hline $\begin{array}{l}\text { Fed'd EEB } 201995 \\
\text { F }\end{array}$ & $\frac{\text { APR U } 82002}{2003 \text { AY }-92003}$ \\
\hline OCT 191999 & \\
\hline Rockd OCB 2A2MM/S & \\
\hline
\end{tabular}

JAN 282000 JAN 092004

FEB $2929 \mathrm{~T} 0$ FEB 92006

i. CCEIVED

JUL 192000

C. BMATH LIBRARY

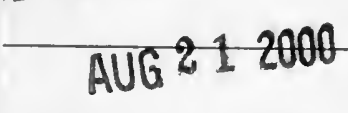

LD 21-40m-10,'65

(F7763s10) 476

General Library

University of California Berkeley 
